\title{
Final Review
}

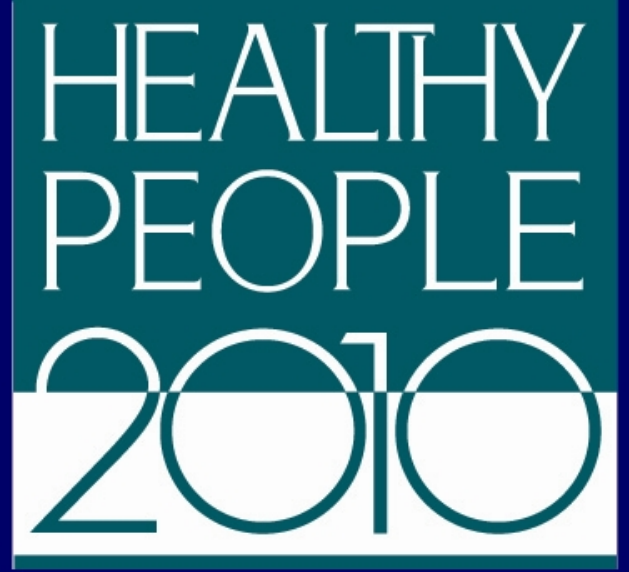




\section{Final Review}

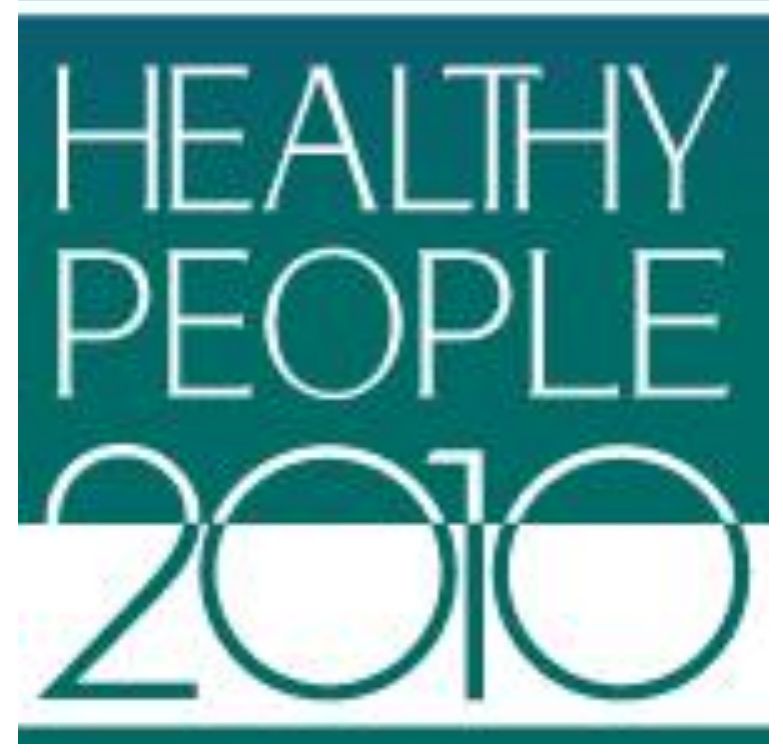




\section{Preface}

The Healthy People 2010 Final Review presents a quantitative end-of-decade assessment of progress in achieving the Healthy People 2010 objectives and goals over the course of the decade. This report was compiled by the National Center for Health Statistics, Centers for Disease Control and Prevention (CDC), with considerable input from the Department of Health and Human Service's lead agencies for the Healthy People initiative. The Healthy People Federal Interagency Workgroup and the Office of Disease Prevention and Health Promotion served in a review capacity.

The Healthy People 2010 Final Review continues the series of profiles of the Nation's health objectives as an integral part of the Department's disease prevention and health promotion initiative for the decade that began in 2000. This initiative was unveiled in January 2000 by the Secretary of the Department of Health and Human Services with the release of Healthy People 2010: Understanding and Improving Health and Objectives for Improving Health. This report presents a summary of progress toward achieving the Healthy People 2010 goals of:

1) Increasing quality and years of healthy life, and

2) Eliminating health disparities.

This publication provides the final tracking data used to present a quantitative assessment of progress for the 969 objectives in the 28 Healthy People 2010 Focus Areas. A summary of progress for the Healthy People 2010 Leading Health Indicators also is presented. This publication incorporates the modifications to objectives from the 2005 Healthy People 2010 Midcourse Review, includes information about the status of each 2010 objective over the course of the decade, and a crosswalk that illustrates how Healthy People 2010 objectives were transitioned to Healthy People 2020. 


\section{Acknowledgements}

Overall responsibility for planning and coordinating the content of the Healthy People 2010 Final Review rested with the Health Promotions Statistics Branch (HPSB), Office of Analysis and Epidemiology, National Center for Health Statistics, under the direction of Rebecca Hines. Production coordination was shared among the following HPSB staff: Lesley Dobrzynski, David Huang, Jeff Pearcy, Cheryl Rose, Kimberly Rosendorf, and Makram Talih.

Production was accomplished by several HPSB working teams which included: Lesley Dobrzynski, Bob Francis, Leda Gurley, David Huang, Elizabeth Jackson, Bruce Jonas, Deepthi Kandi, Insun Kim, Jeff Pearcy, Cheryl Rose, Kimberly Rosendorf, Asel Ryskulova, Makram Talih, Ritu Tuteja, and Jean Williams.

The section discussing the Healthy People 2010 goal of increasing quality and years of healthy life was written by Ritu Tuteja with substantial input from Rebecca Hines, Richard Klein, and Michael Molla.

The section addressing the Healthy People 2010 goal of eliminating health disparities was written by David Huang and Makram Talih, with substantial input from Rebecca Hines and Richard Klein.

Compilation of data used to assess progress of the Healthy People 2010 objectives (the "Progress Chart") was developed by the HPSB research team, particularly Lesley Dobrzynski, Leda Gurley, David Huang, Ken Keppel, Jeff Pearcy, Suzanne Proctor, Zakia Nelson, Ritu Tuteja, Erin Reidy, Asel Ryskulova, and Makram Talih, with substantial input from Rebecca Hines and Richard Klein.

The presentation of health disparities in the Healthy People Final Review was developed by Ken Keppel and Makram Talih with input from Lesley Dobrzynski, Leda Gurley, David Huang, Elizabeth Jackson, Insun Kim, Richard Klein, Zakia Nelson, Jeff Pearcy, Ritu Tuteja, Kimberly Rosendorf, and Asel Ryskulova.

Mary Anne Freedman of Jacaranda Consulting, LLC provided significant support in the drafting process of the Focus Area chapters. In addition, the HPSB analysts each made significant contributions to all aspects of data compilation, verification, and text development for the 28 Focus Areas as follows:

Focus Area 1 (Access to Quality Health Services): David Huang

Focus Area 2 (Arthritis, Osteoporosis, and Chronic Back): Kimberly Rosendorf

Focus Area 3 (Cancer): David Huang

Focus Area 4 (Chronic Kidney Disease): Asel Ryskulova

Focus Area 5 (Diabetes): Lesley Dobrzynski

Focus Area 6 (Disability and Secondary Conditions): Bruce Jonas

Focus Area 7 (Educational and Community-Based Programs): Insun Kim

Focus Area 8 (Environmental Health): Jeff Pearcy 
Focus Area 9 (Family Planning): Ritu Tuteja

Focus Area 10 (Food Safety): Jeff Pearcy

Focus Area 11 (Health Communication): Leda Gurley

Focus Area 12 (Heart Disease and Stroke): Kimberly Rosendorf

Focus Area 13 (HIV): Insun Kim

Focus Area 14 (Immunization and Infectious Diseases): Insun Kim

Focus Area 15 (Injury and Violence Prevention): Kimberly Rosendorf

Focus Area 16 (Maternal, Infant, and Child Health): Elizabeth Jackson

Focus Area 17 (Medical Product Safety): Ritu Tuteja

Focus Area 18 (Mental Health and Mental Disorders): Bruce Jonas

Focus Area 19 (Nutrition and Overweight): Kimberly Rosendorf

Focus Area 20 (Occupational Safety and Health): Jeff Pearcy

Focus Area 21 (Oral Health): Elizabeth Jackson

Focus Area 22 (Physical Activity and Fitness): Asel Ryskulova

Focus Area 23 (Public Health Infrastructure): Kate Brett

Focus Area 24 (Respiratory Diseases): Kimberly Rosendorf

Focus Area 25 (Sexually Transmitted Diseases): Leda Gurley

Focus Area 26 (Substance Abuse): Elizabeth Jackson

Focus Area 27 (Tobacco Use): Lesley Dobrzynski

Focus Area 28 (Vision and Hearing): Asel Ryskulova

Substantial input and technical review were provided by Linda Bilheimer, Rebecca Hines, Richard Klein, Jennifer Madans, and Diane Makuc.

Publication management and editorial review were provided by: Jennifer Reid, Anthony Lipphardt, Tommy Seibert, Barbara Wassell, and Tammy Stewart-Prather.

Publication of the Healthy People 2010 Final Review would not have been possible without the contribution of staff members from the Office of Disease Prevention and Health Promotion, particularly Carter Blakey, Ellis Davis, Jeanette Guyton-Krishnan, Rachel Hayes, Yen Luong, Stacey McBryde, Kathryn McMurry, Holly McPeak, Deb Nichols, Emmeline Ochiai , Geri Tebo, and Kimber Wukitsch, as well as many staff members throughout NCHS, and the Healthy People 2010 lead Federal agencies workgroup members. 


\section{Table of Contents}

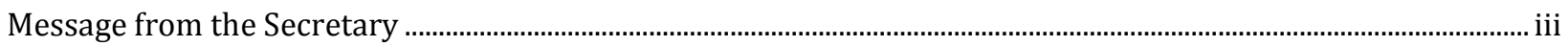

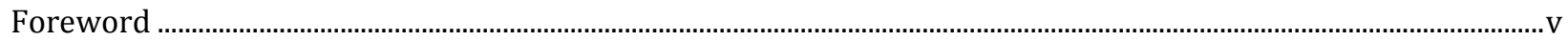

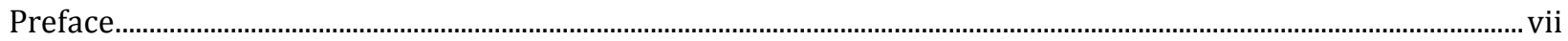

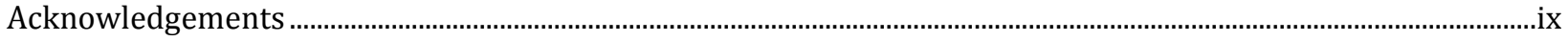

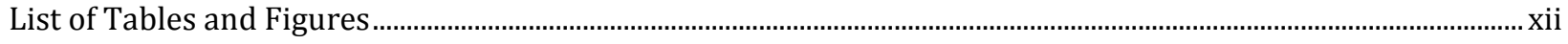

\section{Health of the Nation}

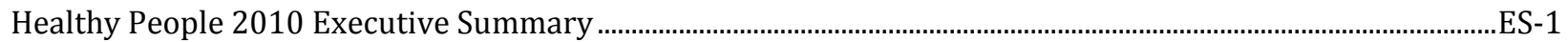

Healthy People 2010 Leading Health Indicators............................................................................................................

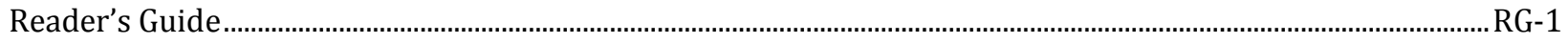

\section{Overview by Focus Area}

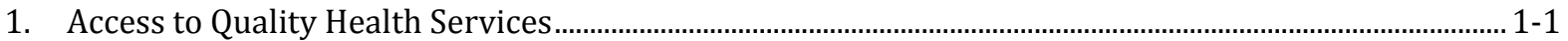

2. Arthritis, Osteoporosis, and Chronic Back Conditions .................................................................................. 2-1

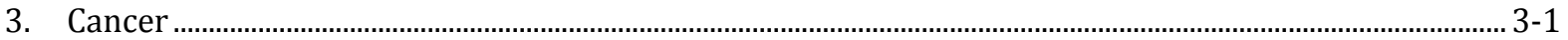

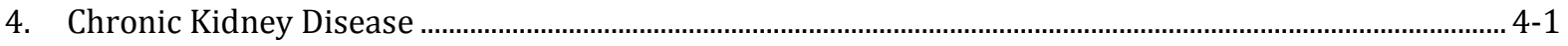

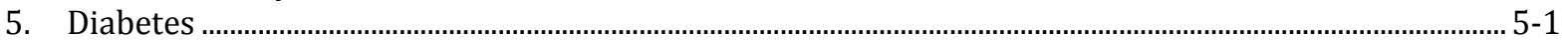

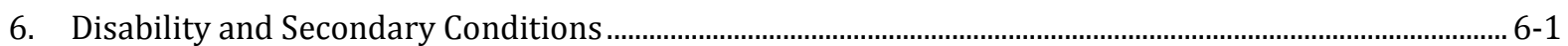

7. Educational and Community-Based Programs ….................................................................................... 7-1

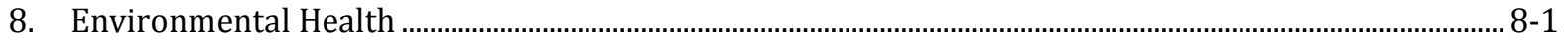

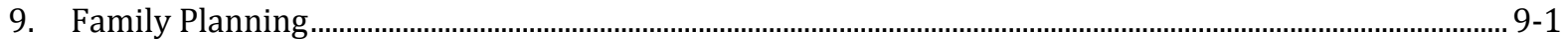

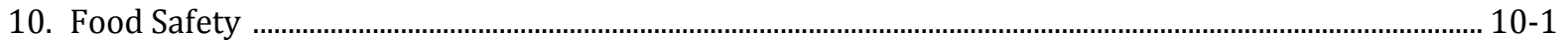

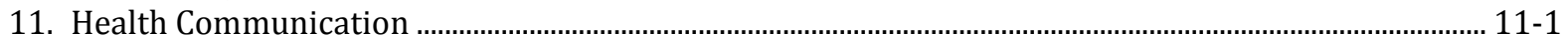

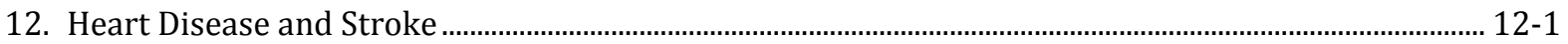

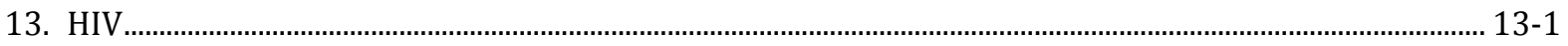

14. Immunization and Infectious Disease ................................................................................................ 14-1

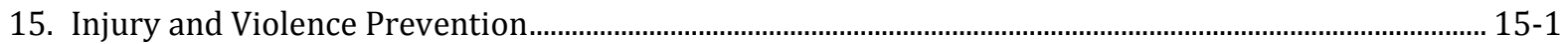

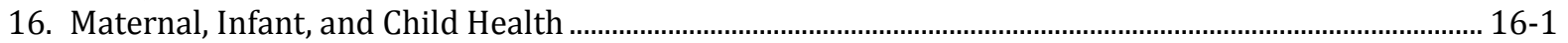

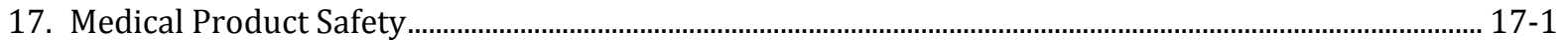

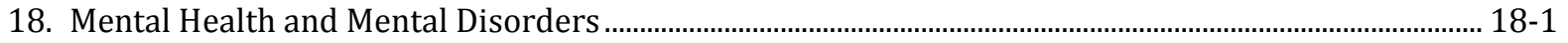

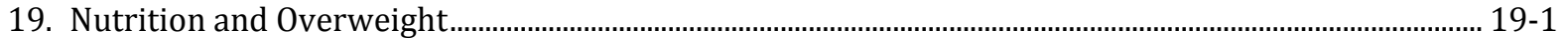

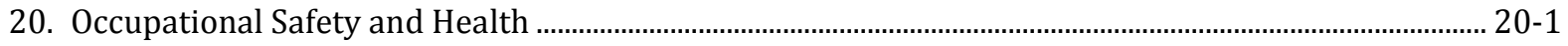

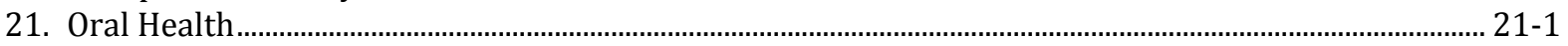

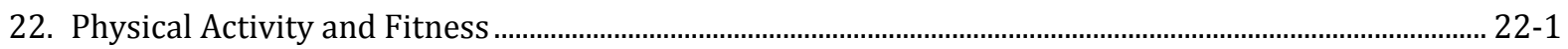

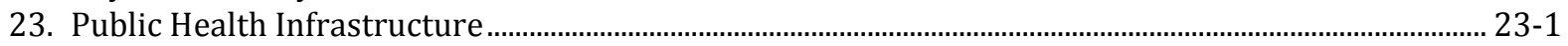

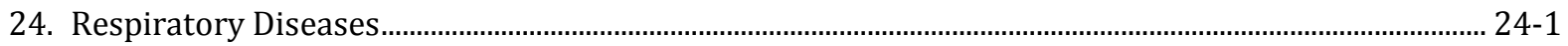

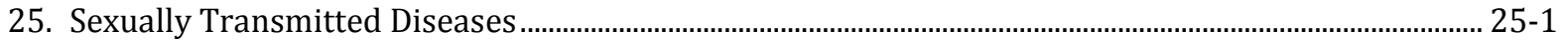

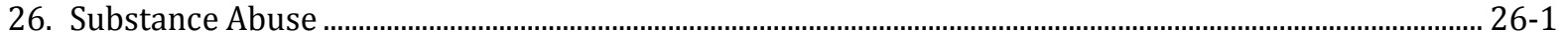

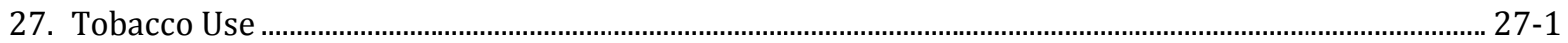

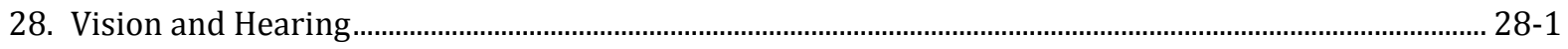

\section{Appendices}

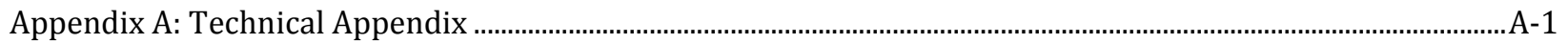

Appendix B: Published Issues of Healthy People Statistical Notes ........................................................................... B-1

Appendix C: Healthy People 2010 Lead Agencies. ............................................................................................................

Appendix D: Crosswalk Between Objectives from Healthy People 2010 to Healthy People 2020.....................D-1

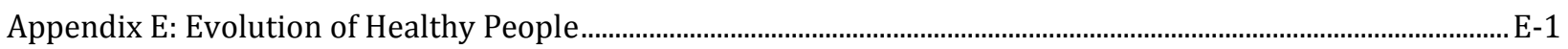




\section{List of Tables and Figures}

\section{Executive Summary}

\section{Tables}

Table ES-1. Healthy People 2010 Objectives: Summary of Progress by Focus Area..............................................ES-10

Table ES-2. Healthy People 2010 Objectives: Summary of Progress for Population Groups..............................ES-13

Table ES-3. Life Expectancy, at Birth and at Age 65, in Years ………………….........................................................ES-15

Table ES-4. Healthy Life Expectancies at Birth, in Years ………………………....................................................ES-19

Table ES-5. Healthy Life Expectancies at Age 65, in Years...........................................................................................ES-21

\section{Figures}

Figure ES-1. Healthy People 2010 Objectives: Status at the Final Review and Summary of Progress toward Target Attainment........................................................................................................................................................ES-9

Figure ES-2. Healthy People 2010 Objectives: Status at the Final Review by Focus Area......................................ES-9

Figure ES-3. Summary of Progress for Objectives with Tracking Data for Each Population Group..................ES-12

Figure ES-4. Expected Years of Life, by Sex and Race, 2006-07 ……………….......................................................ES-15

Figure ES-5. Life Expectancy and Healthy Life Expectancies at Birth, 2006-07 ……………………………….......ES-19

Figure ES-6. Life Expectancy and Healthy Life Expectancies at Age 65, 2006-07 ................................................ES-21

Figure ES-7. Health Disparities at the Most Recent Time Point by Race and Ethnicity ......................................ES-24

Figure ES-8. Changes in Health Disparities from the Baseline to the Most Recent Time Points by Population

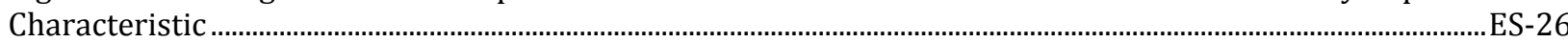

Figure ES-9. Health Disparities at the Most Recent Time Point by Sex....................................................................ES-27

Figure ES-10. Health Disparities at the Most Recent Time Point by Education Level..........................................ES-28

Figure ES-11. Health Disparities at the Most Recent Time Point by Income ..........................................................ES-29

Figure ES-12. Health Disparities at the Most Recent Time Point by Geographic Location ...................................ES-30

Figure ES-13. Health Disparities at the Most Recent Time Point by Disability Status .........................................ES-31

\section{Leading Health Indicators}

Figure LHI-1. Progress Toward Target Attainment for Leading Health Indicators................................................ LHI-16

Figure LHI-2. Health Disparities Table for Leading Health Indicators.

\section{Reader's Guide}

Figure RG-1. Legend for the Health Disparities Table (Figure 2) in each of the Focus Area chapters.

RG-7

\section{Focus Area Chapters}

\section{Chapter 1}

Figure 1-1. Progress Toward Target Attainment for Focus Area 1: Access to Quality Healthy Services.......... 1-15

Figure 1-2. Health Disparities Table for Focus Area 1: Access to Quality Health Services..................................... 1-23

Figure 1-3. Persons with Health Insurance (<65 Years), 2008-Map......................................................................... 1-25 


\section{Chapter 2}

Figure 2-1. Progress Toward Target Attainment for Focus Area 2: Arthritis, Osteoporosis and Chronic Back Conditions

Figure 2-2. Health Disparities Table for Focus Area 2: Arthritis, Osteoporosis, and Chronic Back Conditions ........ 2-12

Figure 2-3. Activity Limitations due to Arthritis-Map

\section{Chapter 3}

Figure 3-1. Progress Toward Target Attainment for Focus Area 3: Cancer

Figure 3-2. Health Disparities Table for Focus Area 3: Cancer

Figure 3-3. Overall Cancer Deaths, 2005-07-Map

Figure 3-4. Pap Test Received within the Past 3 Years (18+ Years), 2008-Map

Figure 3-5. Women Receiving Mammograms within Past 2 Years (40+ Years), 2008-Map

\section{Chapter 4}

Figure 4-1. Progress Toward Target Attainment for Focus Area 4: Chronic Kidney Disease. 4-8

Figure 4-2. Health Disparities Table for Focus Area 4: Chronic Kidney Disease ..................................................... 4-10

Figure 4-3. Dialysis Patients Registered on Kidney Transplant Waiting List ( $<70$ Years), 2007-Map.......... 4-11

Figure 4-4. Cumulative Percent of Persons Receiving a Kidney Transplant Within 3 Years of the Date of Renal Failure ( $<70$ Years), 2005-Map.

\section{Chapter 5}

Figure 5-1. Progress Toward Target Attainment for Focus Area 5: Diabetes. 5-9

Figure 5-2. Health Disparities Table for Focus Area 5: Diabetes

Figure 5-3. Diabetes Prevalence (18+ Years), 2008-Map. $5-14$

\section{Chapter 6}

Figure 6-1. Progress Toward Target Attainment for Focus Area 6: Disability and Secondary Conditions...... 6-11

Figure 6-2. Health Disparities Table for Focus Area 6: Disability and Secondary Conditions 6-14

\section{Chapter 7}

Figure 7-1. Progress Toward Target Attainment for Focus Area 7: Educational and Community-Based Programs 7-13

Figure 7-2. Health Disparities Table for Focus Area 7: Educational and Community Based Programs 7-16

\section{Chapter 8}

Figure 8-1. Progress Toward Target Attainment for Focus Area 8: Environmental Health. 8-19

Figure 8-2. Health Disparities Table for Focus Area 8: Environmental Health 8-29

\section{Chapter 9}

Figure 9-1. Progress Toward Target Attainment for Focus Area 9: Family Planning .. 9-11

Figure 9-2. Health Disparities Table for Focus Area 9: Family Planning 9-16

\section{Chapter 10}

Figure 10-1. Progress Toward Target Attainment for Focus Area 10: Food Safety $10-10$

Figure 10-2. Health Disparities Table for Focus Area 10: Food Safety. 10-14 


\section{Chapter 11}

Figure 11-1. Progress Toward Target Attainment for Focus Area 11: Health Communication.........................11-10

Figure 11-2. Health Disparities Table for Focus Area 11: Health Communication................................................11-13

Figure 11-3. Persons With Internet Access at Home (18+ Years), 2009-Map ..................................................11-14

\section{Chapter 12}

Figure 12-1. Progress Toward Target Attainment for Focus Area 12: Heart Disease and Stroke $.12-11$

Figure 12-2. Health Disparities Table for Focus Area 12: Heart Disease and Stroke 12-14

Figure 12-3. Coronary Heart Disease Deaths, 2005-07-Map.................................................................................12-16

Figure 12-4. Stroke Deaths 2005-07-Map.......................................................................................................12-17

\section{Chapter 13}

Figure 13-1. Progress Toward Target Attainment for Focus Area 13: HIV …………............................................13-12

Figure 13-2. Health Disparities Table for Focus Area 13: HIV ……........................................................................13-15

Figure 13-3. New AIDS cases (13+ years), 2007-Map ……………………………………………………….........13-17

Figure 13-4. HIV Infection Deaths, 2005-07-Map.............................................................................................13-18

\section{Chapter 14}

Figure 14-1. Progress Toward Target Attainment for Focus Area 14: Immunization and Infectious Diseases ...... 14-19

Figure 14-2. Health Disparities Table for Focus Area 14: Immunization and Infectious Diseases ..................14-31 Figure 14-3. Vaccination of Children (19-35 Months)—Three Doses Hepatitis B Vaccine, 2008-Map.......14-34 Figure 14-4. Vaccination of Children (19-35 Months)—One Dose Measles-Mumps-Rubella (MMR) Vaccine, 2008-Map .14-35

Figure 14-5. Universally Recommended Vaccination of Children (19-35 Months) -Four Doses Pneumococcal Conjugate Vaccine, 2008-Map 14-36

\section{Chapter 15}

Figure 15-1. Progress Toward Target Attainment for Focus Area 15: Injury and Violence Prevention.

Figure 15-2. Health Disparities Table for Focus Area 15: Injury and Violence Prevention

Figure 15-3. Deaths from Unintentional Injuries, 2005-07-Map.

Figure 15-4. Motor Vehicle Deaths, 2005-07-Map

\section{Chapter 16}

Figure 16-1. Progress Toward Target Attainment for Focus Area 16: Maternal, Infant, and Child Health ...16-17

Figure 16-2. Health Disparities Table for Focus Area 16: Maternal, Infant, and Child Health 16-24

Figure 16-3. Low Birth Weight (LBW), Infants, 2006-08-Map $16-27$

Figure 16-4. Preterm Live Births-Total ( $<37$ Weeks Gestation), 2006-08-Map $16-28$

\section{Chapter 17}

Figure 17-1. Progress Toward Target Attainment for Focus Area 17: Medical Product Safety $.17-8$ Figure 17-2. Health Disparities Table for Focus Area 17: Medical Product Safety. $17-10$ 


\section{Chapter 18}

Figure 18-1. Progress Toward Target Attainment for Focus Area 18: Mental Health and Mental

Disorders 18-11

Figure 18-2. Health Disparities Table for Focus Area 18: Mental Health and Mental Disorders $.18-13$

Figure 18-3. Suicide, 2005-07-Map. .18-15

\section{Chapter 19}

Figure 19-1. Progress Toward Target Attainment for Focus Area 19: Nutrition and Overweight $19-11$

Figure 19-2. Health Disparities Table for Focus Area 19: Nutrition and Overweight $19-15$

Figure 19-3. Obesity in Adults (20+ Years), 2008-Map

\section{Chapter 20}

Figure 20-1. Progress Toward Target Attainment for Focus Area 20: Occupational Safety and Health.........20-10

Figure 20-2. Health Disparities Table for Focus Area 20: Occupational Safety and Health $20-14$

\section{Chapter 21}

Figure 21-1. Progress Toward Target Attainment for Focus Area 21: Oral Health 21-11

Figure 21-2. Health Disparities Table for Focus Area 21: Oral Health $.21-15$

\section{Chapter 22}

Figure 22-1. Progress Toward Target Attainment for Focus Area 22: Physical Activity and Fitness

Figure 22-2. Health Disparities Table for Focus Area 22: Physical Activity and Fitness $22-12$

Figure 22-3. No Leisure Time Physical Activity among Adults (18+ Years), 2008-Map

\section{Chapter 23}

Figure 23-1. Progress Toward Target Attainment for Focus Area 23: Public Health Infrastructure $23-10$

\section{Chapter 24}

Figure 24-1. Progress Toward Target Attainment for Focus Area 24: Respiratory Diseases. 24-12

Figure 24-2. Health Disparities Table for Focus Area 24: Respiratory Diseases 24-16

Figure 24-3. Deaths from Chronic Obstructive Pulmonary Disease (Excluding Asthma, 45+ Years), 2005-07-Map 24-18

\section{Chapter 25}

Figure 25-1. Progress Toward Target Attainment for Focus Area 25: Sexually Transmitted Diseases ..........25-13

Figure 25-2. Health Disparities Table for Focus Area 25: Sexually Transmitted Diseases ................................25-16

Figure 25-3. Chlamydia Infections (New Cases Per 100,000 Population), 2009-Map.......................................25-18

Figure 25-4. Gonorrhea (New Cases Per 100,000 Population), 2009-Map.

Figure 25-5. Domestic Transmission of Primary and Secondary Syphillis (New Cases Per 100,000 Population), 2009-Map 25-20

\section{Chapter 26}

Figure 26-1. Progress Toward Target Attainment for Focus Area 26: Substance Abuse 26-14

Figure 26-2. Health Disparities Table for Focus Area 26: Substance Abuse. 26-20 
Figure 26-3. Cirrhosis Death Rates (2005-07)—Map

\section{Chapter 27}

Figure 27-1. Progress Toward Target Attainment for Focus Area 27: Tobacco Use..............................................27-13

Figure 27-2. Health Disparities Table for Focus Area 27: Tobacco Use.....................................................................27-19

Figure 27-3. Tobacco Use by Adults—Cigarettes (18+ years), 2008-Map ............................................................27-21

Figure 27-4. Comprehensive Smoke-Free Laws 2009-Map......................................................................................27-22

Figure 27-5. Average Combined Federal and State Excise Taxes on Retail Price of a Standard Pack of

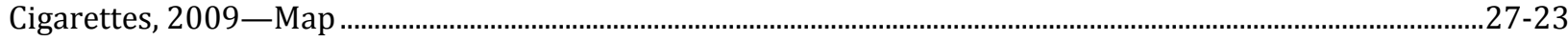

Chapter 28

Figure 28-1. Progress Toward Target Attainment for Focus Area 28: Vision and Hearing .................................28-11

Figure 28-2. Health Disparities Table for Focus Area 28: Vision and Hearing ....................................................28-16 Appendices

Figure A-1. Legend for the Health Disparities Table (Figure 2) in each of the Focus Area chapters.................. A-15 


\section{Final Review}

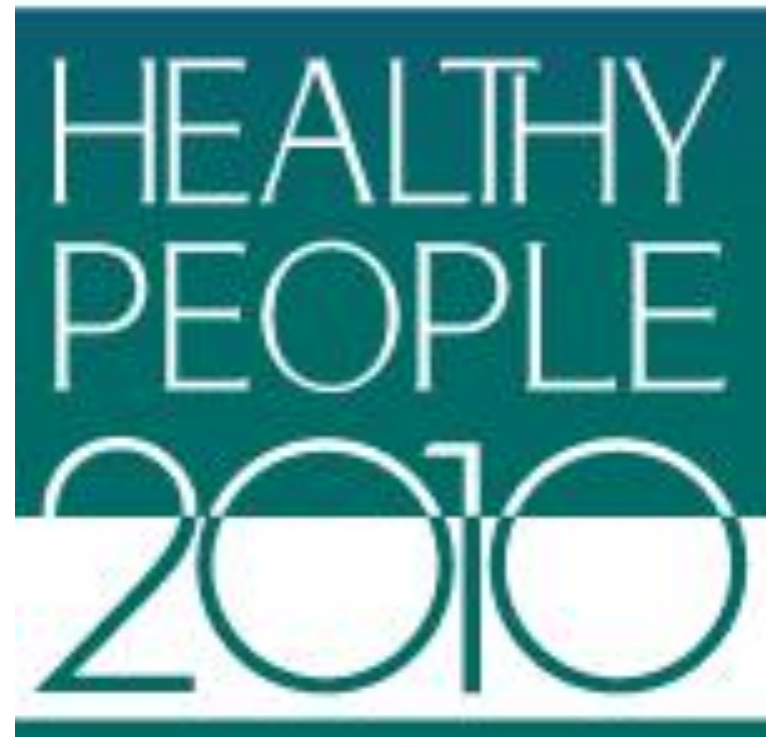

\section{Health of the Nation}




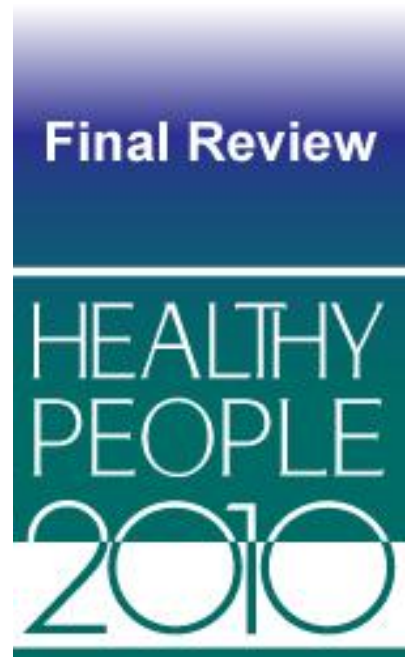

\section{Executive Summary}

\section{Contents}

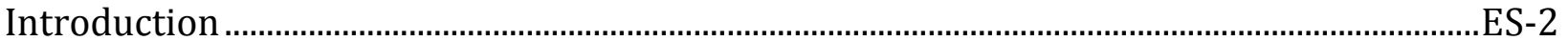

Summary of Progress ................................................................................................................ES-8

Goal 1: Increase Quality and Years of Healthy Life ...............................................................ES-14

Goal 2: Eliminate Health Disparities........................................................................................ES-22

Transitioning to Healthy People 2020: The Decade Ahead .......................................................ES-33

References .................................................................................................................................ES-36 


\section{Healthy People 2010 Final Review: Executive Summary}

\section{Introduction}

\section{History of the Healthy People Initiative}

In setting forth a vision for realizing improved health for all Americans, Healthy People 2010, released in November 2000, identified a set of 10-year health goals and objectives to be achieved during the first decade of the $21^{\text {st }}$ century. Its two overarching goals-to increase quality and years of healthy life and to eliminate health disparities-were supported by specific objectives in 28 Focus Areas. In this way, Healthy People 2010 built on initiatives that had been pursued over the previous few decades, beginning with the publication in 1979 of Healthy People: The Surgeon General's Report on Health Promotion and Disease Prevention [1]. That report led to the initiation of this decade-long, management-by-objective process with the publication in 1980 of Promoting Health/Preventing Disease: Objectives for the Nation [2]. This 1980 initiative was followed in turn by the publication in 1990 of Healthy People 2000: National Health Promotion and Disease Prevention Objectives [3]. Now, Healthy People 2020 will continue these efforts through the second decade of the $21^{\text {st }}$ century. Appendix E provides a summary of the evolution of Healthy People over the past four decades.

\section{Healthy People 2010}

Through Healthy People 2010, the U.S. Department of Health and Human Services (HHS) set out objectives that called for improvements in health status, risk reduction, public and professional awareness of prevention, delivery of health services, protective measures, surveillance, and evaluation, all expressed in specific metrics that allowed the measurement of progress over time toward targets that were to be achieved by the year 2010. Like its predecessors, Healthy People 2010 was developed through a broad collaborative process that drew on the best scientific knowledge available.

Full achievement of the goals and objectives of Healthy People 2010 was predicated on a health system accessible to all Americans that would integrate personal health care and population-based public health activities. The concept of "healthy people in healthy communities," which is the foundation of the initiative, necessitates monitoring and tracking of data on broad-based prevention efforts beyond services provided within physicians' offices, clinics, and hospitals. The concept expands the traditional diseasecentered medical care system to recognize the impact of health promotion and disease prevention efforts based in schools, neighborhoods, workplaces, and families in which people live their daily lives. These are the environments in which a large proportion of preventive action takes place. 
The 28 Focus Areas of Healthy People 2010 were developed by Federal agencies that had the most relevant scientific expertise in each subject area. The development process drew on the collective expertise of the Healthy People Consortium - an alliance which, at the time, encompassed more than 350 national membership organizations and 250 State health, mental health, substance abuse, and environmental agencies. In addition, through a series of regional and national meetings, more than 11,000 public comments on the draft objectives were collected and considered. The Secretary's Council on National Health Promotion and Disease Prevention Objectives for 2010 also provided leadership and advice in the development and implementation of these national health objectives. More information is available from www.healthypeople.gov/2010/data/midcourse/.

\section{Healthy People 2010 Midcourse Review}

Midway through the decade, staff of HHS and other Federal agencies together with experts from across the Nation assessed the status of the national objectives as they had developed over the first half of the decade. This Midcourse Review process involved an examination of trends in data that had become available by January 1, 2005, and it took into account any pertinent new science. The review resulted in changes to some objectives that were made to ensure that Healthy People 2010 remained current and accurate and kept abreast of emerging public health priorities. HHS solicited and considered public comments on these midcourse changes to the Healthy People 2010 objectives. The results of this midcourse assessment were published in the Healthy People 2010 Midcourse Review [4].

\section{Changes to Healthy People 2010 Objectives at the Midcourse Review}

Midcourse changes to Healthy People 2010 objectives encompassed the following: rewordings of objectives; deletion of 66 objectives; additions of new objectives; revisions to baselines and targets; and establishment of baselines and targets for objectives that moved from "developmental" to "measurable," as explained in the next paragraph. Changes were made to reflect the most current science, to reflect the data more accurately, or to provide a more logical or understandable presentation.

To be included in Healthy People 2010, an objective must have a national data source that provides a baseline and at least one additional data point for tracking progress. Some objectives lacked baseline data at the time of their development but had a potential data source and were considered of sufficient national importance to be included in Healthy People. These are called developmental objectives; they provide a vision for a desired outcome or health status. Developmental objectives with no prospect of having a national (baseline) data source were deleted as part of the Midcourse Review. (At the Final Review, 53 developmental objectives that were retained at the Midcourse Review still did not have baseline data.) 


\section{Measuring Healthy People 2010 Progress throughout the Decade}

\section{Progress Reviews}

In addition to the Midcourse Review, progress reviews on the individual Focus Areas were conducted, one each month, until the full cycle of 28 had been completed. Two cycles of these reviews were held during the decade. The progress reviews were formal meetings, chaired by the Assistant Secretary for Health, at which the National Center for Health Statistics (NCHS) within the Centers for Disease Control and Prevention, HHS provided data updates for the Focus Area under review; Federal lead agencies for the Focus Area reported on progress toward achieving Focus Area objectives and initiatives to help in accomplishing that purpose. More information is available from

www.healthypeople.gov/2010/data/PROGRVW/.

\section{DATA2010}

A critical part of Healthy People 2010 is measuring progress toward the targets for the year 2010. The compilation and management of current health data sources are central to assessing and implementing Healthy People 2010 goals and objectives. The data that provide the basis for the Midcourse Review and the Healthy People 2010 final review are available on DATA2010, developed by the Health Promotion Statistics Branch at NCHS. This is an interactive database system that compiles the monitoring data for tracking all the measurable objectives. These are primarily national data; selected state-based data are provided when available. Additional information is available from wonder.cdc.gov/data2010.

\section{Healthy People 2010 Final Review}

The Healthy People 2010 Final Review presents a quantitative summary assessment of progress in achieving the Healthy People 2010 objectives over the course of the decade. This publication, which incorporates the 2005 Midcourse Review modifications to the objectives, provides the most recent data available for the objectives in each of the 28 Focus Areas. A Progress Chart included in each chapter provides a summary display of the progress of each objective for which there were at least two data points available during the decade. Also in each chapter, a Health Disparities Table provides a summary of disparities by race and ethnicity, sex, education level, income, geographic location, and disability status whenever data were available for each objective. Finally, the report includes a summary of progress for the Healthy People 2010 Leading Health Indicators as well as a summary of progress toward achieving the Healthy People 2010 goals of: 1) increasing quality and years of healthy life, and 2) eliminating health disparities. 


\section{Initiatives Related to Healthy People}

\section{Other Departmental Priorities and Healthy People}

As the latest iteration of a long-running initiative, Healthy People 2020 follows the lead of Healthy People 2010 in supporting a wide range of HHS initiatives. Healthy People 2020 aligns with and plays a foundational and/or mutually supportive role with several other major HHS undertakings, including the following:

- The National Prevention and Health Promotion Strategy (NPS) was mandated by the March 23, 2010, Patient Protection and Affordable Care Act. The NPS aims to identify and prioritize national actions to reduce the incidence and burden of the leading causes of death and disability. The NPS aims to move the nation toward a system of health care that features prevention as the cornerstone of care, by concentrating on the underlying drivers of chronic disease. The NPS will promote actions aimed at prevention and healthy development and behavior throughout the stages of life, all of which will be directed toward its primary goal of achieving significant gains in Americans' life expectancy at birth and age 65. The NPS targets reflect those of Healthy People 2020.

- First Lady Michelle Obama's Let's Move! Campaign, begun in 2010, focuses on one ambitious goal: to halt and reverse the epidemic of childhood obesity within one generation, so that children today reach adulthood at a healthy weight. Over the past three decades, childhood obesity rates in America have tripled, and today, nearly one in three children in America are overweight or obese. The Let's Move! initiative focuses on the reform of behavioral factors and environmental factors by promoting active lifestyles and healthy eating through community involvement by way of schools, parents, healthcare providers, and other agents of change. Implementation strategies are now in development for Healthy People 2020 objectives that relate to this initiative and support the Let's Move! goal.

- In July 2010, the White House released the National HIV/AIDS Strategy, the Nation's first-ever comprehensive, coordinated HIV/AIDS roadmap with clear and measurable targets to be achieved by 2015 . Since 1980 , more than 575,000 Americans have lost their lives to AIDS and, currently, more than 1.1 million Americans are living with HIV. Among the 2015 goals of the National Strategy are to: lower the annual number of new infections by 25 percent and to increase from 79 to 90 percent the proportion of people living with HIV who know their serostatus. The objectives encompassed by the Healthy People 2020 HIV Topic Area are consonant with and supportive of these and other goals of the National HIV/AIDS Strategy.

- Inaugurated in 2010 and updated yearly, the National Drug Control Strategy has set policy priorities of reducing prescription drug abuse and drugged driving and of promoting activities to prevent such abuse from occurring. Implementation of the National Strategy is centered in the White House Office of National Drug Control Policy and engages the energies of several other Federal agencies, as well, including the HHS Substance Abuse and Mental Health Services Administration (SAMHSA). SAMHSA is lead agency for the Healthy People 2020 Topic Area on Substance Abuse, which embraces a number of objectives that are directly supportive of the National Strategy. While the Strategy is primarily a blueprint for the Federal Government, it is also proving useful in guiding State and local decisions. 
- The President's Food Safety Working Group was created in 2009 to advise the President on how to upgrade the U.S. food safety system. Chaired jointly by the HHS Secretary and Secretary of Agriculture, the Working Group recommended a public health-focused approach to food safety based on three core principles: prioritizing prevention, strengthening surveillance and enforcement, and improving response and recovery. Taken together, the objectives of the Food Safety Topic Area of Healthy People 2020 all serve to advance these principles.

- The HHS Action Plan to Reduce Racial and Ethnic Health Disparities outlines goals and actions HHS will take to reduce health disparities among racial and ethnic minorities. With the HHS Disparities Action Plan, the Department commits to continuously assessing the impact of all policies and programs on racial and ethnic health disparities. It will promote integrated approaches, evidence-based programs and best practices to reduce these disparities. The HHS Action Plan builds on the strong foundation of the Affordable Care Act and is aligned with programs and initiatives such as the First Lady's Let's Move initiative, the President's National HIV/AIDS Strategy, and Healthy People 2020.

- In November 2010, HHS presented its new Tobacco Control Strategic Action Plan, which seeks to help smokers quit and stop others from starting to use tobacco. One high profile piece of the plan will result in bolder health warnings that must cover the upper half of the front and back of cigarette packages and at least 20 percent of tobacco product advertisements beginning in 2012. In June 2009, the Family Smoking Prevention and Tobacco Control Act had granted the Food and Drug Administration (FDA) the authority to regulate tobacco products. Under the law, the FDA now has sweeping new authorities related to the manufacture, marketing, and sale of tobacco products-authorities covered by a more expansive public health standard than had traditionally been granted to the agency. The objectives of the Healthy People 2020 Topic Area on Tobacco Use provide the data that underpin the Plan and give it direction toward the outcomes we hope to achieve by the end of the decade.

- In February 2010, the U.S. announced a new Global Health Initiative (GHI), which invests $\$ 63$ billion over 6 years to help partner countries improve health outcomes through strengthened health systems and integrated services, with a particular focus on improving the health of women, newborns, and children. Other topics of particular concern in developing countries include HIV/AIDS, malaria, tuberculosis, family planning and reproductive health, nutrition, safety of water supplies, and neglected tropical diseases. The GHI has set a number of targets for accomplishment in assisted countries, for example: reduction of maternal mortality by 30 percent, reduction of under-five mortality rates by 35 percent, reduction of child undernutrition by 30 percent, and prevention of 54 million unintended pregnancies. Healthy People 2020 has a Topic Area on Global Health, new in this decade. 


\section{Guide to Clinical Preventive Services}

The Guide to Clinical Preventive Services includes U.S. Preventive Services Task Force (USPSTF) recommendations on screening, counseling, and preventive medication topics, as well as clinical considerations for each topic. Sponsored since 1998 by the Agency for Healthcare Research and Quality (AHRQ), the USPSTF is an independent panel of experts in primary care and prevention that systematically reviews the evidence of effectiveness and develops recommendations for clinical preventive services. The task force rigorously evaluates clinical research to assess the merits of preventive measures. In the 2010-11 edition of the Guide, the recommended preventive services for adults are in the clinical categories of: cancer; heart, vascular, and respiratory diseases; infectious diseases; injury and violence; mental health conditions and substance abuse; metabolic, nutritional, and endocrine conditions; musculoskeletal conditions; obstetrics and gynecologic conditions; and vision disorders. Recommendations for children and adolescents are given in a separate section. More information is available from www.ahrq.gov/clinic/cps3dix.htm.

\section{Guide to Community Preventive Services}

The Guide to Community Preventive Services serves as a filter for scientific literature on specific health problems that can have a large-scale impact on groups of people who share a common community setting. This guide summarizes what is known about the effectiveness, economic efficiency, and feasibility of interventions to promote community health and prevent disease. The Task Force on Community Preventive Services, an independent decision-making body convened by HHS, makes recommendations for the use of various interventions based on the evidence gathered in rigorous and systematic scientific reviews of published studies conducted by review teams for the guide. The findings from the reviews are published in peer-reviewed journals and also are made available online. Over the last decade or so, the task force has published hundreds of findings across the following topic areas: adolescent health; alcohol; asthma; birth defects; cancer; diabetes; health communication; HIV/AIDS, other STIs and pregnancy; mental health; motor vehicle occupant injury; nutrition; obesity; oral health; physical activity; social environment; tobacco use; vaccines; violence; and worksites. Additional information is available from www.thecommunityguide.org. 


\section{Healthy People 2010 Final Review: Executive Summary}

\section{Summary of Progress}

\section{Healthy People Objectives}

For the end-of-decade assessment of the Healthy People 2010 objectives, the status of 969 specific objectives in 28 subject Focus Areas was assessed. Progress was assessed for objectives using the most recent tracking data available-that is, baseline data and at least one additional data point. For some objectives, although more recent data may have been available, the final Healthy People 2010 data year was selected to be consistent with the baseline year used for the new Healthy People 2020 objectives [5].

The status of the 969 objectives is shown on the left-hand side of Figure ES-1. Based on an evaluation of each objective and comments received from the public as part of the Midcourse Review process, 66 objectives were dropped because data were unavailable or because of a change in the science [6]. Tracking data were unavailable to assess progress for 170 objectives ( $17.5 \%$ of the total), 53 of which lacked baseline data.

Progress can be assessed for 733 objectives with tracking data available, as can be seen in the right-hand side panel of Figure ES-1.

- 170 objectives (23\%) met the Healthy People 2010 targets.

- 349 objectives (48\%) moved toward the Healthy People 2010 targets.

- 39 objectives (5\%) demonstrated no change from the baseline.

- 175 objectives (24\%) moved away from the Healthy People 2010 targets.

In Figure ES- 2 and Table ES-1, similar assessments are shown for each of the 28 Focus Areas. In all Focus Areas there were some objectives that moved toward, met, or exceeded their 2010 targets. For eight Focus Areas, Educational and Community-Based Programs (Focus Area 7), Environmental Health (Focus Area 8), Health Communication (Focus Area 11), Heart Disease and Stroke (Focus Area 12), Immunization and Infectious Diseases (Focus Area 14), Mental Health and Mental Disorders (Focus Area 18), Occupational Safety and Health (Focus Area 20), and Tobacco Use (Focus Area 27) more than 75\% of the objectives moved toward or achieved their targets. The proportion of objectives that could not be assessed was more than 30\% for Access to Quality Health Services (Focus Area 1), Disability and Secondary Conditions (Focus Area 6), Educational and Community-based Programs (Focus Area 7), Environmental Health (Focus Area 8), and Mental Health and Mental Disorders (Focus Area 18). Two Focus Areas, Arthritis, Osteoporosis, and Chronic Back Conditions (Focus Area 2) and Nutrition and Overweight (Focus Area 19), moved toward or achieved less than $25 \%$ of their targets. 
Figure ES-1. Healthy People 2010 Objectives: Status at the Final Review and Summary of Progress toward Target Attainment

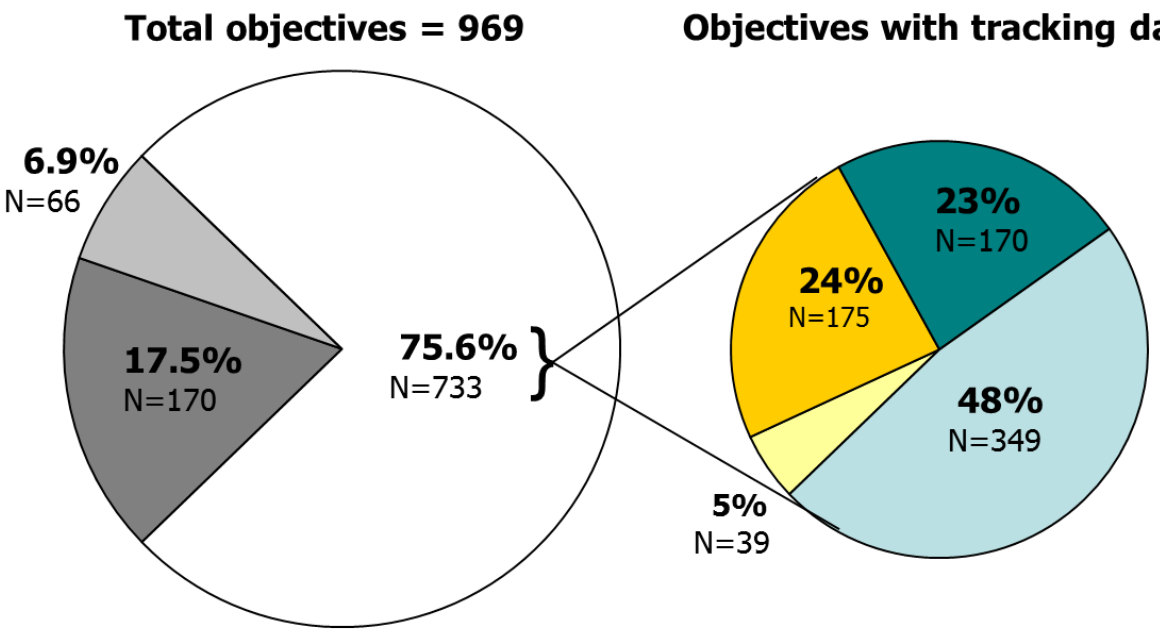

Figure ES-2. Healthy People 2010 Objectives: Status at the Final Review by Focus Area

$$
\mathrm{N}=969 \text { total objectives }
$$

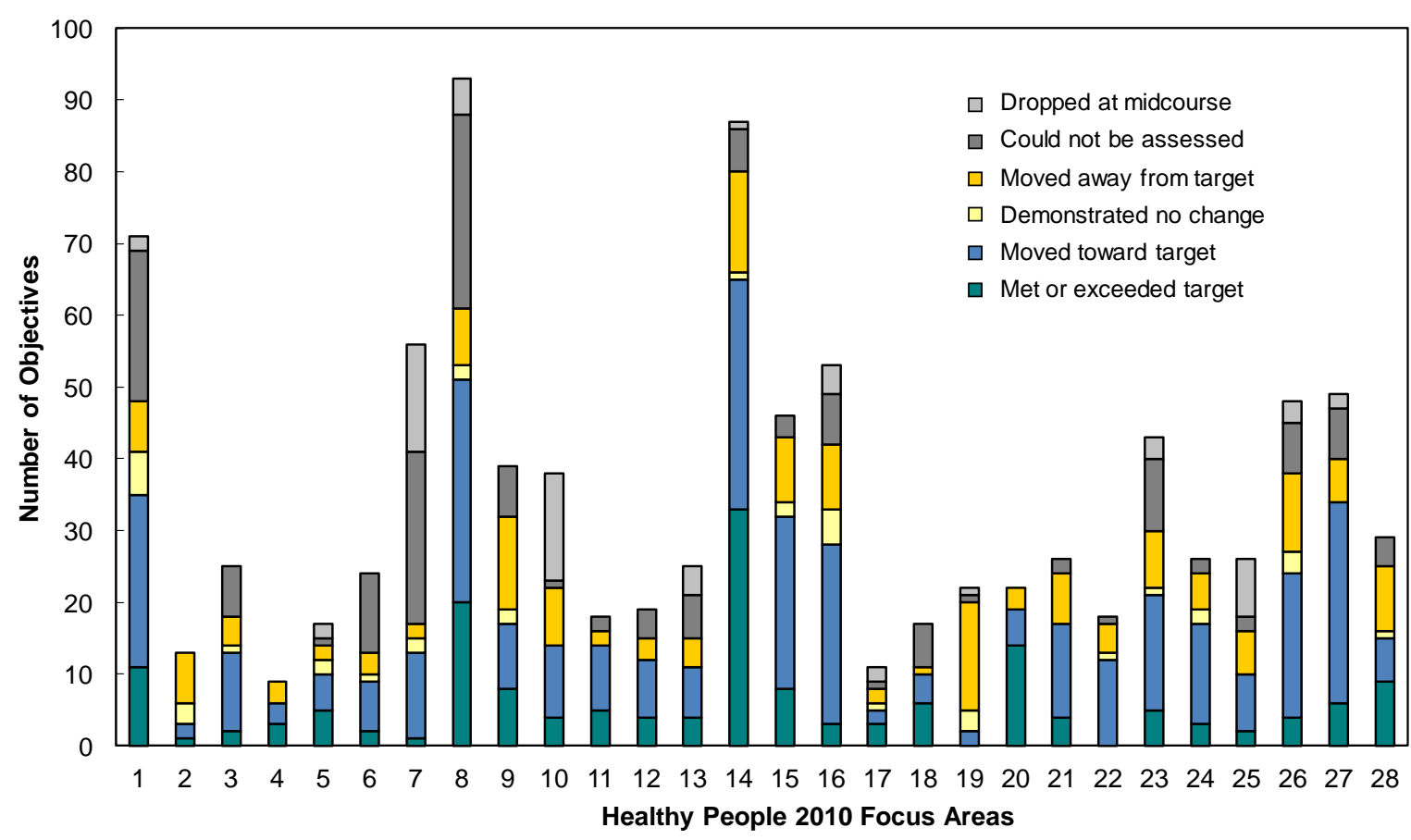


Table ES-1. Healthy People 2010 Objectives: Summary of Progress by Focus Area

\begin{tabular}{|c|c|c|c|c|c|c|c|c|c|}
\hline & Focus area & $\begin{array}{c}\text { Met or } \\
\text { exceeded } \\
\text { target }\end{array}$ & $\begin{array}{c}\text { Moved } \\
\text { toward } \\
\text { target }\end{array}$ & $\begin{array}{c}\text { Demonstrated } \\
\text { no change }\end{array}$ & $\begin{array}{c}\text { Moved } \\
\text { away } \\
\text { from } \\
\text { target }\end{array}$ & Developmental & $\begin{array}{c}\text { No } \\
\text { tracking } \\
\text { data } \\
\text { beyond } \\
\text { baseline }\end{array}$ & $\begin{array}{l}\text { Dropped at } \\
\text { Midcourse }\end{array}$ & Total \\
\hline 1. & Access to quality health services & 11 & 24 & 6 & 7 & 1 & 20 & 2 & 71 \\
\hline 2. & Arthritis, osteoporosis, and chronic back conditions & 1 & 2 & 3 & 7 & 0 & 0 & 0 & 13 \\
\hline 3. & Cancer & 2 & 11 & 1 & 4 & 0 & 7 & 0 & 25 \\
\hline 4. & Chronic kidney disease & 3 & 3 & 0 & 3 & 0 & 0 & 0 & 9 \\
\hline 5. & Diabetes & 5 & 5 & 2 & 2 & 0 & 1 & 2 & 17 \\
\hline 6. & Disability and secondary conditions & 2 & 7 & 1 & 3 & 4 & 7 & 0 & 24 \\
\hline 7. & Educational and community-based programs & 1 & 12 & 2 & 2 & 2 & 22 & 15 & 56 \\
\hline 8. & Environmental health & 21 & 30 & 2 & 8 & 9 & 18 & 5 & 93 \\
\hline 9. & Family planning & 8 & 9 & 2 & 13 & 6 & 1 & 0 & 39 \\
\hline 10. & Food safety & 5 & 11 & 0 & 6 & 1 & 0 & 15 & 38 \\
\hline 11. & Health communication & 5 & 9 & 0 & 2 & 0 & 2 & 0 & 18 \\
\hline 12. & Heart disease and stroke & 4 & 8 & 0 & 3 & 2 & 2 & 0 & 19 \\
\hline 13. & HIV & 4 & 7 & 0 & 4 & 6 & 0 & 4 & 25 \\
\hline 14. & Immunization and infectious diseases & 33 & 32 & 1 & 14 & 2 & 4 & 1 & 87 \\
\hline 15. & Injury and violence prevention & 8 & 24 & 2 & 9 & 0 & 3 & 0 & 46 \\
\hline 16. & Maternal, child, and infant health & 3 & 25 & 5 & 9 & 3 & 4 & 4 & 53 \\
\hline 17. & Medical product safety & 3 & 2 & 1 & 2 & 0 & 1 & 2 & 11 \\
\hline 18. & Mental health and mental disorders & 6 & 4 & 0 & 1 & 0 & 6 & 0 & 17 \\
\hline 19. & Nutrition and overweight & 0 & 2 & 3 & 15 & 0 & 1 & 1 & 22 \\
\hline 20. & Occupational safety and health & 14 & 5 & 0 & 3 & 0 & 0 & 0 & 22 \\
\hline 21. & Oral health & 4 & 13 & 0 & 7 & 0 & 2 & 0 & 26 \\
\hline 22. & Physical activity and fitness & 0 & 12 & 1 & 4 & 0 & 1 & 0 & 18 \\
\hline 23. & Public health infrastructure & 5 & 16 & 1 & 8 & 6 & 4 & 3 & 43 \\
\hline 24. & Respiratory diseases & 3 & 14 & 2 & 5 & 1 & 1 & 0 & 26 \\
\hline 25. & Sexually transmitted diseases & 2 & 8 & 0 & 6 & 1 & 1 & 7 & 25 \\
\hline 26. & Substance abuse & 4 & 20 & 3 & 11 & 5 & 2 & 3 & 48 \\
\hline 27. & Tobacco use & 6 & 28 & 0 & 6 & 4 & 3 & 2 & 49 \\
\hline \multirow[t]{2}{*}{28.} & Vision and hearing & 9 & 6 & 1 & 9 & 0 & 4 & 0 & 29 \\
\hline & Total & 172 & 349 & 39 & 173 & 53 & 117 & 66 & 969 \\
\hline
\end{tabular}




\section{Population Groups}

In Figure ES-3, progress is assessed for specific population groups. This assessment is limited to population-based objectives with tracking data for population groups. It does not include non-population-based objectives such as those based on States, worksites, or those monitored by the number of events. The number of objectives with tracking data varied according to the characteristic and, therefore, the bar's length in the figure below varies for each population group. For Healthy People 2010, most population-based objectives were monitored by race and ethnicity, but the availability of data for specific race and ethnic populations varied. Comparisons by sex were not applicable to all population-based objectives because some applied only to females or males. Geographic location and disability status were optional characteristics included for monitoring selected objectives.

When possible, population-based objectives were also monitored either by education level or by income, as a measure of socio-economic status. Most data systems used in Healthy People 2010 define income as a family's income before taxes. In order to facilitate comparisons among groups and over time, while adjusting for family size and for inflation, Healthy People 2010 categorizes income using the poverty thresholds developed by the U.S. Census Bureau. Thus, the three categories of family income that are primarily used are:

- Poor-below the Federal poverty level

- Near poor-100\% to $199 \%$ of the Federal poverty level

- Middle/high income-200\% or more of the Federal poverty level.

These categories may be overridden by considerations specific to the data system, in which case they are modified as appropriate.

In general, data on educational attainment are presented for persons aged 25 years and over, consistent with guidance given by the U.S. Census Bureau. However, because of the requirements of the different data systems, the age groups used to calculate educational attainment for any specific objective may differ from the age groups used to report the data for other Healthy People 2010 objectives, as well as from select populations within the same objective. The three categories of education level that are primarily used are:

- Less than high school

- High school graduate

- At least some college education.

Further information regarding population groups can be found in Healthy People 2010: General Data Issues, available from: http://www.cdc.gov/nchs/healthy people.htm.

For each select population group, the number of objectives is shown for each of the following: moved away from the target, demonstrated no change, moved toward the target, and met or exceeded the target. Because a single target was set for all population groups, there were some instances where certain population groups had met the Healthy People 2010 target at baseline while other groups had not met the target. 
In general, for each select population group, the number of objectives that moved toward or met the target exceeded the number that moved away from the target. For the American Indian or Alaska Native population, for example, 81 objectives moved toward or exceeded their respective targets while 59 moved away and 11 showed no change between the baseline and the final time points (Table ES-2). For the Native Hawaiian and Other Pacific Islander population, more objectives moved away from the target ( 26 objectives) than moved toward or met the target (21 objectives).

\section{Figure ES-3. Summary of Progress for Objectives with Tracking Data for Each Population Group}

\section{Race and Ethnicity}

American Indian or Alaska Native Asian or Pacific Islander

Asian

Native Hawaiian or Other Pacific Islander

Two or more races

Hispanic

Black non-Hispanic

White non-Hispanic

Sex

Female

Male

Education

Less than high school

High school graduate

At least some college

Income

Poor

Near poor

Middle/high income

Geographic Location

Urban/metropolitan

Ruralinonmetropolitan

Disability Status

Persons with disabilities

Persons without disabilities

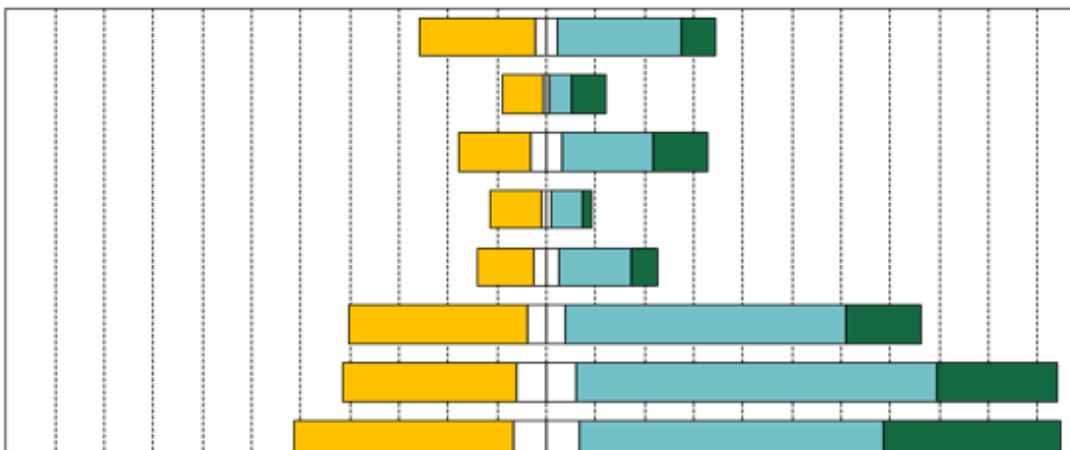

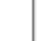


Table ES-2. Healthy People 2010 Objectives: Summary of Progress for Population Groups

\begin{tabular}{|c|c|c|c|c|c|}
\hline Characteristics and Groups & $\begin{array}{c}\text { Moved } \\
\text { away from } \\
\text { target }\end{array}$ & $\begin{array}{l}\text { Demonstrated } \\
\text { no change }\end{array}$ & $\begin{array}{l}\text { Moved } \\
\text { toward } \\
\text { target }\end{array}$ & $\begin{array}{l}\text { Met or } \\
\text { exceeded } \\
\text { target }\end{array}$ & Total \\
\hline \multicolumn{6}{|l|}{ Race and Ethnicity } \\
\hline American Indian or Alaska Native & 59 & 11 & 63 & 18 & 151 \\
\hline Asian or Pacific Islander & 21 & 3 & 11 & 18 & 53 \\
\hline Asian & 37 & 16 & 46 & 28 & 127 \\
\hline Native Hawaiian or Other Pacific Islander & 26 & 5 & 16 & 5 & 52 \\
\hline Two or more races & 29 & 12 & 37 & 14 & 92 \\
\hline Hispanic & 91 & 19 & 143 & 38 & 291 \\
\hline Black non-Hispanic $^{1}$ & 88 & 31 & 183 & 62 & 364 \\
\hline White non-Hispanic ${ }^{1}$ & 112 & 33 & 155 & 90 & 390 \\
\hline \multicolumn{6}{|l|}{ Sex } \\
\hline Female & 96 & 15 & 151 & 67 & 329 \\
\hline Male & 86 & 14 & 169 & 55 & 324 \\
\hline \multicolumn{6}{|l|}{ Education } \\
\hline Less than high school & 37 & 6 & 55 & 8 & 106 \\
\hline High school graduate & 40 & 7 & 50 & 14 & 111 \\
\hline At least some college & 25 & 5 & 45 & 38 & 113 \\
\hline \multicolumn{6}{|l|}{ Income } \\
\hline Poor & 34 & 4 & 47 & 14 & 99 \\
\hline Near poor & 37 & 9 & 33 & 16 & 95 \\
\hline Middle/high income & 28 & 11 & 31 & 31 & 101 \\
\hline \multicolumn{6}{|l|}{ Geographic location } \\
\hline Urban/metropolitan & 13 & 1 & 25 & 5 & 44 \\
\hline Rural/nonmetropolitan & 16 & 4 & 18 & 8 & 46 \\
\hline \multicolumn{6}{|l|}{ Disability status } \\
\hline Persons with disabilities & 28 & 8 & 38 & 11 & 85 \\
\hline Persons without disabilities & 27 & 12 & 39 & 12 & 90 \\
\hline
\end{tabular}

${ }^{1}$ For some objectives, data include persons of Hispanic origin.

The progress for each objective with data beyond the baseline is shown in the Progress Chart in Focus Area chapters of this report. Health disparities between population groups and changes in disparities between the baseline and the most recent time point are examined in the section discussing Goal 2, the elimination of disparities among populations; they also are summarized in the Health Disparities Table in Focus Area chapters. 


\section{Healthy People 2010 Final Review: Executive Summary}

\section{Goal 1: Increase Quality and Years of Healthy Life}

Healthy People 2010: Understanding and Improving Health highlighted the importance of maximizing and increasing both years of life and quality of life in the first overarching goal [6]. Progress toward achieving this goal is currently assessed by measuring life expectancy and three measures of healthy life expectancies: 1) Expected years in good or better health; 2) Expected years free of activity limitations; and 3) Expected years free of selected chronic diseases. These assessments result in the following conclusions:

- Life expectancy improved for the populations that could be assessed throughout the decade.

- Women had longer life expectancy than men, and the white population had a longer life expectancy than the black population.

- Expected years in good or better health and expected years free of activity limitation increased, and expected years free of selected chronic conditions decreased.

- Differences by race and sex were observed in all three healthy life expectancy measures-expected years in good or better health, expected years free of activity limitations, and expected years free of selected chronic diseases.

\section{Life Expectancy}

Life expectancy is the average number of years a hypothetical cohort of people born in a given year could be expected to live based on the age-specific death rates in that year. Since the launch of Healthy People 2010, life expectancy at birth and at age 65 has increased for all populations (Table ES-3 and Figure ES-4). In 2006-07, life expectancy for the total population was 77.8 years, an increase from 76.8 years in 2000-01. Improvements in overall life expectancy reflect improvements in disease-specific death rate objectives within the Healthy People 2010 Focus Areas. Death rates declined for many Healthy People 2010 cause-specific mortality objectives including: female breast cancer (objective 3-3), colorectal cancer (objective 3-5), prostate cancer (objective 3-7), coronary heart disease (objective 12-1), stroke (objective 12-7), cardiovascular disease and diabetes-related deaths among persons with diabetes (objectives 5-6 and 5-7) and HIV (objective 13-14). Even with these improvements, in 2007 U.S. male life expectancy ranked $26^{\text {th }}$ and female life expectancy ranked $25^{\text {th }}$ out of 33 selected countries [7]. 
Between 2000-01 and 2006-07, the percent increase in life expectancy was greater at age 65 years (5.1\%) than at birth (1.3\%). Men (75.3 years) had a lower life expectancy at birth than women (80.3 years), and the black population (73.4 years) had a lower life expectancy at birth than the white population (78.3 years) in 2006-07. However, between 2000-01 and 2006-07, the black population (2.1\%) had a greater relative increase in life expectancy at birth than the white population (1.2\%). Men (1.5\%) also had a greater relative increase in life expectancy at birth than women (1.1\%).

Table ES-3. Life Expectancy, at Birth and at Age 65, in Years

\begin{tabular}{|c|c|c|c|c|c|c|c|}
\hline & & Total & & Black & White & Women & Men \\
\hline \multirow{4}{*}{$\begin{array}{c}\text { Life expectancy, } \\
\text { at birth }\end{array}$} & $2000-01$ & 76.8 & 71.9 & 77.4 & 79.4 & 74.2 \\
\cline { 2 - 8 } & $2002-03$ & 77.0 & 72.2 & 77.5 & 79.5 & 74.4 \\
\cline { 2 - 8 } & $2006-07$ & 77.8 & 73.4 & 78.3 & 80.3 & 75.3 \\
\hline \multirow{4}{*}{$\begin{array}{c}\text { Life expectancy, } \\
\text { at age 65 }\end{array}$} & $2000-01$ & 17.7 & 16.1 & 17.8 & 19.0 & 16.1 \\
\cline { 2 - 8 } & $2002-03$ & 17.9 & 16.4 & 18.0 & 19.1 & 16.4 \\
\cline { 2 - 8 } & $2004-05$ & 18.3 & 16.8 & 18.3 & 19.5 & 16.8 \\
\cline { 2 - 8 } & $2006-07$ & 18.6 & 17.1 & 18.6 & 19.8 & 17.1 \\
\hline
\end{tabular}

Source: National Vital Statistics System (NVSS), NCHS, CDC

Figure ES-4. Expected Years of Life, by Sex and Race, 2006-07

Life expectancy, at birth $\quad \square$ Life expectancy, at age 65

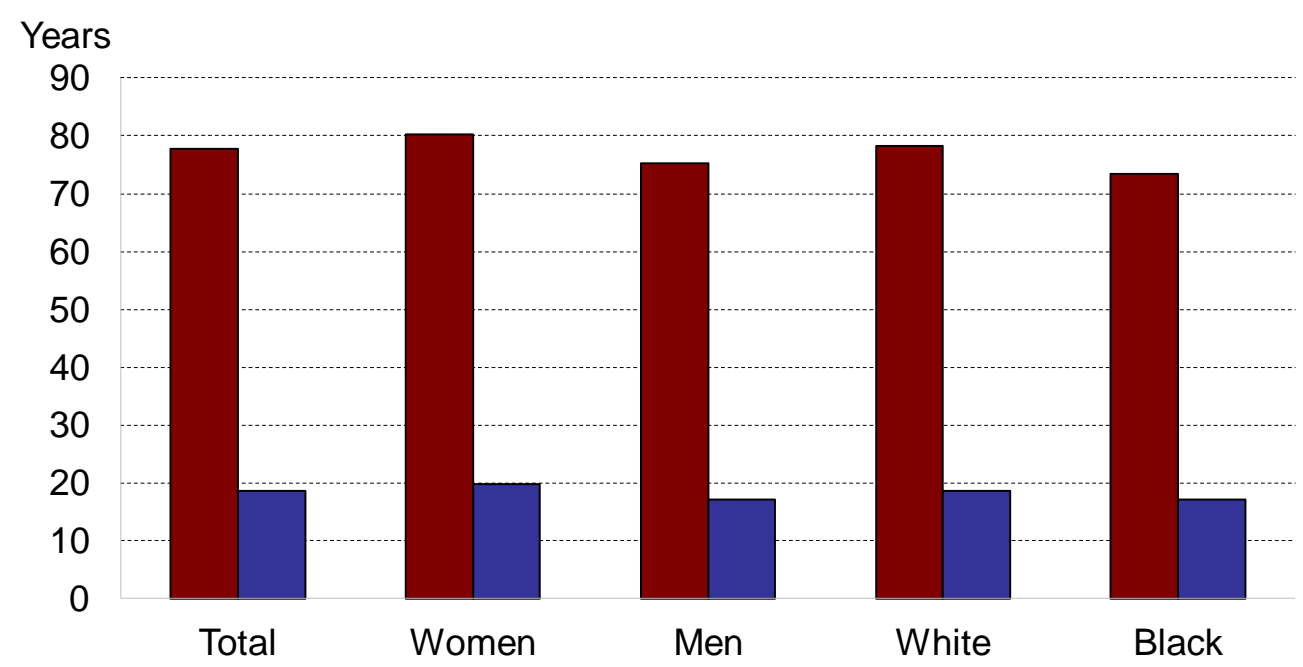

Source: National Vital Statistics System (NVSS), NCHS, CDC. 
Life expectancy for the periods 2000-01 to 2006-07 is not presented for racial and ethnic groups other than the white population and the black population due to data quality problems which prevented the production of reliable U.S. life tables for minority populations, including the Hispanic population, until 2006. Two issues affected the quality of data available for the Hispanic population: misclassification in reporting of race and ethnic origins on U.S. death certificates in comparison with the Census, surveys, and birth certificates; and misstatement of age at the oldest ages in both Census and vital statistics data. Recent research has shown that the classification of race and Hispanic origin on death certificates has improved and that a relatively minor adjustment is required to correct for the effects of the misclassification. In addition, the issue of age misstatement at the oldest ages can be addressed by recent research on Hispanic mortality patterns. Due to the improvement in data quality for the Hispanic population, complete period life tables for the total Hispanic population in 2006 became available in October 2010. However, additional data years for the Hispanic population are not yet available and therefore the Hispanic population is not addressed in this report [8].

Much of the recent gain in life expectancy is concentrated in the older population, the age group with the highest prevalence of functional limitations. As a result, measuring longevity is no longer sufficient to describe the health of a population. Preventing disabling conditions, improving function, relieving physical pain and emotional distress, and maximizing health across the life span have become important public health goals along with increasing life expectancy [9].

\section{Measuring Quality and Years of Healthy Life}

Given the multidimensional nature of health, assessing quality and healthy life is a much more complex process than measuring life expectancy, and the field is evolving. Various measures are used nationally and internationally to measure healthy life. These measures fall into three general categories:

- Self-assessments of overall health status by individuals or their proxies.

- Composite measures that include multiple dimensions of health. Scores on the various dimensions are combined into a single measure using a predetermined algorithm (for example, SF-36, Healthy Days) [11,12].

- Measures that combine death rates and health (where the health indicator can be either of the types described above or an indicator of a single dimension of health). These measures use years as the metric to quantify healthy life (for example, healthy life expectancies, Years of Healthy Life). 
Healthy People 2010: Understanding and Improving Health mentioned several possible measures of population health: "self-perceived" health status; healthy days; and the measure used in Healthy People 2000, Years of Healthy Life (YHL) [6,13]. In response to the need to measure Goal 1 of Healthy People 2010, at the beginning of the decade, NCHS convened a workshop to select measures that best capture the complexity of assessing years of healthy life within the context of Healthy People 2010 [14]. As a result of the workshop, three measures of healthy life expectancies that combine death rates with different measures of health were selected to track progress toward Goal 1 of Healthy People 2010. These healthy life expectancies represent the breadth of recommendations from the workshop. The three new measures are: 1) Expected years in good or better health; 2) Expected years free of activity limitation; and 3) Expected years free of selected chronic diseases. The current set of healthy life expectancies has evolved from the YHL measure, used to track the years and quality of life in Healthy People 2000. YHL combined information about death rates, self-rated health, and activity limitation into a single measure. The current set of healthy life expectancies separate the self-rated health component from the limitation of activities component to better track and understand change over time. For more detail on these measures, see the Technical Appendix.

Data for these three healthy life expectancies were analyzed for the period 2000-01 through 2006-07 for expected years in good or better health and expected years free of activity limitation and for the period 2002-03 through 2006-07 for expected years free of selected chronic diseases. Prevalence data on physician or health professional diagnosed arthritis were unavailable for the years 2000 and 2001; therefore, the expected years free of selected chronic diseases was not analyzed for those years as arthritis is one of the chronic conditions included in the measure. Results of the analysis are mixed with years in good or better health and years free of activity limitation showing an increase while years free of chronic conditions decreased during the decade.

\section{Description of Healthy Life Expectancies for Healthy People 2010}

The healthy life expectancies are calculated using a life-table technique. This technique combines information about average health states and death rates to produce age-specific estimates of expected years of healthy life (see Technical Appendix for details on the methodology).

Expected years in good or better health is defined as the average number of years a person can expect to live in good or better health. This measure assesses healthy life using a single global assessment question which asks a person to rate his or her health as "excellent," "very good," "good," "fair," or "poor". 
Expected years free of activity limitation is defined as the average number of years a person can expect to live free from limitation in activities, the need for assistance in personal or routine care needs, or the need to use special equipment because of health problems.

Expected years free of selected chronic diseases is defined as the average number of years a person can expect to live without being diagnosed by a physician or health professional as having one or more of the following selected conditions for which nationally representative data are available annually: arthritis, asthma, cancer, diabetes, heart disease, high blood pressure, kidney disease, or stroke.

\section{Healthy Life Expectancy at Birth}

Table ES-4 and Figure ES-5 present healthy life expectancies at birth for each of the three measures. Life expectancy is included in the figures for comparison purposes. Based on data from the years 2006-07, individuals in the U.S. could expect to live 69.0 years in good or better health, 66.2 years without activity limitation, and 43.1 years without selected chronic diseases. Expected years in good or better health increased by 0.5 years and expected years free of activity limitations increased 0.7 years between 2000-01 and 2006-07. Expected years free of selected chronic conditions declined by 0.6 years between 2002-03 and 200607.

Women can expect to spend a slightly greater proportion of their lives in fair or poor health, with activity limitations, and with chronic conditions than their male counterparts do. Based on data from years 2006-07, women could expect to live 80.3 years, of which 70.7 years would be in good or better health and 67.8 would be free of activity limitations. Women could, therefore, expect to spend $12 \%$ of their lives in fair or poor health, $16 \%$ with activity limitation, and $46 \%$ with one or more selected chronic diseases. In the years $2006-$ 07 , men could expect to spend $11 \%$ of their lives in fair or poor health, $14 \%$ with activity limitation, and $43 \%$ with one or more selected chronic diseases.

Compared to the white population, the black population could expect to spend a greater proportion of life in an unhealthy status. Based on data from years 2006-07, the black population at birth could expect to spend $16 \%$ of life in fair or poor health, $18 \%$ of life with activity limitation, and $47 \%$ of life with one or more selected chronic diseases. 
Table ES-4. Healthy Life Expectancies at Birth, in Years

\begin{tabular}{|c|c|c|c|c|c|c|}
\hline & & Total & Black & White & Women & Men \\
\hline \multirow{2}{*}{$\begin{array}{c}\text { Expected Years in } \\
\text { Good or Better Health }\end{array}$} & $2000-01$ & 68.5 & 59.8 & 69.7 & 70.2 & 66.6 \\
\cline { 2 - 8 } & $2006-07$ & 69.0 & 61.3 & 70.0 & 70.7 & 67.3 \\
\hline & $2000-01$ & 65.5 & 59.3 & 66.1 & 67.2 & 63.8 \\
\hline $\begin{array}{c}\text { Expected Years free of } \\
\text { activity limitations }\end{array}$ & $2006-07$ & 66.2 & 60.2 & 66.8 & 67.8 & 64.7 \\
\hline & & & & & & \\
\hline $\begin{array}{c}\text { Expected years free of } \\
\text { selected chronic } \\
\text { diseases }\end{array}$ & $2002-03$ & 43.7 & 38.9 & 43.9 & 43.6 & 43.8 \\
\cline { 2 - 8 } & $2006-07$ & 43.1 & 38.6 & 43.4 & 43.5 & 42.7 \\
\hline
\end{tabular}

Source: National Vital Statistics System (NVSS), NCHS, CDC

Figure ES-5. Life Expectancy and Healthy Life Expectancies at Birth, 2006-07

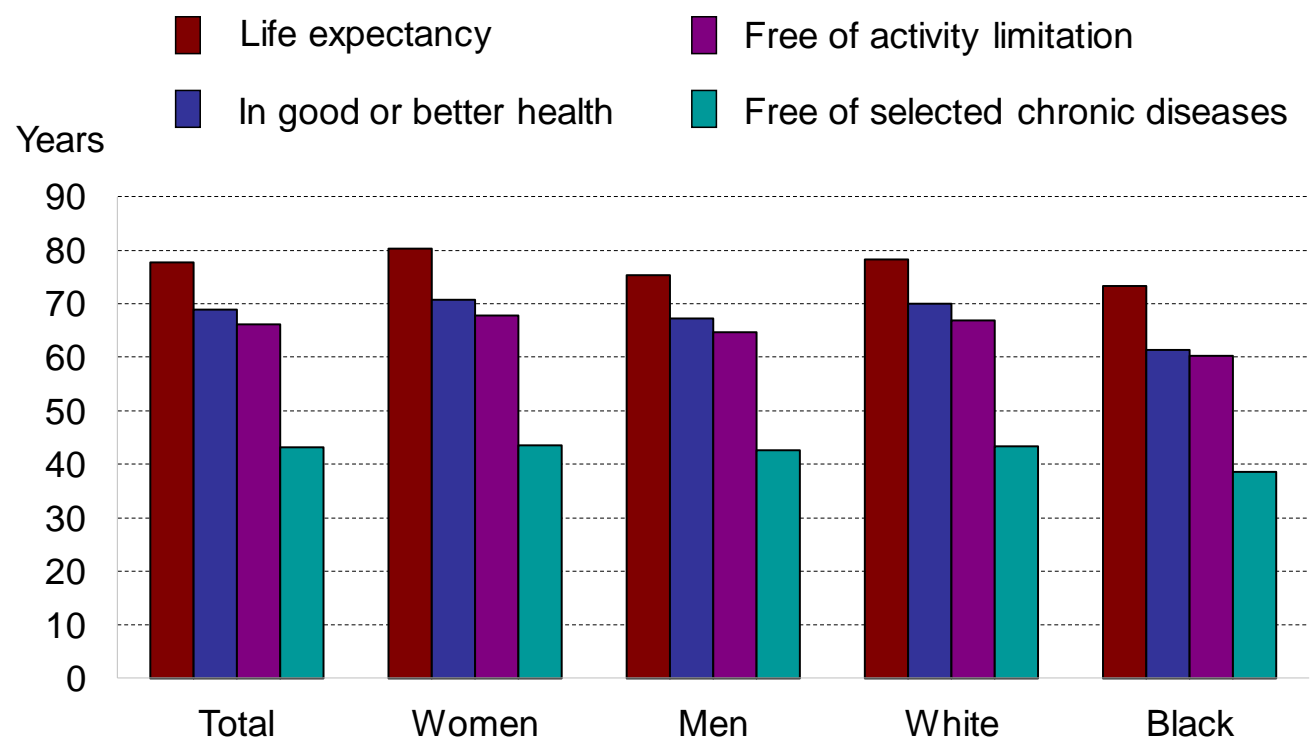

Source: National Health Interview Survey (NHIS), NCHS, CDC; National Vital Statistics System (NVSS), NCHS, CDC. 


\section{Healthy Life Expectancy at Age 65 Years}

Table ES-5 and Figure ES-6 present healthy life expectancies at age 65 years. Based on 2006-07 data, individuals at age 65 years could expect to live an additional 13.7 years in good or better health, 11.8 years without activity limitation, and 2.7 years without one or more selected chronic diseases. Between the years 2000-01 and 2006-07, for those at age 65 years, expected years in good or better health and expected years free of activity limitation increased. Between 2002-03 and 2006-07 expected years free of selected chronic diseases declined.

Similar to the patterns at birth, women at age 65 years could expect to live a greater number of years in a healthy life state, but they would spend a greater proportion of their lives with activity limitation or in fair or poor health. Based on data from years 2006-07, older women could expect to spend $39 \%$ of their remaining lives with activity limitation. Men could expect to spend $33 \%$ of their remaining lives with activity limitation.

It was expected that both older men and older women would spend a large proportion of their remaining lives with selected chronic diseases ( $86 \%$ for men; $86 \%$ for women). Older men and older women were expected to spend similar proportions of their remaining lives in fair or poor health ( $26 \%$ for men; $27 \%$ for women).

Similar to the patterns found at birth, the older black population could expect to spend a greater proportion of remaining life in unhealthy states than the older white population. Based on data from the years 2006-07, the black population aged 65 years could expect to live $39 \%$ of remaining life in fair or poor health, $46 \%$ with activity limitation, and $91 \%$ with selected chronic diseases. Between 2000-01 and 2006-07, the older black population experienced a greater increase in expected years in good or better health than the older white population. There was no statistically significant difference in the expected years free of activity limitation or expected years free of selected chronic conditions between the older black and white populations. 
Table ES-5. Healthy Life Expectancies at Age 65, in years

\begin{tabular}{|c|c|c|c|c|c|c|c|}
\hline & & Total & Black & White & Women & Men \\
\hline \multirow{2}{*}{$\begin{array}{c}\text { Expected Years in Good } \\
\text { or Better Health }\end{array}$} & $2000-01$ & 12.9 & 9.2 & 13.3 & 13.9 & 11.7 \\
\cline { 2 - 8 } & $2006-07$ & 13.7 & 10.5 & 13.9 & 14.5 & 12.6 \\
\hline & $2000-01$ & 11.1 & 8.6 & 11.3 & 11.5 & 10.6 \\
\hline \multirow{2}{*}{$\begin{array}{c}\text { Expected Years free of } \\
\text { activity limitations }\end{array}$} & $2006-07$ & 11.8 & 9.3 & 12.0 & 12.1 & 11.5 \\
\cline { 2 - 8 } & & & & & & \\
\hline \multirow{2}{*}{$\begin{array}{c}\text { Expected Years free of } \\
\text { selected chronic diseases }\end{array}$} & $2002-03$ & 2.8 & 2.0 & 2.9 & 2.9 & 2.7 \\
\cline { 2 - 8 } & $2006-07$ & 2.7 & 1.6 & 2.6 & 2.8 & 2.4 \\
\hline
\end{tabular}

Figure ES-6. Life Expectancy and Healthy Life Expectancies at Age 65, 2006-07

Life expectancy

In good or better health
Free of activity limitation

Free of selected chronic diseases

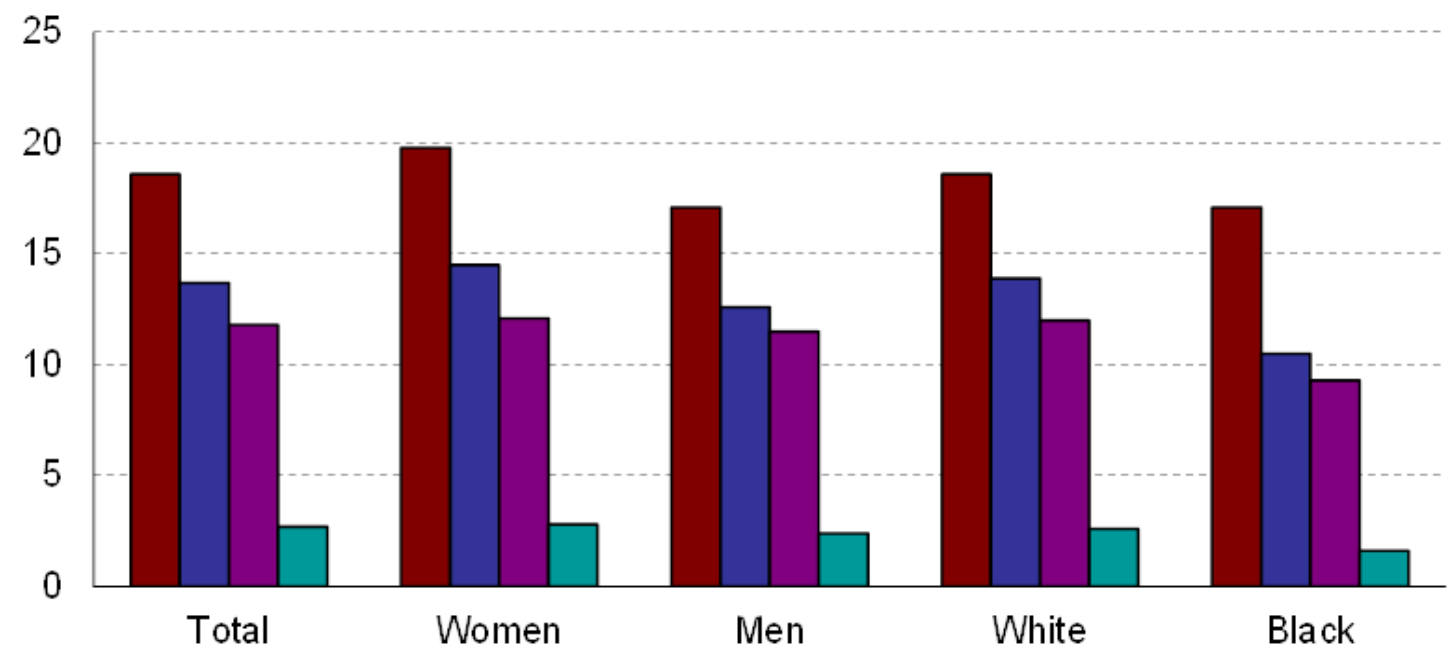

Source: National Health Interview Survey (NHIS), NCHS, CDC; National Vital Statistics System (NVSS), NCHS, CDC. 


\section{Healthy People 2010 Final Review: Executive Summary}

\section{Goal 2: Eliminate Health Disparities}

The second goal of Healthy People 2010 was to eliminate health disparities that occured by race and ethnicity, gender, education, income, geographic location, disability status, or sexual orientation. Findings for specific objectives and populations are presented in 27 of the 28 Focus Area chapters. None of the objectives in Public Health Infrastructure (Focus Area 23) called for population-based data. The findings concerning health disparities among population groups are summarized below.

Substantial health disparities between populations were observed for many Healthy People 2010 objectives. Both increases and decreases in health disparities also were observed for individual populations for specific objectives; however, most of the population-based objectives with data to measure disparities had no change in health disparities.

For specific population characteristics:

- Among 169 objectives with data for racial and ethnic groups, health disparities decreased for 27 objectives and increased for 25 .

- Among 216 objectives with data for males and females, health disparities decreased for 26 objectives and increased for 23 . Females more often had better group rates.

- Among 132 objectives with data for education groups, health disparities decreased for seven objectives and increased for 20 .

- Health disparities among income groups, as well as by geographic location and disability status did not change, with the exception of a few objectives.

There were 469 population-based objectives for which health disparities among populations could be measured. Presented as the second figure in each Focus Area chapter, the Health Disparities Table provides detailed information about health disparities among populations for the objectives in that Focus Area. The Health Disparities Table provides information about the availability of data for each population, the size of health disparities relative to the population group with the best rate for each characteristic, and the magnitude of changes in these disparities between the Healthy People 2010 baseline and the most recent time point for each objective. Data were not available for all populations for each objective, and tracking data were not always available to assess changes in disparity from baseline.

Data were unavailable by sexual orientation for any of the Healthy People 2010 objectives. 
In this Final Review, health disparities are measured using the "best" or most favorable group rate as the reference point. "Best" is used to identify the population group with the most favorable rate among the groups associated with a particular characteristic. "Best" does not imply that no further improvement is called for. Health disparities by race and ethnicity, for example, are measured using the rate for the racial and ethnic population with the best rate as the reference point. Health disparities are measured in relative terms as the percent difference between the rate for each population group and the best group rate for each characteristic. In the measurement of health disparities, objectives are generally expressed in terms of adverse events or conditions, such as death rates, to facilitate comparisons among them. Changes in disparities are measured by subtracting the percent difference from the best group rate at the baseline from the percent difference from the best group rate at the most recent time point. As a result, changes in disparities are measured in percentage points. In addition, when more than two groups are associated with a characteristic (race and ethnicity, education, or income), a summary index is used to describe the average percent difference from the best group in the population overall. The summary index provides a basis for conclusions about changes in the size of the disparities associated with these characteristics. A detailed description of the methods used to measure and evaluate disparities is provided in the Technical Appendix.

\section{Findings Concerning Disparities}

\section{Race and Ethnicity}

Information about health disparities among racial and ethnic populations at the most recent time point based on the Health Disparities Table for each Focus Area is summarized in Figure ES-7. The measurement of health disparities depends on the availability of data for each population. The number of objectives with the data needed to measure health disparities varied from 38 for the Native Hawaiian or Other Pacific Islander population to 354 for the white non-Hispanic population.

\section{American Indian or Alaska Native Population}

The data needed to assess health disparities for the American Indian or Alaska Native population were available for 157 objectives (Figure ES-7). This population had the best group rate for $6 \%$ of these objectives. The American Indian or Alaska Native population had a larger proportion of health disparities in the $100 \%$ or more category than any of the other racial and ethnic populations. The American Indian or Alaska Native population had rates at least twice as high as the best group rate (100\% or more category) for $26 \%$ of these objectives. 
Figure ES-7. Health Disparities at the Most Recent Time Point by Race and Ethnicity

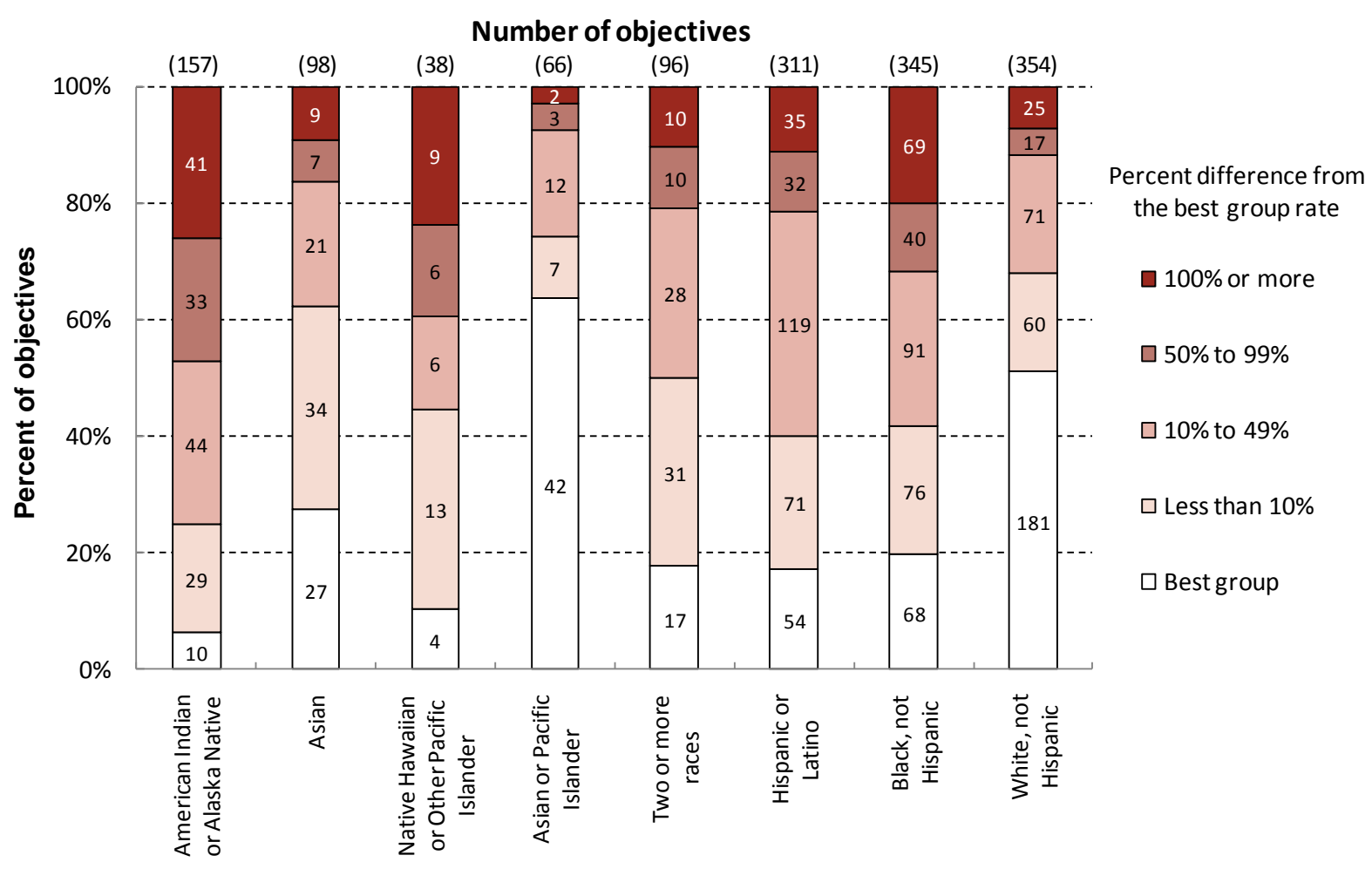

NOTE: "Less than 10\%" includes percent differences that were not statistically significant (when estimates of variability were available).

\section{Asian and Native Hawailian or Other Pacific Islander Populations}

The data needed to assess health disparities for the Asian population (excluding the Native Hawaiian or Other Pacific Islander population) were available for 98 objectives; see Figure ES-7. The Asian population had the best group rate for $28 \%$ of these objectives. This population had rates at least twice as high as the best group rate (100\% or more category) for $9 \%$ of the objectives with available data.

Data for the Native Hawaiian or Other Pacific Islander population were available for 38 objectives (Figure ES-7). This population had a smaller percentage of best group rates $(11 \%)$ and a larger percentage of health disparities of $100 \%$ or more $(24 \%)$ than the Asian population.

Data were available for the combined Asian or Pacific Islander population for 66 objectives (Figure ES-7). This combined population had the best group rate for $64 \%$ of these objectives. The Asian or Pacific Islander population had rates at least twice as high as the best group rate (100\% or more category) for two objectives: cases of hepatitis B in adults 19-24 years (objective 14-3a) and cases of hepatitis A (objective 14-6). 


\section{Two or More Races}

Data for individuals who identified with more than one race were available for 96 objectives (Figure ES-7). The population of persons of two or more races had the best group rate for $18 \%$ of these objectives. This population had rates at least twice as high as the best group rate $(100 \%$ or more category) for $10 \%$ of these objectives.

\section{Hispanic Population}

The data needed to assess health disparities for the Hispanic population were available for 311 objectives (Figure ES-7). The Hispanic population had the best group rate for $17 \%$ of these objectives. This population had rates at least twice as high as the best group rate ( $100 \%$ or more category) for $11 \%$ of these objectives.

\section{Black Non-Hispanic Population}

Data needed to assess health disparities for the black non-Hispanic population (or, in some cases, for the black population, including persons of Hispanic origin) were available for 345 objectives (Figure ES-7). This population had the best group rate for $20 \%$ of these objectives. This population had rates at least twice as high as the best group rate $(100 \%$ or more category) for $20 \%$ of these objectives, including most causes of death in many Focus Areas.

\section{White Non-Hispanic Population}

Data needed to assess health disparities for the white non-Hispanic population (or, in some cases, for the white population, including persons of Hispanic origin) were available for 354 objectives (Figure ES-7). This population had the best group rate for $51 \%$ of these objectives. This population had rates at least twice as high as the best group rate $100 \%$ or more category) for $7 \%$ of these objectives.

\section{Changes in Health Disparities among Racial and Ethnic Groups}

In addition to the findings for specific racial and ethnic population groups, a summary index allows the evaluation of changes in overall health disparities by race and ethnicity over time. There was no change in health disparities among racial and ethnic populations for 111 (69\%) of the 169 objectives with the data to calculate the summary index and assess its change over time. ("No change" includes changes of less than 10 percentage points, regardless of statistical significance, and all changes that were not statistically significant, when estimates of variability were available; see Technical Appendix.) The average percent difference from the best group rate decreased for 27 objectives and increased for 25 objectives (Figure ES-8). 
Figure ES-8. Changes in Health Disparities from the Baseline to the Most Recent Time Points by Population Characteristic

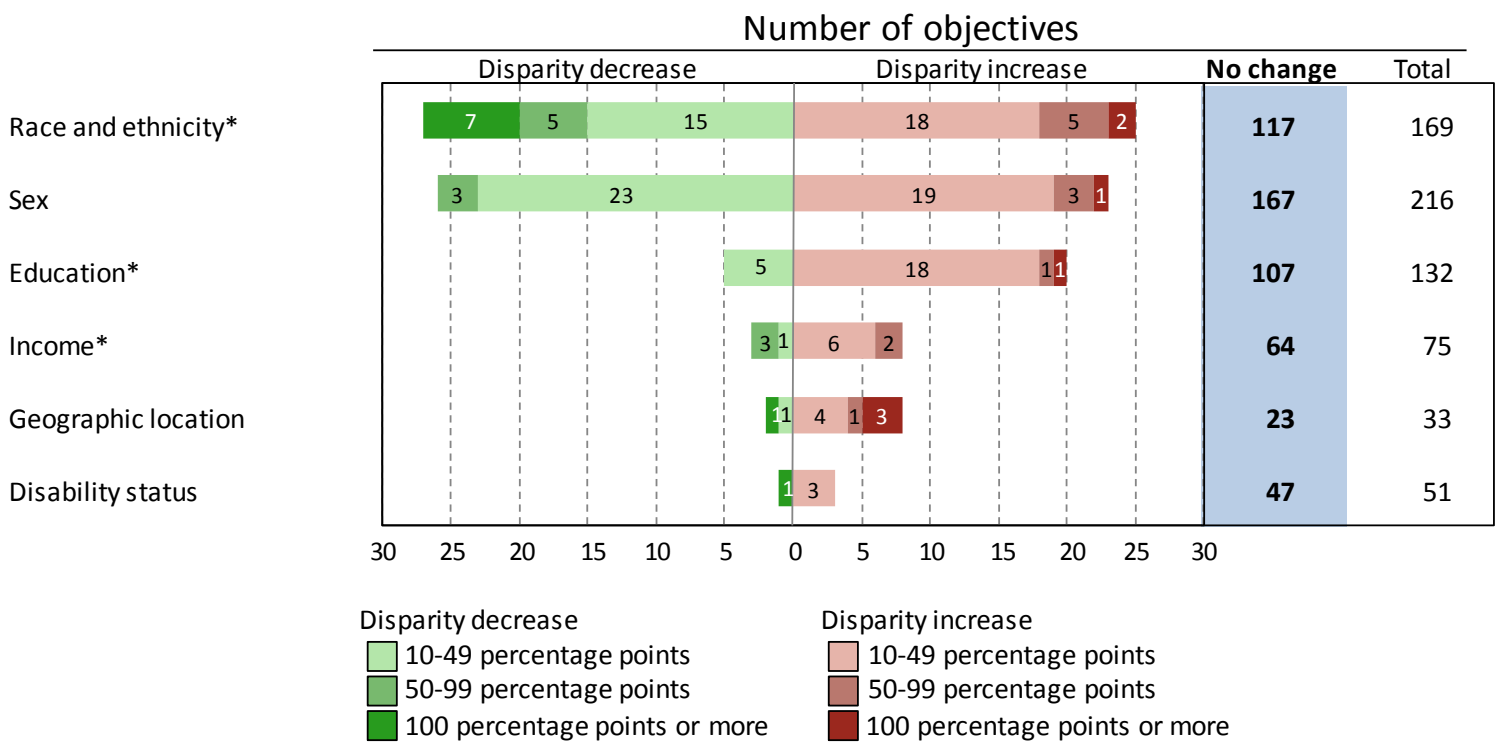

NOTES: Changes in disparity from the baseline to the most recent time points are only shown when they could be assessed. Changes could not be assessed for 54, 82, 4, 3, 10, and 17 objectives by race/ethnicity, sex, education, income, geographic location, and disability status, respectively. "No change" includes: changes of less than 10 percentage points, regardless of statistical significance; and all changes that were not statistically significant, when estimates of variability were available. See Technical Appendix.

* Number of objectives with changes in the summary index as a measure of disparity. Health disparities by income were not included for Focus Area 19 due to data limitations. 


\section{Sex}

Data by sex were available for 318 objectives (Figure ES-9). As noted below, trends in disparity could only be measured for 216 objectives. Health disparities by sex were not relevant to objectives that applied only to females or only to males, including those in Family Planning (Focus Area 9), and a number of objectives in other Focus Areas. Findings concerning health disparities by sex are summarized in Figure ES-9.

Females had the better group rate for $68 \%$ of these objectives, compared with $42 \%$ for males. (Those two percentages, $68 \%$ and $42 \%$, add to over $100 \%$ because there were a number of cases in which the two groups had the same rate; therefore, both were counted as having achieved the best group rate.) Females had a smaller percentage of objectives with rates that were at least twice as high as those for males (100\% or more category).

Figure ES-9. Health Disparities at the Most Recent Time Point by Sex

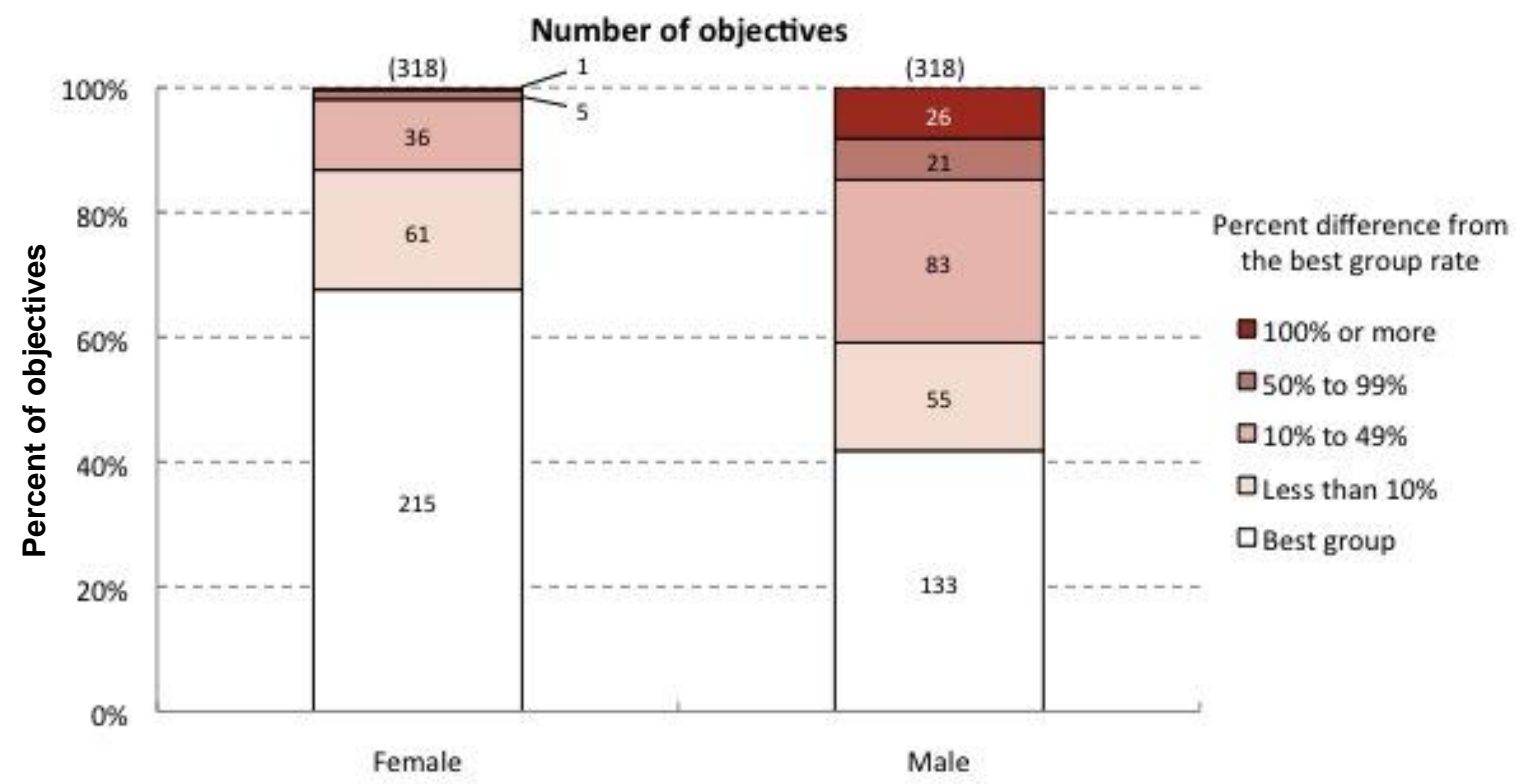

NOTE: "Less than 10\%" includes percent differences that were not statistically significant (when estimates of variability were available).

\section{Changes in Disparities by Sex}

The data needed to evaluate changes over time in health disparities by sex were available for 216 objectives. There was no change in disparity for 167 objectives, or $77 \%$ of the total with data. ("No change" includes changes of less than 10 percentage points, regardless of statistical significance, and all changes that were not statistically significant, when estimates of variability were available; see Technical Appendix.) Disparities decreased for 26 objectives and increased for 23 (Figure ES-8). In addition, there were 33 objectives for which changes in disparities could not be assessed, but were changes in the group with the best rate was observed (e.g., from males to females). 


\section{Education Level}

The data needed to assess health disparities among populations by education level were available for 160 to 161 objectives (Figure ES-10). Education level was not included as a characteristic in all Focus Areas. The population with at least some college education had the best rate for $88 \%$ of the objectives with data by education. The population with less than a high school education and high school graduates had the best group rate for $8 \%$ and $10 \%$ of the objectives with data by education, respectively. There were no objectives for which the disparity between the population with at least some college education and the group with the best rate was $100 \%$ or more. The population that completed high school had rates at least twice as high as the best group rate (100\% or more category) for $18 \%$ of these objectives, and the population with less than a high school education had rates at least twice as high as the best group rate ( $100 \%$ or more category) for $24 \%$ of these objectives.

\section{Figure ES-10. Health Disparities at the Most Recent Time Point by Education Level}

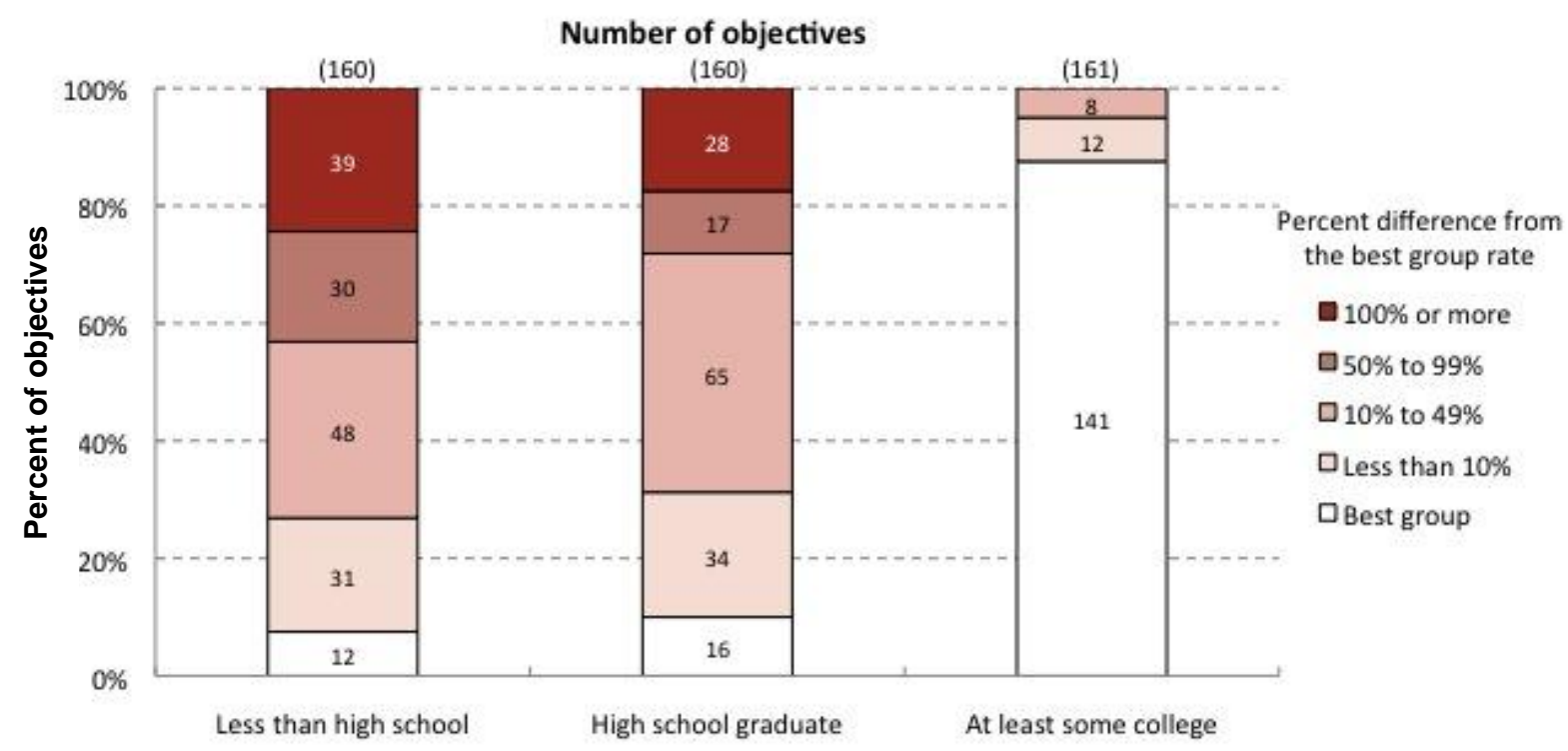

NOTE: "Less than 10\%" includes percent differences that were not statistically significant (when estimates of variability were available).

\section{Changes in Health Disparities by Education Level}

In addition to the findings for individual populations by education level, the summary index permits the evaluation of changes in overall health disparities over time by level of education. There was no change in health disparity among populations by education level for 107 objectives or $81 \%$ of the 132 objectives with the data to calculate the index and assess change over time. ("No change" includes changes of less than 10 percentage points, regardless of statistical significance, and all changes that were not statistically significant, when estimates of variability were available; see Technical Appendix.) The average percent difference from the best group rate decreased for five objectives and increased for 20 (Figure ES-8). There was one increase and no decreases of 100 percentage points or more. 


\section{Income}

Income was not included as a characteristic in all Focus Areas. All of the objectives in Nutrition and Overweight (Focus Area 19) and six objectives in Immunization and Infectious Diseases (Focus Area 14) were excluded from this summary because data by income were available for only two population subgroups (persons with income at or below $130 \%$ of the Federal poverty level, and persons with income above $130 \%$ of the Federal poverty level). This summary is based on 95 to 103 objectives with data for populations by income (Figure ES-11). The population with middle/high income (at or above $200 \%$ or the Federal poverty level) had the best rate for $74 \%$ of the objectives with data by income. The poor (below the Federal poverty level) and near-poor (100-199\% of the Federal poverty level) populations each had the best rate for $21 \%$ and $19 \%$ of their objectives, respectively.

There were no objectives for which the disparities between persons with middle/high incomes and the group with the best rate were $100 \%$ or more. The near-poor population had rates at least twice as high as the best group rate (100\% or more category) for $8 \%$ of the objectives with data. The poor or lowest income population had rates at least twice as high as the best group rate (100\% or more category) for $10 \%$ of the objectives with data.

\section{Figure ES-11. Health Disparities at the Most Recent Time Point by Income}

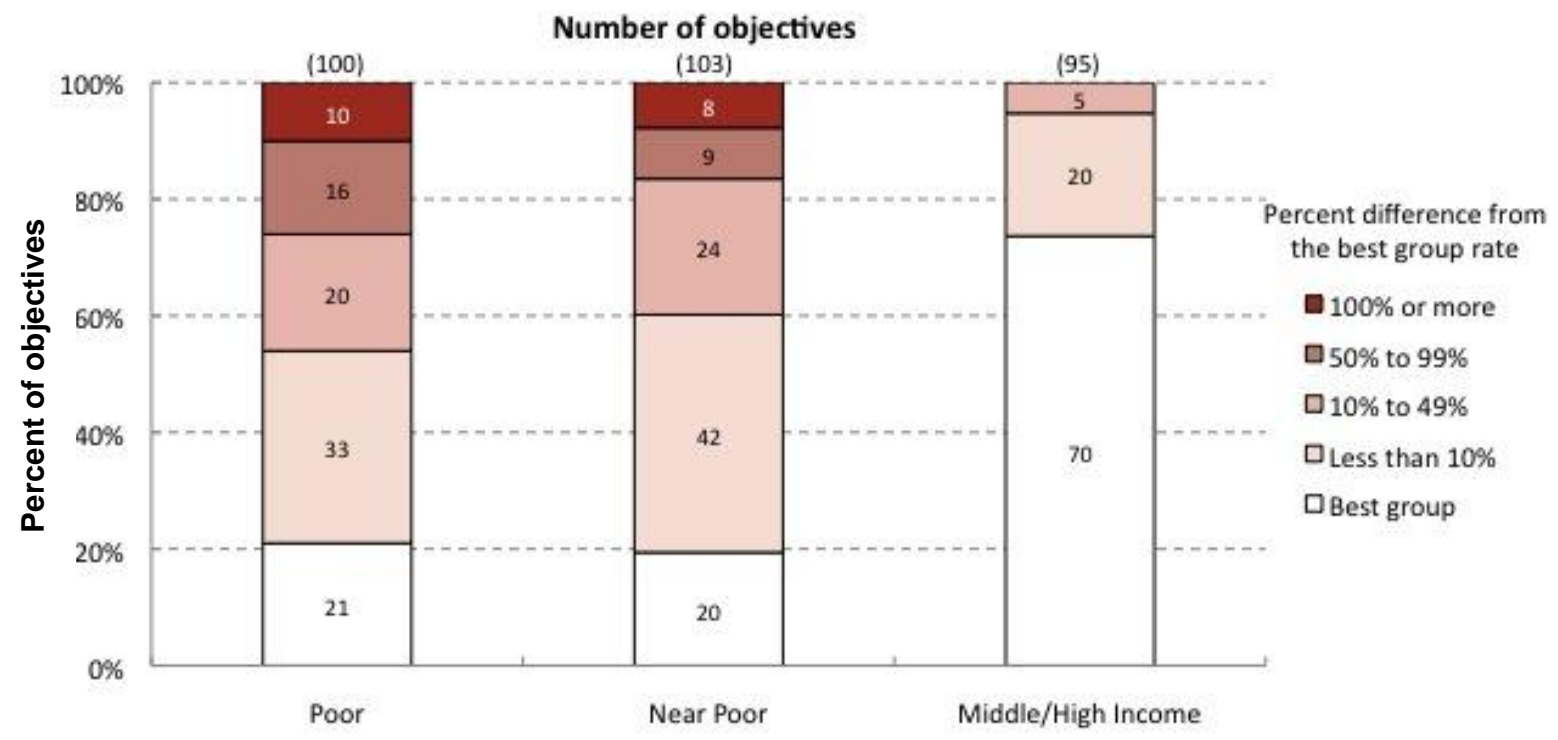

NOTE: "Less than 10\%" includes percent differences that were not statistically significant (when estimates of variability were available).

\section{Changes in Health Disparities by Income}

The summary index enables the evaluation of changes in disparity over time among populations by income. The data needed to evaluate changes in disparity among the populations by income were available for 75 objectives (Figure ES-8). There was little evidence of any change in disparity among populations by income. The average disparity relative to the rate with the best group decreased for three objectives and increased for eight (Figure ES-8). 


\section{Geographic Location}

Geographic location was defined in different ways in Healthy People 2010. For some objectives, the distinction was between urban and rural areas, while for others, the distinction was between metropolitan and nonmetropolitan areas. Findings for health disparities by geographic location for 52 objectives are summarized in Figure ES-12.

Urban or metropolitan areas had the better rate for $71 \%$ of the objectives with data. Urban or metropolitan areas also had more objectives (four objectives) with health disparities of $100 \%$ or more than rural or nonmetropolitan areas (one objective). Rural or nonmetropolitan areas had the better rate for $40 \%$ of these objectives. (Those two percentages, $71 \%$ and $40 \%$, add to over $100 \%$ because there were a number of cases in which the two groups had the same rate; therefore, both were counted as having achieved the best group rate.)

\section{Figure ES-12. Health Disparities at the Most Recent Time Point by Geographic Location}

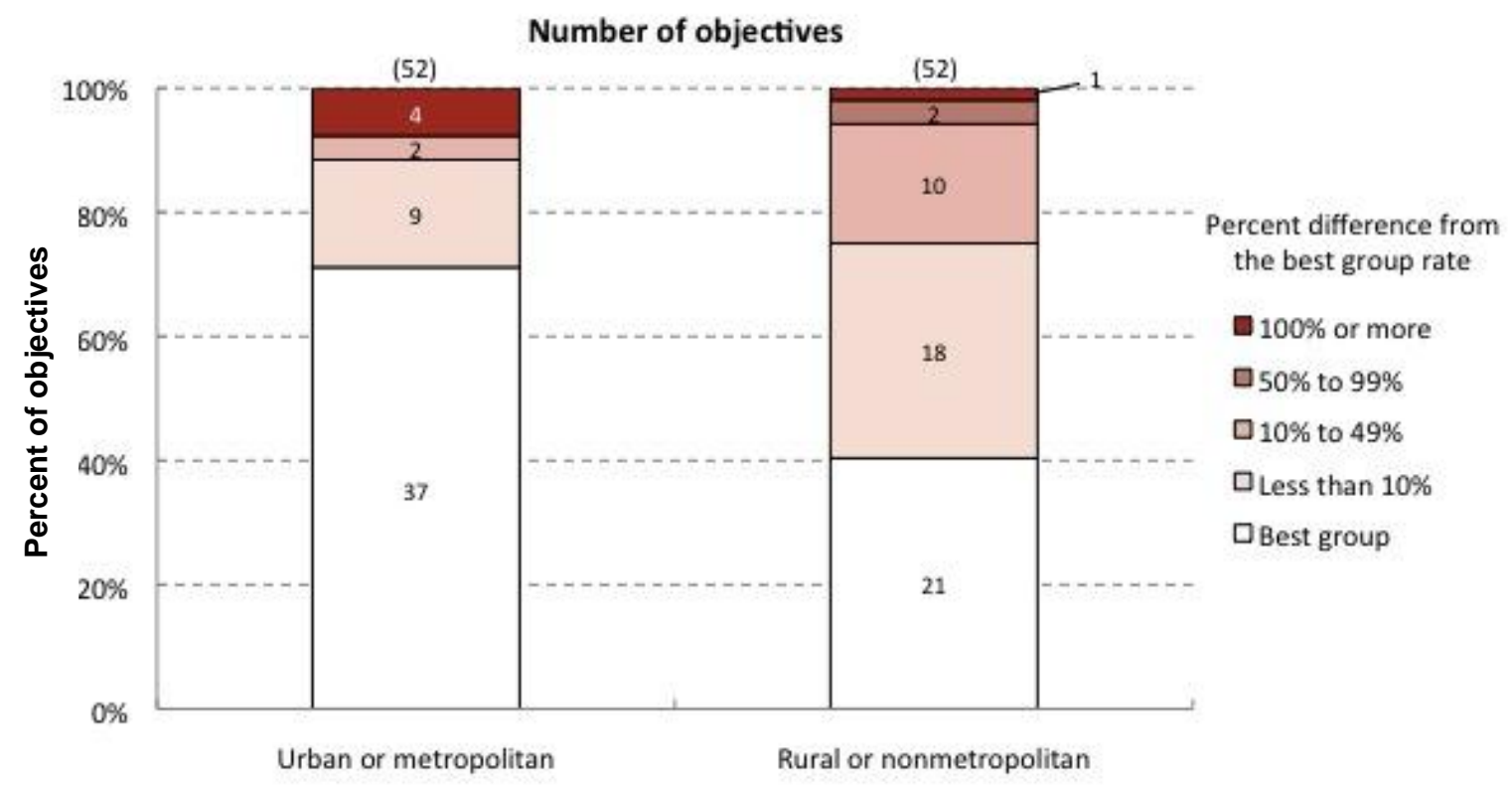

NOTE: "Less than 10\%" includes percent differences that were not statistically significant (when estimates of variability were available).

\section{Changes in Health Disparities by Geographic Location}

The data needed to evaluate changes in health disparities between geographic areas were available for 33 objectives. Health disparities from the better group rate declined for two objectives, and increased for eight (Figure ES-8). 


\section{Disability Status}

Data for persons with disabilities and persons without disabilities for 77 objectives are summarized in Figure ES-13. Persons with disabilities had the better group rate for $42 \%$ of these objectives, and persons without disabilities had the better group rate for $62 \%$. (Those two percentages, $42 \%$ and $62 \%$, add to over $100 \%$ because there were a number of cases in which the two groups had the same rate; therefore, both were counted as having achieved the best group rate.) Persons with disabilities had rates at least twice as high as for persons without disabilities (100\% or more category) for $6 \%$ of these objectives.

\section{Changes in Health Disparities by Disability Status}

The data needed to evaluate changes in health disparities between disability groups were available for 51 objectives (Figure ES-8). There were few changes in disparities by disability status. Health disparities between these populations declined for one objective and increased for three objectives.

Figure ES-13. Health Disparities at the Most Recent Time Point by Disability Status

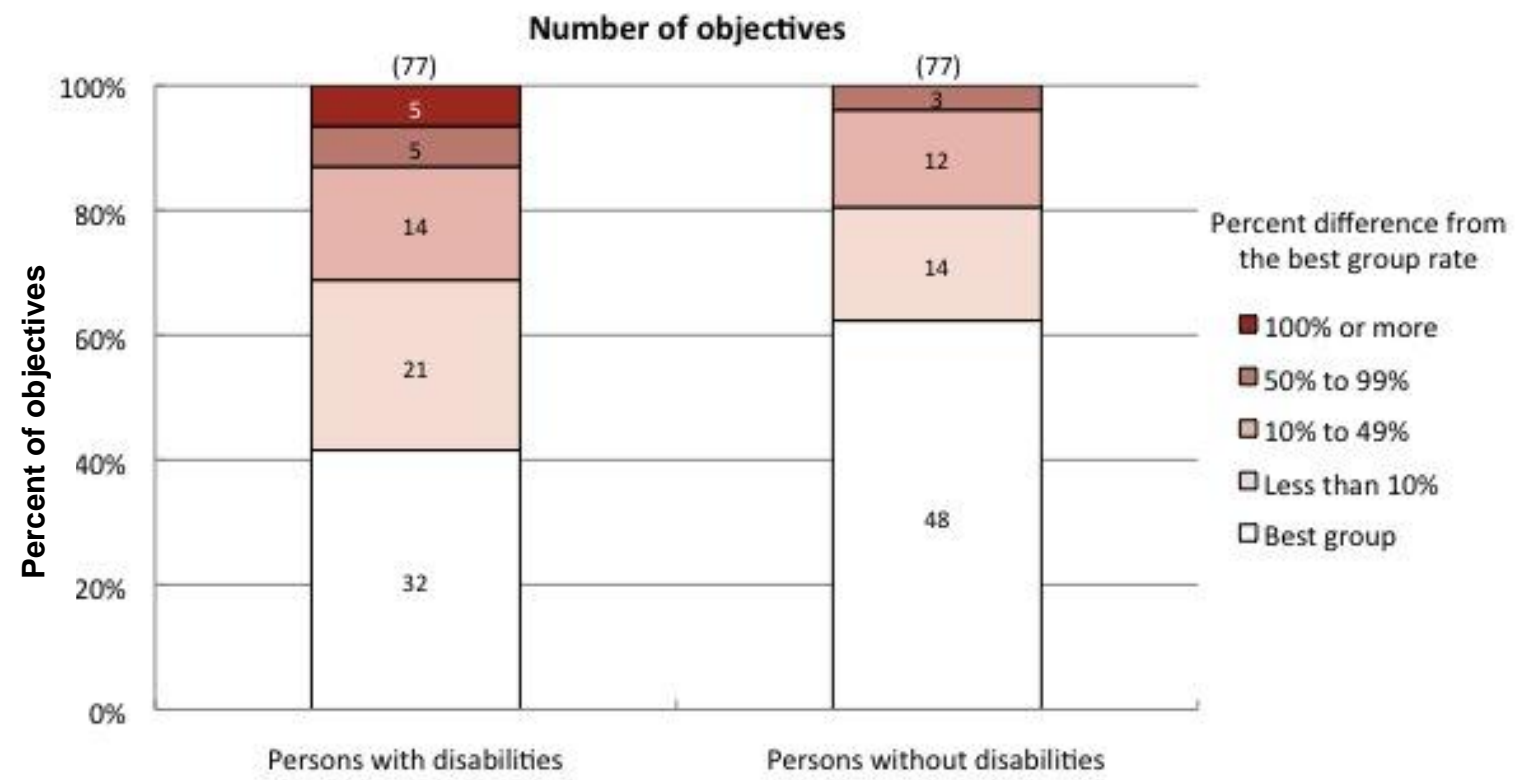

NOTE: "Less than 10\%" includes percent differences that were not statistically significant (when estimates of variability were available). 


\section{Data Limitations}

Several factors limited the number of objectives for which changes in disparities could be assessed. First, the number of years on which this assessment is based varied greatly. Second, this assessment is limited by a lack of data for select populations, such as American Indian or Alaska Native, Asian, Hispanic, and Native Hawaiian or Other Pacific Islander populations.

These findings are also subject to the limitations of the data on which they are based. This assessment is based only on data at the baseline and at the most recent time points; intervening data values were not considered. The findings presented here also are limited by the reliability and validity of information about the persons on which data were based. The reporting of race and income from some data systems was particularly problematic. Assessments of the probability that health disparities or changes in disparities were due to random fluctuations in the data were limited by the lack of estimates of variability for some of the data on which Healthy People 2010 objectives were based. See the Technical Appendix for more information. 


\section{Healthy People 2010 Final Review: Executive Summary}

\section{Transitioning to Healthy People 2020: The Decade Ahead}

In December 2010, HHS launched Healthy People 2020, the successor health promotion initiative for the second decade of the $21^{\text {st }}$ century which builds on the strengths of Healthy People 2010 while breaking new ground in the scope, outreach, and scientific underpinning of the initiative. In contrast with the two goals of Healthy People 2010, Healthy People 2020 is grounded in four overarching goals to:

1) Attain high quality, longer lives free of preventable disease, disability, injury, and premature death.

2) Achieve health equity and eliminate disparities.

3) Create social and physical environments that promote good health for all.

4) Promote quality of life, healthy development, and healthy behaviors across all life stages.

The framework of Healthy People 2020 is organized into 42 Topic Areas (formerly Focus Areas), with 13 new areas added:

- Adolescent Health

- Blood Disorders and Blood Safety

- Dementias, Including Alzheimer's Disease

- Early and Middle Childhood

- Genomics

- Global Health

- Healthcare-Associated Infections

- Health-Related Quality of Life and Well-Being

- Lesbian, Gay, Bisexual, and Transgender Health

- Older Adults

- Preparedness

- Sleep Health

- Social Determinants of Health.

In addition, the 2010 Vision and Hearing Focus Area was split into two separate Topic Areas for 2020: Vision, and Hearing and Other Sensory or Communication Disorders. 
The Healthy People 2020 Topic Areas encompass approximately 1,200 objectives as compared with 969 objectives in Healthy People 2010; 366 objectives have been carried over without change into Healthy People 2020; 358 appear in modified form; 242 have been archived, that is, preserved on inactive but retrievable status on the strength of having at least one data point; and 84 have been discontinued because they had no prospect of acquiring a data source, an improved data source had been identified, or the science had changed. Appendix D, "A Crosswalk Between Objectives From Healthy People 2010 to Healthy People 2020," summarizes the changes between the two decades of objectives.

\section{Innovations of Healthy People 2020}

Healthy People 2020 places a renewed focus on identifying, measuring, tracking, and reducing health disparities using a determinants of health approach. Health status and health behaviors are determined by influences at multiple levels, including personal (i.e., biological, psychological), organizational/institutional, environmental (i.e., both social and physical), and policy levels. Because significant and dynamic inter-relationships exist among these different levels of health determinants, interventions are most likely to be effective when they address determinants at all levels. Historically, many initiatives have focused on individual-level health determinants and interventions. Healthy People 2020 therefore expanded its focus from previous iterations to emphasize tracking and monitoring of health-enhancing social and physical environments. Integrating prevention into the continuum of education - from the earliest ages on-is an integral part of this ecological and determinants approach. Another important innovation in Healthy People 2020 is the expanded population template which will allow a more in-depth analysis of health disparities in comparison with Healthy People 2010.

As with Healthy People 2010, each Healthy People 2020 objective has a:

- Reliable data source

- Baseline measure

- Target for specific improvements to be achieved by the year 2020 .

Draft objectives have been prepared by experts from multiple lead Federal Agencies. The proposed objectives have then been reviewed through a public comment process and by the Healthy People Federal Interagency Workgroup, which used specific selection criteria to choose the final objectives.

Many objectives focus on interventions that are designed to reduce or eliminate illness, disability, and premature death among individuals and communities. Others focus on broader issues such as:

- Eliminating health disparities

- Addressing social determinants of health 
- Improving access to quality health care

- Strengthening public health services

- Improving the availability and dissemination of health-related information.

Over the course of the decade, four foundation health areas will be used to monitor progress toward promoting health, preventing disease and disability, eliminating disparities, and improving quality of life. These broad, cross-cutting areas include:

- General health status, as measured by such factors as life expectancy, healthy life expectancy, years of potential life lost, limitation of activity, chronic disease prevalence, self-assessed health status, and the CDC "Healthy Days Measures."

- Health-related quality of life and well-being, as measured in terms such as physical, mental, and social health-related quality of life; well-being/satisfaction; and participation in common activities.

- Determinants of health, that is, a range of personal, economic, and environmental factors that influence health status, including factors such as biology, genetics, individual behavior, access to health services, and the particular environment(s) in which people may find themselves in the course of life or their daily round.

- Disparities and inequities in health status observed across race/ethnicity, sex, physical and mental ability, and geographical location.

Concurrent with the release of Healthy People 2020, a redesigned website (www.healthypeople.gov) was launched. Replacing the traditional print publication with an interactive website as the main vehicle for dissemination will expand the reach and accessibility of Healthy People and allow users to tailor information to their particular needs and explore evidence-based resources for implementation. Among the new features of the site's contents are the following:

- An index to the Topic Areas and their objectives, with information about each objective's baseline, target, and data source.

- A Determinants of Health section with an animated graphic to illustrate the range of personal, social, economic, and environmental factors that influence health status and often account for health-related disparities among population groups.

- A Stay Connected section with information about signing up for the listserv and links to social networking sites.

Plans for the future include adding capabilities for the website to disseminate researchbased implementation strategies for Topic Areas and objectives and to receive public comments on the objectives during periods set aside for this purpose on an annual basis. 


\section{Healthy People 2010 Final Review: Executive Summary}

\section{References}

1. U.S. Department of Health, Education, and Welfare, Public Health Service. Healthy People: The Surgeon General's Report on Health Promotion and Disease Prevention. Washington, D.C: U.S. Government Printing Office. 1979.

2. U.S. Department of Health, Education, and Welfare, Public Health Service. Promoting Health/Preventing Disease: Objectives for the Nation. Washington, D.C: U.S. Government Printing Office. 1980.

3. U.S. Department of Health and Human Services, Public Health Service. Healthy People 2000: National Health Promotion and Disease Prevention Objectives. Washington, D.C.: U.S. Government Printing Office. 1991.

4. U.S. Department of Health and Human Services. Healthy People 2010 Midcourse Review. Washington, D.C.: U.S. Government Printing Office. 2006.

5. U.S. Department of Health and Human Services. Healthy People 2020 Topics and Objectives. Available from: HealthyPeople.gov.

6. U.S. Department of Health and Human Services. Healthy People 2010. $2^{\text {nd }}$ ed. With Understanding and Improving Health and Objectives for Improving Health. 2 vols. Washington, D.C.: U.S. Government Printing Office. November 2000.

7. National Center for Health Statistics. Health, United States, 2010. With Chartbook on Trends in the Health of Americans. Hyattsville, Maryland: 2010.

8. Arias E. United States life tables by Hispanic origin. National Center for Health Statistics. Vital Health Stat 2(152). 2010.

9. Molla MT, Wagener DK, Madans JH. Summary measures of population health: Methods for calculating healthy life expectancy. Healthy People Statistical Notes, no. 21. Hyattsville, Maryland: National Center for Health Statistics. August 2001.

10. World Health Organization (WHO). The World Health Organization Quality of Life Assessment (WHOQOL). Geneva, Switzerland: WHO, Division of Mental Health. 1995.

11. Centers for Disease Control and Prevention. More information available from www.cdc.gov/hrqol/methods.htm; accessed October 31, 2006.

12. Ware JE, Sherbourne CD. The MOS 36-item Short-Form Health Survey (SF-36): I. Conceptual framework and item selection. Med Care 30, 473-83. 1992.

13. Erickson P, Wilson R, Shannon, I. Years of healthy life. Healthy People Statistical Notes, no. 7. Hyattsville, Maryland: National Center for Health Statistics. 1995.

14. Molla MT, Madans JH, Wagener DK, Crimmins EM. Summary measures of population health: Report of findings on methodologic and data issues. National Center for Health Statistics. Hyattsville, Maryland. 2003. 


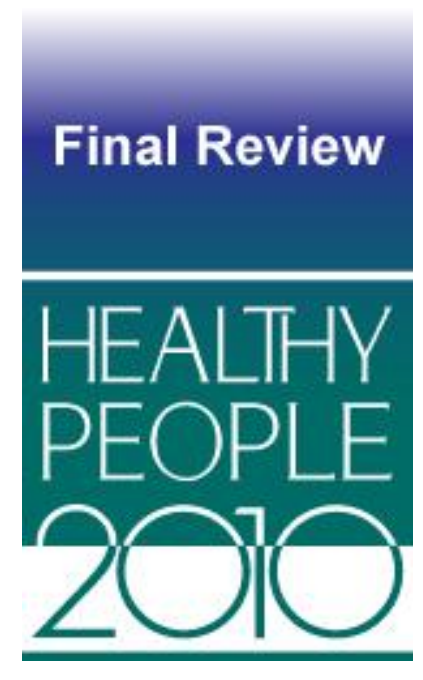

\section{Leading Health Indicators}

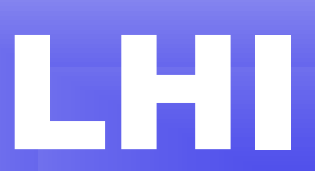

\section{Co-Lead Agencies}

Agency for Healthcare Research and Quality

Agency for Toxic Substances and Disease Registry

Centers for Disease Control and Prevention

Food and Drug Administration

Health Resources and Services Administration

National Institutes of Health

President's Council on Physical Fitness and Sports

Substance Abuse and Mental Health Services Administration

\section{Contents}

Introduction LHI-2

Highlights LHI-3

Summary of Progress. LHI-9

Transition to Healthy People 2020. LHI-10

Data Considerations LHI-11

References and Notes LHI-13

Comprehensive Summary of Objectives LHI-14

Progress Chart. .LHI-16

Health Disparities Table LHI-21 


\section{Introduction}

The objectives used to monitor the Leading Health Indicators are a subset of the Healthy People 2010 objectives that reflect the major public health concerns in the U.S. They were chosen on the basis of their ability to motivate action, the availability of data to measure their progress, and their relevance as broad public health issues. These indicators illuminate individual behaviors, physical and social environmental factors, and important health system issues that greatly affect the health of individuals and communities.

Each of the 10 Leading Health Indicators is monitored through one or more specific Healthy People 2010 objectives. At the launch of Healthy People 2010, the set of Leading Health Indicators included 22 objectives. Six supplemental objectives have been added since, for a total of 28 objectives.

The Leading Health Indicators for Healthy People 2010 are:

- Physical Activity. Two objectives track moderate or vigorous physical activity among adults and vigorous physical activity among adolescents (objectives 22-2 and 22-7, respectively).

- Nutrition and Obesity. Two objectives track obesity in adults and in children and adolescents (objectives 19-2 and 19-3c, respectively).

- Tobacco use. Two objectives monitor cigarette smoking among adults and among adolescents (objectives 27-1a and 27-2b, respectively).

- Substance Abuse. Three objectives track adolescents not using illicit drugs (objective 2610a), adults using illicit drugs (objective 26-10c), and adult binge drinking (objective 2611c).

- Responsible Sexual Behavior. Five objectives monitor condom use by sexually-active unmarried persons (objectives 13-6a and b) and adolescent sexual behavior (objectives 2511a through c). Three supplemental objectives also were included: Condom use among sexually active unmarried males (objective 13-6b); adolescents who had sexual intercourse but not in the past three months (objective 25-11b); and adolescents who used condoms at last intercourse (objective 25-11c).

- Mental Health. Two objectives track suicides (objective 18-1) and treatment of adults with depression (objective 18-9b). (Objective 18-1 is a supplemental objective.)

- Injury and Violence. Two objectives monitor deaths from motor vehicle crashes (objective 15-15a) and homicides (objective 15-32).

- Environmental Quality. Three objectives track exposure to ozone (objective 8-1a), children's exposure to tobacco smoke at home (objective 27-9), and nonsmoker exposure to tobacco smoke (objective 27-10). (Objective 27-9 is a supplemental objective.)

- Immunization. Three objectives monitor fully-immunized young children (objective 1424a) and influenza and pneumonia vaccination of older adults (objectives 14-29a and b, respectively).

- Access to Health Care. Four objectives track persons with health insurance (objective 1-1), persons with a source of ongoing care (objective 1-4a), hospitalizations for pediatric asthma (objective 1-9a), and the receipt of prenatal care beginning in the first trimester (objective 16-6a). (Objective 1-9a is a supplemental objective.) 
All Healthy People tracking data quoted in this chapter, along with technical information and operational definitions for each objective, can be found in the Healthy People 2010 database, DATA2010, available from http://wonder.cdc.gov/data2010/.

More information about the Leading Health Indicators can be found in the following publications:

- Healthy People 2010: Understanding and Improving Health, available from http://www.healthypeople.gov/2010/Document/html/uih/uih bw/uih 4.htm.

- Healthy People 2010 Midcourse Review, available from http://www.healthypeople.gov/2010/data/midcourse/html/default.htm\#FocusAreas.

- Sondik EJ, Huang DT, Klein RJ, Satcher D. Progress Toward the Healthy People 2010 Goals and Objectives. Annu Rev of Public Health 31(1):271-81. 2010.

\section{Highlights}

- Substantial progress [1] was achieved for the Leading Health Indicators during the past decade. Almost two thirds (63\%) of the Leading Health Indicator objectives with data to measure progress moved toward or achieved their Healthy People 2010 targets (Figure LHI-1). However, some health disparities [2] among select populations were observed (Figure LHI-2), as discussed below.

\section{Physical Activity}

- There was little or no progress toward targets for the objectives tracking this Leading Health Indicator. Between 1997 and 2008, the proportion of adults engaging in regular moderate or vigorous physical activity (objective $22-2$ ) remained stable at $32 \%$. The proportion of adolescents engaging in regular vigorous physical activity (objective 22-7) increased by $4.6 \%$ between 1999 and 2009 , from $65 \%$ to $68 \%$, moving toward the Healthy People 2010 target of 85\%; however, that increase was not statistically significant.

\section{Obesity and Overweight}

- Obesity in the U.S. population has increased, moving away from Healthy People 2010 targets. Based on directly measured weight and height, between 1988-94 and 2005-08 the proportion of adults aged 20 years and over who were obese (objective 19-2) rose by $47.8 \%$, from $23 \%$ to $34 \%$ (age adjusted), moving away from the 2010 target of $15 \%$. During the same period, obesity in children and adolescents aged 6-19 years (objective 19-3c) increased by $63.6 \%$, from $11 \%$ to $18 \%$, moving away from the 2010 target of $5 \%$.

\section{Tobacco Use}

Progress was seen for the objectives monitoring this Leading Health Indicator:

- The percentage of adults aged 18 years and over who were current cigarette smokers (objective 27-1a) decreased by $12.5 \%$ between 1998 and 2008, from $24 \%$ to $21 \%$ (age adjusted), moving toward the 2010 target of $12 \%$. However, from 2004 to 2008, the 
proportion of U.S. adults who were current cigarette smokers did not noticeably change. Moreover, health disparities were observed for a number of populations, for example:

- Among educational groups, adults aged 25 years and over with at least some college education had the lowest (best) current cigarette smoking rate, 15\% (age adjusted) in 2008. Adults aged 25 years and over with less than a high school education had a rate of $30 \%$ (age adjusted) in 2008, twice the best group rate [2].

- Adolescent use of cigarettes in the past month (objective 27-2b), decreased by $45.7 \%$, from $35 \%$ in 1999 to $19 \%$ in 2009, moving toward the 2010 target of $16 \%$.

- Among racial and ethnic groups, the black non-Hispanic population had the lowest (best) adolescent cigarette smoking rate, $10 \%$ in 2009 . The rate for the white nonHispanic population was $22 \%$ in 2009 , more than twice the best rate [2].

\section{Substance Abuse}

Progress for this Leading Health Indicator was mixed:

- The proportion of adolescents not using alcohol or illicit drugs in the past month (objective 26-10a) increased by $5.1 \%$ between 2002 and 2008, from $78 \%$ to $82 \%$, moving toward the 2010 target of $91 \%$.

- The proportion of adults using illicit drugs in the past month (objective 26-10c) did not change over the decade. As in 2002, the baseline year for this objective, $7.9 \%$ of adults aged 18 years and over used illicit drugs in the past month in 2008. Similarly, the proportion of adults who engaged in binge drinking in the past month (26-11c) changed very little, increasing by $2.5 \%$ over the same tracking period, from $24.3 \%$ to $24.9 \%$, and moving away from the 2010 target of $13.4 \%$.

\section{Responsible Sexual Behavior}

Four of the five objectives used to monitor this Leading Health Indicator moved toward their targets:

- Condom use among sexually active unmarried persons aged 18-44 years increased, moving toward the 2010 targets of 50\% for females (objective 13-6a) and 54\% for males (objective 13-6b). The proportion of females (or their partners) that used condoms increased by $43.5 \%$ between 1995 and 2006-08, from $23 \%$ to $33 \%$, while the proportion of males (or their partners) who used condoms increased by $4.8 \%$ between 2002 and 2006-08, from $42 \%$ to $44 \%$.

- The proportion of adolescents who had never had sexual intercourse (objective 25-11a) increased by $8.0 \%$ between 1999 and 2009, from 50\% to 54\%, moving toward the 2010 target of $56 \%$.

- The proportion of adolescents who had had sexual intercourse but not in the past three months (objective 25-11b) declined by $3.7 \%$ between 1999 and 2009, from $27 \%$ to $26 \%$, moving away from the target of $30 \%$.

- The proportion of adolescents who used condoms at last intercourse (objective 25-11c) increased by $5.2 \%$ between 1999 and 2009, from 58\% to 61\%, moving toward the 2010 target of $65 \%$. 


\section{Mental Health}

Data to measure progress was available for one of the two objectives used to monitor this Leading Health Indicator, objective 18-1, suicide, which increased over the decade, moving away from the 2010 target. Only baseline data were available for objective 18-9b, treatment for adults with depression.

- The suicide rate (objective 18-1) increased by $7.6 \%$ between 1999 and 2007, from 10.5 to 11.3 per 100,000 population (age adjusted), moving away from the 2010 target of 4.8 per 100,000 . Health disparities were observed for a number of population groups, for example:

- Among racial and ethnic groups, the black non-Hispanic population had the lowest (best) suicide rate, 5.1 per 100,000 population (age adjusted) in 2007. The rates for the American Indian or Alaska Native and the white non-Hispanic populations were 11.5 and 13.5 per 100,000 (age adjusted), respectively. The rate for the American Indian or Alaska Native population was almost two and a half times the best rate (that for the black non-Hispanic population), while the rate for the white nonHispanic population was more than two and a half times the best rate [2].

- The white non-Hispanic population had suicide rates of 12.0 per 100,000 population (age adjusted) in 1999 and 13.5 in 2007, whereas the black nonHispanic population had rates of 5.7 in 1999 and 5.1 in 2007. The disparity between the white non-Hispanic and black non-Hispanic populations increased by 54 percentage points between 1999 and 2007 [3].

- Females had a lower (better) suicide rate than males, 4.7 per 100,000 population (age adjusted) in 2007. The rate for males was 18.4 per 100,000 (age adjusted) in 2007, almost four times the rate for females [2].

- Males had suicide rates of 17.8 per 100,000 population (age adjusted) in 1999 and 18.4 in 2007, whereas females had rates of 4.0 in 1999 and 4.7 in 2007. The disparity between males and females declined by 53 percentage points between 1999 and 2007 [3].

- Among education groups, persons with at least some college education had the lowest (best) suicide rate, 9.9 per 100,000 population (age adjusted) in 2002, whereas high school graduates had a rate of 18.4 per 100,000 (age adjusted) in 2002, almost twice the best group rate [2].

\section{Injury and Violence}

Progress for this Leading Health Indicator was mixed:

- Motor vehicle crash deaths per 100,000 population (objective 15-15a) declined by 6.1\% between 1999 and 2007, from 14.7 to 13.8 (age adjusted), moving toward the 2010 target of 8.0 .

- Among racial and ethnic groups, the Asian or Pacific Islander population had the lowest (best) rate of motor vehicle crash deaths, 7.0 per 100,000 population (age adjusted) in 2007. The American Indian or Alaska Native, black non-Hispanic, and white non-Hispanic populations had rates of 22.5, 14.1, and 14.2 per 100,000 (age adjusted) in 2007, respectively. The rate for the American Indian or Alaska Native population was more than three times the best rate (that for the Asian or Pacific Islander population). The rates for the black non-Hispanic and white non-Hispanic populations were about twice the best rate [2]. 
- The homicide rate (objective 15-32) did not change significantly over the decade. In 1999, the baseline year for this objective, the homicide rate was 6.0 per 100,000 population (age adjusted), compared to a rate of 6.1 in 2007 [1].

- Among racial and ethnic groups, the Asian or Pacific Islander population had the lowest (best) rate of deaths from homicide, 2.3 per 100,000 population (age adjusted) in 2007. The rates for the American Indian or Alaska Native, Hispanic or Latino, and black non-Hispanic populations were 6.5, 6.9, and 21.8 per 100,000 (age adjusted) in 2007, respectively. The rate for the American Indian or Alaska Native population was almost three times the best rate (that for the Asian or Pacific Islander population). The rate for the Hispanic or Latino population was three times the best rate, and the rate for the black non-Hispanic population was about nine and a half times the best rate [2].

- The white non-Hispanic population had the lowest (best) rate of deaths from homicide at baseline, 2.9 deaths per 100.000 (age adjusted) in 1999, while the Asian or Pacific Islander population had the best rate at the most recent data point, 2.3 per 100,000 (age adjusted) in 2007. The black non-Hispanic population had rates of 20.7 and 21.8 per 100,000 (age adjusted) in 1999 and 2007, respectively. Between 1999 and 2007, the disparity between the black non-Hispanic population and the group with the best rate increased by 234 percentage points [3].

- Females had a lower (better) homicide rate than males, 2.5 per 100,000 population (age adjusted) in 2007. The rate for males was 9.6 per 100,000 (age adjusted) in 2007, nearly four times the rate for females [2].

- Among education groups, persons aged 25-64 years with at least some college education had the lowest (best) rate of deaths from homicide, 2.6 per 100,000 population (age adjusted) in 2002. The rates for high school graduates and persons with less than a high school education were 10.5 and 16.0 per 100,000 (age adjusted) in 2002, respectively. High school graduates had a rate that was approximately four times the best group rate (that for persons aged 25-64 years with at least some college education); the rate for persons with less than a high school education was more than six times the best group rate [2].

\section{Environmental Quality}

There was substantial progress for this Leading Health Indicator. Two of the three environmental quality objectives exceeded their 2010 targets:

- The proportion of people living in counties that exceeded NAAQS for ozone (objective 8-1a) declined by $25 \%$ between 1997 and 2010, from 43\% to 36\%, moving toward the 2010 target of $0 \%$. However, the final data year by race and ethnicity was 2004 and, at that time, disparities were observed for a number of population groups:

- Among racial and ethnic groups, the American Indian or Alaska Native population had the lowest (best) rate of living in counties that exceeded NAAQS for ozone (objective 8-1a), 23\% in 2004, whereas the white non-Hispanic, Native Hawaiian or Other Pacific Islander, black non-Hispanic, Hispanic or Latino, and Asian populations had rates of 33\%, 35\%, 43\%, 59\%, and $67 \%$, respectively. 
- The rate for the white non-Hispanic population was almost one and a half times the best rate (that for the American Indian or Alaska Native population); the rate for the Native Hawaiian or Other Pacific Islander population was about one and a half times the best rate; that for the black non-Hispanic population was almost twice the best rate; that for the Hispanic or Latino population was more than two and a half times the best rate; that for the Asian population was nearly three times the best rate [2].

- The rural or nonmetropolitan population had better rates of exposure to ozone (4\% in 1997 and 3\% in 2004) than the urban or metropolitan population (52\% in 1997 and $48 \%$ in 2004). In 2004, the rate for the urban or metropolitan population was 16 times as high as that for the rural or nonmetropolitan population. Between 1997 and 2004, the disparity in ozone exposure between the rural/nonmetropolitan and the urban/metropolitan populations increased by 300 percentage points [3].

- The percentage of children aged six years and under exposed to tobacco smoke at home (objective 27-9) decreased by $70.4 \%$ between 1994 and 2005 , from $27 \%$ to $8 \%$, exceeding the 2010 target of $10 \%$. However, disparities were observed among a number of population groups, for example:

- Among income groups, children aged six years and under living in middle/high income households had the lowest (best) rates of exposure to tobacco smoke at home, $5 \%$ in 2005 , whereas children living in poor or near-poor households had rates of $15 \%$ and $12 \%$ in 2005 , respectively. The rate for children living in poor households was three times the best group rate, while the rate for children living in near-poor households was almost two and a half times the best group rate [2].

- Children living in poor households had rates of exposure to tobacco smoke of $38 \%$ in 1994 and $15 \%$ in 2005; those living in near-poor households had rates of 33\% in 1994 and $12 \%$ in 2005; whereas those living in middle/high income households had rates of $19 \%$ in 1994 and $5 \%$ in 2005. The disparity between children living in poor households and those living in middle/high income households increased by 100 percentage points between 1994 and 2005. During the same period, the disparity between children living in near-poor households and those living in middle/high income households increased by 66 percentage points [3].

- The percentage of nonsmokers aged four years and over exposed to environmental tobacco smoke (objective $27-10$ ) declined by $51.2 \%$ between $1988-94$ and $2005-08$, from $84 \%$ to $41 \%$ (age adjusted), exceeding the 2010 target of $56 \%$.

\section{Immunization}

Progress was seen for the three objectives monitoring this Leading Health Indicator:

- The proportion of young children aged 19-35 months who were fully immunized (objective 14-24a) increased by $6.8 \%$ between 1998 and 2008 , from $73 \%$ to $78 \%$ moving toward the 2010 target of $80 \%$.

- Vaccination of noninstitutionalized high risk persons aged 65 years and over for influenza and pneumonia both increased between 1998 and 2008, moving toward the 2010 targets of $90 \%$. The proportion who had received an influenza vaccination in the past 12 months (objective 14-29a) increased by $4.7 \%$, from $64 \%$ to $67 \%$, and the proportion who had ever received a pneumococcal vaccination (objective $14-29 \mathrm{~b}$ ) increased by $30.4 \%$, from $46 \%$ to $60 \%$ over the tracking period. 


\section{Access to Health Care}

Progress for this Leading Health Indicator was mixed:

- Rates of persons with health insurance (objective 1-1) did not change over the decade. As in 1997, the baseline year for this objective, $83 \%$ of the U.S. population under age 65 years had health insurance coverage in 2008. Disparities were observed for a number of population groups, for example:

- Among racial and ethnic groups, the white non-Hispanic population had the highest (best) rate of health insurance coverage, $88 \%$ in 2008, whereas the American Indian or Alaska Native population and the Hispanic or Latino population had rates of $72 \%$ and $67 \%$ in 2008 , respectively. When expressed as persons without health insurance, the rate for the American Indian or Alaska Native population was more than twice the best rate (that for the white non-Hispanic population). The rate for the Hispanic or Latino population was nearly three times the best rate [2].

- The American Indian or Alaska Native population had health insurance coverage rates of $62 \%$ in 1999 and $72 \%$ in 2008, whereas the white non-Hispanic population had rates of $88 \%$ in both 1999 in 2008 . When rates are expressed in terms of persons without health insurance, the disparity between the American Indian or Alaska Native population and the white non-Hispanic population decreased by 83 percentage points between 1999 and 2008 [2,3].

- Among income groups, the middle/high income population had the highest (best) rate of health insurance coverage, $89 \%$ in 2008, whereas the poor and near-poor populations had rates of $71 \%$ and $69 \%$ in 2008, respectively. When expressed as persons without health insurance, the rate for the poor population was more than two and a half times the best rate (that for the middle/high income population). The rate for the near-poor population was almost three times the best rate [2].

- The poor population had health insurance coverage rates of $66 \%$ in 1997 and $71 \%$ in 2008, whereas the middle/high income population had rates of $90 \%$ in 1997 and $89 \%$ in 2008 . When rates are expressed in terms of persons without health insurance, the disparity between the poor population and the middle/high income population decreased by 76 percentage points between 1997 and 2008 [2,3].

- The proportion of persons with a source of ongoing care (objective 1-4a) declined by $1.1 \%$ between 1998 and 2008, from 87\% to 86\%, moving away from the 2010 target of $96 \%$.

- Among racial and ethnic groups, the white non-Hispanic population had the highest (best) rate, $89 \%$ in 2008, whereas the Hispanic or Latino population had a rate of $77 \%$ in 2008 . When expressed as persons without a specific source of ongoing care, the rate for the Hispanic or Latino population was more than twice the best rate [2].

- Among income groups, the middle/high income population had the highest rate, $90 \%$ in 2008, whereas the poor and near-poor populations had rates of $78 \%$ and $80 \%$ in 2008, respectively. When expressed as persons without a specific source of ongoing care, the rates for the poor and near-poor populations were about twice the best rate [2].

- Hospitalizations for pediatric asthma (objective 1-9a) declined by 35.2\% between 1996 and 2008 , from 23.0 to 14.9 admissions per 100,000 population aged under 18 years, exceeding the 2010 target of 17.3 admissions per 100,000 population. 
- The proportion of pregnant women who began prenatal care in the first trimester (objective 16-6a) increased $1.2 \%$ between 1998 and 2002, from 83\% to 84\%, moving toward the 2010 target of $90 \%$.

- White non-Hispanic women had the highest (best) rate of prenatal care among racial and ethnic populations, $89 \%$ in 2002, whereas the American Indian or Alaska Native, Hispanic or Latino, and black non-Hispanic women had rates of 70\%, 77\%, and $75 \%$, in 2002, respectively. When expressed as women not receiving prenatal care, the rates for American Indian or Alaska Native, Hispanic or Latino, and black non-Hispanic women were more than twice the best rate (that for white nonHispanic women) [2].

- Women aged 20 years and over with at least some college education had the best rate of prenatal care among education groups, $92 \%$ in 2002, whereas high school graduates and women with less than a high school education had rates of $83 \%$ and $72 \%$ in 2002 , respectively, among women aged 20 years and over. When expressed as women aged 20 years and over not receiving prenatal care, the rate for high school graduates was more than twice the best rate; and the rate for women with less than a high school education was three and a half times the best rate [2].

\section{Summary of Progress}

- Figure LHI-1 presents a quantitative assessment of progress [1] in achieving the Healthy People 2010 objectives for Leading Health Indicators. Data to measure progress toward target attainment were available for 27 objectives. Of these:

- Three objectives exceeded their Healthy People 2010 targets (objectives 1-9a, 27-9, and 27-10).

- Fourteen objectives moved toward their targets. A statistically significant difference between the baseline and the final data points was observed for eight of these objectives (14-24a, 14-29a and b, 15-15a, 16-6a, 26-10a, 27-1a, and 27-2b). No significant difference was observed for one objective (22-7); and data to test the significance of the difference were unavailable for five objectives (8-1a, 13-6a and b, and 25-11a and c).

- Three objectives showed no change (objectives 1-1, 22-2, and 26-10c).

- Seven objectives moved away from their targets. A statistically significant difference between the baseline and final data points was observed for four of these objectives (1-4a, 18-1, 19-2, and 19-3c). No significant differences were observed for two objectives (15-32 and 26-11c); and data to test the significance of the difference were unavailable for one objective (25-11b).

- One objective had no data available to measure progress (objective 18-9b).

- Figure LHI-2 displays health disparities [2] for the Leading Health Indicators from the best group rate for each characteristic at the most recent data point. It also displays changes in disparities from baseline to the most recent data point [3]. 
- Twenty-four objectives had statistically significant racial and ethnic health disparities of $10 \%$ or more. In addition, one objective had racial and ethnic health disparities of $10 \%$ or more but lacked data to assess statistical significance. Of these 25 objectives, the white non-Hispanic population had the best rate for 12 objectives (1-1, 1-4a, 14-29a and b, 16-6a, 18-9b, 19-2, 22-2, 22-7, 25-11a and c, and 27-9). The black non-Hispanic population had the best rate for six objectives (13-6a and b, 181, 26-10a, 26-11c, and 27-2b); the Hispanic or Latino population had the best rate for three objectives (26-10c, 27-1a, and 27-10); the Asian or Pacific Islander population had the best rate for two objectives (15-15a and 15-32); the American Indian or Alaska Native population had the best rate for one objective (8-1a); and persons of two or more races had the best rate for one objective (14-24a).

- Females had better rates for 12 of the 14 objectives with statistically significant health disparities of $10 \%$ or more by sex (objectives 1-1, 1-4a, 1-9a, 14-29b, 15-15a, 15-32, 18-1, 18-9b, 26-10c, 26-11c, 27-1a, and 27-10). Males had better rates for the remaining two objectives (22-7 and 25-11c).

- Persons with at least some college education had the best rate for all 12 objectives with statistically significant health disparities of $10 \%$ or more by education (objectives 13-6a and b, 14-29a and b, 15-15a, 15-32, 16-16a, 18-1, 22-2, 26-10c, 27-1a, and 27-10).

- Persons with middle/high incomes had the best rate for seven of the nine objectives with statistically significant health disparities of $10 \%$ or more by income (objectives 1-1, 1-4a, 13-6a, 19-2, 19-3c, 27-1a, and 27-9). Near-poor and poor persons had the best rate for one objective each (14-24a and 26-10a, respectively)

- One objective had a statistically significant health disparity of $10 \%$ or more by geographic location and one had a health disparity of $10 \%$ or more by geographic location but lacked data to assess statistical significance. Persons living in urban or metropolitan areas had a better rate for one (objective 1-1), while persons living in rural or nonmetropolitan areas had a better rate for the other (objective 8-1a).

- Eight objectives had statistically significant health disparities of $10 \%$ or more by disability status. Persons with disabilities (objectives 1-1, 1-4a, and 14-29a and b) and those without disabilities (objectives 13-6a, 19-2, 22-2, 27-1a) each had the best rate for the four of these objectives.

- Health disparities of $100 \%$ or more were observed for some objectives among racial and ethnic populations, as well as by sex, education level, income, and geographic location. Changes in disparities of 50 percentage points or more between the baseline and most recent data points also were observed. Many of these disparities are discussed in the Highlights, above.

\section{Transition to Healthy People 2020}

At the time of this publication, the Leading Health Indicators for Healthy People 2020 had not yet been finalized. Readers are encouraged to reference Appendix D "A Crosswalk Between Objectives From Healthy People 2010 to Healthy People 2020," which summarizes the changes between the two decades of objectives, as well as HealthyPeople.gov for a complete list of Healthy People 2020 topics and objectives. 


\section{Data Considerations}

Beginning in 2003, education data for the mortality objectives 15-15a (motor vehicle crash deaths), 15-32 (homicides), and 18-1 (suicide), and the natality objective 16-6a (prenatal care), from the National Vital Statistics System, were suppressed. The educational attainment item was changed in the new U.S. Standard Certificates of Birth and Death in 2003 to be consistent with the U.S. Census Bureau data and to improve the ability to identify specific types of educational degrees. Many states, however, are still using the 1989 version of the U.S. Standard Certificate of Death, which focuses on highest school grade completed. As a result, educational attainment data collected using the 2003 version are not comparable with data collected using the 1989 version [4].

Data for objective 16-6a (early prenatal care) were based upon the information recorded on birth certificates and also collected by states and local vital records offices. Due to the desire to produce more robust information, the 2003 revision of the standard birth certificate introduced improved standards which produce non-comparable rates [6,7]. For Healthy People 2010, data obtained from the 1997 version of the standard birth certificate was used from baseline through 2002 to track this objective.

The data label used for objective 19-3c "overweight or obesity" in children and adolescents was revised since the Healthy People 2010 Midcourse Review and progress reviews to "obesity" even though the definition (BMI at or above the sex- and age-specific $95^{\text {th }}$ percentile from the 2000 CDC Growth Charts) and interpretation are still the same. This change is consistent with revisions made by the American Academy of Pediatrics, the Institute of Medicine, and other organizations. Strictly speaking, overweight refers to weight in excess of a weight standard which could be due to a greater lean body mass, and obesity refers to excess body fatness. Because the indexes used are based on body mass rather than fatness, the original terminology of "overweight" for children at or above the 95th percentile was intended to clarify that this cut-off point should not be used as diagnostic criteria. Rather, these children may or may not have excess body fat and should, therefore, be screened for obesity. However, because body fat is difficult to measure and the majority of children with BMI at or above the 95th percentile have high adiposity, on a populationwide basis, high weight-for-height can be considered as an adequate indicator of obesity [5].

Education and income are the primary measures of socioeconomic status (SES) in Healthy People 2010. Most data systems used in Healthy People 2010 define income as a family's income before taxes. In order to facilitate comparisons among groups and over time, while adjusting for family size and for inflation, Healthy People 2010 categorizes income using the poverty thresholds developed by the U.S. Census Bureau. Thus, the three categories of family income that are primarily used are:

- Poor-below the Federal poverty level

- Near poor-100\% to $199 \%$ of the Federal poverty level

- Middle/high income-200\% or more of the Federal poverty level. 
These categories may be overridden by considerations specific to the data system, in which case they are modified as appropriate. See Healthy People 2010: General Data Issues, referenced below.

In general, data on educational attainment are presented for persons aged 25 years and over, consistent with guidance given by the U.S. Bureau of the Census. However, because of the requirements of the different data systems, the age groups used to calculate educational attainment for any specific objective may differ from the age groups used to report the data for other Healthy People 2010 objectives, as well as from select populations within the same objective. Therefore, the reader is urged to exercise caution in interpreting the data by educational attainment shown in the Health Disparities Table. See Healthy People 2010: General Data Issues, referenced below.

Additional information on data issues is available from the following sources:

- All Healthy People 2010 tracking data can be found in the Healthy People 2010 database, DATA2010, available from http://wonder.cdc.gov/data2010/.

- Detailed information about the data and data sources used to support these objectives can be found in the Operational Definitions on the DATA2010 website, available from http://wonder.cdc.gov/data2010/focusod.htm.

- More information on statistical issues related to Healthy People tracking and measurement can be found in the Technical Appendix and in Healthy People 2010: General Data Issues, which is available in the Data Issues section of the NCHS Healthy People website under Healthy People 2010. 


\section{References and Notes}

1. Displayed in the Progress Chart (Figure LHI-1), the percent of targeted change achieved expresses the difference between the baseline and the final value relative to the initial difference between the baseline and the Healthy People 2010 target. As such, it is a relative measure of progress toward attaining the Healthy People 2010 target. See the Reader's Guide for more information. When standard errors were available, the difference between the baseline and the final value was tested at the 0.05 level of significance. See the Figure LHI- 1 footnotes, as well as the Technical Appendix, for more detail.

2. Information about disparities among select populations is shown in the Health Disparities Table (Figure LHI-2). Disparity from the best group rate is defined as the percent difference between the best group rate and each of the other group rates for a characteristic. For example, racial and ethnic health disparities are measured as the percent difference between the best racial and ethnic group rate and each of the other racial and ethnic group rates. Similarly, disparities by sex are measured as the percent difference between the better group rate (e.g., female) and the rate for the other group (e.g., male). Some objectives are expressed in terms of favorable events or conditions that are to be increased, while others are expressed in terms of adverse events or conditions that are to be reduced. In order to facilitate comparison of health disparities across different objectives, disparity is measured only in terms of adverse events or conditions. For comparability across objectives, objectives that are expressed in terms of favorable events or conditions are re-expressed using the adverse event or condition for the purpose of computing disparity, but they are not otherwise restated or changed. For example, objective 1-1, to increase the proportion of persons with health insurance (e.g., $72 \%$ of the American Indian or Alaska Native population aged under 65 years had some form of health insurance in 2008), is expressed in terms of the percentage of persons without health insurance (e.g., $100 \%-72 \%=28 \%$ of the American Indian or Alaska Native population aged under 65 years did not have any form of health insurance in 2008) when the disparity from the best group rate is calculated. See the Reader's Guide for more information. When standard errors were available, the difference between the best group rate and each of the other group rates was tested at the 0.05 level of significance. See the Figure LHI-2 footnotes, as well as the Technical Appendix, for more detail.

3. The change in disparity is estimated by subtracting the disparity at baseline from the disparity at the most recent data point and, therefore, is expressed as a change in percentage points. See the Reader's Guide for more information. When standard errors were available, the change in disparity was tested at the 0.05 level of significance. See the Figure LHI-2 footnotes, as well as the Technical Appendix, for more detail.

4. Xu JQ, Kochanek KD, Murphy SL, Tejada-Vera B. Deaths: Final data for 2007. National vital statistics reports; vol 58 no 19. Hyattsville, MD: National Center for Health Statistics. 2010. Available from: http://www.cdc.gov/nchs/data/nvsr/nvsr58/nvsr58 19.pdf.

5. Ogden CL, Flegal KM. Changes in terminology for childhood overweight and obesity. National health statistics reports; no 25. Hyattsville, MD: National Center for Health Statistics. 2010. Available from: http://www.cdc.gov/nchs/data/nhsr/nhsr025.pdf.

6. National Center for Health Statistics. 2003 revision of the U.S. Standard Certificate of Live Birth. 2003. Available from: http://www.cdc.gov/nchs/nvss/vital certificate revisions.htm.

7. National Center for Health Statistics. Report of the panel to evaluate the U.S. Standard Certificates and Reports. National Center for Health Statistics. 2000. Available from: http://www.cdc.gov/nchs/data/dvs/panelreport acc.pdf. 


\section{Comprehensive Summary of Objectives: Leading Health Indicators}

\begin{tabular}{|c|c|c|}
\hline Objective & Description & Data Source \\
\hline $1-1$ & Persons with health insurance ( $<65$ years) & National Health Interview Survey (NHIS), CDC, NCHS. \\
\hline $1-4 a$ & Source of ongoing care & National Health Interview Survey (NHIS), CDC, NCHS. \\
\hline $1-9 \mathrm{a}$ & $\begin{array}{l}\text { Hospitalization for pediatric asthma (admissions per } 10,000 \\
\text { population, }<18 \text { years) }\end{array}$ & Healthcare Cost and Utilization Project (HCUP), AHRQ. \\
\hline $8-1 \mathrm{a}$ & Percent of persons exposed to harmful air pollutants-Ozone & Air Quality System (AQS), Environmental Protection Agency (EPA). \\
\hline $13-6 a$ & $\begin{array}{l}\text { Condom use among sexually active unmarried persons (18-44 years)- } \\
\text { Females }\end{array}$ & National Survey of Family Growth (NSFG), CDC, NCHS. \\
\hline $14-24 a$ & Fully immunized young children 19-35 months & National Immunization Survey (NIS), CDC, NCIRD; NCHS. \\
\hline $14-29 a$ & $\begin{array}{l}\text { Vaccination of noninstitutionalized high-risk older adults-Influenza } \\
\text { vaccine in past } 12 \text { months (age adjusted, } 65+\text { years) }\end{array}$ & National Health Interview Survey (NHIS), CDC, NCHS. \\
\hline $14-29 b$ & $\begin{array}{l}\text { Vaccination of noninstitutionalized high-risk older adults- } \\
\text { Pneumococcal vaccine ever received (age adjusted, } 65+\text { years) }\end{array}$ & National Health Interview Survey (NHIS), CDC, NCHS. \\
\hline $18-1$ & Suicide (age adjusted per 100,000 standard population) & National Vital Statistics System-Mortality (NVSS-M), CDC, NCHS. \\
\hline $18-9 b$ & Treatment for adults with depression (18+ years) & National Comorbidity Survey—Replication (NCS-R), NIH, NIMH. \\
\hline $19-2$ & Obesity in adults (age adjusted, $20+$ years) & $\begin{array}{l}\text { National Health and Nutrition Examination Survey (NHANES), CDC, } \\
\text { NCHS. }\end{array}$ \\
\hline $19-3 c$ & Obesity in children and adolescents 6-19 years & $\begin{array}{l}\text { National Health and Nutrition Examination Survey (NHANES), CDC, } \\
\text { NCHS. }\end{array}$ \\
\hline $22-2$ & $\begin{array}{l}\text { Regular physical activity-Moderate or vigorous (age adjusted, 18+ } \\
\text { years) }\end{array}$ & National Health Interview Survey (NHIS), CDC, NCHS. \\
\hline $22-7$ & Vigorous physical activity in students (grades 9-12) & Youth Risk Behavior Surveillance System (YRBSS), CDC, NCCDPHP. \\
\hline $25-11 \mathrm{a}$ & Students who never had sexual intercourse (grades 9-12) & Youth Risk Behavior Surveillance System (YRBSS), CDC, NCCDPHP. \\
\hline $25-11 b$ & Students who had sexual intercourse, but not in the past 3 months & Youth Risk Behavior Surveillance System (YRBSS), CDC, NCCDPHP. \\
\hline
\end{tabular}




\begin{tabular}{|c|c|c|}
\hline Objective & Description & Data Source \\
\hline & (grades 9-12) & \\
\hline $25-11 c$ & Students who used condoms at last intercourse (grades 9-12) & Youth Risk Behavior Surveillance System (YRBSS), CDC, NCCDPHP. \\
\hline $26-10 \mathrm{a}$ & $\begin{array}{l}\text { Adolescents not using alcohol or illicit drugs in past } 30 \text { days (12-17 } \\
\text { years) }\end{array}$ & National Survey on Drug Use and Health (NSDUH), SAMHSA. \\
\hline $26-10 c$ & Adults using illicit drugs in past 30 days $(18+$ years $)$ & National Survey on Drug Use and Health (NSDUH), SAMHSA. \\
\hline $26-11 c$ & Binge drinking in the past month-Adults $(18+$ years $)$ & National Survey on Drug Use and Health (NSDUH), SAMHSA. \\
\hline $27-1 \mathrm{a}$ & Cigarette use by adults (age adjusted, 18+ years) & National Health Interview Survey (NHIS), CDC, NCHS. \\
\hline $27-2 b$ & Cigarette use in past month by students (grades 9-12) & Youth Risk Behavior Surveillance System (YRBSS), CDC, NCCDPHP. \\
\hline $27-9$ & Exposure to tobacco smoke at home among children ( $\leq 6$ years) & National Health Interview Survey (NHIS), CDC, NCHS. \\
\hline $27-10$ & $\begin{array}{l}\text { Exposure to environmental tobacco smoke among nonsmokers (age } \\
\text { adjusted, } 4+\text { years) }\end{array}$ & $\begin{array}{l}\text { National Health and Nutrition Examination Survey (NHANES), CDC, } \\
\text { NCHS. }\end{array}$ \\
\hline
\end{tabular}


Figure LHI-1. Progress Toward Target Attainment for Leading Health Indicators

Moved away

from target ${ }^{1}$

Moved toward

target

Met or exceeded

target

\section{Physical Activity}

22-2. Regular physical activity-Moderate or vigorous (age adjusted, 18+ years)

22-7. Vigorous physical activity in students (grades 9-12)

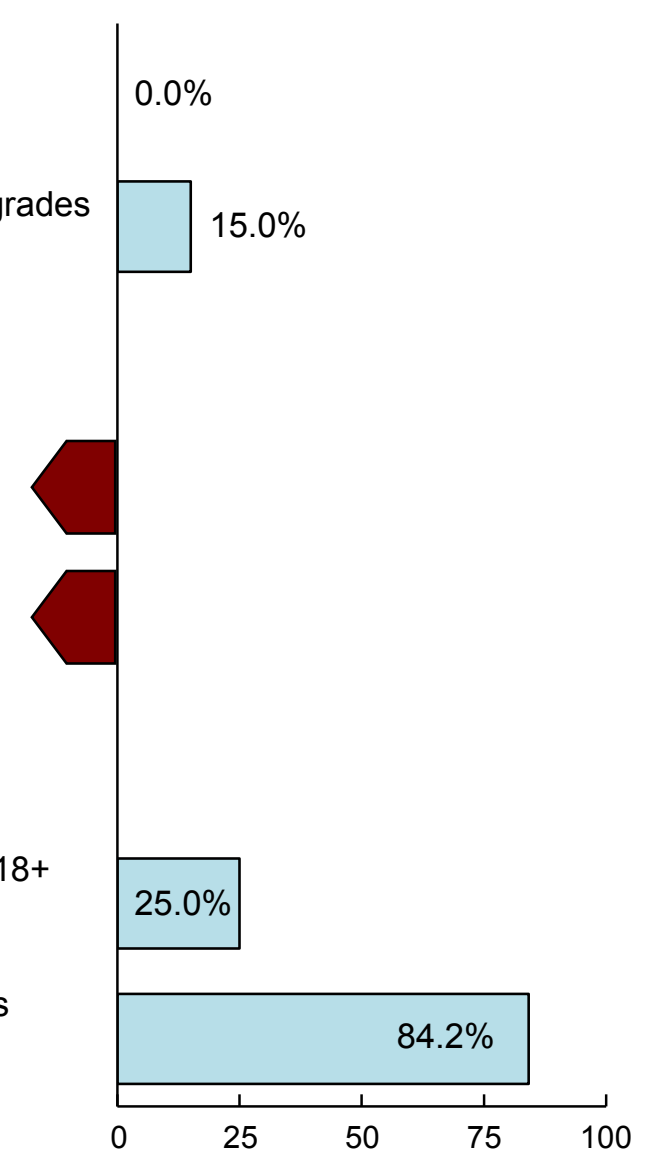

Percent of targeted change achieved ${ }^{5}$

\begin{tabular}{|c|c|c|c|c|c|}
\hline \multirow{2}{*}{$\begin{array}{c}2010 \\
\text { Target }\end{array}$} & \multirow{2}{*}{$\begin{array}{c}\text { Baseline } \\
(\text { Year })\end{array}$} & \multirow{2}{*}{$\begin{array}{c}\text { Final } \\
\text { (Year) }\end{array}$} & \multicolumn{3}{|c|}{ Baseline vs. Final } \\
\cline { 4 - 6 } & Difference $^{2}$ & $\begin{array}{c}\text { Statistically } \\
\text { Significant }^{3}\end{array}$ & $\begin{array}{c}\text { Percent } \\
\text { Change }^{4}\end{array}$ \\
\hline $50 \%$ & $\begin{array}{c}32 \% \\
(1997)\end{array}$ & $\begin{array}{c}32 \% \\
(2008)\end{array}$ & 0 & No & $0.0 \%$ \\
\hline $85 \%$ & $\begin{array}{c}65 \% \\
(1999)\end{array}$ & $\begin{array}{c}68 \% \\
(2009)\end{array}$ & 3 & No & $4.6 \%$ \\
\hline
\end{tabular}

\begin{tabular}{|c|c|c|c|c|c|}
\hline $15 \%$ & $\begin{array}{c}23 \% \\
(1988-94)\end{array}$ & $\begin{array}{c}34 \% \\
(2005-08)\end{array}$ & 11 & Yes & $47.8 \%$ \\
\hline $5 \%$ & $\begin{array}{c}11 \% \\
(1988-94)\end{array}$ & $\begin{array}{c}18 \% \\
(2005-08)\end{array}$ & 7 & Yes & $63.6 \%$ \\
\hline
\end{tabular}

\begin{tabular}{|c|c|c|c|c|c|}
\hline $12 \%$ & $\begin{array}{c}24 \% \\
(1998)\end{array}$ & $\begin{array}{c}21 \% \\
(2008)\end{array}$ & -3 & Yes & $-12.5 \%$ \\
\hline $16 \%$ & $\begin{array}{c}35 \% \\
(1999)\end{array}$ & $\begin{array}{c}19 \% \\
(2009)\end{array}$ & -16 & Yes & $-45.7 \%$ \\
\hline
\end{tabular}

(continued) 
Figure LHI-1. Progress Toward Target Attainment for Leading Health Indicators (continued)

Moved away

from target ${ }^{1}$

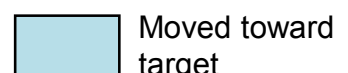

target

Met or exceeded

target

\section{Substance Abuse}

26-10a. Adolescents not using alcohol or illicit drugs in past 30 days (12-17 years)

\section{$30.8 \%$}

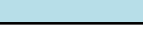

$0.0 \%$ years)

26-11c. Adults binge drinking in the past month $(18+$ years $)$

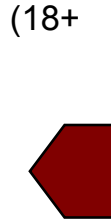

\section{Responsible Sexual Behavior}

13-6. Condom use among sexually active unmarried persons (18-44 years)

a. Females

$37.0 \%$

b. Males*

25-11a. Students who never had sexual intercourse (grades 9-12)

25-11b. Students who had sexual intercourse, but not in the past 3 months (grades 9-12)*

25-11c. Students who used condoms at last intercourse (grades 9-12)*

Percent of targeted change achieved ${ }^{5}$

\begin{tabular}{|c|c|c|c|c|c|}
\hline $50 \%$ & $\begin{array}{c}23 \% \\
(1995)\end{array}$ & $\begin{array}{c}33 \% \\
(2006-08)\end{array}$ & 10 & $\begin{array}{c}\text { Not } \\
\text { tested }\end{array}$ & $43.5 \%$ \\
\hline $54 \%$ & $\begin{array}{c}42 \% \\
(2002)\end{array}$ & $\begin{array}{c}44 \% \\
(2006-08)\end{array}$ & 2 & $\begin{array}{c}\text { Not } \\
\text { tested }\end{array}$ & $4.8 \%$ \\
\hline $56 \%$ & $\begin{array}{c}50 \% \\
(1999)\end{array}$ & $\begin{array}{c}54 \% \\
(2009)\end{array}$ & 4 & $\begin{array}{c}\text { Not } \\
\text { tested }\end{array}$ & $8.0 \%$ \\
\hline $30 \%$ & $\begin{array}{c}27 \% \\
(1999)\end{array}$ & $\begin{array}{c}26 \% \\
(2009)\end{array}$ & -1 & $\begin{array}{c}\text { Not } \\
\text { tested }\end{array}$ & $-3.7 \%$ \\
\hline $65 \%$ & $\begin{array}{c}58 \% \\
(1999)\end{array}$ & $\begin{array}{c}61 \% \\
(2009)\end{array}$ & 3 & $\begin{array}{c}\text { Not } \\
\text { tested }\end{array}$ & $5.2 \%$ \\
\hline
\end{tabular}

\begin{tabular}{|c|c|c|c|c|c|}
\hline \multirow{2}{*}{$\begin{array}{c}2010 \\
\text { Target }\end{array}$} & \multirow{2}{*}{$\begin{array}{c}\text { Baseline } \\
(\text { Year })\end{array}$} & \multirow{2}{*}{$\begin{array}{c}\text { Final } \\
\text { (Year) }\end{array}$} & \multicolumn{3}{|c|}{ Baseline vs. Final } \\
\cline { 4 - 6 } & Difference $^{2}$ & $\begin{array}{c}\text { Statistically } \\
\text { Significant }\end{array}$ & $\begin{array}{c}\text { Percent } \\
\text { Change }^{4}\end{array}$ \\
\hline $91 \%$ & $\begin{array}{c}78 \% \\
(2002)\end{array}$ & $\begin{array}{c}82 \% \\
(2008)\end{array}$ & 4 & Yes & $5.1 \%$ \\
\hline $3.2 \%$ & $\begin{array}{c}7.9 \% \\
(2002)\end{array}$ & $\begin{array}{c}7.9 \% \\
(2008)\end{array}$ & 0 & No & $0.0 \%$ \\
\hline $13.4 \%$ & $\begin{array}{c}24.3 \% \\
(2002)\end{array}$ & $\begin{array}{c}24.9 \% \\
(2008)\end{array}$ & 0.6 & No & $2.5 \%$ \\
\hline
\end{tabular}

(continued)

Page LHI-17
$66.7 \%$

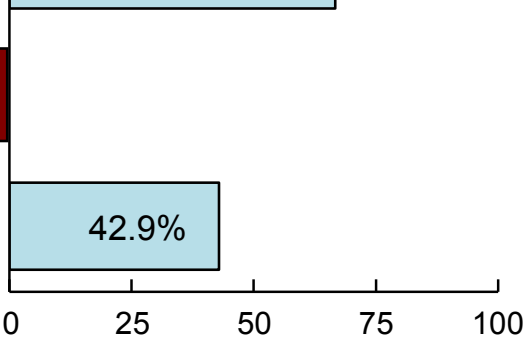

$16.7 \%$

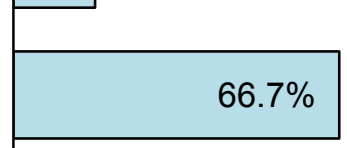


Figure LHI-1. Progress Toward Target Attainment for Leading Health Indicators (continued)

Moved away

from target ${ }^{1}$

Moved toward

target

Met or exceeded

target

\section{Mental Health}

18-1. Suicide (age adjusted, per 100,000 population $)^{\star}$

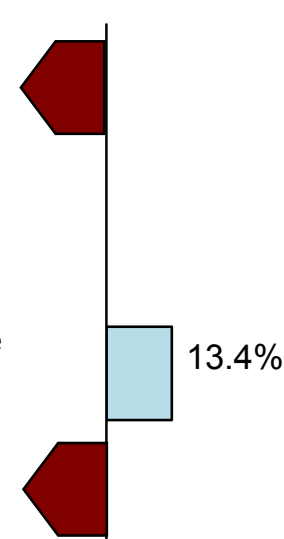

\section{Injury and Violence}

15-15a. Deaths from motor vehicle crashes (age adjusted, per 100,000 population)

15-32. Homicides (age adjusted, per 100,000 population)

\section{Environmental Quality}

8-1a. Percent of persons exposed to ozone

27-9. Exposure to tobacco smoke at home among children $\left(\leq 6\right.$ years) ${ }^{\star}$

27-10. Exposure to environmental tobacco smoke among nonsmokers (age adjusted, 4+ years)

\begin{tabular}{|c|c|c|c|c|c|}
\hline \multirow{2}{*}{$\begin{array}{c}2010 \\
\text { Target }\end{array}$} & \multirow{2}{*}{$\begin{array}{c}\text { Baseline } \\
(\text { Year })\end{array}$} & Final & \multicolumn{3}{|c|}{ Baseline vs. Final } \\
\cline { 4 - 6 } & Year) & Difference $^{2}$ & $\begin{array}{c}\text { Statistically } \\
\text { Significant }^{3}\end{array}$ & $\begin{array}{c}\text { Percent } \\
\text { Change }^{4}\end{array}$ \\
\hline 4.8 & $\begin{array}{c}10.5 \\
(1999)\end{array}$ & $\begin{array}{c}11.3 \\
(2007)\end{array}$ & 0.8 & Yes & $7.6 \%$ \\
\hline
\end{tabular}

\begin{tabular}{|c|c|c|c|c|c|}
\hline 8.0 & $\begin{array}{c}14.7 \\
(1999)\end{array}$ & $\begin{array}{c}13.8 \\
(2007)\end{array}$ & -0.9 & Yes & $-6.1 \%$ \\
\hline 2.8 & $\begin{array}{c}6.0 \\
(1999)\end{array}$ & $\begin{array}{c}6.1 \\
(2007)\end{array}$ & 0.1 & No & $1.7 \%$ \\
\hline
\end{tabular}

\begin{tabular}{|c|c|c|c|c|c|}
\hline $0 \%$ & $\begin{array}{c}43 \% \\
(1997)\end{array}$ & $\begin{array}{c}36 \% \\
(2010)\end{array}$ & -7 & $\begin{array}{c}\text { Not } \\
\text { tested }\end{array}$ & $-16.3 \%$ \\
\hline $10 \%$ & $\begin{array}{c}27 \% \\
(1994)\end{array}$ & $\begin{array}{c}8 \% \\
(2005)\end{array}$ & -19 & Yes & $-70.4 \%$ \\
\hline $56 \%$ & $\begin{array}{c}84 \% \\
(1988-94)\end{array}$ & $\begin{array}{c}41 \% \\
(2005-08)\end{array}$ & -43 & Yes & $-51.2 \%$ \\
\hline
\end{tabular}

(continued)

Percent of targeted change achieved ${ }^{5}$ 
Figure LHI-1. Progress Toward Target Attainment for Leading Health Indicators (continued)

Moved away

from target ${ }^{1}$

Moved toward

target

Met or exceeded

target

\section{Immunization}

14-24a. Fully immunized young children 19-35 months

14-29. Vaccination of noninstitutionalized high-risk older adults (age adjusted, $65+$ years)

a. Influenza vaccine in past 12 months

b. Pneumococcal vaccine ever received

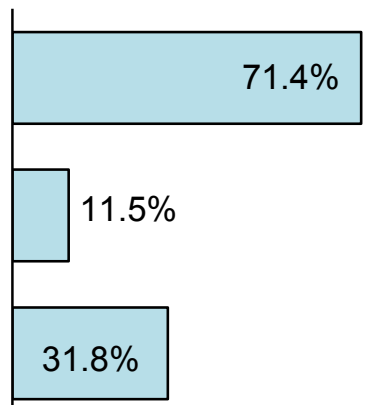

\begin{tabular}{|c|c|c|c|c|c|}
\hline \multirow{2}{*}{$\begin{array}{l}2010 \\
\text { Target }\end{array}$} & \multirow{2}{*}{$\begin{array}{c}\text { Baseline } \\
\text { (Year) }\end{array}$} & \multirow{2}{*}{$\begin{array}{l}\text { Final } \\
\text { (Year) }\end{array}$} & \multicolumn{3}{|c|}{ Baseline vs. Final } \\
\hline & & & Difference $^{2}$ & $\begin{array}{l}\text { Statistically } \\
\text { Significant }^{3}\end{array}$ & $\begin{array}{l}\text { Percent } \\
\text { Change }^{4}\end{array}$ \\
\hline $80 \%$ & $\begin{array}{c}73 \% \\
(1998)\end{array}$ & $\begin{array}{c}78 \% \\
(2008)\end{array}$ & 5 & Yes & $6.8 \%$ \\
\hline $90 \%$ & $\begin{array}{c}64 \% \\
(1998)\end{array}$ & $\begin{array}{c}67 \% \\
(2008)\end{array}$ & 3 & Yes & $4.7 \%$ \\
\hline $90 \%$ & $\begin{array}{c}46 \% \\
(1998)\end{array}$ & $\begin{array}{c}60 \% \\
(2008)\end{array}$ & 14 & Yes & $30.4 \%$ \\
\hline
\end{tabular}

\section{Access to Health Care}

1-1. Persons with health insurance ( $<65$ years $)$

1-4a. Source of ongoing care

1-9a. Hospitalization for pediatric asthma (admissions per 10,000 population, $<18$ years $)^{*}$

16-6a. Prenatal care beginning in first trimester

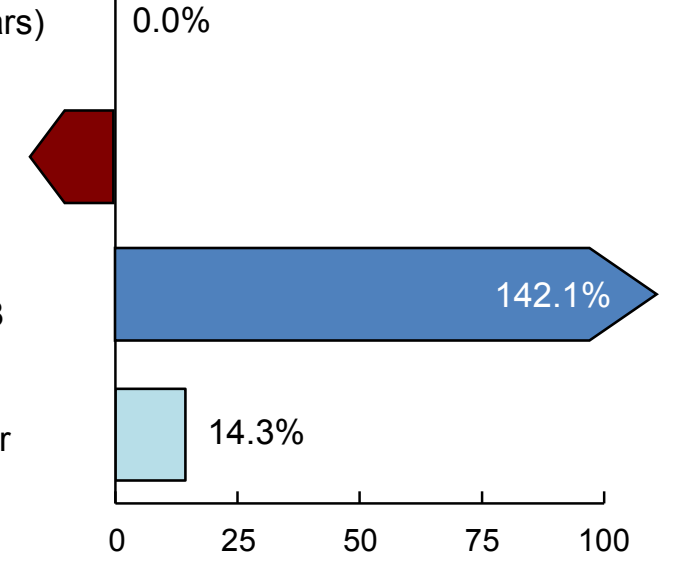

\begin{tabular}{|c|c|c|c|c|c|}
\hline $100 \%$ & $\begin{array}{c}83 \% \\
(1997)\end{array}$ & $\begin{array}{c}83 \% \\
(2008)\end{array}$ & 0 & No & $0.0 \%$ \\
\hline $96 \%$ & $\begin{array}{c}87 \% \\
(1998)\end{array}$ & $\begin{array}{c}86 \% \\
(2008)\end{array}$ & -1 & Yes & $-1.1 \%$ \\
\hline 17.3 & $\begin{array}{c}23.0 \\
(1996)\end{array}$ & $\begin{array}{c}14.9 \\
(2008)\end{array}$ & -8.1 & Yes & $-35.2 \%$ \\
\hline $90 \%$ & $\begin{array}{c}83 \% \\
(1998)\end{array}$ & $\begin{array}{c}84 \% \\
(2002)\end{array}$ & 1 & Yes & $1.2 \%$ \\
\hline
\end{tabular}

Percent of targeted change achieved ${ }^{5}$

(continued) 


\section{Figure LHI-1. Progress Toward Target Attainment for Leading Health Indicators (continued) NOTES}

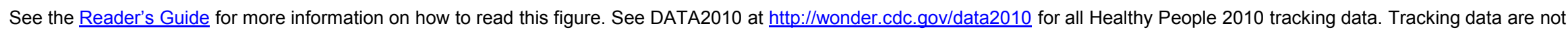
available for objective 18-9b.

\section{FOOTNOTES}

${ }^{1}$ Movement away from target is not quantified using the percent of targeted change achieved. See Technical Appendix for more information.

${ }^{2}$ Difference $=$ Final value - Baseline value. Differences between percents $(\%)$ are measured in percentage points

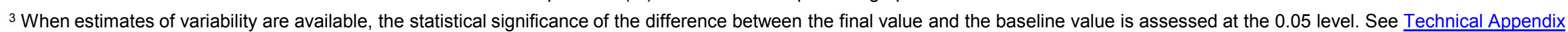
for more information.

${ }^{4}$ Percent change $=\frac{\text { Final value }- \text { Baseline value }}{\text { Baseline value }} \times 100$

${ }^{5}$ Percent of targeted change achieved $=\frac{\text { Final value }- \text { Baseline value }}{\text { Healthy People } 2010 \text { target }- \text { Baseline value }} \times 100$.

* Supplemental measure. See LHI chapter text for more information.

\section{DATA SOURCES}

1-1. National Health Interview Survey (NHIS), CDC, NCHS.

1-4a. National Health Interview Survey (NHIS), CDC, NCHS.

1-9a. Healthcare Cost and Utilization Project (HCUP), AHRQ.

8-1a. Air Quality System (AQS), Environmental Protection Agency (EPA).

13-6a-b. National Survey of Family Growth (NSFG), CDC, NCHS.

14-24a. National Immunization Survey (NIS), CDC, NCIRD; NCHS.

14-29a-b. National Health Interview Survey (NHIS), CDC, NCHS.

15-15a. National Vital Statistics System-Mortality (NVSS-M), CDC, NCHS.

15-32. National Vital Statistics System-Mortality (NVSS-M), CDC, NCHS.

16-6a. National Vital Statistics System-Natality (NVSS-N), CDC, NCHS.

18-1. National Vital Statistics System-Mortality (NVSS-M), CDC, NCHS.

19-2. National Health and Nutrition Examination Survey (NHANES), CDC, NCHS.

19-3c. National Health and Nutrition Examination Survey (NHANES), CDC, NCHS.

22-2. National Health Interview Survey (NHIS), CDC, NCHS.

22-7. Youth Risk Behavior Surveillance System (YRBSS), CDC, NCCDPHP.

25-11a-c. Youth Risk Behavior Surveillance System (YRBSS), CDC, NCCDPHP.

26-10a. National Survey on Drug Use and Health (NSDUH), SAMHSA.

26-10c. National Survey on Drug Use and Health (NSDUH), SAMHSA.

26-11c. National Survey on Drug Use and Health (NSDUH), SAMHSA.

27-1a. National Health Interview Survey (NHIS), CDC, NCHS.

27-2b. Youth Risk Behavior Surveillance System (YRBSS), CDC, NCCDPHP.

27-9. National Health Interview Survey (NHIS), CDC, NCHS.

27-10. National Health and Nutrition Examination Survey (NHANES), CDC, NCHS. 
Figure LHI-2. Health Disparities Table for Leading Health Indicators

Disparities from the best group rate for each characteristic at the most recent data point and changes in disparity from the baseline to the most recent data point.

Population-based objectives
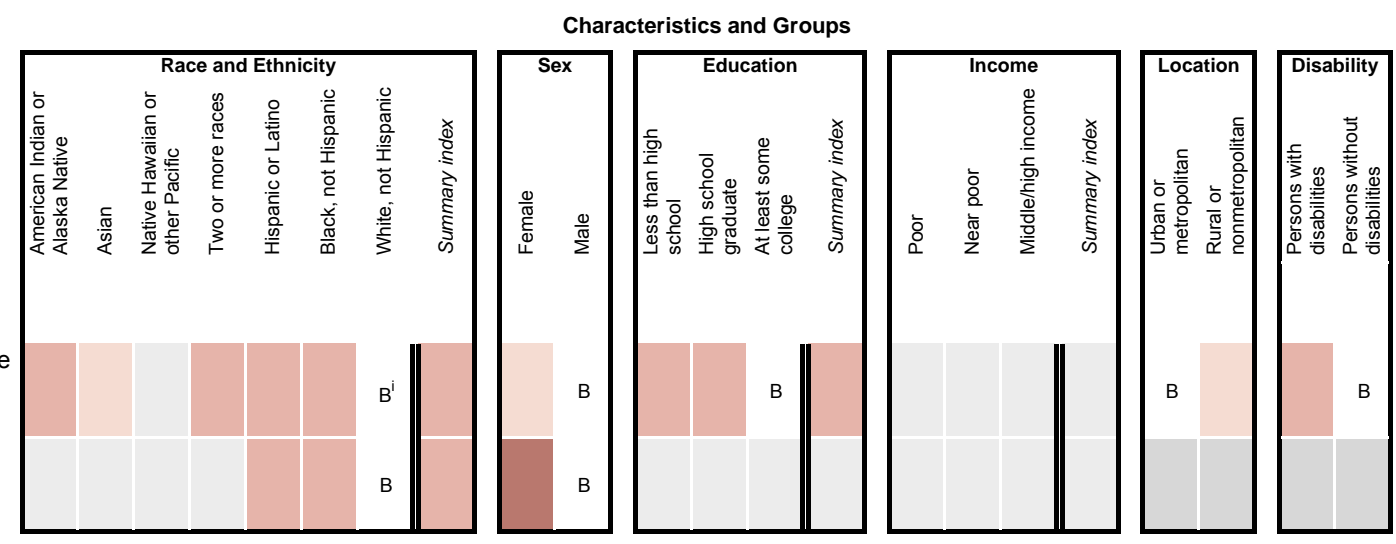

\section{Overweight and Obesity}

19-2. Obesity in adults (age adjusted, 20+ years) $(1988-94,2005-08)^{2,3 *}$

19-3c.

Obesity in children and adolescents 6-19 years $(1988-94,2005-08)^{3 *}$
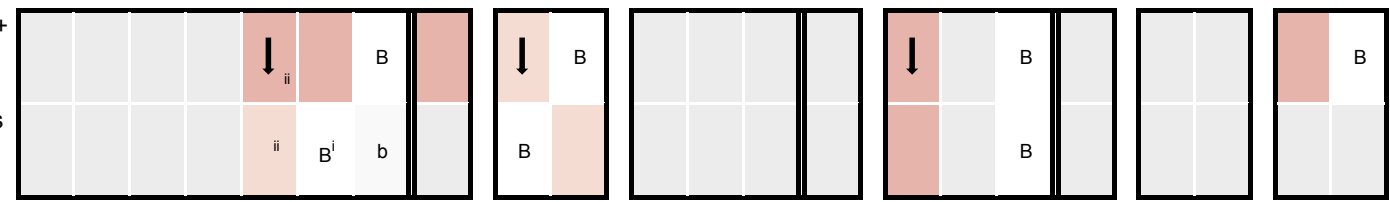

Tobacco Use

27-1a. Cigarette use by adults (age adjusted, 18+ years) $(1998,2008)^{1,}$

27-2b. Cigarette use in past month by students (grades 9-12) (1999, 2009)*
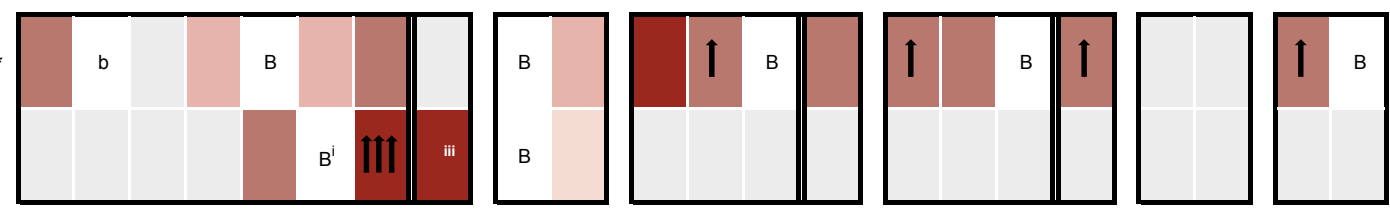

\section{Substance Abuse}

26-10a. Adolescents not using alcohol or illicit drugs in past 30 days (12-17 years) $(2002,2008)^{4 *}$

26-10c. Adults using illicit drugs in past 30 days $(18+$ years $)(2002,2008)^{*}$

26-11c. Adults binge drinking in the past month $(18+$ years $)(2002,2008)^{4 \star}$
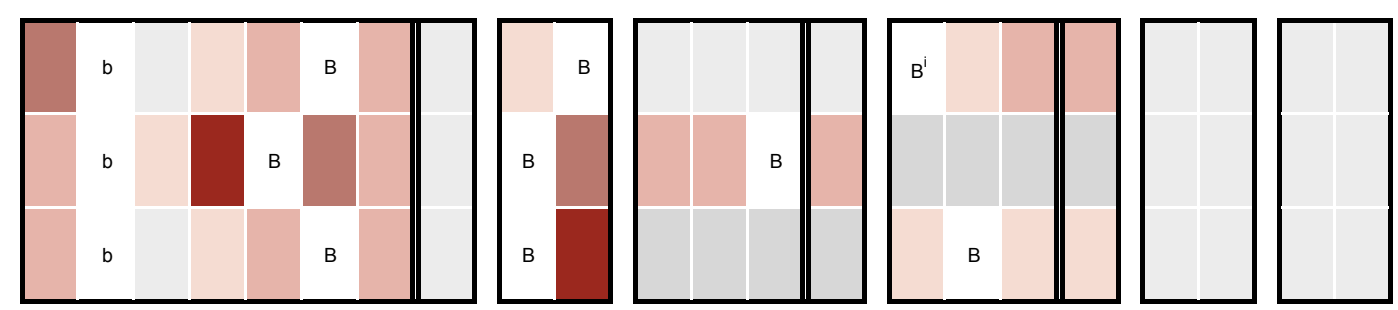

Responsible Sexual Behavior

13-6a. Condom use among sexually active unmarried persons-females (18-44 years) $(1995,2006-08)^{5} \ddagger$

b. Condom use among sexually active unmarried persons-males (18-44 years) $(2002,2006-08)^{5} \ddagger \S$

25-11a. Students who never had sexual intercourse (grades 9-12) (1999, 2009)†

25-11b. Students who had sexual intercourse, but not in the past 3 months (grades 9-12) $(1999,2009) \ddagger \S$

25-11c. Students who used condoms at last intercourse (grades 9-12) (1999, 2009) $\ddagger \S$
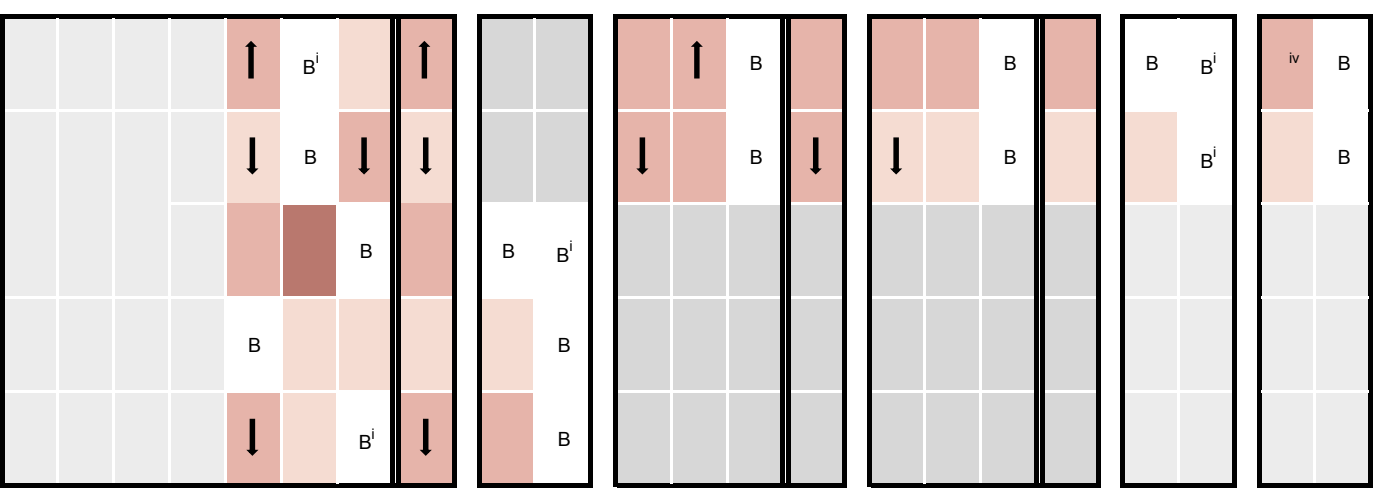

Mental Health

18-1. Suicide (age adjusted, per 100,000 population) $(1999,2007)^{6 *} \S$

18-9b. Treatment for adults with depression $(18+$ years $)(2002)^{*}$
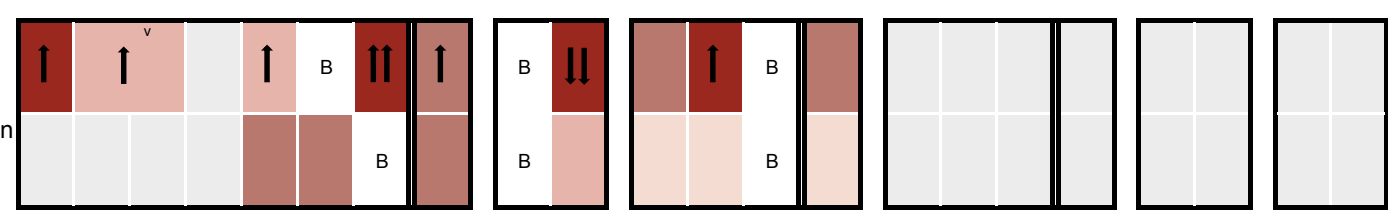

(continued) 
Population-based objectives

\section{Injury and Violence}

15-15a. Deaths from motor vehicle crashes (age adjusted, per 100,000 population) $(1999,2007)^{6 *}$

15-32. Homicides (age adjusted, per 100,000 population) $(1999,2007)^{6 *}$
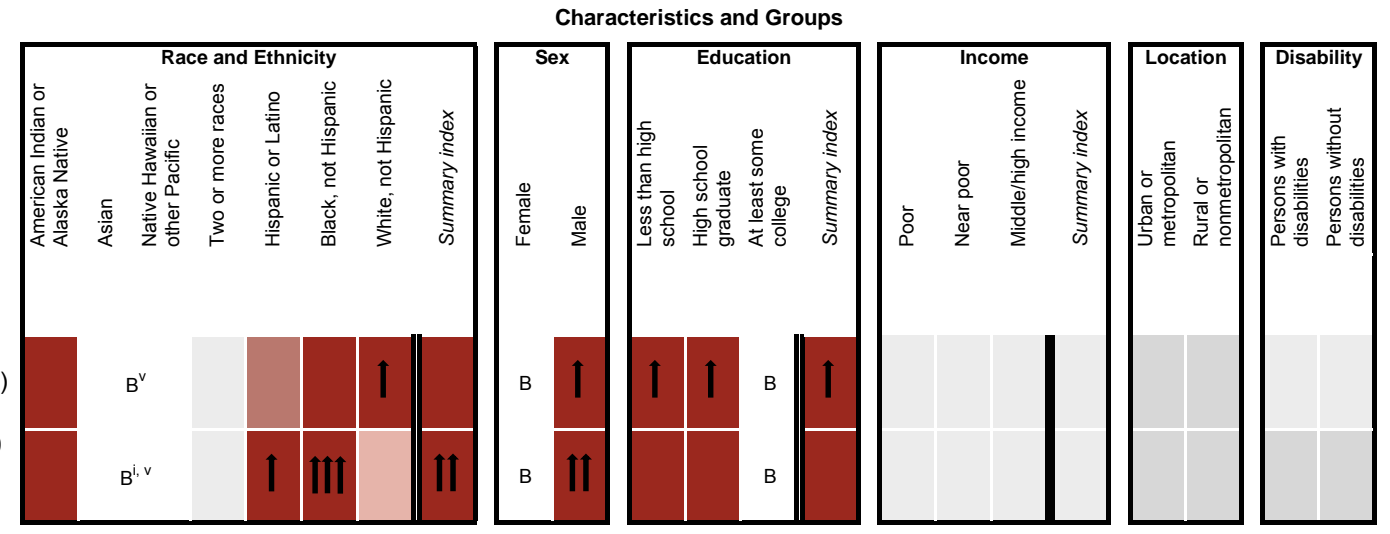

\section{Environmental Quality}

8-1a. Percent of persons exposed to ozone $(1997,2010)^{7} \dagger$

27-9. Exposure to tobacco smoke at home among children [ $\leq 6$ years (yrs)] (1994, $2005)^{8 *} \S$

27-10. Exposure to environmental tobacco smoke among nonsmokers (age adjusted, 4+ yrs) (1988-94, 2005-08)*
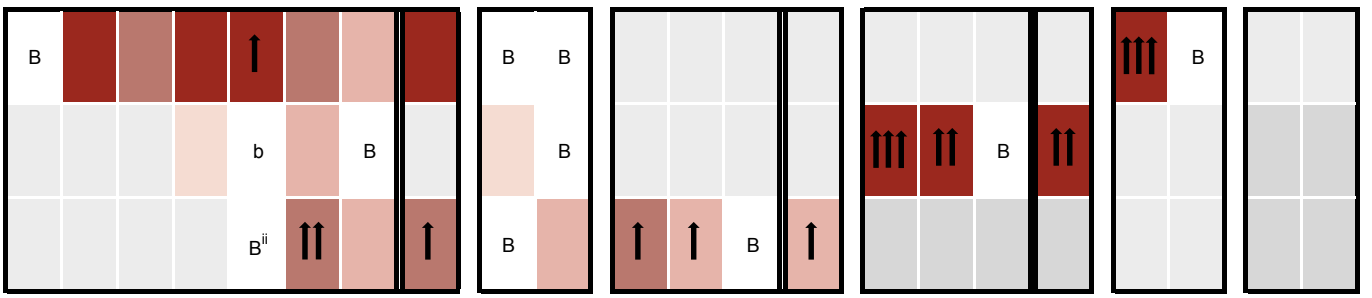

Immunization

14-24a. Fully immunized young children 19-35 months $(1998,2008)^{9,10 *}$

14-29a. Vaccination of noninstitutionalized high-risk older adults-Influenza vaccine in past 12 months (age adjusted, 65+ yrs) $(1998,2008)^{1 *}$

b. Vaccination of noninstitutionalized high-risk older adults-Pneumococcal vaccine ever received (age adjusted, $65+$ yrs) $(1998,2008)^{1 /}$
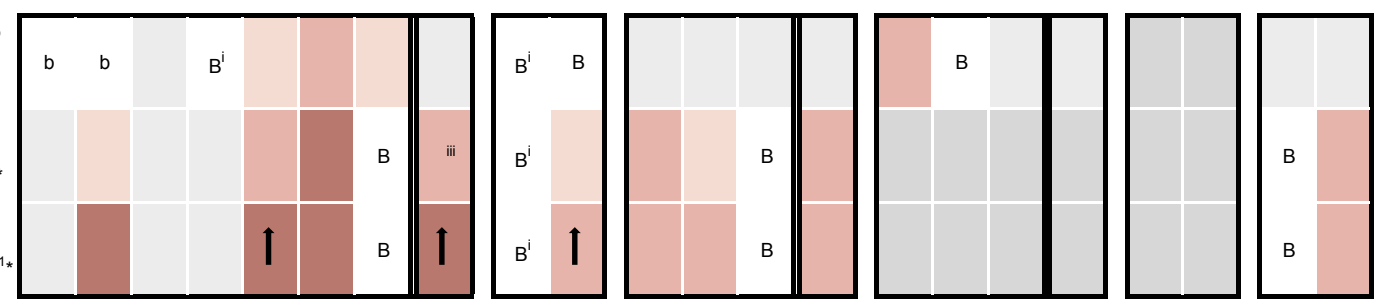

\section{Access to Health Care}

1-1. Persons with health insurance $(<65$ years) $(1997,2008)^{1 *}$

1-4a. Source of ongoing care $(1998,2008)^{1 *}$

1-9a. Hospitalization for pediatric asthma (admissions per 10,000 population, $<18$ years) $(1996,2008)^{\star} \S$

16-6a. Prenatal care beginning in first trimester $(1998,2002)^{*}$
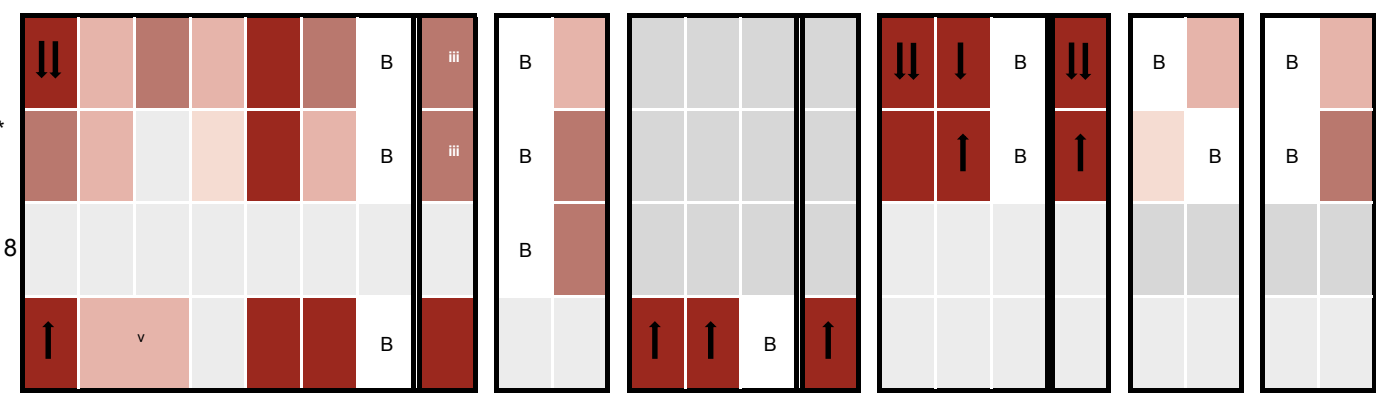

(continued) 


\section{Figure LHI-2. Health Disparities Table for Leading Health Indicators (continued)}

NOTES

See DATA2010 at http://wonder.cdc.gov/data2010 for all Healthy People 2010 tracking data.

Years in parentheses represent the baseline and most recent data years (if available).

Disparity from the best group rate is defined as the percent difference between the best group rate and each of the other group rates for a characteristic (e.g., race and ethnicity). The summary index is the average of these percent differences for a characteristic. Change in disparity is estimated by subtracting the disparity at baseline from the disparity at the most recent data point. Change in the summary index is estimated by subtracting the summary index at baseline from the summary index at the most recent data point. See Technical Appendix for more information.

LEGEND

$\begin{aligned} & \text { The "best" group rate at the most recent } \\ & \text { data point. }\end{aligned}$ B $\begin{aligned} & \text { The group with the best rate for } \\ & \text { specified characteristic. }\end{aligned} \quad$ b $\begin{aligned} & \text { Most favorable group rate for specified } \\ & \text { characteristic, but reliability criterion not met. }\end{aligned}$
$\begin{aligned} & \text { cheliability criterion for best group } \\ & \text { rate not met, or data available for } \\ & \text { only one group. }\end{aligned}$

\begin{tabular}{|c|c|c|c|c|}
\hline \multirow[b]{2}{*}{$\begin{array}{l}\text { Disparity from the best group rate at the } \\
\text { most recent data point. }\end{array}$} & \multicolumn{4}{|c|}{ Percent difference from the best group rate } \\
\hline & $\begin{array}{l}\text { Less than } 10 \% \text {, or difference not } \\
\text { statistically significant (when } \\
\text { estimates of variability are available). }\end{array}$ & $10 \%-49 \%$ & $50 \%-99 \%$ & $100 \%$ or more \\
\hline
\end{tabular}

Increase in disparity (percentage points)

Changes in disparity over time are shown when:

(a) disparities data are available at both baseline and most recent time points;

(b) data are not for the group(s) indicated by "B" or "b" at either time point; and

(c) the change is greater than or equal to 10 percentage points and statistically

significant, or when the change is greater than or equal to 10 percentage

points and estimates of variability were not available. See Technical Appendix.

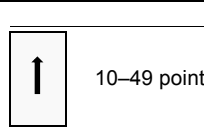

Increase in disparity (percentage points)

L

Availability of Data $\quad$ Data not available.

\begin{tabular}{|l|l|l|l|l} 
Decrease in disparity (percentage points) \\
\hline \hline 10-49 points & II $50-99$ points & III points or more
\end{tabular}

IIT 100 points or more

Characteristic not selected for this objective.

\section{FOOTNOTES}

* Measures of variability were available. Thus, the variability of best group rates was assessed, and statistical significance was tested. Disparities of $10 \%$ or more are displayed when the differences from the best group rate are statistically significant at the 0.05 level. Changes in disparities over time are indicated by arrows when the changes are greater than or equal to 10 percentage points and are statistically significant at the 0.05 level. See Technical Appendix.

$\dagger$ Measures of variability were not available. Thus, the variability of best group rates was not assessed, and statistical significance could not be tested. Nonetheless, disparities and changes in disparities over time are displayed according to their magnitude. See Technical Appendix.

$\ddagger$ Measures of variability were available only for the most recent data. Thus, the variability of best group rates was assessed only for the most recent data, and statistical significance was tested only for the most recent data. Disparities of $10 \%$ or more are displayed when the differences from the best group rate are statistically significant at the 0.05 level. Changes in disparities over time are displayed according to their magnitude, since measures of variability were not available at baseline and therefore statistical significance of changes in disparity could not be tested. See Technical Appendix.

$\S$ Supplemental measure. See LHI chapter text for more information.

1 Baseline data by race and ethnicity are for 1999 .

2 Baseline data by disability status are for 1991-94.

3 Data by income are categorized using only two groups: lower income ( $\leq 130 \%$ of Federal poverty level, displayed under "poor") and higher income (>130\% of Federal poverty level, displayed under "middle/high income").

4 Baseline data by income are for 2005.

5 Data by education level are for persons aged 25-44 years.

6 Most recent data by education level are for 2002 .

7 Most recent data by race and ethnicity, by sex, and by location, are for 2004 .

8 Baseline data by race and ethnicity are for 2005 .

9 Baseline data by race and ethnicity are for 2000

10 Baseline data by income exclude "middle/high income" for comparability with most recent data year.

i The group with the best rate at the most recent data point is different from the group with the best rate at baseline. Both rates met the reliability criterion. See Technical Appendix.

ii Data are for Mexican American.

iii Change in the summary index cannot be assessed. See Technical Appendix.

iv Reliability criterion for best group rate not met, or data available for only one group, at baseline. Change in disparity cannot be assessed. See Technical Appendix.

$\checkmark$ Data are for Asian or Pacific Islander.

\section{DATA SOURCES}

1-1. National Health Interview Survey (NHIS), CDC, NCHS.

1-4a. National Health Interview Survey (NHIS), CDC, NCHS.

1-9a. Healthcare Cost and Utilization Project (HCUP), AHRQ.

8-1a. Air Quality System (AQS), Environmental Protection Agency (EPA).

13-6a-b. National Survey of Family Growth (NSFG), CDC, NCHS.

14-24a. National Immunization Survey (NIS), CDC, NCIRD and NCHS.

14-29a-b. National Health Interview Survey (NHIS), CDC, NCHS.

15-15a. National Vital Statistics System-Mortality (NVSS-M), CDC, NCHS.

15-32. National Vital Statistics System-Mortality (NVSS-M), CDC, NCHS.

16-6a. National Vital Statistics System-Natality (NVSS-N), CDC, NCHS.

18-1. National Vital Statistics System-Mortality (NVSS-M), CDC, NCHS

18-9b. National Comorbidity Survey-Replication (NCS-R), NIH, NIMH.
$19-2$.

$19-3 c$.

$22-2$.

22-7.

25-11a-c.

26-10a.

26-10c.

26-11c.

$27-1 a$.

$27-2 b$.

$27-9$.

27-10.
National Health and Nutrition Examination Survey (NHANES), CDC, NCHS. National Health and Nutrition Examination Survey (NHANES), CDC, NCHS. National Health Interview Survey (NHIS), CDC, NCHS.

Youth Risk Behavior Surveillance System (YRBSS), CDC, NCCDPHP.

Youth Risk Behavior Surveillance System (YRBSS), CDC, NCCDPHP.

National Survey on Drug Use and Health (NSDUH), SAMHSA.

National Survey on Drug Use and Health (NSDUH), SAMHSA.

National Survey on Drug Use and Health (NSDUH), SAMHSA.

National Health Interview Survey (NHIS), CDC, NCHS.

Youth Risk Behavior Surveillance System (YRBSS), CDC, NCCDPHP.

National Health Interview Survey (NHIS), CDC, NCHS.

National Health and Nutrition Examination Survey (NHANES), CDC, NCHS. 


\section{Final Review}

\section{Reader's Guide}

HEALTHY PEOPLE
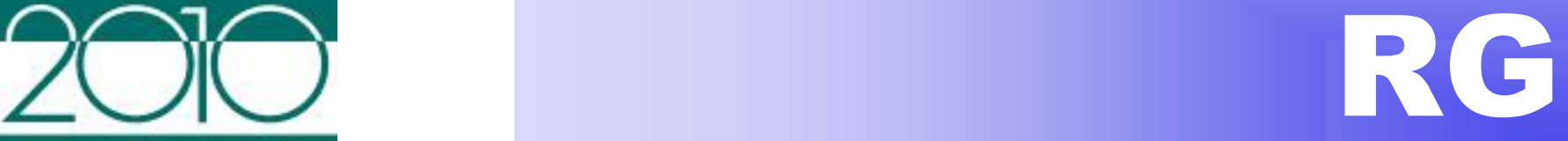

\section{Contents}

Focus Area Chapters.................................................................................................................................... RG-2

Measuring Progress toward the Healthy People 2010 Targets................................................................ RG-3

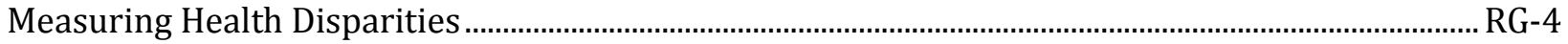

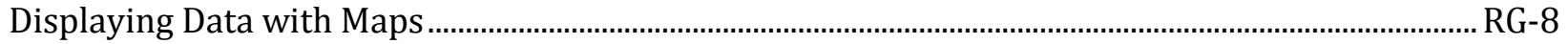

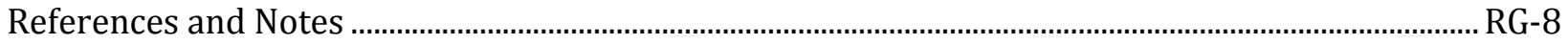




\section{Reader's Guide}

\section{Focus Area Chapters}

Each of the 28 Healthy People 2010 Focus Areas is reviewed in a standalone chapter in the Healthy People 2010 Final Review.

The Focus Area chapter begins with a brief introduction to the Focus Area.

The Highlights section describes the salient findings in relation to progress toward target attainment and to health disparities for selected objectives.

The Summary of Progress section of each chapter provides a more in-depth assessment of progress toward target attainment, and provides the reader with an inventory of objectives that have achieved their Healthy People 2010 targets, moved toward their targets, demonstrated no change, moved away from their targets, or lacked data to assess progress. The Progress Chart, presented as Figure 1 in each Focus Area chapter, displays further quantitative information regarding progress toward target attainment for each objective for which data were available, including the percent of targeted change achieved. See Measuring Progress toward the Healthy People 2010 Targets below.

The Summary of Progress section also discusses progress toward the elimination of health disparities. The Health Disparities Table, presented as Figure 2 in each Focus Area chapter, displays detailed findings in relation to health disparities among select populations for the objectives for which data were available. Objectives based on schools, worksites, states, or those that were measured using the numbers of events are not included in the discussion of health disparities. See Measuring Health Disparities below.

When data are available at the sub-national level, selected objectives are mapped to display spatial variation in percents, rates, or counts. Sub-national data are presented either at the state or Health Service Area (HSA) level. When maps are included they are presented as Figure 3 or higher in each Focus Area chapter. See Displaying Data with Maps below.

Previous Healthy People 2010 publications stated that there were 467 objectives to track progress over the decade. However, many of these objectives consisted of multiple "sub-objectives," each with its own baseline data, data source, and target requiring separate analysis. The total number of objectives and subobjectives upon which the analyses in this report are based is 969 . For the purposes of discussion, both objectives and subobjectives are referred to in this report as objectives given that each receives equal analysis and treatment.

The Transition to Healthy People 2020 section of each chapter describes the framework of the Healthy People 2020 Topic Area(s), and changes and modifications made to the corresponding Healthy People 2010 Focus Area(s) and objectives. Some Healthy People 2010 Focus Areas were split and new Healthy People 2020 Topic Areas were added. As a result, Healthy People 2020 has 42 Topic Areas. Appendix D, "A Crosswalk Between Objectives From Healthy People 2010 to Healthy People 2020," summarizes the changes between the two decades of objectives. 
Each Focus Area chapter in the Healthy People 2010 Final Review concludes with a Data Considerations section and a Comprehensive Summary of Objectives section that lists all objectives in that Focus Area with the corresponding data sources or objective status in those cases where an objective was not retained.

A description of the Progress Chart and a guide to the Health Disparities Table are presented below. The techniques used to develop the charts and tables are discussed in greater detail in the Technical Appendix. Further discussion of the methodological issues involved in the measurement of progress and of health disparities in Healthy People 2010 has also been published [1].

All Healthy People 2010 tracking data are available from http://wonder.cdc.gov/data2010 and are, therefore, not included in this publication.

\section{Measuring Progress toward the Healthy People 2010 Targets}

Progress toward the Healthy People 2010 targets at Final Review is shown in a Progress Chart for each Focus Area (Figure 1). This chart displays the percent of targeted change that was achieved for each objective. Targeted change is the difference between the baseline and the Healthy People 2010 target. The formula for the percent of targeted change achieved is as follows:

$$
\text { Percent of targeted change achieved }=\frac{\text { Final value }- \text { Baseline value }}{\text { Healthy People } 2010 \text { target }- \text { Baseline value }} \times 100 \text {. }
$$

The percent of targeted change achieved expresses the difference between the baseline and the final value relative to the initial difference between the baseline and the Healthy People 2010 target. As such, it is a relative measure of progress toward attaining the Healthy People 2010 target, and it can be used to compare how much of the targeted change has been achieved for an objective relative to other objectives, though care must be exercised in its interpretation. In particular, movement away from the Healthy People 2010 target is not quantified using the percent of targeted change achieved, as it is more meaningful to examine the difference between the final and the baseline values in such cases. See Technical Appendix for more information.

Although the Progress Chart was displayed in previous Healthy People publications, in this report several new columns have been added to provide more in-depth information on the movement that occurred for each objective for which there were at least two data points.

The Progress Chart is divided into three panels. Objective numbers and short descriptions are listed in the left-most panel. The description of an objective includes in parentheses any applicable information regarding the age of the targeted population. Most Healthy People 2010 objectives are measured using proportions, expressed in percents. If the unit of measure for an objective is anything other than a percent (e.g., rate per 100,000 population), then this is also indicated in parentheses as part of the objective description. The percent of targeted change achieved for each objective is displayed in a bar chart in the central panel of the Progress Chart. In the right-most panel of the Progress Chart, a table displays the Healthy People 2010 target, the baseline value and year, the final value and year, the difference between final and baseline values, its statistical significance at the 0.05 level, and the percent change between the final and baseline values. 
The formula for the percent change is as follows:

$$
\text { Percent change between final and baseline values }=\frac{\text { Final value }- \text { Baseline value }}{\text { Baseline value }} \times 100 \text {. }
$$

The percent of targeted change achieved is shown for each objective with data more recent than the baseline. The percent of targeted change achieved is color-coded. Objectives that moved away from the target are in red, moved toward the target are in light blue, and met or exceeded the target are in dark blue. As mentioned earlier, movement away from the Healthy People 2010 target is not quantified using the percent of targeted change achieved in the Progress Chart. Instead, for such objectives, the reader should examine the difference between the final value and the baseline value in order to assess progress. See Technical Appendix.

Objectives for which progress could not be assessed are identified in the notes at the end of the Progress Chart. These notations occur in two general types of situations: (a) the objective was deleted at Midcourse Review, or (b) the objective did not have a baseline, or had a baseline value but no follow up data.

The following observations may be helpful to the interpretation of the percent of targeted change achieved by a specific objective and/or comparisons of progress among multiple objectives:

- The percent of targeted change achieved measures the percent of the difference between the baseline year and the Healthy People 2010 target that was eliminated. For example a value of 25 percent indicates that a quarter of the difference between the baseline and the Healthy People 2010 target was achieved.

- The use and interpretation of the percent of targeted change achieved has limits. It is calculated using only the Healthy People 2010 target, the baseline data point, and the final data point. Furthermore, it does not take into account the number of years that are included nor any fluctuations that may occur during the intervening years. The number of years included, which varies by objective, may also vary within an objective based on population data. See Technical Appendix.

- There are situations in which the percent of targeted change achieved cannot be calculated or does not accurately reflect change in an objective. These situations include instances when the target was met at the baseline, when the amount of targeted change was small relative to the amount of actual change, or when the target was exceeded at baseline. Such situations are footnoted on the applicable charts, and illustrated in the Technical Appendix.

\section{Measuring Health Disparities}

Information about health disparities among select populations is shown in the Health Disparities Table (Figure 2 in each Focus Area chapter). Short descriptions of the population-based objectives are listed along the left side of the chart. The baseline data year(s) are shown in parentheses and, when more recent data were available, the most recent data year(s) are also shown. The description of an objective generally also includes in parentheses any applicable information regarding the underlying measure (e.g., measurement unit) and/or the age of the targeted population. 
Characteristics of the population (race and ethnicity, sex, education, income, geographic location, and disability status) are depicted across the top of the Health Disparities Table. In general, characteristics applicable to each objective were designated in the original Healthy People 2010 document [2].

Characteristics that were not designated for a particular objective are shaded in dark gray. When a characteristic is not applicable for any of the objectives in a Focus Area, it is omitted from the Health Disparities Table for that Focus Area. When data are not available for a designated population or for a particular characteristic, the corresponding boxes are shaded in light gray (see the fourth section of the legend reproduced below). If there are no characteristic-specific data available for an objective, the objective is excluded from the table and referenced in the notes.

Definition. Disparity from the best group rate is defined as the percent difference between the best group rate and each of the other group rates for a characteristic at the most recent data point.

For example, disparities by race and ethnicity are measured as the percent difference between the best racial and ethnic group rate and each of the other racial and ethnic group rates. Similarly, disparities by sex are measured as the percent difference between the better group rate (e.g., female) and the rate for the other group (e.g., male).

Formula. The formula for disparity from the best group rate for a group $\mathrm{G}$ is as follows:

$$
\text { Disparity for group } \mathrm{G}=\frac{\text { Rate for group } \mathrm{G}-\text { Best group rate for characteristic }}{\text { Best group rate for characteristic }} \times 100 \text {. }
$$

Some Healthy People 2010 objectives are expressed in terms of favorable events or conditions that are to be increased, while others are expressed in terms of adverse events or conditions that are to be reduced. In order to facilitate comparison of health disparities across different objectives, disparity is measured only in terms of adverse events or conditions in Healthy People 2010 [1]. Those objectives that are expressed in terms of favorable events or conditions are re-expressed using the adverse event or condition for the purpose of computing disparity, but they are not otherwise restated or changed. See Technical Appendix for more information.

Example. Healthy People 2010 objective 1-1, to increase the proportion of persons with health insurance (e.g., $72 \%$ of the American Indian or Alaska Native population aged under 65 years had some form of health insurance in 2008), is expressed in terms of the percentage of persons without health insurance (e.g., $100 \%-72 \%=28 \%$ of the American Indian or Alaska Native population aged under 65 years did not have any form of health insurance in 2008) when the disparity from the best group rate is calculated.

As a result, the group identified as having the best rate for a given characteristic is always the group with the least adverse event or condition. Thus, disparities defined by the above formula remain nonnegative quantities, equal zero only when the group $\mathrm{G}$ for which disparity is being assessed has rate equal to the best group rate. See Technical Appendix for more information. 
The group with the best or most favorable rate is identified for each characteristic in the Health Disparities Table by a "B." In the few instances when two groups had identical best rates, both groups are identified by a "B." In some cases, the most favorable rate is not sufficiently reliable to be used as the best rate. In these situations, a small letter " $b$ " is included in the cell, and the next most favorable group rate with sufficient reliability is identified with a "B" as the best group. When there is only one group with sufficiently reliable data, a best group is not identified for purposes of measuring disparity, and the cells for all groups with data are left blank in the Health Disparities Table, indicating that disparities could not be assessed. These symbols are described in the first section of the legend that accompanies each of these figures (reproduced below).

A color gradient is used to represent the size of the percent difference from the best group rate for each group at the most recent data point. In some cases, baseline data might be the only data available. The color gradient is shown in the second section of the legend, reproduced below. When measures of variability are available, the variability of best group rates is assessed, and statistical significance is tested. For a given group $\mathrm{G}$ within a characteristic, a disparity of $10 \%$ or more is displayed when the difference from the best group rate (i.e., Rate for group $\mathrm{G}$ - Best group rate) is statistically significant at the 0.05 level. See Technical Appendix.

Change in disparity over time is estimated by subtracting the disparity at baseline from the disparity at the most recent data point. The change is expressed in percentage points: positive differences represent an increase in disparity, and negative differences represent a decrease in disparity. The magnitude of the change is indicated by the number of arrows. (See the third section of the legend reproduced below.) Whenever data are available at both the baseline and most recent time points, changes in disparity over time are shown if the change is greater than or equal to 10 percentage points and statistically significant, or when the change is greater than or equal to 10 percentage points and estimates of variability are not available. See Technical Appendix for a more in-depth discussion.

Footnotes indicate whether statistical testing was performed for either the differences from the best group rate at the most recent data point or the changes in disparities over time.

When there are more than two groups associated with a population characteristic (for example, race and ethnicity, education, and income), a summary index provides a way to determine whether the disparity from the best group rate has increased or decreased on average. The summary index is the average of percent differences between the best group rate and each of the other group rates for a characteristic. These comparisons are made only when disparities data are available for exactly the same groups at the baseline and most recent data points.

The statistical significance of the summary index at the most recent data point and changes in the index over time are assessed when possible. The magnitude of the summary index at the most recent data point, and the magnitude and direction of changes in the summary index over time, are indicated by the color gradient and the arrow symbols, respectively.

More detail on measuring, tracking, and summarizing, health disparities can be found in the Technical Appendix, as well as in a related publication [1]. 


\section{Figure RG-1: Legend for the Health Disparities Table (Figure 2) in each of the Focus Area chapters}

\begin{tabular}{|c|c|c|c|c|c|c|}
\hline \multicolumn{7}{|l|}{ LEGEND } \\
\hline $\begin{array}{l}\text { The "best" group rate at the most } \\
\text { recent data point. }\end{array}$ & B & $\begin{array}{l}\text { The group with the best rate for } \\
\text { specified characteristic. }\end{array}$ & $\mathrm{b}$ & \multicolumn{2}{|c|}{$\begin{array}{l}\text { Most favorable group rate for specified } \\
\text { characteristic, but reliability criterion not met. }\end{array}$} & $\begin{array}{l}\text { Reliability criterion for best } \\
\text { group rate not met, or data } \\
\text { available for only one group }\end{array}$ \\
\hline & \multicolumn{6}{|c|}{ Percent difference from the best group rate } \\
\hline $\begin{array}{l}\text { Disparity from the best group rate at } \\
\text { the most recent data point. }\end{array}$ & & $\begin{array}{l}\text { Less than } 10 \% \text {, or difference not } \\
\text { statistically significant (when } \\
\text { estimates of variability are available). }\end{array}$ & & $10 \%-49 \%$ & $50 \%-99 \%$ & $100 \%$ or more \\
\hline & & & & & parity (percent & \\
\hline \multirow{3}{*}{\multicolumn{3}{|c|}{$\begin{array}{l}\text { Changes in disparity over time are shown when: } \\
\text { (a) disparities data are available at both baseline and most recent time points; } \\
\text { (b) data are not for the group(s) indicated by "B" or "b" at either time point; and } \\
\text { (c) the change is greater than or equal to } 10 \text { percentage points and statistically } \\
\text { significant, or when the change is greater than or equal to } 10 \text { percentage points } \\
\text { and estimates of variability were not available. See Technical Appendix. }\end{array}$}} & & $10-49$ points & 50-99 points & $\begin{array}{l}100 \text { points or } \\
\text { more }\end{array}$ \\
\hline & & & \multicolumn{4}{|c|}{ Decrease in disparity (percentage points) } \\
\hline & & & & 10-49 points & 50-99 points & $\begin{array}{l}100 \text { points or } \\
\text { more }\end{array}$ \\
\hline Availability of Data & & Data not available. & & & \multicolumn{2}{|c|}{ Characteristic not selected for this objective. } \\
\hline
\end{tabular}




\section{Displaying Data with Maps}

When data are available at the sub-national level, selected objectives are mapped to display spatial variation in percents, rates, or counts. Sub-national data are presented either at the state or HSA level. HSAs are defined as "...one or more counties that are relatively self-contained with respect to the provision of routine hospital care" [3]. HSAs are contiguous but may span state boundaries. They frequently contain more than one county with an average of four and maximum of 20 counties. Maps are presented as simple chloropleths and use either a Jenks or modified Jenks classification [4]. A Jenks classification is a method for grouping ordered data in such a way that within-group variance is minimized and between-group variance is maximized. When geographic units (states or HSAs) have values that meet the Healthy People 2010 target, the classification is modified by manually setting the "best" cut-point to the Healthy People 2010 target. The best cutpoint is the highest cut-point for objectives that are expressed in terms of favorable events or conditions that are to be increased, and the lowest cut-point for objectives that are expressed in terms of adverse events or conditions that are to be reduced In some instances where the number of geographic units meeting the target is large a cut-point in the middle of the distribution is set to the target. See Technical Appendix for more information.

\section{References and Notes}

1. Keppel KG, Pearcy JN, Klein RJ. Measuring progress in Healthy People 2010. Statistical Notes, no. 25. Hyattsville, Maryland: National Center for Health Statistics. September 2004.

2. Characteristics for developmental objectives were not included in the original Healthy People 2010 publication, but were added when data sources were identified and the objectives became measurable. Lists of characteristics for all currently measurable objectives can be found in DATA2010, an online database available from: http://wonder.cdc.gov/data2010.

3. Makuc DM, Haglund B, Ingram DD, et.al. Health Service Areas for the United States. National Center for Health Statistics. Vital Health Stat (2)112.1991.

4. Coulson MR. In the matter of class intervals for chloropleth maps: with particular reference to the work of George F Jenks. Cartographica: The International Journal for Geographic Information and Geovisualization. 24(2), 16-39. 2006. 


\section{Final Review}

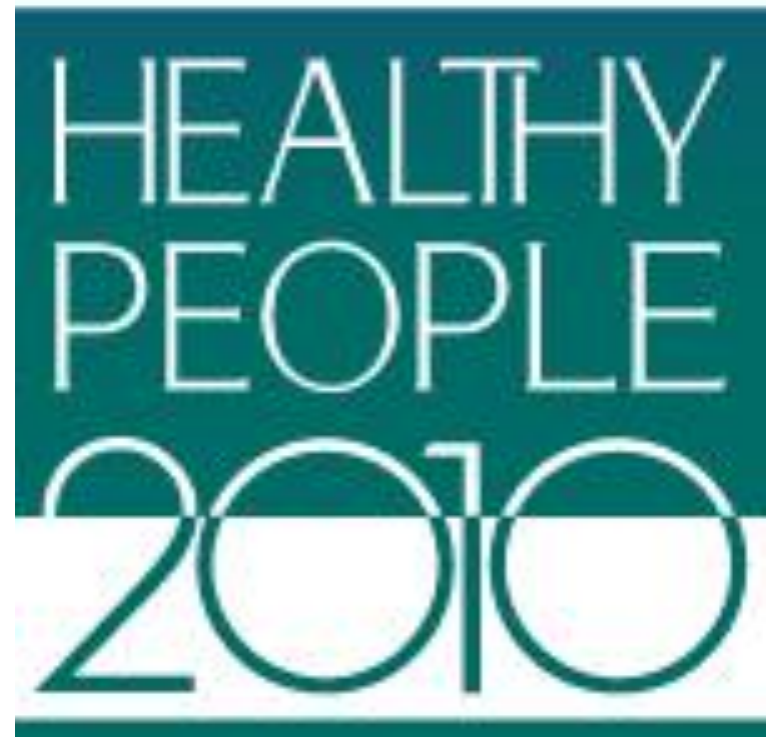

\section{Overview by Focus Area}




\section{Final Review}

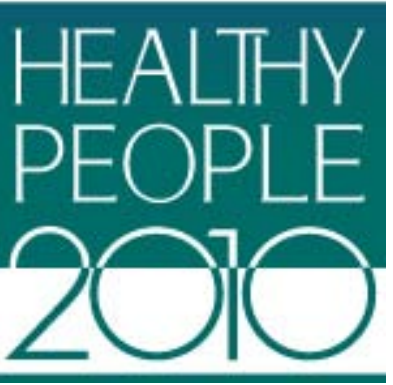

\section{Access to Quality Health Services}

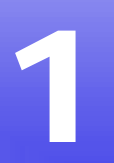

\section{Co-Lead Agencies}

Agency for Healthcare Research and Quality

Health Resources and Services Administration

\section{Contents}

Goal

Highlights

Summary of Progress.

Transition to Healthy People 2020

Data Considerations

References and Notes

Comprehensive Summary of Objectives

Progress Chart

Health Disparities Table

Persons with Health Insurance-Map 


\section{Goal: Improve access to comprehensive, high-quality health care services}

Access to quality health services includes access to primary care, preventive services, and other health care services on a continuum of care in the health care delivery system. The objectives in this chapter monitor progress in four general areas:

- The first section monitors clinical preventive care and includes objectives that track health insurance coverage and counseling about health behaviors.

- Objectives in the second section are concerned with primary care and examine source of ongoing care, having a usual primary care provider, difficulties and delays obtaining needed health care, cultural diversity and racial and ethnic representation in health professions, and hospitalization for ambulatory-care-sensitive conditions.

- Emergency services, including delay or difficulty getting emergency care, rapid prehospital emergency care, trauma care systems, and special needs of children are monitored in the third section.

- The final section tracks the long-term care and rehabilitative services including long term care services and pressure ulcers among nursing home residents.

All Healthy People tracking data quoted in this chapter, along with technical information and operational definitions for each objective, can be found in the Healthy People 2010 database, DATA2010, available from http://wonder.cdc.gov/data2010/.

More information about this Focus Area can be found in the following publications:

- Healthy People 2010: Understanding and Improving Health, available from http://www.healthypeople.gov/2010/Document/tableofcontents.htm\#under.

- Healthy People 2010 Midcourse Review, available from http://www.healthypeople.gov/2010/data/midcourse/html/default.htm\#FocusAreas.

\section{Highlights}

- Substantial progress was achieved in objectives for this Focus Area during the past decade [1]. Seventy-three percent of the Access to Quality Health Services objectives with data to measure progress moved toward or achieved their Healthy People 2010 targets (Figure 11). However, statistically significant health disparities of $10 \%$ or more were observed among racial and ethnic populations and income groups (Figure 1-2) [2].

- Rates of persons with health insurance (objective 1-1) did not change over the decade. As in 1997, the baseline year for this objective, $83 \%$ of the U.S. population under age 65 years had health insurance coverage in 2008. Disparities were observed for a number of population groups, for example: 
- Among racial and ethnic groups, the white non-Hispanic population had the highest (best) rate of health insurance coverage, $88 \%$ in 2008, whereas the American Indian or Alaska Native population and the Hispanic or Latino population had rates of $72 \%$ and $67 \%$ in 2008 , respectively. When expressed as persons without health insurance, the rate for the American Indian or Alaska Native population was more than twice the best group rate (that for the white non-Hispanic population) [2]. The rate for the Hispanic or Latino population was nearly three times the best rate.

- The American Indian or Alaska Native population had health insurance coverage rates of $62 \%$ in 1999 and $72 \%$ in 2008 , whereas the white non-Hispanic population had rates of $88 \%$ in both 1999 in 2008 . When rates are expressed in terms of persons without health insurance, the disparity between the American Indian or Alaska Native population and the white non-Hispanic population decreased by 83 percentage points between 1999 and 2008 [2,3].

- Among income groups, the middle/high income population had the highest (best) rate of health insurance coverage, $89 \%$ in 2008, whereas the poor and near-poor populations had rates of $71 \%$ and $69 \%$ in 2008, respectively. When expressed as persons without health insurance, the rate for the poor population was more than two and a half times the best group rate (that for the middle/high income population) [2]. The rate for the near-poor population was almost three times the best rate.

- The poor population had health insurance coverage rates of $66 \%$ in 1997 and $71 \%$ in 2008, whereas the middle/high income population had rates of $90 \%$ in 1997 and $89 \%$ in 2008 . When rates are expressed in terms of persons without health insurance, the disparity between the poor population and the middle/high income population decreased by 76 percentage points between 1997 and 2008 [2,3].

- Health insurance coverage varied by state. Although no state had achieved the Healthy People 2010 target of total coverage, five states (Connecticut, Hawaii, Iowa, Massachusetts, and Minnesota) had rates of coverage over $88 \%$ in 2008. Texas, at $71 \%$, had the lowest coverage rate (Figure 1-3).

- Statistically significant health disparities of $100 \%$ or more were observed for several other objectives, for example:

- Persons who had a specific source of ongoing care among all ages (objective 1-4a):

- Among racial and ethnic groups, the white non-Hispanic population had the highest (best) rate, $89 \%$ in 2008, whereas the Hispanic or Latino population had a rate of $77 \%$ in 2008 . When expressed as persons without a specific source of ongoing care, the rate for the Hispanic or Latino population was more than twice the best group rate [2].

- Among income groups, the middle/high income population had the highest rate, $90 \%$ in 2008 , whereas the poor and near-poor populations had rates of $78 \%$ and $80 \%$ in 2008 , respectively. When expressed as persons without a specific source of ongoing care, the rates for the poor and near-poor populations were about twice the best group rate [2]. 
- Persons who had a specific source of ongoing care among adults aged 18 years and over (objective 1-4c):

- Among racial and ethnic groups, the white non-Hispanic population had the highest (best) rate, $87 \%$ in 2008, whereas the Hispanic or Latino population had a rate of $69 \%$ in 2008 . When expressed as persons without a specific source of ongoing care, the rate for the Hispanic or Latino population was almost two a half times the best group rate [2].

- Among income groups, the middle/high income population had the best rate, $88 \%$ in 2008, whereas the near-poor and poor populations had rates of $76 \%$ and $71 \%$ in 2008 , respectively. When expressed as persons without a specific source of ongoing care, the rate for the near-poor population was twice the best group rate, while the rate for the poor population was almost two and a half times the best rate [2].

- Persons who delayed or had difficulty in getting emergency medical care (objective 1-10):

- Among racial and ethnic groups, the rate for persons of two or more races (6.7\% in 2001) was about three times the best group rate, that for the white non-Hispanic population $(2.2 \%$ in 2001$)$.

- Among income groups, the rate for the poor population (4.5\% in 2001) was more than twice that of the best group rate, that for the middle/high income population $(2.0 \%$ in 2001$)$.

- The rate for persons with disabilities (5.7\% in 2001) was more than three times that for persons without disabilities (1.8\% in 2001).

\section{Summary of Progress}

- Figure 1-1 presents a quantitative assessment of progress in achieving the Healthy People 2010 objectives for Access to Quality Health Services [1]. Data to measure progress toward target attainment were available for 48 objectives. Of these:

- Eleven objectives (1-7a through d; 1-8b, f, j, n, and r; 1-9a; and 1-12) met or exceeded their Healthy People 2010 targets.

- Twenty-four objectives moved toward their targets. A statistically significant difference between the baseline and the final data points was observed for three of these objectives (1-3c, 1-6, and 1-9c). Data to test the significance of the difference were unavailable for 21 objectives (1-3f; 1-7e and g; 1-8a, d, e, g through i, l, p, q, s, and t; 1-13a, b, e, f, and i; and 1-14a and b).

- Six objectives (1-1; $1-4 \mathrm{~b} ; 1-7 \mathrm{f}$ and $\mathrm{h}$; and $1-8 \mathrm{~m}$ and o) showed no change.

- Seven objectives moved away from their targets. A statistically significant difference between the baseline and final data points was observed for three objectives (1-4a and c, and 1-9b). No significant differences were observed for two objectives (1-5 and 1-16); and data to test the significance of the difference were unavailable for two objectives (1-8c and k).

- One objective (1-3g) remained developmental and 20 objectives (1-3a, b, d, h; 1-10; 1-11a through g; $1-13 c, d, g$, and $h$; and 1-15a through d) had no data available to measure progress [4]. Two objectives (1-2 and 1-3e) were dropped during the decade [5]. 
- Figure 1-2 displays health disparities in Access to Quality Health Services from the best group rate for each characteristic at the most recent data point [2]. It also displays changes in disparities from baseline to the most recent data point [3].

- Of the 10 objectives with statistically significant health disparities of $10 \%$ or more by race and ethnicity, the white non-Hispanic population had the best rate for seven objectives (1-1, 1-3c, 1-4a and c, 1-5, 1-10, and 1-16). The black non-Hispanic population had the best rate for two objectives (1-3a and b) and the Hispanic or Latino population had the best rate for one objective (1-6).

- Females had better rates than males for eight of the nine objectives with statistically significant health disparities of $10 \%$ or more by sex (objectives 1-1, 1-3c, 1-4a and c, $1-5,1-9 a$ and $b$, and 1-16). Males had a better rate than females for the remaining objective (1-6).

- Persons with at least some college education had the best rate for the three objectives with statistically significant health disparities of $10 \%$ or more by education level (objectives 1-3h, 1-5, and 1-10).

- Persons with middle/high incomes had the best rate for all six objectives with statistically significant health disparities of $10 \%$ or more by income (objectives $1-1$, 1-3h, 1-4a and c, 1-6, and 1-10).

- Persons living in rural or nonmetropolitan areas had better rates than persons living in urban or metropolitan areas for two of the three objectives with statistically significant health disparities of $10 \%$ or more by geographic location (objectives 1-4c and 1-5). Persons living in urban or metropolitan areas had a better rate for the third objective (1-1).

- Persons with disabilities had better rates than persons without disabilities for seven of the 10 objectives with statistically significant health disparities of $10 \%$ or more by disability status (objectives 1-1, 1-3a through c, 1-4a and c, and 1-5). Persons without disabilities had better rates for the remaining three objectives (1-3h, 1-6, and 1-10).

- Health disparities of $100 \%$ or more were observed for four objectives: health insurance coverage (objective 1-1), source of ongoing care among all ages and among adults (objective 1-4a and c, respectively), and delay or difficulty in getting emergency care (objective 1-10). These are discussed in the Highlights, above.

- As indicated in the Highlights, above, increases in disparity over time between select population groups and income groups were observed for health insurance coverage.

\section{Transition to Healthy People 2020}

For Healthy People 2020, the Access to Health Services (AHS) Topic Area uses a new organizational approach based on two major components of health services delivery: access to health services and quality of health services. See HealthyPeople.gov for a complete list of Healthy People 2020 topics and objectives. 
Objectives that appear in the Healthy People 2020 AHS Topic Area focus on the first component only, access to health services, whereas objectives that pertain to the second component, quality of health services, have been shifted into the appropriate disease- or condition-specific Topic Area, and are, therefore, spread throughout Healthy People 2020.

The Healthy People 2010 Focus Area name was changed from "Access to Quality Health Services" to "Access to Health Services" for Healthy People 2020 to be consistent with the new organizational structure. In order to capture the objectives that are related to quality of health services, a crosswalk will be created, consisting of objectives found in the other Healthy People 2020 chapters (e.g., cancer screening rates and primary care counseling services) that are aligned with the annual National Health Quality Report (NHQR) [6].

The Healthy People 2020 AHS Topic Area can be grouped into several sections:

- Coverage

- Workforce

- Utilization and Services

- Timeliness.

The differences between the Healthy People 2010 and Healthy People 2020 objectives are summarized below:

- The Healthy People 2020 Access to Health Services Topic Area has a total of 26 objectives, 16 of which are developmental, whereas the Healthy People 2010 Focus Area had 71 objectives [4]. In transitioning to Healthy People 2020, many objectives were dropped due to the shift in Topic Area focus, as well as for data-related issues such as lack of viable data sources and successful attainment of 2010 targets [5].

- Four Healthy People 2010 objectives were retained "as is": health (medical) insurance (objective 1-1), specific source of ongoing care for all ages and for children and adolescents aged 17 years and under (objectives 1-4a and b, respectively), and usual primary care provider (objective 1-5) [7].

- Two Healthy People 2010 objectives were modified [8]. The objective on source of ongoing care for adults 18 years and over (objective 1-4c) was split into adults 18-64 years and adults 65 years and over; and the objective on difficulties or delays in receiving needed health care (objective 1-6) was modified to measure individuals instead of families and split by type of care or service (all, medical care, dental care, and prescription medicines).

- Two Healthy People 2010 objectives, the population covered by basic and advanced life support (objectives 1-11a and b), were reverted to developmental status in 2020 due to a lack of baseline data.

- Three Healthy People 2010 objectives were dropped [5]. Counseling about vehicle restraints and bicycle helmets (objective 1-3e) and prevention of sexually transmitted diseases (objective 1-3g) were dropped. Health insurance coverage for the clinical preventive services objective (1-2) was dropped at the Healthy People 2010 Midcourse Review but then retained as developmental for 2020. 
- The remaining 60 Healthy People 2010 AHS objectives were archived or moved to other Healthy People 2020 Topic Areas, including new Topic Areas related to age groups: Early and Middle Childhood, Adolescent Health, and Older Adults [9]. These objectives cover the following topics: counseling about health behaviors (objectives 1-3a through $\mathrm{d}$, $\mathrm{f}$, and $\mathrm{h}$ ); health professions training on health promotion, disease prevention, and cultural diversity (objectives 1-7a through $\mathrm{h}$ ); racial and ethnic representation in health professions (objectives 1-8a through t); hospitalization for specific conditions (objectives 1-9a through c); emergency care (objectives 1-10 and 1-11c through g); poison control (objectives 1-12); trauma care systems (objectives 1-13a through i); special needs of children (objectives 114a and b); and access to long-term care services (objectives 1-15a through $d$ and 1-16).

- In many cases, objectives were dropped or moved to other Topic Areas due to the revised focus of the AHS Topic Area, while in other cases the target was met or objectives no longer had viable data sources.

- For example, the objective that tracks physician counseling about physical activity (objective 1-3a) was moved into the Healthy People 2020 Physical Activity Topic Area and modified to include objectives on physician counseling and education related to exercise.

- Thirteen new objectives were added to the Healthy People 2020 AHS Topic Area:

- The health insurance coverage objective was expanded from one to three objectives covering medical insurance (retained from Healthy People 2010), dental insurance (developmental), and prescription drug insurance (developmental).

- Four new objectives related to workforce were added. These developmental objectives will track the practicing primary care providers in the following professions: medical doctor, doctor of osteopathy, physician assistant, and nurse practitioner.

- One new developmental objective will track persons who receive appropriate evidence-based clinical services.

- Six new developmental objectives track hospital emergency department visits for which wait time to see an emergency department clinician exceeds the recommended timeframe.

Appendix D, "A Crosswalk Between Objectives from Healthy People 2010 to Healthy People 2020," summarizes the changes between the two decades of objectives, reflecting new knowledge and direction for this area. 


\section{Data Considerations}

Data on health professions training on health promotion, disease prevention, and cultural diversity (objectives 1-7a through $\mathrm{h}$ ) and racial and ethnic representation in health professions (objectives 1$8 a$ through $\mathrm{t}$ ) had definitional issues that resulted in difficulties in interpreting trends for certain objectives during the Healthy People 2010 tracking decade. For example, objectives 1-7e and f used a different survey in 2008 than for the 1999 baseline, which may result in data for those objectives not being comparable over time. The baseline survey data for objectives $1-7 \mathrm{~g}$ and $\mathrm{h}$ did not include the D.N.P. degree as a response option, whereas the 2008 survey data did include that degree. Finally, objectives 1-8a through d, racial and ethnic representation for health professions, do not include data for dental professionals for the final year of data (2009) since those data were not available at the time of publication.

Education and income are the primary measures of socioeconomic status (SES) in Healthy People 2010. Most data systems used in Healthy People 2010 define income as a family's income before taxes. In order to facilitate comparisons among groups and over time, while adjusting for family size and for inflation, Healthy People 2010 categorizes income using the poverty thresholds developed by the U.S. Census Bureau. Thus, the three categories of family income that are primarily used are:

- Poor-below the Federal poverty level

- Near poor-100\% to $199 \%$ of the Federal poverty level

- Middle/high income-200\% or more of the Federal poverty level.

These categories may be overridden by considerations specific to the data system, in which case they are modified as appropriate. See Healthy People 2010: General Data Issues, referenced below.

In general, data on educational attainment are presented for persons aged 25 years and over, consistent with guidance given by the U.S. Bureau of the Census. However, because of the requirements of the different data systems, the age groups used to calculate educational attainment for any specific objective may differ from the age groups used to report the data for other Healthy People 2010 objectives, as well as from select populations within the same objective. Therefore, the reader is urged to exercise caution in interpreting the data by educational attainment shown in the Health Disparities Table. See Healthy People 2010: General Data Issues, referenced below.

Figure 1-3 (Persons with Heath Insurance) presents state-level data from the Behavioral Risk Factor Surveillance System (BRFSS). National data for these objectives come from the National Health Interview Survey (NHIS) and are the basis for setting the targets. BRFSS data may not be comparable to the National data from the NHIS.

Additional information on data issues is available from the following sources:

- All Healthy People 2010 tracking data can be found in the Healthy People 2010 database, DATA2010, available from http://wonder.cdc.gov/data2010/.

- Detailed information about the data and data sources used to support these objectives can be found in the Operational Definitions on the DATA2010 website, available from http://wonder.cdc.gov/data2010/focusod.htm. 
- More information on statistical issues related to Healthy People tracking and measurement can be found in the Technical Appendix and in Healthy People 2010: General Data Issues, which is available in the Data Issues section of the NCHS Healthy People website under Healthy People 2010.

\section{References and Notes}

1. Displayed in the Progress Chart (Figure 1-1), the percent of targeted change achieved expresses the difference between the baseline and the final value relative to the initial difference between the baseline and the Healthy People 2010 target. As such, it is a relative measure of progress toward attaining the Healthy People 2010 target. See the Reader's Guide for more information. When standard errors were available, the difference between the baseline and the final value was tested at the 0.05 level of significance. See the Figure 1-1 footnotes, as well as the Technical Appendix, for more detail.

2. Information about disparities among select populations is shown in the Health Disparities Table (Figure 1-2). Disparity from the best group rate is defined as the percent difference between the best group rate and each of the other group rates for a characteristic. For example, racial and ethnic health disparities are measured as the percent difference between the best racial and ethnic group rate and each of the other racial and ethnic group rates. Similarly, disparities by sex are measured as the percent difference between the better group rate (e.g., female) and the rate for the other group (e.g., male). Some objectives are expressed in terms of favorable events or conditions that are to be increased, while others are expressed in terms of adverse events or conditions that are to be reduced. In order to facilitate comparison of health disparities across different objectives, disparity is measured only in terms of adverse events or conditions. For comparability across objectives, objectives that are expressed in terms of favorable events or conditions are re-expressed using the adverse event or condition for the purpose of computing disparity, but they are not otherwise restated or changed. For example, objective 1-1, to increase the proportion of persons with health insurance (e.g., $72 \%$ of the American Indian or Alaska Native population aged under 65 years had some form of health insurance in 2008), is expressed in terms of the percentage of persons without health insurance (e.g., $100 \%-72 \%=28 \%$ of the American Indian or Alaska Native population aged under 65 years did not have any form of health insurance in 2008) when the disparity from the best group rate is calculated. See the Reader's Guide for more information. When standard errors were available, the difference between the best group rate and each of the other group rates was tested at the 0.05 level of significance. See the Figure 1-2 footnotes, as well as the Technical Appendix, for more detail.

3. The change in disparity is estimated by subtracting the disparity at baseline from the disparity at the most recent data point and, therefore, is expressed as a change in percentage points. See the Reader's Guide for more information. When standard errors were available, the change in disparity was tested at the 0.05 level of significance. See the Figure 1-2 footnotes, as well as the Technical Appendix, for more detail.

4. To be included in Healthy People 2010, an objective must have a national data source that provides a baseline and at least one additional data point for tracking progress. Some objectives lacked baseline data at the time of their development but had a potential data source and were considered of sufficient national importance to be included in Healthy People. These are called "developmental" objectives. When data become available, a developmental objective is moved to measurable status and a Healthy People target can be set.

5. Dropped objectives were not carried forward into Healthy People 2020. These objectives were either developmental or deleted at the Healthy People 2010 Midcourse Review or at another time in Healthy People 2010. 
6. Agency for Healthcare Research and Quality. National Healthcare Quality Report 2010 [Internet]. Washington: Agency for Healthcare Research and Quality; 2010. (AHRQ publication no. 11-0004). Available from: http://www.ahrq.gov/qual/qrdr10.htm.

7. Retained "as is" objectives have no change in the numerator definition or in the denominator definition between the Healthy People 2010 and Healthy People 2020 objectives. These include objectives that were developmental in Healthy People 2010 and are developmental in Healthy People 2020 and for which no numerator or denominator information was available.

8. Modified objectives have some change in the numerator definition or in the denominator definition between the Healthy People 2010 and Healthy People 2020 objectives. These include objectives that went from developmental in Healthy People 2010 to measurable in Healthy People 2020 or vice versa.

9. Archived objectives had at least one data point in Healthy People 2010 but were not carried forward into Healthy People 2020. 


\section{Comprehensive Summary of Objectives: Access to Quality Health Services}

\begin{tabular}{|c|c|c|}
\hline Objective & Description & Data Source or Objective Status \\
\hline $1-1$ & Persons with health insurance ( $<65$ years) & National Health Interview Survey (NHIS), CDC, NCHS. \\
\hline $1-2$ & Health insurance coverage for clinical preventive services & Dropped \\
\hline $1-3 a$ & Counseling about physical activity or exercise (age adjusted, $18+$ years) & National Health Interview Survey (NHIS), CDC, NCHS. \\
\hline $1-3 b$ & Counseling about diet and nutrition (age adjusted, 18+ years) & National Health Interview Survey (NHIS), CDC, NCHS. \\
\hline $1-3 c$ & Counseling about smoking cessation (age adjusted, smokers 18+ years) & National Health Interview Survey (NHIS), CDC, NCHS. \\
\hline $1-3 d$ & Counseling about risky drinking (age adjusted, $18+$ years) & National Health Interview Survey (NHIS), CDC, NCHS. \\
\hline $1-3 e$ & Counseling about childhood injury prevention ( $\leq 17$ years) & Dropped \\
\hline $1-3 f$ & Counseling about unintended pregnancy (females 15-44 years) & National Survey of Family Growth (NSFG), CDC, NCHS. \\
\hline $1-3 g$ & $\begin{array}{l}\text { Counseling about prevention of sexually transmitted diseases (15-44 } \\
\text { years) }\end{array}$ & Developmental \\
\hline $1-3 \mathrm{~h}$ & Counseling about management of menopause (females $45-57$ years) & National Health Interview Survey (NHIS), CDC, NCHS. \\
\hline $1-4 a$ & Source of ongoing care-All ages & National Health Interview Survey (NHIS), CDC, NCHS. \\
\hline $1-4 b$ & Source of ongoing care - Children and adolescents ( $<18$ years) & National Health Interview Survey (NHIS), CDC, NCHS. \\
\hline $1-4 c$ & Source of ongoing care-Adults (18+ years) & National Health Interview Survey (NHIS), CDC, NCHS. \\
\hline $1-5$ & Persons with a usual primary care provider & Medical Expenditure Panel Survey (MEPS), AHRQ. \\
\hline $1-6$ & Difficulties or delays in obtaining needed health care (families) & Medical Expenditure Panel Survey (MEPS), AHRQ. \\
\hline $1-7 \mathrm{a}$ & $\begin{array}{l}\text { Medical doctor (M.D. degree)—Counseling for health promotion and } \\
\text { disease prevention }\end{array}$ & $\begin{array}{l}\text { Liaison Committee on Medical Education (LCME) Annual Medical School } \\
\text { Questionnaire, Association of American Medical Colleges. }\end{array}$ \\
\hline $1-7 b$ & Medical doctor (M.D. degree)—Cultural diversity & $\begin{array}{l}\text { Liaison Committee on Medical Education (LCME) Annual Medical School } \\
\text { Questionnaire, Association of American Medical Colleges. }\end{array}$ \\
\hline $1-7 c$ & $\begin{array}{l}\text { Osteopathic medical doctor (D.O. degree)—Counseling for health } \\
\text { promotion and disease prevention }\end{array}$ & $\begin{array}{l}\text { Annual Report on Osteopathic Medical Education, American Association } \\
\text { of Colleges of Osteopathic Medicine. }\end{array}$ \\
\hline $1-7 d$ & Osteopathic medical doctor (D.O. degree)—Cultural diversity & $\begin{array}{l}\text { Annual Report on Osteopathic Medical Education, American Association } \\
\text { of Colleges of Osteopathic Medicine. }\end{array}$ \\
\hline $1-7 \mathrm{e}$ & $\begin{array}{l}\text { Baccalaureate-level nurse (B.S.N., B.A., or B.S. degree)-Counseling for } \\
\text { health promotion and disease prevention }\end{array}$ & $\begin{array}{l}\text { Special Healthy People Survey of Entry-Level Baccalaureate Nursing } \\
\text { School Curriculum, formerly Survey on Women's Health in the Entry- } \\
\text { Level Baccalaureate Nursing School Curriculum, American Association of } \\
\text { Colleges of Nursing. }\end{array}$ \\
\hline
\end{tabular}




\begin{tabular}{|c|c|c|}
\hline Objective & Description & Data Source or Objective Status \\
\hline $1-7 f$ & $\begin{array}{l}\text { Baccalaureate-level nurse (B.S.N., B.A., or B.S. degree)- Cultural } \\
\text { diversity }\end{array}$ & $\begin{array}{l}\text { Special Healthy People Survey of Entry-Level Baccalaureate Nursing } \\
\text { School Curriculum, formerly Survey on Women's Health in the Entry- } \\
\text { Level Baccalaureate Nursing School Curriculum, American Association of } \\
\text { Colleges of Nursing. }\end{array}$ \\
\hline $1-7 \mathrm{~g}$ & $\begin{array}{l}\text { Nurse Practitioner (M.S., M.S.N., or D.N.P. degree)-Counseling for health } \\
\text { promotion and disease prevention }\end{array}$ & $\begin{array}{l}\text { Collaborative Curriculum Survey, American Association of Colleges of } \\
\text { Nursing and National Organization of Nurse Practitioner Faculties. }\end{array}$ \\
\hline $1-7 \mathrm{~h}$ & Nurse Practitioner (M.S., M.S.N., or D.N.P. degree)—Cultural diversity & $\begin{array}{l}\text { Collaborative Curriculum Survey, American Association of Colleges of } \\
\text { Nursing and National Organization of Nurse Practitioner Faculties. }\end{array}$ \\
\hline $1-8 \mathrm{a}$ & $\begin{array}{l}\text { Racial and ethnic representation in health professions-American Indian } \\
\text { or Alaska Native }\end{array}$ & $\begin{array}{l}\text { Survey of Predoctoral Dental Educational Institutions, American Dental } \\
\text { Association; Profile of Pharmacy Students, American Association of } \\
\text { Colleges of Pharmacy; AAMC Data Book, Association of American Medical } \\
\text { Colleges; Annual Data Report, Association of Schools of Public Health. }\end{array}$ \\
\hline $1-8 b$ & $\begin{array}{l}\text { Racial and ethnic representation in health professions-Asian or Pacific } \\
\text { Islander }\end{array}$ & $\begin{array}{l}\text { Survey of Predoctoral Dental Educational Institutions, American Dental } \\
\text { Association; Profile of Pharmacy Students, American Association of } \\
\text { Colleges of Pharmacy; AMC Data Book, Association of American Medical } \\
\text { Colleges; Annual Data Report, Association of Schools of Public Health. }\end{array}$ \\
\hline $1-8 \mathrm{c}$ & $\begin{array}{l}\text { Racial and ethnic representation in health professions-Black or African } \\
\text { American }\end{array}$ & $\begin{array}{l}\text { Survey of Predoctoral Dental Educational Institutions, American Dental } \\
\text { Association; Profile of Pharmacy Students, American Association of } \\
\text { Colleges of Pharmacy; AAMC Data Book, Association of American Medical } \\
\text { Colleges; Annual Data Report, Association of Schools of Public Health. }\end{array}$ \\
\hline $1-8 d$ & $\begin{array}{l}\text { Racial and ethnic representation in health professions-Hispanic or } \\
\text { Latino }\end{array}$ & $\begin{array}{l}\text { Survey of Predoctoral Dental Educational Institutions, American Dental } \\
\text { Association; Profile of Pharmacy Students, American Association of } \\
\text { Colleges of Pharmacy; AAMC Data Book, Association of American Medical } \\
\text { Colleges; Annual Data Report, Association of Schools of Public Health. }\end{array}$ \\
\hline $1-8 \mathrm{e}$ & $\begin{array}{l}\text { Racial and ethnic representation in Nursing-American Indian or Alaska } \\
\text { Native }\end{array}$ & $\begin{array}{l}\text { Annual Survey of RN (Registered Nurse) Programs, National League for } \\
\text { Nursing, Center for Research in Nursing Education and Community } \\
\text { Health. }\end{array}$ \\
\hline $1-8 f$ & Racial and ethnic representation in Nursing-Asian or Pacific Islander & $\begin{array}{l}\text { Annual Survey of RN (Registered Nurse) Programs, National League for } \\
\text { Nursing, Center for Research in Nursing Education and Community } \\
\text { Health. }\end{array}$ \\
\hline $1-8 \mathrm{~g}$ & Racial and ethnic representation in Nursing-Black or African American & $\begin{array}{l}\text { Annual Survey of RN (Registered Nurse) Programs, National League for } \\
\text { Nursing, Center for Research in Nursing Education and Community } \\
\text { Health. }\end{array}$ \\
\hline
\end{tabular}




\begin{tabular}{|c|c|c|}
\hline Objective & Description & Data Source or Objective Status \\
\hline $1-8 h$ & Racial and ethnic representation in Nursing-Hispanic or Latino & $\begin{array}{l}\text { Annual Survey of RN (Registered Nurse) Programs, National League for } \\
\text { Nursing, Center for Research in Nursing Education and Community } \\
\text { Health. }\end{array}$ \\
\hline $1-8 \mathrm{i}$ & $\begin{array}{l}\text { Racial and ethnic representation in Medicine-American Indian or } \\
\text { Alaska Native }\end{array}$ & $\begin{array}{l}\text { AAMC Data Book: Statistical Information Related to Medical Schools and } \\
\text { Teaching Hospitals, Association of American Medical Colleges. }\end{array}$ \\
\hline $1-8 j$ & Racial and ethnic representation in Medicine-Asian or Pacific Islander & $\begin{array}{l}\text { AAMC Data Book: Statistical Information Related to Medical Schools and } \\
\text { Teaching Hospitals, Association of American Medical Colleges. }\end{array}$ \\
\hline $1-8 \mathrm{k}$ & Racial and ethnic representation in Medicine-Black or African American & $\begin{array}{l}\text { AAMC Data Book: Statistical Information Related to Medical Schools and } \\
\text { Teaching Hospitals, Association of American Medical Colleges. }\end{array}$ \\
\hline $1-81$ & Racial and ethnic representation in Medicine-Hispanic or Latino & $\begin{array}{l}\text { AAMC Data Book: Statistical Information Related to Medical Schools and } \\
\text { Teaching Hospitals, Association of American Medical Colleges. }\end{array}$ \\
\hline $1-8 m$ & $\begin{array}{l}\text { Racial and ethnic representation in Dentistry-American Indian or } \\
\text { Alaska Native }\end{array}$ & $\begin{array}{l}\text { Survey of Predoctoral Dental Educational Institutions, American Dental } \\
\text { Association. }\end{array}$ \\
\hline $1-8 n$ & Racial and ethnic representation in Dentistry-Asian or Pacific Islander & $\begin{array}{l}\text { Survey of Predoctoral Dental Educational Institutions, American Dental } \\
\text { Association. }\end{array}$ \\
\hline $1-80$ & $\begin{array}{l}\text { Racial and ethnic representation in Dentistry-Black or African } \\
\text { American }\end{array}$ & $\begin{array}{l}\text { Survey of Predoctoral Dental Educational Institutions, American Dental } \\
\text { Association. }\end{array}$ \\
\hline $1-8 \mathrm{p}$ & Racial and ethnic representation in Dentistry-Hispanic or Latino & $\begin{array}{l}\text { Survey of Predoctoral Dental Educational Institutions, American Dental } \\
\text { Association. }\end{array}$ \\
\hline $1-8 q$ & $\begin{array}{l}\text { Racial and ethnic representation in Pharmacy-American Indian or } \\
\text { Alaska Native }\end{array}$ & $\begin{array}{l}\text { Profile of Pharmacy Students, American Association of Colleges of } \\
\text { Pharmacy. }\end{array}$ \\
\hline $1-8 \mathrm{r}$ & Racial and ethnic representation in Pharmacy_Asian or Pacific Islander & $\begin{array}{l}\text { Profile of Pharmacy Students, American Association of Colleges of } \\
\text { Pharmacy. }\end{array}$ \\
\hline $1-8 \mathrm{~s}$ & $\begin{array}{l}\text { Racial and ethnic representation in Pharmacy-Black or African } \\
\text { American }\end{array}$ & $\begin{array}{l}\text { Profile of Pharmacy Students, American Association of Colleges of } \\
\text { Pharmacy. }\end{array}$ \\
\hline $1-8 t$ & Racial and ethnic representation in Pharmacy-Hispanic or Latino & $\begin{array}{l}\text { Profile of Pharmacy Students, American Association of Colleges of } \\
\text { Pharmacy. }\end{array}$ \\
\hline $1-9 a$ & $\begin{array}{l}\text { Hospitalization for pediatric asthma (admissions per } 10,000 \text { population, } \\
<18 \text { years) }\end{array}$ & Healthcare Cost and Utilization Project (HCUP), AHRQ. \\
\hline $1-9 b$ & $\begin{array}{l}\text { Hospitalization for uncontrolled diabetes (admissions per 10,000 } \\
\text { population, 18-64 years) }\end{array}$ & $\begin{array}{l}\text { Healthcare Cost and Utilization Project (HCUP), AHRQ. Healthcare Cost } \\
\text { and Utilization Project (HCUP), AHRQ. }\end{array}$ \\
\hline $1-9 c$ & $\begin{array}{l}\text { Hospitalization for immunization-preventable pneumonia or influenza } \\
\text { (admissions per } 10,000 \text { population, } 65+\text { years) }\end{array}$ & $\begin{array}{l}\text { Healthcare Cost and Utilization Project (HCUP), AHRQ. Healthcare Cost } \\
\text { and Utilization Project (HCUP), AHRQ. }\end{array}$ \\
\hline
\end{tabular}




\begin{tabular}{cl}
\hline Objective & \multicolumn{1}{c}{ Description } \\
\hline $1-10$ & Delay or difficulty in getting emergency care (age adjusted, 18+ years) \\
$1-11 \mathrm{a}$ & $\begin{array}{l}\text { Rapid pre-hospital emergency care-Population covered by Basic Life } \\
\text { Support }\end{array}$ \\
$1-11 \mathrm{~b}$ & $\begin{array}{l}\text { Rapid pre-hospital emergency care-Population covered by Advanced } \\
\text { Life Support }\end{array}$ \\
$1-11 \mathrm{c}$ & Rapid pre-hospital emergency care-Population covered by helicopter
\end{tabular}

1-11d Rapid pre-hospital emergency care-Pre-hospital access to online medical control

1-11e Rapid pre-hospital emergency care-Population covered by basic 9-1-1

1-11f Rapid pre-hospital emergency care-Population covered by enhanced 9$1-1$

1-11g Rapid pre-hospital emergency care-Population two-way communication between hospitals

1-12 Single toll-free number for poison control centers

1-13a Trauma care systems (no. States and D.C.)-Presence of active multidisciplinary trauma advisory committee

1-13b Trauma care systems (no. States and D.C.)-Defined process for designing trauma centers

1-13c Trauma care systems (no. States and D.C.)-Use of ACS standards for trauma center verification

1-13d Trauma care systems (no. States and D.C.)-Use of on-site survey teams for trauma center verification

Health Interview Survey (NHIS), CDC, NCHS.

National Assessment of State Trauma System Development, Emergency Medical Services Resources, Disaster Readiness for Mass Casualty Events, HRSA.

National Assessment of State Trauma System Development, Emergency Medical Services Resources, Disaster Readiness for Mass Casualty Events, HRSA.

National Assessment of State Trauma System Development, Emergency Medical Services Resources, Disaster Readiness for Mass Casualty Events, HRSA.

National Assessment of State Trauma System Development, Emergency Medical Services Resources, Disaster Readiness for Mass Casualty Events, HRSA.

National Assessment of State Trauma System Development, Emergency Medical Services Resources, Disaster Readiness for Mass Casualty Events, HRSA.

National Assessment of State Trauma System Development, Emergency Medical Services Resources, Disaster Readiness for Mass Casualty Events, HRSA.

National Assessment of State Trauma System Development, Emergency Medical Services Resources, Disaster Readiness for Mass Casualty Events, HRSA.

American Association of Poison Control Centers Survey, U.S. Poison Control Centers.

Federal Trauma-EMS Systems Program Survey, HRSA.

Federal Trauma-EMS Systems Program Survey, HRSA.

Federal Trauma-EMS Systems Program Survey, HRSA.

Federal Trauma-EMS Systems Program Survey, HRSA. 


\begin{tabular}{|c|c|c|}
\hline Objective & Description & Data Source or Objective Status \\
\hline $1-13 e$ & $\begin{array}{l}\text { Trauma care systems (no. States and D.C.) - Pre-hospital triage criteria } \\
\text { allowing for the bypass of non-designated hospitals }\end{array}$ & Federal Trauma-EMS Systems Program Survey, HRSA. \\
\hline $1-13 f$ & $\begin{array}{l}\text { Trauma care systems (no. States and D.C.)—Standardized inter-hospital } \\
\text { transfer protocols }\end{array}$ & Federal Trauma-EMS Systems Program Survey, HRSA. \\
\hline $1-13 g$ & $\begin{array}{l}\text { Trauma care systems (no. States and D.C.)-Policies describing the types } \\
\text { of patients who should be transferred }\end{array}$ & Federal Trauma-EMS Systems Program Survey, HRSA. \\
\hline $1-13 \mathrm{~h}$ & $\begin{array}{l}\text { Trauma care systems (no. States and D.C.)-Process to monitor and } \\
\text { evaluate trauma system outcomes }\end{array}$ & Federal Trauma-EMS Systems Program Survey, HRSA. \\
\hline $1-13 i$ & Trauma care systems (no. States and D.C.)—Trauma System Plan & Federal Trauma-EMS Systems Program Survey, HRSA. \\
\hline $1-14 a$ & $\begin{array}{l}\text { Special needs of children (no. States and D.C.)—Pediatric protocols for } \\
\text { online medical direction }\end{array}$ & Emergency Medical Services for Children Annual Grantees Survey, HRSA. \\
\hline $1-14 b$ & $\begin{array}{l}\text { Special needs of children (no. States and D.C.) —Pediatric guidelines for } \\
\text { emergency and critical care }\end{array}$ & Emergency Medical Services for Children Annual Grantees Survey, HRSA. \\
\hline $1-15 a$ & $\begin{array}{l}\text { Lack of access to home health care among persons with long-term care } \\
\text { needs (age adjusted, } 65+\text { years) }\end{array}$ & National Health Interview Survey (NHIS), CDC, NCHS. \\
\hline $1-15 b$ & $\begin{array}{l}\text { Lack of access to adult day care among persons with long-term care } \\
\text { needs (age adjusted, 65+ years) }\end{array}$ & National Health Interview Survey (NHIS), CDC, NCHS. \\
\hline $1-15 c$ & $\begin{array}{l}\text { Lack of access to assisted living among persons with long-term care } \\
\text { needs (age adjusted, 65+ years) }\end{array}$ & National Health Interview Survey (NHIS), CDC, NCHS. \\
\hline $1-15 d$ & $\begin{array}{l}\text { Lack of access to nursing home care services among persons with long- } \\
\text { term care needs (aged adjusted, 65+ years) }\end{array}$ & National Health Interview Survey (NHIS), CDC, NCHS. \\
\hline $1-16$ & $\begin{array}{l}\text { Pressure ulcers among nursing home residents (current diagnoses per } \\
1,000 \text { residents) }\end{array}$ & National Nursing Home Survey (NNHS), CDC, NCHS. \\
\hline
\end{tabular}


Figure 1-1. Progress Toward Target Attainment for Focus Area 1: Access to Quality Health Services

Moved away

from target ${ }^{1}$

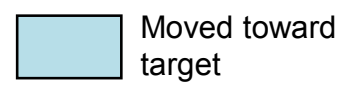

target

Met or exceeded

target

\section{1-1. Persons with health insurance $(<65$ years $)$ \\ 1-3c. Counseling about smoking cessation (age adjusted, smokers $18+$ years) \\ 1-3f. Counseling about unintended pregnancy (females 15-44 years)}

1-4. Source of ongoing care
a. All ages

b. Children and adolescents ( $<18$ years)

C. Adults $(18+$ years $)$

1-5. Persons with a usual primary care provider

1-6. Difficulties or delays in obtaining needed health care (families)

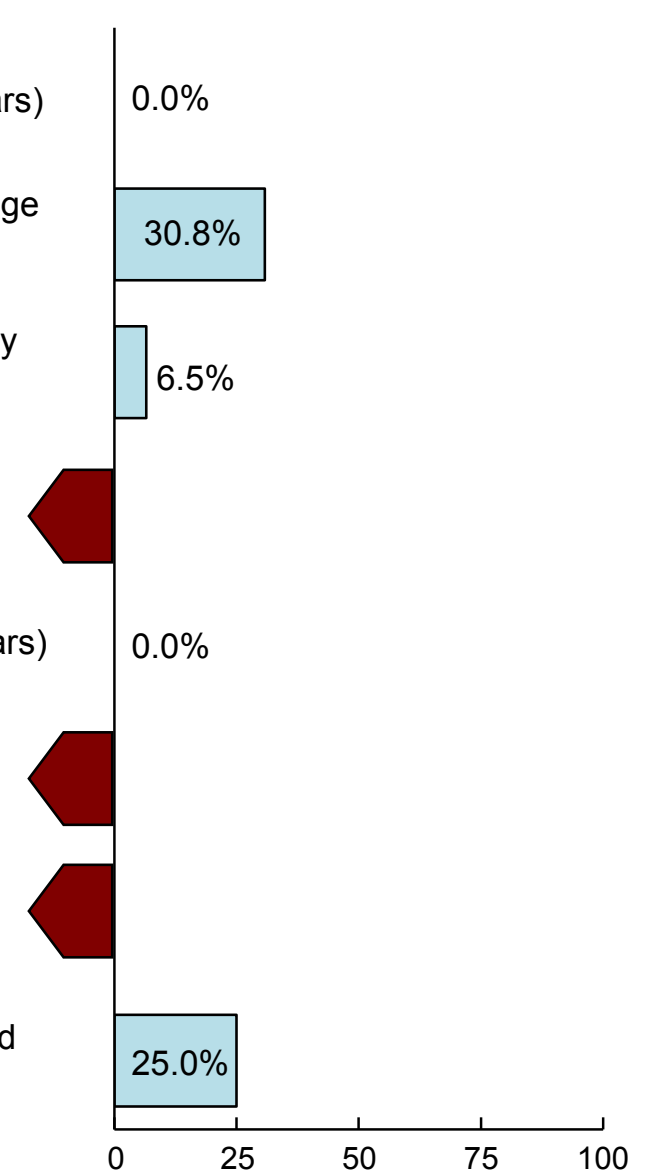

\begin{tabular}{|c|c|c|c|c|c|}
\hline \multirow{2}{*}{$\begin{array}{l}2010 \\
\text { Target }\end{array}$} & \multirow{2}{*}{$\begin{array}{c}\text { Baseline } \\
\text { (Year) }\end{array}$} & \multirow{2}{*}{$\begin{array}{l}\text { Final } \\
\text { (Year) }\end{array}$} & \multicolumn{3}{|c|}{ Baseline vs. Final } \\
\hline & & & Difference $^{2}$ & $\begin{array}{l}\text { Statistically } \\
\text { Significant }^{3}\end{array}$ & $\begin{array}{c}\text { Percent } \\
\text { Change }^{4}\end{array}$ \\
\hline $100 \%$ & $\begin{array}{c}83 \% \\
(1997)\end{array}$ & $\begin{array}{c}83 \% \\
(2008)\end{array}$ & 0 & No & $0.0 \%$ \\
\hline $66 \%$ & $\begin{array}{c}53 \% \\
(2000)\end{array}$ & $\begin{array}{c}57 \% \\
(2005)\end{array}$ & 4 & Yes & $7.5 \%$ \\
\hline $50 \%$ & $\begin{array}{c}19 \% \\
(1995)\end{array}$ & $\begin{array}{c}21 \% \\
(2006-08)\end{array}$ & 2 & $\begin{array}{c}\text { Not } \\
\text { tested }\end{array}$ & $10.5 \%$ \\
\hline $96 \%$ & $\begin{array}{c}87 \% \\
(1998)\end{array}$ & $\begin{array}{c}86 \% \\
(2008)\end{array}$ & -1 & Yes & $-1.1 \%$ \\
\hline $97 \%$ & $\begin{array}{c}94 \% \\
(1998)\end{array}$ & $\begin{array}{c}94 \% \\
(2008)\end{array}$ & 0 & No & $0.0 \%$ \\
\hline $96 \%$ & $\begin{array}{c}85 \% \\
(1998)\end{array}$ & $\begin{array}{c}84 \% \\
(2008)\end{array}$ & -1 & Yes & $-1.2 \%$ \\
\hline $85 \%$ & $\begin{array}{c}77 \% \\
(1996)\end{array}$ & $\begin{array}{c}76 \% \\
(2007)\end{array}$ & -1 & No & $-1.3 \%$ \\
\hline $9 \%$ & $\begin{array}{c}21 \% \\
(2002)\end{array}$ & $\begin{array}{c}18 \% \\
(2007)\end{array}$ & -3 & Yes & $-14.3 \%$ \\
\hline
\end{tabular}

Percent of targeted change achieved ${ }^{5}$ (continued) 
Figure 1-1. Progress Toward Target Attainment for Focus Area 1: Access to Quality Health Services (continued)

Moved away

from target ${ }^{1}$

Moved toward target

Met or exceeded

target

1-7. Medical doctor (M.D. degree)

a. Counseling for health promotion and disease prevention

b. Cultural diversity

Osteopathic medical doctor (D.O. degree)

c. Counseling for health promotion and disease prevention

d. Cultural diversity

Baccalaureate-level nurse (B.S.N., B.A., or B.S. degree)

e. Counseling for health promotion and disease prevention

f. Cultural diversity

Nurse Practitioner (M.S., M.S.N., or D.N.P. degree)

g. Counseling for health promotion and disease prevention

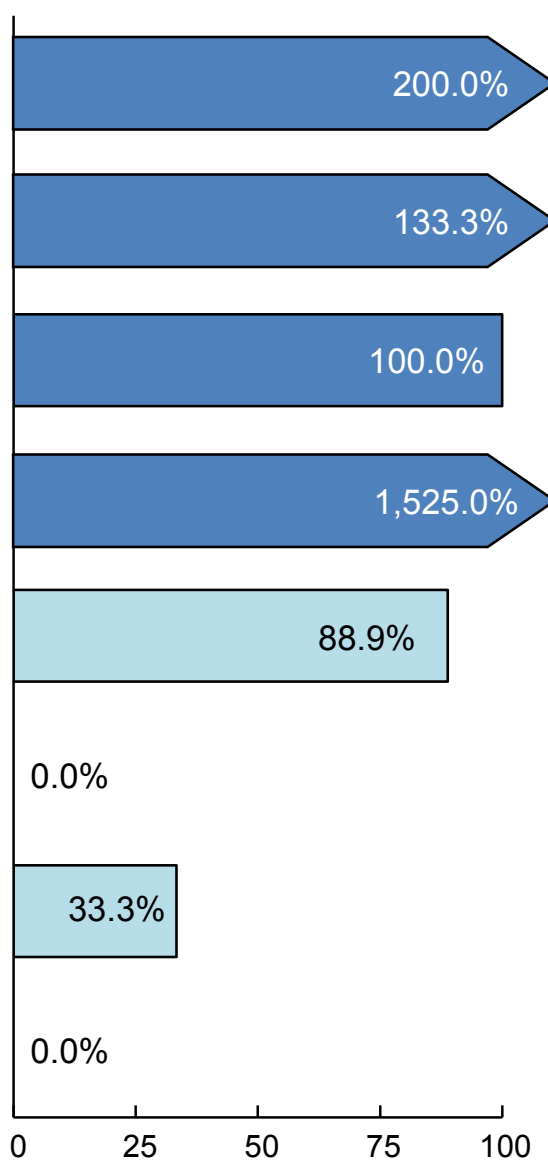

\begin{tabular}{|c|c|c|c|c|c|}
\hline \multirow{2}{*}{$\begin{array}{l}2010 \\
\text { Target }\end{array}$} & \multirow{2}{*}{$\begin{array}{c}\text { Baseline } \\
\text { (Year) }\end{array}$} & \multirow{2}{*}{$\begin{array}{l}\text { Final } \\
\text { (Year) }\end{array}$} & \multicolumn{3}{|c|}{ Baseline vs. Final } \\
\hline & & & Difference $^{2}$ & $\begin{array}{l}\text { Statistically } \\
\text { Significant }\end{array}$ & $\begin{array}{c}\text { Percent } \\
\text { Change }^{4}\end{array}$ \\
\hline $87 \%$ & $\begin{array}{c}79 \% \\
(2003-04)\end{array}$ & $\begin{array}{c}95 \% \\
(2007-08)\end{array}$ & 16 & $\begin{array}{l}\text { Not } \\
\text { tested }\end{array}$ & $20.3 \%$ \\
\hline $96 \%$ & $\begin{array}{c}87 \% \\
(1999-2000)\end{array}$ & $\begin{array}{c}99 \% \\
(2007-08)\end{array}$ & 12 & $\begin{array}{c}\text { Not } \\
\text { tested }\end{array}$ & $13.8 \%$ \\
\hline $100 \%$ & $\begin{array}{c}95 \% \\
(2003-04)\end{array}$ & $\begin{array}{l}100 \% \\
(2009)\end{array}$ & 5 & $\begin{array}{l}\text { Not } \\
\text { tested }\end{array}$ & $5.3 \%$ \\
\hline $39 \%$ & $\begin{array}{c}35 \% \\
(2003-04)\end{array}$ & $\begin{array}{c}96 \% \\
(2009)\end{array}$ & 61 & $\begin{array}{c}\text { Not } \\
\text { tested }\end{array}$ & $174.3 \%$ \\
\hline $100 \%$ & $\begin{array}{l}91 \% \\
(1999)\end{array}$ & $\begin{array}{c}99 \% \\
(2008)\end{array}$ & 8 & $\begin{array}{c}\text { Not } \\
\text { tested }\end{array}$ & $8.8 \%$ \\
\hline $100 \%$ & $\begin{array}{c}98 \% \\
(1999)\end{array}$ & $\begin{array}{c}98 \% \\
(2008)\end{array}$ & 0 & $\begin{array}{c}\text { Not } \\
\text { tested }\end{array}$ & $0.0 \%$ \\
\hline $100 \%$ & $\begin{array}{c}94 \% \\
(2000-01)\end{array}$ & $\begin{array}{c}96 \% \\
(2008)\end{array}$ & 2 & $\begin{array}{l}\text { Not } \\
\text { tested }\end{array}$ & $2.1 \%$ \\
\hline $100 \%$ & $\begin{array}{c}97 \% \\
(2000-01)\end{array}$ & $\begin{array}{l}97 \% \\
(2008)\end{array}$ & 0 & $\begin{array}{l}\text { Not } \\
\text { tested }\end{array}$ & $0.0 \%$ \\
\hline
\end{tabular}

Percent of targeted change achieved 5

(continued) 
Figure 1-1. Progress Toward Target Attainment for Focus Area 1: Access to Quality Health Services (continued)
Moved away
from target ${ }^{1}$
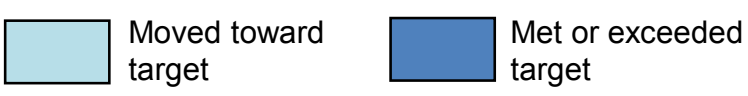

1-8. Racial and ethnic representation in health professions

a. American Indian or Alaska Native

b. Asian or Pacific Islander

c. Black or African American

d. Hispanic or Latino

Racial and ethnic representation in Nursing

e. American Indian or Alaska Native

f. Asian or Pacific Islander

g. Black or African American

h. Hispanic or Latino
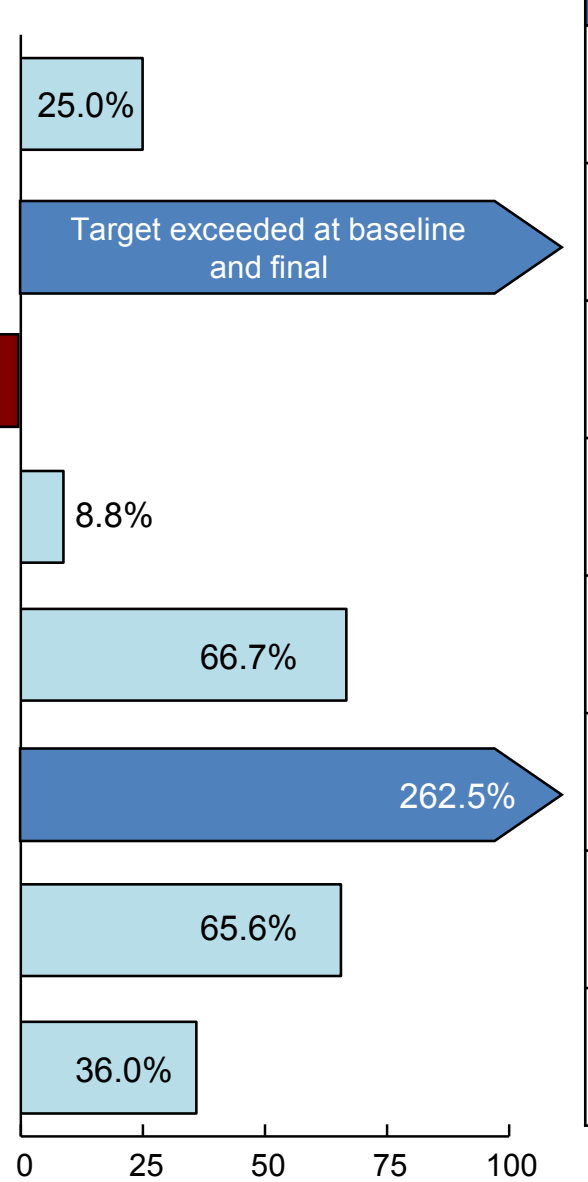

Percent of targeted change achieved ${ }^{5}$

\begin{tabular}{|c|c|c|c|c|c|}
\hline \multirow{2}{*}{$\begin{array}{l}2010 \\
\text { Target }\end{array}$} & \multirow{2}{*}{$\begin{array}{c}\text { Baseline } \\
\text { (Year) }\end{array}$} & \multirow{2}{*}{$\begin{array}{l}\text { Final } \\
\text { (Year) }\end{array}$} & \multicolumn{3}{|c|}{ Baseline vs. Final } \\
\hline & & & Difference $^{2}$ & ${ }_{2} \begin{array}{l}\text { Statistically } \\
\text { Significant }\end{array}$ & $\begin{array}{c}\text { Percent } \\
\text { Change }^{4}\end{array}$ \\
\hline $1.0 \%$ & $\begin{array}{c}0.6 \% \\
(1996-97)\end{array}$ & $\begin{array}{c}0.7 \% \\
(2008-09)\end{array}$ & 0.1 & $\begin{array}{c}\text { Not } \\
\text { tested }\end{array}$ & $16.7 \%$ \\
\hline $4.0 \%$ & $\begin{array}{c}16.3 \% \\
(1996-97)\end{array}$ & $\begin{array}{c}21.2 \% \\
(2008-09)\end{array}$ & 4.9 & $\begin{array}{c}\text { Not } \\
\text { tested }\end{array}$ & $30.1 \%$ \\
\hline $13.0 \%$ & $\begin{array}{c}6.5 \% \\
(1996-97)\end{array}$ & $\begin{array}{c}6.4 \% \\
(2008-09)\end{array}$ & -0.1 & $\begin{array}{c}\text { Not } \\
\text { tested }\end{array}$ & $-1.5 \%$ \\
\hline $12.0 \%$ & $\begin{array}{c}5.2 \% \\
(1996-97)\end{array}$ & $\begin{array}{c}5.8 \% \\
(2008-09)\end{array}$ & 0.6 & $\begin{array}{c}\text { Not } \\
\text { tested }\end{array}$ & $11.5 \%$ \\
\hline $1.0 \%$ & $\begin{array}{c}0.7 \% \\
(1995-96)\end{array}$ & $\begin{array}{c}0.9 \% \\
(2006-07)\end{array}$ & 0.2 & $\begin{array}{l}\text { Not } \\
\text { tested }\end{array}$ & $28.6 \%$ \\
\hline $4.0 \%$ & $\begin{array}{c}3.2 \% \\
(1995-96)\end{array}$ & $\begin{array}{c}5.3 \% \\
(2006-07)\end{array}$ & 2.1 & $\begin{array}{l}\text { Not } \\
\text { tested }\end{array}$ & $65.6 \%$ \\
\hline $13.0 \%$ & $\begin{array}{c}6.9 \% \\
(1995-96)\end{array}$ & $\begin{array}{c}10.9 \% \\
(2006-07)\end{array}$ & 4.0 & $\begin{array}{c}\text { Not } \\
\text { tested }\end{array}$ & $58.0 \%$ \\
\hline $12.0 \%$ & $\begin{array}{c}3.4 \% \\
(1995-96)\end{array}$ & $\begin{array}{c}6.5 \% \\
(2006-07)\end{array}$ & 3.1 & $\begin{array}{c}\text { Not } \\
\text { tested }\end{array}$ & $91.2 \%$ \\
\hline
\end{tabular}

(continued) 
Figure 1-1. Progress Toward Target Attainment for Focus Area 1: Access to Quality Health Services (continued)

Moved away

from target ${ }^{1}$
Met or exceeded

target

1-8. Racial and ethnic representation in Medicine

i. American Indian or Alaska Native

j. Asian or Pacific Islander

k. Black or African American

I. Hispanic or Latino

Racial and ethnic representation in

Dentistry

m. American Indian or Alaska Native

n. Asian or Pacific Islander

o. Black or African American

p. Hispanic or Latino

Moved toward

arget

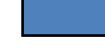


Figure 1-1. Progress Toward Target Attainment for Focus Area 1: Access to Quality Health Services (continued)

Moved away from target ${ }^{1}$
Met or exceeded target

1-8. Racial and ethnic representation in Pharmacy

q. American Indian or Alaska Native

r. Asian or Pacific Islander

s. Black or African American

t. Hispanic or Latino

1-9a. Hospitalization for pediatric asthma

(admissions per 10,000 population, $<18$ years)

1-9b. Hospitalization for uncontrolled diabetes (admissions per 10,000 population, 18-64 years)

1-9c. Hospitalization for immunizationpreventable pneumonia or influenza (admissions per 10,000 population, $65+$ years)

1-12. Single toll-free number for poison control centers
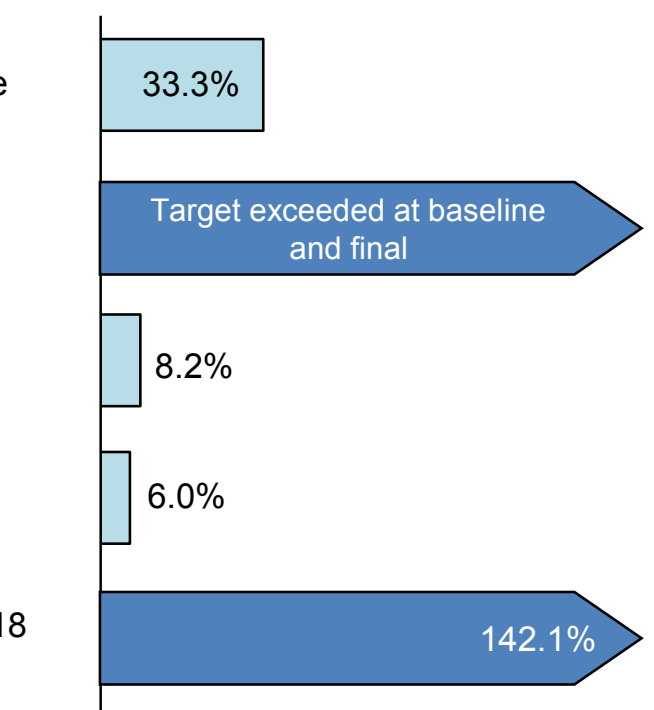

$8.2 \%$
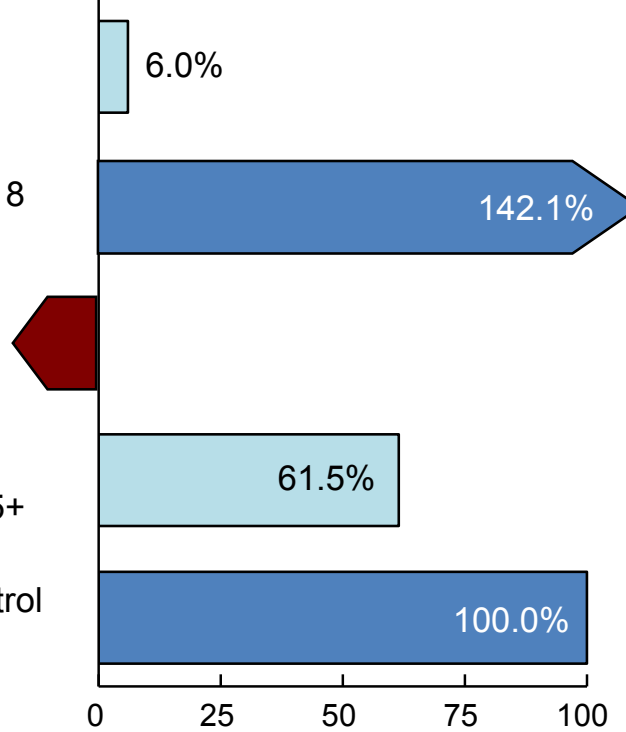

\begin{tabular}{|c|c|c|c|c|c|}
\hline \multirow{2}{*}{$\begin{array}{l}2010 \\
\text { Target }\end{array}$} & \multirow{2}{*}{$\begin{array}{c}\text { Baseline } \\
\text { (Year) }\end{array}$} & \multirow{2}{*}{$\begin{array}{l}\text { Final } \\
\text { (Year) }\end{array}$} & \multicolumn{3}{|c|}{ Baseline vs. Final } \\
\hline & & & Difference $^{2}$ & $\begin{array}{l}\text { Statistically } \\
\text { Significant }^{3}\end{array}$ & $\begin{array}{c}\text { Percent } \\
\text { Change }^{4}\end{array}$ \\
\hline $1.0 \%$ & $\begin{array}{c}0.4 \% \\
(1996-97)\end{array}$ & $\begin{array}{c}0.6 \% \\
(2008-09)\end{array}$ & 0.2 & $\begin{array}{l}\text { Not } \\
\text { tested }\end{array}$ & $50.0 \%$ \\
\hline $4.0 \%$ & $\begin{array}{c}17.5 \% \\
(1996-97)\end{array}$ & $\begin{array}{c}21.2 \% \\
(2008-09)\end{array}$ & 3.7 & $\begin{array}{l}\text { Not } \\
\text { tested }\end{array}$ & $21.1 \%$ \\
\hline $13.0 \%$ & $\begin{array}{c}5.7 \% \\
(1996-97)\end{array}$ & $\begin{array}{c}6.3 \% \\
(2008-09)\end{array}$ & 0.6 & $\begin{array}{c}\text { Not } \\
\text { tested }\end{array}$ & $10.5 \%$ \\
\hline $12.0 \%$ & $\begin{array}{c}3.6 \% \\
(1996-97)\end{array}$ & $\begin{array}{c}4.1 \% \\
(2008-09)\end{array}$ & 0.5 & $\begin{array}{c}\text { Not } \\
\text { tested }\end{array}$ & $13.9 \%$ \\
\hline 17.3 & $\begin{array}{c}23.0 \\
(1996)\end{array}$ & $\begin{array}{c}14.9 \\
(2008)\end{array}$ & -8.1 & Yes & $-35.2 \%$ \\
\hline 5.4 & $\begin{array}{c}7.2 \\
(1996)\end{array}$ & $\begin{array}{c}8.7 \\
(2008)\end{array}$ & 1.5 & Yes & $20.8 \%$ \\
\hline 7.9 & $\begin{array}{c}10.5 \\
(1996)\end{array}$ & $\begin{array}{c}8.9 \\
(2008)\end{array}$ & -1.6 & Yes & $-15.2 \%$ \\
\hline $100 \%$ & $\begin{array}{c}15 \% \\
(1999)\end{array}$ & $\begin{array}{l}100 \% \\
(2005)\end{array}$ & 85 & $\begin{array}{c}\text { Not } \\
\text { tested }\end{array}$ & $566.7 \%$ \\
\hline
\end{tabular}

Percent of targeted change achieved 5

(continued) 
Figure 1-1. Progress Toward Target Attainment for Focus Area 1: Access to Quality Health Services (continued)

Moved away

from target ${ }^{1}$

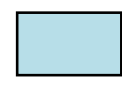

Moved toward

target

Met or exceeded

target

1-13. Trauma care systems (no. States and D.C.)

a. Presence of active multidisciplinary trauma advisory committee

b. Defined process for designing trauma centers

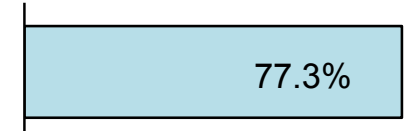

e. Pre-hospital triage criteria allowing for the bypass of non-designated hospitals

f. Standardized inter-hospital transfer protocols

i. Trauma System Plan

1-14. Special needs of children (no. States and D.C.)

a. Pediatric protocols for online medical direction

b. Pediatric guidelines for emergency and critical care

1-16. Pressure ulcers among nursing home residents (current diagnoses per 1,000 residents)

\begin{tabular}{|c|c|c|c|c|c|}
\hline \multirow{2}{*}{$\begin{array}{l}2010 \\
\text { Target }\end{array}$} & \multirow{2}{*}{$\begin{array}{c}\text { Baseline } \\
\text { (Year) }\end{array}$} & \multirow{2}{*}{$\begin{array}{l}\text { Final } \\
\text { (Year) }\end{array}$} & \multicolumn{3}{|c|}{ Baseline vs. Final } \\
\hline & & & Difference $^{2}$ & $\begin{array}{l}\text { Statistically } \\
\text { Significant }^{3}\end{array}$ & $\begin{array}{l}\text { Percent } \\
\text { Change }^{4}\end{array}$ \\
\hline 51 & $\begin{array}{c}29 \\
(2002)\end{array}$ & $\begin{array}{c}46 \\
(2005)\end{array}$ & 17 & $\begin{array}{l}\text { Not } \\
\text { tested }\end{array}$ & $58.6 \%$ \\
\hline 51 & $\begin{array}{c}34 \\
(2002)\end{array}$ & $\begin{array}{c}39 \\
(2005)\end{array}$ & 5 & $\begin{array}{c}\text { Not } \\
\text { tested }\end{array}$ & $14.7 \%$ \\
\hline 51 & $\begin{array}{c}27 \\
(2002)\end{array}$ & $\begin{array}{c}31 \\
(2005)\end{array}$ & 4 & $\begin{array}{l}\text { Not } \\
\text { tested }\end{array}$ & $14.8 \%$ \\
\hline 51 & $\begin{array}{c}23 \\
(2002)\end{array}$ & $\begin{array}{c}31 \\
(2005)\end{array}$ & 8 & $\begin{array}{l}\text { Not } \\
\text { tested }\end{array}$ & $34.8 \%$ \\
\hline 51 & $\begin{array}{c}32 \\
(2002)\end{array}$ & $\begin{array}{c}47 \\
(2005)\end{array}$ & 15 & $\begin{array}{l}\text { Not } \\
\text { tested }\end{array}$ & $46.9 \%$ \\
\hline 51 & $\begin{array}{c}18 \\
(1997)\end{array}$ & $\begin{array}{c}44 \\
(2002)\end{array}$ & 26 & $\begin{array}{c}\text { Not } \\
\text { tested }\end{array}$ & $144.4 \%$ \\
\hline 51 & $\begin{array}{c}11 \\
(1997)\end{array}$ & $\begin{array}{c}41 \\
(2003)\end{array}$ & 30 & $\begin{array}{l}\text { Not } \\
\text { tested }\end{array}$ & $272.7 \%$ \\
\hline 8 & $\begin{array}{c}16 \\
(1997)\end{array}$ & $\begin{array}{c}20 \\
(2004)\end{array}$ & 4 & No & $25.0 \%$ \\
\hline
\end{tabular}

(continued)

Percent of targeted change achieved ${ }^{5}$ 


\section{Figure 1-1. Progress Toward Target Attainment for Focus Area 1: Access to Quality Health Services (continued)}

\section{NOTES}

See the Reader's Guide for more information on how to read this figure. See DATA2010 at http://wonder.cdc.gov/data2010 for all Healthy People 2010 tracking data. Tracking data are not available for objectives 1-3a, 1-3b, 1-3d, 1-3g, 1-3h, 1-10, 1-11a through g, 1-13c, 1-13d, 1-13g, 1-13h, and 1-15a through d. Objectives 1-2 and 1-3e were deleted at the Midcourse Review.

\section{FOOTNOTES}

${ }^{1}$ Movement away from target is not quantified using the percent of targeted change achieved. See Technical Appendix for more information.

${ }^{2}$ Difference $=$ Final value - Baseline value. Differences between percents $(\%)$ are measured in percentage points.

${ }^{3}$ When estimates of variability are available, the statistical significance of the difference between the final value and the baseline value is assessed at the 0.05 level. See Technical Appendix for more information.

${ }^{4}$ Percent change $=\frac{\text { Final value }- \text { Baseline value }}{\text { Baseline value }} \times 100$

${ }^{5}$ Percent of targeted change achieved $=\frac{\text { Final value }- \text { Baseline value }}{\text { Healthy People } 2010 \text { target }- \text { Baseline value }} \times 100$.

\section{DATA SOURCES}

1-1. $\quad$ National Health Interview Survey (NHIS), CDC, NCHS.

1-3c. $\quad$ National Health Interview Survey (NHIS), CDC, NCHS.

1-3f. National Survey of Family Growth (NSFG), CDC, NCHS.

1-4a-c. National Health Interview Survey (NHIS), CDC, NCHS.

1-5-1-6. Medical Expenditure Panel Survey (MEPS), AHRQ.

1-7a-b. Liaison Committee on Medical Education (LCME) Annual Medical School Questionnaire, Association of American Medical Colleges.

1-7c-d. Annual Report on Osteopathic Medical Education, American Association of Colleges of Osteopathic Medicine.

1-7e-f. Special Healthy People Survey of Entry-Level Baccalaureate Nursing School Curriculum, formerly Survey on Women's Health in the Entry-Level Baccalaureate Nursing School Curriculum, American Association of Colleges of Nursing.

1-7g-h. Collaborative Curriculum Survey, American Association of Colleges of Nursing and National Organization of Nurse Practitioner Faculties.

1-8a-d. Survey of Predoctoral Dental Educational Institutions, American Dental Association; Profile of Pharmacy Students, American Association of Colleges of Pharmacy; AAMC Data Book, Association of American Medical Colleges; Annual Data Report, Association of Schools of Public Health.

1-8e-h. Annual Survey of RN (Registered Nurse) Programs, National League for Nursing, Center for Research in Nursing Education and Community Health.

$1-8 \mathrm{i}-\mathrm{I}$.

$1-8 m-p$.

$1-8 \mathrm{q}-\mathrm{t}$.

$1-9 a-c$.

$1-12$.

$1-13 a-b$.

$1-13 e-f$.

$1-13 \mathrm{i}$.

AAMC Data Book: Statistical Information Related to Medical Schools and Teaching Hospitals, Association of American Medical Colleges.

Survey of Predoctoral Dental Educational Institutions, American Dental Association.

Profile of Pharmacy Students, American Association of Colleges of Pharmacy.

Healthcare Cost and Utilization Project (HCUP), AHRQ.

American Association of Poison Control Centers Survey, U.S. Poison Control Centers.

Federal Trauma-EMS Systems Program Survey, HRSA.

Federal Trauma-EMS Systems Program Survey, HRSA.

Federal Trauma-EMS Systems Program Survey, HRSA.

1-14a-b. Emergency Medical Services for Children Annual Grantees Survey, HRSA.

1-16. National Nursing Home Survey (NNHS), CDC, NCHS. 
Figure 1-2. Health Disparities Table for Focus Area 1: Access to Quality Health Services Disparities from the best group rate for each characteristic at the most recent data point and changes in disparity from the baseline to the most recent data point.

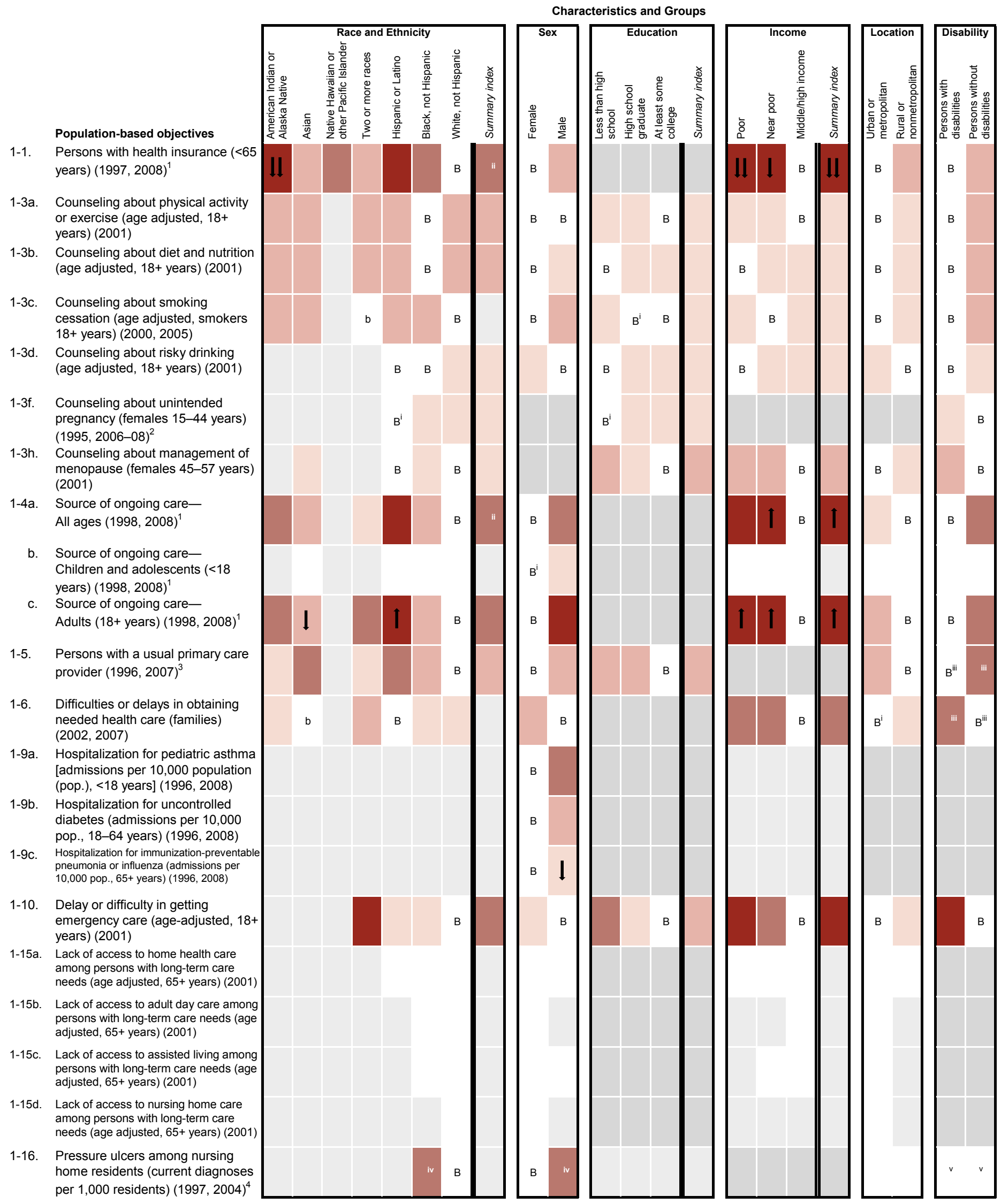

(continued) 


\section{Figure 1-2. Health Disparities Table for Focus Area 1: Access to Quality Health Services (continued)}

NOTES

See DATA2010 at http://wonder.cdc.gov/data2010 for all Healthy People 2010 tracking data. Disparity data are either unavailable or not applicable for objectives 1-3g, 1-7a through h, 1-8a through t, 1-11a through g, 1-12, 1-13a through i, 1-14a and b. Objectives 1-2 and 1-3e were deleted at Midcourse Review.

Years in parentheses represent the baseline and most recent data years (if available).

Disparity from the best group rate is defined as the percent difference between the best group rate and each of the other group rates for a characteristic (e.g., race and ethnicity). The summary index is the average of these percent differences for a characteristic. Change in disparity is estimated by subtracting the disparity at baseline from the disparity at the most recent data point. Change in the summary index is estimated by subtracting the summary index at baseline from the summary index at the most recent data point. See Technical Appendix for more information.

Measures of variability were available for all objectives in this table. Thus, the variability of best group rates was assessed, and statistical significance was tested. Disparities of $10 \%$ or more are displayed when the differences from the best group rate are statistically significant at the 0.05 level. Changes in disparities over time are indicated by arrows when the changes are greater than or equal to 10 percentage points and are statistically significant at the 0.05 level. See Technical Appendix.

\section{LEGEND}

The "best" group rate at the most recent data point.

\section{B The group with the best rate for} specified characteristic.

\section{b}

Most favorable group rate for specified characteristic, but reliability criterion not met.
Reliability criterion for best group rate not met, or data available for only one group.
Disparity from the best group rate at the most recent data point.

Percent difference from the best group rate

Less than $10 \%$, or difference not
statistically significant (when
estimates of variability are available).
$10 \%-49 \%$ $50 \%-99 \%$ $100 \%$ or more Increase in disparity (percentage points)

Changes in disparity over time are shown when:

(a) disparities data are available at both baseline and most recent time points:

(b) data are not for the group(s) indicated by "B" or "b" at either time point; and

(c) the change is greater than or equal to 10 percentage points and statistically significant, or when the change is greater than or equal to 10 percentage

points and estimates of variability were not available. See Technical Appendix.

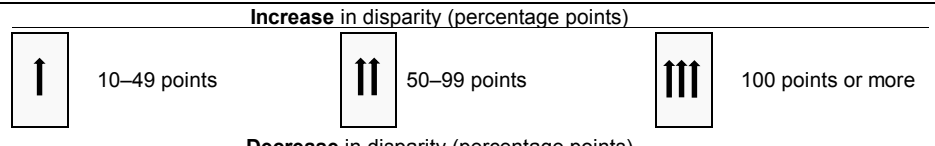

$\longrightarrow$

Availability of Data

Decrease in disparity (percentage points)

\begin{tabular}{|l|l|l|l|}
\hline $10-49$ points & II & II 100 points or more
\end{tabular}

Characteristic not selected for this objective.

FOOTNOTES

1 Baseline data by race and ethnicity are for 1999 .

2 Baseline data by disability status are for 2006-08.

3 Baseline data by race and ethnicity are for 2002 .

4 Baseline data by disability status are for 2004.

i The group with the best rate at the most recent data point is different from the group with the best rate at baseline. Both rates met the reliability criterion. See Technical Appendix.

ii Change in the summary index cannot be assessed. See Technical Appendix.

iii For this objective, only activity limitations are considered as disabilities.

iv Reliability criterion for best group rate not met, or data available for only one group, at baseline. Change in disparity cannot be assessed. See Technical Appendix.

$\checkmark$ For this objective, only severe disabilities are considered as disabilities.

\section{DATA SOURCES}

1-1. National Health Interview Survey (NHIS), CDC, NCHS.

1-3a-d. National Health Interview Survey (NHIS), CDC, NCHS.

1-3f. National Survey of Family Growth (NSFG), CDC, NCHS.

1-3h. National Health Interview Survey (NHIS), CDC, NCHS.

1-4a-c. National Health Interview Survey (NHIS), CDC, NCHS.

1-5-1-6. Medical Expenditure Panel Survey (MEPS), AHRQ.

1-9a-c. Healthcare Cost and Utilization Project (HCUP), AHRQ.

1-10. National Health Interview Survey (NHIS), CDC, NCHS.

1-15a-d. National Health Interview Survey (NHIS), CDC, NCHS.

1-16. National Nursing Home Survey (NNHS), CDC, NCHS. 
Figure 1-3. Persons with Health Insurance (<65 Years) 2008

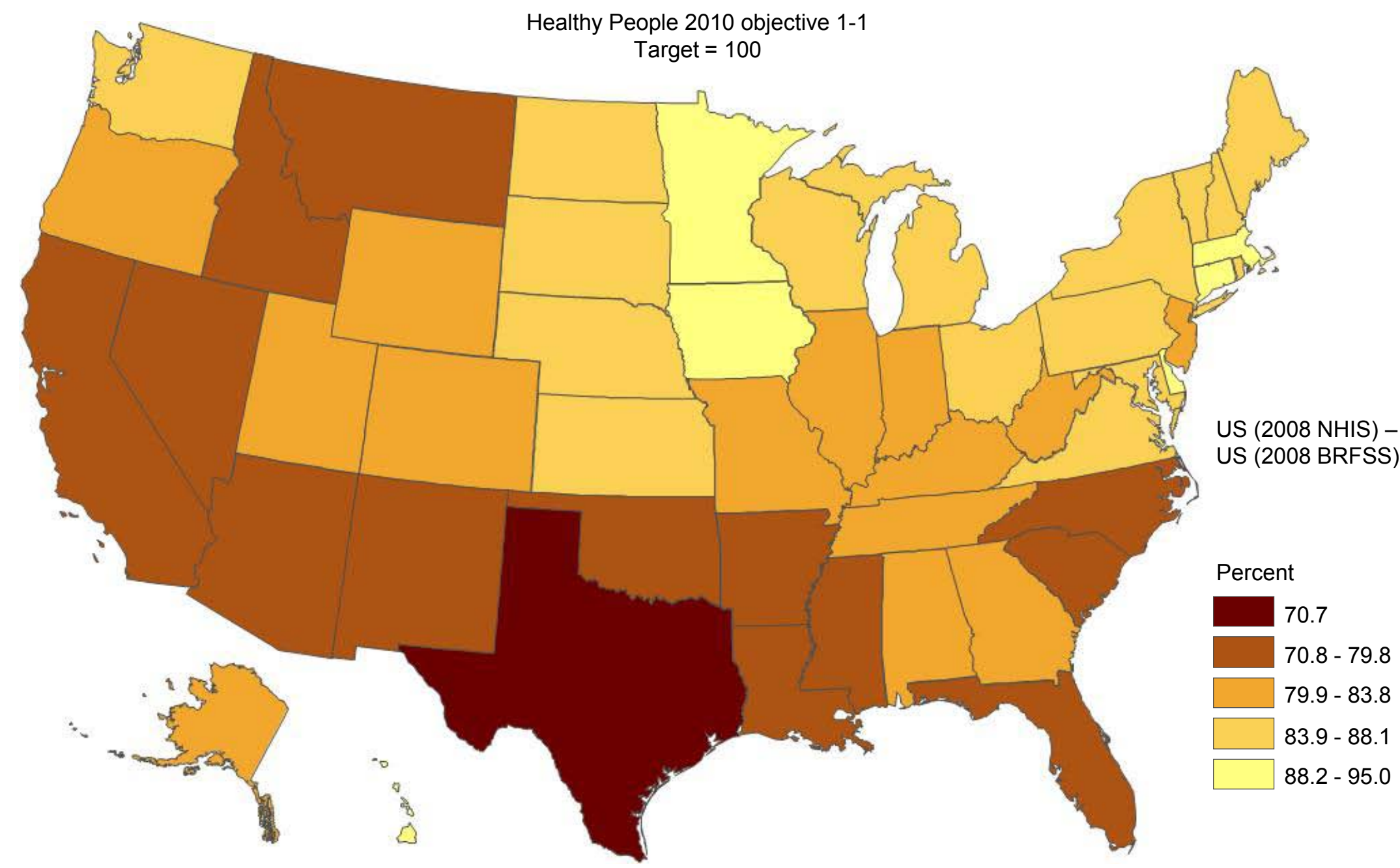

No states met the target

NOTES: Data are age-adjusted to the 2000 standard population. Rates are displayed by a Jenks classification for U.S. states. National data for the objective come from the National Health Interview Survey (NHIS) and is the basis for setting the target. State data from the BRFSS may not be comparable to the national data from the NHIS. Both US rates are shown for comparison purposes.

SOURCE: Behavioral Risk Factor Surveillance System (BRFSS), CDC, NCCDPHP. 


\section{Final Review}

\section{Arthritis, Osteoporosis, and Chronic Back Conditions}

\section{Co-Lead Agencies}

Centers for Disease Control and Prevention

National Institutes of Health

\section{Contents}

Goal.

Highlights

Summary of Progress.

Transition to Healthy People 2020.

Data Considerations

Notes

Comprehensive Summary of Objectives

Progress Chart.

Health Disparities Table

Activity Limitations due to Arthritis-Map. 


\section{Goal: Prevent illness and disability related to arthritis and other rheumatic conditions, osteoporosis, and chronic back conditions}

The objectives in this chapter measure the prevention of illness and disability related to arthritis, osteoporosis, and chronic back conditions. The arthritis objectives track a variety of pain, function, and intervention measures. The osteoporosis objectives track bone mineral density, a measure of the major risk factor for fractures. Hospitalizations for osteoporosis-related vertebral fractures are also monitored. Activity limitation due to chronic back conditions is used to measure the effects of chronic back pain.

All Healthy People tracking data quoted in this chapter, along with technical information and operational definitions for each objective, can be found in the Healthy People 2010 database, DATA2010, available from http://wonder.cdc.gov/data2010/.

More information about this focus area can be found in the following publications:

- Healthy People 2010: Understanding and Improving Health, available from http://www.healthypeople.gov/2010/Document/tableofcontents.htm\#under.

- Healthy People 2010 Midcourse Review, available from http://www.healthypeople.gov/2010/data/midcourse/html/default.htm\#FocusAreas.

\section{Highlights}

- Some progress was made for objectives in this Focus Area during the past decade [1]. Twenty-three percent of the Arthritis, Osteoporosis, and Chronic Back Conditions objectives with data to measure progress moved toward or achieved their Healthy People 2010 targets (Figure 2-1). However, statistically significant health disparities of $100 \%$ or more were observed among education and income groups (Figure 2-2), as discussed below [2].

\section{Arthritis}

- The proportion of overweight and obese adults aged 18 years and over with arthritis who received counseling for weight reduction (objective $2-4$ a) increased by $17.1 \%$ between 2002 and 2006, from 35\% to 41\% (age adjusted), moving toward the Healthy People 2010 target of $46 \%$.

- Statistically significant disparities of $100 \%$ or more were observed in the unemployment rate among adults with arthritis (objective 2-5a).

- Among education groups, persons with at least some college education had the lowest (best) unemployment rate among persons with arthritis aged 25-64 years, $27 \%$ (age-adjusted) in 2008, whereas the rate for persons with less than a high school education was $61 \%$ (age adjusted) in 2008. The rate for the population with less than a high school education was nearly two and a half times the best group rate [2]. 
- Among income groups, the middle/high income population had the lowest (best) unemployment rate among persons aged 18-64 years with arthritis, 23\% (age adjusted) in 2008, whereas the poor and near-poor populations had rates of $69 \%$ and $51 \%$ (age adjusted) in 2008, respectively. The rate for the poor population was three times the best group rate (that for the middle/high income population), while the rate for the near-poor population was more than twice the best rate [2].

- Statistically significant disparities of $100 \%$ or more also were observed in the effect of arthritis on paid work among adults with arthritis (objective 2-5b).

- Among education groups, persons with at least some college education had the lowest (best) rate of effect of arthritis on paid work among persons with arthritis aged 25-64 years, 25\% (age-adjusted) in 2006. The rate for persons with less than a high school education was 53\% (age adjusted) in 2006, more than twice the best group rate [2].

- Among income groups, the middle/high income population had the lowest (best) rate of effect of arthritis on paid work among persons with arthritis aged 18-64 years, $24 \%$ (age adjusted) in 2006 . The poor population had a rate of $58 \%$ (age adjusted) in 2008, almost two and a half times the best group rate [2].

- Activity limitations due to arthritis (objective 2-2) varied by geographic area. In 2007, the states of Connecticut, Delaware, Hawaii, Illinois, Iowa, New Jersey, North Dakota, Pennsylvania, Rhode Island, Virginia, Utah, and Wyoming had rates that met or exceeded the Healthy People 2010 target. The states of Alabama, Alaska, Georgia, Kentucky, Tennessee, and West Virginia had the highest rates (Figure 2-3).

\section{Osteoporosis}

- The prevalence of osteoporosis among adults aged 50 years and over (objective 2-9) declined by $50.0 \%$ between $1988-94$ and $2005-08$, from $12 \%$ to $6 \%$ (age adjusted), exceeding the Healthy People 2010 target of $10 \%$.

\section{Chronic Back Conditions}

- Statistically significant disparities of $100 \%$ or more were observed for activity limitations among adults aged 18 years and over with chronic back conditions (objective 2-11).

- Among racial and ethnic populations, the Hispanic or Latino population had the lowest (best) rate of activity limitations among adults with chronic back conditions, $26 \%$ (age adjusted) in 2008. Persons of two or more races had a rate of $80 \%$ (age adjusted) in 2008, more than three times the best group rate [2].

- Among education groups, persons aged 25 years and over with at least some college had the lowest (best) rate of activity limitations among adults with chronic back conditions, 27\% (age adjusted) in 2008. The rate for persons with less than a high school education was 56\% (age adjusted) in 2008, more than twice the best group rate [2]. 
- Among income groups, the middle/high income population had the lowest (best) rate of activity limitations among adults with chronic back conditions, 22\% (age adjusted) in 2008, whereas the rates for the poor and near-poor populations were $72 \%$ and $49 \%$ (age adjusted) in 2008, respectively. The rate for the poor population was nearly three and a half times the best group rate (that for the middle/high income population), while the rate for the near-poor population was more than twice the best group rate [2].

\section{Summary of Progress}

- Figure 2-1 presents a quantitative assessment of progress in achieving the Healthy People 2010 objectives for Arthritis, Osteoporosis, and Chronic Back Conditions [1]. Data to measure progress toward target attainment were available for all 13 objectives, although most objectives were only monitored over four to six years. Of these:

- One objective (2-9) exceeded the Healthy People 2010 target.

- Two objectives moved toward their targets. A statistically significant difference between the baseline and the final data points was observed for one of these objectives (2-4a). No significant difference was observed for the second objective (2-11).

- Three objectives (2-1, 2-4b, and 2-8) showed no change.

- Seven objectives moved away from their targets. A statistically significant difference between the baseline and the final data points was observed for two of these objectives (2-6 and 2-10). No significant differences were observed for the remaining five objectives (2-2, 2-3, 2-5a and $b$, and 2-7).

- Figure 2-2 displays health disparities in Arthritis, Osteoporosis, and Chronic Back Conditions from the best group rate for each characteristic at the most recent data point [2]. It also displays changes in disparities from baseline to the most recent data point [3].

- Of the seven objectives with statistically significant racial and ethnic health disparities of $10 \%$ or more, the white non-Hispanic population had the best rate for three objectives (2-5b, 2-6, and 2-7). The Hispanic or Latino population had the best rate for two objectives (2-4b and 2-11); and the Asian (objective 2-1) and black nonHispanic (objective 2-4a) populations had the best rate for one objective each.

- Females had better rates than males for three of the four objectives with statistically significant health disparities of $10 \%$ or more by sex (objectives $2-4 \mathrm{a}, 2-4 \mathrm{~b}$, and 2-7). Males had a better rate than females for the fourth objective (2-5a).

- Persons with at least some college education had the best rate for all five of the objectives with statistically significant health disparities of $10 \%$ or more by education level (objectives 2-1, 2-2, 2-5a and b, and 2-11).

- Persons with middle/high incomes had the best rate for five of the six objectives with statistically significant health disparities of $10 \%$ or more by income (objectives $2-1,2-2,2-5 a$ and $b$, and 2-11). The poor and near-poor populations both had the best rate for the sixth objective (2-4a). 
- Health disparities of $100 \%$ or more were observed for three objectives: the unemployment rate among adults with arthritis (objective 2-5a), the effect of arthritis on paid work among adults with arthritis (objective 2-5b), and activity limitations due to chronic back conditions (objective 2-11). These disparities are discussed in the Highlights, above.

\section{Transition to Healthy People 2020}

For Healthy People 2020, the focus of the Arthritis, Osteoporosis, and Chronic Back Conditions Topic Area has been expanded to include more arthritis-specific activity limitations and other health outcomes associated with arthritis and osteoporosis. Consistent with Healthy People 2010, the primary goal of the Healthy People 2020 objectives is to prevent illness and disability related to arthritis and other rheumatic conditions, osteoporosis, and chronic back conditions. See HealthyPeople.gov for a complete list of Healthy People 2020 topics and objectives.

The Healthy People 2020 Arthritis, Osteoporosis, and Chronic Back Conditions Topic Area can be grouped into four sections:

- Arthritis-related pain and impact

- Arthritis health system interventions

- Osteoporosis

- Chronic Back Conditions.

The differences between the Healthy People 2010 objectives and those included in Healthy People 2020 are summarized below:

- The Healthy People 2020 Arthritis, Osteoporosis, and Chronic Back Condition Topic Area has a total of 18 objectives, whereas the Healthy People 2010 Focus Area had 13 objectives.

- Eleven Healthy People 2010 objectives were retained "as is" [4]. Among adults with arthritis, retained objectives tracked joint pain (objective 2-1), activity limitations due to arthritis (objective 2-2), personal care limitations (objective 2-3), counseling for weight reduction (objective 2-4a), counseling for physical activity or exercise (objective 2-4b), unemployment (objective 2-5a), effect of arthritis on paid work (objective 2-5b), and arthritis education (objective 2-8). Other retained objectives include seeing a health care provider for chronic joint symptoms (objective 2-7), prevalence of osteoporosis (objective 2-9), and activity limitations due to chronic back conditions (objective 2-11).

- Two Healthy People 2010 objectives were archived: racial disparity in total knee replacements (objective 2-6) and hospitalization for osteoporosis-associated vertebral fractures (objective 2-10) [5].

- Two objectives (15-28a and b) that track hospitalizations for hip fractures among older adults (separately for females and males) were moved from the Healthy People 2010 Injury and Violence Prevention Focus Area to the Healthy People 2020 Arthritis, Osteoporosis, and Chronic Back Conditions Topic Area. 
- Five new objectives were added to the Healthy People 2020 Arthritis, Osteoporosis, and Chronic Back Conditions Topic Area:

- Four new objectives assess difficulty in performing specific joint-related activities among adults with arthritis: walking a quarter of a mile; walking up 10 steps without resting; stooping, bending or kneeling; and using fingers to grasp or handle small objects.

- A new objective assesses serious psychosocial distress among adults with arthritis.

Appendix D, “A Crosswalk Between Objectives From Healthy People 2010 to Healthy People 2020," summarizes the changes between the two decades of objectives, reflecting new knowledge and direction for this area.

\section{Data Considerations}

Figure 2-3 (Activity Limitations due to Arthritis) presents state-level data from the Behavioral Risk Factor Surveillance System (BRFSS). National data for these objectives come from the National Health Interview Survey (NHIS) and are the basis for setting the targets. BRFSS data may not be comparable to the National data from the NHIS.

Education and income are the primary measures of socioeconomic status (SES) in Healthy People 2010. Most data systems used in Healthy People 2010 define income as a family's income before taxes. In order to facilitate comparisons among groups and over time, while adjusting for family size and for inflation, Healthy People 2010 categorizes income using the poverty thresholds developed by the U.S. Census Bureau. Thus, the three categories of family income that are primarily used are:

- Poor-below the Federal poverty level

- Near poor-100\% to $199 \%$ of the Federal poverty level

- Middle/high income-200\% or more of the Federal poverty level.

These categories may be overridden by considerations specific to the data system, in which case they are modified as appropriate. See Healthy People 2010: General Data Issues, referenced below.

In general, data on educational attainment are presented for persons aged 25 years and over, consistent with guidance given by the U.S. Bureau of the Census. However, because of the requirements of the different data systems, the age groups used to calculate educational attainment for any specific objective may differ from the age groups used to report the data for other Healthy People 2010 objectives, as well as from select populations within the same objective. Therefore, the reader is urged to exercise caution in interpreting the data by educational attainment shown in the Health Disparities Table. See Healthy People 2010: General Data Issues, referenced below, for additional information.

Additional information on data issues is available from the following sources:

- All Healthy People 2010 tracking data can be found in the Healthy People 2010 database, DATA2010, available from http://wonder.cdc.gov/data2010/. 
- Detailed information about the data and data sources used to support these objectives can be found in the Operational Definitions on the DATA2010 website, available from http://wonder.cdc.gov/data2010/focusod.htm.

- More information on statistical issues related to Healthy People tracking and measurement can be found in the Technical Appendix and in Healthy People 2010: General Data Issues, which is available in the Data Issues section of the NCHS Healthy People website under Healthy People 2010.

\section{Notes}

1. Displayed in the Progress Chart (Figure 2-1), the percent of targeted change achieved expresses the difference between the baseline and the final value relative to the initial difference between the baseline and the Healthy People 2010 target. As such, it is a relative measure of progress toward attaining the Healthy People 2010 target. See the Reader's Guide for more information. When standard errors were available, the difference between the baseline and the final value was tested at the 0.05 level of significance. See the Figure 2-1 footnotes, as well as the Technical Appendix for more detail.

2. Information about disparities among select populations is shown in the Health Disparities Table (Figure 2-2). Disparity from the best group rate is defined as the percent difference between the best group rate and each of the other group rates for a characteristic. For example, racial and ethnic health disparities are measured as the percent difference between the best racial and ethnic group rate and each of the other racial and ethnic group rates. Similarly, disparities by sex are measured as the percent difference between the better group rate (e.g., female) and the rate for the other group (e.g., male). Some objectives are expressed in terms of favorable events or conditions that are to be increased, while others are expressed in terms of adverse events or conditions that are to be reduced. In order to facilitate comparison of health disparities across different objectives, disparity is measured only in terms of adverse events or conditions. For comparability across objectives, objectives that are expressed in terms of favorable events or conditions are re-expressed using the adverse event or condition for the purpose of computing disparity, but they are not otherwise restated or changed. For example, objective 1-1, to increase the proportion of persons with health insurance (e.g., $72 \%$ of the American Indian or Alaska Native population aged under 65 years had some form of health insurance in 2008), is expressed in terms of the percentage of persons without health insurance (e.g., $100 \%-72 \%=28 \%$ of the American Indian or Alaska Native population aged under 65 years did not have any form of health insurance in 2008) when the disparity from the best group rate is calculated. See the Reader's Guide for more information. When standard errors were available, the difference between the best group rate and each of the other group rates was tested at the 0.05 level of significance. See the Figure 2-2 footnotes, as well as the Technical Appendix, for more detail.

3. The change in disparity is estimated by subtracting the disparity at baseline from the disparity at the most recent data point and, therefore, is expressed as a change in percentage points. See the Reader's Guide for more information. When standard errors were available, the change in disparity was tested at the 0.05 level of significance. See the Figure 2-2 footnotes, as well as the Technical Appendix, for more detail.

4. Retained "as is" objectives have no change in the numerator definition or in the denominator definition between the Healthy People 2010 and Healthy People 2020 objectives. These include objectives that were developmental in Healthy People 2010 and are developmental in Healthy People 2020 and for which no numerator or denominator information was available.

5. Archived objectives had at least one data point in Healthy People 2010 but were not carried forward into Healthy People 2020. 


\section{Comprehensive Summary of Objectives: Arthritis, Osteoporosis, and Chronic Back Conditions}

\begin{tabular}{|c|c|c|}
\hline Objective & Description & Data Source \\
\hline $2-1$ & $\begin{array}{l}\text { Mean level of joint pain among adults with arthritis (age } \\
\text { adjusted, 18+ years) }\end{array}$ & National Health Interview Survey (NHIS), CDC, NCHS. \\
\hline $2-2$ & Activity limitations due to arthritis (age adjusted, $18+$ years) & National Health Interview Survey (NHIS), CDC, NCHS. \\
\hline $2-3$ & $\begin{array}{l}\text { Personal care limitations in adults with arthritis (age adjusted, } \\
18+\text { years) }\end{array}$ & National Health Interview Survey (NHIS), CDC, NCHS. \\
\hline $2-4 a$ & $\begin{array}{l}\text { Overweight and obese adults with arthritis who receive } \\
\text { counseling for weight reduction (age adjusted, 18+ years) }\end{array}$ & National Health Interview Survey (NHIS), CDC, NCHS. \\
\hline $2-4 b$ & $\begin{array}{l}\text { Adults with arthritis who receive counseling for physical activity } \\
\text { or exercise (age adjusted, } 18+\text { years) }\end{array}$ & National Health Interview Survey (NHIS), CDC, NCHS. \\
\hline $2-5 a$ & $\begin{array}{l}\text { Unemployment rate among adults with arthritis (age adjusted, } \\
\text { 18-64 years) }\end{array}$ & National Health Interview Survey (NHIS), CDC, NCHS. \\
\hline $2-5 b$ & $\begin{array}{l}\text { Effect of arthritis on paid work among adults with arthritis (age } \\
\text { adjusted, 18-64 years) }\end{array}$ & National Health Interview Survey (NHIS), CDC, NCHS. \\
\hline $2-6$ & $\begin{array}{l}\text { Racial disparity in total knee replacement (blacks vs. whites, 65+ } \\
\text { years) }\end{array}$ & Medicare data, CMS. \\
\hline $2-7$ & $\begin{array}{l}\text { Adults with chronic joint symptoms who saw a health care } \\
\text { provider for their symptoms (age adjusted, } 18+\text { years) }\end{array}$ & National Health Interview Survey (NHIS), CDC, NCHS. \\
\hline $2-8$ & $\begin{array}{l}\text { Arthritis education among adults with arthritis (age adjusted, } \\
18+\text { years) }\end{array}$ & National Health Interview Survey (NHIS), CDC, NCHS. \\
\hline $2-9$ & Prevalence of osteoporosis (age adjusted, 50+ years) & $\begin{array}{l}\text { National Health and Nutrition Examination Survey (NHANES), } \\
\text { CDC, NCHS. }\end{array}$ \\
\hline $2-10$ & $\begin{array}{l}\text { Hospitalization for osteoporosis-associated vertebral fractures } \\
\text { (age adjusted per 10,000 standard population, } 65+\text { years) }\end{array}$ & National Hospital Discharge Survey (NHDS), CDC, NCHS. \\
\hline $2-11$ & $\begin{array}{l}\text { Activity limitations due to chronic back conditions (age adjusted } \\
\text { per } 1,000 \text { standard population, } 18+\text { years) }\end{array}$ & National Health Interview Survey (NHIS), CDC, NCHS. \\
\hline
\end{tabular}


Figure 2-1. Progress Toward Target Attainment for Focus Area 2: Arthritis, Osteoporosis and Chronic Back Conditions

Moved away

from target ${ }^{1}$

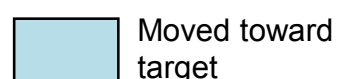

target

Met or exceeded

target

2-1. Mean level of joint pain among adults with arthritis (age adjusted, 18+ years)

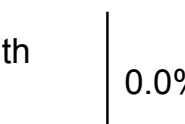

2-2. Activity limitations due to arthritis (age adjusted, 18+ years)

2-3. Personal care limitations in adults with arthritis (age adjusted, 18+ years)

2-4a. Overweight and obese adults with arthritis who receive counseling for weight reduction (age adjusted, 18+ years)

2-4b. Adults with arthritis who receive counseling for physical activity or exercise (age adjusted, $18+$ years)

2-5a. Unemployment rate among adults with arthritis (age adjusted, 18-64 years)

2-5b. Effect of arthritis on paid work among adults with arthritis (age adjusted, 18-64 years)

2-6. Racial disparity in total knee replacement (blacks vs. whites, 65+ years)

\section{$0.0 \%$}

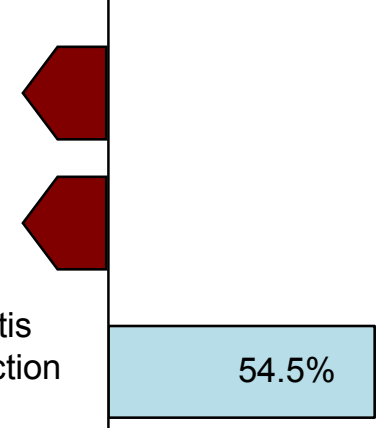

$0.0 \%$

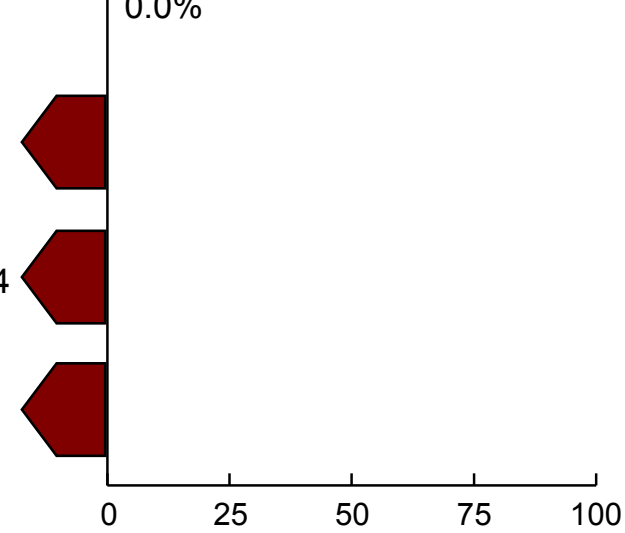

Percent of targeted change achieved ${ }^{5}$

\begin{tabular}{|c|c|c|c|c|c|}
\hline \multirow{2}{*}{$\begin{array}{c}2010 \\
\text { Target }\end{array}$} & \multirow{2}{*}{$\begin{array}{c}\text { Baseline } \\
\text { (Year) }\end{array}$} & \multirow{2}{*}{$\begin{array}{l}\text { Final } \\
\text { (Year) }\end{array}$} & \multicolumn{3}{|c|}{ Baseline vs. Final } \\
\hline & & & Difference ${ }^{2}$ & $\begin{array}{l}\text { Statistically } \\
\text { Significant }^{3}\end{array}$ & $\begin{array}{c}\text { Percent } \\
\text { Change }^{4}\end{array}$ \\
\hline 5.3 & $\begin{array}{c}5.6 \\
(2002)\end{array}$ & $\begin{array}{c}5.6 \\
(2006)\end{array}$ & 0 & No & $0.0 \%$ \\
\hline $33 \%$ & $\begin{array}{c}36 \% \\
(2002)\end{array}$ & $\begin{array}{c}39 \% \\
(2008)\end{array}$ & 3 & No & $8.3 \%$ \\
\hline $1.5 \%$ & $\begin{array}{l}2.1 \% \\
(2002)\end{array}$ & $\begin{array}{c}2.7 \% \\
(2008)\end{array}$ & 0.6 & No & $28.6 \%$ \\
\hline $46 \%$ & $\begin{array}{c}35 \% \\
(2002)\end{array}$ & $\begin{array}{c}41 \% \\
(2006)\end{array}$ & 6 & Yes & $17.1 \%$ \\
\hline $67 \%$ & $\begin{array}{c}52 \% \\
(2002)\end{array}$ & $\begin{array}{c}52 \% \\
(2006)\end{array}$ & 0 & No & $0.0 \%$ \\
\hline $27 \%$ & $\begin{array}{c}33 \% \\
(2002)\end{array}$ & $\begin{array}{c}35 \% \\
(2008)\end{array}$ & 2 & No & $6.1 \%$ \\
\hline $23 \%$ & $\begin{array}{c}30 \% \\
(2002)\end{array}$ & $\begin{array}{c}33 \% \\
(2006)\end{array}$ & 3 & No & $10.0 \%$ \\
\hline $0.0 \%$ & $\begin{array}{l}-35.9 \% \\
(2002)\end{array}$ & $\begin{array}{l}-38.4 \% \\
(2006)\end{array}$ & -2.5 & Yes & $7.0 \%$ \\
\hline
\end{tabular}

(continued) 
Figure 2-1. Progress Toward Target Attainment for Focus Area 2: Arthritis, Osteoporosis and Chronic Back Conditions (continued)

Moved away

from target ${ }^{1}$

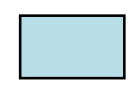

Moved toward

target

Met or exceeded

target

2-7. Adults with chronic joint symptoms who saw a health care provider for their symptoms (age adjusted, 18+ years)

2-8. Arthritis education among adults with arthritis (age adjusted, 18+ years)

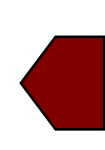

2-9. Prevalence of osteoporosis (age adjusted, $50+$ years)

2-10. Hospitalization for osteoporosis-associated vertebral fractures (age adjusted, per 10,000 population, $65+$ years)

2-11. Activity limitations due to chronic back conditions (age adjusted, per 1,000 population, $18+$ years)

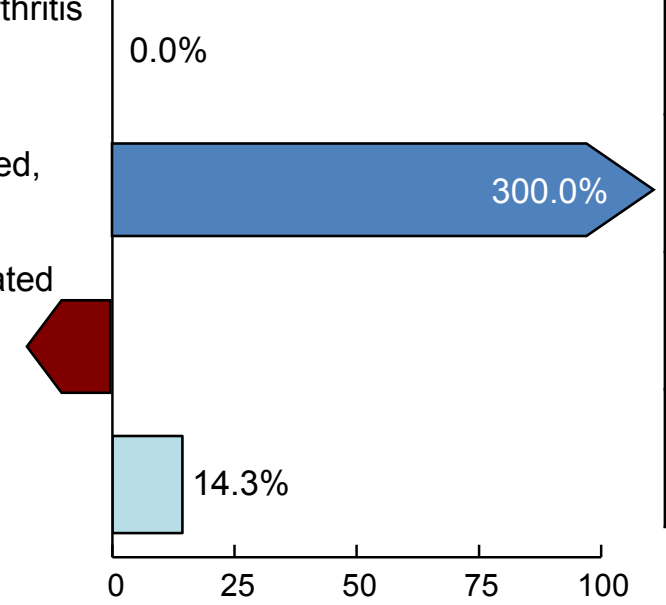

Percent of targeted change achieved ${ }^{5}$

\begin{tabular}{|c|c|c|c|c|c|}
\hline \multirow{2}{*}{$\begin{array}{l}2010 \\
\text { Target }\end{array}$} & \multirow{2}{*}{$\begin{array}{c}\text { Baseline } \\
\text { (Year) }\end{array}$} & \multirow{2}{*}{$\begin{array}{l}\text { Final } \\
\text { (Year) }\end{array}$} & \multicolumn{3}{|c|}{ Baseline vs. Final } \\
\hline & & & Difference $^{2}$ & $\begin{array}{l}\text { Statistically } \\
\text { Significant }^{3}\end{array}$ & $\begin{array}{l}\text { Percent } \\
\text { Change }^{4}\end{array}$ \\
\hline $77 \%$ & $\begin{array}{c}73 \% \\
(2002)\end{array}$ & $\begin{array}{c}72 \% \\
(2008)\end{array}$ & -1 & No & $-1.4 \%$ \\
\hline $13 \%$ & $\begin{array}{c}11 \% \\
(2002)\end{array}$ & $\begin{array}{c}11 \% \\
(2006)\end{array}$ & 0 & No & $0.0 \%$ \\
\hline $10 \%$ & $\begin{array}{c}12 \% \\
(1988-94)\end{array}$ & $\begin{array}{c}6 \% \\
(2005-08)\end{array}$ & -6 & Yes & $-50.0 \%$ \\
\hline 14.0 & $\begin{array}{c}17.5 \\
(1998)\end{array}$ & $\begin{array}{c}23.4 \\
(2007)\end{array}$ & 5.9 & Yes & $33.7 \%$ \\
\hline 25 & $\begin{array}{c}32 \\
(1997)\end{array}$ & $\begin{array}{c}31 \\
(2008)\end{array}$ & -1 & No & $-3.1 \%$ \\
\hline
\end{tabular}

(continued) 


\section{Figure 2-1. Progress Toward Target Attainment for Focus Area 2: Arthritis, Osteoporosis and Chronic Back Conditions}

(continued)

\section{NOTES}

See the Reader's Guide for more information on how to read this figure. See DATA2010 at http://wonder.cdc.gov/data2010 for all Healthy People 2010 tracking data.

\section{FOOTNOTES}

${ }^{1}$ Movement away from target is not quantified using the percent of targeted change achieved. See Technical Appendix for more information.

${ }^{2}$ Difference $=$ Final value - Baseline value. Differences between percents $(\%)$ are measured in percentage points.

${ }^{3}$ When estimates of variability are available, the statistical significance of the difference between the final value and the baseline value is assessed at the 0.05 level. See Technical Appendix for more information.

${ }^{4}$ Percent change $=\frac{\text { Final value }- \text { Baseline value }}{\text { Baseline value }} \times 100$

${ }^{5}$ Percent of targeted change achieved $=\frac{\text { Final value }- \text { Baseline value }}{\text { Healthy People } 2010 \text { target }- \text { Baseline value }} \times 100$.

\section{DATA SOURCES}

2-1-2-3. National Health Interview Survey (NHIS), CDC, NCHS.

2-4a-b. National Health Interview Survey (NHIS), CDC, NCHS.

2-5a-b. $\quad$ National Health Interview Survey (NHIS), CDC, NCHS.

2-6. Medicare data, CMS.

2-7-2-8. National Health Interview Survey (NHIS), CDC, NCHS

2-9. National Health and Nutrition Examination Survey (NHANES), CDC, NCHS.

2-10. National Hospital Discharge Survey (NHDS), CDC, NCHS

2-11. National Health Interview Survey (NHIS), CDC, NCHS. 
Figure 2-2. Health Disparities Table for Focus Area 2: Arthritis, Osteoporosis, and Chronic Back Condition

Disparities from the best group rate for each characteristic at the most recent data point and changes in disparity from the baseline to the most recent data point.

Population-based objectives

2-1. Mean level of joint pain among adults with arthritis (age adjusted, 18+ years) $(2002,2006)$

2-2. Activity limitations due to arthritis (age adjusted, 18+ years) $(2002,2008)$

2-3. Personal care limitations in adults with arthritis (age adjusted, $18+$ years $)(2002,2008)$

2-4a. Overweight and obese adults with arthritis who receive counseling for weight reduction (age adjusted, 18+ years) $(2002,2006)$

2-4b. Adults with arthritis who receive counseling for physical activity or exercise (age adjusted, 18+ years) (2002, 2006)

2-5a. Unemployment rate among adults with arthritis (age adjusted, 18-64 years) $(2002,2008)$

2-5b. Effect of arthritis on paid work among adults with arthritis (age adjusted, 18-64 years) $(2002,2006)$

2-6. Racial disparity in total knee replacement (blacks vs. whites, $65+$ years $)(2000,2006)$

2-7. Adults with chronic joint symptoms who saw a health care provider for their symptoms (age adjusted, 18+ years) $(2002,2008)$

2-8. Arthritis education among adults with arthritis (age adjusted, 18+ years) $(2002,2006)$

2-9. Prevalence of osteoporosis (age adjusted, 50+ years) (1988-94, 2005-08)

2-10. Hospitalization for osteoporosis-associated vertebral fractures (age adjusted, per 10,000 population, 65+ years) $(1998,2007)$

2-11. Activity limitations due to chronic back conditions (age adjusted, per 1,000 population, $18+$ years $)(1997,2008)^{1}$

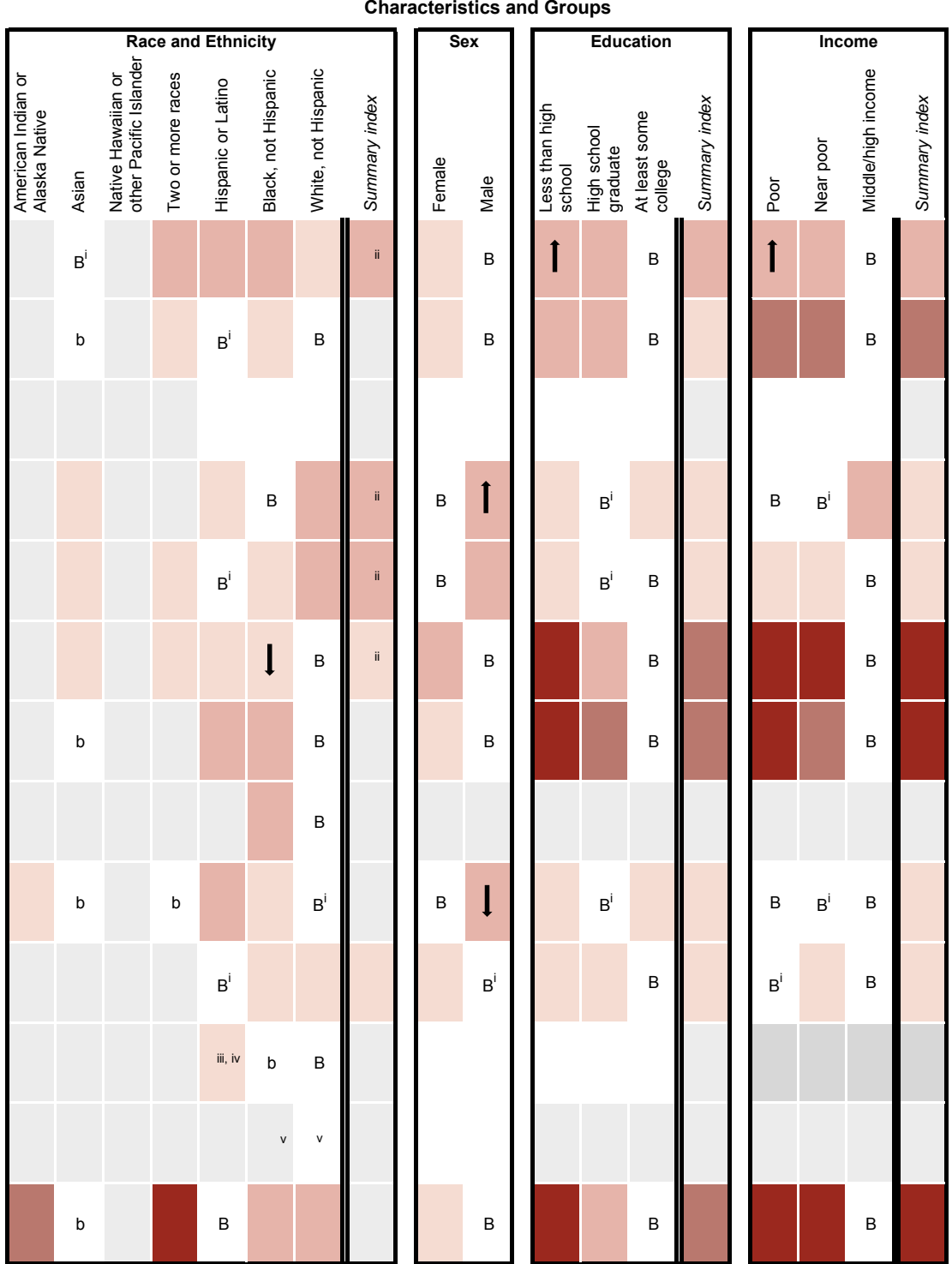

(continued) 


\section{Figure 2-2. Health Disparities Table for Focus Area 2: Arthritis, Osteoporosis, and Chronic Back Condition (continued)}

NOTES

See DATA2010 at http://wonder.cdc.gov/data2010 for all Healthy People 2010 tracking data.

Years in parentheses represent the baseline and most recent data years (if available).

Disparity from the best group rate is defined as the percent difference between the best group rate and each of the other group rates for a characteristic (e.g., race and ethnicity). The summary index is the average of these percent differences for a characteristic. Change in disparity is estimated by subtracting the disparity at baseline from the disparity at the most recent data point. Change in the summary index is estimated by subtracting the summary index at baseline from the summary index at the most recent data point. See Technical Appendix for more information.

Measures of variability were available for all objectives in this table. Thus, the variability of best group rates was assessed, and statistical significance was tested. Disparities of $10 \%$ or more are displayed when the differences from the best group rate are statistically significant at the 0.05 level. Changes in disparities over time are indicated by arrows when the changes are greater than or equal to 10 percentage points and are statistically significant at the 0.05 level. See $\underline{\text { Technical }}$ Appendix.

\section{LEGEND}

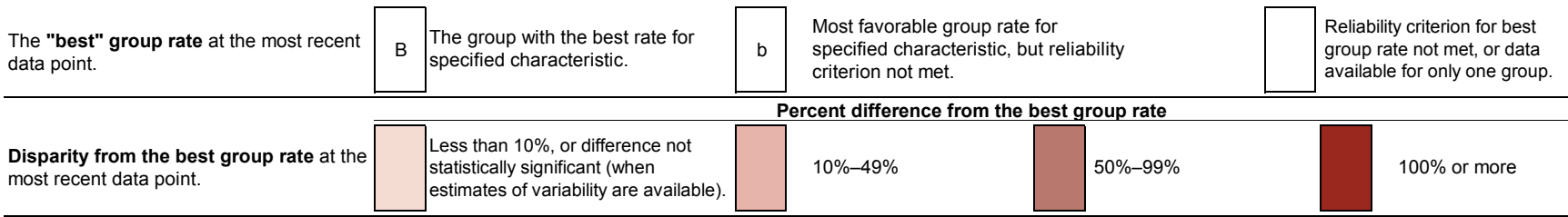

Changes in disparity over time are shown when:

(a) disparities data are available at both baseline and most recent time points;

(b) data are not for the group(s) indicated by "B" or "b" at either time point; and

(c) the change is greater than or equal to 10 percentage points and statistically significant, or when the change is greater than or equal to 10 percentage

points and estimates of variability were not available. See Technical Appendix.

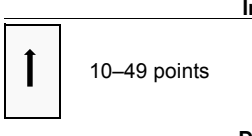

Increase in disparity (percentage points)

\begin{tabular}{llll} 
Availability of Data & Data not available. & Characteristic not selected for this objective. \\
\hline
\end{tabular}

FOOTNOTES

1 Baseline data by race and ethnicity are for 1999.

$\mathrm{i}$ The group with the best rate at the most recent data point is different from the group with the best rate at baseline. Both rates met the reliability criterion. See Technical Appendix.

ii Change in the summary index cannot be assessed. See Technical Appendix.

iii Data are for Mexican American.

iv Reliability criterion for best group rate not met, or data available for only one group, at baseline. Change in disparity cannot be assessed. See Technical Appendix.

$v$ Data include persons of Hispanic origin.

\section{DATA SOURCES}

2-1-2-3. National Health Interview Survey (NHIS), CDC, NCHS.

2-4a-b. National Health Interview Survey (NHIS), CDC, NCHS.

2-5a-b. National Health Interview Survey (NHIS), CDC, NCHS.

2-6. Medicare data, CMS.

2-7-2-8. National Health Interview Survey (NHIS), CDC, NCHS.

2-9. National Health and Nutrition Examination Survey (NHANES), CDC, NCHS.

2-10. National Hospital Discharge Survey (NHDS), CDC, NCHS.

2-11. National Health Interview Survey (NHIS), CDC, NCHS. 
Figure 2-3. Activity Limitations Due to Arthritis - Adults 18+ (2007)

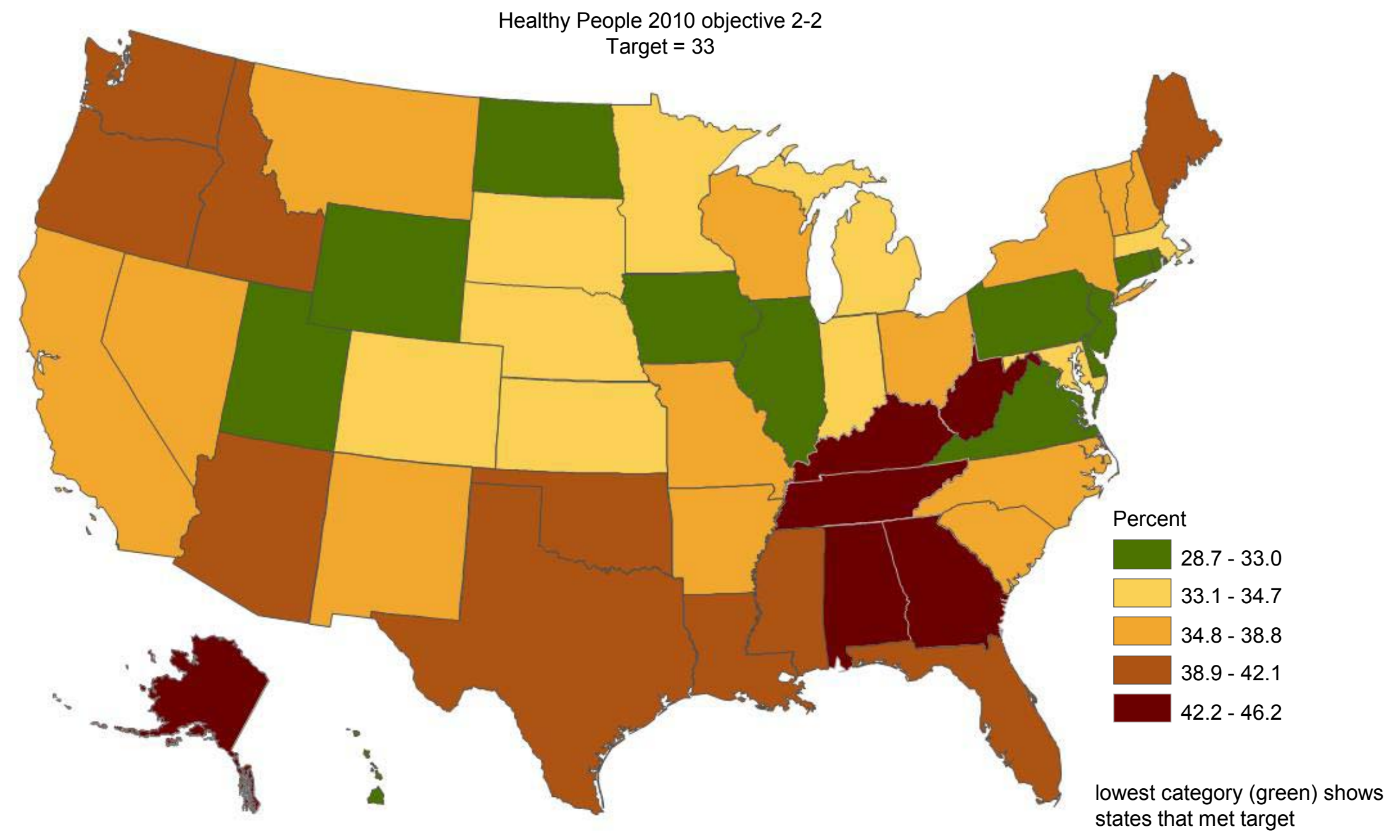

Notes: Data are age-adjusted to the 2000 standard population. Rates are displayed by a modified Jenks classification for U.S. states. National data for the objective come from the National Health Interview Survey (NHIS) and is the basis for setting the target. State data from the BRFSS may not be comparable to the national data from the NHIS.

SOURCE: Behavioral Risk Factor Surveillance System (BRFSS), CDC. 


\section{Final Review}

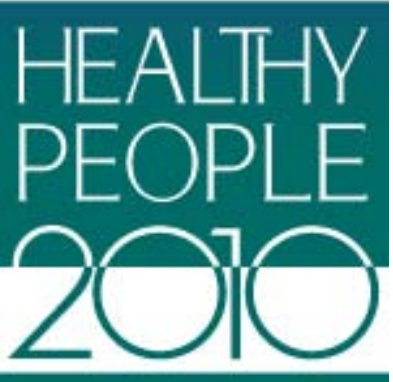

\section{Cancer}

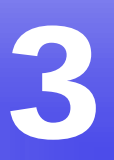

\section{Co-Lead Agencies}

Centers for Disease Control and Prevention

National Institutes of Health

\section{Contents}

Goal

Highlights

Summary of Progress

Transition to Healthy People 2020

Data Considerations

References and Notes

Comprehensive Summary of Objectives

Progress Chart

Health Disparities Table

All Cancer Death Rates-Map

Pap Test within Past Three Years-Map 


\section{Goal: Reduce the number of new cancer cases, as well as the illness, disability, and death caused by cancer}

This chapter includes objectives that track cancer death rates, survival after diagnosis, provider counseling for preventive behaviors such as smoking cessation, limiting sun exposure, the use of effective cancer screening tests, and the availability of statewide cancer registries.

All Healthy People tracking data quoted in this chapter, along with technical information and operational definitions for each objective, can be found in the Healthy People 2010 database, DATA2010, available from http://wonder.cdc.gov/data2010/.

More information about this Focus Area can be found in the following publications:

- Healthy People 2010: Understanding and Improving Health, available from http://www.healthypeople.gov/2010/Document/tableofcontents.htm\#under.

- Healthy People 2010 Midcourse Review, available from http://www.healthypeople.gov/2010/data/midcourse/html/default.htm\#FocusAreas.

\section{Highlights}

- Substantial progress was achieved in objectives for this Focus Area during the past decade [1]. Over $70 \%$ of the Cancer objectives with data to measure progress moved toward or achieved their Healthy People 2010 targets (Figure 3-1). However, for a number of objectives, statistically significant health disparities of $10 \%$ or more were observed among racial and ethnic populations, as well as by sex and education level (Figure 3-2) [2].

- Cancer deaths (objectives 3-1 through 3-8) declined for all cancer mortality objectives except melanoma deaths (objective 3-8). Prostate cancer deaths (objective 3-7) declined by $24.9 \%$ between 1999 and 2007 from 31.1 to 23.5 per 100,000 population (age adjusted), exceeding the 2010 target of 28.2 per 100,000. The overall cancer death rate (objective 3-1) declined by $11.2 \%$ from 200.8 to 178.4 per 100,000 population (age adjusted) over the same tracking period. The melanoma death rate rose by $3.8 \%$ from 2.6 to 2.7 per 100,000 population (age adjusted) over the same tracking period, moving away from the 2010 target of 2.3 per 100,000. Disparities were observed for a number of population groups:

- Among racial and ethnic groups, the Asian or Pacific Islander population had the lowest (best) cancer death rates for five of the eight cancer mortality objectives (31, 3-3 through 3-5, and 3-7). The Hispanic or Latino population had the best group rate for lung cancer (objective 3-2) and oropharyngeal cancer deaths (objective 36 ). The black non-Hispanic population had the best group rate for melanoma deaths (objective 3-8).

- With the exception of melanoma deaths (objective 3-8), the black nonHispanic population had rates that were at least $100 \%$ higher than the best rate for all cancer mortality objectives (objectives 3-1 through 3-8) [2]. 
- The white non-Hispanic population had rates that were at least $100 \%$ higher than the best group rate for four mortality objectives: lung cancer (objective 3-2), female breast cancer (objective 3-3), prostate cancer (objective 3-7), and melanoma (objective 3-8) deaths [2].

- The American Indian or Alaska Native population had a melanoma death rate (1.0 death per 100,000 population in 2007 , age adjusted) that was twice the best group rate (that for the black non-Hispanic population, 0.5 deaths per 100,000 in 2007, age adjusted) [2].

- Females had lower death rates than males for all five non-sex-specific cancer mortality objectives (objectives 3-1, 3-2, 3-5, 3-6, and 3-8). Male rates for oropharyngeal cancer (objective 3-6) and melanoma (objective 3-8) deaths were at least $100 \%$ higher than the female rates.

- Among education groups, persons with at least some college education had the lowest (best) cancer death rates for six of the eight cancer mortality objectives (3-1, 3-2, and 3-4 through 3-7). Persons with less than a high school education had the best rates for female breast cancer (objective 3-3) and melanoma (objective 3-8) deaths. Persons with less than a high school education and high school graduates had rates of lung cancer (objective 3-2), cervical cancer (objective 3-4), and oropharyngeal cancer (objective 3-6) deaths that were at least 100\% higher than the rates for persons with at least some college education.

- Overall cancer mortality (objective 3-1) varied by geographic region. Death rates for the period 2005-07 were lower in the West than they were in the Midwest and Eastern U.S. Many of the health service areas with high death rates were in the South and the Mississippi River Valley (Figure 3-3).

- The proportion of persons aged 50 years and over who had ever received a proctoscopy, colonoscopy, or sigmoidoscopy (objective 3-12b) increased by 48.6\% between 1998 and 2008, from 37\% to 55\%, exceeding the Healthy People 2010 target of 50\%.

- The proportion of women aged 18 years and over who had ever received a Pap test (objective 3-11a) increased by 1.1\% between 1998 and 2008, from $92 \%$ to 93\%, moving toward the Healthy People 2010 target of 97\%. However, the proportion who had been tested within the past three years (objective 3-11b) declined by 3.8\%, from $79 \%$ to $76 \%$, over the same tracking period, moving away from the 2010 target of $90 \%$. Disparities were observed for a number of population groups, for example:

- Among racial and ethnic groups, the populations of white non-Hispanic women and of women of two or more races both had the highest (best) rate of ever receiving a Pap test, 95\% each in 2008, whereas the populations of American Indian or Alaska Native, Hispanic or Latino, and Asian women had rates of $90 \%, 89 \%$, and $79 \%$ in 2008 , respectively. When expressed as women who had never received a Pap test, the rate for American Indian or Alaska Native women was twice the best group rate; the rate for Hispanic or Latino women was more than twice the best group rate; and the rate for Asian women was more than four times the best group rate [2].

- Among education groups, women with at least some college education had the best rate of ever receiving a Pap test, $97 \%$ in 2008, whereas women with less than a high school education had a rate of $91 \%$ in 2008 . When expressed as women who had never received a Pap test, women with less than a high school education had a rate that was three times the best group rate [2]. 
- The proportion of women who received a Pap test within the past three years varied by state. Delaware, Georgia, Massachusetts, and North Carolina had the highest proportions in 2008, whereas Arkansas, Illinois, Louisiana, Oklahoma, and a contiguous group of western states (Idaho, Montana, Nevada, Utah, Wyoming) had the lowest proportions (Figure 3-4). No state met the Healthy People 2010 target.

- Mammogram screening (objective 3-13) did not change between 1998 (baseline) and 2008 (most recent data point); in both years, $67 \%$ of women aged 40 and over had received a mammogram within the past two years, below the Healthy People 2010 target of $70 \%$.

- Most states met the 2010 target for mammograms. Only seven states were below the target in 2008: Alaska, Idaho, Mississippi, Nevada, Oklahoma, Utah, and Wyoming (Figure 3-5).

\section{Summary of Progress}

- Figure 3-1 presents a quantitative assessment of progress in achieving the Healthy People 2010 objectives for Cancer [1]. Data to measure progress toward target attainment were available for 18 objectives. Of these:

- Two objectives (3-7 and 3-12b) exceeded their Healthy People 2010 targets.

- Eleven objectives moved toward their targets. A statistically significant difference between the baseline and the final data points was observed for nine of these objectives (3-1 through 3-6, 3-9b, 3-11, and 3-15). No significant difference was observed for one objective (3-9a) and data to test the significance of the difference were unavailable for one objective (3-14).

- One objective (3-13) showed no change.

- Four objectives moved away from their targets. A statistically significant difference between the baseline and final data points was observed for three of these objectives (3-8, 3-11b, and 3-12a). No significant difference was observed for the remaining objective $(3-10 \mathrm{~h})$.

- Data were unavailable to measure progress for seven objectives (3-10a through g).

- Figure 3-2 displays health disparities in Cancer from the best group rate for each characteristic at the most recent data point [2]. It also displays changes in disparities from baseline to the most recent data point [3].

- Of the 14 objectives with statistically significant racial and ethnic health disparities of $10 \%$ or more, the Asian or Pacific Islander population had the best rate for five objectives (3-1, 3-3 through 3-5, and 3-7), and the white non-Hispanic population for four objectives (3-11a, 3-12b, 3-13, and 3-15). The black non-Hispanic and the Hispanic or Latino populations each had the best rate for three objectives $(3-8,3-$ $11 \mathrm{~b}$, and 3-13; and 3-2, 3-6, and 3-9b, respectively).

- Females had better rates than males for six of the seven objectives with statistically significant health disparities of $10 \%$ or more by sex (objectives 3-1, 3-2, 3-5, 3-6, 38 , and 3-9b). Males had a better rate than females for the objective on ever receiving a proctoscopy, colonoscopy, or sigmoidoscopy (objective 3-12b). 
- Of the 13 objectives with statistically significant health disparities of $10 \%$ or more by education level, persons with at least some college education had the best rate for 11 objectives (3-1, 3-2, 3-4 through 3-7, 3-9b, 3-11a and b, 3-12b, and 3-13). Persons with less than a high school education had the lowest (best) rate for female breast cancer (objective 3-3) and melanoma (objective 3-8) deaths.

- Persons with middle/high incomes had the best rates for all four objectives with statistically significant health disparities of $10 \%$ or more by income (objectives 3 $11 \mathrm{a}$ and $\mathrm{b}, 3-12 \mathrm{~b}$, and 3-13).

- Persons living in urban or metropolitan areas had better rates than those living in rural areas for the two objectives with statistically significant health disparities of $10 \%$ or more by geographic location (objectives $3-11$ b and $3-13$ ).

- Persons without disabilities had better rates than persons with disabilities for two of the three objectives with statistically significant health disparities of $10 \%$ or more by disability status (objectives 3-11b and 3-13). Persons with disabilities had a better rate than persons without disabilities for adults who used protective measures to protect against skin cancer (objective 3-9b).

- Health disparities of $100 \%$ or more were observed for several objectives among racial and ethnic populations, as well as by sex and education level. These are described in the Highlights, above.

\section{Transition to Healthy People 2020}

For Healthy People 2020, the Cancer objectives have been expanded to include a broader range of measures than those presented in Healthy People 2010, reflecting the latest trends in cancer prevention and diagnosis. In addition to objectives on mortality, screening, counseling, survival, and cancer registries, the Healthy People 2020 Cancer Topic Area includes new objectives on cancer incidence, quality of life for cancer survivors, prevalence of sunburn, and use of artificial sources of ultraviolet light for tanning. See HealthyPeople.gov for a complete list of Healthy People 2020 topics and objectives.

The Healthy People 2020 Cancer Topic Area can be grouped into several sections:

- Mortality

- Incidence

- Registries

- Survivorship

- Screening and counseling.

The differences between the Healthy People 2010 objectives and those included in Healthy People 2020 are summarized below:

- The Healthy People 2020 Cancer Topic Area has a total of 27 objectives, five of which are developmental, whereas the Healthy People 2010 Cancer Focus Area had 25 objectives [4]. 
- Seven Healthy People 2010 objectives, including six of the eight cancer mortality objectives $(3-1,3-3,3-4,3-6$ through 3-8) and the objective on adult protection against skin cancer (objective 3-9b), were retained "as is" [5].

- Thirteen Healthy People 2010 objectives were modified to create eleven Healthy People 2020 objectives [6].

- The objectives on lung cancer (objective 3-2) and colorectal cancer (objective 3-5) mortality were revised to match Surveillance Epidemiology and End Results (SEER) cause of death recodes [7].

- The objectives on adolescent protection against skin cancer (objective 3-9a), provider counseling on cancer screening (objectives 3-10f and g), cervical cancer screening (objective 3-11b), mammogram screening (objective 3-13), populationbased cancer registries (objective 3-14), and cancer survivorship (objective 3-15) were all modified to match the most recent available data or the latest screening guidelines.

- The objectives on fecal occult blood test (FOBT) (objective 3-12a) and sigmoidoscopy, colonoscopy, and proctoscopy (objective 3-12b) were combined into one objective on colorectal cancer screening (FOBT, sigmoidoscopy, and colonoscopy) to match the latest screening guidelines.

- Similarly, the objectives on provider counseling for FOBT (objective 3-10d) and sigmoidoscopy, colonoscopy, and proctoscopy (objective 3-10e) were combined into one objective on provider counseling for colorectal cancer screening.

- Five Healthy People 2010 Cancer objectives were either moved to other Healthy People 2020 topic areas or archived [8]. Counseling on smoking cessation (objectives 3-10a through c) and counseling on physical activity (objective 3-10h) were moved to the Healthy People 2020 Tobacco Use and Physical Activity topic areas, respectively. The objective on Pap tests ever received (objective 3-11a) was archived to match the latest screening guidelines.

- Nine new objectives were added to the Healthy People 2020 Cancer Topic Area:

- One developmental objective addresses the physical health-related quality of life of cancer survivors.

- Three new objectives track the incidence of certain cancers, namely invasive colorectal cancer, invasive uterine cervical cancer, and late-stage disease breast cancer.

- One developmental objective addresses the proportion of men who have discussed with their health care provider whether or not to have a prostate-specific antigen (PSA) test to screen for prostate cancer.

- Two new objectives monitor the prevalence of sunburn, one for adolescents and one for adults.

- Two developmental objectives focus on use of artificial sources of ultraviolet light for tanning, one for adolescents and one for adults.

Appendix D, “A Crosswalk Between Objectives From Healthy People 2010 to Healthy People 2020," summarizes the changes between the two decades of objectives, reflecting new knowledge and direction for this area. 


\section{Data Considerations}

Figures 3-4 and 3-5 (Pap tests and Mammograms, respectively) present state-level data from the Behavioral Risk Factor Surveillance System (BRFSS). National data for these objectives come from the National Health Interview Survey (NHIS) and are the basis for setting the targets. BRFSS data may not be comparable to the National data from the NHIS.

Education and income are the primary measures of socioeconomic status (SES) in Healthy People 2010. Most data systems used in Healthy People 2010 define income as a family's income before taxes. In order to facilitate comparisons among groups and over time, while adjusting for family size and for inflation, Healthy People 2010 categorizes income using the poverty thresholds developed by the U.S. Census Bureau. Thus, the three categories of family income that are primarily used are:

- Poor-below the Federal poverty level

- Near poor-100\% to $199 \%$ of the Federal poverty level

- Middle/high income-200\% or more of the Federal poverty level.

These categories may be overridden by considerations specific to the data system, in which case they are modified as appropriate. See Healthy People 2010: General Data Issues, referenced below.

In general, data on educational attainment are presented for persons aged 25 years and over, consistent with guidance given by the U.S. Bureau of the Census. However, because of the requirements of the different data systems, the age groups used to calculate educational attainment for any specific objective may differ from the age groups used to report the data for other Healthy People 2010 objectives, as well as from select populations within the same objective. Therefore, the reader is urged to exercise caution in interpreting the data by educational attainment shown in the Health Disparities Table. See Healthy People 2010: General Data Issues, referenced below.

Beginning in 2003, education data for mortality objectives 3-1 through 3-8 from the National Vital Statistics System have been suppressed. The educational attainment item was changed in the new U.S. Standard Certificate of Death in 2003 to be consistent with the U.S. Census Bureau data and to improve the ability to identify specific types of educational degrees. Many states, however, are still using the 1989 version of the U.S. Standard Certificate of Death, which focuses on highest school grade completed. As a result, educational attainment data collected using the 2003 version are not comparable with data collected using the 1989 version [9].

Additional information on data issues is available from the following sources:

- All Healthy People 2010 tracking data can be found in the Healthy People 2010 database, DATA2010, available from http://wonder.cdc.gov/data2010/.

- Detailed information about the data and data sources used to support these objectives can be found in the Operational Definitions on the DATA2010 website, available from http://wonder.cdc.gov/data2010/focusod.htm. 
- More information on statistical issues related to Healthy People tracking and measurement can be found in the Technical Appendix and in Healthy People 2010: General Data Issues, which is available in the Data Issues section of the NCHS Healthy People website under Healthy People 2010.

\section{References and Notes}

1. Displayed in the Progress Chart (Figure 3-1), the percent of targeted change achieved expresses the difference between the baseline and the final value relative to the initial difference between the baseline and the Healthy People 2010 target. As such, it is a relative measure of progress toward attaining the Healthy People 2010 target. See the Reader's Guide for more information. When standard errors were available, the difference between the baseline and the final value was tested at the 0.05 level of significance. See the Figure 3-1 footnotes, as well as the Technical Appendix, for more detail.

2. Information about disparities among select populations is shown in the Health Disparities Table (Figure 3-2). Disparity from the best group rate is defined as the percent difference between the best group rate and each of the other group rates for a characteristic. For example, racial and ethnic health disparities are measured as the percent difference between the best racial and ethnic group rate and each of the other racial and ethnic group rates. Similarly, disparities by sex are measured as the percent difference between the better group rate (e.g., female) and the rate for the other group (e.g., male). Some objectives are expressed in terms of favorable events or conditions that are to be increased, while others are expressed in terms of adverse events or conditions that are to be reduced. In order to facilitate comparison of health disparities across different objectives, disparity is measured only in terms of adverse events or conditions. For comparability across objectives, objectives that are expressed in terms of favorable events or conditions are re-expressed using the adverse event or condition for the purpose of computing disparity, but they are not otherwise restated or changed. For example, objective 1-1, to increase the proportion of persons with health insurance (e.g., $72 \%$ of the American Indian or Alaska Native population aged under 65 years had some form of health insurance in 2008), is expressed in terms of the percentage of persons without health insurance (e.g., $100 \%-72 \%=28 \%$ of the American Indian or Alaska Native population aged under 65 years did not have any form of health insurance in 2008) when the disparity from the best group rate is calculated. See the Reader's Guide for more information. When standard errors were available, the difference between the best group rate and each of the other group rates was tested at the 0.05 level of significance. See the Figure 3-2 footnotes, as well as the Technical Appendix, for more detail.

3. The change in disparity is estimated by subtracting the disparity at baseline from the disparity at the most recent data point and, therefore, is expressed as a change in percentage points. See the Reader's Guide for more information. When standard errors were available, the change in disparity was tested at the 0.05 level of significance. See the Figure 3-2 footnotes, as well as the Technical Appendix, for more detail.

4. To be included in Healthy People 2010, an objective must have a national data source that provides a baseline and at least one additional data point for tracking progress. Some objectives lacked baseline data at the time of their development but had a potential data source and were considered of sufficient national importance to be included in Healthy People. These are called "developmental" objectives. When data become available, a developmental objective is moved to measurable status and a Healthy People target can be set.

5. Retained "as is" objectives have no change in the numerator definition or in the denominator definition between the Healthy People 2010 and Healthy People 2020 objectives. These include objectives that were developmental in Healthy People 2010 and are developmental in Healthy People 2020 and for which no numerator or denominator information was available. 
6. Modified objectives have some change in the numerator definition or in the denominator definition between the Healthy People 2010 and Healthy People 2020 objectives. These include objectives that went from developmental in Healthy People 2010 to measurable in Healthy People 2020 or vice versa.

7. Cancer mortality data in Healthy People 2020 have been recoded for consistency with cancer incidence and mortality data reported by U.S. Cancer Statistics (USCS), CDC and Surveillance Epidemiology and End Results (SEER), NIH, NCI, resulting in slight changes to definitions for lung and colorectal cancer between Healthy People 2010 and Healthy People 2020. Specifications for the cancer mortality recodes can be found on the SEER website, available from: http://seer.cancer.gov/codrecode.

8. Archived objectives had at least one data point in Healthy People 2010 but were not carried forward into Healthy People 2020.

9. Xu JQ, Kochanek KD, Murphy SL, Tejada-Vera B. Deaths: Final data for 2007. National vital statistics reports; vol 58 no 19. Hyattsville, MD: National Center for Health Statistics. 2010. Available from: http://www.cdc.gov/nchs/data/nvsr/nvsr58/nvsr58 19.pdf. 


\section{Comprehensive Summary of Objectives: Cancer}

\begin{tabular}{|c|c|c|}
\hline Objective & Description & Data Source \\
\hline $3-1$ & Overall cancer deaths (age adjusted per 100,000 standard population) & National Vital Statistics System-Mortality (NVSS-M), CDC, NCHS. \\
\hline $3-2$ & Lung cancer deaths (age adjusted per 100,000 standard population) & National Vital Statistics System-Mortality (NVSS-M), CDC, NCHS. \\
\hline $3-3$ & $\begin{array}{l}\text { Female breast cancer deaths (age adjusted per 100,000 standard } \\
\text { population) }\end{array}$ & National Vital Statistics System-Mortality (NVSS-M), CDC, NCHS. \\
\hline $3-4$ & Cervical cancer deaths (age adjusted per 100,000 standard population) & National Vital Statistics System—Mortality (NVSS-M), CDC, NCHS. \\
\hline $3-5$ & Colorectal cancer deaths (age adjusted per 100,000 standard population) & National Vital Statistics System-Mortality (NVSS-M), CDC, NCHS. \\
\hline $3-6$ & $\begin{array}{l}\text { Oropharyngeal cancer deaths (age adjusted per 100,000 standard } \\
\text { population) }\end{array}$ & National Vital Statistics System—Mortality (NVSS-M), CDC, NCHS. \\
\hline $3-7$ & Prostate cancer deaths (age adjusted per 100,000 standard population) & National Vital Statistics System-Mortality (NVSS-M), CDC, NCHS. \\
\hline 3-8 & Melanoma deaths (age adjusted per 100,000 standard population) & National Vital Statistics System-Mortality (NVSS-M), CDC, NCHS. \\
\hline $3-9 a$ & $\begin{array}{l}\text { Sun exposure and skin cancer-Students who use protective measures } \\
\text { (grades 9-12) }\end{array}$ & Youth Risk Behavior Surveillance System (YRBSS), CDC, NCCDPHP. \\
\hline $3-9 b$ & $\begin{array}{l}\text { Sun exposure and skin cancer-Adults who use protective measures (age } \\
\text { adjusted, 18+ years) }\end{array}$ & National Health Interview Survey (NHIS), CDC, NCHS. \\
\hline $3-10 a$ & Internist counseling about smoking cessation & $\begin{array}{l}\text { Survey of Physicians' Attitudes and Practices in Early Cancer Detection, } \\
\text { American Cancer Society. }\end{array}$ \\
\hline $3-10 b$ & Family physician counseling about smoking cessation & $\begin{array}{l}\text { Survey of Physicians' Attitudes and Practices in Early Cancer Detection, } \\
\text { American Cancer Society. }\end{array}$ \\
\hline $3-10 c$ & Dentist counseling about smoking cessation & Survey of Current Issues in Dentistry, American Dental Association. \\
\hline $3-10 d$ & Primary care provider counseling about blood stool tests & $\begin{array}{l}\text { National Survey of Primary Care Physicians' Recommendations and } \\
\text { Practice for Breast, Cervical, Colorectal, and Lung Cancer Screening, NIH, } \\
\text { NCI. }\end{array}$ \\
\hline $3-10 \mathrm{e}$ & Primary care provider counseling about proctoscopic examinations & $\begin{array}{l}\text { Survey of Physicians' Attitudes and Practices in Early Cancer Detection, } \\
\text { American Cancer Society. }\end{array}$ \\
\hline 3-10f & Primary care provider counseling about mammograms & $\begin{array}{l}\text { National Survey of Primary Care Physicians' Recommendations and } \\
\text { Practice for Breast, Cervical, Colorectal, and Lung Cancer Screening, NIH, } \\
\text { NCI. }\end{array}$ \\
\hline
\end{tabular}




\begin{tabular}{|c|c|c|}
\hline Objective & Description & Data Source \\
\hline $3-10 \mathrm{~g}$ & Primary care provider counseling about Pap tests & $\begin{array}{l}\text { National Survey of Primary Care Physicians' Recommendations and } \\
\text { Practice for Breast, Cervical, Colorectal, and Lung Cancer Screening, NIH, } \\
\text { NCI. }\end{array}$ \\
\hline $3-10 \mathrm{~h}$ & Primary care provider counseling about physical activity & National Ambulatory Medical Care Survey (NAMCS), CDC, NCHS \\
\hline $3-11 \mathrm{a}$ & Pap tests-Ever received (age adjusted, 18+ years) & National Health Interview Survey (NHIS), CDC, NCHS. \\
\hline $3-11 b$ & Pap tests-Received within past 3 years (age adjusted, 18+ years) & National Health Interview Survey (NHIS), CDC, NCHS. \\
\hline $3-12 a$ & $\begin{array}{l}\text { Colorectal cancer screening-Fecal occult blood test (FOBT) within past } \\
2 \text { years (age adjusted, } 50+\text { years) }\end{array}$ & National Health Interview Survey (NHIS), CDC, NCHS. \\
\hline $3-12 b$ & $\begin{array}{l}\text { Colorectal cancer screening_-Proctoscopy, colonoscopy, or } \\
\text { sigmoidoscopy ever received (age adjusted, 50+ years) }\end{array}$ & National Health Interview Survey (NHIS), CDC, NCHS. \\
\hline 3-13 & $\begin{array}{l}\text { Women receiving a mammogram within past } 2 \text { years (age adjusted, } 40+ \\
\text { years) }\end{array}$ & National Health Interview Survey (NHIS), CDC, NCHS. \\
\hline $3-14$ & Statewide cancer registries (no. States and D.C.) & National Program of Cancer Registries (NPCR), CDC, NCCDPHP. \\
\hline 3-15 & Persons living $5+$ years after a diagnosis of cancer & Surveillance, Epidemiology, and End Results (SEER) Program, NIH, NCI. \\
\hline
\end{tabular}


Figure 3-1. Progress Toward Target Attainment for Focus Area 3: Cancer

Moved away

from target ${ }^{1}$

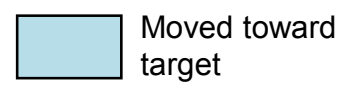

target

Met or exceeded

target

3-1. Overall cancer deaths (age adjusted, per 100,000 population)

3-2. Lung cancer deaths (age adjusted, per 100,000 population)

3-3. Female breast cancer deaths (age adjusted, per 100,000 population)

3-4. Cervical cancer deaths (age adjusted, per 100,000 population)

3-5. Colorectal cancer deaths (age adjusted, per 100,000 population)

3-6. Oropharyngeal cancer deaths (age adjusted, per 100,000 population)

3-7. Prostate cancer deaths (age adjusted, per 100,000 population)

3-8. Melanoma deaths (age adjusted, per 100,000 population)
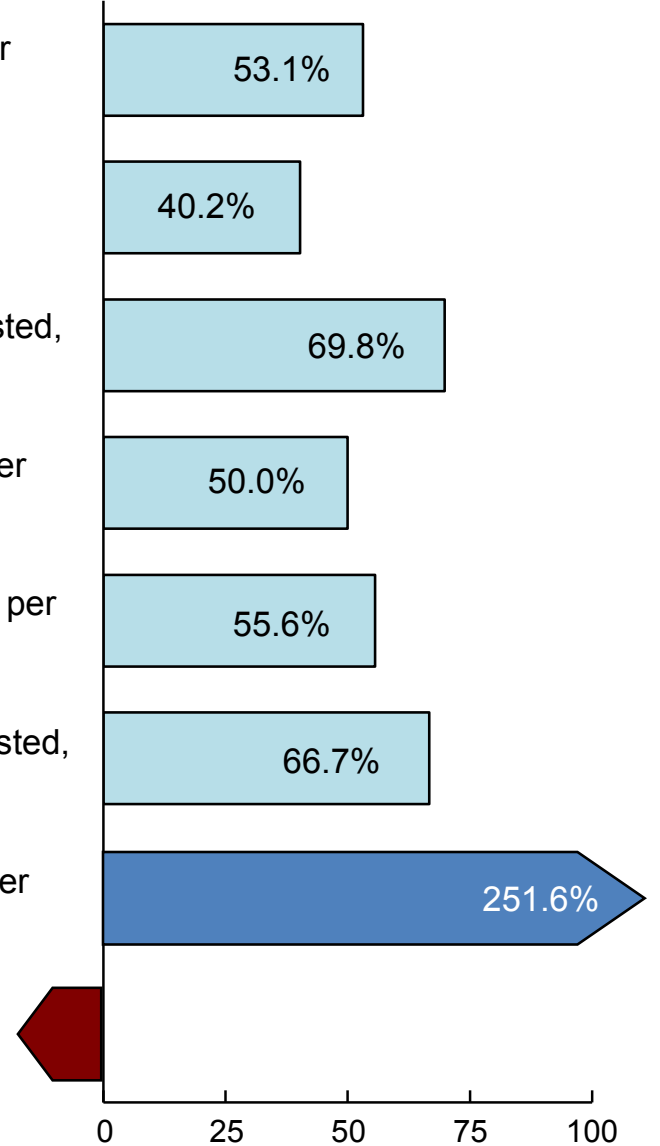

Percent of targeted change achieved 5

\begin{tabular}{|c|c|c|c|c|c|}
\hline \multirow{2}{*}{$\begin{array}{l}2010 \\
\text { Target }\end{array}$} & \multirow{2}{*}{$\begin{array}{l}\text { Baseline } \\
\text { (Year) }\end{array}$} & \multirow{2}{*}{$\begin{array}{l}\text { Final } \\
\text { (Year) }\end{array}$} & \multicolumn{3}{|c|}{ Baseline vs. Final } \\
\hline & & & Difference² & $\begin{array}{l}\text { Statistically } \\
\text { Significant }^{3}\end{array}$ & $\begin{array}{l}\text { Percent } \\
\text { Change }^{4}\end{array}$ \\
\hline 158.6 & $\begin{array}{l}200.8 \\
(1999)\end{array}$ & $\begin{array}{c}178.4 \\
(2007)\end{array}$ & -22.4 & Yes & $-11.2 \%$ \\
\hline 43.3 & $\begin{array}{c}55.5 \\
(1999)\end{array}$ & $\begin{array}{c}50.6 \\
(2007)\end{array}$ & -4.9 & Yes & $-8.8 \%$ \\
\hline 21.3 & $\begin{array}{c}26.6 \\
(1999)\end{array}$ & $\begin{array}{c}22.9 \\
(2007)\end{array}$ & -3.7 & Yes & $-21.1 \%$ \\
\hline 2.0 & $\begin{array}{c}2.8 \\
(1999)\end{array}$ & $\begin{array}{c}2.4 \\
(2007)\end{array}$ & -0.4 & Yes & $-14.3 \%$ \\
\hline 13.7 & $\begin{array}{c}20.9 \\
(1999)\end{array}$ & $\begin{array}{c}16.9 \\
(2007)\end{array}$ & -4.0 & Yes & $-19.1 \%$ \\
\hline 2.4 & $\begin{array}{c}2.7 \\
(1999)\end{array}$ & $\begin{array}{c}2.5 \\
(2007)\end{array}$ & -0.2 & Yes & $-7.4 \%$ \\
\hline 28.2 & $\begin{array}{c}31.1 \\
(1999)\end{array}$ & $\begin{array}{c}23.5 \\
(2007)\end{array}$ & -7.6 & Yes & $-24.9 \%$ \\
\hline 2.3 & $\begin{array}{c}2.6 \\
(2000)\end{array}$ & $\begin{array}{c}2.7 \\
(2007)\end{array}$ & 0.1 & Yes & $3.8 \%$ \\
\hline
\end{tabular}

(continued) 
Figure 3-1. Progress Toward Target Attainment for Focus Area 3: Cancer (continued)

Moved away

from target ${ }^{1}$

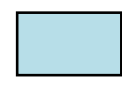

Moved toward

target

Met or exceeded

target

3-9. Sun exposure and skin cancer

a. Students who use protective measures (grades 9-12)

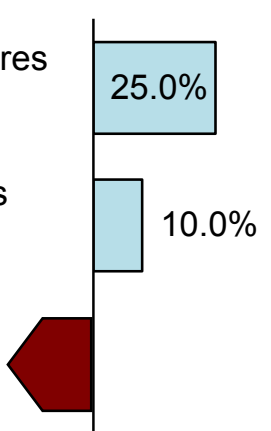

b. Adults who use protective measures (age adjusted, 18+ years)

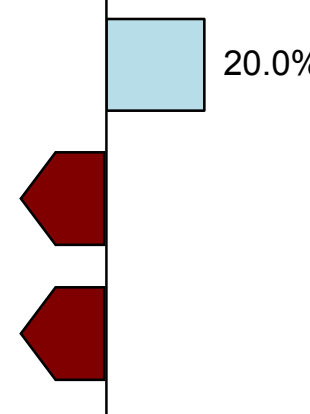

b. Proctoscopy, colonoscopy, or sigmoidoscopy ever received (age adjusted, $50+$ years)

3-13. Women receiving a mammogram within past 2 years (age adjusted, $40+$ years)

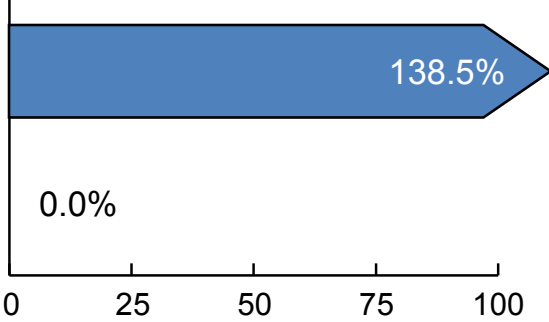

Percent of targeted change achieved ${ }^{5}$

\begin{tabular}{|c|c|c|c|c|c|}
\hline \multirow{2}{*}{$\begin{array}{l}2010 \\
\text { Target }\end{array}$} & \multirow{2}{*}{$\begin{array}{c}\text { Baseline } \\
\text { (Year) }\end{array}$} & \multirow{2}{*}{$\begin{array}{l}\text { Final } \\
\text { (Year) }\end{array}$} & \multicolumn{3}{|c|}{ Baseline vs. Final } \\
\hline & & & Difference 2 & $\begin{array}{l}\text { Statistically } \\
\text { Significant }^{3}\end{array}$ & $\begin{array}{l}\text { Percent } \\
\text { Change }^{4}\end{array}$ \\
\hline $28 \%$ & $\begin{array}{c}24 \% \\
(2005)\end{array}$ & $\begin{array}{c}25 \% \\
(2007)\end{array}$ & 1 & No & $4.2 \%$ \\
\hline $85 \%$ & $\begin{array}{c}65 \% \\
(2005)\end{array}$ & $\begin{array}{c}67 \% \\
(2008)\end{array}$ & 2 & Yes & $3.1 \%$ \\
\hline $85 \%$ & $\begin{array}{c}12 \% \\
(1998)\end{array}$ & $\begin{array}{c}10 \% \\
(2007)\end{array}$ & -2 & No & -16.7 \\
\hline $97 \%$ & $\begin{array}{c}92 \% \\
(1998)\end{array}$ & $\begin{array}{c}93 \% \\
(2008)\end{array}$ & 1 & Yes & $1.1 \%$ \\
\hline $90 \%$ & $\begin{array}{c}79 \% \\
(1998)\end{array}$ & $\begin{array}{c}76 \% \\
(2008)\end{array}$ & -3 & Yes & $-3.8 \%$ \\
\hline $33 \%$ & $\begin{array}{c}24 \% \\
(2000)\end{array}$ & $\begin{array}{c}15 \% \\
(2008)\end{array}$ & -9 & Yes & $-37.5 \%$ \\
\hline $50 \%$ & $\begin{array}{c}37 \% \\
(1998)\end{array}$ & $\begin{array}{c}55 \% \\
(2008)\end{array}$ & 18 & Yes & $48.6 \%$ \\
\hline $70 \%$ & $\begin{array}{c}67 \% \\
(1998)\end{array}$ & $\begin{array}{c}67 \% \\
(2008)\end{array}$ & 0 & No & $0.0 \%$ \\
\hline
\end{tabular}

(continued) 
Figure 3-1. Progress Toward Target Attainment for Focus Area 3: Cancer (continued)

Moved away

from target ${ }^{1}$

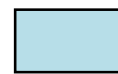

Moved toward

target

Met or exceeded

target

3-14. Statewide cancer registries (no. States and D.C.)

3-15. Persons living 5+ years after a diagnosis of cancer

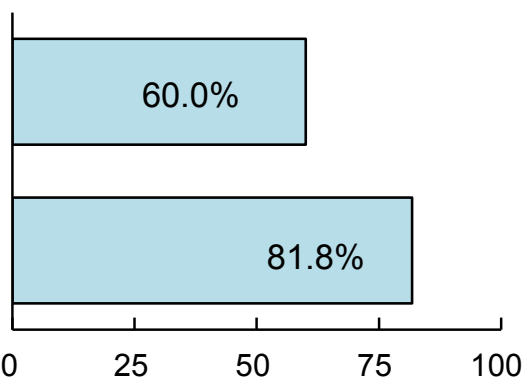

\begin{tabular}{|c|c|c|c|c|c|}
\hline \multirow{2}{*}{$\begin{array}{c}2010 \\
\text { Target }\end{array}$} & \multirow{2}{*}{$\begin{array}{c}\text { Baseline } \\
\text { (Year) }\end{array}$} & Final & \multicolumn{3}{|c|}{ Baseline vs. Final } \\
\cline { 4 - 6 } & Year) & Difference & $\begin{array}{c}\text { Statistically } \\
\text { Significant }\end{array}$ & $\begin{array}{c}\text { Percent } \\
\text { Change }\end{array}$ \\
\hline $45 \%$ & $\begin{array}{c}30 \\
(1999)\end{array}$ & $\begin{array}{c}39 \\
(2006)\end{array}$ & 9 & $\begin{array}{c}\text { Not } \\
\text { tested }\end{array}$ & $30.0 \%$ \\
\hline $70 \%$ & $\begin{array}{c}59 \% \\
(1989-95)\end{array}$ & $\begin{array}{c}68 \% \\
(2000-06)\end{array}$ & 9 & Yes & $15.3 \%$ \\
\hline
\end{tabular}

Percent of targeted change achieved

\section{NOTES}

See the Reader's Guide for more information on how to read this figure. See DATA 2010 at http://wonder.cdc.gov/data2010 for all Healthy People 2010 tracking data. Tracking data are not available for objectives 3-10a through $\mathrm{g}$

\section{FOOTNOTES}

${ }^{1}$ Movement away from target is not quantified using the percent of targeted change achieved. See Technical Appendix for more information.

${ }^{2}$ Difference $=$ Final value - Baseline value. Differences between percents $(\%)$ are measured in percentage points.

${ }^{3}$ When estimates of variability are available, the statistical significance of the difference between the final value and the baseline value is assessed at the 0.05 level. See Technical Appendix for more information.

${ }^{4}$ Percent change $=\frac{\text { Final value }- \text { Baseline value }}{\text { Baseline value }} \times 100$.

${ }^{5}$ Percent of targeted change achieved $=\frac{\text { Final value }- \text { Baseline value }}{\text { Healthy People } 2010 \text { target }- \text { Baseline value }} \times 100$.

\section{DATA SOURCES}

3-1-3-8. National Vital Statistics System-Mortality (NVSS-M), CDC, NCHS.

3-9a. Youth Risk Behavior Surveillance System (YRBSS), CDC, NCCDPHP.

3-9b. National Health Interview Survey (NHIS), CDC, NCHS.

3-10h. National Ambulatory Medical Care Survey (NAMCS), CDC, NCHS

3-11a-b. N N National Health Interview Survey (NHIS), CDC, NCHS. Cancer 3-12a-b. National Health Interview Survey (NHIS), CDC, NCHS.

3-13. National Health Interview Survey (NHIS), CDC, NCHS

3-14. National Program of Cancer Registries (NPCR), CDC, NCCDPHP.

3-15. Surveillance, Epidemiology, and End Results (SEER) Program, NIH, NCl. 
Figure 3-2. Health Disparities Table for Focus Area 3: Cancer

Disparities from the best group rate for each characteristic at the most recent data point and changes in disparity from the baseline to the most recent data point.

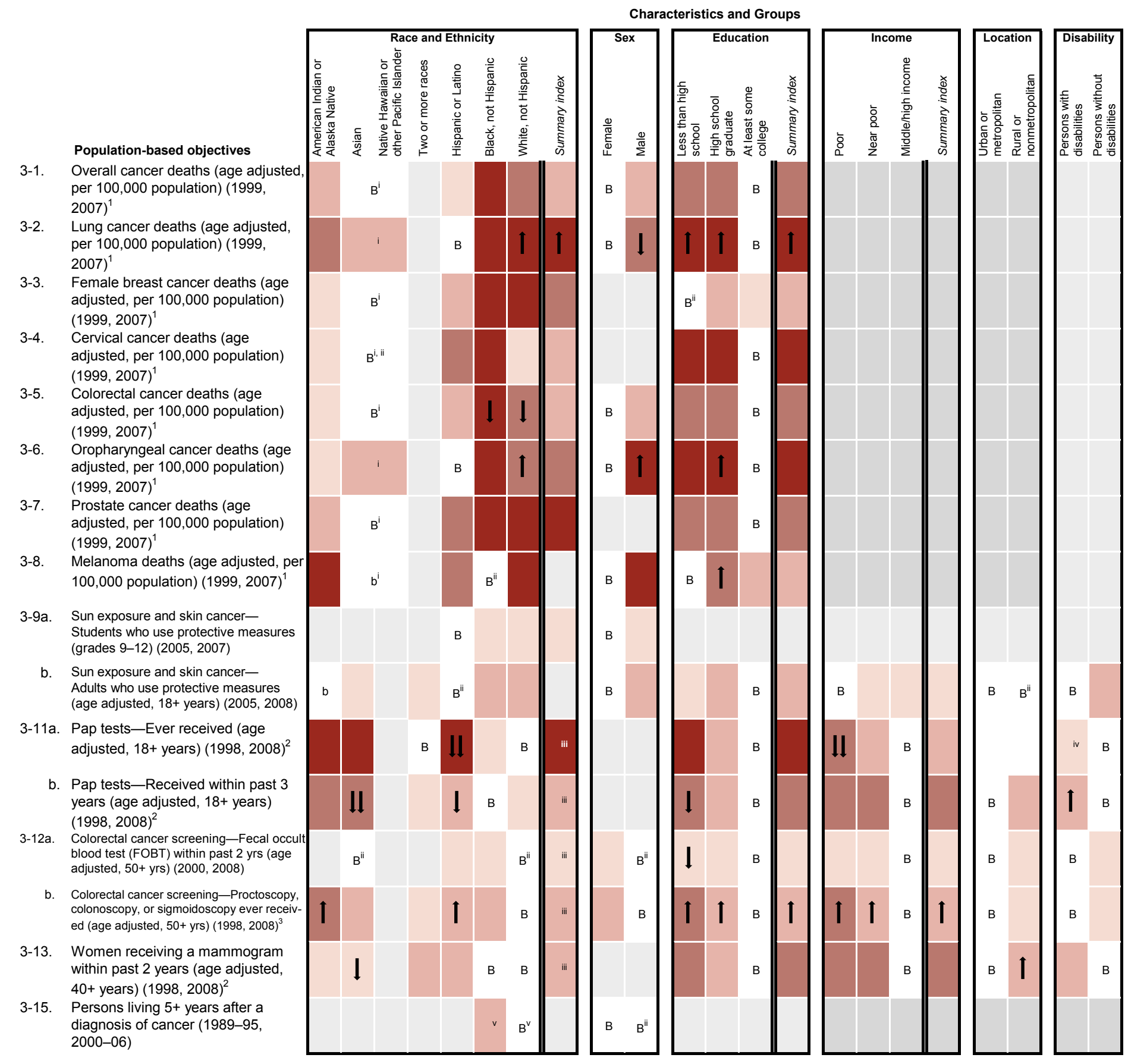

(continued) 


\section{Figure 3-2. Health Disparities Table for Focus Area 3: Cancer (continued)}

\section{NOTES}

See DATA2010 at http://wonder.cdc.gov/data2010 for all Healthy People 2010 tracking data. Disparity data are either unavailable or not applicable for objectives 3-10a through $h$, and 3-14.

Years in parentheses represent the baseline and most recent data years (if available).

Disparity from the best group rate is defined as the percent difference between the best group rate and each of the other group rates for a characteristic (e.g., race and ethnicity). The summary index is the average of these percent differences for a characteristic. Change in disparity is estimated by subtracting the disparity at baseline from the disparity at the most recent data point. Change in the summary index is estimated by subtracting the summary index at baseline from the summary index at the most recent data point. See Technical Appendix for more information.

Measures of variability were available for all objectives in this table. Thus, the variability of best group rates was assessed, and statistical significance was tested. Disparities of $10 \%$ or more are displayed when the differences from the best group rate are statistically significant at the 0.05 level. Changes in disparities over time are indicated by arrows when the changes are greater than or equal to 10 percentage points and are statistically significant at the 0.05 level. See Technical Appendix.

LEGEND

$\begin{aligned} & \text { The "best" group rate at the most recent } \\
& \text { data point. }\end{aligned}$
B $\begin{aligned} & \text { The group with the best rate for } \\
& \text { specified characteristic. }\end{aligned} \quad$ b $\begin{aligned} & \text { Most favorable group rate for specified } \\
& \text { characteristic, but reliability criterion not met. }\end{aligned}$ \begin{tabular}{l}
$\begin{array}{l}\text { Reliability criterion for best } \\
\text { group rate not met, or data } \\
\text { available for only one group. }\end{array}$ \\
\hline
\end{tabular}

\begin{tabular}{ll|l|l|l|l|l|l|l}
\hline & Percent difference from the best group rate \\
$\begin{array}{l}\text { Disparity from the best group rate at the } \\
\text { most recent data point. }\end{array}$ & $\begin{array}{l}\text { Less than } 10 \% \text {, or difference not } \\
\text { statistically significant (when } \\
\text { estimates of variability are available). }\end{array}$ & $10 \%-49 \%$ \\
\hline
\end{tabular}

Increase in disparity (percentage points)

Changes in disparity over time are shown when:

(a) disparities data are available at both baseline and most recent time points;

(b) data are not for the group(s) indicated by "B" or "b" at either time point; and

(b) data are not for the group(s) indicated by "B" or "b" at either time point; and
(c) the change is greater than or equal to 10 percentage points and statistically

significant, or when the change is greater than or equal to 10 percentage

points and estimates of variability were not available. See Technical Appendix.

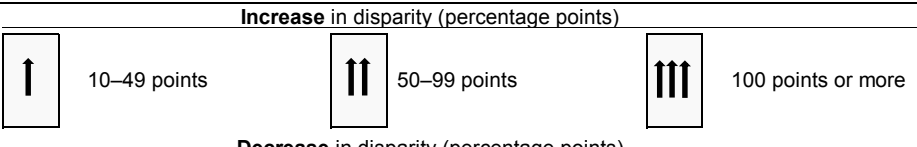

Decrease in disparity (percentage points)

Availability of Data $\quad$ Data not available. $\quad \square$ Characteristic not selected for this objective.

FOOTNOTES

1 Most recent data by education level are for 2002 .

2 Baseline data by race and ethnicity are for 1999.

3 Baseline data by race and ethinicity are for 2000 .

i Data are for Asian or Pacific Islander.

ii The group with the best rate at the most recent data point is different from the group with the best rate at baseline. Both rates met the reliability criterion. See

Technical Appendix.

iii Change in the summary index cannot be assessed. See Technical Appendix.

iv Reliability criterion for best group rate not met, or data available for only one group, at baseline. Change in disparity cannot be assessed. See Technical Appendix.

$\checkmark$ Data include persons of Hispanic origin.

\section{DATA SOURCES}

3-1-3-8. National Vital Statistics System-Mortality (NVSS-M), CDC, NCHS.

3-9a. Youth Risk Behavior Surveillance System (YRBSS), CDC, NCCDPHP.

3-9b. National Health Interview Survey (NHIS), CDC, NCHS.

3-11a-b. National Health Interview Survey (NHIS), CDC, NCHS.

3-12a-b. National Health Interview Survey (NHIS), CDC, NCHS.

3-13. National Health Interview Survey (NHIS), CDC, NCHS.

3-15. Surveillance, Epidemiology, and End Results (SEER) Program, NIH, NCl. 
Figure 3-3. Overall Cancer Deaths (2005-07)

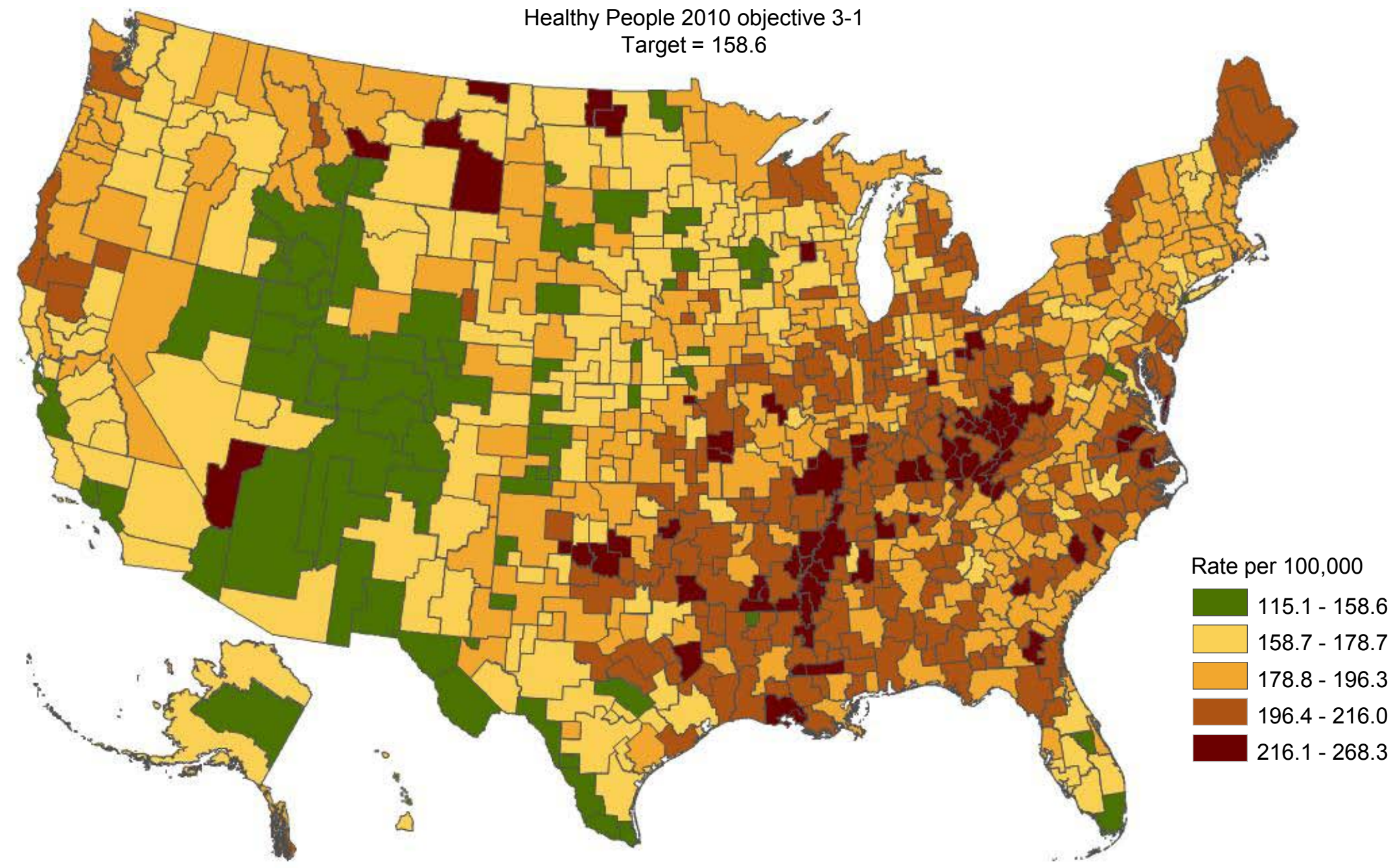

lowest category (green) shows health service areas that met target

NOTES: Data are for ICD-10 codes C00-C97reported as underlying cause. Rates are per 100,000 U. S. Population age-adjusted to the 2000 standard population. Rates are displayed by a modified Jenks classification for U.S. health service area. SOURCE: National Vital Statistics System - Mortality (NVSS-M), CDC, NCHS. 
Figure 3-4. Pap Test Received in the Past 3 Years (18+ Years) 2008

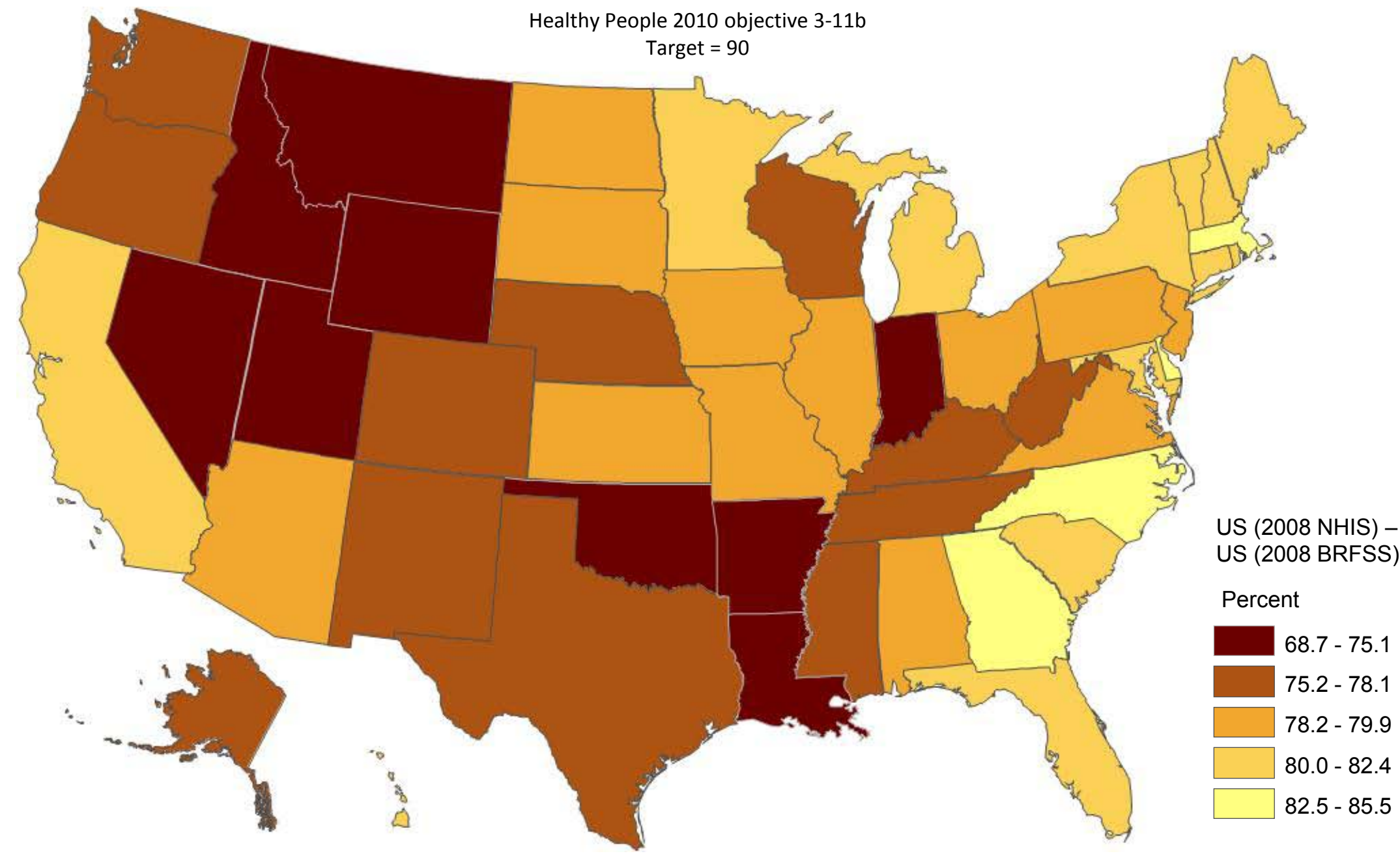

no states met the target

NOTES: Data are age-adjusted to the 2000 standard population. Rates are displayed by a modified Jenks classification for U.S. states. National data for the objective come from the National Health Interview Survey (NHIS) and is the basis for setting the target. State data from the BRFSS may not be comparable to the national data from the NHIS. Both US rates are shown for comparison purposes.

SOURCE: Behavioral Risk Factor Surveillance System (BRFSS), CDC NCCDPHP. 
Figure 3-5. Women Receiving Mammograms within Past 2 Years (40+ Years) 2008

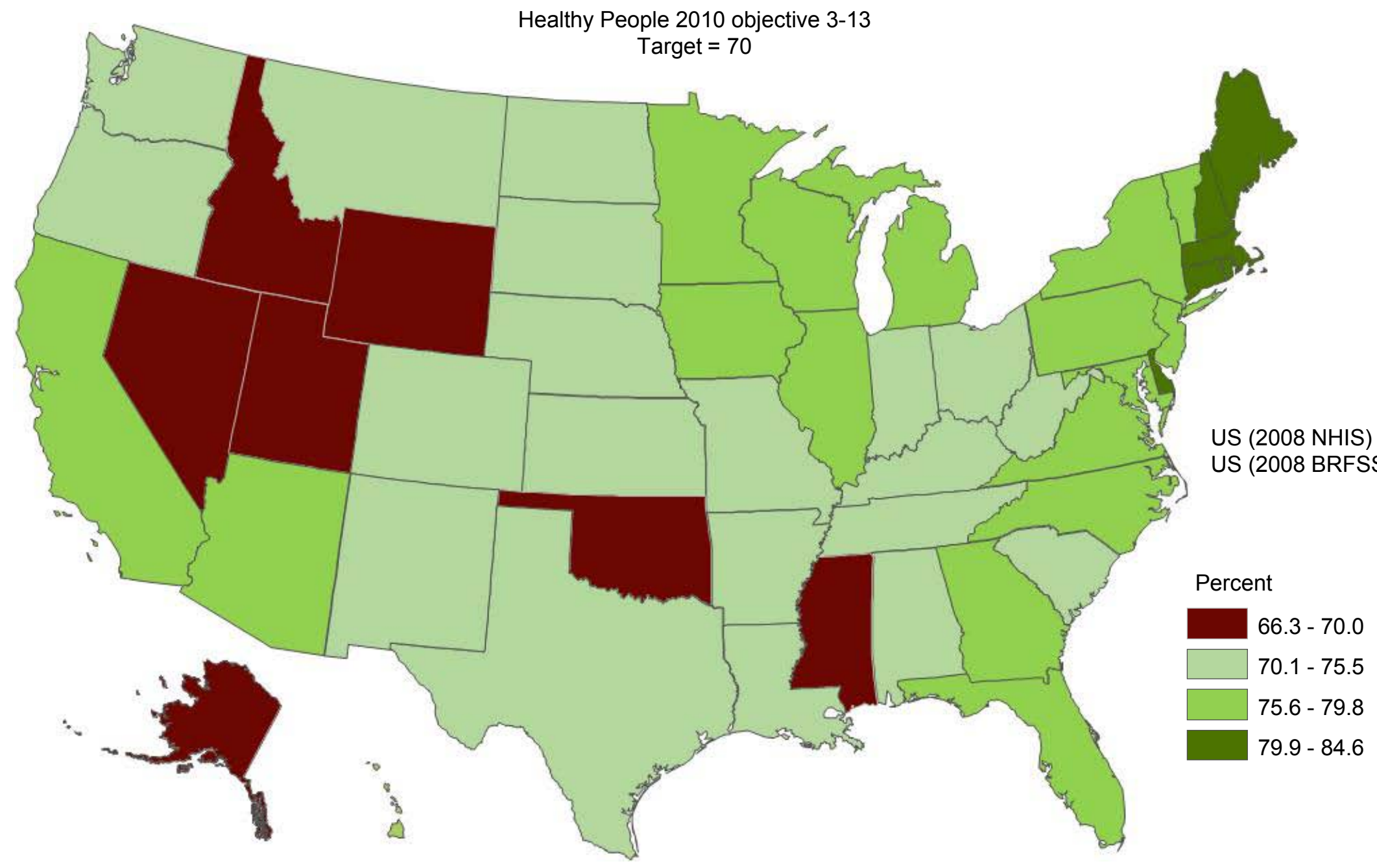

highest categories (green) shows states that met target

NOTES: Data are age-adjusted to the 2000 standard population. Rates are displayed by a modified Jenks classification for U.S. states.

National data for the objective come from the National Health Interview Survey (NHIS) and is the basis for setting the target. State data from the BRFSS may not be comparable to the national data from the NHIS. Both US rates are shown for comparison purposes.

SOURCE: Behavioral Risk Factor Surveillance System (BRFSS), CDC, NCCDPHP. 


\section{Final Review}

\section{Chronic Kidney Disease}

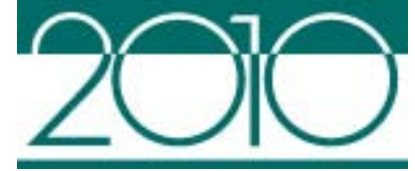

\section{Lead Agency}

National Institutes of Health

\section{Contents}

Goal 4-2

Highlights

Summary of Progress

Transition to Healthy People 2020 4-4

Data Considerations 4-5

References and Notes 4-6

Comprehensive Summary of Objectives 4-7

Progress Chart 4-8

Health Disparities Table 4-10

Kidney Transplant within Three Years-Map. 4-11

Registration for Kidney Transplantation-Map. $4-12$ 


\section{Goal: Reduce new cases of chronic kidney disease and its complications, disability, death, and economic costs}

This chapter includes objectives that monitor new cases of Chronic Kidney Disease (CKD) and End Stage Renal Disease (ESRD), death and disability associated with ESRD, and treatments (including kidney transplantation) for chronic kidney diseases and ESRD and associated conditions.

All tracking data quoted in this chapter, along with technical information and operational definitions, can be found in the Healthy People 2010 database, DATA2010, available from http://wonder.cdc.gov/data2010/.

More information about this Focus Area can be found in the following publications:

- Healthy People 2010: Understanding and Improving Health, available from http://www.healthypeople.gov/2010/Document/tableofcontents.htm\#under.

- Healthy People 2010 Midcourse Review, available from http://www.healthypeople.gov/2010/data/midcourse/html/default.htm\#FocusAreas.

\section{Highlights}

- Substantial progress was achieved for the objectives in this Focus Area over the course of the past decade [1]. Two thirds of the CKD objectives with data to measure progress moved toward or achieved their Healthy People 2010 targets (Figure 4-1). However, most objectives exhibited statistically significant health disparities of $10 \%$ or more by sex and among racial and ethnic population groups (Figure 4-2) [2].

- Cardiovascular disease deaths among persons with chronic kidney failure (objective 4-2) declined by $31.6 \%$ between 1997 and 2008, from 93.7 to 64.1 per 1,000 patient years at risk, exceeding the Healthy People 2010 target of 66.1 per 1,000 patient years at risk.

- New cases of ESRD (objective 4-1) increased by 12.1\% between 1997 and 2008, from 313 to 351 per million population (adjusted for age, gender, and race), moving away from the Healthy People 2010 target of 230 per million population.

- New cases of ESRD due to diabetes (objective 4-7) also increased by 10.9\% between 1997 and 2008, from 138 to 153 per million population (adjusted for age, gender, and race), moving away from the Healthy People 2010 target of 100 per million population.

- The cumulative proportion of persons receiving a kidney transplant within three years of the date of renal failure (objective 4-6) declined by 13.5\% between 1998 and 2005, from $20.0 \%$ to $17.3 \%$, moving away from the Healthy People 2010 target of $29.5 \%$. 
- In 2005, the states of Idaho and Utah, the Upper-Midwest (Minnesota, North and South Dakota, and Wisconsin), and Vermont had the highest cumulative proportions of persons receiving a kidney transplant within three years of the date of renal failure (objective 4-6). These states achieved the Healthy People 2010 target. On the other hand, California, the Southwest (New Mexico, Louisiana, and Texas), and the Southeast (Alabama, Georgia, and North and South Carolina) had the lowest cumulative proportions of persons receiving a kidney transplant within three years of the date of renal failure (Figure 4-3).

- The registration of dialysis patients aged under 70 years for kidney transplantation (objective 4-5) varied by geographic area. In 2007, the states of Delaware, Massachusetts, Pennsylvania, South Dakota, Vermont, and Wisconsin had the highest proportions of patients placed on the transplant waiting list within one year of an ESRD diagnosis. These states achieved the Healthy People 2010 target of 24.8\% (Figure 4-4).

\section{Summary of Progress}

- Figure 4-1 presents a quantitative assessment of progress in achieving the Healthy People 2010 objectives for CKD [1]. Data to measure progress toward target attainment were available for all nine objectives. Of these:

- Three objectives exceeded the Healthy People 2010 targets (objectives 4-2, and 4-8a and b).

- Three objectives moved toward their targets (objectives 4-3, 4-4, and 4-5). A statistically significant difference between the baseline and the final data points was observed for all three of these objectives.

- Three objectives moved away from their targets (objectives 4-1, 4-6, and 4-7). A statistically significant difference between the baseline and final data points was observed for all of these objectives.

- Figure 4-2 provides a quantitative assessment of health disparities in CKD from the best group rate for each characteristic at the most recent data point [2]. It also displays changes in disparities from baseline to the most recent data point [3].

- Of the seven objectives with statistically significant racial and ethnic health disparities of $10 \%$ or more, the Asian population (objectives 4-2 and 4-5), white non-Hispanic population (objectives 4-4 and 4-6), and the population of persons of two or more races (objectives 4-1 and 4-7), each had the best group rate for two objectives. The combined Asian or Pacific Islander population had the best group rate for one objective (4-8a).

- Health disparities of $100 \%$ or more relative to the group with the best rate were observed for two objectives: new cases of ESRD (objective 4-1) and new ESRD cases due to diabetes (objective 4-7).

- Increases in disparities of 100 percentage points or more were observed for the same two objectives.

- Females had better rates than males for two of the three objectives with statistically significant health disparities of $10 \%$ or more by sex (objectives 4-1 and 4-7). Men had a better rate than females for new hemodialysis patients who use arteriovenous fistulas (objective 4-4). 


\section{Transition to Healthy People 2020}

The Healthy People 2020 Chronic Kidney Disease Topic Area features a broader range of objectives than those included in Healthy People 2010. See HealthyPeople.gov for a complete list of Healthy People 2020 topics and objectives.

The Healthy People 2020 objectives can be grouped into several sections:

- CKD process and treatment

- CKD outcomes

- ESRD process and treatment

- ESRD outcomes.

The differences between the Healthy People 2010 and Healthy People 2020 CKD objectives are summarized below:

- The Healthy People 2020 CKD Topic Area has 24 objectives, whereas the Healthy People 2010 Focus Area had nine objectives.

- One Healthy People 2010 objective, new cases of ESRD (objective 4-1), was retained "as is" [4].

- Eight Healthy People 2010 objectives (4-2 through 4-7, and 4-8a and b) were modified [5]. Some were extended to include new measures of CKD and ESRD treatment and outcomes.

- Fifteen new objectives were added to the Healthy People 2020 Topic Area:

- Five CKD and ESRD mortality objectives were added. (Objective 4-2, the Healthy People 2010 objective measuring cardiovascular death in patients with chronic kidney failure, was retained.) The new objectives address the total death and cardiovascular death rates for persons on dialysis; the death rate for dialysis patients within the first three months of initiating therapy; the total and cardiovascular death rates for persons who have had a kidney transplant; and the death rate for persons with CKD.

- Two objectives were added to the Healthy People 2010 objective on arteriovenous fistulas monitoring the use of arteriovenous fistulas and the use of incident catheters.

- Two new objectives focus on improving cardiovascular care in persons with CKD: blood pressure and hyperlipidemia control.

- Objectives measuring the proportion of the U.S. population with CKD and the proportion of persons with CKD who know they have impaired function were added to increase awareness of CKD among health professionals and the general public.

- Three new objectives address recommended medical evaluation and treatment of patients with diabetes and CKD.

- An objective measuring follow-up renal evaluation after acute kidney injury was included to emphasize the importance of timely evaluation in CKD prevention. 
Appendix D, “A Crosswalk Between Objectives From Healthy People 2010 to Healthy People 2020," summarizes the changes between the two decades of objectives, reflecting new knowledge and direction for this area.

\section{Data Considerations}

Many of the objectives in this chapter are tracked using the U.S. Renal Data System (USRDS) data, which uses data collected by the Centers for Medicare \& Medicaid Services. Since 1996, health care providers have been required to provide patient information on all persons with ESRD, regardless of health insurance. Therefore, incidence rates reflect the universe of ESRD cases in the U.S.

There is some lag in reporting new cases of ESRD. As a result, each year's USRDS Annual Data Report includes re-estimates of rates from earlier years [6]. Data for some race/ethnicity groups have not been collected or reported for all years from the Healthy People 2010 baseline to the most recent data point. For example, data in the category "two or more races" for objectives 4-1 and 4-7 were not available until 2006. Therefore, due to the re-estimation method used by the Annual Data Report, data for these groups might not be directly comparable with other race/ethnicity groups.

The USRDS data, data collection procedures, calculation methods, and other technical information are included in its Annual Data Report [6].

Education and income are the primary measures of socioeconomic status (SES) in Healthy People 2010. Most data systems used in Healthy People 2010 define income as a family's income before taxes. In order to facilitate comparisons among groups and over time, while adjusting for family size and for inflation, Healthy People 2010 categorizes income using the poverty thresholds developed by the U.S. Census Bureau. Thus, the three categories of family income that are primarily used are:

- Poor-below the Federal poverty level

- Near poor-100\% to $199 \%$ of the Federal poverty level

- Middle/high income-200\% or more of the Federal poverty level.

These categories may be overridden by considerations specific to the data system, in which case they are modified as appropriate. See Healthy People 2010: General Data Issues, referenced below.

Additional information on data issues is available from the following sources:

- All Healthy People 2010 tracking data can be found in the Healthy People 2010 database, DATA2010, available from http://wonder.cdc.gov/data2010/.

- Detailed information about the data and data sources used to support these objectives can be found in the Operational Definitions on the DATA2010 website, available from http://wonder.cdc.gov/data2010/focusod.htm.

- More information on statistical issues related to Healthy People tracking and measurement can be found in the Technical Appendix and in Healthy People 2010: General Data Issues, which is available in the Data Issues section of the NCHS Healthy People website under Healthy People 2010. 


\section{References and Notes}

1. Displayed in the Progress Chart (Figure 4-1), the percent of targeted change achieved expresses the difference between the baseline and the final value relative to the initial difference between the baseline and the Healthy People 2010 target. As such, it is a relative measure of progress toward attaining the Healthy People 2010 target. See the Reader's Guide for more information. When standard errors were available, the difference between the baseline and the final value was tested at the 0.05 level of significance. See the Figure 4-1 footnotes, as well as the Technical Appendix, for more detail.

2. Information about disparities among select populations is shown in the Health Disparities Table (Figure 4-2). Disparity from the best group rate is defined as the percent difference between the best group rate and each of the other group rates for a characteristic. For example, racial and ethnic health disparities are measured as the percent difference between the best racial and ethnic group rate and each of the other racial and ethnic group rates. Similarly, disparities by sex are measured as the percent difference between the better group rate (e.g., female) and the rate for the other group (e.g., male). Some objectives are expressed in terms of favorable events or conditions that are to be increased, while others are expressed in terms of adverse events or conditions that are to be reduced. In order to facilitate comparison of health disparities across different objectives, disparity is measured only in terms of adverse events or conditions. For comparability across objectives, objectives that are expressed in terms of favorable events or conditions are re-expressed using the adverse event or condition for the purpose of computing disparity, but they are not otherwise restated or changed. For example, objective 1-1, to increase the proportion of persons with health insurance (e.g., $72 \%$ of the American Indian or Alaska Native population aged under 65 years had some form of health insurance in 2008), is expressed in terms of the percentage of persons without health insurance (e.g., $100 \%-72 \%=28 \%$ of the American Indian or Alaska Native population aged under 65 years did not have any form of health insurance in 2008) when the disparity from the best group rate is calculated. See the Reader's Guide for more information. When standard errors were available, the difference between the best group rate and each of the other group rates was tested at the 0.05 level of significance. See the Figure 4-2 footnotes, as well as the Technical Appendix, for more detail.

3. The change in disparity is estimated by subtracting the disparity at baseline from the disparity at the most recent data point and, therefore, is expressed as a change in percentage points. See the Reader's Guide for more information. When standard errors were available, the change in disparity was tested at the 0.05 level of significance. See the Figure 4-2 footnotes, as well as the Technical Appendix, for more detail.

4. Retained "as is" objectives have no change in the numerator definition or in the denominator definition between the Healthy People 2010 and Healthy People 2020 objectives. These include objectives that were developmental in Healthy People 2010 and are developmental in Healthy People 2020 and for which no numerator or denominator information was available.

5. Modified objectives have some change in the numerator definition or in the denominator definition between the Healthy People 2010 and Healthy People 2020 objectives. These include objectives that went from developmental in Healthy People 2010 to measurable in Healthy People 2020 or vice versa.

6. United States Renal Data System, USRDS. 2010 Annual Data Report: Atlas of End-Stage Renal Disease in the United States. Bethesda, MD: National Institutes of Health, National Institute of Diabetes and Digestive and Kidney Diseases, 2010. Available from http://www.usrds.org/adr.htm. 


\section{Comprehensive Summary of Objectives: Chronic Kidney Disease}

\begin{tabular}{|c|c|c|}
\hline Objective & Description & Data Source \\
\hline $4-1$ & $\begin{array}{l}\text { New cases of end-stage renal disease (ESRD) (per million } \\
\text { population, adjusted for age, sex, and race) }\end{array}$ & United States Renal Data System (USRDS), NIH, NIDDK. \\
\hline $4-2$ & $\begin{array}{l}\text { Cardiovascular disease deaths in persons with chronic kidney failure } \\
\text { (per 1,000 patient years at risk) }\end{array}$ & United States Renal Data System (USRDS), NIH, NIDDK. \\
\hline $4-3$ & Pre-ESRD care from a nephrologist & United States Renal Data System (USRDS), NIH, NIDDK. \\
\hline $4-4$ & $\begin{array}{l}\text { New hemodialysis patients who use arteriovenous fistulas (20+ } \\
\text { years) }\end{array}$ & $\begin{array}{l}\text { Centers for Medicare and Medicaid Services Clinical Performance } \\
\text { Measures (CPM) project, CMS. }\end{array}$ \\
\hline $4-5$ & $\begin{array}{l}\text { Dialysis patients registered on kidney transplant waiting list }(<70 \\
\text { years) }\end{array}$ & United States Renal Data System (USRDS), NIH, NIDDK. \\
\hline $4-6$ & $\begin{array}{l}\text { Cumulative percent of persons receiving a kidney transplant within } \\
3 \text { years of the date of renal failure ( }<70 \text { years) }\end{array}$ & United States Renal Data System (USRDS), NIH, NIDDK. \\
\hline $4-7$ & $\begin{array}{l}\text { New cases of ESRD due to diabetes (per million population, adjusted } \\
\text { for age, sex, and race) }\end{array}$ & United States Renal Data System (USRDS), NIH, NIDDK. \\
\hline $4-8 a$ & $\begin{array}{l}\text { Medical evaluation for persons with type } 1 \text { or type } 2 \text { diabetes and } \\
\text { chronic kidney disease }\end{array}$ & $\begin{array}{l}\text { Centers for Medicare and Medicaid Services Standard Analytic Files } \\
\text { (SAF), CMS; U.S. Renal Data System (USRDS), NIH, NIDDK. }\end{array}$ \\
\hline $4-8 b$ & $\begin{array}{l}\text { Medical treatment for persons with type } 1 \text { or type } 2 \text { diabetes and } \\
\text { chronic kidney disease }\end{array}$ & $\begin{array}{l}\text { Centers for Medicare and Medicaid Services Standard Analytic Files } \\
\text { (SAF), CMS; U.S. Renal Data System (USRDS), NIH, NIDDK. }\end{array}$ \\
\hline
\end{tabular}


Figure 4-1. Progress Toward Target Attainment for Focus Area 4: Chronic Kidney Disease

Moved away

from target ${ }^{1}$

Moved toward

target

Met or exceeded

target

4-1. New cases of end-stage renal disease (ESRD) (per million population, adjusted for age, gender, and race)

4-2. Cardiovascular disease deaths in persons with chronic kidney failure (per 1,000 patient years at risk)

4-3. Pre-ESRD care from a nephrologist
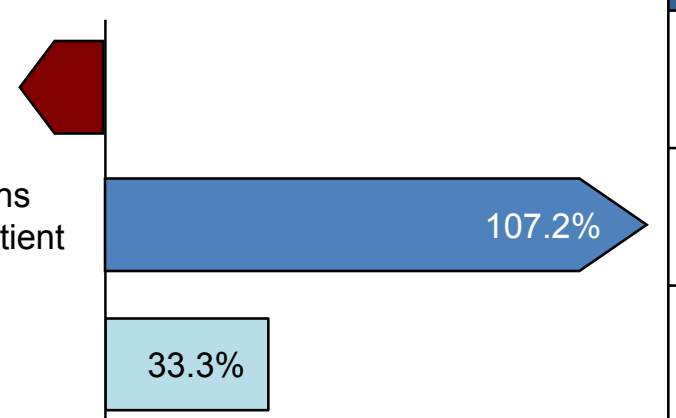

4-4. New hemodialysis patients who use arteriovenous fistulas (20+ years)

4-5. Dialysis patients registered on kidney transplant waiting list ( $<70$ years)

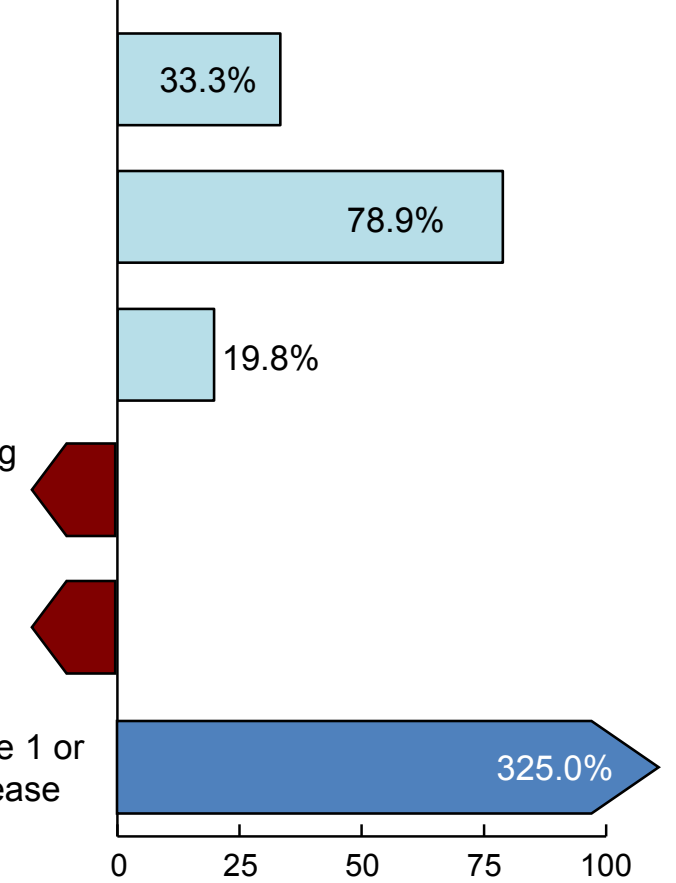

\begin{tabular}{|c|c|c|c|c|c|}
\hline \multirow{2}{*}{$\begin{array}{l}2010 \\
\text { Target }\end{array}$} & \multirow{2}{*}{$\begin{array}{c}\text { Baseline } \\
\text { (Year) }\end{array}$} & \multirow{2}{*}{$\begin{array}{l}\text { Final } \\
\text { (Year) }\end{array}$} & \multicolumn{3}{|c|}{ Baseline vs. Final } \\
\hline & & & Difference $^{2}$ & $\begin{array}{l}\text { Statistically } \\
\text { Significant }^{3}\end{array}$ & $\begin{array}{c}\text { Percent } \\
\text { Change }^{4}\end{array}$ \\
\hline 230 & $\begin{array}{c}313 \\
(1997)\end{array}$ & $\begin{array}{c}351 \\
(2008)\end{array}$ & 38 & Yes & $12.1 \%$ \\
\hline 66.1 & $\begin{array}{c}93.7 \\
(1997)\end{array}$ & $\begin{array}{c}64.1 \\
(2008)\end{array}$ & -29.6 & Yes & $-31.6 \%$ \\
\hline $34 \%$ & $\begin{array}{c}25 \% \\
(2005)\end{array}$ & $\begin{array}{c}28 \% \\
(2008)\end{array}$ & 3 & Yes & $12.0 \%$ \\
\hline $45 \%$ & $\begin{array}{c}26 \% \\
(1998)\end{array}$ & $\begin{array}{c}41 \% \\
(2007)\end{array}$ & 15 & Yes & $57.7 \%$ \\
\hline $24.8 \%$ & $\begin{array}{l}15.2 \% \\
(1998)\end{array}$ & $\begin{array}{l}17.1 \% \\
(2007)\end{array}$ & 1.9 & Yes & $12.5 \%$ \\
\hline $29.5 \%$ & $\begin{array}{l}20.0 \% \\
(1998)\end{array}$ & $\begin{array}{l}17.3 \% \\
(2005)\end{array}$ & -2.7 & Yes & $-13.5 \%$ \\
\hline 100 & $\begin{array}{c}138 \\
(1997)\end{array}$ & $\begin{array}{c}153 \\
(2008)\end{array}$ & 15 & Yes & $10.9 \%$ \\
\hline $25 \%$ & $\begin{array}{c}21 \% \\
(2000)\end{array}$ & $\begin{array}{c}34 \% \\
(2008)\end{array}$ & 13 & Yes & $61.9 \%$ \\
\hline
\end{tabular}

Percent of targeted change achieved 5

(continued) 
Figure 4-1. Progress Toward Target Attainment for Focus Area 4: Chronic Kidney Disease (continued)

Moved away

from target ${ }^{1}$

Moved toward

target

4-8b. Medical treatment for persons with type 1 or type 2 diabetes and chronic kidney disease

Met or exceeded

target
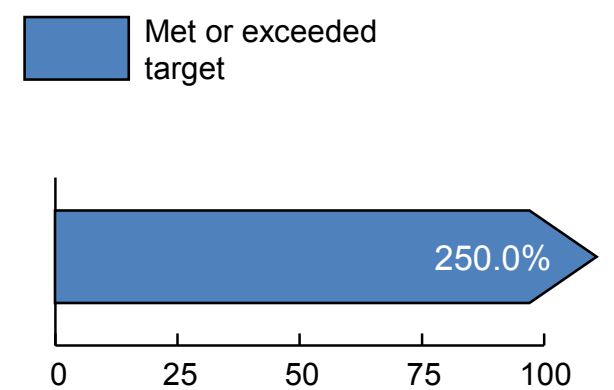

\begin{tabular}{|c|c|c|c|c|c|}
\hline \multirow{2}{*}{$\begin{array}{c}2010 \\
\text { Target }\end{array}$} & \multirow{2}{*}{$\begin{array}{c}\text { Baseline } \\
(\text { Year })\end{array}$} & Final & \multicolumn{3}{|c|}{ Baseline vs. Final } \\
\cline { 4 - 6 } & Year) & Difference $^{2}$ & $\begin{array}{c}\text { Statistically } \\
\text { Significant }\end{array}$ & $\begin{array}{c}\text { Percent } \\
\text { Change }^{4}\end{array}$ \\
\hline $71 \%$ & $\begin{array}{c}69 \% \\
(2000)\end{array}$ & $\begin{array}{c}74 \% \\
(2006)\end{array}$ & 5 & No & $7.2 \%$ \\
\hline
\end{tabular}

Percent of targeted change achieved ${ }^{5}$

NOTES

See the Reader's Guide for more information on how to read this figure. See DATA2010 at http://wonder.cdc.gov/data2010 for all Healthy People 2010 tracking data.

\section{FOOTNOTES}

${ }^{1}$ Movement away from target is not quantified using the percent of targeted change achieved. See Technical Appendix for more information.

${ }^{2}$ Difference $=$ Final value - Baseline value. Differences between percents $(\%)$ are measured in percentage points.

${ }^{3}$ When estimates of variability are available, the statistical significance of the difference between the final value and the baseline value is assessed at the 0.05 level. See Technical Appendix for more information.

${ }^{4}$ Percent change $=\frac{\text { Final value }- \text { Baseline value }}{\text { Baseline value }} \times 100$

${ }^{5}$ Percent of targeted change achieved $=\frac{\text { Final value }- \text { Baseline value }}{\text { Healthy People } 2010 \text { target }- \text { Baseline value }} \times 100$.

DATA SOURCES

4-1-4-3. United States Renal Data System (USRDS), NIH, NIDDK.

4-4. $\quad$ Centers for Medicare and Medicaid Services Clinical Performance Measures (CPM) project, CMS.

4-5-4-7. United States Renal Data System (USRDS), NIH, NIDDK.

4-8a-b. $\quad$ Centers for Medicare and Medicaid Services Standard Analytic Files (SAF), CMS; U.S. Renal Data System (USRDS), NIH, NIDDK. 
Figure 4-2. Health Disparities Table for Focus Area 4: Chronic Kidney Disease

Disparities from the best group rate for each characteristic at the most recent data point and changes in disparity from the baseline to the most recent data point.

\section{Population-based objectives}

4-1. New cases of end-stage renal disease (ESRD) (per million population, adjusted for age, gender, and race) $(1997,2008)^{\S}$

4-2. Cardiovascular disease deaths in persons with chronic kidney failure (per 1,000 patient years at risk) (1997, 2008)

4-3. Pre-ESRD care from a nephrologist $(2005,2008)$

4-4. New hemodialysis patients who use arteriovenous fistulas $(20+$ years $)(1998,2007)$

4-5. Dialysis patients registered on kidney transplant waiting list $(<70$ years) $(1998,2007)$

4-6. Cumulative percent of persons receiving a kidney transplant within 3 years of the date of renal failure (<70 years) $(1998,2005)$

4-7. New cases of ESRD due to diabetes (per million population, adjusted for age, gender, and race) $(1997,2008)^{\S}$

4-8a Medical evaluation for persons with type 1 or type 2 diabetes and chronic kidney disease $(2000,2008)$

4-8b. Medical treatment for persons with type 1 or type 2 diabetes and chronic kidney disease $(2000,2006)$
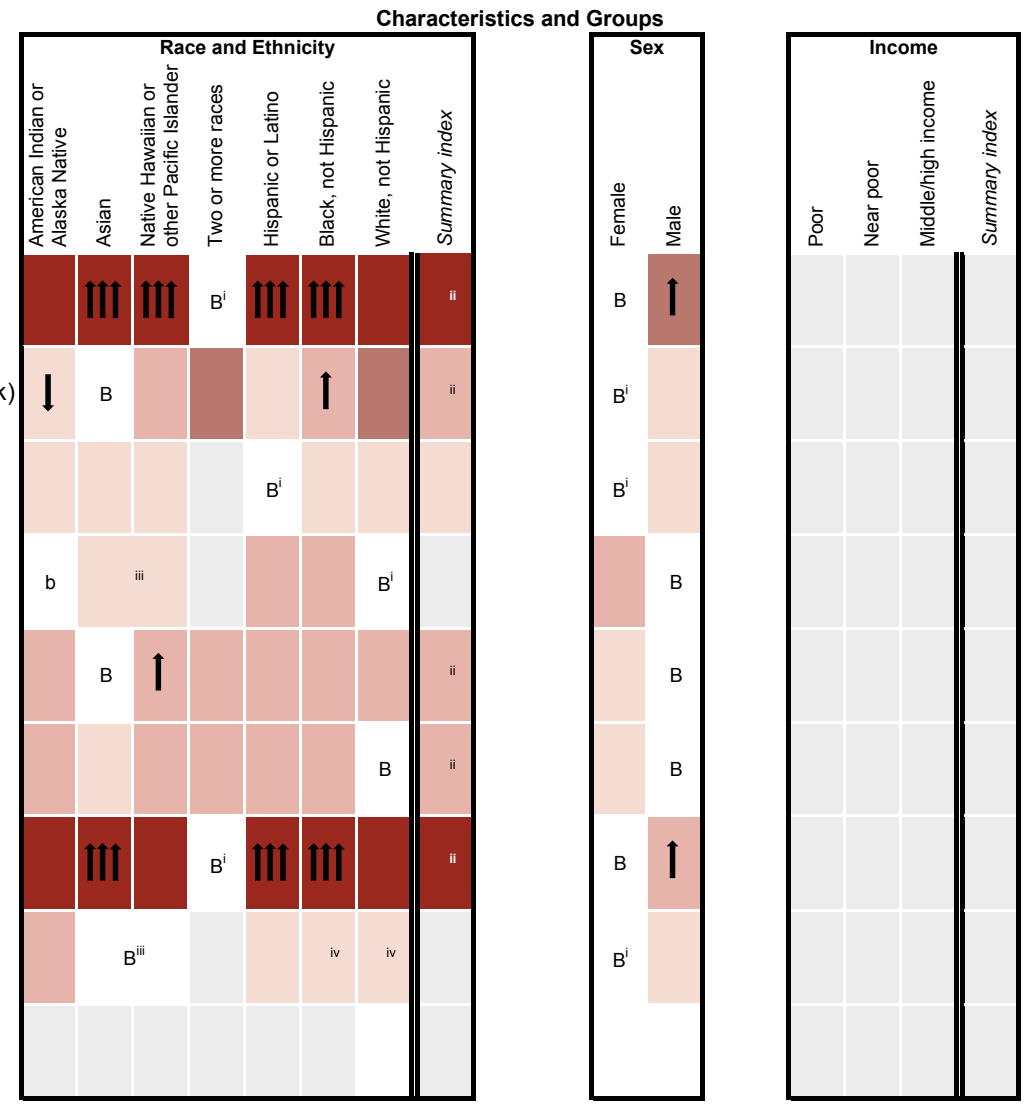

NOTES

See DATA2010 at http://wonder.cdc.gov/data2010 for all Healthy People 2010 tracking data.

Years in parentheses represent the baseline and most recent data years (if available).

Disparity from the best group rate is defined as the percent difference between the best group rate and each of the other group rates for a characteristic (e.g., race and ethnicity). The summary index is the average of these percent differences for a characteristic. Change in disparity is estimated by subtracting the disparity at baseline from the disparity at the most recent data point. Change in the summary index is estimated by subtracting the summary index at baseline from the summary index at the most recent data point. See Technical Appendix for more information.

Measures of variability were available for all objectives in this table. Thus, the variability of best group rates was assessed, and statistical significance was tested. Disparities of $10 \%$ or more are displayed when the differences from the best group rate are statistically significant at the 0.05 level. Changes in disparities over time are indicated by arrows when the changes are greater than or equal to 10 percentage points and are statistically significant at the 0.05 level. See Technical Appendix.

The "best" group rate at the most recent data point.

\section{B The group with the best rate for specified characteristic.}

$$
\begin{aligned}
& \text { Most favorable group rate for specified } \\
& \text { characteristic, but reliability criterion not } \\
& \text { met. }
\end{aligned}
$$

Reliability criterion for best group rate not met, or data available for only one group.

\section{Disparity from the best group rate at the most} recent data point.

\begin{tabular}{|l|l}
\hline \hline $\begin{array}{l}\text { Less than } 10 \%, \text { or difference not } \\
\text { statistically significant (when } \\
\text { estimates of variability are available). }\end{array}$ \\
\hline
\end{tabular}

Percent difference from the best group rate

Changes in disparity over time are shown when:
(a) disparities data are available at both baseline and most recent time points;
(b) data are not for the group(s) indicated by "B" or "b" at either time point; and
(c) the change is greater than or equal to 10 percentage points and statistically
significant, or when the change is greater than or equal to 10 percentage points and
estimates of variability were not available. See Technical Appendix.

§ Data for "Two or more races" were not available until 2006; therefore, these data may not be directly comparable with other groups. See Data Issues section for more information.

i The group with the best rate at the most recent data point is different from the group with the best rate at baseline. Both rates met the reliability criterion. See Technical Appendix.

ii Change in the summary index cannot be assessed. See Technical Appendix.

iii Data are for Asian or Pacific Islander.

iv Data include persons of Hispanic origin

DATA SOURCES

4-1-4-3. United States Renal Data System (USRDS), NIH, NIDDK.

4-4. Centers for Medicare and Medicaid Services Clinical Performance Measures (CPM) proiect, CMS.

4-5-4-7. United States Renal Data System (USRDS), NIH, NIDDK

4-5-4-7. United States Renal Data System (USRDS), NIH, NIDDK.

Chronic Kidney Disease 
Figure 4-3. Dialysis Patients Registered on Kidney Transplant

Waiting List (<70 Years) 2007

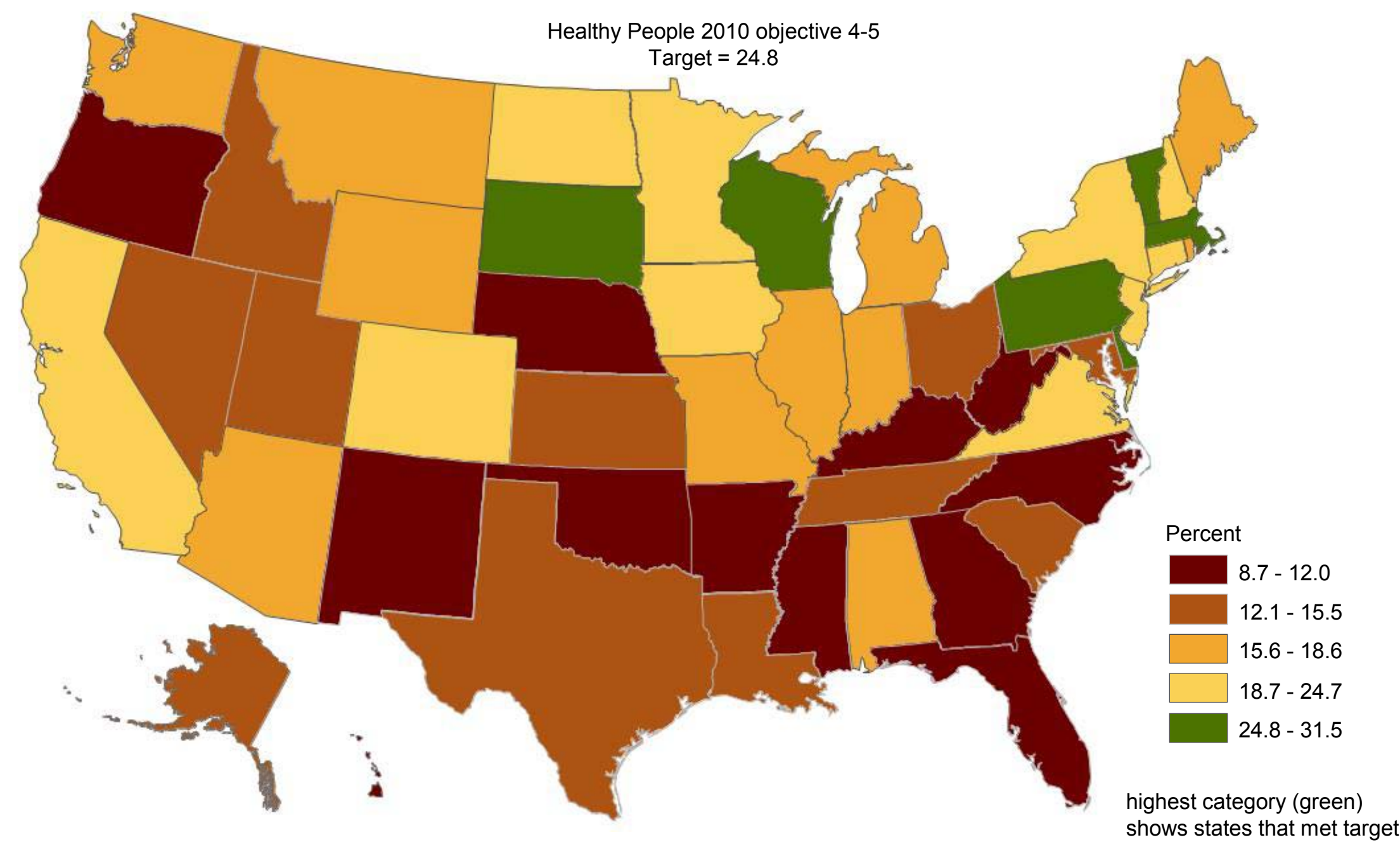

NOTES: Dialysis patients under age 70 years registered on the kidney transplant waiting list within one year of the date of end stage renal disease. Rates are displayed by a modified Jenks classification for U.S. states. The USRDS data, data collection procedures, calculation methods, and other technical information are included in USRDS Annual Data Report, available at http://www.usrds.org/adr.htm . SOURCE: U.S. Renal Data System (USRDS), NIH, NIDDK. 

Failure (<70 Years) 2005

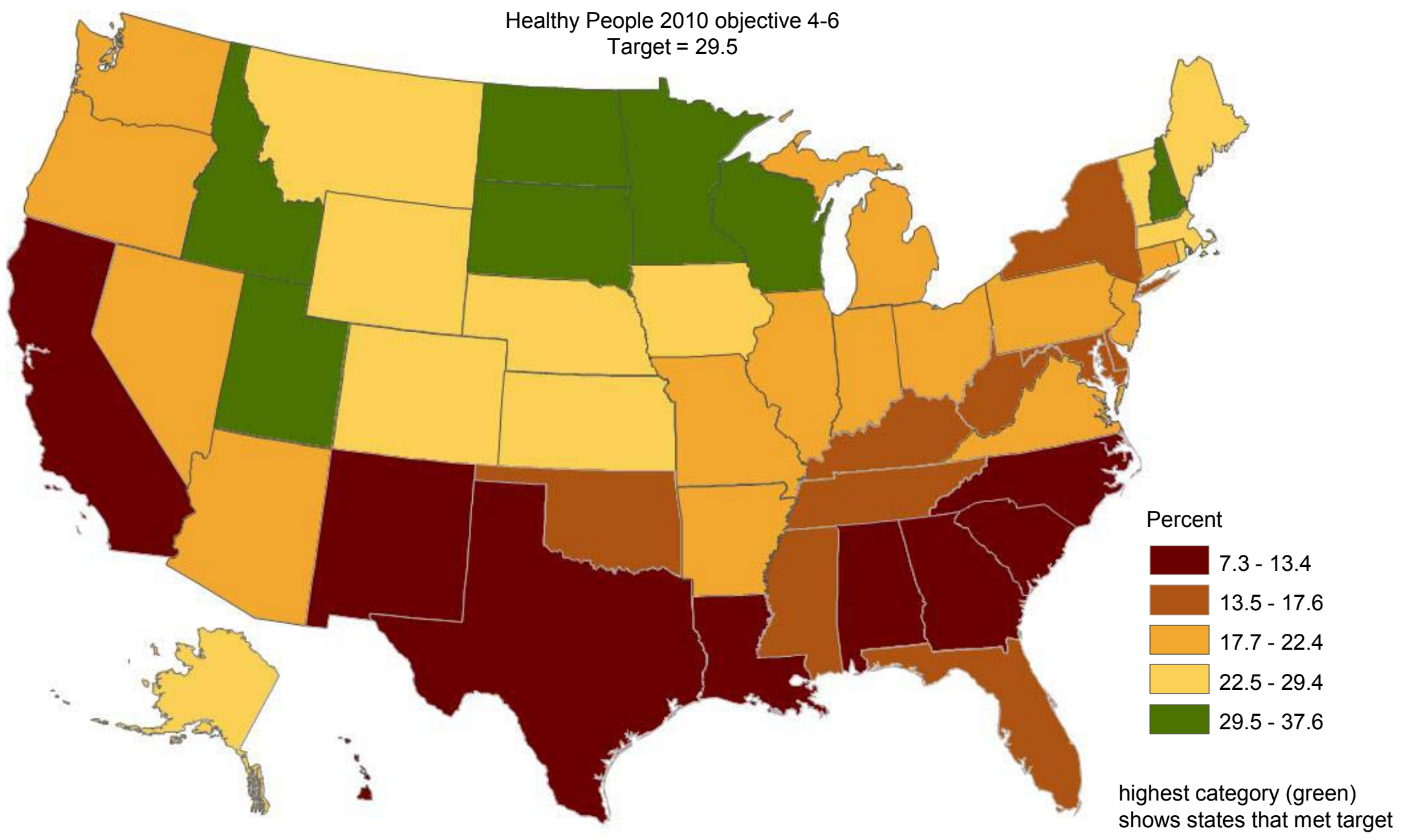

NOTES: Among patients with treated chronic kidney failure who receive a transplant within 3 years of registration on the waiting list. Rates are displayed by a modified Jenks classification for U.S. states. The USRDS data, data collection procedures, calculation methods, and other technical information are included in USRDS Annual Data Report, available at http://www.usrds.org/adr.htm . SOURCE: U.S. Renal Data System (USRDS), NIH, NIDDK. 


\section{Final Review}

\section{Diabetes}

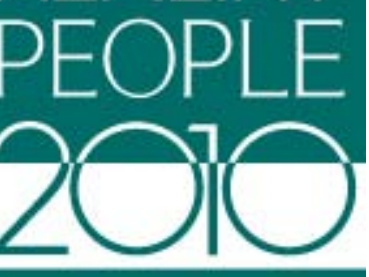

\section{Co-Lead Agencies}

Centers for Disease Control and Prevention

National Institutes of Health, NIDDK

\section{Contents}

Goal

Highlights

Summary of Progress.

Transition to Healthy People 2020

Data Considerations

References and Notes $5-6$

Comprehensive Summary of Objectives $5-8$

Progress Chart

Health Disparities Table

Diabetes Prevalence-Map 


\section{Goal: Through prevention programs, reduce the disease and economic burden of diabetes, and improve the quality of life for all persons who have or are at risk for diabetes}

This chapter includes objectives that track new cases of diabetes, diabetes-related deaths, the diagnosis and treatment of diabetes and related conditions, and diabetes education.

All Healthy People tracking data quoted in this chapter, along with technical information and operational definitions for each objective, can be found in the Healthy People 2010 database, DATA2010, available from http://wonder.cdc.gov/data2010/.

More information about this Focus Area can be found in the following publications:

- Healthy People 2010: Understanding and Improving Health, available from http://www.healthypeople.gov/2010/Document/tableofcontents.htm\#under.

- Healthy People 2010 Midcourse Review, available from http://www.healthypeople.gov/2010/data/midcourse/html/default.htm\#FocusAreas.

\section{Highlights}

- Substantial progress was achieved for the objectives in this Focus Area during the past decade [1]. Seventy-one percent of the Diabetes objectives with data to measure progress moved toward or achieved their Healthy People 2010 targets (Figure 5-1). Most of the health disparities observed by race and ethnicity, sex, education level, and disability status, ranged from $10 \%$ to $99 \%$ in magnitude; larger disparities are discussed below (Figure 5-2) [2].

- The rate of new cases of diabetes (objective 5-2) increased by 45.5\% between 1997-99 and 2006-08, from 5.5 to 8.0 per 1,000 population aged 18-84 years (age adjusted), moving away from the Healthy People 2010 target of 3.8 per 1,000. Disparities were observed for a number of population groups, for example:

- Among education groups, persons with at least some college education had the lowest (best) rate of new cases of diabetes, 6.9 per 1,000 population aged 25-84 years (age adjusted) in 2006-08. Persons with less than a high school education had a rate of 14.0 per 1,000 population aged 25-84 years (age adjusted) in 2006-08. The rate for persons with less than a high school education was about twice the best group rate [2].

- Among disability status groups, persons without disabilities had the lowest (best) rate of new cases of diabetes, 6.3 per 1,000 population aged 18-84 years (age adjusted) in 2006-08. Persons with disabilities had a rate of 18.5 per 1,000 population aged 18-84 years (age adjusted) in 2006-08, nearly three times as high as the best group rate [2].

- The prevalence of diabetes (objective 5-3) increased by 47.5\% between 1997 and 2008, from 40 to 59 per 1,000 population (age adjusted), moving away from the 2010 target of 25 per 1,000. Disparities were observed for a number of population groups, for example: 
- Among racial and ethnic groups, the white non-Hispanic population had the lowest (best) diabetes prevalence rate, 52 per 1,000 population (age adjusted) in 2008, whereas the American Indian or Alaska Native population had a rate of 109 per 1,000 population (age adjusted) in 2008. The rate for the American Indian or Alaska Native population was more than twice the best group rate [2].

- Among disability status groups, persons without disabilities had the lowest (best) diabetes prevalence rate, 43 per 1,000 population (age adjusted) in 2008. Persons with disabilities had a rate of 120 per 1,000 population (age adjusted) in 2008, almost three times the best group rate [2].

- The prevalence of diabetes varied by geographic region. West Virginia and several southern states (Alabama, Georgia, Louisiana, Mississippi Tennessee, and Texas) had the highest rates of diabetes (Figure 5-3).

- The proportion of persons aged 20 years and over with diabetes whose condition had been diagnosed (objective 5-4) increased by $20.3 \%$ between $1988-94$ and $2005-08$, from $64 \%$ to $77 \%$ (age adjusted), moving toward the 2010 target of $78 \%$.

- The diabetes-related death rate among the total population (objective 5-5) declined by $5.2 \%$ between 1999 and 2007, from 77 to 73 per 100,000 population (age adjusted), moving toward the 2010 target of 46 per 100,000. Disparities were observed for a number of population groups, for example:

- Among racial and ethnic groups, the Asian or Pacific Islander population had the lowest (best) rate of diabetes-related deaths, 54 per 100,000 population (age adjusted) in 2007. The black non-Hispanic population had a rate of 127 per 100,000 population (age adjusted) in 2007, nearly two and a half times the best group rate [2].

- The rate of lower extremity amputation in persons with diabetes (objective 5-10) declined by $47.0 \%$ between $1997-99$ and $2005-07$, from 6.6 to 3.5 per 1,000 population (age adjusted), moving toward the 2010 target of 2.9 per 1,000.

- Females had a lower (better) rate of lower extremity amputations than males. The rate for females was 2.2 per 1,000 population (age adjusted) in 2005-07. The rate for males was 4.8 per 1,000 population (age adjusted) in $2005-07$, more than twice the rate for females [2].

- No change was observed in the percentage of persons who received annual foot examinations (objective 5-14) or annual dental examinations (objective 5-15). The percentage of persons aged 18 years and over who received an annual foot examination was 68\% (age adjusted) in both 1998 and 2008. The percentage of persons aged two years and over who had annual dental examinations was 56\% (age adjusted) in both 1997 and 2008. 


\section{Summary of Progress}

- Figure 5-1 presents a quantitative assessment of progress in achieving the Healthy People 2010 objectives for Diabetes [1]. Data to measure progress toward target attainment were available for 14 objectives. Of these:

- Five objectives (5-6, 5-7, 5-11, 5-12, and 5-17) met or exceeded the Healthy People 2010 targets.

- Five objectives moved toward their targets. A statistically significant difference between the baseline and the final data points was observed for four of these objectives (5-1, 5-4, 5-5, and 5-10). No significant difference was observed for the remaining objective (5-13).

- Two objectives (5-14 and 5-15) showed no change.

- Two objectives (5-2 and 5-3) moved away from their targets. A statistically significant difference between the baseline and final data point was observed for both of these objectives.

- Data were unavailable to measure progress for one objective (5-16). Two objectives (5-8 and 5-9) were dropped during the decade [3].

- Figure 5-2 displays health disparities in Diabetes from the best group rate for each characteristic at the most recent data point [2]. It also displays changes in disparities from baseline to the most recent data point [4].

- Of the 11 objectives with statistically significant racial and ethnic health disparities of $10 \%$ or more, the white non-Hispanic population had the only best rate for six objectives (5-1 through 5-3, 5-12, 5-13, and 5-16). The Asian or Pacific Islander population had the best rate for two objectives (5-5 and 5-11) and the Hispanic or Latino population and black non-Hispanic population each had the only best rate for one objective (5-7 and 5-14, respectively). In addition, the black non-Hispanic and white non-Hispanic populations were tied for the best rate for one objective (5-17).

- For all five objectives with statistically significant health disparities of $10 \%$ or more by sex, females had better rates than males (objectives $5-5$ through $5-7,5-10$, and 517).

- Persons with at least some college education had the best rate for nine of the 10 objectives with statistically significant health disparities of $10 \%$ or more by education level (objectives 5-1 through 5-3, 5-5, 5-7, 5-12, and 5-13 through 5-15). Persons with less than a high school education had the best rate for one objective (5-17).

- Persons without disabilities had better rates than persons with disabilities for the two objectives with statistically significant health disparities of $10 \%$ or more by disability status (objectives 5-2 and 5-3; see Highlights).

- Health disparities of $100 \%$ or more were observed for four objectives (5-2, 5-3, 5-5, and 5-10; see Highlights). 


\section{Transition to Healthy People 2020}

For Healthy People 2020, the focus of the Diabetes Topic Area has been expanded to include more objectives on diabetes prevention and control. See HealthyPeople.gov for a complete list of Healthy People 2020 topics and objectives.

The differences between the Healthy People 2010 objectives and those included in Healthy People 2020 are summarized below:

- The Healthy People 2020 Diabetes Topic Area includes 20 objectives, three of which are developmental, whereas the Healthy People 2010 Diabetes Focus Area had 17 objectives, including two objectives (5-8 and 5-9) that were dropped at the Midcourse Review [3,5].

- Nine Healthy People 2010 objectives, including diabetes incidence (objective 5-2), diabetes related deaths (objective 5-5), lower extremity amputations (objective 5-10), annual urinary microalbumin measurement (objective 5-11), A1C test at least two times a year (objective 5-12), annual dilated eye examination (objective 5-13), annual foot examination (objective 5-14), annual dental examination (objective 5-15), and self-blood glucose monitoring (objective 5-17) were retained "as is" [6].

- Two Healthy People 2010 objectives were modified [7]. The objectives tracking diabetes education (objective 5-1) and persons with diagnosed diabetes (objective 5-4) will be measured differently in Healthy People 2020.

- Four Healthy People 2010 objectives were archived: the prevalence of diabetes (objective 53 ), two objectives related to deaths among persons with diabetes (objectives 5-6 and 5-7), and aspirin therapy (objective 5-16) [8].

- Nine new objectives were added to the Healthy People 2020 Diabetes Topic Area:

- Four new objectives on control of diabetes and its complications include the proportion of the diabetic population with hemoglobin A1C test values $>9 \%$, and $\mathrm{A} 1 \mathrm{C}<7 \%$, as well as blood pressure control and cholesterol control among the population with diabetes.

- Three new objectives on diabetes prevention focus on persons at high risk for diabetes with pre-diabetes who report increasing physical activity, trying to lose weight, and reducing fat or calories in diet.

- Two new objectives were added to replace the archived mortality objectives: total mortality among the population with diabetes, and cardiovascular disease deaths in persons with diabetes.

Appendix D, “A Crosswalk Between Objectives From Healthy People 2010 to Healthy People 2020," summarizes the changes between the two decades of objectives, reflecting new knowledge and direction for this area. 


\section{Data Considerations}

Figure 5-3 presents state-level data for diabetes prevalence (objective 5-3) from the Behavioral Risk Factor Surveillance System (BRFSS). National data for this objective comes from the National Health Interview Survey (NHIS) and are the basis for setting targets. BRFSS data may not be comparable to the National data from NHIS. The BRFSS state rates are for the population aged 18 years and over. The NHIS National rate includes all ages.

Beginning in 2003, education data for mortality objectives 5-5, 5-6, and 5-7 from the National Vital Statistics System have been suppressed. The educational attainment item was changed in the new U.S. Standard Certificate of Death in 2003 to be consistent with the U.S. Census Bureau data and to improve the ability to identify specific types of educational degrees. Many states, however, are still using the 1989 version of the U.S. Standard Certificate of Death, which focuses on highest school grade completed. As a result, educational attainment data collected using the 2003 version are not comparable with data collected using the 1989 version [9].

In general, data on educational attainment are presented for persons aged 25 years and over, consistent with guidance given by the U.S. Bureau of the Census. However, because of the requirements of the different data systems, the age groups used to calculate educational attainment for any specific objective may differ from the age groups used to report the data for other Healthy People 2010 objectives, as well as from select populations within the same objective. Therefore, the reader is urged to exercise caution in interpreting the data by educational attainment shown in the Health Disparities Table. See Healthy People 2010: General Data Issues, referenced below.

Additional information on data issues is available from the following sources:

- All Healthy People 2010 tracking data can be found in the Healthy People 2010 database, DATA2010, available from http://wonder.cdc.gov/data2010/.

- Detailed information about the data and data sources used to support these objectives can be found in the Operational Definitions on the DATA2010 website, available from http://wonder.cdc.gov/data2010/focusod.htm.

- More information on statistical issues related to Healthy People tracking and measurement can be found in the Technical Appendix and in Healthy People 2010: General Data Issues, which is available in the Data Issues section of the NCHS Healthy People website under Healthy People 2010.

\section{References and Notes}

1. Displayed in the Progress Chart (Figure 5-1), the percent of targeted change achieved expresses the difference between the baseline and the final value relative to the initial difference between the baseline and the Healthy People 2010 target. As such, it is a relative measure of progress toward attaining the Healthy People 2010 target. See the Reader's Guide for more information. When standard errors were available, the difference between the baseline and the final value was tested at the 0.05 level of significance. See the Figure 5-1 footnotes, as well as the Technical Appendix, for more detail. 
2. Information about disparities among select populations is shown in the Health Disparities Table (Figure 5-2). Disparity from the best group rate is defined as the percent difference between the best group rate and each of the other group rates for a characteristic. For example, racial and ethnic health disparities are measured as the percent difference between the best racial and ethnic group rate and each of the other racial and ethnic group rates. Similarly, disparities by sex are measured as the percent difference between the better group rate (e.g., female) and the rate for the other group (e.g., male). Some objectives are expressed in terms of favorable events or conditions that are to be increased, while others are expressed in terms of adverse events or conditions that are to be reduced. In order to facilitate comparison of health disparities across different objectives, disparity is measured only in terms of adverse events or conditions. For comparability across objectives, objectives that are expressed in terms of favorable events or conditions are re-expressed using the adverse event or condition for the purpose of computing disparity, but they are not otherwise restated or changed. For example, objective 1-1, to increase the proportion of persons with health insurance (e.g., $72 \%$ of the American Indian or Alaska Native population aged under 65 years had some form of health insurance in 2008), is expressed in terms of the percentage of persons without health insurance (e.g., $100 \%-72 \%=28 \%$ of the American Indian or Alaska Native population aged under 65 years did not have any form of health insurance in 2008) when the disparity from the best group rate is calculated. See the Reader's Guide for more information. When standard errors were available, the difference between the best group rate and each of the other group rates was tested at the 0.05 level of significance. See the Figure 5-2 footnotes, as well as the Technical Appendix, for more detail.

3. Dropped objectives were not carried forward into Healthy People 2020. These objectives were either developmental or deleted at the Healthy People 2010 Midcourse Review or at another time in Healthy People 2010.

4. The change in disparity is estimated by subtracting the disparity at baseline from the disparity at the most recent data point and, therefore, is expressed as a change in percentage points. See the Reader's Guide for more information. When standard errors were available, the change in disparity was tested at the 0.05 level of significance. See the Figure 5-2 footnotes, as well as the Technical Appendix, for more detail.

5. To be included in Healthy People 2010, an objective must have a national data source that provides a baseline and at least one additional data point for tracking progress. Some objectives lacked baseline data at the time of their development but had a potential data source and were considered of sufficient national importance to be included in Healthy People. These are called "developmental" objectives. When data become available, a developmental objective is moved to measurable status and a Healthy People target can be set.

6. Retained "as is" objectives have no change in the numerator definition or in the denominator definition between the Healthy People 2010 and Healthy People 2020 objectives. These include objectives that were developmental in Healthy People 2010 and are developmental in Healthy People 2020 and for which no numerator or denominator information was available.

7. Modified objectives have some change in the numerator definition or in the denominator definition between the Healthy People 2010 and Healthy People 2020 objectives. These include objectives that went from developmental in Healthy People 2010 to measurable in Healthy People 2020 or vice versa.

8. Archived objectives had at least one data point in Healthy People 2010 but were not carried forward into Healthy People 2020.

9. Xu JQ Kochanek KD, Murphy SL, Tejada-Vera B. Deaths: Final data for 2007. National vital statistics reports; vol 58 no 19. Hyattsville, MD: National Center for Health Statistics. 2010. Available from: http://www.cdc.gov/nchs/data/nvsr/nvsr58/nvsr58 19.pdf. 


\section{Comprehensive Summary of Objectives: Diabetes}

\begin{tabular}{|c|c|c|}
\hline Objective & Description & Data Source or Objective Status \\
\hline $5-1$ & Diabetes education (age adjusted, 18+ years) & National Health Interview Survey (NHIS), CDC, NCHS. \\
\hline $5-2$ & $\begin{array}{l}\text { New cases of diabetes ( } 3 \text {-year average, age adjusted per } 1,000 \text { standard } \\
\text { population, } 18-84 \text { years) }\end{array}$ & National Health Interview Survey (NHIS), CDC, NCHS. \\
\hline $5-3$ & Prevalence of diabetes (age adjusted per 1,000 standard population) & National Health Interview Survey (NHIS), CDC, NCHS. \\
\hline $5-4$ & $\begin{array}{l}\text { Proportion of persons with diagnosed diabetes (age adjusted, 20+ } \\
\text { years) }\end{array}$ & National Health and Nutrition Examination Survey (NHANES), CDC, NCHS. \\
\hline $5-5$ & Diabetes-related deaths (age adjusted per 100,000 standard population) & National Vital Statistics System-Mortality (NVSS-M), CDC, NCHS. \\
\hline $5-6$ & $\begin{array}{l}\text { Diabetes-related deaths among persons with diabetes (age adjusted per } \\
1,000 \text { standard population) }\end{array}$ & $\begin{array}{l}\text { National Vital Statistics System-Mortality (NVSS-M), CDC, NCHS; National } \\
\text { Health Interview Survey (NHIS), CDC, NCHS. }\end{array}$ \\
\hline $5-7$ & $\begin{array}{l}\text { Cardiovascular disease deaths among persons with diabetes (age } \\
\text { adjusted per 100,000 standard population) }\end{array}$ & $\begin{array}{l}\text { National Vital Statistics System-Mortality (NVSS-M), CDC, NCHS; National } \\
\text { Health Interview Survey (NHIS), CDC, NCHS. }\end{array}$ \\
\hline $5-8$ & Gestational diabetes among pregnant women & Dropped \\
\hline $5-9$ & Foot ulcers among persons with diabetes & Dropped \\
\hline $5-10$ & $\begin{array}{l}\text { Lower extremity amputations in persons with diabetes (3-year average, } \\
\text { age adjusted per 1,000 standard population) }\end{array}$ & $\begin{array}{l}\text { National Hospital Discharge Survey (NHDS), CDC, NCHS; National Health } \\
\text { Interview Survey (NHIS), CDC, NCHS. }\end{array}$ \\
\hline $5-11$ & $\begin{array}{l}\text { Annual urinary microalbumin measurement among Medicare } \\
\text { beneficiaries with diabetes }\end{array}$ & United States Renal Data System (USRDS), NIH, NIDDK. \\
\hline $5-12$ & $\begin{array}{l}\text { A1C Test, at least twice a year among persons with diabetes (age } \\
\text { adjusted, 18+ years) }\end{array}$ & Behavioral Risk Factor Surveillance System (BRFSS), CDC, NCCDPHP. \\
\hline $5-13$ & $\begin{array}{l}\text { Annual dilated eye examinations among persons with diabetes (age } \\
\text { adjusted, 18+ years) }\end{array}$ & National Health Interview Survey (NHIS), CDC, NCHS. \\
\hline $5-14$ & $\begin{array}{l}\text { Annual foot examinations among persons with diabetes (age adjusted, } \\
18+\text { years) }\end{array}$ & Behavioral Risk Factor Surveillance System (BRFSS), CDC, NCCDPHP. \\
\hline $5-15$ & $\begin{array}{l}\text { Annual dental examinations among persons with diabetes (age adjusted, } \\
2+\text { years) }\end{array}$ & National Health Interview Survey (NHIS), CDC, NCHS. \\
\hline $5-16$ & $\begin{array}{l}\text { Aspirin intake } 15+\text { times per month among persons with diabetes (age } \\
\text { adjusted, } 40+\text { years) }\end{array}$ & National Health and Nutrition Examination Survey (NHANES), CDC, NCHS. \\
\hline $5-17$ & $\begin{array}{l}\text { Self-blood-glucose-monitoring at least once daily among persons with } \\
\text { diabetes (age adjusted, 18+ years) }\end{array}$ & Behavioral Risk Factor Surveillance System (BRFSS), CDC, NCCDPHP. \\
\hline
\end{tabular}


Figure 5-1. Progress Toward Target Attainment for Focus Area 5: Diabetes

Moved away

from target ${ }^{1}$

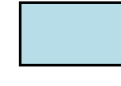

Moved toward

target

Met or exceeded

target

5-1. Diabetes education (age adjusted, 18+ years)

5-2. New cases of diabetes (3-year average, age adjusted, per 1,000 population, 18-84 years)

5-3. Prevalence of diabetes (age adjusted, per 1,000 population)

5-4. Proportion of persons with diagnosed diabetes (age adjusted, 20+ years)

5-5. Diabetes-related deaths (age adjusted, per 100,000 population)

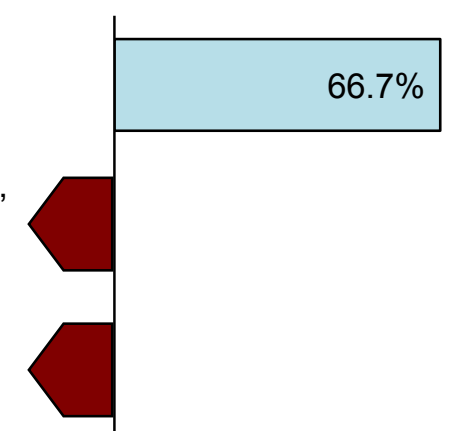

5-6. Diabetes-related deaths among persons with diabetes (age adjusted, per 1,000 population)

5-7. Cardiovascular disease deaths among persons with diabetes (age adjusted, per 100,000 population)

5-10. Lower extremity amputations in persons with diabetes (3-year average, age adjusted, per 1,000 population)

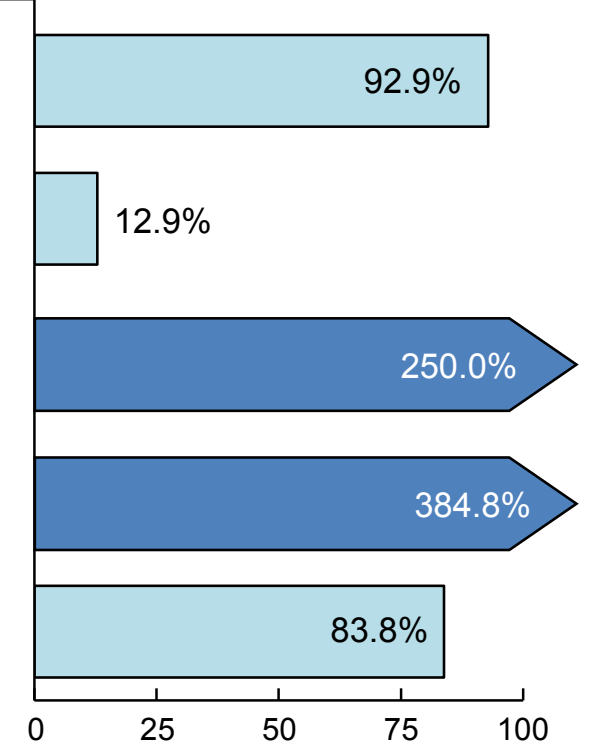

Percent of targeted change achieved ${ }^{5}$

\begin{tabular}{|c|c|c|c|c|c|}
\hline \multirow{2}{*}{$\begin{array}{l}2010 \\
\text { Target }\end{array}$} & \multirow{2}{*}{$\begin{array}{c}\text { Baseline } \\
\text { (Year) }\end{array}$} & \multirow{2}{*}{$\begin{array}{l}\text { Final } \\
\text { (Year) }\end{array}$} & \multicolumn{3}{|c|}{ Baseline vs. Final } \\
\hline & & & Difference $^{2}$ & $\begin{array}{l}\text { Statistically } \\
\text { Significant }\end{array}$ & $\begin{array}{l}\text { Percent } \\
\text { Change }^{4}\end{array}$ \\
\hline $60 \%$ & $\begin{array}{c}45 \% \\
(1998)\end{array}$ & $\begin{array}{c}55 \% \\
(1999)\end{array}$ & 10 & Yes & $22.2 \%$ \\
\hline 3.8 & $\begin{array}{c}5.5 \\
(1997-99)\end{array}$ & $\begin{array}{c}8.0 \\
(2006-08)\end{array}$ & 2.5 & Yes & $45.5 \%$ \\
\hline 25 & $\begin{array}{c}40 \\
(1997)\end{array}$ & $\begin{array}{c}59 \\
(2008)\end{array}$ & 19 & Yes & $47.5 \%$ \\
\hline $78 \%$ & $\begin{array}{c}64 \% \\
(1988-94)\end{array}$ & $\begin{array}{c}77 \% \\
(2005-08)\end{array}$ & 13 & Yes & $20.3 \%$ \\
\hline 46 & $\begin{array}{c}77 \\
(1999)\end{array}$ & $\begin{array}{c}73 \\
(2007)\end{array}$ & -4 & Yes & $-5.2 \%$ \\
\hline 7.8 & $\begin{array}{c}8.8 \\
(1999)\end{array}$ & $\begin{array}{c}6.3 \\
(2007)\end{array}$ & -2.5 & Yes & $-28.4 \%$ \\
\hline 299 & $\begin{array}{c}332 \\
(1999)\end{array}$ & $\begin{array}{c}205 \\
(2007)\end{array}$ & -127 & Yes & $-38.3 \%$ \\
\hline 2.9 & $\begin{array}{c}6.6 \\
(1997-99)\end{array}$ & $\begin{array}{c}3.5 \\
(2005-07)\end{array}$ & -3.1 & Yes & $-47.0 \%$ \\
\hline
\end{tabular}

(continued) 
Figure 5-1. Progress Toward Target Attainment for Focus Area 5: Diabetes (continued)

Moved away

from target ${ }^{1}$

Moved toward

target

Met or exceeded

target

5-11. Annual urinary microalbumin measurement among Medicare beneficiaries with diabetes

5-12. A1C Test, at least twice a year among persons with diabetes (age adjusted, 18+ years)

5-13. Annual dilated eye examinations among persons with diabetes (age adjusted, 18+ years)

5-14. Annual foot examinations among persons with diabetes (age adjusted, 18+ years)

5-15. Annual dental examinations among persons with diabetes (age adjusted, $2+$ years)

5-17. Self-blood-glucose-monitoring at least once daily among persons with diabetes (age adjusted, $18+$ years)

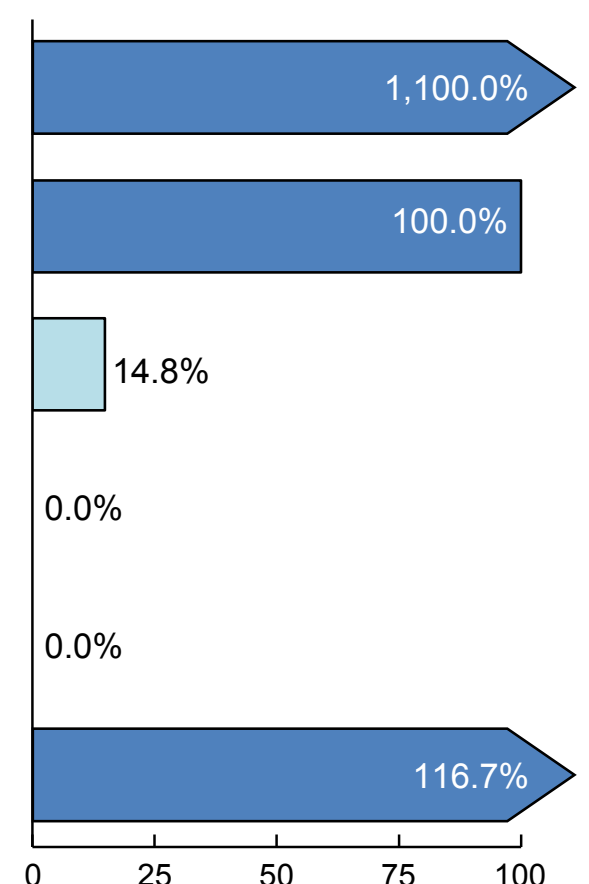

\begin{tabular}{|c|c|c|c|c|c|}
\hline \multirow{2}{*}{$\begin{array}{c}2010 \\
\text { Target }\end{array}$} & \multirow{2}{*}{$\begin{array}{c}\text { Baseline } \\
\text { (Year) }\end{array}$} & \multirow{2}{*}{$\begin{array}{l}\text { Final } \\
\text { (Year) }\end{array}$} & \multicolumn{3}{|c|}{ Baseline vs. Final } \\
\hline & & & Difference $^{2}$ & $\begin{array}{l}\text { Statistically } \\
\text { Significant }^{3}\end{array}$ & $\begin{array}{c}\text { Percent } \\
\text { Change }^{4}\end{array}$ \\
\hline $14 \%$ & $\begin{array}{c}12 \% \\
(2000)\end{array}$ & $\begin{array}{c}34 \% \\
(2007)\end{array}$ & 22 & Yes & $183.3 \%$ \\
\hline $65 \%$ & $\begin{array}{c}59 \% \\
(2000)\end{array}$ & $\begin{array}{c}65 \% \\
(2008)\end{array}$ & 6 & Yes & $10.2 \%$ \\
\hline $76 \%$ & $\begin{array}{c}49 \% \\
(1998)\end{array}$ & $\begin{array}{c}53 \% \\
(2008)\end{array}$ & 4 & No & $8.2 \%$ \\
\hline $91 \%$ & $\begin{array}{c}68 \% \\
(1998)\end{array}$ & $\begin{array}{c}68 \% \\
(2008)\end{array}$ & 0 & No & $0.0 \%$ \\
\hline $71 \%$ & $\begin{array}{c}56 \% \\
(1997)\end{array}$ & $\begin{array}{c}56 \% \\
(2008)\end{array}$ & 0 & No & $0.0 \%$ \\
\hline $61 \%$ & $\begin{array}{c}43 \% \\
(1998)\end{array}$ & $\begin{array}{c}64 \% \\
(2008)\end{array}$ & 21 & Yes & $48.8 \%$ \\
\hline
\end{tabular}

Percent of targeted change achieved ${ }^{5}$

(continued) 


\section{Figure 5-1. Progress Toward Target Attainment for Focus Area 5: Diabetes (continued)}

\section{NOTES}

See the Reader's Guide for more information on how to read this figure. See DATA2010 at http://wonder.cdc.gov/data2010 for all Healthy People 2010 tracking data. Tracking data are not available for objective 5-16. Objectives 5-8 and 5-9 were deleted at the Midcourse Review.

\section{FOOTNOTES}

${ }^{1}$ Movement away from target is not quantified using the percent of targeted change achieved. See Technical Appendix for more information.

${ }^{2}$ Difference $=$ Final value - Baseline value. Differences between percents $(\%)$ are measured in percentage points.

${ }^{3}$ When estimates of variability are available, the statistical significance of the difference between the final value and the baseline value is assessed at the 0.05 level. See $\underline{\text { Technical Appendix }}$ for more information.

${ }^{4}$ Percent change $=\frac{\text { Final value }- \text { Baseline value }}{\text { Baseline value }} \times 100$.
${ }^{5}$ Percent of targeted change achieved $=\frac{\text { Final value }- \text { Baseline value }}{\text { Healthy People } 2010 \text { target }- \text { Baseline value }} \times 100$.

\section{DATA SOURCES}

5-1-5-3. National Health Interview Survey (NHIS), CDC, NCHS.

5-4. National Health and Nutrition Examination Survey (NHANES), CDC, NCHS.

5-5. National Vital Statistics System-Mortality (NVSS-M), CDC, NCHS.

5-6-5-7. National Vital Statistics System-Mortality (NVSS-M), CDC, NCHS; National Health Interview Survey (NHIS), CDC, NCHS.

5-10. National Hospital Discharge Survey (NHDS), CDC, NCHS; National Health Interview Survey (NHIS), CDC, NCHS.

5-11. United States Renal Data System (USRDS), NIH, NIDDK.

5-12. Behavioral Risk Factor Surveillance System (BRFSS), CDC, NCCDPHP.

5-13. National Health Interview Survey (NHIS), CDC, NCHS.

5-14. Behavioral Risk Factor Surveillance System (BRFSS), CDC, NCCDPHP.

5-15. National Health Interview Survey (NHIS), CDC, NCHS.

5-17. Behavioral Risk Factor Surveillance System (BRFSS), CDC, NCCDPHP. 
Figure 5-2. Health Disparities Table for Focus Area 5: Diabetes

Disparities from the best group rate for each characteristic at the most recent data point and changes in disparity from the baseline to the most recent data point.

\begin{abstract}
Population-based objectives
5-1. Diabetes education (age adjusted, 18+ years) (1998 1999) $)^{1}$

5-2. New cases of diabetes (3-year average, age adjusted, per 1,000 population, $18-84$ years) $(1997-99,2006-08)^{2}$

5-3. Prevalence of diabetes (age adjusted, per 1,000 population) $(1997,2008)^{1}$

5-4. Proportion of persons with diagnosed diabetes (age adjusted, 20+ years) $(1988-94,2005-08)^{3}$

5-5. Diabetes-related deaths (age adjusted per 100,000 standard population) $(1999,2007)^{4}$

5-6. Diabetes-related deaths among persons with diabetes (age adjusted, per 1,000 population) (1999, $2007)^{4}$

5-7. Cardiovascular disease deaths among persons with diabetes (age adjusted, per 100,000 population) $(1999,2007)^{4}$

5-10. Lower extremity amputations in persons with diabetes (3-year average, age adjusted, per 1,000 population) (1997-99, 2005-07)

5-11. Annual urinary microalbumin measurement among Medicare beneficiaries with diabetes $(2000,2007)$

5-12. A1C Test, at least twice a year among persons with diabetes (age adjusted, 18+ years) $(2000,2008)^{5}$

5-13. Annual dilated eye examinations among persons with diabetes (age adjusted, 18+ years) $(1998,2008)^{1}$

5-14. Annual foot examinations among persons with diabetes (age adjusted, 18+ years) $(1998,2008)^{5}$

5-15. Annual dental examinations among persons with diabetes (age adjusted, 2+ years) $(1997,2008)^{1}$

5-16. Aspirin intake $15+$ times per month among persons with diabetes (age adjusted, 40+ years) (1999-2002)

5-17. Self-blood-glucose-monitoring at least once daily among persons with diabetes (age adjusted, 18+ years) $(1998,2008)^{5}$
\end{abstract}

Characteristics and Groups

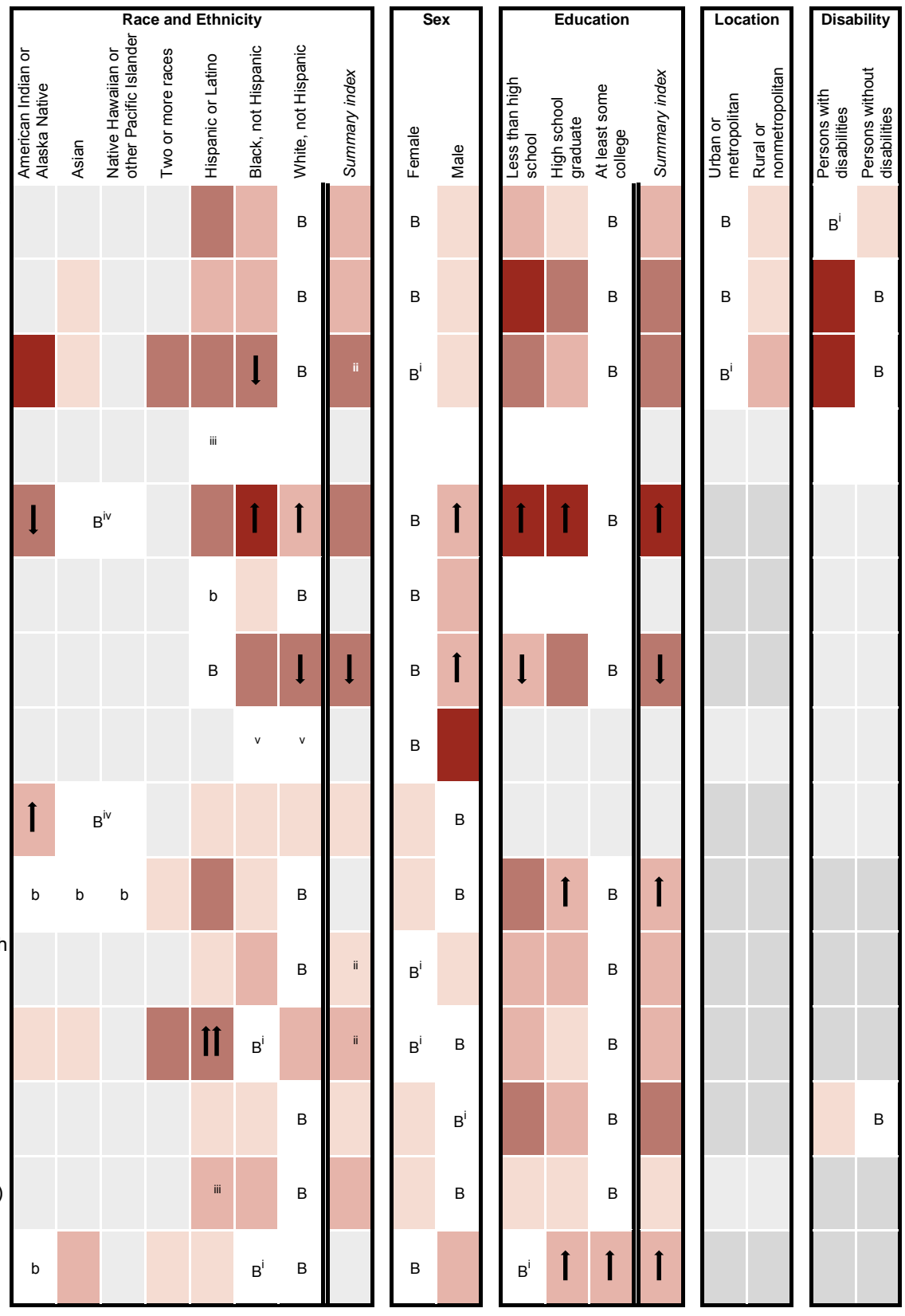

(continued) 
Figure 5-2. Health Disparities Table for Focus Area 5: Diabetes (continued)

NOTES

See DATA2010 at http://wonder.cdc.gov/data2010 for all Healthy People 2010 tracking data. Objectives 5-8 and 5-9 were deleted at Midcourse Review.

Years in parentheses represent the baseline and most recent data years (if available).

Disparity from the best group rate is defined as the percent difference between the best group rate and each of the other group rates for a characteristic (e.g., race and ethnicity). The summary index is the average of these percent differences for a characteristic. Change in disparity is estimated by subtracting the disparity at baseline from the disparity at the most recent data point. Change in the summary index is estimated by subtracting the summary index at baseline from the summary index at the most recent data point. See Technical Appendix for more information.

Measures of variability were available for all objectives in this table. Thus, the variability of best group rates was assessed, and statistical significance was tested. Disparities of $10 \%$ or more are displayed when the differences from the best group rate are statistically significant at the 0.05 level. Changes in disparities over time are indicated by arrows when the changes are greater than or equal to 10 percentage points and are statistically significant at the 0.05 level. See Technical Appendix.

LEGEND

The "best" group rate at the most recent data point. B $\begin{aligned} & \text { The group with the best rate for } \\ & \text { specified characteristic. }\end{aligned}$

Most favorable group rate for specified characteristic, but reliability criterion not met.

Percent difference from the best group rate

\begin{tabular}{|c|c|c|c|c|}
\hline \multirow[b]{2}{*}{$\begin{array}{l}\text { Disparity from the best group rate at the } \\
\text { most recent data point. }\end{array}$} & \multicolumn{4}{|c|}{ Percent difference from the best group rate } \\
\hline & $\begin{array}{l}\text { Less than } 10 \% \text {, or difference not } \\
\text { statistically significant (when } \\
\text { estimates of variability are available). }\end{array}$ & $10 \%-49 \%$ & $50 \%-99 \%$ & $100 \%$ or more \\
\hline
\end{tabular}

Changes in disparity over time are shown when:
(a) disparities data are available at both baseline and most recent time points;
(b) data are not for the group(s) indicated by "B" or "b" at either time point; and
(c) the change is greater than or equal to 10 percentage points and statistically
significant, or when the change is greater than or equal to 10 percentage
points and estimates of variability were not available. See Technical Appendix.

\section{FOOTNOTES}

1 Baseline data by race and ethnicity are for 1999 .

2 Baseline data by race and ethnicity are for 1999-2001.

3 Baseline data by disability status are for 1991-94.

4 Most recent data by education level are for 2002 .

5 Baseline data by race and ethnicity are for 2001.

i The group with the best rate at the most recent data point is different from the group with the best rate at baseline. Both rates met the reliability criterion. See Technical Appendix.

ii Change in the summary index cannot be assessed. See Technical Appendix.

iii Data are for Mexican American.

iv Data are for Asian or Pacific Islander.

$\checkmark$ Data include persons of Hispanic origin.

\section{DATA SOURCES}

5-1-5-3. National Health Interview Survey (NHIS), CDC, NCHS.

5-4. National Health and Nutrition Examination Survey (NHANES), CDC, NCHS.

5-5. National Vital Statistics System-Mortality (NVSS-M), CDC, NCHS.

5-6-5-7. National Vital Statistics System-Mortality (NVSS-M), CDC, NCHS; National Health Interview Survey (NHIS), CDC, NCHS

5-10. National Hospital Discharge Survey (NHDS), CDC, NCHS; National Health Interview Survey (NHIS), CDC, NCHS.

5-11. United States Renal Data System (USRDS), NIH, NIDDK.

5-12. Behavioral Risk Factor Surveillance System (BRFSS), CDC, NCCDPHP.

5-13. National Health Interview Survey (NHIS), CDC, NCHS.

5-14. Behavioral Risk Factor Surveillance System (BRFSS), CDC, NCCDPHP.

5-15. National Health Interview Survey (NHIS), CDC, NCHS.

5-16. National Health and Nutrition Examination Survey (NHANES), CDC, NCHS .

5-17. Behavioral Risk Factor Surveillance System (BRFSS), CDC, NCCDPHP. 
Figure 5-3. Diabetes Prevalence (Aged 18+) 2008

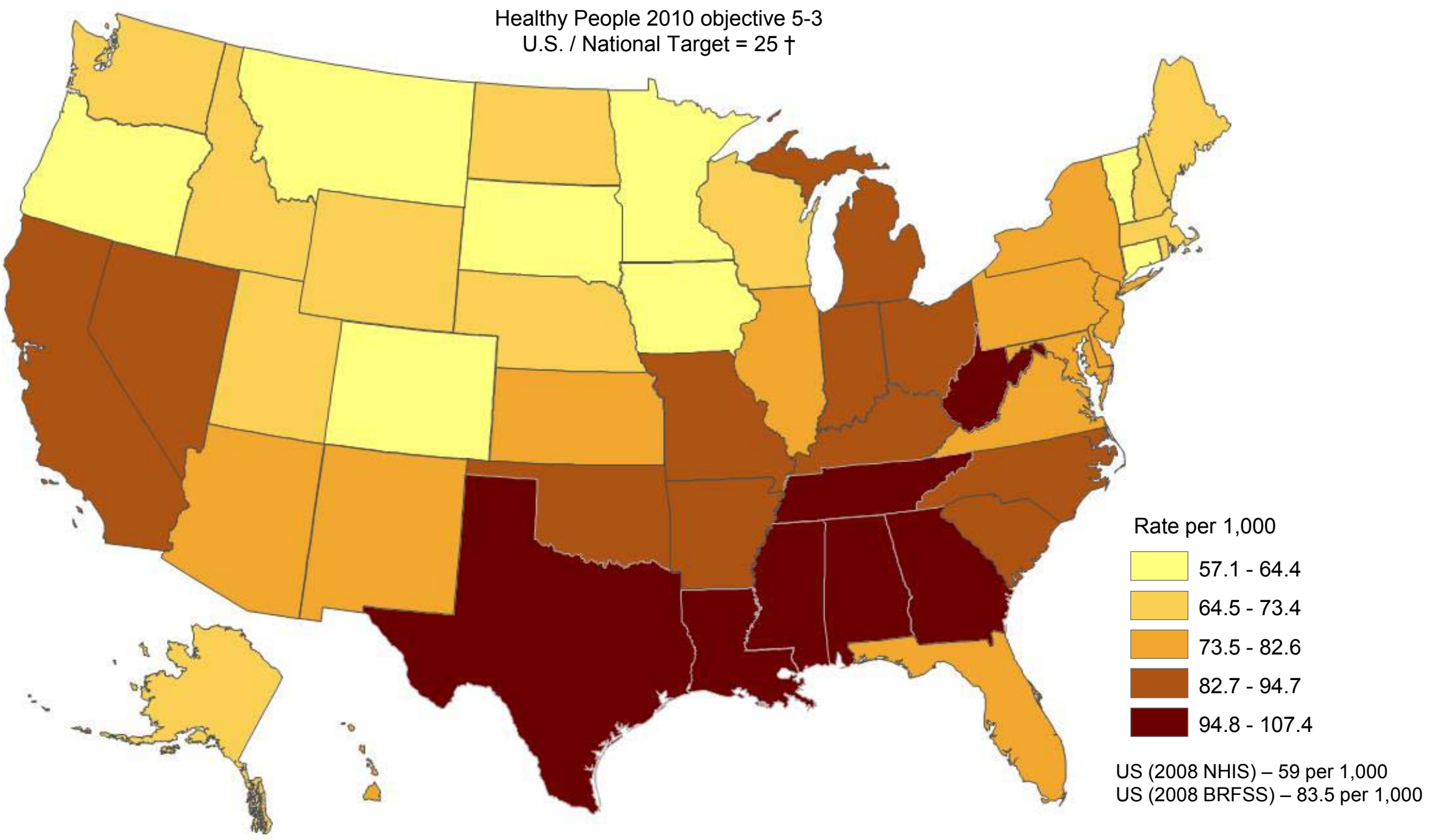

NOTES: Data are age-adjusted to the 2000 standard population. Rates are displayed by a Jenks classification for U.S. states. National data for the objective come from the National Health Interview Survey (NHIS) and is the basis for setting the target †. State data from the BRFSS may not be comparable to the national data from the NHIS. Both US rates are shown for comparison purposes. BRFSS state-based rates are for population 18+, NHIS national rate is for all ages. SOURCE: Behavioral Risk Factor Surveillance System (BRFSS), CDC. 


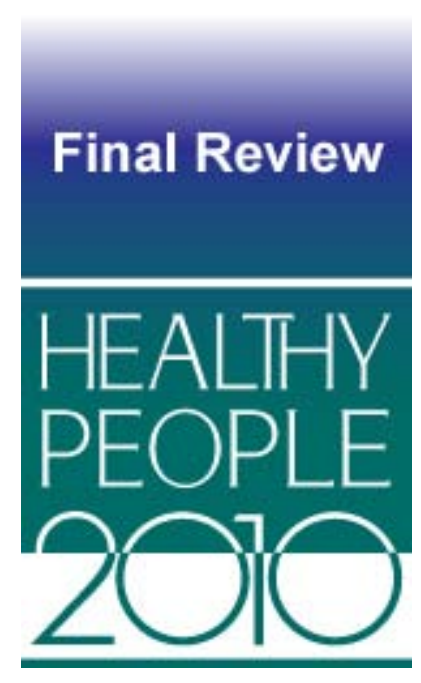

\section{Disability and Sec ondary Conditions}

\section{Co-Lead Agencies}

Centers for Disease Control and Prevention

National Institute on Disability and Rehabilitation Research, U.S. Department of Education

\section{Contents}

Goal 6-2

Highlights 6-2

Summary of Progress. 6-4

Transition to Healthy People 2020 6-5

Data Considerations 6-6

Notes 6-7

Comprehensive Summary of Objectives 6-9

Progress Chart 6-11

Health Disparities Table 6-14 


\section{Goal: Promote the health of people with disabilities, prevent secondary conditions, and eliminate disparities between people with and without disabilities in the U.S. population}

The objectives in this chapter include measures of life satisfaction among people with disabilities, barriers to their participation in everyday aspects of life, and the availability of public health programs to support these individuals and their caregivers. The objectives also track the use of congregate care as well as the availability of surveillance systems that identify persons with disabilities.

All Healthy People tracking data quoted in this chapter, along with technical information and operational definitions for each objective, can be found in the Healthy People 2010 database, DATA2010, available from http://wonder.cdc.gov/data2010/.

More information about this Focus Area can be found in the following publications:

- Healthy People 2010: Understanding and Improving Health, available from http://www.healthypeople.gov/2010/Document/tableofcontents.htm\#under.

- Healthy People 2010 Midcourse Review, available from http://www.healthypeople.gov/2010/data/midcourse/html/default.htm\#FocusAreas.

\section{Highlights}

- Substantial progress was achieved for the objectives in this Focus Area during the past decade [1]. Over two thirds of the Disability and Secondary Conditions objectives with data to measure progress moved toward or achieved their Healthy People 2010 targets (Figure $6-1)$. However, health disparities of $50 \%$ to $99 \%$ were observed among racial and ethnic populations, education groups, and income groups (Figure 6-2), as highlighted below [2].

- The number of states and the District of Columbia (D.C.) with public health surveillance systems for persons with disabilities (objective 6-13a) increased from 14 states in 1999 to 51 in 2009, meeting the 2010 target of 51. During the same time period, the number of states and D.C. with surveillance systems for caregivers of persons with disabilities (objective 6-13e) increased from zero to 51, also meeting the target of 51.

- A statistically significant downward trend was observed during the past decade in the number of adults in congregate care facilities (objective 6-7a) [3]. The number dropped by $36.2 \%$ between 1997 and 2009, from 93,362 to 59,604, moving toward the 2010 target of 46,681 . However, the number of children and young adults in congregate care facilities (objective 6-7b) increased by $11.0 \%$ between 1997 and 2008, from 26,028 to 28,890, moving away from the target of 0 . The proportion of children and youth aged 6-21 years with disabilities who are enrolled in regular education programs (objective 6-9) increased by $28.9 \%$ between $1995-96$ and $2008-09$, from $45 \%$ to $58 \%$, moving toward the 2010 target of $60 \%$. 
- Sadness or depression among children and adolescents aged 4-17 years with disabilities (objective 6-2) decreased by $25.8 \%$ between 1997 and 2007, from 31\% to 23\%, moving toward the 2010 target of $17 \%$.

- The employment rate among adults aged 18-64 years with disabilities (objective 6-8) declined by $14.0 \%$ between 1997 and 2008, from 43\% to 37\%, moving away from the 2010 target of $80 \%$. Disparities were observed for a number of population groups, for example:

- Among racial and ethnic groups, white non-Hispanic adults with disabilities had the highest (best) employment rate, $41 \%$ in 2008, whereas Hispanic or Latino and black non-Hispanic adults with disabilities had rates of $29 \%$ and $27 \%$ in 2008 , respectively. When expressed as unemployment rates among adults with disabilities, the rate for Hispanic or Latino adults was $20 \%$ higher than the best group rate (that for white non-Hispanic adults) and the rate for black non-Hispanic adults was $24 \%$ higher than the best group rate [2].

- Among educational groups, persons with disabilities and at least some college education had the highest (best) employment rate, $52 \%$ in 2008, whereas the rate for persons with disabilities who had less than a high school education was $22 \%$ in 2008. When expressed as unemployment rates among persons with disabilities, the rate for persons with less than a high school education was more than one and a half times the best group rate [2].

- Disparities among racial and ethnic groups were observed for several objectives, for example:

- The white non-Hispanic population had the lowest (best) proportion of persons with disabilities reporting barriers to participation in community activities (objective 6-12d), $11 \%$ in 2002. Persons of two or more races had a rate of $24 \%$ in 2002 , more than twice the best group rate [2].

- The white non-Hispanic population had the highest (best) proportion of persons with disabilities reporting access to health and wellness programs (objective 6-10), $54 \%$ in 2002, whereas the rate for the Hispanic or Latino population was $27 \%$ in 2002. When expressed as persons with disabilities reporting no access to health and wellness programs, the rate for the Hispanic or Latino population was more than one and a half times the best group rate [2].

- The white-non-Hispanic population had the highest (best) proportion of sufficient emotional support among adults with disabilities (objective 6-5), 73\% in 2008, whereas the American Indian or Alaska Native, Asian, and black non-Hispanic populations had rates of 59\%, 58\%, and 58\% in 2008, respectively. When expressed as persons with disabilities without sufficient emotional support, the rates for the American Indian or Alaska Native, Asian, and black non-Hispanic populations were all about one and a half times as high as the best group rate (that for the white nonHispanic population) [2]. 


\section{Summary of Progress}

- Figure 6-1 presents a quantitative assessment of progress in achieving the Healthy People 2010 objectives for Disability and Secondary Conditions [1]. Data to measure progress toward target attainment were available for 13 objectives. Of these:

- Two objectives (6-13a and e) met their Healthy People 2010 targets.

- Seven objectives moved toward their targets. A statistically significant difference between the baseline and the final data points was observed for two of these objectives (6-5 and 6-6). No significant difference was observed for one objective (6$2)$; and data to test the significance of the difference were unavailable for four objectives (6-1, 6-7a, 6-9, and 6-13c).

- One objective showed no change (6-13g).

- Three objectives moved away from their targets. A statistically significant difference between the baseline and final data points was observed for two objectives (6-3 and 6-8); data to test the significance of the difference were unavailable for one objective (6-7b).

- There were four objectives that remained developmental (objectives 6-13b, d, f, and h), and seven that had no additional data available to measure progress (objectives 6-4, 6-10, 6-11, and 6-12a through d) [4].

- Figure 6-2 displays health disparities in Disability and Secondary Conditions from the best group rate for each characteristic at the most recent data point [2]. It also displays changes in disparities from baseline to the most recent data point [5].

- Of the seven objectives with statistically significant racial and ethnic health disparities of $10 \%$ or more, the white non-Hispanic population had the best rate for six objectives (6-4, 6-5, 6-8, 6-10, and 6-12a and d). The Hispanic or Latino population had the best rate for one objective (6-6).

- One health disparity of $100 \%$ or more was observed: barriers to participation in community activities were lowest among the white nonHispanic population; the rate for persons of two or more races was more than twice the best group rate (objective 6-12d; see Highlights).

- Males had better rates for five of the six objectives with statistically significant health disparities of $10 \%$ or more by sex (objectives $6-3,6-8,6-11$, and $6-12 \mathrm{a}$ and d). Females had better rates for the remaining objective (6-4).

- Persons with at least some college education had the best rate for the seven objectives with statistically significant health disparities of $10 \%$ or more by education level (objective 6-3 through 6-6, 6-8, 6-10, and 6-11).

- Health disparities of $50 \%$ to $99 \%$ between persons with less than high school education and persons with at least some college education were observed for five objectives (6-4 through 6-6, 6-8, and 6-10).

- Persons with middle/high incomes had the best rates for the six objectives with statistically significant health disparities of $10 \%$ or more by income (objectives 6-3, 6-4, 6-10, 6-11, and 6-12a and d).

- Health disparities of $50 \%$ to $99 \%$ between low-income (poor) persons and middle/high income persons were observed for four objectives $(6-3,6-4,6$ 10 , and 6-12d). 


\section{Transition to Healthy People 2020}

For Healthy People 2020, the Healthy People 2010 Disability and Secondary Conditions Focus Area was expanded to include a broader range of objectives, with increased emphasis on health determinants. Consequently the Healthy People 2020 Topic Area name was changed from "Disability and Secondary Conditions" to "Disability and Health." See HealthyPeople.gov for a complete list of Healthy People 2020 topics and objectives

The differences between the Healthy People 2010 objectives and those included in Healthy People 2020 objectives are summarized below:

- The Healthy People 2010 Disability and Secondary Conditions Focus Area had 24 objectives whereas Healthy People 2020 Disability and Health Topic Area has a total of 28 objectives.

- Three of the Healthy People 2010 objectives were retained "as is" [6]. These include the inclusion of children and youth in regular education programs, Tribal disability surveillance, and Tribal caregiver surveillance (objectives 6-9, and 6-13b and f).

- Nineteen of the Healthy People 2010 objectives were modified [7].

- Identifying people with disabilities in "surveillance instruments" was reworded to clarify "population data systems" (objective 6-1).

- Two objectives on depressive symptoms among children and adults with disabilities were combined so that the age groups could be better reflected in a demographic template (objectives 6-2 and 6-3).

- Social participation among adults with disabilities was reworded to reflect all ages and a broader range of social activities (objective 6-4).

- Emotional support among adults with disabilities was reworded to include "social" support as well (objective 6-5).

- Two objectives on congregate care among children/youth and adults with disabilities were reworded to reflect residences that serve people instead of facilities with "beds" (objectives 6-7a and b).

- Employment among adults with disabilities was reworded to include youth with disabilities in the new measurement (objective 6-8).

- Two objectives on access to health and wellness programs and not having needed assistive devices and technology were both reworded to reflect barriers (objectives 6-10 and 6-11).

- Four objectives on "reported environmental barriers" to participation in home, school, work, or community activities were reworded to reflect "encountering" barriers" (objectives 6-12a through d).

- Five objectives on state or Tribal health surveillance and health promotion among people with disabilities and their caregivers were reworded to specify state "health departments" with at least "one" program (objectives 6-13c through e, and 6-13g through h). 
- Two Healthy People 2010 objectives were archived [8]. Due to relatively high reported rates and lack of specific public health interventions, life satisfaction among adults with disabilities (objective 6-6) was archived. After meeting the target for several consecutive years, state disability surveillance (objective 6-13a) was archived.

- Nine new objectives were added to the Healthy People 2020 Disability and Health Topic Area. These objectives address:

- Delays in receiving preventive care among persons with disabilities

- Transition planning from pediatric to adult health care for youth with disabilities

- The receipt of appropriate medical care for persons with epilepsy

- Use of inappropriate medications among older adults with disabilities

- Unemployment among persons with disabilities

- Unintentional injury among persons with disabilities

- Early intervention services for children with disabilities

- Master of Public Health (MPH) programs that offer courses in disability and health

- Homes and residential buildings that have visitable features (e.g., no-step entrance to the home).

Appendix D, “A Crosswalk Between Objectives From Healthy People 2010 to Healthy People 2020," summarizes the changes between the two decades of objectives, reflecting new knowledge and direction for this area.

\section{Data Considerations}

Education and income are the primary measures of socioeconomic status (SES) in Healthy People 2010. Most data systems used in Healthy People 2010 define income as a family's income before taxes. In order to facilitate comparisons among groups and over time, while adjusting for family size and for inflation, Healthy People 2010 categorizes income using the poverty thresholds developed by the U.S. Census Bureau. Thus, the three categories of family income that are primarily used are:

- Poor-below the Federal poverty level

- Near poor-100\% to $199 \%$ of the Federal poverty level

- Middle/high income-200\% or more of the Federal poverty level.

These categories may be overridden by considerations specific to the data system, in which case they are modified as appropriate. See Healthy People 2010: General Data Issues, referenced below.

In general, data on educational attainment are presented for persons aged 25 years and over, consistent with guidance given by the U.S. Bureau of the Census. However, because of the requirements of the different data systems, the age groups used to calculate educational attainment for any specific objective may differ from the age groups used to report the data for other Healthy People 2010 objectives, as well as from select populations within the same objective. Therefore, the reader is urged to exercise caution in interpreting the data by educational attainment shown in the Health Disparities Table. See Healthy People 2010: General Data Issues, referenced below. 
Additional information on data issues is available from the following sources:

- All Healthy People 2010 tracking data can be found in the Healthy People 2010 database, DATA2010, available from http://wonder.cdc.gov/data2010/.

- Detailed information about the data and data sources used to support these objectives can be found in the Operational Definitions on the DATA2010 website, available from http://wonder.cdc.gov/data2010/focusod.htm.

- More information on statistical issues related to Healthy People tracking and measurement can be found in the Technical Appendix and in Healthy People 2010: General Data Issues, which is available in the Data Issues section of the NCHS Healthy People website under Healthy People 2010.

\section{Notes}

1. Displayed in the Progress Chart (Figure 6-1), the percent of targeted change achieved expresses the difference between the baseline and the final value relative to the initial difference between the baseline and the Healthy People 2010 target. As such, it is a relative measure of progress toward attaining the Healthy People 2010 target. See the Reader's Guide for more information. When standard errors were available, the difference between the baseline and the final value was tested at the 0.05 level of significance. See the Figure 6-1 footnotes, as well as the Technical Appendix, for more detail.

2. Information about disparities among select populations is shown in the Health Disparities Table (Figure 6-2). Disparity from the best group rate is defined as the percent difference between the best group rate and each of the other group rates for a characteristic. For example, racial and ethnic health disparities are measured as the percent difference between the best racial and ethnic group rate and each of the other racial and ethnic group rates. Similarly, disparities by sex are measured as the percent difference between the better group rate (e.g., female) and the rate for the other group (e.g., male). Some objectives are expressed in terms of favorable events or conditions that are to be increased, while others are expressed in terms of adverse events or conditions that are to be reduced. In order to facilitate comparison of health disparities across different objectives, disparity is measured only in terms of adverse events or conditions. For comparability across objectives, objectives that are expressed in terms of favorable events or conditions are re-expressed using the adverse event or condition for the purpose of computing disparity, but they are not otherwise restated or changed. For example, objective 1-1, to increase the proportion of persons with health insurance (e.g., $72 \%$ of the American Indian or Alaska Native population aged under 65 years had health insurance in 2008), is expressed in terms of the percentage of persons without health insurance (e.g., $100 \%-72 \%=28 \%$ of the American Indian or Alaska Native population aged under 65 years did not have health insurance in 2008) when the disparity from the best group rate is calculated. See the Reader's Guide for more information. When standard errors were available, the difference between the best group rate and each of the other group rates was tested at the 0.05 level of significance. See the Figure 6-2 footnotes, as well as the Technical Appendix, for more detail.

3. The presence of a monotonic increasing or decreasing trend in the underlying measure was tested with the nonparametric Mann-Kendall test, then the slope of a linear trend was estimated with the nonparametric Sen's method. See Technical Appendix for more information.

4. To be included in Healthy People 2010, an objective must have a national data source that provides a baseline and at least one additional data point for tracking progress. Some objectives lacked baseline data at the time of their development but had a potential data source and were considered of sufficient national importance to be included in Healthy People. These are called "developmental" objectives. When data become available, a developmental objective is moved to measurable status and a Healthy People target can be set. 
5. The change in disparity is estimated by subtracting the disparity at baseline from the disparity at the most recent data point and, therefore, is expressed as a change in percentage points. See the Reader's Guide for more information. When standard errors were available, the change in disparity was tested at the 0.05 level of significance. See the Figure 1-2 footnotes, as well as the Technical Appendix, for more detail.

6. Retained "as is" objectives have no change in the numerator definition or in the denominator definition between the Healthy People 2010 and Healthy People 2020 objectives. These include objectives that were developmental in Healthy People 2010 and are developmental in Healthy People 2020 and for which no numerator or denominator information was available.

7. Modified objectives have some change in the numerator definition or in the denominator definition between the Healthy People 2010 and Healthy People 2020 objectives. These include objectives that went from developmental in Healthy People 2010 to measurable in Healthy People 2020 or vice versa.

8. Archived objectives had at least one data point in Healthy People 2010 but were not carried forward into Healthy People 2020. 


\section{Comprehensive Summary of Objectives: Disability and Secondary Conditions}

\begin{tabular}{|c|c|}
\hline Objective & Description \\
\hline $6-1$ & Standard questions to identify people with disabilities in data sets \\
\hline $6-2$ & $\begin{array}{l}\text { Sadness or depression among children and adolescents with disabilities } \\
\text { (4-17 years) }\end{array}$ \\
\hline $6-3$ & $\begin{array}{l}\text { Negative feelings interfering with activities among adults with } \\
\text { disabilities (age adjusted, 18+ years) }\end{array}$ \\
\hline $6-4$ & $\begin{array}{l}\text { Social participation among adults with disabilities (age adjusted, 18+ } \\
\text { years) }\end{array}$ \\
\hline $6-5$ & $\begin{array}{l}\text { Sufficient emotional support among adults with disabilities (age } \\
\text { adjusted, 18+ years) }\end{array}$ \\
\hline $6-6$ & $\begin{array}{l}\text { Satisfaction with life among adults with disabilities (age adjusted, 18+ } \\
\text { years) }\end{array}$ \\
\hline $6-7 a$ & Congregate care of adults with disabilities (number, $22+$ years) \\
\hline $6-7 b$ & $\begin{array}{l}\text { Congregate care of children and young adults with disabilities (number, } \\
\leq 22 \text { years) }\end{array}$ \\
\hline $6-8$ & Employment rate among adults with disabilities (18-64 years) \\
\hline $6-9$ & $\begin{array}{l}\text { Inclusion of children and youth with disabilities in regular education } \\
\text { programs (6-21 years) }\end{array}$ \\
\hline $6-10$ & $\begin{array}{l}\text { Access to health and wellness programs among adults with disabilities } \\
\text { (age adjusted } 18+\text { years) }\end{array}$ \\
\hline $6-11$ & $\begin{array}{l}\text { Lack of assistive devices and technology among adults with disabilities } \\
\text { (age adjusted, 18+ years) }\end{array}$ \\
\hline $6-12 a$ & $\begin{array}{l}\text { Environmental barriers affecting participation in activities at home } \\
\text { among adults with disabilities (age adjusted, 18+ years) }\end{array}$ \\
\hline $6-12 b$ & $\begin{array}{l}\text { Environmental barriers affecting participation in activities at school } \\
\text { among adults with disabilities (age adjusted, 18+ years) }\end{array}$ \\
\hline $6-12 c$ & $\begin{array}{l}\text { Environmental barriers affecting participation in activities at work } \\
\text { among adults with disabilities (age adjusted, 18+ years) }\end{array}$ \\
\hline
\end{tabular}

CDC, NCBDDD.

Data Source or Objective Status

National Health Interview Survey (NHIS), CDC, NCHS.

National Health Interview Survey (NHIS), CDC, NCHS.

National Health Interview Survey (NHIS), CDC, NCHS.

Behavioral Risk Factor Surveillance System (BRFSS), CDC, NCCDPHP.

Behavioral Risk Factor Surveillance System (BRFSS), CDC, NCCDPHP.

Survey of State Developmental Disabilities Directors, University of Minnesota.

Survey of State Developmental Disabilities Directors, University of Minnesota.

National Health Interview Survey (NHIS), CDC, NCHS.

Data Analysis System (DANS), Department of Education.

National Health Interview Survey (NHIS), CDC, NCHS.

National Health Interview Survey (NHIS), CDC, NCHS.

National Health Interview Survey (NHIS), CDC, NCHS.

National Health Interview Survey (NHIS), CDC, NCHS.

National Health Interview Survey (NHIS), CDC, NCHS. 


\begin{tabular}{|c|c|c|}
\hline Objective & Description & Data Source or Objective Status \\
\hline $6-12 d$ & $\begin{array}{l}\text { Environmental barriers affecting participation in community activities } \\
\text { among adults with disabilities (age adjusted, 18+ years }\end{array}$ & National Health Interview Survey (NHIS), CDC, NCHS. \\
\hline $6-13 a$ & Surveillance for persons with disabilities (no. States and D.C.) & CDC, NCDBBB, DH-Team. \\
\hline $6-13 b$ & Surveillance for persons with disabilities (Tribes) & Developmental \\
\hline $6-13 c$ & $\begin{array}{l}\text { Health promotion programs for persons with disabilities (no. States } \\
\text { and D.C.) }\end{array}$ & CDC, NCDBBB, DH-Team. \\
\hline $6-13 d$ & Health promotion programs for persons with disabilities (Tribes) & Developmental \\
\hline $6-13 e$ & Surveillance for caregivers (no. States and D.C.) & CDC, NCDBBB, DH-Team. \\
\hline $6-13 f$ & Surveillance for caregivers (Tribes) & Developmental \\
\hline $6-13 g$ & Health promotion programs for caregivers (no. States and D.C.) & CDC, NCDBBB, DH-Team. \\
\hline $6-13 \mathrm{~h}$ & Health promotion programs for caregivers (Tribes) & Developmental \\
\hline
\end{tabular}


Figure 6-1. Progress Toward Target Attainment for Focus Area 6: Disability and Secondary Conditions

Moved away

from target ${ }^{1}$

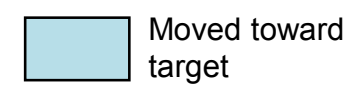

Moved toward

target

Met or exceeded

target

6-1. Standard questions to identify people with disabilities in data sets

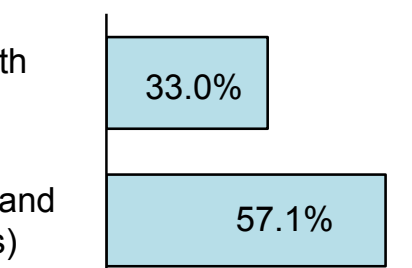

6-2. Sadness or depression among children and adolescents with disabilities (4-17 years)

6-3. Negative feelings interfering with activities among adults with disabilities (age adjusted, 18+ years)

6-5. Sufficient emotional support among adults with disabilities (age adjusted, 18+ years)

6-6. Satisfaction with life among adults with disabilities (age adjusted, 18+ years)

6-7a. Congregate care of adults with disabilities (number, 22+ years)

6-7b. Congregate care of children and youth with disabilities (number, $\leq 22$ years)

6-8. Employment rate among adults with disabilities (18-64 years)

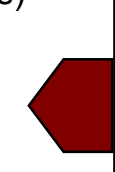

\section{)}

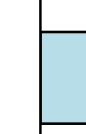

$15.4 \%$

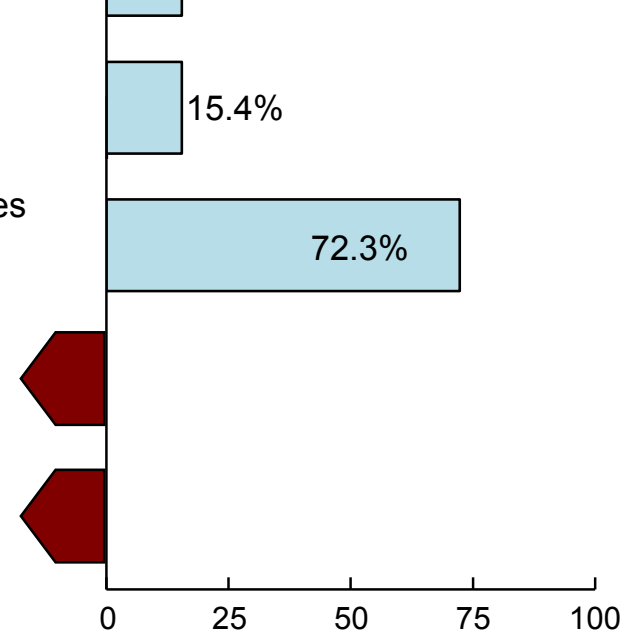

Percent of targeted change achieved ${ }^{5}$

\begin{tabular}{|c|c|c|c|c|c|}
\hline \multirow{2}{*}{$\begin{array}{l}2010 \\
\text { Target }\end{array}$} & \multirow{2}{*}{$\begin{array}{c}\text { Baseline } \\
\text { (Year) }\end{array}$} & \multirow{2}{*}{$\begin{array}{l}\text { Final } \\
\text { (Year) }\end{array}$} & \multicolumn{3}{|c|}{ Baseline vs. Final } \\
\hline & & & Difference $^{2}$ & $\begin{array}{l}\text { Statistically } \\
\text { Significant }^{3}\end{array}$ & $\begin{array}{l}\text { Percent } \\
\text { Change }^{4}\end{array}$ \\
\hline $100 \%$ & $\begin{array}{c}0 \% \\
(1999)\end{array}$ & $\begin{array}{c}33 \% \\
(2009)\end{array}$ & 33 & $\begin{array}{c}\text { Not } \\
\text { tested }\end{array}$ & * \\
\hline $17 \%$ & $\begin{array}{c}31 \% \\
(1997)\end{array}$ & $\begin{array}{c}23 \% \\
(2007)\end{array}$ & -8 & No & $-25.8 \%$ \\
\hline $7 \%$ & $\begin{array}{c}28 \% \\
(1997)\end{array}$ & $\begin{array}{c}32 \% \\
(2008)\end{array}$ & 4 & Yes & $14.3 \%$ \\
\hline $80 \%$ & $\begin{array}{c}67 \% \\
(2005)\end{array}$ & $\begin{array}{c}69 \% \\
(2008)\end{array}$ & 2 & Yes & $3.0 \%$ \\
\hline $97 \%$ & $\begin{array}{c}84 \% \\
(2005)\end{array}$ & $\begin{array}{c}86 \% \\
(2008)\end{array}$ & 2 & Yes & $2.4 \%$ \\
\hline 46,681 & $\begin{array}{l}93,362 \\
(1997)\end{array}$ & $\begin{array}{l}59,604 \\
(2009)\end{array}$ & $-33,758$ & $\begin{array}{l}\text { Not } \\
\text { tested }\end{array}$ & $-36.2 \%$ \\
\hline 0 & $\begin{array}{l}26,028 \\
(1997)\end{array}$ & $\begin{array}{l}28,890 \\
(2008)\end{array}$ & 2,862 & $\begin{array}{c}\text { Not } \\
\text { tested }\end{array}$ & $11.0 \%$ \\
\hline $80 \%$ & $\begin{array}{c}43 \% \\
(1997)\end{array}$ & $\begin{array}{c}37 \% \\
(2008)\end{array}$ & -6 & Yes & $-14.0 \%$ \\
\hline
\end{tabular}

(continued) 
Figure 6-1. Progress Toward Target Attainment for Focus Area 6: Disability and Secondary Conditions (continued)

Moved away

from target ${ }^{1}$

Moved toward

target

Met or exceeded

target

6-9. Inclusion of children and youth with disabilities in regular education programs (621 years)

6-13a. Surveillance for persons with disabilities (no. States and D.C.)

6-13c. Health promotion programs for persons with disabilities (no. States and D.C.)

6-13e. Surveillance for caregivers (no. States and D.C.)

6-13g. Health promotion programs for caregivers (no. States and D.C.)

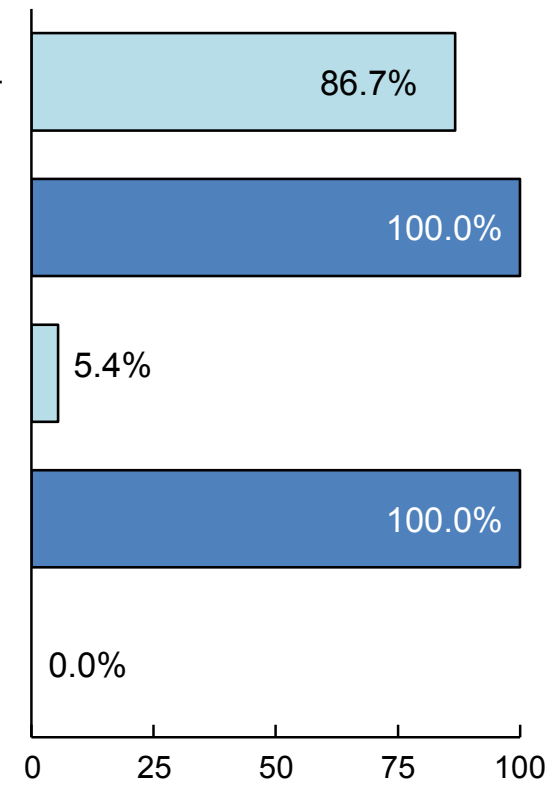

\begin{tabular}{|c|c|c|c|c|c|}
\hline \multirow{2}{*}{$\begin{array}{c}2010 \\
\text { Target }\end{array}$} & \multirow{2}{*}{$\begin{array}{c}\text { Baseline } \\
(\text { Year })\end{array}$} & \multirow{2}{*}{$\begin{array}{c}\text { Final } \\
(\text { Year })\end{array}$} & \multicolumn{3}{|c|}{ Daseline vs. Final } \\
\cline { 4 - 6 } & Difference 2 & $\begin{array}{c}\text { Statistically } \\
\text { Significant }\end{array}$ & $\begin{array}{c}\text { Percent } \\
\text { Change }^{4}\end{array}$ \\
\hline 51 & $\begin{array}{c}45 \% \\
(1995-96)\end{array}$ & $\begin{array}{c}58 \% \\
(2008-09)\end{array}$ & 13 & $\begin{array}{c}\text { Not } \\
\text { tested }\end{array}$ & $28.9 \%$ \\
\hline 51 & $\begin{array}{c}14 \\
(1999)\end{array}$ & $\begin{array}{c}51 \\
(2009)\end{array}$ & 37 & $\begin{array}{c}\text { Not } \\
\text { tested }\end{array}$ & $264.3 \%$ \\
\hline 51 & $\begin{array}{c}14 \\
(1999)\end{array}$ & $\begin{array}{c}(2009) \\
(1999)\end{array}$ & 2 & $\begin{array}{c}\text { Not } \\
\text { tested }\end{array}$ & $14.3 \%$ \\
\hline 51 & $\begin{array}{c}0 \\
(1999)\end{array}$ & $\begin{array}{c}(2009) \\
(2009)\end{array}$ & 51 & $\begin{array}{c}\text { Not } \\
\text { tested }\end{array}$ & $*$ \\
\hline
\end{tabular}

Percent of targeted change achieved 5

(continued) 


\section{Figure 6-1. Progress Toward Target Attainment for Focus Area 6: Disability and Secondary Conditions (continued)}

\section{NOTES}

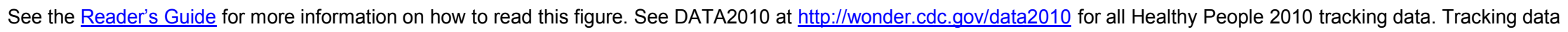
are not available for objectives 6-4, 6-10, 6-11, 6-12a through d, 6-13b, 6-13d, 6-13f, and 6-13h

\section{FOOTNOTES}

${ }^{1}$ Movement away from target is not quantified using the percent of targeted change achieved. See Technical Appendix for more information.

${ }^{2}$ Difference $=$ Final value - Baseline value . Differences between percents (\%) are measured in percentage points.

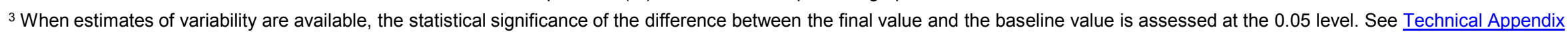
for more information.

\footnotetext{
${ }^{4}$ Percent change $=\frac{\text { Final value }- \text { Baseline value }}{\text { Baseline value }} \times 100$

${ }^{5}$ Percent of targeted change achieved $=\frac{\text { Final value }- \text { Baseline value }}{\text { Healthy People } 2010 \text { target }- \text { Baseline value }} \times 100$.

${ }^{5}$ Percent of targeted change achieved $=\frac{\text { Final value }- \text { Baseline value }}{\text { Healthy People } 2010 \text { target }- \text { Baseline value }} \times 100$.

${ }^{5}$ Percent of targeted change achieved $=\frac{\text { Final value }- \text { Baseline value }}{\text { Healthy People } 2010 \text { target }- \text { Baseline value }} \times 100$.
}

${ }^{*}$ Percent change cannot be calculated. See Technical Appendix for more information.

\section{DATA SOURCES}

6-1. CDC, NCBDDD.

6-2-6-3. National Health Interview Survey (NHIS), CDC, NCHS.

6-5-6-6. Behavioral Risk Factor Surveillance System (BRFSS), CDC, NCCDPHP.

6-7a-b. $\quad$ Survey of State Developmental Disabilities Directors, University of Minnesota.

6-8. National Health Interview Survey (NHIS), CDC, NCHS.

6-9. Data Analysis System (DANS), Department of Education.

6-13a. CDC/NCDBBB/DH-Team.

6-13c. CDC/NCDBBB/DH-Team.

6-13e. CDC/NCDBBB/DH-Team.

6-13g. CDC/NCDBBB/DH-Team. 
Figure 6-2. Health Disparities Table for Focus Area 6: Disability and Secondary Conditions Disparities from the best group rate for each characteristic at the most recent data point and changes in disparity from the baseline to the most recent data point.

\footnotetext{
Population-based objectives

6-2. Sadness or depression among children and adolescents with disabilities (4-17 years) (1997, 2007) $)^{1}$

6-3. Negative feelings interfering with activities among adults with disabilities (age adjusted, 18+ years) $(1997,2008)^{1}$

6-4. Social participation among adults with disabilities (age adjusted, 18+ years) (2001)

6-5. Sufficient emotional support among adults with disabilities (age adjusted, 18+ years) (2005, 2008)

6-6. Satisfaction with life among adults with disabilities (age adjusted, 18+ years) (2005, 2008)

6-8. Employment rate among adults with disabilities (18-64 years) $(1997,2008)^{1}$

6-10. Access to health and wellness programs among adults with disabilities (age adjusted, 18+ years) (2002)

6-11. Lack of assistive devices and technology among adults with disabilities (age adjusted, 18+ years) (2002)

6-12a. Environmental barriers affecting participation in activities at home among adults with disabilities (age adjusted, 18+ years) (2002)

6-12b. Environmental barriers affecting participation in activities at school among adults with disabilities (age adjusted, 18+ years) (2002)

6-12c. Environmental barriers affecting participation in activities at work among adults with disabilities (age adjusted, 18+ years) (2002)

6-12d. Environmental barriers affecting participation in community activities among adults with disabilities (age adjusted, 18+ years) (2002)
}
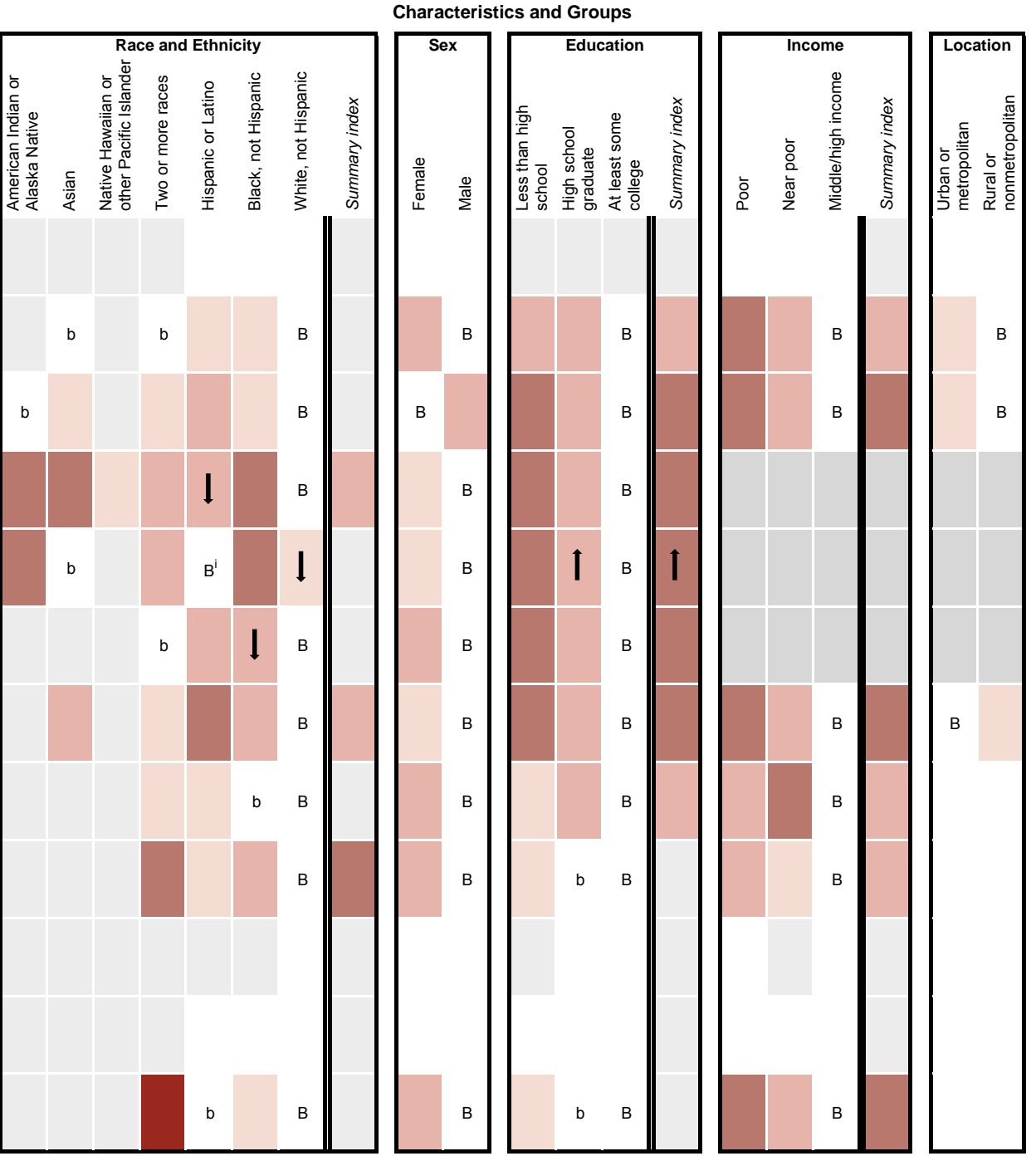

(continued) 


\section{Figure 6-2. Health Disparities Table for Focus Area 6: Disability and Secondary Conditions}

(continued)

NOTES

See DATA2010 at http://wonder.cdc.gov/data2010 for all Healthy People 2010 tracking data. Disparity data are either unavailable or not applicable for objectives 6 1, 6-7a and b, 6-9, and 6-13a through $\mathrm{h}$.

Years in parentheses represent the baseline and most recent data years (if available).

Disparity from the best group rate is defined as the percent difference between the best group rate and each of the other group rates for a characteristic (e.g., race and ethnicity). The summary index is the average of these percent differences for a characteristic. Change in disparity is estimated by subtracting the disparity at baseline from the disparity at the most recent data point. Change in the summary index is estimated by subtracting the summary index at baseline from the summary index at the most recent data point. See Technical Appendix for more information.

Measures of variability were available for all objectives in this table. Thus, the variability of best group rates was assessed, and statistical significance was tested. Disparities of $10 \%$ or more are displayed when the differences from the best group rate are statistically significant at the 0.05 level. Changes in disparities over time are indicated by arrows when the changes are greater than or equal to 10 percentage points and are statistically significant at the 0.05 level. See $\underline{\text { Technical }}$ Appendix.

LEGEND

$\begin{aligned} & \text { The "best" group rate at the most recent } \\ & \text { data point. }\end{aligned}$
B $\begin{aligned} & \text { The group with the best rate for } \\ & \text { specified characteristic. }\end{aligned} \quad$ b $\begin{aligned} & \text { Most favorable group rate for specified } \\ & \text { characteristic, but reliability criterion not met. }\end{aligned}$

\begin{tabular}{l|l|l|l|l|l}
\hline \multicolumn{2}{l}{$\begin{array}{l}\text { Disparity from the best group rate at the } \\
\text { most recent data point. }\end{array}$} & $\begin{array}{l}\text { Less than } 10 \% \text {, or difference not } \\
\text { statistically significant (when } \\
\text { estimates of variability are available). }\end{array}$ & $10 \%-49 \%$ & $50 \%-99 \%$ \\
\hline
\end{tabular}

Changes in disparity over time are shown when:
(a) disparities data are available at both baseline and most recent time points;
(b) data are not for the group(s) indicated by "B" or "b" at either time point; and
(c) the change is greater than or equal to 10 percentage points and statistically
significant, or when the change is greater than or equal to 10 percentage points
and estimates of variability were not available. See Technical Appendix.

FOOTNOTES

1 Baseline data by race and ethnicity are for 1999.

i The group with the best rate at the most recent data point is different from the group with the best rate at baseline. Both rates met the reliability criterion. See Technical Appendix.

\section{DATA SOURCES}

6-2-6-4. National Health Interview Survey (NHIS), CDC, NCHS.

6-5-6-6. Behavioral Risk Factor Surveillance System (BRFSS), CDC, NCCDPHP.

6-8. National Health Interview Survey (NHIS), CDC, NCHS.

6-10-6-11. National Health Interview Survey (NHIS), CDC, NCHS.

6-12a-d. National Health Interview Survey (NHIS), CDC, NCHS. 


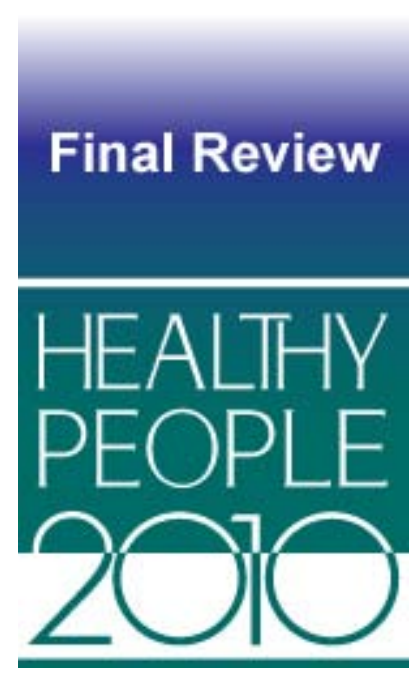

\section{Educational and Community-Based Programs}

\section{Co-Lead Agencies}

Centers for Disease Control and Prevention

Health Services and Resources Administration

\section{Contents}

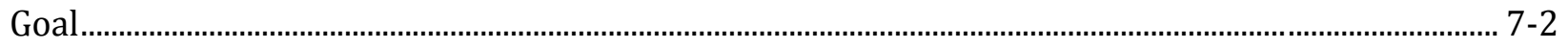

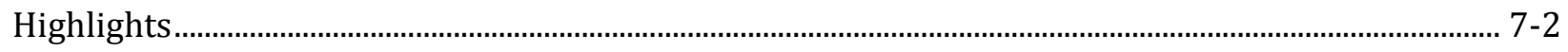

Summary of Progress.......................................................................................................................... 7-3

Transition to Healthy People 2020 ........................................................................................................ 7-4

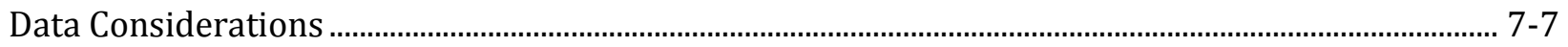

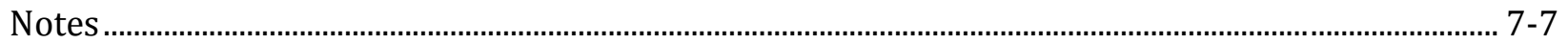

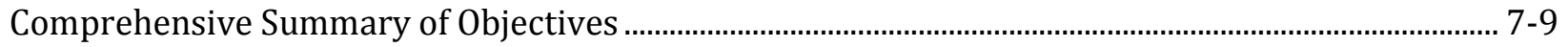

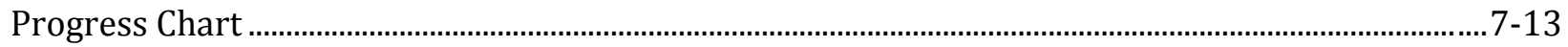

Health Disparities Table ........................................................................................................................ 


\section{Goal: Increase the quality, availability, and effectiveness of educational and community-based programs designed to prevent disease and improve health and quality of life}

This chapter monitors a number of school-related objectives including high school completion, health-related educational programs in schools, and the availability of school nurses. In addition, objectives track health promotion programs in worksites, as well as community-based programs established by local health departments. The number of older adults participating in organized health promotion activities is also monitored.

All Healthy People tracking data quoted in this chapter, along with technical information and operational definitions for each objective, can be found in the Healthy People 2010 database, DATA2010, available from http://wonder.cdc.gov/data2010/.

More information about this Focus Area can be found in the following publications:

- Healthy People 2010: Understanding and Improving Health, available from http://www.healthypeople.gov/2010/Document/tableofcontents.htm\#under.

- Healthy People 2010 Midcourse Review, available from http://www.healthypeople.gov/2010/data/midcourse/html/default.htm\#FocusAreas.

\section{Highlights}

- Substantial progress was achieved for the objectives in this Focus Area during the past decade [1]. Seventy-six percent of the Educational and Community-Based Programs objectives with data to measure progress moved toward or achieved their Healthy People 2010 targets (Figure 7-1). However, statistically significant health disparities were observed by race and ethnicity, sex, and education level, some of which are highlighted below (Figure 7-2) [2].

- The high school completion rate among persons aged 18-24 years (objective 7-1) increased by $4.7 \%$ between 1998 and 2007, from $85 \%$ to $89 \%$, moving toward the Healthy People 2010 target of $90 \%$. Disparities were observed for racial and ethnic population groups as follows:

- Among racial and ethnic groups, the white non-Hispanic population had the highest (best) rate of high school completion, 93\% in 2006, whereas the Hispanic or Latino population, the black non-Hispanic population, and persons of two or more races had rates of $71 \%, 85 \%$, and $90 \%$ in 2006, respectively. When expressed as persons not completing high school, the rate for the Hispanic or Latino population was more than four times the best group rate (that for the white non-Hispanic population) [2]. The rate for the black non-Hispanic population was more than twice the best group rate and the rate for persons of two or more races was nearly one and a half times the best group rate. 
- The proportion of schools with a nurse-to-student ratio of at least one nurse for every 750 students increased for all types of schools (objectives 7-4a through d). Nationally, middle and junior high schools (objective 7-4c) met the 2010 target of 50\% exactly in 2006. There was a $46.2 \%$ increase in the proportion of senior high schools with a 1:750 nurse-to-student ratio (objective 7-4b) between 1994 and 2006, from 26\% to 38\%. Although the proportion of elementary schools with a 1:750 nurse-to-student ratio (objective 7-4d) increased by $7.1 \%$ between 2000 and 2006, from $42 \%$ to $45 \%$, the increase was not statistically significant.

- School health education programs increased in a number of areas. Two examples where such increases were statistically significant are education programs focusing on unintentional injuries (objective 7-2b), which increased by $21.2 \%$ between 1994 and 2006, from $66 \%$ to $80 \%$, and programs addressing violence (objective $7-2 \mathrm{c}$ ), which increased by $32.8 \%$ between 1994 and 2006 , from $58 \%$ to $77 \%$.

\section{Summary of Progress}

- Figure 7-1 presents a quantitative assessment of progress in achieving the Healthy People 2010 objectives for Educational and Community-Based Programs [1]. Data to measure progress toward target attainment were available for 17 objectives. Of these:

- One objective (7-4c, middle and junior high schools with a nurse-to-student ratio of at least one nurse for every 750 students) met the Healthy People 2010 target.

- Twelve objectives moved toward their targets. A statistically significant difference between the baseline and the final data points was observed for six of these objectives (7-1, 7-2a through c, and 7-4a and b). No significant differences were observed for five objectives (7-2d, e, g, and i; and 7-4d); and data to test the significance of the difference were unavailable for one objective (7-3).

- Two objectives (7-2h and j) showed no change.

- Two objectives (7-2f and 7-6) moved away from their targets. A statistically significant difference between the baseline and final data points was observed for one objective (7-6, participation in employer sponsored health promotion activities). No significant difference was observed for the other objective (7-2f, school health education on alcohol and other drug use in middle/junior and senior high schools).

- No data were available to measure progress for the following 39 objectives.

- Two objectives (7-5a and 7-10) remained developmental [3].

- Twenty-two objectives (7-5b through f; 7-11c, g through i, m through o, q through v, y, z, aa; and 7-12) had baseline data only.

- Fifteen objectives (7-7 through 7-9; 7-11a, b, d through f, j through l, p, w, x, and bb) were dropped during the decade [4].

- Figure 7-2 displays health disparities in Educational and Community-Based Programs from the best group rate for each characteristic at the most recent data point [2]. It also displays changes in disparities from baseline to the most recent data point [5]. 
- Three objectives (7-1, 7-3, and 7-12) had racial and ethnic health disparities of $10 \%$ or more. For each of these three objectives, a different group had the best rate, including the white non-Hispanic (objective 7-1), the black non-Hispanic (objective 7-3), and the Asian or Pacific Islander populations (objective 7-12).

- Females had a better rate of high school completion than males (objective 7-1). When expressed as persons not completing high school, the rate for females $(9 \%)$ was significantly lower than the rate for males $(13 \%)$.

\section{Transition to Healthy People 2020}

The Healthy People 2020 Educational and Community-Based Programs Topic Area has expanded from Healthy People 2010 to include objectives that track core clinical prevention and population health content in training of health care professionals. See HealthyPeople.gov for a complete list of Healthy People 2020 topics and objectives.

The Healthy People 2020 Educational and Community-Based Programs Topic Area can be grouped into several sections:

- School Settings

- Worksite Settings

- Health Care Settings

- Community Settings and Select Populations

- Training of Health Care Professionals.

The differences between the Healthy People 2010 objectives and those included in Healthy People 2020 are summarized below:

- The Healthy People 2020 Educational and Community-Based Programs Topic Area has a total of 94 objectives, 18 of which are developmental, whereas the Healthy People 2010 Educational and Community-Based Programs Focus Area had 56 objectives, two of which remained developmental [3].

- Four Healthy People 2010 objectives, including high school completion (objective 7-1), student to nurse ratio in senior high schools and in elementary schools (objective 7-4b and $d$, respectively), and worksite health promotion program in worksites with fewer than 50 employees (objective 7-5a), were retained "as is" [6].

- Twenty Healthy People 2010 objectives were modified [7]:

- School health education objectives (7-2a through $\mathrm{j}$ ) were modified to include elementary schools. Currently, objective 7-2 addresses middle and senior high schools. Adding elementary schools expands this objective to all grades K-12 (elementary, middle, and senior high schools), thus providing comprehensive information on health education in the nation's schools. 
- Student to nurse ratio in all schools (objective 7-4a) was modified to include elementary schools because elementary schools were added to the 2006 School Health Policies and Programs Study (SHPPS). Student to nurse ratio in middle and junior high schools (objective 7-4c) was modified to be limited to middle schools only because the language, "junior high schools," is no longer used in SHPPS.

- Most worksite setting objectives were reverted to developmental status because the data sources used over the last decade are no longer available. New data sources have been identified but currently lack baseline data. The objectives that are now developmental include:

- Culturally appropriate and linguistically competent community health promotion and disease prevention programs in educational and communitybased programs (objective 7-11g)

- Worksite health promotion programs in worksites with 50 or more employees, 50-99, 100-249, 250-749, and 750 or more employees (objectives 7-5b through f, respectively), and employer-sponsored health promotion activities (objective 7-6).

- One Community Settings and Select Populations objective (7-10, community health promotion programs), which was developmental, was modified. The objective expanded to nine objectives addressing population-based primary prevention services in the following priority areas: injury, violence, mental illness, tobacco use, substance abuse, unintended pregnancy, chronic disease programs, nutrition, and physical activity.

- The Healthy People 2010 objective on culturally appropriate and linguistically competent community health promotion programs in educational and communitybased programs (objective 7-11g) was retained as developmental. The data source used in Healthy People 2010 no longer identifies or tracks culturally appropriate or linguistically competent programs, and a new data source is being sought in coordination with the Office of Minority Health within the U.S. Department of Health and Human Services.

- The following 17 objectives were archived [8]:

- School health education in environmental health (objective 7-2j) was archived because information about the topic is no longer collected by the data source (the School Health Policies and Program Study).

- Sixteen community setting and select populations objectives were archived because the data sources used for the past decade no longer collect the data:

- Fifteen objectives that address culturally appropriate and linguistically competent community health promotion and disease prevention programs (objectives 7-11c, h, i, m, n, o, q through v, y, z and aa) were archived because they are no longer tracked by the National Profile of Local Health Departments.

- One objective that addresses older adults who have participated in organized health promotion activities (objective 7-12) was archived because the questions used to collect the data are no longer included on the National Health Interview Survey.

- The following 15 objectives were dropped during the last decade due to either a lack of a national data source or a shift in program priority [4]. 
- All three health care setting objectives: patient and family education (objective 7-7), satisfaction with patient education (objective 7-8), and health care organization sponsorship of community health promotion activities (objective 7-9) were dropped.

- Twelve of the community setting and select populations objectives were dropped due to lack of national data source: culturally appropriate and linguistically competent community health promotion programs (objectives 7-11a, b, d through $\mathrm{f}$, j through l, p, w, x, and bb).

- Sixty-two new objectives were added to the Healthy People 2020 Educational and Community-Based Programs Topic Area:

- Nine developmental objectives address preschools and Early Health Start programs in select priority areas.

- Seven objectives address school health education based on the National Health Education Standards.

- Seven objectives address school health education that promotes personal health and wellness.

- Nine objectives address college and university students who receive information from their institution on select priority health risk behavior areas.

- Thirty new objectives that address the training of health care professionals were added. These include six objectives that focus on training in core clinical prevention and population health content for each of the following professions:

- Doctor of Medicine (M.D.)

- Doctor of Osteopathy (D.0.)

- Undergraduate nursing

- Nurse Practitioner

- Physician Assistant.

The Healthy People 2020 objectives reflect the ongoing importance of Educational and CommunityBased Programs. For objectives that were deleted due to lack of data, the U.S. Department of Health and Human Services and the agencies that serve as the leads for the Healthy People 2020 initiative will consider ways to ensure that these public health issues retain prominence despite the lack of data to monitor them.

Appendix D, “A Crosswalk Between Objectives From Healthy People 2010 to Healthy People 2020,” summarizes the changes between the two decades of objectives, reflecting new knowledge and direction for this area. 


\section{Data Considerations}

Education and income are the primary measures of socioeconomic status (SES) in Healthy People 2010. Most data systems used in Healthy People 2010 define income as a family's income before taxes. In order to facilitate comparisons among groups and over time, while adjusting for family size and for inflation, Healthy People 2010 categorizes income using the poverty thresholds developed by the U.S. Census Bureau. Thus, the three categories of family income that are primarily used are:

- Poor-below the Federal poverty level

- Near poor $-100 \%$ to $199 \%$ of the Federal poverty level

- Middle/high income-200\% or more of the Federal poverty level.

These categories may be overridden by considerations specific to the data system, in which case they are modified as appropriate. See Healthy People 2010: General Data Issues, referenced below.

In general, data on educational attainment are presented for persons aged 25 years and over, consistent with guidance given by the U.S. Bureau of the Census. However, because of the requirements of the different data systems, the age groups used to calculate educational attainment for any specific objective may differ from the age groups used to report the data for other Healthy People 2010 objectives, as well as from select populations within the same objective. Therefore, the reader is urged to exercise caution in interpreting the data by educational attainment shown in the Health Disparities Table. See Healthy People 2010: General Data Issues, referenced below.

Additional information on data issues is available from the following sources:

- All Healthy People tracking data can be found in the Healthy People 2010 database, DATA2010, available from http://wonder.cdc.gov/data2010/.

- Detailed information about the data and data sources used to support these objectives can be found in the Operational Definitions on the DATA2010 website, available from http://wonder.cdc.gov/data2010/focusod.htm.

- More information on statistical issues related to Healthy People tracking and measurement can be found in the Technical Appendix and in Healthy People 2010: General Data Issues, which is available in the Data Issues section of the NCHS Healthy People website under Healthy People 2010.

\section{Notes}

1. Displayed in the Progress Chart (Figure 7-1), the percent of targeted change achieved expresses the difference between the baseline and the final value relative to the initial difference between the baseline and the Healthy People 2010 target. As such, it is a relative measure of progress toward attaining the Healthy People 2010 target. See the Reader's Guide for more information. When standard errors were available, the difference between the baseline and the final value was tested at the 0.05 level of significance. See the Figure 7-1 footnotes, as well as the Technical Appendix, for more detail. 
2. Information about disparities among select populations is shown in the Health Disparities Table (Figure 7-2). Disparity from the best group rate is defined as the percent difference between the best group rate and each of the other group rates for a characteristic. For example, racial and ethnic health disparities are measured as the percent difference between the best racial and ethnic group rate and each of the other racial and ethnic group rates. Similarly, disparities by sex are measured as the percent difference between the better group rate (e.g., female) and the rate for the other group (e.g., male). Some objectives are expressed in terms of favorable events or conditions that are to be increased, while others are expressed in terms of adverse events or conditions that are to be reduced. In order to facilitate comparison of health disparities across different objectives, disparity is measured only in terms of adverse events or conditions. For comparability across objectives, objectives that are expressed in terms of favorable events or conditions are re-expressed using the adverse event or condition for the purpose of computing disparity, but they are not otherwise restated or changed. For example, objective 1-1, to increase the proportion of persons with health insurance (e.g., $72 \%$ of the American Indian or Alaska Native population aged under 65 years had some form of health insurance in 2008), is expressed in terms of the percentage of persons without health insurance (e.g., $100 \%-72 \%=28 \%$ of the American Indian or Alaska Native population aged under 65 years did not have any form of health insurance in 2008) when the disparity from the best group rate is calculated. See the Reader's Guide for more information. When standard errors were available, the difference between the best group rate and each of the other group rates was tested at the 0.05 level of significance. See the Figure 7-2 footnotes, as well as the Technical Appendix, for more detail.

3. To be included in Healthy People 2010, an objective must have a national data source that provides a baseline and at least one additional data point for tracking progress. Some objectives lacked baseline data at the time of their development but had a potential data source and were considered of sufficient national importance to be included in Healthy People. These are called "developmental" objectives. When data become available, a developmental objective is moved to measurable status and a Healthy People target can be set.

4. Dropped objectives were not carried forward into Healthy People 2020. These objectives were either developmental or deleted at the Healthy People 2010 Midcourse Review or at another time in Healthy People 2010.

5. The change in disparity is estimated by subtracting the disparity at baseline from the disparity at the most recent data point and, therefore, is expressed as a change in percentage points. See the Reader's Guide for more information. When standard errors were available, the change in disparity was tested at the 0.05 level of significance. See the Figure 7-2 footnotes, as well as the Technical Appendix, for more detail.

6. Retained "as is" objectives have no change in the numerator definition or in the denominator definition between the Healthy People 2010 and Healthy People 2020 objectives. These include objectives that were developmental in Healthy People 2010 and are developmental in Healthy People 2020 and for which no numerator or denominator information was available.

7. Modified objectives have some change in the numerator definition or in the denominator definition between the Healthy People 2010 and Healthy People 2020 objectives. These include objectives that went from developmental in Healthy People 2010 to measurable in Healthy People 2020 or vice versa.

8. Archived objectives had at least one data point in Healthy People 2010 but were not carried forward into Healthy People 2020. 


\section{Comprehensive Summary of Objectives: Educational and Community-Based Programs}

\begin{tabular}{|c|c|}
\hline Objective & Description \\
\hline $7-1$ & High school completion (18-24 years) \\
\hline $7-2 \mathrm{a}$ & $\begin{array}{l}\text { School health education-All priority areas (middle/junior, senior high } \\
\text { schools) }\end{array}$ \\
\hline $7-2 b$ & $\begin{array}{l}\text { School health education-Unintentional injury (middle/junior, senior } \\
\text { high schools) }\end{array}$ \\
\hline 7-2c & School health education-Violence (middle/junior, senior high schools) \\
\hline $7-2 d$ & School health education—Suicide (middle/junior, senior high schools) \\
\hline $7-2 \mathrm{e}$ & $\begin{array}{l}\text { School health education-Tobacco use and addiction (middle/junior, } \\
\text { senior high schools) }\end{array}$ \\
\hline $7-2 \mathrm{f}$ & $\begin{array}{l}\text { School health education-Alcohol and other drug use (middle/junior, } \\
\text { senior high schools) }\end{array}$ \\
\hline $7-2 \mathrm{~g}$ & $\begin{array}{l}\text { School health education-Unintended pregnancy, HIV/AIDS, and STD } \\
\text { infection (middle/junior, senior high schools) }\end{array}$ \\
\hline $7-2 \mathrm{~h}$ & $\begin{array}{l}\text { School health education-Unhealthy dietary patterns (middle/junior, } \\
\text { senior high schools) }\end{array}$ \\
\hline $7-2 \mathrm{i}$ & $\begin{array}{l}\text { School health education-Inadequate physical activity (middle/junior, } \\
\text { senior high schools) }\end{array}$ \\
\hline $7-2 j$ & $\begin{array}{l}\text { School health education-Environmental health (middle/junior, senior } \\
\text { high schools) }\end{array}$ \\
\hline $7-3$ & Health-risk behavior information for college and university students \\
\hline $7-4 a$ & $\begin{array}{l}\text { School nurse-to-student ratio of at least 1:750-All middle/junior and } \\
\text { senior high schools }\end{array}$ \\
\hline $7-4 b$ & School nurse-to-student ratio of at least 1:750-Senior high schools \\
\hline $7-4 \mathrm{c}$ & $\begin{array}{l}\text { School nurse-to-student ratio of at least 1:750—Middle and junior high } \\
\text { schools }\end{array}$ \\
\hline $7-4 d$ & School nurse-to-student ratio of at least 1:750- Elementary schools \\
\hline $7-5 a$ & $\begin{array}{l}\text { Worksite health promotion programs-Worksites with fewer than } 50 \\
\text { employees }\end{array}$ \\
\hline
\end{tabular}

Data Source or Objective Status
Current Population Survey (CPS), Department of Commerce, Census
Bureau.

School Health Policies and Programs Study (SHPPS), CDC, NCCDPHP.

School Health Policies and Programs Study (SHPPS), CDC, NCCDPHP.

School Health Policies and Programs Study (SHPPS), CDC, NCCDPHP. School Health Policies and Programs Study (SHPPS), CDC, NCCDPHP. School Health Policies and Programs Study (SHPPS), CDC, NCCDPHP.

School Health Policies and Programs Study (SHPPS), CDC, NCCDPHP.

School Health Policies and Programs Study (SHPPS), CDC, NCCDPHP.

School Health Policies and Programs Study (SHPPS), CDC, NCCDPHP.

School Health Policies and Programs Study (SHPPS), CDC, NCCDPHP.

School Health Policies and Programs Study (SHPPS), CDC, NCCDPHP.

National College Health Risk Behavior Survey, CDC, NCCDPHP.

School Health Policies and Programs Study (SHPPS), CDC, NCCDPHP.

School Health Policies and Programs Study (SHPPS), CDC, NCCDPHP. School Health Policies and Programs Study (SHPPS), CDC, NCCDPHP.

School Health Policies and Programs Study (SHPPS), CDC, NCCDPHP. Developmental 


\begin{tabular}{|c|c|c|}
\hline Objective & Description & Data Source or Objective Status \\
\hline $7-5 b$ & $\begin{array}{l}\text { Worksite health promotion programs-Worksites with } 50 \text { or more } \\
\text { employees }\end{array}$ & $\begin{array}{l}\text { National Worksite Health Promotion Survey (NWHPS), Association for } \\
\text { Worksite Health Promotion (AWHP) and OPHS, ODPHP. }\end{array}$ \\
\hline $7-5 c$ & $\begin{array}{l}\text { Worksite health promotion programs-Worksites with } 50 \text { to } 99 \\
\text { employees }\end{array}$ & $\begin{array}{l}\text { National Worksite Health Promotion Survey (NWHPS), Association for } \\
\text { Worksite Health Promotion (AWHP) and OPHS, ODPHP. }\end{array}$ \\
\hline $7-5 d$ & $\begin{array}{l}\text { Worksite health promotion programs-Worksites with } 100 \text { to } 249 \\
\text { employees }\end{array}$ & $\begin{array}{l}\text { National Worksite Health Promotion Survey (NWHPS), Association for } \\
\text { Worksite Health Promotion (AWHP) and OPHS, ODPHP. }\end{array}$ \\
\hline $7-5 e$ & $\begin{array}{l}\text { Worksite health promotion programs-Worksites with } 250 \text { to } 749 \\
\text { employees }\end{array}$ & $\begin{array}{l}\text { National Worksite Health Promotion Survey (NWHPS), Association for } \\
\text { Worksite Health Promotion (AWHP) and OPHS, ODPHP. }\end{array}$ \\
\hline $7-5 f$ & $\begin{array}{l}\text { Worksite health promotion programs-Worksites with } 750 \text { or more } \\
\text { employees }\end{array}$ & $\begin{array}{l}\text { National Worksite Health Promotion Survey (NWHPS), Association for } \\
\text { Worksite Health Promotion (AWHP) and OPHS, ODPHP. }\end{array}$ \\
\hline $7-6$ & $\begin{array}{l}\text { Participation in employer-sponsored health promotion activities (age } \\
\text { adjusted, 18+ years) }\end{array}$ & National Health Interview Survey (NHIS), CDC, NCHS. \\
\hline $7-7$ & Health care organizations that provide patient and family education & Dropped \\
\hline $7-8$ & Satisfaction with patient education & Dropped \\
\hline $7-9$ & $\begin{array}{l}\text { Hospital and managed care organization sponsorship of community } \\
\text { health promotion activities }\end{array}$ & Dropped \\
\hline $7-10$ & $\begin{array}{l}\text { Community health promotion programs addressing Healthy People } 2010 \\
\text { focus areas }\end{array}$ & Developmental \\
\hline $7-11 a$ & $\begin{array}{l}\text { Culturally appropriate and linguistically competent community health } \\
\text { promotion programs-Access to quality health services }\end{array}$ & Dropped \\
\hline $7-11 b$ & $\begin{array}{l}\text { Culturally appropriate and linguistically competent community health } \\
\text { promotion programs-Arthritis, osteoporosis and chronic back } \\
\text { conditions }\end{array}$ & Dropped \\
\hline $7-11 c$ & $\begin{array}{l}\text { Culturally appropriate and linguistically competent community health } \\
\text { promotion programs-Cancer }\end{array}$ & $\begin{array}{l}\text { National Profile of Local Health Departments, National Association of } \\
\text { County and City Health Officials (NACCHO). }\end{array}$ \\
\hline $7-11 d$ & $\begin{array}{l}\text { Culturally appropriate and linguistically competent community health } \\
\text { promotion programs-Chronic kidney disease }\end{array}$ & Dropped \\
\hline $7-11 \mathrm{e}$ & $\begin{array}{l}\text { Culturally appropriate and linguistically competent community health } \\
\text { promotion programs-Diabetes }\end{array}$ & Dropped \\
\hline $7-11 \mathrm{f}$ & $\begin{array}{l}\text { Culturally appropriate and linguistically competent community health } \\
\text { promotion programs-Disability and secondary conditions }\end{array}$ & Dropped \\
\hline
\end{tabular}




\begin{tabular}{|c|c|c|}
\hline Objective & Description & Data Source or Objective Status \\
\hline $7-11 \mathrm{~g}$ & $\begin{array}{l}\text { Culturally appropriate and linguistically competent community health } \\
\text { promotion programs-Educational and community-based programs }\end{array}$ & $\begin{array}{l}\text { National Profile of Local Health Departments, National Association of } \\
\text { County and City Health Officials (NACCHO). }\end{array}$ \\
\hline $7-11 \mathrm{~h}$ & $\begin{array}{l}\text { Culturally appropriate and linguistically competent community health } \\
\text { promotion programs-Environmental health }\end{array}$ & $\begin{array}{l}\text { National Profile of Local Health Departments, National Association of } \\
\text { County and City Health Officials (NACCHO). }\end{array}$ \\
\hline $7-11 \mathrm{i}$ & $\begin{array}{l}\text { Culturally appropriate and linguistically competent community health } \\
\text { promotion programs-Family planning }\end{array}$ & $\begin{array}{l}\text { National Profile of Local Health Departments, National Association of } \\
\text { County and City Health Officials (NACCHO). }\end{array}$ \\
\hline $7-11 j$ & $\begin{array}{l}\text { Culturally appropriate and linguistically competent community health } \\
\text { promotion programs-Food safety }\end{array}$ & Dropped \\
\hline $7-11 \mathrm{k}$ & $\begin{array}{l}\text { Culturally appropriate and linguistically competent community health } \\
\text { promotion programs-Medical product safety }\end{array}$ & Dropped \\
\hline 7-11l & $\begin{array}{l}\text { Culturally appropriate and linguistically competent community health } \\
\text { promotion programs-Health communication }\end{array}$ & Dropped \\
\hline 7-11m & $\begin{array}{l}\text { Culturally appropriate and linguistically competent community health } \\
\text { promotion programs-Heart disease and stroke }\end{array}$ & $\begin{array}{l}\text { National Profile of Local Health Departments, National Association of } \\
\text { County and City Health Officials (NACCHO). }\end{array}$ \\
\hline $7-11 \mathrm{n}$ & $\begin{array}{l}\text { Culturally appropriate and linguistically competent community health } \\
\text { promotion programs-HIV }\end{array}$ & $\begin{array}{l}\text { National Profile of Local Health Departments, National Association of } \\
\text { County and City Health Officials (NACCHO). }\end{array}$ \\
\hline $7-110$ & $\begin{array}{l}\text { Culturally appropriate and linguistically competent community health } \\
\text { promotion programs-Immunization and infectious diseases }\end{array}$ & $\begin{array}{l}\text { National Profile of Local Health Departments, National Association of } \\
\text { County and City Health Officials (NACCHO). }\end{array}$ \\
\hline $7-11 p$ & $\begin{array}{l}\text { Culturally appropriate and linguistically competent community health } \\
\text { promotion programs-Injury and violence prevention }\end{array}$ & Dropped \\
\hline $7-11 q$ & $\begin{array}{l}\text { Culturally appropriate and linguistically competent community health } \\
\text { promotion programs- Maternal, infant (and child) health }\end{array}$ & $\begin{array}{l}\text { National Profile of Local Health Departments, National Association of } \\
\text { County and City Health Officials (NACCHO). }\end{array}$ \\
\hline $7-11 \mathrm{r}$ & $\begin{array}{l}\text { Culturally appropriate and linguistically competent community health } \\
\text { promotion programs-Mental health (and mental disorders) }\end{array}$ & $\begin{array}{l}\text { National Profile of Local Health Departments, National Association of } \\
\text { County and City Health Officials (NACCHO). }\end{array}$ \\
\hline $7-11 \mathrm{~s}$ & $\begin{array}{l}\text { Culturally appropriate and linguistically competent community health } \\
\text { promotion programs-Nutrition and overweight }\end{array}$ & $\begin{array}{l}\text { National Profile of Local Health Departments, National Association of } \\
\text { County and City Health Officials (NACCHO). }\end{array}$ \\
\hline $7-11 t$ & $\begin{array}{l}\text { Culturally appropriate and linguistically competent community health } \\
\text { promotion programs-Occupational safety and health }\end{array}$ & $\begin{array}{l}\text { National Profile of Local Health Departments, National Association of } \\
\text { County and City Health Officials (NACCHO). }\end{array}$ \\
\hline $7-11 u$ & $\begin{array}{l}\text { Culturally appropriate and linguistically competent community health } \\
\text { promotion programs-Oral health }\end{array}$ & $\begin{array}{l}\text { National Profile of Local Health Departments, National Association of } \\
\text { County and City Health Officials (NACCHO). }\end{array}$ \\
\hline $7-11 v$ & $\begin{array}{l}\text { Culturally appropriate and linguistically competent community health } \\
\text { promotion programs-Physical activity and fitness }\end{array}$ & $\begin{array}{l}\text { National Profile of Local Health Departments, National Association of } \\
\text { County and City Health Officials (NACCHO). }\end{array}$ \\
\hline
\end{tabular}




\begin{tabular}{|c|c|c|}
\hline Objective & Description & Data Source or Objective Status \\
\hline $7-11 w$ & $\begin{array}{l}\text { Culturally appropriate and linguistically competent community health } \\
\text { promotion programs-Public health infrastructure }\end{array}$ & Dropped \\
\hline $7-11 x$ & $\begin{array}{l}\text { Culturally appropriate and linguistically competent community health } \\
\text { promotion programs-Respiratory diseases }\end{array}$ & Dropped \\
\hline $7-11 y$ & $\begin{array}{l}\text { Culturally appropriate and linguistically competent community health } \\
\text { promotion programs-Sexually transmitted diseases }\end{array}$ & $\begin{array}{l}\text { National Profile of Local Health Departments, National Association of } \\
\text { County and City Health Officials (NACCHO). }\end{array}$ \\
\hline $7-11 \mathrm{z}$ & $\begin{array}{l}\text { Culturally appropriate and linguistically competent community health } \\
\text { promotion programs-Substance abuse (alcohol and other drugs) }\end{array}$ & $\begin{array}{l}\text { National Profile of Local Health Departments, National Association of } \\
\text { County and City Health Officials (NACCHO). }\end{array}$ \\
\hline 7-11aa & $\begin{array}{l}\text { Culturally appropriate and linguistically competent community health } \\
\text { promotion programs-Tobacco use }\end{array}$ & $\begin{array}{l}\text { National Profile of Local Health Departments, National Association of } \\
\text { County and City Health Officials (NACCHO). }\end{array}$ \\
\hline $7-11 b b$ & $\begin{array}{l}\text { Culturally appropriate and linguistically competent community health } \\
\text { promotion programs-Vision and hearing }\end{array}$ & Dropped \\
\hline $7-12$ & $\begin{array}{l}\text { Participation in community health promotion activities (age adjusted, } \\
65+\text { years) }\end{array}$ & National Health Interview Survey (NHIS), CDC, NHCS. \\
\hline
\end{tabular}


Figure 7-1. Progress Toward Target Attainment for Focus Area 7: Educational and Community-Based Programs

Moved away

from target ${ }^{1}$

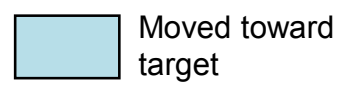

target

Met or exceeded

target

7-1. High school completion (18-24 years)

7-2. School health education

a. All priority areas (middle/junior, senior high schools)

b. Unintentional injury (middle/junior, senior high schools)

c. Violence (middle/junior, senior high schools)

d. Suicide (middle/junior, senior high schools)

e. Tobacco use and addiction (middle/junior, senior high schools)

f. Alcohol and other drug use (middle/junior, senior high schools)

g. Unintended pregnancy, HIVIAIDS, and STD infection (middle/junior, senior high schools)

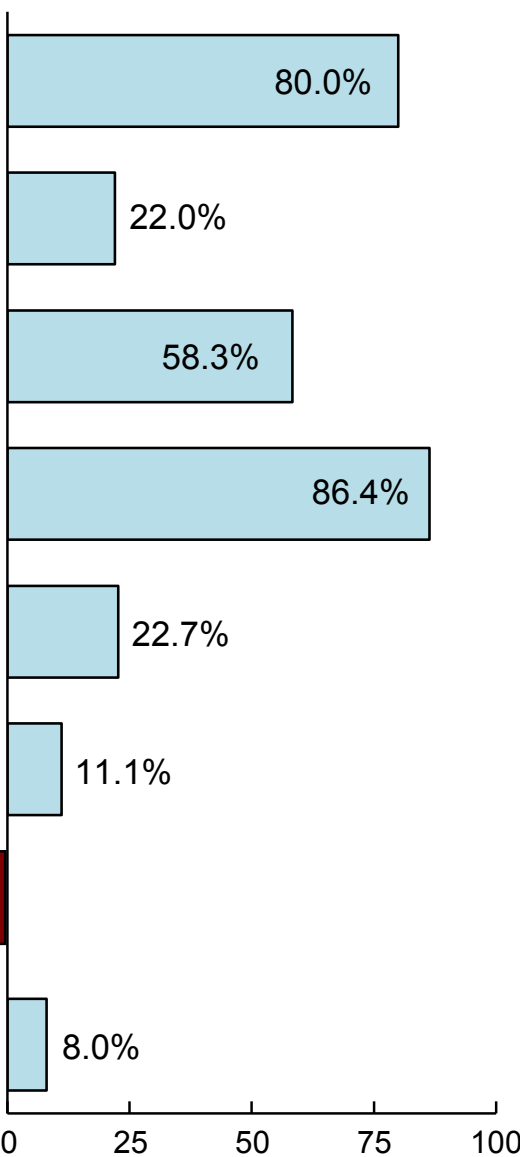

\begin{tabular}{|c|c|c|c|c|c|}
\hline \multirow{2}{*}{$\begin{array}{c}2010 \\
\text { Target }\end{array}$} & \multirow{2}{*}{$\begin{array}{c}\text { Baseline } \\
\text { (Year) }\end{array}$} & \multirow{2}{*}{$\begin{array}{l}\text { Final } \\
\text { (Year) }\end{array}$} & \multicolumn{3}{|c|}{ Baseline vs. Final } \\
\hline & & & Difference ${ }^{2}$ & $\begin{array}{l}\text { Statistically } \\
\text { Significant }^{3}\end{array}$ & $\begin{array}{l}\text { Percent } \\
\text { Change }^{4}\end{array}$ \\
\hline $90 \%$ & $\begin{array}{c}85 \% \\
(1998)\end{array}$ & $\begin{array}{c}89 \% \\
(2007)\end{array}$ & 4 & Yes & $4.7 \%$ \\
\hline $83 \%$ & $\begin{array}{c}33 \% \\
(1994)\end{array}$ & $\begin{array}{c}44 \% \\
(2006)\end{array}$ & 11 & Yes & $33.3 \%$ \\
\hline $90 \%$ & $\begin{array}{c}66 \% \\
(1994)\end{array}$ & $\begin{array}{c}80 \% \\
(2006)\end{array}$ & 14 & Yes & $21.2 \%$ \\
\hline $80 \%$ & $\begin{array}{c}58 \% \\
(1994)\end{array}$ & $\begin{array}{c}77 \% \\
(2006)\end{array}$ & 19 & Yes & $32.8 \%$ \\
\hline $80 \%$ & $\begin{array}{c}58 \% \\
(1994)\end{array}$ & $\begin{array}{c}63 \% \\
(2006)\end{array}$ & 5 & No & $8.6 \%$ \\
\hline $95 \%$ & $\begin{array}{c}86 \% \\
(1994)\end{array}$ & $\begin{array}{c}87 \% \\
(2006)\end{array}$ & 1 & No & $1.2 \%$ \\
\hline $95 \%$ & $\begin{array}{c}90 \% \\
(1994)\end{array}$ & $\begin{array}{c}87 \% \\
(2006)\end{array}$ & -3 & No & $-3.3 \%$ \\
\hline $90 \%$ & $\begin{array}{c}65 \% \\
(1994)\end{array}$ & $\begin{array}{c}67 \% \\
(2006)\end{array}$ & 2 & No & $3.1 \%$ \\
\hline
\end{tabular}

(continued) 
Figure 7-1. Progress Toward Target Attainment for Focus Area 7: Educational and Community-Based Programs (continued)

Moved away from target ${ }^{1}$
Moved toward target

7-2. School health education

h. Unhealthy dietary patterns (middle/junior, senior high schools)

i. Inadequate physical activity (middle/junior, senior high schools)

j. Environmental health (middle/junior, senior high schools)

7-3. Health-risk behavior information for college and university students

7-4. School nurse-to-student ratio of at least 1:750

a. All middle/junior and senior high schools

b. Senior high schools

c. Middle and junior high schools

d. Elementary schools

Met or exceeded target
$68.4 \%$

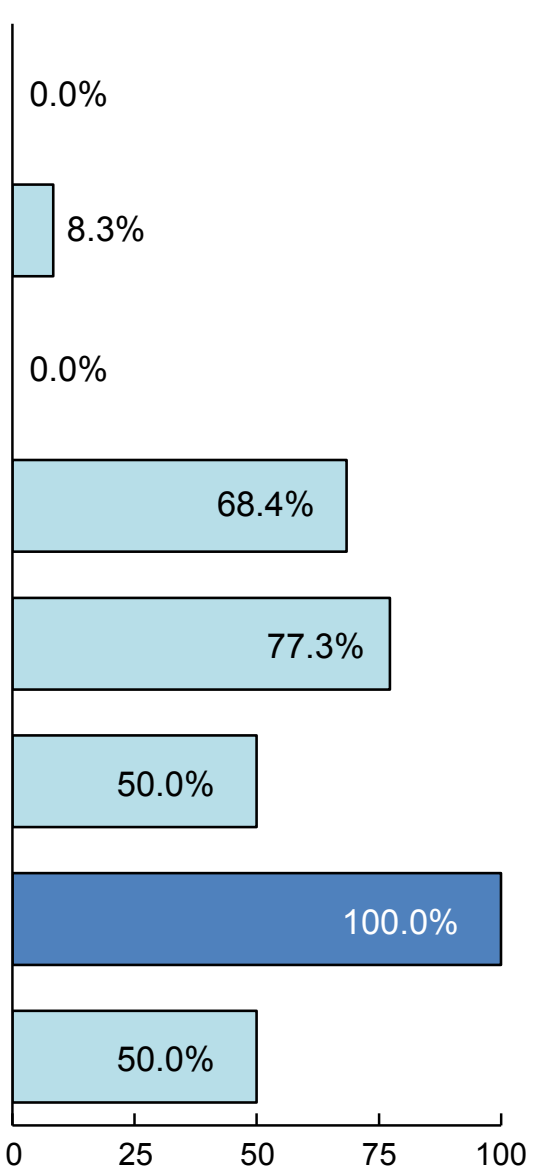

\begin{tabular}{|c|c|c|c|c|c|}
\hline \multirow{2}{*}{$\begin{array}{l}2010 \\
\text { Target }\end{array}$} & \multirow{2}{*}{$\begin{array}{c}\text { Baseline } \\
\text { (Year) }\end{array}$} & \multirow{2}{*}{$\begin{array}{l}\text { Final } \\
\text { (Year) }\end{array}$} & \multicolumn{3}{|c|}{ Baseline vs. Final } \\
\hline & & & Difference ${ }^{2}$ & $\begin{array}{l}\text { Statistically } \\
\text { Significant }^{3}\end{array}$ & $\begin{array}{c}\text { Percent } \\
\text { Change }^{4}\end{array}$ \\
\hline $95 \%$ & $\begin{array}{c}84 \% \\
(1994)\end{array}$ & $\begin{array}{c}84 \% \\
(2006)\end{array}$ & 0 & No & $0.0 \%$ \\
\hline $90 \%$ & $\begin{array}{c}78 \% \\
(1994)\end{array}$ & $\begin{array}{c}79 \% \\
(2006)\end{array}$ & 1 & No & $1.3 \%$ \\
\hline $80 \%$ & $\begin{array}{c}60 \% \\
(1994)\end{array}$ & $\begin{array}{c}60 \% \\
(2000)\end{array}$ & 0 & No & $0.0 \%$ \\
\hline $25 \%$ & $\begin{array}{c}6 \% \\
(1995)\end{array}$ & $\begin{array}{c}19 \% \\
(2008)\end{array}$ & 13 & $\begin{array}{l}\text { Not } \\
\text { tested }\end{array}$ & $216.7 \%$ \\
\hline $50 \%$ & $\begin{array}{c}28 \% \\
(1994)\end{array}$ & $\begin{array}{c}45 \% \\
(2006)\end{array}$ & 17 & Yes & $60.7 \%$ \\
\hline $50 \%$ & $\begin{array}{c}26 \% \\
(1994)\end{array}$ & $\begin{array}{c}38 \% \\
(2006)\end{array}$ & 12 & Yes & $46.2 \%$ \\
\hline $50 \%$ & $\begin{array}{c}32 \% \\
(1994)\end{array}$ & $\begin{array}{c}50 \% \\
(2006)\end{array}$ & 18 & Yes & $56.3 \%$ \\
\hline $48 \%$ & $\begin{array}{c}42 \% \\
(2000)\end{array}$ & $\begin{array}{c}45 \% \\
(2006)\end{array}$ & 3 & No & $7.1 \%$ \\
\hline
\end{tabular}

(continued)

Percent of targeted change achieved ${ }^{5}$ 
Figure 7-1. Progress Toward Target Attainment for Focus Area 7: Educational and Community-Based Programs (continued)

Moved away

from target ${ }^{1}$

Moved toward

target

Met or exceeded

target

7-6. Participation in employer-sponsored health promotion activities (age adjusted, $18+$ years)

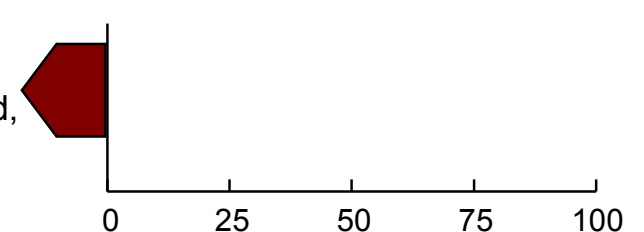

\begin{tabular}{|c|c|c|c|c|c|}
\hline \multirow{2}{*}{$\begin{array}{c}2010 \\
\text { Target }\end{array}$} & \multirow{2}{*}{$\begin{array}{c}\text { Baseline } \\
(\text { Year })\end{array}$} & \multirow{2}{*}{$\begin{array}{c}\text { Final } \\
\text { (Year) }\end{array}$} & \multicolumn{3}{|c|}{ Baseline vs. Final } \\
\cline { 4 - 6 } & Difference $^{2}$ & $\begin{array}{c}\text { Statistically } \\
\text { Significant }\end{array}$ & $\begin{array}{c}\text { Percent } \\
\text { Change }^{4}\end{array}$ \\
\hline $88 \%$ & $\begin{array}{c}67 \% \\
(1994)\end{array}$ & $\begin{array}{c}59 \% \\
(1998)\end{array}$ & -8 & Yes & $-11.9 \%$ \\
\hline
\end{tabular}

\section{Percent of targeted change achieved 5}

\section{NOTES}

See the Reader's Guide for more information on how to read this figure. See DATA2010 at http://wonder.cdc.gov/data2010 for all Healthy People 2010 tracking data. Tracking data are not available for objectives 7-5a through f, 7-10, 7-11c, 7-11g through i, 7-11m through o, 7-11q through v, 7-11y, 7-11z, 7-11aa, and 7-12. Objectives 7-7, 7-8, 7-9, 7-11a, 7-11b, 7-11d through $\mathrm{f}, 7-11 \mathrm{j}$ through I, 7-11p, 7-11w, 7-11x, and 7-11bb were deleted at the Midcourse Review.

\section{FOOTNOTES}

${ }^{1}$ Movement away from target is not quantified using the percent of targeted change achieved. See Technical Appendix for more information.

${ }^{2}$ Difference $=$ Final value - Baseline value. Differences between percents $(\%)$ are measured in percentage points.

${ }^{3}$ When estimates of variability are available, the statistical significance of the difference between the final value and the baseline value is assessed at the 0.05 level. See $\underline{\text { Technical Appendix }}$ for more information.

${ }^{4}$ Percent change $=\frac{\text { Final value }- \text { Baseline value }}{\text { Baseline value }} \times 100$.

${ }^{5}$ Percent of targeted change achieved $=\frac{\text { Final value }- \text { Baseline value }}{\text { Healthy People } 2010 \text { target }- \text { Baseline value }} \times 100$.

\section{DATA SOURCES}

7-1. $\quad$ Current Population Survey (CPS): Department of Commerce, Census Bureau; Department of Labor, Bureau of Labor Statistics.

7-2a-j. School Health Policies and Programs Study (SHPPS), CDC, NCCDPHP.

7-3. National College Health Risk Behavior Survey, CDC, NCCDPHP.

7-4a-d. School Health Policies and Programs Study (SHPPS), CDC, NCCDPHP.

7-6. National Health Interview Survey (NHIS), CDC, NCHS. 
Figure 7-2. Health Disparities Table for Focus Area 7: Educational and Community-Based

Programs

Disparities from the best group rate for each characteristic at the most recent data point and changes in disparity from the baseline to the most recent data point.

7-1. High school completion (18-24 years) $(1998,2007)^{1,2 *}$

7-3. Health-risk behavior information for college and university students $(1995,2008) \dagger$

7-6. Participation in employer-sponsored health promotion activities (age adjusted $18+$ years) $(1994,1998)^{3 *}$

7-12. Participation in community health promotion activities among older adults (age adjusted, 65+ years) $(1998)^{\star}$

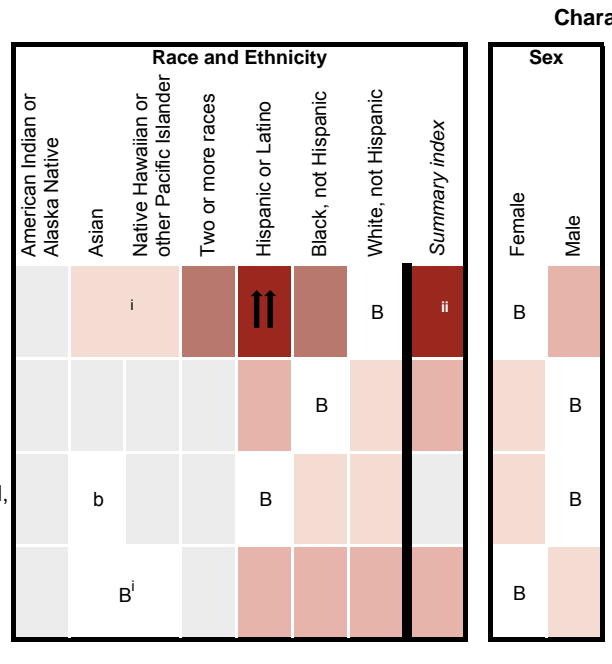

Characteristics and Groups

NOTES

See DATA2010 at http://wonder.cdc.gov/data2010 for all Healthy People 2010 tracking data. Disparity data are either unavailable or not applicable for objectives 7-2a through j, 7-4a through d, 7-5a through f, 7-10, and 7-11c, g, h, i, m, n, o, q through v, y, z, and aa. Objectives 7-7 through 7-9, and 7-11a, b, d, e, f, j, k, I, p, w, x, and bb were deleted at Midcourse Review.

Years in parentheses represent the baseline and most recent data years (if available).

Disparity from the best group rate is defined as the percent difference between the best group rate and each of the other group rates for a characteristic (e.g., race and ethnicity). The summary index is the average of these percent differences for a characteristic. Change in disparity is estimated by subtracting the disparity at baseline from the disparity at the most recent data point. Change in the summary index is estimated by subtracting the summary index at baseline from the summary index at the most recent data point. See Technical Appendix for more information.

LEGEND

$\begin{aligned} & \text { The "best" group rate at the most recent } \\ & \text { data point. }\end{aligned}$
B $\begin{aligned} & \text { The group with the best rate for } \\ & \text { specified characteristic. }\end{aligned} \quad$ b $\begin{aligned} & \text { Most favorable group rate for specified } \\ & \text { characteristic, but reliability criterion not met. }\end{aligned}$

$\begin{aligned} & \text { Disparity from the best group rate at the } \\ & \text { most recent data point. }\end{aligned}$
$\begin{aligned} & \text { Changes in disparity over time are shown when: } \\ & \text { statistically significant (when } \\ & \text { estimates of variability are available). } \\ & \text { (a) disparities data are available at both baseline and most recent time points; } \\ & \text { (b) data are not for the group(s) indicated by "B" or "b" at either time point; and } \\ & \text { (c) the change is greater than or equal to } 10 \text { percentage points and statistically } \\ & \text { significant, or when the change is greater than or equal to 10 percentage } \\ & \text { points and estimates of variability were not available. See Technical Appendix. }\end{aligned}$
Availability of Data

\section{FOOTNOTES}

* Measures of variability were available. Thus, the variability of best group rates was assessed, and statistical significance was tested. Disparities of $10 \%$ or more are displayed when the differences from the best group rate are statistically significant at the 0.05 level. Changes in disparities over time are indicated by arrows when the changes are greater than or equal to 10 percentage points and are statistically significant at the 0.05 level. See Technical Appendix.

$\dagger$ Measures of variability were not available. Thus, the variability of best group rates was not assessed, and statistical significance could not be tested. Nonetheless, disparities and changes in disparities over time are displayed according to their magnitude. See Technical Appendix.

1 Most recent data by race and ethnicity are for 2006 .

2 Baseline data by disability status are for 1995.

3 Baseline data by race and ethnicity are for 1998.

i Data are for Asian or Pacific Islander.

ii Change in the summary index cannot be assessed. See Technical Appendix.

iii The group with the best rate at the most recent data point is different from the group with the best rate at baseline. Both rates met the reliability criterion. See Technical Appendix.

\section{DATA SOURCES}

7-1. Current Population Survey (CPS), Department of Commerce, Census Bureau.

7-3. National College Health Risk Behavior Survey, CDC, NCCDPHP.

7-6. National Health Interview Survey (NHIS), CDC, NCHS.

7-12. National Health Interview Survey (NHIS), CDC, NCHS.

Educational and Community-Based Programs 


\section{Final Review}

\section{Environmental Health}

HEALTHY

PEOPLE

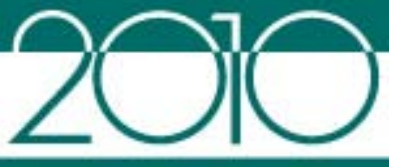

\section{Co-Lead Agencies}

Agency for Toxic Substances and Disease Registry

Centers for Disease Control and Prevention

National Institutes of Health

\section{Contents}

Goal 8-2

Highlights. 8-2

Summary of Progress 8-4

Transition to Healthy People 2020 8-6

Data Considerations 8-9

Notes 8-9

Comprehensive Summary of Objectives 8-11

Progress Chart 8-19

Health Disparities Table 8-29 


\section{Goal: Promote health for all through a healthy environment}

This chapter includes objectives that monitor progress in six general Healthy People areas:

- The Outdoor Air Quality area monitors the proportion of persons exposed to air containing harmful air pollutants.

- The Surface and Ground Water Quality area tracks contaminants in drinking water, fish, and recreational water.

- The Toxics and Waste area monitors exposures to toxic substances and hazardous waste.

- Objectives in the Healthy Homes and Healthy Communities area focus on environmental factors in homes, schools, and worksites.

- The fifth area, Infrastructure and Surveillance, addresses the availability of methods to detect environmental hazards (for example chemical, biological, and other factors that may adversely affect health), exposures to these hazards, and the diseases potentially caused by these hazards.

- Objectives in the final area reflect Global Environmental Health concerns. These objectives address the global burden of disease due to poor water quality, sanitation, and personal and domestic hygiene and the proportion of the population in the U.S.-Mexico border region that has adequate drinking water and sanitation facilities.

All Healthy People tracking data quoted in this chapter, along with technical information and operational definitions for each objective, can be found in the Healthy People 2010 database, DATA2010, available from http://wonder.cdc.gov/data2010/.

More information about this Focus Area can be found in the following publications:

- Healthy People 2010: Understanding and Improving Health, available from http://www.healthypeople.gov/2010/Document/tableofcontents.htm\#under.

- Healthy People 2010 Midcourse Review, available from: http://www.healthypeople.gov/2010/data/midcourse/html/default.htm\#FocusAreas.

\section{Highlights}

- Substantial progress was achieved in objectives for this Focus Area during the past decade [1]. Eighty-four percent of the Environmental Health objectives with data to measure progress moved toward or achieved their Healthy People 2010 targets (Figure 8-1). However, health disparities were observed among racial and ethnic populations in their exposure to harmful air pollutants (Figure 8-2) [2]. Similar disparities were observed between populations residing in urban and rural locations. 
- Between 1997 and 2010, exposure to harmful air pollutants (objective 8-1) declined for all pollutants tracked. The proportion of persons living in counties that exceed National Ambient Air Quality Standards (NAAQS) for carbon monoxide (objective 8-1c) declined from $20 \%$ to $0 \%$; that for nitrogen dioxide (objective $8-1 \mathrm{~d}$ ) declined from $5 \%$ to $0 \%$; that for sulfur dioxide (objective 8-1e) declined from $2 \%$ to $0 \%$; and that for lead (objective 81f) declined from less than $1 \%$ to $0 \%$ in 2010, all meeting the Healthy People 2010 targets of $0 \%$ for those pollutants. While the 2010 targets were not met for ozone (objective 8-1a) and particulate matter (objective 8-1b), air quality for these pollutants improved, declining by $16.3 \%$ and $25 \%$, respectively. The data presented here do not reflect tighter standards that were issued after the targets had been set.

- The proportion of people living in counties that exceeded NAAQS for ozone (objective 8-1a) declined by $25 \%$ between 1997 and 2010, from 43\% to 36\%, moving toward the 2010 target of $0 \%$. However, the final data year by race and ethnicity was 2004 and, at that time, disparities were observed for a number of population groups:

- Among racial and ethnic groups, the American Indian or Alaska Native population had the lowest (best) rate of living in counties that exceeded NAAQS for ozone (objective 8-1a), 23\% in 2004, whereas the white non-Hispanic, Native Hawaiian or Other Pacific Islander, black non-Hispanic, Hispanic or Latino, and Asian populations had rates of 33\%, 35\%, 43\%, 59\%, and 67\%, respectively. The rate for the white non-Hispanic population was almost one and a half times the best group rate (that for the American Indian or Alaska Native population); the rate for the Native Hawaiian or Other Pacific Islander population was about one and a half times the best group rate; the rate for the black non-Hispanic population was almost twice the best group rate; the rate for the Hispanic or Latino population was more than two and a half times the best group rate; and the rate for the Asian population was nearly three times the best group rate [2].

- The rural or nonmetropolitan population had better rates of exposure to ozone (4\% in 1997 and 3\% in 2004) than the urban or metropolitan population (52\% in 1997 and $48 \%$ in 2004). In 2004, the rate for the urban or metropolitan population was 16 times as high as that for the rural or nonmetropolitan population. Between 1997 and 2004, the disparity in ozone exposure between the rural/nonmetropolitan and the urban/metropolitan populations increased by 300 percentage points [3].

- The proportion of people living in counties that exceeded NAAQS for particulate matter (objective 8-1b) declined by 25\% between 1997 and 2010, from $12 \%$ to $9 \%$. However, the final data year by race and ethnicity also was 2004 and, at that time, disparities were observed for a number of population groups.

- Among racial and ethnic groups, the black non-Hispanic population had the lowest (best) rate of particulate matter exposure (objective 8-1b), 6\% in 2004. The American Indian or Alaska Native population had a rate of $13 \%$ in 2004, more than twice the best rate. The Asian and Native Hawaiian or Other Pacific Islander populations each had a rate of $22 \%$ in 2004 , over three and one half times the best rate. The Hispanic or Latino population had a rate of $28 \%$ in 2004 , more than four and one half times the best rate [2]. 
- The rural or nonmetropolitan population had lower (better) rates of exposure to particulate matter (1\% in 1997 and 2004) than the urban or metropolitan population (15\% in 1997 and $13 \%$ in 2004). In 2004, the rate for the urban or metropolitan population was 13 times that of the rural or nonmetropolitan population. Between 1997 and 2004, the disparity in particulate matter between the rural/nonmetropolitan and the urban/metropolitan populations decreased by 200 percentage points [3].

- The use of alternate modes of transportation increased. Trips made by transit (objective 82c) increased by $116.7 \%$ between 1995 and 2009, from $1.8 \%$ to 3.9\%, exceeding the 2010 target of $3.6 \%$. Trips made by walking (objective $8-2 \mathrm{~b}$ ) increased by $92.6 \%$, from $5.4 \%$ to $10.4 \%$, almost achieving the 2010 target of $10.8 \%$. Smaller gains were made for trips by bicycle (objective 8-2a) and telecommuting (objective 8-2d), which increased by $11.1 \%$ and $40 \%$ respectively.

- The proportion of persons served by water systems that met safe drinking water standards (objective 8-5) increased by $9.5 \%$ between 1995 and 2008, from 84\% to 92\%, moving toward the 2010 target of $95 \%$. The number of waterborne disease outbreaks (objective 86) declined by $83.3 \%$ between 1987-96 and 2008, from 6 outbreaks to 1, exceeding the target of 2 outbreaks. However, there was little progress in water conservation (objective 87). Between 1995 and 2005, the daily per capita gallons of domestic water usage declined by only $2 \%$.

- The risks posed by hazardous sites on the National Priority Sites List (objective 8-12a) declined by $11.8 \%$ between 1998 and 2008, from 1,290 to 1,138 sites, exceeding the 2010 target of 1,176 sites.

- Progress was made in exposure to environmental pesticides and chemicals (objectives 8-24 and 8-25). Four of the 15 objectives with data to measure progress met or exceeded their 2010 targets: exposure to propoxur (objective 8-24d) declined from $1.1 \mu \mathrm{g} / \mathrm{gm}$ of creatinine for the $90^{\text {th }}$ percentile of the population aged 6-59 years to below the level of detection $(0.4$ $\mu \mathrm{g}$ ); o-Phenylphenol (objective 8-25g) declined 40\%; diazinon (objective 8-25i) was below the level of detection $(0.58 \mu \mathrm{g})$ in 1999-2000 and $(0.5 \mu \mathrm{g})$ in 2001-02; and mercury in females aged 16-49 years (objective 8-25q) declined by 35.3\%. Eight objectives made progress toward their targets. However, three moved away from their targets, including exposure to chlorpyrifos (objective 8-24c) which increased by $10.8 \%$, cadmium (objective $8-25$ b) which increased by $14.3 \%$, and DDT (objective $8-250$ ) which increased by $1.6 \%$.

\section{Summary of Progress}

- Figure 8-1 presents a quantitative assessment of progress in achieving the Healthy People objectives for Environmental Health. Data to measure progress toward target attainment were available for 61 objectives [1]. Of these:

- Twenty-one objectives met or exceeded the Healthy People 2010 targets (objectives 8-1c through f; 8-2c; 8-6; 8-12a; 8-19; 8-24d; 8-25g, i, and q; 8-27d and e; and 8-30a, e through $\mathrm{i}$, and $\mathrm{l}$ ). 
- Thirty objectives moved toward their targets. A statistically significant difference between the baseline and final data points was observed for one objective (8-22). Data to test the significance of the difference between the baseline and final data points were unavailable for all the remaining objectives $(8-1 \mathrm{a} b$, and $\mathrm{g} ; 8-2 \mathrm{a}, \mathrm{b}$, and d; 8-3 through 5; 8-7; 8-9; 8-13; 8-15; 8-23; 8-24b; 8-25c, e, m, n, p, r, and s; 8-27a through c, i, and o; and 8-29).

- Two objectives showed no change (objectives 8-27g and 8-30b).

- Eight objectives moved away from their targets (objectives 8-10a and b, 8-24c, 8$25 \mathrm{~b}$ and $\mathrm{o}, 8-27 \mathrm{~h}$, and $8-30 \mathrm{j}$ and $\mathrm{l}$ ). Data to test the significance of the difference between the baseline and final data points were unavailable for any of these objectives.

- There were five objectives (8-14a and b, and 8-25j through l) that remained developmental and 21 that had no data available to measure progress (objectives $8-8 \mathrm{a}$ and b; $8-12 \mathrm{~b}$ through d; 8-16a through c; 8-18; 8-20; 8-21; 8-25a, d, f, and h; 8-27f, j and k; and 8-30c and d) [4]. Seven objectives (8-17, 8-24a, 8-25d, 8-27l through $n$, and 8-28) were dropped during the decade [5].

- Figure 8-2 displays health disparities in Environmental Health from the best group rate for each characteristic at the most recent data point [2]. It also displays changes in disparities from baseline to the most recent data point [3].

- One objective (8-22) had statistically significant racial and ethnic health disparities of $10 \%$ or more. Five other objectives (8-1a through c, e, and g) had racial and ethnic health disparities of $10 \%$ or more but lacked data to assess statistical significance. Of these six objectives, the American Indian or Alaska Native, Native Hawaiian or Other Pacific Islander, and black non-Hispanic populations each had the best group rate for one objective. The black non-Hispanic and white non-Hispanic populations were tied for the best group rate for objective 8-1c (exposure to carbon monoxide); persons of two or more races and the black non-Hispanic populations were tied for the best group rate for objective 8-22 (persons in pre-1950s homes tested for lead paint). All racial and ethnic populations except for the Native Hawaiian or Other Pacific Islander population were tied for the best group rate for objective 8-1e (exposure to sulfur dioxide).

- Females had a better rate than males for the one objective with health disparities of $10 \%$ or more by sex (8-1b, exposure to particulate matter).

- Persons living in rural or nonmetropolitan areas had better rates than persons living in urban or metropolitan areas for all four objectives (8-1a through c, and g) with health disparities of $10 \%$ or more by geographic location.

- Several objectives with health disparities of $100 \%$ or more by race and ethnicity and by geographic location were observed. So were objectives with changes in health disparities of 100 percentage points or more over time. These objectives are discussed in the Highlights, above. 


\section{Transition to Healthy People 2020}

The Healthy People 2020 Environmental Health Topic Area has fewer objectives than those included in Healthy People 2010. See HealthyPeople.gov for a complete list of Healthy People 2020 topics and objectives.

The Healthy People 2020 objectives can be grouped into several sections:

- Outdoor air quality

- Water quality

- Toxics and waste

- Healthy homes and healthy communities

- Infrastructure and surveillance.

The differences between the Healthy People 2010 and Healthy People 2020 objectives are summarized below:

- The Healthy People 2020 Environmental Health Topic Area has a total of 67 objectives, whereas the Healthy People 2010 Environmental Health Focus Area had 93 objectives.

- Twenty-seven Healthy People 2010 objectives were retained "as is" [6].

- Increase the proportion of persons served by community water systems who receive a supply of drinking water that meets the regulations of the Safe Drinking Water Act (objective 8-5).

- Reduce waterborne disease outbreaks arising from water intended for drinking among persons served by community water systems (objective 8-6).

- Eliminate elevated blood lead levels in children (objective 8-11).

- Minimize the risks to human health and the environment posed by hazardous sites: National Priority List sites (objective 8-12a).

- Reduce pesticide exposures that result in visits to a health care facility (objective 813).

- Increase recycling of municipal solid waste (objective 8-15).

- Reduce the proportion of occupied housing units that have moderate or severe physical problems (objective 8-22).

- Reduce exposure to pesticides as measured by urine concentrations of metabolites:

- Paranitrophenol (methyl parathion and parathions) (objective 8-24b)

- 3,4,6-trichloro-2-pyridinol (chlorpyrifos) (objective 8-24c).

- Reduce exposure to selected environmental chemicals in the population, as measured by blood and urine concentrations of the substances or their metabolites:

- Arsenic (objective 8-25a)

- Cadmium (objective 8-25b)

- Lead (objective 8-25c)

- Mercury, children aged 1-5 years (objective 8-25e)

- Mercury, females aged 16-49 years (objective 8-25q)

- Chlordane (Oxychlordane) (objective 8-25m) 
- DDT (DDE) (objective 8-25o)

- beta-hexacyclochlorohexane or beta-HCH (objective 8-25p)

- cis-and trans-Permethrin (objective 8-25h)

- Dioxins (objective 8-25k).

- Improve the utility, awareness, and use of existing information systems for environmental health (objective 8-26).

- Increase the number of territories, tribes, and states (including the District of Columbia) that monitor diseases or conditions that can be caused by exposure to environmental hazards:

- Lead poisoning (objective 8-27a)

- Pesticide poisoning (objective 8-27b)

- Mercury poisoning (objective 8-27c)

- Arsenic poisoning (objective 8-27d)

- Cadmium poisoning (objective 8-27e)

- Acute chemical poisoning (objective 8-27g)

- Carbon monoxide poisoning (objective 8-27h).

- Twenty-one Healthy People 2010 objectives were modified, expanded, and retained, resulting in 35 objectives in Healthy People 2020 [7].

- In Healthy People 2010 there were seven objectives (8-1a through g) that tracked air quality separately for each of six criteria air pollutants (ozone, carbon monoxide, nitrogen dioxide, particulate matter, sulfur dioxide, and lead), and the total population exposed to any of these. In Healthy People 2020 air quality is tracked by a single objective (Air Quality Index), which is a composite measure of criteria air pollutants.

- The objectives (8-2a through d) to increase use of alternative modes of transportation for work commutes to reduce motor vehicle emissions and improve the nation's air quality, has a new, more timely data source.

- The objective to reduce air toxic emissions to decrease the risk of adverse health effects caused by airborne toxics (objective 8-4) was split into three objectives by source type.

- The objective for school policies to protect against environmental hazards (objective 8-20) was split into nine objectives to separately track specific policies.

- Other objectives had changes in operational definition.

- Thirty-six Healthy People 2010 objectives were archived [8]. These include objectives addressing: cleaner alternative fuels (objective 8-3); water bodies safe for fishing and recreation (objectives 8-8a and b); fish consumption advisories (objectives 8-10a and b); risks posed by hazardous sites (objectives $8-12$ b through $\mathrm{d}$ ); indoor allergens (objectives 8$16 \mathrm{a}$ and $\mathrm{b}$ ); proportion of persons living in homes tested for radon (objective 8-18); disaster preparedness plans, protocols, and exercises (objective 8-21); exposure to pesticides (objectives 8-24d, and 8-25f, g, i, n, r, and s); monitoring environmentally related diseases (objectives 8-27f, i through $\mathrm{k}$, and o); and water quality in the U.S.-Mexico border region (objectives 8-30a through l). 
- In general, these objectives were archived because the data source could not produce consistent, comparable data. In the case of cleaner alternative fuels, it was not clear what negative externalities would be associated with the increased use of these fuels. Objectives related to monitoring exposure to environmental chemicals were archived because the measures used to monitor them were below the limits of detection, or because the public health concern could be tracked by a related chemical or was not deemed a significant public health concern by CDC.

- Nine objectives were dropped, either due to the lack of a data source, because data was never produced by the data source, the measure was consistently below the level of detection, or it was an inadequate environmental marker [5].

- Production-related waste released by the business sector (objective 8-14a)

- Office building air quality - Number that are managed using good indoor air quality practices (objective 8-17)

- Exposure to pesticides-Urine concentrations in $\mu \mathrm{g} / \mathrm{g}$ creatinine-1-naphthol (carbaryl) (aged 6 years and over) (objective 8-24a)

- Exposure to pesticides, heavy metals, and selected environmental chemicalsManganese (objective 8-25d)

- Exposure to pesticides, heavy metals, and selected environmental chemicalsFurans (objective 8-25l)

- Monitoring environmentally related diseases-Skin cancer (objective 8-27l)

- Monitoring environmentally related diseases-Malignant melanoma (objective 8$27 \mathrm{~m}$ )

- Monitoring environmentally related diseases-Other skin cancer (objective 8-27n)

- Local agencies using surveillance data for vector control (objective 8-28).

- Five new objectives were added for Healthy People 2020.

- Exposure to potential endocrine disruptors-Bisphenol A

- Exposure to potential endocrine disruptors-Perchlorate

- Exposure to potential endocrine disruptors-Mono-n-butyl phthalate

- Exposure to potential endocrine disruptors-BDE 47, (2,2',4,4'-tetrabromodiphenyl ether)

- Reduce the number of new schools sited within 500 feet of an interstate or federal or state highway.

Appendix D, “A Crosswalk Between Objectives From Healthy People 2010 to Healthy People 2020,” summarizes the changes between the two decades of objectives, reflecting new knowledge and direction for this area. 


\section{Data Considerations}

Education and income are the primary measures of socioeconomic status (SES) in Healthy People 2010. Most data systems used in Healthy People 2010 define income as a family's income before taxes. In order to facilitate comparisons among groups and over time, while adjusting for family size and for inflation, Healthy People 2010 categorizes income using the poverty thresholds developed by the U.S. Census Bureau. Thus, the three categories of family income that are primarily used are:

- Poor-below the Federal poverty level

- Near poor-100\% to $199 \%$ of the Federal poverty level

- Middle/high income-200\% or more of the Federal poverty level.

These categories may be overridden by considerations specific to the data system, in which case they are modified as appropriate. See Healthy People 2010: General Data Issues, referenced below.

Information on data issues is available from the following sources:

- All Healthy People 2010 tracking data can be found in the Healthy People 2010 database, DATA2010, available from http://wonder.cdc.gov/data2010/.

- Detailed information about the data and data sources used to support these objectives can be found in the Operational Definitions on the DATA2010 website, available from http://wonder.cdc.gov/data2010/focusod.htm.

- More information on statistical issues related to Healthy People tracking and measurement can be found in the Technical Appendix and in Healthy People 2010: General Data Issues, which is available in the Data Issues section of the NCHS Healthy People website under Healthy People 2010.

\section{Notes}

1. Displayed in the Progress Chart (Figure 8-1), the percent of targeted change achieved expresses the difference between the baseline and the final value relative to the initial difference between the baseline and the Healthy People 2010 target. As such, it is a relative measure of progress toward attaining the Healthy People 2010 target. See the Reader's Guide for more information. When standard errors were available, the difference between the baseline and the final value was tested at the 0.05 level of significance. See the Figure 8-1 footnotes, as well as the Technical Appendix, for more detail.

2. Information about disparities among select populations is shown in the Health Disparities Table (Figure 8-2). Disparity from the best group rate is defined as the percent difference between the best group rate and each of the other group rates for a characteristic. For example, racial and ethnic health disparities are measured as the percent difference between the best racial and ethnic group rate and each of the other racial and ethnic group rates. Similarly, disparities by sex are measured as the percent difference between the better group rate (e.g., female) and the rate for the other group (e.g., male). Some objectives are expressed in terms of favorable events or conditions that are to be increased, while others are expressed in terms of adverse events or conditions that are to be reduced. In order to facilitate comparison of health disparities across different objectives, disparity is measured only in terms of adverse events or conditions. For comparability across objectives, objectives that are expressed in terms of favorable events or conditions are re-expressed using the adverse event or condition for the purpose of computing disparity, but they are not otherwise 
restated or changed. For example, objective 1-1, to increase the proportion of persons with health insurance (e.g., $72 \%$ of the American Indian or Alaska Native population aged under 65 years had some form of health insurance in 2008), is expressed in terms of the percentage of persons without health insurance (e.g., $100 \%-72 \%=28 \%$ of the American Indian or Alaska Native population aged under 65 years did not have any form of health insurance in 2008) when the disparity from the best group rate is calculated. See the Reader's Guide for more information. When standard errors were available, the difference between the best group rate and each of the other group rates was tested at the 0.05 level of significance. See the Figure 8-2 footnotes, as well as the Technical Appendix, for more detail

3. The change in disparity is estimated by subtracting the disparity at baseline from the disparity at the most recent data point and, therefore, is expressed as a change in percentage points. See the Reader's Guide for more information. When standard errors were available, the change in disparity was tested at the 0.05 level of significance. See the Figure 8-2 footnotes, as well as the Technical Appendix, for more detail.

4. To be included in Healthy People 2010, an objective must have a national data source that provides a baseline and at least one additional data point for tracking progress. Some objectives lacked baseline data at the time of their development but had a potential data source and were considered of sufficient national importance to be included in Healthy People. These are called "developmental" objectives. When data become available, a developmental objective is moved to measurable status and a Healthy People target can be set.

5. Dropped objectives were not carried forward into Healthy People 2020. These objectives were either developmental or deleted at the Healthy People 2010 Midcourse Review or at another time in Healthy People 2010.

6. Retained "as is" objectives have no change in the numerator definition or in the denominator definition between the Healthy People 2010 and Healthy People 2020 objectives. These include objectives that were developmental in Healthy People 2010 and are developmental in Healthy People 2020 and for which no numerator or denominator information was available.

7. Modified objectives have some change in the numerator definition or in the denominator definition between the Healthy People 2010 and Healthy People 2020 objectives. These include objectives that went from developmental in Healthy People 2010 to measurable in Healthy People 2020 or vice versa.

8. Archived objectives had at least one data point in Healthy People 2010 but were not carried forward into Healthy People 2020. 


\section{Comprehensive Summary of Objectives: Environmental Health}

\begin{tabular}{|c|c|c|}
\hline Objective & Description & Data Source or Objective Status \\
\hline $8-1 \mathrm{a}$ & Percent of persons exposed to harmful air pollutants-Ozone & Air Quality System (AQS), Environmental Protection Agency (EPA). \\
\hline $8-1 b$ & $\begin{array}{l}\text { Percent of persons exposed to harmful air pollutants-Particulate } \\
\text { matter }(\leq 10 \mu \mathrm{m} \text { in diameter })\end{array}$ & Air Quality System (AQS), Environmental Protection Agency (EPA). \\
\hline $8-1 c$ & $\begin{array}{l}\text { Percent of persons exposed to harmful air pollutants-Carbon } \\
\text { monoxide }\end{array}$ & Air Quality System (AQS), Environmental Protection Agency (EPA). \\
\hline $8-1 d$ & Percent of persons exposed to harmful air pollutants-Nitrogen dioxide & Air Quality System (AQS), Environmental Protection Agency (EPA). \\
\hline $8-1 e$ & Percent of persons exposed to harmful air pollutants-Sulfur dioxide & Air Quality System (AQS), Environmental Protection Agency (EPA). \\
\hline $8-1 f$ & Percent of persons exposed to harmful air pollutants-Lead & Air Quality System (AQS), Environmental Protection Agency (EPA). \\
\hline $8-1 g$ & Number of persons (thousands) exposed to any harmful air pollutants & Air Quality System (AQS), Environmental Protection Agency (EPA). \\
\hline $8-2 a$ & Alternative modes of transportation-Trips made by bicycling & $\begin{array}{l}\text { National Household Travel Survey (NHTS), formerly Nationwide } \\
\text { Personal Transportation Survey (NPTS), Department of Transportation } \\
\text { (DOT). }\end{array}$ \\
\hline $8-2 b$ & Alternative modes of transportation-Trips made by walking & $\begin{array}{l}\text { National Household Travel Survey (NHTS), formerly Nationwide } \\
\text { Personal Transportation Survey (NPTS), Department of Transportation } \\
\text { (DOT). }\end{array}$ \\
\hline $8-2 c$ & Alternative modes of transportation-Trips made by transit & $\begin{array}{l}\text { National Household Travel Survey (NHTS), formerly Nationwide } \\
\text { Personal Transportation Survey (NPTS), Department of Transportation } \\
\text { (DOT). }\end{array}$ \\
\hline $8-2 d$ & Alternative modes of transportation-Persons who telecommute & $\begin{array}{l}\text { National Household Travel Survey (NHTS), formerly Nationwide } \\
\text { Personal Transportation Survey (NPTS), Department of Transportation } \\
\text { (DOT). }\end{array}$ \\
\hline $8-3$ & Cleaner alternative fuels & $\begin{array}{l}\text { Alternatives to Traditional Transportation Fuels, Department of } \\
\text { Energy, (DOE). }\end{array}$ \\
\hline $8-4$ & Airborne toxins (million tons) & $\begin{array}{l}\text { National Emissions Inventory (NEI), Environmental Protection Agency } \\
\text { (EPA). }\end{array}$ \\
\hline $8-5$ & Safe drinking water & $\begin{array}{l}\text { Potable Water Surveillance System (PWSS) and Safe Drinking Water } \\
\text { Information System (SDWIS), Environmental Protection Agency (EPA). }\end{array}$ \\
\hline $8-6$ & Waterborne disease outbreaks (average no. per year) & State Reporting Systems, CDC, NCID. \\
\hline
\end{tabular}




\begin{tabular}{|c|c|c|}
\hline Objective & Description & Data Source or Objective Status \\
\hline $8-7$ & $\begin{array}{l}\text { Water conservation (gallons of domestic water usage per capita per } \\
\text { day) }\end{array}$ & $\begin{array}{l}\text { Estimated Use of Water in the United States, Department of Interior } \\
\text { (DOI). }\end{array}$ \\
\hline $8-8 a$ & Water bodies safe for fishing and recreation-Rivers and streams & $\begin{array}{l}\text { National Water Quality Inventory, U.S Environmental Protection } \\
\text { Agency (EPA), Office of Water (OW), Office of Wetlands, Oceans, and } \\
\text { Watersheds (OWOW). }\end{array}$ \\
\hline $8-8 b$ & $\begin{array}{l}\text { Water bodies safe for fishing and recreation-Lakes, ponds, and } \\
\text { reservoirs }\end{array}$ & $\begin{array}{l}\text { National Water Quality Inventory, U.S Environmental Protection } \\
\text { Agency (EPA), Office of Water (OW), Office of Wetlands, Oceans, and } \\
\text { Watersheds (OWOW). }\end{array}$ \\
\hline $8-9$ & $\begin{array}{l}\text { Beach open and safe for swimming (percent of days during beach } \\
\text { season) }\end{array}$ & $\begin{array}{l}\text { Beaches Environmental Assessment, Closure and Health Program } \\
\text { (BEACH), Environmental Protection Agency (EPA). }\end{array}$ \\
\hline $8-10 \mathrm{a}$ & Fish consumption advisories-Rivers & $\begin{array}{l}\text { National Listing of Fish Advisories, Environmental Protection Agency } \\
\text { (EPA). }\end{array}$ \\
\hline $8-10 b$ & Fish consumption advisories - Lakes & $\begin{array}{l}\text { National Listing of Fish Advisories, Environmental Protection Agency } \\
\text { (EPA). }\end{array}$ \\
\hline $8-11$ & Elevated blood lead levels in children $1-5$ years $(\geq 10 \mu \mathrm{g} / \mathrm{dL})$ & $\begin{array}{l}\text { National Health and Nutrition Examination Survey (NHANES), CDC, } \\
\text { NCHS. }\end{array}$ \\
\hline $8-12 \mathrm{a}$ & Risks posed by hazardous sites-National Priority List sites & $\begin{array}{l}\text { Comprehensive Environmental Response and Cleanup Liability } \\
\text { Information System (CERCLIS), Environmental Protection Agency } \\
\text { (EPA). }\end{array}$ \\
\hline $8-12 b$ & $\begin{array}{l}\text { Risks posed by hazardous sites-Resource Conservation and Recovery } \\
\text { Act facilities }\end{array}$ & $\begin{array}{l}\text { Resource Conservation Recovery Act Info (RCRAInfo), Environmental } \\
\text { Protection (EPA), Office of Solid Waste and Emergency Response } \\
\text { (OSWER), Office of Solid Waste (OSW). }\end{array}$ \\
\hline $8-12 c$ & $\begin{array}{l}\text { Risks posed by hazardous sites-Leaking underground storage } \\
\text { facilities }\end{array}$ & $\begin{array}{l}\text { Environmental Protection (EPA), Office of Solid Waste and Emergency } \\
\text { Response (OSWER), Office of Underground Storage Tanks (OUST). }\end{array}$ \\
\hline $8-12 d$ & Risks posed by hazardous sites-Brownfield properties & $\begin{array}{l}\text { Environmental Protection (EPA), Office of Solid Waste and Emergency } \\
\text { Response (OSWER), Office of Brownfields Cleanup and Redevelopment } \\
\text { (OBCR). }\end{array}$ \\
\hline $8-13$ & $\begin{array}{l}\text { Pesticide exposures resulting in visits to a health care facility (no. of } \\
\text { visits per year) }\end{array}$ & $\begin{array}{l}\text { National Poison Data System (NPDS) (formerly the Toxic Exposure } \\
\text { Surveillance System [TESS]), American Association of Poison Control } \\
\text { Centers. }\end{array}$ \\
\hline $8-14 a$ & $\begin{array}{l}\text { Production-related waste released by the business sector (per unit of } \\
\text { production) }\end{array}$ & Developmental \\
\hline
\end{tabular}




\begin{tabular}{|c|c|c|}
\hline Objective & Description & Data Source or Objective Status \\
\hline $8-14 b$ & $\begin{array}{l}\text { Toxic chemicals released by the business sector (per unit of } \\
\text { production) }\end{array}$ & Dropped \\
\hline $8-15$ & Recycled municipal solid waste (percent of total municipal solid waste) & $\begin{array}{l}\text { Municipal Solid Waste in The United States, Environmental Protecti } \\
\text { Agency (EPA). }\end{array}$ \\
\hline $8-16 a$ & Indoor allergens - Group 1 dust mite allergens $>2 \mu \mathrm{g} / \mathrm{g}$ of dust in bed & $\begin{array}{l}\text { National Survey of Lead and Allergens in Housing, NIH, NIEHS, and } \\
\text { Department of Housing and Urban Development (HUD). }\end{array}$ \\
\hline $8-16 b$ & Indoor allergens - Group 1 dust mite allergens $>10 \mu \mathrm{g} / \mathrm{g}$ of dust in bed & $\begin{array}{l}\text { National Survey of Lead and Allergens in Housing, NIH, NIEHS, and t } \\
\text { Department of Housing and Urban Development (HUD). }\end{array}$ \\
\hline $8-16 c$ & $\begin{array}{l}\text { Indoor allergens-German cockroach allergens }>0.1 \text { unit/g of dust in } \\
\text { the bed }\end{array}$ & $\begin{array}{l}\text { National Survey of Lead and Allergens in Housing, NIH, NIEHS, and } \\
\text { Department of Housing and Urban Development (HUD). }\end{array}$ \\
\hline $8-17$ & $\begin{array}{l}\text { Office buildings that are managed using good indoor air quality } \\
\text { practices (number) }\end{array}$ & Dropped \\
\hline $8-18$ & Proportion of persons living in homes tested for radon (age adjusted) & National Health Interview Survey (NHIS), CDC, NCHS. \\
\hline 8-19 & Radon-resistant new home construction (number) & $\begin{array}{l}\text { National Association of Home Builders Research Center Survey, } \\
\text { National Association of Home Builders. }\end{array}$ \\
\hline $8-20$ & School policies to protect against environmental hazards & School Health Policies and Programs Study (SHPPS), CDC, NCCDPHF \\
\hline $8-21$ & $\begin{array}{l}\text { Disaster preparedness plans, protocols, and exercises (no. States and } \\
\text { D.C.) }\end{array}$ & $\begin{array}{l}\text { Association of State and Territorial Health Officials (ASTHO); CDC, } \\
\text { Division of State and Local Readiness, (DSLR). }\end{array}$ \\
\hline $8-22$ & $\begin{array}{l}\text { Proportion of persons in pre-1950s homes tested for lead-based paint } \\
\text { (age adjusted, 18+ years) }\end{array}$ & National Health Interview Survey (NHIS), CDC, NCHS. \\
\hline $8-23$ & $\begin{array}{l}\text { Substandard housing (percent of homes with moderate or severe } \\
\text { physical problems) }\end{array}$ & $\begin{array}{l}\text { American Housing Survey (AHS), Department of Commerce, Census } \\
\text { Bureau. }\end{array}$ \\
\hline $8-24 a$ & $\begin{array}{l}\text { Pesticide exposure-Urine concentrations ( } \mu \mathrm{g} / \mathrm{g} \text { creatinine, 6-59 } \\
\text { years) }-1 \text { naphthol (carbaryl) ( } \mu \mathrm{g} / \mathrm{g} \text { creatinine) }\end{array}$ & Dropped \\
\hline $8-24 b$ & $\begin{array}{l}\text { Pesticide exposure-Urine concentrations ( } \mu \mathrm{g} / \mathrm{g} \text { creatinine, } 6-59 \\
\text { years)-Paranitrophenol (methyl parathion and parathions) ( } \mu \mathrm{g} / \mathrm{g} \\
\text { creatinine) }\end{array}$ & $\begin{array}{l}\text { National Report on Human Exposure To Environmental Chemicals, } \\
\text { CDC, NCEH; National Health and Nutrition Examination Survey } \\
\text { (NHANES), CDC, NCHS. }\end{array}$ \\
\hline $8-24 c$ & $\begin{array}{l}\text { Pesticide exposure-Urine concentrations ( } \mu \mathrm{g} / \mathrm{g} \text { creatinine, } 6-59 \\
\text { years)-3, 5, 6-trichloro-2-pyridinol (chlorpyrifos) ( } \mu \mathrm{g} / \mathrm{g} \text { creatinine) }\end{array}$ & $\begin{array}{l}\text { National Report on Human Exposure To Environmental Chemicals, } \\
\text { CDC, NCEH; National Health and Nutrition Examination Survey } \\
\text { (NHANES), CDC, NCHS. }\end{array}$ \\
\hline $8-24 d$ & $\begin{array}{l}\text { Pesticide exposure-Urine concentrations ( } \mu \mathrm{g} / \mathrm{g} \text { creatinine, 6-59 } \\
\text { years)-Isopropoxyphenol (propoxur) ( } \mu \mathrm{g} / \mathrm{g} \text { creatinine) }\end{array}$ & $\begin{array}{l}\text { National Report on Human Exposure To Environmental Chemicals, } \\
\text { CDC, NCEH; National Health and Nutrition Examination Survey } \\
\text { (NHANES), CDC, NCHS. }\end{array}$ \\
\hline
\end{tabular}




\begin{tabular}{|c|c|c|}
\hline Objective & Description & Data Source or Objective Status \\
\hline $8-25 a$ & Exposure to Arsenic & $\begin{array}{l}\text { National Report on Human Exposure To Environmental Chemicals, } \\
\text { CDC, NCEH; National Health and Nutrition Examination Survey } \\
\text { (NHANES), CDC, NCHS. }\end{array}$ \\
\hline $8-25 b$ & Exposure to Cadmium-Blood concentration $(\mu \mathrm{g} / \mathrm{L}$ blood $)$ & $\begin{array}{l}\text { National Report on Human Exposure To Environmental Chemicals, } \\
\text { CDC, NCEH; National Health and Nutrition Examination Survey } \\
\text { (NHANES), CDC, NCHS. }\end{array}$ \\
\hline $8-25 c$ & Exposure to Lead-Blood concentration $(\mu \mathrm{g} / \mathrm{L}$ blood $)$ & $\begin{array}{l}\text { National Report on Human Exposure To Environmental Chemicals, } \\
\text { CDC, NCEH; National Health and Nutrition Examination Survey } \\
\text { (NHANES), CDC, NCHS. }\end{array}$ \\
\hline $8-25 d$ & Exposure to Manganese & Dropped \\
\hline $8-25 e$ & Mercury in children 1-5 years-Blood concentration ( $\mu \mathrm{g} / \mathrm{L}$ blood) & $\begin{array}{l}\text { National Report on Human Exposure To Environmental Chemicals, } \\
\text { CDC, NCEH; National Health and Nutrition Examination Survey } \\
\text { (NHANES), CDC, NCHS. }\end{array}$ \\
\hline $8-25 f$ & Exposure to 2, 4-D ( $\mu \mathrm{g} / \mathrm{g}$ creatinine) & $\begin{array}{l}\text { National Report on Human Exposure To Environmental Chemicals, } \\
\text { CDC, NCEH; National Health and Nutrition Examination Survey } \\
\text { (NHANES), CDC, NCHS. }\end{array}$ \\
\hline $8-25 g$ & Exposure to o-Phenylphenol-Urine concentration ( $\mu \mathrm{g} / \mathrm{g}$ creatinine) & $\begin{array}{l}\text { National Report on Human Exposure To Environmental Chemicals, } \\
\text { CDC, NCEH; National Health and Nutrition Examination Survey } \\
\text { (NHANES), CDC, NCHS. }\end{array}$ \\
\hline $8-25 h$ & Exposure to cis-and-trans-Permethrin & Developmental \\
\hline $8-25 i$ & Exposure to Diazinon-Urine concentration ( $\mu \mathrm{g} / \mathrm{g}$ creatinine) & $\begin{array}{l}\text { National Report on Human Exposure To Environmental Chemicals, } \\
\text { CDC, NCEH; National Health and Nutrition Examination Survey } \\
\text { (NHANES), CDC, NCHS. }\end{array}$ \\
\hline $8-25 j$ & Exposure to Polychlorinated biphenyls & Developmental \\
\hline $8-25 \mathrm{k}$ & Exposure to Dioxins & Developmental \\
\hline $8-251$ & Exposure to Furans & Dropped \\
\hline $8-25 m$ & $\begin{array}{l}\text { Exposure to Chlordane/Oxychlordane-Serum concentration (ng/g } \\
\text { lipid) }\end{array}$ & $\begin{array}{l}\text { National Report on Human Exposure To Environmental Chemicals, } \\
\text { CDC, NCEH; National Health and Nutrition Examination Survey } \\
\text { (NHANES), CDC, NCHS. }\end{array}$ \\
\hline $8-25 n$ & Exposure to Dieldrin-Serum concentration (ng/g lipid) & $\begin{array}{l}\text { National Report on Human Exposure To Environmental Chemicals, } \\
\text { CDC, NCEH; National Health and Nutrition Examination Survey } \\
\text { (NHANES), CDC, NCHS. }\end{array}$ \\
\hline
\end{tabular}




\begin{tabular}{ll}
\hline Objective & \\
\hline $8-250$ & Exposure to DDT/DDE-Serum concentration (ng/g lipid) \\
$8-25 p$ & Exposure to Lindane/beta-HCH-Serum concentration (ng/g lipid) \\
$8-25 q$ & $\begin{array}{l}\text { Exposure to Mercury in females } 16-49 \text { years-Blood concentration } \\
(\mu \mathrm{g} / \mathrm{L})\end{array}$
\end{tabular}

8-25r Exposure to Chlordane/trans-Nonachlor-Serum concentration (ng/g lipid)

8-25s Exposure to Chlordane/Heptachlor epoxide-Serum concentration (ng/g lipid)

8-26 Information systems used for public health (no. States)

8-27a Monitoring environmentally related diseases (no. States and D.C.)Lead poisoning

8-27b Monitoring environmentally related diseases (no. States and D.C.) Pesticide poisoning

8-27c Monitoring environmentally related diseases (no. States and D.C.)Mercury poisoning

8-27d Monitoring environmentally related diseases (no. States and D.C.) Arsenic poisoning

8-27e Monitoring environmentally related diseases (no. States and D.C.) Cadmium poisoning

8-27f Monitoring environmentally related diseases (no. States and D.C.) Methemoglobinemia

8-27g Monitoring environmentally related diseases (no. States and D.C.)Acute chemical poisoning by nonmedicinal chemicals not identified above
Data Source or Objective Status

National Report on Human Exposure To Environmental Chemicals,

CDC, NCEH; National Health and Nutrition Examination Survey

(NHANES), CDC, NCHS.

National Report on Human Exposure To Environmental Chemicals, CDC, NCEH; National Health and Nutrition Examination Survey (NHANES), CDC, NCHS.

National Report on Human Exposure To Environmental Chemicals, CDC, NCEH; National Health and Nutrition Examination Survey (NHANES), CDC, NCHS.

National Report on Human Exposure To Environmental Chemicals, CDC, NCEH; National Health and Nutrition Examination Survey (NHANES), CDC, NCHS.

National Report on Human Exposure To Environmental Chemicals, CDC, NCEH; National Health and Nutrition Examination Survey (NHANES), CDC, NCHS.

National Environmental Public Health Tracking Network (EPHT), CDC, NCEH.

State Reportable Conditions Assessment (SRCA), Council of State and Territorial Epidemiologists (CSTE).

State Reportable Conditions Assessment (SRCA), Council of State and Territorial Epidemiologists (CSTE).

State Reportable Conditions Assessment (SRCA), Council of State and Territorial Epidemiologists (CSTE).

State Reportable Conditions Assessment (SRCA), Council of State and Territorial Epidemiologists (CSTE).

State Reportable Conditions Assessment (SRCA), Council of State and Territorial Epidemiologists (CSTE).

Periodic surveys, Public Health Foundation (PHF) and Council of State and Territorial Epidemiologist (CSTE).

State Reportable Conditions Assessment (SRCA), Council of State and Territorial Epidemiologists (CSTE). 


\begin{tabular}{|c|c|c|}
\hline Objective & Description & Data Source or Objective Status \\
\hline $8-27 \mathrm{~h}$ & $\begin{array}{l}\text { Monitoring environmentally related diseases (no. States and D.C.)- } \\
\text { Carbon monoxide poisoning }\end{array}$ & $\begin{array}{l}\text { State Reportable Conditions Assessment (SRCA), Council of State and } \\
\text { Territorial Epidemiologists (CSTE). }\end{array}$ \\
\hline $8-27 \mathrm{i}$ & $\begin{array}{l}\text { Monitoring environmentally related diseases (no. States and D.C.)- } \\
\text { Asthma }\end{array}$ & $\begin{array}{l}\text { State Reportable Conditions Assessment (SRCA), Council of State and } \\
\text { Territorial Epidemiologists (CSTE). }\end{array}$ \\
\hline $8-27 j$ & $\begin{array}{l}\text { Monitoring environmentally related diseases (no. States and D.C.)- } \\
\text { Hyperthermia }\end{array}$ & $\begin{array}{l}\text { Periodic surveys, Public Health Foundation (PHF) and Council of State } \\
\text { and Territorial Epidemiologist (CSTE). }\end{array}$ \\
\hline $8-27 k$ & $\begin{array}{l}\text { Monitoring environmentally related diseases (no. States and D.C.)- } \\
\text { Hypothermia }\end{array}$ & $\begin{array}{l}\text { Periodic surveys, Public Health Foundation (PHF) and Council of State } \\
\text { and Territorial Epidemiologist (CSTE). }\end{array}$ \\
\hline $8-271$ & $\begin{array}{l}\text { Monitoring environmentally related diseases (no. States and D.C.)- } \\
\text { Skin cancer }\end{array}$ & Dropped \\
\hline $8-27 \mathrm{~m}$ & $\begin{array}{l}\text { Monitoring environmentally related diseases (no. States and D.C.)- } \\
\text { Malignant melanoma }\end{array}$ & Dropped \\
\hline $8-27 n$ & $\begin{array}{l}\text { Monitoring environmentally related diseases (no. States and D.C.)- } \\
\text { Other skin cancer }\end{array}$ & Dropped \\
\hline $8-270$ & $\begin{array}{l}\text { Monitoring environmentally related diseases (no. States and D.C.)- } \\
\text { Birth defects }\end{array}$ & $\begin{array}{l}\text { State Reportable Conditions Assessment (SRCA), Council of State and } \\
\text { Territorial Epidemiologists (CSTE). }\end{array}$ \\
\hline 8-28 & Local agencies using surveillance data for vector control & Dropped \\
\hline $8-29$ & Global burden of disease (no. deaths in thousands) & Global Burden of Disease Project, World Health Organization (WHO). \\
\hline $8-30 \mathrm{a}$ & $\begin{array}{l}\text { Proportion of population in U.S.-Mexico border region with wastewater } \\
\text { sewer service-Ciudad Acuna }\end{array}$ & $\begin{array}{l}\text { Environmental Protection Agency (EPA), Office of Water (OW), Office of } \\
\text { Wastewater Management (OWM); Mexico's Comision Nacional de Agua; } \\
\text { State and Local Health Departments; American Water Works } \\
\text { Association; Rural Water Association; U.S.-Mexican Border Health } \\
\text { Association. }\end{array}$ \\
\hline $8-30 b$ & $\begin{array}{l}\text { Proportion of population in U.S.-Mexico border region with wastewater } \\
\text { sewer service-Matamoros }\end{array}$ & $\begin{array}{l}\text { Environmental Protection Agency (EPA), Office of Water (OW), Office of } \\
\text { Wastewater Management (OWM); Mexico's Comision Nacional de Agua; } \\
\text { State and Local Health Departments; American Water Works } \\
\text { Association; Rural Water Association; U.S.-Mexican Border Health } \\
\text { Association. }\end{array}$ \\
\hline $8-30 c$ & $\begin{array}{l}\text { Proportion of population in U.S.-Mexico border region with wastewater } \\
\text { sewer service-Mexicali }\end{array}$ & $\begin{array}{l}\text { Environmental Protection Agency (EPA), Office of Water (OW), Office of } \\
\text { Wastewater Management (OWM); Mexico's Comision Nacional de Agua; } \\
\text { State and Local Health Departments; American Water Works } \\
\text { Association; Rural Water Association; U.S.-Mexican Border Health } \\
\text { Association. }\end{array}$ \\
\hline
\end{tabular}




\begin{tabular}{ll}
\hline Objective & \multicolumn{1}{c}{ Description } \\
\hline 8-30d & $\begin{array}{l}\text { Proportion of population in U.S.-Mexico border region with wastewater } \\
\text { sewer service-Nogales, Sonora }\end{array}$ \\
8-30e & $\begin{array}{l}\text { Proportion of population in U.S.-Mexico border region with wastewater } \\
\text { sewer service-Piedras Negras }\end{array}$
\end{tabular}
sewer service-Piedras Negras

8-30f Proportion of population in U.S.-Mexico border region with wastewater sewer service-Reynosa

Proportion of population in U.S.-Mexico border region with wastewater treatment service-Ciudad Acuna

Environmental Protection Agency (EPA), Office of Water (OW), Office of Wastewater Management (OWM); Mexico's Comision Nacional de Agua; State and Local Health Departments; American Water Works Association; Rural Water Association; U.S.-Mexican Border Health Association.

Environmental Protection Agency (EPA), Office of Water (OW), Office of Wastewater Management (OWM); Mexico's Comision Nacional de Agua; State and Local Health Departments; American Water Works Association; Rural Water Association; U.S.-Mexican Border Health Association.

Environmental Protection Agency (EPA), Office of Water (OW), Office of Wastewater Management (OWM); Mexico's Comision Nacional de Agua; State and Local Health Departments; American Water Works Association; Rural Water Association; U.S.-Mexican Border Health Association.

Environmental Protection Agency (EPA), Office of Water (OW), Office of Wastewater Management (OWM); Mexico's Comision Nacional de Agua; State and Local Health Departments; American Water Works Association; Rural Water Association; U.S.-Mexican Border Health Association.

Environmental Protection Agency (EPA), Office of Water (OW), Office of Wastewater Management (OWM); Mexico's Comision Nacional de Agua; State and Local Health Departments; American Water Works Association; Rural Water Association; U.S.-Mexican Border Health Association.

8-30i Proportion of population in U.S.-Mexico border region with wastewater treatment service-Mexicali

Environmental Protection Agency (EPA), Office of Water (OW), Office of Wastewater Management (OWM); Mexico's Comision Nacional de Agua; State and Local Health Departments; American Water Works Association; Rural Water Association; U.S.-Mexican Border Health Association. 


\begin{tabular}{llc}
\hline Objective & \multicolumn{1}{c}{ Description } & Data Source or Objective Status \\
\hline $8-30 \mathrm{j}$ & Proportion of population in U.S.-Mexico border region with wastewater & Environmental Protection Agency (EPA), Office of Water (OW), Office of
\end{tabular}
treatment service-Nogales, Sonora

Wastewater Management (OWM); Mexico's Comision Nacional de Agua;

State and Local Health Departments; American Water Works

Association; Rural Water Association; U.S.-Mexican Border Health

Association.

8-30k Proportion of population in U.S.-Mexico border region with wastewater treatment service-Piedras Negras

Environmental Protection Agency (EPA), Office of Water (OW), Office of Wastewater Management (OWM); Mexico's Comision Nacional de Agua; State and Local Health Departments; American Water Works

Association; Rural Water Association; U.S.-Mexican Border Health Association.

8-301 Proportion of population in U.S.-Mexico border region with wastewater treatment service-Reynosa

Environmental Protection Agency (EPA), Office of Water (OW), Office of Wastewater Management (OWM); Mexico's Comision Nacional de Agua; State and Local Health Departments; American Water Works Association; Rural Water Association; U.S.-Mexican Border Health Association. 
Figure 8-1. Progress Toward Target Attainment for Focus Area 8: Environmental Health

Moved away

from target ${ }^{1}$
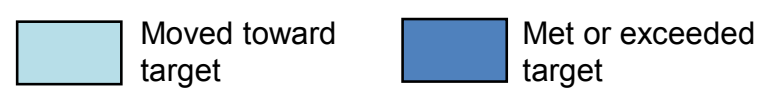

8-1. Percent of persons exposed to harmful air pollutants
a. Ozone
c. Carbon monoxide
d. Nitrogen dioxide
e. Sulfur dioxide

b. Particulate matter $(\leq 10 \mu \mathrm{m}$ in diameter $)$

f. Lead

8-1g. Number of persons (thousands) exposed to any harmful air pollutants

8-2. Alternative modes of transportation

a. Trips made by bicycling

target

\begin{tabular}{|c|c|c|c|c|c|}
\hline \multirow{2}{*}{$\begin{array}{l}2010 \\
\text { Target }\end{array}$} & \multirow{2}{*}{$\begin{array}{c}\text { Baseline } \\
\text { (Year) }\end{array}$} & \multirow{2}{*}{$\begin{array}{l}\text { Final } \\
\text { (Year) }\end{array}$} & \multicolumn{3}{|c|}{ Baseline vs. Final } \\
\hline & & & Difference $^{2}$ & $\begin{array}{l}\text { Statistically } \\
\text { Significant }{ }^{3}\end{array}$ & $\begin{array}{c}\text { Percent } \\
\text { Change }^{4}\end{array}$ \\
\hline $0 \%$ & $\begin{array}{c}43 \% \\
(1997)\end{array}$ & $\begin{array}{c}36 \% \\
(2010)\end{array}$ & -7 & $\begin{array}{l}\text { Not } \\
\text { tested }\end{array}$ & $-16.3 \%$ \\
\hline $0 \%$ & $\begin{array}{c}12 \% \\
(1997)\end{array}$ & $\begin{array}{c}9 \% \\
(2010)\end{array}$ & -3 & $\begin{array}{c}\text { Not } \\
\text { tested }\end{array}$ & $-25 \%$ \\
\hline $0 \%$ & $\begin{array}{c}20 \% \\
(1997)\end{array}$ & $\begin{array}{c}0 \% \\
(2010)\end{array}$ & -20 & $\begin{array}{c}\text { Not } \\
\text { tested }\end{array}$ & $-100 \%$ \\
\hline $0 \%$ & $\begin{array}{c}5 \% \\
(1997)\end{array}$ & $\begin{array}{c}0 \% \\
(2010)\end{array}$ & -5 & $\begin{array}{c}\text { Not } \\
\text { tested }\end{array}$ & $-100 \%$ \\
\hline $0 \%$ & $\begin{array}{c}2 \% \\
(1997)\end{array}$ & $\begin{array}{c}0 \% \\
(2010)\end{array}$ & -2 & $\begin{array}{l}\text { Not } \\
\text { tested }\end{array}$ & $-100 \%$ \\
\hline $0 \%$ & $\begin{array}{c}<1 \% \\
(1997)\end{array}$ & $\begin{array}{c}0 \% \\
(2010)\end{array}$ & $>-1$ & $\begin{array}{l}\text { Not } \\
\text { tested }\end{array}$ & $-100 \%$ \\
\hline 0 & $\begin{array}{c}137,019 \\
(1997)\end{array}$ & $\begin{array}{c}107,991 \\
(2010)\end{array}$ & $-29,028$ & $\begin{array}{c}\text { Not } \\
\text { tested }\end{array}$ & $-21.2 \%$ \\
\hline $1.8 \%$ & $\begin{array}{c}0.9 \% \\
(1995)\end{array}$ & $\begin{array}{l}1.0 \% \\
(2009)\end{array}$ & 0.1 & $\begin{array}{c}\text { Not } \\
\text { tested }\end{array}$ & $11.1 \%$ \\
\hline
\end{tabular}

(continued)

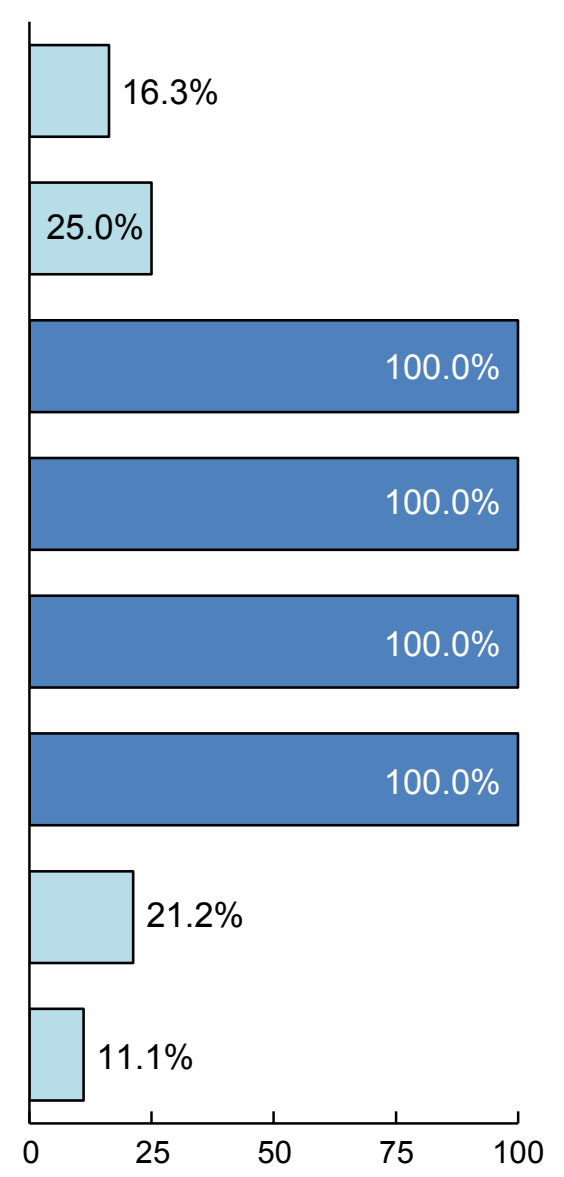

Per targeted change achieved 
Figure 8-1. Progress Toward Target Attainment for Focus Area 8: Environmental Health (continued)

Moved away

from target ${ }^{1}$

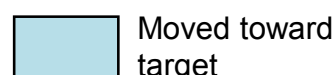

target

8-2. Alternative modes of transportation

b. Trips made by walking

c. Trips made by transit

d. Persons who telecommute

8-3. Cleaner alternative fuels

8-4. $\quad$ Airborne toxins (million tons)

8-5. Safe drinking water

8-6. Waterborne disease outbreaks (average no. per year)

8-7. Water conservation (gallons of domestic water usage per capita per day)
Met or exceeded

target

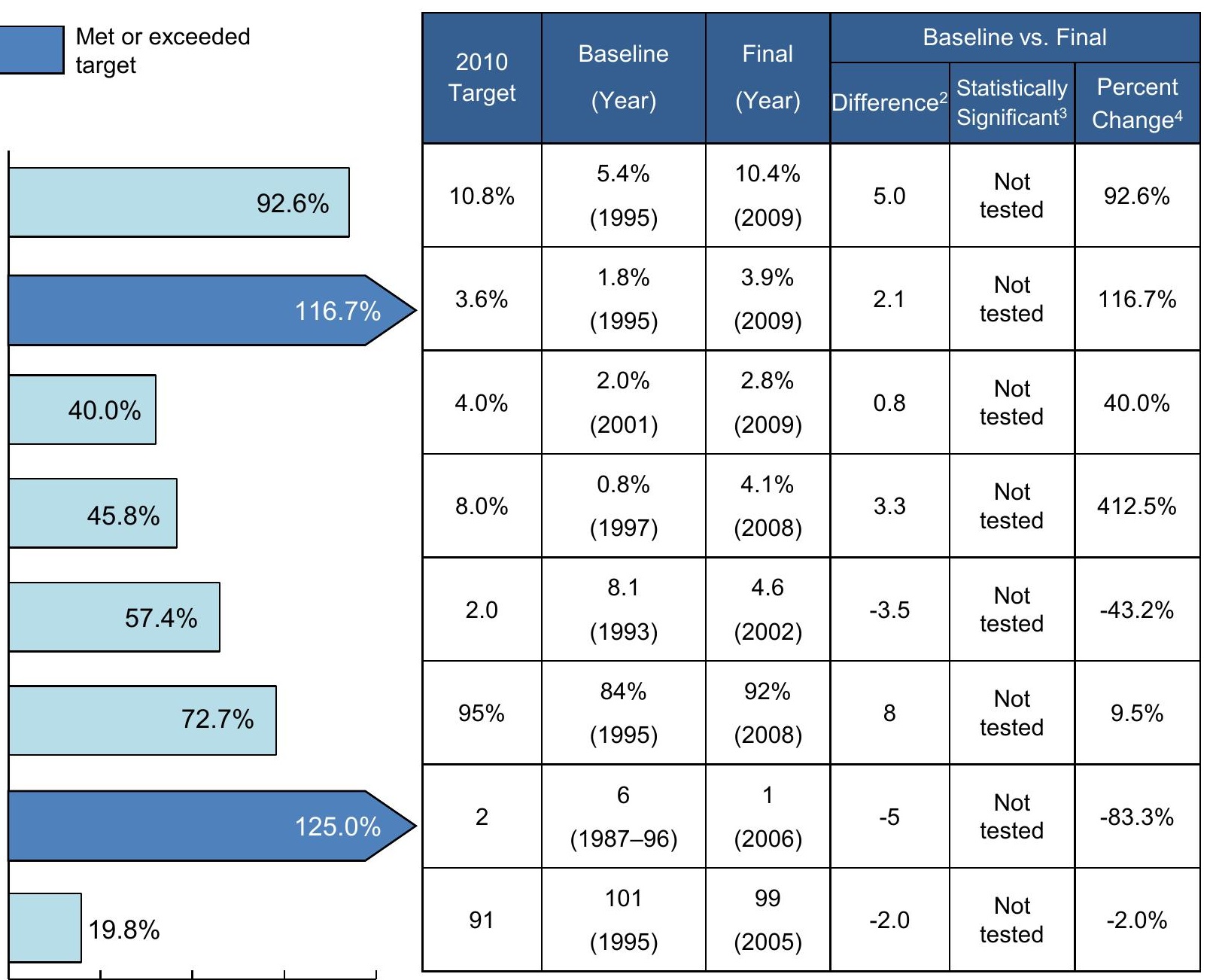

(continued) 
Figure 8-1. Progress Toward Target Attainment for Focus Area 8: Environmental Health (continued)

Moved away

from target ${ }^{1}$

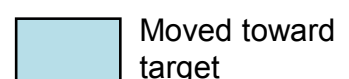

target

Met or exceeded

target

8-9. Beach open and safe for swimming (percent of days during beach season)

8-10. Fish consumption advisories

a. Rivers

b. Lakes

8-12a. Risks posed by hazardous sites-National Priority List sites

8-13. Pesticide exposures resulting in visits to a health care facility (no. of visits per year)

8-15. Recycled municipal solid waste (percent of total municipal solid waste)

8-19. Radon-resistant new home construction (number)

8-22. Proportion of persons in pre-1950s homes tested for lead-based paint (age adjusted, $18+$ years)
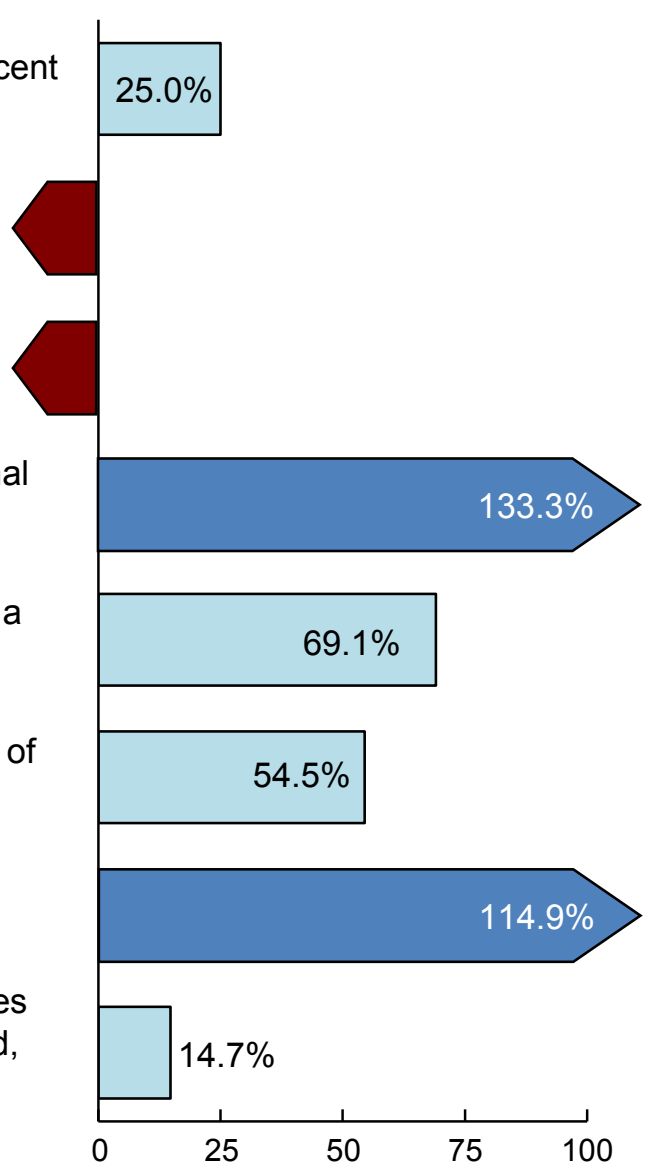

Percent of targeted change achieved ${ }^{5}$

\begin{tabular}{|c|c|c|c|c|c|}
\hline \multirow{2}{*}{$\begin{array}{l}2010 \\
\text { Target }\end{array}$} & \multirow{2}{*}{$\begin{array}{c}\text { Baseline } \\
\text { (Year) }\end{array}$} & \multirow{2}{*}{$\begin{array}{l}\text { Final } \\
\text { (Year) }\end{array}$} & \multicolumn{3}{|c|}{ Baseline vs. Final } \\
\hline & & & Difference $^{2}$ & $\begin{array}{l}\text { Statistically } \\
\text { Significant }^{3}\end{array}$ & $\begin{array}{l}\text { Percent } \\
\text { Change }^{4}\end{array}$ \\
\hline $98 \%$ & $\begin{array}{c}94 \% \\
(2002)\end{array}$ & $\begin{array}{c}95 \% \\
(2008)\end{array}$ & 1 & $\begin{array}{l}\text { Not } \\
\text { tested }\end{array}$ & $1.1 \%$ \\
\hline $13.8 \%$ & $\begin{array}{l}15.3 \% \\
(2002)\end{array}$ & $\begin{array}{c}24 \% \\
(2004)\end{array}$ & 8.7 & $\begin{array}{c}\text { Not } \\
\text { tested }\end{array}$ & $56.9 \%$ \\
\hline $29.6 \%$ & $\begin{array}{l}32.9 \% \\
(2002)\end{array}$ & $\begin{array}{c}35 \% \\
(2004)\end{array}$ & 2.1 & $\begin{array}{c}\text { Not } \\
\text { tested }\end{array}$ & $6.4 \%$ \\
\hline 1,176 & $\begin{array}{l}1,290 \\
(1998)\end{array}$ & $\begin{array}{l}1,138 \\
(2008)\end{array}$ & -152 & $\begin{array}{c}\text { Not } \\
\text { tested }\end{array}$ & $-11.8 \%$ \\
\hline 11,398 & $\begin{array}{l}22,933 \\
(1997)\end{array}$ & $\begin{array}{l}14,963 \\
(2008)\end{array}$ & -7970 & $\begin{array}{c}\text { Not } \\
\text { tested }\end{array}$ & $-34.8 \%$ \\
\hline $38 \%$ & $\begin{array}{c}27 \% \\
(1996)\end{array}$ & $\begin{array}{c}33 \% \\
(2008)\end{array}$ & 6 & $\begin{array}{c}\text { Not } \\
\text { tested }\end{array}$ & $22.2 \%$ \\
\hline 978,750 & $\begin{array}{c}652,500 \\
(1997)\end{array}$ & $\begin{array}{c}1,027,500 \\
(2007)\end{array}$ & 375,000 & $\begin{array}{c}\text { Not } \\
\text { tested }\end{array}$ & $57.5 \%$ \\
\hline $50 \%$ & $\begin{array}{c}16 \% \\
(1998)\end{array}$ & $\begin{array}{c}21 \% \\
(2002)\end{array}$ & 5 & Yes & $31.3 \%$ \\
\hline
\end{tabular}

(continued) 
Figure 8-1. Progress Toward Target Attainment for Focus Area 8: Environmental Health (continued)

Moved away from target ${ }^{1}$
Moved toward target
Met or exceeded target

8-23. Substandard housing (percent of homes with moderate or severe physical problems)

8-24. Pesticide exposure-Urine concentrations ( $\mu \mathrm{g} / \mathrm{g}$ creatinine, $6-59$ years)

b. Paranitrophenol (methyl parathion and parathions) ( $\mu \mathrm{g} / \mathrm{g}$ creatinine)

c. 3, 5, 6-trichloro-2-pyridinol (chlorpyrifos) ( $\mu \mathrm{g} / \mathrm{g}$ creatinine)

d. Isopropoxyphenol (propoxur) ( $\mu \mathrm{g} / \mathrm{g}$ creatinine)

8-25. Exposure to

b. Cadmium-Blood concentration $(\mu \mathrm{g} / \mathrm{L}$ blood $)$

c. Lead-Blood concentration ( $\mu \mathrm{g} / \mathrm{L}$ blood)

e. Mercury in children 1-5 years-Blood concentration $(\mu \mathrm{g} / \mathrm{L}$ blood)

g. o-Phenylphenol-Urine concentration ( $\mu \mathrm{g} / \mathrm{g}$ creatinine)

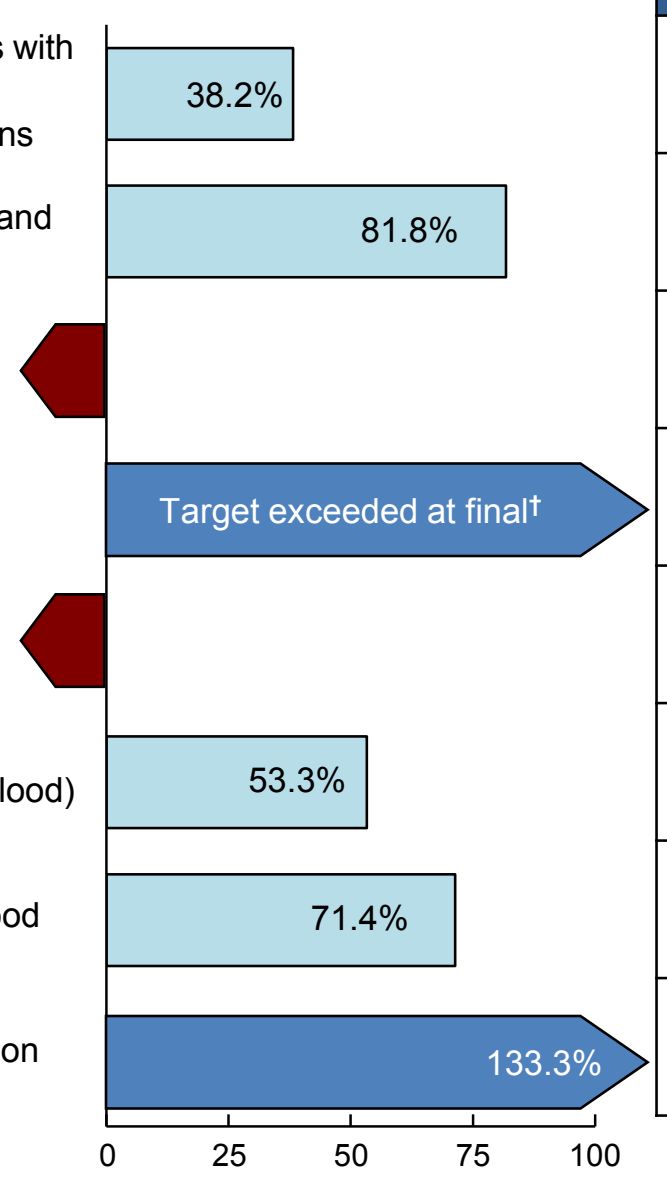

Percent of targeted change achieved ${ }^{5}$

\begin{tabular}{|c|c|c|c|c|c|}
\hline \multirow{2}{*}{$\begin{array}{l}2010 \\
\text { Target }\end{array}$} & \multirow{2}{*}{$\begin{array}{c}\text { Baseline } \\
\text { (Year) }\end{array}$} & \multirow{2}{*}{$\begin{array}{l}\text { Final } \\
\text { (Year) }\end{array}$} & \multicolumn{3}{|c|}{ Baseline vs. Final } \\
\hline & & & Difference & $\begin{array}{l}\text { Statistically } \\
\text { Significant }\end{array}$ & $\begin{array}{c}\text { Percent } \\
\text { Change }^{4}\end{array}$ \\
\hline $3.1 \%$ & $\begin{array}{c}6.5 \% \\
(1995)\end{array}$ & $\begin{array}{l}5.2 \% \\
(2007)\end{array}$ & -1.3 & $\begin{array}{l}\text { Not } \\
\text { tested }\end{array}$ & $-20.0 \%$ \\
\hline 2.7 & $\begin{array}{c}3.8 \\
(1988-94)\end{array}$ & $\begin{array}{c}2.9 \\
(2001-02)\end{array}$ & -0.9 & $\begin{array}{l}\text { Not } \\
\text { tested }\end{array}$ & $-23.7 \%$ \\
\hline 5.8 & $\begin{array}{c}8.3 \\
(1988-94)\end{array}$ & $\begin{array}{c}9.2 \\
(2001-02)\end{array}$ & 0.9 & $\begin{array}{c}\text { Not } \\
\text { tested }\end{array}$ & $10.8 \%$ \\
\hline 1.1 & $\begin{array}{c}1.6 \\
(1988-94)\end{array}$ & $\begin{array}{c}\text { BLOD }^{6} \\
(2001-02)\end{array}$ & * & $\begin{array}{c}\text { Not } \\
\text { tested }\end{array}$ & * \\
\hline 1.0 & $\begin{array}{c}1.4 \\
(1999-00)\end{array}$ & $\begin{array}{c}1.6 \\
(2003-04)\end{array}$ & 0.2 & $\begin{array}{c}\text { Not } \\
\text { tested }\end{array}$ & $14.3 \%$ \\
\hline 3.5 & $\begin{array}{c}5.0 \\
(1988-94)\end{array}$ & $\begin{array}{c}4.2 \\
(2003-04)\end{array}$ & -0.8 & $\begin{array}{c}\text { Not } \\
\text { tested }\end{array}$ & $-16.0 \%$ \\
\hline 1.6 & $\begin{array}{c}2.3 \\
(1999-00)\end{array}$ & $\begin{array}{c}1.8 \\
(2003-04)\end{array}$ & -0.5 & $\begin{array}{l}\text { Not } \\
\text { tested }\end{array}$ & $-21.7 \%$ \\
\hline 2.1 & $\begin{array}{c}3.0 \\
(1999-00)\end{array}$ & $\begin{array}{c}1.8 \\
(2001-02)\end{array}$ & -1.2 & $\begin{array}{c}\text { Not } \\
\text { tested }\end{array}$ & $-40.0 \%$ \\
\hline
\end{tabular}

(continued) 
Figure 8-1. Progress Toward Target Attainment for Focus Area 8: Environmental Health (continued)

Moved away from target ${ }^{1}$
Moved toward target

(1)

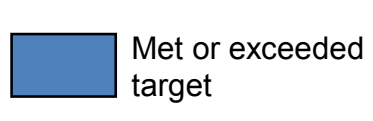

8-25. Exposure to

i. Diazinon-Urine concentration $(\mu \mathrm{g} / \mathrm{g}$ creatinine)

m. Chlordane/Oxychlordane-Serum concentration ( $\mathrm{ng} / \mathrm{g}$ lipid)

n. Dieldrin-Serum concentration (ng/g lipid)

o. DDT/DDE-Serum concentration (ng/g lipid)

p. Lindane/beta- $\mathrm{HCH}-$ Serum concentration ( $\mathrm{ng} / \mathrm{g}$ lipid)

q. Mercury in females 16-49 yearsBlood concentration $(\mu \mathrm{g} / \mathrm{L})$

r. Chlordane/trans-Nonachlor-Serum concentration ( $\mathrm{ng} / \mathrm{g}$ lipid)

s. Chlordane/Heptachlor epoxide-Serum concentration (ng/g lipid)
Met or exceeded

\begin{tabular}{|c|c|c|c|c|c|}
\hline \multirow{2}{*}{$\begin{array}{c}2010 \\
\text { Target }\end{array}$} & \multirow{2}{*}{$\begin{array}{c}\text { Baseline } \\
\text { (Year) }\end{array}$} & \multirow{2}{*}{$\begin{array}{l}\text { Final } \\
\text { (Year) }\end{array}$} & \multicolumn{3}{|c|}{ Baseline vs. Final } \\
\hline & & & Difference ${ }^{2}$ & $\begin{array}{l}\text { Statistically } \\
\text { Significant }^{3}\end{array}$ & $\begin{array}{c}\text { Percent } \\
\text { Change }^{4}\end{array}$ \\
\hline $\mathrm{BLOD}^{6}$ & $\begin{array}{c}\text { BLOD }^{6} \\
(1999-00)\end{array}$ & $\begin{array}{c}\text { BLOD }^{6} \\
(2001-02)\end{array}$ & $\mathrm{BLOD}^{6}$ & $\begin{array}{c}\text { Not } \\
\text { tested }\end{array}$ & * \\
\hline 31.4 & $\begin{array}{c}44.8 \\
(1999-00)\end{array}$ & $\begin{array}{c}37.7 \\
(2003-04)\end{array}$ & -7.1 & $\begin{array}{c}\text { Not } \\
\text { tested }\end{array}$ & $-15.8 \%$ \\
\hline 14.2 & $\begin{array}{c}20.3 \\
(2001-02)\end{array}$ & $\begin{array}{c}19.0 \\
(2003-04)\end{array}$ & -1.3 & $\begin{array}{c}\text { Not } \\
\text { tested }\end{array}$ & $-6.4 \%$ \\
\hline 1,281 & $\begin{array}{c}1,830 \\
(1999-00)\end{array}$ & $\begin{array}{c}1,860 \\
(2003-04)\end{array}$ & 30 & $\begin{array}{c}\text { Not } \\
\text { tested }\end{array}$ & $1.6 \%$ \\
\hline 48.2 & $\begin{array}{c}68.9 \\
(1999-00)\end{array}$ & $\begin{array}{c}56.5 \\
(2003-04)\end{array}$ & -12.4 & $\begin{array}{c}\text { Not } \\
\text { tested }\end{array}$ & $-18.0 \%$ \\
\hline 5.0 & $\begin{array}{c}7.1 \\
(1999-00)\end{array}$ & $\begin{array}{c}4.6 \\
(2001-02)\end{array}$ & -2.5 & $\begin{array}{c}\text { Not } \\
\text { tested }\end{array}$ & $-35.2 \%$ \\
\hline 55.6 & $\begin{array}{c}79.4 \\
(1999-00)\end{array}$ & $\begin{array}{c}68.3 \\
(2003-04)\end{array}$ & -11.1 & $\begin{array}{c}\text { Not } \\
\text { tested }\end{array}$ & $-14.0 \%$ \\
\hline 16.8 & $\begin{array}{c}24.0 \\
(1999-00)\end{array}$ & $\begin{array}{c}18.9 \\
(2003-04)\end{array}$ & -5.1 & $\begin{array}{c}\text { Not } \\
\text { tested }\end{array}$ & $-21.3 \%$ \\
\hline
\end{tabular}

(continued)

Percent of targeted change achieved ${ }^{5}$

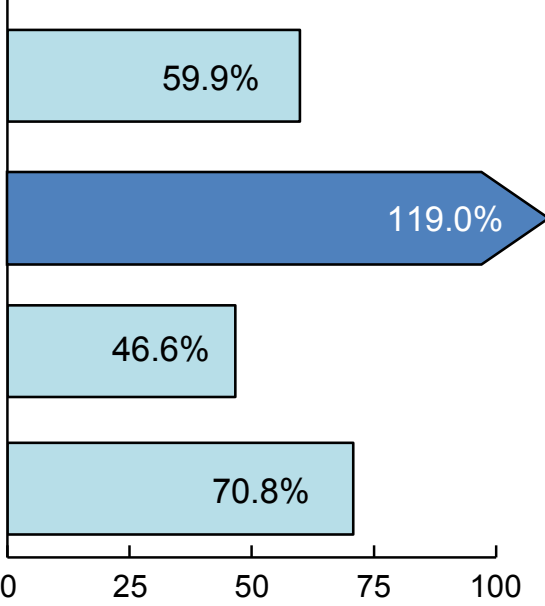


Figure 8-1. Progress Toward Target Attainment for Focus Area 8: Environmental Health (continued)

Moved away

from target ${ }^{1}$

Moved toward

Met or exceeded

target

8-26. Information systems used for public health (no. States)

8-27. Monitoring environmentally related diseases (no. States and D.C.)

a. Lead poisoning

b. Pesticide poisoning

c. Mercury poisoning

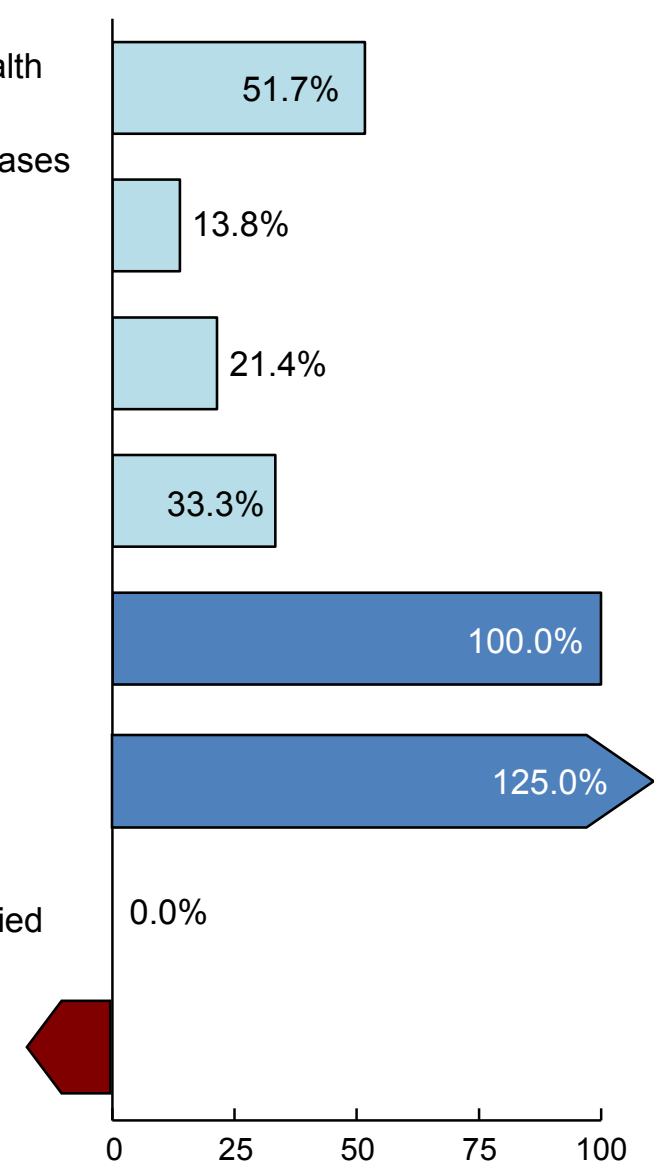

d. Arsenic poisoning

e. Cadmium poisoning

g. Acute chemical poisoning by nonmedicinal chemicals not identified above

h. Carbon monoxide poisoning

\begin{tabular}{|c|c|c|c|c|c|}
\hline \multirow{2}{*}{$\begin{array}{l}2010 \\
\text { Target }\end{array}$} & \multirow{2}{*}{$\begin{array}{c}\text { Baseline } \\
\text { (Year) }\end{array}$} & \multirow{2}{*}{$\begin{array}{l}\text { Final } \\
\text { (Year) }\end{array}$} & \multicolumn{3}{|c|}{ Baseline vs. Final } \\
\hline & & & Difference ${ }^{2}$ & $\begin{array}{l}\text { Statistically } \\
\text { Significant }{ }^{3}\end{array}$ & $\begin{array}{l}\text { Percent } \\
\text { Change }^{4}\end{array}$ \\
\hline 30 & $\begin{array}{c}1 \\
(2008)\end{array}$ & $\begin{array}{c}16 \\
(2010)\end{array}$ & 15 & $\begin{array}{l}\text { Not } \\
\text { tested }\end{array}$ & $1,500.0 \%$ \\
\hline 51 & $\begin{array}{c}22 \\
(2007)\end{array}$ & $\begin{array}{c}26 \\
(2010)\end{array}$ & 4 & $\begin{array}{c}\text { Not } \\
\text { tested }\end{array}$ & $18.2 \%$ \\
\hline 25 & $\begin{array}{c}11 \\
(2007)\end{array}$ & $\begin{array}{c}14 \\
(2009)\end{array}$ & 3 & $\begin{array}{c}\text { Not } \\
\text { tested }\end{array}$ & $27.3 \%$ \\
\hline 20 & $\begin{array}{c}14 \\
(2007)\end{array}$ & $\begin{array}{c}16 \\
(2010)\end{array}$ & 2 & $\begin{array}{l}\text { Not } \\
\text { tested }\end{array}$ & $14.3 \%$ \\
\hline 15 & $\begin{array}{c}12 \\
(2007)\end{array}$ & $\begin{array}{c}15 \\
(2010)\end{array}$ & 3 & $\begin{array}{c}\text { Not } \\
\text { tested }\end{array}$ & $25.0 \%$ \\
\hline 15 & $\begin{array}{c}11 \\
(2007)\end{array}$ & $\begin{array}{c}16 \\
(2010)\end{array}$ & 5 & $\begin{array}{l}\text { Not } \\
\text { tested }\end{array}$ & $45.5 \%$ \\
\hline 15 & $\begin{array}{c}9 \\
(2008)\end{array}$ & $\begin{array}{c}9 \\
(2009)\end{array}$ & 0 & $\begin{array}{c}\text { Not } \\
\text { tested }\end{array}$ & $0.0 \%$ \\
\hline 51 & $\begin{array}{c}10 \\
(2007)\end{array}$ & $\begin{array}{c}9 \\
(2009)\end{array}$ & -1 & $\begin{array}{l}\text { Not } \\
\text { tested }\end{array}$ & $-10.0 \%$ \\
\hline
\end{tabular}

Percent of targeted change achieved ${ }^{5}$

(continued) 
Figure 8-1. Progress Toward Target Attainment for Focus Area 8: Environmental Health (continued)

Moved away

from target ${ }^{1}$

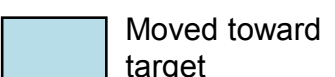

target

8-27. Monitoring environmentally related diseases

(no. States and D.C.)

i. Asthma

o. Birth defects

8-29. Global burden of disease (no. deaths in thousands)

8-30. Proportion of population in U.S.-Mexico border region with wastewater sewer service

a. Ciudad Acuna

b. Matamoros

e. Piedras Negras

f. Reynosa

8-30. Proportion of population in U.S.-Mexico border region with wastewater treatment service

g. Ciudad Acuna
Met or exceeded

target

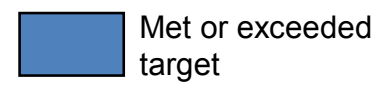

$$
\mid
$$

\begin{tabular}{|c|c|c|c|c|c|}
\hline \multirow{2}{*}{$\begin{array}{l}2010 \\
\text { Target }\end{array}$} & \multirow{2}{*}{$\begin{array}{c}\text { Baseline } \\
\text { (Year) }\end{array}$} & \multirow{2}{*}{$\begin{array}{l}\text { Final } \\
\text { (Year) }\end{array}$} & \multicolumn{3}{|c|}{ Baseline vs. Final } \\
\hline & & & Difference $^{2}$ & $\begin{array}{l}\text { Statistically } \\
\text { Significant }^{3}\end{array}$ & $\begin{array}{l}\text { Percent } \\
\text { Change }\end{array}$ \\
\hline 25 & $\begin{array}{c}2 \\
(2007)\end{array}$ & $\begin{array}{c}3 \\
(2009)\end{array}$ & 1 & $\begin{array}{l}\text { Not } \\
\text { tested }\end{array}$ & $50.0 \%$ \\
\hline 51 & $\begin{array}{c}6 \\
(2007)\end{array}$ & $\begin{array}{c}8 \\
(2009)\end{array}$ & 2 & $\begin{array}{l}\text { Not } \\
\text { tested }\end{array}$ & $33.3 \%$ \\
\hline $2,135.0$ & $\begin{array}{c}2,668.2 \\
(1990)\end{array}$ & $\begin{array}{r}2,200.0 \\
(2004)\end{array}$ & -468.2 & $\begin{array}{c}\text { Not } \\
\text { tested }\end{array}$ & $-17.5 \%$ \\
\hline $49 \%$ & $\begin{array}{c}39 \% \\
(1997)\end{array}$ & $\begin{array}{c}75 \% \\
(2002)\end{array}$ & 36 & $\begin{array}{c}\text { Not } \\
\text { tested }\end{array}$ & $92.3 \%$ \\
\hline $57 \%$ & $\begin{array}{c}47 \% \\
(1997)\end{array}$ & $\begin{array}{c}47 \% \\
(2002)\end{array}$ & 0 & $\begin{array}{l}\text { Not } \\
\text { tested }\end{array}$ & $0.0 \%$ \\
\hline $90 \%$ & $\begin{array}{c}80 \% \\
(1997)\end{array}$ & $\begin{array}{l}100 \% \\
(2002)\end{array}$ & 20 & $\begin{array}{c}\text { Not } \\
\text { tested }\end{array}$ & $25.0 \%$ \\
\hline $67 \%$ & $\begin{array}{c}57 \% \\
(1997)\end{array}$ & $\begin{array}{c}75 \% \\
(2002)\end{array}$ & 18 & $\begin{array}{l}\text { Not } \\
\text { tested }\end{array}$ & $31.6 \%$ \\
\hline $10 \%$ & $\begin{array}{c}0 \% \\
(1997)\end{array}$ & $\begin{array}{c}89 \% \\
(2009)\end{array}$ & 89 & $\begin{array}{l}\text { Not } \\
\text { tested }\end{array}$ & * \\
\hline
\end{tabular}

(continued) 
Figure 8-1. Progress Toward Target Attainment for Focus Area 8: Environmental Health (continued)

Moved away

from target ${ }^{1}$
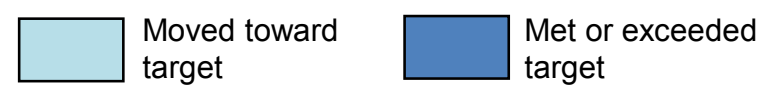

8-30. Proportion of population in U.S.-Mexico border region with wastewater treatment service

h. Matamoros

i. Mexicali

j. Nogales, Sonora

k. Piedras Negras

I. Reynosa

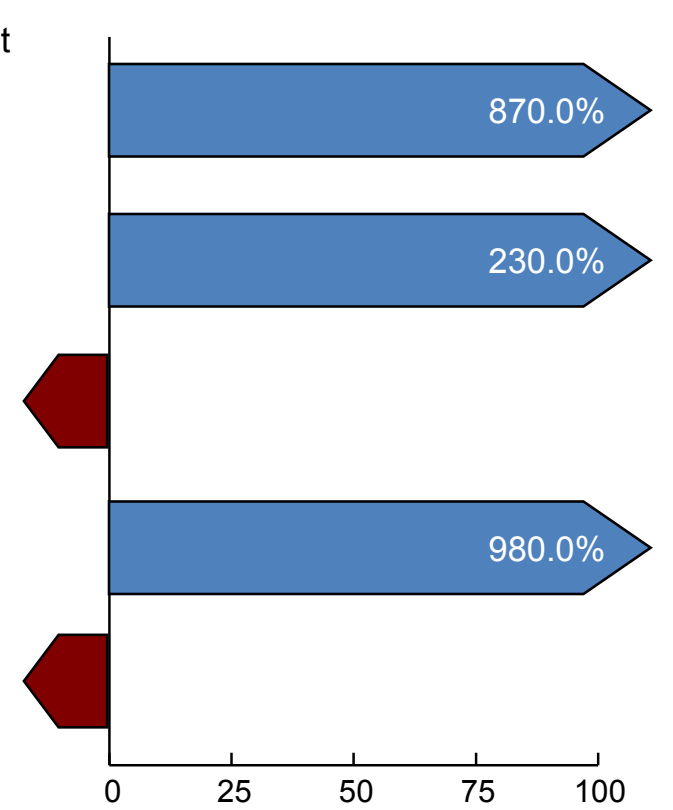

\begin{tabular}{|c|c|c|c|c|c|}
\hline \multirow{2}{*}{$\begin{array}{l}2010 \\
\text { Target }\end{array}$} & \multirow{2}{*}{$\begin{array}{c}\text { Baseline } \\
\text { (Year) }\end{array}$} & \multirow{2}{*}{$\begin{array}{l}\text { Final } \\
\text { (Year) }\end{array}$} & \multicolumn{3}{|c|}{ Baseline vs. Final } \\
\hline & & & Difference $^{2}$ & $\begin{array}{l}\text { Statistically } \\
\text { Significant }^{3}\end{array}$ & $\begin{array}{l}\text { Percent } \\
\text { Change }^{4}\end{array}$ \\
\hline $10 \%$ & $\begin{array}{c}0 \% \\
(1997)\end{array}$ & $\begin{array}{c}87 \% \\
(2010)\end{array}$ & 87 & $\begin{array}{c}\text { Not } \\
\text { tested }\end{array}$ & * \\
\hline $82 \%$ & $\begin{array}{c}72 \% \\
(1997)\end{array}$ & $\begin{array}{c}95 \% \\
(2010)\end{array}$ & 23 & $\begin{array}{c}\text { Not } \\
\text { tested }\end{array}$ & $31.9 \%$ \\
\hline $100 \%$ & $\begin{array}{l}100 \% \\
(1997)\end{array}$ & $\begin{array}{c}89 \% \\
(2010)\end{array}$ & -11 & $\begin{array}{c}\text { Not } \\
\text { tested }\end{array}$ & $-11.0 \%$ \\
\hline $10 \%$ & $\begin{array}{c}0 \% \\
(1997)\end{array}$ & $\begin{array}{c}98 \% \\
(2010)\end{array}$ & 98 & $\begin{array}{c}\text { Not } \\
\text { tested }\end{array}$ & * \\
\hline $100 \%$ & $\begin{array}{l}100 \% \\
(1997)\end{array}$ & $\begin{array}{c}89 \% \\
(2010)\end{array}$ & -11 & $\begin{array}{c}\text { Not } \\
\text { tested }\end{array}$ & $-11.0 \%$ \\
\hline
\end{tabular}

Percent of targeted change achieved 5

(continued) 


\section{Figure 8-1. Progress Toward Target Attainment for Focus Area 8: Environmental Health (continued)}

\section{NOTES}

See the Reader's Guide for more information on how to read this figure. See DATA2010 at http://wonder.cdc.gov/data2010 for all Healthy People 2010 tracking data. Tracking data are not available for objectives 8-8a, 8-8b, 8-12b through d, 8-14a, 8-14b, 8-16a through c, 8-17, 8-18, 8-20, 8-21, 8-25a, 8-25d, 8-25f, 8-25h, 8-25j through I, 8-27f, 8-27j, 8-27k, 8-30c, and 8-30d. Final tracking data for objective 8-11 are not statistically reliable. Objectives 8-24a, 8-27l through $\mathrm{n}$, and 8-28 were deleted at the Midcourse Review.

\section{FOOTNOTES}

${ }^{1}$ Movement away from target is not quantified using the percent of targeted change achieved. See Technical Appendix for more information.

${ }^{2}$ Difference $=$ Final value - Baseline value. Differences between percents (\%) are measured in percentage points.

${ }^{3}$ When estimates of variability are available, the statistical significance of the difference between the final value and the baseline value is assessed at the 0.05 level. See Technical Appendix for more information.

${ }^{4}$ Percent change $=\frac{\text { Final value }- \text { Baseline value }}{\text { Baseline value }} \times 100$.

\footnotetext{
${ }^{5}$ Percent of targeted change achieved $=\frac{\text { Final value }- \text { Baseline value }}{\text { Healthy People } 2010 \text { target }- \text { Baseline value }} \times 100$.

${ }^{6}$ Below level of detection (BLOD)

* Difference and/or percent change cannot be calculated. See Technical Appendix for more information.

† Percent of targeted change cannot be calculated. See Technical Appendix for more information.
}

\section{DATA SOURCES}

8-1a-g. $\quad$ Air Quality System (AQS), Environmental Protection Agency (EPA).

8-2a-d. National Household Travel Survey (NHTS), formerly Nationwide Personal Transportation Survey (NPTS), Department of Transportation (DOT).

$8-3$.

$8-4$.

$8-5$.

8-6.

8-7.

8-9.

8-10a-b.

$8-12 a$.

8-13.

8-15.

$8-19$.

8-22.

Alternatives to Traditional Transportation Fuels, Department of Energy, (DOE)

National Emissions Inventory (NEI), Environmental Protection Agency (EPA).

Potable Water Surveillance System (PWSS) and Safe Drinking Water Information System (SDWIS), Environmental Protection Agency (EPA).

State Reporting Systems, CDC, NCID.

Estimated Use of Water in the United States, Department of Interior (DOI).

Beaches Environmental Assessment, Closure and Health Program (BEACH), Environmental Protection Agency (EPA).

National Listing of Fish Advisories, Environmental Protection Agency (EPA).

Comprehensive Environmental Response and Cleanup Liability Information System (CERCLIS), Environmental Protection Agency (EPA).

Toxic Exposure Surveillance System (TESS), American Association of Poison Control Centers.

Municipal Solid Waste in The United States, Environmental Protection Agency (EPA).

National Association of Home Builders Research Center Survey, National Association of Home Builders.

National Health Interview Survey (NHIS), CDC, NCHS.

American Housing Survey (AHS), Department of Commerce, Census Bureau. 
Figure 8-1. Progress Toward Target Attainment for Focus Area 8: Environmental Health (continued)

\section{DATA SOURCES (continued)}

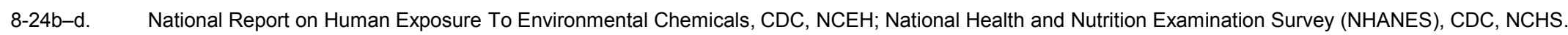
National Report on Human Exposure To Environmental Chemicals, CDC, NCEH; National Health and Nutrition Examination Survey (NHANES), CDC, NCHS. 8-25e. 8-25g. 8-25i. $8-25 m-s$. $8-26$. 8-27a-e. $8-27 \mathrm{~g}-\mathrm{i}$. $8-270$.

8-29.

8-30a-b.

8-30e-l. National Report on Human Exposure To Environmental Chemicals, CDC, NCEH; National Health and Nutrition Examination Survey (NHANES), CDC, NCHS. National Report on Human Exposure To Environmental Chemicals, CDC, NCEH; National Health and Nutrition Examination Survey (NHANES), CDC, NCHS. National Report on Human Exposure To Environmental Chemicals, CDC, NCEH; National Health and Nutrition Examination Survey (NHANES), CDC, NCHS. National Report on Human Exposure To Environmental Chemicals, CDC, NCEH; National Health and Nutrition Examination Survey (NHANES), CDC, NCHS. National Environmental Public Health Tracking Network (EPHT), CDC, NCEH.

State Reportable Conditions Assessment (SRCA), Council of State and Territorial Epidemiologists (CSTE).

State Reportable Conditions Assessment (SRCA), Council of State and Territorial Epidemiologists (CSTE).

State Reportable Conditions Assessment (SRCA), Council of State and Territorial Epidemiologists (CSTE).

Global Burden of Disease Project, World Health Organization (WHO).

Environmental Protection Agency (EPA), Office of Water (OW), Office of Wastewater Management (OWM); Mexico's Comision Nacional de Agua; State and Local Health Departments; American Water Works Association; Rural Water Association; U.S.-Mexican Border Health Association.

Environmental Protection Agency (EPA), Office of Water (OW), Office of Wastewater Management (OWM); Mexico's Comision Nacional de Agua; State and Local Health Departments; American Water Works Association; Rural Water Association; U.S.-Mexican Border Health Association. 
Figure 8-2. Health Disparities Table for Focus Area 8: Environmental Health Disparities from the best group rate for each characteristic at the most recent data point and changes in disparity from the baseline to the most recent data point.

Population-based objectives

8-1a. Percent of persons exposed to harmful air pollutantsOzone $(1997,2010)^{1} \dagger$

b. Percent of persons exposed to harmful air pollutantsParticulate matter $\left(\leq 10 \mu \mathrm{m}\right.$ in diameter) $(1997,2010)^{1} \dagger$

c. Percent of persons exposed to harmful air pollutantsCarbon monoxide $(1997,2010)^{1} \dagger$

d. Percent of persons exposed to harmful air pollutantsNitrogen dioxide $(1997,2010)^{1} \dagger$

e. Percent of persons exposed to harmful air pollutantsSulfur dioxide $(1997,2010)^{1} \dagger$

f. Percent of persons exposed to harmful air pollutantsLead $(1997,2010)^{1} \dagger$

8-1g. Number of persons (thousands) exposed to any harmful air pollutants $(1997,2010)^{1} \dagger$

8-11. Elevated blood lead levels in children $1-5$ years $(\geq 10$ $\mu \mathrm{g} / \mathrm{dL})(1991-94,2005-08)^{*}$

8-18. Proportion of persons living in homes tested for radon (age adjusted) (1998)*

8-22. Proportion of persons in pre-1950s homes tested for leadbased paint (age adjusted, 18+ years) $(1998,2002)^{2 *}$
Characteristics and Groups

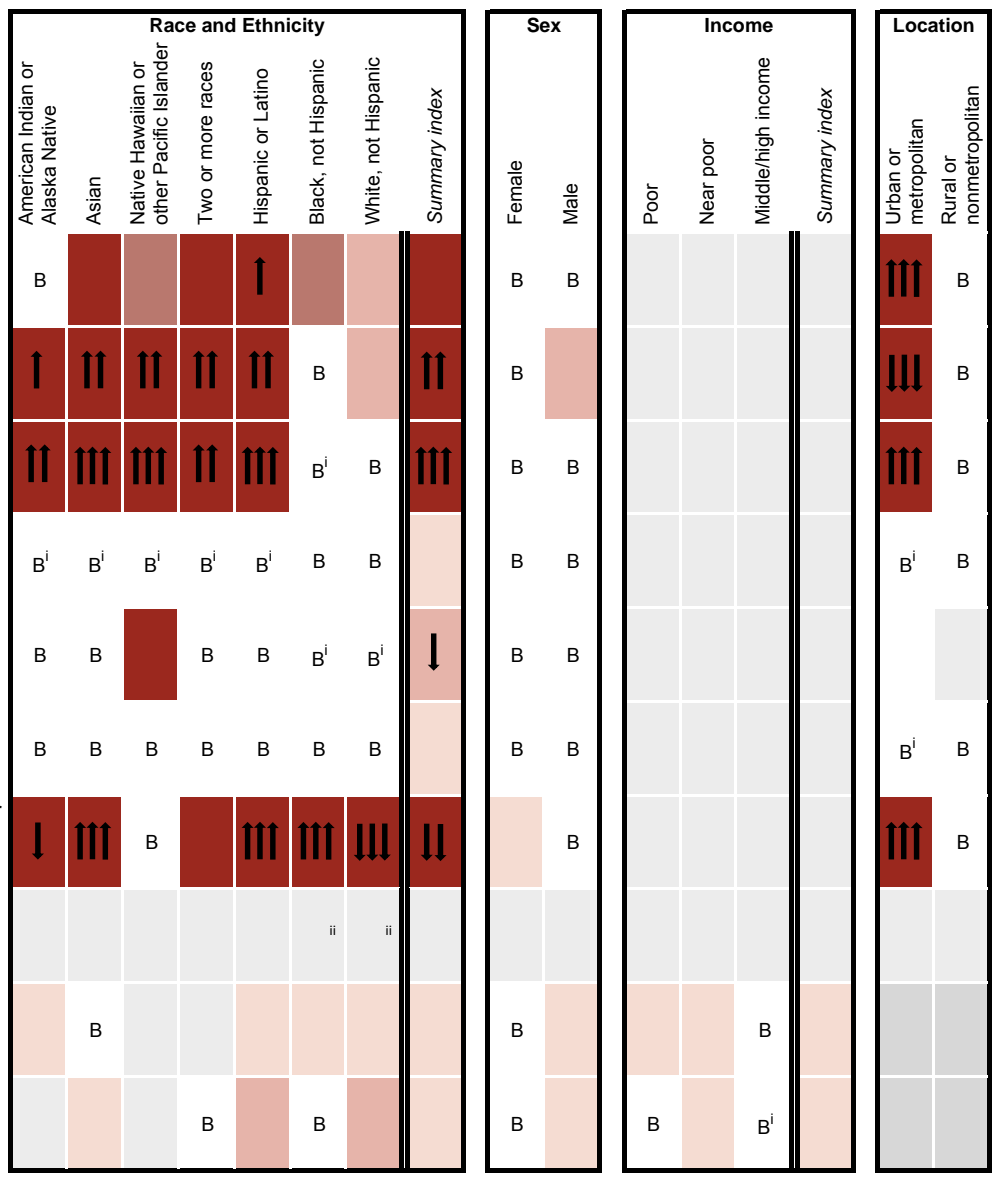

(continued) 
Figure 8-2. Health Disparities Table for Focus Area 8: Environmental Health (continued)

NOTES

See DATA2010 at http://wonder.cdc.gov/data2010 for all Healthy People 2010 tracking data. Disparity data are either unavailable or not applicable for objectives 8-2a through d, 8-3 through 8-7, 8-8a and b, 8-9, 8-10a and b, 8-12a through d, 8-13, 8-14a and b, 8-15, 8-16a through c, 8-17, 8-19 through 8-21, 8-23, 8-24b through d, 8-25a through s, 8-26, 8-27a through k, 8-27o, 8-29, and 8-30a through I. Objectives 8-24a, 8-27I through n, and 8-28 were deleted at Midcourse Review.

Years in parentheses represent the baseline and most recent data years (if available).

Disparity from the best group rate is defined as the percent difference between the best group rate and each of the other group rates for a characteristic (e.g., race and ethnicity). The summary index is the average of these percent differences for a characteristic. Change in disparity is estimated by subtracting the disparity at baseline from the disparity at the most recent data point. Change in the summary index is estimated by subtracting the summary index at baseline from the summary index at the most recent data point. See Technical Appendix for more information.

LEGEND

\begin{tabular}{|c|c|c|c|c|c|}
\hline $\begin{array}{l}\text { The "best" group rate at the most recent } \\
\text { data point. }\end{array}$ & B & $\begin{array}{l}\text { The group with the best rate for } \\
\text { specified characteristic. }\end{array}$ & b & $\begin{array}{l}\text { Most favorable group rate for specified } \\
\text { characteristic, but reliability criterion not } \\
\text { met. }\end{array}$ & $\begin{array}{l}\text { Reliability criterion for best } \\
\text { group rate not met, or data } \\
\text { available for only one group. }\end{array}$ \\
\hline
\end{tabular}

\begin{tabular}{ll|l|l|l|l}
\hline $\begin{array}{l}\text { Disparity from the best group rate at the } \\
\text { most recent data point. }\end{array}$ & $\begin{array}{l}\text { Less than 10\%, or difference not } \\
\text { statistically significant (when } \\
\text { estimates of variability are available). }\end{array}$ & $10 \%-49 \%$ & Percent difference from the best group rate \\
\hline
\end{tabular}

Changes in disparity over time are shown when:
(a) disparities data are available at both baseline and most recent time points;
(b) data are not for the group(s) indicated by "B" or "b" at either time point; and
(c) the change is greater than or equal to 10 percentage points and statistically
significant, or when the change is greater than or equal to 10 percentage
points and estimates of variability were not available. See Technical Appendix.

FOOTNOTES

* Measures of variability were available. Thus, the variability of best group rates was assessed, and statistical significance was tested. Disparities of $10 \%$ or more are displayed when the differences from the best group rate are statistically significant at the 0.05 level. Changes in disparities over time are indicated by arrows when the changes are greater than or equal to 10 percentage points and are statistically significant at the 0.05 level. See $\underline{T e c h n i c a l ~ A p p e n d i x}$.

† Measures of variability were not available. Thus, the variability of best group rates was not assessed, and statistical significance could not be tested.

Nonetheless, disparities and changes in disparities over time are displayed according to their magnitude. See Technical Appendix.

1 Most recent data by race and ethnicity, by sex, and by location, are for 2004.

2 Baseline data by race and ethnicity are for 2002.

$i$ The group with the best rate at the most recent data point is different from the group with the best rate at baseline. Both rates met the reliability criterion. See Technical Appendix.

ii Data are for Mexican American.

\section{DATA SOURCES}

8-1a-g. Air Quality System (AQS), Environmental Protection Agency (EPA).

8-11. National Health and Nutrition Examination Survey (NHANES), CDC, NCHS.

8-18. National Health Interview Survey (NHIS), CDC, NCHS.

8-22. National Health Interview Survey (NHIS), CDC, NCHS. 


\section{Final Review}

\section{Family Planning}

PEOPLE

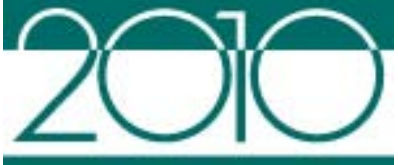

\section{Lead Agency}

Office of Population Affairs

\section{Contents}

Goal. 9-2

Highlights 9-2

Summary of Progress. 9-3

Transition to Healthy People 2020 $9-4$

Data Considerations $9-6$

References and Notes $9-6$

Comprehensive Summary of Objectives 9-8

Progress Chart 9-11

Health Disparities Table 9-16 


\section{Goal: Improve family planning and spacing and prevent unintended pregnancy}

This chapter includes objectives that track intended pregnancies, birth spacing, infertility, and adolescent pregnancies. Contraceptive use and family planning clinic visits among adolescents and persons at risk of unintended pregnancy are also monitored, and so is instruction on reproductive health issues for adolescents.

All Healthy People tracking data quoted in this chapter, along with technical information and operational definitions for each objective, can be found in the Healthy People 2010 database, (DATA2010), available from http://wonder.cdc.gov/data2010/.

More information about this focus area can be found in the following publications:

- Healthy People 2010: Understanding and Improving Health, available from http://www.healthypeople.gov/2010/Document/tableofcontents.htm\#under.

- Healthy People 2010 Midcourse Review, available from http://www.healthypeople.gov/2010/data/midcourse/html/default.htm\#FocusAreas.

\section{Highlights}

- Progress was achieved in objectives for this Focus Area during the past decade [1]. About half (53\%) of the Family Planning objectives with data to measure progress moved toward or achieved their Healthy People 2010 targets (Figure 9-1). However, health disparities among racial and ethnic population groups, as well as by income and by disability status, were observed (Figure 9-2), some of which are highlighted below [2].

- While several Family Planning objectives did not meet the Healthy People 2010 targets overall, some objectives met or even exceeded their targets for certain population groups. For example, in order for intended pregnancy (objective 9-1), considered as the principal objective of the Family Planning Focus Area, to have met the 2010 target, this objective would have had to increase from $52 \%$ to $70 \%$. This targeted increase was not achieved, and no progress was made during the decade overall. Nonetheless, married women did meet the 2010 target: $73 \%$ of their pregnancies were intended in 2002. Differential progress by marital status and income continues to be observed.

- Contraceptive failure, the proportion of women aged 15-44 years who experienced pregnancy within 12 months of continuous contraceptive use (objective 9-4), declined by $20 \%$ between 1995 and 2002, from 15\% to 12\%, moving toward the 2010 target of $8 \%$. Although the 2010 target was not met overall, middle/high income women almost met the target with an $8.4 \%$ failure rate in 2002 . However, the failure rate for poor women was $20 \%$, which is almost double the overall population rate of $12 \%$. Moreover, health disparities by income increased, as seen next [3]. 
- Middle/high income women had the lowest (best) rates of contraceptive failure among income groups, $12 \%$ in 1995 and $8.4 \%$ in 2002; whereas near-poor women had rates of $17 \%$ in 1995 and $18 \%$ in 2002, and poor women had rates of $26 \%$ in 1995 and $20 \%$ in 2002. In 2002, the rate for near-poor women was more than twice the best group rate (that for middle/high income women), while the rate for poor women was almost two and a half times the best group rate [2]. Between 1995 and 2002 , the disparity between near-poor and middle/high income women increased by 83 percentage points [3].

- Among racial and ethnic groups, white non-Hispanic women had the lowest (best) rate of contraceptive failure, $10 \%$ in 2002 . The rate for black non-Hispanic women was $21 \%$ in 2002, more than twice that of white non-Hispanic women [2].

- Adolescent pregnancy among females aged 15-17 years (objective 9-7) declined by 37\% between 1996 and 2005, from 63 to 40 per 1,000 females, moving toward the 2010 target of 39 per 1,000 .

- Among racial and ethnic groups, white non-Hispanic females aged 15-17 had the lowest (best) adolescent pregnancy rate, 22 per 1,000 in 2005. Hispanic or Latino and black non-Hispanic females aged 15-17 had rates of 85 and 88 per 1,000 in 2005 , respectively. The rate for Hispanic or Latino females aged 15-17 was almost four times the best group rate (that for white non-Hispanic females aged 15-17), while the rate for black non-Hispanic females aged 15-17 was four times the best group rate [2].

- White non-Hispanic females aged 15-17 had adolescent pregnancy rates of 40 per 1,000 in 1996 and 22 per 1,000 in 2005, whereas Hispanic or Latino females aged 15-17 had rates of 109 per 1,000 in 1996 and 80 per 1,000 in 2005. Between 1996 and 2005, the disparity between Hispanic or Latino and white non-Hispanic females aged 15-17 increased by 91 percentage points [3].

\section{Summary of Progress}

- Figure 9-1 presents a quantitative assessment of progress in achieving the Healthy People 2010 objectives for Family Planning [1]. Data to measure progress toward target attainment were available for 32 objectives. Of these:

- Eight objectives (9-8a; 9-10c through h; and 9-11i) met or exceeded the Healthy People 2010 targets.

- Nine objectives moved toward their targets. A statistically significant difference between the baseline and the final data points was observed for one of these objectives (9-9a). No significant differences were observed for two objectives (9-11a and o); and data to test the significance of the difference were unavailable for the remaining six objectives $(9-4,9-7,9-8 b, 9 b, 9-10 b$, and 9-12).

- Two objectives (9-6a and 9-11k) showed no change.

- Thirteen objectives moved away from their targets. A statistically significant difference between the baseline and final data points was observed for two of these objectives (9-3 and 9-6b). No significant differences were observed for eight objectives (9-6c; 9-10a; and 9-11b through d, j, l, and p). Data to test the significance of the difference were unavailable for three objectives (9-1, 9-2 and 9-5). 
- Six objectives (9-11e through h, m, and n) remained developmental [4].

- Data were unavailable to measure progress for one objective (9-13).

- Figure 9-2 displays health disparities in Family Planning from the best group rate for each characteristic at the most recent data point [2]. It also displays changes in disparities from baseline to the most recent data point [3].

- Statistically significant racial and ethnic health disparities of $10 \%$ or more were observed for seven objectives (9-6b; 9-10c and d; and 9-11j through $\mathrm{l}$, and p); three additional objectives (9-1, 9-4 and 9-7) had racial and ethnic health disparities of $10 \%$ or more but no data to assess statistical significance. Of these 10 objectives, the white non-Hispanic population had the best group rate for eight objectives $(9-1 ; 9-4$; 9-7; 9-10c and d; and 9-11j through l). The Hispanic or Latino population and the black non-Hispanic population each had the best group rate for the two remaining objectives (9-6b and 9-11p).

- Statistically significant health disparities of $10 \%$ or more by income were observed for five objectives (9-2; 9-3; and 9-11c, d, and k); two additional objectives (9-1 and 9-4) had a health disparity of $10 \%$ or more by income but no data to assess statistical significance. Persons with middle/high incomes had the best group rate for all seven of these objectives.

- Two objectives (9-4 and 9-7) had health disparities of $100 \%$ or more among racial and ethnic populations and/or income groups, as well as changes in disparities of 50 percentage points or more over time. These disparities are discussed in the Highlights, above.

\section{Transition to Healthy People 2020}

The focus of the Healthy People 2020 Family Planning Topic Area-increasing the proportion of pregnancies that are intended, improving pregnancy planning and spacing, and preventing unintended pregnancy-is consistent with that of the Healthy People 2010 Focus Area. As publicly funded family planning services prevent 1.94 million unintended pregnancies, including 400,000 teen pregnancies each year, new objectives addressing services provided by publicly funded family planning clinics have been added to the Healthy People 2020 Topic Area [5]. See HealthyPeople.gov for a complete list of Healthy People 2020 topics and objectives.

The Healthy People 2020 Family Planning topic area can be grouped into four sections:

- Proportion of pregnancies that are intended and the rate of adolescent pregnancy

- Receipt of reproductive health services

- Effective use of contraception for pregnancy prevention and protection against disease

- Receipt of education on prevention of sexually transmitted diseases (STD) and unwanted pregnancy.

The differences between the Healthy People 2010 and Healthy People 2020 objectives are summarized below: 
- The Healthy People 2020 Family Planning Topic Area has 40 objectives, one of which is developmental, whereas the Healthy People 2010 Focus Area had 39 objectives, six of which were developmental [4].

- Twenty-one Healthy People 2010 objectives (9-1; 9-4; 9-9a and b; 9-11a through p; and 913) were retained "as is" [6]. These include objectives that focus on the proportion of pregnancies that are intended, contraceptive failure within 12 months of continuous use, abstinence among adolescents insurance coverage for contraceptive supplies and services, and reproductive health prevention education. Data are not shown in the DATA2010 database for six of these objectives (including formal and informal instruction on HIV/AIDS prevention and formal instruction on sexually transmitted diseases) but data are available in Healthy People 2020.

- One Healthy People 2010 objective, the rate of adolescent pregnancy (objective 9-7), was retained "as is" for ages 15-17 in Healthy People 2020 [6]. An additional objective on adolescent pregnancy also was added to Healthy People 2020 and focuses on ages 18-19.

- Thirteen Healthy People 2010 objectives (9-2; 9-3; 9-5; 9-8a and b; and 9-10a through h) were modified, including the objectives on birth spacing, contraceptive use among females at risk of unintended pregnancy, emergency contraception, abstinence before age 15, pregnancy prevention and STD protection $[7,8]$.

- One objective (9-12) addressing problems in becoming pregnant and maintaining a pregnancy was modified and moved to the Maternal, Infant, and Child Health Topic Area [7].

- Three Healthy People 2010 objectives (9-6a through c) that focused on male involvement in pregnancy prevention were archived [9]. Other objectives on male involvement in family planning are spread throughout the Healthy People 2020 Topic Area.

- Five new objectives were added to the Healthy People 2020 Topic Area:

- One new objective tracks the proportion of publicly funded family planning clinics that offer a full range of FDA-approved contraceptive methods on-site.

- Two new objectives track the proportion of sexually active persons who receive reproductive health services.

- One new objective monitors the number of states that set the income eligibility level for Medicaid-covered family planning services to at least the same level used to determine eligibility for Medicaid-covered pregnancy-related care.

- One new objective tracks the proportion of females in need of publicly-supported contraceptive services and supplies who receive those services and supplies.

Appendix D, “A Crosswalk Between Objectives From Healthy People 2010 to Healthy People 2020," summarizes the changes between the two decades of objectives, reflecting new knowledge and direction for this area. 


\section{Data Considerations}

Education and income are the primary measures of socioeconomic status (SES) in Healthy People 2010. Most data systems used in Healthy People 2010 define income as a family's income before taxes. In order to facilitate comparisons among groups and over time, while adjusting for family size and for inflation, Healthy People 2010 categorizes income using the poverty thresholds developed by the U.S. Census Bureau. Thus, the three categories of family income that are primarily used are:

- Poor-below the Federal poverty level

- Near poor-100\% to $199 \%$ of the Federal poverty level

- Middle/high income-200\% or more of the Federal poverty level.

These categories may be overridden by considerations specific to the data system, in which case they are modified as appropriate. See Healthy People 2010: General Data Issues, referenced below.

Information on data issues is available from the following sources:

- All Healthy People 2010 tracking data can be found in the Healthy People 2010 database, DATA2010, available from http://wonder.cdc.gov/data2010/.

- Detailed information about the data and data sources used to support these objectives can be found in the Operational Definitions on the DATA2010 website, available from http://wonder.cdc.gov/data2010/focusod.htm.

- More information on statistical issues related to Healthy People tracking and measurement can be found in the Technical Appendix and in Healthy People 2010: General Data Issues, which is available in the Data Issues section of the NCHS Healthy People website under Healthy People 2010.

\section{References and Notes}

1. Displayed in the Progress Chart (Figure 9-1), the percent of targeted change achieved expresses the difference between the baseline and the final value relative to the initial difference between the baseline and the Healthy People 2010 target. As such, it is a relative measure of progress toward attaining the Healthy People 2010 target. See the Reader's Guide for more information. When standard errors were available, the difference between the baseline and the final value was tested at the 0.05 level of significance. See the Figure 9-1 footnotes, as well as the Technical Appendix, for more detail.

2. Information about disparities among select populations is shown in the Health Disparities Table (Figure 9-2). Disparity from the best group rate is defined as the percent difference between the best group rate and each of the other group rates for a characteristic. For example, racial and ethnic health disparities are measured as the percent difference between the best racial and ethnic group rate and each of the other racial and ethnic group rates. Similarly, disparities by sex are measured as the percent difference between the better group rate (e.g., female) and the rate for the other group (e.g., male). Some objectives are expressed in terms of favorable events or conditions that are to be increased, while others are expressed in terms of adverse events or conditions that are to be reduced. In order to facilitate comparison of health disparities across different objectives, disparity is measured only in terms of adverse events or conditions. For comparability across objectives, objectives that are expressed in terms of favorable events or conditions are re-expressed using the adverse event or condition for the purpose of computing disparity, but they are not otherwise 
restated or changed. For example, objective 1-1, to increase the proportion of persons with health insurance (e.g., $72 \%$ of the American Indian or Alaska Native population aged under 65 years had some form of health insurance in 2008), is expressed in terms of the percentage of persons without health insurance (e.g., $100 \%-72 \%=28 \%$ of the American Indian or Alaska Native population aged under 65 years did not have any form of health insurance in 2008) when the disparity from the best group rate is calculated. See the Reader's Guide for more information. When standard errors were available, the difference between the best group rate and each of the other group rates was tested at the 0.05 level of significance. See the Figure 9-2 footnotes, as well as the Technical Appendix, for more detail.

3. The change in disparity is estimated by subtracting the disparity at baseline from the disparity at the most recent data point and, therefore, is expressed as a change in percentage points. See the Reader's Guide for more information. When standard errors were available, the change in disparity was tested at the 0.05 level of significance. See the Figure 9-2 footnotes, as well as the Technical Appendix, for more detail.

4. To be included in Healthy People 2010, an objective must have a national data source that provides a baseline and at least one additional data point for tracking progress. Some objectives lacked baseline data at the time of their development but had a potential data source and were considered of sufficient national importance to be included in Healthy People. These are called "developmental" objectives. When data become available, a developmental objective is moved to measurable status and a Healthy People target can be set.

5. Guttmacher Institute. In brief: Facts on publicly funded contraceptive services in the United States. Washington; Guttmacher Institute. April 2010. Available from: http://www.guttmacher.org/pubs/fb_contraceptive_serv.pdf

6. Retained "as is" objectives have no change in the numerator definition or in the denominator definition between the Healthy People 2010 and Healthy People 2020 objectives. These include objectives that were developmental in Healthy People 2010 and are developmental in Healthy People 2020 and for which no numerator or denominator information was available.

7. Modified objectives have some change in the numerator definition or in the denominator definition between the Healthy People 2010 and Healthy People 2020 objectives. These include objectives that went from developmental in Healthy People 2010 to measurable in Healthy People 2020 or vice versa.

8. Objectives 9-8a and b (abstinence before age 15) are defined as abstinence by age 15 in Healthy People 2020.

9. Archived objectives had at least one data point in Healthy People 2010 but were not carried forward into Healthy People 2020. 


\section{Comprehensive Summary of Objectives: Family Planning}

\begin{tabular}{|c|c|}
\hline Objective & Description \\
\hline $9-1$ & Intended pregnancy (females 15-44 years) \\
\hline $9-2$ & $\begin{array}{l}\text { Births occurring within } 24 \text { months of a previous birth (females 15-44 } \\
\text { years) }\end{array}$ \\
\hline $9-3$ & $\begin{array}{l}\text { Contraceptive use-Females at risk of unintended pregnancy (15-44 } \\
\text { years) }\end{array}$ \\
\hline $9-4$ & $\begin{array}{l}\text { Contraceptive failure within } 12 \text { months of continuous use-Females } \\
\text { experiencing pregnancy (15-44 years) }\end{array}$ \\
\hline $9-5$ & Emergency contraception provided by family planning agencies \\
\hline $9-6 a$ & $\begin{array}{l}\text { Involvement in pregnancy prevention among unmarried males } 15-24 \\
\text { years-Family planning clinic visit with female partner in last } 12 \\
\text { months }\end{array}$ \\
\hline $9-6 b$ & $\begin{array}{l}\text { Involvement in pregnancy prevention among unmarried males } 15-24 \\
\text { years-Family planning clinic visit for himself in last } 12 \text { months }\end{array}$ \\
\hline $9-6 c$ & $\begin{array}{l}\text { Involvement in pregnancy prevention among unmarried males 15-24 } \\
\text { years-Advice/counseling from a doctor on birth control in last } 12 \\
\text { months }\end{array}$ \\
\hline $9-7$ & Adolescent pregnancy (per 1,000 population, $15-17$ years) \\
\hline $9-8 \mathrm{a}$ & Abstinence before age 15 years-Females (15-19 years) \\
\hline $9-8 b$ & Abstinence before age 15 years-Males (15-19 years) \\
\hline $9-9 a$ & Abstinence among adolescents $15-17$ years-Females \\
\hline $9-9 b$ & Abstinence among adolescents $15-17$ years-Males \\
\hline
\end{tabular}

National Survey of Family Growth (NSFG), CDC, NCHS; National Vital Statistics System-Natality (NVSS-N), CDC, NCHS; Abortion Provider Survey, Guttmacher Institute; Abortion Surveillance Data, CDC, NCCDPHP.

National Survey of Family Growth (NSFG), CDC, NCHS.

National Survey of Family Growth (NSFG), CDC, NCHS.

National Survey of Family Growth (NSFG), CDC, NCHS; Abortion Patient Survey, Guttmacher Institute.

Guttmacher Institute.

National Survey of Family Growth (NSFG), CDC, NCHS.

National Survey of Family Growth (NSFG), CDC, NCHS.

National Survey of Family Growth (NSFG), CDC, NCHS.

National Survey of Family Growth (NSFG), CDC, NCHS; National Vital Statistics System-Natality (NVSS-N), CDC, NCHS; Abortion Provider Survey, Guttmacher Institute; Abortion Surveillance Data, CDC, NCCDPHP.

National Survey of Family Growth (NSFG), CDC, NCHS.

National Survey of Adolescent Males (NSAM), Urban Institute; National Survey of Family Growth (NSFG), CDC, NCHS.

National Survey of Family Growth (NSFG), CDC, NCHS.

National Survey of Adolescent Males (NSAM), Urban Institute; National Survey of Family Growth (NSFG), CDC, NCHS. 


\begin{tabular}{|c|c|c|}
\hline Objective & Description & Data Source or Objective Status \\
\hline $9-10 a$ & $\begin{array}{l}\text { Pregnancy prevention and STD protection in unmarried adolescents } \\
15-17 \text { years - Condom use (partner) at first intercourse, females }\end{array}$ & National Survey of Family Growth (NSFG), CDC, NCHS. \\
\hline $9-10 b$ & $\begin{array}{l}\text { Pregnancy prevention and STD protection in unmarried adolescents } \\
15-17 \text { years - Condom use at first intercourse, males }\end{array}$ & $\begin{array}{l}\text { National Survey of Adolescent Males (NSAM), Urban Institute; National } \\
\text { Survey of Family Growth (NSFG), CDC, NCHS. }\end{array}$ \\
\hline $9-10 c$ & $\begin{array}{l}\text { Pregnancy prevention and STD protection in unmarried adolescents } \\
15-17 \text { years-Condom use (partner) and hormonal method use at first } \\
\text { intercourse, females }\end{array}$ & National Survey of Family Growth (NSFG), CDC, NCHS. \\
\hline $9-10 d$ & $\begin{array}{l}\text { Pregnancy prevention and STD protection in unmarried adolescents } \\
15-17 \text { years-Condom use and hormonal method (partner) at first } \\
\text { intercourse, males }\end{array}$ & $\begin{array}{l}\text { National Survey of Adolescent Males (NSAM), Urban Institute; National } \\
\text { Survey of Family Growth (NSFG), CDC, NCHS. }\end{array}$ \\
\hline $9-10 \mathrm{e}$ & $\begin{array}{l}\text { Pregnancy prevention and STD protection in unmarried adolescents } \\
15-17 \text { years-Condom use (partner) at last intercourse, females }\end{array}$ & National Survey of Family Growth (NSFG), CDC, NCHS. \\
\hline $9-10 \mathrm{f}$ & $\begin{array}{l}\text { Pregnancy prevention and STD protection in unmarried adolescents } \\
15-17 \text { years-Condom use at last intercourse, males }\end{array}$ & $\begin{array}{l}\text { National Survey of Adolescent Males (NSAM), Urban Institute; National } \\
\text { Survey of Family Growth (NSFG), CDC, NCHS. }\end{array}$ \\
\hline $9-10 \mathrm{~g}$ & $\begin{array}{l}\text { Pregnancy prevention and STD protection in unmarried adolescents } \\
15-17 \text { years-Condom use (partner) and hormonal method at last } \\
\text { intercourse, females }\end{array}$ & National Survey of Family Growth (NSFG), CDC, NCHS. \\
\hline $9-10 \mathrm{~h}$ & $\begin{array}{l}\text { Pregnancy prevention and STD protection in unmarried adolescents } \\
15-17 \text { years-Condom use and hormonal method (partner) at last } \\
\text { intercourse, males }\end{array}$ & $\begin{array}{l}\text { National Survey of Adolescent Males (NSAM), Urban Institute; National } \\
\text { Survey of Family Growth (NSFG), CDC, NCHS. }\end{array}$ \\
\hline $9-11 a$ & $\begin{array}{l}\text { Reproductive health prevention education among young adults 15-19 } \\
\text { years-Formal education on abstinence, females }\end{array}$ & National Survey of Family Growth (NSFG), CDC, NCHS. \\
\hline $9-11 b$ & $\begin{array}{l}\text { Reproductive health prevention education among young adults 15-19 } \\
\text { years-Formal education on abstinence, males }\end{array}$ & National Survey of Family Growth (NSFG), CDC, NCHS. \\
\hline $9-11 c$ & $\begin{array}{l}\text { Reproductive health prevention education among young adults 15-19 } \\
\text { years-Formal education on birth control methods, females }\end{array}$ & National Survey of Family Growth (NSFG), CDC, NCHS. \\
\hline $9-11 d$ & $\begin{array}{l}\text { Reproductive health prevention education among young adults 15-19 } \\
\text { years-Formal education on birth control methods, males }\end{array}$ & National Survey of Family Growth (NSFG), CDC, NCHS. \\
\hline $9-11 \mathrm{e}$ & $\begin{array}{l}\text { Reproductive health prevention education among young adults 15-19 } \\
\text { years-Formal education on HIV/AIDS prevention, females }\end{array}$ & Developmental \\
\hline $9-11 f$ & $\begin{array}{l}\text { Reproductive health prevention education among young adults 15-19 } \\
\text { years-Formal education on HIV/AIDS prevention, males }\end{array}$ & Developmental \\
\hline
\end{tabular}




\begin{tabular}{|c|c|}
\hline Objective & Description \\
\hline $9-11 \mathrm{~g}$ & $\begin{array}{l}\text { Reproductive health prevention education among young adults 15-19 } \\
\text { years-Formal education on sexually transmitted diseases, females }\end{array}$ \\
\hline $9-11 \mathrm{~h}$ & $\begin{array}{l}\text { Reproductive health prevention education among young adults 15-19 } \\
\text { years-Formal education on sexually transmitted diseases, males }\end{array}$ \\
\hline $9-11 i$ & $\begin{array}{l}\text { Reproductive health prevention education among young adults 15-19 } \\
\text { years-Informal education on abstinence, females }\end{array}$ \\
\hline 9-11j & $\begin{array}{l}\text { Reproductive health prevention education among young adults 15-19 } \\
\text { years-Informal education on abstinence, males }\end{array}$ \\
\hline $9-11 \mathrm{k}$ & $\begin{array}{l}\text { Reproductive health prevention education among young adults 15-19 } \\
\text { years-Informal education on birth control methods, females }\end{array}$ \\
\hline 9-11l & $\begin{array}{l}\text { Reproductive health prevention education among young adults 15-19 } \\
\text { years-Informal education on birth control methods, males }\end{array}$ \\
\hline $9-11 \mathrm{~m}$ & $\begin{array}{l}\text { Reproductive health prevention education among young adults 15-19 } \\
\text { years-Informal education on HIV/AIDS prevention, females }\end{array}$ \\
\hline $9-11 n$ & $\begin{array}{l}\text { Reproductive health prevention education among young adults 15-19 } \\
\text { years-Informal education on HIV/AIDS prevention, males }\end{array}$ \\
\hline $9-110$ & $\begin{array}{l}\text { Reproductive health prevention education among young adults 15-19 } \\
\text { years-Informal education on sexually transmitted diseases, females }\end{array}$ \\
\hline $9-11 p$ & $\begin{array}{l}\text { Reproductive health prevention education among young adults 15-19 } \\
\text { years-Informal education on sexually transmitted diseases, males }\end{array}$ \\
\hline $9-12$ & $\begin{array}{l}\text { Problems in becoming pregnant and maintaining a pregnancy-Wives } \\
\text { of married couples (15-44 years) }\end{array}$ \\
\hline $9-13$ & Insurance coverage for contraceptive supplies and services \\
\hline
\end{tabular}

Developmental

Developmental

National Survey of Family Growth (NSFG), CDC, NCHS.

National Survey of Family Growth (NSFG), CDC, NCHS.

National Survey of Family Growth (NSFG), CDC, NCHS.

National Survey of Family Growth (NSFG), CDC, NCHS.

Developmental

Developmental

National Survey of Family Growth (NSFG), CDC, NCHS.

National Survey of Family Growth (NSFG), CDC, NCHS.

National Survey of Family Growth (NSFG), CDC, NCHS.

The Alan Guttmacher Institute. 
Figure 9-1. Progress Toward Target Attainment for Focus Area 9: Family Planning

Moved away

from target ${ }^{1}$
Moved toward target
Met or exceeded

target

9-1. Intended pregnancy (females 15-44 years)

9-2. Births occurring within 24 months of a previous birth (females 15-44 years)

9-3. Contraceptive use-Females at risk of unintended pregnancy (15-44 years)

9-4. Contraceptive failure within 12 months of continuous use-Females experiencing pregnancy (15-44 years)

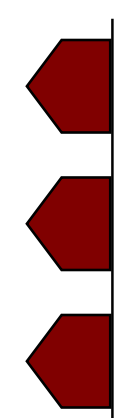

9-5. Emergency contraception provided by family planning agencies

9-6. Involvement in pregnancy prevention among unmarried males 15-24 years

a. Family planning clinic visit with female partner in last 12 months

b. Family planning clinic visit for himself in last 12 months

c. Advice/counseling from a doctor on birth control in last 12 months

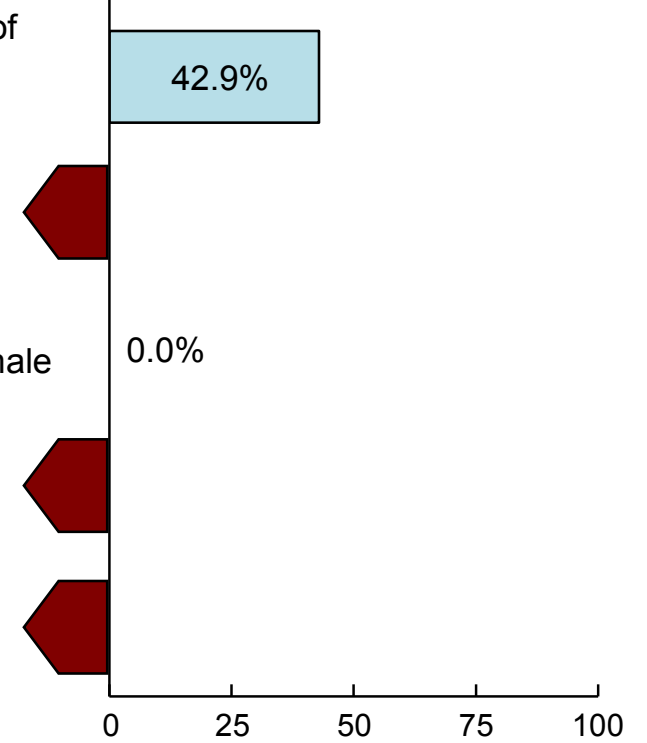

\begin{tabular}{|c|c|c|c|c|c|}
\hline \multirow{2}{*}{$\begin{array}{l}2010 \\
\text { Target }\end{array}$} & \multirow{2}{*}{$\begin{array}{c}\text { Baseline } \\
\text { (Year) }\end{array}$} & \multirow{2}{*}{$\begin{array}{l}\text { Final } \\
\text { (Year) }\end{array}$} & \multicolumn{3}{|c|}{ Baseline vs. Final } \\
\hline & & & Difference ${ }^{2}$ & $\begin{array}{l}\text { Statistically } \\
\text { Significant }^{3}\end{array}$ & $\begin{array}{l}\text { Percent } \\
\text { Change }^{4}\end{array}$ \\
\hline $70 \%$ & $\begin{array}{c}52 \% \\
(1995)\end{array}$ & $\begin{array}{l}51 \% \\
2002\end{array}$ & -1 & $\begin{array}{c}\text { Not } \\
\text { tested }\end{array}$ & $-1.9 \%$ \\
\hline $6 \%$ & $\begin{array}{c}11 \% \\
(1995)\end{array}$ & $\begin{array}{c}16 \% \\
(2006-08)\end{array}$ & 5 & $\begin{array}{c}\text { Not } \\
\text { tested }\end{array}$ & $45.5 \%$ \\
\hline $100 \%$ & $\begin{array}{c}93 \% \\
(1995)\end{array}$ & $\begin{array}{c}89 \% \\
(2006-08)\end{array}$ & -4 & Yes & $-4.3 \%$ \\
\hline $8 \%$ & $\begin{array}{c}15 \% \\
(1995)\end{array}$ & $\begin{array}{c}12 \% \\
(2002)\end{array}$ & -3 & $\begin{array}{l}\text { Not } \\
\text { tested }\end{array}$ & $-20 \%$ \\
\hline $90 \%$ & $\begin{array}{c}80 \% \\
(1999)\end{array}$ & $\begin{array}{c}79 \% \\
(2003)\end{array}$ & -1 & $\begin{array}{c}\text { Not } \\
\text { tested }\end{array}$ & $-1.3 \%$ \\
\hline $22 \%$ & $\begin{array}{c}21 \% \\
(2002)\end{array}$ & $\begin{array}{c}21 \% \\
(2006-08)\end{array}$ & 0 & No & $0.0 \%$ \\
\hline $37 \%$ & $\begin{array}{c}31 \% \\
(2002)\end{array}$ & $\begin{array}{c}25 \% \\
(2006-08)\end{array}$ & -6 & Yes & $-19.4 \%$ \\
\hline $37 \%$ & $\begin{array}{c}21 \% \\
(2002)\end{array}$ & $\begin{array}{c}20 \% \\
(2006-08)\end{array}$ & -1 & No & $-4.8 \%$ \\
\hline
\end{tabular}

Percent of targeted change achieved ${ }^{5}$

(continued) 
Figure 9-1. Progress Toward Target Attainment for Focus Area 9: Family Planning (continued)

Moved away

from target ${ }^{1}$

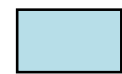

Moved toward

target

Met or exceeded

target

9-7. Adolescent pregnancy (per 1,000 population 15-17 years)

9-8. Abstinence before age 15 years

a. Females (15-19 years)

b. Males (15-19 years)

9-9. Abstinence among adolescents 15-17 years

a. Females

b. Males

9-10. Pregnancy prevention and STD protection in unmarried adolescents 15-17 years

a. Condom use (partner) at first intercourse, females

b. Condom use at first intercourse, males

c. Condom use (partner) and hormonal method use at first intercourse, females

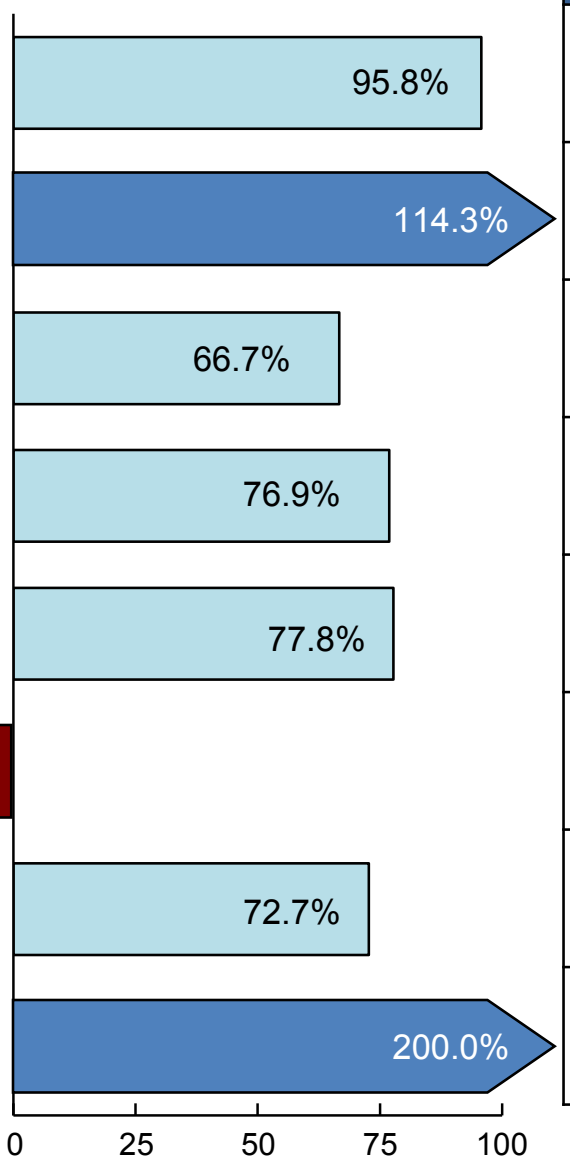

Percent of targeted change achieved $^{5}$

\begin{tabular}{|c|c|c|c|c|c|}
\hline \multirow{2}{*}{$\begin{array}{c}2010 \\
\text { Target }\end{array}$} & \multirow{2}{*}{$\begin{array}{c}\text { Baseline } \\
\text { (Year) }\end{array}$} & \multirow{2}{*}{$\begin{array}{l}\text { Final } \\
\text { (Year) }\end{array}$} & \multicolumn{3}{|c|}{ Baseline vs. Final } \\
\hline & & & Difference $^{2}$ & $\begin{array}{l}\text { Statistically } \\
\text { Significant }^{3}\end{array}$ & $\begin{array}{l}\text { Percent } \\
\text { Change }\end{array}$ \\
\hline 39 & $\begin{array}{c}63 \\
(1996)\end{array}$ & $\begin{array}{c}40 \\
(2005)\end{array}$ & -23 & $\begin{array}{c}\text { Not } \\
\text { tested }\end{array}$ & $-36.5 \%$ \\
\hline $88 \%$ & $\begin{array}{c}81 \% \\
(1995)\end{array}$ & $\begin{array}{c}89 \% \\
(2006-08)\end{array}$ & 8 & $\begin{array}{l}\text { Not } \\
\text { tested }\end{array}$ & $9.9 \%$ \\
\hline $88 \%$ & $\begin{array}{c}79 \% \\
(1995)\end{array}$ & $\begin{array}{c}85 \% \\
(2006-08)\end{array}$ & 6 & $\begin{array}{c}\text { Not } \\
\text { tested }\end{array}$ & $7.6 \%$ \\
\hline $75 \%$ & $\begin{array}{c}62 \% \\
(1995)\end{array}$ & $\begin{array}{c}72 \% \\
(2006-08)\end{array}$ & 10 & Yes & $16.1 \%$ \\
\hline $75 \%$ & $\begin{array}{c}57 \% \\
(1995)\end{array}$ & $\begin{array}{c}71 \% \\
(2006-08)\end{array}$ & 14 & $\begin{array}{c}\text { Not } \\
\text { tested }\end{array}$ & $24.6 \%$ \\
\hline $75 \%$ & $\begin{array}{c}69 \% \\
(1995)\end{array}$ & $\begin{array}{c}62 \% \\
(2006-08)\end{array}$ & -7 & No & $-10.1 \%$ \\
\hline $83 \%$ & $\begin{array}{c}72 \% \\
(1995)\end{array}$ & $\begin{array}{c}80 \% \\
(2006-08)\end{array}$ & 8 & $\begin{array}{c}\text { Not } \\
\text { tested }\end{array}$ & $11.1 \%$ \\
\hline $9 \%$ & $\begin{array}{c}7 \% \\
(1995)\end{array}$ & $\begin{array}{c}11 \% \\
(2006-08)\end{array}$ & 4 & No & $57.1 \%$ \\
\hline
\end{tabular}

(continued) 
Figure 9-1. Progress Toward Target Attainment for Focus Area 9: Family Planning (continued)

Moved away

from target ${ }^{1}$
Moved toward target
Met or exceeded

target

9-10. Pregnancy prevention and STD protection in unmarried adolescents $15-17$ years

d. Condom use and hormonal method (partner) at first intercourse, males

e. Condom use (partner) at last intercourse, females

f. Condom use at last intercourse, males

g. Condom use (partner) and hormonal method at last intercourse, females

h. Condom use and hormonal method (partner) at last intercourse, males

9-11. Reproductive health prevention education among young adults $15-19$ years

a. Formal education on abstinence, females

b. Formal education on abstinence, males

c. Formal education on birth control methods, females

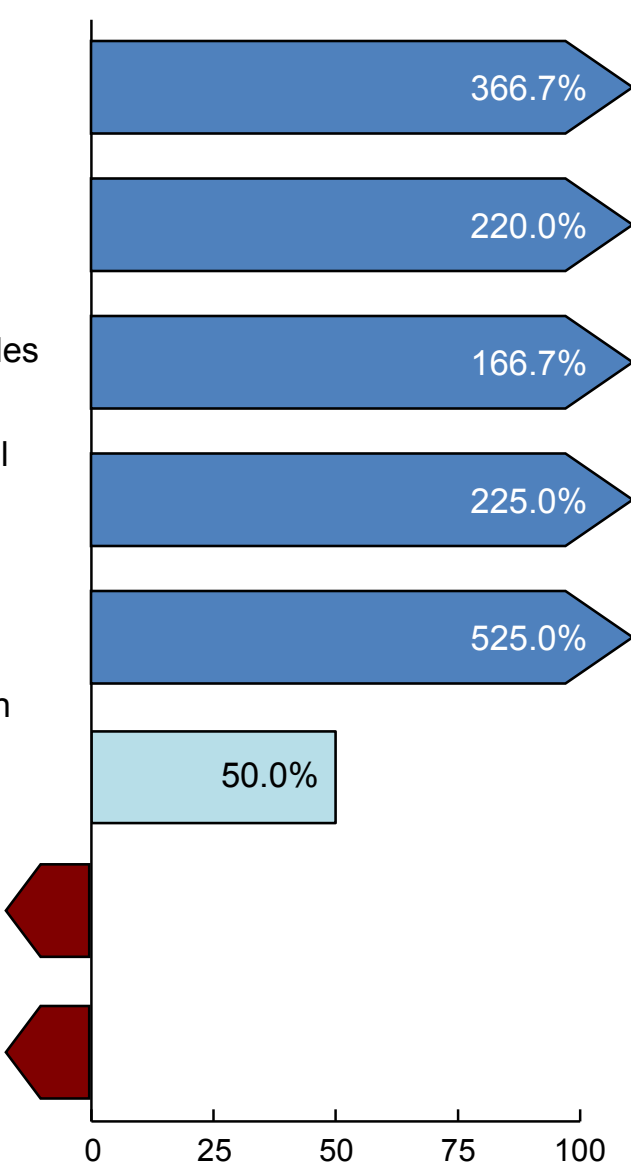

\begin{tabular}{|c|c|c|c|c|c|}
\hline \multirow{2}{*}{$\begin{array}{l}2010 \\
\text { Target }\end{array}$} & \multirow{2}{*}{$\begin{array}{c}\text { Baseline } \\
\text { (Year) }\end{array}$} & \multirow{2}{*}{$\begin{array}{l}\text { Final } \\
\text { (Year) }\end{array}$} & \multicolumn{3}{|c|}{ Baseline vs. Final } \\
\hline & & & Difference & $\begin{array}{l}\text { Statistically } \\
\text { Significant }^{3}\end{array}$ & $\begin{array}{l}\text { Percent } \\
\text { Change }^{4}\end{array}$ \\
\hline $11 \%$ & $\begin{array}{c}8 \% \\
(1995)\end{array}$ & $\begin{array}{c}19 \% \\
(2006-08)\end{array}$ & 11 & $\begin{array}{c}\text { Not } \\
\text { tested }\end{array}$ & $137.5 \%$ \\
\hline $49 \%$ & $\begin{array}{c}39 \% \\
(1995)\end{array}$ & $\begin{array}{c}61 \% \\
(2006-08)\end{array}$ & 22 & Yes & $56.4 \%$ \\
\hline $79 \%$ & $\begin{array}{c}70 \% \\
(1995)\end{array}$ & $\begin{array}{c}85 \% \\
(2006-08)\end{array}$ & 15 & $\begin{array}{c}\text { Not } \\
\text { tested }\end{array}$ & $21.4 \%$ \\
\hline $11 \%$ & $\begin{array}{c}7 \% \\
(1995)\end{array}$ & $\begin{array}{c}16 \% \\
(2006-08)\end{array}$ & 9 & Yes & $128.6 \%$ \\
\hline $20 \%$ & $\begin{array}{c}16 \% \\
(1995)\end{array}$ & $\begin{array}{c}37 \% \\
(2006-08)\end{array}$ & 21 & $\begin{array}{c}\text { Not } \\
\text { tested }\end{array}$ & $131.3 \%$ \\
\hline $88 \%$ & $\begin{array}{c}86 \% \\
(2002)\end{array}$ & $\begin{array}{c}87 \% \\
(2006-08)\end{array}$ & 1 & No & $1.2 \%$ \\
\hline $85 \%$ & $\begin{array}{c}83 \% \\
(2002)\end{array}$ & $\begin{array}{c}81 \% \\
(2006-08)\end{array}$ & -2 & No & $-2.4 \%$ \\
\hline $73 \%$ & $\begin{array}{c}70 \% \\
(2002)\end{array}$ & $\begin{array}{c}69 \% \\
(2006-08)\end{array}$ & -1 & No & $-1.4 \%$ \\
\hline
\end{tabular}

Percent of targeted change achieved ${ }^{5}$ 
Figure 9-1. Progress Toward Target Attainment for Focus Area 9: Family Planning (continued)
Moved away
from target ${ }^{1}$
Moved toward
target
Met or exceeded
target

9-11. Reproductive health prevention education among young adults 15-19 years

d. Formal education on birth control methods, males

i. Informal education on abstinence, females

j. Informal education on abstinence, males

k. Informal education on birth control methods, females

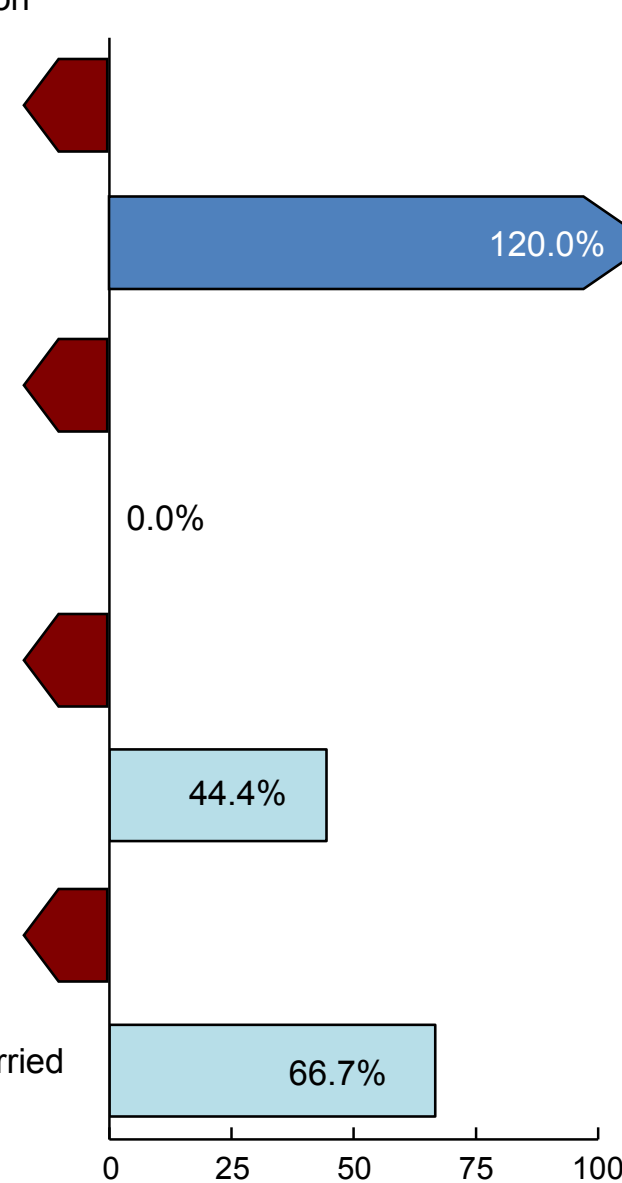

\begin{tabular}{|c|c|c|c|c|c|}
\hline \multirow{2}{*}{$\begin{array}{l}2010 \\
\text { Target }\end{array}$} & \multirow{2}{*}{$\begin{array}{c}\text { Baseline } \\
\text { (Year) }\end{array}$} & \multirow{2}{*}{$\begin{array}{l}\text { Final } \\
\text { (Year) }\end{array}$} & \multicolumn{3}{|c|}{ Baseline vs. Final } \\
\hline & & & Difference $^{2}$ & $\begin{array}{l}\text { Statistically } \\
\text { Significant }^{3}\end{array}$ & $\begin{array}{l}\text { Percent } \\
\text { Change }^{4}\end{array}$ \\
\hline $70 \%$ & $\begin{array}{c}66 \% \\
(2002)\end{array}$ & $\begin{array}{c}62 \% \\
(2006-08)\end{array}$ & -4 & No & $-6.1 \%$ \\
\hline $62 \%$ & $\begin{array}{c}57 \% \\
(2002)\end{array}$ & $\begin{array}{c}63 \% \\
(2006-08)\end{array}$ & 6 & Yes & $10.5 \%$ \\
\hline $49 \%$ & $\begin{array}{c}45 \% \\
(2002)\end{array}$ & $\begin{array}{c}42 \% \\
(2006-08)\end{array}$ & -3 & No & $-6.7 \%$ \\
\hline $57 \%$ & $\begin{array}{c}51 \% \\
(2002)\end{array}$ & $\begin{array}{c}51 \% \\
(2006-08)\end{array}$ & 0 & No & $0.0 \%$ \\
\hline $38 \%$ & $\begin{array}{c}33 \% \\
(2002)\end{array}$ & $\begin{array}{c}31 \% \\
(2006-08)\end{array}$ & -2 & No & $-6.1 \%$ \\
\hline $60 \%$ & $\begin{array}{c}51 \% \\
(2002)\end{array}$ & $\begin{array}{c}55 \% \\
(2006-08)\end{array}$ & 4 & No & $7.8 \%$ \\
\hline $57 \%$ & $\begin{array}{c}52 \% \\
(2002)\end{array}$ & $\begin{array}{c}49 \% \\
(2006-08)\end{array}$ & -3 & No & $-5.8 \%$ \\
\hline $10 \%$ & $\begin{array}{c}13 \% \\
(1995)\end{array}$ & $\begin{array}{c}11 \% \\
(2006-08)\end{array}$ & -2 & $\begin{array}{l}\text { Not } \\
\text { tested }\end{array}$ & $-15.4 \%$ \\
\hline
\end{tabular}

Percent of targeted change achieved ${ }^{5}$

(continued) 


\section{Figure 9-1. Progress Toward Target Attainment for Focus Area 9: Family Planning (continued)}

\section{NOTES}

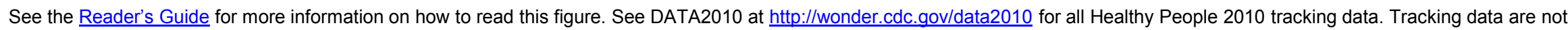
available for objectives 9-11e through $\mathrm{h}, 9-11 \mathrm{~m}, 9-11 \mathrm{n}$, and 9-13.

\section{FOOTNOTES}

${ }^{1}$ Movement away from target is not quantified using the percent of targeted change achieved. See Technical Appendix for more information.

2 Difference $=$ Final value - Baseline value. Differences between percents (\%) are measured in percentage points.

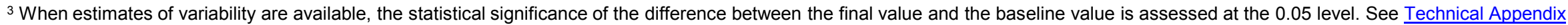
for more information.

${ }^{4}$ Percent change $=\frac{\text { Final value }- \text { Baseline value }}{\text { Baseline value }} \times 100$.

\footnotetext{
${ }^{5}$ Percent of targeted change achieved $=\frac{\text { Final value }- \text { Baseline value }}{\text { Heathy }}$

Healthy People 2010 target - Baseline value

DATA SOURCES
}

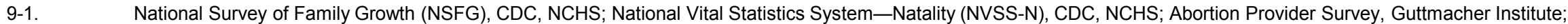
Abortion Surveillance Data, CDC, NCCDPHP.

9-2-9-3. National Survey of Family Growth (NSFG), CDC, NCHS

$9-4$.

$9-5$.

$9-6 a-c$

$\begin{array}{ll}\text { 9-7. } & \text { National Survey of Family Growth (NSFG), CDC, NCHS; } \\ \text { 9-8a. } & \text { National Survey of Family Growth (NSFG), CDC, NCHS. }\end{array}$

National Survey of Family Growth (NSFG), CDC, NCHS; Abortion Patient Survey, Guttmacher Institute.

Guttmacher Institute.

National Survey of Family Growth (NSFG), CDC, NCHS.

9-8b. National Survey of Adolescent Males (NSAM), Urban Institute; National Survey of Family Growth (NSFG), CDC, NCHS.

9-9a. National Survey of Family Growth (NSFG), CDC, NCHS.

9-9b. National Survey of Adolescent Males (NSAM), Urban Institute; National Survey of Family Growth (NSFG), CDC, NCHS.

9-10a. National Survey of Family Growth (NSFG), CDC, NCHS.

9-10b. National Survey of Adolescent Males (NSAM), Urban Institute; National Survey of Family Growth (NSFG), CDC, NCHS.

9-10c. National Survey of Family Growth (NSFG), CDC, NCHS.

9-10d. National Survey of Adolescent Males (NSAM), Urban Institute; National Survey of Family Growth (NSFG), CDC, NCHS.

9-10e. National Survey of Family Growth (NSFG), CDC, NCHS

9-10f. National Survey of Adolescent Males (NSAM), Urban Institute; National Survey of Family Growth (NSFG), CDC, NCHS.

9-10g. National Survey of Family Growth (NSFG), CDC, NCHS.

9-10h. National Survey of Adolescent Males (NSAM), Urban Institute; National Survey of Family Growth (NSFG), CDC, NCHS.

9-11a-d. National Survey of Family Growth (NSFG), CDC, NCHS.

9-11i-I. National Survey of Family Growth (NSFG), CDC, NCHS

9-110-p. National Survey of Family Growth (NSFG), CDC, NCHS.

9-12. National Survey of Family Growth (NSFG), CDC, NCHS. 
Figure 9-2. Health Disparities Table for Focus Area 9: Family Planning

Disparities from the best group rate for each characteristic at the most recent data point and changes in disparity from the baseline to the most recent data point.

Population-based objectives

9-1. Intended pregnancy (females $15-44$ years) $(1995,2002) \dagger$

9-2. Births occurring within 24 months of a previous birth (females 15-44 years) $(1995,2006-08)^{1} \ddagger$

9-3. Contraceptive use-Females at risk of unintended pregnancy $\left(15-44\right.$ years) $(1995,2006-08)^{1 *}$

9-4. Contraceptive failure within 12 months of continuous use-Females experiencing pregnancy $(15-44$ years $)(1995,2002) \dagger$

9-6a. Involvement in pregnancy prevention among unmarried males 15-24 years-Family planning clinic visit with female partner in last 12 months $(2002,2006-08)^{*}$

b. Involvement in pregnancy prevention among unmarried males 15-24 years-Family planning clinic visit for himself in last 12 months $(2002,2006-08)^{*}$

c. Involvement in pregnancy prevention among unmarried males 15-24 years-Advice/counseling from a doctor on birth control in last 12 months $(2002,2006-08)^{*}$

9-7. Adolescent pregnancy (per 1,000 population, 15-17 years) (1996, 2005)†

9-8a. Abstinence before age 15 years-Females (15-19 years) (1995, 2006-08) $)^{1} \ddagger$

b. Abstinence before age 15 years-Males (15-19 years) (1995, 2006-08) $)^{1,2} \ddagger$

9-9a. Abstinence among adolescents 15-17 years-Females (1995, $2006-08)^{1 *}$

b. Abstinence among adolescents 15-17 years-Males (1995, $2006-08)^{1,2} \ddagger$

9-10a. Pregnancy prevention and STD protection in unmarried adolescents 15-17 years-Condom use (partner) at first intercourse, females $(1995,2006-08)^{1 *}$

b. Pregnancy prevention and STD protection in unmarried adolescents 15-17 years-Condom use at first intercourse, males $(1995,2006-08)^{1,2} \ddagger$

c. Pregnancy prevention and STD protection in unmarried adolescents 15-17 years-Condom use (partner) and hormonal method use at first intercourse, females $(1995,2006-08)^{1 *}$

d. Pregnancy prevention and STD protection in unmarried adolescents 15-17 years-Condom use and hormonal method (partner) at first intercourse, males $(1995,2006-08)^{1,2} \ddagger$

e. Pregnancy prevention and STD protection in unmarried adolescents 15-17 years-Condom use (partner) at last intercourse, females $(1995,2006-08)^{1 *}$

f. Pregnancy prevention and STD protection in unmarried adolescents 15-17 years-Condom use at last intercourse, males $(1995,2006-08)^{1,2} \ddagger$

g. Pregnancy prevention and STD protection in unmarried adolescents 15-17 years-Condom use (partner) and hormonal method at last intercourse, females $(1995,2006-08)^{1 *}$

h. Pregnancy prevention and STD protection in unmarried adolescents 15-17 years-Condom use and hormonal method (partner) at last intercourse, males $(1995,2006-08)^{1,2} \ddagger$
Characteristics and Groups

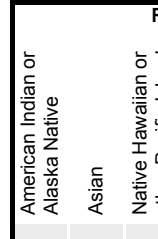

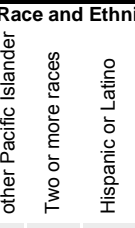

\section{icity}

告

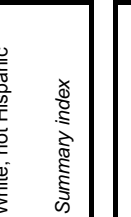

Disability

亏ั

B

$B^{\text {ii }}$

B

B

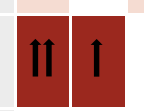

B

$B^{i i}$

$B^{i i}$

$B^{\mathrm{ii}}$
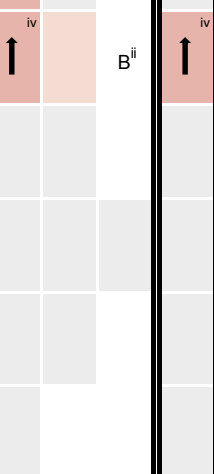


\section{Population-based objectives}

9-11a. Reproductive health prevention education among young adults 15-19 years-Formal education on abstinence, females (2002, 2006-08)*

b. Reproductive health prevention education among young adults 15-19 years-Formal education on abstinence, males (2002, 2006-08)*

c. Reproductive health prevention education among young adults 15-19 years -Formal education on birth control methods, females $(2002,2006-08)^{*}$

d. Reproductive health prevention education among young adults 15-19 years-Formal education on birth control methods, males $(2002,2006-08)^{*}$

9-11i. Reproductive health prevention education among young adults 15-19 years-Informal education on abstinence, females (2002, 2006-08)*

j. Reproductive health prevention education among young adults 15-19 years-Informal education on abstinence, males (2002, 2006-08)*

k. Reproductive health prevention education among young adults 15-19 years-Informal education on birth control methods, females $(2002,2006-08)^{*}$

I. Reproductive health prevention education among young adults 15-19 years-Informal education on birth control methods, males $(2002,2006-08)^{*}$

9-110. Reproductive health prevention education among young adults 15-19 years-Informal education on sexually transmitted diseases, females $(2002,2006-08)^{*}$

p. Reproductive health prevention education among young adults 15-19 years-Informal education on sexually transmitted diseases, males (2002, 2006-08)*

9-12. Problems in becoming pregnant and maintaining a pregnancy-Wives of married couples (15-44 years) (1995, 2006-08) $)^{1} \ddagger$
Characteristics and Groups

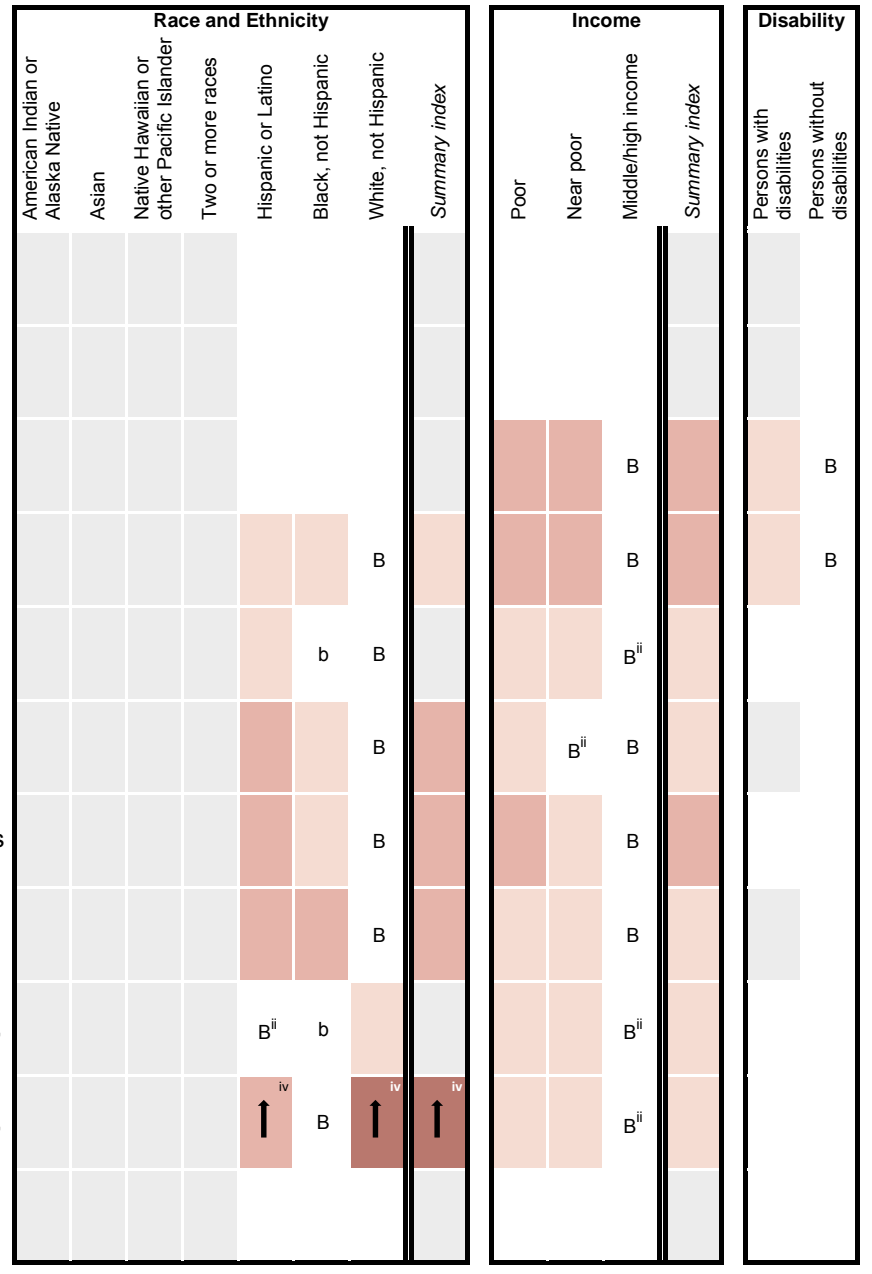

(continued) 


\section{Figure 9-2. Health Disparities Table for Focus Area 9: Family Planning (continued)}

NOTES

See DATA2010 at http://wonder.cdc.gov/data2010 for all Healthy People 2010 tracking data. Disparity data are either unavailable or not applicable for objectives 9-5, 9-11e through $\mathrm{h}, 9-11 \mathrm{~m}$ and $\mathrm{n}$, and 9-13.

Years in parentheses represent the baseline and most recent data years (if available).

Disparity from the best group rate is defined as the percent difference between the best group rate and each of the other group rates for a characteristic (e.g., race and ethnicity). The summary index is the average of these percent differences for a characteristic. Change in disparity is estimated by subtracting the disparity at baseline from the disparity at the most recent data point. Change in the summary index is estimated by subtracting the summary index at baseline from the summary index at the most recent data point. See Technical Appendix for more information.

\section{LEGEND}

\begin{tabular}{|c|c|c|c|c|c|}
\hline $\begin{array}{l}\text { The "best" group rate at the most recent } \\
\text { data point. }\end{array}$ & B & $\begin{array}{l}\text { The group with the best rate for } \\
\text { specified characteristic. }\end{array}$ & $b$ & $\begin{array}{l}\text { Most favorable group rate for specified } \\
\text { characteristic, but reliability criterion not } \\
\text { met. }\end{array}$ & $\begin{array}{l}\text { Reliability criterion for best } \\
\text { group rate not met, or data } \\
\text { available for only one group. }\end{array}$ \\
\hline
\end{tabular}

\begin{tabular}{|c|c|c|c|c|}
\hline \multirow[b]{2}{*}{$\begin{array}{l}\text { Disparity from the best group rate at the } \\
\text { most recent data point. }\end{array}$} & \multicolumn{4}{|c|}{ Percent difference from the best group rate } \\
\hline & $\begin{array}{l}\text { Less than } 10 \% \text {, or difference not } \\
\text { statistically significant (when } \\
\text { estimates of variability are available). }\end{array}$ & $10 \%-49 \%$ & $50 \%-99 \%$ & $100 \%$ or more \\
\hline
\end{tabular}

Changes in disparity over time are shown when:
(a) disparities data are available at both baseline and most recent time points;
(b) data are not for the group(s) indicated by "B" or "b" at either time point; and
(c) the change is greater than or equal to 10 percentage points and statistically
significant, or when the change is greater than or equal to 10 percentage
points and estimates of variability were not available. See Technical Appendix.

\section{FOOTNOTES}

* Measures of variability were available. Thus, the variability of best group rates was assessed, and statistical significance was tested. Disparities of $10 \%$ or more are displayed when the differences from the best group rate are statistically significant at the 0.05 level. Changes in disparities over time are indicated by arrows when the changes are greater than or equal to 10 percentage points and are statistically significant at the 0.05 level. See Technical Appendix.

$\dagger$ Measures of variability were not available. Thus, the variability of best group rates was not assessed, and statistical significance could not be tested. Nonetheless, disparities and changes in disparities over time are displayed according to their magnitude. See Technical Appendix.

¥ Measures of variability were available only for the most recent data. Thus, the variability of best group rates was assessed only for the most recent data, and statistical significance was tested only for the most recent data. Disparities of $10 \%$ or more are displayed when the differences from the best group rate are statistically significant at the 0.05 level. Changes in disparities over time are displayed according to their magnitude, since measures of variability were not available at baseline and therefore statistical significance of changes in disparity could not be tested. See Technical Appendix.

1 Baseline data by disability status are for 2002. Measures of variability were available for disability, see footnote * above.

2 Baseline data by income are for 2002. Measures of variability were available for income, see footnote * above.

i Data include persons of Hispanic origin.

ii The group with the best rate at the most recent data point is different from the group with the best rate at baseline. Both rates met the reliability criterion. See Technical Appendix.

iii Reliability criterion for best group rate not met, or data available for only one group, at baseline. Change in disparity cannot be assessed. See Technical Appendix

iv At baseline, persons reported only one race or reported more than one race and identified one primary race. Therefore, disparities at the most recent and the baseline data points may not be directly comparable.

$\checkmark$ Change in the summary index cannot be assessed. See Technical Appendix.

\section{DATA SOURCES}

9-1. National Survey of Family Growth (NSFG), CDC, NCHS; National Vital Statistics System—Natality (NVSS-N), CDC, NCHS; Abortion Provider Survey, Guttmacher Institute; Abortion Surveillance Data, CDC, NCCDPHP.

9-2-9-3. National Survey of Family Growth (NSFG), CDC, NCHS.

9-4. National Survey of Family Growth (NSFG), CDC, NCHS; Abortion Patient Survey, Guttmacher Institute.

9-6a-c. National Survey of Family Growth (NSFG), CDC, NCHS.

9-7. National Survey of Family Growth (NSFG), CDC, NCHS; National Vital Statistics System—Natality (NVSS-N), CDC, NCHS; Abortion Provider Survey, Guttmacher Institute; Abortion Surveillance Data, CDC, NCCDPHP.

9-8a. National Survey of Family Growth (NSFG), CDC, NCHS.

9-8b. National Survey of Adolescent Males (NSAM), Urban Institute; National Survey of Family Growth (NSFG), CDC, NCHS.

9-9a. National Survey of Family Growth (NSFG), CDC, NCHS.

9-9b. National Survey of Adolescent Males (NSAM), Urban Institute; National Survey of Family Growth (NSFG), CDC, NCHS.

9-10a. National Survey of Family Growth (NSFG), CDC, NCHS.

9-10b. National Survey of Adolescent Males (NSAM), Urban Institute; National Survey of Family Growth (NSFG), CDC, NCHS.

9-10c. National Survey of Family Growth (NSFG), CDC, NCHS.

9-10d. National Survey of Adolescent Males (NSAM), Urban Institute; National Survey of Family Growth (NSFG), CDC, NCHS

9-10e. National Survey of Family Growth (NSFG), CDC, NCHS.

9-10f. National Survey of Adolescent Males (NSAM), Urban Institute; National Survey of Family Growth (NSFG), CDC, NCHS.

9-10g. National Survey of Family Growth (NSFG), CDC, NCHS.

9-10h. National Survey of Adolescent Males (NSAM), Urban Institute; National Survey of Family Growth (NSFG), CDC, NCHS.

9-11a-d. National Survey of Family Growth (NSFG), CDC, NCHS.

9-11i-I. National Survey of Family Growth (NSFG), CDC, NCHS.

9-110-p. National Survey of Family Growth (NSFG), CDC, NCHS.

9-12. National Survey of Family Growth (NSFG), CDC, NCHS. 


\section{Final Review}

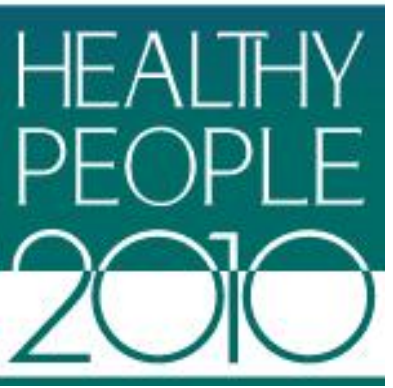

\section{Food Safety}

10

\section{Co-Lead Agencies}

Food and Drug Administration

Food Safety and Inspection Service, U.S. Department of Agriculture

\section{Contents}

Goal 10-2

Highlights. 10-2

Summary of Progress. 10-3

Transition to Healthy People 2020 10-4

Data Considerations 10-5

Notes 10-6

Comprehensive Summary of Objectives $10-8$

Progress Chart $10-10$

Health Disparities Table 10-14 


\section{Goal: Reduce foodborne illnesses}

This chapter addresses the rate of disease caused by microorganisms transmitted commonly by food, such as Salmonella and Campylobacter. Specific objectives monitor new cases of infections caused by important foodborne pathogens as well as the food-safety practices of consumers and of retail food establishments.

All Healthy People tracking data quoted in this chapter, along with technical information and operational definitions for each objective, can be found in the Healthy People 2010 database, DATA2010, available from http://wonder.cdc.gov/data2010/.

More information about this Focus Area can be found in the following publications:

- Healthy People 2010: Understanding and Improving Health, available from http://www.healthypeople.gov/2010/Document/tableofcontents.htm\#under.

- Healthy People 2010 Midcourse Review, available from http://www.healthypeople.gov/2010/data/midcourse/html/default.htm\#FocusAreas.

\section{Highlights}

- Substantial progress was achieved in objectives for this Focus Area during the past decade [1]. Almost three-quarters (73\%) of the Food Safety Focus Area objectives moved toward or achieved their Healthy People 2010 targets (Figure 10-1). With the exception of one objective (10-5), data on health disparities by race and ethnicity were unavailable [2]. However, most objectives exhibited heath disparities of $10 \%$ or more by sex (Figure 10-2).

- Statistically significant downward trends were observed in the rates of foodborne infections from Campylobacter species and Shiga toxin-producing Escherichia coli 0157 (STEC 0157) (objectives 10-1a and b) [3]. Between 1997 and 2009, rate of Campylobacter species infections decreased by $47.6 \%$, from 24.6 to 12.9 per 100,000 population, moving toward the Healthy People 2010 target of 12.3 per 100,000; the rate of STEC 0157 infections decreased by $52.4 \%$, from 2.1 to 1.0 per 100,000 population, meeting the 2010 target of 1.0 per 100,000. In addition, the rate of Listeria monocytogenes infections (objective 10-1c) declined by $36.2 \%$ between 1997 and 2009 , from 0.47 to 0.30 , moving toward the 2010 target of 0.24 per 100,000 population.

- The proportion of non-Typhi Salmonella from humans (percent of isolates) resistant to gentamicin (objective 10-3c) and ampicillin (objective 10-3d) declined. Gentamicinresistant isolates declined by $51.7 \%$ between 1997 and 2008 , from $2.9 \%$ to $1.4 \%$, exceeding the 2010 target of $2.9 \%$. Ampicillin-resistant isolates declined by $49.2 \%$ between 1997 and 2008 , from $18.3 \%$ to $9.3 \%$, exceeding the 2010 target of $18.3 \%$.

- Progress in outbreaks of foodborne infections was mixed. Outbreaks due to Salmonella serotype Enteritidis (objective 10-2b) decreased by 44.9\% between 1997 and 2008, from 49 to 27 , achieving $88.0 \%$ of the 2010 target of 24 outbreaks. However, outbreaks due to Escherichia coli 0157:H7 (objective 10-2a) increased between 1997 and 2008, from 10 to 32 outbreaks, moving away from the 2010 target of five outbreaks. 
- Consumer food safety practices (objective 10-5) improved by 4.2\% between 1998 and 2006 , from $72 \%$ to $75 \%$, moving toward the 2010 target of $79 \%$ of the population following safe food practices.

- Food safety practices in retail establishments (objectives 10-6a through i) increased in all nine categories, moving toward or exceeding the 2010 targets.

- Safe retail food preparation in meat and poultry departments (objective 10-6g) increased by $8.6 \%$ between 1998 and 2008, from $81 \%$ to $88 \%$, exceeding the 2010 target of $86 \%$.

- Safe retail food preparation in produce departments (objective 10-6h) increased by $10.5 \%$ between 1998 and 2008, from $76 \%$ to $84 \%$, exceeding the 2010 target of $82 \%$.

\section{Summary of Progress}

- Figure 10-1 presents a quantitative assessment of progress in achieving the Healthy People 2010 objectives for Food Safety [1]. Data to measure progress toward target attainment were available for 22 objectives. Of these:

- Five objectives met or exceeded their Healthy People 2010 targets (objectives 10$1 b, 10-3 c$ and $d$, and 10-6f and g).

- Eleven objectives moved toward their targets. A statistically significant difference between the baseline and the final data points was observed for one of these objectives (10-5, consumer food safety practices). No significant differences were observed for seven objectives (10-6a through $\mathrm{f}$, and i); and data to test the significance of the difference were unavailable for three objectives $(10-1 \mathrm{a}$ and $\mathrm{c}$, and 10-2b).

- Six objectives moved away from their targets. No statistically significant differences between the baseline and final data points were observed for one objective (10-4b). Data to test the significance of the difference were unavailable for five objectives (10-1d and f, 10-2a, and 10-3a and b).

- Sixteen objectives were dropped during the decade (objectives 10-1e and g, 10-3e through p, 10-4a, and 10-7) [4].

- Figure 10-2 displays health disparities in the Food Safety Focus Area objectives from the best group rate for each characteristic at the most recent data point [2]. It also displays changes in disparities from baseline to the most recent data point [5].

- The white non-Hispanic population had the best group rate for one objective with statistically significant racial and ethnic disparities of $10 \%$ or more (objective 10-5).

- One objective had statistically significant health disparities by sex of $10 \%$ or more (objective 10-5). Four additional objectives had health disparities by sex of $10 \%$ or more but lacked data to measure variability (objectives 10-1a through c, and f). Males were the better group for three of these five objectives (10-1b, c, and f). Females were the better group for two objectives (10-1a and 10-5). 


\section{Transition to Healthy People 2020}

The Healthy People 2020 Food Safety Topic Area has fewer objectives than those included in Healthy People 2010. See HealthyPeople.gov for a complete list of Healthy People 2020 topics and objectives.

The Healthy People 2020 objectives can be grouped into several sections:

- Food-related infections

- Antimicrobial resistance

- Consumer food safety practices

- Retail food safety practices.

The differences between the Healthy People 2010 and Healthy People 2020 objectives are summarized below:

- The Healthy People 2020 Food Safety Topic Area has 28 objectives, whereas the Healthy People 2010 Focus Area had 38 objectives.

- Eight Healthy People 2010 objectives were retained "as is" [6].

- Infections caused by microorganisms transmitted commonly by food:

- Campylobacter species (objective 10-1a)

- Shiga toxin-producing Escherichia coli 0157 (objective 10-1b)

- Listeria monocytogenes (objective 10-1c)

- Salmonella species (objective 10-1d)

- Cases of post-diarrheal hemolytic uremic syndrome (HUS) in persons under 5 years of age (HUS) (objective 10-1f).

- Non-Typhi Salmonella from humans (percent of isolates) resistant to:

- Gentamicin (objective 10-3c)

- Ampicillin (objective 10-3d).

- Severe allergic reactions to food among adults aged 18 years and over with food allergy diagnosis (objective 10-4b).

- Three Healthy People 2010 objectives were modified and expanded to six Healthy People 2020 objectives [7]. Non-Typhi Salmonella from humans (percent of isolates) resistant to fluoroquinolones (objective 10-3a) and third-generation cephalosporins (objective 10-3b) will be tracked in Healthy People 2020 with nalidixic acid and ceftriaxone, respectively. The consumer food safety practices objective (10-5) was modified by subdividing the existing composite objective into four discrete objectives to track specific consumer practices.

- Nine objectives for improving food safety practices in retail and foodservice establishments were measurable in Healthy People 2010 but are developmental in Healthy People 2020 (objectives 10-6a through i) [8]. Actual measures and targets for improvement will be modified but will continue to be based on observed levels of compliance in select retail establishment types.

- Two Healthy People 2010 objectives were archived: Outbreaks of food borne infections due to Escherichia coli 0157:H7 (objective 10-2a) and Salmonella serotype Enteritidis (objective 10-2b) [9]. 
- Sixteen Healthy People 2010 objectives were dropped during the decade [4]. Two of these were determined not to be a significant public health concern (objectives 10-1e and 10-7). One did not have a national data source (objective 10-1g). Twelve objectives (10-3e through p) were dependent upon data from a regulatory program of the U.S. Department of Agriculture's Food Safety and Inspection Service that was not designed to estimate prevalence and, therefore, could not be used to establish measurable objectives. One objective (10-4a, food allergy deaths) was dropped because the data source did not reliably track the actual number of cases of anaphylaxis mortality.

- Nine new objectives were added for Healthy People 2020. These will track the number of infections caused by Vibrio species and Yersinia species; the number of outbreak-associated infections caused by food commodity group for beef, dairy, fruits and nuts, leafy vegetables, and poultry; prevention of Non-Typhi Salmonella occurring in humans (percent of isolates) resistant to three or more classes of antimicrobial agents; and prevention of Campylobacter jejuni from occurring in humans (percent of isolates) resistant to erythromycin.

Appendix D “A Crosswalk Between Objectives From Healthy People 2010 to Healthy People 2020," summarizes the changes between the two decades of objectives, reflecting new knowledge and direction for this area.

\section{Data Considerations}

Education and income are the primary measures of socioeconomic status (SES) in Healthy People 2010. Most data systems used in Healthy People 2010 define income as a family's income before taxes. In order to facilitate comparisons among groups and over time, while adjusting for family size and for inflation, Healthy People 2010 categorizes income using the poverty thresholds developed by the U.S. Census Bureau. Thus, the three categories of family income that are primarily used are:

- Poor-below the Federal poverty level

- Near poor-100\% to $199 \%$ of the Federal poverty level

- Middle/high income-200\% or more of the Federal poverty level.

These categories may be overridden by considerations specific to the data system, in which case they are modified as appropriate. See Healthy People 2010: General Data Issues, referenced below.

In general, data on educational attainment are presented for persons aged 25 years and over, consistent with guidance given by the U.S. Bureau of the Census. However, because of the requirements of the different data systems, the age groups used to calculate educational attainment for any specific objective may differ from the age groups used to report the data for other Healthy People 2010 objectives, as well as from select populations within the same objective. Therefore, the reader is urged to exercise caution in interpreting the data by educational attainment shown in the Health Disparities Table. See Healthy People 2010: General Data Issues, referenced below, for additional information. 
Additional information on data issues is available from the following sources:

- All Healthy People 2010 tracking data can be found in the Healthy People 2010 database, DATA2010, available from http://wonder.cdc.gov/data2010/.

- Detailed information about the data and data sources used to support these objectives can be found in the Operational Definitions on the DATA2010 website, available from http://wonder.cdc.gov/data2010/focusod.htm.

- More information on statistical issues related to Healthy People tracking and measurement can be found in the Technical Appendix and in Healthy People 2010: General Data Issues, which is available in the Data Issues section of the NCHS Healthy People website under Healthy People 2010.

\section{Notes}

1. Displayed in the Progress Chart (Figure 10-1), the percent of targeted change achieved expresses the difference between the baseline and the final value relative to the initial difference between the baseline and the Healthy People 2010 target. As such, it is a relative measure of progress toward attaining the Healthy People 2010 target. See the Reader's Guide for more information. When standard errors were available, the difference between the baseline and the final value was tested at the 0.05 level of significance. See the Figure 10-1 footnotes, as well as the Technical Appendix, for more detail.

2. Information about disparities among select populations is shown in the Health Disparities Table (Figure 10-2). Disparity from the best group rate is defined as the percent difference between the best group rate and each of the other group rates for a characteristic. For example, racial and ethnic health disparities are measured as the percent difference between the best racial and ethnic group rate and each of the other racial and ethnic group rates. Similarly, disparities by sex are measured as the percent difference between the better group rate (e.g., female) and the rate for the other group (e.g., male). Some objectives are expressed in terms of favorable events or conditions that are to be increased, while others are expressed in terms of adverse events or conditions that are to be reduced. In order to facilitate comparison of health disparities across different objectives, disparity is measured only in terms of adverse events or conditions. For comparability across objectives, objectives that are expressed in terms of favorable events or conditions are re-expressed using the adverse event or condition for the purpose of computing disparity, but they are not otherwise restated or changed. For example, objective 1-1, to increase the proportion of persons with health insurance (e.g., $72 \%$ of the American Indian or Alaska Native population aged under 65 years had some form of health insurance in 2008), is expressed in terms of the percentage of persons without health insurance (e.g., $100 \%-72 \%=28 \%$ of the American Indian or Alaska Native population aged under 65 years did not have any form of health insurance in 2008) when the disparity from the best group rate is calculated. See the Reader's Guide for more information. When standard errors were available, the difference between the best group rate and each of the other group rates was tested at the 0.05 level of significance. See the Figure 10-2 footnotes, as well as the Technical Appendix, for more detail.

3. The presence of a monotonic increasing or decreasing trend in the underlying measure was tested with the nonparametric Mann-Kendall test, then the slope of a linear trend was estimated with the nonparametric Sen's method. See Technical Appendix for more information.

4. Dropped objectives were not carried forward into Healthy People 2020. These objectives were either developmental or deleted at the Healthy People 2010 Midcourse Review or at another time in Healthy People 2010. 
5. The change in disparity is estimated by subtracting the disparity at baseline from the disparity at the most recent data point and, therefore, is expressed as a change in percentage points. See the Reader's Guide for more information. When standard errors were available, the change in disparity was tested at the 0.05 level of significance. See the Figure 10-2 footnotes, as well as the Technical Appendix, for more detail.

6. Retained "as is" objectives have no change in the numerator definition or in the denominator definition between the Healthy People 2010 and Healthy People 2020 objectives. These include objectives that were developmental in Healthy People 2010 and are developmental in Healthy People 2020 and for which no numerator or denominator information was available.

7. Modified objectives have some change in the numerator definition or in the denominator definition between the Healthy People 2010 and Healthy People 2020 objectives. These include objectives that went from developmental in Healthy People 2010 to measurable in Healthy People 2020 or vice versa.

8. To be included in Healthy People 2010, an objective must have a national data source that provides a baseline and at least one additional data point for tracking progress. Some objectives lacked baseline data at the time of their development but had a potential data source and were considered of sufficient national importance to be included in Healthy People. These are called "developmental" objectives. When data become available, a developmental objective is moved to measurable status and a Healthy People target can be set.

9. Archived objectives had at least one data point in Healthy People 2010 but were not carried forward into Healthy People 2020. 


\section{Comprehensive Summary of Objectives: Food Safety}

\begin{tabular}{|c|c|c|}
\hline Objective & Description & Data Source or Objective Status \\
\hline $10-1 \mathrm{a}$ & $\begin{array}{l}\text { Foodborne infections-Campylobacter species (per 100,000 } \\
\text { population) }\end{array}$ & $\begin{array}{l}\text { Foodborne Disease Active Surveillance Network (FoodNet): CDC, } \\
\text { NCEZID }\end{array}$ \\
\hline $10-1 b$ & $\begin{array}{l}\text { Foodborne infections-Escherichia coli 0157:H7 (per 100,000 } \\
\text { population) }\end{array}$ & $\begin{array}{l}\text { Foodborne Disease Active Surveillance Network (FoodNet): CDC, } \\
\text { NCEZID. }\end{array}$ \\
\hline $10-1 c$ & $\begin{array}{l}\text { Foodborne infections-Listeria monocytogenes (per 100,000 } \\
\text { population) }\end{array}$ & $\begin{array}{l}\text { Foodborne Disease Active Surveillance Network (FoodNet): CDC, } \\
\text { NCEZID. }\end{array}$ \\
\hline $10-1 d$ & Foodborne infections-Salmonella species (per 100,000 population) & $\begin{array}{l}\text { Foodborne Disease Active Surveillance Network (FoodNet): CDC, } \\
\text { NCEZID. }\end{array}$ \\
\hline $10-1 \mathrm{e}$ & $\begin{array}{l}\text { Foodborne infections: - Cyclospora cayetanensis (per } 100.000 \\
\text { population) }\end{array}$ & Dropped \\
\hline $10-1 \mathrm{f}$ & $\begin{array}{l}\text { Foodborne infections-Cases of postdiarrheal hemolytic uremic } \\
\text { syndrome (HUS) (per } 100,000 \text { population }<5 \text { years) }\end{array}$ & $\begin{array}{l}\text { Foodborne Disease Active Surveillance Network (FoodNet): CDC, } \\
\text { NCEZID. }\end{array}$ \\
\hline $10-1 \mathrm{~g}$ & Foodborne infections-Congenital Toxoplasma gondii & Dropped \\
\hline $10-2 \mathrm{a}$ & Outbreaks of foodborne infections-Escherichia coli 0157:H7 & Foodborne Disease Outbreak Surveillance System, CDC, NCEZID. \\
\hline $10-2 b$ & Outbreaks of foodborne infections-Salmonella serotype Enteritidis & Foodborne Disease Outbreak Surveillance System, CDC, NCEZID. \\
\hline $10-3 a$ & $\begin{array}{l}\text { Non-Typhi Salmonella from humans (percent of isolates) resistant to } \\
\text { fluoroquinolones }\end{array}$ & $\begin{array}{l}\text { National Antimicrobial Resistance Monitoring System: Enteric } \\
\text { Bacteria- (NARMS), CDC, NCEZID. }\end{array}$ \\
\hline $10-3 b$ & $\begin{array}{l}\text { Non-Typhi Salmonella from humans (percent of isolates) resistant to } \\
\text { third-generation cephalosporins }\end{array}$ & $\begin{array}{l}\text { National Antimicrobial Resistance Monitoring System: Enteric } \\
\text { Bacteria- (NARMS), CDC, NCEZID. }\end{array}$ \\
\hline $10-3 c$ & $\begin{array}{l}\text { Non-Typhi Salmonella from humans (percent of isolates) resistant to } \\
\text { gentamicin }\end{array}$ & $\begin{array}{l}\text { National Antimicrobial Resistance Monitoring System: Enteric } \\
\text { Bacteria- (NARMS), CDC, NCEZID }\end{array}$ \\
\hline $10-3 d$ & $\begin{array}{l}\text { Non-Typhi Salmonella from humans (percent of isolates) resistant to } \\
\text { ampicillin }\end{array}$ & $\begin{array}{l}\text { National Antimicrobial Resistance Monitoring System: Enteric } \\
\text { Bacteria- (NARMS), CDC, NCEZID }\end{array}$ \\
\hline $10-3 e$ & $\begin{array}{l}\text { Non-Typhi Salmonella from cattle at slaughter (percent of isolates) } \\
\text { resistant to fluoroquinolones }\end{array}$ & Dropped \\
\hline $10-3 f$ & $\begin{array}{l}\text { Non-Typhi Salmonella from cattle at slaughter (percent of isolates) } \\
\text { resistant to third-generation cephalosporins }\end{array}$ & Dropped \\
\hline $10-3 g$ & $\begin{array}{l}\text { Non-Typhi Salmonella from cattle at slaughter (percent of isolates) } \\
\text { resistant to gentamicin }\end{array}$ & Dropped \\
\hline $10-3 h$ & $\begin{array}{l}\text { Non-Typhi Salmonella from cattle at slaughter (percent of isolates) } \\
\text { resistant to ampicillin }\end{array}$ & Dropped \\
\hline
\end{tabular}




\begin{tabular}{|c|c|c|}
\hline Objective & Description & Data Source or Objective Status \\
\hline $10-3 i$ & $\begin{array}{l}\text { Non-Typhi Salmonella from poultry at slaughter (percent of isolates) } \\
\text { resistant to fluoroquinolones }\end{array}$ & Dropped \\
\hline $10-3 j$ & $\begin{array}{l}\text { Non-Typhi Salmonella from poultry at slaughter (percent of isolates) } \\
\text { resistant to third-generation cephalosporins }\end{array}$ & Dropped \\
\hline $10-3 \mathrm{k}$ & $\begin{array}{l}\text { Non-Typhi Salmonella from poultry at slaughter (percent of isolates) } \\
\text { resistant to gentamicin }\end{array}$ & Dropped \\
\hline $10-31$ & $\begin{array}{l}\text { Non-Typhi Salmonella from poultry at slaughter (percent of isolates) } \\
\text { resistant to ampicillin }\end{array}$ & Dropped \\
\hline $10-3 n$ & $\begin{array}{l}\text { Non-Typhi Salmonella from swine at slaughter (percent of isolates) } \\
\text { resistant to third-generation cephalosporins }\end{array}$ & Dropped \\
\hline $10-30$ & $\begin{array}{l}\text { Non-Typhi Salmonella from swine at slaughter (percent of isolates) } \\
\text { resistant to gentamicin }\end{array}$ & Dropped \\
\hline $10-5$ & Consumer food safety practices $(18+$ years $)$ & $\begin{array}{l}\text { Food Safety Survey (FSS), FDA, CFSAN; and Department of } \\
\text { Agriculture (USDA). }\end{array}$ \\
\hline $10-6 a$ & Safe retail food preparation-Hospitals & Retail Food Database of Foodborne Illness Risk Factors, FDA, CFSAN. \\
\hline $10-6 b$ & Safe retail food preparation-Nursing homes & Retail Food Database of Foodborne Illness Risk Factors, FDA, CFSAN. \\
\hline $10-6 c$ & Safe retail food preparation-Elementary schools & Retail Food Database of Foodborne Illness Risk Factors, FDA, CFSAN. \\
\hline $10-6 d$ & Safe retail food preparation-Fast food restaurants & Retail Food Database of Foodborne Illness Risk Factors, FDA, CFSAN. \\
\hline $10-6 e$ & Safe retail food preparation-Full-service restaurants & Retail Food Database of Foodborne Illness Risk Factors, FDA, CFSAN. \\
\hline $10-6 f$ & Safe retail food preparation—Deli departments & Retail Food Database of Foodborne Illness Risk Factors, FDA, CFSAN. \\
\hline $10-6 g$ & Safe retail food preparation-Meat/poultry departments & Retail Food Database of Foodborne Illness Risk Factors, FDA, CFSAN. \\
\hline $10-6 h$ & Safe retail food preparation—Produce departments & Retail Food Database of Foodborne Illness Risk Factors, FDA, CFSAN. \\
\hline
\end{tabular}


Figure 10-1. Progress Toward Target Attainment for Focus Area 10: Food Safety

Moved away

from target ${ }^{1}$

Moved toward

target

Met or exceeded

target

10-1. Foodborne infections (per 100,000 population)

a. Campylobacter species

b. Escherichia coli O157:H7

c. Listeria monocytogenes

d. Salmonella species

f. Cases of postdiarrheal hemolytic uremic syndrome (HUS)

( $<5$ years)

10-2. Outbreaks of foodborne infections

a. Escherichia coli O157:H7

b. Salmonella serotype Enteritidis

10-3. Non-Typhi Salmonella from humans (percent of isolates) resistant to:

a. fluoroquinolones

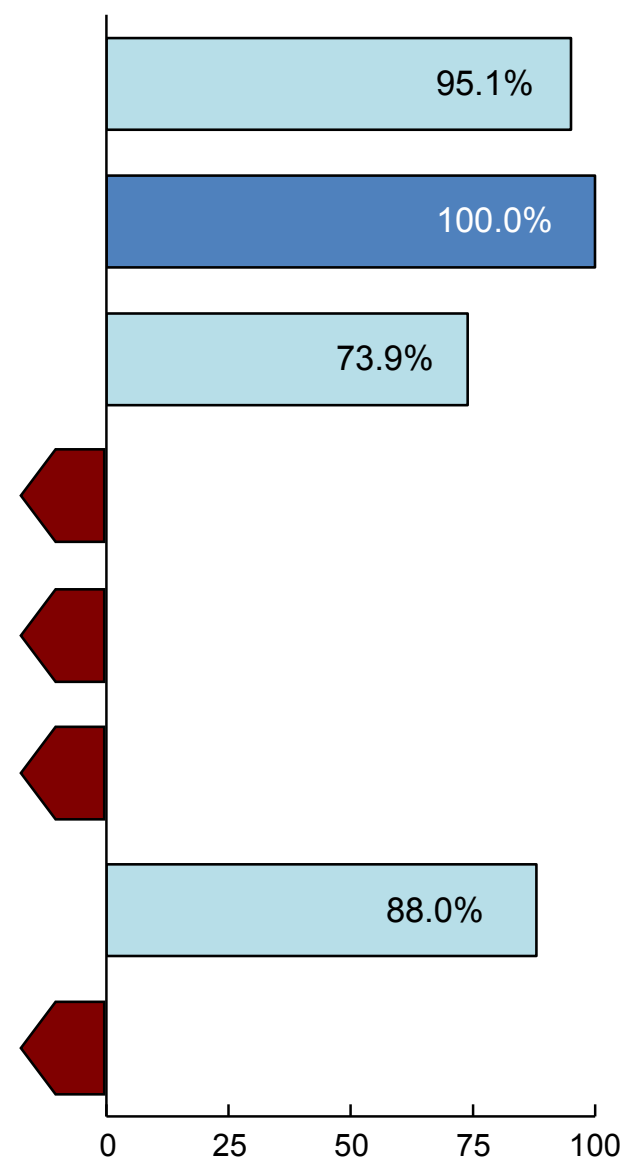

\begin{tabular}{|c|c|c|c|c|c|}
\hline \multirow{2}{*}{$\begin{array}{l}2010 \\
\text { Target }\end{array}$} & \multirow{2}{*}{$\begin{array}{c}\text { Baseline } \\
\text { (Year) }\end{array}$} & \multirow{2}{*}{$\begin{array}{l}\text { Final } \\
\text { (Year) }\end{array}$} & \multicolumn{3}{|c|}{ Baseline vs. Final } \\
\hline & & & Difference $^{2}$ & $\begin{array}{l}\text { Statistically } \\
\text { Significant }^{3}\end{array}$ & $\begin{array}{c}\text { Percent } \\
\text { Change }^{4}\end{array}$ \\
\hline 12.3 & $\begin{array}{c}24.6 \\
(1997)\end{array}$ & $\begin{array}{c}12.9 \\
(2009)\end{array}$ & -11.7 & $\begin{array}{c}\text { Not } \\
\text { tested }\end{array}$ & $-47.6 \%$ \\
\hline 1.0 & $\begin{array}{c}2.1 \\
(1997)\end{array}$ & $\begin{array}{c}1.0 \\
(2009)\end{array}$ & -1.1 & $\begin{array}{c}\text { Not } \\
\text { tested }\end{array}$ & $-52.4 \%$ \\
\hline 0.24 & $\begin{array}{c}0.47 \\
(1997)\end{array}$ & $\begin{array}{c}0.30 \\
(2009)\end{array}$ & -0.17 & $\begin{array}{c}\text { Not } \\
\text { tested }\end{array}$ & $-36.2 \%$ \\
\hline 6.8 & $\begin{array}{c}13.6 \\
(1997)\end{array}$ & $\begin{array}{c}15.0 \\
(2009)\end{array}$ & 1.4 & $\begin{array}{c}\text { Not } \\
\text { tested }\end{array}$ & 10.3 \\
\hline 0.90 & $\begin{array}{c}1.80 \\
(2000)\end{array}$ & $\begin{array}{c}2.03 \\
(2006)\end{array}$ & 0.23 & $\begin{array}{l}\text { Not } \\
\text { tested }\end{array}$ & $12.8 \%$ \\
\hline 5 & $\begin{array}{c}10 \\
(1997)\end{array}$ & $\begin{array}{c}32 \\
(2008)\end{array}$ & 22 & $\begin{array}{c}\text { Not } \\
\text { tested }\end{array}$ & $220.0 \%$ \\
\hline 24 & $\begin{array}{c}49 \\
(1997)\end{array}$ & $\begin{array}{c}27 \\
(2008)\end{array}$ & -22 & $\begin{array}{l}\text { Not } \\
\text { tested }\end{array}$ & $-44.9 \%$ \\
\hline $0.0 \%$ & $\begin{array}{c}0.0 \% \\
(1997)\end{array}$ & $\begin{array}{c}0.1 \% \\
(2008)\end{array}$ & 0.1 & $\begin{array}{l}\text { Not } \\
\text { tested }\end{array}$ & * \\
\hline
\end{tabular}

Percent of targeted change achieved 5

(continued) 
Figure 10-1. Progress Toward Target Attainment for Focus Area 10: Food Safety (continued)

Moved away

from target ${ }^{1}$

Moved toward

target

Met or exceeded

target

10-3. Non-Typhi Salmonella from humans (percent of isolates) resistant to:

b. Third-generation cephalosporins

c. Gentamicin

d. Ampicillin

10-4b. Severe allergic reactions to food among adults with food allergy diagnosis (18+ years)

10-5. Consumer food safety practices $(18+$ years $)$

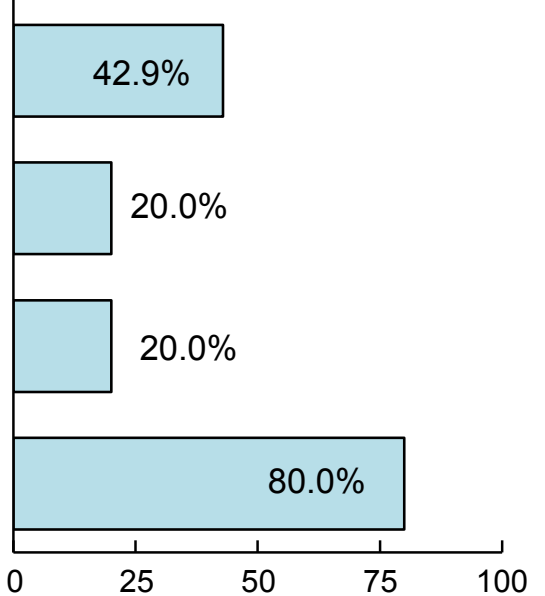

\begin{tabular}{|c|c|c|c|c|c|}
\hline \multirow{2}{*}{$\begin{array}{l}2010 \\
\text { Target }\end{array}$} & \multirow{2}{*}{$\begin{array}{c}\text { Baseline } \\
\text { (Year) }\end{array}$} & \multirow{2}{*}{$\begin{array}{l}\text { Final } \\
\text { (Year) }\end{array}$} & \multicolumn{3}{|c|}{ Baseline vs. Final } \\
\hline & & & Difference ${ }^{2}$ & $\begin{array}{l}\text { Statistically } \\
\text { Significant }^{3}\end{array}$ & $\begin{array}{c}\text { Percent } \\
\text { Change }^{4}\end{array}$ \\
\hline $0.1 \%$ & $\begin{array}{c}0.1 \% \\
(1997)\end{array}$ & $\begin{array}{c}0.3 \% \\
(2008)\end{array}$ & 0.2 & $\begin{array}{l}\text { Not } \\
\text { tested }\end{array}$ & $200.0 \%$ \\
\hline $2.9 \%$ & $\begin{array}{c}2.9 \% \\
(1997)\end{array}$ & $\begin{array}{c}1.4 \% \\
(2008)\end{array}$ & -1.5 & $\begin{array}{c}\text { Not } \\
\text { tested }\end{array}$ & $-51.7 \%$ \\
\hline $18.3 \%$ & $\begin{array}{l}18.3 \% \\
(1997)\end{array}$ & $\begin{array}{l}9.3 \% \\
(2008)\end{array}$ & -9 & $\begin{array}{c}\text { Not } \\
\text { tested }\end{array}$ & $-49.2 \%$ \\
\hline $21 \%$ & $\begin{array}{c}26 \% \\
(2001)\end{array}$ & $\begin{array}{c}29 \% \\
(2006)\end{array}$ & 3 & No & $11.5 \%$ \\
\hline $79 \%$ & $\begin{array}{c}72 \% \\
(1998)\end{array}$ & $\begin{array}{c}75 \% \\
(2006)\end{array}$ & 3 & Yes & $4.2 \%$ \\
\hline $85 \%$ & $\begin{array}{c}80 \% \\
(1998)\end{array}$ & $\begin{array}{c}81 \% \\
(2008)\end{array}$ & 1 & No & $1.3 \%$ \\
\hline $87 \%$ & $\begin{array}{c}82 \% \\
(1998)\end{array}$ & $\begin{array}{c}83 \% \\
(2008)\end{array}$ & 1 & No & $1.2 \%$ \\
\hline $85 \%$ & $\begin{array}{c}80 \% \\
(1998)\end{array}$ & $\begin{array}{c}84 \% \\
(2008)\end{array}$ & 4 & No & $5.0 \%$ \\
\hline
\end{tabular}

Percent of targeted change achieved ${ }^{5}$

(continued) 
Figure 10-1. Progress Toward Target Attainment for Focus Area 10: Food Safety (continued)

Moved away

from target ${ }^{1}$

Moved toward

target

Met or exceeded

target

10-6. Safe retail food preparation

d. Fast food restaurants

e. Full-service restaurants

f. Deli departments

g. Meat/poultry departments

h. Produce departments

i. Seafood departments

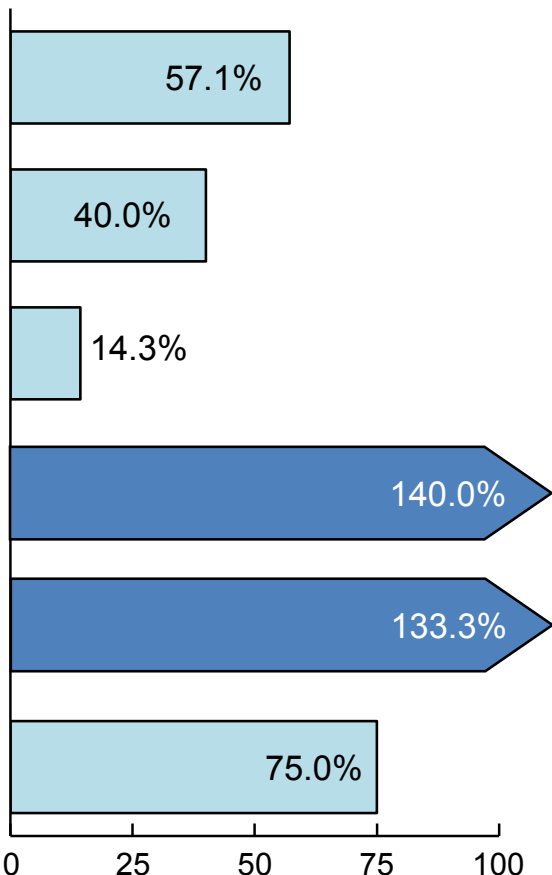

\begin{tabular}{|c|c|c|c|c|c|}
\hline \multirow{2}{*}{$\begin{array}{l}2010 \\
\text { Target }\end{array}$} & \multirow{2}{*}{$\begin{array}{c}\text { Baseline } \\
\text { (Year) }\end{array}$} & \multirow{2}{*}{$\begin{array}{l}\text { Final } \\
\text { (Year) }\end{array}$} & \multicolumn{3}{|c|}{ Baseline vs. Final } \\
\hline & & & Difference ${ }^{2}$ & $\begin{array}{l}\text { Statistically } \\
\text { Significant }^{3}\end{array}$ & $\begin{array}{c}\text { Percent } \\
\text { Change }^{4}\end{array}$ \\
\hline $81 \%$ & $\begin{array}{c}74 \% \\
(1998)\end{array}$ & $\begin{array}{c}78 \% \\
(2008)\end{array}$ & 4 & No & $5.4 \%$ \\
\hline $70 \%$ & $\begin{array}{c}60 \% \\
(1998)\end{array}$ & $\begin{array}{c}64 \% \\
(2008)\end{array}$ & 4 & No & $6.7 \%$ \\
\hline $80 \%$ & $\begin{array}{c}73 \% \\
(1998)\end{array}$ & $\begin{array}{c}74 \% \\
(2008)\end{array}$ & 1 & No & $1.4 \%$ \\
\hline $86 \%$ & $\begin{array}{c}81 \% \\
(1998)\end{array}$ & $\begin{array}{c}88 \% \\
(2008)\end{array}$ & 7 & No & $8.6 \%$ \\
\hline $82 \%$ & $\begin{array}{c}76 \% \\
(1998)\end{array}$ & $\begin{array}{c}84 \% \\
(2008)\end{array}$ & 8 & No & $10.5 \%$ \\
\hline $87 \%$ & $\begin{array}{c}83 \% \\
(1998)\end{array}$ & $\begin{array}{c}86 \% \\
(2008)\end{array}$ & 3 & No & $3.6 \%$ \\
\hline
\end{tabular}

Percent of targeted change achieved ${ }^{5}$

(continued) 
Figure 10-1. Progress Toward Target Attainment for Focus Area 10: Food Safety (continued)

\section{NOTES}

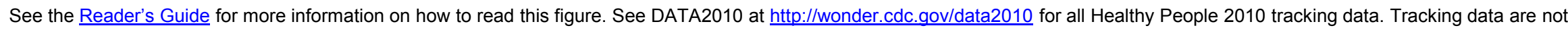
available for objective 10-4a. Objectives 10-1e, 10-1g, 10-3e through $\mathrm{p}$, and 10-7 were deleted at the Midcourse Review.

\section{FOOTNOTES}

${ }^{1}$ Movement away from target is not quantified using the percent of targeted change achieved. See Technical Appendix for more information.

2 Difference $=$ Final value - Baseline value. Differences between percents (\%) are measured in percentage points.

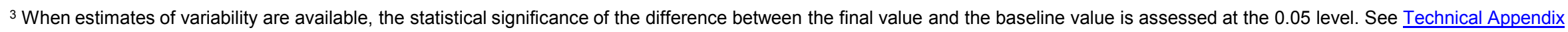
for more information.

${ }^{4}$ Percent change $=\frac{\text { Final value }- \text { Baseline value }}{\text { Baseline value }} \times 100$.

${ }^{5}$ Percent of targeted change achieved $=\frac{\text { Final value }- \text { Baseline value }}{\text { Healthy People } 2010 \text { target }- \text { Baseline value }} \times 100$.

* Percent change cannot be calculated. See Technical Appendix for more information.

\section{DATA SOURCES}

10-1a-d. Foodborne Disease Active Surveillance Network (FoodNet): CDC, NCPDCID; FDA, CFSAN; Department of Agriculture (USDA); State agencies.

10-1f. Foodborne Disease Active Surveillance Network (FoodNet): CDC, NCPDCID; FDA, CFSAN; Department of Agriculture (USDA); State agencies.

10-2a-b. Foodborne Disease Outbreak Surveillance System, CDC, NCID.

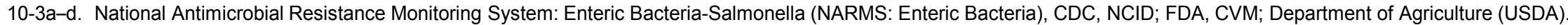
Foodborne Disease Active Surveillance Network (FoodNet): CDC, NCPDCID; FDA, CFSAN; USDA; State agencies.

10-4b. Food Safety Survey (FSS), FDA, CFSAN; and Department of Agriculture (USDA)

10-5. Food Safety Survey (FSS), FDA, CFSAN; and Department of Agriculture (USDA).

10-6a-i. Retail Food Database of Foodborne Illness Risk Factors, FDA, CFSAN. 
Disparities from the best group rate for each characteristic at the most recent data point and changes in disparity from the baseline to the most recent data point.

Population-based objectives

10-1a. Foodborne infections-Campylobacter species (per 100,000 population) (1997, 2009)†

b. Foodborne infections-Escherichia coli O157:H7 (per 100,000 population) (1997, 2009)†

c. Foodborne infections-Listeria monocytogenes (per 100,000 population) $(1997,2009) \dagger$

d. Foodborne infections-Salmonella species (per 100,000 population) $(1997,2009) \dagger$

f. Foodborne infections-Cases of postdiarrheal hemolytic uremic syndrome (HUS) (per 100,000 population $<5$ years $)(2000,2006) \dagger$

10-4b. Severe allergic reactions to food among adults with food allergy diagnosis (18+ years) $(2001$, 2006)*

10-5. Consumer food safety practices $(18+$ years $)$ $(1998,2006)$

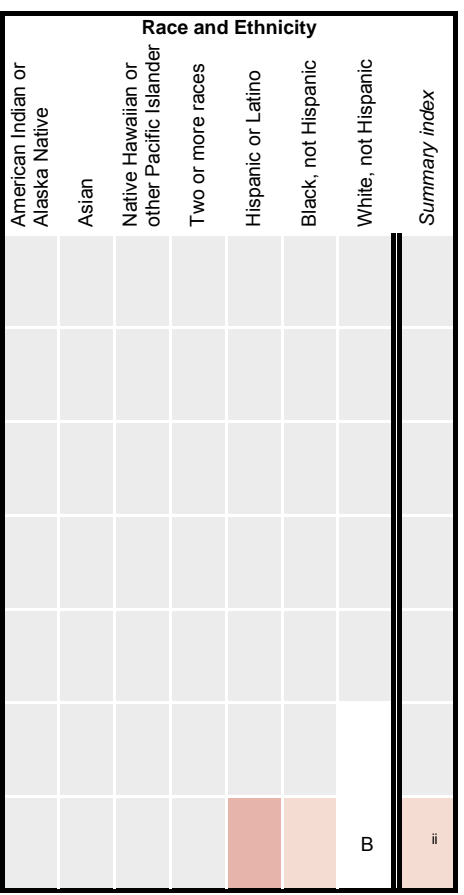

Characteristics and Groups

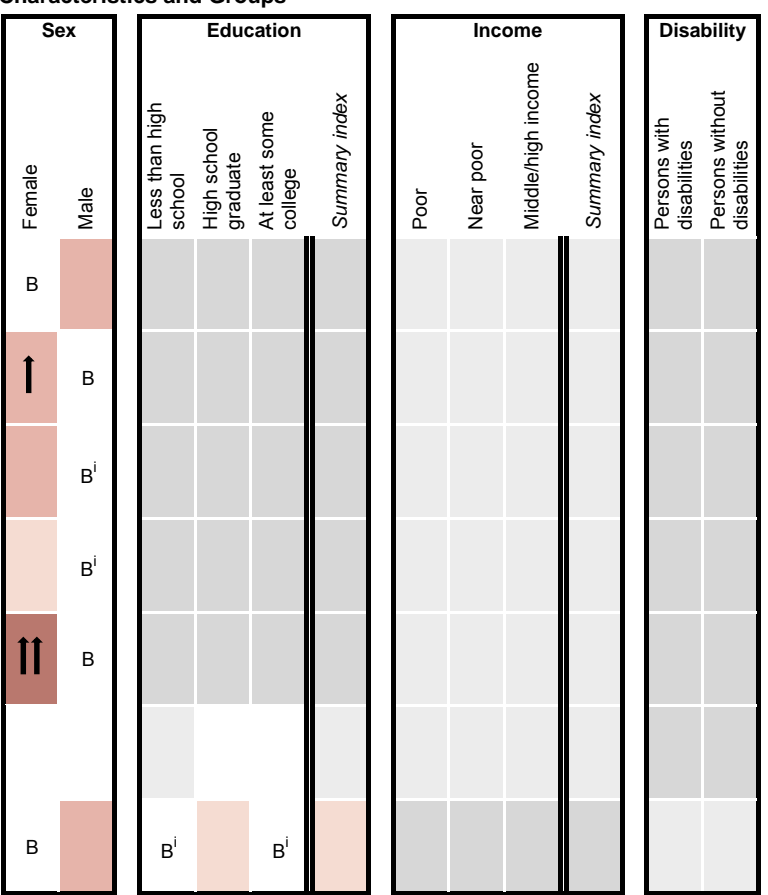

NOTES

See DATA2010 at http://wonder.cdc.gov/data2010 for all Healthy People 2010 tracking data. Disparity data are either unavailable or not applicable for objectives 10-2a and b, 10-3a through d, 10-4a, and 10-6a through i. Objectives 10-1e and g, 10-3e through p, and 10-7, were deleted at Midcourse Review.

Years in parentheses represent the baseline and most recent data years (if available).

Disparity from the best group rate is defined as the percent difference between the best group rate and each of the other group rates for a characteristic (e.g., race and ethnicity). The summary index is the average of these percent differences for a characteristic. Change in disparity is estimated by subtracting the disparity at baseline from the disparity at the most recent data point. Change in the summary index is estimated by subtracting the summary index at baseline from the summary index at the most recent data point. See Technical Appendix for more information.

\begin{tabular}{l|l|l|l|l}
\cline { 2 - 3 } The "best" group rate at the most recent data point. & B & $\begin{array}{l}\text { The group with the best rate } \\
\text { for specified characteristic. }\end{array}$
\end{tabular}$\quad$ b $\begin{aligned} & \text { Most favorable group rate for specified } \\
& \text { characteristic, but reliability criterion not met. }\end{aligned}$

\begin{tabular}{|c|c|c|c|c|}
\hline \multirow[b]{2}{*}{$\begin{array}{l}\text { Disparity from the best group rate at the most } \\
\text { recent data point. }\end{array}$} & \multicolumn{4}{|c|}{ Percent difference from the best group rate } \\
\hline & $\begin{array}{l}\text { Less than } 10 \% \text {, or difference not } \\
\text { statistically significant (when } \\
\text { estimates of variability are available). }\end{array}$ & $10 \%-49 \%$ & $50 \%-99 \%$ & $\begin{array}{l}100 \% \text { or } \\
\text { more }\end{array}$ \\
\hline
\end{tabular}

Changes in disparity over time are shown when:

(a) disparities data are available at both baseline and most recent time points

(b) data are not for the group(s) indicated by "B" or "b" at either time point; and

(c) the change is greater than or equal to 10 percentage points and statistically

significant, or when the change is greater than or equal to 10 percentage points and

estimates of variability were not available. See Technical Appendix.

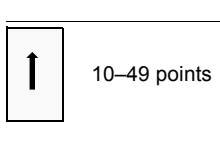

Increase in disparity (percentage points)

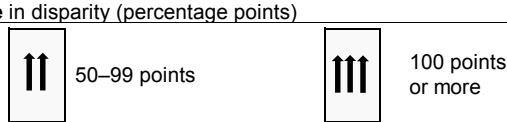

Decrease in disparity (percentage points)

\begin{tabular}{l|l|l|l|l|l}
100 points & 10-49 points
\end{tabular}

Availability of Data

* Measures of variability were available. Thus, the variability of best group rates was assessed, and statistical significance was tested. Disparities of $10 \%$ or more are displayed when the differences from the best group rate are statistically significant at the 0.05 level. Changes in disparities over time are indicated by arrows when the changes are greater than or equal to 10 percentage points and are statistically significant at the 0.05 level. See Technical Appendix.

† Measures of variability were not available. Thus, the variability of best group rates was not assessed, and statistical significance could not be tested. Nonetheless, disparities and changes in disparities over time are displayed according to their magnitude. See Technical Appendix.

$\mathrm{i}$ The group with the best rate at the most recent data point is different from the group with the best rate at baseline. Both rates met the reliability criterion. See Technical Appendix.

ii Change in the summary index cannot be assessed. See Technical Appendix.

DATA SOURCES

10-1a-d. Foodborne Disease Active Surveillance Network (FoodNet): CDC, NCPDCID; FDA, CFSAN; Department of Agriculture (USDA); State agencies.

10-1f. Foodborne Disease Active Surveillance Network (FoodNet): CDC, NCPDCID; FDA, CFSAN; Department of Agriculture (USDA); State agencies.

10-4b. Food Safety Survey (FSS), FDA, CFSAN; and Department of Agriculture (USDA)

10-5. Food Safety Survey (FSS), FDA, CFSAN; and Department of Agriculture (USDA)

Food Safety 


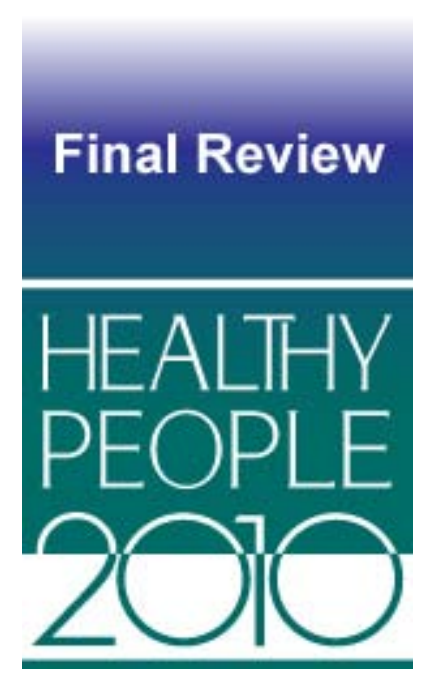

\section{Health Communication}

11

\section{Lead Agency}

Office of Disease Prevention and Health Promotion

\section{Contents}

Goal.

Highlights.

Summary of Progress.

Transition to Healthy People 2020 .

Data Considerations

Notes

Comprehensive Summary of Objectives

Progress Chart

Health Disparities Table

Persons with Internet Access at Home-Map 


\section{Goal: Use communication strategically to improve health}

The objectives in this chapter monitor the availability of Internet access, health literacy, and the characteristics of health communication campaigns and health-related websites. The number of centers for excellence in health communication and patient perception of health provider communication skills are also tracked.

All Healthy People tracking data quoted in this chapter, along with technical information and operational definitions for each objective, can be found in the Healthy People 2010 database, DATA2010, available from http://wonder.cdc.gov/data2010/.

More information about this focus area can be found in the following publications:

- Healthy People 2010: Understanding and Improving Health, available from http://www.healthypeople.gov/2010/Document/tableofcontents.htm\#under.

- Healthy People 2010 Midcourse Review, available from http://www.healthypeople.gov/2010/data/midcourse/html/default.htm\#FocusAreas.

\section{Highlights}

- Substantial progress was achieved in objectives for this Focus Area during the past decade [1]. Fourteen of the 16 Health Communication objectives with data to measure progress moved toward or achieved their Healthy People 2010 targets (Figure 11-1). However, health disparities of $10 \%$ or more were observed for a number of population groups (Figure 11-2), some of which are highlighted below [2].

- The proportion of adults with Internet access at home (objective 11-1) increased by $165.4 \%$ between 1998 and 2009, from 26\% to 69\%, moving toward the Healthy People 2010 target of $80 \%$.

- Among racial and ethnic groups, the Asian population had the highest (best) rate of Internet access at home, $80 \%$ in 2009, whereas the American Indian or Alaska Native, Hispanic or Latino, and black non-Hispanic populations each had a rate of $53 \%$ in 2009 . When expressed as persons without Internet access at home, the rates for these three populations were almost two and a half times the best group rate (that for the Asian population) [2].

- Among education groups, persons with at least some college education had the highest (best) rates of Internet access at home, 31\% in 1998 and $82 \%$ in 2009, whereas high school graduates had rates of $16 \%$ in 1998 and $57 \%$ in 2009 , and persons with less than a high school education had rates of $5 \%$ in 1998 and 32\% in 2009.

- In 2009, when expressed as persons without Internet access at home, the rate for high school graduates was almost two and a half times the best group rate (that for persons with at least some college education), while the rate for persons with less than a high school education was nearly four times the best group rate [2]. 
- Between 1998 and 2009, the disparity between high school graduates and persons with at least some college education (the group with the best rate) increased by 117 percentage points, while the disparity between persons with less than a high school education and the group with the best rate increased by 240 percentage points [3].

- Internet access at home varied by geographic area. In 2009, the proportion of adults with Internet access at home was highest in the states of Alaska, Connecticut, Massachusetts, New Hampshire, New Jersey, Oregon, Utah, and Washington. The states with the lowest proportion of adults with Internet access at home were Alabama, Arkansas, Mississippi, and South Carolina (Figure 11-3).

- The proportion of health websites disclosing information that could be used to assess the quality of the site (objectives 11-4a through g) increased for all categories. The Healthy People 2010 targets were exceeded for four objectives:

- The proportion of websites that disclosed their purpose, uses, and limitations (objective $11-4$ b) increased by $20.0 \%$ between 2006 and 2009 , from $35 \%$ to $42 \%$, exceeding the target of $40 \%$.

- The proportion that disclosed their privacy policies (objective 11-4d) increased by $13.3 \%$ between 2006 and 2009 , from $75 \%$ to $85 \%$, exceeding the target of $80 \%$.

- The proportion that provided user feedback options (objective 11-4e) increased by $49.2 \%$ between 2006 and 2009 , from $59 \%$ to $88 \%$, exceeding the target of $64 \%$.

- The proportion of websites that met at least three of the six disclosure criteria (objective $11-4 \mathrm{~g}$ ) increased by $116.7 \%$ between 2006 and 2009, from $24 \%$ to 52\%, exceeding the target of $29 \%$.

- Health disparities of $100 \%$ or more in the proportion of persons with below basic health literacy skills (objective 11-2b) were observed for a number of population groups:

- Among racial and ethnic groups, the white non-Hispanic population had the lowest (best) rate of persons with below basic health literacy, 9\% in 2003, whereas the black non-Hispanic, American Indian or Alaska Native, and Hispanic or Latino populations had rates of $24 \%, 25 \%$, and $41 \%$ in 2003 , respectively. The rate for the black non-Hispanic population was more than two and a half times the best rate (that for the white non-Hispanic population); the rate for the American Indian or Alaska Native population was almost three times the best rate; and the rate for the Hispanic or Latino population was more than four and a half times the best rate [2].

- Among education groups, persons with at least some college education had the lowest (best) rate of persons below basic health literacy, 5\% in 2003. High school graduates and persons with less than a high school education had rates of $15 \%$ and $54 \%$ in 2003, respectively. The rate for high school graduates was three times the best group rate (that for persons with at least some college education), while the rate for persons with less than a high school education was nearly 11 times the best group rate [2].

- Persons without disabilities had a lower (better) rate of persons below basic health literacy than persons with disabilities, $10 \%$ in 2003 . The rate for persons with disabilities was $23 \%$ in 2003, nearly two and a half times the rate for persons without disabilities [2]. 


\section{Summary of Progress}

- Figure 11-1 presents a quantitative assessment of progress in achieving the Healthy People 2010 objectives for Health Communication. Data to measure progress toward target attainment were available for 16 objectives [1]. Of these:

- Five objectives (11-4b, d, e, and g; and 11-5) exceeded the Healthy People 2010 targets.

- Nine objectives moved toward their targets. A statistically significant difference between the baseline and the final data points was observed for six of these objectives (11-1; 11-4c and f; and 11-6a, c, and d). No significant differences were observed for two objectives (11-4a and 11-6b); and data to test the significance of the difference were unavailable for one objective (11-3c).

- Two objectives moved away from their targets (objectives 11-3a and b). Data to test the significance of the difference between the baseline and the final data points were unavailable for either of these objectives.

- Data were unavailable to measure progress for two objectives (11-2a and b).

- Figure 11-2 displays health disparities in Health Communication from the best group rate for each characteristic at the most recent data point [2]. It also displays changes in disparities from baseline to the most recent data point [3].

- Of the seven objectives with statistically significant racial and ethnic health disparities of $10 \%$ or more, the black non-Hispanic population had the best rate for four objectives (11-6a through d). The Asian population had the best rate for two objectives (11-1 and 11-2b) and the white non-Hispanic population had the best rate for one objective (11-2b).

- Males had better rates than females for two of the three objectives with statistically significant health disparities of $10 \%$ or more by sex (objectives 11-1 and 11-6a). Females had a better rate for objective 11-2b.

- Of the seven objectives with statistically significant health disparities of $10 \%$ or more by education level, high school graduates had the best rate for three objectives (11-6a, c, and d), persons with at least some college education also had the best rate for three objectives (11-1a, and 11-2a and b). The population of high school graduates and the population of persons with at least some college education both had the best group rate for one objective (persons reporting their health care providers explained things so they could understand; objective 11-6b).

- Residents of urban or metropolitan areas had the better group rate for the one objective with statistically significant health disparities of $10 \%$ or more by geographic location (persons with Internet access at home; objective 11-1).

- Persons without disabilities had the better group rate for all three objectives with statistically significant health disparities of $10 \%$ or more by disability status (objectives 11-2b, and 11-6b and c).

- As discussed in the Highlights, above, health disparities of $100 \%$ or more were observed for two objectives (11-1 and 11-2b). Changes in health disparities of 100 percentage points or more were observed for one objective (11-1). 


\section{Transition to Healthy People 2020}

For Healthy People 2020, the focus of the Health Communication and Health Information Technology (IT) objectives has been expanded from those presented in Healthy People 2010 to include more objectives that are shaped by the communication processes and the information technology that people interact with every day. The Healthy People 2010 Focus Area name was changed from Health Communication to Health Communication and Health IT to strategically combine health IT tools and effective health communication processes. See HealthyPeople.gov for a complete list of Healthy People 2020 topics and objectives.

The Healthy People 2020 Health Communication and Health IT objectives are geared toward:

- Providing personalized self-management tools and resources

- Building social support networks

- Delivering accurate, accessible, and actionable health information that is targeted or tailored

- Facilitating the meaningful use of health IT and exchange of health information among health care and public health professionals

- Enabling quick and informed action to health risks and public health emergencies

- Increasing health literacy skills

- Providing new opportunities to connect with culturally diverse and hard-to-reach populations

- Providing a trained workforce for the design of programs and interventions that result in healthier behaviors

- Increasing Internet and mobile access.

The differences between the Healthy People 2010 and Healthy People 2020 objectives are summarized below:

- The Healthy People 2020 Health Communication and Health IT Area has a total of 24 objectives, 10 of which are developmental, whereas the Healthy People 2010 Health Communication Focus Area had 18 objectives [4].

- Four Healthy People 2010 objectives were retained "as is" [5]. These objectives address patient reports of health care provider communication skills (objectives 11-6a through d).

- Two Healthy People 2010 objectives were modified [6]. These objectives include Internet access at home (objective 11-1) and health websites that disclose at least three of select criteria (objective 11-4g) [6]. 
- Twelve Healthy People 2010 objectives were archived [7]. These objectives include: persons with proficient health literacy (objective 11-2a); persons with below basic health literacy (objective 11-2b); health communication campaigns sponsored by the U.S. Department of Health and Human Services that include formative evaluations (objective 113a); health communication campaigns sponsored by the U.S. Department of Health and Human Services that include process evaluation (objective 11-3b); health websites that disclose the identity of the responsible persons or organizations (objective 11-4a); health websites that disclose the purpose, uses and limitations of the sites (objective 11-4b); health websites that disclose content development practices and policies on the sites (objective 11-4c); health websites that disclose privacy policy and protection on the sites (objective 11-4d); health websites that disclose user feedback and evaluation on the sites (objective 11-4e); health websites that disclose content creation on the sites (objective 114f); and the number of Centers for Excellence in Health Communication (objective 11-5).

- Seventeen new objectives were added to the Healthy People 2020 Topic Area:

- Three new health literacy objectives monitor the proportion of persons who report that their health care provider always give them easy-to-understand instructions about what to do to take care of their illness or health condition; that their health care provider always ask them to describe how they will follow the instructions; and that their health care providers' office always offers help in filling out a form.

- Three new social marketing objectives track the proportion of state health departments using social marketing in health promotion and disease prevention programs; schools of public health and accredited master of public health (MPH) programs that offer one or more courses in social marketing; and schools of public health and accredited MPH programs that offer workforce development activities in social marketing for public health practitioners.

- Two new Internet access objectives track the proportion of persons with broadband access to the Internet and the proportion of persons who use mobile devices.

- Two new electronic personal health management tools objectives target the proportion of persons who use the Internet to keep track of personal health information, such as care received, test results, or upcoming medical appointments; and persons who use the Internet to communicate with their provider.

- The remaining seven new objectives track the proportion of:

- Persons who report that their health care providers always involve them in decisions about their health care as much as they want

- Patients whose doctor recommends personalized health information resources to help them manage their health

- Adults who report having friends or family members whom they talk with about their health

- Online health information seekers who report easily accessing health information

- Medical practices that use electronic health records

- Meaningful users of health IT

- Crisis and emergency risk messages intended to protect the public's health that demonstrate the use of best practices. 
The Healthy People 2020 objectives continue to reflect the importance of the use of health communication strategies and health IT to improve population health outcomes and health care quality, and to achieve health equity. For objectives that were deleted due to lack of data, the U.S. Department of Health and Human Services and the agencies that serve as the leads for the Healthy People 2020 initiative will consider ways to ensure that these public health issues retain prominence despite their current lack of data.

Appendix D, “A Crosswalk Between Objectives From Healthy People 2010 to Healthy People 2020,” summarizes the changes between the two decades of objectives, reflecting new knowledge and direction for this area.

\section{Data Considerations}

In general, data on educational attainment are presented for persons aged 25 years and over, consistent with guidance given by the U.S. Bureau of the Census. However, because of the requirements of the different data systems, the age groups used to calculate educational attainment for any specific objective may differ from the age groups used to report the data for other Healthy People 2010 objectives, as well as from select populations within the same objective. Therefore, the reader is urged to exercise caution in interpreting the data by educational attainment shown in the Health Disparities Table. See Healthy People 2010: General Data Issues, referenced below.

Additional information on data issues is available from the following sources:

- All Healthy People 2010 tracking data can be found in the Healthy People 2010 database, DATA2010, available from http://wonder.cdc.gov/data2010/.

- Detailed information about the data and data sources used to support these objectives can be found in the Operational Definitions on the DATA2010 website, available from http://wonder.cdc.gov/data2010/focusod.htm.

- More information on statistical issues related to Healthy People tracking and measurement can be found in the Technical Appendix and in Healthy People 2010: General Data Issues, which is available in the Data Issues section of the NCHS Healthy People website under Healthy People 2010.

\section{Notes}

1. Displayed in the Progress Chart (Figure 11-1), the percent of targeted change achieved expresses the difference between the baseline and the final value relative to the initial difference between the baseline and the Healthy People 2010 target. As such, it is a relative measure of progress toward attaining the Healthy People 2010 target. See the Reader's Guide for more information. When standard errors were available, the difference between the baseline and the final value was tested at the 0.05 level of significance. See the Figure 11-1 footnotes, as well as the Technical Appendix, for more detail. 
2. Information about disparities among select populations is shown in the Health Disparities Table (Figure 11-2). Disparity from the best group rate is defined as the percent difference between the best group rate and each of the other group rates for a characteristic. For example, racial and ethnic health disparities are measured as the percent difference between the best racial and ethnic group rate and each of the other racial and ethnic group rates. Similarly, disparities by sex are measured as the percent difference between the better group rate (e.g., female) and the rate for the other group (e.g., male). Some objectives are expressed in terms of favorable events or conditions that are to be increased, while others are expressed in terms of adverse events or conditions that are to be reduced. In order to facilitate comparison of health disparities across different objectives, disparity is measured only in terms of adverse events or conditions. For comparability across objectives, objectives that are expressed in terms of favorable events or conditions are re-expressed using the adverse event or condition for the purpose of computing disparity, but they are not otherwise restated or changed. For example, objective 1-1, to increase the proportion of persons with health insurance (e.g., $72 \%$ of the American Indian or Alaska Native population aged under 65 years had some form of health insurance in 2008), is expressed in terms of the percentage of persons without health insurance (e.g., $100 \%-72 \%=28 \%$ of the American Indian or Alaska Native population aged under 65 years did not have any form of health insurance in 2008) when the disparity from the best group rate is calculated. See the Reader's Guide for more information. When standard errors were available, the difference between the best group rate and each of the other group rates was tested at the 0.05 level of significance. See the Figure 11-2 footnotes, as well as the Technical Appendix, for more detail.

3. The change in disparity is estimated by subtracting the disparity at baseline from the disparity at the most recent data point and, therefore, is expressed as a change in percentage points. See the Reader's Guide for more information. When standard errors were available, the change in disparity was tested at the 0.05 level of significance. See the Figure 11-2 footnotes, as well as the Technical Appendix, for more detail.

4. To be included in Healthy People 2010, an objective must have a national data source that provides a baseline and at least one additional data point for tracking progress. Some objectives lacked baseline data at the time of their development but had a potential data source and were considered of sufficient national importance to be included in Healthy People. These are called "developmental" objectives. When data become available, a developmental objective is moved to measurable status and a Healthy People target can be set.

5. Retained "as is" objectives have no change in the numerator definition or in the denominator definition between the Healthy People 2010 and Healthy People 2020 objectives. These include objectives that were developmental in Healthy People 2010 and are developmental in Healthy People 2020 and for which no numerator or denominator information was available.

6. Modified objectives have some change in the numerator definition or in the denominator definition between the Healthy People 2010 and Healthy People 2020 objectives. These include objectives that went from developmental in Healthy People 2010 to measurable in Healthy People 2020 or vice versa.

7. Archived objectives had at least one data point in Healthy People 2010 but were not carried forward into Healthy People 2020. 


\section{Comprehensive Summary of Objectives: Health Communication}

\begin{tabular}{|c|c|}
\hline Objective & Description \\
\hline $11-1$ & Persons with Internet access at home $(18+$ years $)$ \\
\hline $11-2 \mathrm{a}$ & Persons with proficient health literacy (16+ years) \\
\hline $11-2 b$ & Persons with below basic health literacy $(16+$ years $)$ \\
\hline $11-3 a$ & $\begin{array}{l}\text { DHHS-sponsored health communication campaigns that include formative } \\
\text { evaluation }\end{array}$ \\
\hline $11-3 b$ & $\begin{array}{l}\text { DHHS-sponsored health communication campaigns that include process } \\
\text { evaluation }\end{array}$ \\
\hline $11-3 c$ & $\begin{array}{l}\text { DHHS-sponsored health communication campaigns that include outcome } \\
\text { evaluation }\end{array}$ \\
\hline $11-4 \mathrm{a}$ & Health websites that disclose identity persons/organization responsible \\
\hline $11-4 b$ & Health websites that disclose purpose/uses/limitations \\
\hline $11-4 c$ & Health websites that disclose content development practices/policies \\
\hline $11-4 d$ & Health websites that disclose privacy policy/protection \\
\hline $11-4 \mathrm{e}$ & Health websites that disclose user feedback/evaluation \\
\hline $11-4 f$ & Health websites that disclose content creation/updating \\
\hline $11-4 \mathrm{~g}$ & Health websites that disclose three or more of the above criteria \\
\hline $11-5$ & Centers for excellence in health communication \\
\hline $11-6 a$ & $\begin{array}{l}\text { Patients reporting doctors or other health providers always listen carefully } \\
\text { to them }(18+\text { years })\end{array}$ \\
\hline $11-6 b$ & $\begin{array}{l}\text { Patients reporting doctors or other health providers always explain things } \\
\text { so they can understand }(18+\text { years })\end{array}$ \\
\hline $11-6 c$ & $\begin{array}{l}\text { Patients reporting doctors or other health providers always show respect } \\
\text { for what they have to say }(18+\text { years })\end{array}$ \\
\hline $11-6 d$ & $\begin{array}{l}\text { Patients reporting doctors or other health providers always spend enough } \\
\text { time with them }(18+\text { years })\end{array}$ \\
\hline
\end{tabular}

Data Source

Current Population Survey (CPS): Department of Commerce, Census

Bureau; Department of Labor, Bureau of Labor Statistics.

National Assessment of Health Literacy (NAAL), National Center for Education Statistics (NCES), U.S. Department of Education.

National Assessment of Health Literacy (NAAL), National Center for Education Statistics (NCES), U.S. Department of Education.

DHHS, Office of Disease Prevention and Health Promotion.

DHHS, Office of Disease Prevention and Health Promotion.

DHHS, Office of Disease Prevention and Health Promotion.

DHHS, Office of Disease Prevention and Health Promotion.

DHHS, Office of Disease Prevention and Health Promotion.

DHHS, Office of Disease Prevention and Health Promotion.

DHHS, Office of Disease Prevention and Health Promotion.

DHHS, Office of Disease Prevention and Health Promotion.

DHHS, Office of Disease Prevention and Health Promotion.

DHHS, Office of Disease Prevention and Health Promotion.

NIH, NCI.

Medical Expenditure Panel Survey (MEPS), AHRQ.

Medical Expenditure Panel Survey (MEPS), AHRQ.

Medical Expenditure Panel Survey (MEPS), AHRQ.

Medical Expenditure Panel Survey (MEPS), AHRQ. 
Figure 11-1. Progress Toward Target Attainment for Focus Area 11: Health Communication

Moved away

from target ${ }^{1}$

Moved toward

target

Met or exceeded

target

11-1. Persons with Internet access at home (18+ years)

11-3. DHHS-sponsored health communication campaigns that include

a. Formative evaluation

b. Process evaluation

c. Outcome evaluation

11-4. Health websites that disclose

a. Identity (persons/organization responsible) ${ }^{5}$

b. Purpose/uses/limitations

c. Content development practices/policies ${ }^{5}$

d. Privacy policy/protection

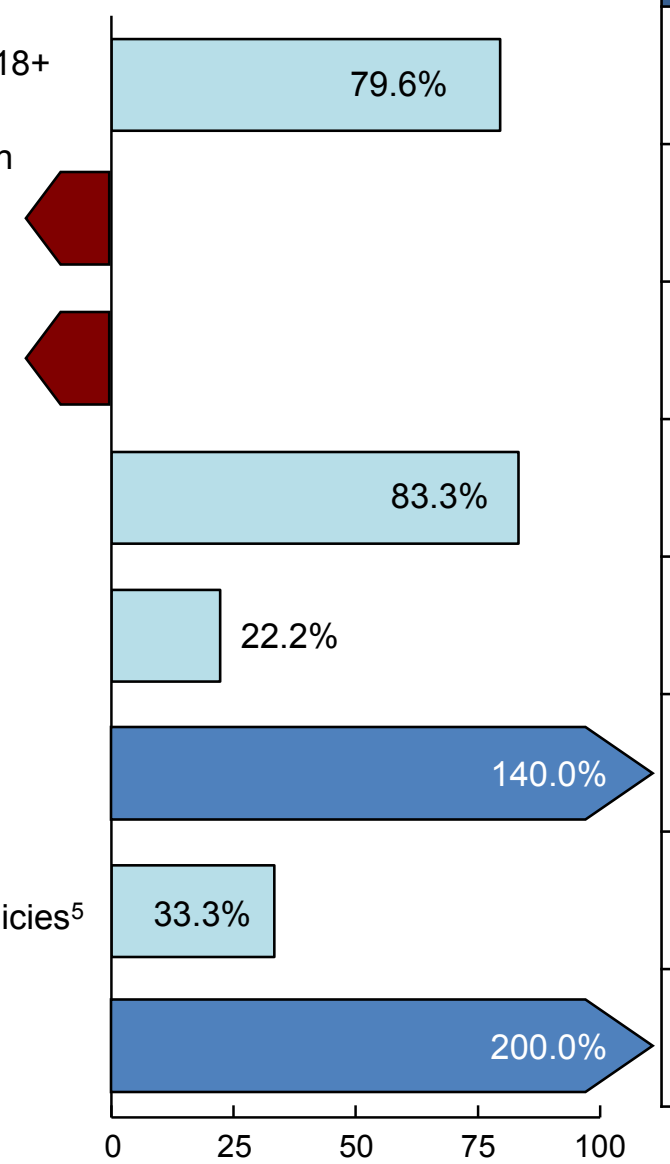

Percent of targeted change achieved 6

\begin{tabular}{|c|c|c|c|c|c|}
\hline \multirow{2}{*}{$\begin{array}{l}2010 \\
\text { Target }\end{array}$} & \multirow{2}{*}{$\begin{array}{c}\text { Baseline } \\
\text { (Year) }\end{array}$} & \multirow{2}{*}{$\begin{array}{l}\text { Final } \\
\text { (Year) }\end{array}$} & \multicolumn{3}{|c|}{ Baseline vs. Final } \\
\hline & & & Difference ${ }^{2}$ & $\begin{array}{l}\text { Statistically } \\
\text { Significant }^{3}\end{array}$ & $\begin{array}{l}\text { Percent } \\
\text { Change }\end{array}$ \\
\hline $80 \%$ & $\begin{array}{c}26 \% \\
(1998)\end{array}$ & $\begin{array}{c}69 \% \\
(2009)\end{array}$ & 43 & Yes & $165.4 \%$ \\
\hline $100 \%$ & $\begin{array}{c}95 \% \\
(2005)\end{array}$ & $\begin{array}{c}80 \% \\
(2009)\end{array}$ & -15 & $\begin{array}{c}\text { Not } \\
\text { tested }\end{array}$ & $-15.8 \%$ \\
\hline $89 \%$ & $\begin{array}{c}81 \% \\
(2005)\end{array}$ & $\begin{array}{c}68 \% \\
(2009)\end{array}$ & -13 & $\begin{array}{l}\text { Not } \\
\text { tested }\end{array}$ & $-16.0 \%$ \\
\hline $65 \%$ & $\begin{array}{c}59 \% \\
(2005)\end{array}$ & $\begin{array}{c}64 \% \\
(2009)\end{array}$ & 5 & $\begin{array}{c}\text { Not } \\
\text { tested }\end{array}$ & $8.5 \%$ \\
\hline $19 \%$ & $\begin{array}{c}10 \% \\
(2006)\end{array}$ & $\begin{array}{c}12 \% \\
(2009)\end{array}$ & 2 & No & $20.0 \%$ \\
\hline $40 \%$ & $\begin{array}{c}35 \% \\
(2006)\end{array}$ & $\begin{array}{c}42 \% \\
(2009)\end{array}$ & 7 & No & $20.0 \%$ \\
\hline $10 \%$ & $\begin{array}{c}1 \% \\
(2006)\end{array}$ & $\begin{array}{c}4 \% \\
(2009)\end{array}$ & 3 & Yes & $300.0 \%$ \\
\hline $80 \%$ & $\begin{array}{c}75 \% \\
(2006)\end{array}$ & $\begin{array}{c}85 \% \\
(2009)\end{array}$ & 10 & No & $13.3 \%$ \\
\hline
\end{tabular}

(continued) 
Figure 11-1. Progress Toward Target Attainment for Focus Area 11: Health Communication (continued)

Moved away

from target ${ }^{1}$

Moved toward

target

Met or exceeded

target

11-4 Health websites that disclose

e. User feedback/evaluation

f. Content creation/updating ${ }^{5}$

g. Three or more of the above criteria

11-5. Centers for excellence in health communication

11-6. Patients (18+ years) reporting doctors or other health providers always

a. Listen carefully to them

b. Explain things so they can understand

c. Show respect for what they have to say

d. Spend enough time with them

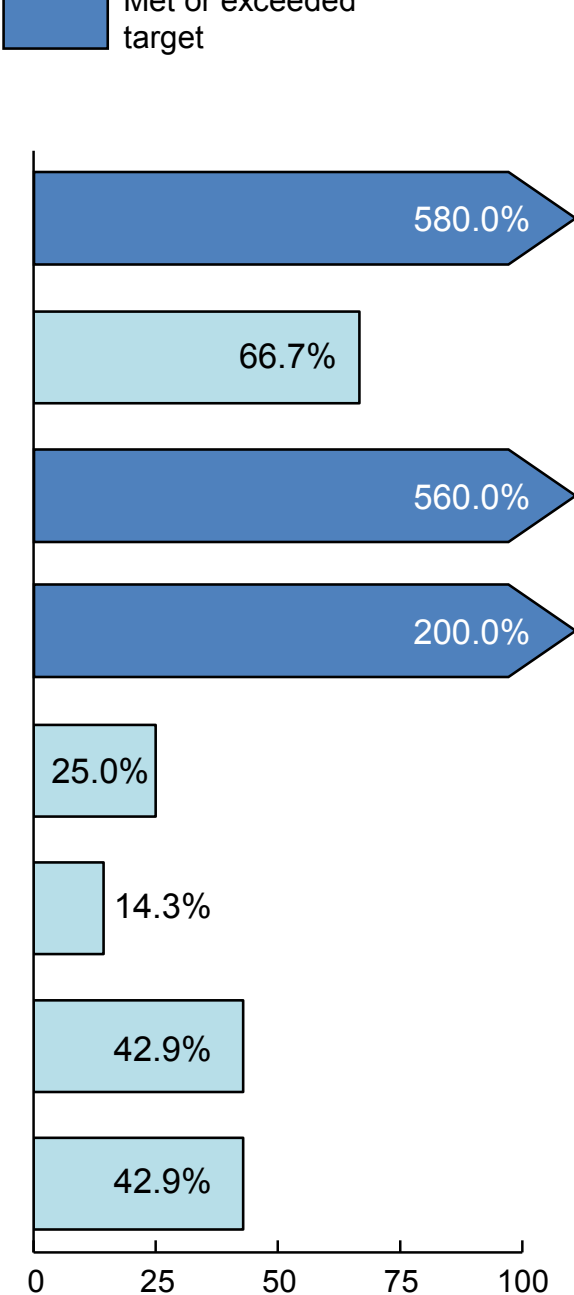

\begin{tabular}{|c|c|c|c|c|c|}
\hline \multirow{2}{*}{$\begin{array}{l}2010 \\
\text { Target }\end{array}$} & \multirow{2}{*}{$\begin{array}{c}\text { Baseline } \\
\text { (Year) }\end{array}$} & \multirow{2}{*}{$\begin{array}{l}\text { Final } \\
\text { (Year) }\end{array}$} & \multicolumn{3}{|c|}{ Baseline vs. Final } \\
\hline & & & Difference ${ }^{2}$ & $\begin{array}{l}\text { Statistically } \\
\text { Significant }^{3}\end{array}$ & $\begin{array}{l}\text { Percent } \\
\text { Change }^{4}\end{array}$ \\
\hline $64 \%$ & $\begin{array}{c}59 \% \\
(2006)\end{array}$ & $\begin{array}{c}88 \% \\
(2009)\end{array}$ & 29 & Yes & $49.2 \%$ \\
\hline $10 \%$ & $\begin{array}{c}1 \% \\
(2006)\end{array}$ & $\begin{array}{c}7 \% \\
(2009)\end{array}$ & 6 & Yes & $600.0 \%$ \\
\hline $29 \%$ & $\begin{array}{c}24 \% \\
(2006)\end{array}$ & $\begin{array}{c}52 \% \\
(2009)\end{array}$ & 28 & $\begin{array}{c}\text { Not } \\
\text { tested }\end{array}$ & $116.7 \%$ \\
\hline 6 & $\begin{array}{c}4 \\
(2003)\end{array}$ & $\begin{array}{c}8 \\
(2006)\end{array}$ & 4 & $\begin{array}{c}\text { Not } \\
\text { tested }\end{array}$ & $100.0 \%$ \\
\hline $65 \%$ & $\begin{array}{c}57 \% \\
(2000)\end{array}$ & $\begin{array}{c}59 \% \\
(2007)\end{array}$ & 2 & Yes & $3.5 \%$ \\
\hline $66 \%$ & $\begin{array}{c}59 \% \\
(2000)\end{array}$ & $\begin{array}{c}60 \% \\
(2007)\end{array}$ & 1 & No & $1.7 \%$ \\
\hline $66 \%$ & $\begin{array}{c}59 \% \\
(2000)\end{array}$ & $\begin{array}{c}62 \% \\
(2007)\end{array}$ & 3 & Yes & $5.1 \%$ \\
\hline $53 \%$ & $\begin{array}{c}46 \% \\
(2000)\end{array}$ & $\begin{array}{c}49 \% \\
(2007)\end{array}$ & 3 & Yes & $6.5 \%$ \\
\hline
\end{tabular}

Percent of targeted change achieved ${ }^{6}$

(continued) 


\section{Figure 11-1. Progress Toward Target Attainment for Focus Area 11: Health Communication (continued)}

\section{NOTES}

See the Reader's Guide for more information on how to read this figure. See DATA2010 at http://wonder.cdc.gov/data2010 for all Healthy People 2010 tracking data. Tracking data are not available for objectives $11-2 \mathrm{a}$ and $11-2 \mathrm{~b}$

\section{FOOTNOTES}

${ }^{1}$ Movement away from target is not quantified using the percent of targeted change achieved. See Technical Appendix for more information.

${ }^{2}$ Difference $=$ Final value - Baseline value. Differences between percents $(\%)$ are measured in percentage points.

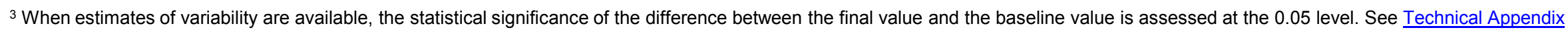
for more information.

${ }^{4}$ Percent change $=\frac{\text { Final value }- \text { Baseline value }}{\text { Baseline value }} \times 100$.

${ }^{5}$ Baseline data are statistically unreliable. Values are shown to allow assessment of full criteria set. Refer to Operational Definition for more information.

${ }^{6}$ Percent of targeted change achieved $=\frac{\text { Final value }- \text { Baseline value }}{\text { Healthy People } 2010 \text { target }- \text { Baseline value }} \times 100$.

\section{DATA SOURCES}

11-1. Current Population Survey (CPS): Department of Commerce, Census Bureau; Department of Labor, Bureau of Labor Statistics.

11-3a-c. DHHS, Office of Disease Prevention and Health Promotion.

11-4a-g. DHHS, Office of Disease Prevention and Health Promotion.

11-5. $\mathrm{NIH}, \mathrm{NCl}$.

11-6a-d. Medical Expenditure Panel Survey (MEPS), AHRQ. 
Figure 11-2. Health Disparities Table for Focus Area 11: Health Communication

Disparities from the best group rate for each characteristic at the most recent data point and changes in disparity from the baseline to the most recent data point.

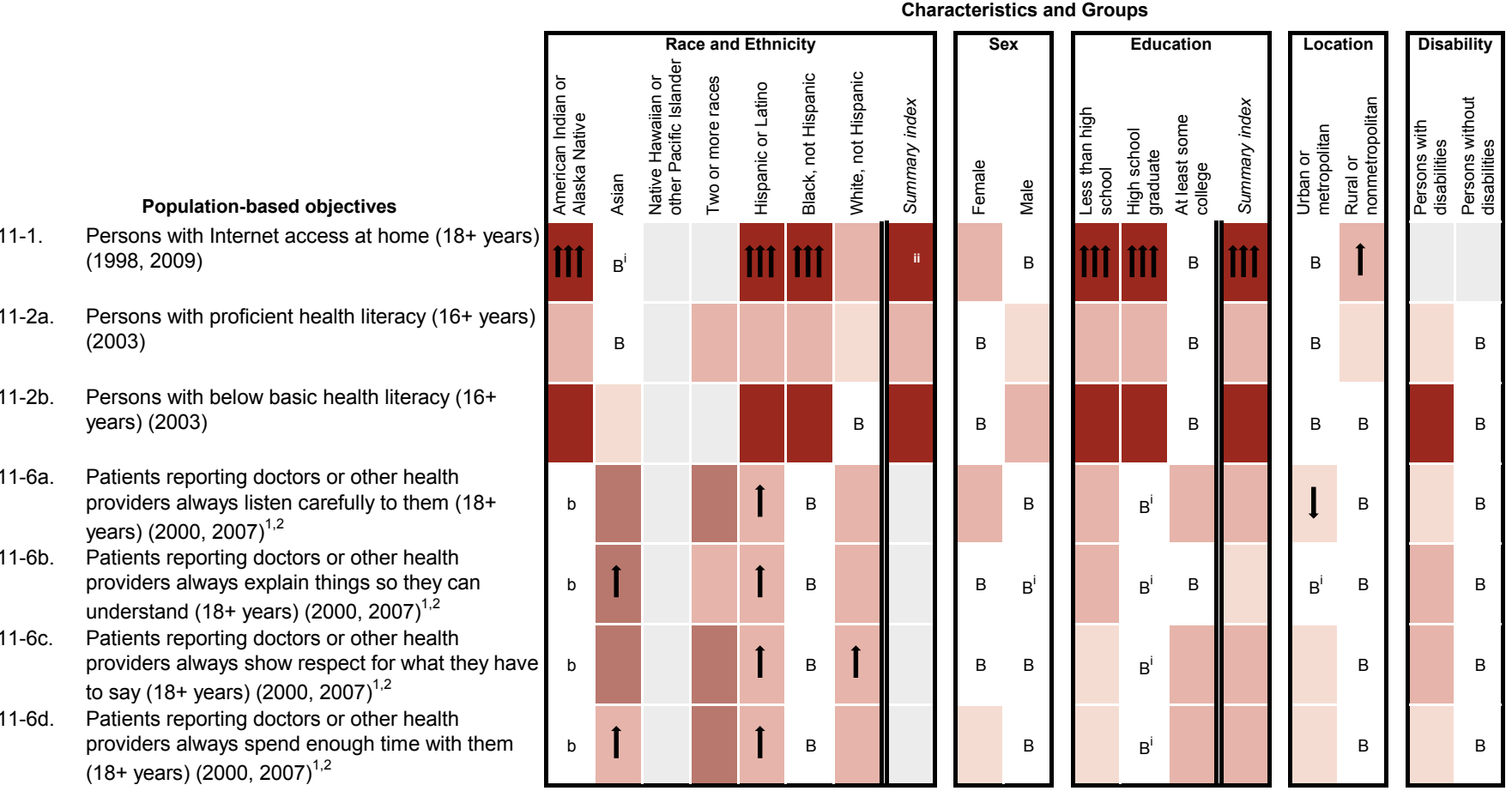

NOTES

See DATA2010 at http://wonder.cdc.gov/data2010 for all Healthy People 2010 tracking data. Disparity data are either unavailable or not applicable for objectives 11-3a through c, 11-4a through $\mathrm{g}$, and 11-5.

Years in parentheses represent the baseline and most recent data years (if available).

Disparity from the best group rate is defined as the percent difference between the best group rate and each of the other group rates for a characteristic (e.g., race and ethnicity). The summary index is the average of these percent differences for a characteristic. Change in disparity is estimated by subtracting the disparity at baseline from the disparity at the most recent data point. Change in the summary index is estimated by subtracting the summary index at baseline from the summary index at the most recent data point. See Technical Appendix for more information.

Measures of variability were available for all the objectives in this table. Thus, the variability of best group rates was assessed, and statistical significance was tested. Disparities of $10 \%$ or more are displayed when the differences from the best group rate are statistically significant at the 0.05 level. Changes in disparities over time are indicated by arrows when the changes are greater than or equal to 10 percentage points and are statistically significant at the 0.05 level. See Technical Appendix.

LEGEND

\begin{tabular}{l|l|l|l|l} 
The "best" group rate at the most recent data point. & B $\begin{array}{l}\text { The group with the best rate for } \\
\text { specified characteristic. }\end{array} \quad \begin{array}{l}\text { Most favorable group rate for specified } \\
\text { characteristic, but reliability criterion not } \\
\text { met. }\end{array}$
\end{tabular}

\begin{tabular}{|c|c|c|c|c|}
\hline \multirow[b]{2}{*}{$\begin{array}{l}\text { Disparity from the best group rate at the most recent } \\
\text { data point. }\end{array}$} & \multicolumn{4}{|c|}{ Percent difference from the best group rate } \\
\hline & $\begin{array}{l}\text { Less than } 10 \% \text {, or difference not } \\
\text { statistically significant (when } \\
\text { estimates of variability are available). }\end{array}$ & $10 \%-49 \%$ & $50 \%-99 \%$ & $\begin{array}{l}100 \% \text { or } \\
\text { more }\end{array}$ \\
\hline
\end{tabular}

Changes in disparity over time are shown when:

(a) disparities data are available at both baseline and most recent time points;

(b) data are not for the group(s) indicated by "B" or "b" at either time point; and

(c) the change is greater than or equal to 10 percentage points and statistically significant, or

when the change is greater than or equal to 10 percentage points and estimates of variability

were not available. See Technical Appendix.

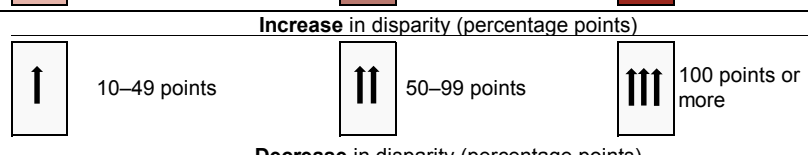

Availability of Data

Data not available

Characteristic not selected for this objective.

\section{FOOTNOTES}

1 Baseline data by race and ethnicity are for 2002 .

2 Most recent data by disability status are for 2004 .

$\mathrm{i}$ The group with the best rate at the most recent data point is different from the group with the best rate at baseline. Both rates met the reliability criterion. See Technical Appendix.

ii Change in the summary index cannot be assessed. See Technical Appendix.

DATA SOURCES

11-1. Current Population Survey (CPS), Department of Commerce, Census Bureau.

11-2a-b. National Assessment of Health Literacy (NAAL), Department of Education, National Center for Education Statistics (NCES).

11-6a-d. Medical Expenditure Panel Survey (MEPS), AHRQ.

Health Communication

Page 11-13 
Figure 11-3. Persons with Internet Access at Home (18+ years) 2009

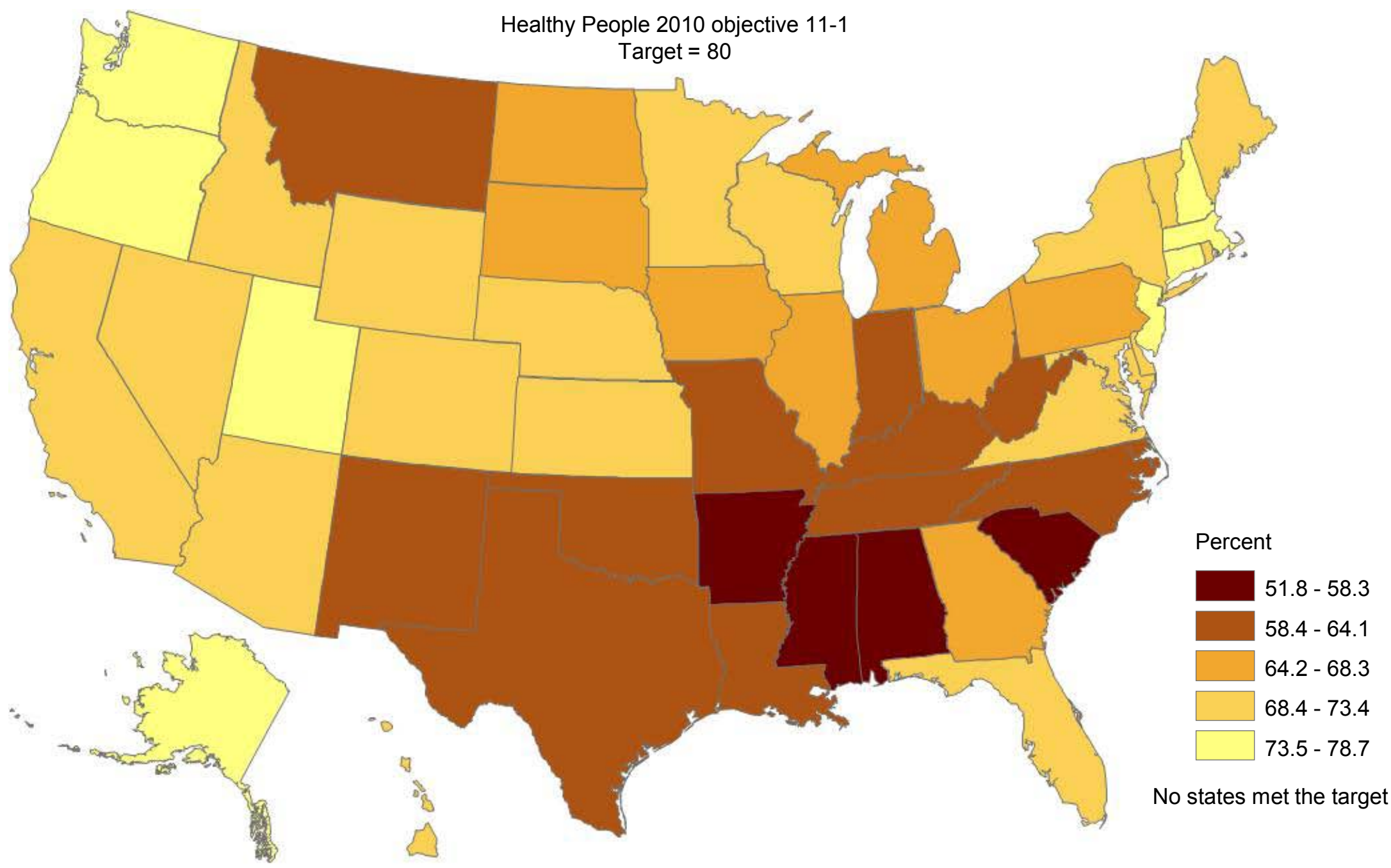

Notes: Rates are displayed by a Jenks classification for U.S. states.

SOURCE: Current Population Survey (CPS), U.S. Department of Commerce, Bureau of the Census. 


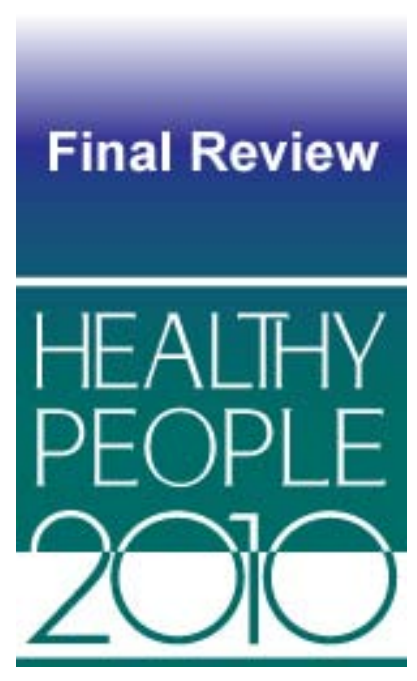

\section{Heart Disease and Stroke}

\section{Co-Lead Agencies}

Centers for Disease Control and Prevention

National Institutes of Health

\section{Contents}

Goal.

Highlights

Summary of Progress

Transition to Healthy People 2020

Data Considerations

References and Notes

Comprehensive Summary of Objectives

Progress Chart

Health Disparities Table

Coronary Heart Disease Death Rates-Map.

Stroke Death Rates-Map 


\section{Goal: Improve cardiovascular health and quality of life through the prevention of risk factors; detection and treatment of risk factors; early identification and treatment of heart attacks and strokes; and prevention of recurrent cardiovascular events}

This chapter includes objectives for the Focus Area that monitors coronary heart disease (CHD) and stroke deaths, heart failure hospitalizations, risk factors for heart disease and stroke, knowledge of heart attack and stroke symptoms and response, and the availability of treatment options.

All Healthy People tracking data quoted in this chapter, along with technical information and operational definitions for each objective, can be found in the Healthy People 2010 database, DATA2010, available from http://wonder.cdc.gov/data2010/.

More information about this Focus Area can be found in the following publications:

- Healthy People 2010: Understanding and Improving Health, available from http://www.healthypeople.gov/2010/Document/tableofcontents.htm\#under.

- Healthy People 2010 Midcourse Review, available from http://www.healthypeople.gov/2010/data/midcourse/html/default.htm\#FocusAreas.

\section{Highlights}

- Substantial progress was achieved for the majority of objectives in this Focus Area over the last decade [1]. Two-thirds of the Heart Disease and Stroke objectives with data to monitor progress moved toward or achieved their Healthy People 2010 targets. However, for three objectives, the change was opposite the direction of the target (Figure 12-1). Moreover, health disparities of $50 \%$ or more among racial and ethnic populations and education groups were observed (Figure 12-2), as highlighted below [2].

- The CHD death rate (objective 12-1) declined by 35.4\% between 1999 and 2007, from 195 to 126 deaths per 100,000 population (age adjusted), exceeding the Healthy People 2010 target of 156 deaths per 100,000. Disparities were observed for a number of population groups, for example:

- Among racial and ethnic groups, the Asian or Pacific Islander population had the lowest (best) CHD mortality rate, 71 deaths per 100,000 population (age adjusted) in 2007, whereas the black non-Hispanic population had a rate of 153 deaths per 100,000 (age adjusted) in 2007. The rate for the black non-Hispanic population was more than twice the best group rate [2]. 
- Among education groups, persons aged 25-64 years with at least some college education had the lowest (best) CHD mortality rate, 30 deaths per 100,000 population (age adjusted) in 2002. Persons aged 25-64 years with less than a high school education had a rate of 83 deaths per 100,000 (age adjusted) in 2002, and high school graduates in the same age group had a rate of 71 deaths per 100,000 (age adjusted) in 2002. The rate for persons with less than a high school education was almost three times the best group rate, while the rate for high school graduates was almost two and a half times the best group rate [2].

- While the Healthy People 2010 target for CHD deaths was largely met throughout the U.S., there remained geographic pockets with higher rates along the OhioMississippi River Basin, a geographic region commonly referred to as the "Coronary Valley" (Figure 12-3).

- The stroke death rate (objective 12-7) declined by 32.3\% between 1999 and 2007, from 62 to 42 deaths per 100,000 (age adjusted), exceeding the 2010 target of 50 deaths per 100,000 . Disparities were observed for a number of population groups, for example:

- Among racial and ethnic groups, the American Indian or Alaska Native population had the lowest (best) rate, 30 deaths per 100,000 population (age adjusted) in 2007. The black non-Hispanic population had a rate of 62 deaths per 100,000 (age adjusted) in 2007, more than twice the best group rate [2].

- Among education groups, persons aged 25-64 years with at least some college education had the lowest (best) stroke death rate, 7 deaths per 100,000 population (age adjusted) in 2002. Persons aged 25-64 years with less than a high school education had a rate of 21 deaths per 100,000 (age adjusted) in 2002, and high school graduates in the same age group had a rate of 16 deaths per 100,000 (age adjusted) in 2002. The rate for persons with less than a high school education was three times the best group rate, while the rate for high school graduates was almost two and a half times the best group rate [2].

- While the Healthy People 2010 target for stroke deaths was largely met throughout the U.S., there remained geographic pockets with higher rates in the Southeast, a geographic region commonly referred to as the "Stroke Belt" (Figure 12-4).

- Hospitalization rates for congestive heart failure among persons aged 65-74 years (objective 12-6a) declined by $35.6 \%$ between 1997 and 2007, from 13.2 to 8.5 hospitalizations per 1,000, moving toward the 2010 target of 6.5 hospitalizations per 1,000 . Congestive heart failure hospitalizations among persons aged 75-84 years (objective 126b), declined by $26.2 \%$ between 1997 and 2007, from 26.7 to 19.7 hospitalizations per 1,000, moving toward the 2010 target of 13.5 hospitalizations per 1,000; and among persons aged 85 years and over (objective 12-6c), hospitalizations declined by $37.6 \%$, from 52.7 to 32.9 per 1,000 , moving toward the 2010 target of 26.5 hospitalizations per 1,000 .

- In the 65-74 years age group (objective 12-6a), the white population had the lowest (best) rate among racial and ethnic groups, 5.9 hospitalizations per 1,000 population in 2007. The black population had a rate of 14.0 per 1,000 in 2007, nearly two and a half times the best group rate [2].

- In the 75-84 years age group (objective 12-6b), the white population also had the lowest (best) rate among racial and ethnic groups, 14.8 hospitlaizations per 1,000 population in 2007. The black population had a rate of 25.9 per 1,000 in 2007, almost twice the best group rate [2]. 
- The proportion of persons aged 18 years and over who had their blood pressure measured in the past two years and who know their blood pressure level (objective 12-12) increased by $1.1 \%$ between 1998 and 2008, from $90 \%$ to $91 \%$ (age adjusted), moving toward the Healthy People 2010 target of $95 \%$. Disparities were observed for a number of population groups, for example:

- Among racial and ethnic groups, the white non-Hispanic and black non-Hispanic populations had the highest (best) rate of blood pressure monitoring, 92\% (age adjusted) in 2008, whereas the Hispanic or Latino population had a rate of $82 \%$ (age adjusted) in 2008. When expressed as persons who do no monitoring, the rate for the Hispanic or Latino population was more than twice the best group rate [2].

- Among education groups, persons aged 25 years and over with at least some college education had the highest (best) rate of blood pressure monitoring, 94\% in 2008, whereas persons aged 25 years and over with less than a high school education had a rate of $83 \%$ in 2008 . When expressed as persons who do no monitoring, the rate for persons with less than a high school education was almost three times the best group rate [2].

- Persons with less than a high school education had blood pressure monitoring rates of $84 \%$ in 1998 and $83 \%$ in 2008, whereas persons with at least some college education had rates of $93 \%$ in 1998 and $94 \%$ in 2008. When rates are expressed in terms of persons who do no monitoring, the disparity between persons without a high school education and those with at least some college education decreased by 55 percentage points between 1998 and 2008 [2,3].

- Mean total blood cholesterol levels among persons aged 20 years and over (objective 1213) declined by 3.9\% between 1988-94 and 2005-08, from 206 to $198 \mathrm{mg} / \mathrm{dL}$ (age adjusted), exceeding the 2010 target of $199 \mathrm{mg} / \mathrm{dL}$. During the same period, the proportion of persons aged 20 years and over with high blood cholesterol levels (objective 12-14) fell by $28.6 \%$, from $21 \%$ to $15 \%$ (age adjusted), exceeding the 2010 target of $17 \%$.

- The proportion of persons aged 18 years and over who were aware of the symptoms of a heart attack and the importance of calling 911 (objective 12-2) declined by $11.9 \%$ between 2001 and 2008, from $42 \%$ to 37\% (age adjusted), moving away from the 2010 target of $47 \%$.

- The proportion of persons aged 18 years and over who were aware of the symptoms of a stroke (objective 12-8) declined by $10.0 \%$ between 2001 and 2009 , from $60 \%$ to $54 \%$ (age adjusted), moving away from the 2010 target of $65 \%$.

- The prevalence of hypertension among persons aged 18 years and over (objective 12-9) increased by $20.0 \%$ between $1988-94$ and $2005-08$, from $25 \%$ to $30 \%$ (age adjusted), moving away from the 2010 target of $14 \%$. Disparities were observed for a number of population groups, for example:

- Among racial and ethnic populations, the Mexican American population had the lowest (best) rate, $26 \%$ (age adjusted) in 2005-08. The rate for the black nonHispanic population was $42 \%$ (age adjusted) in 2005-08, more than one and a half times the best group rate [2]. 


\section{Summary of Progress}

- Figure 12-1 presents a quantitative assessment of progress in achieving the Healthy People 2010 objectives for Heart Disease and Stroke [1]. Data to measure progress toward target attainment were available for 15 objectives. Of these:

- Four objectives (12-1, 12-7, 12-13, and 12-14) exceeded their Healthy People 2010 targets.

- Eight objectives (12-4, 12-6a through c, 12-10 through 12-12, and 12-15) moved toward their targets. A statistically significant difference between the baseline and the final data points was observed for each of these objectives.

- Three objectives (12-2, 12-8, and 12-9) moved away from their targets. A statistically significant difference between the baseline and final data points was observed for each of these objectives.

- Two objectives (12-5 and 12-16) remained developmental and two objectives (12-3a and b) had no data available to measure progress [4].

- Figure 12-2 displays health disparities in Heart Disease and Stroke from the best group rate for each characteristic at the most recent data point [2]. It also displays changes in disparities from baseline to the most recent data point [3].

- Of the 11 objectives with statistically significant racial and ethnic health disparities of $10 \%$ or more, the white non-Hispanic population had the only best rate for three objectives $(12-2,12-8$, and 12-10) and the white population (including persons of Hispanic origin) had the best rate for two objectives (12-6a and b). The American Indian or Alaska Native (objective 12-7), Asian or Pacific Islander (objective 12-1), Asian (objective 12-15), Mexican American (objective 12-9) and black non-Hispanic populations (objective 12-14) each had the only best rate for one objective. The black non-Hispanic and white non-Hispanic populations were tied for the best group rate for one objective (12-12).

- Females had better rates than males for eight of the nine objectives with statistically significant health disparities of $10 \%$ or more by sex (objectives $12-1,12-2,12-6$ a and $b, 12-8,12-10,12-12$, and 12-15). Males had a better rate for one objective (1214).

- Persons with at least some college education had the best rates for all seven of the objectives with statistically significant health disparities of $10 \%$ or more by education level (objectives 12-1, 12-2, 12-4, 12-7, 12-8, 12-12, and 12-15).

- Persons with middle/high incomes had the best rate for both of the objectives with statistically significant health disparities of $10 \%$ or more by income (objectives $12-9$ and 12-10).

- Persons without disabilities had a better rate than persons with disabilities for two of the three objectives with statistically significant health disparities of $10 \%$ or more by disability status (objectives 12-8 and 12-9). Persons with disabilities had a better rate for the other objective (12-10).

- There were several objectives with health disparities of $100 \%$ or more. Most of these are discussed in the Highlights, above. 


\section{Transition to Healthy People 2020}

The goal of the Healthy People 2020 Heart Disease and Stroke Topic Area is consistent with the Healthy People 2010 goal (stated on page 12-2, above). The Healthy People 2020 objectives expand upon the prevalence, treatment, and control of individual heart disease and stroke risk factors and also include an overall measure of cardiovascular health that takes into account the status and interaction of all major cardiovascular disease (CVD) risk factors to generate a composite CVD risk score. The objectives also plan to monitor rehabilitation following heart attack and stroke. See HealthyPeople.gov for a complete list of Healthy People 2020 topics and objectives.

The Healthy People 2020 Heart Disease and Stroke Topic Area can be grouped into five sections:

- Prevention of risk factors

- Detection and treatment of risk factors

- Early identification and treatment of heart attack and strokes

- Prevention of recurrent cardiovascular events

- Cross-cutting.

The differences between the Healthy People 2010 objectives and those included in Healthy People 2020 objectives are summarized below:

- The Healthy People 2020 Heart Disease and Stroke Topic Area has a total of 49 objectives, 31 of which are developmental [4]. The Healthy People 2010 Heart Disease and Stroke Focus Area had 19 objectives, two of which were developmental.

- Ten Healthy People 2010 objectives were retained "as is" [5].

- Nine objectives were retained as measurable: coronary heart disease deaths (objective 12-1), stroke deaths (objective 12-7), knowledge of stroke symptoms (objective 12-8), hypertension prevalence (objective 12-9), blood pressure control (objective 12-10), blood pressure monitoring (objective 12-12), mean total cholesterol (objective 12-13), prevalence of high cholesterol (objective 12-14), and cholesterol screening (objective 12-15).

- One objective, adults with CHD who have their LDL-cholesterol at or below the recommended level (objective 12-16), was retained as developmental.

- Eight Healthy People 2010 objectives were modified to create 12 Healthy People 2020 objectives [6].

- Three objectives for congestive heart failure hospitalizations among older adults aged 65-74, 75-84, and 85 years and over (objectives 12-6a through c) were expanded to heart failure hospitalizations.

- Fibrinolytic therapy within an hour of symptom onset and percutaneous intervention therapy within 90 minutes of symptom onset of heart attack (objectives 12-3a and b) were modified to within 30 and 90 minutes of hospital arrival, respectively. 
- Training in cardiopulmonary resuscitation (CPR) in the past year (objective 12-4) and timely electrical shock therapy for out-of-hospital cardiac arrest (objective 125 ) were combined into one developmental objective. The resulting Healthy People 2020 objective addresses appropriate bystander response to, and emergency medical services for, out-of hospital cardiac arrest.

- Taking action to help control blood pressure (objective 12-11) was divided into six objectives. There are five developmental objectives for hypertension regarding meeting recommended guidelines for body mass index (BMI), saturated fat consumption, sodium intake, physical activity, and moderate alcohol consumption as well as one objective for prescribed antihypertensive medication use among adults with hypertension.

- One Healthy People 2010 objective (objective 12-2) that tracks knowledge of heart attack symptoms and importance of calling 911 was retained "as is" and also modified to create three Healthy People 2020 objectives. The other two objectives separately track the two knowledge components.

- Twenty-four new objectives, all of which are developmental, were added to the Healthy People 2020 Heart Disease and Stroke Topic Area:

- A new objective tracking overall cardiovascular health.

- A new objective monitoring hypertension prevalence among children and adolescents.

- Five new objectives for prehypertension regarding meeting recommended guidelines for body mass index (BMI), saturated fat consumption, sodium intake, physical activity, and moderate alcohol consumption.

- Eight new objectives on cholesterol lowering management advice and adherence: diet, physical activity, weight control, and prescribed drug therapy.

- Three new objectives on aspirin use for CVD risk reduction.

- Two new rehabilitation objectives for heart attack and stroke survivors.

- Four new stroke objectives including knowledge of stroke symptoms and the importance of calling 911, knowledge of the importance of calling 911 for stroke, acute reperfusion therapy within three hours of symptom onset for stroke patients, and adults who have had a stroke who have their LDL-cholesterol at or below recommended levels.

Six new objectives that address incidence, case fatality, and recurrence rates for both heart attacks and strokes were proposed but not included in the Healthy People 2020 Heart Disease and Stroke Topic Area due to lack of national data sources.

Appendix D, “A Crosswalk Between Objectives from Healthy People 2010 to Healthy People 2020," summarizes the changes between the two decades of objectives, reflecting new knowledge and direction for this area. 


\section{Data Considerations}

Education and income are the primary measures of socioeconomic status (SES) in Healthy People 2010. Most data systems used in Healthy People 2010 define income as a family's income before taxes. In order to facilitate comparisons among groups and over time, while adjusting for family size and for inflation, Healthy People 2010 categorizes income using the poverty thresholds developed by the U.S. Census Bureau. Thus, the three categories of family income that are primarily used are:

- Poor-below the Federal poverty level

- Near poor $-100 \%$ to $199 \%$ of the Federal poverty level

- Middle/high income-200\% or more of the Federal poverty level.

These categories may be overridden by considerations specific to the data system, in which case they are modified as appropriate. See Healthy People 2010: General Data Issues, referenced below.

Beginning in 2003, education data for CHD and stroke deaths (objectives 12-1 and 12-7) from the National Vital Statistics System were suppressed. The educational attainment item was changed in the new U.S. Standard Certificate of Death in 2003 to be consistent with the U.S. Census Bureau data and to improve the ability to identify specific types of educational degrees. Many states, however, are still using the 1989 version of the U.S. Standard Certificate of Death, which focuses on highest school grade completed. As a result, educational attainment data collected using the 2003 version are not comparable with data collected using the 1989 version [7].

In general, data on educational attainment are presented for persons aged 25 years and over, consistent with guidance given by the U.S. Bureau of the Census. However, because of the requirements of the different data systems, the age groups used to calculate educational attainment for any specific objective may differ from the age groups used to report the data for other Healthy People 2010 objectives, as well as from select populations within the same objective. Therefore, the reader is urged to exercise caution in interpreting the data by educational attainment shown in the Health Disparities Table. See Healthy People 2010: General Data Issues, referenced below.

Additional information on data issues is available from the following sources:

- All Healthy People 2010 tracking data can be found in the Healthy People 2010 database, DATA2010, available from http://wonder.cdc.gov/data2010/.

- Detailed information about the data and data sources used to support these objectives can be found in the Operational Definitions on the DATA2010 website, available from http://wonder.cdc.gov/data2010/focusod.htm.

- More information on statistical issues related to Healthy People tracking and measurement can be found in the Technical Appendix and in Healthy People 2010: General Data Issues, which is available in the Data Issues section of the NCHS Healthy People website under Healthy People 2010. 


\section{References and Notes}

1. Displayed in the Progress Chart (Figure 12-1), the percent of targeted change achieved expresses the difference between the baseline and the final value relative to the initial difference between the baseline and the Healthy People 2010 target. As such, it is a relative measure of progress toward attaining the Healthy People 2010 target. See the Reader's Guide for more information. When standard errors were available, the difference between the baseline and the final value was tested at the 0.05 level of significance. See the Figure 12-1 footnotes, as well as the Technical Appendix for more detail.

2. Information about disparities among select populations is shown in the Health Disparities Table (Figure 12-2). Disparity from the best group rate is defined as the percent difference between the best group rate and each of the other group rates for a characteristic. For example, racial and ethnic health disparities are measured as the percent difference between the best racial and ethnic group rate and each of the other racial and ethnic group rates. Similarly, disparities by sex are measured as the percent difference between the better group rate (e.g., female) and the rate for the other group (e.g., male). Some objectives are expressed in terms of favorable events or conditions that are to be increased, while others are expressed in terms of adverse events or conditions that are to be reduced. In order to facilitate comparison of health disparities across different objectives, disparity is measured only in terms of adverse events or conditions. For comparability across objectives, objectives that are expressed in terms of favorable events or conditions are re-expressed using the adverse event or condition for the purpose of computing disparity, but they are not otherwise restated or changed. For example, objective 1-1, to increase the proportion of persons with health insurance (e.g., $72 \%$ of the American Indian or Alaska Native population aged under 65 years had some form of health insurance in 2008), is expressed in terms of the percentage of persons without health insurance (e.g., $100 \%-72 \%=28 \%$ of the American Indian or Alaska Native population aged under 65 years did not have any form of health insurance in 2008) when the disparity from the best group rate is calculated. See the Reader's Guide for more information. When standard errors were available, the difference between the best group rate and each of the other group rates was tested at the 0.05 level of significance. See the Figure 12-2 footnotes, as well as the Technical Appendix, for more detail.

3. The change in disparity is estimated by subtracting the disparity at baseline from the disparity at the most recent data point and, therefore, is expressed as a change in percentage points. See the Reader's Guide for more information. When standard errors were available, the change in disparity was tested at the 0.05 level of significance. See the Figure 12-2 footnotes, as well as the Technical Appendix, for more detail.

4. To be included in Healthy People 2010, an objective must have a national data source that provides a baseline and at least one additional data point for tracking progress. Some objectives lacked baseline data at the time of their development but had a potential data source and were considered of sufficient national importance to be included in Healthy People. These are called "developmental" objectives. When data become available, a developmental objective is moved to measurable status and a Healthy People target can be set.

5. Retained "as is" objectives have no change in the numerator definition or in the denominator definition between the Healthy People 2010 and Healthy People 2020 objectives. These include objectives that were developmental in Healthy People 2010 and are developmental in Healthy People 2020 and for which no numerator or denominator information was available.

6. Modified objectives have some change in the numerator definition or in the denominator definition between the Healthy People 2010 and Healthy People 2020 objectives. These include objectives that went from developmental in Healthy People 2010 to measurable in Healthy People 2020 or vice versa.

7. Xu JQ, Kochanek KD, Murphy SL, Tejada-Vera B. Deaths: Final data for 2007. National vital statistics reports; vol 58 no 19. Hyattsville, MD: National Center for Health Statistics. 2010. Available from: http://www.cdc.gov/nchs/data/nvsr/nvsr58/nvsr58 19.pdf. 


\section{Comprehensive Summary of Objectives: Heart Disease and Stroke}

\begin{tabular}{|c|c|c|}
\hline Objective & Description & Data Source or Objective Status \\
\hline $12-1$ & $\begin{array}{l}\text { Coronary heart disease (CHD) deaths (age adjusted per 100,000 } \\
\text { standard population) }\end{array}$ & National Vital Statistics System-Mortality (NVSS-M), CDC, NCHS. \\
\hline $12-2$ & $\begin{array}{l}\text { Knowledge of heart attack symptoms and importance of calling } 911 \\
\text { (age adjusted, } 20+\text { years) }\end{array}$ & National Health Interview Survey (NHIS), CDC, NCHS. \\
\hline $12-3 a$ & Fibrinolytics within an hour of symptom onset & $\begin{array}{l}\text { National Registry of Myocardial Infarction (NRMI-4), National Acute } \\
\text { Myocardial Infarction Project, CMS }\end{array}$ \\
\hline $12-3 b$ & Percutaneous intervention (PCI) within 90 minutes of symptom onset & $\begin{array}{l}\text { National Registry of Myocardial Infarction (NRMI-4), National Acute } \\
\text { Myocardial Infarction Project, CMS }\end{array}$ \\
\hline $12-4$ & $\begin{array}{l}\text { Training in cardiopulmonary resuscitation (CPR) in past year (age } \\
\text { adjusted, } 20+\text { years) }\end{array}$ & National Health Interview Survey (NHIS), CDC, NCHS. \\
\hline $12-5$ & Timely electrical shock therapy for out-of-hospital cardiac arrest & Developmental. \\
\hline $12-6 a$ & $\begin{array}{l}\text { Congestive heart failure hospitalizations }-65-74 \text { years (per } 1,000 \\
\text { population) }\end{array}$ & National Hospital Discharge Survey (NHDS), CDC, NCHS. \\
\hline $12-6 b$ & $\begin{array}{l}\text { Congestive heart failure hospitalizations }-75-84 \text { years (per } 1,000 \\
\text { population) }\end{array}$ & National Hospital Discharge Survey (NHDS), CDC, NCHS. \\
\hline $12-6 c$ & $\begin{array}{l}\text { Congestive heart failure hospitalizations-85+ years (per 1,000 } \\
\text { population) }\end{array}$ & National Hospital Discharge Survey (NHDS), CDC, NCHS. \\
\hline $12-7$ & Stroke deaths (age adjusted per 100,000 standard population) & National Vital Statistics System-Mortality (NVSS-M), CDC, NCHS. \\
\hline $12-8$ & Knowledge of stroke symptoms (age adjusted, $20+$ years) & National Health Interview Survey (NHIS), CDC, NCHS. \\
\hline $12-9$ & High blood pressure (age adjusted, 18+ years) & National Health and Nutrition Examination Survey (NHANES), CDC, NCHS. \\
\hline $12-10$ & High blood pressure control (age adjusted, 18+ years) & National Health and Nutrition Examination Survey (NHANES), CDC, NCHS. \\
\hline $12-11$ & Taking action to help control blood pressure (age adjusted, 18+ years) & National Health Interview Survey (NHIS), CDC, NCHS. \\
\hline $12-12$ & $\begin{array}{l}\text { Adults who had their blood pressure measured in past } 2 \text { years and } \\
\text { know their blood pressure level (age adjusted, } 18+\text { years) }\end{array}$ & National Health Interview Survey (NHIS), CDC, NCHS. \\
\hline $12-13$ & Mean total blood cholesterol levels (mg/dL, age adjusted, 20+ years) & National Health and Nutrition Examination Survey (NHANES), CDC, NCHS. \\
\hline $12-14$ & High blood cholesterol levels (age adjusted, 20+ years) & National Health and Nutrition Examination Survey (NHANES), CDC, NCHS. \\
\hline $12-15$ & Blood cholesterol screening in past 5 years (age adjusted, $18+$ years) & National Health Interview Survey (NHIS), CDC, NCHS. \\
\hline $12-16$ & $\begin{array}{l}\text { Adults with CHD who have their LDL-cholesterol at or below the } \\
\text { recommended level }\end{array}$ & Developmental. \\
\hline
\end{tabular}


Figure 12-1. Progress Toward Target Attainment for Focus Area 12: Heart Disease and Stroke

Moved away

from target ${ }^{1}$

Moved toward

target

Met or exceeded

target

12-1. Coronary heart disease (CHD) deaths (age adjusted, per 100,000 population)

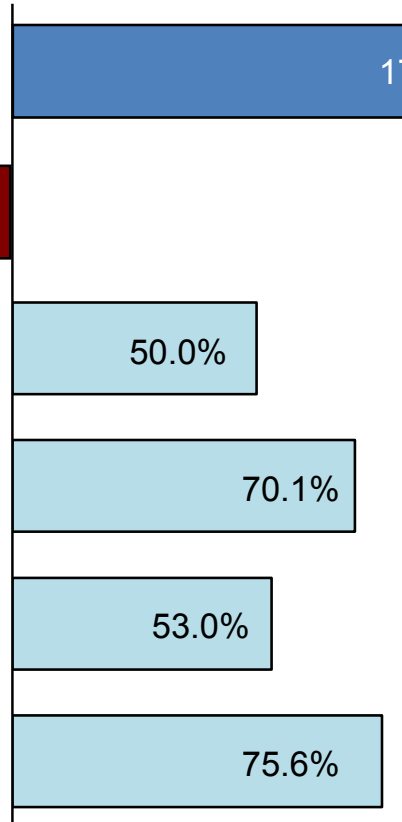

c. $85+$ years

12-7. Stroke deaths (age adjusted, per 100,000 population)

12-8. Knowledge of stroke symptoms (age adjusted, 20+ years)

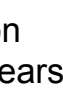

12-6. Congestive heart failure hospitalizations (per 1,000 population)

a. $65-74$ years

b. $75-84$ years

\begin{tabular}{|c|c|c|c|c|c|}
\hline \multirow{2}{*}{$\begin{array}{l}2010 \\
\text { Target }\end{array}$} & \multirow{2}{*}{$\begin{array}{c}\text { Baseline } \\
\text { (Year) }\end{array}$} & \multirow{2}{*}{$\begin{array}{l}\text { Final } \\
\text { (Year) }\end{array}$} & \multicolumn{3}{|c|}{ Baseline vs. Final } \\
\hline & & & Difference $^{2}$ & $\begin{array}{l}\text { Statistically } \\
\text { Significant }{ }^{3}\end{array}$ & $\begin{array}{l}\text { Percent } \\
\text { Change }^{4}\end{array}$ \\
\hline 156 & $\begin{array}{c}195 \\
(1999)\end{array}$ & $\begin{array}{c}126 \\
(2007)\end{array}$ & -69 & Yes & -35.4 \\
\hline $47 \%$ & $\begin{array}{c}42 \% \\
(2001)\end{array}$ & $\begin{array}{c}37 \% \\
(2008)\end{array}$ & -5 & Yes & $-11.9 \%$ \\
\hline $12 \%$ & $\begin{array}{c}8 \% \\
(2001)\end{array}$ & $\begin{array}{c}10 \% \\
(2008)\end{array}$ & 2 & Yes & $25.0 \%$ \\
\hline 6.5 & $\begin{array}{c}13.2 \\
(1997)\end{array}$ & $\begin{array}{c}8.5 \\
(2007)\end{array}$ & -4.7 & Yes & $-35.6 \%$ \\
\hline 13.5 & $\begin{array}{c}26.7 \\
(1997)\end{array}$ & $\begin{array}{c}19.7 \\
(2007)\end{array}$ & -7.0 & Yes & $-26.2 \%$ \\
\hline 26.5 & $\begin{array}{c}52.7 \\
(1997)\end{array}$ & $\begin{array}{c}32.9 \\
(2007)\end{array}$ & -19.8 & Yes & $-37.6 \%$ \\
\hline 50 & $\begin{array}{c}62 \\
(1999)\end{array}$ & $\begin{array}{c}42 \\
(2007)\end{array}$ & -20 & Yes & $-32.3 \%$ \\
\hline $65 \%$ & $\begin{array}{c}60 \% \\
(2001)\end{array}$ & $\begin{array}{c}54 \% \\
(2009)\end{array}$ & -6 & Yes & $-10.0 \%$ \\
\hline
\end{tabular}


Figure 12-1. Progress Toward Target Attainment for Focus Area 12: Heart Disease and Stroke (continued)

Moved away

from target ${ }^{1}$

Moved toward

target

Met or exceeded

target

12-9. High blood pressure (age adjusted, 18+ years)

12-10. High blood pressure control (age adjusted, $18+$ years

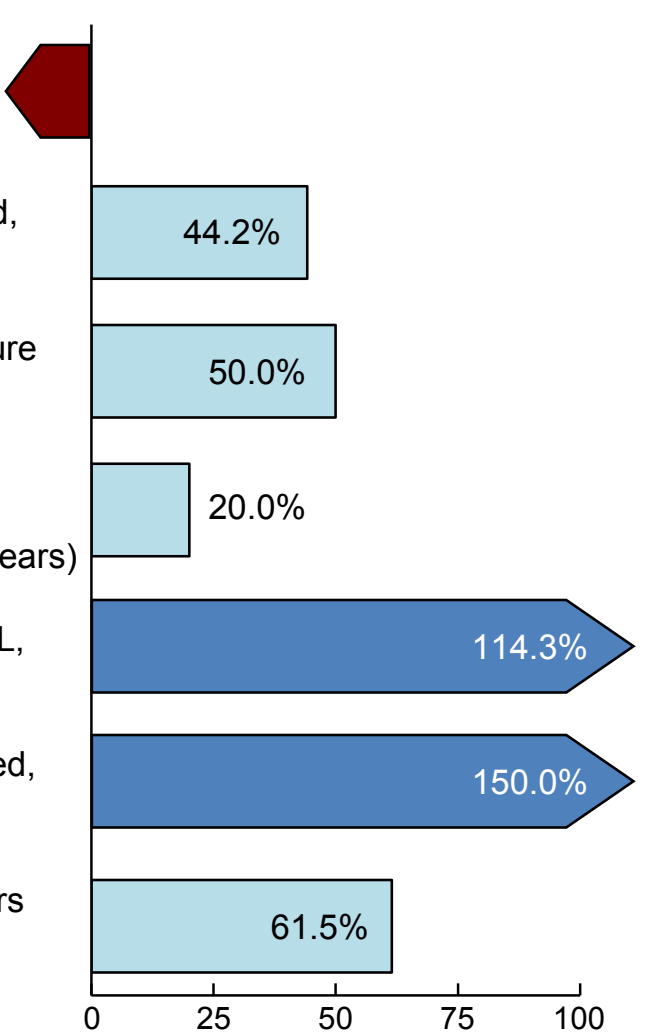

12-11. Taking action to help control blood pressure (age adjusted, 18+ years)

12-12. Adults who had their blood pressure measured in past 2 years and know their blood pressure level (age adjusted, 18+ years)

12-13. Mean total blood cholesterol levels $(\mathrm{mg} / \mathrm{dL}$, age adjusted, $20+$ years)

12-14. High blood cholesterol levels (age adjusted, $20+$ years)

12-15. Blood cholesterol screening in past 5 years (age adjusted, 18+ years)

\begin{tabular}{|c|c|c|c|c|c|}
\hline \multirow{2}{*}{$\begin{array}{l}2010 \\
\text { Target }\end{array}$} & \multirow{2}{*}{$\begin{array}{c}\text { Baseline } \\
\text { (Year) }\end{array}$} & \multirow{2}{*}{$\begin{array}{l}\text { Final } \\
\text { (Year) }\end{array}$} & \multicolumn{3}{|c|}{ Baseline vs. Final } \\
\hline & & & Difference $^{2}$ & $\begin{array}{l}\text { Statistically } \\
\text { Significant }\end{array}$ & $\begin{array}{l}\text { Percent } \\
\text { Change }^{4}\end{array}$ \\
\hline $14 \%$ & $\begin{array}{c}25 \% \\
(1988-94)\end{array}$ & $\begin{array}{c}30 \% \\
(2005-08)\end{array}$ & 5 & Yes & $20.0 \%$ \\
\hline $68 \%$ & $\begin{array}{c}25 \% \\
(1988-94)\end{array}$ & $\begin{array}{c}44 \% \\
(2005-08)\end{array}$ & 19 & Yes & $76.0 \%$ \\
\hline $98 \%$ & $\begin{array}{c}84 \% \\
(1998)\end{array}$ & $\begin{array}{c}91 \% \\
(2008)\end{array}$ & 7 & Yes & $8.3 \%$ \\
\hline $95 \%$ & $\begin{array}{c}90 \% \\
(1998)\end{array}$ & $\begin{array}{c}91 \% \\
(2008)\end{array}$ & 1 & Yes & $1.1 \%$ \\
\hline 199 & $\begin{array}{c}206 \\
(1988-94)\end{array}$ & $\begin{array}{c}198 \\
(2005-08)\end{array}$ & -8 & Yes & $-3.9 \%$ \\
\hline $17 \%$ & $\begin{array}{c}21 \% \\
(1988-94)\end{array}$ & $\begin{array}{c}15 \% \\
(2005-08)\end{array}$ & -6 & Yes & $-28.6 \%$ \\
\hline $80 \%$ & $\begin{array}{c}67 \% \\
(1998)\end{array}$ & $\begin{array}{c}75 \% \\
(2008)\end{array}$ & 8 & Yes & $11.9 \%$ \\
\hline
\end{tabular}

Percent of targeted change achieved

(continued) 


\section{Figure 12-1. Progress Toward Target Attainment for Focus Area 12: Heart Disease and Stroke (continued)}

\section{NOTES}

See the Reader's Guide for more information on how to read this figure. See DATA2010 at http://wonder.cdc.gov/data2010 for all Healthy People 2010 tracking data. Tracking data are not available for objectives 12-3a, 12-3b, 12-5 and 12-16.

\section{FOOTNOTES}

${ }^{1}$ Movement away from target is not quantified using the percent of targeted change achieved. See Technical Appendix for more information.

${ }^{2}$ Difference $=$ Final value - Baseline value. Differences between percents $(\%)$ are measured in percentage points.

${ }^{3}$ When estimates of variability are available, the statistical significance of the difference between the final value and the baseline value is assessed at the 0.05 level. See $\underline{\text { Technical Appendix }}$ for more information.

${ }^{4}$ Percent change $=\frac{\text { Final value }- \text { Baseline value }}{\text { Baseline value }} \times 100$.
${ }^{5}$ Percent of targeted change achieved $=\frac{\text { Final value }- \text { Baseline value }}{\text { Healthy People } 2010 \text { target }- \text { Baseline value }} \times 100$.

\section{DATA SOURCES}

12-1. National Vital Statistics System-Mortality (NVSS-M), CDC, NCHS.

12-2. National Health Interview Survey (NHIS), CDC, NCHS.

12-4. National Health Interview Survey (NHIS), CDC, NCHS.

12-6a-c. National Hospital Discharge Survey (NHDS), CDC, NCHS.

12-7. National Vital Statistics System-Mortality (NVSS-M), CDC, NCHS.

12-8. $\quad$ National Health Interview Survey (NHIS), CDC, NCHS.

12-9-12-10. National Health and Nutrition Examination Survey (NHANES), CDC, NCHS.

12-11-12-12. National Health Interview Survey (NHIS), CDC, NCHS.

12-13-12-14. National Health and Nutrition Examination Survey (NHANES), CDC, NCHS.

12-15. National Health Interview Survey (NHIS), CDC, NCHS. 
Figure 12-2. Health Disparities Table for Focus Area 12: Heart Disease and Stroke Disparities from the best group rate for each characteristic at the most recent data point and changes in disparity from the baseline to the most recent data point.

Population-based objectives

12-1. Coronary heart disease (CHD) deaths (age adjusted, per 100,000 population) $(1999,2007)^{1 *}$

12-2. Knowledge of heart attack symptoms and importance of calling 911 (age adjusted, $20+$ years) $(2001,2008)^{\star}$

12-3a. Fibrinolytics within an hour of symptom onset (2000-04)†

12-3b. Percutaneous intervention (PCl) within 90 minutes of symptom onset (2000-04)†

12-4. Training in cardiopulmonary resuscitation (CPR) in past year (age adjusted, 20+ years) $(2001,2008)^{\star}$

12-6a. Congestive heart failure hospitalizations-65-74 years (per 1,000 population) $(1997,2007)^{*}$

b. Congestive heart failure hospitalizations-75-84 years (per 1,000 population) $(1997,2007)^{*}$

c. Congestive heart failure hospitalizations - 85+ years (per 1,000 population) $(1997,2007)^{*}$

12-7. Stroke deaths (age adjusted, per 100,000 population) $(1999,2007)^{1 *}$

12-8. Knowledge of stroke symptoms (age adjusted, 20+ years) $(2001,2009)^{*}$

12-9. High blood pressure (BP) (age adjusted, 18+ years) (1988-94, 2005-08) $)^{2 *}$

12-10. High BP control (age adjusted, 18+ years) $(1988-94,2005-08)^{2 *}$

12-11. Taking action to help control BP (age adjusted, 18+ years) $(1998,2008)^{3 *}$

12-12. Adults who had their $\mathrm{BP}$ measured in past 2 years and know their BP level (age adjusted, 18+ years) $(1998,2008)^{3 *}$

12-13. Mean total blood cholesterol levels (mg/dL, age adjusted, $20+$ years) (1988-94, 2005-08) 2*

12-14. High blood cholesterol levels (age adjusted, 20+ years) (1988-94, 2005-08) 2* $^{2}$

12-15. Blood cholesterol screening in past 5 years (age adjusted, $18+$ years) $(1998,2008)^{3 *}$

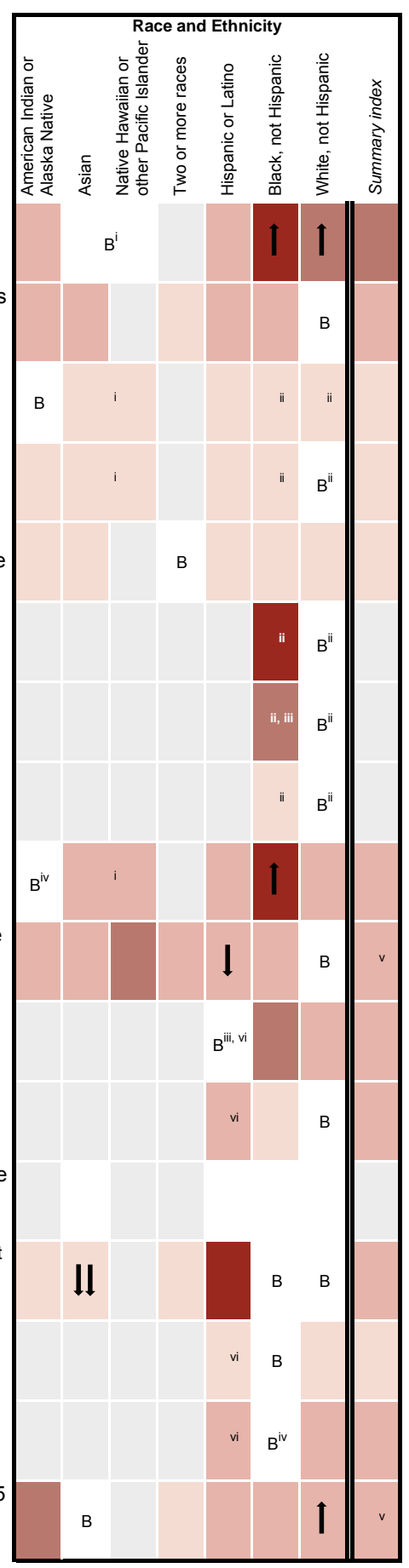

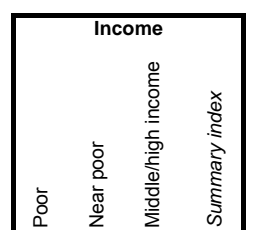
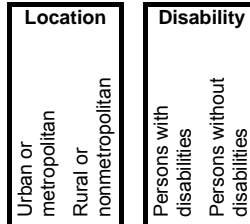

B

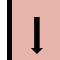

1
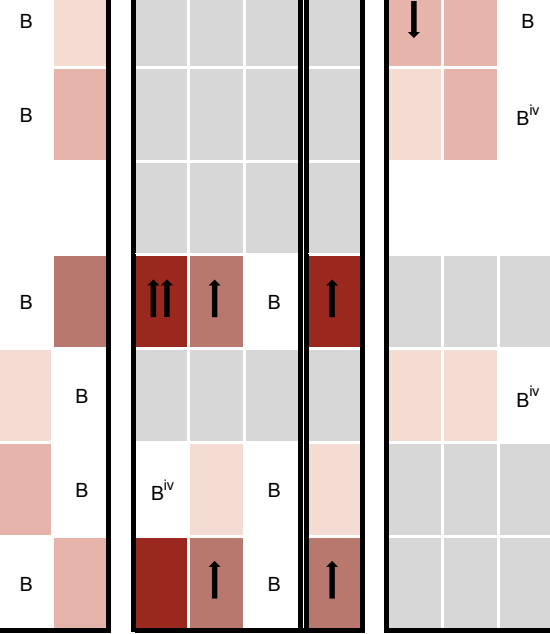

в 1 
Figure 12-2. Health Disparities Table for Focus Area 12: Heart Disease and Stroke (continued)

NOTES

See DATA2010 at http://wonder.cdc.gov/data2010 for all Healthy People 2010 tracking data. Disparity data are either unavailable or not applicable for objectives $12-5$ and 12-16.

Years in parentheses represent the baseline and most recent data years (if available).

Disparity from the best group rate is defined as the percent difference between the best group rate and each of the other group rates for a characteristic (e.g., race and ethnicity). The summary index is the average of these percent differences for a characteristic. Change in disparity is estimated by subtracting the disparity at baseline from the disparity at the most recent data point. Change in the summary index is estimated by subtracting the summary index at baseline from the summary index at the most recent data point. See Technical Appendix for more information.

LEGEND

The "best" group rate at the most recent data point.

B The group with the best rate for specified characteristic.

Most favorable group rate for specified characteristic, but reliability criterion no met.

Percent difference from the best group rate

\begin{tabular}{l} 
Disparity from the best group rate at the $\begin{array}{l}\text { Less than } 10 \% \text {, or difference not } \\
\text { most recent data point. } \\
\text { estimates of variability are available). }\end{array}$ \\
\hline estimen of ariant
\end{tabular}

Changes in disparity over time are shown when:

(a) disparities data are available at both baseline and most recent time points;

(b) data are not for the group(s) indicated by "B" or "b" at either time point; and

(c) the change is greater than or equal to 10 percentage points and statistically

significant, or when the change is greater than or equal to 10 percentage

points and estimates of variability were not available. See Technical Appendix.

$\begin{array}{ll}10 \%-49 \% & 50 \%-99 \%\end{array}$

Reliability criterion for best group rate not met, or data available for only one group.

\begin{tabular}{ll|l|l|l|} 
& & & & \\
Availability of Data & Data not available. & Characteristic not selected for this objective. \\
\hline
\end{tabular}

\section{FOOTNOTES}

* Measures of variability were available. Thus, the variability of best group rates was assessed, and statistical significance was tested. Disparities of $10 \%$ or more are displayed when the differences from the best group rate are statistically significant at the 0.05 level. Changes in disparities over time are indicated by arrows when the changes are greater than or equal to 10 percentage points and are statistically significant at the 0.05 level. See Technical Appendix.

† Measures of variability were not available. Thus, the variability of best group rates was not assessed, and statistical significance could not be tested. Nonetheless, disparities and changes in disparities over time are displayed according to their magnitude. See Technical Appendix.

1 Most recent data by education level are for 2002.

2 Baseline data by disability status are for 1991-94.

3 Baseline data by race and ethnicity are for 2003 .

i Data are for Asian or Pacific Islander.

ii Data include persons of Hispanic origin.

iii Reliability criterion for best group rate not met, or data available for only one group, at baseline. Change in disparity cannot be assessed. See Technical Appendix.

iv The group with the best rate at the most recent data point is different from the group with the best rate at baseline. Both rates met the reliability criterion. See Technical Appendix.

v Change in the summary index cannot be assessed. See Technical Appendix.

vi Data are for Mexican American.

\section{DATA SOURCES}

12-1. National Vital Statistics System-Mortality (NVSS-M), CDC, NCHS

12-2. National Health Interview Survey (NHIS), CDC, NCHS.

12- National Registry of Myocardial Infarction (NRMI-4), National Acute Myocardial Infarction Project, CMS

$3 a-b$.

$12-4$.

$12-$

$6 a-c$.

12-7.

$12-8$.

12-9- National Health and Nutrition Examination Survey (NHANES), CDC, NCHS

12-10.

12-11- National Health Interview Survey (NHIS), CDC, NCHS.

$12-12$.

12-13- National Health and Nutrition Examination Survey (NHANES), CDC, NCHS.

$12-14$

12-15. National Health Interview Survey (NHIS), CDC, NCHS. 
Figure 12-3. Coronary Heart Disease Deaths (2005-07)

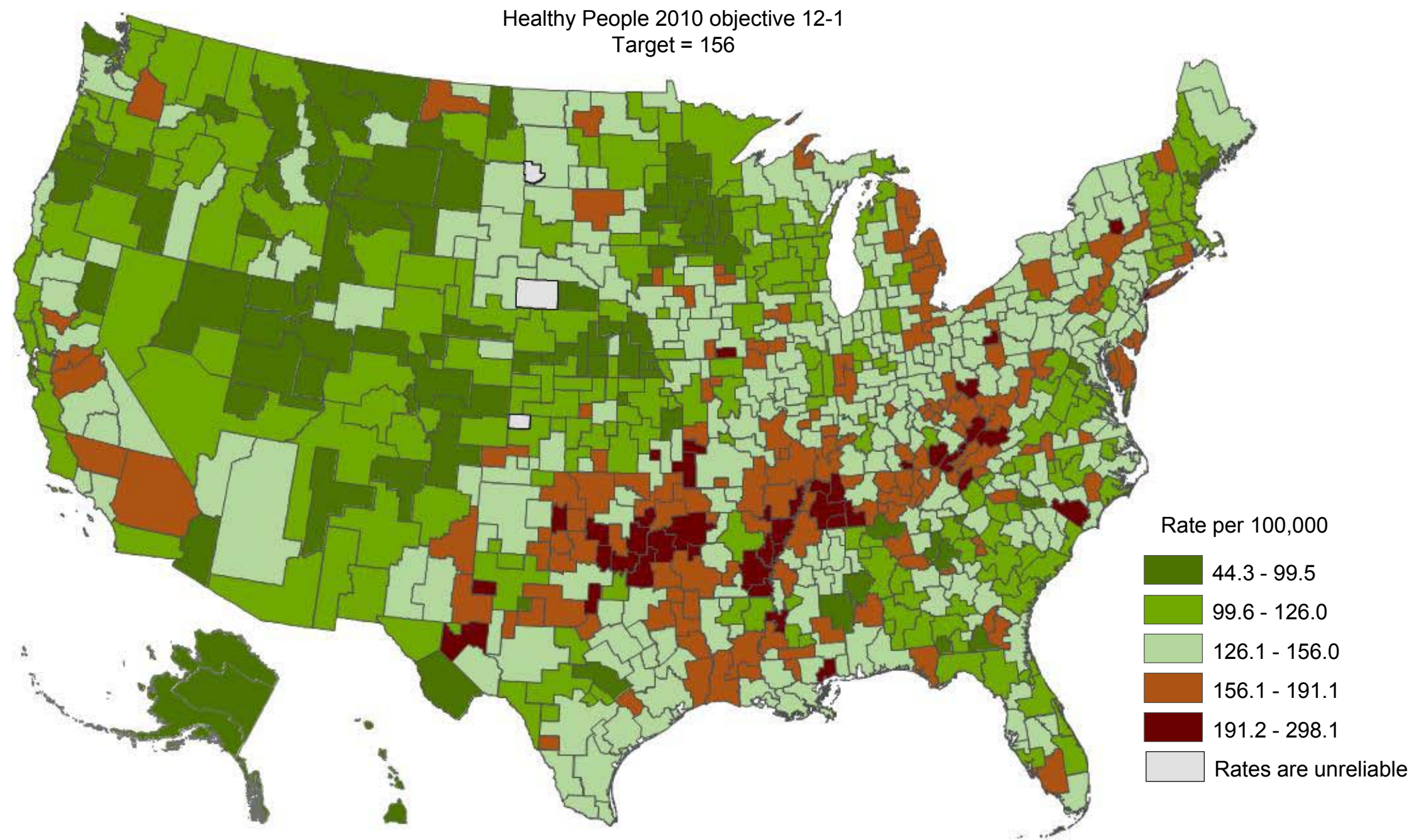

lowest categories (green) show health service areas that met target

NOTES: Data are for ICD-10 codes I20-I25reported as underlying cause. Rates are per 100,000 U. S. Population age-adjusted to the 2000 standard population. Rates are displayed by a modified Jenks classification for U.S. health service area.

SOURCE: National Vital Statistics System - Mortality, CDC, NCHS. 
Figure 12-4. Stroke Deaths (2005-07)

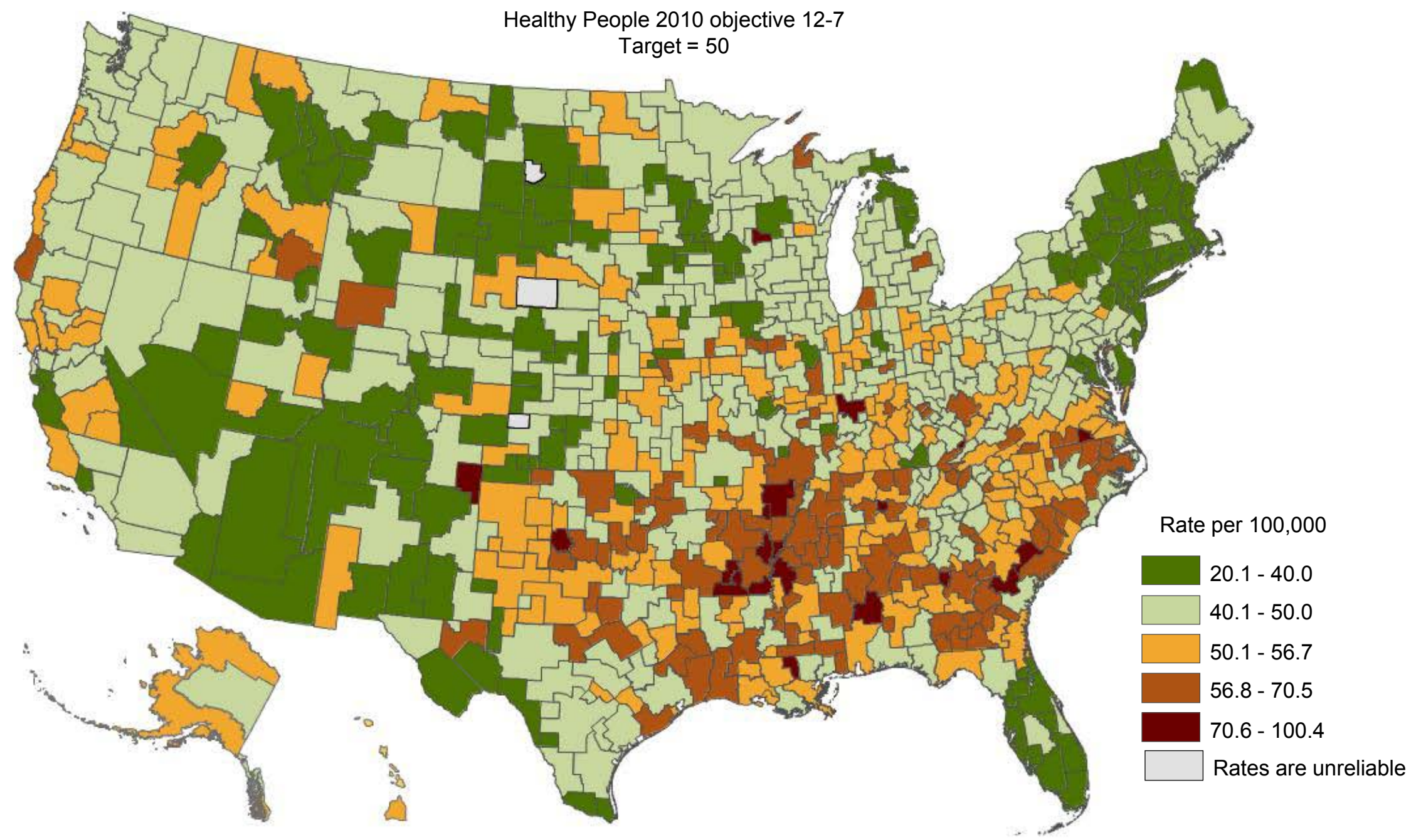

lowest categories (green) show health service areas that met target

NOTES: Data are for ICD-10 codes 160-I69reported as underlying cause. Rates are per 100,000 U. S. Population age-adjusted to the 2000 standard population. Rates are displayed by a modified Jenks classification for U.S. health service area. SOURCE: National Vital Statistics System - Mortality, CDC, NCHS. 


\section{Final Review}
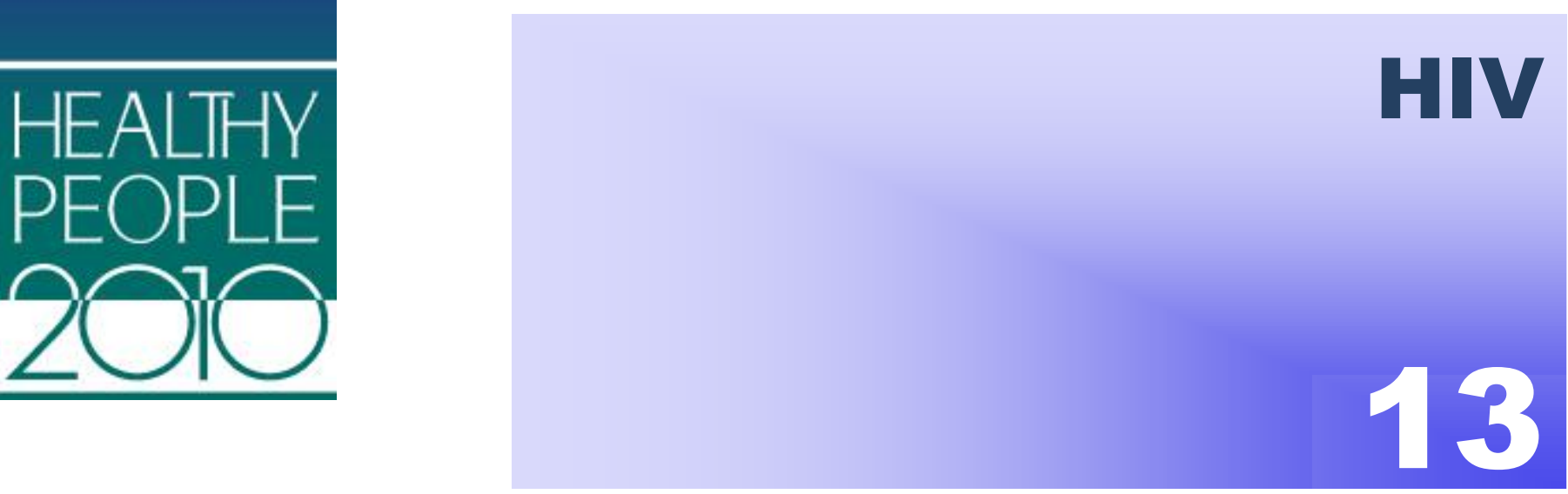

\section{Co-Lead Agencies}

Centers for Disease Control and Prevention

Health Resources and Services Administration

\section{Contents}

Goal

Highlights

Summary of Progress.

Transition to Healthy People 2020

Data Considerations

References and Notes

Comprehensive Summary of Objectives

Progress Chart

Health Disparities Table

New AIDS Cases-Map

HIV Infection Deaths-Map. 


\section{Goal: Prevent human immunodeficiency virus (HIV) infection and its related illness and death}

The objectives in this chapter track cases of HIV infection and acquired immunodeficiency syndrome (AIDS), HIV/AIDS deaths, HIV/AIDS prevention and HIV/AIDS testing.

All Healthy People 2010 tracking data quoted in this chapter, along with technical information and operational definitions for each objective, can be found in the Healthy People 2010 database, DATA2010, available from http://wonder.cdc.gov/data2010/.

More information about this Focus Area can be found in the following publications:

- Healthy People 2010: Understanding and Improving Health, available from http://www.healthypeople.gov/2010/Document/tableofcontents.htm\#under.

- Healthy People 2010 Midcourse Review, available from http://www.healthypeople.gov/2010/data/midcourse/html/default.htm\#FocusAreas.

\section{Highlights}

- Substantial progress was achieved in objectives for this Focus Area during the past decade [1]. Over $70 \%$ of the HIV objectives with data to measure progress moved toward or achieved their Healthy People 2010 targets (Figure 13-1). However, health disparities were observed among racial and ethnic population groups, as well as by sex, education level, income, and disability status (Figure 13-2), as discussed below [2].

- A statistically significant downward trend was observed in the rate of new AIDS diagnoses among persons aged 13 years and over (objective 13-1) [3]. The rate decreased by $20.7 \%$ between 1998 and 2007, from 18.4 to 14.6 new cases per 100,000 population, moving toward the Healthy People 2010 target of 0.9 per 100,000 population.

- Among racial and ethnic groups, the Asian or Pacific Islander population had the lowest (best) rate of AIDS diagnoses, 4.7 new cases per 100,000 population in 2007. The rate for the Hispanic or Latino population, 20.9 new cases per 100,000 in 2007, was almost four and a half times the best rate; the rate for the black non-Hispanic population, 58.6 per 100,000 in 2007, was over 12 times the best rate [2].

- Females had a lower (better) rate of annual AIDS diagnoses than males, 7.6 per 100,000 population in 2007. Males had a rate of 21.9 per 100,000 in 2007, almost three times the rate for females [2].

- The rate of annual AIDS diagnoses varied by state. In 2007, Alaska, the Central and Midwest states, Maine, New Hampshire, Vermont, and West Virginia, had the lowest rates. The District of Columbia, with 154.6 new cases per 100,000 population, had the highest rate (Figure 13-3).

- The annual number of new AIDS diagnoses attributed to male-to-male sexual contact among adults and adolescents aged 13 years and over (objective 13-2) increased by $0.7 \%$ between 1998 and 2007, from 16,882 to 16,992, moving away from the 2010 target of 12,661. 
- The number of new cases of AIDS among persons aged 13 years and over who injected drugs (objective 13-3) decreased by 47.1\% between 1998 and 2007, from 11,514 to 6,093, exceeding the 2010 target of 8,636.

- The number of new cases of AIDS among adult and adolescent males, aged 13 years and over, who had sex with men and who injected drugs (objective 13-4) declined by $36.1 \%$ between 1998 and 2007, from 2,751 to 1,759, exceeding the 2010 target of 2,064.

- HIV-infection deaths (objective 13-14) declined by 30.2\% between 1999 and 2007, from 5.3 to 3.7 deaths per 100,000 population (age adjusted), moving toward the 2010 target of 0.7 deaths per 100,000 population.

- Among racial and ethnic groups, the white non-Hispanic population had the best rates of HIV-infection deaths, 2.3 per 100,000 population (age adjusted) in 1999 and 1.5 in 2007. The Hispanic or Latino population had a rate of 4.1 in 2007, while the black non-Hispanic population had rates of 24.0 in 1999 and 17.8 in 2007.

- In 2007, the HIV infection death rate for the Hispanic or Latino population was more than two and a half times the best rate (that for the white nonHispanic population), while the rate for the black non-Hispanic population was almost 12 times the best rate [2].

- Between 1999 and 2007, the disparity between the black non-Hispanic and white non-Hispanic populations increased by 143 percentage points [4].

- Females had lower (better) HIV-infection death rates than males, 2.5 deaths per 100,000 population (age adjusted) in 1999 and 2.1 in 2007. Males had rates of 8.2 in 1999 and 5.4 in 2007. In 2007, the rate for males was more than two and a half times that for females. Between 1999 and 2007, the disparity between males and females declined by 71 percentage points [4].

- The HIV-infection death rates varied by state. Among those states with reliable data for the period 2005-07, the HIV-infection death rates for Delaware, Florida, Georgia, Louisiana, Maryland, Mississippi, New York, and South Carolina ranged from 4.6 to 9.3 deaths per 100,000 population (age adjusted). The District of Columbia, with an HIV-infection death rate of 34.9 per 100,000 (age adjusted), had the highest rate (Figure 13-4).

- A statistically significant upward trend was observed in the proportion of HIV-infected persons surviving three or more years after an AIDS diagnosis (objective 13-16) [3]. The proportion increased by $12.8 \%$ between 1998 and 2006, from $78 \%$ to $88 \%$, exceeding the 2010 target of $86 \%$.

- A statistically significant downward trend was observed in the number of perinatallyacquired AIDS diagnoses (objective 13-17b) [3]. The number declined by $88.5 \%$ between 1998 and 2007, from 243 to 28 new cases, exceeding the 2010 target of 75 new cases. 
- HIV testing of tuberculosis patients aged 25-44 years (objective 13-11) increased by 19.7\% between 1998 and 2008, from 61\% to 73\%, moving toward the 2010 target of $89 \%$.

- Among racial and ethnic groups, the black non-Hispanic population had the highest (best) rate of HIV testing among tuberculosis patients aged 25-44 years, 88\% in 2008, whereas the Asian, the Native Hawaiian or Other Pacific Islander, and the Hispanic or Latino populations had rates of $61 \%, 61 \%$, and $69 \%$ in 2008 , respectively. When expressed in terms of patients who were not tested for HIV, the rates for the Asian and the Native Hawaiian or Other Pacific Islander populations were almost three and a half times the best rate (that for the black non-Hispanic population), while the rate for the Hispanic or Latino population was more than two and a half times the best rate [2].

\section{Summary of Progress}

- Figure 13-1 presents a quantitative assessment of progress in achieving the Healthy People 2010 objectives for HIV [1]. Data to measure progress toward target attainment were available for 15 objectives. Of these:

- Four objectives exceeded their Healthy People 2010 targets (objectives 13-3, 13-4, 13-16, and 13-17b).

- Seven objectives moved toward their targets. A statistically significant difference between the baseline and the final data points was observed for one of these objectives (13-14). Data were unavailable to test the significance of the difference for the remaining six objectives (13-1, 13-6a and b, 13-11, and 13-13d and f).

- Four objectives moved away from their targets (objectives 13-2,13-8, and 13-13c and e). Data were unavailable to test the significance of the difference between the baseline and the final data points for all of these objectives.

- Six objectives (13-5, 13-13a and b, 13-15, 13-17a, and 13-18) remained developmental and four objectives $(13-7,13-9,13-10$ and 13-12) were dropped during the decade [5,6].

- Figure 13-2 displays health disparities from the best group rate for each characteristic at the most recent data point [2]. It also displays changes in disparities from baseline to the most recent data point [4].

- Statistically significant health disparities of $10 \%$ or more by race and ethnicity were observed for three objectives. Health disparities of $10 \%$ or more by race and ethnicity were observed for seven additional objectives, though their significance could not be tested. Of these 10 objectives, the black non-Hispanic population had the best rate for four objectives (13-6a and b, 13-11, and 13-13f). The Asian or Pacific Islander (objective 13-1), the Hispanic or Latino (objective 13-13d), the American Indian or Alaska Native (objective 13-13e), and the white non-Hispanic (objective 13-14) populations each had the only best rate for one objective. The Asian or Pacific Islander and Hispanic or Latino populations were tied for the best rate for one objective (13-13c), while the Asian or Pacific Islander and white nonHispanic populations were tied for the best rate for another (objective 13-16). 
- One objective had statistically significant health disparities of $10 \%$ or more by sex and three objectives had health disparities of $10 \%$ or more by sex but no data to assess significance. Of these four objectives, females had better rates than males for two (objectives 13-1 and 13-14) and males had better rates than females for the other two (objectives 13-11 and 13-13c).

- Persons with at least some college education had the best rates for all three of the objectives with statistically significant health disparities of $10 \%$ or more by education level (objectives 13-6a and b, and 13-14).

- Persons with middle/high incomes had the best rate for the one objective (13-6a) with statistically significant health disparities of $10 \%$ or more by income.

- Persons without disabilities had a better rate than persons with disabilities for the one objective (13-6a) with statistically significant health disparities of $10 \%$ or more by disability status.

- Several objectives exhibited health disparities of $100 \%$ or more, and some had changes in disparities of 50 percentage points or more over time. Many of these are discussed in the Highlights, above.

\section{Transition to Healthy People 2020}

For Healthy People 2020, the focus of the HIV objectives has expanded to focus more on HIV testing among populations at increased risk of HIV infection. The general terminology has transitioned from the term HIV/AIDS to HIV. The term HIV focuses on persons diagnosed with HIV infection regardless of their stage of disease. Nevertheless, AIDS diagnoses are still tracked for selected objectives. See HealthyPeople.gov for a complete list of Healthy People 2020 topics and objectives.

The Healthy People 2020 HIV Topic Area can be grouped into several sections:

- Diagnosis of HIV Infection and AIDS

- Medical Healthcare, Survival, and Death after Diagnosis of HIV Infection and AIDS

- HIV Testing

- HIV Prevention.

The differences between the Healthy People 2010 objectives and those included in Healthy People 2020 objectives are summarized below:

- The Healthy People 2020 HIV Topic Area has a total of 23 objectives, seven of which are developmental, whereas the Healthy People 2010 HIV Focus Area had 25 objectives of which six were developmental and four were dropped at the Midcourse Review [5,6].

- Ten Healthy People 2010 objectives were retained "as is" [7]. These include,

- Eight measurable objectives: new AIDS cases (objective 13-1), AIDS among men who have sex with men (objective 13-2), AIDS among persons who inject drugs (objective 13-3), HIV counseling and education for persons in substance abuse treatment (objective 13-8), HIV testing in tuberculosis patients (objective 13-11), HIV-infection deaths (objective 13-14), HIV infected persons surviving three or more years after a diagnosis of AIDS (objective 13-16), and perinatally-acquired AIDS (objective 13-17b). 
- Two developmental objectives: new HIV/AIDS cases (objective 13-5) and new HIV infections diagnosed before progression to AIDS (objective 13-15).

- Ten Healthy People 2010 objectives were modified to create five Healthy People 2020 objectives [8]:

- Two developmental and four measurable objectives addressing treatment according to guidelines among HIV-infected persons (objectives 13-13a through f) were combined to create one developmental objective in Healthy People 2020.

- Objective 13-7, measuring the number of HIV-positive persons who know their serostatus, was deleted during the Healthy People 2010 Midcourse Review. It was reinstated in Healthy People 2020, as a measurable objective.

- Condom use among females and males (objectives 13-6a and b, respectively) was modified to expand the age group of the target population from 18-44 years to 1544 years.

- Perinatally-acquired HIV/AIDS diagnosed (objective 13-17a) was modified to monitor HIV only. This objective is still developmental.

- One Healthy People 2010 objective, AIDS among men who have sex with men and who inject drugs (objective 13-4), was archived [9].

- Four Healthy People 2010 objectives were dropped [6]. HIV/AIDS, STD, and TB education in state prisons (objective 13-9), HIV counseling and testing in state prisons (objective 13-10), and screening for STDs and immunization for hepatitis B (objective 13-12) were dropped at the Midcourse Review. One developmental objective, HIV/AIDS diagnosed in adolescent and young females aged 13-24 years (objective 13-18) was dropped due to lack of a data source.

- Eight new objectives were added to the Healthy People 2020 HIV Topic Area:

- Five new objectives, including HIV transmission among adolescents and adults, new AIDS cases among adolescent and adult heterosexuals, HIV testing among adolescents and adults, HIV testing among pregnant women, and HIV testing among adolescents and young adults, were added as measurable objectives.

- Three new objectives, including new (incident) HIV infections among adolescents and adults, HIV testing among men who have sex with men, and the proportion of men who have sex with men who reported unprotected anal sex in the past 12 months, were added as developmental objectives.

Appendix D “A Crosswalk Between Objectives from Healthy People 2010 to Healthy People 2020," summarizes the changes between the two decades of objectives, reflecting new knowledge and direction for this area. 


\section{Data Considerations}

The HIV/AIDS Surveillance System - the data source for many Health People 2010 HIV objectiveswas renamed the HIV Surveillance System in 2008, highlighting the focus on diagnosis of HIV infection regardless of the person's stage of disease. Data in the HIV/AIDS Surveillance System are continually updated, and new records are added as they are reported. For this reason, data for any given year may change over time. All data points for HIV objectives monitored through the HIV Surveillance System are updated annually, often resulting in revisions of baselines and targets.

Education and income are the primary measures of socioeconomic status (SES) in Healthy People 2010. Most data systems used in Healthy People 2010 define income as a family's income before taxes. In order to facilitate comparisons among groups and over time, while adjusting for family size and for inflation, Healthy People 2010 categorizes income using the poverty thresholds developed by the U.S. Census Bureau. Thus, the three categories of family income that are primarily used are:

- Poor-below the Federal poverty level

- Near poor-100\% to $199 \%$ of the Federal poverty level

- Middle/high income-200\% or more of the Federal poverty level.

These categories may be overridden by considerations specific to the data system, in which case they are modified as appropriate. See Healthy People 2010: General Data Issues, referenced below.

Beginning in 2003, education data for the mortality objective 13-14 (HIV-infection deaths) from the National Vital Statistics System have been suppressed. The educational attainment item was changed in the new U.S. Standard Certificate of Death in 2003 to be consistent with the U.S. Census Bureau data and to improve the ability to identify specific types of educational degrees. However, many states are still using the 1989 version of the U.S. Standard Certificate of Death, which focuses on highest school grade completed. As a result, educational attainment data collected using the 2003 version are not comparable with data collected using the 1989 version [10].

In general, data on educational attainment are presented for persons aged 25 years and over, consistent with guidance given by the U.S. Bureau of the Census. However, because of the requirements of the different data systems, the age groups used to calculate educational attainment for any specific objective may differ from the age groups used to report the data for other Healthy People 2010 objectives, as well as from select populations within the same objective. Therefore, the reader is urged to exercise caution in interpreting the data by educational attainment shown in the Health Disparities Table. See Healthy People 2010: General Data Issues, referenced below.

Additional information on data issues is available from the following sources:

- All Healthy People 2010 tracking data can be found in the Healthy People 2010 database, DATA2010, available from http://wonder.cdc.gov/data2010/.

- Detailed information about the data and data sources used to support these objectives can be found in the Operational Definitions on the DATA2010 website, available from http://wonder.cdc.gov/data2010/focusod.htm. 
- More information on statistical issues related to Healthy People tracking and measurement can be found in the Technical Appendix and in Healthy People 2010: General Data Issues, which is available in the Data Issues section of the NCHS Healthy People website under Healthy People 2010.

\section{References and Notes}

1. Displayed in the Progress Chart (Figure 13-1), the percent of targeted change achieved expresses the difference between the baseline and the final value relative to the initial difference between the baseline and the Healthy People 2010 target. As such, it is a relative measure of progress toward attaining the Healthy People 2010 target. See the Reader's Guide for more information. When standard errors were available, the difference between the baseline and the final value was tested at the 0.05 level of significance. See the Figure 13-1 footnotes, as well as the Technical Appendix, for more detail.

2. Information about disparities among select populations is shown in the Health Disparities Table (Figure 13-2). Disparity from the best group rate is defined as the percent difference between the best group rate and each of the other group rates for a characteristic. For example, racial and ethnic health disparities are measured as the percent difference between the best racial and ethnic group rate and each of the other racial and ethnic group rates. Similarly, disparities by sex are measured as the percent difference between the better group rate (e.g. female) and the rate for the other group (e.g. male). Some objectives are expressed in terms of favorable events or conditions that are to be increased, while others are expressed in terms of adverse events or conditions that are to be reduced. In order to facilitate comparison of health disparities across different objectives, disparity is measured only in terms of adverse events or conditions. For comparability across objectives, objectives that are expressed in terms of favorable events or conditions are re-expressed using the adverse event or condition for the purpose of computing disparity, but they are not otherwise restated or changed. For example, objective 1-1, to increase the proportion of persons with health insurance (e.g., $72 \%$ of the American Indian or Alaska Native population aged under 65 years had some form of health insurance in 2008), is expressed in terms of the percentage of persons without health insurance (e.g., $100 \%-72 \%=28 \%$ of the American Indian or Alaska Native population aged under 65 years did not have any form of health insurance in 2008) when the disparity from the best group rate is calculated. See the Reader's Guide for more information. When standard errors were available, the difference between the best group rate and each of the other group rates was tested at the 0.05 level of significance. See the Figure 13-2 footnotes, as well as the Technical Appendix, for more detail.

3. The presence of a monotonic increasing or decreasing trend in the underlying measure was tested with the nonparametric Mann-Kendall test, then the slope of a linear trend was estimated with the nonparametric Sen's method. See Technical Appendix for more information.

4. The change in disparity is estimated by subtracting the disparity at baseline from the disparity at the most recent data point and, therefore, is expressed as a change in percentage points. See the Reader's Guide for more information. When standard errors were available, the change in disparity was tested at the 0.05 level of significance. See the Figure 13-2 footnotes, as well as the Technical Appendix, for more detail.

5. To be included in Healthy People 2010, an objective must have a national data source that provides a baseline and at least one additional data point for tracking progress. Some objectives lacked baseline data at the time of their development but had a potential data source and were considered of sufficient national importance to be included in Healthy People. These are called "developmental" objectives. When data become available, a developmental objective is moved to measurable status and a Healthy People target can be set. 
6. Dropped objectives were not carried forward into Healthy People 2020. These objectives were either developmental or deleted at the Healthy People 2010 Midcourse Review or at another time in Healthy People 2010.

7. Retained "as is" objectives have no change in the numerator definition or in the denominator definition between the Healthy People 2010 and Healthy People 2020 objectives. These include objectives that were developmental in Healthy People 2010 and are developmental in Healthy People 2020 and for which no numerator or denominator information was available.

8. Modified objectives have some change in the numerator definition or in the denominator definition between the Healthy People 2010 and Healthy People 2020 objectives. These include objectives that went from developmental in Healthy People 2010 to measurable in Healthy People 2020 or vice versa.

9. Archived objectives had at least one data point in Healthy People 2010 but were not carried forward into Healthy People 2020.

10. Xu JQ Kochanek KD, Murphy SL, Tejada-Vera B. Deaths: Final data for 2007. National vital statistics reports; vol 58 no 19. Hyattsville, MD: National Center for Health Statistics. 2010.. Available from: http://www.cdc.gov/nchs/data/nvsr/nvsr58/nvsr58 19.pdf 


\section{Comprehensive Summary of Objectives: HIV}

\begin{tabular}{|c|c|c|}
\hline Objective & Description & Data Source or Objective Status \\
\hline $13-1$ & New AIDS cases (per 100,000 population, $13+$ years) & HIV Surveillance System, CDC, NCHHSTP. \\
\hline $13-2$ & AIDS among men who have sex with men (no. new cases, $13+$ years) & HIV Surveillance System, CDC, NCHHSTP. \\
\hline $13-3$ & AIDS among persons who inject drugs (no. new cases, 13+ years) & HIV Surveillance System, CDC, NCHHSTP. \\
\hline $13-4$ & $\begin{array}{l}\text { AIDS among men who have sex with men and who inject drugs (no. } \\
\text { new cases, } 13+\text { years) }\end{array}$ & HIV Surveillance System, CDC, NCHHSTP. \\
\hline $13-5$ & New HIV/AIDS cases diagnosed among adolescents and adults & Developmental \\
\hline $13-6 a$ & $\begin{array}{l}\text { Condom use among sexually active unmarried persons (18-44 years)- } \\
\text { Females }\end{array}$ & National Survey of Family Growth (NSFG), CDC, NCHS. \\
\hline $13-6 b$ & $\begin{array}{l}\text { Condom use among sexually active unmarried persons (18-44 years)- } \\
\text { Males }\end{array}$ & National Survey of Family Growth (NSFG), CDC, NCHS. \\
\hline $13-7$ & Knowledge of serostatus-Among HIV positive persons & Dropped \\
\hline $13-8$ & HIV counseling and education for persons in substance abuse treatment & $\begin{array}{l}\text { Baseline: Uniform Facility Data Set (UFDS), SAMHSA; Final: National } \\
\text { Survey of Substance Abuse Treatment Services (N-SSATS), SAMHSA. }\end{array}$ \\
\hline $13-9$ & HIV/AIDS, STD, and TB education in State prisons & Dropped \\
\hline $13-10$ & HIV counseling and testing in State prisons & Dropped \\
\hline $13-11$ & HIV testing in TB patients ( $25-44$ years) & National TB Surveillance System, CDC, NCHHSTP. \\
\hline $13-12$ & $\begin{array}{l}\text { Screening for STDs and immunization for hepatitis B-Among HIV } \\
\text { counselees }(18+\text { years })\end{array}$ & Dropped \\
\hline $13-13 a$ & $\begin{array}{l}\text { Treatment according to guidelines-Viral load testing among HIV- } \\
\text { infected persons }(13+\text { years })\end{array}$ & Developmental \\
\hline $13-13 b$ & $\begin{array}{l}\text { Treatment according to guidelines-Tuberculin skin testing (TST) } \\
\text { among HIV-infected persons }(13+\text { years })\end{array}$ & Developmental \\
\hline $13-13 c$ & Any antiretroviral therapy among HIV-infected persons $(13+$ years $)$ & $\begin{array}{l}\text { Adult and Adolescent Spectrum of HIV Disease (ASD) Surveillance } \\
\text { Project, CDC, NCHHSTP. }\end{array}$ \\
\hline $13-13 d$ & $\begin{array}{l}\text { Highly active antiretroviral therapy (HAART) among HIV-infected } \\
\text { persons }(13+\text { years })\end{array}$ & $\begin{array}{l}\text { Adult and Adolescent Spectrum of HIV Disease (ASD) Surveillance } \\
\text { Project, CDC, NCHHSTP. }\end{array}$ \\
\hline $13-13 e$ & $\begin{array}{l}\text { Pneumocystis carinii pneumonia (PCP) prophylaxis among HIV-infected } \\
\text { persons }(13+\text { years })\end{array}$ & $\begin{array}{l}\text { Adult and Adolescent Spectrum of HIV Disease (ASD) Surveillance } \\
\text { Project, CDC, NCHHSTP. }\end{array}$ \\
\hline
\end{tabular}




\begin{tabular}{|c|c|c|}
\hline Objective & Description & Data Source or Objective Status \\
\hline $13-13 f$ & $\begin{array}{l}\text { Mycobacterium avium complex (MAC) prophylaxis among HIV-infected } \\
\text { persons }(13+\text { years })\end{array}$ & $\begin{array}{l}\text { Adult and Adolescent Spectrum of HIV Disease (ASD) Surveillance } \\
\text { Project, CDC, NCHHSTP. }\end{array}$ \\
\hline $13-14$ & HIV-infection deaths (age adjusted per 100,000 standard population) & National Vital Statistics System-Mortality (NVSS-M), CDC, NCHS. \\
\hline $13-15$ & New HIV infection diagnosed before progression to AIDS & Developmental \\
\hline $13-16$ & HIV infected persons surviving $3+$ years after diagnosis of AIDS & HIV Surveillance System, CDC, NCHHSTP. \\
\hline $13-17 a$ & Perinatally acquired HIV/AIDS diagnosed (no. new cases) & Developmental \\
\hline $13-17 b$ & Perinatally acquired AIDS (no. new cases) & HIV Surveillance System, CDC, NCHHSTP. \\
\hline $13-18$ & HIV/AIDS diagnosed in adolescent and young females (13-24 years) & Developmental \\
\hline
\end{tabular}


Figure 13-1. Progress Toward Target Attainment for Focus Area 13: HIV

Moved away

from target ${ }^{1}$

Moved toward

target

Met or exceeded

target

13-1. New AIDS cases (per 100,000 population $13+$ years)

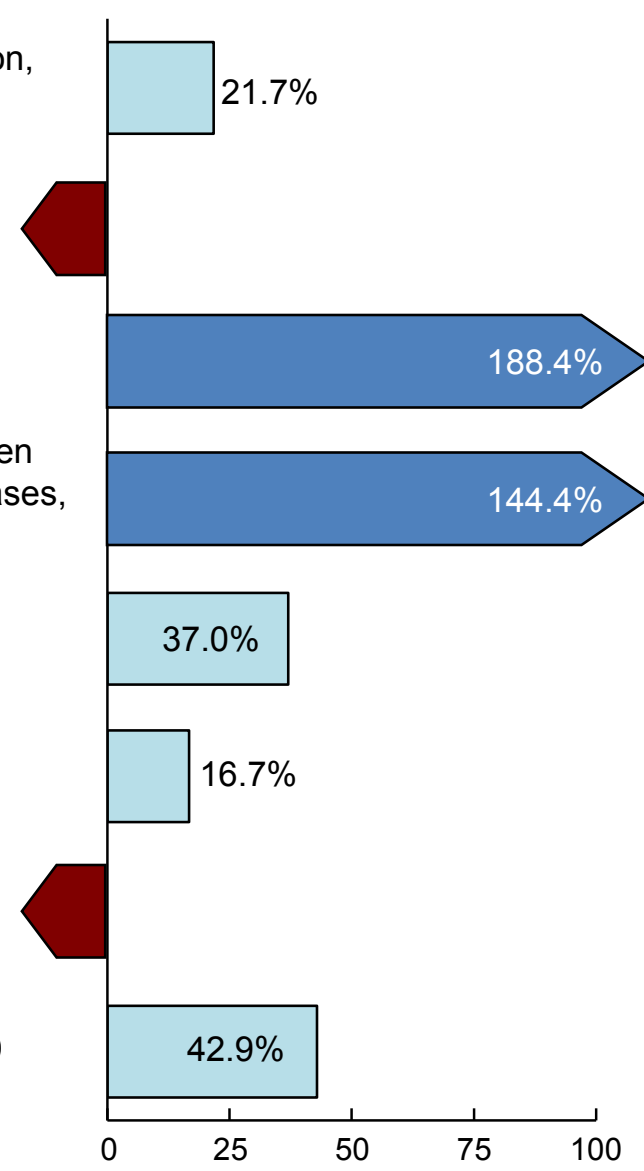

\begin{tabular}{|c|c|c|c|c|c|}
\hline \multirow{2}{*}{$\begin{array}{l}2010 \\
\text { Target }\end{array}$} & \multirow{2}{*}{$\begin{array}{c}\text { Baseline } \\
\text { (Year) }\end{array}$} & \multirow{2}{*}{$\begin{array}{l}\text { Final } \\
\text { (Year) }\end{array}$} & \multicolumn{3}{|c|}{ Baseline vs. Final } \\
\hline & & & Difference ${ }^{2}$ & $\begin{array}{l}\text { Statistically } \\
\text { Significant }^{3}\end{array}$ & $\begin{array}{l}\text { Percent } \\
\text { Change }^{4}\end{array}$ \\
\hline 0.9 & $\begin{array}{c}18.4 \\
(1998)\end{array}$ & $\begin{array}{c}14.6 \\
(2007)\end{array}$ & -3.8 & $\begin{array}{c}\text { Not } \\
\text { tested }\end{array}$ & $-20.7 \%$ \\
\hline 12,661 & $\begin{array}{l}16,882 \\
(1998)\end{array}$ & $\begin{array}{l}16,992 \\
(2007)\end{array}$ & 110 & $\begin{array}{c}\text { Not } \\
\text { tested }\end{array}$ & $0.7 \%$ \\
\hline 8,636 & $\begin{array}{l}11,514 \\
(1998)\end{array}$ & $\begin{array}{l}6,093 \\
(2007)\end{array}$ & $-5,421$ & $\begin{array}{c}\text { Not } \\
\text { tested }\end{array}$ & $-47.1 \%$ \\
\hline 2,064 & $\begin{array}{l}2,751 \\
(1998)\end{array}$ & $\begin{array}{l}1,759 \\
(2007)\end{array}$ & -992 & $\begin{array}{c}\text { Not } \\
\text { tested }\end{array}$ & $-36.1 \%$ \\
\hline $50 \%$ & $\begin{array}{c}23 \% \\
(1995)\end{array}$ & $\begin{array}{c}33 \% \\
(2006-08)\end{array}$ & 10 & $\begin{array}{c}\text { Not } \\
\text { tested }\end{array}$ & $43.5 \%$ \\
\hline $54 \%$ & $\begin{array}{c}42 \% \\
(2002)\end{array}$ & $\begin{array}{c}44 \% \\
(2006-08)\end{array}$ & 2 & $\begin{array}{l}\text { Not } \\
\text { tested }\end{array}$ & $4.8 \%$ \\
\hline $70 \%$ & $\begin{array}{c}58 \% \\
(1997)\end{array}$ & $\begin{array}{c}54 \% \\
(2008)\end{array}$ & -4 & $\begin{array}{c}\text { Not } \\
\text { tested }\end{array}$ & $-6.9 \%$ \\
\hline $89 \%$ & $\begin{array}{c}61 \% \\
(1998)\end{array}$ & $\begin{array}{c}73 \% \\
(2008)\end{array}$ & 12 & $\begin{array}{c}\text { Not } \\
\text { tested }\end{array}$ & $19.7 \%$ \\
\hline
\end{tabular}

Percent of targeted change achieved 5

(continued) 
Figure 13-1. Progress Toward Target Attainment for Focus Area 13: HIV (continued)

Moved away

from target ${ }^{1}$

Moved toward

target

Met or exceeded

target

13-13c. Any antiretroviral therapy among HIVinfected persons $(13+$ years $)$

13-13d. Highly active antiretroviral therapy (HAART) among HIV-infected persons (13+ years)

13-13e. Pneumocystis carinii pneumonia (PCP) prophylaxis among HIV-infected persons $(13+$ years $)$

13-13f. Mycobacterium avium complex (MAC) prophylaxis among HIV-infected persons $(13+$ years $)$

13-14. HIV-infection deaths (age adjusted, per 100,000 population)

13-16. HIV infected persons surviving $3+$ years after a diagnosis of AIDS

13-17b. Perinatally acquired AIDS (number of new cases)
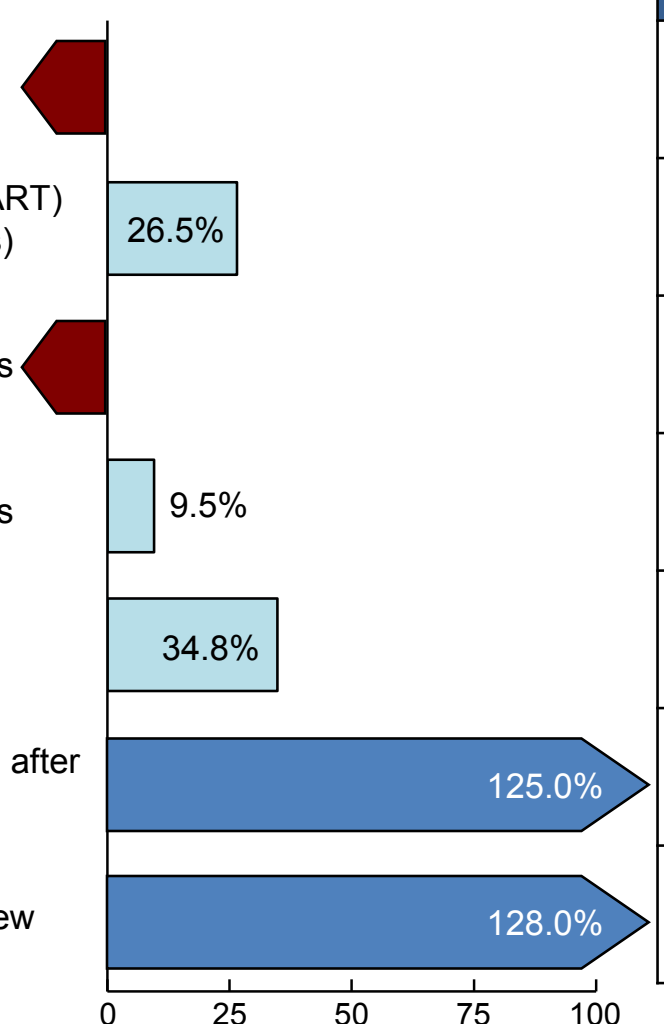

\begin{tabular}{|c|c|c|c|c|c|}
\hline \multirow{2}{*}{$\begin{array}{l}2010 \\
\text { Target }\end{array}$} & \multirow{2}{*}{$\begin{array}{c}\text { Baseline } \\
\text { (Year) }\end{array}$} & \multirow{2}{*}{$\begin{array}{l}\text { Final } \\
\text { (Year) }\end{array}$} & \multicolumn{3}{|c|}{ Baseline vs. Final } \\
\hline & & & Difference $^{2}$ & $\begin{array}{l}\text { Statistically } \\
\text { Significant }^{3}\end{array}$ & $\begin{array}{l}\text { Percent } \\
\text { Change }^{4}\end{array}$ \\
\hline $95 \%$ & $\begin{array}{c}85 \% \\
(1997)\end{array}$ & $\begin{array}{c}84 \% \\
(2003)\end{array}$ & -1 & $\begin{array}{c}\text { Not } \\
\text { tested }\end{array}$ & $-1.2 \%$ \\
\hline $95 \%$ & $\begin{array}{c}61 \% \\
(1997)\end{array}$ & $\begin{array}{c}70 \% \\
(2003)\end{array}$ & 9 & $\begin{array}{l}\text { Not } \\
\text { tested }\end{array}$ & $14.8 \%$ \\
\hline $95 \%$ & $\begin{array}{c}81 \% \\
(1997)\end{array}$ & $\begin{array}{c}68 \% \\
(2003)\end{array}$ & -13 & $\begin{array}{l}\text { Not } \\
\text { tested }\end{array}$ & $-16.0 \%$ \\
\hline $95 \%$ & $\begin{array}{c}53 \% \\
(1997)\end{array}$ & $\begin{array}{c}57 \% \\
(2003)\end{array}$ & 4 & $\begin{array}{l}\text { Not } \\
\text { tested }\end{array}$ & $7.5 \%$ \\
\hline 0.7 & $\begin{array}{c}5.3 \\
(1999)\end{array}$ & $\begin{array}{c}3.7 \\
(2007)\end{array}$ & -1.6 & Yes & $-30.2 \%$ \\
\hline $86 \%$ & $\begin{array}{c}78 \% \\
(1998)\end{array}$ & $\begin{array}{c}88 \% \\
(2006)\end{array}$ & 10 & $\begin{array}{c}\text { Not } \\
\text { tested }\end{array}$ & $12.8 \%$ \\
\hline 75 & $\begin{array}{c}243 \\
(1998)\end{array}$ & $\begin{array}{c}28 \\
(2007)\end{array}$ & -215 & $\begin{array}{c}\text { Not } \\
\text { tested }\end{array}$ & $-88.5 \%$ \\
\hline
\end{tabular}

Percent of targeted change achieved ${ }^{5}$ 
Figure 13-1. Progress Toward Target Attainment for Focus Area 13: HIV (continued)

\section{NOTES}

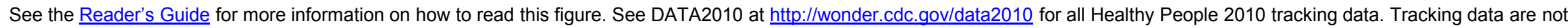
available for objective 13-5, 13-13a, 13-13b, 13-15, 13-17a, and 13-18. Objectives 13-7, 13-9, 13-10, and 13-12 were deleted at the Midcourse Review.

\section{FOOTNOTES}

${ }^{1}$ Movement away from target is not quantified using the percent of targeted change achieved. See Technical Appendix for more information.

${ }^{2}$ Difference $=$ Final value - Baseline value. Differences between percents (\%) are measured in percentage points.

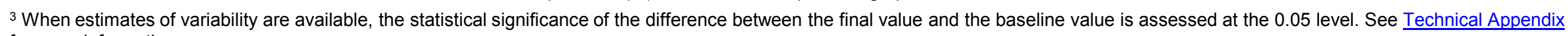
for more information.

${ }^{4}$ Percent change $=\frac{\text { Final value }- \text { Baseline value }}{\text { Baseline value }} \times 100$.
${ }^{5}$ Percent of targeted change achieved $=\frac{\text { Final value }- \text { Baseline value }}{\text { Healthy People } 2010 \text { target }- \text { Baseline value }} \times 100$.

\section{DATA SOURCES}

13-1-13-4. HIV Surveillance System, CDC, NCHHSTP.

$\begin{array}{ll}13-6 a-b . & \text { National Survey of Family Growth (NSFG), CDC, NCHS. } \\ 13-8 . & \text { Baseline: Uniform Facility Data Set (UFDS), SAMHSA; Final }\end{array}$

13-8.

13-11.

13-13c-f.

13-14.

13-16.

Baseline: Uniform Facility Data Set (UFDS), SAMHSA; Final: National Survey of Substance Abuse Treatment Services (N-SSATS), SAMHSA.

National TB Surveillance System, CDC, NCHHSTP.

Adult and Adolescent Spectrum of HIV Disease (ASD) Surveillance Project, CDC, NCHHSTP.

National Vital Statistics System-Mortality (NVSS-M), CDC, NCHS.

HIV Surveillance System, CDC, NCHHSTP.

13-17b. HIV Surveillance System, CDC, NCHHSTP. 
Figure 13-2. Health Disparities Table for Focus Area 13: HIV

Disparities from the best group rate for each characteristic at the most recent data point and changes in disparity from the baseline to the most recent data point.

Characteristics and Groups

Population-based objectives

13-1. New AIDS cases (per 100,000 population, $13+$ years $)(1998$ 2007)†

13-6a. Condom use among sexually active unmarried persons-females (18-44 years) $(1995,2006-08)^{1} \ddagger$

b. Condom use among sexually active unmarried persons-males (18-44 years) $(2002,2006-08)^{1} \ddagger$

13-11. HIV testing in TB patients (25-44 years) $(1998,2008) \dagger$

13-13c. Any antiretroviral therapy among HIV-infected persons (13+ years) (1997, 2003)†

13-13d. Highly active antiretroviral therapy (HAART) among HIV-infected persons $(13+$ years $)(1997,2003) \dagger$

13-13e. Pneumocystis carinii pneumonia (PCP) prophylaxis among HIV-infected persons $(13+$ years $)(1997,2003) \dagger$

13-13f. Mycobacterium avium complex (MAC) prophylaxis among HIV-infected persons $(13+$ years $)(1997,2003) \dagger$

13-14. HIV-infection deaths (age adjusted per 100,000 population) (1999, $2007)^{2,3 *}$

13-16. HIV infected persons surviving 3+ years after a diagnosis of AIDS (1998, 2006)†

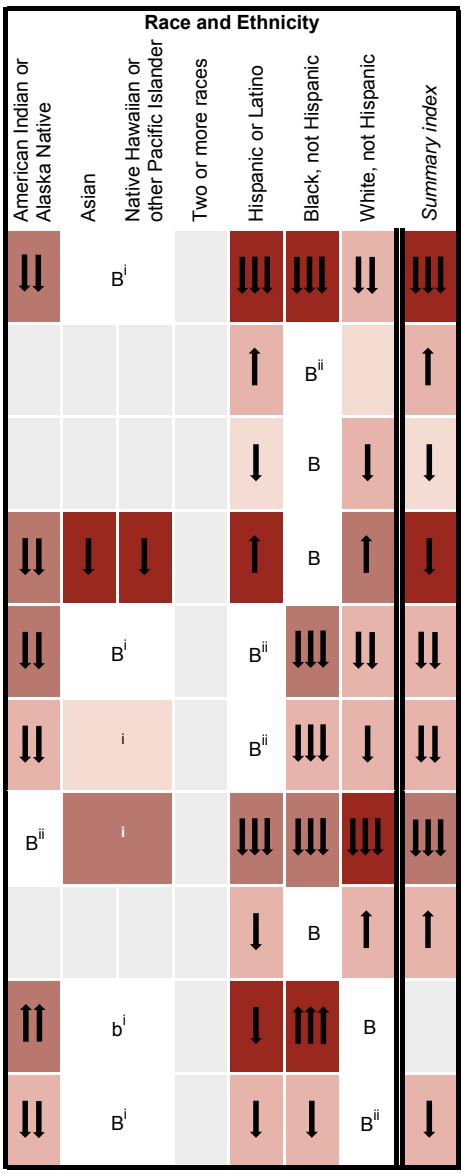

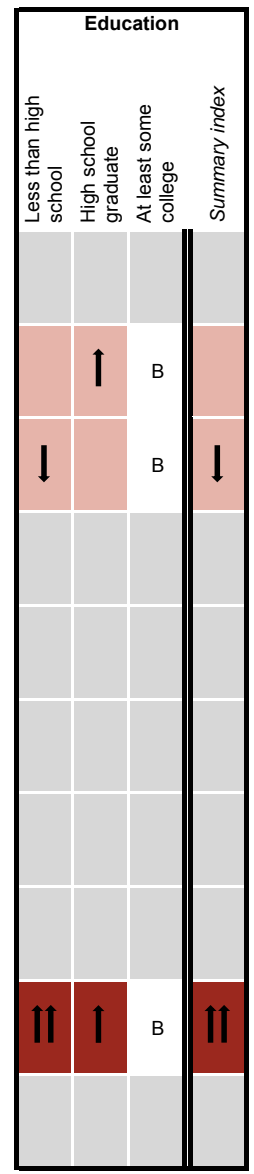

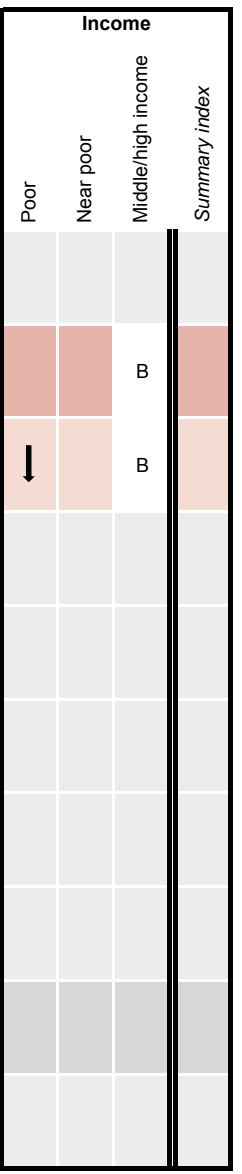

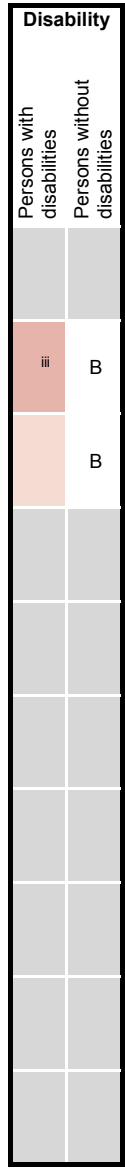

(continued) 
Figure 13-2. Health Disparities Table for Focus Area 13: HIV (continued)

NOTES

See DATA2010 at http://wonder.cdc.gov/data2010 for all Healthy People 2010 tracking data. Disparity data are either unavailable or not applicable for objectives $13-2$ through 13-5, 13-8, 13-13a and b, 13-15, 13-17a and b, and 13-18. Objectives 13-7, 13-9, 13-10, and 13-12 were deleted at Midcourse Review.

Years in parentheses represent the baseline and most recent data years (if available).

Disparity from the best group rate is defined as the percent difference between the best group rate and each of the other group rates for a characteristic (e.g., race and ethnicity). The summary index is the average of these percent differences for a characteristic. Change in disparity is estimated by subtracting the disparity at baseline from the disparity at the most recent data point. Change in the summary index is estimated by subtracting the summary index at baseline from the summary index at the most recent data point. See Technical Appendix for more information.

LEGEND

$\begin{aligned} & \text { The "best" group rate at the most recent } \\ & \text { data point. }\end{aligned}$ B $\begin{aligned} & \text { The group with the best rate for } \\ & \text { specified characteristic. }\end{aligned} \quad$ b $\begin{aligned} & \text { Most favorable group rate for specified characteristic, } \\ & \text { but reliability criterion not met. }\end{aligned}$
$\begin{aligned} & \text { Reliability criterion for best group } \\ & \text { rate not met, or data available for } \\ & \text { only one group. }\end{aligned}$

\begin{tabular}{|c|c|c|c|c|}
\hline \multirow[b]{2}{*}{$\begin{array}{l}\text { Disparity from the best group rate at the } \\
\text { most recent data point. }\end{array}$} & \multicolumn{4}{|c|}{ Percent difference from the best group rate } \\
\hline & $\begin{array}{l}\text { Less than } 10 \% \text {, or difference not } \\
\text { statistically significant (when } \\
\text { estimates of variability are available). }\end{array}$ & $10 \%-49 \%$ & $50 \%-99 \%$ & $100 \%$ or more \\
\hline
\end{tabular}

Changes in disparity over time are shown when:

(a) disparities data are available at both baseline and most recent time points; (b) data are not for the group(s) indicated by "B" or "b" at either time point; and (c) the change is greater than or equal to 10 percentage points and statistically significant, or when the change is greater than or equal to 10 percentage significant, or when the change is greater than or equal to 10 percentage
points and estimates of variability were not available. See Technical Appendix.

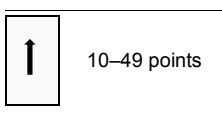

Increase in disparity (percentage points)

\begin{tabular}{|c|c|c|c|c|}
\hline points and estimates & ble. See Technical Appendix. & 10-49 points & 50-99 points & 100 points or more \\
\hline Availability of Data & Data not available. & & Characteristic & objective. \\
\hline
\end{tabular}

\section{FOOTNOTES}

* Measures of variability were available. Thus, the variability of best group rates was assessed, and statistical significance was tested. Disparities of $10 \%$ or more are displayed when the differences from the best group rate are statistically significant at the 0.05 level. Changes in disparities over time are indicated by arrows when the changes are greater than or equal to 10 percentage points and are statistically significant at the 0.05 level. See $\underline{\text { Technical Appendix. }}$

† Measures of variability were not available. Thus, the variability of best group rates was not assessed, and statistical significance could not be tested. Nonetheless, disparities and changes in disparities over time are displayed according to their magnitude. See Technical Appendix.

$\ddagger$ Measures of variability were available only for the most recent data. Thus, the variability of best group rates was assessed only for the most recent data, and statistical significance was tested only for the most recent data. Disparities of $10 \%$ or more are displayed when the differences from the best group rate are statistically significant at the 0.05 level. Changes in disparities over time are displayed according to their magnitude, since measures of variability were not available at baseline and therefore statistical significance of changes in disparity could not be tested. See Technical Appendix.

1 Data by education level are for persons aged $25-44$ years.

2 Data by education level are for persons aged 25-64 years.

3 Most recent data by education level is for 2002

i Data are for Asian or Pacific Islander.

ii The group with the best rate at the most recent data point is different from the group with the best rate at baseline. Both rates met the reliability criterion. See Technical Appendix.

iii Reliability criterion for best group rate not met, or data available for only one group, at baseline. Change in disparity cannot be assessed. See Technical Appendix.

DATA SOURCES

13-1. HIV Surveillance System, CDC, NCHHSTP.

13-6a-b. National Survey of Family Growth (NSFG), CDC, NCHS.

13-11. National TB Surveillance System, CDC, NCHHSTP.

13-13c-f. Adult and Adolescent Spectrum of HIV Disease (ASD) Surveillance Project, CDC, NCHHSTP.

13-14. National Vital Statistics System-Mortality (NVSS-M), CDC, NCHS

13-16. HIV Surveillance System, CDC, NCHHSTP. 
Figure 13-3. New AIDS Cases (13+ Years) 2007

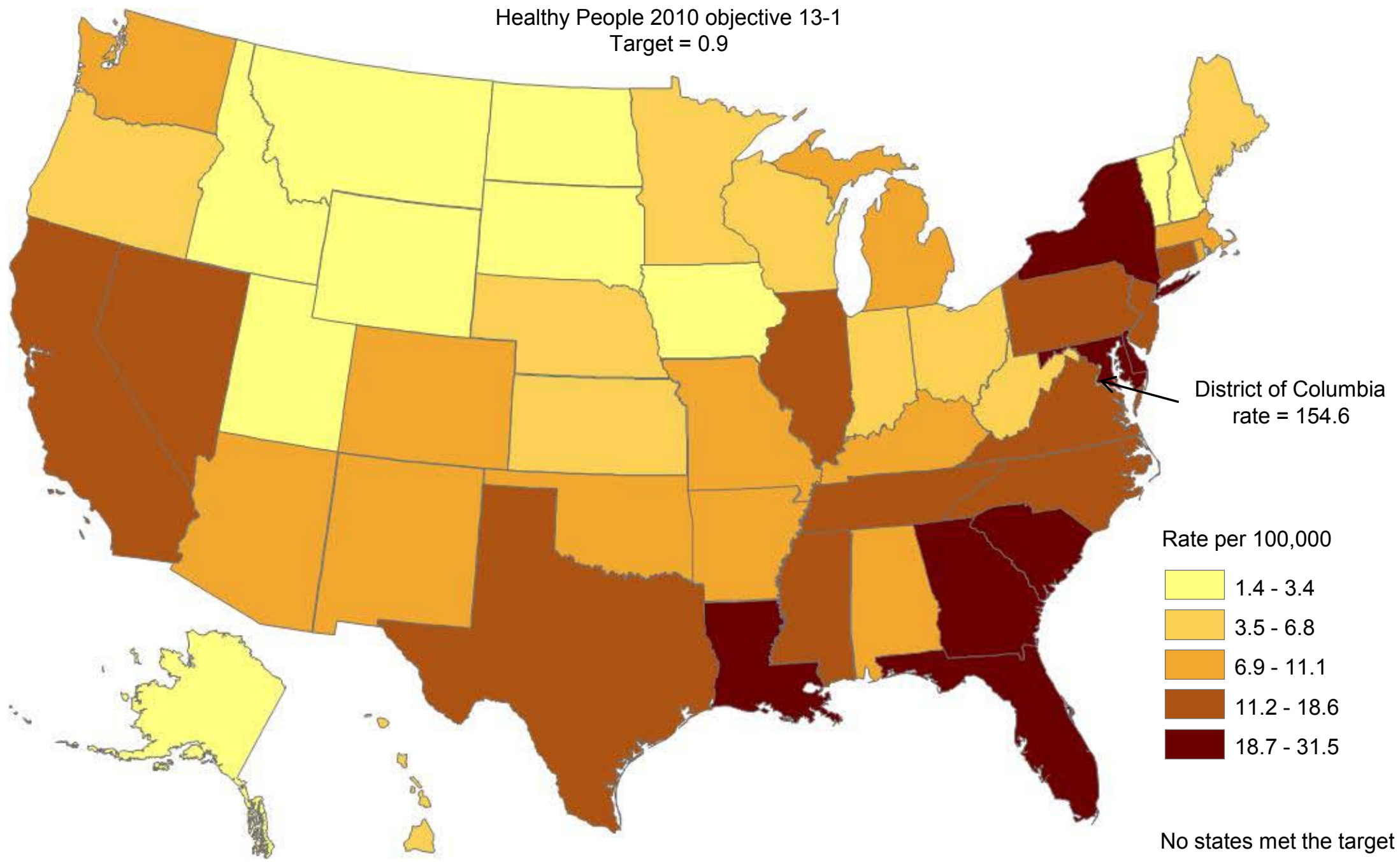

NOTES: Data are age-adjusted to the 2000 standard population. Rates are displayed by a Jenks classification for U.S. states. SOURCE: HIV Surveillance System, CDC, NCHHSTP. 
Figure 13-4. HIV Infection Deaths 2005-07

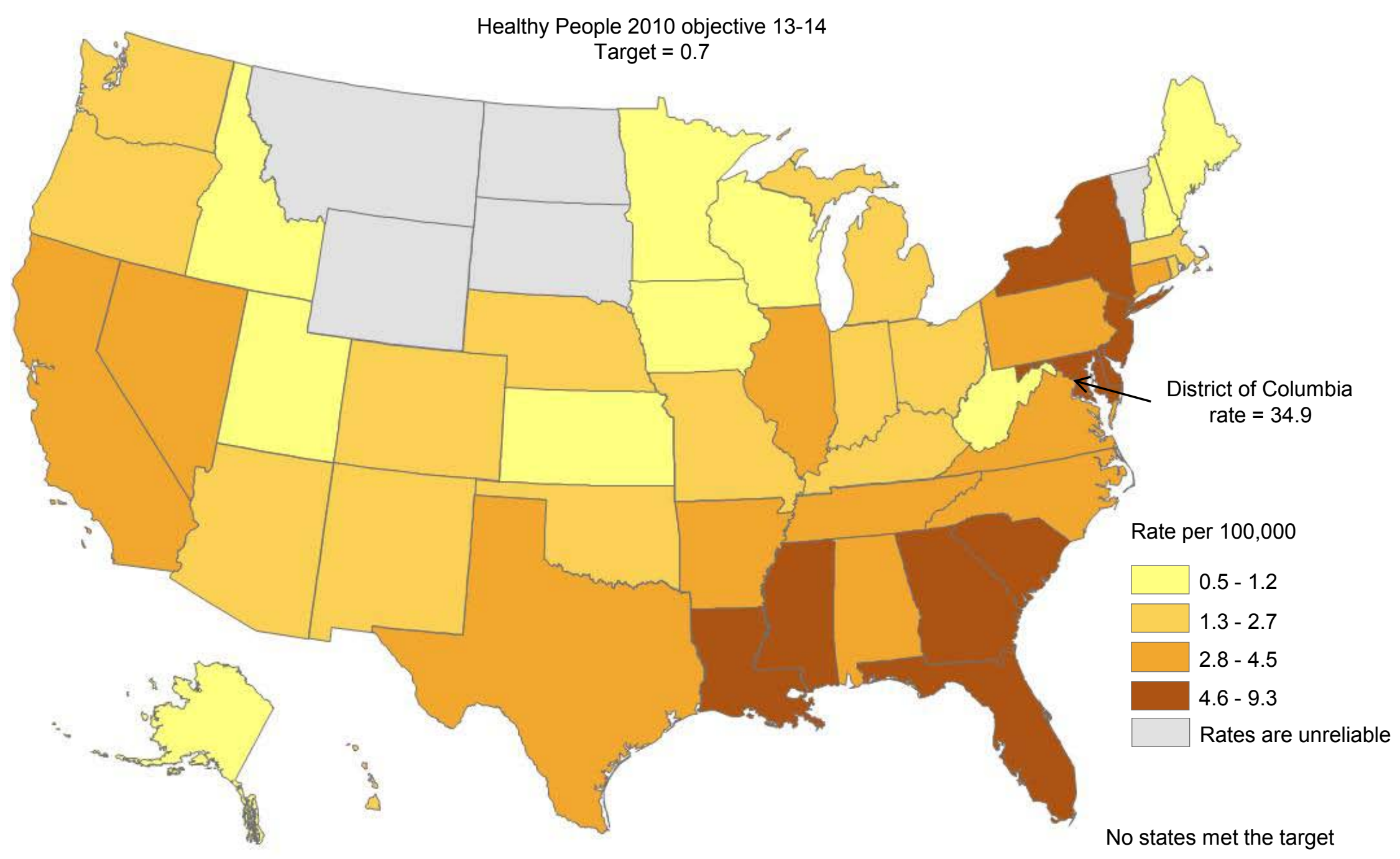

NOTES: Data are for ICD-10 codes B20-B24 reported as underlying cause. Rates age-adjusted to the 2000 standard population. Rates are displayed by a Jenks classification for U.S. states.

SOURCE:National Vital Statistics System - Mortality (NVSS-M), CDC, NCHS. 


\section{Immunization and Infectious Diseases}

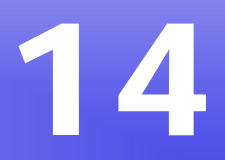

\section{Lead Agency}

Centers for Disease Control and Prevention

\section{Contents}

Goal.

Highlights.

Summary of Progress.

Transition to Healthy People 2020

Data Considerations

Notes

Comprehensive Summary of Objectives

Progress Chart

Health Disparities Table

Vaccination of children 19-35 months: Three doses of hepatitis B (Hep B) vaccine-Map.......... 14-34

Vaccination of children 19-35 months: One dose Measles-Mumps-Rubella (MMR) vaccine-Map

Vaccination of children 19-35 months: Four doses pneumococcal conjugate vaccine (PCV)—Map 


\section{Goal: Prevent disease, disability, and death from infectious diseases, including vaccine-preventable diseases}

The 87 objectives in this chapter cover five general areas in immunization and infectious diseases:

- Diseases preventable through universal vaccination. This area includes objectives monitoring progress in the reduction of vaccine-preventable diseases such as polio, pertussis, rubella, and hepatitis B.

- Diseases preventable through targeted vaccination. The objectives in this area address diseases affecting high-risk populations or certain endemic areas that can be prevented through targeted vaccination.

- Infectious diseases and emerging antimicrobial resistance. The objectives in this area focus on conditions such as tuberculosis and its treatment, hepatitis $\mathrm{C}$, and hospitalacquired infections.

- Vaccine coverage and strategy. These objectives address immunization rates for children, adolescents, and adults.

- Vaccine safety. These objectives address the monitoring of adverse outcomes to vaccination.

All Healthy People 2010 tracking data quoted in this chapter, along with technical information and operational definitions for each objective, can be found in the Healthy People 2010 database,

DATA2010, available from http://wonder.cdc.gov/data2010/.

More information about this Focus Area can be found in the following publications:

- Healthy People 2010: Understanding and Improving Health, available from http://www.healthypeople.gov/2010/Document/tableofcontents.htm\#under.

- Healthy People 2010 Midcourse Review, available from http://www.healthypeople.gov/2010/data/midcourse/html/default.htm\#FocusAreas.

\section{Highlights}

- Substantial progress was achieved in objectives for this Focus Area during the past decade [1]. Over eighty percent of the Immunization and Infectious Diseases objectives with data to measure progress moved toward or achieved their Healthy People 2010 targets (Figure 141). However, health disparities of $10 \%$ or more were observed among select population groups (Figure 14-2), some of which are highlighted below [2].

\section{Diseases preventable through universal vaccination}

Most of the objectives in this area moved toward or achieved the Healthy People 2010 targets. 
- New cases of hepatitis B among children aged 2-18 years (objective 14-1d) declined by $92.9 \%$ between 1997 and 2008, from 708 to 50 cases, moving toward the 2010 target of 7 cases.

- Cases of rubella (objective 14-1i) declined by 97.3\% between 1998 and 2008, from 364 to 10 cases, moving toward the 2010 target of 0 cases. Similarly, cases of varicella (chicken pox) among persons aged under 18 years (objective 14-1k) declined by $73.7 \%$ between 1999 and 2008, from 2,229,000 to 586,000 cases, moving toward the 2010 target of 223,000 cases.

- The prevalence of hepatitis B in adults (objectives 14-3a through g) declined for all age groups and high-risk groups. The number of cases of hepatitis B among injection drug users (objective 14-3d) declined by 80.3\% between 1997 and 2008, from 7,135 to 1,408 cases, exceeding the 2010 target of 1,784 cases.

\section{Hepatitis B-Persons aged 19-24 years (objective 14-3a)}

- Among racial and ethnic populations, the Hispanic or Latino population had the lowest (best) rate of hepatitis B for persons aged 19-24 years, 1.4 cases per 100,000 population in 2008. The Asian or Pacific Islander, American Indian or Alaska Native, and black non-Hispanic populations had hepatitis B rates of 3.1, 5.1 and 5.7 cases per 100,000 in 2008, respectively.

- The rate for the Asian or Pacific Islander population was more than twice the best group rate (that for the Hispanic or Latino population); the rate for the American Indian or Alaska Native population was more than three and a half times the best group rate; and the rate for the black non-Hispanic population was more than four times the best group rate [2].

- The white non-Hispanic population had the best rate in 1997 (7.7 cases per 100,000 population), while the Hispanic or Latino population had the best rate in 2008 (1.4 cases per 100,000). The rates for the Asian or Pacific Islander population were 33.7 cases per 100,000 in 1997 and 3.1 in 2008. Between 1997 and 2008, the disparity between the Asian or Pacific Islander population and the group with the best rate declined by 216 percentage points [3].

\section{Hepatitis B-Persons aged 25-39 years (objective 14-3b)}

- The Asian or Pacific Islander population had the lowest (best) rate of hepatitis B for persons aged 25-39 years, 2.7 cases per 100,000 population in 2008. The rate for the white non-Hispanic population, 2.7 cases per 100,000 in 2008, was twice the best group rate; the American Indian or Alaska Native population had a rate of 9.7 cases per 100,000 in 2008, more than three and a half times the best group rate; and the rate for the black non-Hispanic population, 10.7 cases per 100,000 in 2008, was about four times the best group rate [2].

Hepatitis B-Persons aged 40 years and over (objective 14-3c)

- The Asian or Pacific Islander population also had the lowest (best) rate of hepatitis B for persons aged 40 years and over, 4.0 cases per 100,000 population in 2008 . The American Indian or Alaska Native and black non-Hispanic populations had rates of 10.3 and 13.2 cases per 100,000 in 2008 , respectively. 
- The rate for the American Indian or Alaska Native population was more than two and a half times the best group rate (that for the Asian or Pacific Islander population), while the rate for the black non-Hispanic population was almost three and a half times the best group rate [2].

- Among persons aged 40 years and over, the hepatitis B rate for males was twice the rate for females, 9.5 and 4.6 cases per 100,000 population in 2008 , respectively [2].

\section{Hepatitis B-All age groups (objectives 14-3a through c)}

- Between 1997 and 2008, the disparity in hepatitis B rates between the American Indian or Alaska Native population and the population with the best rate increased by at least 100 percentage points for all age groups (objectives 14-3a through c) [3].

- The incidence of bacterial meningitis in young children aged 1-23 months (objective 14-4) declined by $34.6 \%$ between 1998 and 2008 , from 13.0 to 8.5 cases per 100,000 population, exceeding the 2010 target of 8.6 per 100,000 .

- The incidence of invasive pneumococcal infections among young children and older adults (objective 14-5a and b) declined between 1997 and 2008.

- Among children aged under 5 years (objective 14-5a), the incidence of invasive pneumococcal infections decreased by $74.0 \%$ between 1997 and 2008, from 77 to 20 new cases per 100,000 population, exceeding the 2010 target of 46 per 100,000.

- Among adults aged 65 years and over (objective 14-5b), the incidence of invasive pneumococcal infections decreased by 33.9\% between 1997 and 2008, from 62 to 41 new cases per 100,000 population, exceeding the 2010 target of 42 per 100,000.

- The incidence of penicillin-resistant pneumococcal infections among young children under 5 years of age (objective 14-5c) declined by 56.3\% between 1997 and 2008, from 16 to 7 new cases per 100,000, moving toward the 2010 target of 6 per 100,000.

\section{Diseases preventable through targeted vaccination}

Two objectives in this area exceeded their Healthy People 2010 targets.

- The incidence of hepatitis A (objective 14-6) declined by $92.0 \%$ between 1997 and 2008, from 11.2 to 0.9 new cases per 100,000 population, exceeding the Healthy People 2010 target of 4.3 per 100,000 .

- Among racial and ethnic groups, the black non-Hispanic population had the lowest (best) rate of hepatitis A, 0.4 new cases per 100,000 population in 2008 . The rate for the Asian or Pacific Islander population was 1.3 new cases per 100,000 in 2008, almost three and a half times the best group rate [2]. The rate for Hispanic or Latino population was 1.0 new cases per 100,000 in 2008 , two and a half times the best group rate.

- The Asian or Pacific Islander population had the best group rate in 1997 (4.4 new cases per 100,000 ), while the black non-Hispanic population had the best group rate in 2008 (0.4 per 100,000). The Hispanic or Latino population had rates of 23.4 per 100,000 in 1997 and 1.0 in 2008. Between 1997 and 2008, the disparity between the Hispanic or Latino population and the best group declined by 282 percentage points [3]. 
- The incidence of meningococcal disease (objective 14-7) declined by 69.2\% between 1997 and 2008, from 1.3 to 0.4 new cases per 100,000 population, exceeding the Healthy People 2010 target of 1.0 per 100,000 .

\section{Infectious diseases and emerging antimicrobial resistance}

Many objectives in this area moved toward their 2010 targets over the past decade.

- The following objectives exceeded the Healthy People 2010 targets:

- The incidence of hepatitis C (objective 14-9) decreased by $88.0 \%$ between 1997 and 2007, from 2.5 to 0.3 new cases per 100,000 population, exceeding the target of 1.0 .

- Treatment for high-risk persons with latent tuberculosis infection (objective 14-13) increased by $51.1 \%$ between 2000 and 2007 , from $45 \%$ to $68 \%$, exceeding the target of $57 \%$.

- Invasive early onset group B streptococcal disease (objective 14-16) declined by $70.0 \%$ between 1996 and 2008, from 1.0 to 0.3 cases per 1,000 live births, exceeding the target of 0.5 .

- Peptic ulcer hospitalizations (objective 14-17) decreased by 39.4\% between 1998 and 2007, from 71 to 43 hospitalizations per 100,000 population (age adjusted), exceeding the target of 46 .

- Antibiotics prescribed for ear infections in children aged under 5 years (objective 14-18) declined by $29.0 \%$ between 1996-97 and 2006-07, from 69 to 49 courses per 100 population, exceeding the target of 56.

- Hospital-acquired infections among adult and infant intensive care patients (objectives 14-20a through e) declined for all categories, exceeding the targets.

- The incidence of tuberculosis (TB; objective 14-11) decreased by $36.4 \%$ between 1998 and 2008, from 6.6 to 4.2 new cases per 100,000 population, moving toward the 2010 target of 1.0 per 100,000 .

- Among racial and ethnic groups, the white non-Hispanic population had the lowest (best) rates of new TB cases, 1.5 per 100,000 population in 1998 and 1.1 in 2008. All other racial and ethnic populations with data to measure disparity had rates that were at least $100 \%$ higher than the best rate [2]. The rates for the Hispanic or Latino population were 12.6 new cases per 100,000 population in 1998, and 8.1 in 2008 . Between 1998 and 2008, the disparity between the Hispanic or Latino and the white non-Hispanic populations increased by 189 percentage points [3].

\section{Vaccination coverage and strategy}

Many of the vaccination coverage objectives either achieved or made substantial progress toward their Healthy People 2010 targets.

- Targets for the vaccination of children aged 19-35 months were exceeded for the Haemophilus influenza type b (Hib), hepatitis B (Hep B), measles-mumps-rubella (MMR), polio, and varicella vaccines (objectives $14-22 \mathrm{~b}$ through $\mathrm{f}$, respectively). Vaccination rates for pneumococcal conjugate vaccine (PCV) (objective 14-22g) increased by $300.0 \%$ between 2002 and 2008, from 20\% to 80\%, moving toward the 2010 target of $90 \%$. 
- All but three states (Montana, Nevada, and Washington) had achieved the $90 \%$ target for Hep B vaccination (objective 14-22c) in 2008 (Figure 14-3).

- Thirty-eight states had achieved the $90 \%$ target for MMR vaccination. MMR vaccination rates for the rest of the U.S. were all above 85\% (Figure 14-4).

- Vaccination rates for PCV, one of the newest vaccines, still varied among states. Only one state, Connecticut, had achieved the 90\% target for PCV (objective 14-22g) in 2008. The rates were lowest, at or below 70\%, in Nevada, Oklahoma, and Wyoming (Figure 14-5).

- The vaccination targets for children in day care (objective 14-23) were met for diphtheriatetanus-acellular pertussis (DTap), MMR, polio, and Hib vaccines (objectives 14-23a, b, c, and $\mathrm{l}$, respectively).

- The proportion of private providers who measured childhood vaccine coverage levels (objective 14-25b) tripled between 1999 and 2009, from $11 \%$ to 33\%, moving toward the 2010 target of $55 \%$.

- The percentage of children aged under 6 years who participated in population-based immunization registries (objective 14-26) increased by $257.1 \%$ between 1999 and 2008, from $21 \%$ to $75 \%$, exceeding the 2010 target of $62 \%$.

- Targets for the vaccination of adolescents aged 13-15 years were exceeded for Hep B and MMR vaccines (objective 14-27a and $b$, respectively). The proportion of adolescents in this age group who had received a varicella vaccination (objective $14-27 \mathrm{~d}$ ) increased by $91.1 \%$ between 1997 and 2008, from 45\% to 86\%, moving toward the 2010 target of $90 \%$. However, the receipt of a tetanus-diphtheria (Td) booster (objective 14-27c) decreased $23.7 \%$ between 1997 and 2008, from 93\% to 71\%, moving away from the target. The Combined Tetanus, Diptheria and Pertussis vaccine (Tdap) was introduced in 2006, leading to the decline in $\mathrm{Td}$ administration. However, overall tetanus booster vaccination (either through Td or Tdap) had been increasing over time.

\section{Vaccine safety}

- One objective in this area, the proportion of Vaccine Adverse Event Reports (VAERS) that were submitted electronically (objective 14-31b) increased by $112.5 \%$ between 2003 and 2009 , from $16 \%$ to $34 \%$, exceeding the 2010 target of $30 \%$. 


\section{Summary of Progress}

- Figure 14-1 presents a quantitative assessment of progress in achieving the Healthy People 2010 objectives for Immunization and Infectious Diseases [1]. Data to measure progress toward target attainment were available for 80 objectives. Of these:

- Thirty-three objectives met or exceeded the Healthy People 2010 targets (objectives 14-1a, b, and h; 14-3d; 14-4; 14-5a and b; 14-6; 14-7; 14-9; 14-13; 14-16 through 14-18; 14-20a through e; 14-22b through f; 14-23a through c, and l; 14-26; 14-27a and b; 14-30a; and 14-31b).

- Thirty-two objectives moved toward their targets. A statistically significant difference between the baseline and the final data points was observed for eight of these objectives (14-1k, 14-19, 14-24a, 14-27d, and 14-29a through d). No significant difference was observed for one objective (14-22a); and data to test the significance of the difference were unavailable for 23 objectives $(14-1 \mathrm{~d}, \mathrm{f}, \mathrm{i}$, and $\mathrm{j}$; $14-2 ; 14-3 a$, through $\mathrm{c}$, and e through g; 14-5c; 14-10 through 14-12; 14-22g; $14-$ 25b; 14-28a and b; 14-29f and g; 14-30b; and 14-31a).

- One objective (14-25a) showed no change.

- Fourteen objectives moved away from their targets. A statistically significant difference between the baseline and final data points was observed for one objective (14-27c). Data to test the significance of the difference were unavailable for 13 objectives (14-1c, e, and g; 14-5d; 14-8; 14-21; 14-23f through j; 14-28c; and 1429e).

- Two objectives (14-22h and 14-24b) remained developmental and four objectives (14-14; and 14-23d, e, and k) had no follow-up data available to measure progress [4]. One objective (14-15) was dropped during the decade [5].

- Figure 14-2 displays health disparities in Immunization and Infectious Diseases from the best group rate for each characteristic at the most recent data point [2]. It also displays changes in disparities from baseline to the most recent data point [3].

- Ten objectives had statistically significant racial and ethnic health disparities of $10 \%$ or more. An additional 11 objectives had racial and ethnic health disparities of $10 \%$ or more but lacked data to test significance. Of these 21 objectives, the white nonHispanic population had the only best rate for eight objectives $(14-11 ; 14-22 \mathrm{~g}$; 14 27d; and 14-29a, b, and e through g), while the white population (including persons of Hispanic origin) had the best rate for five objectives (14-5a through c, 14-7, and 14-16). The Asian or Pacific Islander (objectives 14-3b and c), Hispanic or Latino (objectives 14-3a and 14-22f), and black non-Hispanic (objectives 14-6 and 14-12) populations each had the only best rate for two objectives. Persons of two or more races had the best group rate for one objective (14-24a). The Hispanic or Latino and white non-Hispanic populations both had the best rate for one objective (14-24a).

- Five objectives had statistically significant health disparities of $10 \%$ or more by sex. Eleven additional objectives had health disparities of $10 \%$ or more by sex, but lacked data to test significance. Females had the better rates for 15 of these 16 objectives (14-3a through c; 14-4; 14-5a, c, and d; 14-6; 14-8; 14-11; 14-12; 14-18; 14-22d; and 14-29b and c). Males had the better rate for the remaining objective $(14-27 a)$. 
- Persons with at least some college education had the best group rate for all four objectives (14-29a through c, and g) with statistically significant health disparities of $10 \%$ or more by education level.

- Persons living in an urban or metropolitan area had better rates than persons living in rural or nonmetropolitan areas for all three objectives (14-27a, c, and d) with statistically significant health disparities of $10 \%$ or more by geographic location.

- Persons with disabilities had better rates than persons without disabilities for four of the five objectives (14-29a through d) with statistically significant health disparities of $10 \%$ or more by disability status, while persons without disabilities had the better rate for the remaining objective (14-29e).

- Racial and ethnic health disparities of $100 \%$ or more, as well as changes in disparities of 100 percentage points or more over time, were observed for several objectives. Most of these are discussed in the Highlights, above.

\section{Transition to Healthy People 2020}

For Healthy People 2020, the focus of the Immunization and Infectious Diseases Topic Area was expanded to include vaccinations against seasonal influenza in more defined segments of the population. Also, the Healthy People 2010 objectives were modified to better address healthcareacquired infections. See HealthyPeople.gov for a complete list of Healthy People 2020 topics and objectives.

The Healthy People 2020 Immunization and Infectious Diseases Topic Area can be grouped into several sections:

- Diseases Preventable Through Universal Vaccination

- Diseases Preventable Through Targeted Vaccination

- Infectious Diseases and Emerging Antimicrobial Resistance

- Vaccination Coverage and Strategies

- Surveillance and Monitoring.

The differences between the Healthy People 2010 and Healthy People 2020 objectives are summarized below:

- The Healthy People 2020 Immunization and Infectious Disease Topic Area has a total of 77 objectives, five of which are developmental, whereas the Healthy People 2010 Immunization and Infectious Disease Focus Area had 87 objectives, of which two were developmental.

- Twenty-nine Healthy People 2010 objectives were retained "as is" [6].

- Diseases preventable through universal vaccination. Nine objectives were retained, including: Congenital Rubella Syndrome (CRS) (objective 14-1a), measles (objective 14-1e), mumps (objective 14-1f), polio wild-type virus (objective 14-1h), rubella (objective 14-1i), varicella (objective 14-1k), chronic hepatitis B perinatal infections (objective 14-2), new invasive pneumococcal infections in children under five years (objective 14-5a), and new invasive pneumococcal infections in persons aged 65 years and over (objective 14-5b). 
- Diseases preventable through targeted vaccination. The two retained objectives in this category are new hepatitis A (objective 14-6) and new meningococcal disease (objective 14-7).

- Infectious diseases and emerging antimicrobial resistance. The following six objectives were retained: TB (objective 14-11), curative therapy for TB (objective 14-12), treatment for latent TB (objective 14-13), group B streptococcal disease among newborns (objective 14-16), antibiotic misuse for ear infections (objective 14-18), and antibiotic misuse for common cold (objective 14-19).

- Vaccination coverage and strategies. Twelve objectives were retained:

- Six childhood vaccination objectives: four doses DTaP (objective 14-22a), three doses Hep B (objective 14-22c), one dose MMR (objective 14-22d), three doses polio (objective 14-22e), one dose varicella (objective 14-22f), and four doses PCV (objective 14-22g)

- Public health and private providers who measure childhood vaccination coverage levels (objectives 14-25a and b)

- Population-based immunization registries for children under six years (objective 14-26)

- Hep B vaccination among occupationally exposed workers (objective 1428c); pneumococcal vaccination among noninstitutionalized adults aged 65 years and over (objective 14-29b); and pneumococcal vaccination among noninstitutionalized high-risk adults aged 18-64 years (objective 14-29d).

Thirty Healthy People 2010 objectives were modified to created 28 Healthy People 2020 objectives [7].

- Diseases preventable through universal vaccination. Ten objectives were modified:

- Hib among children aged under 5 years (objective 14-1c) and new hepatitis B cases among persons aged 2-18 years (objective 14-1d) were modified due to new measurement units.

- The target population for pertussis (objective 14-1g) was changed from children aged under 7 years in Healthy People 2010 to children aged under 1 year in Healthy People 2020.

- Three hepatitis B infection objectives among high-risk adults aged 19-24, 25-39, and 40 years and over (objectives 14-3a through c) were consolidated into one for adults aged 19 years and over.

- Hepatitis B infection among injection drug users and men who have sex with men (objectives 14-3d and f) were modified because of changes in the case definition.

- Invasive penicillin-resistant pneumococcal infections among children aged under 5 years and adults aged 65 years and over (objectives 14-5c and d) were modified because of changes in the case definition.

- Infectious Diseases and Emerging Antimicrobial Resistance. Two objectives were modified: the data source for Hepatitis C (objective 14-9) was changed, and timely laboratory confirmation of tuberculosis cases (objective 14-14) was modified due to a change in the measurement unit and the data source. 
- Vaccination Coverage and Strategies. Seventeen objectives were modified:

- Vaccination with three doses Hib (objective 14-22b) was modified due to a change in the data collection method.

- The dosage for influenza vaccination among children aged 6-23 months (objective 14-22h) was modified from one dose for the developmental Healthy People 2010 objective to 1-2 doses, depending on age appropriateness, for the measurable Healthy People 2020 objective.

- All five vaccination objectives for kindergarten (DTaP, MMR, polio, Hep B, and varicella; objectives 14-23f through j, respectively) were modified to exclude children in licensed day care settings.

- Complete vaccination coverage among children (objective 14-24a) was updated to be consistent with the current guidelines established by the Advisory Committee for Immunization Practices. The revised series reflects a recommendation of: at least four doses of DTaP, at least three doses of polio, at least one dose of MMR, at least three or four doses of Hib (depending on vaccine brand), at least three doses of Hep B, at least one dose of varicella, and at least four doses of PCV.

- Among teens aged 13-15 years, the tetanus and diphtheria (Td) booster (objective 14-27c) was changed to the combined tetanus-diphtheriaacellular-pertussis (Tdap) booster, and the dosage was changed for the varicella vaccine from one or more (objective 14-27d) to two doses.

- Two Hep B vaccination objectives among high-risk adults including longterm hemodialysis patients and men who have sex with men (objectives 1428a and b) were reverted to developmental status [4].

- The data source for influenza and pneumococcal vaccination among institutionalized adults (objective 14-29f) was changed in Healthy People 2020. All four influenza vaccination objectives (14-29a, c, e, and g) were modified to conform to a new definition of seasonal flu.

- Vaccine Safety. Active surveillance for vaccine safety via large linked databases (objective 14-31a) was modified to address the scientific knowledge on vaccine safety and adverse events. This objective is developmental in Healthy People 2020.

Twenty-five Healthy People 2010 objectives were archived [8].

- Diseases preventable through universal vaccination. Five objectives were archived: diphtheria (objective 14-1b) and tetanus (objective 14-1j) among persons aged under 35 years, hepatitis B among heterosexually active persons (objective 143e), hepatitis B among occupationally exposed workers (objective 14-3g), and bacterial meningitis in young children (objective 14-4).

- Diseases preventable through targeted vaccination. One objective (14-8), Lyme disease, was archived because it was dependent on the availability of the vaccine for Lyme disease which was pulled off the market by the manufacturer.

- Infectious diseases and emerging antimicrobial resistance. Seven objectives were archived due to changes in program priorities: identification of persons with chronic hepatitis C (objective 14-10); hospitalizations for peptic ulcer (objective 1417); four hospital intensive care unit-acquired infections objectives (objectives 14$20 \mathrm{a}$, and c through e); and antimicrobial use in intensive care unit (objective 14-21). 
- Vaccination Coverage and Strategies. Seven vaccination (DTaP, MMR, polio, Hep $\mathrm{B}$, varicella, PCV, and Hib) objectives for day care (objectives 14-23a through e, and $14-23 \mathrm{k}$ and $\mathrm{l}$ ) were archived due to the lack of a data source; and two vaccination objectives for teens (Hep B and MMR, objectives 14-27a and b) were archived.

- Vaccine safety. Three objectives were archived: vaccine-associated paralytic polio (objective 14-30a), febrile seizures following pertussis vaccines (objective 14-30b), and the number of vaccine adverse event reports (VAERS) that are submitted electronically (objective 14-31b).

- Prevention services for international travelers (objective 14-15) was dropped at the Midcourse Review and the developmental objective intended to track adolescents aged 1315 years who received the recommended vaccines (objective 14-24b) was dropped at the end of the decade due to lack of data sources [5].

- Twenty new objectives were added to the Healthy People 2020 Immunization and Infectious Diseases Topic Area:

- Diseases preventable through universal vaccination. One objective, pertussis among adolescents, was added.

- Vaccination coverage and strategies. Fifteen new objectives were added to this section: two doses hepatitis A vaccine, a birth dose of Hep B vaccine, two or three doses of rotavirus vaccine, one dose meningococcal vaccine (MCV) among adolescents, three doses human papillomavirus vaccine (HPV) among female adolescents, five influenza (flu) vaccine objectives, zoster (shingles) vaccination, Hep B vaccination among injection drug users (developmental), the number of states collecting kindergarten vaccination records using minimum standards, and State participation in the Immunization Information System for adolescent vaccination. An objective that measures zero doses of vaccine among children aged 19-35 months was also added.

- Infectious diseases and emerging antimicrobial resistance. Four new objectives were added: electronic surveillance of rabies, influenza-virus resistance to antiviral agents, awareness of hepatitis $\mathrm{C}$ infection status and hepatitis $\mathrm{B}$ testing within minority communities experiencing health disparities (developmental).

- One objective, central line-associated bloodstream infection among intensive care unit patients (objective 14-20b), was moved to the Healthcare-Associated Infections Topic Area.

Appendix D, “A Crosswalk Between Objectives From Healthy People 2010 to Healthy People 2020," summarizes the changes between the two decades of objectives, reflecting new knowledge and direction for this area.

\section{Data Considerations}

The data source used to track the four vaccination coverage objectives among adolescents, (objectives 14-27a through d) was the National Health Interview Survey for data years between 1997 and 2003. Starting in 2006, the data source was the newly implemented National Immunization Survey-Teen (NIS-Teen). 
Education and income are the primary measures of socioeconomic status (SES) in Healthy People 2010. Most data systems used in Healthy People 2010 define income as a family's income before taxes. In order to facilitate comparisons among groups and over time, while adjusting for family size and for inflation, Healthy People 2010 categorizes income using the poverty thresholds developed by the U.S. Census Bureau. Thus, the three categories of family income that are primarily used are:

- Poor-below the Federal poverty level

- Near poor-100\% to $199 \%$ of the Federal poverty level

- Middle/high income-200\% or more of the Federal poverty level.

These categories may be overridden by considerations specific to the data system, in which case they are modified as appropriate. See Healthy People 2010: General Data Issues, referenced below.

In general, data on educational attainment are presented for persons aged 25 years and over, consistent with guidance given by the U.S. Bureau of the Census. However, because of the requirements of the different data systems, the age groups used to calculate educational attainment for any specific objective may differ from the age groups used to report the data for other Healthy People 2010 objectives, as well as from select populations within the same objective. Therefore, the reader is urged to exercise caution in interpreting the data by educational attainment shown in the Health Disparities Table. See Healthy People 2010: General Data Issues, referenced below.

Additional information on data issues is available from the following sources:

- All Healthy People 2010 tracking data can be found in the Healthy People 2010 database, (DATA2010), available from http://wonder.cdc.gov/data2010/

- Detailed information about the data and data sources used to support these objectives can be found in the Operational Definitions on the DATA2010 website, available from http://wonder.cdc.gov/data2010/focusod.htm.

- More information on statistical issues related to Healthy People tracking and measurement can be found in the Technical Appendix and in Healthy People 2010: General Data Issues, which is available in the Data Issues section of the NCHS Healthy People website under Healthy People 2010.

\section{Notes}

1. Displayed in the Progress Chart (Figure 14-1), the percent of targeted change achieved expresses the difference between the baseline and the final value relative to the initial difference between the baseline and the Healthy People 2010 target. As such, it is a relative measure of progress toward attaining the Healthy People 2010 target. See the Reader's Guide for more information. When standard errors were available, the difference between the baseline and the final value was tested at the 0.05 level of significance. See the Figure 14-1 footnotes, as well as the Technical Appendix, for more detail. 
2. Information about disparities among select populations is shown in the Health Disparities Table (Figure 14-2). Disparity from the best group rate is defined as the percent difference between the best group rate and each of the other group rates for a characteristic. For example, racial and ethnic health disparities are measured as the percent difference between the best racial and ethnic group rate and each of the other racial and ethnic group rates. Similarly, disparities by sex are measured as the percent difference between the better group rate (e.g., female) and the rate for the other group (e.g., male). Some objectives are expressed in terms of favorable events or conditions that are to be increased, while others are expressed in terms of adverse events or conditions that are to be reduced. In order to facilitate comparison of health disparities across different objectives, disparity is measured only in terms of adverse events or conditions. For comparability across objectives, objectives that are expressed in terms of favorable events or conditions are re-expressed using the adverse event or condition for the purpose of computing disparity, but they are not otherwise restated or changed. For example, objective 1-1, to increase the proportion of persons with health insurance (e.g., $72 \%$ of the American Indian or Alaska Native population aged under 65 years had some form of health insurance in 2008), is expressed in terms of the percentage of persons without health insurance (e.g., $100 \%-72 \%=28 \%$ of the American Indian or Alaska Native population aged under 65 years did not have any form of health insurance in 2008) when the disparity from the best group rate is calculated. See the Reader's Guide for more information. When standard errors were available, the difference between the best group rate and each of the other group rates was tested at the 0.05 level of significance. See the Figure 14-2 footnotes, as well as the Technical Appendix, for more detail.

3. The change in disparity is estimated by subtracting the disparity at baseline from the disparity at the most recent data point and, therefore, is expressed as a change in percentage points. See the Reader's Guide for more information. When standard errors were available, the change in disparity was tested at the 0.05 level of significance. See the Figure 14-2 footnotes, as well as the Technical Appendix, for more detail.

4. To be included in Healthy People 2010, an objective must have a national data source that provides a baseline and at least one additional data point for tracking progress. Some objectives lacked baseline data at the time of their development but had a potential data source and were considered of sufficient national importance to be included in Healthy People. These are called "developmental" objectives. When data become available, a developmental objective is moved to measurable status and a Healthy People target can be set.

5. Dropped objectives were not carried forward into Healthy People 2020. These objectives were either developmental or deleted at the Healthy People 2010 Midcourse Review or at another time in Healthy People 2010.

6. Retained "as is" objectives have no change in the numerator definition or in the denominator definition between the Healthy People 2010 and Healthy People 2020 objectives. These include objectives that were developmental in Healthy People 2010 and are developmental in Healthy People 2020 and for which no numerator or denominator information was available.

7. Modified objectives have some change in the numerator definition or in the denominator definition between the Healthy People 2010 and Healthy People 2020 objectives. These include objectives that went from developmental in Healthy People 2010 to measurable in Healthy People 2020 or vice versa.

8. Archived objectives had at least one data point in Healthy People 2010 but were not carried forward into Healthy People 2020. 


\section{Comprehensive Summary of Objectives: Immunization and Infectious Disease}

\begin{tabular}{|c|c|}
\hline Objective & Description \\
\hline $14-1 \mathrm{a}$ & $\begin{array}{l}\text { Vaccine-preventable diseases-Congenital rubella syndrome (no. cases, } \\
<1 \text { year) }\end{array}$ \\
\hline $14-1 b$ & Vaccine-preventable diseases-Diphtheria (no. cases, <35 years) \\
\hline $14-1 c$ & $\begin{array}{l}\text { Vaccine-preventable diseases-Haemophilus influenza type b and } \\
\text { unknown (no. cases, }<5 \text { years) }\end{array}$ \\
\hline $14-1 d$ & Vaccine-preventable diseases-Hepatitis B (no. cases, 2-18 years) \\
\hline $14-1 \mathrm{e}$ & Vaccine-preventable diseases-Measles (no. cases) \\
\hline $14-1 \mathrm{f}$ & Vaccine-preventable diseases-Mumps (no. cases) \\
\hline $14-1 g$ & Vaccine-preventable diseases-Pertussis (no. cases, $<7$ years) \\
\hline $14-1 \mathrm{~h}$ & Vaccine-preventable diseases-Polio (wild-type virus) (no. cases) \\
\hline $14-1 \mathrm{i}$ & Vaccine-preventable diseases-Rubella (no. cases) \\
\hline $14-1 j$ & Vaccine-preventable diseases-Tetanus (no. cases, $<35$ years) \\
\hline $14-1 \mathrm{k}$ & $\begin{array}{l}\text { Vaccine-preventable diseases-Varicella (chicken pox) (no. cases in } \\
\text { thousands, }<18 \text { years) }\end{array}$ \\
\hline $14-2$ & $\begin{array}{l}\text { Perinatal hepatitis B infections in infants and young children (no. cases, } \\
1-24 \text { months) }\end{array}$ \\
\hline $14-3 a$ & Hepatitis B in adults $19-24$ years (cases per 100,000 ) \\
\hline $14-3 b$ & Hepatitis B in adults 25-39 years (cases per 100,000) \\
\hline $14-3 c$ & Hepatitis B in adults $40+$ years (cases per 100,000 ) \\
\hline $14-3 d$ & Hepatitis B in injection drug users (no. cases) \\
\hline $14-3 e$ & Hepatitis B in heterosexually active persons (no. cases) \\
\hline $14-3 f$ & Hepatitis B in men who have sex with men (no. cases) \\
\hline $14-3 g$ & Hepatitis B in occupationally exposed workers (no. cases) \\
\hline $14-4$ & $\begin{array}{l}\text { Bacterial meningitis in young children (new cases per } 100,000 \\
\text { population, 1-23 months) }\end{array}$ \\
\hline $14-5 a$ & $\begin{array}{l}\text { Invasive pneumococcal infections-Children (new cases per 100,000 } \\
\text { population, }<5 \text { years) }\end{array}$ \\
\hline $14-5 b$ & $\begin{array}{l}\text { Invasive pneumococcal infections-Adults (new cases per } 100,000 \\
\text { population, } 65+\text { years) }\end{array}$ \\
\hline
\end{tabular}

National Congenital Rubella Syndrome Registry (NCRSR), CDC, NCIRD.

National Notifiable Diseases Surveillance System (NNDSS), CDC, NCPHI. National Notifiable Disease Surveillance System (NNDSS), CDC, NCPHI; Active Bacterial Core Surveillance (ABCs), CDC, NCIRD.

National Notifiable Diseases Surveillance System (NNDSS), CDC, NCPHI. National Notifiable Diseases Surveillance System (NNDSS), CDC, NCPHI. National Notifiable Diseases Surveillance System (NNDSS), CDC, NCPHI. National Notifiable Diseases Surveillance System (NNDSS), CDC, NCPHI. National Notifiable Diseases Surveillance System (NNDSS), CDC, NCPHI. National Notifiable Diseases Surveillance System (NNDSS), CDC, NCPHI. National Notifiable Diseases Surveillance System (NNDSS), CDC, NCPHI. National Health Interview Survey (NHIS), CDC, NCHS.

Perinatal Hepatitis B Prevention Program, CDC, NCHHSTP; National Vital Statistics System-Natality (NVSS-N), CDC, NCHS.

National Notifiable Diseases Surveillance System (NNDSS), CDC, NCPHI. National Notifiable Diseases Surveillance System (NNDSS), CDC, NCPHI. National Notifiable Diseases Surveillance System (NNDSS), CDC, NCPHI. National Notifiable Diseases Surveillance System (NNDSS), CDC, NCPHI. National Notifiable Diseases Surveillance System (NNDSS), CDC, NCPHI. National Notifiable Diseases Surveillance System (NNDSS), CDC, NCPHI. National Notifiable Diseases Surveillance System (NNDSS), CDC, NCPHI. Active Bacterial Core Surveillance (ABCs), CDC, NCIRD.

Active Bacterial Core Surveillance (ABCs), CDC, NCIRD.

Active Bacterial Core Surveillance (ABCs), CDC, NCIRD. 


\begin{tabular}{|c|c|c|}
\hline Objective & Description & Data Source or Objective Status \\
\hline $14-5 c$ & $\begin{array}{l}\text { Penicillin-resistant invasive pneumococcal infections-Children (new } \\
\text { cases per } 100,000 \text { population, }<5 \text { years) }\end{array}$ & Active Bacterial Core Surveillance (ABCs), CDC, NCIRD. \\
\hline $14-5 d$ & $\begin{array}{l}\text { Penicillin-resistant invasive pneumococcal infections-Adults (new } \\
\text { cases per } 100,000 \text { population, } 65+\text { years) }\end{array}$ & Active Bacterial Core Surveillance (ABCs), CDC, NCIRD. \\
\hline $14-6$ & Hepatitis A (new cases per 100,000 population) & National Notifiable Diseases Surveillance System (NNDSS), CDC, NCPHI. \\
\hline $14-7$ & Meningococcal disease (new cases per 100,000 population) & $\begin{array}{l}\text { Active Bacterial Core Surveillance (ABCs), CDC, NCIRD; National } \\
\text { Notifiable Diseases Surveillance System (NNDSS), CDC, NCPHI. }\end{array}$ \\
\hline $14-8$ & Lyme disease in endemic States (new cases per 100,000 population) & National Notifiable Diseases Surveillance System (NNDSS), CDC, NCPHI. \\
\hline $14-9$ & Hepatitis C (new cases per 100,000 population) & Sentinel Counties Study of Viral Hepatitis, CDC, NCHHSTP. \\
\hline $14-10$ & States reporting chronic HCV infection (no. States) & $\begin{array}{l}\text { State health department databases of persons with HCV infection; } \\
\text { National Health and Nutrition Examination Survey (NHANES), CDC, } \\
\text { NCHS. }\end{array}$ \\
\hline $14-11$ & Tuberculosis (new cases per 100,000 population) & National TB Surveillance System, CDC, NCHHSTP. \\
\hline $14-12$ & Curative therapy for tuberculosis & National TB Surveillance System, CDC, NCHHSTP. \\
\hline $14-13$ & Treatment for high-risk persons with latent tuberculosis infection & $\begin{array}{l}\text { Aggregate Reports for Tuberculosis Program Evaluation, CDC, } \\
\text { NCHHSTP. }\end{array}$ \\
\hline $14-14$ & $\begin{array}{l}\text { Timely laboratory confirmation of tuberculosis cases-Average } \\
\text { number of days to report } 75 \% \text { of cases }\end{array}$ & Survey of State Public Health Laboratories, CDC, NCHSTP. \\
\hline 14-15 & Prevention services for international travelers & Dropped \\
\hline $14-16$ & $\begin{array}{l}\text { Invasive early onset group B streptococcal disease (per 1,000 live } \\
\text { births) }\end{array}$ & $\begin{array}{l}\text { National Notifiable Disease Surveillance System (NNDSS), CDC, NCPHI; } \\
\text { Active Bacterial Core Surveillance (ABCs), CDC, NCIRD. }\end{array}$ \\
\hline $14-17$ & Peptic ulcer hospitalizations (age adjusted, per 100,000 population) & National Hospital Discharge Survey (NHDS), CDC, NCHS. \\
\hline $14-18$ & $\begin{array}{l}\text { Antibiotics prescribed for ear infections in children (courses prescribed } \\
\text { per } 100 \text { population, }<5 \text { years) }\end{array}$ & $\begin{array}{l}\text { National Ambulatory Medical Care Survey (NAMCS), CDC, NCHS; } \\
\text { National Hospital Ambulatory Medical Care Survey (NHAMCS), CDC, } \\
\text { NCHS. }\end{array}$ \\
\hline $14-19$ & $\begin{array}{l}\text { Antibiotics prescribed for common cold (courses prescribed per } \\
100,000 \text { population) }\end{array}$ & $\begin{array}{l}\text { National Ambulatory Medical Care Survey (NAMCS), CDC, NCHS; } \\
\text { National Hospital Ambulatory Medical Care Survey (NHAMCS), CDC, } \\
\text { NCHS. }\end{array}$ \\
\hline $14-20 \mathrm{a}$ & $\begin{array}{l}\text { Hospital-acquired infections among adult intensive care patients- } \\
\text { Catheter-associated urinary tract infection (per 1,000 days use) }\end{array}$ & $\begin{array}{l}\text { Baseline: National Noscomial Infections Surveillance System (NNIS), } \\
\text { CDC, NCPDCID; Final: National Healthcare Safety Network (NHSN), CDC, } \\
\text { NCPDCID. }\end{array}$ \\
\hline
\end{tabular}




\begin{tabular}{|c|c|}
\hline Objective & Description \\
\hline $14-20 b$ & $\begin{array}{l}\text { Hospital-acquired infections among adult intensive care-Central line- } \\
\text { associated bloodstream infection (per 1,000 days use) }\end{array}$ \\
\hline $14-20 c$ & $\begin{array}{l}\text { Hospital-acquired infections among adult intensive care patients- } \\
\text { Ventilator-associated pneumonia (per } 1,000 \text { days use) }\end{array}$ \\
\hline $14-20 d$ & $\begin{array}{l}\text { Hospital-acquired infections among infants in intensive care weighing } \\
\leq 1,000 \text { grams at birth-Central line-associated bloodstream infection } \\
\text { (per 1,000 days use) }\end{array}$ \\
\hline $14-20 \mathrm{e}$ & $\begin{array}{l}\text { Hospital-acquired infections among infants in intensive care weighing } \\
\leq 1,000 \text { grams at birth-Ventilator-associated pneumonia (per 1,000 } \\
\text { days use) }\end{array}$ \\
\hline $14-21$ & $\begin{array}{l}\text { Antimicrobial use in intensive care units (daily doses per } 1,000 \text { patient } \\
\text { days) }\end{array}$ \\
\hline $14-22 \mathrm{a}$ & $\begin{array}{l}\text { Vaccination of children 19-35 months-4 doses diphtheria-tetanus- } \\
\text { acellular pertussis (DTaP) vaccine }\end{array}$ \\
\hline $14-22 b$ & $\begin{array}{l}\text { Vaccination of children 19-35 months }-3 \text { doses Haemophilus influenza } \\
\text { type b (Hib) vaccine }\end{array}$ \\
\hline $14-22 c$ & $\begin{array}{l}\text { Vaccination of children 19-35 months-3 doses hepatitis B (Hep B) } \\
\text { vaccine }\end{array}$ \\
\hline $14-22 d$ & $\begin{array}{l}\text { Vaccination of children } 19-35 \text { months }-1 \text { dose measles-mumps-rubella } \\
\text { (MMR) vaccine }\end{array}$ \\
\hline $14-22 \mathrm{e}$ & Vaccination of children $19-35$ months -3 doses polio vaccine \\
\hline $14-22 f$ & Vaccination of children $19-35$ months -1 dose varicella vaccine \\
\hline $14-22 \mathrm{~g}$ & $\begin{array}{l}\text { Vaccination of children } 19-35 \text { months }-4 \text { doses pneumococcal } \\
\text { conjugate vaccine (PCV) }\end{array}$ \\
\hline $14-22 \mathrm{~h}$ & Vaccination of children $6-23$ months -1 dose influenza vaccine \\
\hline $14-23 a$ & $\begin{array}{l}\text { Vaccine coverage of children in day care-Diphtheria-tetanus-acellular } \\
\text { pertussis (DTaP) vaccine }\end{array}$ \\
\hline $14-23 b$ & $\begin{array}{l}\text { Vaccine coverage of children in day care-Measles-mumps-rubella } \\
\text { (MMR) vaccine }\end{array}$ \\
\hline $14-23 c$ & Vaccine coverage of children in day care-Polio vaccine \\
\hline
\end{tabular}

Baseline: National Noscomial Infections Surveillance System (NNIS),

CDC, NCPDCID; Final: National Healthcare Safety Network (NHSN), CDC, NCPDCID.

Baseline: National Noscomial Infections Surveillance System (NNIS), CDC, NCPDCID; Final: National Healthcare Safety Network (NHSN), CDC, NCPDCID.

Baseline: National Noscomial Infections Surveillance System (NNIS), CDC, NCPDCID; Final: National Healthcare Safety Network (NHSN), CDC, NCPDCID.

Baseline: National Noscomial Infections Surveillance System (NNIS), CDC, NCPDCID; Final: National Healthcare Safety Network (NHSN), CDC, NCPDCID.

National Noscomial Infections Surveillance System (NNIS), CDC, NCPDCID.

National Immunization Survey (NIS): CDC, NCIRD; CDC, NCHS.

National Immunization Survey (NIS): CDC, NCIRD; CDC, NCHS.

National Immunization Survey (NIS): CDC, NCIRD; CDC, NCHS.

National Immunization Survey (NIS): CDC, NCIRD; CDC, NCHS.

National Immunization Survey (NIS): CDC, NCIRD; CDC, NCHS.

National Immunization Survey (NIS): CDC, NCIRD; CDC, NCHS.

National Immunization Survey (NIS): CDC, NCIRD; CDC, NCHS.

Developmental

Annual Immunization Assessment Reports, CDC, NCIRD.

Annual Immunization Assessment Reports, CDC, NCIRD.

Annual Immunization Assessment Reports, CDC, NCIRD. 


\begin{tabular}{|c|c|c|}
\hline Objective & Description & Data Source or Objective Status \\
\hline $14-23 d$ & Vaccine coverage of children in day care-Hepatitis B (Hep B) vaccine & Day Care and Head Start Assessment Reports, CDC, NCIRD. \\
\hline $14-23 e$ & Vaccine coverage of children in day care-Varicella vaccine & Day Care and Head Start Assessment Reports, CDC, NCIRD. \\
\hline $14-23 f$ & $\begin{array}{l}\text { Vaccine coverage of children in kindergarten-Diphtheria-tetanus- } \\
\text { acellular pertussis (DTaP) vaccine }\end{array}$ & School Immunization Assessment Survey, CDC, NCIRD. \\
\hline $14-23 g$ & $\begin{array}{l}\text { Vaccine coverage of children in kindergarten-Measles-mumps-rubella } \\
\text { (MMR) vaccine }\end{array}$ & School Immunization Assessment Survey, CDC, NCIRD. \\
\hline $14-23 j$ & Vaccine coverage of children in kindergarten-Varicella vaccine & School Immunization Assessment Survey, CDC, NCIRD. \\
\hline $14-23 \mathrm{k}$ & $\begin{array}{l}\text { Vaccine coverage of children in daycare-Pneumococcal conjugate } \\
\text { vaccine (PCV) }\end{array}$ & Day Care and Head Start Assessment Reports, CDC, NCIRD. \\
\hline 14-23l & $\begin{array}{l}\text { Vaccine coverage of children in licensed daycare facilities- } \\
\text { Haemophilus influenzae type b (Hib) vaccine }\end{array}$ & Day Care and Head Start Assessment Program, CDC, NCIRD. \\
\hline $14-25 b$ & $\begin{array}{l}\text { Providers who measure childhood vaccination coverage levels-Private } \\
\text { providers }\end{array}$ & Annual Immunization Assessment Reports, CDC, NCIRD. \\
\hline $14-26$ & $\begin{array}{l}\text { Children }<6 \text { years participating in population-based immunization } \\
\text { registries }\end{array}$ & Annual Immunization Assessment Reports, CDC, NCIRD. \\
\hline $14-27 a$ & $\begin{array}{l}\text { Vaccination coverage among adolescents } 13-15 \text { years-3+ doses } \\
\text { hepatitis B (Hep B) vaccine }\end{array}$ & $\begin{array}{l}\text { Baseline: National Health Interview Survey (NHIS), CDC, NCHS. Final: } \\
\text { National Immunization Survey-Teen (NIS-Teen), CDC, NCIRD and } \\
\text { CDC, NCHS. }\end{array}$ \\
\hline $14-27 b$ & $\begin{array}{l}\text { Vaccination coverage among adolescents } 13-15 \text { years }-2+\text { doses } \\
\text { measles-mumps-rubella (MMR) vaccine }\end{array}$ & $\begin{array}{l}\text { Baseline: National Health Interview Survey (NHIS), CDC, NCHS. Final: } \\
\text { National Immunization Survey-Teen (NIS-Teen), CDC, NCIRD and } \\
\text { CDC, NCHS. }\end{array}$ \\
\hline $14-27 c$ & $\begin{array}{l}\text { Vaccination coverage among adolescents } 13-15 \text { years }-1+\text { doses } \\
\text { tetanus-diptheria booster }\end{array}$ & $\begin{array}{l}\text { Baseline: National Health Interview Survey (NHIS), CDC, NCHS. Final: } \\
\text { National Immunization Survey-Teen (NIS-Teen), CDC, NCIRD and } \\
\text { CDC, NCHS. }\end{array}$ \\
\hline
\end{tabular}




\begin{tabular}{|c|c|c|}
\hline Objective & Description & Data Source or Objective Status \\
\hline $14-27 d$ & $\begin{array}{l}\text { Vaccination coverage among adolescents } 13-15 \text { years-1+ doses } \\
\text { varicella (excluding adolescents who have had varicella) }\end{array}$ & $\begin{array}{l}\text { Baseline: National Health Interview Survey (NHIS), CDC, NCHS. Final: } \\
\text { National Immunization Survey-Teen (NIS-Teen), CDC, NCIRD and } \\
\text { CDC, NCHS. }\end{array}$ \\
\hline $14-28 a$ & $\begin{array}{l}\text { Hepatitis B vaccination among high-risk groups-Long-term } \\
\text { hemodialysis patients }\end{array}$ & Annual Survey of Chronic Hemodialysis Centers: CDC, NCHHSTP; CMS. \\
\hline $14-28 b$ & $\begin{array}{l}\text { Hepatitis B (Hep B) vaccination among high-risk groups-Men who } \\
\text { have sex with men }\end{array}$ & Young Men's Survey, CDC, NCHHSTP. \\
\hline $14-28 c$ & $\begin{array}{l}\text { Hepatitis B (Hep B) vaccination among high-risk groups- } \\
\text { Occupationally exposed workers }\end{array}$ & Periodic vaccine coverage surveys, CDC, NCPDCID. \\
\hline $14-29 a$ & $\begin{array}{l}\text { Vaccination of noninstitutionalized high-risk older adults-Influenza } \\
\text { vaccine in past } 12 \text { months (age adjusted, } 65+\text { years) }\end{array}$ & National Health Interview Survey (NHIS), CDC, NCHS. \\
\hline $14-29 b$ & $\begin{array}{l}\text { Vaccination of noninstitutionalized high-risk older adults- } \\
\text { Pneumococcal vaccine ever received (age adjusted, 65+ years) }\end{array}$ & National Health Interview Survey (NHIS), CDC, NCHS. \\
\hline $14-29 c$ & $\begin{array}{l}\text { Vaccination of noninstitutionalized high-risk adults-Influenza vaccine } \\
\text { in past } 12 \text { months (age adjusted, 18-64 years) }\end{array}$ & National Health Interview Survey (NHIS), CDC, NCHS. \\
\hline $14-29 d$ & $\begin{array}{l}\text { Vaccination of noninstitutionalized high-risk adults-Pneumococcal } \\
\text { vaccine ever received (age adjusted, 18-64 years) }\end{array}$ & National Health Interview Survey (NHIS), CDC, NCHS. \\
\hline $14-29 e$ & $\begin{array}{l}\text { Vaccination of adults in long-term care or nursing homes-Influenza } \\
\text { vaccine in past } 12 \text { months (age adjusted, 18+ years) }\end{array}$ & National Nursing Home Survey (NNHS), CDC, NCHS. \\
\hline $14-29 f$ & $\begin{array}{l}\text { Vaccination of adults in long-term care or nursing homes- } \\
\text { Pneumococcal vaccine ever received (age adjusted, 18+ years) }\end{array}$ & National Nursing Home Survey (NNHS), CDC, NCHS. \\
\hline $14-29 \mathrm{~g}$ & $\begin{array}{l}\text { Vaccination of health care workers-Influenza vaccine in past } 12 \\
\text { months (age adjusted, 18-64 years) }\end{array}$ & National Health Interview Survey (NHIS), CDC, NCHS. \\
\hline $14-30 \mathrm{a}$ & $\begin{array}{l}\text { Adverse events from vaccinations-Associated paralytic polio } \\
\text { (number) }\end{array}$ & National Notifiable Diseases Surveillance System (NNDSS), CDC, NCPHI. \\
\hline $14-30 b$ & $\begin{array}{l}\text { Adverse events from vaccinations-Febrile seizures caused by } \\
\text { pertussis vaccines (number) }\end{array}$ & $\begin{array}{l}\text { Vaccine Adverse Event Reporting System (VAERS) CDC, OD; FDA. } \\
\text { Vaccine Safety Datalink (VSD), CDC, OD. }\end{array}$ \\
\hline $14-31 a$ & $\begin{array}{l}\text { Active surveillance for vaccine safety via large linked databases } \\
\text { (number in millions) }\end{array}$ & Vaccine Safety Datalink (VSD), CDC, OD. \\
\hline $14-31 b$ & Vaccine Adverse Event Reports (VAERS) submitted electronically & Vaccine Adverse Event Reporting System (VAERS): CDC, OD; FDA. \\
\hline
\end{tabular}


Figure 14-1. Progress Toward Target Attainment for Focus Area 14: Immunization and Infectious Diseases

Moved away

from target ${ }^{1}$

Moved toward

target

Met or exceeded

target

14-1. Vaccine-preventable diseases (no. cases)

a. Congenital rubella syndrome $(<1$ year $)$

b. Diphtheria ( $<35$ years)

c. Haemophilus influenza type b and unknown $(<5$ years)

d. Hepatitis B (2-18 years)

e. Measles

f. Mumps

g. Pertussis ( $<7$ years)

h. Polio (wild-type virus)

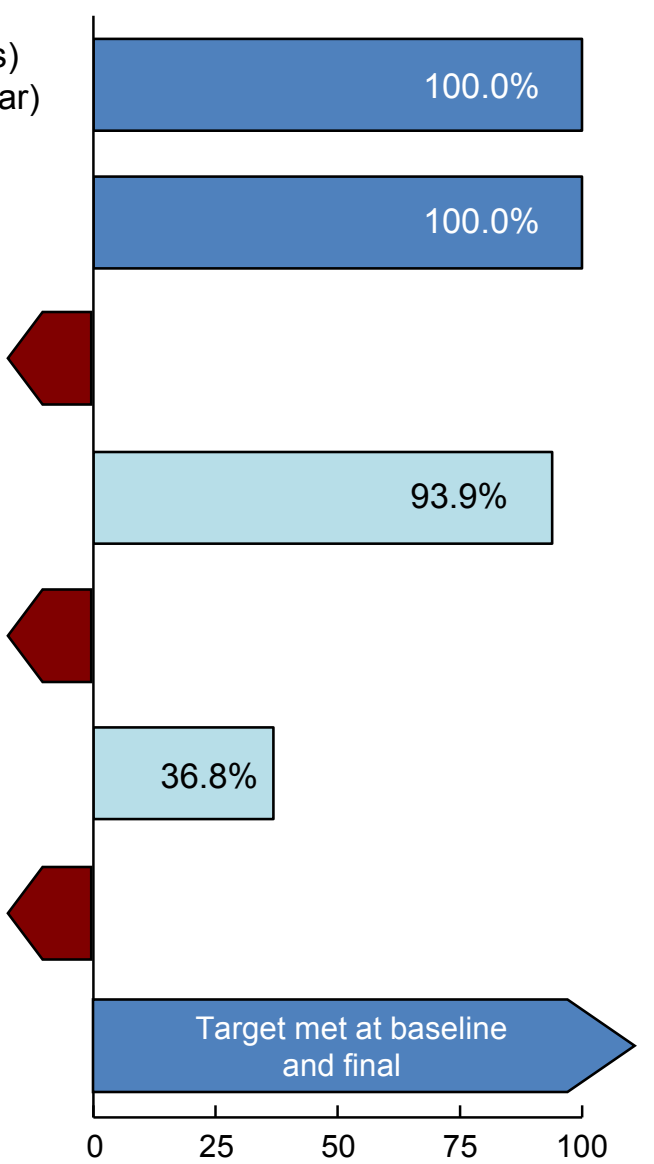

\begin{tabular}{|c|c|c|c|c|c|}
\hline \multirow{2}{*}{$\begin{array}{l}2010 \\
\text { Target }\end{array}$} & \multirow{2}{*}{$\begin{array}{c}\text { Baseline } \\
\text { (Year) }\end{array}$} & \multirow{2}{*}{$\begin{array}{l}\text { Final } \\
\text { (Year) }\end{array}$} & \multicolumn{3}{|c|}{ Baseline vs. Final } \\
\hline & & & | Difference ${ }^{2}$ & $\begin{array}{l}\text { Statistically } \\
\text { Significant }^{3}\end{array}$ & $\begin{array}{c}\text { Percent } \\
\text { Change }^{4}\end{array}$ \\
\hline 0 & $\begin{array}{c}7 \\
(1998)\end{array}$ & $\begin{array}{c}0 \\
(2008)\end{array}$ & -7 & $\begin{array}{c}\text { Not } \\
\text { tested }\end{array}$ & $-100.0 \%$ \\
\hline 0 & $\begin{array}{c}1 \\
(1998)\end{array}$ & $\begin{array}{c}0 \\
(2008)\end{array}$ & -1 & $\begin{array}{c}\text { Not } \\
\text { tested }\end{array}$ & $-100.0 \%$ \\
\hline 0 & $\begin{array}{c}163 \\
(1998)\end{array}$ & $\begin{array}{c}193 \\
(2008)\end{array}$ & 30 & $\begin{array}{c}\text { Not } \\
\text { tested }\end{array}$ & $18.4 \%$ \\
\hline 7 & $\begin{array}{c}708 \\
(1997)\end{array}$ & $\begin{array}{c}50 \\
(2008)\end{array}$ & -658 & $\begin{array}{c}\text { Not } \\
\text { tested }\end{array}$ & $-92.9 \%$ \\
\hline 0 & $\begin{array}{c}74 \\
(1998)\end{array}$ & $\begin{array}{c}115 \\
(2008)\end{array}$ & 41 & $\begin{array}{c}\text { Not } \\
\text { tested }\end{array}$ & $55.4 \%$ \\
\hline 0 & $\begin{array}{c}666 \\
(1998)\end{array}$ & $\begin{array}{c}421 \\
(2008)\end{array}$ & -245 & $\begin{array}{c}\text { Not } \\
\text { tested }\end{array}$ & $-36.8 \%$ \\
\hline 2,000 & $\begin{array}{l}3,417 \\
(1998)\end{array}$ & $\begin{array}{l}4,166 \\
(2008)\end{array}$ & 749 & $\begin{array}{c}\text { Not } \\
\text { tested }\end{array}$ & $21.9 \%$ \\
\hline 0 & $\begin{array}{c}0 \\
(1998)\end{array}$ & $\begin{array}{c}0 \\
(2008)\end{array}$ & 0 & $\begin{array}{c}\text { Not } \\
\text { tested }\end{array}$ & * \\
\hline
\end{tabular}

Percent of targeted change achieved ${ }^{5}$

(continued) 
Figure 14-1. Progress Toward Target Attainment for Focus Area 14: Immunization and Infectious Diseases (continued)

Moved away

from target ${ }^{1}$

Moved toward

target

Met or exceeded

target

14-1. Vaccine-preventable diseases (no. cases)

i. Rubella

j. Tetanus ( $<35$ years $)$

k. Varicella (chicken pox) (no. cases in thousands, $<18$ years)

14-2. Perinatal hepatitis $B$ infections in infants and young children (no. cases, 1-24 months)

14-3. Hepatitis B (cases per 100,000)

a. Adults 19-24 years

b. Adults $25-39$ years

c. Adults $40+$ years

14-3d. Hepatitis B (no. cases)

d. Injection drug users

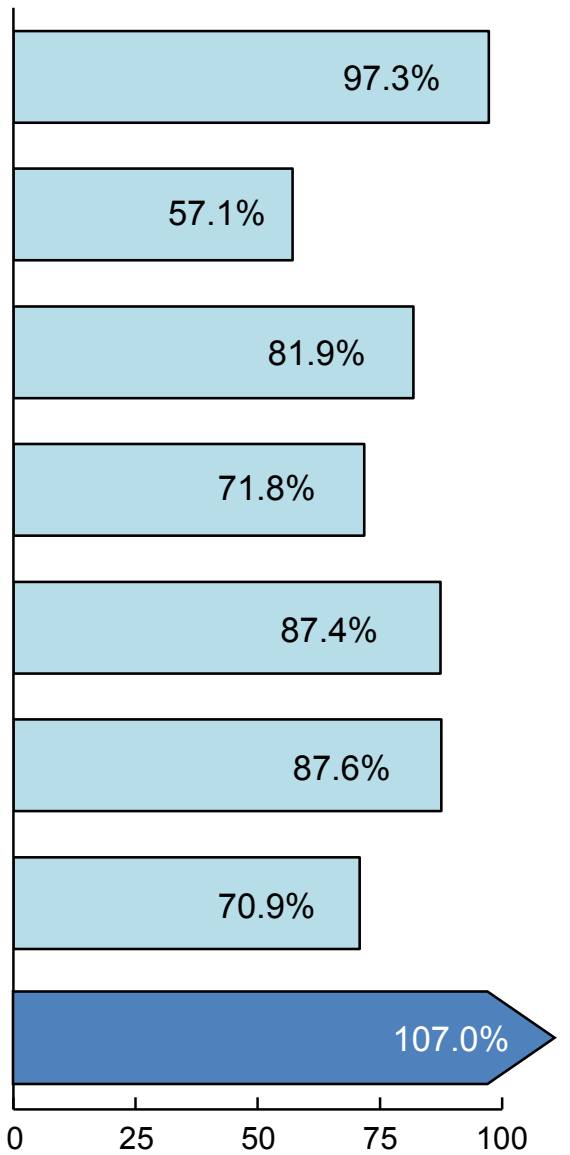

\begin{tabular}{|c|c|c|c|c|c|}
\hline \multirow{2}{*}{$\begin{array}{l}2010 \\
\text { Target }\end{array}$} & \multirow{2}{*}{$\begin{array}{c}\text { Baseline } \\
\text { (Year) }\end{array}$} & \multirow{2}{*}{$\begin{array}{l}\text { Final } \\
\text { (Year) }\end{array}$} & \multicolumn{3}{|c|}{ Baseline vs. Final } \\
\hline & & & | Difference ${ }^{2}$ & $\begin{array}{l}\text { Statistically } \\
\text { Significant }^{3}\end{array}$ & $\begin{array}{c}\text { Percent } \\
\text { Change }^{4}\end{array}$ \\
\hline 0 & $\begin{array}{c}364 \\
(1998)\end{array}$ & $\begin{array}{c}10 \\
(2008)\end{array}$ & -354 & $\begin{array}{c}\text { Not } \\
\text { tested }\end{array}$ & $-97.3 \%$ \\
\hline 0 & $\begin{array}{c}14 \\
(1998)\end{array}$ & $\begin{array}{c}6 \\
(2008)\end{array}$ & -8 & $\begin{array}{c}\text { Not } \\
\text { tested }\end{array}$ & $-57.1 \%$ \\
\hline 223 & $\begin{array}{l}2,229 \\
(1999)\end{array}$ & $\begin{array}{c}586 \\
(2008)\end{array}$ & $-1,643$ & Yes & $-73.7 \%$ \\
\hline 400 & $\begin{array}{l}1,682 \\
(1995)\end{array}$ & $\begin{array}{c}761 \\
(2008)\end{array}$ & -921 & $\begin{array}{c}\text { Not } \\
\text { tested }\end{array}$ & $-54.8 \%$ \\
\hline 1.8 & $\begin{array}{c}18.5 \\
(1997)\end{array}$ & $\begin{array}{c}3.9 \\
(2008)\end{array}$ & -14.6 & $\begin{array}{c}\text { Not } \\
\text { tested }\end{array}$ & $-78.9 \%$ \\
\hline 5.2 & $\begin{array}{c}20.5 \\
(1997)\end{array}$ & $\begin{array}{c}7.1 \\
(2008)\end{array}$ & -13.4 & $\begin{array}{c}\text { Not } \\
\text { tested }\end{array}$ & $-65.4 \%$ \\
\hline 3.7 & $\begin{array}{c}14.7 \\
(1997)\end{array}$ & $\begin{array}{c}6.9 \\
(2008)\end{array}$ & -7.8 & $\begin{array}{c}\text { Not } \\
\text { tested }\end{array}$ & $-53.1 \%$ \\
\hline 1,784 & $\begin{array}{l}7,135 \\
(1997)\end{array}$ & $\begin{array}{l}1,408 \\
(2008)\end{array}$ & $-5,727$ & $\begin{array}{c}\text { Not } \\
\text { tested }\end{array}$ & $-80.3 \%$ \\
\hline
\end{tabular}

Percent of targeted change achieved ${ }^{5}$

(continued) 
Figure 14-1. Progress Toward Target Attainment for Focus Area 14: Immunization and Infectious Diseases (continued)

Moved away from target ${ }^{1}$

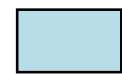

Moved toward

target

Met or exceeded

target

14-3. Hepatitis B (no. cases)

e. Heterosexually active persons

f. Men who have sex with men

g. Occupationally exposed workers

14-4. Bacterial meningitis in young children (new cases per 100,000 population, 1-23 months)

14-5. Invasive pneumococcal infections (new cases per 100,000 population)

a. Children ( $<5$ years)

b. Adults $(65+$ years $)$

c. Penicillin-resistant-Children $(<5$ years $)$

d. Penicillin-resistant-Adults (65+ years)

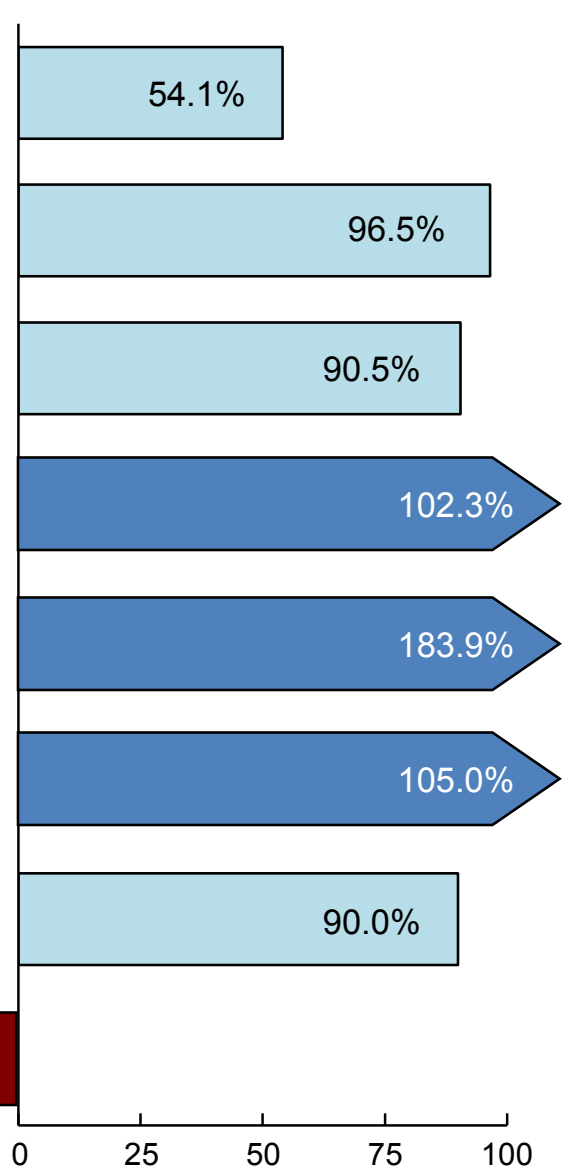

Percent of targeted change achieved ${ }^{5}$

\begin{tabular}{|c|c|c|c|c|c|}
\hline \multirow{2}{*}{$\begin{array}{l}2010 \\
\text { Target }\end{array}$} & \multirow{2}{*}{$\begin{array}{c}\text { Baseline } \\
\text { (Year) }\end{array}$} & \multirow{2}{*}{$\begin{array}{l}\text { Final } \\
\text { (Year) }\end{array}$} & \multicolumn{3}{|c|}{ Baseline vs. Final } \\
\hline & & & Difference $^{2}$ & $\begin{array}{l}\text { Statistically } \\
\text { Significant }^{3}\end{array}$ & $\begin{array}{c}\text { Percent } \\
\text { Change }^{4}\end{array}$ \\
\hline 1,223 & $\begin{array}{l}15,021 \\
(1997)\end{array}$ & $\begin{array}{l}7,563 \\
(2008)\end{array}$ & $-7,458$ & $\begin{array}{c}\text { Not } \\
\text { tested }\end{array}$ & $-49.7 \%$ \\
\hline 1,302 & $\begin{array}{l}5,209 \\
(1997)\end{array}$ & $\begin{array}{l}1,439 \\
(2008)\end{array}$ & $-3,770$ & $\begin{array}{c}\text { Not } \\
\text { tested }\end{array}$ & $-72.4 \%$ \\
\hline 60 & $\begin{array}{c}239 \\
(1997)\end{array}$ & $\begin{array}{c}77 \\
(2008)\end{array}$ & -162 & $\begin{array}{c}\text { Not } \\
\text { tested }\end{array}$ & $-67.8 \%$ \\
\hline 8.6 & $\begin{array}{c}13.0 \\
(1998)\end{array}$ & $\begin{array}{c}8.5 \\
(2008)\end{array}$ & -4.5 & $\begin{array}{l}\text { Not } \\
\text { tested }\end{array}$ & $-34.6 \%$ \\
\hline 46 & $\begin{array}{c}77 \\
(1997)\end{array}$ & $\begin{array}{c}20 \\
(2008)\end{array}$ & -57 & $\begin{array}{c}\text { Not } \\
\text { tested }\end{array}$ & $-74.0 \%$ \\
\hline 42 & $\begin{array}{c}62 \\
(1997)\end{array}$ & $\begin{array}{c}41 \\
(2008)\end{array}$ & -21 & $\begin{array}{l}\text { Not } \\
\text { tested }\end{array}$ & $-33.9 \%$ \\
\hline 6 & $\begin{array}{c}16 \\
(1997)\end{array}$ & $\begin{array}{c}7 \\
(2008)\end{array}$ & -9 & $\begin{array}{c}\text { Not } \\
\text { tested }\end{array}$ & $-56.3 \%$ \\
\hline 7 & $\begin{array}{c}8 \\
(1997)\end{array}$ & $\begin{array}{c}10 \\
(2008)\end{array}$ & 2 & $\begin{array}{c}\text { Not } \\
\text { tested }\end{array}$ & $25.0 \%$ \\
\hline
\end{tabular}

(continued) 
Figure 14-1. Progress Toward Target Attainment for Focus Area 14: Immunization and Infectious Diseases (continued)

Moved away

from target ${ }^{1}$

Moved toward

14-6. Hepatitis A (new cases per 100,000 population)

14-7. Meningococcal disease (new cases per 100,000 population)

14-8. Lyme disease in endemic States (new cases per 100,000 population)

14-9. Hepatitis C (new cases per 100,000 population)

14-10. States reporting chronic HCV infection (no. States)

14-11. Tuberculosis (new cases per 100,000 population)

14-12. Curative therapy for tuberculosis

14-13. Treatment for high-risk persons with latent tuberculosis infection

Met or exceeded

target

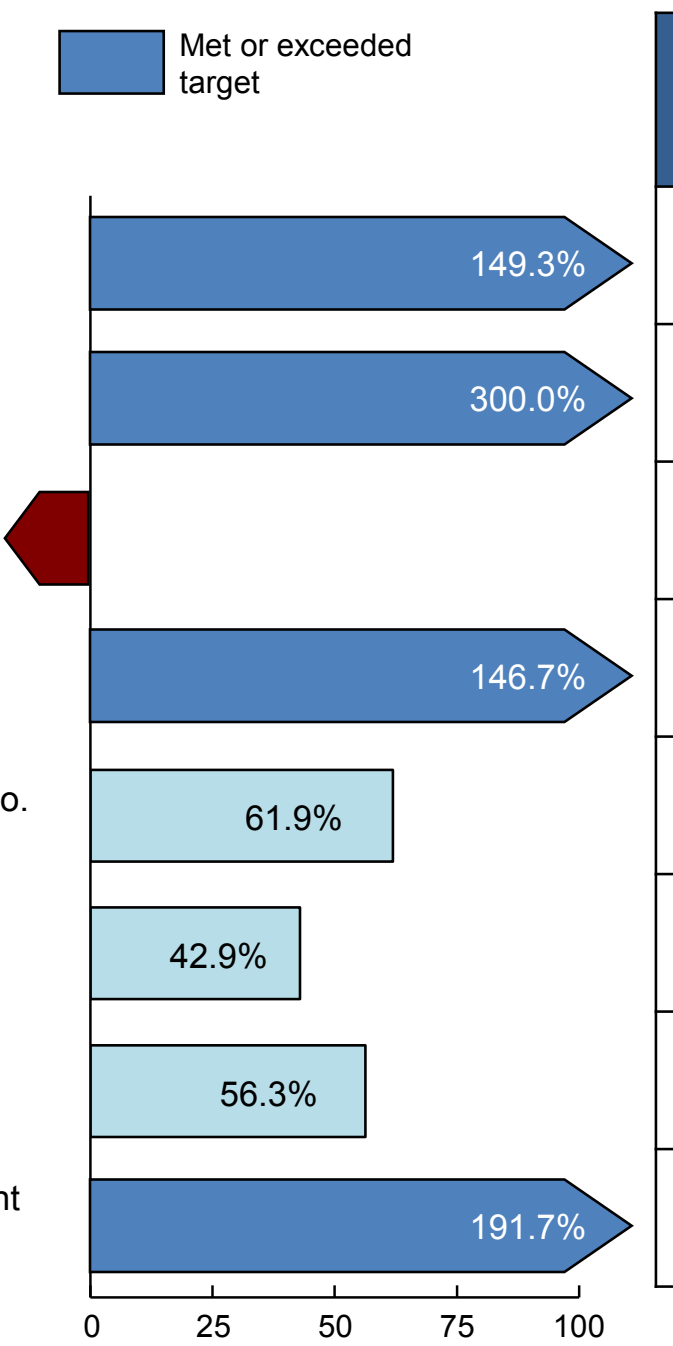

Percent of targeted change achieved ${ }^{5}$

\begin{tabular}{|c|c|c|c|c|c|}
\hline \multirow{2}{*}{$\begin{array}{l}2010 \\
\text { Target }\end{array}$} & \multirow{2}{*}{$\begin{array}{c}\text { Baseline } \\
\text { (Year) }\end{array}$} & \multirow{2}{*}{$\begin{array}{l}\text { Final } \\
\text { (Year) }\end{array}$} & \multicolumn{3}{|c|}{ Baseline vs. Final } \\
\hline & & & Difference $^{2}$ & $\begin{array}{l}\text { Statistically } \\
\text { Significant }^{3}\end{array}$ & $\begin{array}{l}\text { Percent } \\
\text { Change }^{4}\end{array}$ \\
\hline 4.3 & $\begin{array}{c}11.2 \\
(1997)\end{array}$ & $\begin{array}{c}0.9 \\
(2008)\end{array}$ & -10.3 & $\begin{array}{l}\text { Not } \\
\text { tested }\end{array}$ & $-92.0 \%$ \\
\hline 1.0 & $\begin{array}{c}1.3 \\
(1997)\end{array}$ & $\begin{array}{c}0.4 \\
(2008)\end{array}$ & -0.9 & $\begin{array}{l}\text { Not } \\
\text { tested }\end{array}$ & $-69.2 \%$ \\
\hline 9.7 & $\begin{array}{c}17.4 \\
(1992-96)\end{array}$ & $\begin{array}{c}50.1 \\
(2008)\end{array}$ & 32.7 & $\begin{array}{c}\text { Not } \\
\text { tested }\end{array}$ & $187.9 \%$ \\
\hline 1.0 & $\begin{array}{c}2.5 \\
(1997)\end{array}$ & $\begin{array}{c}0.3 \\
(2007)\end{array}$ & -2.2 & $\begin{array}{c}\text { Not } \\
\text { tested }\end{array}$ & $-88.0 \%$ \\
\hline 40 & $\begin{array}{c}19 \\
(2003)\end{array}$ & $\begin{array}{c}32 \\
(2008)\end{array}$ & 13 & $\begin{array}{c}\text { Not } \\
\text { tested }\end{array}$ & $68.4 \%$ \\
\hline 1.0 & $\begin{array}{c}6.6 \\
(1998)\end{array}$ & $\begin{array}{c}4.2 \\
(2008)\end{array}$ & -2.4 & $\begin{array}{c}\text { Not } \\
\text { tested }\end{array}$ & $-36.4 \%$ \\
\hline $90 \%$ & $\begin{array}{c}74 \% \\
(1996)\end{array}$ & $\begin{array}{c}83 \% \\
(2007)\end{array}$ & 9 & $\begin{array}{l}\text { Not } \\
\text { tested }\end{array}$ & $12.2 \%$ \\
\hline $57 \%$ & $\begin{array}{c}45 \% \\
(2000)\end{array}$ & $\begin{array}{c}68 \% \\
(2007)\end{array}$ & 23 & $\begin{array}{c}\text { Not } \\
\text { tested }\end{array}$ & $51.1 \%$ \\
\hline
\end{tabular}

(continued) 
Figure 14-1. Progress Toward Target Attainment for Focus Area 14: Immunization and Infectious Diseases (continued)

Moved away from target ${ }^{1}$

Moved toward target

Met or exceeded target

14-16. Invasive early onset group B streptococcal disease (per 1,000 live births)

14-17. Peptic ulcer hospitalizations (age adjusted, per 100,000 population)

14-18. Antibiotics prescribed for ear infections in children (courses prescribed per 100 population, $<5$ years)

14-19. Antibiotics prescribed for common cold (courses prescribed per 100,000 population)

14-20. Hospital-acquired infections among adult intensive care patients (per 1,000 days use)

a. Catheter-associated urinary tract infection

b. Central line-associated bloodstream infection

c. Ventilator-associated pneumonia

Hospital-acquired infections among infants in intensive care weighing $\leq 1,000$ grams at birth

d. Central line-associated bloodstream infection (per 1,000 days use)

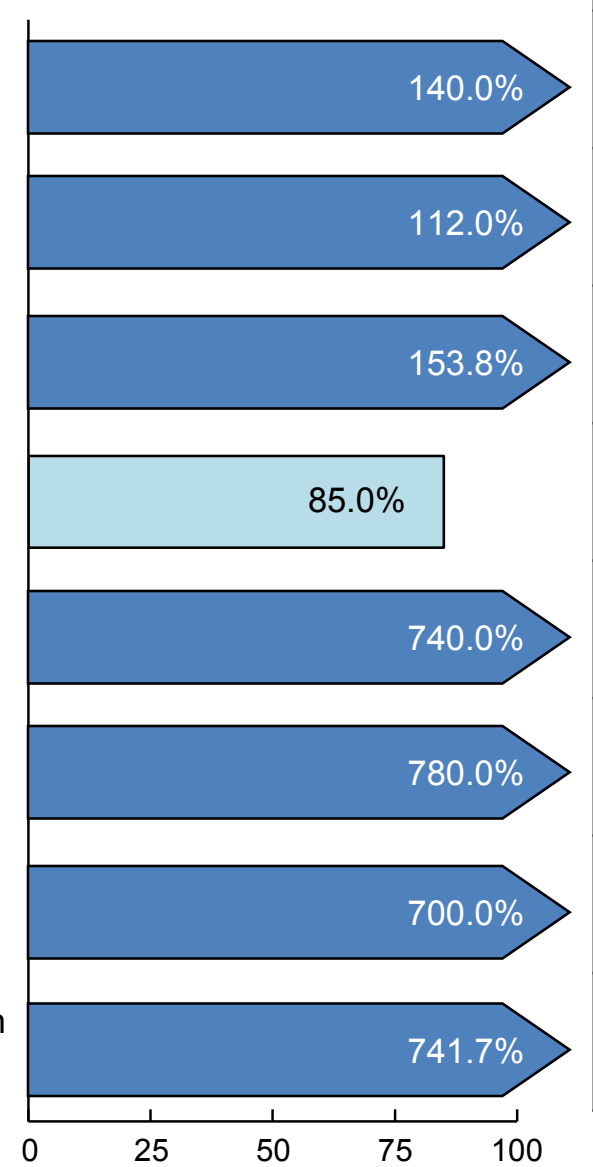

\begin{tabular}{|c|c|c|c|c|c|}
\hline \multirow{2}{*}{$\begin{array}{l}2010 \\
\text { Target }\end{array}$} & \multirow{2}{*}{$\begin{array}{c}\text { Baseline } \\
\text { (Year) }\end{array}$} & \multirow{2}{*}{$\begin{array}{l}\text { Final } \\
\text { (Year) }\end{array}$} & \multicolumn{3}{|c|}{ Baseline vs. Final } \\
\hline & & & Difference $^{2}$ & $\begin{array}{l}\text { Statistically } \\
\text { Significant }^{3}\end{array}$ & $\begin{array}{l}\text { Percent } \\
\text { Change }^{4}\end{array}$ \\
\hline 0.5 & $\begin{array}{c}1.0 \\
(1996)\end{array}$ & $\begin{array}{c}0.3 \\
(2008)\end{array}$ & -0.7 & $\begin{array}{l}\text { Not } \\
\text { tested }\end{array}$ & $-70.0 \%$ \\
\hline 46 & $\begin{array}{c}71 \\
(1998)\end{array}$ & $\begin{array}{c}43 \\
(2007)\end{array}$ & -28 & Yes & $-39.4 \%$ \\
\hline 56 & $\begin{array}{c}69 \\
(1996-97)\end{array}$ & $\begin{array}{c}49 \\
(2006-07)\end{array}$ & -20 & Yes & $-29.0 \%$ \\
\hline 1,268 & $\begin{array}{c}2,535 \\
(1996-97)\end{array}$ & $\begin{array}{c}1.458 \\
(2006-07)\end{array}$ & $-1,077$ & Yes & $-42.5 \%$ \\
\hline 5.0 & $\begin{array}{c}5.5 \\
(1995-98)\end{array}$ & $\begin{array}{c}1.8 \\
(2009)\end{array}$ & -3.7 & $\begin{array}{c}\text { Not } \\
\text { tested }\end{array}$ & $-67.3 \%$ \\
\hline 5.0 & $\begin{array}{c}5.5 \\
(1995-98)\end{array}$ & $\begin{array}{c}1.6 \\
(2009)\end{array}$ & -3.9 & $\begin{array}{c}\text { Not } \\
\text { tested }\end{array}$ & $-70.9 \%$ \\
\hline 5.3 & $\begin{array}{c}5.9 \\
(2002-03)\end{array}$ & $\begin{array}{c}1.7 \\
(2009)\end{array}$ & -4.2 & $\begin{array}{c}\text { Not } \\
\text { tested }\end{array}$ & $-71.2 \%$ \\
\hline 11.0 & $\begin{array}{c}12.2 \\
(1995-98)\end{array}$ & $\begin{array}{c}3.3 \\
(2009)\end{array}$ & -8.9 & $\begin{array}{c}\text { Not } \\
\text { tested }\end{array}$ & $-73.0 \%$ \\
\hline
\end{tabular}

Percent of targeted change achieved ${ }^{5}$

(continued) 
Figure 14-1. Progress Toward Target Attainment for Focus Area 14: Immunization and Infectious Diseases (continued)

Moved away

from target ${ }^{1}$

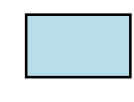

Moved toward

target

Met or exceeded

target

14-20. Hospital-acquired infections among infants in intensive care weighing $\leq 1,000$ grams at birth

e. Ventilator-associated pneumonia (per 1,000 days use)

14-21. Antimicrobial use in intensive care units (daily doses per 1,000 patient days)

14-22. Vaccination of children 19-35 months

a. 4 doses diphtheria-tetanus-acellular pertussis (DTaP) vaccine

b. 3 doses Haemophilus influenza type $b$ (Hib) vaccine

c. 3 doses hepatitis B (Hep B) vaccine

d. 1 dose measles-mumps-rubella (MMR) vaccine

e. 3 doses polio vaccine

f. 1 dose varicella vaccine
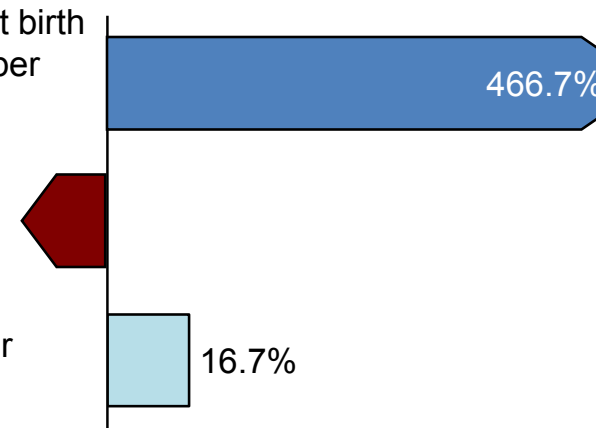

$16.7 \%$

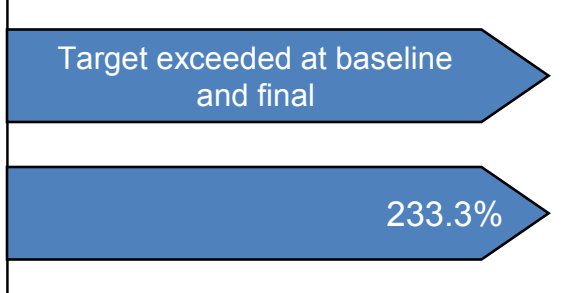

Target exceeded at baseline and final

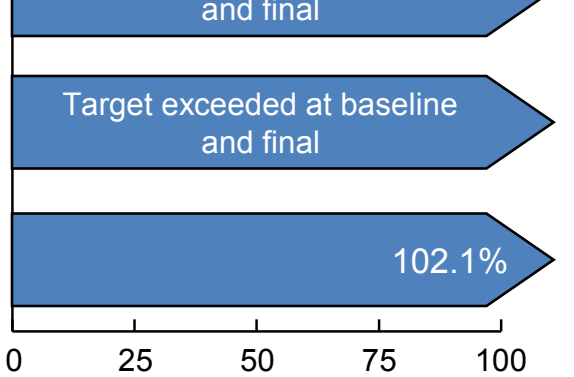

Percent of targeted change achieved ${ }^{5}$

\begin{tabular}{|c|c|c|c|c|c|}
\hline \multirow{2}{*}{$\begin{array}{c}2010 \\
\text { Target }\end{array}$} & \multirow{2}{*}{$\begin{array}{c}\text { Baseline } \\
\text { (Year) }\end{array}$} & \multirow{2}{*}{$\begin{array}{l}\text { Final } \\
\text { (Year) }\end{array}$} & \multicolumn{3}{|c|}{ Baseline vs. Final } \\
\hline & & & Difference ${ }^{2}$ & $\begin{array}{l}\text { Statistically } \\
\text { Significant }^{3}\end{array}$ & $\begin{array}{l}\text { Percent } \\
\text { Change }^{4}\end{array}$ \\
\hline 2.7 & $\begin{array}{c}3.0 \\
(2002-03)\end{array}$ & $\begin{array}{c}1.6 \\
(2009)\end{array}$ & -1.4 & $\begin{array}{l}\text { Not } \\
\text { tested }\end{array}$ & $-46.7 \%$ \\
\hline 85.1 & $\begin{array}{c}106.4 \\
(1996-2003)\end{array}$ & $\mid \begin{array}{c}108.3 \\
(1996-2004)\end{array}$ & 1.9 & $\begin{array}{l}\text { Not } \\
\text { tested }\end{array}$ & $1.8 \%$ \\
\hline $90 \%$ & $\begin{array}{c}84 \% \\
(1998)\end{array}$ & $\begin{array}{c}85 \% \\
(2008)\end{array}$ & 1 & No & $1.2 \%$ \\
\hline $90 \%$ & $\begin{array}{c}93 \% \\
(1998)\end{array}$ & $\begin{array}{l}91 \% \\
(2008)\end{array}$ & -2 & Yes & $-2.2 \%$ \\
\hline $90 \%$ & $\begin{array}{c}87 \% \\
(1998)\end{array}$ & $\begin{array}{c}94 \% \\
(2008)\end{array}$ & 7 & Yes & $8.0 \%$ \\
\hline $90 \%$ & $\begin{array}{c}92 \% \\
(1998)\end{array}$ & $\begin{array}{c}92 \% \\
(2008)\end{array}$ & 0 & No & $0.0 \%$ \\
\hline $90 \%$ & $\begin{array}{c}91 \% \\
(1998)\end{array}$ & $\begin{array}{c}94 \% \\
(2008)\end{array}$ & 3 & Yes & $3.3 \%$ \\
\hline $90 \%$ & $\begin{array}{c}43 \% \\
(1998)\end{array}$ & $\begin{array}{c}91 \% \\
(2008)\end{array}$ & 48 & Yes & $111.6 \%$ \\
\hline
\end{tabular}

(continued) 
Figure 14-1. Progress Toward Target Attainment for Focus Area 14: Immunization and Infectious Diseases (continued)

Moved away

from target ${ }^{1}$

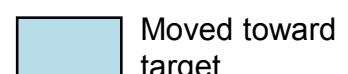

target

Met or exceeded

target

14-22. Vaccination of children 19-35 months

g. 4 doses pneumococcal conjugate vaccine (PCV)

14-23. Vaccine coverage of children in day care

a. Diphtheria-tetanus-acellular pertussis (DTaP) vaccine

b. Measles-mumps-rubella (MMR) vaccine

c. Polio vaccine

Vaccine coverage of children in kindergarten

f. Diphtheria-tetanus-acellular pertussis (DTaP) vaccine

g. Measles-mumps-rubella (MMR) vaccine

h. Polio vaccine

i. Hepatitis B (Hep B) vaccine

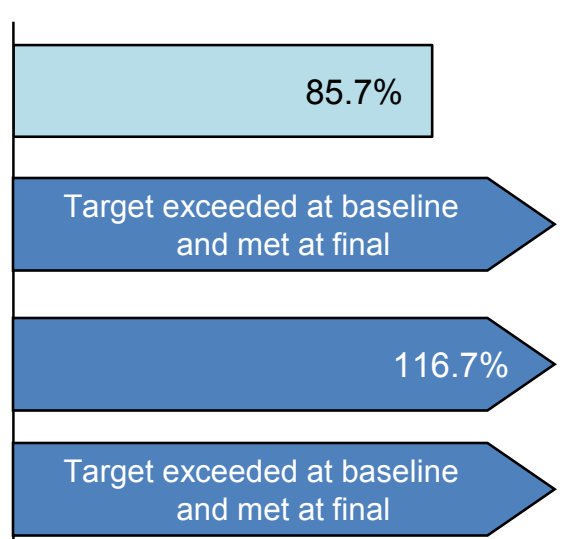

\begin{tabular}{|c|c|c|c|c|c|}
\hline \multirow{2}{*}{$\begin{array}{l}2010 \\
\text { Target }\end{array}$} & \multirow{2}{*}{$\begin{array}{c}\text { Baseline } \\
\text { (Year) }\end{array}$} & \multirow{2}{*}{$\begin{array}{l}\text { Final } \\
\text { (Year) }\end{array}$} & \multicolumn{3}{|c|}{ Baseline vs. Final } \\
\hline & & & Difference ${ }^{2}$ & $\begin{array}{l}\text { Statistically } \\
\text { Significant }^{3}\end{array}$ & $\begin{array}{c}\text { Percent } \\
\text { Change }^{4}\end{array}$ \\
\hline $90 \%$ & $\begin{array}{c}20 \% \\
(2002)\end{array}$ & $\begin{array}{c}80 \% \\
(2008)\end{array}$ & 60 & $\begin{array}{l}\text { Not } \\
\text { tested }\end{array}$ & $300.0 \%$ \\
\hline $95 \%$ & $\begin{array}{c}96 \% \\
(1997-98)\end{array}$ & $\begin{array}{c}95 \% \\
(2000)\end{array}$ & -1 & $\begin{array}{l}\text { Not } \\
\text { tested }\end{array}$ & $-1.0 \%$ \\
\hline $95 \%$ & $\begin{array}{c}89 \% \\
(1997-98)\end{array}$ & $\begin{array}{c}96 \% \\
(2000)\end{array}$ & 7 & $\begin{array}{c}\text { Not } \\
\text { tested }\end{array}$ & $7.9 \%$ \\
\hline $95 \%$ & $\begin{array}{c}96 \% \\
(1997-98)\end{array}$ & $\begin{array}{c}95 \% \\
(2000)\end{array}$ & -1 & $\begin{array}{c}\text { Not } \\
\text { tested }\end{array}$ & $-1.0 \%$ \\
\hline $95 \%$ & $\begin{array}{c}95 \% \\
(2002-03)\end{array}$ & $\begin{array}{c}93 \% \\
(2008)\end{array}$ & -2 & $\begin{array}{l}\text { Not } \\
\text { tested }\end{array}$ & $-2.1 \%$ \\
\hline $95 \%$ & $\begin{array}{c}96 \% \\
(2002-03)\end{array}$ & $\begin{array}{c}92 \% \\
(2008)\end{array}$ & -4 & $\begin{array}{l}\text { Not } \\
\text { tested }\end{array}$ & $-4.2 \%$ \\
\hline $95 \%$ & $\begin{array}{c}96 \% \\
(2002-03)\end{array}$ & $\begin{array}{c}94 \% \\
(2008)\end{array}$ & -2 & $\begin{array}{l}\text { Not } \\
\text { tested }\end{array}$ & $-2.1 \%$ \\
\hline $95 \%$ & $\begin{array}{c}96 \% \\
(2002-03)\end{array}$ & $\begin{array}{c}94 \% \\
(2008)\end{array}$ & -2 & $\begin{array}{l}\text { Not } \\
\text { tested }\end{array}$ & $-2.1 \%$ \\
\hline
\end{tabular}

(continued)

Percent of targeted change achieved ${ }^{5}$ 
Figure 14-1. Progress Toward Target Attainment for Focus Area 14: Immunization and Infectious Diseases (continued)

$\begin{aligned} & \text { Moved away } \\ & \text { from target }\end{aligned}{ }^{1}$
$\begin{aligned} & \text { Moved toward } \\ & \text { target }\end{aligned} \quad \square \quad \begin{aligned} & \text { Met or exceeded } \\ & \text { target }\end{aligned}$

14-23. Vaccine coverage of children in kindergarten j. Varicella vaccine

Vaccine coverage of children in licensed daycare facilities

I. Haemophilus influenzae type b (Hib) vaccine

14-24a. Fully immunized young children 19-35 months

14-25. Providers who measure childhood vaccination coverage levels

a. Public health providers

b. Private providers

14-26. Children $<6$ years participating in populationbased immunization registries

14-27. Vaccination coverage among adolescents 13-15 years

a. 3+ doses hepatitis B (Hep B) vaccine

b. 2+ doses measles-mumps-rubella (MMR) vaccine

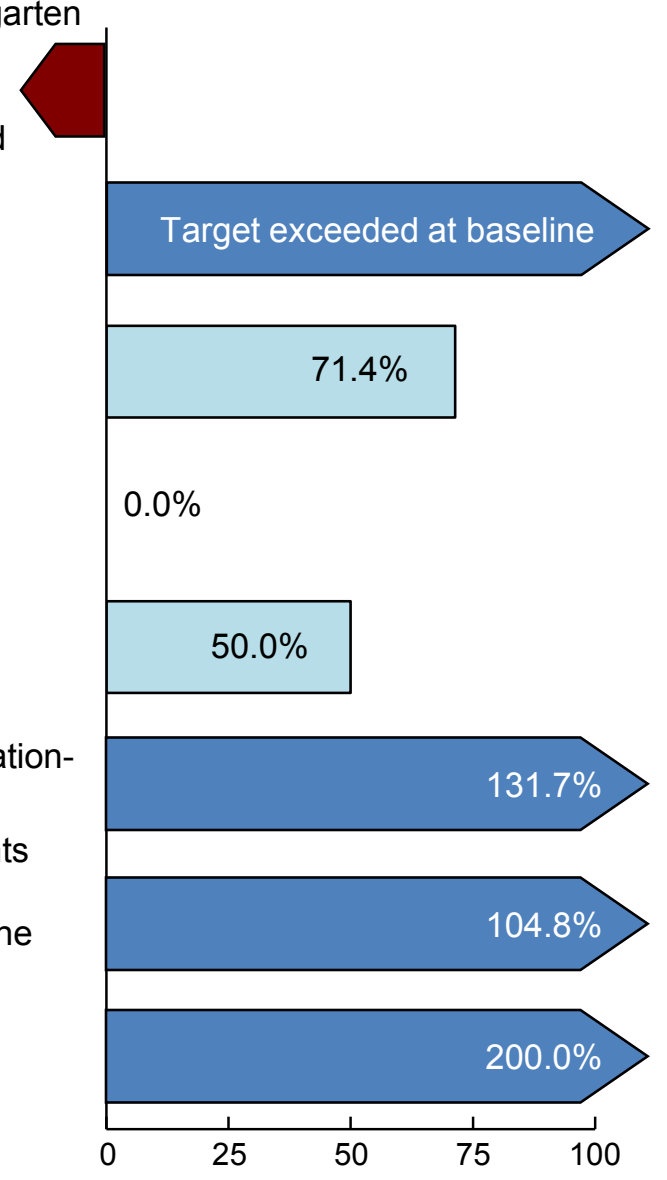

\begin{tabular}{|c|c|c|c|c|c|}
\hline \multirow{2}{*}{$\begin{array}{l}2010 \\
\text { Target }\end{array}$} & \multirow{2}{*}{$\begin{array}{c}\text { Baseline } \\
\text { (Year) }\end{array}$} & \multirow{2}{*}{$\begin{array}{l}\text { Final } \\
\text { (Year) }\end{array}$} & \multicolumn{3}{|c|}{ Baseline vs. Final } \\
\hline & & & Difference $^{2}$ & $\begin{array}{l}\text { Statistically } \\
\text { Significant }^{3}\end{array}$ & $\begin{array}{c}\text { Percent } \\
\text { Change }^{4}\end{array}$ \\
\hline $95 \%$ & $\begin{array}{c}93 \% \\
(2002-03)\end{array}$ & $\begin{array}{c}91 \% \\
(2008)\end{array}$ & -2 & $\begin{array}{c}\text { Not } \\
\text { tested }\end{array}$ & $-2.2 \%$ \\
\hline $90 \%$ & $\begin{array}{c}94 \% \\
(2003-04)\end{array}$ & $N / A^{6}$ & $N / A^{6}$ & $N / A^{6}$ & $N / A^{6}$ \\
\hline $80 \%$ & $\begin{array}{c}73 \% \\
(1998)\end{array}$ & $\begin{array}{c}78 \% \\
(2008)\end{array}$ & 5 & Yes & $6.8 \%$ \\
\hline $55 \%$ & $\begin{array}{c}40 \% \\
(1999)\end{array}$ & $\begin{array}{c}40 \% \\
(2009)\end{array}$ & 0 & $\begin{array}{c}\text { Not } \\
\text { tested }\end{array}$ & $0.0 \%$ \\
\hline $55 \%$ & $\begin{array}{c}11 \% \\
(1999)\end{array}$ & $\begin{array}{c}33 \% \\
(2009)\end{array}$ & 22 & $\begin{array}{c}\text { Not } \\
\text { tested }\end{array}$ & $200.0 \%$ \\
\hline $62 \%$ & $\begin{array}{c}21 \% \\
(1999)\end{array}$ & $\begin{array}{c}75 \% \\
(2008)\end{array}$ & 54 & $\begin{array}{c}\text { Not } \\
\text { tested }\end{array}$ & $257.1 \%$ \\
\hline $90 \%$ & $\begin{array}{c}48 \% \\
(1997)\end{array}$ & $\begin{array}{c}92 \% \\
(2008)\end{array}$ & 44 & Yes & $91.7 \%$ \\
\hline $90 \%$ & $\begin{array}{c}89 \% \\
(1997)\end{array}$ & $\begin{array}{c}91 \% \\
(2008)\end{array}$ & 2 & Yes & $2.2 \%$ \\
\hline
\end{tabular}

Percent of targeted change achieved ${ }^{5}$

(continued) 
Figure 14-1. Progress Toward Target Attainment for Focus Area 14: Immunization and Infectious Diseases (continued)

Moved away

from target ${ }^{1}$

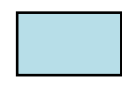

Moved toward

target

Met or exceeded

target

14-27. Vaccination coverage among adolescents 13-15 years

c. 1+ doses tetanus-diptheria booster

d. 1+ doses varicella (excluding adolescents who have had varicella)

14-28. Hepatitis B vaccination among high-risk groups

a. Long-term hemodialysis patients

b. Men who have sex with men
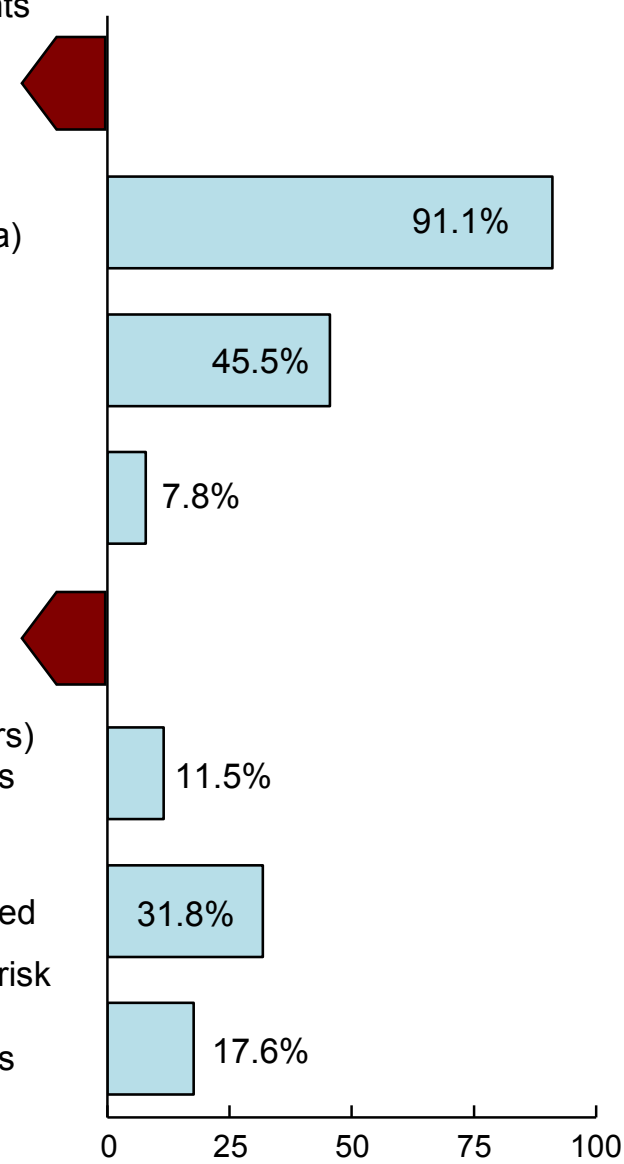

\begin{tabular}{|c|c|c|c|c|c|}
\hline \multirow{2}{*}{$\begin{array}{c}2010 \\
\text { Target }\end{array}$} & \multirow{2}{*}{$\begin{array}{c}\text { Baseline } \\
\text { (Year) }\end{array}$} & \multirow{2}{*}{$\begin{array}{l}\text { Final } \\
\text { (Year) }\end{array}$} & \multicolumn{3}{|c|}{ Baseline vs. Final } \\
\hline & & & Difference $^{2}$ & $\begin{array}{l}\text { Statistically } \\
\text { Significant }^{3}\end{array}$ & $\begin{array}{c}\text { Percent } \\
\text { Change }^{4}\end{array}$ \\
\hline $90 \%$ & $\begin{array}{c}93 \% \\
(1997)\end{array}$ & $\begin{array}{c}71 \% \\
(2008)\end{array}$ & -22 & Yes & $-23.7 \%$ \\
\hline $90 \%$ & $\begin{array}{c}45 \% \\
(1997)\end{array}$ & $\begin{array}{c}86 \% \\
(2008)\end{array}$ & 41 & Yes & $91.1 \%$ \\
\hline $90 \%$ & $\begin{array}{c}35 \% \\
(1995)\end{array}$ & $\begin{array}{c}60 \% \\
(2001)\end{array}$ & 25 & $\begin{array}{c}\text { Not } \\
\text { tested }\end{array}$ & $71.4 \%$ \\
\hline $60 \%$ & $\begin{array}{c}9 \% \\
(1994-99)\end{array}$ & $\begin{array}{c}13 \% \\
(1998-00)\end{array}$ & 4 & $\begin{array}{l}\text { Not } \\
\text { tested }\end{array}$ & $44.4 \%$ \\
\hline $93 \%$ & $\begin{array}{c}67 \% \\
(1995)\end{array}$ & $\begin{array}{c}64 \% \\
(2008)\end{array}$ & -3 & $\begin{array}{l}\text { Not } \\
\text { tested }\end{array}$ & $-4.5 \%$ \\
\hline $90 \%$ & $\begin{array}{c}64 \% \\
(1998)\end{array}$ & $\begin{array}{c}67 \% \\
(2008)\end{array}$ & 3 & Yes & $4.7 \%$ \\
\hline $90 \%$ & $\begin{array}{c}46 \% \\
(1998)\end{array}$ & $\begin{array}{c}60 \% \\
(2008)\end{array}$ & 14 & Yes & $30.4 \%$ \\
\hline $60 \%$ & $\begin{array}{c}26 \% \\
(1998)\end{array}$ & $\begin{array}{c}32 \% \\
(2008)\end{array}$ & 6 & Yes & $23.1 \%$ \\
\hline
\end{tabular}

Percent of targeted change achieved 5 (continued) 
Figure 14-1. Progress Toward Target Attainment for Focus Area 14: Immunization and Infectious Diseases (continued)

Moved away

from target ${ }^{1}$
Moved toward target

Met or exceeded

target

14-29. Vaccination of noninstitutionalized high-risk adults (age adjusted, 18-64 years)

d. Pneumococcal vaccine ever received

Vaccination of adults in long-term care or nursing homes (age adjusted, 18+ years)

e. Influenza vaccine in past 12 months

f. Pneumococcal vaccine ever received

Vaccination of health care workers (age adjusted, 18-64 years)

g. Influenza vaccine in past 12 months

14-30. Adverse events from vaccinations (number)

a. Associated paralytic polio

b. Febrile seizures caused by pertussis vaccines

14-31a. Active surveillance for vaccine safety via large linked databases (number in millions)

14-31b. Vaccine Adverse Events Reports (VAERS) submitted electronically
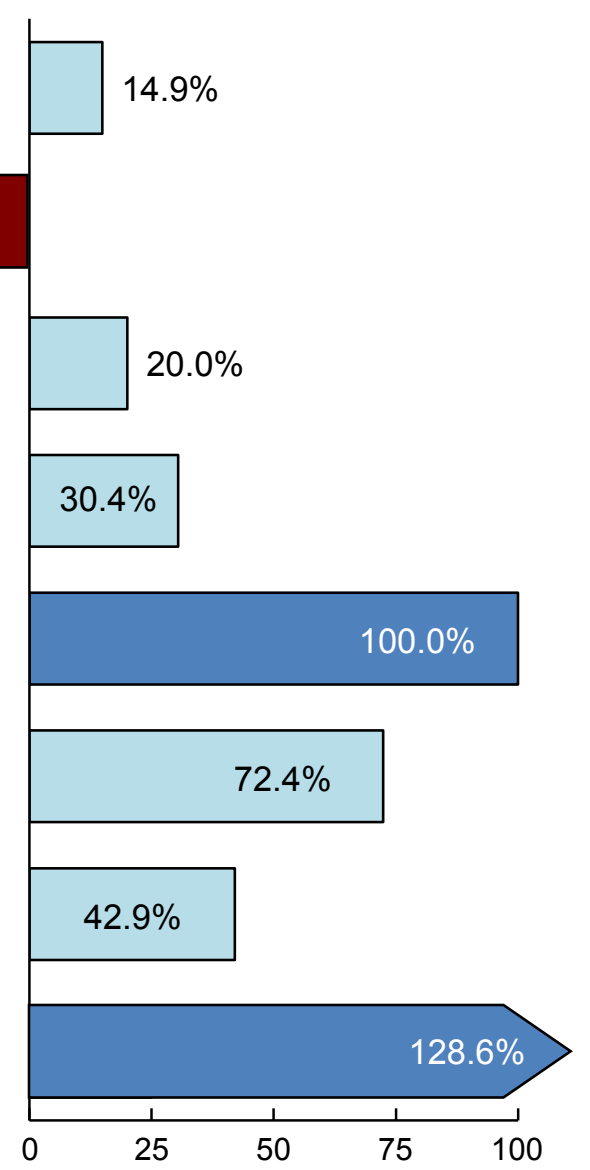

Percent of targeted change achieved ${ }^{5}$

\begin{tabular}{|c|c|c|c|c|c|}
\hline \multirow{2}{*}{$\begin{array}{l}2010 \\
\text { Target }\end{array}$} & \multirow{2}{*}{$\begin{array}{c}\text { Baseline } \\
\text { (Year) }\end{array}$} & \multirow{2}{*}{$\begin{array}{l}\text { Final } \\
\text { (Year) }\end{array}$} & \multicolumn{3}{|c|}{ Baseline vs. Final } \\
\hline & & & Difference $^{2}$ & $\begin{array}{l}\text { Statistically } \\
\text { Significant }^{3}\end{array}$ & $\begin{array}{c}\text { Percent } \\
\text { Change }^{4}\end{array}$ \\
\hline $60 \%$ & $\begin{array}{c}13 \% \\
(1998)\end{array}$ & $\begin{array}{c}20 \% \\
(2008)\end{array}$ & 7 & Yes & $53.8 \%$ \\
\hline $90 \%$ & $\begin{array}{c}59 \% \\
(1997)\end{array}$ & $\begin{array}{c}57 \% \\
(2004)\end{array}$ & -2 & $\begin{array}{l}\text { Not } \\
\text { tested }\end{array}$ & $-3.4 \%$ \\
\hline $90 \%$ & $\begin{array}{c}25 \% \\
(1997)\end{array}$ & $\begin{array}{c}38 \% \\
(2004)\end{array}$ & 13 & $\begin{array}{c}\text { Not } \\
\text { tested }\end{array}$ & $52.0 \%$ \\
\hline $60 \%$ & $\begin{array}{c}37 \% \\
(2000)\end{array}$ & $\begin{array}{c}44 \% \\
(2008)\end{array}$ & 7 & $\begin{array}{l}\text { Not } \\
\text { tested }\end{array}$ & $18.9 \%$ \\
\hline 0 & $\begin{array}{c}5 \\
(1997)\end{array}$ & $\begin{array}{c}0 \\
(2006)\end{array}$ & -5 & $\begin{array}{c}\text { Not } \\
\text { tested }\end{array}$ & $-100.0 \%$ \\
\hline 57 & $\begin{array}{c}115 \\
(1998)\end{array}$ & $\begin{array}{c}73 \\
(2006)\end{array}$ & -42 & $\begin{array}{l}\text { Not } \\
\text { tested }\end{array}$ & $-36.5 \%$ \\
\hline 13 & $\begin{array}{c}6 \\
(1999)\end{array}$ & $\begin{array}{c}9 \\
(2009)\end{array}$ & 3 & $\begin{array}{c}\text { Not } \\
\text { tested }\end{array}$ & $50.0 \%$ \\
\hline $30 \%$ & $\begin{array}{c}16 \% \\
(2003)\end{array}$ & $\begin{array}{c}34 \% \\
(2009)\end{array}$ & 18 & $\begin{array}{c}\text { Not } \\
\text { tested }\end{array}$ & $112.5 \%$ \\
\hline
\end{tabular}

(continued) 


\section{Figure 14-1. Progress Toward Target Attainment for Focus Area 14: Immunization and Infectious Diseases (continued)}

\section{NOTES}

See the Reader's Guide for more information on how to read this figure. See DATA2010 at http://wonder.cdc.gov/data2010 for all Healthy People 2010 tracking data. Tracking data are not available for objectives 14-14, 14-22h, 14-23d, 14-23e, 14-23k, and 14-24b. Objective 14-15 was deleted at the Midcourse Review.

\section{FOOTNOTES}

${ }^{1}$ Movement away from target is not quantified using the percent of targeted change achieved. See Technical Appendix for more information.

${ }^{2}$ Difference $=$ Final value - Baseline value. Differences between percents $(\%)$ are measured in percentage points.

${ }^{3}$ When estimates of variability are available, the statistical significance of the difference between the final value and the baseline value is assessed at the 0.05 level. See Technical Appendix for more information.

${ }^{4}$ Percent change $=\frac{\text { Final value }- \text { Baseline value }}{\text { Baseline value }} \times 100$.

${ }^{5}$ Percent of targeted change achieved $=\frac{\text { Final value }- \text { Baseline value }}{\text { Healthy People } 2010 \text { target }- \text { Baseline value }} \times 100$.

${ }^{6}$ Data beyond the baseline are not available; difference, statistical significance, and percent change cannot be calculated. See Technical Appendix for more information.

* Percent change cannot be calculated. See Technical Appendix for more information.

\section{DATA SOURCES}

14-1a. National Congenital Rubella Syndrome Registry (NCRSR), CDC, NCIRD.

14-1b. National Notifiable Diseases Surveillance System (NNDSS), CDC, NCPHI.

14-1c. National Notifiable Disease Surveillance System (NNDSS), CDC, NCPHI; Active Bacterial Core Surveillance (ABCs), CDC, NCIRD.

14-1d-j. National Notifiable Diseases Surveillance System (NNDSS), CDC, NCPHI.

14-1k. National Health Interview Survey (NHIS), CDC, NCHS.

14-2. Perinatal Hepatitis B Prevention Program, CDC, NCHHSTP; National Vital Statistics System-Natality (NVSS-N), CDC, NCHS.

14-3a-g. National Notifiable Diseases Surveillance System (NNDSS), CDC, NCPHI.

14-4. Active Bacterial Core Surveillance (ABCs), CDC, NCIRD.

14-5a-d. Active Bacterial Core Surveillance (ABCs), CDC, NCIRD.

14-6. National Notifiable Diseases Surveillance System (NNDSS), CDC, NCPHI

14-7. Active Bacterial Core Surveillance (ABCs), CDC, NCIRD; National Notifiable Diseases Surveillance System (NNDSS), CDC, NCPHI.

14-8. National Notifiable Diseases Surveillance System (NNDSS), CDC, NCPHI

14-9. Sentinel Counties Study of Viral Hepatitis, CDC, NCHHSTP.

14-10.

14-11-12.

14-13.

14-16.

State health department databases of persons with HCV infection; National Health and Nutrition Examination Survey (NHANES), CDC, NCHS.

National TB Surveillance System, CDC, NCHHSTP.

Aggregate Reports for Tuberculosis Program Evaluation, CDC, NCHHSTP.

National Notifiable Disease Surveillance System (NNDSS), CDC, NCPHI; Active Bacterial Core Surveillance (ABCs), CDC, NCIRD. 
Figure 14-1. Progress Toward Target Attainment for Focus Area 14: Immunization and Infectious Diseases (continued)

\section{DATA SOURCES (continued)}

14-17. National Hospital Discharge Survey (NHDS), CDC, NCHS.

14-18-19. National Ambulatory Medical Care Survey (NAMCS), CDC, NCHS; National Hospital Ambulatory Medical Care Survey (NHAMCS), CDC, NCHS.

14-20a-e. Baseline: National Noscomial Infections Surveillance System (NNIS), CDC, NCPDCID; Final: National Healthcare Safety Network (NHSN), CDC, NCPDCID

14-21. National Noscomial Infections Surveillance System (NNIS), CDC, NCPDCID.

14-22a-g. National Immunization Survey (NIS): CDC, NCIRD; CDC, NCHS.

14-23a-c. Annual Immunization Assessment Reports, CDC, NCIRD.

14-23f-j. School Immunization Assessment Survey, CDC, NCIRD.

14-23l. Day Care and Head Start Assessment Program, CDC, NCIRD.

14-24a. National Immunization Survey (NIS): CDC, NCIRD; CDC, NCHS.

14-25a-b. Annual Immunization Assessment Reports, CDC, NCIRD.

14-26. Annual Immunization Assessment Reports, CDC, NCIRD.

14-27a-d. Baseline: National Health Interview Survey (NHIS), CDC, NCHS. Final: National Immunization Survey-Teen (NIS-Teen), CDC, NCIRD and CDC, NCHS.

14-28a. Annual Survey of Chronic Hemodialysis Centers: CDC, NCHHSTP; CMS.

14-28b. Young Men's Survey, CDC, NCHHSTP.

14-28c. Periodic vaccine coverage surveys, CDC, NCPDCID.

14-29a-d. National Health Interview Survey (NHIS), CDC, NCHS.

14-29e-g. National Nursing Home Survey (NNHS), CDC, NCHS.

14-30a. National Notifiable Diseases Surveillance System (NNDSS), CDC, NCPHI.

14-30b. Vaccine Adverse Event Reporting System (VAERS) CDC, OD; FDA. Vaccine Safety Datalink (VSD), CDC, OD.

14-31a. Vaccine Safety Datalink (VSD), CDC, OD.

14-31b. Vaccine Adverse Event Reporting System (VAERS): CDC, OD; FDA. 
Figure 14-2. Health Disparities Table for Focus Area 14: Immunization and Infectious

Diseases

Disparities from the best group rate for each characteristic at the most recent data point and changes in disparity from the baseline to the most recent data point.

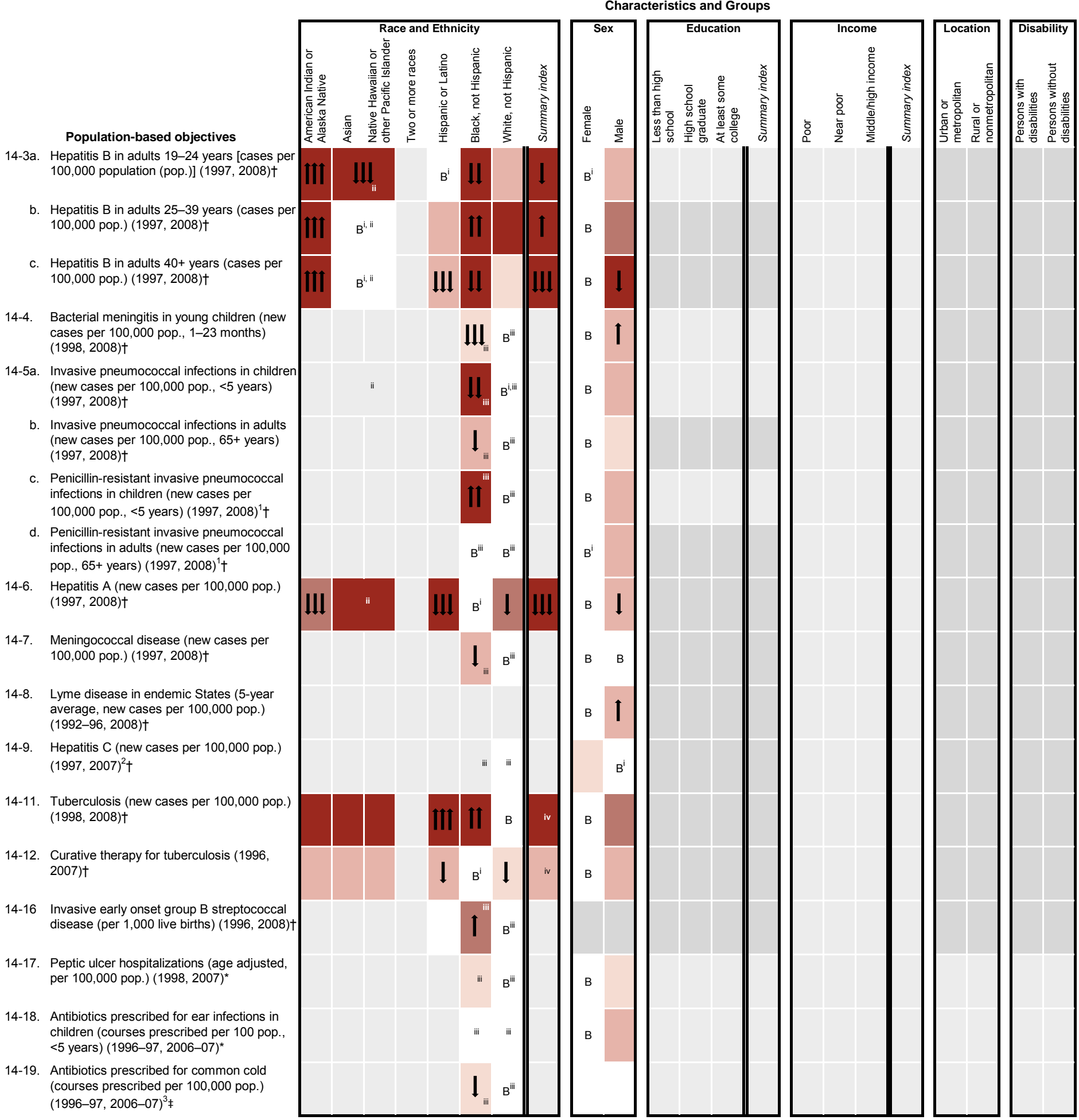

(continued) 
Figure 14-2. Health Disparities Table for Focus Area 14: Immunization and Infectious Diseases (continued)

Population-based objectives

14-22a. Vaccination of children 19-35 months4 doses diphtheria-tetanus-acellular pertussis (DTaP) vaccine $(1998,2008)^{4,5} \ddagger$

b. Vaccination of children 19-35 months3 doses Haemophilus influenza type b $(\mathrm{Hib})$ vaccine $(1998,2008)^{4,5} \ddagger$

c. Vaccination of children 19-35 months3 doses hepatitis B (Hep B) vaccine (1998 $2008)^{4,5} \ddagger$

d. Vaccination of children 19-35 months1 dose measles-mumps-rubella (MMR) vaccine $(1998,2008)^{4,5} \ddagger$

e. Vaccination of children 19-35 months3 doses polio vaccine $(1998,2008)^{4,5} \ddagger$

f. Vaccination of children $19-35$ months1 dose varicella vaccine $(1998,2008)^{4,5} \ddagger$

g. Vaccination of children 19-35 months4 doses pneumococcal conjugate vaccine (PCV) $(2002,2008)^{6 *}$

14-24a. Fully immunized young children 19-35 months $(1998,2008)^{4,5 *}$

14-27a. Vaccination coverage among adolescents 13-15 years- $3+$ doses of hepatitis B (Hep B) vaccine $(1997,2008)^{7,8 *}$

b. Vaccination coverage among adolescents 13-15 years-2+ doses of measles, mumps, rubella (MMR) vaccine $(1997,2008)^{7,8 *}$

c. Vaccination coverage among adolescents 13-15 years- $1+$ doses of tetanus-diptheria booster $(1997,2008)^{7,8 *}$

d. Vaccination coverage among adolescents 13-15 years - $1+$ doses of varicella vaccine (exclud. those with varicella) $(1997,2008)^{7,8 *}$

14-29a. Vaccination of noninstitutionalized high-risk older adults-Influenza vaccine in past 12 months (age adjusted, $65+\mathrm{yrs})(1998,2008)^{7 *}$

b. Vaccination of noninstitutionalized high-risk older adults-Pneumococcal vaccine ever received (age adjusted, 65+ yrs) $(1998,2008)^{7 \star}$

c. Vaccination of noninstitutionalized high-risk adults-Influenza vaccine in past 12 months (age adjusted, 18-64 yrs) $(1998,2008)^{7 *}$

d. Vaccination of noninstitutionalized high-risk older adults-Pneumococcal vaccine ever received (age adjusted, 18-64 yrs) $(1998,2008)^{7}$

e. Vaccination of adults in long-term care or nursing homes-Influenza vaccine in past 12 months (age adjusted, $18+\mathrm{yrs})(1997,2004)^{9} \ddagger$

f. Vaccination of adults in long-term care or nursing homes-Pneumococcal vaccine ever received (age adjusted, $18+\mathrm{yrs})(1997,2004)^{9} \ddagger$

g. Vaccination of health care workersInfluenza vaccine in past 12 months (age adjusted, 18-64 years) $(2000,2008)^{*}$

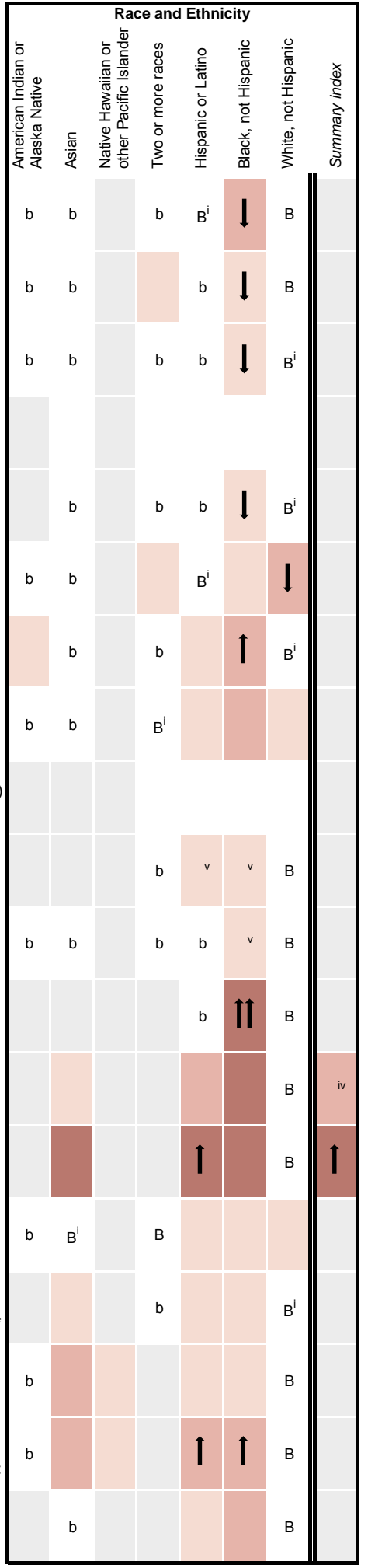

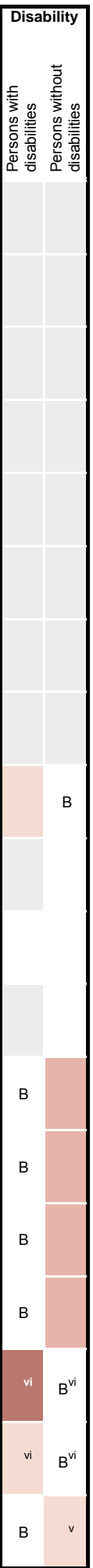

(continued) 


\section{Figure 14-2. Health Disparities Table for Focus Area 14: Immunization and Infectious Diseases (continued)}

NOTES

See DATA2010 at http://wonder.cdc.gov/data2010 for all Healthy People 2010 tracking data. Disparity data are either unavailable or not applicable for objectives 14-1a through k, 14-2, 14-3d through g, 14-10, 14-13, 14-14, 14-20a through e, 14-21, 14-22h, 14-23a through I, 14-24b, 14-25a and b, 14-26, 14-28a through c, 14-30a and b, and 14-31a and b. Objective 14-15 was deleted at Midcourse Review.

Years in parentheses represent the baseline and most recent data years (if available).

Disparity from the best group rate is defined as the percent difference between the best group rate and each of the other group rates for a characteristic (e.g., race and ethnicity). The summary index is the average of these percent differences for a characteristic. Change in disparity is estimated by subtracting the disparity at baseline from the disparity at the most recent data point. Change in the summary index is estimated by subtracting the summary index at baseline from the summary index at the most recent data point. See Technical Appendix for more information.

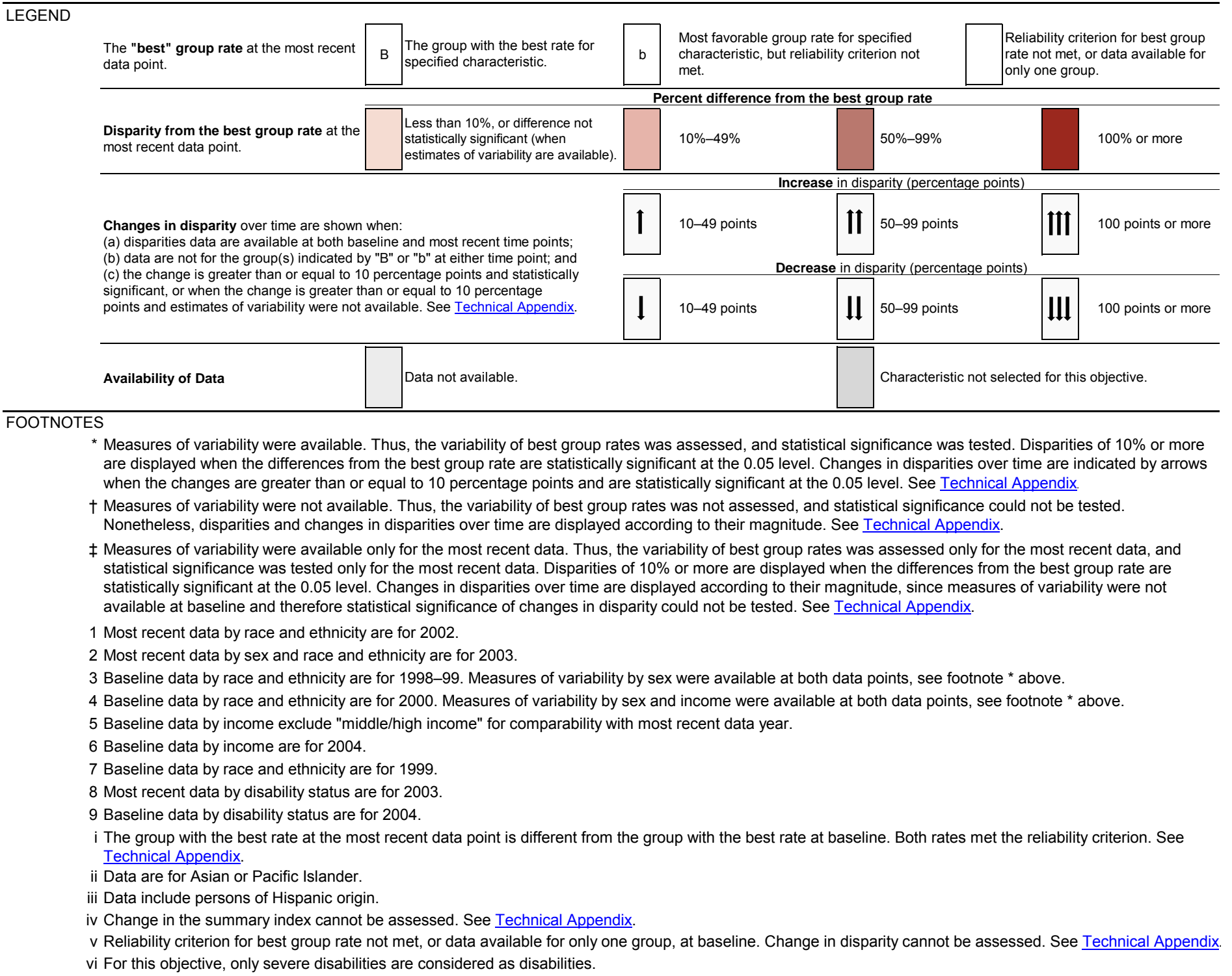

DATA SOURCES

14-3a-c. National Notifiable Diseases Surveillance System (NNDSS), CDC, NCPHI.

14-4. $\quad$ Active Bacterial Core Surveillance (ABCs), CDC, NCIRD.

14-5a-d. Active Bacterial Core Surveillance (ABCs), CDC, NCIRD.

14-6. National Notifiable Diseases Surveillance System (NNDSS), CDC, NCPHI.

14-7. Active Bacterial Core Surveillance (ABCs), CDC, NCIRD; National Notifiable Diseases Surveillance System (NNDSS), CDC, NCPHI.

14-8. $\quad$ National Notifiable Diseases Surveillance System (NNDSS), CDC, NCPHI.

14-9. Sentinel Counties Study of Viral Hepatitis, CDC, NCHHSTP.

14-11-14-12. National TB Surveillance System, CDC, NCHHSTP.

14-16. National Notifiable Disease Surveillance System (NNDSS), CDC, NCPHI; Active Bacterial Core Surveillance (ABCs), CDC, NCIRD.

14-17. National Hospital Discharge Survey (NHDS), CDC, NCHS.

14-18-14-19. National Ambulatory Medical Care Survey (NAMCS), CDC, NCHS; National Hospital Ambulatory Medical Care Survey (NHAMCS), CDC, NCHS.

14-22a-g. National Immunization Survey (NIS), CDC, NCIRD and NCHS.

14-24a. National Immunization Survey (NIS), CDC, NCIRD and NCHS.

14-27a-d. Baseline: National Health Interview Survey (NHIS), CDC, NCHS. Final: National Immunization Survey-Teen (NIS-Teen), CDC, NCIRD and NCHS.

14-29a-d. National Health Interview Survey (NHIS), CDC, NCHS.

14-29e-f. National Nursing Home Survey (NNHS), CDC, NCHS.

14-29g. National Health Interview Survey (NHIS), CDC, NCHS

Immunization and Infectious Diseases 
Figure 14-3. Vaccination of Children (19 to 35 months) -

3 Doses Hepatitis B (HepB) Vaccine 2008

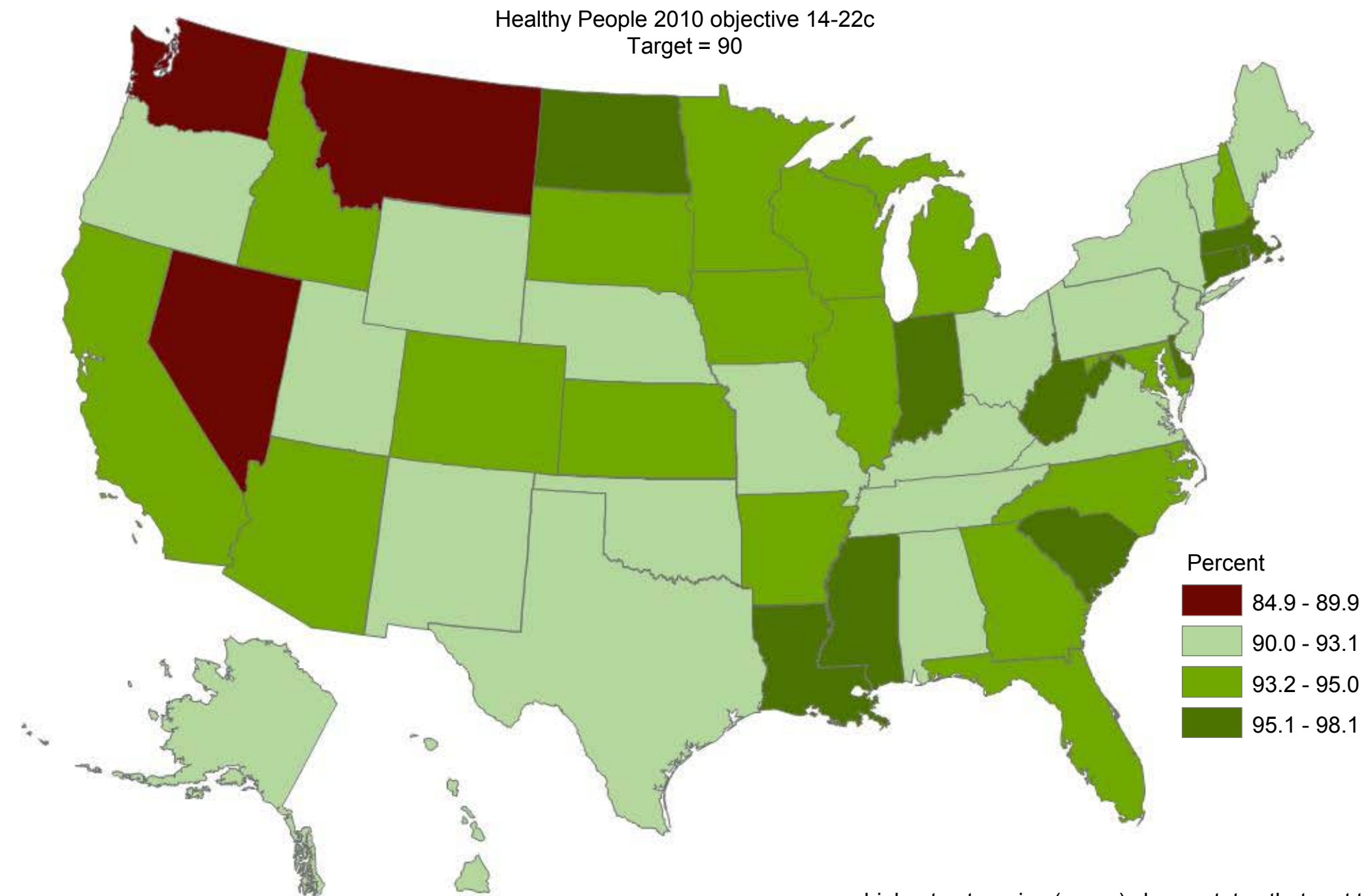

highest categories (green) shows states that met target

NOTES: Rates are displayed by a modified Jenks classification for U.S. states.

SOURCE: National Immunization Survey (NIS), CDC, NCIRD 
Figure 14-4. Vaccination of Children (19 to 35 months) -

1 Dose Measles-Mumps-Rubella (MMR) Vaccine 2008

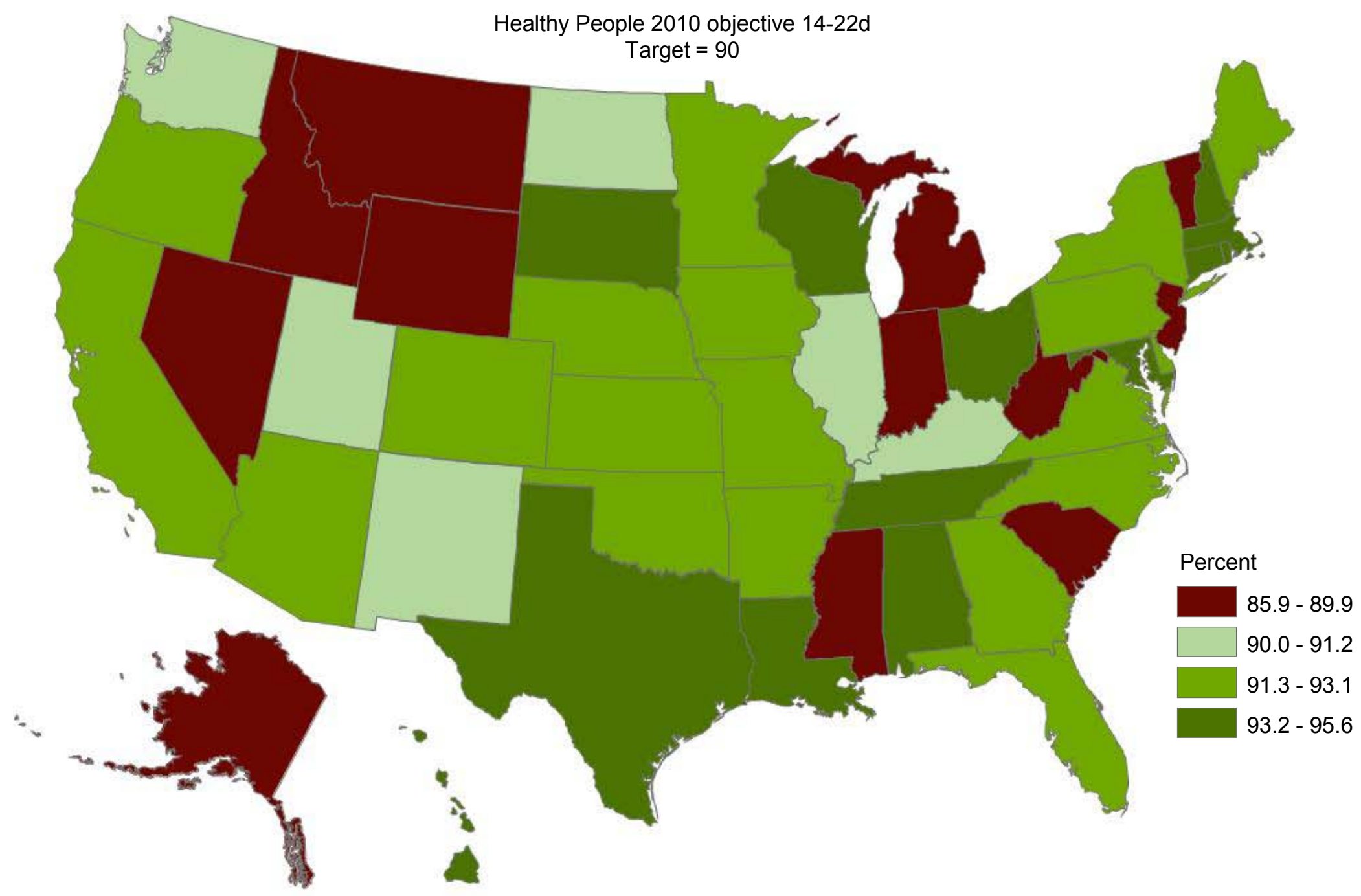

highest categories (green) shows states that met target

NOTES: Rates are displayed by a modified Jenks classification for U.S. states.

SOURCE: National Immunization Survey (NIS), CDC, NCIRD 
Figure 14-5. Universally Recommended Vaccination of Children (19 to 35 months) 4 Doses Pneumococcal Conjugate Vaccine 2008

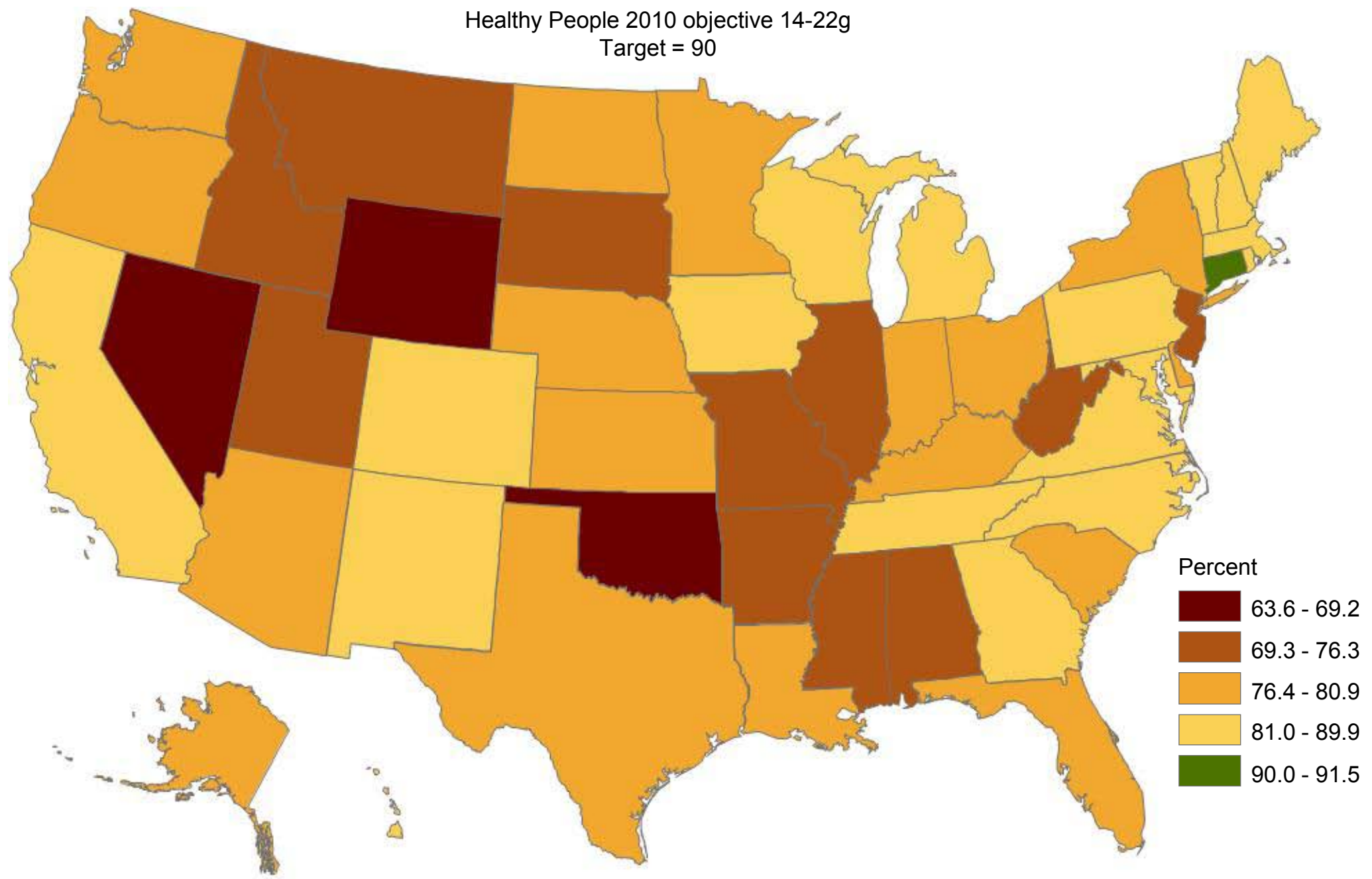

highest category (green) shows states that met target

NOTES: Rates are displayed by a modified Jenks classification for U.S. states.

SOURCE: National Immunization Survey (NIS), CDC, NCIRD and NCHS. 


\section{Injury and Violence}

Prevention
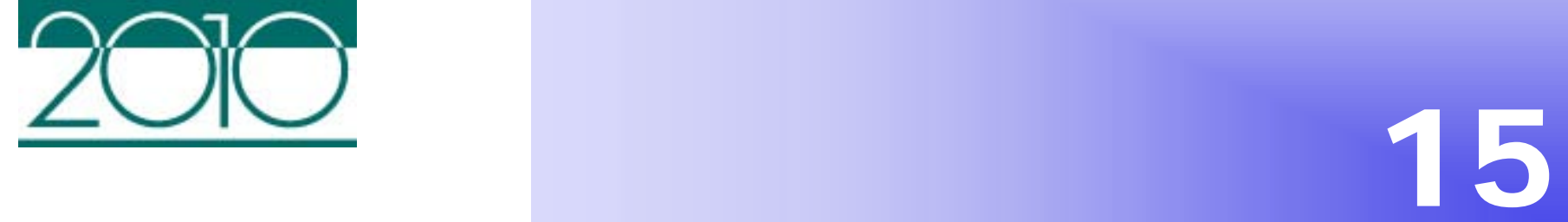

\section{Lead Agency}

Centers for Disease Control and Prevention

\section{Contents}

Goal.

Highlights.

Summary of Progress.

Transition to Healthy People 2020

Data Considerations

References and Notes

Comprehensive Summary of Objectives

Progress Chart

Health Disparities Table

Unintentional Injury Death Rates-Map 


\section{Goal: Reduce injuries, disabilities, and deaths due to unintentional injuries and violence}

The objectives in this chapter monitor progress in three major areas:

- Injuries. This area includes objectives that track deaths and nonfatal injuries caused by both accidents (unintentional) and violence such as traumatic brain injuries, poisoning, and suffocation. The availability of surveillance systems to track injury-related incidents and deaths are also monitored.

- Unintentional injuries. This area includes objectives that track deaths and nonfatal injuries caused by accidents such as motor vehicle-related injuries, falls, drownings, and residential fire deaths. Individual behaviors and state laws for unintentional injury prevention are also monitored.

- Violence. The objectives in this area track deaths and injuries from violent acts such as homicide, child maltreatment, and sexual assault.

All Healthy People tracking data quoted in this chapter, along with technical information and operational definitions for each objective, can be found in the Healthy People 2010 database, DATA2010, available from http://wonder.cdc.gov/data2010/.

More information about this Focus Area can be found in the following publications:

- Healthy People 2010: Understanding and Improving Health, available from http://www.healthypeople.gov/2010/Document/tableofcontents.htm\#under.

- Healthy People 2010 Midcourse Review, available from http://www.healthypeople.gov/2010/data/midcourse/html/default.htm\#FocusAreas.

\section{Highlights}

- Substantial progress was achieved in objectives for this Focus Area during the past decade [1]. Seventy-four percent of the Injury and Violence Prevention objectives with data to monitor progress moved toward or exceeded their Healthy People 2010 targets (Figure 151). Statistically significant health disparities were observed among racial and ethnic populations, as well as by sex, education level, and geographic location (Figure 15-2), as highlighted below [2].

\section{Injuries}

- The poisoning death rate (objective 15-8) increased by $84.5 \%$ between 1999 and 2007, from 7.1 to 13.1 per 100,000 population (age adjusted), moving away from the Healthy People 2010 target of 1.5 per 100,000 population. 
- Among racial and ethnic groups, the Asian or Pacific Islander population had the lowest (best) rates of poisoning deaths: 1.6 deaths per 100,000 population (age adjusted) in 1999 and 2.5 in 2007. The Hispanic or Latino population had rates of 5.9 per 100,000 (age adjusted) in 1999 and 6.9 in 2007; the black non-Hispanic population had rates of 8.2 in 1999 and 10.6 in 2007; and the white non-Hispanic population had rates of 7.3 in 1999 and 15.8 in 2007.

- In 2007, the rate for the Hispanic or Latino population was almost three times the best group rate (that for the Asian or Pacific Islander population); the rate for the black non-Hispanic population was more than four times the best group rate; and the rate for the white non-Hispanic population was almost six and a half times the best group rate [2].

- Between 1999 and 2007, the disparity in the poisoning death rate between the Hispanic or Latino population and the Asian or Pacific Islander population (group with the best rate) decreased by 93 percentage points [3]; similarly, the disparity between the black non-Hispanic and the Asian or Pacific Islander population decreased by 89 percentage points; and the disparity between the white non-Hispanic and the Asian or Pacific Islander population increased by 176 percentage points.

- Among education groups, persons aged 25-64 years with at least some college education had the lowest (best) poisoning death rate, 7.9 deaths per 100,000 population (age adjusted) in 2002. Persons with less than a high school education had a rate of 25.8 deaths per 100,000 population (age adjusted) in 2002, almost three and a half times the best group rate (that for persons with at least some college education) [2]. High school graduates had a rate of 22.4 per 100,000 (age adjusted) in 2002, almost three times the best group rate.

\section{Unintentional Injuries}

- The unintentional injury death rate (objective 15-13) increased by $13.3 \%$ between 1999 and 2007, from 35.3 to 40.0 per 100,000 population (age adjusted), moving away from the Healthy People 2010 target of 17.1 per 100,000 population.

- Among racial and ethnic groups, the Asian or Pacific Islander population had the lowest (best) rate of unintentional injury deaths, 17.0 per 100,000 population (age adjusted) in 2007. The American Indian or Alaska Native, black non-Hispanic, and white non-Hispanic populations had rates of 55.7, 37.6, and 43.0 deaths per 100,000 population (aged adjusted) in 2007, respectively. The rate for the American Indian or Alaska Native population was almost three and a half times the best group rate (that for the Asian or Pacific Islander population); the rate for the black nonHispanic population was more than twice the best group rate; and the rate for the white non-Hispanic population was about two and a half times the best rate [2].

- Females had a lower (better) unintentional injury death rate than males, 25.8 deaths per 100,000 population (age adjusted) in 2007 . The rate for males, 55.8 per 100,000 (age adjusted) in 2007, was more than twice the rate for females [2]. 
- Among education groups, persons aged 25-64 years with at least some college education had the lowest (best) unintentional injury death rate, 18.3 deaths per 100,000 population (age adjusted) in 2002. Persons with less than a high school education had a rate of 61.0 per 100,000 (age adjusted) in 2002, almost three and a half times the best group rate; while high school graduates had a rate of 50.0 per 100,000 (age adjusted) in 2002, more than two and a half times the best rate [2].

- Unintentional injury death rates varied by geographic area. In 2005-07, there were clusters of elevated rates in Appalachian West Virginia, Kentucky, and Tennessee, the Lower Mississippi Delta, and the Mountain West (Figure 15-3).

- The motor vehicle crash death rates decreased, moving toward the 2010 targets. Motor vehicle crash deaths per 100,000 population (objective 15-15a) declined by $6.1 \%$ between 1999 and 2007, from 14.7 to 13.8 (age adjusted), moving toward the target of 8.0. Motor vehicle crash deaths per 100 million vehicle miles travelled (objective 15-15b) declined by $18.8 \%$ between 1998 and 2008, from 1.6 to 1.3, moving toward the target of 0.8 .

- The disparity patterns for motor vehicle crash death rates (objective 15-15a) were very similar to those for unintentional injury death rates, described above. Among racial and ethnic groups, the Asian or Pacific Islander population had the lowest (best) rate, 7.0 per 100,000 population (age adjusted) in 2007. The American Indian or Alaska Native, black non-Hispanic, and white non-Hispanic populations had rates of 22.5, 14.1, and 14.2 per 100,000 (age adjusted) in 2007, respectively. The rate for the American Indian or Alaska Native population was more than three times the best group rate; the rates for the black non-Hispanic and white non-Hispanic populations were about twice the best group rate [2].

- Females had a lower (better) motor vehicle crash death rate than males, 7.9 per 100,000 population (age adjusted) in 2007. The rate for males, 19.9 per 100,000 (age adjusted) in 2007, was approximately two and a half times that for females [2].

- Among education groups, persons aged 25-64 years with at least some college education had the lowest (best) rate of motor vehicle crash deaths, 8.4 per 100,000 population (age adjusted) in 2002. High school graduates and persons with less than a high school education had rates of 22.3 and 26.0 per 100,000 (age adjusted) in 2002 , respectively. The rate for high school graduates was more than two and a half times the best group rate, while the rate for persons with less than a high school education was more than three times the best group rate [2].

- Motor vehicle death rates varied by geographic area. In 2005-07, there were clusters of higher rates in Kentucky, South Florida, and the Mountain West. Several areas met the Healthy People 2010 target (Figure 15-4).

- The nonfatal motor vehicle crash-related injury rate (objective 15-17) declined by $34.7 \%$ between 1998 and 2008, from 1,181 to 771 per 100,000 population, exceeding the 2010 target of 933 per 100,000 population.

- The use of safety belts (objective 15-19) increased by $25.4 \%$ between 1999 and 2009, from $67 \%$ to $84 \%$, moving toward the 2010 target of $89 \%$. However, there was no improvement in the use of child restraints (objective 15-20). As in 2002, the baseline year for this objective, in $2009,88 \%$ of children aged seven years and under were properly restrained in child safety seats. The number of states that had adopted graduated driver licensing laws (objective 15-22) increased from 23 states in 1999 to 50 states (including the District of Columbia) in 2009, moving toward the target of 51 (50 states and the District of Columbia). 
- The residential fire death rate (objective 15-25) declined by $10.0 \%$ between 1999 and 2007, from 1.0 to 0.9 per 100,000 population (age adjusted), moving toward the 2010 target of 0.2 per 100,000 .

- The use of smoke alarms in residences also increased. The proportion of persons living in residences with functioning alarms on every floor (objective 15-26a) and the proportion of residences with functioning alarms on every floor (objective 15-26b) both increased by $3.4 \%$ between 1998 and 2003, from 88\% to 91\% (age adjusted for objective 15-26a), moving toward the Healthy People 2010 targets of $100 \%$.

- The death rate from unintentional falls (objective 15-27) increased by 45.8\% between 1999 and 2007 , from 4.8 to 7.0 per 100,000 population (age adjusted), moving away from the 2010 target of 3.3 per 100,000 .

- Among racial and ethnic groups, the black non-Hispanic population had the lowest (best) rates of deaths from unintentional falls, 3.5 per 100,000 population (age adjusted) in both 1999 and 2007. The rates for the white non-Hispanic population were 5.0 per 100,000 (age adjusted) in 1999 and 7.6 in 2007. In 2007, the rate for the white non-Hispanic population was more than twice that of the black nonHispanic population [2]. Between 1999 and 2007, the disparity between the white non-Hispanic and black non-Hispanic populations increased by 74 percentage points [3].

- Among education groups, persons aged 25-64 years with at least some college education had the lowest (best) death rate from unintentional falls, 1.3 per 100,000 population (age adjusted) in 2002. The rates for high school graduates and persons with less than a high school education were 2.6 and 3.1 per 100,000 (age adjusted) in 2002, respectively. The rate for high school graduates was twice the best group rate, while the rate for persons with less than a high school education was almost two and a half times the best group rate [2].

- Hospitalization rates for hip fractures among women and men aged 65 years and over (objectives 15-28a and b) each decreased by $22 \%$ between 1998 and 2007, from 1,055.8 to 823.5 per 100,000 population (age adjusted) for women and from 592.7 to 464.9 per 100,000 (age adjusted) for men. The hospitalization rate for females moved toward the 2010 target of 416.0 per 100,000 , whereas the rate for males exceeded the target 474.0 per 100,000 .

\section{Violence}

- The homicide rate did not change significantly over the decade. In 1999, the baseline year for this objective (15-32), the homicide rate was 6.0 per 100,000 population (age adjusted), compared to a rate of 6.1 in 2007 [1].

- Among racial and ethnic groups, the Asian or Pacific Islander population had the lowest (best) rate of deaths from homicide, 2.3 per 100,000 population (age adjusted) in 2007. The rates for the American Indian or Alaska Native, Hispanic or Latino, and black non-Hispanic populations were 6.5, 6.9, and 21.8 per 100,000 (age adjusted) in 2007, respectively. The rate for the American Indian or Alaska Native population was almost three times the best group rate; the rate for the Hispanic or Latino population was three times the best group rate; and the rate for the black non-Hispanic population was about nine and a half times the best group rate [2]. 
- The white non-Hispanic population had the lowest (best) rate of deaths from homicide at baseline, 2.9 deaths per 100.000 (age adjusted) in 1999, while the Asian or Pacific Islander population had the best rate at the most recent data point, 2.3 per 100,000 (age adjusted) in 2007. The black non-Hispanic population had rates of 20.7 and 21.8 per 100,000 (age adjusted) in 1999 and 2007, respectively. Between 1999 and 2007, the disparity between the black non-Hispanic population and the group with the best rate increased by 234 percentage points [3].

- Females had a lower (better) homicide rate than males, 2.5 per 100,000 population (age adjusted) in 2007. The rate for males was 9.6 per 100,000 (age adjusted) in 2007, nearly four times the rate for females [2].

- Among education groups, persons aged 25-64 years with at least some college education had the lowest (best) rate of deaths from homicide, 2.6 per 100,000 population (age adjusted) in 2002. The rates for high school graduates and persons with less than a high school education were 10.5 and 16.0 per 100,000 (age adjusted) in 2002, respectively. High school graduates had a rate that was approximately four times the best group rate; the rate for persons with less than a high school education was more than six times the best group rate [2].

- Physical assault by intimate partners (objective 15-34) decreased by 36.1\% between 1998 and 2009 , from 3.6 to 2.3 per 1,000 population aged 12 years and over, exceeding the 2010 target of 2.7 per 1,000 population.

- Rape or attempted rape (objective 15-35) declined by 66.7\% between 1998 and 2009, from 0.9 to 0.3 per 1,000 population aged 12 years and over, exceeding the 2010 target of 0.8 per 1,000 population.

- Sexual assault other than rape (objective 15-36) declined by 66.7\% between 1998 and 2009 , from 0.6 to 0.2 per 1,000 population aged 12 years and over, exceeding the 2010 target of 0.4 per 1,000 population.

- Physical assaults (objective 15-37) declined by 47.6\% between 1998 and 2008, from 31.1 to 16.3 per 1,000 population aged 12 years and over, moving toward the 2010 target of 13.6 per 1,000 population.

- Physical fighting among students in grades $9-12$ (objective $15-38$ ) declined by $13.9 \%$ between 1999 and 2009, from 36\% to 31\%, exceeding the 2010 target of 32\%.

\section{Summary of Progress}

- Figure 15-1 presents a quantitative assessment of progress in achieving the Healthy People 2010 objectives for Injury and Violence Prevention [1]. Data to measure progress toward target attainment were available for 43 objectives. Of these:

- Eight objectives $(15-12,15-17,15-28 b, 15-33 a, 15-34$ through 15-36, and 15-38) met or exceeded their Healthy People 2010 targets.

- Twenty-four objectives moved toward their targets. A statistically significant difference between the baseline and the final data points was observed for eight of these objectives (15-15a, 15-19, 15-25, 15-26a and b, 15-28a, 15-29, and 15-37). No significant differences were observed for nine objectives $(15-2,15-3,15-5,15-7,15-$ $14,15-18,15-21,15-30$, and 15-39); and data to test significance were unavailable for seven objectives $(15-6,15-10,15-11,15-15 b, 15-16,15-22$, and 15-24). 
- Two objectives (15-20 and 15-31a) showed no change.

- Nine objectives moved away from their targets. A statistically significant difference between the baseline and final data points was observed for six of these objectives $(15-1,15-8,15-9,15-13,15-27$, and 15-31b). No significant differences were observed for two objectives (15-31c and 15-32); and data to test the significance of the difference were unavailable for one objective (15-33b).

- Three objectives (15-4 and 15-23a and b) had no data available to measure progress.

- Figure 15-2 displays health disparities in Injury and Violence Prevention from the best group rate for each characteristic at the most recent data point [2]. It also displays changes in disparities from baseline to the most recent data point [3].

- Of the 14 objectives with statistically significant racial and ethnic health disparities of $10 \%$ or more, the Asian or Pacific Islander population had the only best rate for five objectives $(15-3,15-8,15-13,15-15 a$, and 15-32). The white non-Hispanic population had the best rate for four objectives (15-4, 15-26a, 15-37, and 15-38) and the white population had the best rate for one objective (15-12). The Hispanic or Latino population had the only best rate for two objectives (15-25 and 15-29). The black non-Hispanic population had the best rate for one objective (15-27). The Asian or Pacific Islander and Hispanic or Latino populations both had the best rate for one objective (15-9). The Asian population had the best rate for one objective (15-16) with racial and ethnic health disparities of $10 \%$ or more, but without available data to assess statistical significance.

- Females had the better group rate for all 16 of the objectives with statistically significant health disparities of $10 \%$ or more by sex (objectives 15-1, 15-3, 15-4, 15$8,15-9,15-12$ through $15-14,15-15 a, 15-25,15-27,15-29,15-32$, and 15-37 through 15-39) and one objective with health disparities of $10 \%$ or more by sex that lacked data to assess statistical significance (objective 15-16). Males had the better rate for one objective with health disparities of $10 \%$ or more by sex that lacked data to assess statistical significance (objective 15-33a).

- Persons with at least some college education had the best rate for 10 of the 11 objectives with statistically significant health disparities of $10 \%$ or more by education level (objectives 15-3, 15-8, 15-9, 15-13, 15-15a, 15-25, 15-26a, 15-27, 15-29, and 15-32). High school graduates had the best rate for one objective (objective 15-4).

- Persons living in urban or metropolitan areas had the better rate for the one objective with statistically significant health disparities of $10 \%$ or more by geographic location (objective 15-29).

- Health disparities of $100 \%$ or more among racial and ethnic populations, by sex, and by education level were observed for a number of objectives in this Focus Area. Changes in disparities over time also were observed. Many of these disparities are discussed in the Highlights, above. 


\section{Transition to Healthy People 2020}

For Healthy People 2020, the focus of the Injury and Violence Prevention objectives has been expanded to include a broader range of types of injuries and violence and improved strategies for prevention, surveillance, and reducing the consequences of injuries. The Injury and Violence Prevention objectives primarily assess the rates of unintentional and violence-related injuries of varying severity, prevention including individual behaviors and state laws, and surveillance systems. See HealthyPeople.gov for a complete list of Healthy People 2020 topics and objectives.

The Healthy People 2020 Injury and Violence Prevention Topic Area can be grouped into three sections:

- Injury Prevention

- Unintentional Injury Prevention

- Violence Prevention.

The differences between the Healthy People 2010 objectives and those included in Healthy People 2020 are summarized below:

- The Healthy People 2020 Injury and Violence Prevention Topic Area has a total of 65 objectives, nine of which are developmental, whereas the Healthy People 2010 Injury and Violence Prevention Focus Area had 46 objectives [4].

- Twenty-two Healthy People 2010 objectives were retained "as is" [5]. These include: nonfatal traumatic brain injury hospitalizations (objective 15-1); nonfatal spinal cord injury hospitalizations (objective 15-2); firearm-related deaths (objective 15-3); nonfatal firearmrelated injuries (objective 15-5); unintentional injury deaths (objective 15-13); nonfatal unintentional injuries (objective 15-14); motor vehicle crash-related deaths per population (objective 15-15a); motor vehicle crash-related deaths per vehicle miles traveled (objective 15-15b); pedestrian deaths (objective 15-16); nonfatal motor vehicle crash-related injuries (objective 15-17); nonfatal pedestrian injuries (objective 15-18); safety belt use (objective 15-19); motorcycle helmet use (objective 15-21); states with bicycle helmet laws (objective 15-24); residential fire deaths (objective 15-25); unintentional drowning deaths (objective 15-29); schools requiring students to wear appropriate protective gear in physical education (objective 15-31a) and intramural activities or physical activity clubs (objective 15-31c); homicides (objective 15-32); physical assaults (objective 15-37); physical fighting among adolescents (objective 15-38); and weapon carrying by adolescents on school property (objective 15-39).

- Thirteen Healthy People 2010 objectives were modified to create 19 Healthy People 2020 objectives, five of which are developmental $[4,6]$.

- The threshold for the developmental objective on state-level child fatality review for deaths due to external causes (objective 15-6) was decreased from $100 \%$ to $90 \%$ of such deaths.

- A more reliable data source was selected for nonfatal poisonings (objective 15-7).

- Deaths from suffocation (objective 15-9) was modified to create three Healthy People 2020 objectives to track only those that are unintentional among all persons, infants, and older adults (the age groups at highest risk). 
- Emergency department and hospital discharge surveillance of ICD-9-CM external cause of injury codes (objectives 15-10 and 15-11) were modified to better assess how well states are performing.

- Emergency department visits for injuries (objective 15-12) was modified to eliminate the double counting of the more severe injuries that will be tracked by the new objectives for nonfatal injury hospitalizations and injury deaths.

- Child restraints use (objective 15-20) was modified to separately accommodate the four age specific guidelines for types of child restraints.

- States with graduated driver licensing laws (objective 15-22) was modified to track those states with laws rated as "good" based on the criteria set by the Insurance Institute for Highway Safety.

- Maltreatment of children (objective 15-33a) was modified to track only nonfatal maltreatment and avoid overlap with the child maltreatment fatalities objective (1533b). The methodology for counting cases also was revised for both objectives.

- The data source for physical assault by intimate partners, rape or attempted rape, and sexual assault other than rape (objectives 15-34 through 15-36), was changed to enable the prevalence of such violence to be monitored within a health context rather than a crime context, allowing for greater disclosure. Sexual assault other than rape (objective 15-36) was divided into two Healthy People 2020 objectives to separately track abusive sexual contact other than rape or attempted rape and noncontact sexual abuse. The Healthy People 2020 data source is new and, therefore, these four objectives are developmental.

- Two Healthy People 2010 objectives were retained "as is" and also modified to create six Healthy People 2020 objectives [5,6]:

- Poisoning deaths among all ages (objective 15-8) was retained. This objective also was modified to create three other Healthy People 2020 objectives: 1) poisoning deaths among adults aged 35-54 years (the age group at highest risk); and unintentional or undetermined poisoning deaths among 2) all persons and 3) adults aged 35-54 years.

- Deaths from unintentional falls (objective 15-27) was retained. This objective also was modified to track unintentional fall-related deaths among older adults 65 years and over, the age group at highest risk.

- Seven Healthy People 2010 objectives were archived [7]. Objectives tracking improper firearm storage in homes (objective 15-4), regular bicycle helmet use among children and adults (objectives 15-23a and b), persons living in residences with functional smoke alarms on every floor (objective 15-26a), and residences with functional smoke alarms on every floor (objective 15-26b) were deleted due to the lack of an ongoing national data source. The objective regarding emergency department visits for dog bites (objective 15-30) was deleted due to the low rate and lack of effective prevention programs. The objective on schools requiring students to wear appropriate protective gear in interscholastic sports (objective 15-31b) was deleted because it was near the maximum achievement level.

- Two objectives that track hospitalizations for hip fractures among older adults (separately for females and males, objectives 15-28a and b) were moved from the Healthy People 2010 Injury and Violence Prevention Focus Area to the Healthy People 2020 Arthritis, Osteoporosis, and Chronic Back Topic Area. 
- Eighteen new objectives, four of which are developmental, were added to the Healthy People 2020 Injury and Violence Prevention Topic Area:

- Eight new injury objectives include fatal injuries, hospitalizations visits for nonfatal injuries, fatal traumatic brain injuries, emergency department visits for nonfatal traumatic brain injuries, fatal spinal cord injuries, a developmental objective on state-level child fatality review for sudden and unexpected infant deaths, population with trauma care access, and land mass with trauma care access.

- Two new unintentional injury objectives include pedalcyclist deaths and sports and recreation injuries.

- Eight new violence objectives include nonfatal physical assault injuries, bullying among adolescents, nonfatal intentional self harm injuries, children's exposure to violence, three developmental objectives on types of intimate partner violence (sexual violence, psychological abuse, and stalking), and states with national violent death reporting systems.

Appendix D, “A Crosswalk Between Objectives from Healthy People 2010 to Healthy People 2020," summarizes the changes between the two decades of objectives, reflecting new knowledge and direction for this area.

\section{Data Considerations}

Beginning in 2003, education data for mortality objectives 15-3, 15-8, 15-9, 15-13, 15-15a, 15-25, 15-27, 15-29, and 15-32 from the National Vital Statistics System were suppressed. The educational attainment item was changed in the new U.S. Standard Certificate of Death in 2003 to be consistent with the U.S. Census Bureau data and to improve the ability to identify specific types of educational degrees. Many states, however, are still using the 1989 version of the U.S. Standard Certificate of Death, which focuses on highest school grade completed. As a result, educational attainment data collected using the 2003 version are not comparable with data collected using the 1989 version [8].

In general, data on educational attainment are presented for persons aged 25 years and over, consistent with guidance given by the U.S. Bureau of the Census. However, because of the requirements of the different data systems, the age groups used to calculate educational attainment for any specific objective may differ from the age groups used to report the data for other Healthy People 2010 objectives, as well as from select populations within the same objective. Therefore, the reader is urged to exercise caution in interpreting the data by educational attainment shown in the Health Disparities Table. See Healthy People 2010: General Data Issues, referenced below. 
The age-adjusted rate of initial emergency department visits for injuries (objective 15-12) was significantly lower in 2007 (91 visits per 1,000 population) than in 2006 (108 visits per 1,000 population); see DATA2010. In contrast, a flat trend in the rate of initial emergency department visits for injuries was observed between 2001 and 2006 [9]. Some of the observed decrease in the rate from 2006 to 2007 may be related to a modification in the data collection instrument. In 2007, the checkbox for "initial visit for problem" under the "episode of care" item in the National Hospital Ambulatory Medical Care Survey patient record form was reinstated. This item was used in 1992 and 2001-04, but removed from the patient record in 2005. A proxy for initial visits was imputed in 2005-06. The item was restored in 2007. The percentage of unknown or blank responses was higher in 2007 than in 2001-04.

The rate of child maltreatment (objective 15-33a) was significantly lower in 2007-09 than in 200006 , even though the rates were relatively stable during these two separate time periods. Between 2006 and 2007, the rate of victimization dropped from 12.1 to 10.6 per 1,000 children, which was a change of $12 \%$. This decrease can be attributed to several factors including the increase in children who received an "other" disposition which is mostly used for low- or medium-risk cases, the decrease in the percentage of children who received a substantiated or indicated disposition, and the decrease in the number of children who received an investigation or assessment. It is not possible to tell whether this decrease indicates a trend until more data are collected [10].

Additional information on data issues is available from the following sources:

- All Healthy People 2010 tracking data can be found in the Healthy People 2010 database, DATA2010, available from http://wonder.cdc.gov/data2010/.

- Detailed information about the data and data sources used to support these objectives can be found in the Operational Definitions on the DATA2010 website, available from http://wonder.cdc.gov/data2010/focusod.htm.

- More information on statistical issues related to Healthy People tracking and measurement can be found in the Technical Appendix and in Healthy People 2010: General Data Issues, which is available in the Data Issues section of the NCHS Healthy People website under Healthy People 2010.

\section{References and Notes}

1. Displayed in the Progress Chart (Figure 15-1), the percent of targeted change achieved expresses the difference between the baseline and the final value relative to the initial difference between the baseline and the Healthy People 2010 target. As such, it is a relative measure of progress toward attaining the Healthy People 2010 target. See the Reader's Guide for more information. When standard errors were available, the difference between the baseline and the final value was tested at the 0.05 level of significance. See the Figure 15-1 footnotes, as well as the Technical Appendix for more detail.

2. Information about disparities among select populations is shown in the Health Disparities Table (Figure 15-2). Disparity from the best group rate is defined as the percent difference between the best group rate and each of the other group rates for a characteristic. For example, racial and ethnic health disparities are measured as the percent difference between the best racial and ethnic group rate and each of the other racial and ethnic group rates. Similarly, disparities by sex are measured as 
the percent difference between the better group rate (e.g., female) and the rate for the other group (e.g., male). Some objectives are expressed in terms of favorable events or conditions that are to be increased, while others are expressed in terms of adverse events or conditions that are to be reduced. In order to facilitate comparison of health disparities across different objectives, disparity is measured only in terms of adverse events or conditions. For comparability across objectives, objectives that are expressed in terms of favorable events or conditions are re-expressed using the adverse event or condition for the purpose of computing disparity, but they are not otherwise restated or changed. For example, objective 1-1, to increase the proportion of persons with health insurance (e.g., $72 \%$ of the American Indian or Alaska Native population aged under 65 years had some form of health insurance in 2008), is expressed in terms of the percentage of persons without health insurance (e.g., $100 \%-72 \%=28 \%$ of the American Indian or Alaska Native population aged under 65 years did not have any form of health insurance in 2008) when the disparity from the best group rate is calculated. See the Reader's Guide for more information. When standard errors were available, the difference between the best group rate and each of the other group rates was tested at the 0.05 level of significance. See the Figure 15-2 footnotes, as well as the Technical Appendix, for more detail.

3. The change in disparity is estimated by subtracting the disparity at baseline from the disparity at the most recent data point and, therefore, is expressed as a change in percentage points. See the Reader's Guide for more information. When standard errors were available, the change in disparity was tested at the 0.05 level of significance. See the Figure 15-2 footnotes, as well as the Technical Appendix, for more detail.

4. To be included in Healthy People 2010, an objective must have a national data source that provides a baseline and at least one additional data point for tracking progress. Some objectives lacked baseline data at the time of their development but had a potential data source and were considered of sufficient national importance to be included in Healthy People. These are called "developmental" objectives. When data become available, a developmental objective is moved to measurable status and a Healthy People target can be set.

5. Retained "as is" objectives have no change in the numerator definition or in the denominator definition between the Healthy People 2010 and Healthy People 2020 objectives. These include objectives that were developmental in Healthy People 2010 and are developmental in Healthy People 2020 and for which no numerator or denominator information was available.

6. Modified objectives have some change in the numerator definition or in the denominator definition between the Healthy People 2010 and Healthy People 2020 objectives. These include objectives that went from developmental in Healthy People 2010 to measurable in Healthy People 2020 or vice versa.

7. Archived objectives had at least one data point in Healthy People 2010 but were not carried forward into Healthy People 2020.

8. Xu JQ, Kochanek KD, Murphy SL, Tejada-Vera B. Deaths: Final data for 2007. National vital statistics reports; vol 58 no 19. Hyattsville, MD: National Center for Health Statistics. 2010. Available from: http://www.cdc.gov/nchs/data/nvsr/nvsr58/nvsr58 19.pdf.

9. The presence of a monotonic increasing or decreasing trend in the underlying measure was tested with the nonparametric Mann-Kendall test, then the slope of a linear trend was estimated with the nonparametric Sen's method. See Technical Appendix for more information.

10. U.S. Department of Health and Human Services, Administration for Children and Families, Administration on Children, Youth and Families, Children's Bureau. Child Maltreatment 2009. Washington, DC; 2010. Available from: http://www.acf.hhs.gov/programs/cb/stats research/index.htm\#can 


\section{Comprehensive Summary of Objectives: Injury and Violence Prevention}

\begin{tabular}{|c|c|c|}
\hline Objective & Description & Data Source \\
\hline $15-1$ & $\begin{array}{l}\text { Nonfatal traumatic brain injury hospitalizations (age adjusted } \\
\text { per 100,000 standard population) }\end{array}$ & National Hospital Discharge Survey (NHDS), CDC, NCHS. \\
\hline $15-2$ & $\begin{array}{l}\text { Nonfatal spinal cord injury hospitalizations (age adjusted per } \\
100,000 \text { standard population) }\end{array}$ & National Hospital Discharge Survey (NHDS), CDC, NCHS. \\
\hline $15-3$ & $\begin{array}{l}\text { Firearm-related deaths (age adjusted per 100,000 standard } \\
\text { population) }\end{array}$ & $\begin{array}{l}\text { National Vital Statistics System-Mortality (NVSS-M), CDC, } \\
\text { NCHS. }\end{array}$ \\
\hline $15-4$ & $\begin{array}{l}\text { Persons in homes with improperly stored firearms (loaded and } \\
\text { unlocked) (age adjusted, 18+ years) }\end{array}$ & National Health Interview Survey (NHIS), CDC, NCHS. \\
\hline $15-5$ & Nonfatal firearm-related injuries (per 100,000 population) & $\begin{array}{l}\text { National Electronic Injury Surveillance System (NEISS), } \\
\text { Consumer Product Safety Commission (CPSC). }\end{array}$ \\
\hline $15-6$ & $\begin{array}{l}\text { State-level child fatality review for deaths due to external causes } \\
\text { ( } \leq 17 \text { years, no. states and D.C.) }\end{array}$ & $\begin{array}{l}\text { Michigan Public Health Institute; National Vital Statistics } \\
\text { System-Mortality (NVSS-M), CDC, NCHS. }\end{array}$ \\
\hline $15-7$ & $\begin{array}{l}\text { Emergency department visits for nonfatal poisonings (age } \\
\text { adjusted per 100,000 standard population) }\end{array}$ & $\begin{array}{l}\text { National Hospital Ambulatory Medical Care Survey (NHAMCS), } \\
\text { CDC, NCHS. }\end{array}$ \\
\hline $15-8$ & $\begin{array}{l}\text { Deaths from poisoning (age adjusted per 100,000 standard } \\
\text { population) }\end{array}$ & $\begin{array}{l}\text { National Vital Statistics System-Mortality (NVSS-M), CDC, } \\
\text { NCHS. }\end{array}$ \\
\hline $15-9$ & $\begin{array}{l}\text { Deaths from suffocation (age adjusted per 100,000 standard } \\
\text { population) }\end{array}$ & $\begin{array}{l}\text { National Vital Statistics System-Mortality (NVSS-M), CDC, } \\
\text { NCHS. }\end{array}$ \\
\hline $15-10$ & $\begin{array}{l}\text { Emergency department routine collection of ICD-9-CM external } \\
\text { causes of injury codes (no. states and D.C.) }\end{array}$ & $\begin{array}{l}\text { External Cause of Injury Survey, American Public Health } \\
\text { Association (APHA). }\end{array}$ \\
\hline $15-11$ & $\begin{array}{l}\text { Hospital discharge mandated use of ICD-9-CM external causes of } \\
\text { injury codes (no. states and D.C.) }\end{array}$ & $\begin{array}{l}\text { External Cause of Injury Survey, American Public Health } \\
\text { Association (APHA). }\end{array}$ \\
\hline $15-12$ & $\begin{array}{l}\text { Initial emergency department visits for injuries (age adjusted } \\
\text { per 1,000 standard population) }\end{array}$ & $\begin{array}{l}\text { National Hospital Ambulatory Medical Care Survey (NHAMCS), } \\
\text { CDC, NCHS. }\end{array}$ \\
\hline $15-13$ & $\begin{array}{l}\text { Deaths from unintentional injuries (age adjusted per 100,000 } \\
\text { standard population) }\end{array}$ & $\begin{array}{l}\text { National Vital Statistics System-Mortality (NVSS-M), CDC, } \\
\text { NCHS. }\end{array}$ \\
\hline $15-14$ & $\begin{array}{l}\text { Nonfatal unintentional injuries (age adjusted per 100,000 } \\
\text { standard population) }\end{array}$ & $\begin{array}{l}\text { National Electronic Injury Surveillance System - All Injury } \\
\text { Program (NEISS-AIP), CDC, NCIPC; Consumer Product Safety } \\
\text { Commission (CPSC). }\end{array}$ \\
\hline
\end{tabular}




\begin{tabular}{|c|c|c|}
\hline Objective & Description & Data Source \\
\hline $15-15 a$ & $\begin{array}{l}\text { Deaths from motor vehicle crashes-Age adjusted per } 100,000 \\
\text { standard population }\end{array}$ & $\begin{array}{l}\text { National Vital Statistics System-Mortality (NVSS-M), CDC, } \\
\text { NCHS. }\end{array}$ \\
\hline $15-15 b$ & $\begin{array}{l}\text { Deaths from motor vehicle crashes-Per } 100 \text { million vehicle } \\
\text { miles traveled }\end{array}$ & $\begin{array}{l}\text { Fatality Analysis Reporting System (FARS), Department of } \\
\text { Transportation (DOT). }\end{array}$ \\
\hline $15-16$ & Pedestrian deaths on public roads (per 100,000 population) & $\begin{array}{l}\text { Fatality Analysis Reporting System (FARS), Department of } \\
\text { Transportation (DOT). }\end{array}$ \\
\hline $15-17$ & $\begin{array}{l}\text { Nonfatal motor vehicle crash-related injuries on public roads } \\
\text { (per } 100,000 \text { population) }\end{array}$ & $\begin{array}{l}\text { General Estimates System (GES), Department of Transportation } \\
\text { (DOT). }\end{array}$ \\
\hline $15-18$ & $\begin{array}{l}\text { Nonfatal pedestrian injuries on public roads (per 100,000 } \\
\text { population) }\end{array}$ & $\begin{array}{l}\text { General Estimates System (GES), Department of Transportation } \\
\text { (DOT). }\end{array}$ \\
\hline $15-19$ & Safety belt use & $\begin{array}{l}\text { National Occupant Protection Use Survey (NOPUS), Department } \\
\text { of Transportation (DOT). }\end{array}$ \\
\hline $15-20$ & Child restraint use ( $\leq 7$ years $)$ & $\begin{array}{l}\text { National Occupant Protection Use Survey (NOPUS), Department } \\
\text { of Transportation (DOT). }\end{array}$ \\
\hline $15-21$ & Motorcycle helmet use & $\begin{array}{l}\text { National Occupant Protection Use Survey (NOPUS), Department } \\
\text { of Transportation (DOT). }\end{array}$ \\
\hline $15-22$ & Graduated driver licensing laws (no. states and D.C.) & $\begin{array}{l}\text { U.S. Licensing Systems for Young Drivers, Insurance Institute for } \\
\text { Highway Safety. }\end{array}$ \\
\hline $15-23 a$ & Regular bicycle helmet use-Children (1-15 years) & $\begin{array}{l}\text { National Bike Helmet Survey, Consumer Product Safety } \\
\text { Commission (CPSC). }\end{array}$ \\
\hline $15-23 b$ & Regular bicycle helmet use-Adults (16+ years) & $\begin{array}{l}\text { National Bike Helmet Survey, Consumer Product Safety } \\
\text { Commission (CPSC). }\end{array}$ \\
\hline $15-24$ & Bicycle helmet laws for riders $<15$ years (no. states and D.C.) & Bicycle Helmet Safety Institute. \\
\hline $15-25$ & $\begin{array}{l}\text { Residential fire deaths (age adjusted per 100,000 standard } \\
\text { population) }\end{array}$ & $\begin{array}{l}\text { National Vital Statistics System-Mortality (NVSS-M), CDC, } \\
\text { NCHS. }\end{array}$ \\
\hline $15-26 a$ & $\begin{array}{l}\text { Functional smoke alarms in residences-Persons living in } \\
\text { residences with alarms on every floor (age adjusted) }\end{array}$ & National Health Interview Survey (NHIS), CDC, NCHS. \\
\hline $15-26 b$ & $\begin{array}{l}\text { Functional smoke alarms in residences-Proportion of } \\
\text { residences with alarms on every floor }\end{array}$ & National Health Interview Survey (NHIS), CDC, NCHS. \\
\hline $15-27$ & $\begin{array}{l}\text { Deaths from unintentional falls (age adjusted per 100,000 } \\
\text { standard population) }\end{array}$ & $\begin{array}{l}\text { National Vital Statistics System-Mortality (NVSS-M), CDC, } \\
\text { NCHS. }\end{array}$ \\
\hline
\end{tabular}




\begin{tabular}{|c|c|c|}
\hline Objective & Description & Data Source \\
\hline $15-28 \mathrm{a}$ & $\begin{array}{l}\text { Hospitalizations for hip fractures (age adjusted per 100,000 } \\
\text { standard population, } 65+\text { years) - Females }\end{array}$ & National Hospital Discharge Survey (NHDS), CDC, NCHS. \\
\hline $15-28 b$ & $\begin{array}{l}\text { Hospitalizations for hip fractures (age adjusted per 100,000 } \\
\text { standard population, } 65+\text { years) -Males }\end{array}$ & National Hospital Discharge Survey (NHDS), CDC, NCHS. \\
\hline $15-29$ & $\begin{array}{l}\text { Unintentional drownings (age adjusted per 100,000 standard } \\
\text { population) }\end{array}$ & $\begin{array}{l}\text { National Vital Statistics System-Mortality (NVSS-M), CDC, } \\
\text { NCHS. }\end{array}$ \\
\hline $15-30$ & $\begin{array}{l}\text { Emergency department visits for dog bite injuries (age adjusted } \\
\text { per } 100,000 \text { standard population) }\end{array}$ & $\begin{array}{l}\text { National Hospital Ambulatory Medical Care Survey (NHAMCS), } \\
\text { CDC, NCHS. }\end{array}$ \\
\hline $15-31 \mathrm{a}$ & $\begin{array}{l}\text { Schools requiring students to wear appropriate protective } \\
\text { gear-Physical education }\end{array}$ & $\begin{array}{l}\text { School Health Policies and Programs Study (SHPPS), CDC, } \\
\text { NCCDPHP. }\end{array}$ \\
\hline $15-31 b$ & $\begin{array}{l}\text { Schools requiring students to wear appropriate protective } \\
\text { gear-Interscholastic sports }\end{array}$ & $\begin{array}{l}\text { School Health Policies and Programs Study (SHPPS), CDC, } \\
\text { NCCDPHP. }\end{array}$ \\
\hline $15-31 c$ & $\begin{array}{l}\text { Schools requiring students to wear appropriate protective } \\
\text { gear-Intramural activities or physical activity clubs }\end{array}$ & $\begin{array}{l}\text { School Health Policies and Programs Study (SHPPS), CDC, } \\
\text { NCCDPHP. }\end{array}$ \\
\hline $15-32$ & Homicides (age adjusted per 100,000 standard population) & $\begin{array}{l}\text { National Vital Statistics System-Mortality (NVSS-M), CDC, } \\
\text { NCHS. }\end{array}$ \\
\hline $15-33 a$ & Maltreatment of children (per 1,000 population, $<18$ years) & National Child Abuse and Neglect Data System (NCANDS), ACF. \\
\hline $15-33 b$ & $\begin{array}{l}\text { Child maltreatment fatalities (per 100,000 population, }<18 \\
\text { years) }\end{array}$ & National Child Abuse and Neglect Data System (NCANDS), ACF. \\
\hline $15-34$ & $\begin{array}{l}\text { Physical assault by intimate partners (per } 1,000 \text { population, } 12+ \\
\text { years) }\end{array}$ & $\begin{array}{l}\text { National Crime Victimization Survey (NCVS), Department of } \\
\text { Justice (DOJ), Bureau of Justice Statistics (BJS). }\end{array}$ \\
\hline $15-35$ & Rape or attempted rape (per 1,000 population, $12+$ years) & $\begin{array}{l}\text { National Crime Victimization Survey (NCVS), Department of } \\
\text { Justice (DOJ), Bureau of Justice Statistics (BJS). }\end{array}$ \\
\hline $15-36$ & Sexual assault other than rape (per 1,000 population, $12+$ years) & $\begin{array}{l}\text { National Crime Victimization Survey (NCVS), Department of } \\
\text { Justice (DOJ), Bureau of Justice Statistics (BJS). }\end{array}$ \\
\hline $15-37$ & Physical assaults (per 1,000 population, $12+$ years) & $\begin{array}{l}\text { National Crime Victimization Survey (NCVS), Department of } \\
\text { Justice (DOJ), Bureau of Justice Statistics (BJS). }\end{array}$ \\
\hline $15-38$ & Physical fighting among students (grades 9-12) & $\begin{array}{l}\text { Youth Risk Behavior Surveillance System (YRBSS), CDC, } \\
\text { NCCDPHP. }\end{array}$ \\
\hline $15-39$ & Weapon carrying by students on school property (grades 9-12) & $\begin{array}{l}\text { Youth Risk Behavior Surveillance System (YRBSS), CDC, } \\
\text { NCCDPHP. }\end{array}$ \\
\hline
\end{tabular}


Figure 15-1. Progress Toward Target Attainment for Focus Area 15: Injury and Violence Prevention

Moved away

from target ${ }^{1}$
Moved toward

target
Met or exceeded

target

15-1. Nonfatal traumatic brain injury hospitalizations (age adjusted. per 100,000 population)

15-2. Nonfatal spinal cord injury hospitalizations (age adjusted, per 100,000 population)

15-3. Firearm-related deaths (age adjusted, per 100,000 population)

15-5. Nonfatal firearm-related injuries (per 100,000 population)

15-6. State-level child fatality review for deaths due to external causes ( $\leq 17$ years, no. States and D.C.)

15-7. Emergency department visits for nonfatal poisonings (age adjusted, per 100,000 population)

15-8. Deaths from poisoning (age adjusted, per 100,000 population)

15-9. Deaths from suffocation (age adjusted, per 100,000 population)

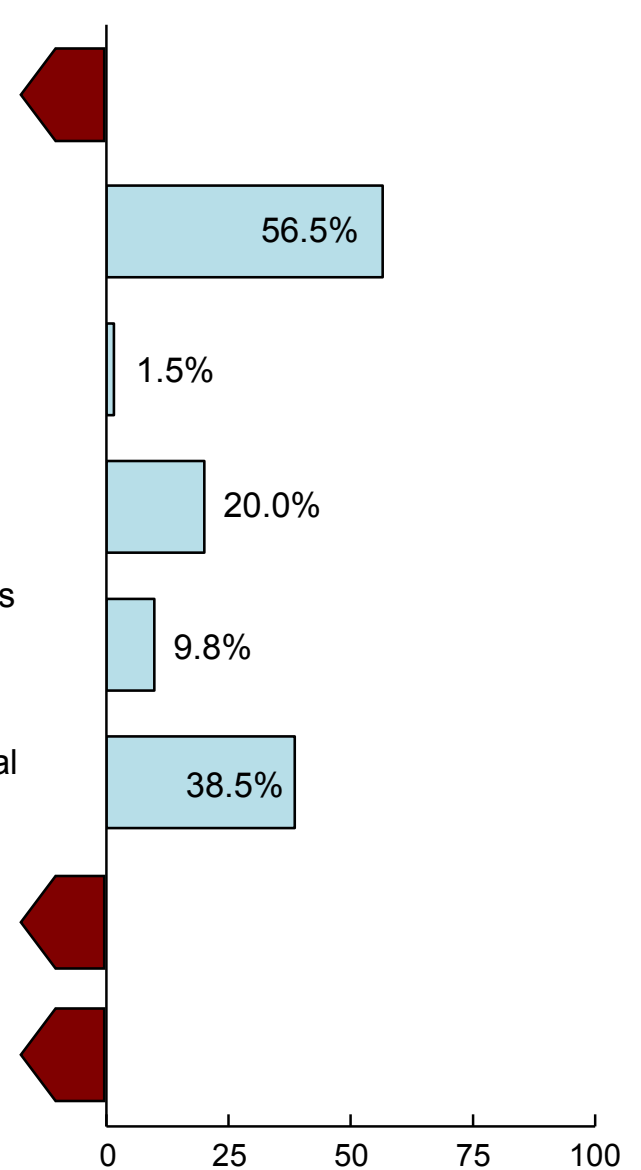

\begin{tabular}{|c|c|c|c|c|c|}
\hline \multirow{2}{*}{$\begin{array}{l}2010 \\
\text { Target }\end{array}$} & \multirow{2}{*}{$\begin{array}{c}\text { Baseline } \\
\text { (Year) }\end{array}$} & \multirow{2}{*}{$\begin{array}{l}\text { Final } \\
\text { (Year) }\end{array}$} & \multicolumn{3}{|c|}{ Baseline vs. Final } \\
\hline & & & Difference ${ }^{2}$ & $\begin{array}{l}\text { Statistically } \\
\text { Significant }^{3}\end{array}$ & $\begin{array}{l}\text { Percent } \\
\text { Change }^{4}\end{array}$ \\
\hline 53.6 & $\begin{array}{c}71.7 \\
(1998)\end{array}$ & $\begin{array}{c}85.6 \\
(2007)\end{array}$ & 13.9 & Yes & $19.4 \%$ \\
\hline 2.6 & $\begin{array}{c}4.9 \\
(1998)\end{array}$ & $\begin{array}{c}3.6 \\
(2007)\end{array}$ & -1.3 & No & $-26.5 \%$ \\
\hline 3.6 & $\begin{array}{c}10.3 \\
(1999)\end{array}$ & $\begin{array}{c}10.2 \\
(2007)\end{array}$ & -0.1 & No & $-1.0 \%$ \\
\hline 9.1 & $\begin{array}{c}23.6 \\
(1997)\end{array}$ & $\begin{array}{c}20.7 \\
(2007)\end{array}$ & -2.9 & No & $-12.3 \%$ \\
\hline 51 & $\begin{array}{c}10 \\
(2000)\end{array}$ & $\begin{array}{c}14 \\
(2007)\end{array}$ & 4 & $\begin{array}{c}\text { Not } \\
\text { tested }\end{array}$ & $40.0 \%$ \\
\hline 288.6 & $\begin{array}{l}343.6 \\
(1997)\end{array}$ & $\begin{array}{l}322.4 \\
(2007)\end{array}$ & -21.2 & No & $-6.2 \%$ \\
\hline 1.5 & $\begin{array}{c}7.1 \\
(1999)\end{array}$ & $\begin{array}{c}13.1 \\
(2007)\end{array}$ & 6 & Yes & $84.5 \%$ \\
\hline 3.3 & $\begin{array}{c}4.2 \\
(1999)\end{array}$ & $\begin{array}{c}4.9 \\
(2007)\end{array}$ & 0.7 & Yes & $16.7 \%$ \\
\hline
\end{tabular}

Percent of targeted change achieved ${ }^{5}$ 
Figure 15-1. Progress Toward Target Attainment for Focus Area 15: Injury and Violence Prevention (continued)

Moved away

from target ${ }^{1}$
Moved toward target

15-10. Emergency department routine collection of ICD-9-CM external causes of injury codes (no. States and D.C.)

15-11. Hospital discharge mandated use of ICD-9$\mathrm{CM}$ external causes of injury codes (no. States and D.C.)

15-12. Initial emergency department visits for injuries (age adjusted, per 1,000 population)

15-13. Deaths from unintentional injuries (age adjusted, per 100,000 population)

15-14. Nonfatal unintentional injuries (age adjusted, per 100,000 population)

15-15. Deaths from motor vehicle crashes

a. Age adjusted, per 100,000 population

b. Per 100 million vehicle miles traveled

15-16. Pedestrian deaths on public roads (per 100,000 population)

Met or exceeded

target

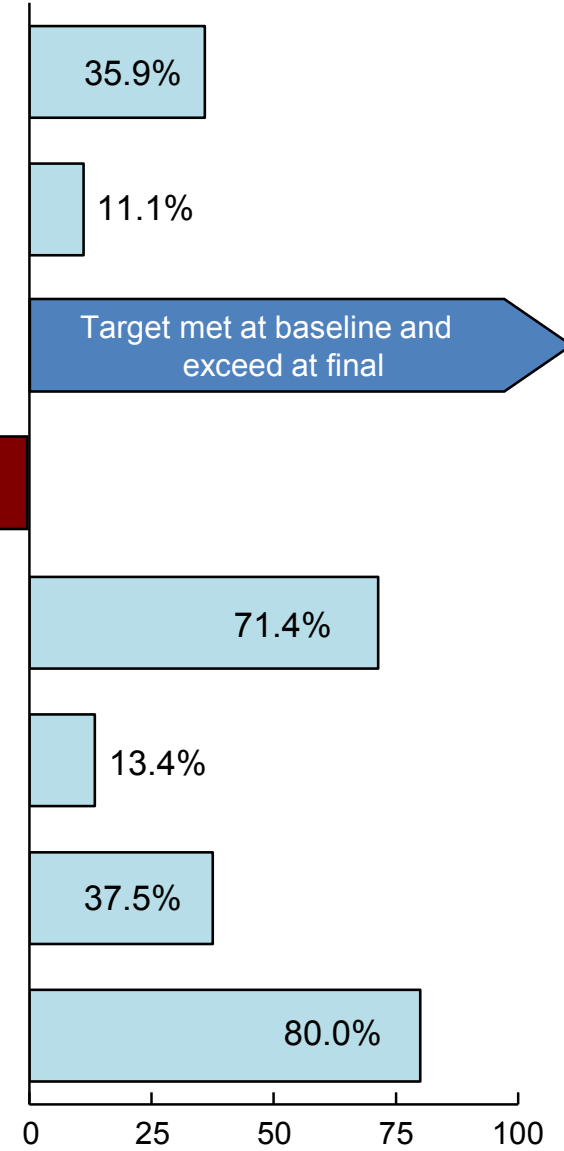

\begin{tabular}{|c|c|c|c|c|c|}
\hline \multirow{2}{*}{$\begin{array}{l}2010 \\
\text { Target }\end{array}$} & \multirow{2}{*}{$\begin{array}{c}\text { Baseline } \\
\text { (Year) }\end{array}$} & \multirow{2}{*}{$\begin{array}{l}\text { Final } \\
\text { (Year) }\end{array}$} & \multicolumn{3}{|c|}{ Baseline vs. Final } \\
\hline & & & Difference ${ }^{2}$ & $\begin{array}{l}\text { Statistically } \\
\text { Significant }^{3}\end{array}$ & $\begin{array}{l}\text { Percent } \\
\text { Change }^{4}\end{array}$ \\
\hline 51 & $\begin{array}{c}12 \\
(1998)\end{array}$ & $\begin{array}{c}26 \\
(2007)\end{array}$ & 14 & $\begin{array}{c}\text { Not } \\
\text { tested }\end{array}$ & $116.7 \%$ \\
\hline 51 & $\begin{array}{c}24 \\
(1998)\end{array}$ & $\begin{array}{c}27 \\
(2007)\end{array}$ & 3 & $\begin{array}{c}\text { Not } \\
\text { tested }\end{array}$ & $12.5 \%$ \\
\hline 107 & $\begin{array}{c}107 \\
(2001)\end{array}$ & $\begin{array}{c}91 \\
(2007)\end{array}$ & -16 & Yes & $-15.0 \%$ \\
\hline 17.1 & $\begin{array}{c}35.3 \\
(1999)\end{array}$ & $\begin{array}{c}40.0 \\
(2007)\end{array}$ & 4.7 & Yes & $13.3 \%$ \\
\hline $9,000.0$ & $\begin{array}{l}9,767.4 \\
(2000)\end{array}$ & $\begin{array}{l}9,219.3 \\
(2008)\end{array}$ & -548.1 & No & $-5.6 \%$ \\
\hline 8.0 & $\begin{array}{c}14.7 \\
(1999)\end{array}$ & $\begin{array}{c}13.8 \\
(2007)\end{array}$ & -0.9 & Yes & $-6.1 \%$ \\
\hline 0.8 & $\begin{array}{c}1.6 \\
(1998)\end{array}$ & $\begin{array}{c}1.3 \\
(2008)\end{array}$ & -0.3 & $\begin{array}{c}\text { Not } \\
\text { tested }\end{array}$ & $-18.8 \%$ \\
\hline 1.4 & $\begin{array}{c}1.9 \\
(1998)\end{array}$ & $\begin{array}{c}1.5 \\
(2008)\end{array}$ & -0.4 & $\begin{array}{c}\text { Not } \\
\text { tested }\end{array}$ & $-21.1 \%$ \\
\hline
\end{tabular}

Percent of targeted change achieved 5 (continued) 
Figure 15-1. Progress Toward Target Attainment for Focus Area 15: Injury and Violence Prevention (continued)

Moved away

from target ${ }^{1}$

Moved toward

target

15-17. Nonfatal motor vehicle crash-related injuries on public roads (per 100,000 population)

15-18. Nonfatal pedestrian injuries on public roads (per 100,000 population)

15-19. Safety belt use

15-20. Child restraint use ( $\leq 7$ years)

15-21. Motorcycle helmet use

15-22. Graduated driver licensing laws (no. States and D.C.)

15-24. Bicycle helmet laws for riders $<15$ years (no. States and D.C.)

15-25. Residential fire deaths (age adjusted, per 100,000 population)

Met or exceeded

target

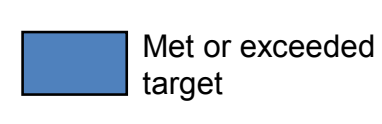$$
\begin{array}{|}
\hline \\
\hline \\
\hline
\end{array}
$$
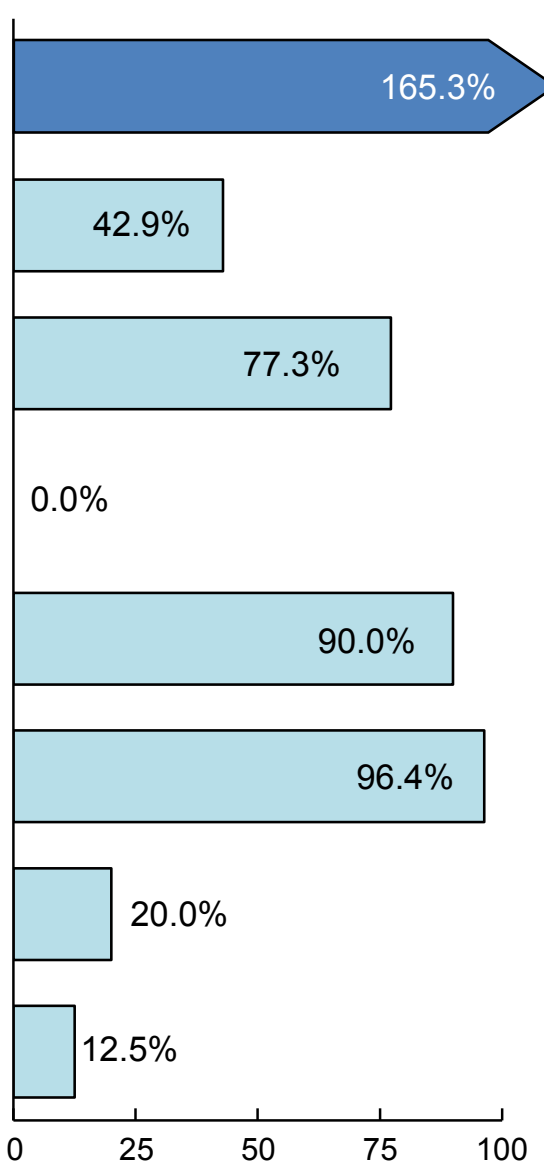

\begin{tabular}{|c|c|c|c|c|c|}
\hline \multirow{2}{*}{$\begin{array}{l}2010 \\
\text { Target }\end{array}$} & \multirow{2}{*}{$\begin{array}{c}\text { Baseline } \\
\text { (Year) }\end{array}$} & \multirow{2}{*}{$\begin{array}{l}\text { Final } \\
\text { (Year) }\end{array}$} & \multicolumn{3}{|c|}{ Baseline vs. Final } \\
\hline & & & Difference ${ }^{2}$ & $\begin{array}{l}\text { Statistically } \\
\text { Significant }^{3}\end{array}$ & $\begin{array}{l}\text { Percent } \\
\text { Change }^{4}\end{array}$ \\
\hline 933 & $\begin{array}{l}1,181 \\
(1998)\end{array}$ & $\begin{array}{c}771 \\
(2008)\end{array}$ & -410 & Yes & $-34.7 \%$ \\
\hline 19 & $\begin{array}{c}26 \\
(1998)\end{array}$ & $\begin{array}{c}23 \\
(2008)\end{array}$ & -3 & No & $-11.5 \%$ \\
\hline $89 \%$ & $\begin{array}{c}67 \% \\
(1999)\end{array}$ & $\begin{array}{c}84 \% \\
(2009)\end{array}$ & 17 & Yes & $25.4 \%$ \\
\hline $100 \%$ & $\begin{array}{c}88 \% \\
(2002)\end{array}$ & $\begin{array}{c}88 \% \\
(2009)\end{array}$ & 0 & No & $0.0 \%$ \\
\hline $68 \%$ & $\begin{array}{c}58 \% \\
(2002)\end{array}$ & $\begin{array}{c}67 \% \\
(2009)\end{array}$ & 9 & No & $15.5 \%$ \\
\hline 51 & $\begin{array}{c}23 \\
(1999)\end{array}$ & $\begin{array}{c}50 \\
(2009)\end{array}$ & 27 & $\begin{array}{c}\text { Not } \\
\text { tested }\end{array}$ & $117.4 \%$ \\
\hline 51 & $\begin{array}{c}11 \\
(1999)\end{array}$ & $\begin{array}{c}19 \\
(2009)\end{array}$ & 8 & $\begin{array}{c}\text { Not } \\
\text { tested }\end{array}$ & $72.7 \%$ \\
\hline 0.2 & $\begin{array}{c}1.0 \\
(1999)\end{array}$ & $\begin{array}{c}0.9 \\
(2007)\end{array}$ & -0.1 & Yes & $-10.0 \%$ \\
\hline
\end{tabular}

Percent of targeted change achieved ${ }^{5}$ (continued)

Page 15-18 
Figure 15-1. Progress Toward Target Attainment for Focus Area 15: Injury and Violence Prevention (continued)

Moved away

from target ${ }^{1}$

Moved toward

target

Met or exceeded

target

15-26. Functional smoke alarms in residences

a. Persons living in residences with alarms on every floor (age adjusted)

b. Proportion of residences with alarms on every floor

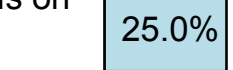

15-27. Deaths from unintentional falls (age adjusted, per 100,000 population)

15-28. Hospitalizations for hip fractures (age adjusted, per 100,000 population, $65+$ years)

a. Females

b. Males

15-29. Unintentional drownings (age adjusted per 100,000 standard population)

15-30. Emergency department visits for dog bite injuries (age adjusted, per 100,000 population)

15-31. Schools requiring students to wear appropriate protective gear

a. Physical education
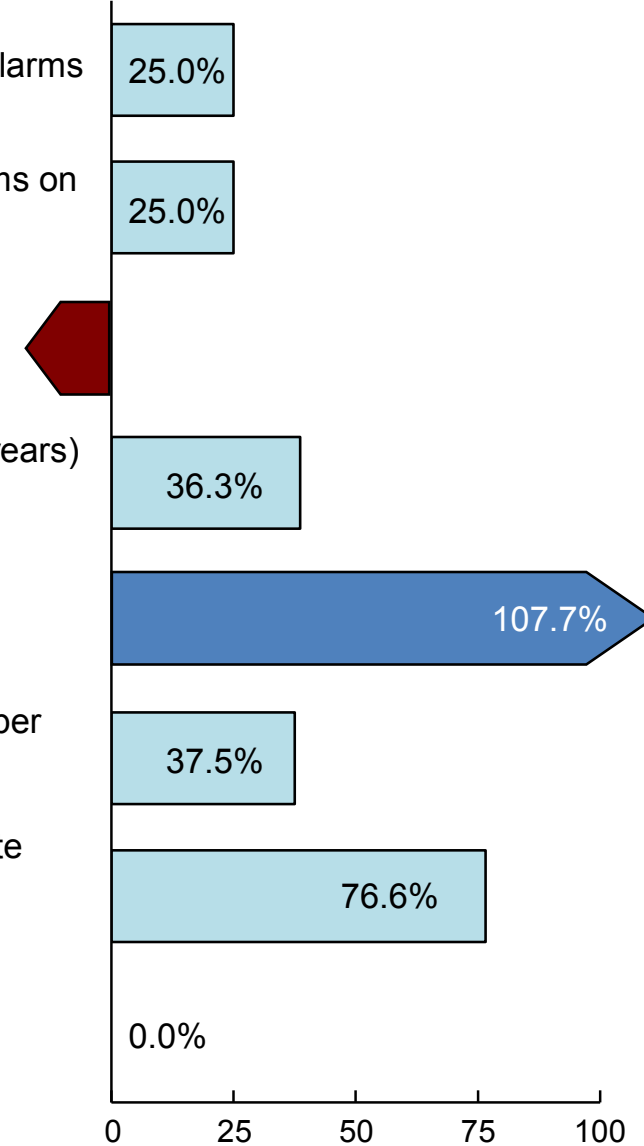

\begin{tabular}{|c|c|c|c|c|c|}
\hline \multirow{2}{*}{$\begin{array}{l}2010 \\
\text { Target }\end{array}$} & \multirow{2}{*}{$\begin{array}{c}\text { Baseline } \\
\text { (Year) }\end{array}$} & \multirow{2}{*}{$\begin{array}{l}\text { Final } \\
\text { (Year) }\end{array}$} & \multicolumn{3}{|c|}{ Baseline vs. Final } \\
\hline & & & Difference $^{2}$ & $\begin{array}{l}\text { Statistically } \\
\text { Significant }^{3}\end{array}$ & $\begin{array}{l}\text { Percent } \\
\text { Change }^{4}\end{array}$ \\
\hline $100 \%$ & $\begin{array}{c}88 \% \\
(1998)\end{array}$ & $\begin{array}{c}91 \% \\
(2003)\end{array}$ & 3 & Yes & $3.4 \%$ \\
\hline $100 \%$ & $\begin{array}{c}88 \% \\
(1998)\end{array}$ & $\begin{array}{c}91 \% \\
(2003)\end{array}$ & 3 & Yes & $3.4 \%$ \\
\hline 3.3 & $\begin{array}{c}4.8 \\
(1999)\end{array}$ & $\begin{array}{c}7.0 \\
(2007)\end{array}$ & 2.2 & Yes & $45.8 \%$ \\
\hline 416.0 & $\begin{array}{l}1,055.8 \\
(1998)\end{array}$ & $\begin{array}{l}823.5 \\
(2007)\end{array}$ & -232.3 & Yes & $-22.0 \%$ \\
\hline 474.0 & $\begin{array}{l}592.7 \\
(1998)\end{array}$ & $\begin{array}{l}464.9 \\
(2007)\end{array}$ & -127.8 & No & $-21.6 \%$ \\
\hline 0.7 & $\begin{array}{c}1.5 \\
(1999)\end{array}$ & $\begin{array}{c}1.2 \\
(2007)\end{array}$ & -0.3 & Yes & $-20.0 \%$ \\
\hline 113.0 & $\begin{array}{l}150.2 \\
(1997)\end{array}$ & $\begin{array}{l}121.7 \\
(2007)\end{array}$ & -28.5 & No & $-19.0 \%$ \\
\hline $85 \%$ & $\begin{array}{c}77 \% \\
(2000)\end{array}$ & $\begin{array}{c}77 \% \\
(2006)\end{array}$ & 0 & No & $0.0 \%$ \\
\hline
\end{tabular}

Percent of targeted change achieved 5

(continued) 
Figure 15-1. Progress Toward Target Attainment for Focus Area 15: Injury and Violence Prevention (continued)

Moved away

from target ${ }^{1}$

Moved toward

target

Met or exceeded

target

15-31. Schools requiring students to wear appropriate protective gear

b. Interscholastic sports

c. Intramural activities or physical activity clubs

15-32. Homicides (age adjusted, per 100,000 population)

15-33a. Maltreatment of children (per 1,000 population, $<18$ years)

15-33b. Child maltreatment fatalities (per 100,000 population, $<18$ years)

15-34. Physical assault by intimate partners (per 1,000 population, $12+$ years)

15-35. Rape or attempted rape (per 1,000 population, $12+$ years)

15-36. Sexual assault other than rape (per 1,000 population, $12+$ years)

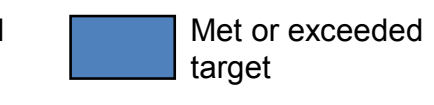

\begin{tabular}{|c|c|c|c|c|c|}
\hline \multirow{2}{*}{$\begin{array}{l}2010 \\
\text { Target }\end{array}$} & \multirow{2}{*}{$\begin{array}{c}\text { Baseline } \\
\text { (Year) }\end{array}$} & \multirow{2}{*}{$\begin{array}{l}\text { Final } \\
\text { (Year) }\end{array}$} & \multicolumn{3}{|c|}{ Baseline vs. Final } \\
\hline & & & Difference ${ }^{2}$ & $\begin{array}{l}\text { Statistically } \\
\text { Significant }^{3}\end{array}$ & $\begin{array}{l}\text { Percent } \\
\text { Change }^{4}\end{array}$ \\
\hline $100 \%$ & $\begin{array}{c}98 \% \\
(2000)\end{array}$ & $\begin{array}{c}94 \% \\
(2006)\end{array}$ & -4 & Yes & $-4.1 \%$ \\
\hline $97 \%$ & $\begin{array}{c}88 \% \\
(2000)\end{array}$ & $\begin{array}{c}86 \% \\
(2006)\end{array}$ & -2 & No & $-2.3 \%$ \\
\hline 2.8 & $\begin{array}{c}6.0 \\
(1999)\end{array}$ & $\begin{array}{c}6.1 \\
(2007)\end{array}$ & 0.1 & No & $1.7 \%$ \\
\hline 10.2 & $\begin{array}{c}12.7 \\
(1998)\end{array}$ & $\begin{array}{c}10.1 \\
(2009)\end{array}$ & -2.6 & $\begin{array}{c}\text { Not } \\
\text { tested }\end{array}$ & $-20.5 \%$ \\
\hline 1.5 & $\begin{array}{c}1.7 \\
(1998)\end{array}$ & $\begin{array}{c}2.4 \\
(2009)\end{array}$ & 0.7 & $\begin{array}{c}\text { Not } \\
\text { tested }\end{array}$ & $41.2 \%$ \\
\hline 2.7 & $\begin{array}{c}3.6 \\
(1998)\end{array}$ & $\begin{array}{c}2.3 \\
(2009)\end{array}$ & -1.3 & Yes & $-36.1 \%$ \\
\hline 0.8 & $\begin{array}{c}0.9 \\
(1998)\end{array}$ & $\begin{array}{c}0.3 \\
(2009)\end{array}$ & -0.6 & Yes & $-66.7 \%$ \\
\hline 0.4 & $\begin{array}{c}0.6 \\
(1998)\end{array}$ & $\begin{array}{c}0.2 \\
(2009)\end{array}$ & -0.4 & Yes & $-66.7 \%$ \\
\hline
\end{tabular}

Percent of targeted change achieved ${ }^{5}$

(continued) 
Figure 15-1. Progress Toward Target Attainment for Focus Area 15: Injury and Violence Prevention (continued)

Moved away

from target ${ }^{1}$

Moved toward

target

Met or exceeded

target

15-37. Physical assaults (per 1,000 population, 12+ years)

15-38. Physical fighting among students (grades 9-12)

15-39. Weapon carrying by students on school property (grades 9-12)

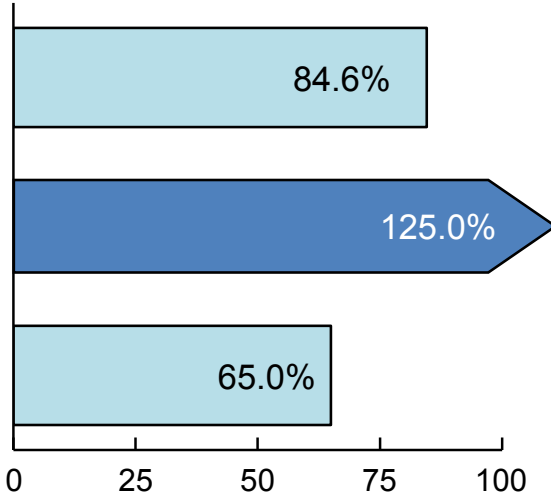

\begin{tabular}{|c|c|c|c|c|c|}
\hline \multirow{2}{*}{$\begin{array}{c}2010 \\
\text { Target }\end{array}$} & \multirow{2}{*}{$\begin{array}{c}\text { Baseline } \\
(\text { Year })\end{array}$} & \multirow{2}{*}{$\begin{array}{c}\text { Final } \\
(\text { Year })\end{array}$} & \multicolumn{3}{|c|}{ Baseline vs. Final } \\
\cline { 4 - 6 } & Difference & & $\begin{array}{c}\text { Statistically } \\
\text { Significant }^{3}\end{array}$ & $\begin{array}{c}\text { Percent } \\
\text { Change }^{4}\end{array}$ \\
\hline 13.6 & $\begin{array}{c}31.1 \\
(1998)\end{array}$ & $\begin{array}{c}16.3 \\
(2008)\end{array}$ & -14.8 & Yes & $-47.6 \%$ \\
\hline $32 \%$ & $\begin{array}{c}36 \% \\
(1999)\end{array}$ & $\begin{array}{c}31 \% \\
(2009)\end{array}$ & -5 & Yes & $-13.9 \%$ \\
\hline $4.9 \%$ & $\begin{array}{c}6.9 \% \\
(1999)\end{array}$ & $\begin{array}{c}5.6 \% \\
(2009)\end{array}$ & -1.3 & No & $-18.8 \%$ \\
\hline
\end{tabular}

Percent of targeted change achieved ${ }^{5}$

\section{NOTES}

See the Reader's Guide for more information on how to read this figure. See DATA2010 at http://wonder.cdc.gov/data2010 for all Healthy People 2010 tracking data. Tracking data are not available for objectives 15-4, 15-23a, and 15-23b.

\section{FOOTNOTES}

${ }^{1}$ Movement away from target is not quantified using the percent of targeted change achieved. See Technical Appendix for more information.

${ }^{2}$ Difference $=$ Final value - Baseline value. Differences between percents (\%) are measured in percentage points.

${ }^{3}$ When estimates of variability are available, the statistical significance of the difference between the final value and the baseline value is assessed at the 0.05 level. See Technical Appendix for more information.

${ }^{4}$ Percent change $=\frac{\text { Final value }- \text { Baseline value }}{\text { Baseline value }} \times 100$

${ }^{5}$ Percent of targeted change achieved $=\frac{\text { Final value }- \text { Baseline value }}{\text { Healthy People } 2010 \text { target }- \text { Baseline value }} \times 100$. 
Figure 15-1. Progress Toward Target Attainment for Focus Area 15: Injury and Violence Prevention (continued)

\section{DATA SOURCES}

15-1-15-2. National Hospital Discharge Survey (NHDS), CDC, NCHS.

15-3. National Vital Statistics System-Mortality (NVSS-M), CDC, NCHS.

15-5. National Electronic Injury Surveillance System (NEISS), Consumer Product Safety Commission (CPSC).

15-6. Michigan Public Health Institute; National Vital Statistics System—Mortality (NVSS-M), CDC, NCHS.

15-7. National Hospital Ambulatory Medical Care Survey (NHAMCS), CDC, NCHS.

15-8-9. National Vital Statistics System-Mortality (NVSS-M), CDC, NCHS.

15-10-15-11. External Cause of Injury Survey, American Public Health Association (APHA).

15-12. National Hospital Ambulatory Medical Care Survey (NHAMCS), CDC, NCHS.

15-13. National Vital Statistics System-Mortality (NVSS-M), CDC, NCHS.

15-14. National Electronic Injury Surveillance System-All Injury Program (NEISS-AIP): CDC, NCIPC; Consumer Product Safety Commission (CPSC).

15-15a. National Vital Statistics System-Mortality (NVSS-M), CDC, NCHS.

15-15b. Fatality Analysis Reporting System (FARS), Department of Transportation (DOT).

15-16. Fatality Analysis Reporting System (FARS), Department of Transportation (DOT).

15-17-15-18. General Estimates System (GES), Department of Transportation (DOT).

15-19-15-21. National Occupant Protection Use Survey (NOPUS), Department of Transportation (DOT).

15-22. U.S. Licensing Systems for Young Drivers, Insurance Institute for Highway Safety.

15-24. Bicycle Helmet Safety Institute.

15-25. National Vital Statistics System-Mortality (NVSS-M), CDC, NCHS.

15-26a-b. National Health Interview Survey (NHIS), CDC, NCHS.

15-27. National Vital Statistics System-Mortality (NVSS-M), CDC, NCHS.

15-28a-b. National Hospital Discharge Survey (NHDS), CDC, NCHS.

15-29. National Vital Statistics System-Mortality (NVSS-M), CDC, NCHS.

15-30. National Hospital Ambulatory Medical Care Survey (NHAMCS), CDC, NCHS

15-31a-c. School Health Policies and Programs Study (SHPPS), CDC, NCCDPHP.

15-32. National Vital Statistics System-Mortality (NVSS-M), CDC, NCHS.

15-33a-b. National Child Abuse and Neglect Data System (NCANDS), ACF.

15-34-15-37. National Crime Victimization Survey (NCVS), Department of Justice (DOJ), Bureau of Justice Statistics (BJS).

15-38-15-39. Youth Risk Behavior Surveillance System (YRBSS), CDC, NCCDPHP. 
Figure 15-2. Health Disparities Table for Focus Area 15: Injury and Violence

Disparities from the best group rate for each characteristic at the most recent data point and changes in disparity from the baseline to the most recent data point.

Population-based objectives

15-1. Nonfatal traumatic brain injury hospitalizations (age adjusted, per 100,000 population) $(1998,2007)^{\star}$

15-2. Nonfatal spinal cord injury hospitalizations (age adjusted, per 100,000 population) $(1998,2007)^{*}$

15-3. Firearm-related deaths (age adjusted, per 100,000 population) $(1999,2007)^{1 *}$

15-4. Persons in homes with impoperly stored firearms (loaded and unlocked) (age adjusted, 18+ years) (1998)*

15-5. Nonfatal firearm-related injuries (per 100,000 population) $(1997,2007)^{*}$

15-7. Emergency department visits for nonfatal poisonings (age adjusted, per 100,000 population) $(1997,2007)^{\star}$

15-8. Deaths from poisoning (age adjusted, per 100,000 population) $(1999,2007)^{1 *}$

15-9. Deaths from suffocation (age adjusted, per 100,000 population) $(1999,2007)^{1 *}$

15-12. Initial emergency department visits for injuries (age adjusted, per 1,000 population) $(2001,2007)^{*}$

15-13. Deaths from unintentional injuries (age adjusted, per $100,000$ population $)(1999,2007)^{1 *}$

15-14. Nonfatal unintentional injuries (age adjusted, per 100,000 population) $(2000,2008)^{*}$

15-15a. Deaths from motor vehicle crashes (age adjusted, per 100,000 population) $(1999,2007)^{1 *}$

15-16. Pedestrian deaths on public roads (per 100,000 population) $(1998,2008)^{2} \dagger$

15-17. Nonfatal motor vehicle crash-related injuries on public roads (per 100,000 population) $(1998,2008)^{*}$

15-18. Nonfatal pedestrian injuries on public roads (per 100,000 population) $(1998,2008)^{\star}$

15-25. Residential fire deaths (age adjusted, per 100,000 population) $(1999,2007)^{1 *}$

15-26a. Persons living in residences with functional smoke alarms on every floor (age adjusted) $(1998,2003)^{3 *}$

15-27. Deaths from unintentional falls (age adjusted, per 100,000 population) $(1999,2007)^{1 *}$

15-28a. Hospitalizations for hip fractures-Females (age adjusted, per 100,000 population, $65+$ years $)(1998,2007)^{*}$

b. Hospitalizations for hip fractures-Males (age adjusted, per 100,000 population, $65+$ years $)(1998,2007)^{\star}$

15-29. Unintentional drownings (age adjusted, per 100,000 population) $(1999,2007)^{1 *}$

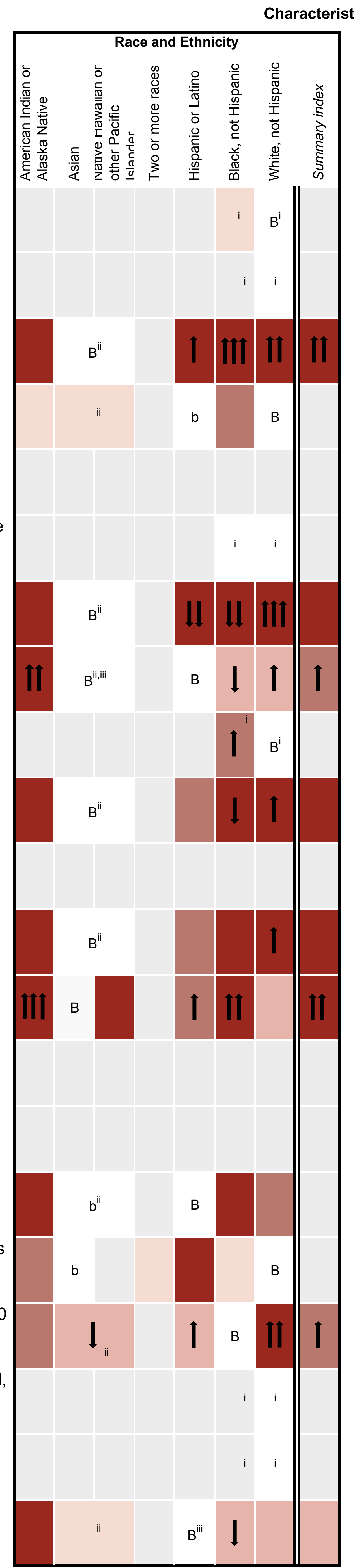

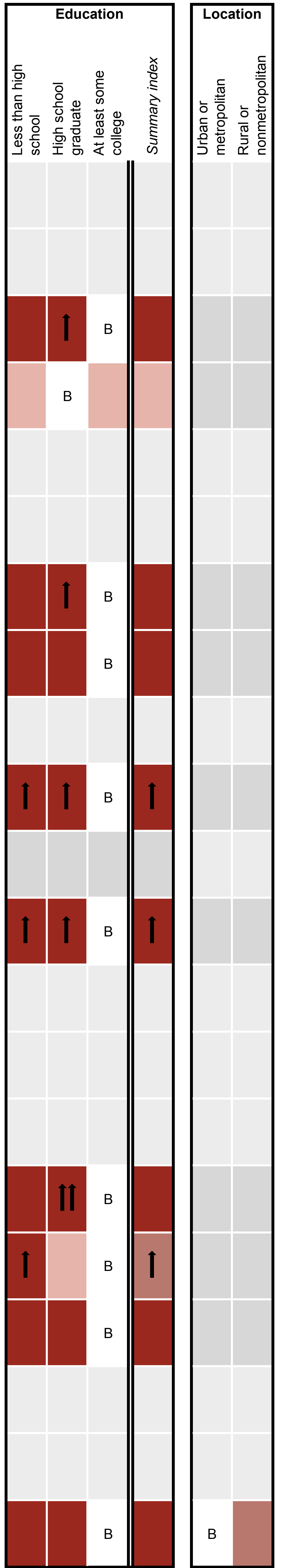

(continued) 
Figure 15-2. Health Disparities Table for Focus Area 15: Injury and Violence Prevention (continued)

Population-based objectives

15-30. Emergency department visits for dog bite injuries (age adjusted, per 100,000 population) $(1997,2007)^{\star}$

15-32. Homicides (age adjusted, per 100,000 population) (1999, $2007)^{1 *}$

15-33a. Maltreatment of children (per 1,000 population, $<18$ years) (1998, 2009)†

15-33b. Child maltreatment fatalities (per 100,000 population, $<18$ years) $(1998,2009) \dagger$

15-34. Physical assault by intimate partners (per 1,000 population, $12+$ years $)(1998,2009)^{4 *}$

15-35. Rape or attempted rape (per 1,000 population, $12+$ years) $(1998,2009)^{4 *}$

15-36. Sexual assault other than rape (per 1,000 population, 12+ years) $(1998,2009)^{4,5 *}$

15-37. Physical assaults (per 1,000 population, 12+ years) (1998, $2008)^{4 *}$

15-38. Physical fighting among students (grades 9-12) (1999, 2009)*

15-39. Weapon carrying by students on school property (grades 9-12) $(1999,2009)^{*}$

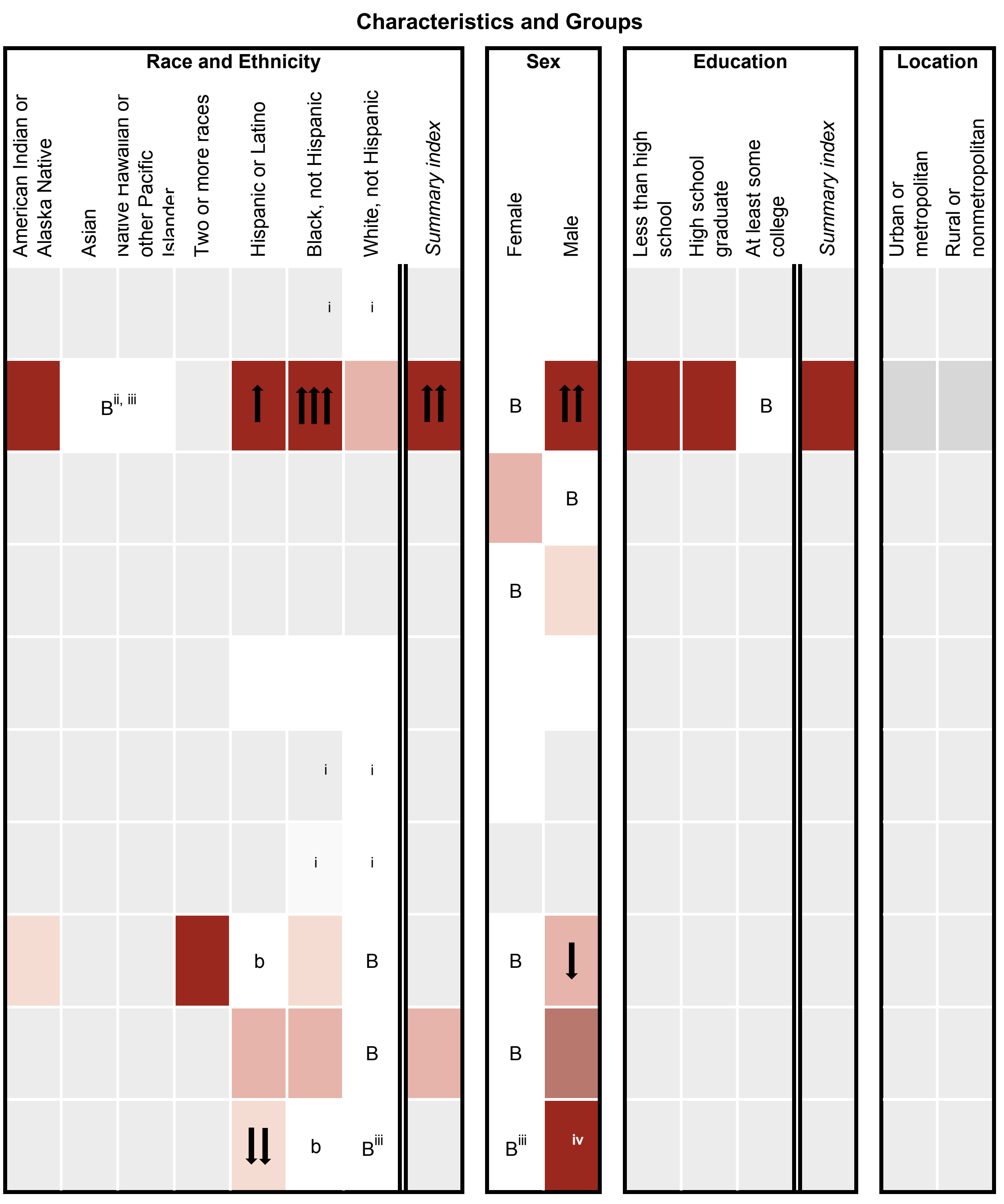

(continued) 
See DATA2010 at http://wonder.cdc.gov/data2010 for all Healthy People 2010 tracking data. Disparity data are either unavailable or not applicable for objectives 15 . $6,15-10,15-11,15-15 b, 15-19$ through 15-22, 15-23a and b, 15-24, 15-26b, and 15-31a through $c$.

Years in parentheses represent the baseline and most recent data years (if available).

Disparity from the best group rate is defined as the percent difference between the best group rate and each of the other group rates for a characteristic (e.g., race and ethnicity). The summary index is the average of these percent differences for a characteristic. Change in disparity is estimated by subtracting the disparity at baseline from the disparity at the most recent data point. Change in the summary index is estimated by subtracting the summary index at baseline from the summary index at the most recent data point. See Technical Appendix for more information.

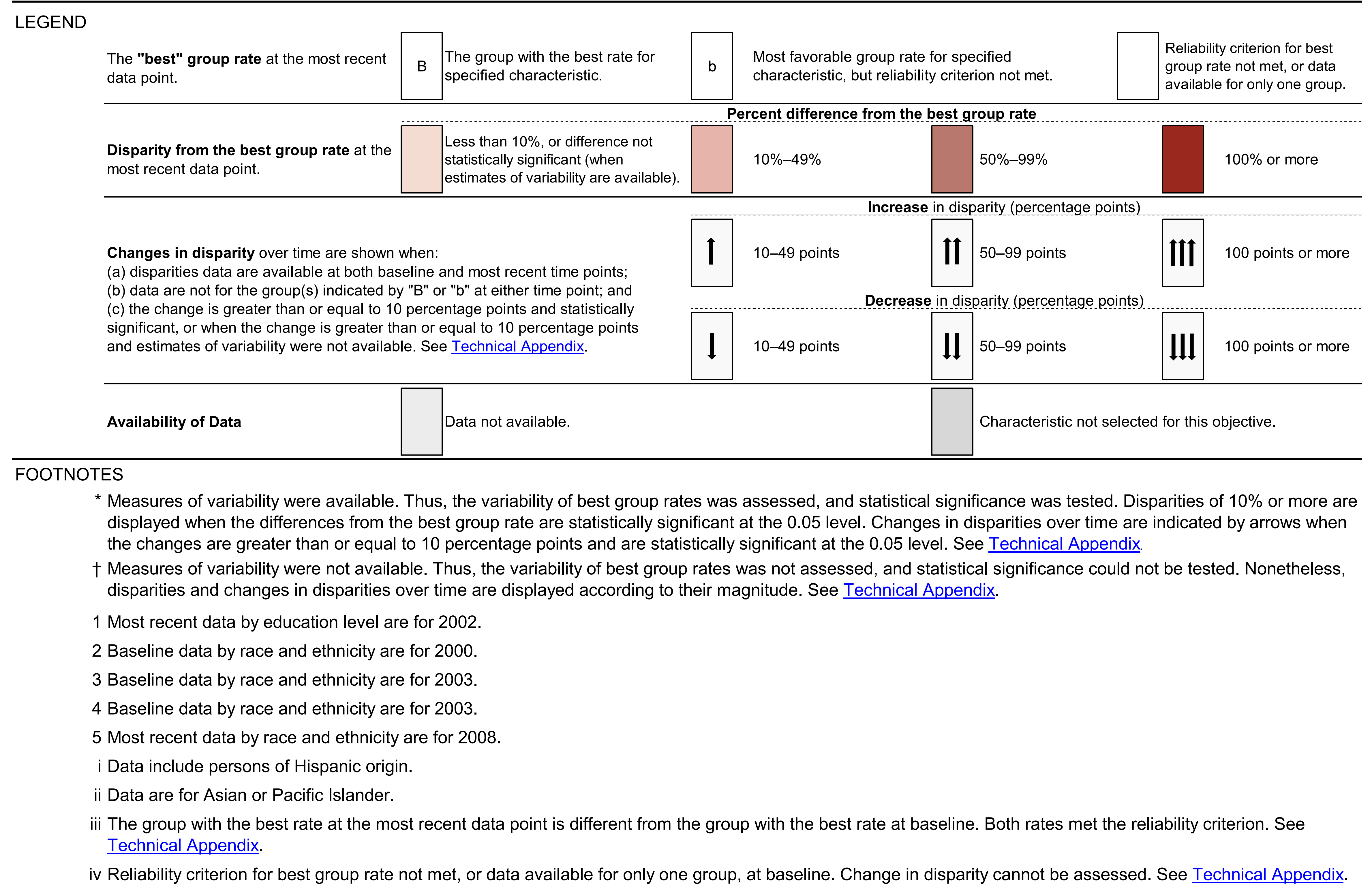

\section{DATA SOURCES}

15-1-15-2. National Hospital Discharge Survey (NHDS), CDC, NCHS.

15-3. National Vital Statistics System-Mortality (NVSS-M), CDC, NCHS.

15-4. National Health Interview Survey (NHIS), CDC, NCHS.

15-5. National Electronic Injury Surveillance System (NEISS), Consumer Product Safety Commission (CPSC).

15-7. National Hospital Ambulatory Medical Care Survey (NHAMCS), CDC, NCHS.

15-8-15-9. National Vital Statistics System-Mortality (NVSS-M), CDC, NCHS.

15-12. National Hospital Ambulatory Medical Care Survey (NHAMCS), CDC, NCHS.

15-13. National Vital Statistics System-Mortality (NVSS-M), CDC, NCHS.

15-14. National Electronic Injury Surveillance System-All Injury Program (NEISS-AIP): CDC, NCIPC; Consumer Product Safety Commission (CPSC).

15-15a. National Vital Statistics System-Mortality (NVSS-M), CDC, NCHS.

15-16. Fatality Analysis Reporting System (FARS), Department of Transportation (DOT).

15-17-15-18. General Estimates System (GES), Department of Transportation (DOT).

15-25. National Vital Statistics System-Mortality (NVSS-M), CDC, NCHS.

15-26a. National Health Interview Survey (NHIS), CDC, NCHS.

15-27. National Vital Statistics System-Mortality (NVSS-M), CDC, NCHS.

15-28a-b. National Hospital Discharge Survey (NHDS), CDC, NCHS.

15-29. National Vital Statistics System-Mortality (NVSS-M), CDC, NCHS.

15-30. National Hospital Ambulatory Medical Care Survey (NHAMCS), CDC, NCHS.

15-32. National Vital Statistics System-Mortality (NVSS-M), CDC, NCHS.

15-33a-b. National Child Abuse and Neglect Data System (NCANDS), Administration on Children, Youth and Families, Administration for Children and Families (ACF), Children's Bureau. 15-34-15-37. National Crime Victimization Survey (NCVS), Department of Justice (DOJ), Bureau of Justice Statistics (BJS).

15-38-15-39. Youth Risk Behavior Surveillance System (YRBSS), CDC, NCCDPHP. 
Figure 15-3. Deaths from Unintentional Injuries 2005-07

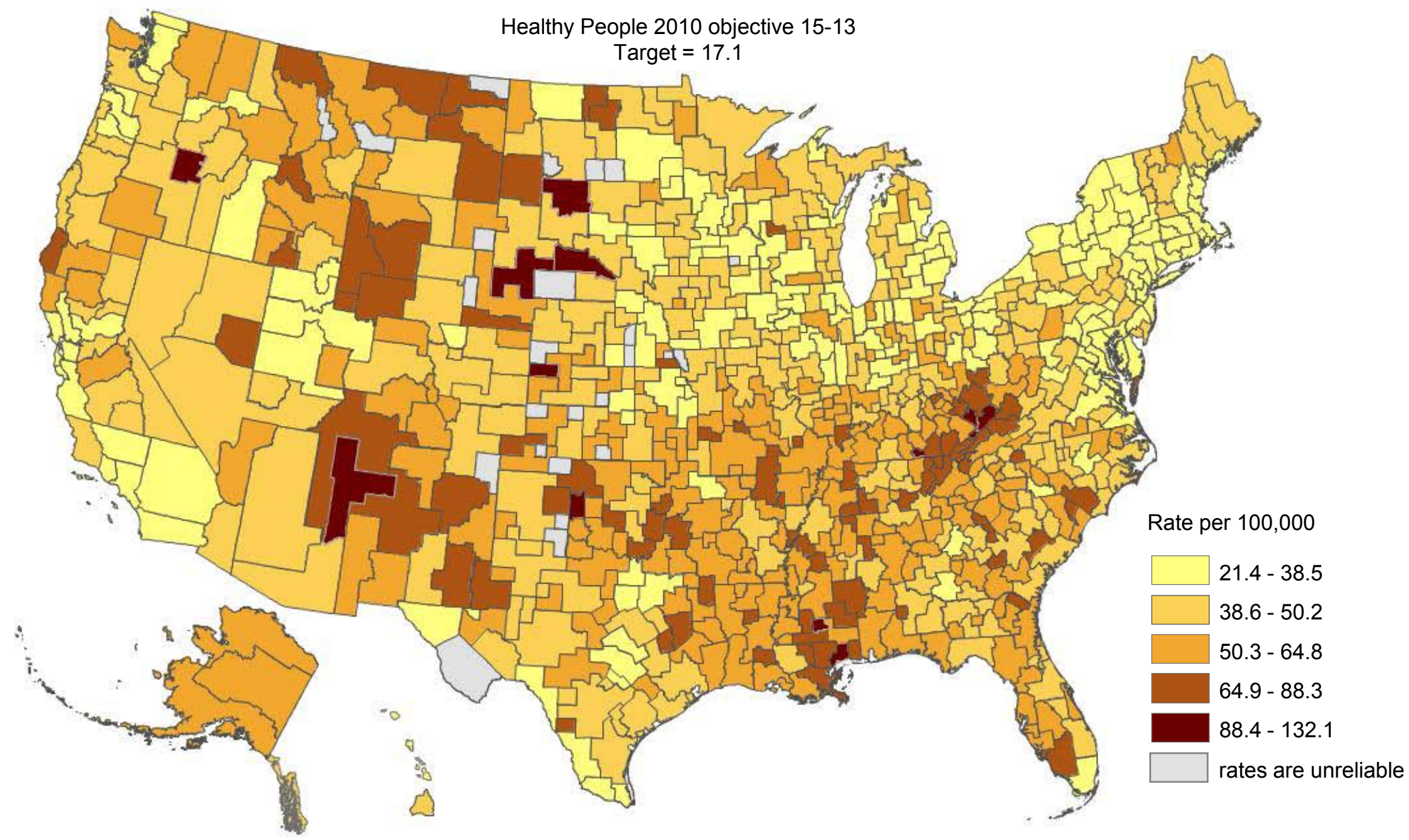

no health service areas met target

NOTES: Data are for ICD-10 codes V01-X59, Y85-Y86 reported as underlying cause. Rates are per 100,000 U. S. Population age-adjusted to the 2000 standard population. Rates are displayed by a Jenks classification for U.S. health service area. SOURCE: National Vital Statistics System - Mortality, CDC, NCHS. 
Figure 15-4. Motor Vehicle Deaths 2005-07

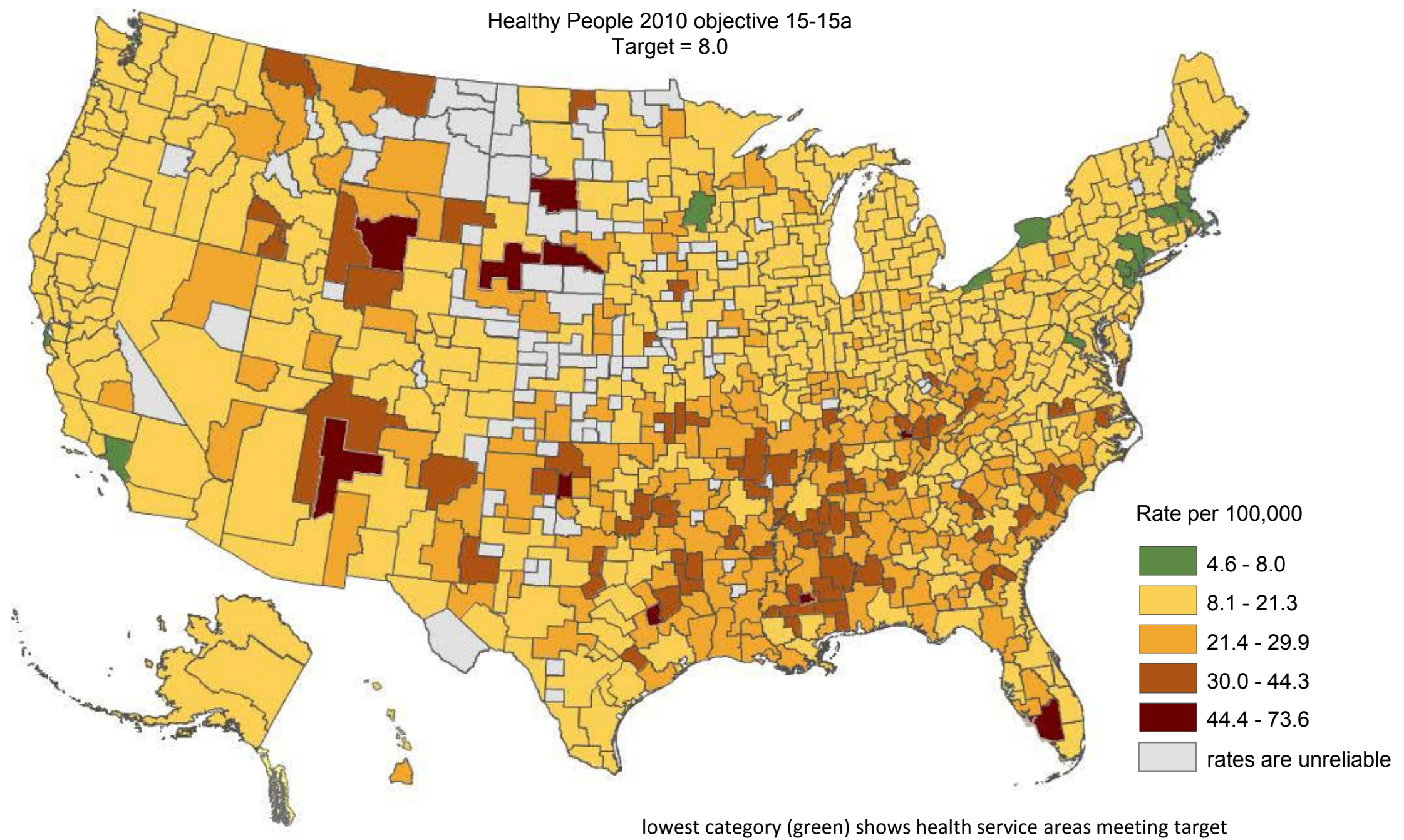

NOTES: Data are for ICD-10 codes V02-V04 (.1- .9), V09.2, V12-V14 (.3-.9), V19 (.4-.6), V20-V28 (.3-.9), V29 (.4-.9), V30-V39 (.4-.9), V40-V49 (.4-.9), V50-V59 (.4-.9), V60-V69 (.4-.9), V70-V79 (.4-.9), V80 (.3-.5), V81.1, V82.1, V83-V86 (.0-.3), V87 (.0-.8), V89.2 reported as underlying cause.

Rates are per 100,000 U. S. Population age-adjusted to the 2000 standard population. Rates are displayed by modified Jenks classification for U. S. health service area.

SOURCE: National Vital Statistics System - Mortality, CDC, NCHS. 


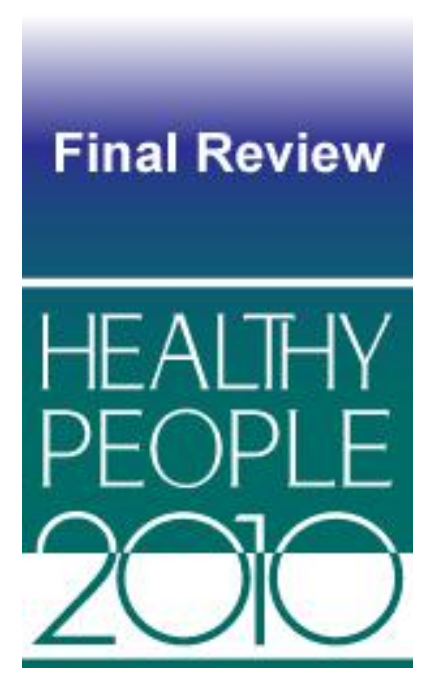

\section{Maternal, Infant, and Child Health}

\section{Lead Agency}

Centers for Disease Control and Prevention

Health Resources and Services Administration

\section{Contents}

Goal $16-2$

Highlights... 16-2

Summary of Progress. 16-6

Transition to Healthy People 2020. 16-7

Data Considerations $16-11$

References and Notes 16-12

Comprehensive Summary of Objectives $16-14$

Progress Chart. 16-17

Health Disparities Table 16-24

Low Birth Weight (LBW), Infants, 2006-08-Map 16-27

Preterm Live Births-Total (<37 Completed Weeks Gestation), 2006-08-Map $16-28$ 


\section{Goal: Improve the health and well-being of women, infants, children, and families}

The objectives in this chapter address a wide range of conditions related to the health and quality of life for mothers, infants, and children. These include infant and child deaths, congenital anomalies, pregnancy-related illness, low birth weight and preterm deliveries, prenatal care, breastfeeding, newborn screenings, and availability of medical homes for children with special health care needs.

All Healthy People tracking data quoted in this chapter, along with technical information and operational definitions for each objective, can be found in the Healthy People 2010 database, DATA2010, available from http://wonder.cdc.gov/data2010/.

More information about this focus area can be found in the following publications:

- Healthy People 2010: Understanding and Improving Health, available from http://www.healthypeople.gov/2010/Document/tableofcontents.htm\#under.

- Healthy People 2010 Midcourse Review, available from http://www.healthypeople.gov/2010/data/midcourse/html/default.htm\#FocusAreas.

\section{Highlights}

- Substantial progress was achieved in objectives for this Focus Area during the past decade [1]. Two-thirds (66.7\%) of the Maternal, Infant, and Child Health objectives with data to measure progress moved toward or achieved their Healthy People 2010 targets (Figure 161). However, health disparities of $100 \%$ or more among racial and ethnic population groups, as well as by sex and education level also were observed (Figure 16-2) [2].

- The fetal, perinatal, infant, neonatal, and postneonatal mortality rates (objectives 16-1a through 16-1e) declined over the decade, moving toward their 2010 targets. Between 1997 and 2005, the fetal mortality rate (deaths to fetuses of 20 weeks or more gestation per 1,000 live births and fetal deaths, objective 16-1a) decreased by $8.8 \%$, from 6.8 to 6.2 , moving toward the 2010 target of 4.1 . The infant mortality rate (deaths to infants aged less than one year per 1,000 live births, objective 16-1c) declined by $6.9 \%$ between 1998 and 2006, from 7.2 to 6.7, moving toward the 2010 target of 4.5 .

- Among racial and ethnic groups, the Asian or Pacific Islander population had the lowest (best) death rates for all of these objectives (16-1a through e). Disparities of $100 \%$ or more in all these objectives were observed for the black non-Hispanic population compared to the group with the best rate. For example:

- In 2005, the fetal mortality rate for the Asian or Pacific Islander population (objective 16-1a) was 4.8 fetal deaths per 1,000 live births plus fetal deaths, whereas the rate for the black non-Hispanic population was 11.1, almost two and a half times that for the Asian or Pacific Islander population [2].

- Similarly, the infant mortality rate for the Asian or Pacific Islander population (objective 16-1c) was 4.5 per 1,000 live births in 2006, whereas the rate for the black non-Hispanic population was 13.4, about three times that for the Asian or Pacific Islander population [2]. 
- The American Indian or Alaska Native population also experienced large disparities in infant (objective 16-1c) and postneonatal mortality rates (objective 16-1e; deaths to infants aged 28 days to less than one year per 1,000 live births). In 2006, the American Indian or Alaska Native population had an infant mortality rate of 8.3 , almost twice the Asian or Pacific Islander population rate of 4.5. The American Indian or Alaska Native population also had a postneonatal mortality rate of 4.0 per 1,000 live births, almost three times the Asian or Pacific Islander population rate of 1.4. The postneonatal mortality rate for the black non-Hispanic population (4.4 per 1,000 live births) was more than three times that for the Asian or Pacific Islander population [2].

- Among education groups, infants of mothers aged 20 years and over with at least some college education had the lowest (best) postneonatal mortality rate (objective 16-1e), 1.4 postneonatal deaths per 1,000 live births in 2002. Infants whose mothers had less than a high school education had a rate of 3.3 postneonatal deaths per 1,000 live births in 2002, almost two and a half times the best group rate [2].

- Deaths from sudden infant death syndrome (SIDS; objective 16-1h) among infants less than one year of age declined by $17.9 \%$ between 1999 and 2006, from 0.67 to 0.55 deaths per 1,000 live births, moving toward the 2010 target of 0.23 . The proportion of infants aged less than eight months who were placed to sleep on their backs (objective 16-13) increased by $105.6 \%$ between 1996 and 2009 , from $36 \%$ to $74 \%$, exceeding the target of $70 \%$. Placing infants to sleep on their backs is considered one of the best ways to reduce risk of SIDS [3].

- Among racial and ethnic groups, the Hispanic or Latino population had the lowest (best) SIDS death rate, 0.27 deaths per 1,000 live births in 2006. In 2006, the white non-Hispanic population had a SIDS death rate of 0.56 per 1,000 live births, more than twice the best group rate (that for the Hispanic or Latino population); the black non-Hispanic population had a rate of 1.05 per 1,000 live births, almost four times the best rate; and the rate for the American Indian or Alaska Native population was 1.19 per 1,000 live births, almost four and a half times the best group rate [2].

- Among education groups, infants of mothers with at least some college education had the lowest (best) rate of SIDS death in 2002, 0.27 per 1,000 live births. In 2002, the rate for infants whose mothers were high school graduates was 0.69 , about two and a half times the best group rate. The rate for infants whose mothers had less than a high school education was 0.86 , more than three times the best rate [2].

- Death rates among children and adolescents (objectives 16-2a and b, and 16-3a and b) declined by $11 \%$ to $21 \%$ between 1998 and 2007, moving toward the 2010 targets. Yet the death rate for young adults aged 20-24 years (objective 16-3c) increased by $6.0 \%$, from 92.7 to 98.3 deaths per 100,000 population, moving away from the 2010 target of 41.5.

- In 2007, among racial and ethnic groups, the Asian or Pacific Islander population had the lowest (best) mortality rates for all age groups, although the rate for children aged 5-9 years (objective 16-2b) did not meet the reliability criterion for the best group rate. Therefore, the white non-Hispanic population was considered to have the best rate for the purpose of racial and ethnic disparity comparisons for this objective; see Figure 16-2 footnotes. 


\section{Mortality-Children aged 1-4 years (objective 16-2a)}

- The Asian or Pacific Islander population had the lowest (best) mortality rate among children aged 1-4 years, 21.7 deaths per 1,000 population in 2007, whereas the American Indian or Alaska Native and black non-Hispanic populations had rates of 54.9 and 43.7 per 1,000 in 2007, respectively. The rate for the American Indian or Alaska Native population was about two and a half times the best rate, while that for the black non-Hispanic population was about twice the best rate [2].

\section{Mortality-Adolescents aged 10-14 years (objective 16-3a)}

- The Asian or Pacific Islander population had the lowest (best) mortality rate among adolescents aged $10-14$ years, 12.3 deaths per 100,000 population in 2007 . The black non-Hispanic population had a rate of 24.6 per 100,000 in 2007 , twice the best rate [2].

\section{Mortality-Adolescents aged 15-19 years (objective 16-3b)}

- In 2007, the Asian or Pacific Islander population had the lowest (best) mortality rate among adolescents aged 15-19 years, 32.7 deaths per 100,000 population, whereas the American Indian or Alaska Native and black non-Hispanic populations had rates of 86.5 and 85.7 deaths per 100,000 , respectively, more than two and a half times the best rate [2].

\section{Mortality-Young adults aged 20-24 years (objective 16-3c)}

- The Asian or Pacific Islander population had the lowest (best) mortality rates among young adults aged 20-24 years, 41.6 deaths per 100,00 population in 1998 and 53.2 in 2007. The American Indian or Alaska Native population had rates of 127.6 in 1998 and 120.7 in 2007, while the black non-Hispanic population had rates of 163.4 in 1998 and 142.2 in 2007.

- In 2007, the rate for the American Indian or Alaska Native population was almost two and a half times the best rate (that for the Asian or Pacific Islander population); and the rate for the black non-Hispanic population was more than two and a half times the best rate [2].

- Between 1998 and 2007, the disparity between the American Indian or Alaska Native population and the Asian or Pacific Islander population (group with the best rate) decreased by 80 percentage points, while the disparity between the black non-Hispanic population and the Asian or Pacific Islander population decreased by 125 percentage points [4].

\section{Mortality-Females aged 15-19 and 20-24 years (objectives 16-3b and c)}

- Females aged 15-19 and 20-24 years had lower (better) death rates than males, 35.7 and 48.4 deaths per 100,000 population, respectively in 2007 . The rate for males aged 15-19 years was 86.8 per 100,000, almost two and a half times the rate for females. The rate for males aged 20-24 years was 145.2 per 100,000, three times the rate for females [2]. 
- Cesarean births to low risk women increased between 1998 and 2007. During this period the proportion of cesarean births to low risk women who had not had a previous cesarean (objective 16-9a) rose by $44.4 \%$, from $18 \%$ to $26 \%$, moving away from the 2010 target of $15 \%$. The proportion of repeat cesarean births (objective $16-9 \mathrm{~b}$ ) increased by $26.4 \%$, from $72 \%$ to $91 \%$, moving away from the 2010 target of $63 \%$.

- Between 1998 and 2007, the proportion of live births that were low birth weight (under 2,500 grams, objective 16-10a) and very low birth weight (under 1,500 grams, objective 1610b) increased by $7.9 \%$ (from $7.6 \%$ to $8.2 \%$ ) and $7.1 \%$ (from $1.4 \%$ to $1.5 \%$ ), respectively, moving away from the 2010 targets of $5.0 \%$ and $0.9 \%$.

- Among racial and ethnic groups, the Hispanic or Latino population had the lowest (best) rate of low birth weight births, 6.9\% in 2007. The black non-Hispanic population had a rate of $13.9 \%$ in 2007 , about twice the best rate. The Asian or Pacific Islander population had the lowest (best) rate of very low birth weight births, $1.1 \%$ in 2007 . The rate of very low birth weight births for the black nonHispanic population was $3.2 \%$ in 2007 , almost three times the best rate [2].

- Low birth weight birth rates varied by geographic region. In 2006-08, the proportions of low birth weight infants born in the Southeast and Mountain West were higher than the proportions of low birth weight infants born in the Northwest, Midwest, and Northeast regions of the U.S. A few geographic areas met the 2010 target of 5.0 low birth weight births per 1,000 live births (Figure 16-3).

- Between 1998 and 2007, the proportion of live births that were preterm (less than 37 completed weeks of gestation, objective $16-11 \mathrm{a}$ ) increased by $9.5 \%$, from $11.6 \%$ to $12.7 \%$, moving away from the 2010 target of $7.6 \%$.

- Preterm births varied by geographic region. In 2006-08, the proportion of preterm infants born in the Southeast was higher than the proportion of preterm infants born in the Northwest, Midwest, or Northeast regions of the U.S. (Figure 16-4).

- The proportion of non-pregnant women aged 15-44 years who consumed at least $400 \mu \mathrm{g}$ of folic acid (objective 16-16a) increased by 14.3\% between 1991-94 and 2005-06, from 21\% to $24 \%$, moving toward the 2010 target of $80 \%$. The median red blood cell (RBC) folate level (objective 16-16b) among non-pregnant women aged 15-44 years increased by $58.2 \%$ between 1988-94 and 2005-06, from 158 to $250 \mathrm{ng} / \mathrm{ml}$, exceeding the 2010 target of 220 . Between 1996 and 2007, the rate of spina bifida and other neural tube defects (objective 16-15) decreased by $20.0 \%$, from 60 to 48 new cases per 100,000 live births, moving toward the 2010 target of 30 per 100,000 .

- The proportion of mothers who breastfed their infants (objectives 16-19a through e) increased for every category, moving toward the 2010 targets. The largest increase was observed for the proportion of mothers who breastfed their infants at one year after birth (objective 16-19c). Between 2000 and 2006, the proportion increased by $43.8 \%$, from $16 \%$ to $23 \%$, moving toward the 2010 target of $25 \%$. 


\section{Summary of Progress}

- Figure 16-1 presents a quantitative assessment of progress in achieving the Healthy People 2010 objectives for Maternal, Infant, and Child Health [1]. Data to measure progress toward target attainment were available for 42 objectives. Of these:

- Three objectives (16-13, 16-14c, and 16-16b) exceeded their 2010 targets.

- Twenty-five objectives moved toward their targets. A statistically significant difference between the baseline and the final data points was observed for 19 of these objectives (16-1a through e; 16-1g and h; 16-2a and b; 16-3a and b; 16-6a and b; 16-17c; and 16-19a through e). No significant differences were observed for three objectives (16-4, 16-5a, and 16-21); and data to test the significance of the difference were unavailable for three objectives (16-8, 16-15, and 16-16a).

- Five objectives (16-1f; 16-11c; and 16-17a, b, and d) showed no change.

- Nine objectives moved away from their targets. A statistically significant difference between the baseline and final data points was observed for seven of these objectives (16-3c; 16-9a and b; 16-10a and b; and 16-11a and b). No significant differences were observed for two objectives (16-14a and $b$ ).

- Three objectives (16-12, and 16-20a and b) remained developmental [5]. Data were unavailable to measure progress for four objectives $(16-7,16-18,16-22$, and 16-23). Four objectives (16-5b and c, 16-14d, and 16-20c) were dropped during the decade [6].

- Figure 16-2 displays health disparities in Maternal, Infant, and Child Health from the best group rate for each characteristic at the most recent data point [2]. It also displays changes in disparities from baseline to the most recent data point [4].

- Thirty-three objectives had statistically significant racial and ethnic health disparities of $10 \%$ or more, and four additional objectives had racial and ethnic health disparities of $10 \%$ or more but lacked data to assess statistical significance. Of these 37 objectives, the Asian or Pacific Islander population had the only best rate for 13 objectives (16-1b through f; 16-2a; 16-3a through c; 16-10b; and 16-11a through c). The white non-Hispanic population had the only best rate for 11 objectives (16-2b, 16-4, 16-5a, 16-6a and b, 16-7, 16-13, 16-14a, 16-14c, and 16-16a and $b)$. The Asian population had the only best rate for four objectives (16-19b through e). The Hispanic or Latino population had the only best rate for three objectives (16-1h, 16-10a, and 16-19a). And the black non-Hispanic and American Indian or Alaska Native population each had the only best rate for one objective (1615 and 16-9a, respectively). The white non Hispanic and Hispanic or Latino populations both had the best rate for two objectives (16-1g and 16-18). The Asian or Pacific Islander and white non-Hispanic populations both had the best rate for one objective (16-1a). And the Asian or Pacific Islander and Hispanic or Latino populations both had the best rate for one objective (16-17c).

- Twelve objectives had statistically significant health disparities of $10 \%$ or more by sex. Females had better rates for 11 of these 12 objectives (16-1b through 1e; 16-1h; 16-2a and b; 16-3a through c; and 16-14a). Males had the better rate for the remaining objective (16-10a). 
- Twenty-three objectives had statistically significant health disparities of $10 \%$ or more by education level (16-1a through $h ; 16-4 ; 16-6 a$ and $b ; 16-10 a$ and $b ; 16-11 a$ through c; 16-16a and b; 16-17c; and 16-19a through d), and one additional objective (16-7) had a health disparity of $10 \%$ or more by education level but lacked data to assess statistical significance. Persons with at least some college education had the best rate for each of these 24 objectives.

- Persons without disabilities had the better rate for the one objective (16-16a) with statistically significant health disparities of $10 \%$ or more by disability status.

- Twenty objectives had racial and ethnic health disparities of $100 \%$ or more. Three objectives had health disparities of $100 \%$ or more by sex, and six objectives had health disparities of $100 \%$ or more by education level. Changes in disparity between the baseline and most recent points were observed for several objectives. Many of these disparities are discussed in the Highlights, above.

\section{Transition to Healthy People 2020}

For Healthy People 2020, the Maternal, Infant, and Child Health Topic Area continues to address a wide range of conditions, health behaviors, and health systems indicators that affect the health, wellness, and quality of life of women, infants, children, and families. See HealthyPeople.gov for a complete list of Healthy People 2020 topics and objectives.

The Healthy People 2020 Maternal, Infant, and Child Health objectives can be grouped into seven sections:

- Morbidity and mortality

- Pregnancy health and behaviors

- Preconception health behaviors

- Postpartum health and behaviors

- Infant care

- Disability and other impairments

- Health services.

The transition from Healthy People 2010 to Healthy People 2020 objectives is summarized below:

- The Healthy People 2020 Maternal, Infant, and Child Health Topic Area has 71 objectives, 10 of which are developmental, whereas the Healthy People 2010 Focus Area had 53 objectives, three of which were developmental [5].

- Thirty Healthy People 2010 objectives, including 13 mortality objectives (16-1a through h; 16-2a and b; and 16-3a through c), five morbidity objectives (16-5a, 16-10a and b, and 16$11 \mathrm{a}$ and $\mathrm{c}$ ), five breastfeeding objectives (16-19a through e), three obstetrical/infant care objectives (16-9a and b, and 16-22), one folic acid intake objective (16-16a), two objectives on abstinence from illicit drugs and alcohol during pregnancy (objectives 16-17a and d), and one objective on VLBW infants born at level III facilities (objective 16-8) were retained "as is" [7]. 
- One Healthy People 2010 objective (16-14a), children diagnosed with mental retardation, was archived [8].

- Four Healthy People 2010 objectives were dropped during the decade due to the lack of a national data source [6]. The dropped objectives include hospitalization for ectopic pregnancies (objective 16-5b), hospitalizations for postpartum complications including depression (objective 16-5c), children diagnosed with epilepsy (objective 16-14d), and enrollment of infants with disorders diagnosed through newborn bloodspot screening in appropriate service interventions (objective 16-20c).

- Eighteen Healthy People 2010 objectives were modified to create 24 Healthy People 2020 objectives [9]:

- Maternal mortality statistics are based upon the information recorded on death certificates and collected by state and local vital records offices. Due to concerns about data quality in the ascertainment of maternal mortality, the 2003 revision of the standard death certificate introduced improved data quality but produced rates that are not comparable to rates produced using the 1989 version of the death certificate [10]. For Healthy People 2010, data obtained from the 1989 version of the standard death certificate were used from baseline through 2002 to track this objective (16-4). The Healthy People 2020 objective will be tracked with data from the 2003 standard death certificate.

- Objectives on prenatal care (objective 16-6a and b) and maternal smoking during pregnancy (objective 16-17c) were derived from information recorded on birth certificates and also collected by states and local vital records offices. Due to the desire to produce more robust information, the 2003 revision of the standard birth certificate introduced improved data quality but produced rates that are not comparable to rates produced using the 1989 version of the birth certificate $[11,12]$. For Healthy People 2010, data obtained from the 1989 version of the standard birth certificate were used from baseline through 2002 to track these objectives. The Healthy People 2020 objectives will be tracked with data from the 2003 standard birth certificate.

- The objective on maternal weight gain during pregnancy (objective 16-12) remained developmental throughout the tracking period for Healthy People 2010. New data from the 2003 revision of the standard birth certificate will be used to track this objective in Healthy People 2020.

- In Healthy People 2010, only one national data point could be obtained to monitor the proportion of pregnant women who attended childbirth classes (objective 16-7). The data system used to track this objective is being changed for Healthy People 2020 in the effort to provide trend data.

- Preterm birth at 32-36 weeks gestation (objective 16-11b) was revised to include an additional objective to monitor the rate of late preterm birth (live births at 34-36 weeks gestation) in addition to live births at 32-33 weeks gestation, live births at less than 32 weeks gestation, and total preterm births. This change was made in recognition of evidence showing that late preterm infants, those born between 34 and 36 completed weeks of gestation, comprise over $70 \%$ of all preterm births and account for almost all of the increase in the U.S. preterm birth rate over the past two decades [13]. These new reporting categories are consistent with reports on birth outcomes [14]. 
- Fetal alcohol syndrome (objective 16-18) is tracked with data from the Fetal Alcohol Syndrome Surveillance Network (FASSNet). For Healthy People 2010, the overall prevalence was determined using data from four of the five funded states for the birth years 1995 through 1997 [15]. When the FASSNet cooperative agreement ended, seven programs from eight states were funded under a different agreement to conduct prevention and surveillance of fetal alcohol syndrome. Colorado was the only state to be funded under both FASSNet and the new agreement. Since the remaining funded states did not include the FASSNet states, the data used to monitor the Healthy People 2020 objective are not comparable to the Healthy People 2010 data.

- In 2002, the National Institute on Alcohol Abuse and Alcoholism revised the definition of binge drinking for women from drinking five or more alcoholic beverages at the same time or within a couple of hours of each other to four or more alcoholic beverages [16]. For Healthy People 2010, binge drinking during pregnancy (objective 16-17b) is tracked with the original definition. Healthy People 2020 will track binge drinking with the revised definition.

- Data for newborn bloodspot screening and follow-up was never obtained to track the two Healthy People 2010 objectives (16-20a and b). In Healthy People 2020, the data source was changed, and the objectives were modified so that they could be tracked. An additional objective that tracks annual assessments of services was also added.

- The Healthy People 2010 infant sleep position objective (16-13) was tracked using data from the National Infant Sleep Position Study. The Pregnancy Risk Assessment Monitoring System is used to measure infant sleep position for the Healthy People 2020 objective. The new data source will monitor the percentage of infants who are placed to sleep on their backs.

- The Healthy People 2010 objective monitoring the rate of cerebral palsy in children (objective 16-14b) was revised to track the proportion of children with cerebral palsy born at low birth weight (less than 2,500 grams). The scope was shifted because cerebral palsy is the most common motor disability in childhood affecting approximately 1.5 to 3.3 per 1,000 live births [17]. The inverse relationship between increased risk of cerebral palsy and being born at lower birth weights has been consistently well supported over time [18].

- The Healthy People 2010 objective that tracked the average age at which autism spectrum disorders were identified in children (objective 16-14c) was revised into three Healthy People 2020 objectives: the proportion of young children screened for autism spectrum disorders (ASD) by 24 months of age, the proportion of children with an ASD with a first evaluation by 36 months of age, and the proportion of children with an ASD enrolled in special services by 48 months of age.

- The Healthy People 2010 objective that tracked the occurrence of spina bifida and other neural tube defects (objective 16-15) was revised into two Healthy People 2020 objectives: the rate of anencephaly diagnosed in infants and the rate of spina bifida diagnosed in infants. 
- The Healthy People 2010 objective that tracked the median red blood cell (RBC) folate concentration in non-pregnant women (objective 16-16b) was revised for Healthy People 2020 to track the proportion of non-pregnant women with low RBC folate levels. The Healthy People 2010 target for this objective was exceeded; the revision reflects a continued interest in monitoring women at greatest risk suboptimal RBC folate concentrations.

- The Healthy People 2010 objective that tracked the rate of hospitalization for sickle cell disease in black or African American children (objective 16-21) was moved to the Blood Disorders and Blood Safety Topic Area for Healthy People 2020.

- The Healthy People 2010 objective addressing the proportion of children with special health care needs under 18 years of age who receive their care in familycentered, comprehensive, coordinated systems (objective 16-23) was divided into two objectives: children less than 11 years and children aged 12-17 years.

- Seventeen new objectives, seven of which are developmental, were added to the Healthy People 2020 Topic Area:

- Two new objectives that track infant deaths; one from sudden unexpected infant deaths (SUID) and the other is for infants diagnosed with Down syndrome.

- Six new objectives relate to preconception care services and health behaviors prior to pregnancy:

- Discussed preconception health with a health professional

- Took multivitamins/folic acid

- Did not smoke

- Did not drink

- Had a healthy weight

- Used contraception to plan pregnancy.

- Two new objectives (targeting men and women, individually) will track impaired fecundity.

- Two new objectives will track postpartum health and behaviors: the relapse of smoking among women who quit smoking during pregnancy and the proportion of women giving birth who attend a postpartum care visit with a health care professional.

- Three new objectives will target infant care:

- Employers that have worksite lactation support programs

- Breastfed newborns not given formula within the first 2 days of life

- Births in facilities that provide recommended care for lactating mothers and their babies.

- Two new objectives will track children with developmental delays. The first will track the child's age at first evaluation and the second will track the child's age when enrolled in special services.

Appendix D, “A Crosswalk Between Objectives From Healthy People 2010 to Healthy People 2020," summarizes the changes between the two decades of objectives, reflecting new knowledge and direction for this area. 


\section{Data Considerations}

Education and income are the primary measures of socioeconomic status (SES) in Healthy People 2010. Most data systems used in Healthy People 2010 define income as a family's income before taxes. In order to facilitate comparisons among groups and over time, while adjusting for family size and for inflation, Healthy People 2010 categorizes income using the poverty thresholds developed by the U.S. Census Bureau. Thus, the three categories of family income that are primarily used are:

- Poor-below the Federal poverty level

- Near poor-100\% to $199 \%$ of the Federal poverty level

- Middle/high income-200\% or more of the Federal poverty level.

These categories may be overridden by considerations specific to the data system, in which case they are modified as appropriate. See Healthy People 2010: General Data Issues, referenced below.

In general, data on educational attainment are presented for persons aged 25 years and over, consistent with guidance given by the U.S. Bureau of the Census. However, because of the requirements of the different data systems, the age groups used to calculate educational attainment for any specific objective may differ from the age groups used to report the data for other Healthy People 2010 objectives, as well as from select populations within the same objective. Therefore, the reader is urged to exercise caution in interpreting the data by educational attainment shown in the Health Disparities Table. See Healthy People 2010: General Data Issues, referenced below.

Beginning in 2003, education data from the National Vital Statistics System have been suppressed. The educational attainment item was changed in the new U.S. Standard Certificates for Births, Deaths, and Fetal Death in 2003 to be consistent with the U.S. Census Bureau data and to improve the ability to identify specific types of educational degrees. Many states, however, are still using the 1989 version of the U.S. Standard Certificates, which focuses on highest school grade completed. As a result, educational attainment data collected using the 2003 version are not comparable with data collected using the 1989 version [19].

Additional information on data issues is available from the following sources:

- All Healthy People 2010 tracking data can be found in the Healthy People 2010 database, DATA2010, available from http://wonder.cdc.gov/data2010/.

- Detailed information about the data and data sources used to support these objectives can be found in the Operational Definitions on the DATA2010 website, available from http://wonder.cdc.gov/data2010/focusod.htm.

- More information on statistical issues related to Healthy People tracking and measurement can be found in the Technical Appendix and in Healthy People 2010: General Data Issues, which is available in the Data Issues section of the NCHS Healthy People website under Healthy People 2010. 


\section{References and Notes}

1. Displayed in the Progress Chart (Figure 16-1), the percent of targeted change achieved expresses the difference between the baseline and the final value relative to the initial difference between the baseline and the Healthy People 2010 target. As such, it is a relative measure of progress toward attaining the Healthy People 2010 target. See the Reader's Guide for more information. When standard errors were available, the difference between the baseline and the final value was tested at the 0.05 level of significance. See the Figure 16-1 footnotes, as well as the Technical Appendix, for more detail.

2. Information about disparities among select populations is shown in the Health Disparities Table (Figure 16-2). Disparity from the best group rate is defined as the percent difference between the best group rate and each of the other group rates for a characteristic. For example, racial and ethnic health disparities are measured as the percent difference between the best racial and ethnic group rate and each of the other racial and ethnic group rates. Similarly, disparities by sex are measured as the percent difference between the better group rate (e.g., female) and the rate for the other group (e.g., male). Some objectives are expressed in terms of favorable events or conditions that are to be increased, while others are expressed in terms of adverse events or conditions that are to be reduced. In order to facilitate comparison of health disparities across different objectives, disparity is measured only in terms of adverse events or conditions. For comparability across objectives, objectives that are expressed in terms of favorable events or conditions are re-expressed using the adverse event or condition for the purpose of computing disparity, but they are not otherwise restated or changed. For example, objective 1-1, to increase the proportion of persons with health insurance (e.g., $72 \%$ of the American Indian or Alaska Native population aged under 65 years had some form of health insurance in 2008), is expressed in terms of the percentage of persons without health insurance (e.g., $100 \%-72 \%=28 \%$ of the American Indian or Alaska Native population aged under 65 years did not have any form of health insurance in 2008) when the disparity from the best group rate is calculated. See the Reader's Guide for more information. When standard errors were available, the difference between the best group rate and each of the other group rates was tested at the 0.05 level of significance. See the Figure 16-2 footnotes, as well as the Technical Appendix, for more detail.

3. More information about infant sleep positions and SIDS can be found from the Back to Sleep Campaign website: http://www.nichd.nih.gov/publications/pubs/safe sleep gen.cfm.

4. The change in disparity is estimated by subtracting the disparity at baseline from the disparity at the most recent data point and, therefore, is expressed as a change in percentage points. See the Reader's Guide for more information. When standard errors were available, the change in disparity was tested at the 0.05 level of significance. See the Figure 16-2 footnotes, as well as the Technical Appendix, for more detail.

5. To be included in Healthy People 2010, an objective must have a national data source that provides a baseline and at least one additional data point for tracking progress. Some objectives lacked baseline data at the time of their development but had a potential data source and were considered of sufficient national importance to be included in Healthy People. These are called "developmental" objectives. When data become available, a developmental objective is moved to measurable status and a Healthy People target can be set.

6. Dropped objectives were not carried forward into Healthy People 2020. These objectives were either developmental or deleted at the Healthy People 2010 Midcourse Review or at another time in Healthy People 2010.

7. Retained "as is" objectives have no change in the numerator definition or in the denominator definition between the Healthy People 2010 and Healthy People 2020 objectives. These include objectives that were developmental in Healthy People 2010 and are developmental in Healthy People 2020 and for which no numerator or denominator information was available. 
8. Archived objectives had at least one data point in Healthy People 2010 but were not carried forward into Healthy People 2020.

9. Modified objectives have some change in the numerator definition or in the denominator definition between the Healthy People 2010 and Healthy People 2020 objectives. These include objectives that went from developmental in Healthy People 2010 to measurable in Healthy People 2020 or vice versa.

10. Chang J, Elam-Evans LD, Berg CJ, Herndon J, Flowers L, Seed KA, Syverson CJ. Pregnancy-related mortality surveillance-United States, 1991-1999. In: CDC surveillance summaries (February 21). MMWR 52(SS-2):1-8. 2003. Available from: http://www.cdc.gov/mmwr/pdf/ss/ss5202.pdf.

11. National Center for Health Statistics. 2003 revision of the U.S. Standard Certificate of Live Birth. Hyattsville, MD: National Center for Health Statistics. 2003. Available from: http://www.cdc.gov/nchs/nvss/vital certificate revisions.htm.

12. National Center for Health Statistics. Report of the panel to evaluate the U.S. Standard Certificates and Reports. Hyattsville, MD: National Center for Health Statistics. 2000. Available from: http://www.cdc.gov/nchs/data/dvs/panelreport acc.pdf.

13. More information can be found from the March of Dimes website: http://www.marchofdimes.com/.

14. Hamilton BE, Martin JA, Ventura SJ. Births: Preliminary data for 2007. National vital statistics reports; vol 57 no 12. Hyattsville, MD: National Center for Health Statistics. 2009. Available from: http://www.cdc.gov/nchs/data/nvsr/nvsr57/nvsr57 12.pdf.

15. Meaney FJ, Miller LA. A comparison of fetal alcohol syndrome surveillance network and birth defects surveillance methodology in determining prevalence rates of fetal alcohol syndrome. Birth Defects Res A Clin Mol Teratol 67:819-21. 2003. DOI: 10.1002/bdra.10122.

16. NIAAA Newsletter, NIH Publication No. 04-5346. Available from: http://pubs.niaaa.nih.gov/publications/Newsletter/winter2004/Newsletter Number3.pdf

17. Pakula A, Van Naarden-Braun K, Yeargin-Allsopp M. Epidemiology and Classification of Cerebral Palsy, Phys Med Rehab 20(3):425-52. 2009, Aug.

18. Wu YW, Xing G, Fuentes-Afflick GE, Danielson B, Smith LH, Gilbert WM. Racial, Ethnic, and Socioeconomic Disparities in the Prevalence of Cerebral Palsy. Pediatrics 127(3):e674-81. 2011. Published ahead of print February 21, 2011. DOI:10.1542/peds.2010-1656.

19. Xu JQ Kochanek KD, Murphy SL, Tejada-Vera B. Deaths: Final data for 2007. National vital statistics reports; vol 58 no 19. Hyattsville, MD: National Center for Health Statistics. 2010. Available from: http://www.cdc.gov/nchs/data/nvsr/nvsr58/nvsr58 19.pdf. 


\section{Comprehensive Summary of Objectives: Maternal, Infant, and Child Health}

\begin{tabular}{|c|c|c|}
\hline Objective & Description & Data Source or Objective Status \\
\hline $16-1 \mathrm{a}$ & $\begin{array}{l}\text { Fetal deaths }(20+\text { weeks gestation, per 1,000 live births plus fetal } \\
\text { deaths) }\end{array}$ & $\begin{array}{l}\text { National Vital Statistics System-Fetal Death and Natality (NVSS-FD, } \\
\text { NVSS-N), CDC, NCHS. }\end{array}$ \\
\hline $16-1 b$ & $\begin{array}{l}\text { Perinatal deaths ( } 28 \text { weeks gestation to }<7 \text { days after birth, per } 1,000 \\
\text { live births plus fetal deaths) }\end{array}$ & $\begin{array}{l}\text { National Vital Statistics System-Fetal Death, Mortality and Natality } \\
\text { (NVSS-FD, NVSS-M, NVSS-N), CDC, NCHS. }\end{array}$ \\
\hline $16-1 c$ & All Infant deaths (<1 year, per 1,000 live births) & $\begin{array}{l}\text { National Vital Statistics System-Mortality and Natality (NVSS-M, } \\
\text { NVSS-N), CDC, NCHS. }\end{array}$ \\
\hline $16-1 d$ & Neonatal deaths (<28 days, per 1,000 live births) & $\begin{array}{l}\text { National Vital Statistics System-Mortality and Natality (NVSS-M, } \\
\text { NVSS-N), CDC, NCHS. }\end{array}$ \\
\hline $16-1 e$ & Postneonatal deaths (28 days to $<1$ year, per 1,000 live births) & $\begin{array}{l}\text { National Vital Statistics System-Mortality and Natality (NVSS-M, } \\
\text { NVSS-N), CDC, NCHS. }\end{array}$ \\
\hline $16-1 f$ & Infant deaths due to birth defects ( $<1$ year, per 1,000 live births) & $\begin{array}{l}\text { National Vital Statistics System-Mortality and Natality (NVSS-M, } \\
\text { NVSS-N), CDC, NCHS. }\end{array}$ \\
\hline $16-1 \mathrm{~g}$ & $\begin{array}{l}\text { Infant deaths due to congenital heart defects ( }<1 \text { year, per 1,000 live } \\
\text { births) }\end{array}$ & $\begin{array}{l}\text { National Vital Statistics System-Mortality and Natality (NVSS-M, } \\
\text { NVSS-N), CDC, NCHS. }\end{array}$ \\
\hline $16-1 \mathrm{~h}$ & $\begin{array}{l}\text { Infant deaths due to sudden infant death syndrome (SIDS) }(<1 \text { year, per } \\
1,000 \text { live births) }\end{array}$ & $\begin{array}{l}\text { National Vital Statistics System-Mortality and Natality (NVSS-M, } \\
\text { NVSS-N), CDC, NCHS. }\end{array}$ \\
\hline $16-2 a$ & Child deaths $-1-4$ years (per 100,000 population) & National Vital Statistics System-Mortality (NVSS-M), CDC, NCHS. \\
\hline $16-2 b$ & Child deaths $-5-9$ years (per 100,000 population) & National Vital Statistics System-Mortality (NVSS-M), CDC, NCHS. \\
\hline $16-3 a$ & $\begin{array}{l}\text { Adolescent and young adult deaths }-10-14 \text { years (per 100,000 } \\
\text { population) }\end{array}$ & National Vital Statistics System-Mortality (NVSS-M), CDC, NCHS. \\
\hline $16-3 b$ & $\begin{array}{l}\text { Adolescent and young adult deaths }-15-19 \text { years (per 100,000 } \\
\text { population) }\end{array}$ & National Vital Statistics System-Mortality (NVSS-M), CDC, NCHS. \\
\hline $16-3 c$ & $\begin{array}{l}\text { Adolescent and young adult deaths }-20-24 \text { years (per } 100,000 \\
\text { population) }\end{array}$ & National Vital Statistics System-Mortality (NVSS-M), CDC, NCHS. \\
\hline $16-4$ & Maternal deaths (per 100,000 live births) & $\begin{array}{l}\text { National Vital Statistics System-Mortality and Natality (NVSS-M, } \\
\text { NVSS-N), CDC, NCHS. }\end{array}$ \\
\hline $16-5 a$ & $\begin{array}{l}\text { Maternal complications during hospitalized labor and delivery (per } 100 \\
\text { deliveries) }\end{array}$ & National Hospital Discharge Survey (NHDS), CDC, NCHS. \\
\hline $16-5 b$ & $\begin{array}{l}\text { Maternal illness and complications due to pregnancy-Hospitalizations } \\
\text { for ectopic pregnancies }\end{array}$ & Dropped \\
\hline
\end{tabular}




\begin{tabular}{|c|c|c|}
\hline Objective & Description & Data Source or Objective Status \\
\hline $16-5 c$ & $\begin{array}{l}\text { Maternal illness and complications due to pregnancy-Hospitalizations } \\
\text { for postpartum complications, including depression }\end{array}$ & Dropped \\
\hline $16-6 a$ & Prenatal care-Beginning in first trimester & National Vital Statistics System-Natality (NVSS-N), CDC, NCHS. \\
\hline $16-6 b$ & Prenatal care-Early and adequate & National Vital Statistics System-Natality (NVSS-N), CDC, NCHS. \\
\hline $16-7$ & Childbirth class attendance-Pregnant women who attend & $\begin{array}{l}\text { National Survey of Early Childhood Health (NSECH): HRSA, MCHB; CDC, } \\
\text { NCHS. }\end{array}$ \\
\hline $16-8$ & Very low birth weight infants born at level III hospitals & Title V Reporting System, HRSA. \\
\hline $16-9 a$ & Cesarean births-No prior cesarean birth & National Vital Statistics System-Natality (NVSS-N), CDC, NCHS. \\
\hline $16-9 b$ & Cesarean births-Prior cesarean birth & National Vital Statistics System-Natality (NVSS-N), CDC, NCHS. \\
\hline $16-10 \mathrm{a}$ & Low birth weight (LBW), infants $(<2,500$ grams) & National Vital Statistics System-Natality (NVSS-N), CDC, NCHS. \\
\hline $16-10 \mathrm{~b}$ & Very low birth weight (VLBW), infants $(<1,500$ grams) & National Vital Statistics System-Natality (NVSS-N), CDC, NCHS. \\
\hline $16-11 \mathrm{a}$ & Preterm live births-Total ( $<37$ weeks gestation) & National Vital Statistics System-Natality (NVSS-N), CDC, NCHS. \\
\hline $16-11 b$ & Preterm live births-32-36 weeks gestation & National Vital Statistics System-Natality (NVSS-N), CDC, NCHS. \\
\hline $16-11 c$ & Preterm live births $-<32$ weeks gestation & National Vital Statistics System-Natality (NVSS-N), CDC, NCHS. \\
\hline $16-12$ & Recommended weight gain during pregnancy & Developmental \\
\hline $16-13$ & Infants put to sleep on their backs $(<8$ months $)$ & National Infant Sleep Position Study (NISP), NIH, NICHD. \\
\hline $16-14 a$ & $\begin{array}{l}\text { Developmental disabilities-Mental retardation-IQ } \leq 70 \text { (per 10,000 } \\
\text { population, Metropolitan Atlanta, } 8 \text { years) }\end{array}$ & $\begin{array}{l}\text { Metropolitan Atlanta Development Disabilities Surveillance Program } \\
\text { (MADDSP), CDC, NCBDDD. }\end{array}$ \\
\hline $16-14 b$ & $\begin{array}{l}\text { Developmental disabilities-Cerebral palsy (per } 10,000 \text { population, } \\
\text { Metropolitan Atlanta, } 8 \text { years) }\end{array}$ & $\begin{array}{l}\text { Metropolitan Atlanta Development Disabilities Surveillance Program } \\
\text { (MADDSP), CDC, NCBDDD. }\end{array}$ \\
\hline $16-14 c$ & $\begin{array}{l}\text { Developmental disabilities-Age at first identification of autism } \\
\text { spectrum disorder (in months, Metropolitan Atlanta, } 8 \text { years) }\end{array}$ & $\begin{array}{l}\text { Metropolitan Atlanta Development Disabilities Surveillance Program } \\
\text { (MADDSP), CDC, NCBDDD. }\end{array}$ \\
\hline $16-14 d$ & Epilepsy-Metropolitan Atlanta & Dropped \\
\hline $16-15$ & $\begin{array}{l}\text { Spina bifida and other neural tube defects (new cases per 100,000 live } \\
\text { births) }\end{array}$ & National Birth Defects Prevention Network (NBDPN), CDC, NCBDDD. \\
\hline $16-16 a$ & $\begin{array}{l}\text { Folic acid consumption } \geq 400 \mu g \text { daily by nonpregnant women }(15-44 \\
\text { years) }\end{array}$ & $\begin{array}{l}\text { National Health and Nutrition Examination Survey (NHANES), CDC, } \\
\text { NCHS. }\end{array}$ \\
\hline $16-16 b$ & $\begin{array}{l}\text { Median red blood cell (RBC) folate level among nonpregnant women } \\
\text { (ng/ml, 15-44 years) }\end{array}$ & $\begin{array}{l}\text { National Health and Nutrition Examination Survey (NHANES), CDC, } \\
\text { NCHS. }\end{array}$ \\
\hline $16-17 \mathrm{a}$ & Pregnant women abstaining from alcohol in past month (15-44 years) & National Survey on Drug Use and Health (NSDUH), SAMHSA. \\
\hline
\end{tabular}




\begin{tabular}{|c|c|c|}
\hline Objective & Description & Data Source or Objective Status \\
\hline $16-17 b$ & $\begin{array}{l}\text { Pregnant women abstaining from binge drinking in past month (15-44 } \\
\text { years) }\end{array}$ & National Survey on Drug Use and Health (NSDUH), SAMHSA. \\
\hline $16-17 c$ & Pregnant women abstaining from cigarette smoking during pregnancy & National Vital Statistics System-Natality (NVSS-N), CDC, NCHS. \\
\hline $16-17 d$ & $\begin{array}{l}\text { Pregnant women abstaining from illicit drugs in past month (15-44 } \\
\text { years) }\end{array}$ & National Survey on Drug Use and Health (NSDUH), SAMHSA. \\
\hline $16-18$ & Fetal alcohol syndrome (cases per 1,000 live births) & Fetal Alcohol Syndrome Surveillance System (FASSNet), CDC, NCBDDD. \\
\hline $16-19 a$ & Breastfeeding-Ever & National Immunization Survey (NIS), CDC, NCIRD and NCHS. \\
\hline $16-19 b$ & Breastfeeding-At 6 months & National Immunization Survey (NIS), CDC, NCIRD and NCHS. \\
\hline $16-19 c$ & Breastfeeding-At 1 year & National Immunization Survey (NIS), CDC, NCIRD and NCHS. \\
\hline $16-19 d$ & Exclusive breastfeeding-Through 3 months & National Immunization Survey (NIS), CDC, NCIRD and NCHS. \\
\hline $16-19 \mathrm{e}$ & Exclusive breastfeeding-Through 6 months & National Immunization Survey (NIS), CDC, NCIRD and NCHS. \\
\hline $16-20 \mathrm{a}$ & Newborn bloodspot screening-State-mandated conditions & Developmental \\
\hline $16-20 b$ & $\begin{array}{l}\text { Newborn bloodspot screening-Timely follow-up diagnostic testing for } \\
\text { screening positives }\end{array}$ & Developmental \\
\hline $16-20 c$ & $\begin{array}{l}\text { Newborn bloodspot screening-Timely enrollment of infant with } \\
\text { diagnosed disorders in appropriate service interventions }\end{array}$ & Dropped \\
\hline $16-21$ & $\begin{array}{l}\text { Hospital discharges for sickle cell disease (per } 100,000 \text { black or African } \\
\text { American children, } \leq 9 \text { years) }\end{array}$ & National Hospital Discharge Survey (NHDS), CDC, NCHS. \\
\hline $16-22$ & Medical homes for children with special health care needs & $\begin{array}{l}\text { National Survey of Children with Special Health Care Needs (NS- } \\
\text { CSHCN), CDC, NCHS. }\end{array}$ \\
\hline $16-23$ & Service systems for children with special health care needs & $\begin{array}{l}\text { National Survey of Children with Special Health Care Needs (NS- } \\
\text { CSHCN), CDC, NCHS. }\end{array}$ \\
\hline
\end{tabular}


Figure 16-1. Progress Toward Target Attainment for Focus Area 16: Maternal, Infant, and Child Health

Moved away

from target ${ }^{1}$

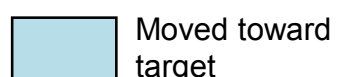

target

Met or exceeded

target

16-1a. Fetal deaths (20+ weeks gestation, per 1,000 live births plus fetal deaths)

16-1b. Perinatal deaths (28 weeks gestation to $<7$ days after birth, per 1,000 live births plus fetal deaths)

16-1c. All Infant deaths (<1 year, per 1,000 live births)

16-1d. Neonatal deaths (<28 days, per 1,000 live births)

16-1e. Postneonatal deaths ( 28 days to $<1$ year, per 1,000 live births)

16-1f. Infant deaths due to birth defects (<1 year, per 1,000 live births)

16-1g. Infant deaths due to congenital heart defects (<1 year, per 1,000 live births)

16-1h. Infant deaths due to sudden infant death syndrome (SIDS) $(<1$ year, per 1,000 live births)

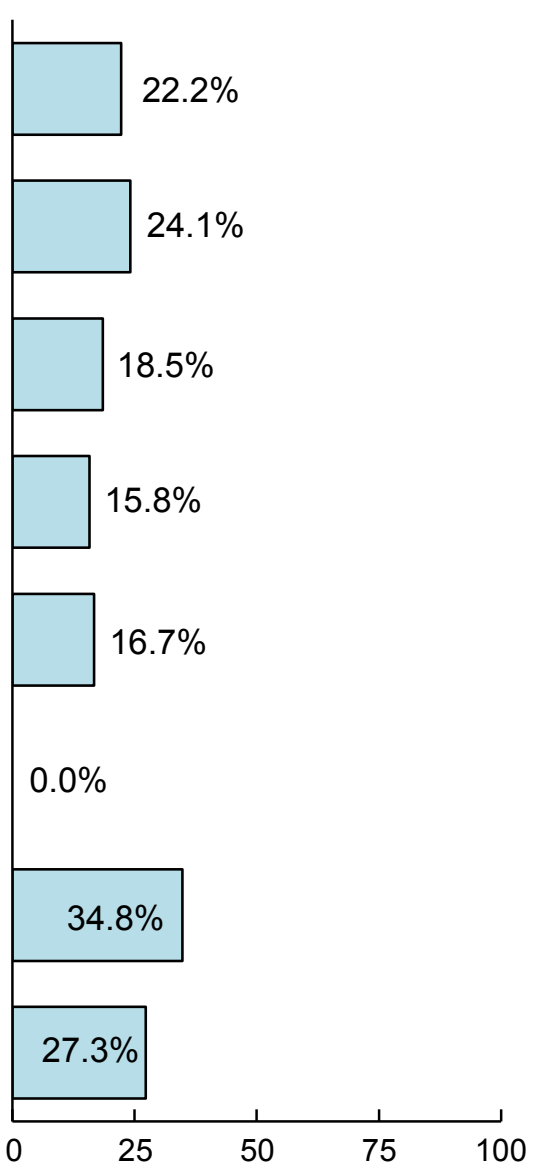

\begin{tabular}{|c|c|c|c|c|c|}
\hline \multirow{2}{*}{$\begin{array}{l}2010 \\
\text { Target }\end{array}$} & \multirow{2}{*}{$\begin{array}{c}\text { Baseline } \\
\text { (Year) }\end{array}$} & \multirow{2}{*}{$\begin{array}{l}\text { Final } \\
\text { (Year) }\end{array}$} & \multicolumn{3}{|c|}{ Baseline vs. Final } \\
\hline & & & Difference ${ }^{2}$ & $\begin{array}{l}\text { Statistically } \\
\text { Significant }^{3}\end{array}$ & $\begin{array}{l}\text { Percent } \\
\text { Change }^{4}\end{array}$ \\
\hline 4.1 & $\begin{array}{c}6.8 \\
(1997)\end{array}$ & $\begin{array}{c}6.2 \\
(2005)\end{array}$ & -0.6 & Yes & $-8.8 \%$ \\
\hline 4.4 & $\begin{array}{c}7.3 \\
(1997)\end{array}$ & $\begin{array}{c}6.6 \\
(2005)\end{array}$ & -0.7 & Yes & $-9.6 \%$ \\
\hline 4.5 & $\begin{array}{c}7.2 \\
(1998)\end{array}$ & $\begin{array}{c}6.7 \\
(2006)\end{array}$ & -0.5 & Yes & $-6.9 \%$ \\
\hline 2.9 & $\begin{array}{c}4.8 \\
(1998)\end{array}$ & $\begin{array}{c}4.5 \\
(2006)\end{array}$ & -0.3 & Yes & $-6.2 \%$ \\
\hline 1.2 & $\begin{array}{c}2.4 \\
(1998)\end{array}$ & $\begin{array}{c}2.2 \\
(2006)\end{array}$ & -0.2 & Yes & $-8.3 \%$ \\
\hline 0.7 & $\begin{array}{c}1.4 \\
(1999)\end{array}$ & $\begin{array}{c}1.4 \\
(2006)\end{array}$ & 0.0 & No & $0.0 \%$ \\
\hline 0.23 & $\begin{array}{c}0.46 \\
(1999)\end{array}$ & $\begin{array}{c}0.38 \\
(2006)\end{array}$ & -0.08 & Yes & $-17.4 \%$ \\
\hline 0.23 & $\begin{array}{c}0.67 \\
(1999)\end{array}$ & $\begin{array}{c}0.55 \\
(2006)\end{array}$ & 0.12 & Yes & -17.9 \\
\hline
\end{tabular}

Percent of targeted change achieved ${ }^{5}$ (continued) 
Figure 16-1. Progress Toward Target Attainment for Focus Area 16: Maternal, Infant, and Child Health (continued)

Moved away

from target ${ }^{1}$

Moved toward

target

Met or exceeded

target

16-2. Child deaths (per 100,000 population)

a. 1-4 years

b. 5-9 years

16-3. Adolescent and young adult deaths (per 100,000 population)

a. 10-14 years

b. $15-19$ years

C. 20-24 years

16-4. Maternal deaths (per 100,000 live births)

16-5a. Maternal complications during hospitalized labor and delivery (per 100 deliveries)

16-6. Prenatal care

a. Beginning in first trimester

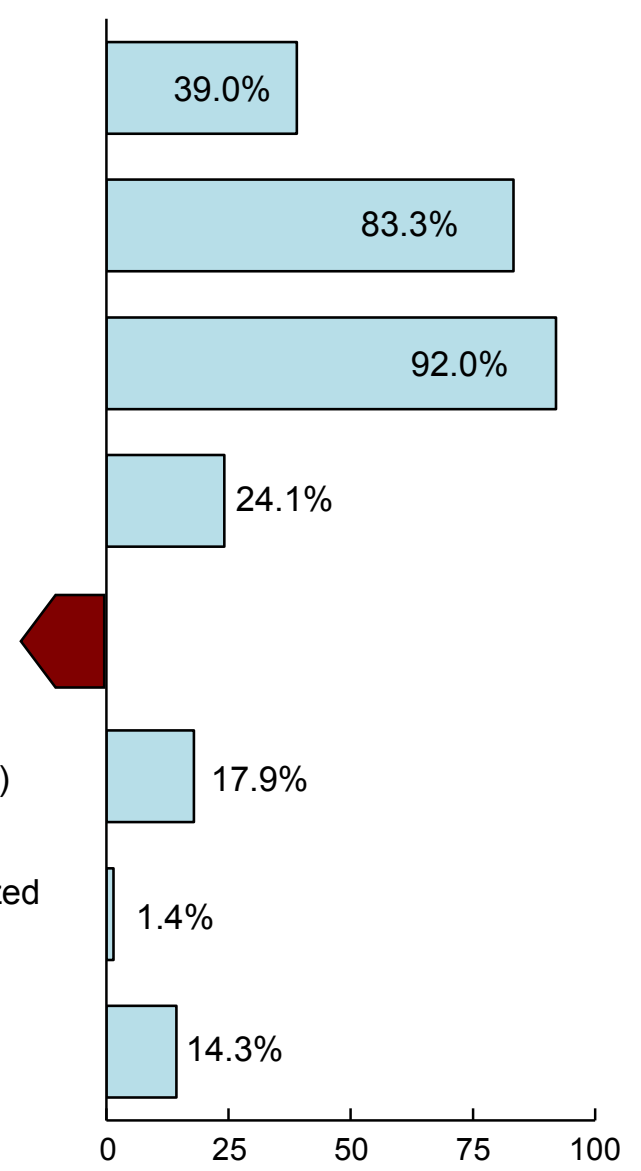

\begin{tabular}{|c|c|c|c|c|c|}
\hline \multirow{2}{*}{$\begin{array}{l}2010 \\
\text { Target }\end{array}$} & \multirow{2}{*}{$\begin{array}{c}\text { Baseline } \\
\text { (Year) }\end{array}$} & \multirow{2}{*}{$\begin{array}{l}\text { Final } \\
\text { (Year) }\end{array}$} & \multicolumn{3}{|c|}{ Baseline vs. Final } \\
\hline & & & Difference ${ }^{2}$ & $\begin{array}{l}\text { Statistically } \\
\text { Significant }^{3}\end{array}$ & $\begin{array}{l}\text { Percent } \\
\text { Change }^{4}\end{array}$ \\
\hline 20.0 & $\begin{array}{c}34.1 \\
(1998)\end{array}$ & $\begin{array}{c}28.6 \\
(2007)\end{array}$ & -5.5 & Yes & $-16.1 \%$ \\
\hline 13.0 & $\begin{array}{c}17.2 \\
(1998)\end{array}$ & $\begin{array}{c}13.7 \\
(2007)\end{array}$ & -3.5 & Yes & $-20.3 \%$ \\
\hline 16.5 & $\begin{array}{c}21.5 \\
(1998)\end{array}$ & $\begin{array}{c}16.9 \\
(2007)\end{array}$ & -4.6 & Yes & $-21.4 \%$ \\
\hline 38.0 & $\begin{array}{c}69.5 \\
(1998)\end{array}$ & $\begin{array}{c}61.9 \\
(2007)\end{array}$ & -7.6 & Yes & $-10.9 \%$ \\
\hline 41.5 & $\begin{array}{c}92.7 \\
(1998)\end{array}$ & $\begin{array}{c}98.3 \\
(2007)\end{array}$ & 5.6 & Yes & $6.0 \%$ \\
\hline 4.3 & $\begin{array}{c}9.9 \\
(1999)\end{array}$ & $\begin{array}{c}8.9 \\
(2002)\end{array}$ & -1.0 & No & $-10.1 \%$ \\
\hline 24.0 & $\begin{array}{c}31.2 \\
(1998)\end{array}$ & $\begin{array}{c}31.1 \\
(2007)\end{array}$ & -0.1 & No & $-0.3 \%$ \\
\hline $90 \%$ & $\begin{array}{c}83 \% \\
(1998)\end{array}$ & $\begin{array}{c}84 \% \\
(2002)\end{array}$ & 1 & Yes & $1.2 \%$ \\
\hline
\end{tabular}

Percent of targeted change achieved ${ }^{5}$ (continued)

Page 16-18 
Figure 16-1. Progress Toward Target Attainment for Focus Area 16: Maternal, Infant, and Child Health (continued)

Moved away

from target ${ }^{1}$

Moved toward target

Met or exceeded

target

16-6. Prenatal care

b. Early and adequate

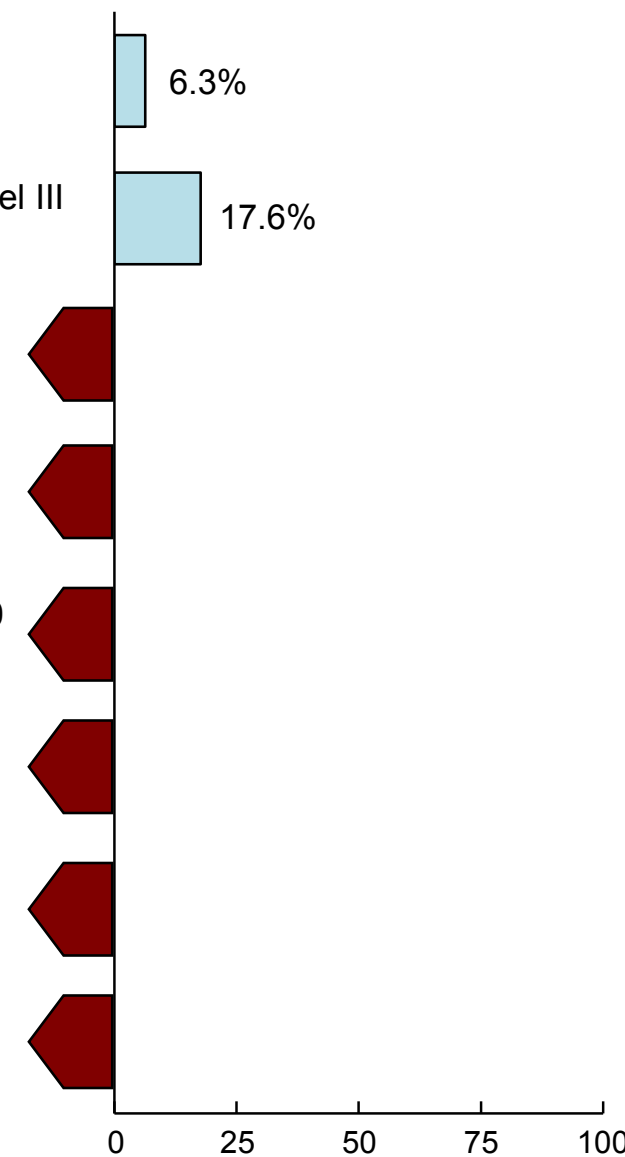

Percent of targeted change achieved ${ }^{5}$

16-8. Very low birth weight infants born at level III hospitals

16-9. Cesarean births

a. No prior cesarean birth

b. Prior cesarean birth

16-10a. Low birth weight (LBW), infants $(<2,500$ grams)

16-10b. Very low birth weight (VLBW), infants $(<1,500$ grams $)$

16-11. Preterm live births

a. Total (<37 weeks gestation)

b. $32-36$ weeks gestation

\begin{tabular}{|c|c|c|c|c|c|}
\hline \multirow{2}{*}{$\begin{array}{l}2010 \\
\text { Target }\end{array}$} & \multirow{2}{*}{$\begin{array}{c}\text { Baseline } \\
\text { (Year) }\end{array}$} & \multirow{2}{*}{$\begin{array}{l}\text { Final } \\
\text { (Year) }\end{array}$} & \multicolumn{3}{|c|}{ Baseline vs. Final } \\
\hline & & & Difference $^{2}$ & $\begin{array}{l}\text { Statistically } \\
\text { Significant }^{3}\end{array}$ & $\begin{array}{l}\text { Percent } \\
\text { Change }^{4}\end{array}$ \\
\hline $90 \%$ & $\begin{array}{c}74 \% \\
(1998)\end{array}$ & $\begin{array}{c}75 \% \\
(2002)\end{array}$ & 1 & Yes & $1.4 \%$ \\
\hline $90 \%$ & $\begin{array}{c}73 \% \\
(1996-97)\end{array}$ & $\begin{array}{c}76 \% \\
(2008)\end{array}$ & 3 & $\begin{array}{l}\text { Not } \\
\text { tested }\end{array}$ & $4.1 \%$ \\
\hline $15 \%$ & $\begin{array}{c}18 \% \\
(1998)\end{array}$ & $\begin{array}{c}26 \% \\
(2007)\end{array}$ & 8 & Yes & $44.4 \%$ \\
\hline $63 \%$ & $\begin{array}{c}72 \% \\
(1998)\end{array}$ & $\begin{array}{c}91 \% \\
(2007)\end{array}$ & 19 & Yes & $26.4 \%$ \\
\hline $5.0 \%$ & $\begin{array}{c}7.6 \% \\
(1998)\end{array}$ & $\begin{array}{l}8.2 \% \\
(2007)\end{array}$ & 0.6 & Yes & $7.9 \%$ \\
\hline $0.9 \%$ & $\begin{array}{c}1.4 \% \\
(1998)\end{array}$ & $\begin{array}{c}1.5 \% \\
(2007)\end{array}$ & 0.1 & Yes & $7.1 \%$ \\
\hline $7.6 \%$ & $\begin{array}{l}11.6 \% \\
(1998)\end{array}$ & $\begin{array}{l}12.7 \% \\
(2007)\end{array}$ & 1.1 & Yes & $9.5 \%$ \\
\hline $6.4 \%$ & $\begin{array}{c}9.6 \% \\
(1998)\end{array}$ & $\begin{array}{l}10.6 \% \\
(2007)\end{array}$ & 1.0 & Yes & $10.4 \%$ \\
\hline
\end{tabular}

(continued) 
Figure 16-1. Progress Toward Target Attainment for Focus Area 16: Maternal, Infant, and Child Health (continued)

Moved away

from target ${ }^{1}$

Moved toward

target

Met or exceeded

target

16-11. Preterm live births

c. $<32$ weeks gestation

16-13. Infants put to sleep on their backs ( $<8$ months)

16-14. Developmental disabilities (Metropolitan Atlanta, 8 years)

a. Mental retardation-IQ $\leq 70$ (per 10,000 population)

b. Cerebral palsy (per 10,000 population)

c. Age at first identification of autism spectrum disorder (in months)

16-15. Spina bifida and other neural tube defects (new cases per 100,000 live births)

16-16a. Folic acid consumption $\geq 400 \mu$ gaily by nonpregnant women (15-44 years)

16-16b. Median red blood cell (RBC) folate level among nonpregnant women $(\mathrm{ng} / \mathrm{ml}, 15$ 44 years)

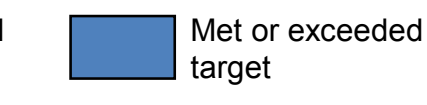

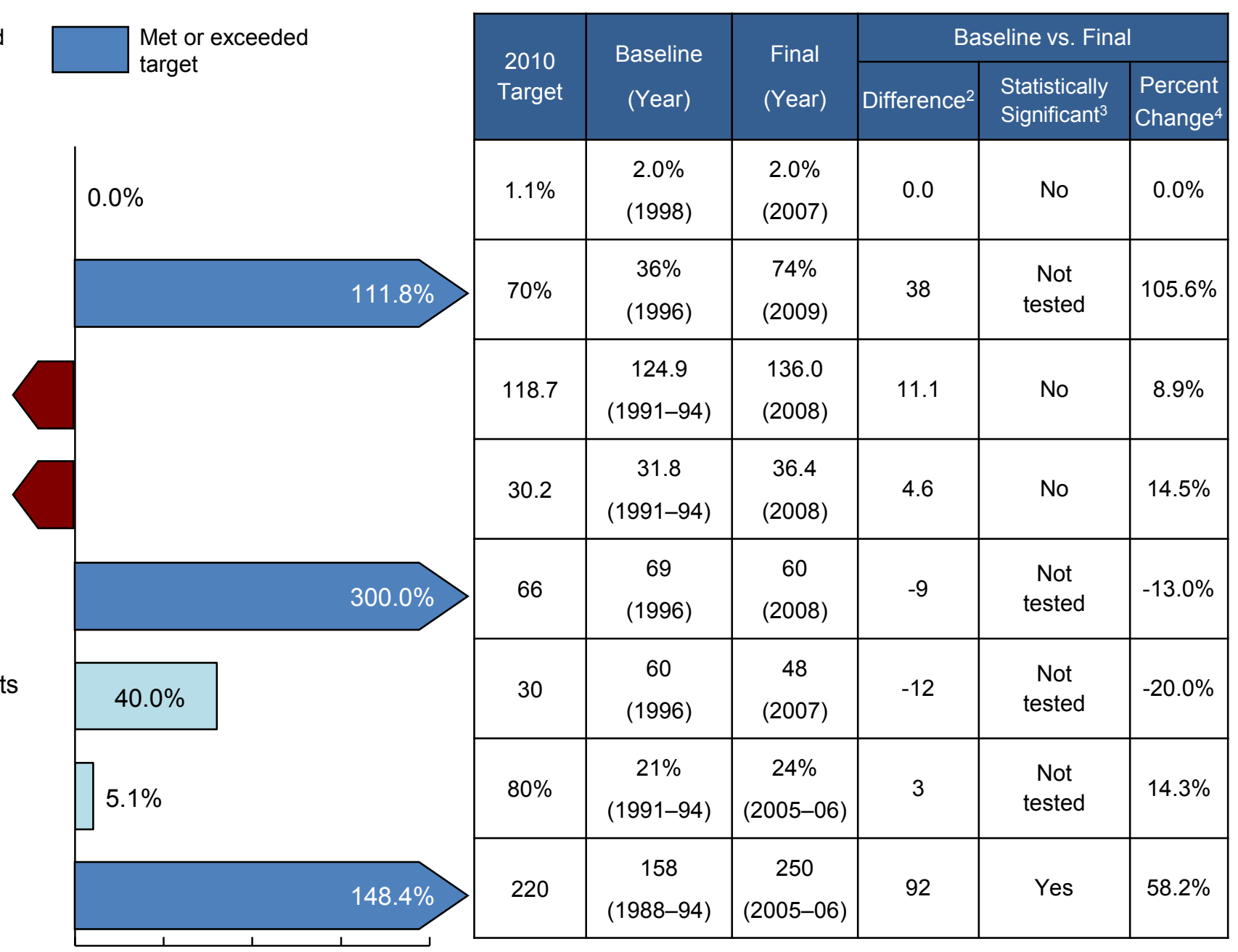

Percent of targeted change achieved ${ }^{5}$

(continued) 
Figure 16-1. Progress Toward Target Attainment for Focus Area 16: Maternal, Infant, and Child Health (continued)

Moved away

from target ${ }^{1}$

Moved toward

target

Met or exceeded

target

16-17. Pregnant women abstaining from

a. Alcohol in past month (15-44 years)

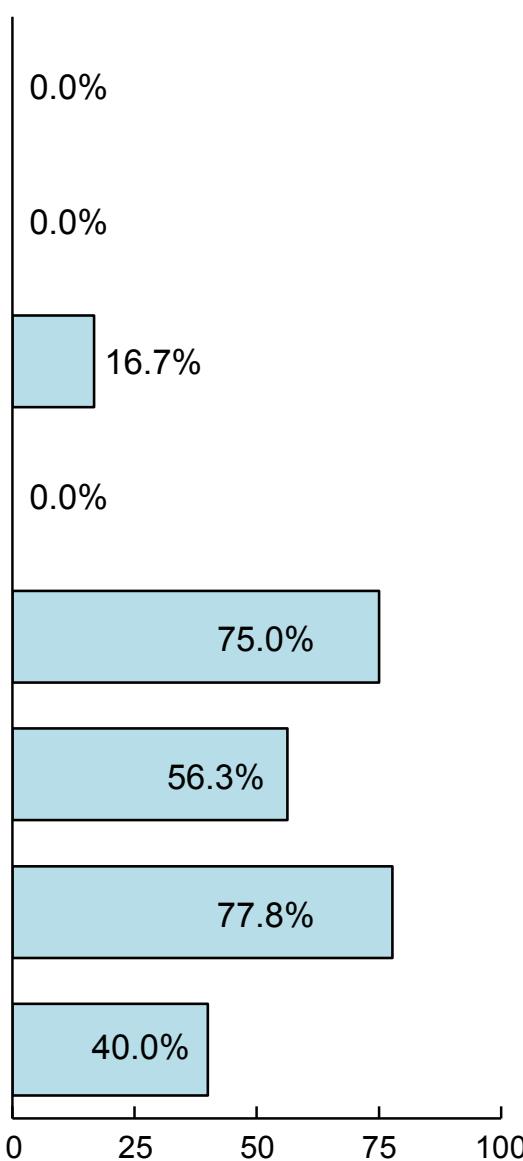

\begin{tabular}{|c|c|c|c|c|c|}
\hline \multirow{2}{*}{$\begin{array}{l}2010 \\
\text { Target }\end{array}$} & \multirow{2}{*}{$\begin{array}{c}\text { Baseline } \\
\text { (Year) }\end{array}$} & \multirow{2}{*}{$\begin{array}{l}\text { Final } \\
\text { (Year) }\end{array}$} & \multicolumn{3}{|c|}{ Baseline vs. Final } \\
\hline & & & Difference & $\begin{array}{l}\text { Statistically } \\
\text { Significant }^{3}\end{array}$ & $\begin{array}{l}\text { Percent } \\
\text { Change }^{4}\end{array}$ \\
\hline $95 \%$ & $\begin{array}{c}90 \% \\
(2002-03)\end{array}$ & $\begin{array}{c}90 \% \\
(2008-09)\end{array}$ & 0 & No & $0.0 \%$ \\
\hline $100 \%$ & $\begin{array}{c}96 \% \\
(2002-03)\end{array}$ & $\begin{array}{c}96 \% \\
(2008-09)\end{array}$ & 0 & No & $0.0 \%$ \\
\hline $99 \%$ & $\begin{array}{c}87 \% \\
(1998)\end{array}$ & $\begin{array}{c}89 \% \\
(2002)\end{array}$ & 2 & Yes & $2.3 \%$ \\
\hline $100 \%$ & $\begin{array}{c}96 \% \\
(2002-03)\end{array}$ & $\begin{array}{c}96 \% \\
(2008-09)\end{array}$ & 0 & No & $0.0 \%$ \\
\hline $75 \%$ & $\begin{array}{c}71 \% \\
(2000)\end{array}$ & $\begin{array}{c}74 \% \\
(2006)\end{array}$ & 3 & Yes & $4.2 \%$ \\
\hline $50 \%$ & $\begin{array}{c}34 \% \\
(2000)\end{array}$ & $\begin{array}{c}43 \% \\
(2006)\end{array}$ & 9 & Yes & $26.5 \%$ \\
\hline $25 \%$ & $\begin{array}{c}16 \% \\
(2000)\end{array}$ & $\begin{array}{c}23 \% \\
(2006)\end{array}$ & 7 & Yes & $43.8 \%$ \\
\hline $40 \%$ & $\begin{array}{c}30 \% \\
(2003)\end{array}$ & $\begin{array}{c}34 \% \\
(2006)\end{array}$ & 4 & Yes & $13.3 \%$ \\
\hline
\end{tabular}

d. Through 3 months

Percent of targeted change achieved ${ }^{5}$

(continued) 
Figure 16-1. Progress Toward Target Attainment for Focus Area 16: Maternal, Infant, and Child Health (continued)

Moved away from target ${ }^{1}$

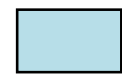

Moved toward

target

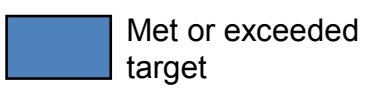

16-19. Exclusive breastfeeding

e. Through 6 months

16-21. Hospital discharges for sickle cell disease (per 100,000 black or African American children, $\leq 9$ years)

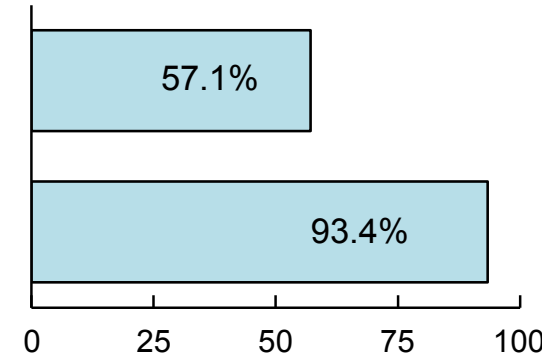

\begin{tabular}{|c|c|c|c|c|c|}
\hline \multirow{2}{*}{$\begin{array}{l}2010 \\
\text { Target }\end{array}$} & \multirow{2}{*}{$\begin{array}{c}\text { Baseline } \\
\text { (Year) }\end{array}$} & \multirow{2}{*}{$\begin{array}{l}\text { Final } \\
\text { (Year) }\end{array}$} & \multicolumn{3}{|c|}{ Baseline vs. Final } \\
\hline & & & Difference $^{2}$ & $\begin{array}{l}\text { Statistically } \\
\text { Significant }^{3}\end{array}$ & $\begin{array}{l}\text { Percent } \\
\text { Change }\end{array}$ \\
\hline $17 \%$ & $\begin{array}{c}10 \% \\
(2003)\end{array}$ & $\begin{array}{c}14 \% \\
(2006)\end{array}$ & 4 & Yes & $40.0 \%$ \\
\hline 182.2 & $\begin{array}{c}227.8 \\
(1995-99)\end{array}$ & $\begin{array}{c}185.2 \\
(2003-07)\end{array}$ & -42.6 & No & $-18.7 \%$ \\
\hline
\end{tabular}

Percent of targeted change achieved ${ }^{5}$

\section{NOTES}

See the Reader's Guide for more information on how to read this figure. See DATA2010 at http://wonder.cdc.gov/data2010 for all Healthy People 2010 tracking data. Tracking data are not available for objectives 16-7, 16-12, 16-18, 16-20a, 16-20b, 16-22, and 16-23. Objectives 16-5b, 16-5c, 16-14d, and 16-20c were deleted at the Midcourse Review.

\section{FOOTNOTES}

${ }^{1}$ Movement away from target is not quantified using the percent of targeted change achieved. See Technical Appendix for more information.

${ }^{2}$ Difference $=$ Final value - Baseline value. Differences between percents $(\%)$ are measured in percentage points.

${ }^{3}$ When estimates of variability are available, the statistical significance of the difference between the final value and the baseline value is assessed at the 0.05 level. See Technical Appendix for more information.

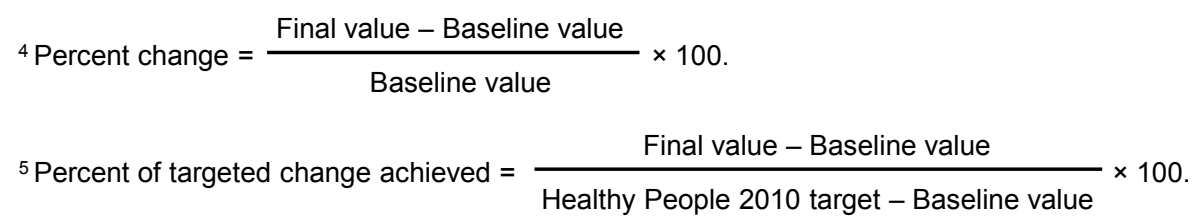


Figure 16-1. Progress Toward Target Attainment for Focus Area 16: Maternal, Infant, and Child Health (continued)

\section{DATA SOURCES}

16-1a. National Vital Statistics System-Fetal Death and Natality (NVSS-FD, NVSS-N), CDC, NCHS.

16-1b. National Vital Statistics System-Fetal Death, Mortality and Natality (NVSS-FD, NVSS-M, NVSS-N), CDC, NCHS.

16-1c-h. National Vital Statistics System-Mortality and Natality (NVSS-M, NVSS-N), CDC, NCHS.

16-2a-b. National Vital Statistics System-Mortality (NVSS-M), CDC, NCHS.

16-3a-C. National Vital Statistics System-Mortality (NVSS-M), CDC, NCHS.

16-4. National Vital Statistics System-Mortality and Natality (NVSS-M, NVSS-N), CDC, NCHS.

16-5a. National Hospital Discharge Survey (NHDS), CDC, NCHS.

16-6a-b. $\quad$ National Vital Statistics System-Natality (NVSS-N), CDC, NCHS.

16-8. Title V Reporting System, HRSA.

16-9a-b. National Vital Statistics System-Natality (NVSS-N), CDC, NCHS.

16-10a-b. National Vital Statistics System-Natality (NVSS-N), CDC, NCHS.

16-11a-c. National Vital Statistics System-Natality (NVSS-N), CDC, NCHS.

16-13. National Infant Sleep Position Study (NISP), NIH, NICHD.

16-14a-c. Metropolitan Atlanta Development Disabilities Surveillance Program (MADDSP), CDC, NCBDDD.

16-15. National Birth Defects Prevention Network (NBDPN), CDC, NCBDDD.

16-16a-b. National Health and Nutrition Examination Survey (NHANES), CDC, NCHS.

16-17a-b. National Survey on Drug Use and Health (NSDUH), SAMHSA.

16-17c. National Vital Statistics System-Natality (NVSS-N), CDC, NCHS

16-17d. National Survey on Drug Use and Health (NSDUH), SAMHSA.

16-19a-e. National Immunization Survey (NIS), CDC, NCIRD and NCHS.

16-21. National Hospital Discharge Survey (NHDS), CDC, NCHS 
Figure 16-2. Health Disparities Table for Focus Area 16: Maternal, Infant, and Child Health

Disparities from the best group rate for each characteristic at the most recent data point and changes in disparity from the baseline to the most recent data point.

Characteristics and Groups

Population-based objectives

16-1a. Fetal deaths $(20+$ weeks gestation, per 1,000 live births plus fetal deaths) $(1997,2005)^{1,2,3 *}$

16-1b. Perinatal deaths ( 28 weeks gestation to $<7$ days after birth, per 1,000 live births plus fetal deaths) (1997, $2005)^{1,2,3 \star}$

16-1c. All Infant deaths (<1 year, per 1,000 live births) (1998, $2006)^{1,2,3 *}$

16-1d. Neonatal deaths (<28 days, per 1,000 live births) (1998, $2006)^{1,2,3 *}$

16-1e. Postneonatal deaths ( 28 days to $<1$ year, per 1,000 live births) $(1998,2006)^{1,2,3 \text { * }}$

16-1f. Infant deaths due to birth defects ( $<1$ year, per 1,000 live births) $(1999,2006)^{1,2,3 *}$

16-1g. Infant deaths due to congenital heart defects $(<1$ year, per 1,000 live births) $(1999,2006)^{1,2,3 *}$

16-1h. Infant deaths due to sudden infant death syndrome (SIDS) $\left(<1\right.$ year, per 1,000 live births) $(1999,2006)^{1,2,3 \text { * }}$

16-2a. Child deaths-1-4 years (per 100,000 population) (1998, $2007)^{\star}$

b. Child deaths - 5-9 years (per 100,000 population) (1998, 2007)*

16-3a. Adolescent and young adults deaths $-10-14$ years (per $100,000$ population $)(1998,2007)^{\star}$

b. Adolescent and young adult deaths-15-19 years (per 100,000 population) $(1998,2007)^{\star}$

c. Adolescent and young adult deaths-20-24 years (per 100,000 population) $(1998,2007)^{\star}$

16-4. Maternal deaths (per 100,000 live births) $(1999,2002)$

16-5a. Maternal complications during hospitalized labor and delivery (per 100 deliveries) $(1998,2007)^{\star}$

16-6a. Prenatal care-Beginning in first trimester $(1998,2002)^{\star}$

b. Prenatal care-Early and adequate $(1998,2002)^{*}$

16-7. Childbirth class attendance among pregnant women (2000)†

16-9a. Cesarean births-No prior cesarean birth (1998, $2007)^{1,2,3 *}$

b. Cesarean births-Prior cesarean birth $(1998,2007)^{1,2,3 *}$

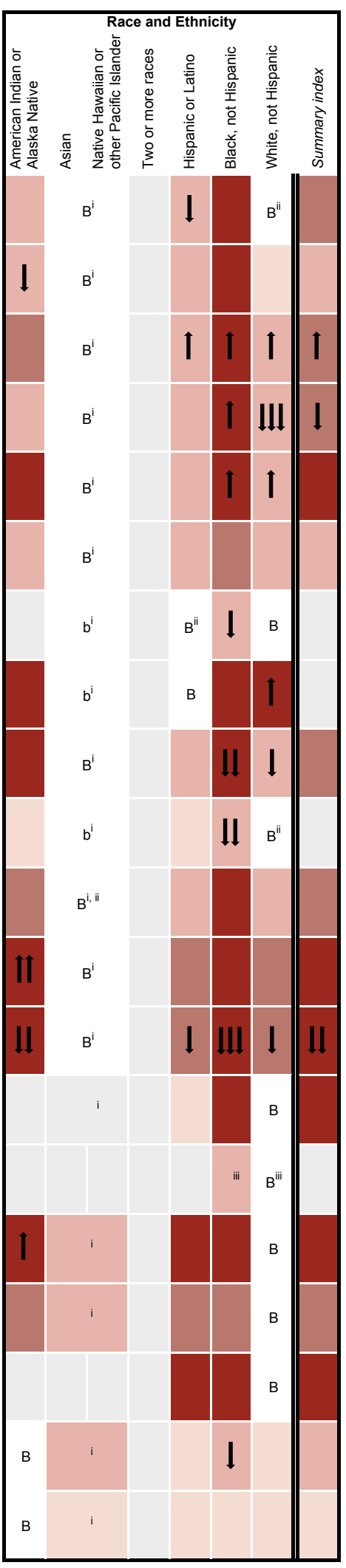

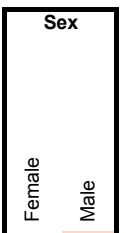

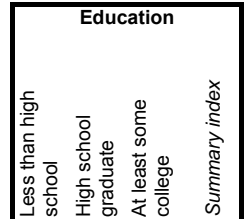

B

B

B

B

B

$B$

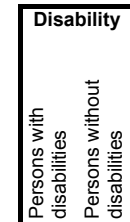

B

B

B

B

B

B

B $B^{i i}$

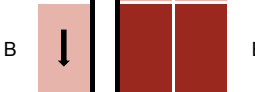

B

B

B

B

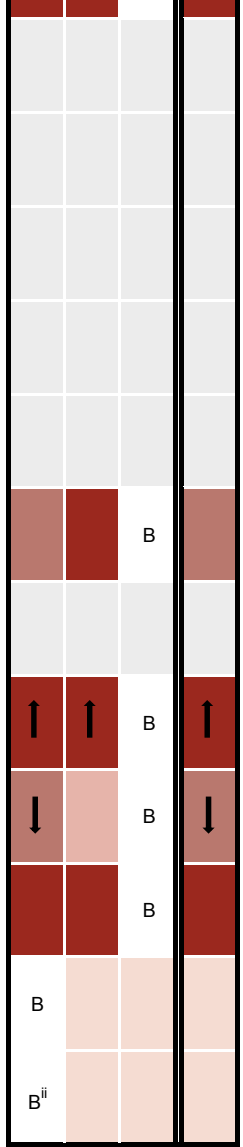

(continued) 
Figure 16-2. Health Disparities Table for Focus Area 16: Maternal, Infant, and Child Health (continued)

\section{Population-based objectives}

16-10a. Low birth weight (LBW), infants (<2,500 grams) (1998, $2007)^{1,2,3 *}$

16-10b. Very low birth weight (VLBW), infants $(<1,500$ grams $)$ $(1998,2007)^{1,2,3 *}$

16-11a. Preterm live births-Total (<37 weeks gestation) (1998 $2007)^{1,2,3 *}$

b. Preterm live births-32-36 weeks gestation (1998, $2007)^{1,2,3 *}$

c. Preterm live births - <32 weeks gestation (1998, $2007)^{1,2,3 *}$

16-13. Infants put to sleep on their backs (<8 months) (1996, 2009)†

16-14a. Developmental disabilities-Mental retardation-IQ $\leq 70$ (per 10,000 population, Metropolitan Atlanta, 8 years) $(1991-94,2008)^{\star}$

b. Developmental disabilities-Cerebral palsy (per 10,000 population, Metropolitan Atlanta, 8 years) (1991-94, 2008)*

c. Developmental disabilities-Age at first identification of autism spectrum disorder (in months, Metropolitan Atlanta, 8 years) $(1996,2008) \dagger$

16-15. Spina bifida and other neural tube defects (new cases per 100,000 live births) $(1996,2007)^{4 *}$

16-16a. Folic acid consumption $\geq 400 \mu$ g daily by nonpregnant women (15-44 years) $(1991-94,2005-06)^{5} \ddagger$

16-16b. Median red blood cell (RBC) folate level among nonpregnant women $(\mathrm{ng} / \mathrm{ml}, 15-44$ years) (1988-94, 2005-06 $)^{6 *}$

16-17a. Pregnant women abstaining from alcohol in past month (15-44 years) $(2002-03,2008-09)^{*}$

b. Pregnant women abstaining from binge drinking in past month (15-44 years) $(2002-03,2008-09)^{\star}$

c. Pregnant women abstaining from cigarette smoking during pregnancy $(1998,2002)^{\star}$

d. Pregnant women abstaining from illicit drugs in past month (15-44 years) $(2002-03,2008-09)^{\star}$

16-18. Fetal alcohol syndrome (cases per 1,000 live births) (1995-97)†

16-19a. Breastfeeding-Ever $(2000,2006)^{2,3 *}$

b. Breastfeeding-At 6 months $(2000,2006)^{2,3 *}$

c. Breastfeeding-At 1 year $(2000,2006)^{2,3 *}$

16-19d. Exclusive breastfeeding-Through 3 months (2003, $2006)^{2,3 *}$

e. Exclusive breastfeeding-Through 6 months (2003, $2006)^{2,3 *}$

Maternal, Infant, and Child Health

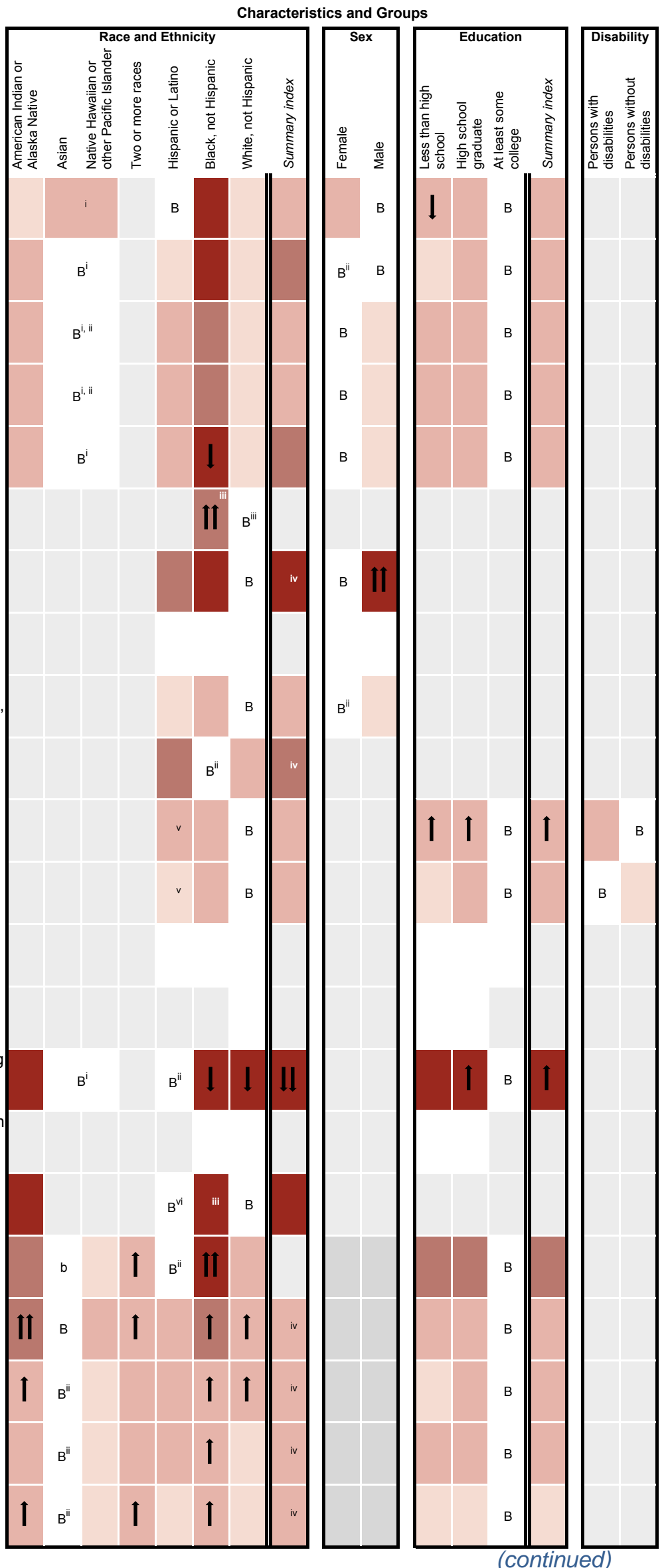

Page 16-25 


\section{Population-based objectives}

16-21. Hospital discharges for sickle cell disease (per 100,000 black or African American children, $\leq 9$ years) (1995-99, 2003-07)*

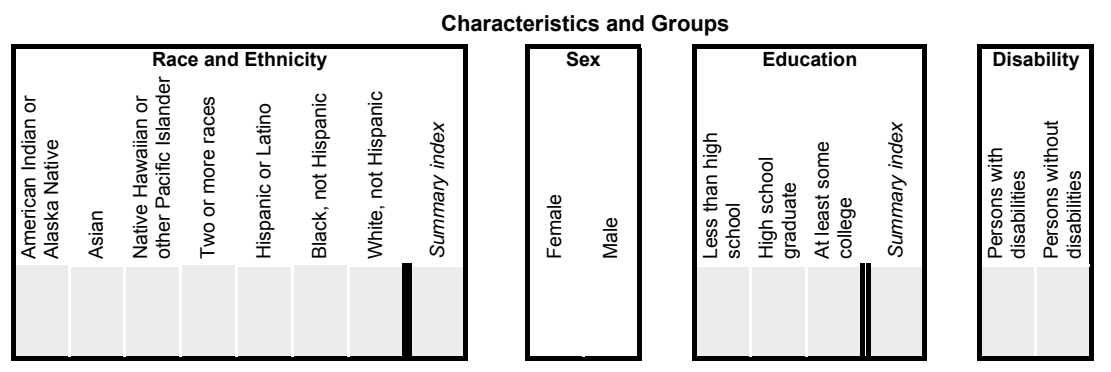

NOTES

See DATA2010 at http://wonder.cdc.gov/data2010 for all Healthy People 2010 tracking data. Disparity data are either unavailable or not applicable for objectives 16-8, 16-12, 16-20a and b, 16-22, and 16-23. Objectives 16-5b and c, 16-14d, and 16-20c, were deleted at Midcourse Review.

Years in parentheses represent the baseline and most recent data years (if available).

Disparity from the best group rate is defined as the percent difference between the best group rate and each of the other group rates for a characteristic (e.g., race and ethnicity). The summary index is the average of these percent differences for a characteristic. Change in disparity is estimated by subtracting the disparity at baseline from the disparity at the most recent data point. Change in the summary index is estimated by subtracting the summary index at baseline from the summary index at the most recent data point. See Technical Appendix for more information.

LEGEND

\begin{tabular}{l|l|l|l|l|l|l|l}
\hline $\begin{array}{l}\text { The "best" group rate at the most recent } \\
\text { data point. }\end{array}$ & B $\begin{array}{l}\text { The group with the best rate for } \\
\text { specified characteristic. }\end{array}$ & $\begin{array}{l}\text { Most favorable group rate for specified } \\
\text { characteristic, but reliability criterion not met. }\end{array}$ \\
\hline
\end{tabular}

\begin{tabular}{|c|c|c|c|c|}
\hline \multirow[b]{2}{*}{$\begin{array}{l}\text { Disparity from the best group rate at the } \\
\text { most recent data point. }\end{array}$} & \multicolumn{4}{|c|}{ Percent difference from the best group rate } \\
\hline & $\begin{array}{l}\text { Less than } 10 \% \text {, or difference not } \\
\text { statistically significant (when } \\
\text { estimates of variability are available). }\end{array}$ & $10 \%-49 \%$ & $50 \%-99 \%$ & $100 \%$ or more \\
\hline
\end{tabular}

Changes in disparity over time are shown when:

(a) disparities data are available at both baseline and most recent time points;

(b) data are not for the group(s) indicated by "B" or "b" at either time point; and

(c) the change is greater than or equal to 10 percentage points and statistically

significant, or when the change is greater than or equal to 10 percentage

points and estimates of variability were not available. See Technical Appendix.

\begin{tabular}{|l|l|l|l|l} 
Increase in disparity (percentage points) \\
10-49 points
\end{tabular}

Availability of Data $\quad$ Data not available.

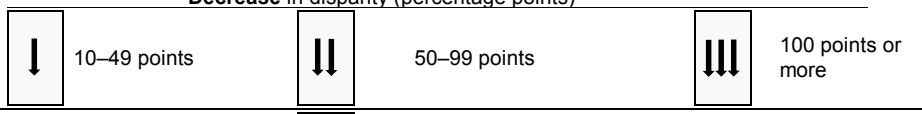

\section{OTES}

* Measures of variability were available. Thus, the variability of best group rates was assessed, and statistical significance was tested. Disparities of $10 \%$ or more are displayed when the differences from the best group rate are statistically significant at the 0.05 level. Changes in disparities over time are indicated by arrows when the changes are greater than or equal to 10 percentage points and are statistically significant at the 0.05 level. See Technical Appendix.

† Measures of variability were not available. Thus, the variability of best group rates was not assessed, and statistical significance could not be tested. Nonetheless, disparities and changes in disparities over time are displayed according to their magnitude. See Technical Appendix.

‡ Measures of variability for data by education level and disability status were available only for the most recent data. Thus, the variability of best group rates was assessed only for the most recent data, and statistical significance was tested only for the most recent data. Disparities of $10 \%$ or more are displayed when the differences from the best group rate are statistically significant at the 0.05 level. Changes in disparities over time are displayed according to their magnitude, since measures of variability were not available at baseline and therefore statistical significance of changes in disparity could not be tested. See Technical Appendix.

1 Most recent data by education level are for 2002 .

2 Data by education level are for the mother.

3 Data by race and ethnicity are for the mother.

4 Baseline data by race and ethnicity are for 1998.

5 Baseline data by race and ethnicity are for 2001-02. Measures of variability were available by race and ethnicity for $2001-02$, see footnote * above.

6 Baseline data by disability status are for 1991-94.

i Data are for Asian or Pacific Islander.

ii The group with the best rate at the most recent data point is different from the group with the best rate at baseline. Both rates met the reliability criterion. See Technical Appendix.

iii Data include persons of Hispanic origin.

iv Change in the summary index cannot be assessed. See Technical Appendix.

$\checkmark$ Data are for Mexican American.

vi Data exclude black of Hispanic origin.

DATA SOURCES

16-1a. National Vital Statistics System—Fetal Death and Natality (NVSS-FD, NVSS-N), CDC, 16-11a-c. National Vital Statistics System-Natality (NVSS-N), CDC, NCHS. NCHS.

16-1b. National Vital Statistics System—Fetal Death, Mortality, and Natality (NVSS-FD, NVSS- 16-13. M, NVSS-N), CDC, NCHS.

16-1c-h. National Vital Statistics System-Mortality and Natality (NVSS-M, NVSS-N), CDC, NCHS.

16-2a-b. National Vital Statistics System-Mortality (NVSS-M), CDC, NCHS.

16-3a-c. National Vital Statistics System-Mortality (NVSS-M), CDC, NCHS.

16-4. National Vital Statistics System-Mortality and Natality (NVSS-M, NVSS-N), CDC NCHS.

16-5a. National Hospital Discharge Survey (NHDS), CDC, NCHS.

16-6a-b. National Vital Statistics System-Natality (NVSS-N), CDC, NCHS.

16-7. National Survey of Early Childhood Health (NSECH): HRSA, MCHB; CDC, NCHS

16-9a-b. National Vital Statistics System-Natality (NVSS-N), CDC, NCHS.

16-10a-b. National Vital Statistics System-Natality (NVSS-N), CDC, NCHS.

Maternal, Infant, and Child Health

16-14a-c. Metropolitan Atlanta Development Disabilities Surveillance Program (MADDSP), CDC, NCBDDD

16-15. National Birth Defects Prevention Network (NBDPN), CDC, NCBDDD.

16-16a-b. National Health and Nutrition Examination Survey (NHANES), CDC, NCHS.

16-17a-b. National Survey on Drug Use and Health (NSDUH), SAMHSA.

16-17c. National Vital Statistics System-Natality (NVSS-N), CDC, NCHS.

16-17d. National Survey on Drug Use and Health (NSDUH), SAMHSA.

16-18. Fetal Alcohol Syndrome Surveillance System (FASSNet), CDC, NCBDDD.

16-19a. National Immunization Survey (NIS), CDC, NCIRD and NCHS

16-21. National Hospital Discharge Survey (NHDS), CDC, NCHS. 
Figure 16-3. Low Birth Weight (LBW), Infants 2006-08

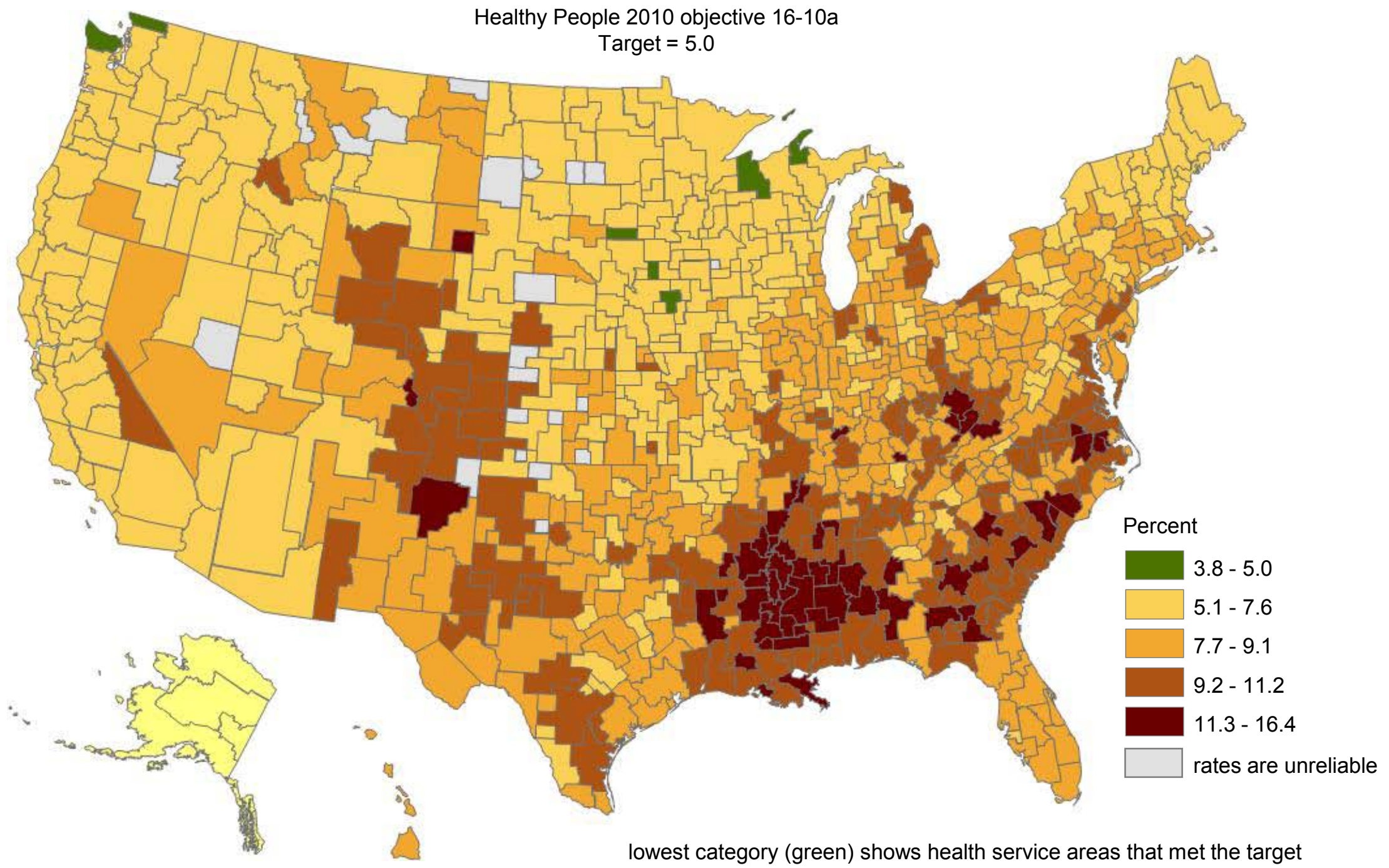

NOTES: Data are for low (<2,500 grams) birth weight births as a percent of all live births Rates are displayed by modified Jenks classification for U. S. health service area. SOURCE: National Vital Statistics System - Natality, CDC, NCHS. 
Figure 16-4. Preterm Live Births Total (<37 Weeks Gestation) 2006-08

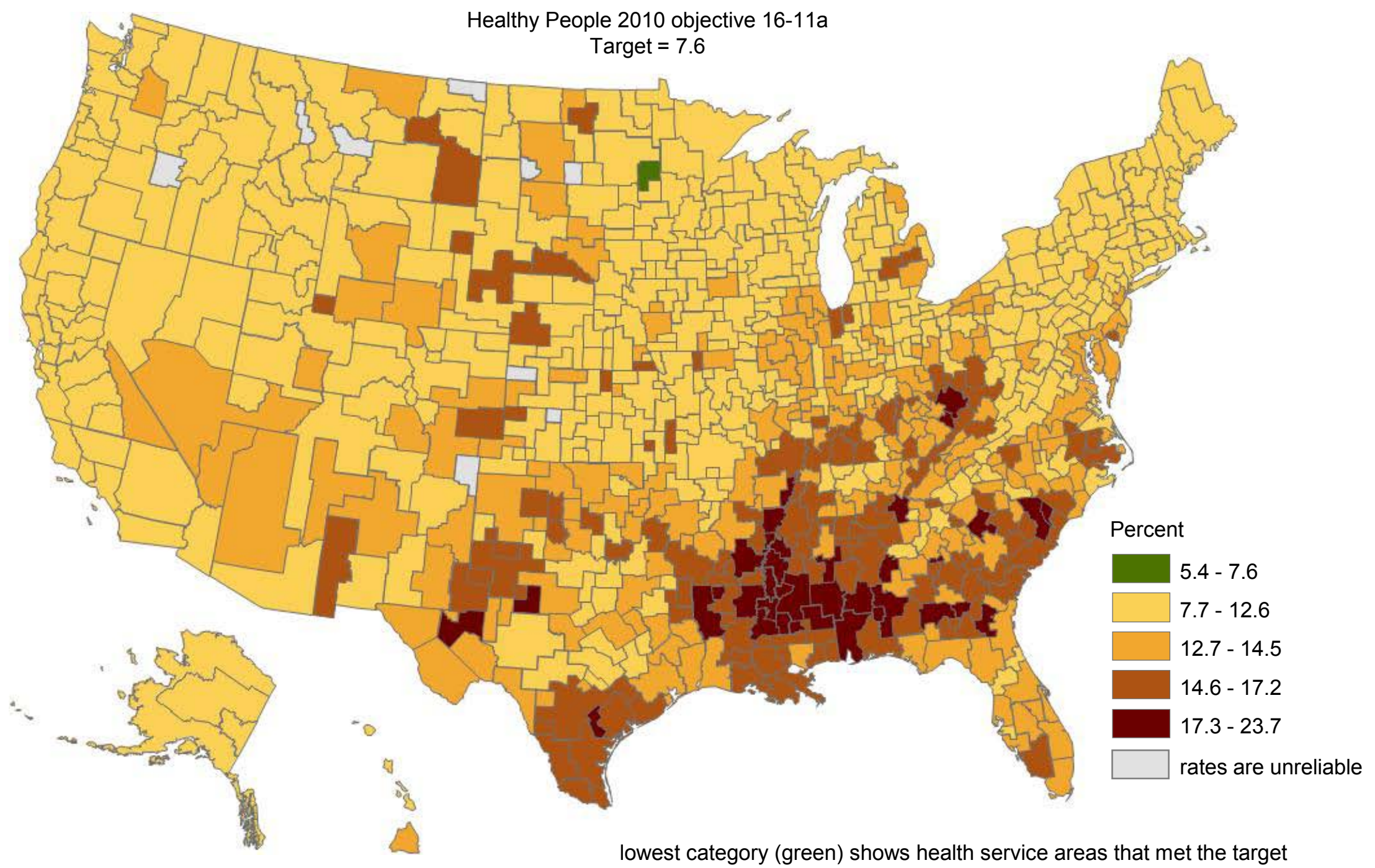

NOTES: Data are for preterm births (<37 weeks gestations) as a percent of all live births Rates are displayed by modified Jenks classification for U. S. health service area. SOURCE: National Vital Statistics System - Natality, CDC, NCHS. 


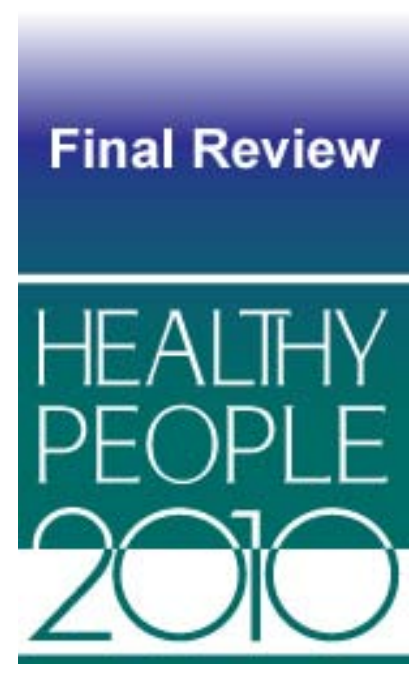

\section{Medical Product} Safety

\section{Lead Agency}

Food and Drug Administration

\section{Contents}

Goal.

Highlights.

Summary of Progress.

Transition to Healthy People 2020. 17-3

Data Considerations 17-5

Notes 17-5

Comprehensive Summary of Objectives 17-7

Progress Chart 17-8

Health Disparities Table 17-9 


\section{Goal: Ensure the safe and effective use of medical products}

The objectives in this chapter track the use of electronic medical records and prescription services, patient receipt of counseling about prescriptions, and availability of surveillance systems to monitor adverse drug reactions.

All Healthy People tracking data quoted in this chapter, along with technical information and operational definitions for each objective, can be found in the Healthy People 2010 database, DATA2010, available from http://wonder.cdc.gov/data1010/.

More information about this Focus Area can be found in the following publications:

- Healthy People 2010: Understanding and Improving Health, available from http://www.healthypeople.gov/2010/Document/tableofcontents.htm\#under.

- Healthy People 2010 Midcourse Review, available from http://www.healthypeople.gov/2010/data/midcourse/html/default.htm\#FocusAreas.

\section{Highlights}

- Some progress was achieved in objectives for this Focus Area during the past decade [1]. Five of the eight Medical Product Safety objectives with data to measure progress (objectives 17-2a through 17-2d, and 17-5a) moved toward or achieved their Healthy People 2010 targets (Figure 17-1).

- The use of electronic medical records by health care providers in health care organizations (objective $17-2 \mathrm{a}$ ) increased by $166.7 \%$ between 2000 and 2007 , from $12 \%$ to $32 \%$, exceeding the 2010 target of $18 \%$.

- The use of computerized prescriber order entry in general and children's hospitals (objective 17-2c) increased by 533.3\% between 2003 and 2010, from 3\% to 19\%, exceeding the 2010 target of $4 \%$. Similarly, the use of computerized prescriber order entry in urban acute care facilities (objective 17-2d) increased by $76.9 \%$ between 2007 and 2009, from $13 \%$ to $23 \%$, exceeding the 2010 target of $20 \%$.

- The monitoring of adverse events associated with medical practice (objective 17-1a) declined by $25.6 \%$ between 1999 and 2009, from 82\% to 61\%, moving away from the 2010 target of $90 \%$.

- Blood donations among adults aged 18 years and over (objective 17-6) remained stable. Six percent (age-adjusted) of the U.S. population donated blood in 2008, showing no change from 1998. This objective did not meet the 2010 target of $8 \%$. 


\section{Summary of Progress}

- Figure 17-1 presents a quantitative assessment of progress in achieving the Healthy People 2010 objectives for Medical Product Safety [1]. Data to measure progress toward target attainment were available for eight objectives. Of these:

- Three objectives (17-2a, c and d) met or exceeded their Healthy People 2010 targets.

- Two objectives (17-2b and 17-5a) moved toward their targets, but data to test the significance of the difference between the baseline and final data points were unavailable.

- One objective (17-6) showed no change.

- Two objectives (17-1a and 17-5b) moved away from their targets, but data to test the significance of the difference between the baseline and final data points were unavailable.

- One objective (17-4) had no data available to measure progress and two objectives (17-1b and 17-3) were dropped during the decade [2].

- Figure 17-2 displays health disparities in Medical Product Safety from the best group rate for each characteristic at the most recent data point [3]. It also displays changes in disparities from baseline to the most recent data point [4].

- Disparity data were available for only one objective (17-6, blood donations). This objective showed no health disparities of $10 \%$ or more by race and ethnicity, sex, education level, or disability status.

\section{Transition to Healthy People 2020}

The range of topics covered in the Medical Product Safety chapter for Healthy People 2020 has been narrowed in comparison to the objectives presented in Healthy People 2010. The Healthy People 2010 Focus Area covered various topics such as electronic medical record use, blood donation, oral counseling on prescription medications and adverse medical events. In contrast, the Healthy People 2020 Topic Area focuses on overall improvement of patient treatment and appropriate use of medical products including drugs, biological products and medical devices. The Healthy People 2020 objectives reflect strong scientific support for safe use of medical products, which promotes better health among Americans. See HealthyPeople.gov for a complete list of Healthy People 2020 topics and objectives.

Objectives in the Healthy People 2020 Medical Product Safety Topic Area fall into two major categories:

- Monitoring of adverse medical events

- Safe and effective treatment of pain. 
The differences between the Healthy People 2010 objectives and those included in Healthy People 2020 objectives are summarized below:

- The Healthy People 2020 Medical Product Safety Topic Area has a total of 11 objectives, five of which are developmental [5]. The Healthy People 2010 Medical Product Safety Focus Area also had 11 objectives.

- Two Healthy People 2010 objectives were retained "as is" [6]. Monitoring and analyzing adverse medical events within healthcare organizations and blood donations (objective 171a) was retained. The blood donation objective (objective 17-6) was also retained, but was moved to the Blood Safety Topic Area.

- Two Healthy People 2010 objectives were deleted at the Midcourse Review due to a lack of tracking data [2]. The objectives on adverse medical events associated with medical devices (objective 17-1b) and provider review of medications taken by patients and those with chronic conditions (objective 17-3) were dropped.

- Seven Healthy People 2010 objectives were archived [7].

- Three objectives that dealt with receipt of oral counseling and useful information regarding prescription medications were archived due to a lack of national data in the future (objectives 17-4, 17-5a, and 17-5b).

- Four objectives that refer to electronic medical record use (objectives 17-2a through 17-2d) in Healthy People 2010 were also archived as the Medical Product Safety Topic Area is no longer focusing on that issue.

- While the Healthy People 2010 objectives on electronic medical record use have all been archived there is a new objective on monitoring the proportion of medical practices that use electronic health records in the Healthy People 2020 Health Communication and Health Information Technology Topic Area.

- Nine new objectives were added to the Healthy People 2020 Medical Product Safety Topic Area:

- Four objectives to track the safe and effective treatment of pain have been added. These objectives track patients suffering from untreated pain due to a lack of access to pain treatment, the number of non-FDA approved pain medication on the market, and serious injuries and death from pain medications.

- Another new objective tracks the use of safe and effective medical products associated with predictive biomarkers.

- Four objectives were added to measure the number of emergency department visits for common, preventable adverse events from medication.

Appendix D, "A Crosswalk Between Objectives from Healthy People 2010 to Healthy People 2020," summarizes the changes between the two decades of objectives, reflecting new knowledge and direction for this area. 


\section{Data Considerations}

In general, data on educational attainment are presented for persons aged 25 years and over, consistent with guidance given by the U.S. Bureau of the Census. However, because of the requirements of the different data systems, the age groups used to calculate educational attainment for any specific objective may differ from the age groups used to report the data for other Healthy People 2010 objectives, as well as from select populations within the same objective. Therefore, the reader is urged to exercise caution in interpreting the data by educational attainment shown in the Health Disparities Table. See Healthy People 2010: General Data Issues, referenced below.

Additional information on data issues is available from the following sources:

- All Healthy People 2010 tracking data can be found in the Healthy People 2010 database, DATA2010, available from http://wonder.cdc.gov/data2010/.

- Detailed information about the data and data sources used to support these objectives can be found in the Operational Definitions on the DATA2010 website, available from http://wonder.cdc.gov/data2010/focusod.htm.

- More information on statistical issues related to Healthy People tracking and measurement can be found in the Technical Appendix and in Healthy People 2010: General Data Issues, which is available in the Data Issues section of the NCHS Healthy People website under Healthy People 2010.

\section{Notes}

1. Displayed in the Progress Chart (Figure 17-1), the percent of targeted change achieved expresses the difference between the baseline and the final value relative to the initial difference between the baseline and the Healthy People 2010 target. As such, it is a relative measure of progress toward attaining the Healthy People 2010 target. See the Reader's Guide for more information. When standard errors were available, the difference between the baseline and the final value was tested at the 0.05 level of significance. See the Figure 17-1 footnotes, as well as the Technical Appendix, for more detail.

2. Dropped objectives were not carried forward into Healthy People 2020. These objectives were either developmental or deleted at the Healthy People 2010 Midcourse Review or at another time in Healthy People 2010.

3. Information about disparities among select populations is shown in the Health Disparities Table (Figure 17-2). Disparity from the best group rate is defined as the percent difference between the best group rate and each of the other group rates for a characteristic. For example, racial and ethnic health disparities are measured as the percent difference between the best racial and ethnic group rate and each of the other racial and ethnic group rates. Similarly, disparities by sex are measured as the percent difference between the better group rate (e.g., female) and the rate for the other group (e.g., male). Some objectives are expressed in terms of favorable events or conditions that are to be increased, while others are expressed in terms of adverse events or conditions that are to be reduced. In order to facilitate comparison of health disparities across different objectives, disparity is measured only in terms of adverse events or conditions. For comparability across objectives, objectives that are expressed in terms of favorable events or conditions are re-expressed using the adverse event or condition for the purpose of computing disparity, but they are not otherwise restated or changed. For example, objective 1-1, to increase the proportion of persons with health 
insurance (e.g., $72 \%$ of the American Indian or Alaska Native population aged under 65 years had some form of health insurance in 2008), is expressed in terms of the percentage of persons without health insurance (e.g., $100 \%-72 \%=28 \%$ of the American Indian or Alaska Native population aged under 65 years did not have any form of health insurance in 2008) when the disparity from the best group rate is calculated. See the Reader's Guide for more information. When standard errors were available, the difference between the best group rate and each of the other group rates was tested at the 0.05 level of significance. See the Figure 17-2 footnotes, as well as the Technical Appendix, for more detail.

4. The change in disparity is estimated by subtracting the disparity at baseline from the disparity at the most recent data point and, therefore, is expressed as a change in percentage points. See the Reader's Guide for more information. When standard errors were available, the change in disparity was tested at the 0.05 level of significance. See the Figure 17-2 footnotes, as well as the Technical Appendix, for more detail.

5. To be included in Healthy People 2010, an objective must have a national data source that provides a baseline and at least one additional data point for tracking progress. Some objectives lacked baseline data at the time of their development but had a potential data source and were considered of sufficient national importance to be included in Healthy People. These are called "developmental" objectives. When data become available, a developmental objective is moved to measurable status and a Healthy People target can be set.

6. Retained "as is" objectives have no change in the numerator definition or in the denominator definition between the Healthy People 2010 and Healthy People 2020 objectives. These include objectives that were developmental in Healthy People 2010 and are developmental in Healthy People 2020 and for which no numerator or denominator information was available.

7. Archived objectives had at least one data point in Healthy People 2010 but were not carried forward into Healthy People 2020. 


\section{Comprehensive Summary of Objectives: Medical Product Safety}

\begin{tabular}{|c|c|c|}
\hline Objective & Description & Data Source or Objective Status \\
\hline $17-1 \mathrm{a}$ & $\begin{array}{l}\text { Monitoring and analyzing adverse events associated with } \\
\text { medical therapies }\end{array}$ & $\begin{array}{l}\text { National Survey of Pharmacy Practice in Acute Care Settings; } \\
\text { American Society of Health Systems Pharmacists. }\end{array}$ \\
\hline $17-1 b$ & $\begin{array}{l}\text { Monitoring of adverse medical events associated with medical } \\
\text { devices }\end{array}$ & Dropped \\
\hline $17-2 \mathrm{a}$ & $\begin{array}{l}\text { Electronic medical record use by health care providers in health } \\
\text { care organizations }\end{array}$ & $\begin{array}{l}\text { Annual Health Care Information and Management Systems } \\
\text { Society Leadership Survey, Healthcare Information and } \\
\text { Management Systems Society. }\end{array}$ \\
\hline $17-2 b$ & $\begin{array}{l}\text { Electronic medical record use by pharmacists in managed care } \\
\text { and integrated health systems }\end{array}$ & $\begin{array}{l}\text { National Survey of Ambulatory Care Responsibilities of } \\
\text { Pharmacists in Managed Care and Integrated Health Systems, } \\
\text { American Society of Health Systems Pharmacists. }\end{array}$ \\
\hline $17-2 c$ & $\begin{array}{l}\text { Computerized prescriber order entry use by general and } \\
\text { children's hospitals }\end{array}$ & $\begin{array}{l}\text { National Survey of Pharmacy Practice in Hospital Settings, } \\
\text { American Society of Health Systems Pharmacists. }\end{array}$ \\
\hline $17-2 d$ & $\begin{array}{l}\text { Computerized prescriber order entry use by urban acute care } \\
\text { facilities }\end{array}$ & The Leapfrog Group Hospital Patient Safety Survey. \\
\hline $17-3$ & $\begin{array}{l}\text { Provider review of medications taken by older patients and } \\
\text { those with chronic conditions }\end{array}$ & Dropped \\
\hline $17-4$ & $\begin{array}{l}\text { Receipt of useful information about prescriptions from } \\
\text { pharmacies }\end{array}$ & $\begin{array}{l}\text { Evaluation of Written Prescription Information Provided In } \\
\text { Community Pharmacies, Food and Drug Administration. }\end{array}$ \\
\hline $17-5 a$ & Oral counseling about medications from prescribers & $\begin{array}{l}\text { National Survey of Prescription Medicine Information Received } \\
\text { by Consumers, FDA. }\end{array}$ \\
\hline $17-5 b$ & Oral counseling about medications from pharmacists & $\begin{array}{l}\text { National Survey of Prescription Medicine Information Received } \\
\text { by Consumers, FDA. }\end{array}$ \\
\hline $17-6$ & Blood donations (age adjusted, 18+ years) & National Health Interview Survey (NHIS), CDC, NCHS. \\
\hline
\end{tabular}


Figure 17-1. Progress Toward Target Attainment for Focus Area 17: Medical Product Safety

Moved away

from target ${ }^{1}$

Moved toward

target

Met or exceeded

target

17-1a. Monitoring and analyzing adverse events associated with medical therapies

17-2. Electronic medical record use by

a. Health care providers in health care organizations

b. Pharmacists in managed care and integrated health systems

Computerized prescriber order entry use by

c. General and children's hospitals

d. Urban acute care facilities

17-5. Oral counseling about medications from

a. Prescribers

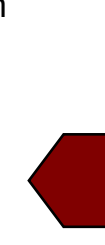

17-6. Blood donations (age adjusted, 18+ years)

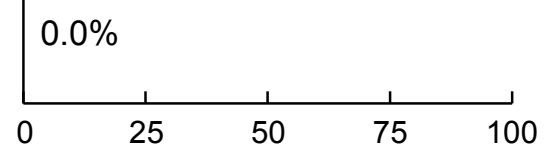

\begin{tabular}{|c|c|c|c|c|c|}
\hline \multirow{2}{*}{$\begin{array}{l}2010 \\
\text { Target }\end{array}$} & \multirow{2}{*}{$\begin{array}{c}\text { Baseline } \\
\text { (Year) }\end{array}$} & \multirow{2}{*}{$\begin{array}{l}\text { Final } \\
\text { (Year) }\end{array}$} & \multicolumn{3}{|c|}{ Baseline vs. Final } \\
\hline & & & Difference $^{2}$ & $\begin{array}{l}\text { Statistically } \\
\text { Significant }\end{array}$ & $\begin{array}{l}\text { Percent } \\
\text { Change }^{4}\end{array}$ \\
\hline $90 \%$ & $\begin{array}{c}82 \% \\
(1998)\end{array}$ & $\begin{array}{c}61 \% \\
(2009)\end{array}$ & -21 & $\begin{array}{c}\text { Not } \\
\text { tested }\end{array}$ & $-25.6 \%$ \\
\hline $18 \%$ & $\begin{array}{c}12 \% \\
(2000)\end{array}$ & $\begin{array}{c}32 \% \\
(2007)\end{array}$ & 20 & $\begin{array}{c}\text { Not } \\
\text { tested }\end{array}$ & $166.7 \%$ \\
\hline $46 \%$ & $\begin{array}{c}31 \% \\
(1999)\end{array}$ & $\begin{array}{c}33 \% \\
(2001)\end{array}$ & 2 & $\begin{array}{c}\text { Not } \\
\text { tested }\end{array}$ & $6.5 \%$ \\
\hline $4 \%$ & $\begin{array}{c}3 \% \\
(2003)\end{array}$ & $\begin{array}{c}19 \% \\
(2010)\end{array}$ & 16 & $\begin{array}{l}\text { Not } \\
\text { tested }\end{array}$ & $533.3 \%$ \\
\hline $20 \%$ & $\begin{array}{c}13 \% \\
(2007)\end{array}$ & $\begin{array}{c}23 \% \\
(2009)\end{array}$ & 10 & $\begin{array}{c}\text { Not } \\
\text { tested }\end{array}$ & $76.9 \%$ \\
\hline $95 \%$ & $\begin{array}{c}24 \% \\
(1998)\end{array}$ & $\begin{array}{c}26 \% \\
(2004)\end{array}$ & 2 & $\begin{array}{c}\text { Not } \\
\text { tested }\end{array}$ & $8.3 \%$ \\
\hline $95 \%$ & $\begin{array}{c}14 \% \\
(1998)\end{array}$ & $\begin{array}{c}6 \% \\
(2004)\end{array}$ & -8 & $\begin{array}{l}\text { Not } \\
\text { tested }\end{array}$ & $-57.1 \%$ \\
\hline $8 \%$ & $\begin{array}{c}6 \% \\
(1998)\end{array}$ & $\begin{array}{c}6 \% \\
(2008)\end{array}$ & 0 & No & $0.0 \%$ \\
\hline
\end{tabular}

Percent of targeted change achieved ${ }^{5}$

(continued) 


\section{Figure 17-1. Progress Toward Target Attainment for Focus Area 17: Medical Product Safety (continued)}

\section{NOTES}

See the Reader's Guide for more information on how to read this figure. See DATA2010 at http://wonder.cdc.gov/data2010 for all Healthy People 2010 tracking data. Tracking data are not available for objective 17-4. Objectives $17-1 \mathrm{~b}$ and $17-3$ were deleted at the Midcourse Review.

\section{FOOTNOTES}

${ }^{1}$ Movement away from target is not quantified using the percent of targeted change achieved. See Technical Appendix for more information.

${ }^{2}$ Difference $=$ Final value - Baseline value. Differences between percents $(\%)$ are measured in percentage points.

${ }^{3}$ When estimates of variability are available, the statistical significance of the difference between the final value and the baseline value is assessed at the 0.05 level. See $\underline{\text { Technical Appendix }}$ for more information.

\footnotetext{
${ }^{4}$ Percent change $=\frac{\text { Final value }- \text { Baseline value }}{\text { Baseline value }} \times 100$

${ }^{5}$ Percent of targeted change achieved $=\frac{\text { Final value }- \text { Baseline value }}{\text { Healthy People } 2010 \text { target }- \text { Baseline value }} \times 100$.
}

\section{DATA SOURCES}

17-1a. National Survey of Pharmacy Practice in Acute Care Settings; American Society of Health Systems Pharmacists.

17-2a. Annual Health Care Information and Management Systems Society Leadership Survey, Healthcare Information and Management Systems Society.

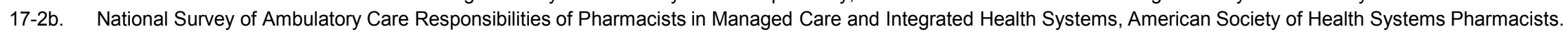

17-2c. National Survey of Pharmacy Practice in Hospital Settings, American Society of Health Systems Pharmacists.

17-2d. The Leapfrog Group Hospital Patient Safety Survey.

17-5a-b. National Survey of Prescription Medicine Information Received by Consumers, FDA

17-6. National Health Interview Survey (NHIS), CDC, NCHS. 
Figure 17-2. Health Disparities Table for Focus Area 17: Medical Product Safety Disparities from the best group rate for each characteristic at the most recent data point and changes in disparity from the baseline to the most recent data point.

Characteristics and Groups

Population-based objectives

17-6. Blood donations (age adjusted, 18+ years) $(1998,2008)^{1}$

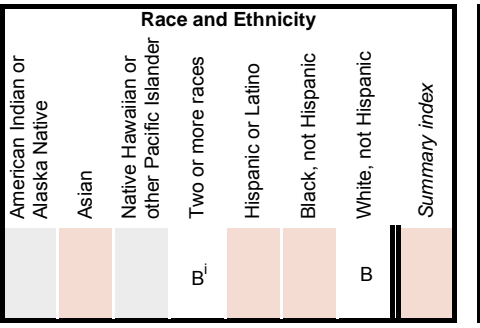

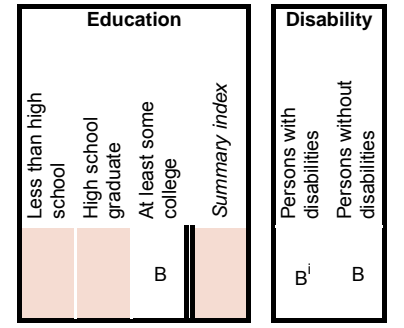

NOTES

See DATA2010 at http://wonder.cdc.gov/data2010 for all Healthy People 2010 tracking data. Disparity data are either unavailable or not applicable for objectives 17-1a, 17-2a through d, 17-4, and 17-5a and b. Objectives 17-1b and 17-3 were deleted at Midcourse Review.

Years in parentheses represent the baseline and most recent data years (if available).

Disparity from the best group rate is defined as the percent difference between the best group rate and each of the other group rates for a characteristic (e.g., race and ethnicity). The summary index is the average of these percent differences for a characteristic. Change in disparity is estimated by subtracting the disparity at baseline from the disparity at the most recent data point. Change in the summary index is estimated by subtracting the summary index at baseline from the summary index at the most recent data point. See Technical Appendix for more information.

Measures of variability were available for the objective in this table. Thus, the variability of best group rates was assessed, and statistical significance was tested Disparities of $10 \%$ or more are displayed when the differences from the best group rate are statistically significant at the 0.05 level. Changes in disparities over time are indicated by arrows when the changes are greater than or equal to 10 percentage points and are statistically significant at the 0.05 level. See Technical Appendix.

LEGEND

$\begin{aligned} & \text { The "best" group rate at the most recent } \\
& \text { data point. }\end{aligned}$
B $\begin{aligned} & \text { The group with the best rate for } \\
& \text { specified characteristic. }\end{aligned} \quad \mathrm{b} \quad \begin{aligned} & \text { Most favorable group rate for specified } \\
& \text { characteristic, but reliability criterion not met. }\end{aligned}$ \begin{tabular}{l}
$\begin{array}{l}\text { Reliability criterion for best } \\
\text { group rate not met, or data } \\
\text { available for only one group. }\end{array}$ \\
\hline
\end{tabular}

\begin{tabular}{ll|l|l|l|l}
\hline $\begin{array}{l}\text { Disparity from the best group rate at the } \\
\text { most recent data point. }\end{array}$ & $\begin{array}{l}\text { Less than 10\%, or difference not } \\
\text { statistically significant (when } \\
\text { estimates of variability are available). }\end{array}$ & $10 \%-49 \%$ & $50 \%-99 \%$ \\
\hline
\end{tabular}

Changes in disparity over time are shown when:

(a) disparities data are available at both baseline and most recent time points;

(b) data are not for the group(s) indicated by "B" or "b" at either time point; and

(c) the change is greater than or equal to 10 percentage points and statistically

significant, or when the change is greater than or equal to 10 percentage

points and estimates of variability were not available. See Technical Appendix.

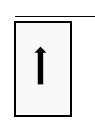

10-49 points

Increase in disparity (percentage points)

L

Availability of Data

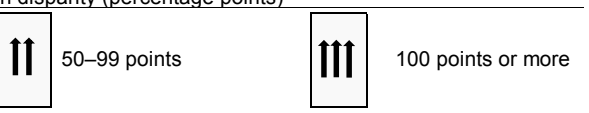

Decrease in disparity (percentage points)

I 10-49 points

FOOTNOTES

1 Baseline data by race and ethnicity are for 1999.

i The group with the best rate at the most recent data point is different from the group with the best rate at baseline. Both rates met the reliability criterion. See Technical Appendix.

\section{DATA SOURCES}

17-6. National Health Interview Survey (NHIS), CDC, NCHS. 


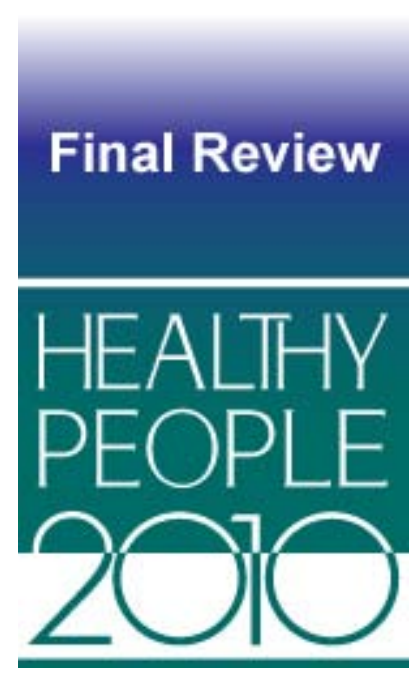

\section{Mental Health and Mental Disorders}

18

\section{Co-Lead Agencies}

National Institutes of Health

Substance Abuse and Mental Health Services Administration

\section{Contents}

Goal $18-2$

Highlights 18-2

Summary of Progress. $18-4$

Transition to Healthy People 2020 18-5

Data Considerations 18-6

References and Notes 18-7

Comprehensive Summary of Objectives 18-9

Progress Chart 18-10

Health Disparities Table 18-13

Suicide-Map 18-15 


\section{Goal: Improve mental health and ensure access to appropriate, quality, mental health services}

The objectives in this chapter monitor a broad range of mental health disorders, behaviors and problems as well as the availability of a variety of community-based and other treatment programs for persons in need of mental health services.

All Healthy People tracking data quoted in this chapter, along with technical information and operational definitions for each objective, can be found in the Healthy People 2010 database, DATA2010, available from http://wonder.cdc.gov/data2010/.

More information about this Focus Area can be found in the following publications:

- Healthy People 2010: Understanding and Improving Health, available from http://www.healthypeople.gov/2010/Document/tableofcontents.htm\#under.

- Healthy People 2010 Midcourse Review, available from http://www.healthypeople.gov/2010/data/midcourse/html/default.htm\#FocusAreas.

\section{Highlights}

- Substantial progress was achieved in objectives for this Focus Area during the past decade [1]. All but one of the 11 Mental Health and Mental Disorders objectives with data to measure progress moved toward or achieved their Healthy People 2010 targets (Figure 181). However, health disparities persisted among racial and ethnic populations, as well as by sex and education level [2]. As discussed below, health disparities of $50 \%$ or more were observed for a number of objectives (Figure 18-2).

- The suicide rate (objective 18-1) increased by 7.6\% between 1999 and 2007, from 10.5 to 11.3 per 100,000 population (age adjusted), moving away from the 2010 target of 4.8 per 100,000. Disparities were observed for a number of population groups, for example:

- Among racial and ethnic groups, the black non-Hispanic population had the lowest (best) suicide rate, 5.1 per 100,000 population (age adjusted) in 2007. The rates for the American Indian or Alaska Native and the white non-Hispanic populations were 11.5 and 13.5 per 100,000 (age adjusted), respectively. The rate for the American Indian or Alaska Native population was almost two and a half times the best group rate (that for the black non-Hispanic population), while the white non-Hispanic rate was more than two and a half times the best group rate [2].

- The white non-Hispanic population had suicide rates of 12.0 per 100,000 population (age adjusted) in 1999 and 13.5 in 2007, whereas the black nonHispanic population had rates of 5.7 in 1999 and 5.1 in 2007. The disparity between the white non-Hispanic and black non-Hispanic populations increased by 54 percentage points between 1999 and 2007 [3].

- Females had a lower (better) suicide rate than males, 4.7 per 100,000 population (age adjusted) in 2007. The rate for males was 18.4 per 100,000 (age adjusted) in 2007, almost four times the rate for females [2]. 
- Males had suicide rates of 17.8 per 100,000 population (age adjusted) in 1999 and 18.4 in 2007, whereas females had rates of 4.0 in 1999 and 4.7 in 2007. The disparity between males and females declined by 53 percentage points between 1999 and 2007 [3].

- Among education groups, persons with at least some college education had the lowest (best) suicide rate, 9.9 per 100,000 population (age adjusted) in 2002, whereas high school graduates had a rate of 18.4 per 100,000 (age adjusted) in 2002, almost twice the best group rate [2].

- Suicide rates varied by geographic region. In 2005-07, the suicide rate was generally higher in the western U.S. than in the rest of the country (Figure 18-3).

- Suicide attempts by students in grades 9-12 that required medical attention (objective 182) decreased by $26.9 \%$ between 1999 and 2009, from 2.6\% to 1.9\%, moving toward the 2010 target of $1.0 \%$.

- Six objectives exceeded their 2010 targets:

- The proportion of homeless adults aged 18 years and over with mental health problems who received mental health services (objective 18-3) increased by $85.2 \%$ between 2000 and 2009, from $27 \%$ to $50 \%$, exceeding the target of $30 \%$.

- The proportion of adolescents (students in grades 9-12) who engaged in disordered eating to control their weight (objective 18-5) declined by $26.3 \%$ between 2001 and 2009 , from $19 \%$ to $14 \%$, exceeding the target of $16 \%$. Disparities were observed for some population groups, for example:

- Boys had a lower (better) rate of disordered eating than girls, 10\% in 2009, whereas girls had a rate of $19 \%$ in 2009, almost twice the rate for boys.

- The proportion of primary care facilities that provided mental health treatment (objective 18-6) increased by $12.9 \%$ between 2000 and 2009, from $62 \%$ to $70 \%$, exceeding the target of $68 \%$.

- The proportion of children aged 4-17 years with mental health problems who received treatment (objective 18-7) increased by $15.0 \%$ between 2000 and 2008, from $60 \%$ to $69 \%$, exceeding the target of $67 \%$.

- The proportion of juvenile residential facilities that screened new admissions for mental health problems (objective 18-8) increased by $16.0 \%$ between 2000 and 2006 , from $50 \%$ to $58 \%$, exceeding the target of $55 \%$.

- The proportion of counties served by community-based jail diversion programs for adults with serious mental illness (objective 18-11) more than doubled between 2004 and 2010 , from $6.9 \%$ to $14.1 \%$, exceeding the target of $7.6 \%$.

- Racial and ethnic health disparities were observed in the treatment of adults for serious mental illness, depression, and schizophrenia (objectives 18-9a through c).

- White non-Hispanic adults aged 18 years and over had the highest (best) rate of treatment for serious mental illness (objective 18-9a), 68\% in 2002, whereas Hispanic or Latino and black non-Hispanic adults had rates of $45 \%$ and $51 \%$ in 2002 , respectively. When expressed as persons not receiving treatment, the rate for Hispanic or Latino adults was more than one and a half times the best group rate (that for white non-Hispanic adults) [2]. The rate for black non-Hispanic adults was about one and a half times the best group rate. 
- White non-Hispanic adults also had the highest (best) rate of treatment for depression (objective 18-9b), 63\% in 2002, whereas Hispanic or Latino and black non-Hispanic adults had rates of $42 \%$ and $43 \%$ in 2002 , respectively. When expressed as persons not receiving treatment, the rate for Hispanic or Latino adults was more than one and a half times the best group rate (that for white non-Hispanic adults), while the rate for black non-Hispanic adults was about one and a half times the best group rate [2].

- Similarly, white non-Hispanic adults had the highest (best) rate of treatment for schizophrenia (objective 18-9c), 63\% in 2002, whereas Hispanic or Latino and black non-Hispanic adults had rates of $42 \%$ and $41 \%$ in 2002 , respectively. When expressed as persons not receiving treatment, the rates for both groups (Hispanic or Latino and black non-Hispanic adults) were more than one and a half times the best group rate [2].

- Males had a higher (better) rate of employment than females for persons with serious mental illness (objective 18-4), 60\% vs. 46\% in 2002. When expressed as persons with serious mental illness who were unemployed, the rate for females was almost one and a half times that for males [2].

\section{Summary of Progress}

- Figure 18-1 presents a quantitative assessment of progress in achieving the Healthy People 2010 objectives for Mental Health and Mental Disorders [1]. Data to measure progress toward target attainment were available for 11 objectives. Of these:

- Six objectives (18-3, 18-5 through 18-8, 18-11) exceeded their Healthy People 2010 targets.

- Four objectives moved toward their targets. A statistically significant difference between the baseline and the final data points was observed for one of these objectives (18-2). Data to test the significance of the difference were unavailable for the other three objectives (18-12 through 18-14).

- One objective moved away from the 2010 target. A statistically significant difference between the baseline and final data points was observed for this objective (18-1).

- Six objectives had no data available to measure progress (objectives 18-4, 18-9a through d, and 18-10).

- Figure 18-2 displays health disparities in Mental Health and Mental Disorders from the best group rate for each characteristic at the most recent data point [2]. It also displays changes in disparities from baseline to the most recent data point [3].

- Five objectives had statistically significant racial and ethnic health disparities of $10 \%$ or more (objectives 18-1, 18-5, and 18-9a, b, and d), and one objective had racial and ethnic health disparities of $10 \%$ or more but lacked data to assess statistical significance (objective 18-9c). Of these six objectives, the white nonHispanic population had the best rate for five objectives (18-5, and 18-9a through d), and the black non-Hispanic population had the best rate for one objective (18-1). 
- Health disparities of 50\% to 99\% between the white non-Hispanic (best rate) population and the other racial and ethnic populations with data (the Hispanic or Latino and the black non-Hispanic populations) were observed for three treatment-related objectives: the proportion of adults with mental disorders who received treatment for serious mental illness (objective 189a), depression (objective 18-9b), and schizophrenia (objective 18-9c); see Highlights, above.

- Females had better rates than males for three of the five objectives with statistically significant health disparities of $10 \%$ or more by sex (objectives 18-1, and 18-9a and b). Males had better rates for the remaining two objectives $(18-4,18-5)$. Females also had a better rate of treatment for schizophrenia (objective 18-9c), the one objective with health disparities of $10 \%$ or more by sex that lacked data to assess statistical significance.

- Four objectives had statistically significant health disparities of $10 \%$ or more by education level (objectives 18-1, 18-4, and 18-9a and d), and one objective (18-9c) had health disparities of $10 \%$ or more by education level but lacked data to assess statistical significance. Persons with at least some college education had the best rates for three of these five objectives (18-1, 18-4, and 18-9d). High school graduates had the best rates for two objectives (18-9a and c).

- The disparities for objective 18-1, suicide, are discussed in the Highlights, above.

\section{Transition to Healthy People 2020}

The focus of the Mental Health and Mental Disorders Healthy People 2020 Topic Area continues to include the broad range of objectives presented in Healthy People 2010. Two objectives were added to the Topic Area, as noted below. See HealthyPeople.gov for a complete list of Healthy People 2020 topics and objectives.

The differences between the Healthy People 2010 objectives and those included in Healthy People 2020 objectives are summarized below:

- The Healthy People 2020 Mental Health and Mental Disorders Topic Area has a total of 16 objectives, whereas the Healthy People 2010 Mental Health and Mental Disorders Focus Area had 17 objectives.

- Eleven Healthy People 2010 objectives (18-1 through 18-3, 18-5 through 18-8, and 18-11 through 18-14) were retained "as is" [4].

- Three Healthy People 2010 objectives were modified [5]. Data sources had not been available for objectives addressing adults with mental health problems who received treatment (objectives 18-9a and b), adults with co-occurring substance abuse and mental health problems who received treatment (objective 18-10), and adults with serious mental illness who were employed (objective 18-4). These objectives have been modified and will be tracked through the National Survey on Drug Use and Health.

- Two treatment objectives were archived due to lack of data: adults with generalized anxiety disorder who receive treatment (objective 18-9d), and adults with schizophrenia who received treatment (objective 18-9c) [6]. 
- Two new objectives were added to the Healthy People 2020 Topic Area. These objectives address:

- The proportion of persons who experience major depressive episode

- Depression screening by primary care providers.

Appendix D, "A Crosswalk Between Objectives from Healthy People 2010 to Healthy People 2020," summarizes the changes between the two decades of objectives, reflecting new knowledge and direction for this area.

\section{Data Considerations}

Education and income are the primary measures of socioeconomic status (SES) in Healthy People 2010. Most data systems used in Healthy People 2010 define income as a family's income before taxes. In order to facilitate comparisons among groups and over time, while adjusting for family size and for inflation, Healthy People 2010 categorizes income using the poverty thresholds developed by the U.S. Census Bureau. Thus, the three categories of family income that are primarily used are:

- Poor-below the Federal poverty level

- Near poor-100\% to $199 \%$ of the Federal poverty level

- Middle/high income-200\% or more of the Federal poverty level.

These categories may be overridden by considerations specific to the data system, in which case they are modified as appropriate. See Healthy People 2010: General Data Issues, referenced below.

Beginning in 2003, education data for mortality objective 18-1 (suicide) from the National Vital Statistics System have been suppressed. The educational attainment item was changed in the new U.S. Standard Certificate of Death in 2003 to be consistent with the U.S. Census Bureau data and to improve the ability to identify specific types of educational degrees. Many states, however, are still using the 1989 version of the U.S. Standard Certificate of Death, which focuses on highest school grade completed. As a result, educational attainment data collected using the 2003 version are not comparable with data collected using the 1989 version [7].

In general, data on educational attainment are presented for persons aged 25 years and over, consistent with guidance given by the U.S. Bureau of the Census. However, because of the requirements of the different data systems, the age groups used to calculate educational attainment for any specific objective may differ from the age groups used to report the data for other Healthy People 2010 objectives, as well as from select populations within the same objective. Therefore, the reader is urged to exercise caution in interpreting the data by educational attainment shown in the Health Disparities Table. See Healthy People 2010: General Data Issues, referenced below.

Additional information on data issues is available from the following sources:

- All Healthy People 2010 tracking data can be found in the Healthy People 2010 database, DATA2010, available from http://wonder.cdc.gov/data2010/. 
- Detailed information about the data and data sources used to support these objectives can be found in the Operational Definitions on the DATA2010 website, available from http://wonder.cdc.gov/data2010/focusod.htm.

- More information on statistical issues related to Healthy People tracking and measurement can be found in the Technical Appendix and in Healthy People 2010: General Data Issues, which is available in the Data Issues section of the NCHS Healthy People website under Healthy People 2010.

\section{References and Notes}

1. Displayed in the Progress Chart (Figure 18-1), the percent of targeted change achieved expresses the difference between the baseline and the final value relative to the initial difference between the baseline and the Healthy People 2010 target. As such, it is a relative measure of progress toward attaining the Healthy People 2010 target. See the Reader's Guide for more information. When standard errors were available, the difference between the baseline and the final value was tested at the 0.05 level of significance. See the Figure 18-1 footnotes, as well as the Technical Appendix, for more detail.

2. Information about disparities among select populations is shown in the Health Disparities Table (Figure 18-2). Disparity from the best group rate is defined as the percent difference between the best group rate and each of the other group rates for a characteristic. For example, racial and ethnic health disparities are measured as the percent difference between the best racial and ethnic group rate and each of the other racial and ethnic group rates. Similarly, disparities by sex are measured as the percent difference between the better group rate (e.g., female) and the rate for the other group (e.g., male). Some objectives are expressed in terms of favorable events or conditions that are to be increased, while others are expressed in terms of adverse events or conditions that are to be reduced. In order to facilitate comparison of health disparities across different objectives, disparity is measured only in terms of adverse events or conditions. For comparability across objectives, objectives that are expressed in terms of favorable events or conditions are re-expressed using the adverse event or condition for the purpose of computing disparity, but they are not otherwise restated or changed. For example, objective 1-1, to increase the proportion of persons with health insurance (e.g., $72 \%$ of the American Indian or Alaska Native population aged under 65 years had some form of health insurance in 2008), is expressed in terms of the percentage of persons without health insurance (e.g., $100 \%-72 \%=28 \%$ of the American Indian or Alaska Native population aged under 65 years did not have any form of health insurance in 2008) when the disparity from the best group rate is calculated. See the Reader's Guide for more information. When standard errors were available, the difference between the best group rate and each of the other group rates was tested at the 0.05 level of significance. See the Figure 18-2 footnotes, as well as the Technical Appendix, for more detail.

3. The change in disparity is estimated by subtracting the disparity at baseline from the disparity at the most recent data point and, therefore, is expressed as a change in percentage points. See the Reader's Guide for more information. When standard errors were available, the change in disparity was tested at the 0.05 level of significance. See the Figure 18-2 footnotes, as well as the Technical Appendix, for more detail.

4. Retained "as is" objectives have no change in the numerator definition or in the denominator definition between the Healthy People 2010 and Healthy People 2020 objectives. These include objectives that were developmental in Healthy People 2010 and are developmental in Healthy People 2020 and for which no numerator or denominator information was available. 
5. Modified objectives have some change in the numerator definition or in the denominator definition between the Healthy People 2010 and Healthy People 2020 objectives. These include objectives that went from developmental in Healthy People 2010 to measurable in Healthy People 2020 or vice versa.

6. Archived objectives had at least one data point in Healthy People 2010 but were not carried forward into Healthy People 2020.

7. Xu JQ, Kochanek KD, Murphy SL, Tejada-Vera B. Deaths: Final data for 2007. National vital statistics reports; vol 58 no 19. Hyattsville, MD: National Center for Health Statistics. 2010. Available from: http://www.cdc.gov/nchs/data/nvsr/nvsr58/nvsr58 19.pdf. 


\section{Comprehensive Summary of Objectives: Mental Health and Mental Disorders}

\begin{tabular}{|c|c|c|}
\hline Objective & Description & Data Source \\
\hline $18-1$ & Suicide (age adjusted per 100,000 standard population) & National Vital Statistics System-Mortality (NVSS-M), CDC, NCHS. \\
\hline $18-2$ & $\begin{array}{l}\text { Suicide attempts by students that required medical attention (grades } \\
9-12 \text { ) }\end{array}$ & Youth Risk Behavior Surveillance System (YRBSS), CDC, NCCDPHP. \\
\hline $18-3$ & $\begin{array}{l}\text { Homeless adults with mental health problems who receive mental } \\
\text { health services }(18+\text { years })\end{array}$ & $\begin{array}{l}\text { Projects for Assistance in Transition from Homelessness (PATH) } \\
\text { Annual Application, SAMHSA. }\end{array}$ \\
\hline $18-4$ & Employment of persons with serious mental illness (SMI) (18+ years) & National Comorbidity Survey-Replication (NCS-R), NIH, NIMH. \\
\hline $18-5$ & Students engaging in disordered eating (grades 9-12) & Youth Risk Behavior Surveillance System (YRBSS), CDC, NCCDPHP. \\
\hline $18-6$ & Primary care facilities that provide mental health treatment & Uniform Data System (UDS), HRSA. \\
\hline $18-7$ & Treatment for children with mental health problems (4-17 years) & National Health Interview Survey (NHIS), CDC, NCHS. \\
\hline $18-8$ & $\begin{array}{l}\text { Juvenile residential facilities that screen admissions for mental health } \\
\text { problems }\end{array}$ & $\begin{array}{l}\text { Juvenile Residential Facility Census (JRFC), National Center for Juvenile } \\
\text { Justice. }\end{array}$ \\
\hline $18-9 \mathrm{a}$ & Treatment for adults with serious mental illness (SMI) (18+ years) & National Comorbidity Survey—Replication (NCS-R), NIH, NIMH. \\
\hline $18-9 b$ & Treatment for adults with depression (18+ years) & National Comorbidity Survey-Replication (NCS-R), NIH, NIMH. \\
\hline $18-9 c$ & Treatment for adults with schizophrenia $(18+$ years $)$ & Epidemiologic Catchment Area (ECA) Program, NIH, NIMH \\
\hline $18-9 d$ & Treatment for adults with generalized anxiety disorder (18+ years) & National Comorbidity Survey-Replication (NCS-R), NIH, NIMH. \\
\hline $18-10$ & $\begin{array}{l}\text { Treatment for co-occurring substance abuse and mental disorders (18+ } \\
\text { years) }\end{array}$ & National Comorbidity Survey-Replication (NCS-R), NIH, NIMH. \\
\hline $18-11$ & $\begin{array}{l}\text { Community-based jail diversion programs for adults with serious } \\
\text { mental illness (SMI) }\end{array}$ & Mental Health Courts Survey (MHCS), SAMHSA. \\
\hline $18-12$ & $\begin{array}{l}\text { State tracking of consumer satisfaction with mental health services (no. } \\
\text { States and D.C.) }\end{array}$ & Uniform Reporting System (URS), SAMHSA. \\
\hline $18-13$ & $\begin{array}{l}\text { State mental health plans addressing cultural competence (no. States } \\
\text { and D.C.) }\end{array}$ & $\begin{array}{l}\text { State Mental Health Agency Profiling System, National Association of } \\
\text { State Mental Health Program Directors, National Research Institute; } \\
\text { SAMHSA, CMHS. }\end{array}$ \\
\hline $18-14$ & $\begin{array}{l}\text { State mental health plans addressing care of elderly persons (no. States } \\
\text { and D.C.) }\end{array}$ & $\begin{array}{l}\text { State Mental Health Agency Profiling System, National Association of } \\
\text { State Mental Health Program Directors, National Research Institute; } \\
\text { SAMHSA, CMHS. }\end{array}$ \\
\hline
\end{tabular}


Figure 18-1. Progress Toward Target Attainment for Focus Area 18: Mental Health and Mental Disorders

Moved away

from target ${ }^{1}$

Moved toward

target

Met or exceeded

target

18-1. Suicide (age adjusted, per 100,000 population)

18-2. Suicide attempts by students that required medical attention (grades 9-12)

18-3. Homeless adults with mental health problems who receive mental health services $(18+$ years $)$

18-5. Students engaging in disordered eating (grades 9-12)

18-6. Primary care facilities that provide mental health treatment

18-7. Treatment for children with mental health problems (4-17 years)

18-8. Juvenile residential facilities that screen admissions for mental health problems

18-11. Community-based jail diversion programs for adults with serious mental illness (SMI)
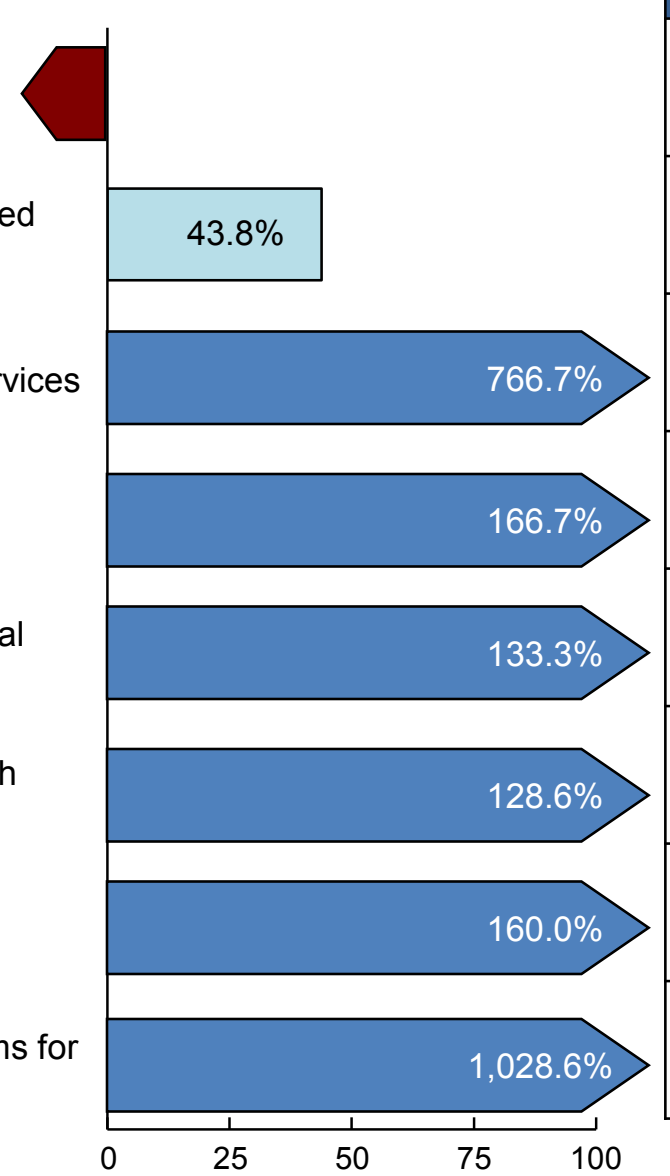

\begin{tabular}{|c|c|c|c|c|c|}
\hline \multirow{2}{*}{$\begin{array}{l}2010 \\
\text { Target }\end{array}$} & \multirow{2}{*}{$\begin{array}{c}\text { Baseline } \\
\text { (Year) }\end{array}$} & \multirow{2}{*}{$\begin{array}{l}\text { Final } \\
\text { (Year) }\end{array}$} & \multicolumn{3}{|c|}{ Baseline vs. Final } \\
\hline & & & Difference ${ }^{2}$ & $\begin{array}{l}\text { Statistically } \\
\text { Significant }^{3}\end{array}$ & $\begin{array}{l}\text { Percent } \\
\text { Change }^{4}\end{array}$ \\
\hline 4.8 & $\begin{array}{c}10.5 \\
(1999)\end{array}$ & $\begin{array}{c}11.3 \\
(2007)\end{array}$ & 0.8 & Yes & $7.6 \%$ \\
\hline $1.0 \%$ & $\begin{array}{c}2.6 \% \\
(1999)\end{array}$ & $\begin{array}{c}1.9 \% \\
(2009)\end{array}$ & -0.7 & Yes & $-26.9 \%$ \\
\hline $30 \%$ & $\begin{array}{c}27 \% \\
(2000)\end{array}$ & $\begin{array}{c}50 \% \\
(2009)\end{array}$ & 23 & $\begin{array}{c}\text { Not } \\
\text { tested }\end{array}$ & $85.2 \%$ \\
\hline $16 \%$ & $\begin{array}{c}19 \% \\
(2001)\end{array}$ & $\begin{array}{c}14 \% \\
(2009)\end{array}$ & -5 & Yes & $-26.3 \%$ \\
\hline $68 \%$ & $\begin{array}{c}62 \% \\
(2000)\end{array}$ & $\begin{array}{c}70 \% \\
(2009)\end{array}$ & 8 & $\begin{array}{c}\text { Not } \\
\text { tested }\end{array}$ & $12.9 \%$ \\
\hline $67 \%$ & $\begin{array}{c}60 \% \\
(2001)\end{array}$ & $\begin{array}{c}69 \% \\
(2008)\end{array}$ & 9 & Yes & $15.0 \%$ \\
\hline $55 \%$ & $\begin{array}{c}50 \% \\
(2000)\end{array}$ & $\begin{array}{c}58 \% \\
(2006)\end{array}$ & 8 & $\begin{array}{c}\text { Not } \\
\text { tested }\end{array}$ & $16.0 \%$ \\
\hline $7.6 \%$ & $\begin{array}{c}6.9 \% \\
(2004)\end{array}$ & $\begin{array}{l}14.1 \% \\
(2010)\end{array}$ & 7.2 & $\begin{array}{c}\text { Not } \\
\text { tested }\end{array}$ & $104.3 \%$ \\
\hline
\end{tabular}

Percent of targeted change achieved ${ }^{5}$

(continued) 
Figure 18-1. Progress Toward Target Attainment for Focus Area 18: Mental Health and Mental Disorders (continued)

Moved away

from target ${ }^{1}$

Moved toward

target

Met or exceeded

target

18-12. State tracking of consumer satisfaction with mental health services (no. States and D.C.)

18-13. State mental health plans addressing cultural competence (no. States and D.C.)

18-14. State mental health plans addressing care of elderly persons (no. States and D.C.)

$12.1 \%$

\begin{tabular}{|c|c|c|c|c|c|}
\hline \multirow{2}{*}{$\begin{array}{c}2010 \\
\text { Target }\end{array}$} & \multirow{2}{*}{$\begin{array}{c}\text { Baseline } \\
\text { (Year) }\end{array}$} & \multirow{2}{*}{$\begin{array}{l}\text { Final } \\
\text { (Year) }\end{array}$} & \multicolumn{3}{|c|}{ Baseline vs. Final } \\
\hline & & & Difference $^{2}$ & $\begin{array}{l}\text { Statistically } \\
\text { Significant }^{3}\end{array}$ & $\begin{array}{c}\text { Percent } \\
\text { Change }^{4}\end{array}$ \\
\hline 51 & $\begin{array}{c}34 \\
(2002)\end{array}$ & $\begin{array}{c}50 \\
(2009)\end{array}$ & 16 & $\begin{array}{l}\text { Not } \\
\text { tested }\end{array}$ & $47.1 \%$ \\
\hline 32 & $\begin{array}{c}29 \\
(2004)\end{array}$ & $\begin{array}{c}30 \\
(2009)\end{array}$ & 1 & $\begin{array}{l}\text { Not } \\
\text { tested }\end{array}$ & $3.4 \%$ \\
\hline 51 & $\begin{array}{c}18 \\
(2001)\end{array}$ & $\begin{array}{c}22 \\
(2009)\end{array}$ & 4 & $\begin{array}{l}\text { Not } \\
\text { tested }\end{array}$ & $22.2 \%$ \\
\hline
\end{tabular}

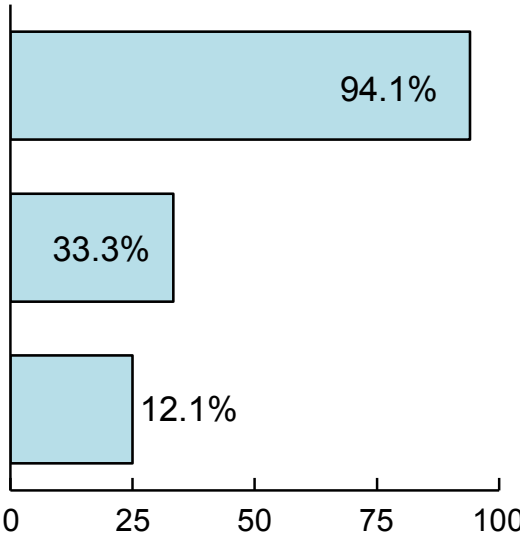

100

Percent of targeted change achieved 5

NOTES

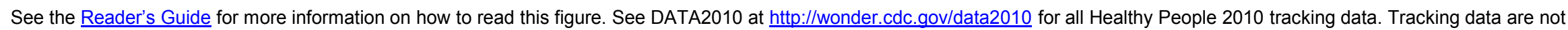
available for objectives 18-4, 18-9a through d, and 18-10.

\section{FOOTNOTES}

${ }^{1}$ Movement away from target is not quantified using the percent of targeted change achieved. See Technical Appendix for more information.

${ }^{2}$ Difference $=$ Final value - Baseline value. Differences between percents $(\%)$ are measured in percentage points

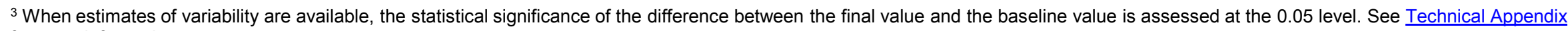
for more information.

${ }^{4}$ Percent change $=\frac{\text { Final value }- \text { Baseline value }}{\text { Baseline value }} \times 100$.

${ }^{5}$ Percent of targeted change achieved $=\frac{\text { Final value }- \text { Baseline value }}{\text { Healthy People } 2010 \text { target }- \text { Baseline value }} \times 100$. 
Figure 18-1. Progress Toward Target Attainment for Focus Area 18: Mental Health and Mental Disorders (continued)

\section{DATA SOURCES}

18-1. National Vital Statistics System—Mortality (NVSS-M), CDC, NCHS.

18-2. Youth Risk Behavior Surveillance System (YRBSS), CDC, NCCDPHP.

18-3. Projects for Assistance in Transition from Homelessness (PATH) Annual Application, SAMHSA.

18-5. Youth Risk Behavior Surveillance System (YRBSS), CDC, NCCDPHP.

18-6. Uniform Data System (UDS), HRSA.

18-7. National Health Interview Survey (NHIS), CDC, NCHS.

18-8. Juvenile Residential Facility Census (JRFC), National Center for Juvenile Justice.

18-11. Mental Health Courts Survey (MHCS), SAMHSA.

18-12. Uniform Reporting System (URS), SAMHSA.

18-13-18-14. State Mental Health Agency Profiling System, National Association of State Mental Health Program Directors, National Research Institute; SAMHSA, CMHS. 
Figure 18-2. Health Disparities Table for Focus Area 18: Mental Health and Mental

\section{Disorders}

Disparities from the best group rate for each characteristic at the most recent data point and changes in disparity from the baseline to the most recent data point.

Population-based objectives

18-1. Suicide (age adjusted, per 100,000 population) $(1999,2007)^{1 \star}$

18-2. Suicide attempts by students that required medical attention (grades 9-12) (1999, 2009)*

18-4. Employment of adults with serious mental illness (SMI) $\left(18+\right.$ years) $(2002)^{*}$

18-5. Students engaging in disordered eating (grades 9-12) $(2001,2009)^{\star}$

18-7. Treatment for children with mental health problems (4-17 years) $(2001,2008)^{*}$

18-9a. Treatment for adults with serious mental illness (SMI) $(18+$ years $)(2002)^{*}$

18-9b. Treatment for adults with depression (18+ years) $(2002)^{*}$

18-9c. Treatment for adults with schizophrenia (18+ years) (1984)†

18-9d. Treatment for adults with generalized anxiety disorder (18+ years) $(2002)^{\star}$

18-10. Treatment for co-occurring substance abuse and mental disorders $\left(18+\right.$ years) $(2002)^{\star}$

Characteristics and Groups
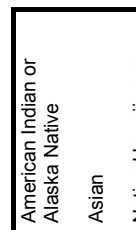

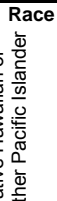

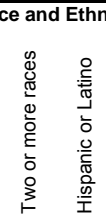

\section{nicity}

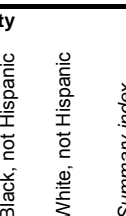

$\sqrt{1}$

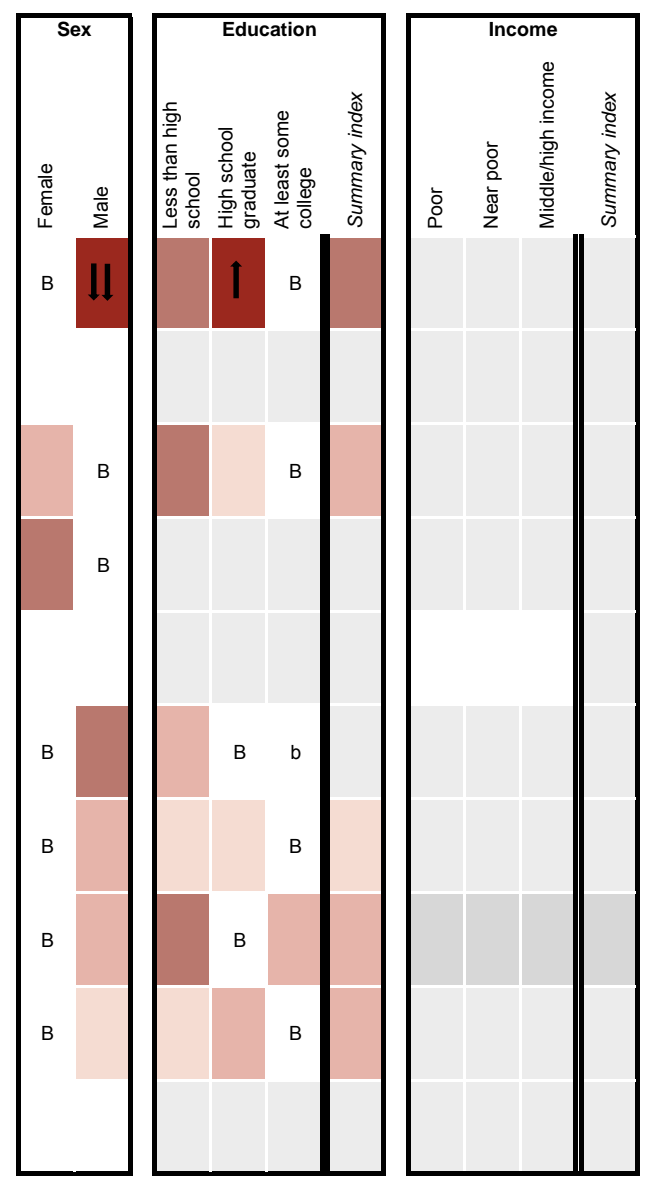

(continued) 


\section{Figure 18-2. Health Disparities Table for Focus Area 18: Mental Health and Mental Disorders (continued)}

NOTES

See DATA2010 at http://wonder.cdc.gov/data2010 for all Healthy People 2010 tracking data. Disparity data are either unavailable or not applicable for objectives $18-3,18-6,18-8$, and 18-11 through 18-14.

Years in parentheses represent the baseline and most recent data years (if available).

Disparity from the best group rate is defined as the percent difference between the best group rate and each of the other group rates for a characteristic (e.g., race and ethnicity). The summary index is the average of these percent differences for a characteristic. Change in disparity is estimated by subtracting the disparity at baseline from the disparity at the most recent data point. Change in the summary index is estimated by subtracting the summary index at baseline from the summary index at the most recent data point. See Technical Appendix for more information.

\section{LEGEND}

The "best" group rate at the most recent data point. B $\begin{aligned} & \text { The group with the best rate for } \\ & \text { specified characteristic. }\end{aligned}$

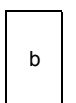

Most favorable group rate for specified

characteristic, but reliability criterion not met.

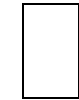

Reliability criterion for best group rate not met, or data available for only one group.

Disparity from the best group rate at the
most recent data point.
Shanges in disparity over time are shown when:
statistically significant (when
estimates of variability are available).
(b) data are not for the group(s) indicated by "B" or "b" at either time point; and
(c) the change is greater than or equal to 10 percentage points and statistically
significant, or when the change is greater than or equal to 10 percentage
points and estimates of variability were not available. See Technical Appendix.

\section{FOOTNOTES}

* Measures of variability were available. Thus, the variability of best group rates was assessed, and statistical significance was tested. Disparities of $10 \%$ or more are displayed when the differences from the best group rate are statistically significant at the 0.05 level. Changes in disparities over time are indicated by arrows when the changes are greater than or equal to 10 percentage points and are statistically significant at the 0.05 level. See Technical Appendix.

† Measures of variability were not available. Thus, the variability of best group rates was not assessed, and statistical significance could not be tested. Nonetheless, disparities and changes in disparities over time are displayed according to their magnitude. See Technical Appendix.

1 Most recent data by education level are for 2002 .

i Data are for Asian or Pacific Islander.

ii Change in the summary index cannot be assessed. See Technical Appendix.

\section{DATA SOURCES}

18-1. National Vital Statistics System-Mortality (NVSS-M), CDC, NCHS

18-2. Youth Risk Behavior Surveillance System (YRBSS), CDC, NCCDPHP.

18-4. National Comorbidity Survey-Replication (NCS-R), NIH, NIMH.

18-5. Youth Risk Behavior Surveillance System (YRBSS), CDC, NCCDPHP.

18-7. National Health Interview Survey (NHIS), CDC, NCHS.

18-9a-b. National Comorbidity Survey-Replication (NCS-R), NIH, NIMH.

18-9c. Epidemiologic Catchment Area (ECA) Program, NIH, NIMH.

18-9d. National Comorbidity Survey-Replication (NCS-R), NIH, NIMH.

18-10. National Comorbidity Survey-Replication (NCS-R), NIH, NIMH. 
Figure 18-3. Suicide 2005-07

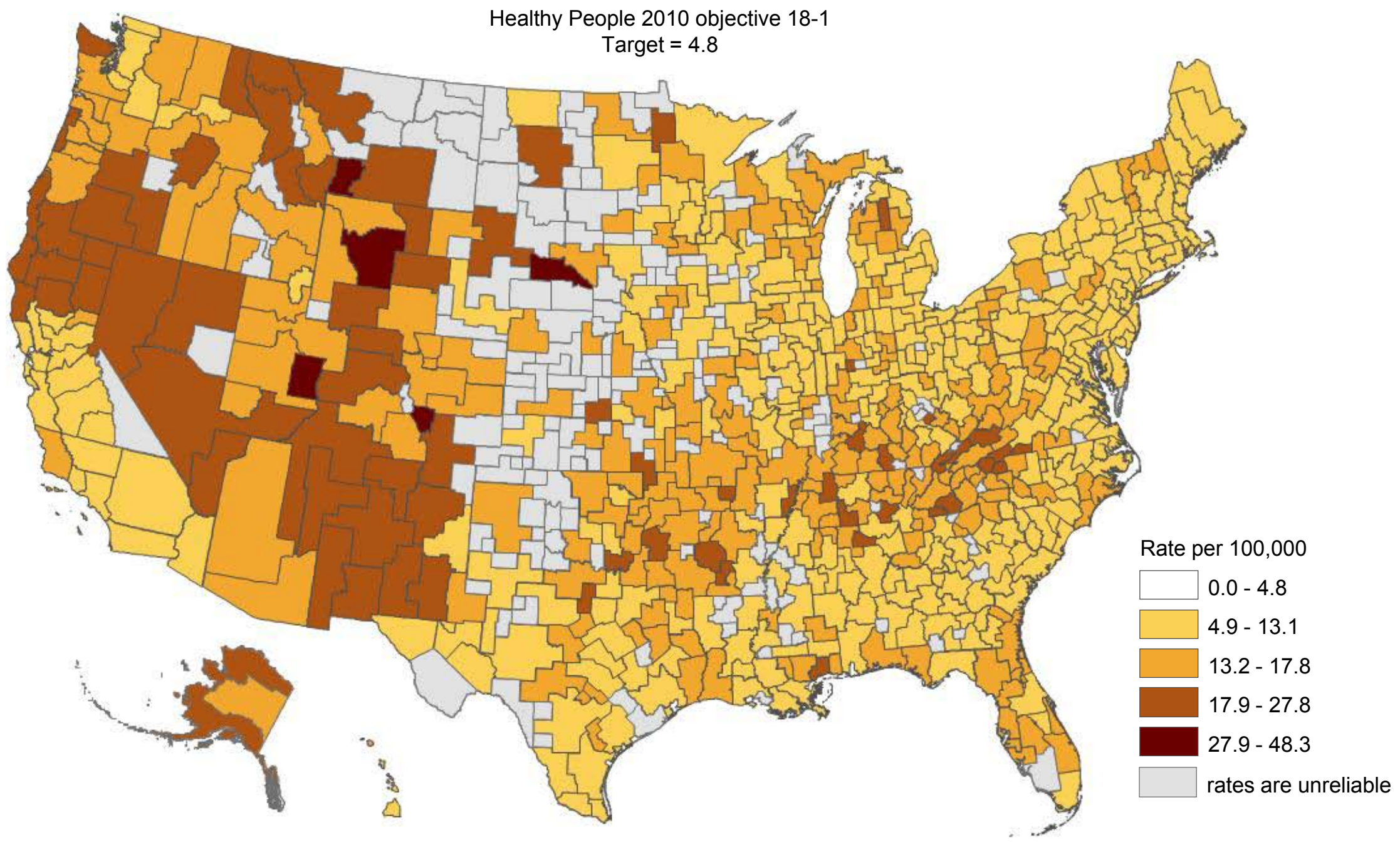

no health service areas with a reliable rate met the target

NOTES: Data are for ICD-10 codes X60-X84, Y87.0 reported as underlying cause. Rates are per 100,000 U. S. Population age-adjusted to the 2000 standard population. Rates are displayed by a Jenks classification for U.S. health service area. SOURCE: National Vital Statistics System - Mortality, CDC, NCHS. 


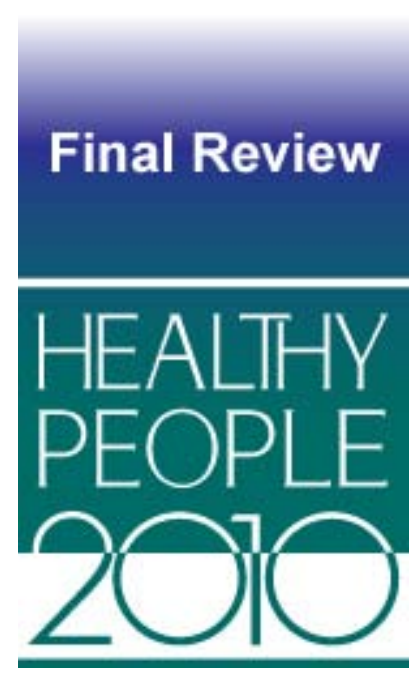

\section{Nutrition and Overweight}

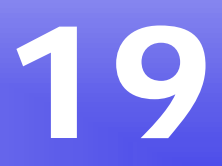

\section{Co-Lead Agencies}

Food and Drug Administration

National Institutes of Health

\section{Contents}

Goal

Highlights

Summary of Progress

Transition to Healthy People 2020

Data Considerations

References and Notes

Comprehensive Summary of Objectives

Progress Chart

Health Disparities Table

Obesity in Adults-Map. 


\section{Goal: Promote health and reduce chronic disease associated with diet and weight}

The objectives in this chapter monitor trends in overweight and obesity, growth retardation, the consumption of various types of foods and nutrients, iron deficiency, diet and nutrition counseling, and food security (access to food).

All Healthy People tracking data quoted in this chapter, along with technical information and operational definitions for each objective, can be found in the Healthy People 2010 database, DATA2010, available from http://wonder.cdc.gov/data2010/.

More information about this Focus Area can be found in the following publications:

- Healthy People 2010: Understanding and Improving Health, available from http://www.healthypeople.gov/2010/Document/tableofcontents.htm\#under.

- Healthy People 2010 Midcourse Review, available from http://www.healthypeople.gov/2010/data/midcourse/html/default.htm\#FocusAreas.

\section{Highlights}

- Almost no progress was made toward the Healthy People 2010 targets for objectives in this Focus Area [1]. Only one Nutrition and Overweight objective (19-11, calcium intake) showed significant positive movement (Figure 19-1). In addition, statistically significant health disparities were observed among racial and ethnic populations, as well as by sex, income, and disability status (Figure 19-2), some of which are discussed below [2].

- The proportion of adults aged 20 years and over who were at a healthy weight based on directly measured weight and height (objective 19-1) decreased by $26.2 \%$ between 1988 94 and $2005-08$, from $42 \%$ to $31 \%$ (age adjusted), moving away from the Healthy People 2010 target of $60 \%$.

- Obesity in the U.S. population has increased, moving away from Healthy People 2010 targets. Based on directly measured weight and height, between 1988-94 and 2005-08 the proportion of adults aged 20 years and over who were obese (objective 19-2) rose by $47.8 \%$, from $23 \%$ to $34 \%$ (age adjusted), moving away from the 2010 target of $15 \%$. During the same period, obesity increased by $54.5 \%$ in children aged $6-11$ years, from $11 \%$ to $17 \%$, (objective $19-3 \mathrm{a}$ ) and by $63.6 \%$ in adolescents aged $12-19$ years, from $11 \%$ to $18 \%$ (objective 19-3b), moving away from the 2010 targets of $5 \%$.

- Obesity in adults varied by geographic area. Based on self-reported weight and height, in 2008, Colorado, Connecticut, the District of Columbia, Rhode Island, and Massachusetts had the lowest obesity rates, whereas Alabama, Mississippi, Oklahoma, South Carolina, and West Virginia had the highest rates (Figure 19-3).

- The proportion of persons with healthful eating patterns, objectives 19-5 through 19-11, showed little change. These objectives remained well below their 2010 targets. One objective did show some positive progress: calcium intake among persons aged two years and over (objective 19-11) increased by $35.5 \%$ between $1988-94$ and $2003-04$, from $31 \%$ to $42 \%$ (age adjusted), moving toward the Healthy People 2010 target of $74 \%$. 
- Food security among U.S. households (objective 19-18) declined by 3.4\% between 1995 and 2008 , from $88 \%$ to $85 \%$, moving away from the 2010 target of $94 \%$. Disparities were observed for a number of population groups, for example:

- Among racial and ethnic groups, white non-Hispanic households had the highest (best) rate of food security, 89\% in 2008, whereas black non-Hispanic, Hispanic or Latino, and American Indian or Alaska Native households had rates of 74\%, 73\%, and $77 \%$ in 2008, respectively. When expressed as households with food insecurity, the rates for black non-Hispanic and Hispanic or Latino households were almost two and a half times the best rate (that for white non-Hispanic households), while the rate for American Indian or Alaska Native households was more than twice the best rate (Figure 19-2) [2].

\section{Summary of Progress}

- Figure 19-1 presents a quantitative assessment of progress in achieving the Healthy People 2010 objectives for Nutrition and Overweight. Data to measure progress toward target attainment were available for 20 objectives [1]. Of these:

- Two objectives moved toward their 2010 targets. A statistically significant difference between the baseline and the final data points was observed for one of these objectives (19-11). No significant difference was observed for the other objective (19-5).

- Three objectives (19-4, 19-6, and 19-12a) showed no change.

- Fifteen objectives moved away from their targets. A statistically significant difference between the baseline and final data points was observed for nine objectives (19-1, 19-2, 19-3a through c, 19-7, 19-12c, 19-17, and 19-18). No significant differences were observed for five objectives (19-8 through 19-10, 19$12 \mathrm{~b}$, and 19-14); and data to test the significance of the difference were unavailable for one objective (19-13).

- One objective (19-16) had no follow-up data available to measure progress and one objective (19-15) was dropped during the decade [3].

- Figure 19-2 displays health disparities in Nutrition and Overweight from the best group rate for each characteristic at the most recent data point [2]. It also displays changes in disparities from baseline to the most recent data point [4].

- Statistically significant health disparities of $10 \%$ or more by race and ethnicity were observed for 10 objectives. Health disparities of $10 \%$ or more by race and ethnicity were observed for two additional objectives, though data to test their statistical significance were unavailable. Of these 12 objectives, the white non-Hispanic population had the best rate for six objectives (19-1, 19-2, 19-11, 19-12c, 19-13, and 19-18). The black non-Hispanic and American Indian or Alaska Native populations had the best rate for one objective each (objectives 19-3a and 19-4, respectively). The Mexican American population had the best rate for four objectives (19-5 and 19-8 through 19-10). 
- Females had better rates than males for two of the three objectives with statistically significant health disparities of $10 \%$ or more by sex (objectives 19-1 and 19-10). Males had a better rate for the third objective (19-11). Females also had a better rate for one objective with a disparity of $10 \%$ or more by sex for which statistical significance could not be assessed (objective 19-4).

- Persons whose income was above $130 \%$ of the poverty threshold (Federal poverty level; see Data Considerations section below) had better rates than persons whose income was below $130 \%$ of the poverty threshold for six of the seven objectives with statistically significant health disparities of $10 \%$ or more by income (objectives 19-2, 19-3b and c, 19-11, 19-12c, and 19-18). Persons whose income was below $130 \%$ of the poverty threshold had a better rate for the seventh objective (19-9).

- Persons without disabilities had a better rate than persons with disabilities for the one objective with statistically significant health disparities of $10 \%$ or more by disability status (objective 19-2).

\section{Transition to Healthy People 2020}

For Healthy People 2020, the focus of the Nutrition and Weight Status objectives was expanded to include a broader range of policies and environmental factors that support eating a healthful diet and maintaining a healthy body weight in settings such as schools, worksites, health care organizations, and communities. In addition, the wording and definitions of the food and nutrient consumption objectives have been revised to be applicable to the 2010 Dietary Guidelines for Americans (DGA) and minimize the need to revise the objectives with subsequent updates [5]. The Healthy People 2010 Focus Area name was changed from "Nutrition and Overweight" to "Nutrition and Weight Status" to better describe the range of weight-related objectives. The Nutrition and Weight Status objectives primarily assess individual behaviors regarding the consumption of healthful diets and achievement and maintenance of healthy body weights, and the policies and environments that support these behaviors. See HealthyPeople.gov for a complete list of Healthy People 2020 topics and objectives.

The Healthy People 2020 Nutrition and Weight Status Topic Area can be grouped into six sections:

- Healthier Food Access

- Healthcare and Worksite Settings

- Weight Status

- Food Insecurity

- Food and Nutrient Consumption

- Iron Deficiency.

The differences between the Healthy People 2010 objectives and those included in Healthy People 2020 are summarized below: 
- The Healthy People 2020 Nutrition and Weight Status Topic Area has a total of 38 objectives, seven of which are developmental [6]. The Healthy People 2010 Nutrition and Overweight Focus Area had 22 objectives, one of which was dropped at Midcourse Review [3].

- Six Healthy People 2010 objectives were retained "as is" [7]. These objectives include: healthy weight in adults (objective 19-1), obesity in adults (objective 19-2), obesity in children (separately assessed for those aged 6-11 years and 12-19 years; objectives 19-3a and b, respectively), iron deficiency in pregnant females (objective 19-14), and the complement to food security (i.e., food insecurity) among U.S. households (objective 19-18).

- Four Healthy People 2010 objectives were archived or dropped [3,8]. Growth retardation in low-income children (objective 19-4), anemia in low-income pregnant females (objective 19-13), and total fat intake (objective 19-9) were archived. The meals and snacks at school objective (19-15) was deleted at Midcourse Review due to the lack of a national data source.

- Twelve Healthy People 2010 objectives were modified to create 13 Healthy People 2020 objectives [9].

- The age group tracked for obesity in children (objective 19-3c) was expanded from 6-19 years to $2-19$ years.

- Three Food Consumption objectives for fruits (objective 19-5), vegetables (objective 19-6), and grains (objective 19-7) were changed to create four objectives that are applicable to the 2010 DGA and assess mean intake. The objective for vegetables was divided into two objectives to separately monitor the quantity and variety of vegetables consumed.

- Three Nutrient Consumption objectives, including the percentage of calories from saturated fat (objective 19-8), total sodium (objective 19-10), and total calcium (objective 19-11), were also changed to assess mean intake rather than the percent of the population meeting the DGA, to allow population groups' progress to be assessed in meeting DGA recommendations over the decade without the need to modify the objectives with future releases of the DGA.

- The model used to determine iron deficiency was changed from the ferritin model to the body iron stores model for three iron deficiency objectives (19-12a through c) assessed among young children (aged 1-2 years and 3-4 years) and non-pregnant females.

- The objective (19-16) on worksite nutrition and weight management classes or counseling was moved back to developmental status until more current data become available.

- The objective (19-17) tracking physician office visits that include nutrition counseling or education for patients with cardiovascular disease, diabetes, or hyperlipidemia was modified to include additional diagnostic information from the patient record.

- Nineteen new objectives, six of which are developmental, were added to the Healthy People 2020 Nutrition and Weight Status Topic Area [6]:

- Five new objectives tracking healthier food access include state nutrition standards for child care, schools not offering calorically sweetened beverages, school requirements for fruit and vegetable availability, state-level incentive policies for food retail, and a developmental objective on access to food retail outlets that sell a variety of foods that are encouraged by the DGA. 
- Four new healthcare objectives include primary care physicians who assess child and adult patients' body mass index (BMI), physician office visits with weight reduction, nutrition or physical activity counseling or education for obese patients, and nutrition counseling for all patients.

- Six new weight status objectives include obesity among children aged 2-5 years, and five developmental objectives on inappropriate weight gain among youth and adults.

- A new food security objective tracks very low food security among children.

- Three new nutrient consumption objectives focus on the percentage of calories from solid fats, added sugars, and both.

Appendix D, “A Crosswalk Between Objectives From Healthy People 2010 to Healthy People 2020," summarizes the changes between the two decades of objectives, reflecting new knowledge and direction for this area.

\section{Data Considerations}

State level rates for obesity among adults (objective 19-2) in Figure 19-3 are based on self-reported weight and height data from Behavioral Risk Factory Surveillance System (BRFSS) telephone interviews and may not be comparable to national rates in Figures 19-1 and 19-2, which are based on directly measured weight and height from National Health and Nutrition Examination Survey (NHANES). BMI estimates derived from self-reported height and weight tend to be lower than those derived from measured height and weight due to underreporting of weight and over-reporting of height [10]. However, BRFSS data is still useful in assessing geographic differences and changes over time.

The data label used for objectives 19-3a through c "overweight or obesity" in children and adolescents, was revised since the Healthy People 2010 Midcourse Review and progress reviews to "obesity" even though the definition (BMI at or above the sex-and age-specific 95th percentile from the 2000 CDC Growth Charts) and interpretation are still the same. This terminology change in NCHS and other CDC publications is consistent with revisions made by the American Academy of Pediatrics, the Institute of Medicine, and other organizations. Because the indexes used are based on body mass rather than fatness, the original terminology of "overweight" for children at or above the 95th percentile was intended to clarify that this cut-off point should not be used as diagnostic criteria. Rather, these children may or may not have excess body fat and should, therefore, be screened for obesity. However, because body fat is difficult to measure and the majority of children with BMI at or above the 95th percentile have high adiposity, on a population-wide basis, high weight-for-height can be considered as an indicator for obesity [11].

Education and income are the primary measures of socioeconomic status (SES) in Healthy People 2010. Most data systems used in Healthy People 2010 define income as a family's income before taxes. In order to facilitate comparisons among groups and over time, while adjusting for family size and for inflation, Healthy People 2010 categorizes income using the poverty thresholds developed by the U.S. Census Bureau. Thus, the three categories of family income that are primarily used are: 
- Poor-below the Federal poverty level

- Near poor-100\% to $199 \%$ of the Federal poverty level

- Middle/high income-200\% or more of the Federal poverty level.

These categories may be overridden by considerations specific to the data system, in which case they are modified as appropriate. See Healthy People 2010: General Data Issues, referenced below.

Additional information on data issues is available from the following sources:

- All Healthy People 2010 tracking data can be found in the Healthy People 2010 database, DATA2010, available from http://wonder.cdc.gov/data2010/.

- Detailed information about the data and data sources used to support these objectives can be found in the Operational Definitions on the DATA2010 website, available from http://wonder.cdc.gov/data2010/focusod.htm.

- More information on statistical issues related to Healthy People tracking and measurement can be found in the Technical Appendix and in Healthy People 2010: General Data Issues, which is available in the Data Issues section of the NCHS Healthy People website under Healthy People 2010.

\section{References and Notes}

1. Displayed in the Progress Chart (Figure 19-1), the percent of targeted change achieved expresses the difference between the baseline and the final value relative to the initial difference between the baseline and the Healthy People 2010 target. As such, it is a relative measure of progress toward attaining the Healthy People 2010 target. See the Reader's Guide for more information. When standard errors were available, the difference between the baseline and the final value was tested at the 0.05 level of significance. See the Figure 19-1 footnotes, as well as the Technical Appendix, for more detail.

2. Information about disparities among select populations is shown in the Health Disparities Table (Figure 19-2). Disparity from the best group rate is defined as the percent difference between the best group rate and each of the other group rates for a characteristic. For example, racial and ethnic health disparities are measured as the percent difference between the best racial and ethnic group rate and each of the other racial and ethnic group rates. Similarly, disparities by sex are measured as the percent difference between the better group rate (e.g., female) and the rate for the other group (e.g., male). Some objectives are expressed in terms of favorable events or conditions that are to be increased, while others are expressed in terms of adverse events or conditions that are to be reduced. In order to facilitate comparison of health disparities across different objectives, disparity is measured only in terms of adverse events or conditions. For comparability across objectives, objectives that are expressed in terms of favorable events or conditions are re-expressed using the adverse event or condition for the purpose of computing disparity, but they are not otherwise restated or changed. For example, objective 1-1, to increase the proportion of persons with health insurance (e.g., $72 \%$ of the American Indian or Alaska Native population aged under 65 years had some form of health insurance in 2008), is expressed in terms of the percentage of persons without health insurance (e.g., $100 \%-72 \%=28 \%$ of the American Indian or Alaska Native population aged under 65 years did not have any form of health insurance in 2008) when the disparity from the best group rate is calculated See the Reader's Guide for more information. When standard errors were available, the difference between the best group rate and each of the other group rates was tested at the 0.05 level of significance. See the Figure 19-2 footnotes, as well as the Technical Appendix, for more detail. 
3. Dropped objectives were not carried forward into Healthy People 2020. These objectives were either developmental or deleted at the Healthy People 2010 Midcourse Review or at another time in Healthy People 2010.

4. The change in disparity is estimated by subtracting the disparity at baseline from the disparity at the most recent data point and, therefore, is expressed as a change in percentage points. See the Reader's Guide for more information. When standard errors were available, the change in disparity was tested at the 0.05 level of significance. See the Figure 19-2 footnotes, as well as the Technical Appendix, for more detail.

5. US Department of Health and Human Services and US Department of Agriculture (USDA). Dietary guidelines for Americans, 2010. 7th ed. Washington: US Government Printing Office, 2011 Jan. Available from http://www.health.gov/dietaryguidelines/.

6. To be included in Healthy People 2010, an objective must have a national data source that provides a baseline and at least one additional data point for tracking progress. Some objectives lacked baseline data at the time of their development but had a potential data source and were considered of sufficient national importance to be included in Healthy People. These are called "developmental" objectives. When data become available, a developmental objective is moved to measurable status and a Healthy People target can be set.

7. Retained "as is" objectives have no change in the numerator definition or in the denominator definition between the Healthy People 2010 and Healthy People 2020 objectives. These include objectives that were developmental in Healthy People 2010 and are developmental in Healthy People 2020 and for which no numerator or denominator information was available.

8. Archived objectives had at least one data point in Healthy People 2010 but were not carried forward into Healthy People 2020.

9. Modified objectives have some change in the numerator definition or in the denominator definition between the Healthy People 2010 and Healthy People 2020 objectives. These include objectives that went from developmental in Healthy People 2010 to measurable in Healthy People 2020 or vice versa.

10. Gorber SC, Tremblay M, Moher D, Gorber B. A comparison of direct vs.self-report measures for assessing height, weight and body mass index: a systematic review. Obes Rev 8(4):307-26. 2007 Jul.

11. Ogden CL, Flegal KM. Changes in terminology for childhood overweight and obesity. National health statistics reports; no 25. Hyattsville, MD: National Center for Health Statistics. 2010. Available from http://www.cdc.gov/nchs/data/nhsr/nhsr025.pdf. 


\section{Comprehensive Summary of Objectives: Nutrition and Overweight}

\begin{tabular}{|c|c|c|}
\hline Objective & Description & Data Source or Objective Status \\
\hline $19-1$ & Healthy weight in adults (age adjusted, $20+$ years) & $\begin{array}{l}\text { National Health and Nutrition Examination Survey (NHANEs } \\
\text { CDC, NCHS. }\end{array}$ \\
\hline $19-2$ & Obesity in adults (age adjusted, $20+$ years) & $\begin{array}{l}\text { National Health and Nutrition Examination Survey (NHANES } \\
\text { CDC, NCHS. }\end{array}$ \\
\hline $19-3 a$ & Obesity-Children 6-11 years & $\begin{array}{l}\text { National Health and Nutrition Examination Survey (NHANES } \\
\text { CDC, NCHS. }\end{array}$ \\
\hline $19-3 b$ & Obesity-Adolescents $12-19$ years & $\begin{array}{l}\text { National Health and Nutrition Examination Survey (NHANES } \\
\text { CDC, NCHS. }\end{array}$ \\
\hline $19-3 c$ & Obesity_Children and adolescents 6-19 years & $\begin{array}{l}\text { National Health and Nutrition Examination Survey (NHANES } \\
\text { CDC, NCHS. }\end{array}$ \\
\hline $19-4$ & Growth retardation in low-income children $(<5$ years $)$ & $\begin{array}{l}\text { Pediatric Nutrition Surveillance System (PedNss), CDC, } \\
\text { NCCDPHP. }\end{array}$ \\
\hline $19-5$ & Fruit intake-At least two daily servings (age adjusted, $2+$ years) & $\begin{array}{l}\text { Baseline: Continuing Survey of Food Intake by Individuals } \\
\text { (CSFII), Department of Agriculture (USDA). Final: National } \\
\text { Health and Nutrition Examination Survey (NHANES): CDC, } \\
\text { NCHS; USDA, ARS. }\end{array}$ \\
\hline $19-6$ & $\begin{array}{l}\text { Vegetable intake-At least three daily servings with at least } 1 / 3 \\
\text { dark green or orange (age adjusted, } 2+\text { years) }\end{array}$ & $\begin{array}{l}\text { Baseline: Continuing Survey of Food Intake by Individuals } \\
\text { (CSFII), Department of Agriculture (USDA). Final: National } \\
\text { Health and Nutrition Examination Survey (NHANES): CDC, } \\
\text { NCHS; USDA, ARS. }\end{array}$ \\
\hline $19-7$ & $\begin{array}{l}\text { Grain product intake-At least six daily servings with at least } \\
\text { three being whole grains (age adjusted, } 2+\text { years) }\end{array}$ & $\begin{array}{l}\text { Baseline: Continuing Survey of Food Intake by Individuals } \\
\text { (CSFII), Department of Agriculture (USDA). Final: National } \\
\text { Health and Nutrition Examination Survey (NHANES): CDC, } \\
\text { NCHS; USDA, ARS. }\end{array}$ \\
\hline $19-8$ & $\begin{array}{l}\text { Saturated fat intake-Less than } 10 \% \text { of caloric intake (age } \\
\text { adjusted, } 2+\text { years) }\end{array}$ & $\begin{array}{l}\text { Baseline: Continuing Survey of Food Intake by Individuals } \\
\text { (CSFII), Department of Agriculture (USDA). Final: National } \\
\text { Health and Nutrition Examination Survey (NHANES): CDC, } \\
\text { NCHS; USDA, ARS. }\end{array}$ \\
\hline
\end{tabular}




\begin{tabular}{|c|c|}
\hline Objective & Description \\
\hline $19-9$ & $\begin{array}{l}\text { Total fat intake-No more than } 30 \% \text { of caloric intake (age } \\
\text { adjusted, } 2+\text { years) }\end{array}$ \\
\hline $19-10$ & $\begin{array}{l}\text { Total sodium intake-No more than 2,400 mg daily (age } \\
\text { adjusted, } 2+\text { years) }\end{array}$ \\
\hline $19-11$ & $\begin{array}{l}\text { Total calcium intake-At or above recommended level (age } \\
\text { adjusted, } 2+\text { years) }\end{array}$ \\
\hline $19-12 a$ & Iron deficiency-Children $1-2$ years \\
\hline $19-12 b$ & Iron deficiency-Children 3-4 years \\
\hline $19-12 c$ & Iron deficiency-Nonpregnant females $12-49$ years \\
\hline $19-13$ & Anemia in low-income pregnant females in their third trimester \\
\hline $19-14$ & Iron deficiency in pregnant females \\
\hline $19-15$ & $\begin{array}{l}\text { Meals and snacks at school-Children and Adolescents (6-19 } \\
\text { years) }\end{array}$ \\
\hline $19-16$ & $\begin{array}{l}\text { Worksite nutrition and weight management classes or } \\
\text { counseling }\end{array}$ \\
\hline $19-17$ & $\begin{array}{l}\text { Physician office visits with nutrition counseling for patients with } \\
\text { cardiovascular disease, diabetes, or hyperlipidemia (age } \\
\text { adjusted, } 20+\text { years) }\end{array}$ \\
\hline $19-18$ & Food security among U.S. households \\
\hline
\end{tabular}

Baseline: Continuing Survey of Food Intake by Individuals (CSFII), Department of Agriculture (USDA). Final: National Health and Nutrition Examination Survey (NHANES): CDC, NCHS; USDA, ARS.

National Health and Nutrition Examination Survey (NHANES): CDC, NCHS; USDA, ARS.

National Health and Nutrition Examination Survey (NHANES): CDC, NCHS; USDA, ARS.

National Health and Nutrition Examination Survey (NHANES), CDC, NCHS.

National Health and Nutrition Examination Survey (NHANES), CDC, NCHS.

National Health and Nutrition Examination Survey (NHANES), CDC, NCHS.

Pregnancy Nutrition Surveillance System, CDC, NCCDPHP.

National Health and Nutrition Examination Survey (NHANES), CDC, NCHS.

Dropped

National Worksite Health Promotion Survey (NWHPS), Partnership for Prevention and OPHS, ODPHP.

National Ambulatory Medical Care Survey (NAMCS), CDC, NCHS.

Current Population Survey (CPS): Department of Commerce,

Census Bureau; Department of Labor, Bureau of Labor Statistics. 
Figure 19-1. Progress Toward Target Attainment for Focus Area 19: Nutrition and Overweight

Moved away

from target ${ }^{1}$

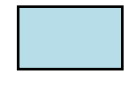

Moved toward

target

Met or exceeded

target

19-1. Healthy weight in adults (age adjusted $20+$ years)

19-2. Obesity in adults (age adjusted, 20+ years)

19-3. Obesity

a. Children 6-11 years

b. Adolescents 12-19 years

C. Children and adolescents 6-19 years

19-4. Growth retardation in low-income children ( $<5$ years)

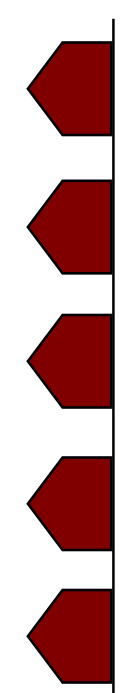

9-5. Fruit intake-At least two daily servings (age adjusted, 2+ years)

19-6. Vegetable intake-At least three daily servings with at least $1 / 3$ dark green or orange (age adjusted, $2+$ years)

\begin{tabular}{|c|c|c|c|c|c|}
\hline \multirow{2}{*}{$\begin{array}{c}2010 \\
\text { Target }\end{array}$} & \multirow{2}{*}{$\begin{array}{c}\text { Baseline } \\
\text { (Year) }\end{array}$} & \multirow{2}{*}{$\begin{array}{l}\text { Final } \\
\text { (Year) }\end{array}$} & \multicolumn{3}{|c|}{ Baseline vs. Final } \\
\hline & & & Difference $^{2}$ & $\begin{array}{l}\text { Statistically } \\
\text { Significant }^{3}\end{array}$ & $\begin{array}{l}\text { Percent } \\
\text { Change }^{4}\end{array}$ \\
\hline $60 \%$ & $\begin{array}{c}42 \% \\
(1988-94)\end{array}$ & $\begin{array}{c}31 \% \\
(2005-08)\end{array}$ & -11 & Yes & $-26.2 \%$ \\
\hline $15 \%$ & $\begin{array}{c}23 \% \\
(1988-94)\end{array}$ & $\begin{array}{c}34 \% \\
(2005-08)\end{array}$ & 11 & Yes & $47.8 \%$ \\
\hline $5 \%$ & $\begin{array}{c}11 \% \\
(1988-94)\end{array}$ & $\begin{array}{c}17 \% \\
(2005-08)\end{array}$ & 6 & Yes & $54.5 \%$ \\
\hline $5 \%$ & $\begin{array}{c}11 \% \\
(1988-94)\end{array}$ & $\begin{array}{c}18 \% \\
(2005-08)\end{array}$ & 7 & Yes & $63.6 \%$ \\
\hline $5 \%$ & $\begin{array}{c}11 \% \\
(1988-94)\end{array}$ & $\begin{array}{c}18 \% \\
(2005-08)\end{array}$ & 7 & Yes & $63.6 \%$ \\
\hline $4 \%$ & $\begin{array}{c}6 \% \\
(1997)\end{array}$ & $\begin{array}{c}6 \% \\
(2009)\end{array}$ & 0 & $\begin{array}{c}\text { Not } \\
\text { tested }\end{array}$ & $0.0 \%$ \\
\hline $75 \%$ & $\begin{array}{c}39 \% \\
(1994-96)\end{array}$ & $\begin{array}{c}40 \% \\
(2003-04)\end{array}$ & 1 & No & $2.6 \%$ \\
\hline $50 \%$ & $\begin{array}{c}4 \% \\
(1994-96)\end{array}$ & $\begin{array}{c}4 \% \\
(2003-04)\end{array}$ & 0 & No & $0.0 \%$ \\
\hline
\end{tabular}

(continued) 
Figure 19-1. Progress Toward Target Attainment for Focus Area 19: Nutrition and Overweight (continued)

Moved away

from target ${ }^{1}$
Moved toward target
Met or exceeded

target

19-7. Grain product intake-At least six daily servings with at least three being whole grains (age adjusted, 2+ years)

19-8. Saturated fat intake-Less than $10 \%$ of caloric intake (age adjusted, $2+$ years)

19-9. Total fat intake-No more than $30 \%$ of caloric intake (age adjusted, $2+$ years)

19-10. Total sodium intake-No more than 2,400 mg daily (age adjusted, 2+ years)

19-11. Total calcium intake-At or above recommended level (age adjusted, 2+ years)

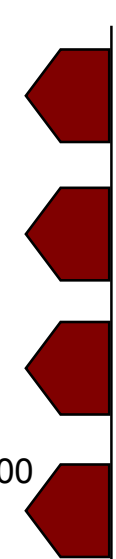

19-12. Iron deficiency

a. Children 1-2 years

b. Children 3-4 years

c. Nonpregnant females $12-49$ years

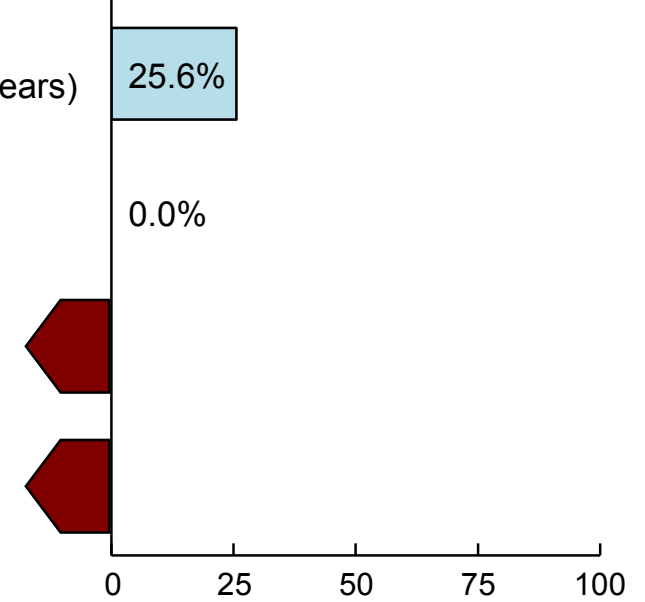

\begin{tabular}{|c|c|c|c|c|c|}
\hline \multirow{2}{*}{$\begin{array}{l}2010 \\
\text { Target }\end{array}$} & \multirow{2}{*}{$\begin{array}{c}\text { Baseline } \\
\text { (Year) }\end{array}$} & \multirow{2}{*}{$\begin{array}{l}\text { Final } \\
\text { (Year) }\end{array}$} & \multicolumn{3}{|c|}{ Baseline vs. Final } \\
\hline & & & Difference ${ }^{2}$ & $\begin{array}{l}\text { Statistically } \\
\text { Significant }^{3}\end{array}$ & $\begin{array}{l}\text { Percent } \\
\text { Change }^{4}\end{array}$ \\
\hline $50 \%$ & $\begin{array}{c}4 \% \\
(1994-96)\end{array}$ & $\begin{array}{c}3 \% \\
(2003-04)\end{array}$ & -1 & Yes & $-25.0 \%$ \\
\hline $75 \%$ & $\begin{array}{c}36 \% \\
(1994-96)\end{array}$ & $\begin{array}{c}34 \% \\
(2005-08)\end{array}$ & -2 & No & $-5.6 \%$ \\
\hline $75 \%$ & $\begin{array}{c}33 \% \\
(1994-96)\end{array}$ & $\begin{array}{c}31 \% \\
(2005-08)\end{array}$ & -2 & No & $-6.1 \%$ \\
\hline $65 \%$ & $\begin{array}{c}15 \% \\
(1988-94)\end{array}$ & $\begin{array}{c}13 \% \\
(2003-04)\end{array}$ & -2 & No & $-13.3 \%$ \\
\hline $74 \%$ & $\begin{array}{c}31 \% \\
(1988-94)\end{array}$ & $\begin{array}{c}42 \% \\
(2003-04)\end{array}$ & 11 & Yes & $35.5 \%$ \\
\hline $5 \%$ & $\begin{array}{c}9 \% \\
(1988-94)\end{array}$ & $\begin{array}{c}9 \% \\
(1999-02)\end{array}$ & 0 & No & $0.0 \%$ \\
\hline $1 \%$ & $\begin{array}{c}4 \% \\
(1988-94)\end{array}$ & $\begin{array}{c}6 \% \\
(2003-06)\end{array}$ & 2 & No & $50.0 \%$ \\
\hline $7 \%$ & $\begin{array}{c}11 \% \\
(1988-94)\end{array}$ & $\begin{array}{c}16 \% \\
(2003-06)\end{array}$ & 5 & Yes & $45.5 \%$ \\
\hline
\end{tabular}

Percent of targeted change achieved ${ }^{5}$

(continued) 
Figure 19-1. Progress Toward Target Attainment for Focus Area 19: Nutrition and Overweight (continued)

Moved away from target ${ }^{1}$
Moved toward target
Met or exceeded

target

19-13. Anemia in low-income pregnant females in their third trimester

19-14. Iron deficiency in pregnant females

19-17. Physician office visits with nutrition counseling for patients with cardiovascular disease, diabetes, or hyperlipidemia (age adjusted, 20+ years)

19-18. Food security among U.S. households

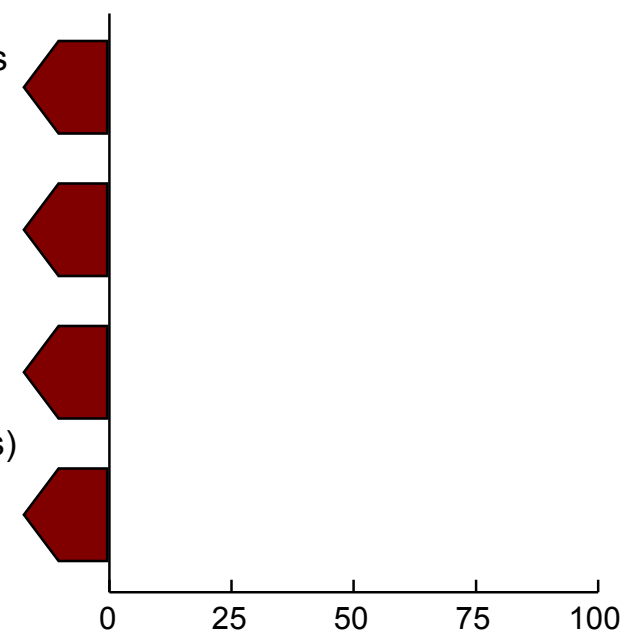

\begin{tabular}{|c|c|c|c|c|c|}
\hline \multirow{2}{*}{$\begin{array}{c}2010 \\
\text { Target }\end{array}$} & \multirow{2}{*}{$\begin{array}{c}\text { Baseline } \\
(\text { Year }\end{array}$} & \multirow{2}{*}{$\begin{array}{c}\text { Final } \\
(\text { Year })\end{array}$} & \multicolumn{3}{|c|}{ Baseline vs. Final } \\
\cline { 4 - 6 } & Difference & & $\begin{array}{c}\text { Statistically } \\
\text { Significant }^{3}\end{array}$ & $\begin{array}{c}\text { Percent } \\
\text { Change }^{4}\end{array}$ \\
\hline $9 \%$ & $\begin{array}{c}29 \% \\
(1996)\end{array}$ & $\begin{array}{c}34 \% \\
(2009)\end{array}$ & 5 & $\begin{array}{c}\text { Not } \\
\text { tested }\end{array}$ & $17.2 \%$ \\
\hline $75 \%$ & $\begin{array}{c}14 \% \\
(1999-02)\end{array}$ & $\begin{array}{c}16 \% \\
(2003-06)\end{array}$ & 2 & No & $14.3 \%$ \\
\hline $94 \%$ & $\begin{array}{c}88 \% \\
(1999)\end{array}$ & $\begin{array}{c}28 \% \\
(2007)\end{array}$ & -14 & Yes & $-33.3 \%$ \\
\hline
\end{tabular}

\section{Percent of targeted change achieved}

\section{NOTES}

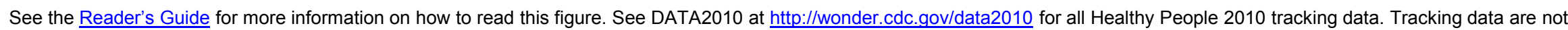
available for objective 19-16. Objective 19-15 was deleted at the Midcourse Review.

\section{FOOTNOTES}

${ }^{1}$ Movement away from target is not quantified using the percent of targeted change achieved. See Technical Appendix for more information.

${ }^{2}$ Difference $=$ Final value - Baseline value. Differences between percents $(\%)$ are measured in percentage points.

${ }^{3}$ When estimates of variability are available, the statistical significance of the difference between the final value and the baseline value is assessed at the 0.05 level. See Technical Appendix for more information.

${ }^{4}$ Percent change $=\frac{\text { Final value }- \text { Baseline value }}{\text { Baseline value }} \times 100$.

${ }^{5}$ Percent of targeted change achieved $=\frac{\text { Final value }- \text { Baseline value }}{\text { Healthy People } 2010 \text { target }- \text { Baseline value }} \times 100$. 
Figure 19-1. Progress Toward Target Attainment for Focus Area 19: Nutrition and Overweight (continued)

\section{DATA SOURCES}

19-1-19-2. National Health and Nutrition Examination Survey (NHANES), CDC, NCHS.

19-3a-c. National Health and Nutrition Examination Survey (NHANES), CDC, NCHS.

19-4. Pediatric Nutrition Surveillance System (PedNss), CDC, NCCDPHP.

19-5-19-9. Baseline: Continuing Survey of Food Intake by Individuals (CSFII), Department of Agriculture (USDA). Final: National Health and Nutrition Examination Survey (NHANES), $\mathrm{CDC}, \mathrm{NCHS}$.

19-10-19-11. National Health and Nutrition Examination Survey (NHANES), CDC, NCHS.

19-12a-c. National Health and Nutrition Examination Survey (NHANES), CDC, NCHS.

19-13. Pregnancy Nutrition Surveillance System, CDC, NCCDPHP.

19-14. National Health and Nutrition Examination Survey (NHANES), CDC, NCHS.

19-17. National Ambulatory Medical Care Survey (NAMCS), CDC, NCHS.

19-18. Current Population Survey (CPS): Department of Commerce, Census Bureau; Department of Labor, Bureau of Labor Statistics. 
Figure 19-2. Health Disparities Table for Focus Area 19: Nutrition and Overweight Disparities from the best group rate for each characteristic at the most recent data point and changes in disparity from the baseline to the most recent data point.

Population-based objectives

19-1. Healthy weight in adults (age adjusted, 20+ years) $(1988-94,2005-08)^{1}$ *

19-2. Obesity in adults (age adjusted, 20+ years) (1988-94, 2005-08) ${ }^{1 *}$

19-3a. Obesity—Children 6-11 years (1988-94, 2005-08)*

b. Obesity-Adolescents 12-19 years (1988-94, 2005-08)*

c. Obesity-Children and adolescents 6-19 years (1988-94, 2005-08)*

19-4. Growth retardation in low-income children ( $<5$ years) $(1997,2009) \dagger$

19-5. Fruit intake-At least two daily servings (age adjusted, 2+ years) $(1994-96,2003-04)^{\star}$

19-6. Vegetable intake-At least three daily servings with at least $1 / 3$ dark green or orange (age adjusted, $2+$ years) (1994-96, 2003-04)*

19-7. Grain product intake-At least six daily servings with at least three being whole grains (age adjusted, $2+$ years) (1994-96, 2003-04)*

19-8. Saturated fat intake-Less than $10 \%$ of caloric intake (age adjusted, 2+ years) (1994-96, 2005-08)*

19-9. Total fat intake-No more than $30 \%$ of caloric intake (age adjusted, 2+ years) (1994-96, 2005-08)*

19-10. Total sodium intake-No more than $2,400 \mathrm{mg}$ daily (age adjusted, 2+ years) $(1988-94,2003-04)^{1 *}$

19-11. Total calcium intake-At or above recommended level (age adjusted, 2+ years) (1988-94, 2003-04) ${ }^{1 *}$

19-12a. Iron deficiency_Children 1-2 years (1988-94, 1999-02)*

b. Iron deficiency-Children 3-4 years $(1988-94,2003-06)^{2 *}$

c. Iron deficiency-Nonpregnant females 12-49 years $(1988-94,2003-06)^{1 *}$

19-13. Anemia in low-income pregnant females in their third trimester $(1996,2009) \dagger$

19-14. Iron deficiency in pregnant females $(1999-02,2003-06)^{*}$

19-17. Physician office visits with nutrition counseling for patients with cardiovascular disease, diabetes, or hyperlipidemia (age adjusted, 20+ years) $(1997,2007)^{*}$

19-18. Food security among U.S. households $(1995,2008)^{3,4 *}$

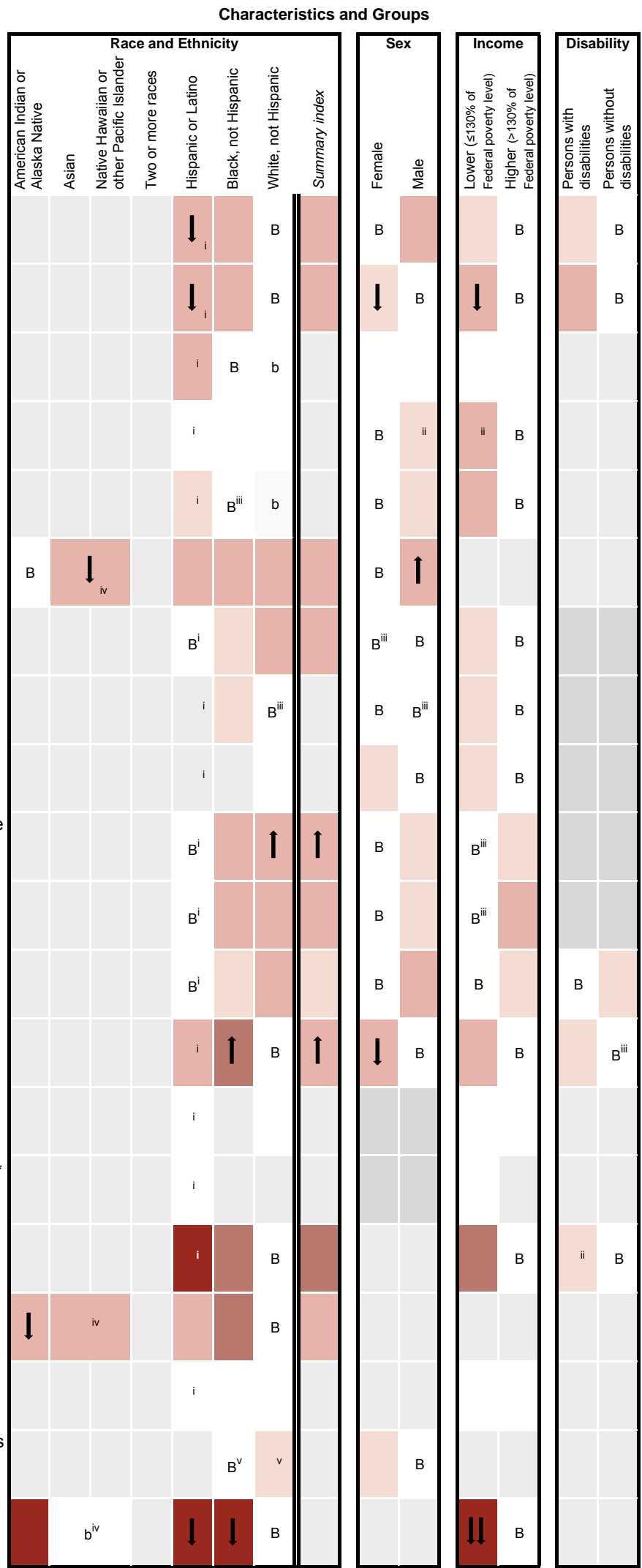

(continued) 


\section{Figure 19-2. Health Disparities Table for Focus Area 19: Nutrition and Overweight (continued)}

NOTES

See DATA2010 at http://wonder.cdc.gov/data2010 for all Healthy People 2010 tracking data. Disparity data are either unavailable or not applicable for objective 19-16. Objective 19-15 was deleted at Midcourse Review.

Years in parentheses represent the baseline and most recent data years (if available).

Disparity from the best group rate is defined as the percent difference between the best group rate and each of the other group rates for a characteristic (e.g., race and ethnicity). The summary index is the average of these percent differences for a characteristic. Change in disparity is estimated by subtracting the disparity at baseline from the disparity at the most recent data point. Change in the summary index is estimated by subtracting the summary index at baseline from the summary index at the most recent data point. See Technical Appendix for more information.

LEGEND

\begin{tabular}{|c|c|c|c|c|c|}
\hline $\begin{array}{l}\text { The "best" group rate at the most recent } \\
\text { data point. }\end{array}$ & B & $\begin{array}{l}\text { The group with the best rate for } \\
\text { specified characteristic. }\end{array}$ & b & $\begin{array}{l}\text { Most favorable group rate for } \\
\text { specified characteristic, but reliability } \\
\text { criterion not met. }\end{array}$ & $\begin{array}{l}\text { Reliability criterion for best } \\
\text { group rate not met, or data } \\
\text { available for only one group }\end{array}$ \\
\hline
\end{tabular}
Percent difference from the best group rate

Disparity from the best group rate at the most recent data point.

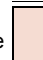

Less than $10 \%$, or difference not statistically significant (when estimates of variability are available)
$50 \%-99 \%$ $10 \%-49 \%$

$50 \%-99 \%$ Increase in disparity (percentage points)

Changes in disparity over time are shown when: (a) disparities data are available at both baseline and most recent time points; (b) data are not for the group(s) indicated by "B" or "b" at either time point; and (c) the change is greater than or equal to 10 percentage points and statistically significant, or when the change is greater than or equal to 10 percentage points and estimates of variability were not available. See Technical Appendix.
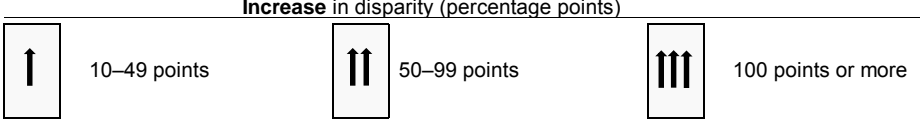
Decrease in disparity (percentage points)

\begin{tabular}{|c|c|c|c|}
\hline 1 & 10-49 points & 50-99 points & 100 points or more \\
\hline
\end{tabular}
Data not available Characteristic not selected for this objective.

\section{FOOTNOTES}

* Measures of variability were available. Thus, the variability of best group rates was assessed, and statistical significance was tested. Disparities of $10 \%$ or more are displayed when the differences from the best group rate are statistically significant at the 0.05 level. Changes in disparities over time are indicated by arrows when the changes are greater than or equal to 10 percentage points and are statistically significant at the 0.05 level. See Technical Appendix.

† Measures of variability were not available. Thus, the variability of best group rates was not assessed, and statistical significance could not be tested. Nonetheless, disparities and changes in disparities over time are displayed according to their magnitude. See Technical Appendix.

1 Baseline data by disability status are for 1991-94

2 Most recent data by race and ethnicity are for 1988-94.

3 Baseline data for American Indian or Alaska Native are based on years 1995-97.

4 Most recent data for American Indian or Alaska Native are based on years 2006-08.

i Data are for Mexican American.

ii Reliability criterion for best group rate not met, or data available for only one group, at baseline. Change in disparity cannot be assessed. See $\underline{\text { Technical }}$ Appendix.

iii The group with the best rate at the most recent data point is different from the group with the best rate at baseline. Both rates met the reliability criterion. See Technical Appendix.

iv Data are for Asian or Pacific Islander.

v Data include persons of Hispanic origin.

\section{DATA SOURCES}

19-1-19-2. National Health and Nutrition Examination Survey (NHANES), CDC, NCHS.

19-3a-c. National Health and Nutrition Examination Survey (NHANES), CDC, NCHS

19-4. Pediatric Nutrition Surveillance System (PedNss), CDC, NCCDPHP.

19-5-19-9. Baseline: Continuing Survey of Food Intake by Individuals (CSFII), Department of Agriculture (USDA). Final: National Health and Nutrition Examination Survey (NHANES), CDC, NCHS.

19-10-19-11. National Health and Nutrition Examination Survey (NHANES), CDC, NCHS.

19-12a-c. National Health and Nutrition Examination Survey (NHANES), CDC, NCHS

19-13. Pregnancy Nutrition Surveillance System, CDC, NCCDPHP.

19-14. National Health and Nutrition Examination Survey (NHANES), CDC, NCHS.

19-17. National Ambulatory Medical Care Survey (NAMCS), CDC, NCHS.

19-18. Current Population Survey (CPS): Department of Commerce, Census Bureau; Department of Labor, Bureau of Labor Statistics. 
Figure 19-3. Obesity in Adults (20+ Years) 2008

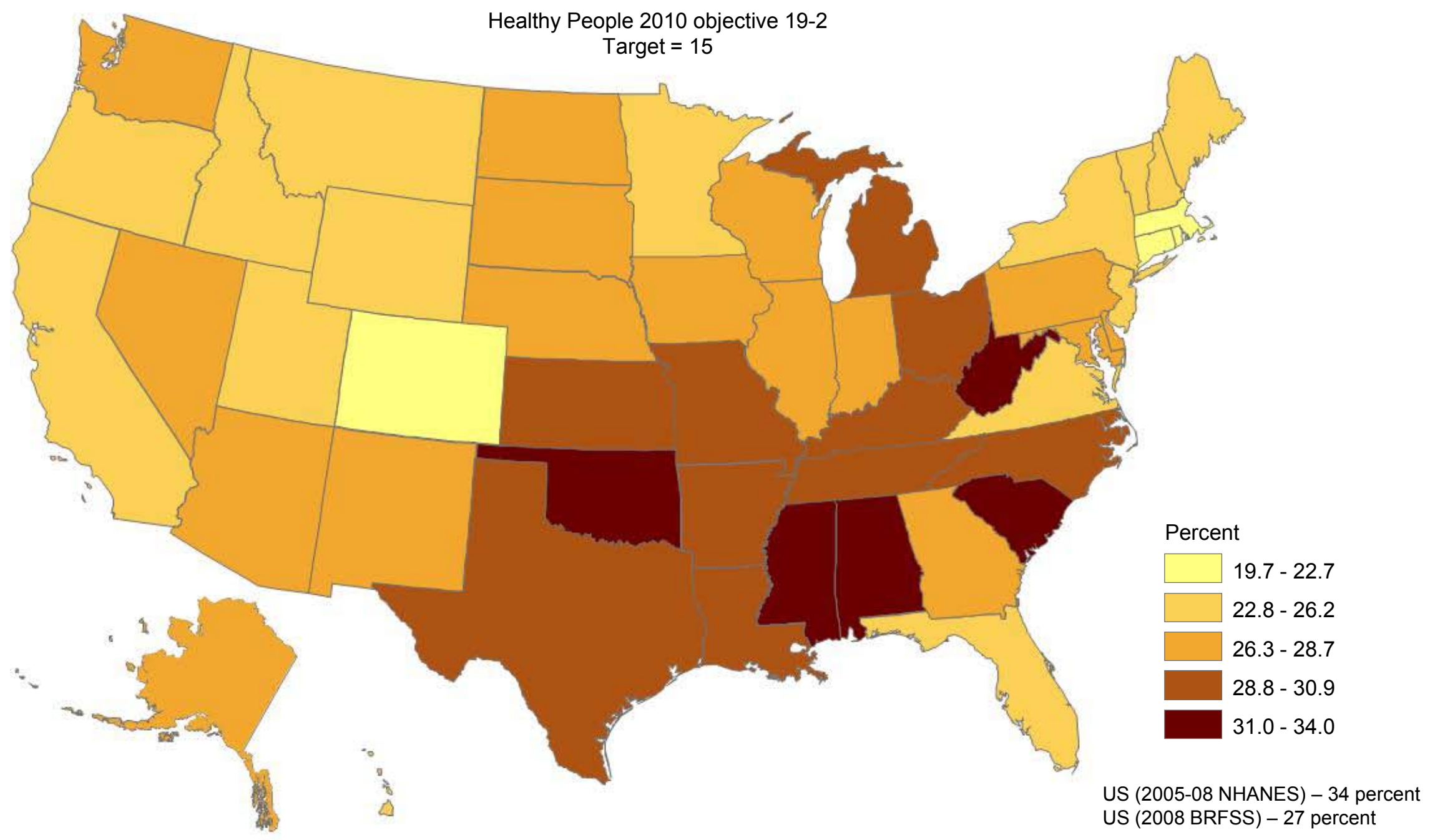

No states met the target

Notes: Data are age-adjusted to the 2000 standard population. Rates are displayed by a Jenks classification for U.S. states. National data for the objective are based on measured height and weight from the National Health and Nutrition Examination Survey (NHANES) and are the basis for setting the target. State data from the BRFSS are based on self-reported weight and height and may not be comparable to the national data from the NHANES. SOURCE: Behavioral Risk Factor Surveillance System (BRFSS), CDC. 


\section{Final Review}

\section{Occupational Safety and Health}

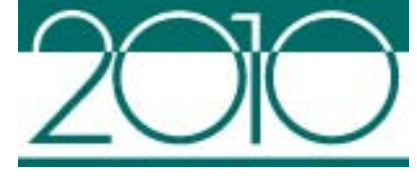

20

\section{Lead Agency}

Centers for Disease Control and Prevention

\section{Contents}

Goal.

Highlights.

Summary of Progress.

Transition to Healthy People 2020

Data Considerations

Notes

Comprehensive Summary of Objectives 20-8

Progress Chart 20-10

Health Disparities Table 


\section{Goal: Promote the health and safety of people at work through prevention and early intervention}

The objectives in the chapter track work-related deaths, injuries, and selected health conditions, for example, hearing loss, elevated blood lead levels, and worksite stress reduction.

All Healthy People tracking data quoted in this chapter, along with technical information and operational definitions for each objective, can be found in the Healthy People 2010 database, (DATA2010), available from http://wonder.cdc.gov/data2010/.

More information about this Focus Area can be found in the following publications:

- Healthy People 2010: Understanding and Improving Health, available from http://www.healthypeople.gov/2010/Document/tableofcontents.htm\#under.

- Healthy People 2010 Midcourse Review, available from http://www.healthypeople.gov/2010/data/midcourse/html/default.htm\#FocusAreas.

\section{Highlights}

- Substantial progress was achieved for objectives in this Focus Area during the past decade [1]. Almost two-thirds (64\%) of the Occupational Safety and Health objectives achieved their Healthy People 2010 targets. Only three objectives (20-1d through e, and 20-9) did not make progress toward the 2010 targets (Figure 20-1). Health disparities among racial and ethnic groups and by sex were observed for one objective (Figure 20-2) [2].

- Work-related injury deaths among workers aged 16 years and over in all industries (objective 20-1a) declined by $26.7 \%$ between 1998 and 2009, from 4.5 to 3.3 deaths per 100,000 workers, moving toward the 2010 target of 3.2 deaths per 100,000 workers. Workrelated injury deaths among workers aged 16 years and over in mining (objective $20-1 \mathrm{~b}$ ) declined by $46.2 \%$ between 1998 and 2009 , from 23.6 to 12.7 per 100,000 , exceeding the 2010 target of 16.5, Similarly, work-related injury deaths among workers aged 16 years and over in construction (objective 20-1c) declined by 33.1\%, from 14.5 to 9.7 per 100,000, exceeding the target of 10.1 .

- Female workers had a lower (better) rate of work-related injury deaths in all industries than male workers, 0.6 deaths per 100,000 in 2009 . The rate for male workers, 5.5 deaths per 100,000, was more than nine times the rate for female workers [2].

- Work-related injuries per 100 full-time workers (objective 20-2) declined for all industry groups (objectives 20-2a through g), exceeding the 2010 targets. Work-related injuries in all industries (objective 20-2a) declined by $45.2 \%$ between 1998 and 2009, from 6.2 to 3.4 injuries per 100 full-time workers, exceeding the 2010 target of 4.3. Similar results were observed for individual industry sectors (e.g., construction, health services). Statistically significant downward trends in injuries were observed between the baseline (1997 or 1998) and 2009 for all industry sectors [3]. 
- Work-related homicides among workers aged 16 years and over (objective 20-5) declined by $20 \%$ between 1998 and 2006, from 0.5 to 0.4 per 100,000 workers, meeting the 2010 target of 0.4 . Work-related assaults among workers aged 16 years and over (objective 20-6) declined by $66.4 \%$ between 1998 and 2009, from 1.10 to 0.37 per 100 workers, exceeding the 2010 target of 0.78 .

\section{Summary of Progress}

- Figure 20-1 presents a quantitative assessment of progress in achieving the Healthy People 2010 objectives for Occupational Safety and Health [1]. Data to measure progress toward target attainment were available for 22 objectives. Of these:

- Fourteen objectives (20-1b and c, 20-2a through g, 20-3, 20-5, 20-6, 20-8, and 2011) met or exceeded their Healthy People 2010 targets.

- Five objectives moved toward their targets. A statistically significant difference between the baseline and the final data points was observed for one of these objectives (20-7). Data to test the significance of the difference were unavailable for four objectives (20-1a, 20-2h, 20-4, and 20-10).

- Three objectives (20-1d, 20-1e, and 20-9) moved away from their targets, but data to test the significance of the difference between the baseline and the final data points were unavailable for these objectives.

- Figure 20-2 displays health disparities in Occupational Safety and Health from the best group rate for each characteristic at the most recent data point [2]. It also displays changes in disparities from baseline to the most recent data point [4]. Health disparities data were only available for objective 20-1a, work-related injury deaths for all industries:

- The black non-Hispanic population had a lower rate of work-related injury deaths than the Hispanic or Latino or the white non-Hispanic populations.

- Females had a lower rate than males of work-related injury deaths.

\section{Transition to Healthy People 2020}

The Healthy People 2020 Occupational Safety and Health Topic Area consists of fewer objectives than those included in Healthy People 2010. See HealthyPeople.gov for a complete list of Healthy People 2020 topics and objectives.

The Healthy People 2020 objectives can be grouped into several sections:

- Work related fatalities

- Work related injuries

- Objectives that target specific occupational hazards.

The differences between the Healthy People 2010 and Healthy People 2020 objectives are summarized below: 
- The Healthy People 2020 Occupational Safety and Health Topic Area has 16 objectives, whereas the Healthy People 2010 Focus Area had 22 objectives.

- Eleven Healthy People 2010 objectives were retained "as is" [5].

- Reduce deaths from work-related injuries. The titles of two of these five objectives were modified: deaths from work-related injury-Transportation (objective 20-1d) was changed to Transportation and warehousing; and deaths from work-related injury-Agriculture, forestry, and fishing (objective 20-1e) was changed to Agriculture, forestry, fishing, and hunting.

- Reduce the rate of injury and illness cases involving days away from work due to overexertion or repetitive motion (objective 20-3).

- Reduce pneumoconiosis deaths (objective 20-4).

- Reduce deaths from work-related homicides (objective 20-5).

- Reduce the proportion of persons who have elevated blood lead concentrations from work exposures (objective 20-7).

- Reduce occupational skin diseases or disorders among full-time workers (objective 20-8).

- Reduce new cases of work-related, noise-induced hearing loss (objective 20-11).

- Ten Healthy People 2010 objectives were modified [6].

- Objectives for non-fatal work-related injuries were reduced to a single objective tracking all industries (objectives 20-2a through 20-2g).

- The targeted population for adolescent workers was expanded from 15-17 years to 15-19 years of age (objective $20-2 \mathrm{~h}$ ).

- The objective to reduce work-related assaults will be tracked with a new data source (objective 20-6).

- The objective to increase the proportion of employees who have access to workplace programs that prevent or reduce employee stress will be tracked with a new data source (objective 20-9).

- Seven Healthy People 2010 objectives were archived [7]. Non-fatal work-related injury rates by specific industry sector were not substantially different than the overall rate. Existing data sources for needle stick injuries were not adequate to accurately track the objective because there is no single national sharps injury surveillance system. Another limitation is underreporting of injuries. It is estimated that approximately half of exposures are reported, with reporting rates varying by occupational group.

- Reduce non-fatal work-related injuries:

- Construction (objective 20-2b)

- Health Services (objective 20-2c)

- Agriculture, Forestry, and Fishing (objective 20-2d)

- Transportation (objective 20-2e)

- Mining (objective 20-2f)

- Manufacturing (objective 20-2g).

- Reduce occupational needle stick injuries among hospital-based health care workers (objective 20-10). 
- One new objective was added to track work-related injuries treated in emergency departments.

Appendix D, “A Crosswalk Between Objectives From Healthy People 2010 to Healthy People 2020," summarizes the changes between the two decades of objectives, reflecting new knowledge and direction for this area.

\section{Data Considerations}

A number of objectives in this Focus Area are tracked through the data sources maintained by the Bureau of Labor Statistics. Work-related injury deaths (objective 20-1) are monitored through the Census of Fatal Occupational Injuries and non-fatal injuries and illnesses are tracked through the Survey of Occupational Injuries and Illnesses. Over the past decade, industry data from these two sources were classified according to several different classification systems. From 1998 to 2002, data were classified using the 1987 Standard Industrial Classification (SIC) Manual. Data from 2003 to 2008 were classified using the 2002 North American Industry Classification System (NAICS). Industry data after 2008 are classified using the 2007 NAICS. The substantial differences between the SIC and NAICS result in breaks in series. From 1998 to 2005 rates were employment-based, whereas from 2006 to 2009 rates were hours-based. Effective January 1, 2002, the Occupational Safety and Health Administration (OSHA) revised its requirements for recording occupational injuries and illnesses. Due to the changes in classification systems and the revised OSHA reporting requirements, users are urged to use caution when examining trend data for the past decade.

Education and income are the primary measures of socioeconomic status (SES) in Healthy People 2010. Most data systems used in Healthy People 2010 define income as a family's income before taxes. In order to facilitate comparisons among groups and over time, while adjusting for family size and for inflation, Healthy People 2010 categorizes income using the poverty thresholds developed by the U.S. Census Bureau. Thus, the three categories of family income that are primarily used are:

- Poor-below the Federal poverty level

- Near poor-100\% to $199 \%$ of the Federal poverty level

- Middle/high income-200\% or more of the Federal poverty level.

These categories may be overridden by considerations specific to the data system, in which case they are modified as appropriate. See Healthy People 2010: General Data Issues, referenced below.

Additional information on data issues is available from the following sources:

- All Healthy People 2010 tracking data can be found in the Healthy People 2010 database, (DATA2010), available from http://wonder.cdc.gov/data2010/.

- Detailed information about the data and data sources used to support these objectives can be found in the Operational Definitions on the DATA2010 website, available from http://wonder.cdc.gov/data2010/focusod.htm. 
- More information on statistical issues related to Healthy People tracking and measurement can be found in the Technical Appendix and in Healthy People 2010: General Data Issues, which is available in the Data Issues section of the NCHS Healthy People website under Healthy People 2010.

\section{Notes}

1. Displayed in the Progress Chart (Figure 20-1), the percent of targeted change achieved expresses the difference between the baseline and the final value relative to the initial difference between the baseline and the Healthy People 2010 target. As such, it is a relative measure of progress toward attaining the Healthy People 2010 target. See the Reader's Guide for more information. When standard errors were available, the difference between the baseline and the final value was tested at the 0.05 level of significance. See the Figure 20-1 footnotes, as well as the Technical Appendix, for more detail.

2. Information about disparities among select populations is shown in the Health Disparities Table (Figure 20-2). Disparity from the best group rate is defined as the percent difference between the best group rate and each of the other group rates for a characteristic. For example, racial and ethnic health disparities are measured as the percent difference between the best racial and ethnic group rate and each of the other racial and ethnic group rates. Similarly, disparities by sex are measured as the percent difference between the better group rate (e.g., female) and the rate for the other group (e.g., male). Some objectives are expressed in terms of favorable events or conditions that are to be increased, while others are expressed in terms of adverse events or conditions that are to be reduced. In order to facilitate comparison of health disparities across different objectives, disparity is measured only in terms of adverse events or conditions. For comparability across objectives, objectives that are expressed in terms of favorable events or conditions are re-expressed using the adverse event or condition for the purpose of computing disparity, but they are not otherwise restated or changed. For example, objective 1-1, to increase the proportion of persons with health insurance (e.g., $72 \%$ of the American Indian or Alaska Native population aged under 65 years had some form of health insurance in 2008), is expressed in terms of the percentage of persons without health insurance (e.g., $100 \%-72 \%=28 \%$ of the American Indian or Alaska Native population aged under 65 years did not have any form of health insurance in 2008) when the disparity from the best group rate is calculated. See the Reader's Guide for more information. When standard errors were available, the difference between the best group rate and each of the other group rates was tested at the 0.05 level of significance. See the Figure 20-2 footnotes, as well as the Technical Appendix, for more detail.

3. The presence of a monotonic increasing or decreasing trend in the underlying measure was tested with the nonparametric Mann-Kendall test, then the slope of a linear trend was estimated with the nonparametric Sen's method. See Technical Appendix for more information.

4. The change in disparity is estimated by subtracting the disparity at baseline from the disparity at the most recent data point and, therefore, is expressed as a change in percentage points. See the Reader's Guide for more information. When standard errors were available, the change in disparity was tested at the 0.05 level of significance. See the Figure 20-2 footnotes, as well as the Technical Appendix, for more detail.

5. Retained "as is" objectives have no change in the numerator definition or in the denominator definition between the Healthy People 2010 and Healthy People 2020 objectives. These include objectives that were developmental in Healthy People 2010 and are developmental in Healthy People 2020 and for which no numerator or denominator information was available. 
6. Modified objectives have some change in the numerator definition or in the denominator definition between the Healthy People 2010 and Healthy People 2020 objectives. These include objectives that went from developmental in Healthy People 2010 to measurable in Healthy People 2020 or vice versa.

7. Archived objectives had at least one data point in Healthy People 2010 but were not carried forward into Healthy People 2020. 


\section{Comprehensive Summary of Objectives: Occupational Safety and Health}

\begin{tabular}{|c|c|c|}
\hline Objective & Description & Data Source \\
\hline $20-1 \mathrm{a}$ & $\begin{array}{l}\text { Work-related injury deaths-All industries (per 100,000 workers, 16+ } \\
\text { years) }\end{array}$ & $\begin{array}{l}\text { Census of Fatal Occupational Injuries (CFOI), Department of Labor } \\
\text { (DOL), Bureau of Labor Statistics (BLS). }\end{array}$ \\
\hline $20-1 b$ & Work-related injury deaths-Mining (per 100,000 workers, $16+$ years) & $\begin{array}{l}\text { Census of Fatal Occupational Injuries (CFOI), Department of Labor } \\
\text { (DOL), Bureau of Labor Statistics (BLS). }\end{array}$ \\
\hline $20-1 c$ & $\begin{array}{l}\text { Work-related injury deaths-Construction (per 100,000 workers, 16+ } \\
\text { years) }\end{array}$ & $\begin{array}{l}\text { Census of Fatal Occupational Injuries (CFOI), Department of Labor } \\
\text { (DOL), Bureau of Labor Statistics (BLS). }\end{array}$ \\
\hline $20-1 d$ & $\begin{array}{l}\text { Work-related injury deaths-Transportation (per 100,000 workers, } \\
16+\text { years) }\end{array}$ & $\begin{array}{l}\text { Census of Fatal Occupational Injuries (CFOI), Department of Labor } \\
\text { (DOL), Bureau of Labor Statistics (BLS). }\end{array}$ \\
\hline $20-1 e$ & $\begin{array}{l}\text { Work-related injury deaths-Agriculture, forestry, and fishing (per } \\
100,000 \text { workers, } 16+\text { years) }\end{array}$ & $\begin{array}{l}\text { Census of Fatal Occupational Injuries (CFOI), Department of Labor } \\
\text { (DOL), Bureau of Labor Statistics (BLS). }\end{array}$ \\
\hline $20-2 a$ & Work-related injuries-All industries (per 100 full-time workers) & $\begin{array}{l}\text { Survey of Occupational Injuries and Illnesses (SOII), Department of } \\
\text { Labor (DOL), Bureau of Labor Statistics (BLS). }\end{array}$ \\
\hline $20-2 b$ & Work-related injuries-Construction (per 100 full-time workers) & $\begin{array}{l}\text { Survey of Occupational Injuries and Illnesses (SOII), Department of } \\
\text { Labor (DOL), Bureau of Labor Statistics (BLS). }\end{array}$ \\
\hline $20-2 c$ & Work-related injuries-Health services (per 100 full-time workers) & $\begin{array}{l}\text { Survey of Occupational Injuries and Illnesses (SOII), Department of } \\
\text { Labor (DOL), Bureau of Labor Statistics (BLS). }\end{array}$ \\
\hline $20-2 d$ & $\begin{array}{l}\text { Work-related injuries-Agricultural, forestry, and fishing (per } 100 \text { full- } \\
\text { time workers) }\end{array}$ & $\begin{array}{l}\text { Survey of Occupational Injuries and Illnesses (SOII), Department of } \\
\text { Labor (DOL), Bureau of Labor Statistics (BLS). }\end{array}$ \\
\hline $20-2 \mathrm{e}$ & Work-related injuries-Transportation (per 100 full-time workers) & $\begin{array}{l}\text { Survey of Occupational Injuries and Illnesses (SOII), Department of } \\
\text { Labor (DOL), Bureau of Labor Statistics (BLS). }\end{array}$ \\
\hline $20-2 \mathrm{f}$ & Work-related injuries-Mining (per 100 full-time workers) & $\begin{array}{l}\text { Survey of Occupational Injuries and Illnesses (SOII), Department of } \\
\text { Labor (DOL), Bureau of Labor Statistics (BLS). }\end{array}$ \\
\hline $20-2 g$ & Work-related injuries-Manufacturing (per 100 full-time workers) & $\begin{array}{l}\text { Survey of Occupational Injuries and Illnesses (SOII), Department of } \\
\text { Labor (DOL), Bureau of Labor Statistics (BLS). }\end{array}$ \\
\hline $20-2 \mathrm{~h}$ & $\begin{array}{l}\text { Work-related injuries among adolescent workers (per } 100 \text { full-time } \\
\text { workers, 15-17 years) }\end{array}$ & $\begin{array}{l}\text { National Electronic Injury Surveillance System (NEISS): Consumer } \\
\text { Product Safety Commission (CPSC); CDC, NIOSH. }\end{array}$ \\
\hline $20-3$ & $\begin{array}{l}\text { Overexertion or repetitive motion injuries (per 100,000 full-time } \\
\text { workers) }\end{array}$ & $\begin{array}{l}\text { Survey of Occupational Injuries and Illnesses (SOII), Department of } \\
\text { Labor (DOL), Bureau of Labor Statistics (BLS). }\end{array}$ \\
\hline $20-4$ & Pneumoconiosis deaths (number, $15+$ years) & $\begin{array}{l}\text { National Occupational Respiratory Mortality System (NORMS), CDC, } \\
\text { NIOSH. }\end{array}$ \\
\hline
\end{tabular}




\begin{tabular}{|c|c|c|}
\hline Objective & Description & Data Source \\
\hline $20-5$ & Work-related homicides (per 100,000 workers, $16+$ years) & $\begin{array}{l}\text { Census of Fatal Occupational Injuries (CFOI), Department of Labor } \\
\text { (DOL), Bureau of Labor Statistics (BLS). }\end{array}$ \\
\hline $20-6$ & Work-related assaults (per 100 workers, $16+$ years) & $\begin{array}{l}\text { National Crime Victimization Survey (NCVS), Department of Justice } \\
\text { (DOJ), Bureau of Justice Statistics (BJS). }\end{array}$ \\
\hline $20-7$ & $\begin{array}{l}\text { Elevated blood lead levels }-\geq 25 \mu \mathrm{g} / \mathrm{dL} \text { (per 100,000 employed, 16+ } \\
\text { years) }\end{array}$ & $\begin{array}{l}\text { Adult Blood Lead Epidemiology and Surveillance Program (ABLES), } \\
\text { CDC, NIOSH. }\end{array}$ \\
\hline $20-8$ & $\begin{array}{l}\text { Occupational skin diseases or disorders (per 100,000 full-time } \\
\text { workers) }\end{array}$ & $\begin{array}{l}\text { Survey of Occupational Injuries and Illnesses (SOII), Department of } \\
\text { Labor (DOL), Bureau of Labor Statistics (BLS). }\end{array}$ \\
\hline $20-9$ & Worksite stress reduction programs-Worksites with $50+$ employees & $\begin{array}{l}\text { National Survey of Worksite Health Promotion Activities (NSWHP), } \\
\text { Association for Worksite Health Promotion (AWHP) and OPHS, ODPHP. }\end{array}$ \\
\hline $20-10$ & $\begin{array}{l}\text { Needlestick injuries among hospital-based health care workers } \\
\text { (thousands) }\end{array}$ & $\begin{array}{l}\text { National Surveillance System for Hospital Health Care Workers (NaSH), } \\
\text { CDC, NCPDCID, and Exposure Prevention Information Network } \\
\text { (EPINet), International Health Care Worker Safety Center, University of } \\
\text { Virginia. }\end{array}$ \\
\hline $20-11$ & $\begin{array}{l}\text { Noise-induced hearing loss, work-related (per 10,000 full-time } \\
\text { workers) }\end{array}$ & $\begin{array}{l}\text { Survey of Occupational Injuries and Illnesses (SOII), Department of } \\
\text { Labor (DOL), Bureau of Labor Statistics (BLS). }\end{array}$ \\
\hline
\end{tabular}


Figure 20-1. Progress Toward Target Attainment for Focus Area 20: Occupational Safety and Health

Moved away from target ${ }^{1}$

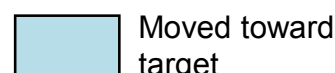
target

Met or exceeded target

20-1. Work-related injury deaths (per 100,000 workers, $16+$ years)

a. All industries

b. Mining

c. Construction

d. Transportation

e. Agriculture, forestry, and fishing

20-2. Work-related injuries (per 100 full-time workers)

a. All industries

b. Construction

c. Health services

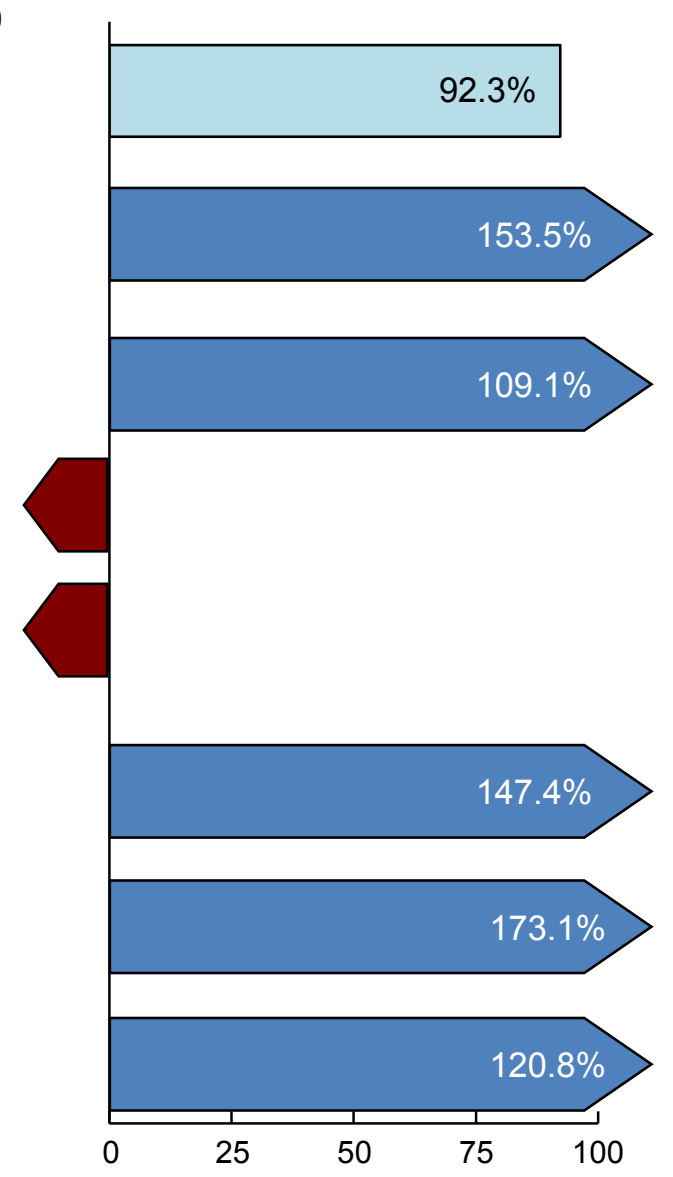

Percent of targeted change achieved ${ }^{5}$

\begin{tabular}{|c|c|c|c|c|c|}
\hline \multirow{2}{*}{$\begin{array}{l}2010 \\
\text { Target }\end{array}$} & \multirow{2}{*}{$\begin{array}{c}\text { Baseline } \\
\text { (Year) }\end{array}$} & \multirow{2}{*}{$\begin{array}{l}\text { Final } \\
\text { (Year) }\end{array}$} & \multicolumn{3}{|c|}{ Baseline vs. Final } \\
\hline & & & Difference ${ }^{2}$ & $\begin{array}{l}\text { Statistically } \\
\text { Significant }{ }^{3}\end{array}$ & $\begin{array}{l}\text { Percent } \\
\text { Change }\end{array}$ \\
\hline 3.2 & $\begin{array}{c}4.5 \\
(1998)\end{array}$ & $\begin{array}{c}3.3 \\
(2009)\end{array}$ & -1.2 & $\begin{array}{c}\text { Not } \\
\text { tested }\end{array}$ & $-26.7 \%$ \\
\hline 16.5 & $\begin{array}{c}23.6 \\
(1998)\end{array}$ & $\begin{array}{c}12.7 \\
(2009)\end{array}$ & -10.9 & $\begin{array}{c}\text { Not } \\
\text { tested }\end{array}$ & $-46.2 \%$ \\
\hline 10.1 & $\begin{array}{c}14.5 \\
(1998)\end{array}$ & $\begin{array}{c}9.7 \\
(2009)\end{array}$ & -4.8 & $\begin{array}{c}\text { Not } \\
\text { tested }\end{array}$ & $-33.1 \%$ \\
\hline 8.3 & $\begin{array}{c}11.8 \\
(1998)\end{array}$ & $\begin{array}{c}12.1 \\
(2009)\end{array}$ & 0.3 & $\begin{array}{c}\text { Not } \\
\text { tested }\end{array}$ & $2.5 \%$ \\
\hline 16.3 & $\begin{array}{c}23.3 \\
(1998)\end{array}$ & $\begin{array}{c}26.0 \\
(2009)\end{array}$ & 2.7 & $\begin{array}{c}\text { Not } \\
\text { tested }\end{array}$ & $11.6 \%$ \\
\hline 4.3 & $\begin{array}{c}6.2 \\
(1998)\end{array}$ & $\begin{array}{c}3.4 \\
(2009)\end{array}$ & -2.8 & Yes & $-45.2 \%$ \\
\hline 6.1 & $\begin{array}{c}8.7 \\
(1998)\end{array}$ & $\begin{array}{c}4.2 \\
(2009)\end{array}$ & -4.5 & Yes & $-51.7 \%$ \\
\hline 5.5 & $\begin{array}{c}7.9 \\
(1997)\end{array}$ & $\begin{array}{c}5.0 \\
(2009)\end{array}$ & -2.9 & $\begin{array}{c}\text { Not } \\
\text { tested }\end{array}$ & $-36.7 \%$ \\
\hline
\end{tabular}

(continued) 
Figure 20-1. Progress Toward Target Attainment for Focus Area 20: Occupational Safety and Health (continued)

Moved away

from target ${ }^{1}$

Moved toward

Met or exceeded

target

20-2. Work-related injuries (per 100 full-time workers)

d. Agricultural, forestry, and fishing

e. Transportation

f. Mining

g. Manufacturing

20-2h. Work-related injuries among adolescent workers (per 100 full-time workers, 15-17 years)

20-3. Overexertion or repetitive motion injuries (per 100,000 full-time workers)

20-4. Pneumoconiosis deaths (number, 15+ years)

20-5. Work-related homicides (per 100,000 workers, $16+$ years)

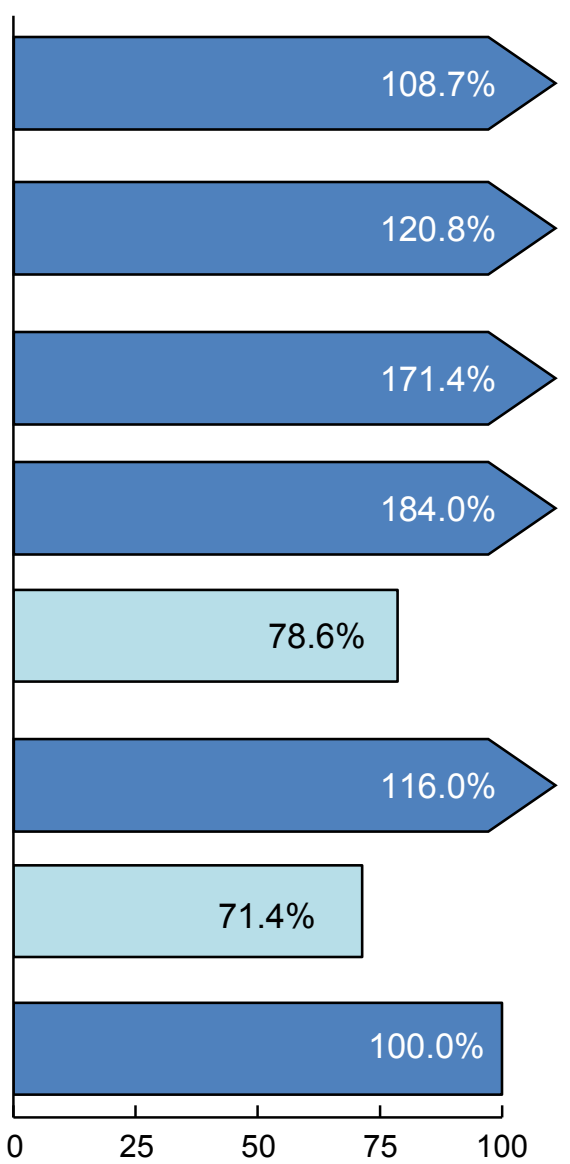

\begin{tabular}{|c|c|c|c|c|c|}
\hline \multirow{2}{*}{$\begin{array}{l}2010 \\
\text { Target }\end{array}$} & \multirow{2}{*}{$\begin{array}{c}\text { Baseline } \\
\text { (Year) }\end{array}$} & \multirow{2}{*}{$\begin{array}{l}\text { Final } \\
\text { (Year) }\end{array}$} & \multicolumn{3}{|c|}{ Baseline vs. Final } \\
\hline & & & Difference $^{2}$ & $\begin{array}{l}\text { Statistically } \\
\text { Significant }^{3}\end{array}$ & $\begin{array}{c}\text { Percent } \\
\text { Change }^{4}\end{array}$ \\
\hline 5.3 & $\begin{array}{c}7.6 \\
(1998)\end{array}$ & $\begin{array}{c}5.1 \\
(2009)\end{array}$ & -2.5 & Yes & $-32.9 \%$ \\
\hline 5.5 & $\begin{array}{c}7.9 \\
(1997)\end{array}$ & $\begin{array}{c}5.0 \\
(2009)\end{array}$ & -2.9 & $\begin{array}{l}\text { Not } \\
\text { tested }\end{array}$ & $-36.7 \%$ \\
\hline 3.3 & $\begin{array}{c}4.7 \\
(1998)\end{array}$ & $\begin{array}{c}2.3 \\
(2009)\end{array}$ & -2.4 & Yes & $-51.1 \%$ \\
\hline 6.0 & $\begin{array}{c}8.5 \\
(1998)\end{array}$ & $\begin{array}{c}3.9 \\
(2009)\end{array}$ & -4.6 & Yes & $-54.1 \%$ \\
\hline 3.5 & $\begin{array}{c}4.9 \\
(1998)\end{array}$ & $\begin{array}{c}3.8 \\
(2008)\end{array}$ & -1.1 & $\begin{array}{c}\text { Not } \\
\text { tested }\end{array}$ & $-22.4 \%$ \\
\hline 338 & $\begin{array}{c}675 \\
(1997)\end{array}$ & $\begin{array}{c}284 \\
(2009)\end{array}$ & -391 & $\begin{array}{c}\text { Not } \\
\text { tested }\end{array}$ & $-57.9 \%$ \\
\hline 1,900 & $\begin{array}{l}2,928 \\
(1997)\end{array}$ & $\begin{array}{l}2,194 \\
(2007)\end{array}$ & -734 & $\begin{array}{l}\text { Not } \\
\text { tested }\end{array}$ & $-25.1 \%$ \\
\hline 0.4 & $\begin{array}{c}0.5 \\
(1998)\end{array}$ & $\begin{array}{c}0.4 \\
(2006)\end{array}$ & -0.1 & $\begin{array}{l}\text { Not } \\
\text { tested }\end{array}$ & $-20.0 \%$ \\
\hline
\end{tabular}

Percent of targeted change achieved ${ }^{5}$

(continued) 
Figure 20-1. Progress Toward Target Attainment for Focus Area 20: Occupational Safety and Health (continued)

Moved away

from target ${ }^{1}$

Moved toward

target

Met or exceeded

target

20-6. Work-related assaults (per 100 workers, 16+ years)

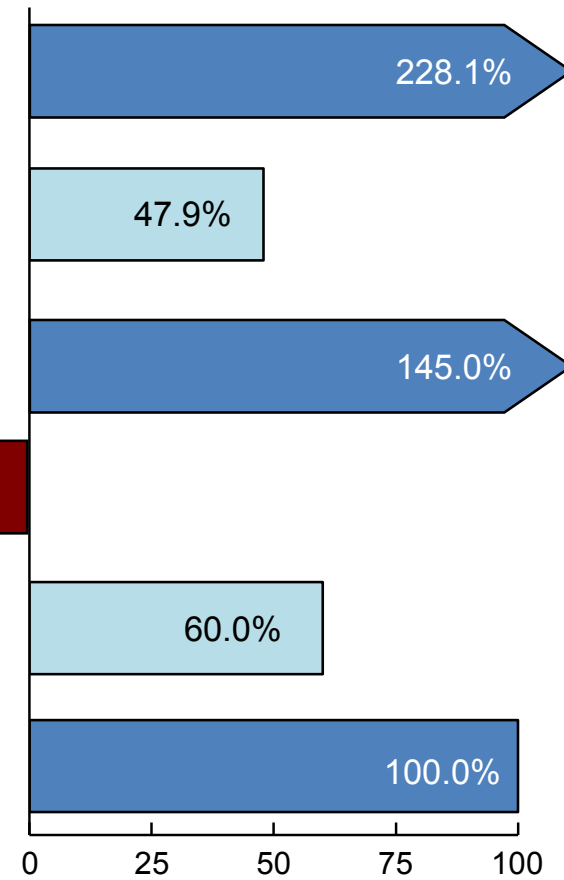

\begin{tabular}{|c|c|c|c|c|c|}
\hline \multirow{2}{*}{$\begin{array}{c}2010 \\
\text { Target }\end{array}$} & \multirow{2}{*}{$\begin{array}{c}\text { Baseline } \\
\text { (Year) }\end{array}$} & \multirow{2}{*}{$\begin{array}{l}\text { Final } \\
\text { (Year) }\end{array}$} & \multicolumn{3}{|c|}{ Baseline vs. Final } \\
\hline & & & Difference ${ }^{2}$ & $\begin{array}{l}\text { Statistically } \\
\text { Significant }^{3}\end{array}$ & $\begin{array}{c}\text { Percent } \\
\text { Change }^{4}\end{array}$ \\
\hline 0.78 & $\begin{array}{c}1.10 \\
(1998)\end{array}$ & $\begin{array}{c}0.37 \\
(2009)\end{array}$ & -0.73 & $\begin{array}{l}\text { Not } \\
\text { tested }\end{array}$ & $-66.4 \%$ \\
\hline 0.0 & $\begin{array}{c}12.1 \\
(1998)\end{array}$ & $\begin{array}{c}6.3 \\
(2009)\end{array}$ & -5.8 & Yes & $-47.9 \%$ \\
\hline 47 & $\begin{array}{c}67 \\
(1997)\end{array}$ & $\begin{array}{c}38 \\
(2009)\end{array}$ & -29 & $\begin{array}{l}\text { Not } \\
\text { tested }\end{array}$ & $-43.3 \%$ \\
\hline $50 \%$ & $\begin{array}{c}37 \% \\
(1992)\end{array}$ & $\begin{array}{c}25 \% \\
(2004)\end{array}$ & -12 & $\begin{array}{c}\text { Not } \\
\text { tested }\end{array}$ & $-32.4 \%$ \\
\hline 269 & $\begin{array}{c}384 \\
(1998)\end{array}$ & $\begin{array}{c}315 \\
(2000)\end{array}$ & -69 & $\begin{array}{l}\text { Not } \\
\text { tested }\end{array}$ & $-18.0 \%$ \\
\hline 2.2 & $\begin{array}{c}3.2 \\
(2004)\end{array}$ & $\begin{array}{c}2.2 \\
(2009)\end{array}$ & -1.0 & Yes & $-31.3 \%$ \\
\hline
\end{tabular}

Percent of targeted change achieved ${ }^{5}$

(continued) 


\section{Figure 20-1. Progress Toward Target Attainment for Focus Area 20: Occupational Safety and Health (continued)}

\section{NOTES}

See the Reader's Guide for more information on how to read this figure. See DATA2010 at http://wonder.cdc.gov/data2010 for all Healthy People 2010 tracking data.

\section{FOOTNOTES}

${ }^{1}$ Movement away from target is not quantified using the percent of targeted change achieved. See Technical Appendix for more information.

${ }^{2}$ Difference $=$ Final value - Baseline value. Differences between percents $(\%)$ are measured in percentage points.

${ }^{3}$ When estimates of variability are available, the statistical significance of the difference between the final value and the baseline value is assessed at the 0.05 level. See Technical Appendix for more information.

${ }^{4}$ Percent change $=\frac{\text { Final value }- \text { Baseline value }}{\text { Baseline value }} \times 100$

${ }^{5}$ Percent of targeted change achieved $=\frac{\text { Final value }- \text { Baseline value }}{\text { Healthy People } 2010 \text { target }- \text { Baseline value }} \times 100$.

\section{DATA SOURCES}

20-1a-e. Census of Fatal Occupational Injuries (CFOI), Department of Labor (DOL), Bureau of Labor Statistics (BLS).

20-2a-g. Survey of Occupational Injuries and IIInesses (SOII), Department of Labor (DOL), Bureau of Labor Statistics (BLS).

20-2h. National Electronic Injury Surveillance System (NEISS): Consumer Product Safety Commission (CPSC); CDC, NIOSH.

20-3. Survey of Occupational Injuries and IIInesses (SOII), Department of Labor (DOL), Bureau of Labor Statistics (BLS).

20-4. National Occupational Respiratory Mortality System (NORMS), CDC, NIOSH.

20-5. Census of Fatal Occupational Injuries (CFOI), Department of Labor (DOL), Bureau of Labor Statistics (BLS).

20-6. National Crime Victimization Survey (NCVS), Department of Justice (DOJ), Bureau of Justice Statistics (BJS).

20-7. Adult Blood Lead Epidemiology and Surveillance Program (ABLES), CDC, NIOSH.

20-8. Survey of Occupational Injuries and IIInesses (SOII), Department of Labor (DOL), Bureau of Labor Statistics (BLS).

20-9. National Survey of Worksite Health Promotion Activities (NSWHP), Association for Worksite Health Promotion (AWHP) and OPHS, ODPHP

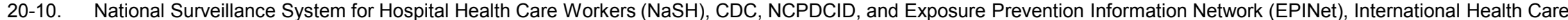
Worker Safety Center, University of Virginia.

20-11. Survey of Occupational Injuries and IIInesses (SOII), Department of Labor (DOL), Bureau of Labor Statistics (BLS). 
Figure 20-2. Health Disparities Table for Focus Area 20: Occupational Safety and Health

Disparities from the best group rate for each characteristic at the most recent data point and changes in disparity from the baseline to the most recent data point.

Population-based objectives

20-1a. Work-related injury deaths-All industries (per 100,000 workers, 16+ years) $(1998,2009)^{1} \dagger$

20-2h. Work-related injuries among adolescent workers (per 100 full-time workers, 15-17 years) $(1998,2008) \ddagger$

20-6. Work-related assaults (per 100 workers, $16+$ years $)(1998,2009)^{1,2 *}$

Characteristics and Groups

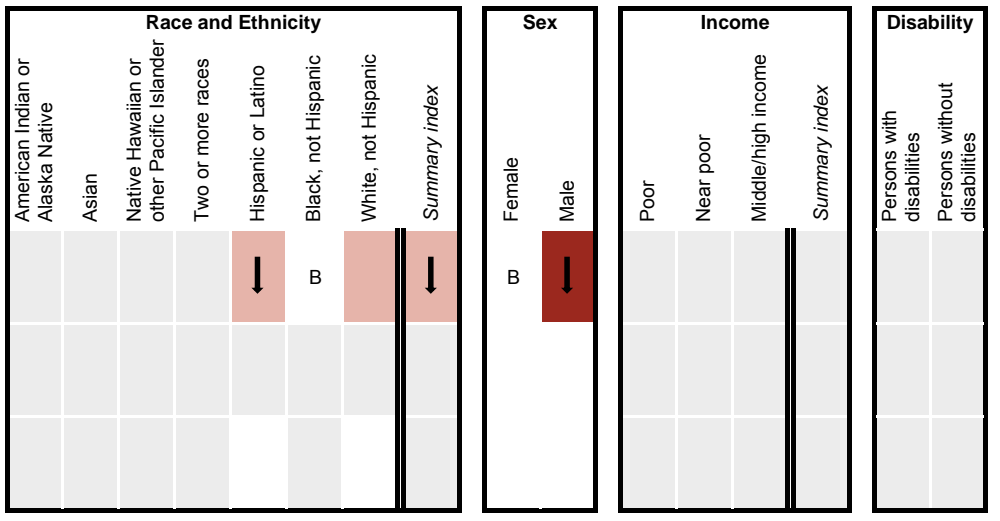

NOTES

See DATA2010 at http://wonder.cdc.gov/data2010 for all Healthy People 2010 tracking data. Disparity data are either unavailable or not applicable for objectives 20-1b through e, 20-2a through g, 20-3 through 20-5, and 20-7 through 20-11.

Years in parentheses represent the baseline and most recent data years (if available).

Disparity from the best group rate is defined as the percent difference between the best group rate and each of the other group rates for a characteristic (e.g., race and ethnicity). The summary index is the average of these percent differences for a characteristic. Change in disparity is estimated by subtracting the disparity at baseline from the disparity at the most recent data point. Change in the summary index is estimated by subtracting the summary index at baseline from the summary index at the most recent data point. See Technical Appendix for more information.

LEGEND

\begin{tabular}{l|l|l|l|l|l|l|}
$\begin{array}{l}\text { The "best" group rate at the most recent } \\
\text { data point. }\end{array}$ & B $\begin{array}{l}\text { The group with the best rate for } \\
\text { specified characteristic. }\end{array} \quad \begin{array}{l}\text { Most favorable group rate for specified } \\
\text { characteristic, but reliability criterion not met. }\end{array}$ \\
\hline
\end{tabular}

\begin{tabular}{ll|l|l|l|l|l|l}
\hline $\begin{array}{l}\text { Disparity from the best group rate at the } \\
\text { most recent data point. }\end{array}$ & $\begin{array}{l}\text { Less than } 10 \% \text {, or difference not } \\
\text { statistically significant (when } \\
\text { estimates of variability are available). }\end{array}$ & $10 \%-49 \%$ & $50 \%-99 \%$ \\
\hline
\end{tabular}

Changes in disparity over time are shown when:
(a) disparities data are available at both baseline and most recent time points;
(b) data are not for the group(s) indicated by "B" or "b" at either time point; and
(c) the change is greater than or equal to 10 percentage points and statistically
significant, or when the change is greater than or equal to 10 percentage
points and estimates of variability were not available. See Technical Appendix.

\section{FOOTNOTES}

* Measures of variability were available. Thus, the variability of best group rates was assessed, and statistical significance was tested. Disparities of $10 \%$ or more are displayed when the differences from the best group rate are statistically significant at the 0.05 level. Changes in disparities over time are indicated by arrows when the changes are greater than or equal to 10 percentage points and are statistically significant at the 0.05 level. See Technical Appendix.

† Measures of variability were not available. Thus, the variability of best group rates was not assessed, and statistical significance could not be tested. Nonetheless, disparities and changes in disparities over time are displayed according to their magnitude. See Technical Appendix.

$\ddagger$ Measures of variability were available only for the most recent data. Thus, the variability of best group rates was assessed only for the most recent data, and statistical significance was tested only for the most recent data. Disparities of $10 \%$ or more are displayed when the differences from the best group rate are statistically significant at the 0.05 level. Changes in disparities over time are displayed according to their magnitude, since measures of variability were not available at baseline and therefore statistical significance of changes in disparity could not be tested. See Technical Appendix.

1 Baseline data by race and ethnicity are for 2000

2 Baseline data by sex are for 2000 .

\section{DATA SOURCES}

20-1a. Census of Fatal Occupational Injuries (CFOI), Department of Labor (DOL), Bureau of Labor Statistics (BLS).

20-2h. National Electronic Injury Surveillance System (NEISS): Consumer Product Safety Commission (CPSC); CDC, NIOSH.

20-6. National Crime Victimization Survey (NCVS), Department of Justice (DOJ), Bureau of Justice Statistics (BJS). 


\section{Final Review}

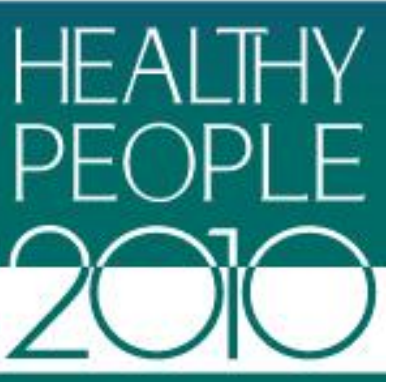

\section{Oral Health}

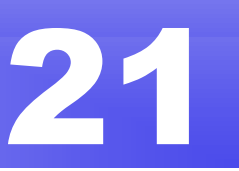

\section{Co-Lead Agencies}

Centers for Disease Control and Prevention

Health Resources and Services Administration

Indian Health Service

National Institutes of Health

\section{Contents}

Goal.

Highlights

Summary of Progress

Transition to Healthy People 2020

Data Considerations

Notes

Comprehensive Summary of Objectives

Progress Chart

Health Disparities Table 


\section{Goal: Prevent and control oral and craniofacial diseases, conditions, and injuries and improve access to related services}

The objectives in this chapter track dental caries, tooth loss, periodontal disease, and untreated dental decay. Preventive measures such as annual dental visits, the use of dental sealants, fluoridation programs, and the availability of school and community-based dental services are also monitored.

All Healthy People tracking data quoted in this chapter, along with technical information and operational definitions for each objective, can be found in the Healthy People 2010 database, DATA2010, available from http://wonder.cdc.gov/data2010/.

More information about this focus area can be found in the following publications:

- Healthy People 2010: Understanding and Improving Health, available from http://www.healthypeople.gov/2010/Document/tableofcontents.htm\#under.

- Healthy People 2010 Midcourse Review, available from http://www.healthypeople.gov/2010/data/midcourse/html/default.htm\#FocusAreas.

\section{Highlights}

- Substantial progress was achieved in objectives for this Focus Area during the past decade [1]. Seventy-one percent of the Oral Health objectives with data to measure progress moved toward or achieved their Healthy People 2010 targets (Figure 21-1). However, health disparities among racial and ethnic population groups, as well as by sex and by education level, were observed (Figure 21-2), some of which are highlighted below [2].

- Dental caries experience in the primary teeth of children aged 2-4 years (objective 21-1a) increased by $33.3 \%$ between $1988-94$ and $1999-2004$, from $18 \%$ to $24 \%$, moving away from the Healthy People 2010 target of $11 \%$.

- Dental caries experience in permanent teeth of adolescents aged 15 years (objective 21-1c) decreased by $8.2 \%$ between $1988-94$ and $1999-2004$, from $61 \%$ to $56 \%$, moving toward the 2010 target of $51 \%$.

- Untreated dental decay in permanent teeth of adolescents aged 15 years (objective 21-2c) decreased by $10.0 \%$ between $1988-94$ and $1999-2004$, from $20 \%$ to $18 \%$, moving toward the 2010 target of $15 \%$.

- The proportion of adults aged 35-44 years with untreated dental decay (objective 21-2d) increased by $3.7 \%$ between $1988-94$ and $1999-2004$ from $27 \%$ to $28 \%$, moving away from the 2010 target of $15 \%$. However, the change was not statistically significant

- Among education groups, persons with at least some college education had the lowest (best) rate of untreated dental decay, 18\% in 1999-2004. Persons with less than a high school education had a rate of 50\% in 1999-2004, almost three times the best group rate [2]. 
- The proportion of adults aged 35-44 years with no permanent tooth loss due to caries or periodontal disease (objective 21-3) increased by $26.7 \%$ between 1988-94 and 1999-2004, from $30 \%$ to $38 \%$, moving toward the 2010 target of $40 \%$. During the same period, the proportion of adults aged 65-74 years who experienced complete tooth loss (objective 214) declined by $17.2 \%$, from $29 \%$ to $24 \%$, moving toward the 2010 target of $22 \%$.

- The proportion of children aged 8 years and of adolescents aged 14 years (objectives 21-8a and $b$, respectively) who had a dental sealant on at least one molar increased by $39.1 \%$ and $40.0 \%$, from $23 \%$ to $32 \%$ and $15 \%$ to $21 \%$, respectively, between $1988-94$ and $1999-2004$, moving toward their 2010 targets of $50 \%$.

- Several objectives met or exceeded their 2010 targets:

- The proportion of school-based health centers providing dental sealants as part of an oral health program (objective 21-13a) doubled, increasing from $12 \%$ in 200102 to $24 \%$ in 2007-08, and exceeding the 2010 target of $15 \%$.

- The proportion of community-based health centers with an oral health component (objective 21-14) increased by $44.2 \%$ between 1997 and 2007 , from $52 \%$ to $75 \%$, meeting the 2010 target of $75 \%$.

- The number of State and local dental programs directed by public health professionals (objective 21-17a) increased by 38.5\% between 2003 and 2009, from 39 to 54 programs, exceeding the 2010 target of 41 programs.

- The number of Indian Health Service and Tribal dental programs directed by public health professionals (objective 21-17b) increased from 9 programs in 2003 to 10 in 2006. The target for this objective (9 programs) was met at baseline and exceeded at the final data point.

\section{Summary of Progress}

- Figure 21-1 presents a quantitative assessment of progress in achieving the Healthy People 2010 objectives for Oral Health [1]. Data to measure progress toward target attainment were available for 24 objectives. Of these:

- Four objectives (21-13a, 21-14, and 21-17a and b) met or exceeded their 2010 targets.

- Thirteen objectives moved toward their targets. A statistically significant difference between the baseline and the final data points was observed for five of these objectives $(21-3,21-4,21-5 b, 21-7$, and 21-12). No significant differences were observed for four objectives (21-1c, 21-2c, and 21-8a and b); and data to test the significance of the difference were unavailable for four objectives $(21-9,21-13 \mathrm{~b}, 21$ 15, and 21-16).

- Seven objectives moved away from their targets. A statistically significant difference between the baseline and final data points was observed for one objective (21-1a). No significant differences were observed for five objectives (21-1b; 21-2a, b, and d; and 21-10); and data to test the significance of the difference were unavailable for one objective (21-6).

- Data were unavailable to measure progress for two objectives (21-5a and 21-11). 
- Figure 21-2 displays health disparities in Oral Health from the best group rate for each characteristic at the most recent data point [2]. It also displays changes in disparities from baseline to the most recent data point [3].

- Nine objectives had statistically significant racial and ethnic health disparities of $10 \%$ or more (objectives 21- $1 \mathrm{a}$ and $\mathrm{b}, 21-2 \mathrm{~d}, 21-3,21-5 \mathrm{~b}, 21-7,21-8 \mathrm{a}$ and $\mathrm{b}$, and 21 10). Two additional objectives (21-5a and 21-6) had racial and ethnic health disparities of $10 \%$ or more, but lacked data to assess statistical significance. The white non-Hispanic population had the only best group rate for 10 of these objectives; while the Hispanic or Latino and white non-Hispanic populations both had the best group rate for objective 21-6.

- Four objectives had statistically significant health disparities of $10 \%$ or more by sex (objectives 21-1a, 21-2d, 21-3, and 21-10), and two objectives (21-5a and 21-6) had health disparities of $10 \%$ or more by sex but lacked data to assess statistical significance. Females had the better rate for five of these six objectives (21-1a, 212d, 21-5a, 21-6, and 21-10). Males had a better rate for the sixth objective (21-3).

- Nine objectives had statistically significant health disparities of $10 \%$ or more by education level (objectives 21-1b and c, 21-2d, 21-3, 21-5b, 21-7, 21-8a and b, and 21-10), and one objective (21-5a) had health disparities of $10 \%$ or more by education level but lacked data to assess statistical significance. Persons with at least some college education had the best group rate for all 10 of these objectives.

- Two objectives had health disparities of $100 \%$ or more by education level (objectives 21-2d and 21-5b), and one objective (21-5b) had a change in disparity by education level over time of 100 percentage points or more [3].

\section{Transition to Healthy People 2020}

For Healthy People 2020, the focus of the Oral Health objectives has expanded to include a broader range of health behaviors, interventions to reduce tooth decay, improved methods of monitoring oral diseases and conditions, and programs that provide preventive oral health services at the community and national levels. See HealthyPeople.gov for a complete list of Healthy People 2020 topics and objectives.

The Healthy People 2020 objectives for Oral Health can be grouped into several sections:

- Oral health of children and adolescents

- Oral health of adults

- Access to preventive services

- Oral health interventions

- Monitoring, surveillance systems

- Public health infrastructure.

The differences between the Healthy People 2010 and Healthy People 2020 objectives are summarized below: 
- The Healthy People 2020 Oral Health Topic Area has 33 objectives, five of which are developmental, whereas the Healthy People 2010 Oral Health Focus Area had 26 objectives [4].

- Seven Healthy People 2010 objectives were retained "as is" [5]. These objectives include: untreated dental decay in adults (objective 21-2d), complete tooth loss in older adults (objective 21-4), community water fluoridation (objective 21-9), annual dental visits (objective 21-10), dental care and sealants provided in school-based health centers (objectives 21-13a and b), and community-based health centers with an oral health component (objective 21-14).

- Two Healthy People 2010 objectives were archived [6].

- The methodology for assessing gingival bleeding used at baseline was modified over the course of the decade and a new definition has yet to be defined. Therefore, gingivitis in adults (objective 21-5a) will not be tracked in Healthy People 2020.

- Use of the oral health care system by nursing home residents has not been collected in a national survey since baseline. Therefore, the objective for residents in longterm facilities (objective 21-11) was archived due to lack of national data.

- Seventeen Healthy People 2010 objectives were modified to create 18 Healthy People 2020 objectives [7].

- The age groups tracked for the following objectives were modified and expanded: dental caries experience and untreated dental decay in children and adolescents (objectives 21-1a through c, and 21-2a through c), dental sealants in children and adolescents (objectives 21-8a and b), permanent tooth loss in adults (objective 213 ), and destructive periodontal diseases in adults (objective 21-5b).

- The data sources for early detection of oral and pharyngeal cancers (objective 21-6) and oral and pharyngeal cancer screening by a dental professional (objective 21-7) were modified. In addition, the age group tracked for oral and pharyngeal cancer screening was expanded. This objective is developmental in Healthy People 2020 [4].

- The definition of an annual preventive dental services visit for low-income youth (objective 21-12) has been changed. The Healthy People 2010 objective defines a preventive visit as one in which the patient received 1) an examination, 2) a dental sealant, 3) a fluoride treatment, 4) a dental prophylaxis, or 5) an X-ray examination. The Healthy People 2020 objective defines a preventive visit as one in which the patient received 1) a dental sealant, 2) a fluoride treatment, or 3) a dental prophylaxis. In addition, the age group tracked was changed from 2-19 years to 218 years.

- The objective tracking the number of states that record and refer children and youth diagnosed with a cleft lip or palate (objective 21-15) has been expanded to two developmental objectives, tracking the registry and referral of such children separately.

- The definitions of state oral and craniofacial surveillance systems (objective 21-16) and state and local dental health programs directed by public health professionals (objective 21-17a) were modified. 
- The number of Indian Health Service and Tribal dental programs that serve jurisdictions of 30,000 or more persons and are directed by a public health professional (objective 21-17b) has increased to 33: one headquarter or national program, 20 field programs, and 12 regional or area offices.

- Eight new objectives, two of which are developmental were added to the Healthy People 2020 Topic Area [4].

- One new objective tracks the proportion of patients who receive oral health at federally qualified health centers. Two objectives track untreated dental decay: adults aged 65-74 years with coronal caries in at least one tooth, and adults aged 75 years and over with root surface caries in at least one tooth.

- Two developmental prevention objectives focus on counseling to reduce tobacco use and encourage smoking cessation and referral for glycemic control by dental professionals.

- One new objective tracks sealant placement on primary molars for children aged 35 years.

- One new objective expands the scope of services tracked in school-based health centers to include fluoride treatments.

- One new objective tracks the expansion of existing infrastructure by monitoring the increase of oral health prevention and care services provided by local health departments.

Appendix D “A Crosswalk Between Objectives From Healthy People 2010 to Healthy People 2020," summarizes the changes between the two decades of objectives, reflecting new knowledge and direction for this area.

\section{Data Considerations}

Education and income are the primary measures of socioeconomic status (SES) in Healthy People 2010. Most data systems used in Healthy People 2010 define income as a family's income before taxes. In order to facilitate comparisons among groups and over time, while adjusting for family size and for inflation, Healthy People 2010 categorizes income using the poverty thresholds developed by the U.S. Census Bureau. Thus, the three categories of family income that are primarily used are:

- Poor-below the Federal poverty level

- Near poor-100\% to $199 \%$ of the Federal poverty level

- Middle/high income-200\% or more of the Federal poverty level.

These categories may be overridden by considerations specific to the data system, in which case they are modified as appropriate. See Healthy People 2010: General Data Issues, referenced below. 
In general, data on educational attainment are presented for persons aged 25 years and over, consistent with guidance given by the U.S. Bureau of the Census. However, because of the requirements of the different data systems, the age groups used to calculate educational attainment for any specific objective may differ from the age groups used to report the data for other Healthy People 2010 objectives, as well as from select populations within the same objective. Therefore, the reader is urged to exercise caution in interpreting the data by educational attainment shown in the Health Disparities Table. See Healthy People 2010: General Data Issues, referenced below.

Additional information on data issues is available from the following sources:

- All Healthy People 2010 tracking data can be found in the Healthy People 2010 database, DATA2010, available from http://wonder.cdc.gov/data2010/.

- Detailed information about the data and data sources used to support these objectives can be found in the Operational Definitions on the DATA2010 website, available from http://wonder.cdc.gov/data2010/focusod.htm.

- More information on statistical issues related to Healthy People tracking and measurement can be found in the Technical Appendix and in Healthy People 2010: General Data Issues, which is available in the Data Issues section of the NCHS Healthy People website under Healthy People 2010.

\section{Notes}

1. Displayed in the Progress Chart (Figure 21-1), the percent of targeted change achieved expresses the difference between the baseline and the final value relative to the initial difference between the baseline and the Healthy People 2010 target. As such, it is a relative measure of progress toward attaining the Healthy People 2010 target. See the Reader's Guide for more information. When standard errors were available, the difference between the baseline and the final value was tested at the 0.05 level of significance. See the Figure 21-1 footnotes, as well as the Technical Appendix, for more detail.

2. Information about disparities among select populations is shown in the Health Disparities Table (Figure 21-2). Disparity from the best group rate is defined as the percent difference between the best group rate and each of the other group rates for a characteristic. For example, racial and ethnic health disparities are measured as the percent difference between the best racial and ethnic group rate and each of the other racial and ethnic group rates. Similarly, disparities by sex are measured as the percent difference between the better group rate (e.g., female) and the rate for the other group (e.g., male). Some objectives are expressed in terms of favorable events or conditions that are to be increased, while others are expressed in terms of adverse events or conditions that are to be reduced. In order to facilitate comparison of health disparities across different objectives, disparity is measured only in terms of adverse events or conditions. For comparability across objectives, objectives that are expressed in terms of favorable events or conditions are re-expressed using the adverse event or condition for the purpose of computing disparity, but they are not otherwise restated or changed. For example, objective 1-1, to increase the proportion of persons with health insurance (e.g., $72 \%$ of the American Indian or Alaska Native population aged under 65 years had some form of health insurance in 2008), is expressed in terms of the percentage of persons without health insurance (e.g., $100 \%-72 \%=28 \%$ of the American Indian or Alaska Native population aged under 65 years did not have any form of health insurance in 2008) when the disparity from the best group rate is calculated. See the Reader's Guide for more information. When standard errors were available, the difference between the best group rate and each of the other group rates was tested at the 0.05 level of significance. See the Figure 21-2 footnotes, as well as the Technical Appendix, for more detail. 
3. The change in disparity is estimated by subtracting the disparity at baseline from the disparity at the most recent data point and, therefore, is expressed as a change in percentage points. See the Reader's Guide for more information. When standard errors were available, the change in disparity was tested at the 0.05 level of significance. See the Figure 21-2 footnotes, as well as the Technical Appendix, for more detail.

4. To be included in Healthy People 2010, an objective must have a national data source that provides a baseline and at least one additional data point for tracking progress. Some objectives lacked baseline data at the time of their development but had a potential data source and were considered of sufficient national importance to be included in Healthy People. These are called "developmental" objectives. When data become available, a developmental objective is moved to measurable status and a Healthy People target can be set.

5. Retained "as is" objectives have no change in the numerator definition or in the denominator definition between the Healthy People 2010 and Healthy People 2020 objectives. These include objectives that were developmental in Healthy People 2010 and are developmental in Healthy People 2020 and for which no numerator or denominator information was available.

6. Archived objectives had at least one data point in Healthy People 2010 but were not carried forward into Healthy People 2020.

7. Modified objectives have some change in the numerator definition or in the denominator definition between the Healthy People 2010 and Healthy People 2020 objectives. These include objectives that went from developmental in Healthy People 2010 to measurable in Healthy People 2020 or vice versa. 


\section{Comprehensive Summary of Objectives: Oral Health}

\begin{tabular}{|c|c|c|}
\hline Objective & Description & Data Source \\
\hline $21-1 \mathrm{a}$ & Dental caries experience-Primary teeth-Young children (2-4 years) & $\begin{array}{l}\text { National Health and Nutrition Examination Survey (NHANES), CDC, } \\
\text { NCHS. }\end{array}$ \\
\hline $21-1 b$ & $\begin{array}{l}\text { Dental caries experience-Primary or permanent teeth-Children (6-8 } \\
\text { years) }\end{array}$ & $\begin{array}{l}\text { National Health and Nutrition Examination Survey (NHANES), CDC, } \\
\text { NCHS. }\end{array}$ \\
\hline $21-1 c$ & Dental caries experience-Permanent teeth-Adolescents (15 years) & $\begin{array}{l}\text { National Health and Nutrition Examination Survey (NHANES), CDC, } \\
\text { NCHS. }\end{array}$ \\
\hline $21-2 \mathrm{a}$ & Untreated dental decay-Primary teeth-Young children (2-4 years) & $\begin{array}{l}\text { National Health and Nutrition Examination Survey (NHANES), CDC, } \\
\text { NCHS }\end{array}$ \\
\hline $21-2 b$ & $\begin{array}{l}\text { Untreated dental decay-Primary or permanent teeth-Children (6-8 } \\
\text { years) }\end{array}$ & $\begin{array}{l}\text { National Health and Nutrition Examination Survey (NHANES), CDC, } \\
\text { NCHS }\end{array}$ \\
\hline $21-2 c$ & Untreated dental decay-Permanent teeth-Adolescents (15 years) & $\begin{array}{l}\text { National Health and Nutrition Examination Survey (NHANES), CDC, } \\
\text { NCHS }\end{array}$ \\
\hline $21-2 d$ & Untreated dental decay-Adults (35-44 years) & $\begin{array}{l}\text { National Health and Nutrition Examination Survey (NHANES), CDC, } \\
\text { NCHS }\end{array}$ \\
\hline $21-3$ & $\begin{array}{l}\text { No permanent tooth loss due to caries or periodontal disease in adults } \\
\text { (35-44 years) }\end{array}$ & $\begin{array}{l}\text { National Health and Nutrition Examination Survey (NHANES), CDC, } \\
\text { NCHS. }\end{array}$ \\
\hline $21-4$ & Complete tooth loss in older adults (65-74 years) & $\begin{array}{l}\text { National Health and Nutrition Examination Survey (NHANES), CDC, } \\
\text { NCHS. }\end{array}$ \\
\hline $21-5 a$ & Gingivitis in adults (35-44 years) & $\begin{array}{l}\text { National Health and Nutrition Examination Survey (NHANES), CDC, } \\
\text { NCHS. }\end{array}$ \\
\hline $21-5 b$ & Destructive periodontal disease in adults ( $35-44$ years) & $\begin{array}{l}\text { National Health and Nutrition Examination Survey (NHANES), CDC, } \\
\text { NCHS; Oral Health Survey of Native Americans, 1999-2000, IHS. }\end{array}$ \\
\hline $21-6$ & Early detection of oral and pharyngeal cancers & Surveillance, Epidemiology, and End Results (SEER) Program, NIH, NCI. \\
\hline $21-7$ & $\begin{array}{l}\text { Annual examinations for oral and pharyngeal cancers in adults (age } \\
\text { adjusted, } 40+\text { years) }\end{array}$ & National Health Interview Survey (NHIS), CDC, NCHS. \\
\hline $21-8 a$ & Dental sealants-Children (8 years) & $\begin{array}{l}\text { National Health and Nutrition Examination Survey (NHANES), CDC, } \\
\text { NCHS. }\end{array}$ \\
\hline $21-8 b$ & Dental sealants-Adolescents (14 years) & $\begin{array}{l}\text { National Health and Nutrition Examination Survey (NHANES), CDC, } \\
\text { NCHS. }\end{array}$ \\
\hline
\end{tabular}




\begin{tabular}{|c|c|c|}
\hline Objective & Description & Data Source \\
\hline $21-9$ & Population served by optimally fluoridated community water & CDC Fluoridation Census, CDC, NCCDPHP. \\
\hline $21-10$ & Annual dental visits (age adjusted, $2+$ years) & Medical Expenditure Panel Survey (MEPS), AHRQ. \\
\hline $21-11$ & Use of oral health care system by residents in long-term care facilities & National Nursing Home Survey (NNHS), CDC, NCHS. \\
\hline $21-12$ & $\begin{array}{l}\text { Annual preventive dental services for low-income children and } \\
\text { adolescents (<19 years) }\end{array}$ & Medical Expenditure Panel Survey (MEPS), AHRQ. \\
\hline $21-13 a$ & $\begin{array}{l}\text { School-based health centers with oral health component-Dental } \\
\text { sealants }\end{array}$ & $\begin{array}{l}\text { School-Based Health Care Census, National Assembly of School Based } \\
\text { Health Care. }\end{array}$ \\
\hline $21-13 b$ & School-based health centers with oral health component-Dental care & $\begin{array}{l}\text { School-Based Health Care Census, National Assembly of School Based } \\
\text { Health Care. }\end{array}$ \\
\hline $21-14$ & Community-based health centers with oral health components & HRSA, Bureau of Primary Health Care. \\
\hline $21-15$ & $\begin{array}{l}\text { Recording and referral of children and youth with cleft lip or palate (no. } \\
\text { States and D.C.) }\end{array}$ & $\begin{array}{l}\text { Annual Synopses of State and Territorial Dental Public Health } \\
\text { Programs, Association of State and Territorial Dental Directors } \\
\text { (ASTDD). }\end{array}$ \\
\hline $21-16$ & $\begin{array}{l}\text { Oral and craniofacial State-based surveillance systems (no. States and } \\
\text { D.C.) }\end{array}$ & $\begin{array}{l}\text { Annual Synopses of State and Territorial Dental Public Health } \\
\text { Programs, Association of State and Territorial Dental Directors } \\
\text { (ASTDD). }\end{array}$ \\
\hline $21-17 a$ & State and local dental programs directed by public health professionals & Association of State and Territorial Dental Directors (ASTDD). \\
\hline $21-17 b$ & $\begin{array}{l}\text { Indian Health Service and Tribal dental programs directed by public } \\
\text { health professionals }\end{array}$ & IHS, Division of Oral Health. \\
\hline
\end{tabular}


Figure 21-1. Progress Toward Target Attainment for Focus Area 21: Oral Health

Moved away

from target ${ }^{1}$
Moved toward target

Met or exceeded

target

21-1. Dental caries experience

a. Primary teeth-Young children ( $2-4$ years)

b. Primary or permanent teethChildren (6-8 years)

c. Permanent teeth-Adolescents (15 years)

$50.0 \%$

21-2. Untreated dental decay

a. Primary teeth-Young children (2-4 years)

b. Primary or permanent teethChildren (6-8 years)

c. Permanent teeth-Adolescents (15 years)

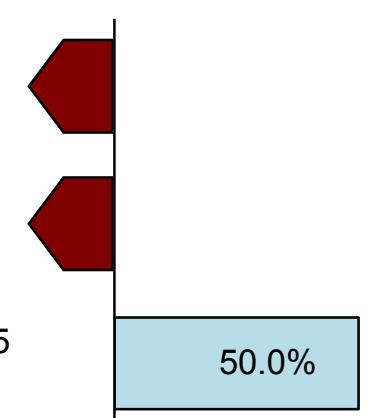

$40.0 \%$

d. Adults (35-44 years)

21-3. No permanent tooth loss due to caries or periodontal disease in adults (35-44 years)
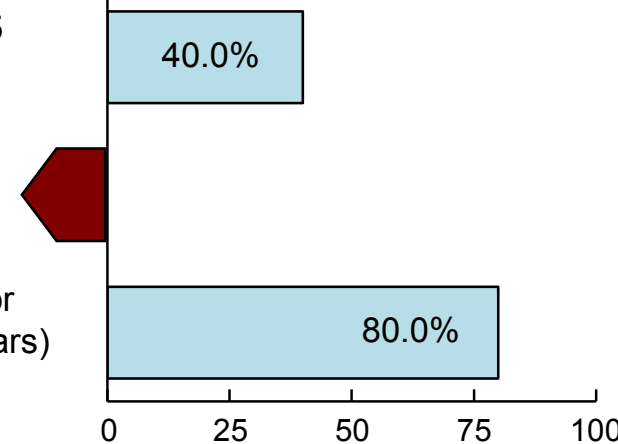

\begin{tabular}{|c|c|c|c|c|c|}
\hline \multirow{2}{*}{$\begin{array}{l}2010 \\
\text { Target }\end{array}$} & \multirow{2}{*}{$\begin{array}{c}\text { Baseline } \\
\text { (Year) }\end{array}$} & \multirow{2}{*}{$\begin{array}{l}\text { Final } \\
\text { (Year) }\end{array}$} & \multicolumn{3}{|c|}{ Baseline vs. Final } \\
\hline & & & Difference ${ }^{2}$ & $\begin{array}{l}\text { Statistically } \\
\text { Significant }^{3}\end{array}$ & $\begin{array}{l}\text { Percent } \\
\text { Change }^{4}\end{array}$ \\
\hline $11 \%$ & $\begin{array}{c}18 \% \\
(1988-94)\end{array}$ & $\begin{array}{c}24 \% \\
(1999-2004)\end{array}$ & 6 & Yes & $33.3 \%$ \\
\hline $42 \%$ & $\begin{array}{c}52 \% \\
(1988-94)\end{array}$ & $\begin{array}{c}53 \% \\
(1999-2004)\end{array}$ & 1 & No & $1.9 \%$ \\
\hline $51 \%$ & $\begin{array}{c}61 \% \\
(1988-94)\end{array}$ & $\begin{array}{c}56 \% \\
(1999-2004)\end{array}$ & -5 & No & $-8.2 \%$ \\
\hline $9 \%$ & $\begin{array}{c}16 \% \\
(1988-94)\end{array}$ & $\begin{array}{c}19 \% \\
(1999-2004)\end{array}$ & 3 & No & $18.8 \%$ \\
\hline $21 \%$ & $\begin{array}{c}28 \% \\
(1988-94)\end{array}$ & $\begin{array}{c}29 \% \\
(1999-2004)\end{array}$ & 1 & No & $3.6 \%$ \\
\hline $15 \%$ & $\begin{array}{c}20 \% \\
(1988-94)\end{array}$ & $\begin{array}{c}18 \% \\
(1999-2004)\end{array}$ & -2 & No & $-10.0 \%$ \\
\hline $15 \%$ & $\begin{array}{c}27 \% \\
(1988-94)\end{array}$ & $\begin{array}{c}28 \% \\
(1999-2004)\end{array}$ & 1 & No & $3.7 \%$ \\
\hline $40 \%$ & $\begin{array}{c}30 \% \\
(1988-94)\end{array}$ & $\begin{array}{c}38 \% \\
(1999-2004)\end{array}$ & 8 & Yes & $26.7 \%$ \\
\hline
\end{tabular}

Percent of targeted change achieved ${ }^{5}$

(continued) 
Figure 21-1. Progress Toward Target Attainment for Focus Area 21: Oral Health (continued)

Moved away

from target ${ }^{1}$

Moved toward

target

Met or exceeded

target

21-4. Complete tooth loss in older adults $(65-74$ years)

21-5b. Destructive periodontal disease in adults (35-44 years)

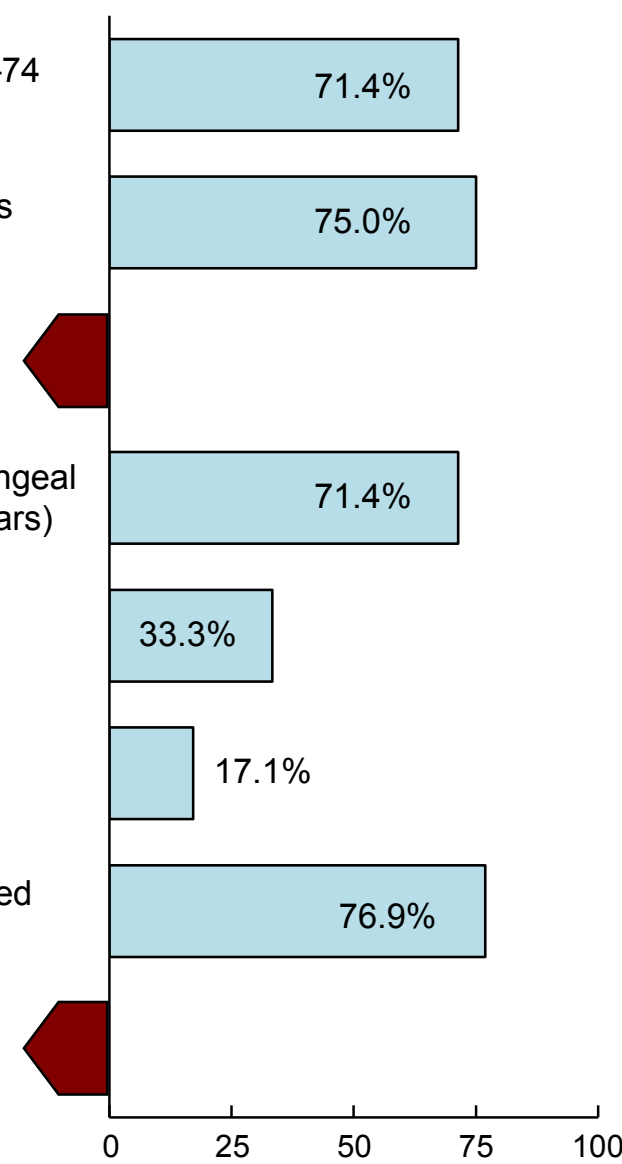

\begin{tabular}{|c|c|c|c|c|c|}
\hline \multirow{2}{*}{$\begin{array}{l}2010 \\
\text { Target }\end{array}$} & \multirow{2}{*}{$\begin{array}{c}\text { Baseline } \\
\text { (Year) }\end{array}$} & \multirow{2}{*}{$\begin{array}{l}\text { Final } \\
\text { (Year) }\end{array}$} & \multicolumn{3}{|c|}{ Baseline vs. Final } \\
\hline & & & Difference $^{2}$ & $\begin{array}{l}\text { Statistically } \\
\text { Significant }^{3}\end{array}$ & $\begin{array}{l}\text { Percent } \\
\text { Change }^{4}\end{array}$ \\
\hline $22 \%$ & $\begin{array}{c}29 \% \\
(1988-94)\end{array}$ & $\begin{array}{c}24 \% \\
(1999-2004)\end{array}$ & -5 & Yes & $-17.2 \%$ \\
\hline $14 \%$ & $\begin{array}{c}22 \% \\
(1988-94)\end{array}$ & $\begin{array}{c}16 \% \\
(1999-2004)\end{array}$ & -6 & Yes & $-27.3 \%$ \\
\hline $51 \%$ & $\begin{array}{c}36 \% \\
(1992-95)\end{array}$ & $\begin{array}{c}33 \% \\
(2006)\end{array}$ & -3 & $\begin{array}{c}\text { Not } \\
\text { tested }\end{array}$ & $-8.3 \%$ \\
\hline $20 \%$ & $\begin{array}{c}13 \% \\
(1998)\end{array}$ & $\begin{array}{c}18 \% \\
(2008)\end{array}$ & 5 & Yes & $38.5 \%$ \\
\hline $50 \%$ & $\begin{array}{c}23 \% \\
(1988-94)\end{array}$ & $\begin{array}{c}32 \% \\
(1999-2004)\end{array}$ & 9 & No & $39.1 \%$ \\
\hline $50 \%$ & $\begin{array}{c}15 \% \\
(1988-94)\end{array}$ & $\begin{array}{c}21 \% \\
(1999-2004)\end{array}$ & 6 & No & $40.0 \%$ \\
\hline $75 \%$ & $\begin{array}{c}62 \% \\
(1992)\end{array}$ & $\begin{array}{c}72 \% \\
(2008)\end{array}$ & 10 & $\begin{array}{c}\text { Not } \\
\text { tested }\end{array}$ & $16.1 \%$ \\
\hline $56 \%$ & $\begin{array}{c}44 \% \\
(1996)\end{array}$ & $\begin{array}{c}43 \% \\
(2008)\end{array}$ & -1 & No & $-2.3 \%$ \\
\hline
\end{tabular}

Percent of targeted change achieved ${ }^{5}$

(continued) 
Figure 21-1. Progress Toward Target Attainment for Focus Area 21: Oral Health (continued)

Moved away

from target ${ }^{1}$

Moved toward

target

Met or exceeded

target

21-12. Annual preventive dental services for lowincome children and adolescents ( $<19$ years)

21-13. School-based health centers with oral health component

a. Dental sealants

b. Dental care

21-14. Community-based health centers with oral health components

21-15. Recording and referral of children and youth with cleft lip or palate (no. States and D.C.)

21-16. Oral and craniofacial State-based surveillance systems (no. States and D.C.)

21-17a. State and local dental programs directed by public health professionals

21-17b. Indian Health Service and Tribal dental programs directed by public health professionals

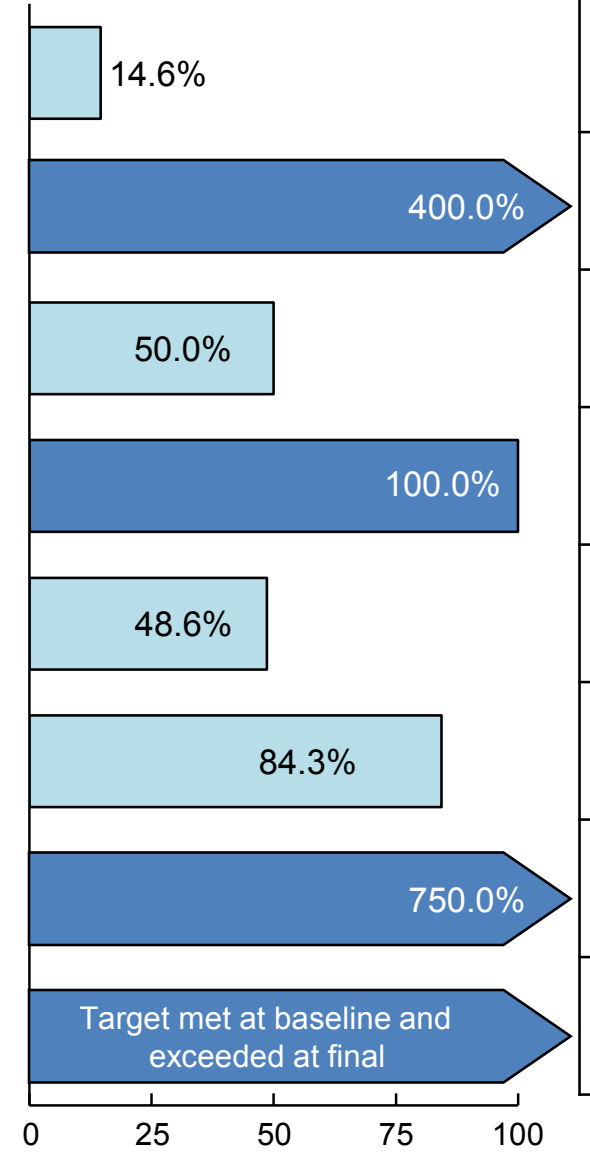

\begin{tabular}{|c|c|c|c|c|c|}
\hline \multirow{2}{*}{$\begin{array}{c}2010 \\
\text { Target }\end{array}$} & \multirow{2}{*}{$\begin{array}{c}\text { Baseline } \\
\text { (Year) }\end{array}$} & \multirow{2}{*}{$\begin{array}{l}\text { Final } \\
\text { (Year) }\end{array}$} & \multicolumn{3}{|c|}{ Baseline vs. Final } \\
\hline & & & Difference $^{2}$ & $\begin{array}{l}\text { Statistically } \\
\text { Significant }^{3}\end{array}$ & $\begin{array}{l}\text { Percent } \\
\text { Change }^{4}\end{array}$ \\
\hline $66 \%$ & $\begin{array}{c}25 \% \\
(1996)\end{array}$ & $\begin{array}{c}31 \% \\
(2008)\end{array}$ & 6 & Yes & $24.0 \%$ \\
\hline $15 \%$ & $\begin{array}{c}12 \% \\
(2001-02)\end{array}$ & $\begin{array}{c}24 \% \\
(2007-08)\end{array}$ & 12 & $\begin{array}{c}\text { Not } \\
\text { tested }\end{array}$ & $100.0 \%$ \\
\hline $11 \%$ & $\begin{array}{c}9 \% \\
(2001-02)\end{array}$ & $\begin{array}{c}10 \% \\
(2007-08)\end{array}$ & 1 & $\begin{array}{l}\text { Not } \\
\text { tested }\end{array}$ & $11.1 \%$ \\
\hline $75 \%$ & $\begin{array}{c}52 \% \\
(1997)\end{array}$ & $\begin{array}{c}75 \% \\
(2007)\end{array}$ & 23 & $\begin{array}{c}\text { Not } \\
\text { tested }\end{array}$ & $44.2 \%$ \\
\hline 51 & $\begin{array}{c}16 \\
(2003)\end{array}$ & $\begin{array}{c}33 \\
(2009)\end{array}$ & 17 & $\begin{array}{c}\text { Not } \\
\text { tested }\end{array}$ & $106.3 \%$ \\
\hline 51 & $\begin{array}{c}0 \\
(1999)\end{array}$ & $\begin{array}{c}43 \\
(2010)\end{array}$ & 43 & $\begin{array}{l}\text { Not } \\
\text { tested }\end{array}$ & * \\
\hline 41 & $\begin{array}{c}39 \\
(2003)\end{array}$ & $\begin{array}{c}54 \\
(2009)\end{array}$ & 15 & $\begin{array}{l}\text { Not } \\
\text { tested }\end{array}$ & $38.5 \%$ \\
\hline 9 & $\begin{array}{c}9 \\
(2003)\end{array}$ & $\begin{array}{c}10 \\
(2006)\end{array}$ & 1 & $\begin{array}{c}\text { Not } \\
\text { tested }\end{array}$ & $11.1 \%$ \\
\hline
\end{tabular}

Percent of targeted change achieved ${ }^{5}$

(continued) 


\section{Figure 21-1. Progress Toward Target Attainment for Focus Area 21: Oral Health (continued)}

\section{NOTES}

See the Reader's Guide for more information on how to read this figure. See DATA2010 at http://wonder.cdc.gov/data2010 for all Healthy People 2010 tracking data. Tracking data are not available for objectives $21-5 a$ and $21-11$

\section{FOOTNOTES}

${ }^{1}$ Movement away from target is not quantified using the percent of targeted change achieved. See Technical Appendix for more information.

${ }^{2}$ Difference $=$ Final value - Baseline value. Differences between percents (\%) are measured in percentage points.

${ }^{3}$ When estimates of variability are available, the statistical significance of the difference between the final value and the baseline value is assessed at the 0.05 level. See Technical Appendix for more information.

${ }^{4}$ Percent change $=\frac{\text { Final value }- \text { Baseline value }}{\text { Baseline value }} \times 100$

${ }^{5}$ Percent of targeted change achieved $=\frac{\text { Final value }- \text { Baseline value }}{\text { Healthy People } 2010 \text { target }- \text { Baseline value }} \times 100$.

${ }^{*}$ Percent change cannot be calculated. See Technical Appendix for more information.

\section{DATA SOURCES}

21-1a-c. National Health and Nutrition Examination Survey (NHANES), CDC, NCHS.

21-2a-d. National Health and Nutrition Examination Survey (NHANES), CDC, NCHS

21-3-21-4. National Health and Nutrition Examination Survey (NHANES), CDC, NCHS.

21-5b. National Health and Nutrition Examination Survey (NHANES), CDC, NCHS.

21-6. Surveillance, Epidemiology, and End Results (SEER) Program, $\mathrm{NIH}, \mathrm{NCl}$.

21-7. National Health Interview Survey (NHIS), CDC, NCHS.

21-8a-b. National Health and Nutrition Examination Survey (NHANES), CDC, NCHS.

21-9. CDC Fluoridation Census, CDC, NCCDPHP.

21-10. Medical Expenditure Panel Survey (MEPS), AHRQ.

21-12. Medical Expenditure Panel Survey (MEPS), AHRQ.

21-13a-b. School-Based Health Care Census, National Assembly of School Based Health Care.

21-14. HRSA, Bureau of Primary Health Care.

21-15-21-16. Annual Synopses of State and Territorial Dental Public Health Programs, Association of State and Territorial Dental Directors (ASTDD).

21-17a. Association of State and Territorial Dental Directors (ASTDD).

21-17b. IHS, Division of Oral Health. 
Figure 21-2. Health Disparities Table for Focus Area 21: Oral Health Disparities from the best group rate for each characteristic at the most recent data point and changes in disparity from the baseline to the most recent data point.

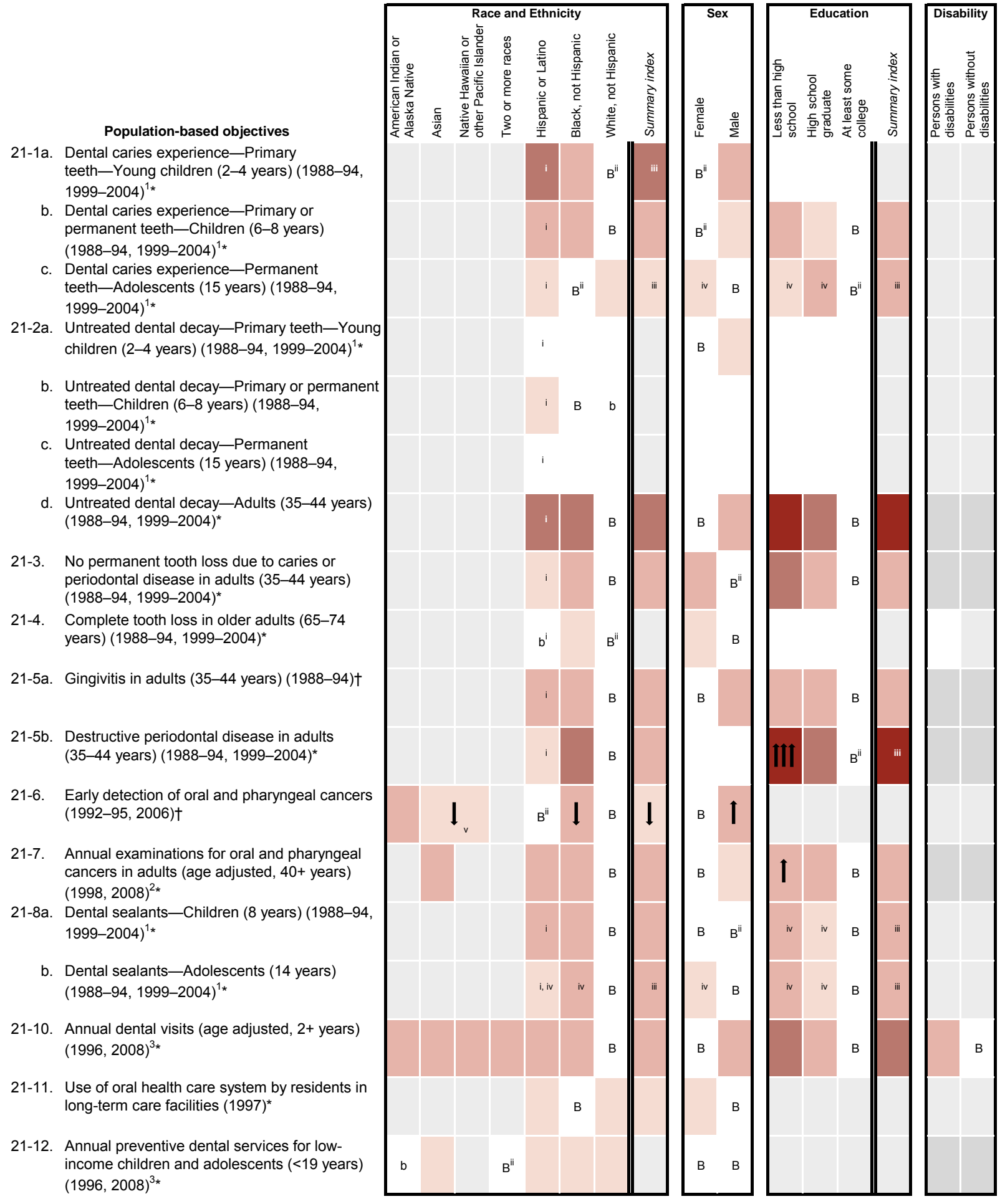

(continued) 


\section{Figure 21-2. Health Disparities Table for Focus Area 21: Oral Health (continued)}

\section{NOTES}

See DATA2010 at http://wonder.cdc.gov/data2010 for all Healthy People 2010 tracking data. Disparity data are either unavailable or not applicable for objectives 21-9, 21-13a and b, 21-14 through 21-16, and 21-17a and b.

Years in parentheses represent the baseline and most recent data years (if available)

Disparity from the best group rate is defined as the percent difference between the best group rate and each of the other group rates for a characteristic (e.g. race and ethnicity). The summary index is the average of these percent differences for a characteristic. Change in disparity is estimated by subtracting the disparity at baseline from the disparity at the most recent data point. Change in the summary index is estimated by subtracting the summary index at baseline from the summary index at the most recent data point. See Technical Appendix for more information.

\section{LEGEND}

$\begin{aligned} & \text { The "best" group rate at the most recent } \\ & \text { data point. }\end{aligned}$
B $\begin{aligned} & \text { The group with the best rate for } \\ & \text { specified characteristic. }\end{aligned} \quad$ b $\begin{aligned} & \text { Most favorable group rate for specified } \\ & \text { characteristic, but reliability criterion not } \\ & \text { met. }\end{aligned}$

\begin{tabular}{|c|c|c|c|c|}
\hline \multirow[b]{2}{*}{$\begin{array}{l}\text { Disparity from the best group rate at the } \\
\text { most recent data point. }\end{array}$} & \multicolumn{4}{|c|}{ Percent difference from the best group rate } \\
\hline & $\begin{array}{l}\text { Less than } 10 \% \text {, or difference not } \\
\text { statistically significant (when } \\
\text { estimates of variability are available). }\end{array}$ & $10 \%-49 \%$ & $50 \%-99 \%$ & $100 \%$ or more \\
\hline
\end{tabular}

Increase in disparity (percentage points)

Changes in disparity over time are shown when:

(a) disparities data are available at both baseline and most recent time points;

(b) data are not for the group(s) indicated by "B" or "b" at either time point; and

(c) the change is greater than or equal to 10 percentage points and statistically

significant, or when the change is greater than or equal to 10 percentage

points and estimates of variability were not available. See Technical Appendix.
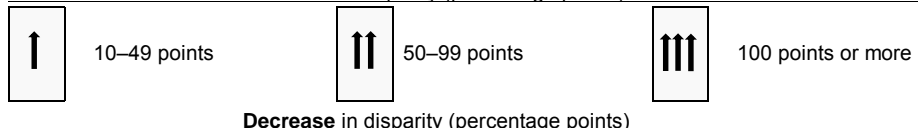

Decrease in disparity (percentage points)

\begin{tabular}{lll}
\hline Availability of Data & Data not available. & $\square$ Characteristic not selected for this objective. \\
\hline
\end{tabular}

\section{FOOTNOTES}

* Measures of variability were available. Thus, the variability of best group rates was assessed, and statistical significance was tested. Disparities of $10 \%$ or more are displayed when the differences from the best group rate are statistically significant at the 0.05 level. Changes in disparities over time are indicated by arrows when the changes are greater than or equal to 10 percentage points and are statistically significant at the 0.05 level. See Technical Appendix.

† Measures of variability were not available. Thus, the variability of best group rates was not assessed, and statistical significance could not be tested. Nonetheless, disparities and changes in disparities over time are displayed according to their magnitude. See Technical Appendix.

1 Data by education level are for the head of household

2 Baseline data by race and ethnicity are for 2008.

3 Baseline data by race and ethnicity are for 2002 .

i Data are for Mexican American.

ii The group with the best rate at the most recent data point is different from the group with the best rate at baseline. Both rates met the reliability criterion. See Technical Appendix.

iii Change in the summary index cannot be assessed. See Technical Appendix.

iv Reliability criterion for best group rate not met, or data available for only one group, at baseline. Change in disparity cannot be assessed. See Technical Appendix.

v Data are for Asian or Pacific Islander.

\section{DATA SOURCES}

21-1a-c. National Health and Nutrition Examination Survey (NHANES), CDC, NCHS.

21-2a-d. National Health and Nutrition Examination Survey (NHANES), CDC, NCHS

21-3-21-4. National Health and Nutrition Examination Survey (NHANES), CDC, NCHS.

21-5a-b. National Health and Nutrition Examination Survey (NHANES), CDC, NCHS.

21-6. Surveillance, Epidemiology, and End Results (SEER) Program, $\mathrm{NIH}, \mathrm{NCl}$.

21-7. National Health Interview Survey (NHIS), CDC, NCHS.

21-8a-b. National Health and Nutrition Examination Survey (NHANES), CDC, NCHS.

21-10. Medical Expenditure Panel Survey (MEPS), AHRQ.

21-11. National Nursing Home Survey (NNHS), CDC, NCHS.

21-12. Medical Expenditure Panel Survey (MEPS), AHRQ. 


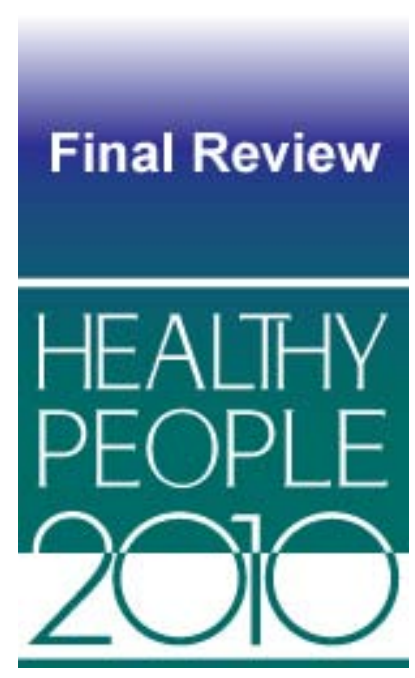

\section{Physical Activity and Fitness}

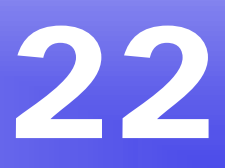

\section{Co-Lead Agencies}

Centers for Disease Control and Prevention

President's Council on Physical Fitness and Sports

\section{Contents}

Goal

Highlights

Summary of Progress

Transition to Healthy People 2020

Data Considerations

References and Notes

Comprehensive Summary of Objectives

Progress Chart

Health Disparities Table

No Leisure Time Physical Activity Among Adults-Map 


\section{Goal: Improve health, fitness, and quality of life through daily physical activity}

This chapter includes objectives that track participation in physical activities, access to physical activity and fitness programs, and the availability of physical activity facilities at schools and worksites.

All Healthy People tracking data quoted in this chapter, along with technical information and operational definitions can be found in the Healthy People 2010 database, DATA2010, available from http://wonder.cdc.gov/data2010/.

More information about this Focus Area can be found in the following publications:

- Healthy People 2010: Understanding and Improving Health, available from http://www.healthypeople.gov/2010/Document/tableofcontents.htm\#under.

- Healthy People 2010 Midcourse Review, available from http://www.healthypeople.gov/2010/data/midcourse/html/default.htm\#FocusAreas.

\section{Highlights}

- Substantial progress was achieved in objectives for this Focus Area during the past decade. About $70 \%$ of the Physical Activity and Fitness objectives with data to measure progress moved toward their Healthy People 2010 targets (Figure 22-1) [1]. However, almost all of the objectives in this Focus Area exhibited statistically significant health disparities among select populations [2]. These disparities ranged from $10 \%$ to $49 \%$ in magnitude, with the exception of two objectives (discussed below) which displayed statistically significant disparities of $50 \%$ or more (Figure $22-2$ ).

- Television viewing by students in grades 9-12 (objective 22-11) declined. The percentage of students who watched television for two or fewer hours a day increased by $17.5 \%$ between 1999 and 2009, from 57\% to 67\%, moving toward the Healthy People 2010 target of 75\%. Disparities were observed for a number of population groups, for example:

- Among racial and ethnic groups, white non-Hispanic students had the highest (best) rate of television viewing for two or fewer hours a day, $75 \%$ in 2009 , whereas Hispanic or Latino students and black non-Hispanic students had rates of $58 \%$ and $44 \%$ in 2009 , respectively. When expressed as viewing television for more than two hours a day, the rate for Hispanic or Latino students was more than one and onehalf times the best group rate (that for white non-Hispanic students). The rate for black non-Hispanic students was more than twice the best group rate.

- The proportion of adults who did not participate in any form of leisure time physical activity (objective 22-1) decreased by $9 \%$ between 1997 and 2008, from 40\% to 36\%, moving toward the 2010 target of $20 \%$. 
- Among education groups, persons with at least some college education had the lowest (best) rate of no leisure time physical activity, $27 \%$ in 2008. Persons with less than a high school education had a rate of $59 \%$ in 2008 , more than twice the best group rate.

- Participation in leisure time physical activity varied by geographic region. Colorado, Minnesota, Oregon, Vermont, and Washington had the lowest rates of nonparticipation in 2008. These states achieved the Healthy People 2010 target. Kentucky, West Virginia, and a contiguous group of southern states (Alabama, Arkansas, Louisiana, Mississippi, Oklahoma, and Texas) had the highest rates (Figure 22-3).

\section{Summary of Progress}

- Figure 22-1 presents a quantitative assessment of progress in achieving the Healthy People 2010 objectives for Physical Activity and Fitness [1]. Data to measure progress toward target attainment were available for 17 objectives.

- None of the objectives in this Focus Area achieved the Healthy People 2010 targets.

- Twelve objectives moved toward their targets. A statistically significant difference between the baseline and the final data points was observed for five of these objectives $(22-1,22-4,22-11$, and 22-14a and b). No significant differences were observed for seven objectives (22-3, 22-5 through 22-7, 22-8a, 22-9, and 22-10).

- One objective showed no change (objective 22-2).

- Four objectives moved away from their targets. A statistically significant difference between the baseline and final data point was observed for two objectives $(22-8 \mathrm{~b}$ and 22-15a). No significant differences were observed for the other two objectives (22-12 and 22-15b).

- Data were unavailable to measure progress for one objective (22-13); this objective had baseline data only.

- Figure 22-2 displays health disparities in Physical Activity and Fitness from the best group rate for each characteristic at the most recent data point [2]. It also displays changes in disparities from baseline to the most recent data point [3].

- Of the 10 objectives with statistically significant health disparities of $10 \%$ or more among racial and ethnic populations, the white non-Hispanic population had the best rate for seven objectives (22-1 through 22-4, 22-6, 22-7, and 22-11); the Hispanic or Latino population had the best rate for two objectives (22-9 and 22-10); and persons of two or more races had the best rate for one objective (22-5).

- Health disparities of $100 \%$ or more were observed for one objective (22-11, student television viewing); see Highlights, above.

- Males had better rates than females for the four objectives with statistically significant health disparities of $10 \%$ or more by sex (objectives $22-1,22-4,22-7$, and 22-10). 
- Persons with at least some college education had the best rates for five of the six objectives with statistically significant health disparities of $10 \%$ or more by education level (objectives 22-1 through 22-4, and 22-5). Persons with less than a high school education had the best rate for one objective (22-14a).

- Residents of urban or metropolitan areas had better rates than residents of rural areas for the three objectives with statistically significant health disparities of $10 \%$ or more by geographic location (objectives 22-1, and 22-14a and b).

- Persons without disabilities had better rates than persons with disabilities for the four objectives with statistically significant health disparities of $10 \%$ or more by disability status (objectives 22-1 through 22-4).

\section{Transition to Healthy People 2020}

For Healthy People 2020, the focus of the Physical Activity objectives has been expanded to include a broader range of activities than those included in Healthy People 2010. The Healthy People 2010 Focus Area name was changed from "Physical Activity and Fitness" to "Physical Activity" to be consistent with 2008 Physical Activity Guidelines for Americans [4]. With the exception of muscle strengthening, the objectives primarily assess aerobic physical activity behaviors and the environments and policies that support being physically active. See HealthyPeople.gov for a complete list of Healthy People 2020 topics and objectives.

The Healthy People 2020 Physical Activity Topic Area can be grouped into several sections:

- Aerobic physical activity and muscular strengthening activity in adults

- Aerobic physical activity and muscular strengthening activity in children and adolescents

- Physical education and school recess

- Access to school physical activity facilities

- Environmental policies enhancing physical activity opportunities

- Physical activity policies in child care setting

- Physical counseling related to physical activity.

The differences between the Healthy People 2010 and Healthy People 2020 objectives are summarized below:

- The Healthy People 2020 Physical Activity Topic Area has a total of 36 objectives, 10 of which are developmental, whereas the Healthy People 2010 Physical Activity and Fitness Focus Area had 18 objectives [5].

- Five Healthy People 2010 objectives, including no leisure time physical activity (objective 22-1), physical education requirement (objectives 22-8a and b), participation in daily physical education in schools (objective 22-9), and access to physical activities in school facilities (objective 22-12), were retained "as is" [6]. 
- Six Healthy People 2010 objectives were modified (objectives 22-2 through 22-4, 22-6, 227, and 22-11) [7]. These physical activity and muscle strengthening activity in adults and adolescents objectives were changed to reflect 2008 Federal Physical Activity Guidelines recommendations.

- Five objectives addressing worksite physical activity programs (objective 22-13), walking and bicycling for transportation (objectives 22-14a and b, and 22-15a and b), were returned to developmental status due to a lack of baseline data [5].

- Two objectives were archived [8]. The flexibility objective (22-5) was archived due to a change in the physical activity guideline recommendations. The physical activity in physical education class measure (objective 22-10) was archived because it lacked a national data source.

- The objective that tracks physician counseling about physical activity (objective 1-3a) was moved from Healthy People 2010 Focus Area "Access to Quality Health Services" into the Healthy People 2020 Physical Activity Topic Area and modified to include two objectives on physician counseling or education related to exercise.

- Seventeen new objectives were added to the Healthy People 2020 Physical Activity Topic Area:

- Seven new objectives address physical activity programs for children and adolescents objectives: time for recess, regularly scheduled school recess, daily physical education in elementary schools, and physical activity policies in child care settings.

- The television viewing objective (22-11) was expanded to include seven new objectives of other types of screen time such as computer use, video and computer games among children and adolescents aged less than 17 years.

- Three new Healthy People 2020 environment objectives include measures to track legislative policies that enhance access and availability of physical activity opportunities.

Appendix D, "A Crosswalk Between Objectives From Healthy People 2010 to Healthy People 2020," summarizes the changes between the two decades of objectives, reflecting new knowledge and direction for this area. 


\section{Data Considerations}

Figure 22-3 (no leisure time physical activity among adults) presents state-level data from the Behavioral Risk Factor Surveillance System (BRFSS). National data for this objective comes from the National Health Interview Survey (NHIS) and is the basis for setting the target. BRFSS data may not be comparable to the National data from the NHIS.

In general, data on educational attainment are presented for persons aged 25 years and over, consistent with guidance given by the U.S. Bureau of the Census. However, because of the requirements of the different data systems, the age groups used to calculate educational attainment for any specific objective may differ from the age groups used to report the data for other Healthy People 2010 objectives, as well as from select populations within the same objective. Therefore, the reader is urged to exercise caution in interpreting the data by educational attainment shown in the Health Disparities Table. See Healthy People 2010: General Data Issues, referenced below.

Additional information on data issues is available from the following sources:

- All Healthy People 2010 tracking data can be found in the Healthy People 2010 database, DATA2010, available from http://wonder.cdc.gov/data2010/

- Detailed information about the data and data sources used to support these objectives can be found in the Operational Definitions on the DATA2010 website, available from http://wonder.cdc.gov/data2010/focusod.htm.

- More information on statistical issues related to Healthy People tracking and measurement can be found in the Technical Appendix and in Healthy People 2010: General Data Issues, which is available in the Data Issues section of the NCHS Healthy People website under Healthy People 2010.

\section{References and Notes}

1. Displayed in the Progress Chart (Figure 22-1), the percent of targeted change achieved expresses the difference between the baseline and the final value relative to the initial difference between the baseline and the Healthy People 2010 target. As such, it is a relative measure of progress toward attaining the Healthy People 2010 target. See the Reader's Guide for more information. When standard errors were available, the difference between the baseline and the final value was tested at the 0.05 level of significance. See the Figure 22-1 footnotes, as well as the Technical Appendix, for more detail.

2. Information about disparities among select populations is shown in the Health Disparities Table (Figure 22-2). Disparity from the best group rate is defined as the percent difference between the best group rate and each of the other group rates for a characteristic. For example, racial and ethnic health disparities are measured as the percent difference between the best racial and ethnic group rate and each of the other racial and ethnic group rates. Similarly, disparities by sex are measured as the percent difference between the better group rate (e.g., female) and the rate for the other group (e.g., male). Some objectives are expressed in terms of favorable events or conditions that are to be increased, while others are expressed in terms of adverse events or conditions that are to be reduced. In order to facilitate comparison of health disparities across different objectives, disparity is measured only in terms of adverse events or conditions. For comparability across objectives, objectives that are expressed in terms of favorable events or conditions are re-expressed using the 
adverse event or condition for the purpose of computing disparity, but they are not otherwise restated or changed. For example, objective 1-1, to increase the proportion of persons with health insurance (e.g., $72 \%$ of the American Indian or Alaska Native population aged under 65 years had some form of health insurance in 2008), is expressed in terms of the percentage of persons without health insurance (e.g., $100 \%-72 \%=28 \%$ of the American Indian or Alaska Native population aged under 65 years did not have any form of health insurance in 2008) when the disparity from the best group rate is calculated. See the Reader's Guide for more information. When standard errors were available, the difference between the best group rate and each of the other group rates was tested at the 0.05 level of significance. See the Figure 22-2 footnotes, as well as the Technical Appendix, for more detail.

3. The change in disparity is estimated by subtracting the disparity at baseline from the disparity at the most recent data point and, therefore, is expressed as a change in percentage points. See the Reader's Guide for more information. When standard errors were available, the change in disparity was tested at the 0.05 level of significance. See the Figure 22-2 footnotes, as well as the Technical Appendix, for more detail.

4. 2008 Physical Activity Guidelines for Americans. Washington, DC: US Department of Health and Human Services (USDHHS); 2008. Available from: http://www.health.gov/PAGuidelines/

5. To be included in Healthy People 2010, an objective must have a national data source that provides a baseline and at least one additional data point for tracking progress. Some objectives lacked baseline data at the time of their development but had a potential data source and were considered of sufficient national importance to be included in Healthy People. These are called "developmental" objectives. When data become available, a developmental objective is moved to measurable status and a Healthy People target can be set.

6. Retained "as is" objectives have no change in the numerator definition or in the denominator definition between the Healthy People 2010 and Healthy People 2020 objectives. These include objectives that were developmental in Healthy People 2010 and are developmental in Healthy People 2020 and for which no numerator or denominator information was available.

7. Modified objectives have some change in the numerator definition or in the denominator definition between the Healthy People 2010 and Healthy People 2020 objectives. These include objectives that went from developmental in Healthy People 2010 to measurable in Healthy People 2020 or vice versa.

8. Archived objectives had at least one data point in Healthy People 2010 but were not carried forward into Healthy People 2020. 


\section{Comprehensive Summary of Objectives: Physical Activity and Fitness}

\begin{tabular}{|c|c|c|}
\hline Objective & Description & Data Source \\
\hline $22-1$ & No leisure-time physical activity (age adjusted, 18+ years) & National Health Interview Survey (NHIS), CDC, NCHS. \\
\hline $22-2$ & Regular physical activity-Moderate or vigorous (age adjusted, $18+$ years) & National Health Interview Survey (NHIS), CDC, NCHS. \\
\hline $22-3$ & Regular physical activity_-Vigorous (age adjusted, 18+ years) & National Health Interview Survey (NHIS), CDC, NCHS. \\
\hline $22-4$ & Regular muscle-strengthening activity (age adjusted, 18+ years) & National Health Interview Survey (NHIS), CDC, NCHS. \\
\hline $22-5$ & Flexibility training (age adjusted, 18+ years) & National Health Interview Survey (NHIS), CDC, NCHS. \\
\hline $22-6$ & Moderate physical activity in students (grades 9-12) & Youth Risk Behavior Surveillance System (YRBSS), CDC, NCCDPHP. \\
\hline $22-7$ & Vigorous physical activity in students (grades 9-12) & Youth Risk Behavior Surveillance System (YRBSS), CDC, NCCDPHP. \\
\hline $22-8 \mathrm{a}$ & Physical education requirement in middle and junior high schools & School Health Policies and Programs Study (SHPPS), CDC, NCCDPHP. \\
\hline $22-8 b$ & Physical education requirement in senior high schools & School Health Policies and Programs Study (SHPPS), CDC, NCCDPHP. \\
\hline $22-9$ & Student participation in daily physical education in schools (grades 9-12) & Youth Risk Behavior Surveillance System (YRBSS), CDC, NCCDPHP. \\
\hline $22-10$ & Student physical activity in physical education class (grades 9-12) & Youth Risk Behavior Surveillance System (YRBSS), CDC, NCCDPHP. \\
\hline $22-11$ & Student television viewing-At most 2 hours per school day (grades 9-12) & Youth Risk Behavior Surveillance System (YRBSS), CDC, NCCDPHP. \\
\hline $22-12$ & Access to school physical activity facilities during non-school time & School Health Policies and Programs Study (SHPPS), CDC, NCCDPHP. \\
\hline $22-13$ & Worksite physical activity and fitness programs & $\begin{array}{l}\text { National Worksite Health Promotion Survey (NWHPS), Association } \\
\text { for Worksite Health Promotion (AWHP and OPHS, ODPHP. }\end{array}$ \\
\hline $22-14 a$ & $\begin{array}{l}\text { Walking for transportation-Adults-Trips } \leq 1 \text { mile (age adjusted, } 18+ \\
\text { years) }\end{array}$ & $\begin{array}{l}\text { National Household Travel Survey (NHTS), formerly Nationwide } \\
\text { Personal Transportation Survey (NPTS), Department of } \\
\text { Transportation (DOT). }\end{array}$ \\
\hline $22-14 b$ & $\begin{array}{l}\text { Walking for transportation-Children and adolescents-Trips to school } \leq 1 \\
\text { mile }(5-15 \text { years })\end{array}$ & $\begin{array}{l}\text { National Household Travel Survey (NHTS), formerly Nationwide } \\
\text { Personal Transportation Survey (NPTS), Department of } \\
\text { Transportation (DOT). }\end{array}$ \\
\hline $22-15 a$ & $\begin{array}{l}\text { Bicycling for transportation-Adults-Trips } \leq 5 \text { miles (age adjusted, } 18+ \\
\text { years ) }\end{array}$ & $\begin{array}{l}\text { National Household Travel Survey (NHTS), formerly Nationwide } \\
\text { Personal Transportation Survey (NPTS), Department of } \\
\text { Transportation (DOT). }\end{array}$ \\
\hline $22-15 b$ & $\begin{array}{l}\text { Walking for transportation-Children and adolescents-Trips to school } \leq 2 \\
\text { miles (5-15 years) }\end{array}$ & $\begin{array}{l}\text { National Household Travel Survey (NHTS), formerly Nationwide } \\
\text { Personal Transportation Survey (NPTS), Department of } \\
\text { Transportation (DOT). }\end{array}$ \\
\hline
\end{tabular}


Figure 22-1. Progress Toward Target Attainment for Focus Area 22: Physical Activity and Fitness

Moved away

from target ${ }^{1}$

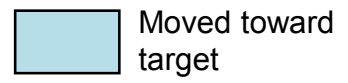

target

Met or exceeded

target

22-1. No leisure-time physical activity (age adjusted, $18+$ years)

22-2. Regular physical activity-Moderate or vigorous (age adjusted, 18+ years)

22-3. Regular physical activity-Vigorous (age adjusted, $18+$ years)

22-4. Regular muscle-strengthening activity (age adjusted, 18+ years)

22-5. Flexibility training (age adjusted, 18+ years)

22-6. Moderate physical activity in students (grades 9-12)

22-7. Vigorous physical activity in students (grades 9-12)

22-8a. Physical education requirement in middle and junior high schools

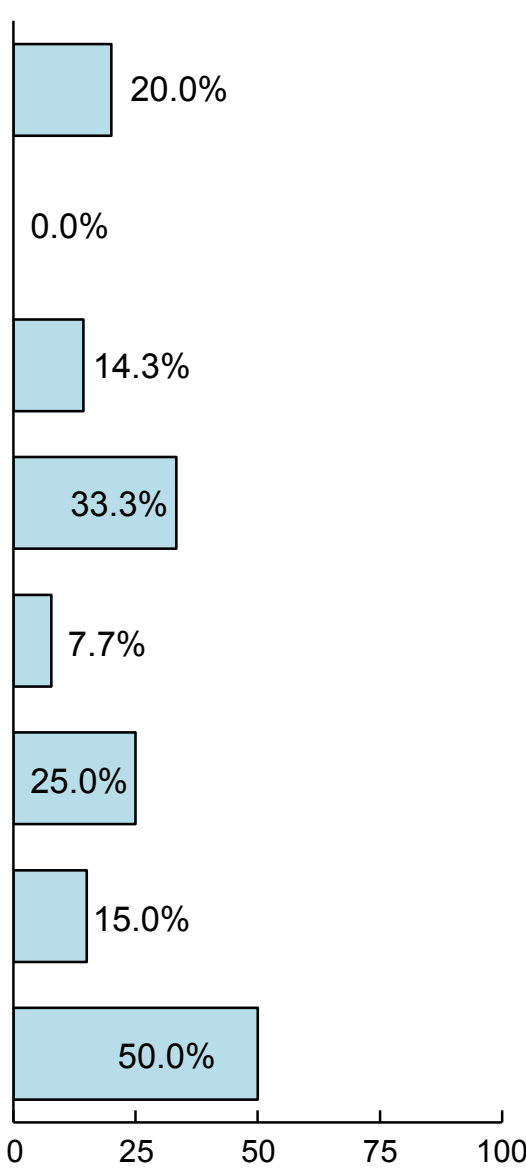

\begin{tabular}{|c|c|c|c|c|c|}
\hline \multirow{2}{*}{$\begin{array}{l}2010 \\
\text { Target }\end{array}$} & \multirow{2}{*}{$\begin{array}{c}\text { Baseline } \\
\text { (Year) }\end{array}$} & \multirow{2}{*}{$\begin{array}{l}\text { Final } \\
\text { (Year) }\end{array}$} & \multicolumn{3}{|c|}{ Baseline vs. Final } \\
\hline & & & Difference $^{2}$ & $\begin{array}{l}\text { Statistically } \\
\text { Significant }^{3}\end{array}$ & $\begin{array}{c}\text { Percent } \\
\text { Change }^{4}\end{array}$ \\
\hline $20 \%$ & $\begin{array}{c}40 \% \\
(1997)\end{array}$ & $\begin{array}{c}36 \% \\
(2008)\end{array}$ & -4 & Yes & $-10.0 \%$ \\
\hline $50 \%$ & $\begin{array}{c}32 \% \\
(1997)\end{array}$ & $\begin{array}{c}32 \% \\
(2008)\end{array}$ & 0 & No & $0.0 \%$ \\
\hline $30 \%$ & $\begin{array}{c}23 \% \\
(1997)\end{array}$ & $\begin{array}{c}24 \% \\
(2008)\end{array}$ & 1 & No & $4.3 \%$ \\
\hline $30 \%$ & $\begin{array}{c}18 \% \\
(1998)\end{array}$ & $\begin{array}{c}22 \% \\
(2008)\end{array}$ & 4 & Yes & $22.2 \%$ \\
\hline $43 \%$ & $\begin{array}{c}30 \% \\
(1998)\end{array}$ & $\begin{array}{c}31 \% \\
(2001)\end{array}$ & 1 & No & $3.3 \%$ \\
\hline $35 \%$ & $\begin{array}{c}27 \% \\
(1999)\end{array}$ & $\begin{array}{c}29 \% \\
(2009)\end{array}$ & 2 & No & $7.4 \%$ \\
\hline $85 \%$ & $\begin{array}{c}65 \% \\
(1999)\end{array}$ & $\begin{array}{c}68 \% \\
(2009)\end{array}$ & 3 & No & $4.6 \%$ \\
\hline $9.4 \%$ & $\begin{array}{l}6.4 \% \\
(2000)\end{array}$ & $\begin{array}{c}7.9 \% \\
(2006)\end{array}$ & 1.5 & No & $23.4 \%$ \\
\hline
\end{tabular}

Percent of targeted change achieved ${ }^{5}$ (continued) 
Figure 22-1. Progress Toward Target Attainment for Focus Area 22: Physical Activity and Fitness (continued)

Moved away

from target ${ }^{1}$

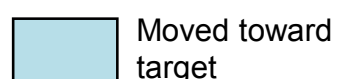

target

Met or exceeded

target

22-8b. Physical education requirement in senior high schools

22-9. Student participation in daily physical education in schools (grades 9-12)

22-10. Student physical activity in physical education class (grades 9-12)

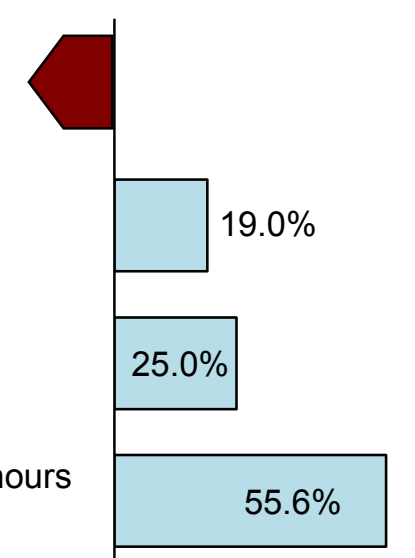

22-11. Student television viewing-At most 2 hours per school day (grades 9-12)

22-12. Access to school physical activity facilities during non-school time

22-14. Walking for transportation

a. Adults-Trips $\leq 1$ mile (age adjusted, $18+$ years)

b. Children and adolescents-Trips to school $\leq 1$ mile (5-15 years)

22-15. Bicycling for transportation

a. Adults-Trips $\leq 5$ miles (age adjusted, 18+ years)

\begin{tabular}{|c|c|c|c|c|c|}
\hline \multirow{2}{*}{$\begin{array}{l}2010 \\
\text { Target }\end{array}$} & \multirow{2}{*}{$\begin{array}{c}\text { Baseline } \\
\text { (Year) }\end{array}$} & \multirow{2}{*}{$\begin{array}{l}\text { Final } \\
\text { (Year) }\end{array}$} & \multicolumn{3}{|c|}{ Baseline vs. Final } \\
\hline & & & Difference $^{2}$ & $\begin{array}{l}\text { Statistically } \\
\text { Significant }^{3}\end{array}$ & $\begin{array}{c}\text { Percent } \\
\text { Change }^{4}\end{array}$ \\
\hline $14.5 \%$ & $\begin{array}{l}5.8 \% \\
(2000)\end{array}$ & $\begin{array}{l}2.1 \% \\
(2006)\end{array}$ & -3.7 & Yes & $-63.8 \%$ \\
\hline $50 \%$ & $\begin{array}{c}29 \% \\
(1999)\end{array}$ & $\begin{array}{c}33 \% \\
(2009)\end{array}$ & 4 & No & $13.8 \%$ \\
\hline $50 \%$ & $\begin{array}{c}38 \% \\
(1999)\end{array}$ & $\begin{array}{c}41 \% \\
(2009)\end{array}$ & 3 & No & $7.9 \%$ \\
\hline $75 \%$ & $\begin{array}{c}57 \% \\
(1999)\end{array}$ & $\begin{array}{c}67 \% \\
(2009)\end{array}$ & 10 & Yes & $17.5 \%$ \\
\hline $50 \%$ & $\begin{array}{c}35 \% \\
(2000)\end{array}$ & $\begin{array}{c}29 \% \\
(2006)\end{array}$ & -6 & No & $-17.1 \%$ \\
\hline $25 \%$ & $\begin{array}{c}17 \% \\
(1995)\end{array}$ & $\begin{array}{c}21 \% \\
(2001)\end{array}$ & 4 & Yes & $23.5 \%$ \\
\hline $50 \%$ & $\begin{array}{c}31 \% \\
(1995)\end{array}$ & $\begin{array}{c}36 \% \\
(2001)\end{array}$ & 5 & Yes & $16.1 \%$ \\
\hline $2.0 \%$ & $\begin{array}{c}0.6 \% \\
(1995)\end{array}$ & $\begin{array}{l}0.4 \% \\
(2001)\end{array}$ & -0.2 & Yes & $-33.3 \%$ \\
\hline
\end{tabular}

(continued) 
Figure 22-1. Progress Toward Target Attainment for Focus Area 22: Physical Activity and Fitness (continued)

Moved away

from target ${ }^{1}$

Moved toward

target

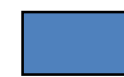

Met or exceeded

target

22-15. Bicycling for transportation

b. Children and adolescents-Trips to school $\leq 2$ miles (5-15 years)

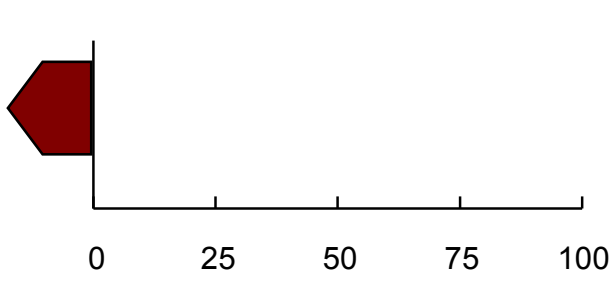

\begin{tabular}{|c|c|c|c|c|c|}
\hline \multirow{2}{*}{$\begin{array}{l}2010 \\
\text { Target }\end{array}$} & \multirow{2}{*}{$\begin{array}{c}\text { Baseline } \\
\text { (Year) }\end{array}$} & \multirow{2}{*}{$\begin{array}{l}\text { Final } \\
\text { (Year) }\end{array}$} & \multicolumn{3}{|c|}{ Baseline vs. Final } \\
\hline & & & Difference $^{2}$ & $\begin{array}{l}\text { Statistically } \\
\text { Significant }^{3}\end{array}$ & $\begin{array}{l}\text { Percent } \\
\text { Change }\end{array}$ \\
\hline $5.0 \%$ & $\begin{array}{c}2.4 \% \\
(1995)\end{array}$ & $\begin{array}{c}1.5 \% \\
(2001)\end{array}$ & -0.9 & No & $-37.5 \%$ \\
\hline
\end{tabular}

\section{Percent of targeted change achieved ${ }^{5}$}

\section{NOTES}

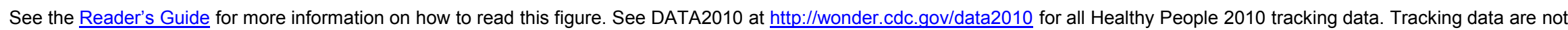
available for objective 22-13.

\section{FOOTNOTES}

${ }^{1}$ Movement away from target is not quantified using the percent of targeted change achieved. See Technical Appendix for more information.

${ }^{2}$ Difference $=$ Final value - Baseline value. Differences between percents $(\%)$ are measured in percentage points

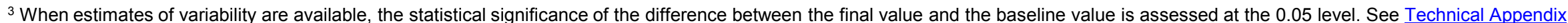
for more information.

${ }^{4}$ Percent change $=\frac{\text { Final value }- \text { Baseline value }}{\text { Baseline value }} \times 100$.

${ }^{5}$ Percent of targeted change achieved $=\frac{\text { Final value }- \text { Baseline value }}{\text { Healthy People } 2010 \text { target }- \text { Baseline value }} \times 100$.

\section{DATA SOURCES}

22-1-22-5. National Health Interview Survey (NHIS), CDC, NCHS.

22-6-22-7. Youth Risk Behavior Surveillance System (YRBSS), CDC, NCCDPHP.

22-8a-b. School Health Policies and Programs Study (SHPPS), CDC, NCCDPHP

22-9-22-11. Youth Risk Behavior Surveillance System (YRBSS), CDC, NCCDPHP.

22-12. School Health Policies and Programs Study (SHPPS), CDC, NCCDPHP

22-14a-b. National Household Travel Survey (NHTS), formerly Nationwide Personal Transportation Survey (NPTS), Department of Transportation (DOT).

22-15a-b. National Household Travel Survey (NHTS), formerly Nationwide Personal Transportation Survey (NPTS), Department of Transportation (DOT). 
Figure 22-2. Health Disparities Table for Focus Area 22: Physical Activity and Fitness Disparities from the best group rate for each characteristic at the most recent data point and changes in disparity from the baseline to the most recent data point.

\section{2-1. No leisure-time physical activity $\quad$ (age adusted, $18+$ to (age adjusted, 18+ years) $(1997,2008)^{1}$}

22-2. Regular physical activity-Moderate or vigorous (age adjusted, $18+$ years) $(1997,2008)^{1}$

22-3. Regular physical activity-Vigorous (age adjusted, $18+$ years) $(1997,2008)^{1}$

22-4. Regular muscle-strengthening activity (age adjusted, 18+ years) $(1998,2008)^{1}$

22-5. Flexibility training (age adjusted, $18+$ years) $(1998,2001)^{2}$

22-6. Moderate physical activity in students (grades 9-12) (1999, 2009)

22-7. Vigorous physical activity in students (grades 9-12) $(1999,2009)$

22-9. Student participation in daily physical education in schools (grades 9-12) (1999, 2009)

22-10. Student physical activity in physical education class (grades 9-12) (1999, 2009)

22-11. Student television viewing-At most 2 hours per school day (grades 9-12) $(1999,2009)$

22-14a. Walking for transportation—Adults

- Trips $\leq 1$ mile (age adjusted, $18+$ years) $(1995,2001)$

b. Walking for transportation-Children and adolescents -Trips to school $\leq 1$ mile $(5-15$ years) $(1995,2001)$

22-15a. Bicycling for transportation-Adults

-Trips $\leq 5$ miles (age adjusted, 18+ years) $(1995,2001)$

b. Bicycling for transportation-Children and adolescents -Trips to school $\leq 2$ miles $\left(5-15\right.$ years) $(1995,2001)^{3}$

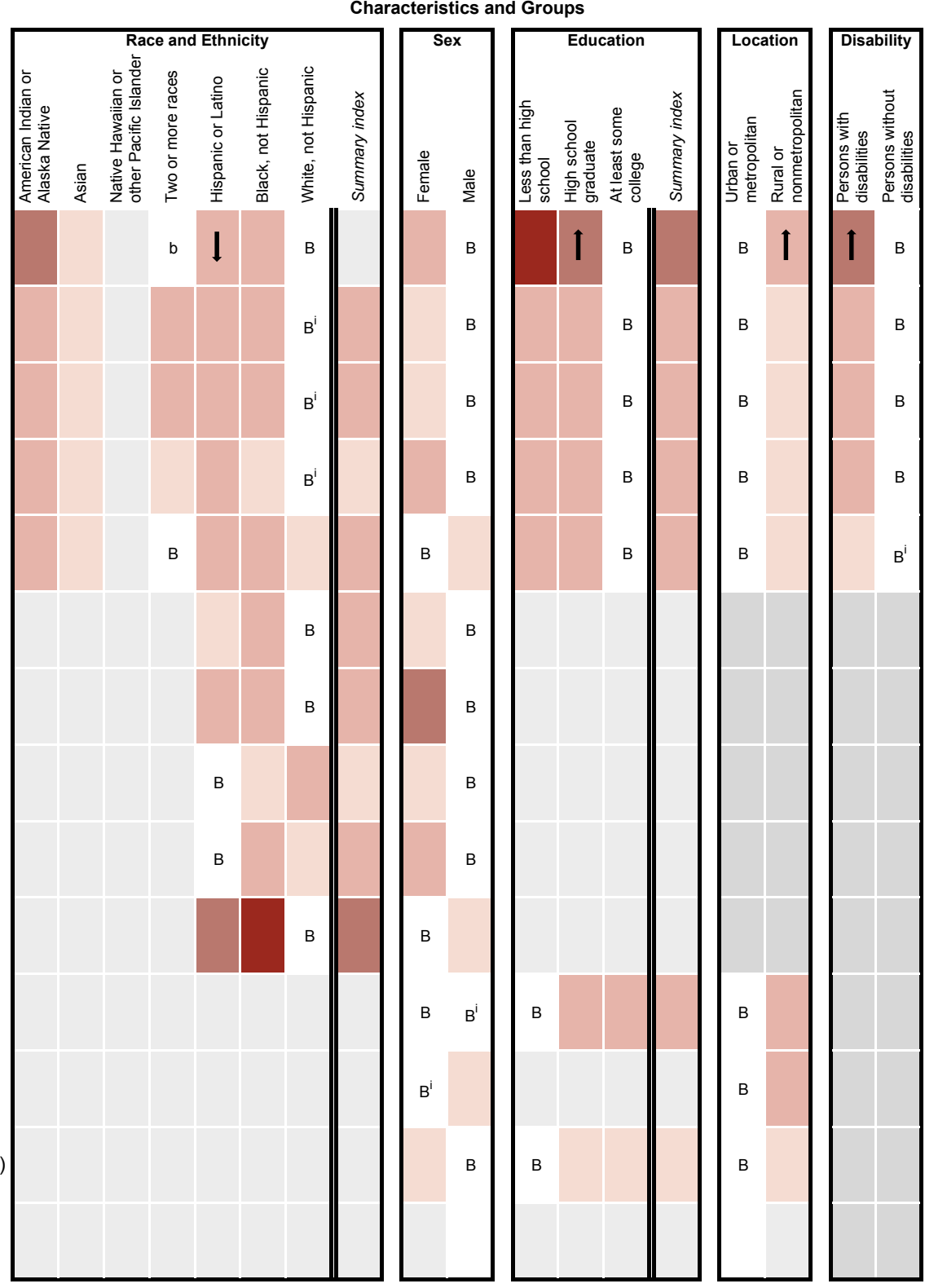




\section{Figure 22-2. Health Disparities Table for Focus Area 22: Physical Activity and Fitness (continued)}

NOTES

See DATA2010 at http://wonder.cdc.gov/data2010 for all Healthy People 2010 tracking data. Disparity data are either unavailable or not applicable for objectives 22-8a and b, 22-12, and 22-13.

Years in parentheses represent the baseline and most recent data years (if available).

Disparity from the best group rate is defined as the percent difference between the best group rate and each of the other group rates for a characteristic (e.g., race and ethnicity). The summary index is the average of these percent differences for a characteristic. Change in disparity is estimated by subtracting the disparity at baseline from the disparity at the most recent data point. Change in the summary index is estimated by subtracting the summary index at baseline from the summary index at the most recent data point. See Technical Appendix for more information.

Measures of variability were available for all objectives in this table. Thus, the variability of best group rates was assessed, and statistical significance was tested. Disparities of $10 \%$ or more are displayed when the differences from the best group rate are statistically significant at the 0.05 level. Changes in disparities over time are indicated by arrows when the changes are greater than or equal to 10 percentage points and are statistically significant at the 0.05 level. See Technical Appendix.

LEGEND

\begin{tabular}{|c|c|c|c|c|c|}
\hline $\begin{array}{l}\text { The "best" group rate at the most recent } \\
\text { data point. }\end{array}$ & B & $\begin{array}{l}\text { The group with the best rate for } \\
\text { specified characteristic. }\end{array}$ & $b$ & $\begin{array}{l}\text { Most favorable group rate for specified } \\
\text { characteristic, but reliability criterion not } \\
\text { met. }\end{array}$ & $\begin{array}{l}\text { Reliability criterion for best } \\
\text { group rate not met, or data } \\
\text { available for only one group. }\end{array}$ \\
\hline & \multicolumn{5}{|c|}{ Percent difference from the best group rate } \\
\hline $\begin{array}{l}\text { Disparity from the best group rate at the } \\
\text { most recent data point. }\end{array}$ & & $\begin{array}{l}\text { Less than } 10 \% \text {, or difference not } \\
\text { statistically significant (when } \\
\text { estimates of variability are available). }\end{array}$ & & $10 \%-49 \%$ & $100 \%$ or more \\
\hline
\end{tabular}

Changes in disparity over time are shown when:
(a) disparities data are available at both baseline and most recent time points;
(b) data are not for the group(s) indicated by "B" or "b" at either time point; and
(c) the change is greater than or equal to 10 percentage points and statistically
significant, or when the change is greater than or equal to 10 percentage
points and estimates of variability were not available. See Technical Appendix.

FOOTNOTES

1 Baseline data by race and ethnicity are for 1999.

2 Baseline data by race and ethnicity are for 2001.

3 Most current data by location are for 1995.

$i$ The group with the best rate at the most recent data point is different from the group with the best rate at baseline. Both rates met the reliability criterion. See

Technical Appendix.

\section{DATA SOURCES}

22-1-22-5. National Health Interview Survey (NHIS), CDC, NCHS.

22-6-22-7. Youth Risk Behavior Surveillance System (YRBSS), CDC, NCCDPHP

22-9-22-11. Youth Risk Behavior Surveillance System (YRBSS), CDC, NCCDPHP

22-14a-b. National Household Travel Survey (NHTS), formerly Nationwide Personal Transportation Survey (NPTS), Department of Transportation (DOT)

22-15a-b. National Household Travel Survey (NHTS), formerly Nationwide Personal Transportation Survey (NPTS), Department of Transportation (DOT) 
Figure 22-3. No Leisure Time Physical Activity Among Adults (18+ Years) 2008

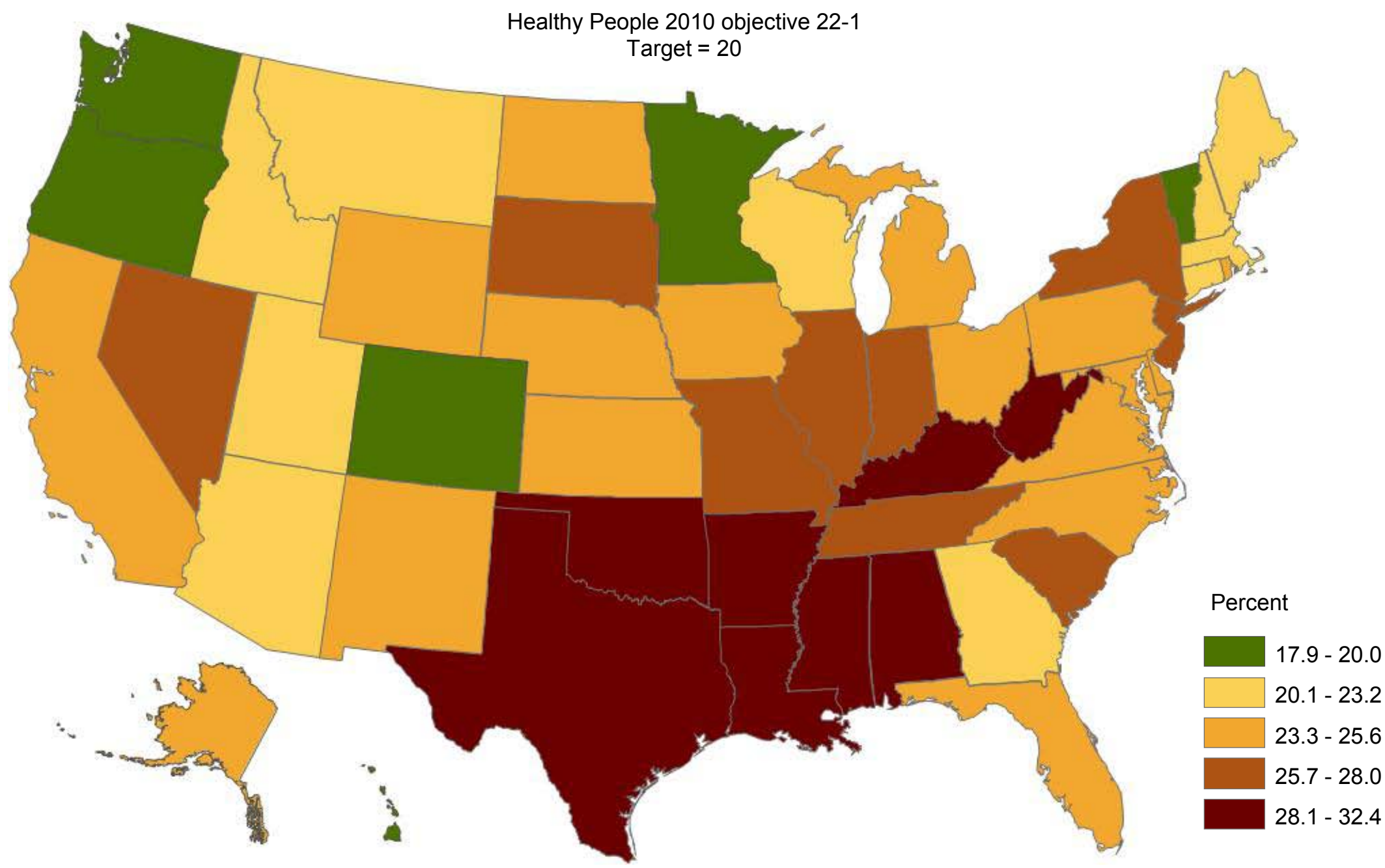

lowest category (green) shows states that met target

NOTES: Data are age-adjusted to the 2000 standard population. Rates are displayed by a modified Jenks classification for U.S. states. National data for the objective come from the National Health Interview Survey (NHIS) and is the basis for setting the target. State data from the BRFSS may not be comparable to the national data from the NHIS.

SOURCE: Behavioral Risk Factor Surveillance System (BRFSS), CDC.

Physical Activity and Fitness 


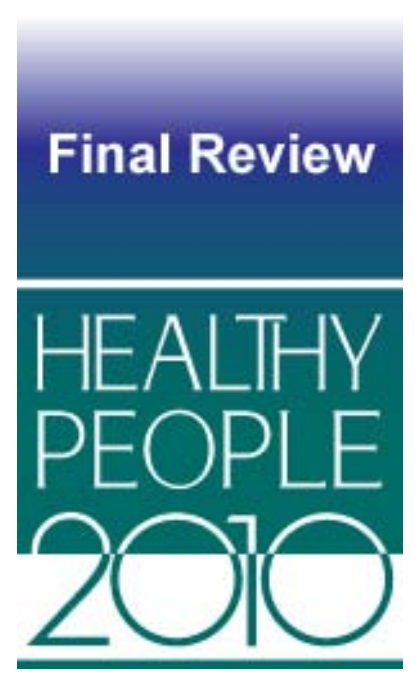

\section{Public Health Infrastructure}

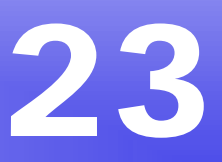

\section{Co-Lead Agencies}

Centers for Disease Control and Prevention

Health Resources and Services Administration

\section{Contents}

Goal.

Highlights

Summary of Progress

Transition to Healthy People 2020

Data Considerations

References and Notes

Comprehensive Summary of Objectives

Progress Chart 


\section{Goal: Ensure that Federal, Tribal, State, and local health agencies have the infrastructure to provide essential public health services effectively}

The Public Health Infrastructure Focus Area supports the goals and objectives of all other Focus Areas, particularly those that address preparedness and prevention, the management of chronic disease, and those that emphasize healthy behavioral choices. The Public Health Infrastructure objectives encompass Tribal, rural, and urban populations. They focus on four components: data and information systems, workforce, public health organizations, and prevention research.

All Healthy People tracking data quoted in this chapter, along with technical information and operational definitions for each objective, can be found in the Healthy People 2010 database, DATA2010, available from http://wonder.cdc.gov/data2010/.

More information about this Focus Area can be found in the following publications:

- Healthy People 2010: Understanding and Improving Health, available from http://www.healthypeople.gov/2010/Document/tableofcontents.htm\#under.

- Healthy People 2010 Midcourse Review, available from http://www.healthypeople.gov/2010/data/midcourse/html/default.htm\#FocusAreas.

\section{Highlights}

- Over two-thirds of the Public Health Infrastructure objectives were developmental when Healthy People 2010 was first published [1,2]. During the past decade, data sources were identified for many of these objectives, allowing them to be tracked and monitored. Since a number of objectives were revised and others added, the counts of objectives are not strictly comparable. As of this Final Review, 14\% of the objectives remain developmental, and $7 \%$ were dropped during the decade [3].

- Substantial progress was achieved in objectives for this Focus Area during the past decade, although the tracking period for a number of objectives was five years or less [4]. Seventy percent of the Public Health Infrastructure objectives with data to measure progress moved toward or achieved their Healthy People 2010 targets (see Figure 23-1).

- The timely release of Healthy People 2010 data increased over the decade. The proportion of objectives measured by major data systems from which data were released within one year of the end of data collection (objective 23-7) increased by $83.3 \%$ between 2000 and 2009 , from $36 \%$ to $66 \%$, moving toward the Healthy People 2010 target of $100 \%$.

- The National Public Health Performance Standards Program assesses the public health system's capacity to perform essential services (objectives 23-11a through d). 
- The use of performance standards by State public health systems (objective 23-11a) increased by $177.8 \%$ between 2004 and 2009, from 9 to 25 states, moving toward the 2010 target of 35 states. The use of standards by local public health systems (objective 23-11b) more than doubled between 2004 and 2009 , from $12 \%$ to $28 \%$, moving toward the target of $50 \%$.

- During the same period, there was a small increase in the number of states meeting the optimal performance standards (objective 23-11c), from $0 \%$ to $4 \%$, moving toward the target of $50 \%$. The proportion of local public health systems meeting the standards (objective 23-11d) increased by $52.8 \%$ between 2004 and 2009, from $36 \%$ to $55 \%$, exceeding the 2010 target of $50 \%$.

- The percent of State epidemiologists with formal training in epidemiology (objective 2314a) increased by $50 \%$ between 2001 and 2008, from $58 \%$ to $87 \%$, exceeding the target of $80 \%$.

\section{Summary of Progress}

- Figure 23-1 presents a quantitative assessment of progress in achieving the Healthy People 2010 objectives for Public Health Infrastructure [4]. Data to measure progress toward target attainment were available for 30 objectives. Of these:

- Five objectives (23-2a, c and d; 23-11d; and 23-14a) met or exceeded their Healthy People 2010 targets.

- Sixteen objectives (23-4; 23-6; 23-7; 23-11a through c; 23-13c, e, g, i, j, and k; 23-14c and d; and 23-15a and b) moved toward their targets. Data to test the significance of the difference between the baseline and the final data points were unavailable for all of these objectives.

- One objective (23-3) showed no change.

- Eight objectives moved away from their targets. A statistically significant difference between the baseline and final data points was observed for one objective (23-8b). Data to test the significance of the difference were unavailable for the other seven objectives (23-12c and d, and 23-13a, b, d, f, and h).

- Six objectives (23-2b, 23-8a, 23-10a, 23-12a, 23-14b, and 23-17) remained developmental and four objectives $(23-9,23-10 \mathrm{~b}$ and $\mathrm{c}$, and $23-12 \mathrm{~b})$ had no data available to measure progress [1]. Three objectives (23-1, 23-5, and 23-16) were dropped during the decade [3]. 


\section{Transition to Healthy People 2020}

The objectives in the Healthy People 2020 Public Health Infrastructure Topic Area continue to be anchored in the provision of essential services. See HealthyPeople.gov for a complete list of Healthy People 2020 topics and objectives. In addition, the Healthy People 2020 objectives focus more on education of the workforce and improvement of health departments than in Healthy People 2010. Public health law and public health systems research objectives proved to be difficult to measure for Healthy People 2010 and therefore have been dropped, at least until appropriate measures can be identified.

The Healthy People 2020 objectives can be grouped into three sections:

- Workforce

- Data and information systems

- Public health organizations.

The differences between the Healthy People 2010 objectives and Healthy People 2020 are summarized below:

- The Healthy People 2020 Public Health Infrastructure Topic Area has 44 objectives, 19 of which are developmental, whereas the Healthy People 2010 Focus Area had 43 objectives, including six that were still developmental at the end of the decade [1].

- Fifteen Healthy People 2010 objectives with data were retained "as is" [5]. These include: the use of core competencies in job descriptions at local health agencies and in public health curricula (objectives 23-8b and 23-9), the use of performance standards in local public health systems (objective 23-11b), provision or assurance of comprehensive laboratory services to support essential public health services (objectives 23-13a through k), and the formal training of State epidemiologists (objective 23-14a). An additional four objectives that address Tribal health agency activities (objectives 23-8a, 23-10a, 23-12a, and 23-14b) were also retained "as is" in developmental status [1].

- Ten Healthy People 2010 objectives were modified [6]. A new data source is being sought for monitoring the provision of continuing education to public health workers (objectives 23-10b and c); health improvement plans implemented at the state and local levels (objectives 23-12b through d) will be tracked and counted if conducted within the last five, rather than three, years; and the mechanisms for measuring several other objectives (23-4, 23-6, 23-7, and 23-14c and d) will be changed.

- Eleven Healthy People 2010 objectives were archived [7]. Three of these (objectives 23-1, 23-5, and 23-16) had been dropped at the Midcourse Review [3]. Of the remaining eight, the availability of health indicator data was considered complete (objectives 23-2a, c, and d); geo-coding of major data systems (objective 23-3) could not expand further than it had during the previous decade; the meeting of national performance standards (objective 23$11 \mathrm{c}$ and d) will be subsumed into the accreditation objective; and public health law and public health systems research (objectives 23-15a and b) cannot be adequately measured at this time. 
- Sixteen new objectives were added to the Healthy People 2020 Topic Area:

- Five new objectives measure availability of public health programs at the community college and undergraduate levels, as well as the uniformity of undergraduate programs in public health that incorporate core competencies in their curriculum.

- Objectives that track the levels of government expected to incorporate Core Competencies for Public Health Professions into job descriptions and performance evaluations have been expanded to include Federal and State public health agencies. Local boards of health were added to the objective targeting public health system assessment.

- Three objectives monitoring the number of states using the most recent edition of the U.S. Standard Certificates to collect vital statistics data were added.

- Objectives that track the quality and quantity of Healthy People 2020 data were expanded.

- A public health laboratory systems performance objective was added.

- Objectives addressing public health agency quality improvement and accreditation of State, Tribal and local health departments were also added.

The Healthy People 2020 objectives reflect the ever-present importance of public health infrastructure to effectively provide essential public health services. For objectives that were deleted due to lack of data, the U.S. Department of Health and Human Services and the agencies that serve as the leads for the Healthy People 2020 initiative will consider ways to ensure that these public health issues retain prominence despite the lack of data to track them.

Appendix D, “A Crosswalk Between Objectives From Healthy People 2010 to Healthy People 2020,” summarizes the changes between the two decades of objectives, reflecting new knowledge and direction for this area.

\section{Data Considerations}

Data collection and data analysis has been a challenge for measuring the Tribal objectives in the Public Health Infrastructure Focus Area due to the diversity of services, methods of service delivery, and data collection and measurement.

The data source used to measure continuing education of public health workers (objectives $23-10 \mathrm{~b}$ and c) was a national survey of registered nurses. These data were used to characterize all public health workers but only include one type of such employee. Furthermore, the survey was discontinued in 2000 .

The Epidemiology Capacity Assessment, conducted by the Council of State and Territorial Epidemiologists, was the data source for tracking State epidemiologic services (objectives 23-14a and c). States were asked if they had adequate epidemiologic capacity to provide the four essential public health services. The National Profile of Local Health Departments, conducted by the National Association of County and City Health Departments, was the data source for tracking epidemiology 
services provided through local public health agencies (objective 23-14d). Respondents were asked to indicate which organization provided epidemiology and surveillance services in six categories. Agencies responding that services were provided by the local health department only, another local government agency only, a State agency only, or a non-government organization only, were categorized as providing adequate epidemiologic services. Because of the different definitions of adequate services used, the data for State and local health departments should not be compared.

Additional information on data issues is available from the following sources:

- All Healthy People 2010 tracking data can be found in the Healthy People 2010 database, DATA2010, available from http://wonder.cdc.gov/data2010/.

- Detailed information about the data and data sources used to support these objectives can be found in the Operational Definitions on the DATA2010 website, available from http://wonder.cdc.gov/data2010/focusod.htm.

- More information on statistical issues related to Healthy People tracking and measurement can be found in the Technical Appendix and in Healthy People 2010: General Data Issues, which is available in the Data Issues section of the NCHS Healthy People website under Healthy People 2010.

\section{References and Notes}

1. To be included in Healthy People 2010, an objective must have a national data source that provides a baseline and at least one additional data point for tracking progress. Some objectives lacked baseline data at the time of their development but had a potential data source and were considered of sufficient national importance to be included in Healthy People. These are called "developmental" objectives. When data become available, a developmental objective is moved to measurable status and a Healthy People target can be set.

2. U.S. Department of Health and Human Services. Healthy People 2010. $2^{\text {nd }}$ ed. With Understanding and Improving Health and Objectives for Improving Health. 2 vols. Washington, DC: U.S. Government Printing Office, November 2000.

3. Dropped objectives were not carried forward into Healthy People 2020. These objectives were either developmental or deleted at the Healthy People 2010 Midcourse Review or at another time in Healthy People 2010.

4. Displayed in the Progress Chart (Figure 23-1), the percent of targeted change achieved expresses the difference between the baseline and the final value relative to the initial difference between the baseline and the Healthy People 2010 target. As such, it is a relative measure of progress toward attaining the Healthy People 2010 target. See the Reader's Guide for more information. When standard errors were available, the difference between the baseline and the final value was tested at the 0.05 level of significance. See the Figure 23-1 footnotes, as well as the Technical Appendix, for more detail.

5. Retained "as is" objectives have no change in the numerator definition or in the denominator definition between the Healthy People 2010 and Healthy People 2020 objectives. These include objectives that were developmental in Healthy People 2010 and are developmental in Healthy People 2020 and for which no numerator or denominator information was available.

6. Modified objectives have some change in the numerator definition or in the denominator definition between the Healthy People 2010 and Healthy People 2020 objectives. These include objectives that went from developmental in Healthy People 2010 to measurable in Healthy People 2020 or vice versa.

7. Archived objectives had at least one data point in Healthy People 2010 but were not carried forward into Healthy People 2020. 


\section{Comprehensive Summary of Objectives: Public Health Infrastructure}

\begin{tabular}{|c|c|c|}
\hline Objective & Description & Data Source or Objective Status \\
\hline $23-1$ & Public health employee access to the Internet & Dropped \\
\hline $23-2 a$ & Health-related indicator data available-National & Assessment of Objective Data Availability (AODA), CDC, NCHS. \\
\hline $23-2 b$ & Health-related indicator data available—Tribal & Developmental \\
\hline $23-2 c$ & Health-related indicator data available-State & Assessment of Objective Data Availability (AODA), CDC, NCHS. \\
\hline $23-2 d$ & Health-related indicator data available—Local & Assessment of Objective Data Availability (AODA), CDC, NCHS. \\
\hline $23-3$ & Use of geo-coding in major health data systems & Assessment of Objective Data Availability (AODA), CDC, NCHS. \\
\hline $23-4$ & Data for all population groups in Healthy People 2010 objectives & Assessment of Objective Data Availability (AODA), CDC, NCHS. \\
\hline $23-5$ & Data for Leading Health Indicators & Dropped \\
\hline $23-6$ & Healthy People 2010 objectives tracked at least every three years & Assessment of Objective Data Availability (AODA), CDC, NCHS. \\
\hline $23-7$ & $\begin{array}{l}\text { Release of data on Healthy People } 2010 \text { objectives within one year of } \\
\text { collection }\end{array}$ & Assessment of Objective Data Availability (AODA), CDC, NCHS. \\
\hline $23-8 \mathrm{a}$ & $\begin{array}{l}\text { Tribal agencies with core competencies in job descriptions and } \\
\text { performance evaluations }\end{array}$ & Developmental \\
\hline $23-8 b$ & $\begin{array}{l}\text { Local agencies with core competencies in job descriptions and } \\
\text { performance evaluations }\end{array}$ & $\begin{array}{l}\text { National Profile of Local Health Departments, National Association of } \\
\text { County and City Health Officials (NACCHO). }\end{array}$ \\
\hline $23-9$ & Core competencies in public health curricula & $\begin{array}{l}\text { Public Health Competencies Survey, Council on Linkages, American } \\
\text { Schools of Public Health, Association of Teachers of Preventive Medicine } \\
\text { and the Quad Council }\end{array}$ \\
\hline $23-10 a$ & Continuing education-Tribal public health personnel & Developmental \\
\hline $23-10 b$ & Continuing education-State public health personnel & $\begin{array}{l}\text { National Sample Survey of Registered Nurses, HRSA, Bureau of Health } \\
\text { Professionals }\end{array}$ \\
\hline $23-10 c$ & Continuing education-Local public health personnel & $\begin{array}{l}\text { National Sample Survey of Registered Nurses, HRSA, Bureau of Health } \\
\text { Professionals }\end{array}$ \\
\hline 23-11a & Use of performance standards-State public health systems (no. States) & National Public Health Performance Standards Program, CDC, OCPHP. \\
\hline $23-11 b$ & Use of performance standards-Local public health systems & National Public Health Performance Standards Program, CDC, OCPHP. \\
\hline $23-11 c$ & Met performance standards-State public health systems & National Public Health Performance Standards Program, CDC, OCPHP. \\
\hline 23-11d & Met performance standards_Local public health systems & National Public Health Performance Standards Program, CDC, OCPHP. \\
\hline $23-12 a$ & Health improvement plans-Tribal health agencies & Developmental \\
\hline
\end{tabular}




\begin{tabular}{|c|c|c|}
\hline Objective & Description & Data Source or Objective Status \\
\hline $23-12 b$ & Health improvement plans-State health agencies & $\begin{array}{l}\text { Salary Survey of State and Territorial Health Officials, Association of State } \\
\text { and Territorial Health Officials (ASTHO) }\end{array}$ \\
\hline $23-12 c$ & Health improvement plans-Local health agencies & $\begin{array}{l}\text { National Profile of Local Health Departments, National Association of } \\
\text { County and City Health Officials (NACCHO). }\end{array}$ \\
\hline $23-12 d$ & Health improvement plans_-Local plan linked to State plan & $\begin{array}{l}\text { National Profile of Local Health Departments, National Association of } \\
\text { County and City Health Officials (NACCHO). }\end{array}$ \\
\hline $23-13 a$ & $\begin{array}{l}\text { Public health laboratory services (States and D.C.) - Disease prevention } \\
\text { control and surveillance }\end{array}$ & $\begin{array}{l}\text { Comprehensive Laboratory Services Survey, Association of Public Health } \\
\text { Laboratories (APHL). }\end{array}$ \\
\hline $23-13 b$ & $\begin{array}{l}\text { Public health laboratory services (States and D.C.) - Integrated data } \\
\text { management }\end{array}$ & $\begin{array}{l}\text { Comprehensive Laboratory Services Survey, Association of Public Health } \\
\text { Laboratories (APHL). }\end{array}$ \\
\hline $23-13 c$ & $\begin{array}{l}\text { Public health laboratory services (States and D.C.)-Reference and } \\
\text { specialized testing }\end{array}$ & $\begin{array}{l}\text { Comprehensive Laboratory Services Survey, Association of Public Health } \\
\text { Laboratories (APHL). }\end{array}$ \\
\hline $23-13 d$ & $\begin{array}{l}\text { Public health laboratory services (States and D.C.) -Environmental } \\
\text { health and protection }\end{array}$ & $\begin{array}{l}\text { Comprehensive Laboratory Services Survey, Association of Public Health } \\
\text { Laboratories (APHL). }\end{array}$ \\
\hline $23-13 e$ & Public health laboratory services (States and D.C.)—Food safety & $\begin{array}{l}\text { Comprehensive Laboratory Services Survey, Association of Public Health } \\
\text { Laboratories (APHL). }\end{array}$ \\
\hline $23-13 f$ & $\begin{array}{l}\text { Public health laboratory services (States and D.C.)-Laboratory } \\
\text { improvement and regulation }\end{array}$ & $\begin{array}{l}\text { Comprehensive Laboratory Services Survey, Association of Public Health } \\
\text { Laboratories (APHL). }\end{array}$ \\
\hline $23-13 g$ & Public health laboratory services (States and D.C.)-Policy development & $\begin{array}{l}\text { Comprehensive Laboratory Services Survey, Association of Public Health } \\
\text { Laboratories (APHL). }\end{array}$ \\
\hline $23-13 h$ & Public health laboratory services (States and D.C.) -Emergency response & $\begin{array}{l}\text { Comprehensive Laboratory Services Survey, Association of Public Health } \\
\text { Laboratories (APHL). }\end{array}$ \\
\hline $23-13 i$ & $\begin{array}{l}\text { Public health laboratory services (States and D.C.) - Public health related } \\
\text { research }\end{array}$ & $\begin{array}{l}\text { Comprehensive Laboratory Services Survey, Association of Public Health } \\
\text { Laboratories (APHL). }\end{array}$ \\
\hline $23-13 j$ & $\begin{array}{l}\text { Public health laboratory services (States and D.C.) - Training and } \\
\text { education }\end{array}$ & $\begin{array}{l}\text { Comprehensive Laboratory Services Survey, Association of Public Health } \\
\text { Laboratories (APHL). }\end{array}$ \\
\hline $23-13 \mathrm{k}$ & $\begin{array}{l}\text { Public health laboratory services (States and D.C.)-Partnerships and } \\
\text { communication }\end{array}$ & $\begin{array}{l}\text { Comprehensive Laboratory Services Survey, Association of Public Health } \\
\text { Laboratories (APHL). }\end{array}$ \\
\hline $23-14 a$ & $\begin{array}{l}\text { Provide or assure comprehensive epidemiology services-State } \\
\text { epidemiologists with formal training }\end{array}$ & $\begin{array}{l}\text { Epidemiology Capacity Assessment, Council of State and Territorial } \\
\text { Epidemiologists (CSTE). }\end{array}$ \\
\hline $23-14 b$ & $\begin{array}{l}\text { Provide or assure comprehensive epidemiology services-Tribal } \\
\text { agencies }\end{array}$ & Developmental \\
\hline
\end{tabular}




\begin{tabular}{|c|c|c|}
\hline Objective & Description & Data Source or Objective Status \\
\hline $23-14 c$ & Provide or assure comprehensive epidemiology services-State agencies & $\begin{array}{l}\text { Epidemiology Capacity Assessment, Council of State and Territorial } \\
\text { Epidemiologists (CSTE). }\end{array}$ \\
\hline $23-14 d$ & Provide or assure comprehensive epidemiology services_Local agencies & $\begin{array}{l}\text { National Profile of Local Health Departments, National Association of } \\
\text { County and City Health Officials (NACCHO). }\end{array}$ \\
\hline $23-15 a$ & $\begin{array}{l}\text { Evaluation of public health laws-Turning Point Model State Public } \\
\text { Health Act (no. States and D.C.) }\end{array}$ & $\begin{array}{l}\text { Center for Public Health Law and the Public's Health, Georgetown } \\
\text { University Law Center and Johns Hopkins Bloomberg School of Public } \\
\text { Health. }\end{array}$ \\
\hline $23-15 b$ & $\begin{array}{l}\text { Evaluation of public health laws-Model State Emergency Powers Act } \\
\text { (no. States and D.C.) }\end{array}$ & $\begin{array}{l}\text { Center for Public Health Law and the Public's Health, Georgetown } \\
\text { University Law Center and Johns Hopkins Bloomberg School of Public } \\
\text { Health. }\end{array}$ \\
\hline $23-16$ & Data on public health expenditures & Dropped \\
\hline $23-17$ & Population-based prevention research & Developmental \\
\hline
\end{tabular}


Figure 23-1. Progress Toward Target Attainment for Focus Area 23: Public Health Infrastructure

Moved away

from target ${ }^{1}$

Moved toward

target

Met or exceeded

target

23-2. Health-related indicator data available

a. National

c. State

d. Local

23-3. Use of geocoding in major health data systems

23-4. Data for all population groups in Healthy People 2010 objectives

23-6. Healthy People 2010 objectives tracked at least every three years

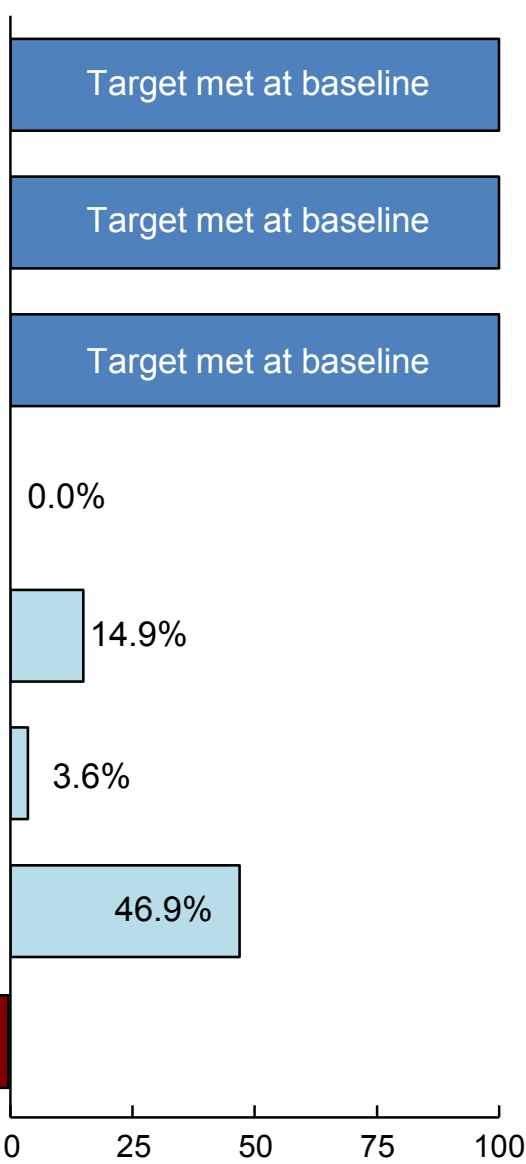

\begin{tabular}{|c|c|c|c|c|c|}
\hline \multirow{2}{*}{$\begin{array}{l}2010 \\
\text { Target }\end{array}$} & \multirow{2}{*}{$\begin{array}{c}\text { Baseline } \\
\text { (Year) }\end{array}$} & \multirow{2}{*}{$\begin{array}{l}\text { Final } \\
\text { (Year) }\end{array}$} & \multicolumn{3}{|c|}{ Baseline vs. Final } \\
\hline & & & Difference $^{2}$ & $\begin{array}{l}\text { Statistically } \\
\text { Significant }^{3}\end{array}$ & $\begin{array}{c}\text { Percent } \\
\text { Change }^{4}\end{array}$ \\
\hline $100 \%$ & $\begin{array}{l}100 \% \\
(2008)\end{array}$ & $N / A^{5}$ & $N / A^{5}$ & $N / A^{5}$ & $N / A^{5}$ \\
\hline $100 \%$ & $\begin{array}{l}100 \% \\
(2008)\end{array}$ & $N / A^{5}$ & $N / A^{5}$ & $N / A^{5}$ & $N / A^{5}$ \\
\hline $100 \%$ & $\begin{array}{l}100 \% \\
(2008)\end{array}$ & $N / A^{5}$ & $N / A^{5}$ & $N / A^{5}$ & $N / A^{5}$ \\
\hline $100 \%$ & $\begin{array}{c}50 \% \\
(2000)\end{array}$ & $\begin{array}{c}50 \% \\
(2009)\end{array}$ & 0 & $\begin{array}{c}\text { Not } \\
\text { tested }\end{array}$ & $0.0 \%$ \\
\hline $100 \%$ & $\begin{array}{c}13 \% \\
(2004)\end{array}$ & $\begin{array}{c}26 \% \\
(2008)\end{array}$ & 13 & $\begin{array}{c}\text { Not } \\
\text { tested }\end{array}$ & $100.0 \%$ \\
\hline $100 \%$ & $\begin{array}{c}44 \% \\
(2004)\end{array}$ & $\begin{array}{c}46 \% \\
(2008)\end{array}$ & 2 & $\begin{array}{l}\text { Not } \\
\text { tested }\end{array}$ & $4.5 \%$ \\
\hline $100 \%$ & $\begin{array}{c}36 \% \\
(2000)\end{array}$ & $\begin{array}{c}66 \% \\
(2009)\end{array}$ & 30 & $\begin{array}{c}\text { Not } \\
\text { tested }\end{array}$ & $83.3 \%$ \\
\hline $31 \%$ & $\begin{array}{c}21 \% \\
(2005)\end{array}$ & $\begin{array}{c}15 \% \\
(2008)\end{array}$ & -6 & Yes & $-28.6 \%$ \\
\hline
\end{tabular}

Percent of targeted change achieved ${ }^{7}$ (continued) 
Figure 23-1. Progress Toward Target Attainment for Focus Area 23: Public Health Infrastructure (continued)

Moved away

from target ${ }^{1}$

Moved toward target

Met or exceeded

target

23-11. Use of performance standards

a. State public health systems (no. States)

b. Local public health systems

Met performance standards

c. State public health systems (no. States)

d. Local public health systems

23-12. Health improvement plans

c. Local health agencies

d. Local plan linked to State plan

23-13. Public health laboratory services (States and D.C.)

a. Disease prevention control and surveillance

b. Integrated data management

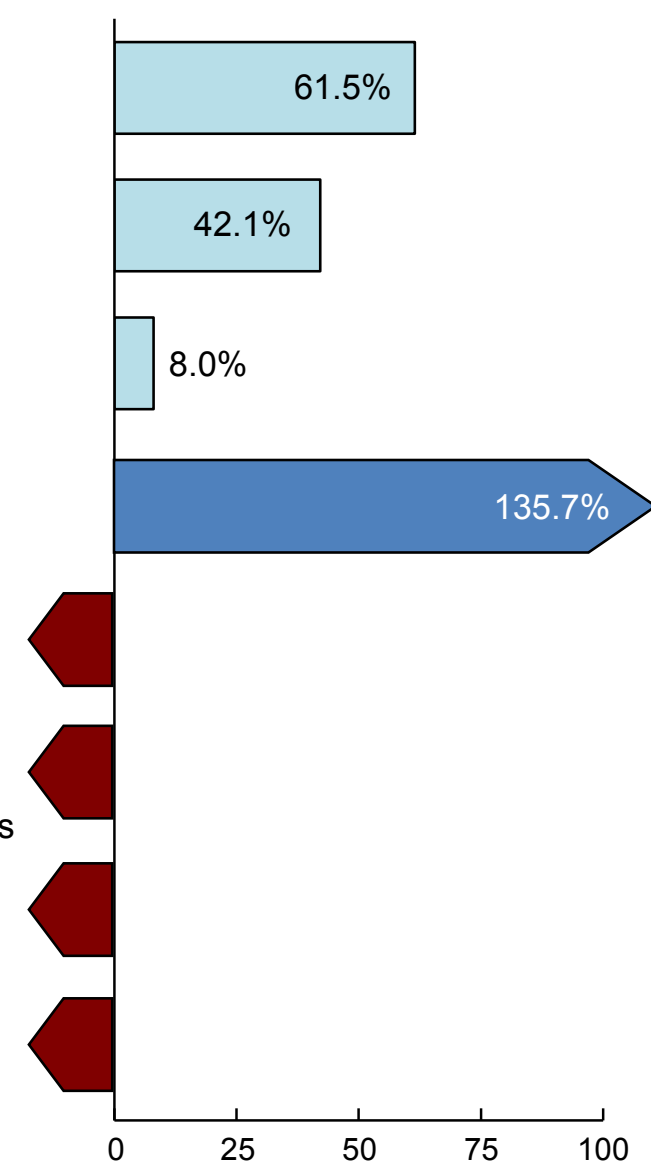

\begin{tabular}{|c|c|c|c|c|c|}
\hline \multirow{2}{*}{$\begin{array}{l}2010 \\
\text { Target }\end{array}$} & \multirow{2}{*}{$\begin{array}{c}\text { Baseline } \\
\text { (Year) }\end{array}$} & \multirow{2}{*}{$\begin{array}{l}\text { Final } \\
\text { (Year) }\end{array}$} & \multicolumn{3}{|c|}{ Baseline vs. Final } \\
\hline & & & Difference $^{2}$ & $\begin{array}{l}\text { Statistically } \\
\text { Significant }^{3}\end{array}$ & $\begin{array}{c}\text { Percent } \\
\text { Change }^{4}\end{array}$ \\
\hline 35 & $\begin{array}{c}9 \\
(2004)\end{array}$ & $\begin{array}{c}25 \\
(2009)\end{array}$ & 16 & $\begin{array}{c}\text { Not } \\
\text { tested }\end{array}$ & $177.8 \%$ \\
\hline $50 \%$ & $\begin{array}{c}12 \% \\
(2004)\end{array}$ & $\begin{array}{c}28 \% \\
(2009)\end{array}$ & 16 & $\begin{array}{c}\text { Not } \\
\text { tested }\end{array}$ & $133.3 \%$ \\
\hline $50 \%$ & $\begin{array}{c}0 \% \\
(2004)\end{array}$ & $\begin{array}{c}4 \% \\
(2009)\end{array}$ & 4 & $\begin{array}{c}\text { Not } \\
\text { tested }\end{array}$ & $N / A^{6}$ \\
\hline $50 \%$ & $\begin{array}{c}36 \% \\
(2004)\end{array}$ & $\begin{array}{c}55 \% \\
(2009)\end{array}$ & 19 & $\begin{array}{c}\text { Not } \\
\text { tested }\end{array}$ & $52.8 \%$ \\
\hline $80 \%$ & $\begin{array}{c}53 \% \\
(1999)\end{array}$ & $\begin{array}{c}49 \% \\
(2008)\end{array}$ & -4 & $\begin{array}{c}\text { Not } \\
\text { tested }\end{array}$ & $-7.5 \%$ \\
\hline $41 \%$ & $\begin{array}{c}37 \% \\
(2005)\end{array}$ & $\begin{array}{c}33 \% \\
(2008)\end{array}$ & -4 & $\begin{array}{c}\text { Not } \\
\text { tested }\end{array}$ & $-10.8 \%$ \\
\hline $100 \%$ & $\begin{array}{c}98 \% \\
(2006)\end{array}$ & $\begin{array}{c}88 \% \\
(2008)\end{array}$ & -10 & $\begin{array}{c}\text { Not } \\
\text { tested }\end{array}$ & $-10.2 \%$ \\
\hline $73 \%$ & $\begin{array}{c}59 \% \\
(2006)\end{array}$ & $\begin{array}{c}55 \% \\
(2008)\end{array}$ & -4 & $\begin{array}{c}\text { Not } \\
\text { tested }\end{array}$ & $-6.8 \%$ \\
\hline
\end{tabular}

Percent of targeted change achieved ${ }^{7}$

(continued) 
Figure 23-1. Progress Toward Target Attainment for Focus Area 23: Public Health Infrastructure (continued)

Moved away

from target ${ }^{1}$
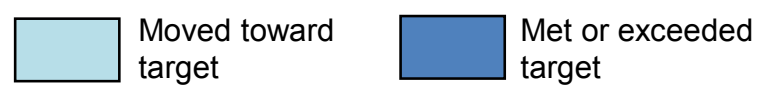

23-13. Public health laboratory services (States and

D.C.)

C. Reference and specialized testing

d. Environmental health and protection

e. Food safety

f. Laboratory improvement and regulation

g. Policy development

h. Emergency response

i. Public health related research

j. Training and education

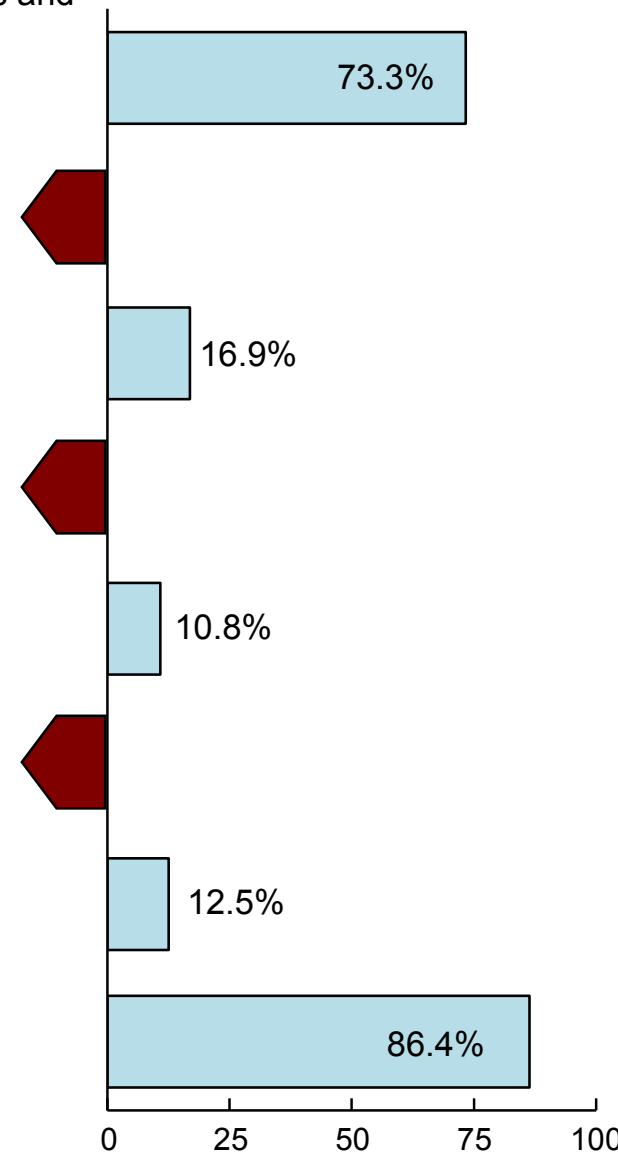

Percent of targeted change achieved ${ }^{7}$

\begin{tabular}{|c|c|c|c|c|c|}
\hline \multirow{2}{*}{$\begin{array}{c}2010 \\
\text { Target }\end{array}$} & \multirow{2}{*}{$\begin{array}{c}\text { Baseline } \\
\text { (Year) }\end{array}$} & \multirow{2}{*}{$\begin{array}{l}\text { Final } \\
\text { (Year) }\end{array}$} & \multicolumn{3}{|c|}{ Baseline vs. Final } \\
\hline & & & Difference $^{2}$ & $\begin{array}{l}\text { Statistically } \\
\text { Significant }^{3}\end{array}$ & $\begin{array}{c}\text { Percent } \\
\text { Change }^{4}\end{array}$ \\
\hline $82 \%$ & $\begin{array}{c}67 \% \\
(2006)\end{array}$ & $\begin{array}{c}78 \% \\
(2008)\end{array}$ & 11 & $\begin{array}{l}\text { Not } \\
\text { tested }\end{array}$ & $16.4 \%$ \\
\hline $100 \%$ & $\begin{array}{c}57 \% \\
(2006)\end{array}$ & $\begin{array}{c}55 \% \\
(2008)\end{array}$ & -2 & $\begin{array}{l}\text { Not } \\
\text { tested }\end{array}$ & $-3.5 \%$ \\
\hline $100 \%$ & $\begin{array}{c}17 \% \\
(2006)\end{array}$ & $\begin{array}{c}31 \% \\
(2008)\end{array}$ & 14 & $\begin{array}{l}\text { Not } \\
\text { tested }\end{array}$ & $82.4 \%$ \\
\hline $50 \%$ & $\begin{array}{c}46 \% \\
(2006)\end{array}$ & $\begin{array}{c}41 \% \\
(2008)\end{array}$ & -5 & $\begin{array}{l}\text { Not } \\
\text { tested }\end{array}$ & $-10.9 \%$ \\
\hline $100 \%$ & $\begin{array}{c}63 \% \\
(2006)\end{array}$ & $\begin{array}{c}67 \% \\
(2008)\end{array}$ & 4 & $\begin{array}{l}\text { Not } \\
\text { tested }\end{array}$ & $6.3 \%$ \\
\hline $100 \%$ & $\begin{array}{c}72 \% \\
(2006)\end{array}$ & $\begin{array}{c}61 \% \\
(2008)\end{array}$ & -11 & $\begin{array}{l}\text { Not } \\
\text { tested }\end{array}$ & $-15.3 \%$ \\
\hline $50 \%$ & $\begin{array}{c}26 \% \\
(2006)\end{array}$ & $\begin{array}{c}29 \% \\
(2008)\end{array}$ & 3 & $\begin{array}{l}\text { Not } \\
\text { tested }\end{array}$ & $11.5 \%$ \\
\hline $50 \%$ & $\begin{array}{c}28 \% \\
(2006)\end{array}$ & $\begin{array}{c}47 \% \\
(2008)\end{array}$ & 19 & $\begin{array}{l}\text { Not } \\
\text { tested }\end{array}$ & $65.9 \%$ \\
\hline
\end{tabular}

(continued) 
Figure 23-1. Progress Toward Target Attainment for Focus Area 23: Public Health Infrastructure (continued)
Moved away
from target ${ }^{1}$
Moved toward
Met or exceeded
target

23-13. Public health laboratory services (States and

D.C.)

k. Partnerships and communication

23-14. Provide or assure comprehensive epidemiology services

a. State epidemiologists with formal training

c. State agencies

d. Local agencies

23-15. Evaluation of public health laws

a. Turning Point Model State Public Health Act (no. States and D.C.)

b. Model State Emergency Powers Act (no. States and D.C.)

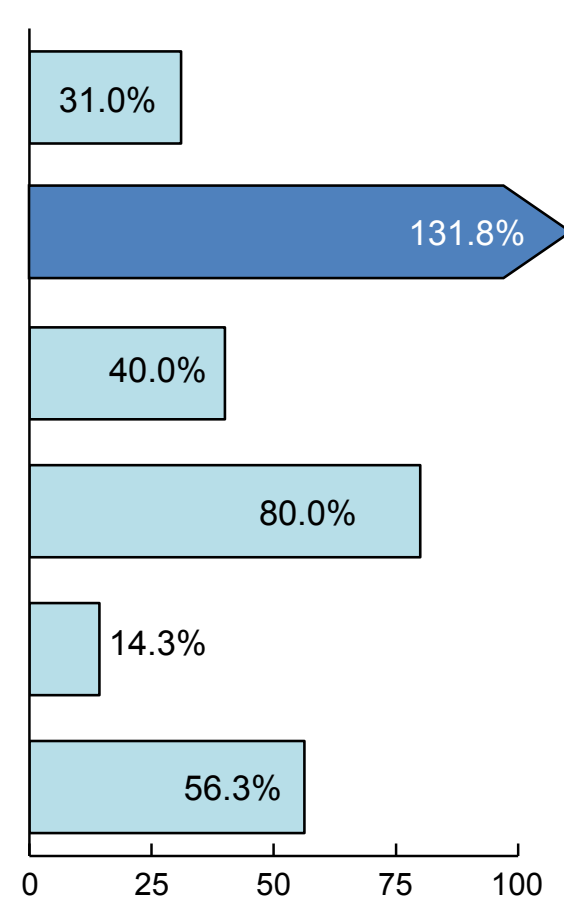

\begin{tabular}{|c|c|c|c|c|c|}
\hline \multirow{2}{*}{$\begin{array}{c}2010 \\
\text { Target }\end{array}$} & \multirow{2}{*}{$\begin{array}{c}\text { Baseline } \\
\text { (Year) }\end{array}$} & \multirow{2}{*}{$\begin{array}{l}\text { Final } \\
\text { (Year) }\end{array}$} & \multicolumn{3}{|c|}{ Baseline vs. Final } \\
\hline & & & Difference ${ }^{2}$ & $\begin{array}{l}\text { Statistically } \\
\text { Significant }^{3}\end{array}$ & $\begin{array}{l}\text { Percent } \\
\text { Change }^{4}\end{array}$ \\
\hline $81 \%$ & $\begin{array}{c}52 \% \\
(2006)\end{array}$ & $\begin{array}{c}61 \% \\
(2008)\end{array}$ & 9 & $\begin{array}{c}\text { Not } \\
\text { tested }\end{array}$ & $17.3 \%$ \\
\hline $80 \%$ & $\begin{array}{c}58 \% \\
(2001)\end{array}$ & $\begin{array}{c}87 \% \\
(2009)\end{array}$ & 29 & $\begin{array}{c}\text { Not } \\
\text { tested }\end{array}$ & $50.0 \%$ \\
\hline $15 \%$ & $\begin{array}{c}10 \% \\
(2004)\end{array}$ & $\begin{array}{c}12 \% \\
(2009)\end{array}$ & 2 & $\begin{array}{c}\text { Not } \\
\text { tested }\end{array}$ & $20.0 \%$ \\
\hline $57 \%$ & $\begin{array}{c}52 \% \\
(2005)\end{array}$ & $\begin{array}{c}56 \% \\
(2008)\end{array}$ & 4 & $\begin{array}{l}\text { Not } \\
\text { tested }\end{array}$ & $7.7 \%$ \\
\hline 51 & $\begin{array}{c}30 \\
(2003)\end{array}$ & $\begin{array}{c}33 \\
(2007)\end{array}$ & 3 & $\begin{array}{l}\text { Not } \\
\text { tested }\end{array}$ & $10.0 \%$ \\
\hline 51 & $\begin{array}{c}35 \\
(2003)\end{array}$ & $\begin{array}{c}44 \\
(2006)\end{array}$ & 9 & $\begin{array}{c}\text { Not } \\
\text { tested }\end{array}$ & $25.7 \%$ \\
\hline
\end{tabular}

Percent of targeted change achieved ${ }^{7}$

(continued) 


\section{Figure 23-1. Progress Toward Target Attainment for Focus Area 23: Public Health Infrastructure (continued)}

\section{NOTES}

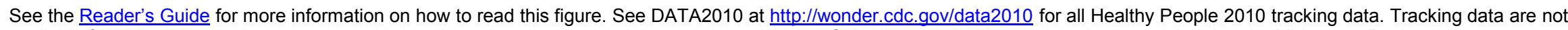
available for objectives 23-2b, 23-8a, 23-9, 23-10a through c, 23-12a, 23-12b, 23-14b, and 23-17. Objectives 23-1, 23-5, and 23-16 were deleted at the Midcourse Review.

\section{FOOTNOTES}

${ }^{1}$ Movement away from target is not quantified using the percent of targeted change achieved. See Technical Appendix for more information.

${ }^{2}$ Difference $=$ Final value - Baseline value. Differences between percents (\%) are measured in percentage points.

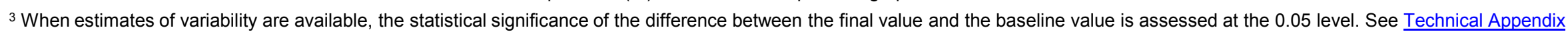
for more information.

${ }^{4}$ Percent change $=\frac{\text { Final value }- \text { Baseline value }}{\text { Baseline value }} \times 100$

${ }^{5}$ Data beyond the baseline are not available; difference, statistical significance, and percent change cannot be calculated. See Technical Appendix for more information.

${ }^{6}$ Percent change cannot be calculated. See Technical Appendix for more information.

${ }^{7}$ Percent of targeted change achieved $=\frac{\text { Final value }- \text { Baseline value }}{\text { Healthy People } 2010 \text { target }- \text { Baseline value }} \times 100$.

\section{DATA SOURCES}

23-2a. Assessment of Objective Data Availability (AODA), CDC, NCHS.

23-2c-d. Assessment of Objective Data Availability (AODA), CDC, NCHS.

23-3-23-4. Assessment of Objective Data Availability (AODA), CDC, NCHS.

23-6-23-7. Assessment of Objective Data Availability (AODA), CDC, NCHS.

23-8b. National Profile of Local Health Departments, National Association of County and City Health Officials (NACCHO).

23-11a-d. National Public Health Performance Standards Program, CDC, OCPHP.

23-12c-d. National Profile of Local Health Departments, National Association of County and City Health Officials (NACCHO).

23-13a-k. Comprehensive Laboratory Services Survey, Association of Public Health Laboratories (APHL).

23-14a. Epidemiology Capacity Assessment, Council of State and Territorial Epidemiologists (CSTE).

23-14c. Epidemiology Capacity Assessment, Council of State and Territorial Epidemiologists (CSTE).

23-14d. National Profile of Local Health Departments, National Association of County and City Health Officials (NACCHO).

23-15a-b. Center for Public Health Law and the Public's Health, Georgetown University Law Center and Johns Hopkins Bloomberg School of Public Health. 


\section{Final Review}

\section{Respiratory Diseases}
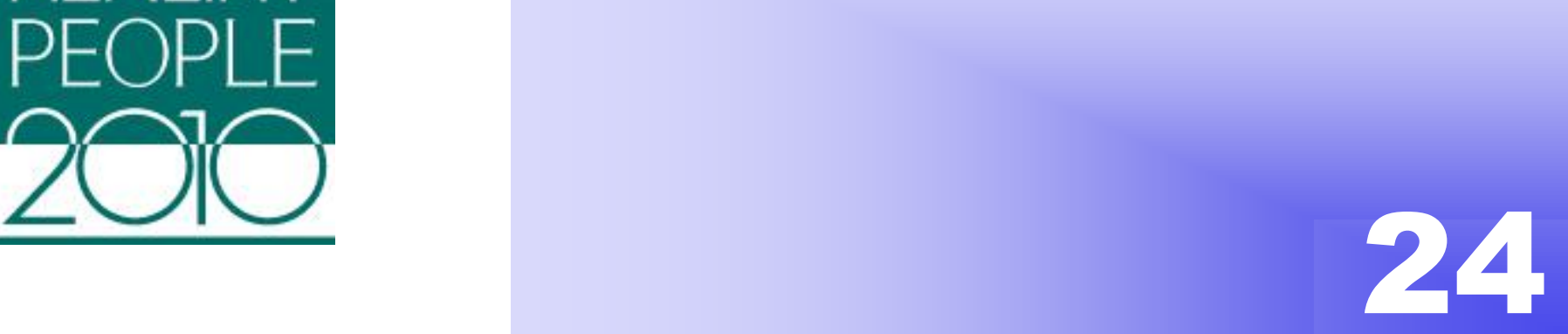

\section{Co-Lead Agencies}

Centers for Disease Control and Prevention

National Institutes of Health

\section{Contents}

Goal

Highlights

Summary of Progress.

Transition to Healthy People 2020.

Data Considerations

References and Notes

Comprehensive Summary of Objectives

Progress Chart

Health Disparities Table

Chronic Obstructive Pulmonary Disease Death Rates-Map. 


\section{Goal: Promote respiratory health through better prevention, detection, treatment, and education efforts}

The objectives in this chapter track deaths, hospitalizations, and lost school or work days due to asthma; appropriate asthma care; and State-based asthma surveillance systems. Chronic obstructive pulmonary disease (COPD) deaths and activity limitations due to chronic lung and breathing problems are also monitored, as are issues related to persons with sleep apnea.

All Healthy People tracking data quoted in this chapter, along with technical information and operational definitions for each objective, can be found in the Healthy People 2010 database, DATA2010, available from http://wonder.cdc.gov/data2010/.

More information about this Focus Area can be found in the following publications:

- Healthy People 2010: Understanding and Improving Health, available from http://www.healthypeople.gov/2010/Document/tableofcontents.htm\#under.

- Healthy People 2010 Midcourse Review, available from http://www.healthypeople.gov/2010/data/midcourse/html/default.htm\#FocusAreas.

\section{Highlights}

- Substantial progress was achieved in objectives for this Focus Area during the past decade [1]. Seventy-one percent of the Respiratory Diseases objectives with data to monitor progress moved toward or achieved their Healthy People 2010 targets (Figure 24-1). However, statistically significant health disparities were observed for some objectives by race and ethnicity, as well as by sex, education level, and income [2]. Disparities of $50 \%$ or more remained for severe outcomes such as asthma and COPD deaths (Figure 24-2), as discussed below.

- The asthma death rate declined among adolescents and adults, especially at older ages. Between 1999 and 2007, asthma deaths among persons aged 15-34 years (objective 24-1c) declined by $28.6 \%$, from 5.6 to 4.0 deaths per million population, moving toward the Healthy People 2010 target of 1.9 deaths per million; asthma deaths among persons aged 15-34 years (objective 24-1c) declined by $29.0 \%$, from 15.5 to 11.0 deaths per million population, moving toward the 2010 target of 8.0 deaths per million; and asthma deaths among persons aged 65 years and over (objective 24-1e) declined by $37.7 \%$, from 69.5 to 43.3 deaths per million population, exceeding the 2010 target of 47.0 deaths per million.

- Among adolescents and adults aged 15-34 years (objective 24-1c), the black nonHispanic population had an asthma death rate of 11.5 deaths per million in 2007, approximately four times the rate for the white non-Hispanic population (2.9 deaths per million in 2007) [2]. Among persons aged 35-64 years in 2007, the asthma death rate for the black non-Hispanic population (34.0 deaths per million) was more than four times that of the white non-Hispanic population (8.3 deaths per million). The asthma death rates among adults aged 65 years and over (objective 24-1e) for the Asian or Pacific Islander and the black non-Hispanic populations were 63.9 and 63.8 deaths per million in 2007, respectively, more than one and a half times the rate for the white non-Hispanic population (40.9 deaths per million in 2007). 
- Females aged 65 years and over (objective 24-1e) had asthma death rates of 84.2 deaths per million in 1999 and 55.0 in 2007, whereas males had rates of 48.4 in 1999 and 27.2 in 2007. In 2007, the asthma death rate for females was approximately twice the rate for males [2]. Between 1999 and 2007, the disparity between females and males increased by 28 percentage points [3].

- Adults aged 35-64 years (objective 24-1d) with at least some college education had the lowest (best) asthma death rate among education groups, 7.6 deaths per million in 2002, whereas high school graduates and persons with less than a high school education had rates of 20.5 and 25.7 deaths per million in 2002, respectively. The rate for high school graduates was more than two and a half times the best group rate, while the rate for persons with less than a high school education was almost three and a half times the best group rate [2].

- The asthma hospitalization rates for persons 65 years and over (objective 24-2c) increased by $42.9 \%$ between 1998 and 2007, from 17.7 to 25.3 hospitalizations per 10,000 population (age adjusted), moving away from the 2010 target of 11.0 hospitalizations per 10,000.

- The proportion of persons with asthma who received assistance in reducing exposure to environmental risk factors (objective 24-7f) increased by $18.6 \%$ between 2002 and 2008, from $43 \%$ to $51 \%$ (age adjusted), exceeding the 2010 target of $50 \%$.

- The number of states (including D.C.) with state-based asthma surveillance systems (objective 24-8) increased from 19 states in 2003 to 36 states in 2009, exceeding the 2010 target of 25 states.

- The rates of activity limitations due to chronic lung and breathing problems (objective 249) for poor and near-poor persons aged 45 years and over (5.1\% in 2008, age adjusted) were more than three times the rate for middle/high income persons $(1.6 \%$ in 2008 , age adjusted) [2]. Between 1997 and 2008, the disparity between near-poor persons (3.8\% in 1997, age adjusted; $5.1 \%$ in 2008) and persons with middle/high incomes (1.8\% in 1997 , age adjusted; $1.6 \%$ in 2008) increased by 108 percentage points [3].

- Deaths from COPD among persons aged 45 years and over (objective 24-10) declined by $9.3 \%$ between 1999 and 2007, from 123.9 to 112.4 deaths per 100,000 population (age adjusted), moving toward the 2010 target of 62.3 deaths per 100,000 .

- Among racial and ethnic groups, the Asian or Pacific Islander population had the lowest (best) death rate for COPD: 47.6 deaths per 100,000 (age adjusted) in 1999 and 33.9 in 2007. The American Indian or Alaska Native population had COPD death rates of 91.8 per 100,000 (age adjusted) in 1999 and 83.8 in 2007; the black nonHispanic population had rates of 83.4 per 100,000 (age adjusted) in 1999 and 73.8 in 2007; and the white non-Hispanic population had rates of 133.1 per 100,000 (age adjusted) in 1999 and 124.8 in 2007.

- In 2007, the rate for the American Indian or Alaska Native population was about two and a half times the best group rate (that for the Asian or Pacific Islander population); the black non-Hispanic population's rate was more than twice the best group rate; and the rate for the white non-Hispanic population was more than three and a half times the best group rate [2]. 
- Between 1999 and 2007, the disparity in COPD death rates between the American Indian or Alaska Native population and the Asian or Pacific Islander population (group with the best rate) increased by 54 percentage points [3]. During the same period, the disparity between the white nonHispanic population and the Asian or Pacific Islander population increased by 89 percentage points.

- Persons aged 45-64 years with at least some college education had the lowest (best) COPD mortality rate (6.9 deaths per 100,000 in 2002, age adjusted) among education groups. The rate for high school graduates, 28.4 deaths per 100,000 (age adjusted) in 2002, was more than four times the best group rate; while the rate for persons with less than a high school education, 49.7 deaths per 100,000 (age adjusted) in 2002, was more than seven times the best group rate [2].

- COPD death rates vary by geographic region. In 2005-07, the highest rates were observed in the Ohio River Valley, the Great Plains, and Northern California (Figure 24-3). A few areas met the 2010 target.

\section{Summary of Progress}

- Figure 24-1 presents a quantitative assessment of progress in achieving the Healthy People 2010 objectives for Respiratory Diseases [1]. Data to measure progress toward target attainment were available for 24 objectives. Of these:

- Three objectives (24-1e, 24-7f, and 24-8) met or exceeded their Healthy People 2010 targets.

- Fourteen objectives moved toward their targets. A statistically significant difference between the baseline and the final data points was observed for four of these objectives (24-1c and d, 24-3b, and 24-10). No significant differences were observed for nine objectives (24-1b, 24-2a and b, 24-3a and c, 24-4, 24-5, 24-7d, and 24-9); and data to test the significance of the difference were unavailable for one objective (24-12).

- Two objectives (24-7a and c) showed no change.

- Five objectives moved away from their targets. A statistically significant difference between the baseline and final data point was observed for one objective (24-2c). No significant difference was observed for the remaining four objectives (24-1a, 246, 24-7b, and 24-7e).

- One objective (24-11b) remained developmental [4].

- Follow-up data were unavailable to measure progress for one objective (24-11a). 
- Figure 24-2 displays health disparities in Respiratory Diseases from the best group rate for each characteristic at the most recent data point [2]. It also displays changes in disparities from baseline to the most recent data point [3].

- Of the 11 objectives with statistically significant health disparities of $10 \%$ or more by race and ethnicity, the white non-Hispanic population had the best rate for six objectives (24-1c through e, 24-4, 24-7d, and 24-11a) and the white population (including persons of Hispanic origin) had the best rate for one objective (24-3b). The black non-Hispanic population had the best rate for three objectives (24-6, and 24-7a and c), and the Asian or Pacific Islander population had the best rate for one objective (24-10).

- Males had better rates than females for four of the eight objectives with statistically significant health disparities of $10 \%$ or more by sex (objectives $24-1 \mathrm{~d}$ and e, $24-3 \mathrm{~b}$, and 24-9); females had better rates than males for the other four objectives (24-1c, 24-7f, 24-10, and 24-11a).

- Persons with at least some college education had the best rate for both of the objectives with statistically significant health disparities of $10 \%$ or more by education level (objectives 24-1d and 24-10).

- Persons with middle/high incomes had the best rate for the three objectives with statistically significant health disparities of $10 \%$ or more by income (objectives 24 7c and d, and 24-9).

- Several objectives had health disparities of $100 \%$ or more. Many of these are discussed in the Highlights, above.

\section{Transition to Healthy People 2020}

The Healthy People 2010 Respiratory Diseases Focus Area was divided into two Healthy People 2020 Topic Areas: Respiratory Diseases and Sleep Health. Sleep's contribution to public health is best communicated as a separate topic area because sleep is a fundamental biological requirement for health that cross-cuts many topic areas and there are many non-respiratory causes of disordered sleep. For Healthy People 2020, the Respiratory Diseases objectives have been expanded to include additional National Asthma Education and Prevention Program (NAEPP) guidelines for asthma care and indicators of the burden of COPD. The Respiratory Diseases objectives primarily assess the burden of asthma and COPD and related measures on prevention, detection, treatment, and education efforts. Sleep Health has added objectives tracking sufficient sleep. The Sleep Health objectives are focused on adequate sleep and treatment of sleep disorders as well as the impact of fatigue on motor vehicle crashes. See HealthyPeople.gov for a complete list of Healthy People 2020 topics and objectives.

The Healthy People 2020 Respiratory Diseases Topic Area can be grouped into two sections:

- Asthma

- Chronic Obstructive Pulmonary Disease. 
The differences between the Healthy People 2010 objectives and those included in Healthy People 2020 are summarized below:

- The Healthy People 2020 Respiratory Diseases Topic Area has a total of 27 objectives, four of which are developmental [4]. The Sleep Topic Area has a total of four objectives. The Healthy People 2010 Respiratory Diseases Focus Area had 26 objectives, one of which was developmental.

- Sixteen Healthy People 2010 objectives were retained "as is" [5]. These include: asthma deaths, separately assessed for persons aged 35-64 years (objective 24-1d), and persons aged 65 years and over (objective 24-1e); hospitalizations for asthma, separately assessed for persons under age 5 years (objective 24-2a), persons aged 5-64 years (objective 24-2b), and persons aged 65 years and over (objective 24-2c); emergency department visits for asthma, separately assessed for persons under age 5 years (objective 24-3a), persons aged 5-64 years (objective 24-3b), and persons aged 65 years and over (objective 24-3c); activity limitations among persons with asthma (objective 24-4); patient education among persons with asthma (objective 24-6); five objectives tracking NAEPP guidelines for asthma care, namely receipt of written asthma plans from health care provider (objective 24-7a), proper use instructions with inhalers (objective 24-7b), education on early signs, symptoms and response to asthma episodes (objective 24-7c), appropriate medication regimens for asthma care (objective 24-7d), and assistance in reducing exposure to environmental risks for asthma (objective 24-7f); and COPD deaths (objective 24-10).

- Two Healthy People 2010 Respiratory Diseases objectives were archived or dropped [6,7]. Long-term management care after hospitalization for asthma (objective 24-7e) was archived due to a lack of reliable data. The developmental objective (objective 24-11b) on long-term medical management for persons with symptoms of obstructive sleep apnea was dropped due to the lack of a national data source.

- Six Healthy People 2010 Respiratory Diseases objectives were modified to create five Healthy People 2020 Respiratory Disease objectives [8].

- Three asthma deaths objectives among persons under age 5 years (objective 24-1a), 5-14 years (objective 24-1b), and 15-34 years (objective 24-1c) were combined into one objective for persons 35 years and under.

- The objective tracking the average number of school or work days lost due to asthma (objective 24-5) was divided into two separate objectives for children and adults and modified to assess the percentage of persons that miss school days or work days due to asthma, respectively.

- The objective tracking states with asthma surveillance systems (objective 24-8) was expanded to also count territories and the definition was modified to include recipients of either of two funding sources that require asthma surveillance in addition to states and territories participating in a detailed asthma survey.

- The objective tracking activity limitations due to chronic lung and breathing problems (objective 24-9) was modified to target adults with COPD instead of adults with activity limitations. 
- Six new objectives were added to the Healthy People 2020 Respiratory Diseases Topic Area:

- Three new developmental NAEPP asthma care objectives: routine annual follow-up visits for asthma, annual medical assessment of asthma control, and consultation on any work related causes of asthma.

- Two new health care utilization objectives for COPD: hospitalizations and emergency department visits.

- A new developmental objective tracking the diagnosis of underlying COPD.

- Two objectives were moved to the new Sleep Health Topic Area including persons with symptoms of obstructive sleep apnea who seek medical evaluation (objective 24-11a) and motor vehicle crashes due to drowsy driving (objective 24-12). The motor vehicle crash objective was modified to include all crashes, not just those involving driver fatalities.

- Two new objectives were added to the Healthy People 2020 Sleep Health Topic Area:

- Sufficient sleep among students in grades 9-12

- Sufficient sleep among adults.

Appendix D "A Crosswalk Between Objectives From Healthy People 2010 to Healthy People 2020," summarizes the changes between the two decades of objectives, reflecting new knowledge and direction for this area.

\section{Data Considerations}

Education and income are the primary measures of socioeconomic status (SES) in Healthy People 2010. Most data systems used in Healthy People 2010 define income as a family's income before taxes. In order to facilitate comparisons among groups and over time, while adjusting for family size and for inflation, Healthy People 2010 categorizes income using the poverty thresholds developed by the U.S. Census Bureau. Thus, the three categories of family income that are primarily used are:

- Poor-below the Federal poverty level

- Near poor-100\% to $199 \%$ of the Federal poverty level

- Middle/high income-200\% or more of the Federal poverty level.

These categories may be overridden by considerations specific to the data system, in which case they are modified as appropriate. See Healthy People 2010: General Data Issues, referenced below.

Beginning in 2003, education data for asthma and COPD deaths (objectives 24-1c and d and 24-10) from the National Vital Statistics System have been suppressed. The educational attainment item was changed in the new U.S. Standard Certificate of Death in 2003 to be consistent with the U.S. Census Bureau data and to improve the ability to identify specific types of educational degrees. Many states, however, are still using the 1989 version of the U.S. Standard Certificate of Death, which focuses on highest school grade completed. As a result, educational attainment data collected using the 2003 version are not comparable with data collected using the 1989 version [9]. 
In general, data on educational attainment are presented for persons aged 25 years and over, consistent with guidance given by the U.S. Bureau of the Census. However, because of the requirements of the different data systems, the age groups used to calculate educational attainment for any specific objective may differ from the age groups used to report the data for other Healthy People 2010 objectives, as well as from select populations within the same objective. Therefore, the reader is urged to exercise caution in interpreting the data by educational attainment shown in the Health Disparities Table. See Healthy People 2010: General Data Issues, referenced below.

Additional information on data issues is available from the following sources:

- All Healthy People 2010 tracking data can be found in the Healthy People 2010 database, DATA2010, available from http://wonder.cdc.gov/data2010/.

- Detailed information about the data and data sources used to support these objectives can be found in the Operational Definitions on the DATA2010 website, available from http://wonder.cdc.gov/data2010/focusod.htm.

- More information on statistical issues related to Healthy People tracking and measurement can be found in the Technical Appendix and in Healthy People 2010: General Data Issues, which is available in the Data Issues section of the NCHS Healthy People website under Healthy People 2010.

\section{References and Notes}

1. Displayed in the Progress Chart (Figure 24-1), the percent of targeted change achieved expresses the difference between the baseline and the final value relative to the initial difference between the baseline and the Healthy People 2010 target. As such, it is a relative measure of progress toward attaining the Healthy People 2010 target. See the Reader's Guide for more information. When standard errors were available, the difference between the baseline and the final value was tested at the 0.05 level of significance. See the Figure 24-1 footnotes, as well as the Technical Appendix, for more detail.

2. Information about disparities among select populations is shown in the Health Disparities Table (Figure 24-2). Disparity from the best group rate is defined as the percent difference between the best group rate and each of the other group rates for a characteristic. For example, racial and ethnic health disparities are measured as the percent difference between the best racial and ethnic group rate and each of the other racial and ethnic group rates. Similarly, disparities by sex are measured as the percent difference between the better group rate (e.g., female) and the rate for the other group (e.g., male). Some objectives are expressed in terms of favorable events or conditions that are to be increased, while others are expressed in terms of adverse events or conditions that are to be reduced. In order to facilitate comparison of health disparities across different objectives, disparity is measured only in terms of adverse events or conditions. For comparability across objectives, objectives that are expressed in terms of favorable events or conditions are re-expressed using the adverse event or condition for the purpose of computing disparity, but they are not otherwise restated or changed. For example, objective 1-1, to increase the proportion of persons with health insurance (e.g., $72 \%$ of the American Indian or Alaska Native population aged under 65 years had some form of health insurance in 2008), is expressed in terms of the percentage of persons without health insurance (e.g., $100 \%-72 \%=28 \%$ of the American Indian or Alaska Native population aged under 65 years did not have any form of health insurance in 2008) when the disparity from the best group rate is calculated. See the Reader's Guide for more information. When standard errors were available, the difference between the best group rate and each of the other group rates was tested at the 0.05 level of significance. See the Figure 24-2 footnotes, as well as the Technical Appendix, for more detail. 
3. The change in disparity is estimated by subtracting the disparity at baseline from the disparity at the most recent data point and, therefore, is expressed as a change in percentage points. See the Reader's Guide for more information. When standard errors were available, the change in disparity was tested at the 0.05 level of significance. See the Figure 24-2 footnotes, as well as the Technical Appendix, for more detail.

4. To be included in Healthy People 2010, an objective must have a national data source that provides a baseline and at least one additional data point for tracking progress. Some objectives lacked baseline data at the time of their development but had a potential data source and were considered of sufficient national importance to be included in Healthy People. These are called "developmental" objectives. When data become available, a developmental objective is moved to measurable status and a Healthy People target can be set.

5. Retained "as is" objectives have no change in the numerator definition or in the denominator definition between the Healthy People 2010 and Healthy People 2020objectives. These include objectives that were developmental in Healthy People 2010 and are developmental in Healthy People 2020 and for which no numerator or denominator information was available.

6. Archived objectives had at least one data point in Healthy People 2010 but were not carried forward into Healthy People 2020.

7. Dropped objectives were not carried forward into Healthy People 2020. These objectives were either developmental or deleted at the Healthy People 2010 Midcourse Review or at another time in Healthy People 2010.

8. Modified objectives have some change in the numerator definition or in the denominator definition between the Healthy People 2010 and Healthy People 2020 objectives. These include objectives that went from developmental in Healthy People 2010 to measurable in Healthy People 2020 or vice versa.

9. Xu JQ, Kochanek KD, Murphy SL, Tejada-Vera B. Deaths: Final data for 2007. National vital statistics reports; vol 58 no 19. Hyattsville, MD: National Center for Health Statistics. 2010. Available from: http://www.cdc.gov/nchs/data/nvsr/nvsr58/nvsr58 19.pdf. 


\section{Comprehensive Summary of Objectives: Respiratory Diseases}

\begin{tabular}{|c|c|c|}
\hline Objective & Description & Data Source or Objective Status \\
\hline $24-1 \mathrm{a}$ & Deaths from asthma-Children $<5$ years (per million population) & $\begin{array}{l}\text { National Vital Statistics System-Mortality (NVSS-M), CDC, } \\
\text { NCHS. }\end{array}$ \\
\hline $24-1 b$ & $\begin{array}{l}\text { Deaths from asthma-Children and adolescents 5-14 years (per } \\
\text { million population) }\end{array}$ & $\begin{array}{l}\text { National Vital Statistics System-Mortality (NVSS-M), CDC, } \\
\text { NCHS. }\end{array}$ \\
\hline $24-1 c$ & $\begin{array}{l}\text { Deaths from asthma-Adolescents and adults } 15-34 \text { years (per } \\
\text { million population) }\end{array}$ & $\begin{array}{l}\text { National Vital Statistics System-Mortality (NVSS-M), CDC, } \\
\text { NCHS. }\end{array}$ \\
\hline $24-1 d$ & $\begin{array}{l}\text { Deaths from asthma-Adults 35-64 years (per million } \\
\text { population) }\end{array}$ & $\begin{array}{l}\text { National Vital Statistics System-Mortality (NVSS-M), CDC, } \\
\text { NCHS. }\end{array}$ \\
\hline $24-1 e$ & $\begin{array}{l}\text { Deaths from asthma-Older adults } 65+\text { years (per million } \\
\text { population) }\end{array}$ & $\begin{array}{l}\text { National Vital Statistics System-Mortality (NVSS-M), CDC, } \\
\text { NCHS. }\end{array}$ \\
\hline $24-2 a$ & $\begin{array}{l}\text { Hospitalizations for asthma-Children }<5 \text { years (per 10,000 } \\
\text { population) }\end{array}$ & National Hospital Discharge Survey (NHDS), CDC, NCHS. \\
\hline $24-2 b$ & $\begin{array}{l}\text { Hospitalizations for asthma-Children and adults 5-64 years } \\
\text { (age adjusted per 10,000 standard population) }\end{array}$ & National Hospital Discharge Survey (NHDS), CDC, NCHS. \\
\hline $24-2 c$ & $\begin{array}{l}\text { Hospitalizations for asthma-Older adults } 65+\text { years (age } \\
\text { adjusted per 10,000 standard population) }\end{array}$ & National Hospital Discharge Survey (NHDS), CDC, NCHS. \\
\hline $24-3 a$ & $\begin{array}{l}\text { Emergency department visits for asthma-Children }<5 \text { years } \\
\text { (per 10,000 population) }\end{array}$ & $\begin{array}{l}\text { National Hospital Ambulatory Medical Care Survey (NHAMCS), } \\
\text { CDC, NCHS. }\end{array}$ \\
\hline $24-3 b$ & $\begin{array}{l}\text { Emergency department visits for asthma-Children and adults } \\
5-64 \text { years (per } 10,000 \text { population) }\end{array}$ & $\begin{array}{l}\text { National Hospital Ambulatory Medical Care Survey (NHAMCS), } \\
\text { CDC, NCHS. }\end{array}$ \\
\hline $24-3 c$ & $\begin{array}{l}\text { Emergency department visits for asthma-Older adults } 65+ \\
\text { years (per 10,000 population) }\end{array}$ & $\begin{array}{l}\text { National Hospital Ambulatory Medical Care Survey (NHAMCS), } \\
\text { CDC, NCHS. }\end{array}$ \\
\hline $24-4$ & Activity limitations among persons with asthma (age adjusted) & National Health Interview Survey (NHIS), CDC, NCHS. \\
\hline $24-5$ & $\begin{array}{l}\text { School or work days missed by persons with asthma, due to } \\
\text { asthma (5-64 years) }\end{array}$ & National Health Interview Survey (NHIS), CDC, NCHS. \\
\hline $24-6$ & Patient education among persons with asthma (age adjusted) & National Health Interview Survey (NHIS), CDC, NCHS. \\
\hline $24-7 a$ & $\begin{array}{l}\text { Persons with asthma receiving written asthma plans from health } \\
\text { care provider (age adjusted) }\end{array}$ & National Health Interview Survey (NHIS), CDC, NCHS. \\
\hline
\end{tabular}




\begin{tabular}{|c|c|}
\hline Objective & Description \\
\hline $24-7 b$ & $\begin{array}{l}\text { Persons with asthma receiving proper-use instructions with } \\
\text { prescribed inhalers (age adjusted) }\end{array}$ \\
\hline 24-7c & $\begin{array}{l}\text { Persons with asthma receiving education on early signs, } \\
\text { symptoms and responses to asthma episodes (age adjusted) }\end{array}$ \\
\hline $24-7 d$ & $\begin{array}{l}\text { Persons with asthma receiving medication regimens that } \\
\text { prevent need for }>1 \text { beta agonist inhalation canister per month } \\
\text { (age adjusted) }\end{array}$ \\
\hline $24-7 e$ & $\begin{array}{l}\text { Persons with asthma receiving long-term management care after } \\
\text { hospitalization due to asthma (age adjusted) }\end{array}$ \\
\hline $24-7 f$ & $\begin{array}{l}\text { Persons with asthma receiving assistance in reducing exposure } \\
\text { to environmental risk factors (age adjusted) }\end{array}$ \\
\hline $24-8$ & State-based asthma surveillance systems (no. States) \\
\hline $24-9$ & $\begin{array}{l}\text { Activity limitations due to chronic lung and breathing problems } \\
\text { (age adjusted, } 45+\text { years) }\end{array}$ \\
\hline $24-10$ & $\begin{array}{l}\text { Deaths from chronic obstructive pulmonary disease (COPD, } \\
\text { excluding asthma) (age adjusted per 100,000 standard } \\
\text { population, } 45+\text { years) }\end{array}$ \\
\hline 24-11a & $\begin{array}{l}\text { Medical evaluation for persons with symptoms of obstructive } \\
\text { sleep apnea (age adjusted, aged } 20 \text { years and over) }\end{array}$ \\
\hline $24-11 b$ & $\begin{array}{l}\text { Long-term medical management among persons with symptoms } \\
\text { of obstructive sleep apnea }\end{array}$ \\
\hline $24-12$ & $\begin{array}{l}\text { Drivers involved in fatal motor vehicle crashes due to excessive } \\
\text { sleepiness }\end{array}$ \\
\hline
\end{tabular}

Data Source or Objective Status

National Health Interview Survey (NHIS), CDC, NCHS.

National Health Interview Survey (NHIS), CDC, NCHS.

National Health Interview Survey (NHIS), CDC, NCHS.

National Health Interview Survey (NHIS), CDC, NCHS.

Behavioral Risk Factor Surveillance System (BRFSS), CDC, NCCDPHP.

National Health Interview Survey (NHIS), CDC, NCHS.

National Vital Statistics System-Mortality (NVSS-M), CDC, NCHS.

National Health and Nutrition Examination Survey (NHANES), CDC, NCHS.

Developmental

Fatality Analysis Reporting System (FARS), Department of Transportation (DOT). 
Figure 24-1. Progress Toward Target Attainment for Focus Area 24: Respiratory Diseases

Moved away

from target ${ }^{1}$

Moved toward

target

Met or exceeded

target

24-1. Deaths from asthma (per million population)
a. Children $<5$ years

b. Children and adolescents 5-14 years

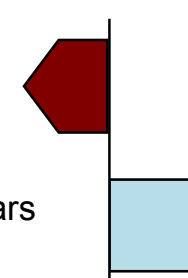

c. Adolescents and adults 15-34 years

d. Adults 35-64 years

e. Older adults $65+$ years

24-2. Hospitalizations for asthma

a. Children $<5$ years (per 10,000 population)

b. Children and adults 5-64 years (age adjusted, per 10,000 population)

c. Older adults $65+$ years (age adjusted, per 10,000 population)

\begin{tabular}{|c|c|c|c|c|c|}
\hline \multirow{2}{*}{$\begin{array}{c}2010 \\
\text { Target }\end{array}$} & \multirow{2}{*}{$\begin{array}{c}\text { Baseline } \\
\text { (Year) }\end{array}$} & \multirow{2}{*}{$\begin{array}{l}\text { Final } \\
\text { (Year) }\end{array}$} & \multicolumn{3}{|c|}{ Baseline vs. Final } \\
\hline & & & Difference $^{2}$ & $\begin{array}{l}\text { Statistically } \\
\text { Significant }^{3}\end{array}$ & $\begin{array}{l}\text { Percent } \\
\text { Change }^{4}\end{array}$ \\
\hline 0.9 & $\begin{array}{c}1.7 \\
(1999)\end{array}$ & $\begin{array}{c}2.2 \\
(2007)\end{array}$ & 0.5 & No & $29.4 \%$ \\
\hline 0.9 & $\begin{array}{c}3.1 \\
(1999)\end{array}$ & $\begin{array}{c}2.7 \\
(2007)\end{array}$ & -0.4 & No & $-12.9 \%$ \\
\hline 1.9 & $\begin{array}{c}5.6 \\
(1999)\end{array}$ & $\begin{array}{c}4.0 \\
(2007)\end{array}$ & -1.6 & Yes & $-28.6 \%$ \\
\hline 8.0 & $\begin{array}{c}15.5 \\
(1999)\end{array}$ & $\begin{array}{c}11.0 \\
(2007)\end{array}$ & -4.5 & Yes & $-29.0 \%$ \\
\hline 47.0 & $\begin{array}{c}69.5 \\
(1999)\end{array}$ & $\begin{array}{c}43.3 \\
(2007)\end{array}$ & -26.2 & Yes & $-37.7 \%$ \\
\hline 25.0 & $\begin{array}{c}45.6 \\
(1998)\end{array}$ & $\begin{array}{c}41.4 \\
(2007)\end{array}$ & -4.2 & No & $-9.2 \%$ \\
\hline 7.7 & $\begin{array}{c}12.5 \\
(1998)\end{array}$ & $\begin{array}{c}11.1 \\
(2007)\end{array}$ & -1.4 & No & $-11.2 \%$ \\
\hline 11.0 & $\begin{array}{c}17.7 \\
(1998)\end{array}$ & $\begin{array}{c}25.3 \\
(2007)\end{array}$ & 7.6 & Yes & $42.9 \%$ \\
\hline
\end{tabular}

$18.2 \%$

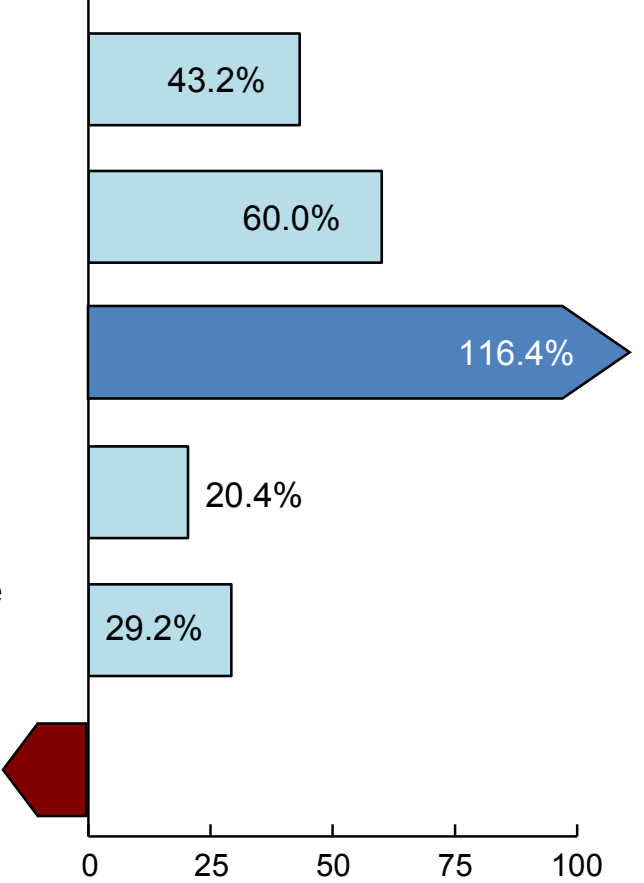

Percent of targeted change achieved ${ }^{5}$

(continued) 
Figure 24-1. Progress Toward Target Attainment for Focus Area 24: Respiratory Diseases (continued)

Moved away

from target ${ }^{1}$

Moved toward

target

Met or exceeded

target

24-3. Emergency department visits for asthma

a. Children $<5$ years (per 10,000 population)

b. Children and adults 5-64 years (per 10,000 population)

c. Older adults $65+$ years (per 10,000 population)

24-4. Activity limitations among persons with asthma (age adjusted)

24-5. School or work days missed by persons with asthma, due to asthma (5-64 years)

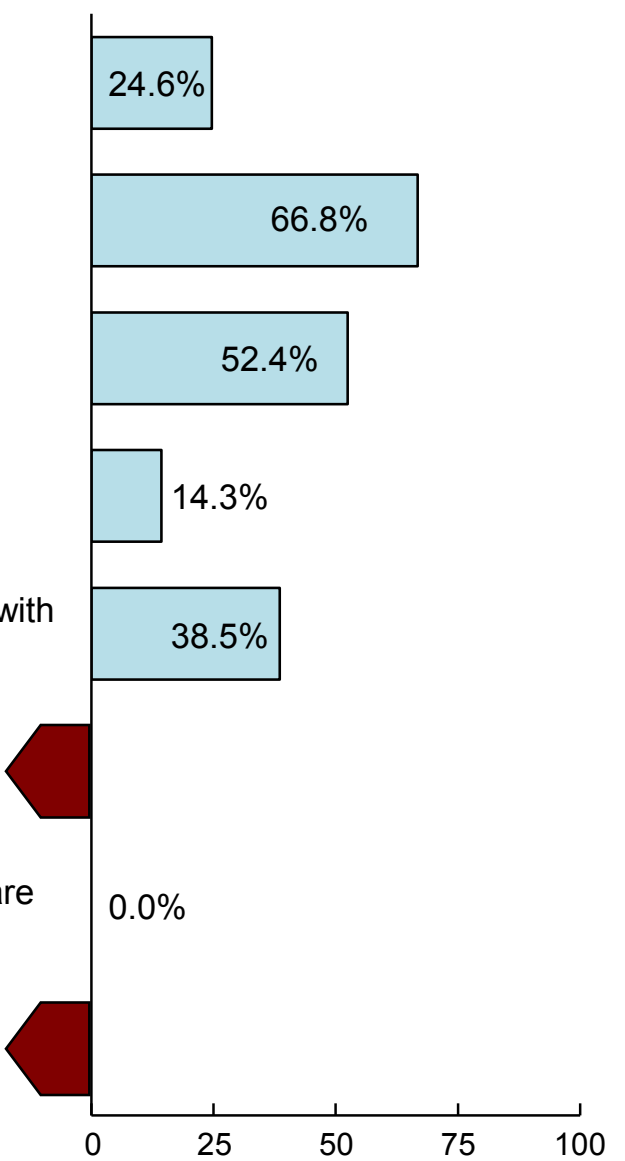

\begin{tabular}{|c|c|c|c|c|c|}
\hline \multirow{2}{*}{$\begin{array}{l}2010 \\
\text { Target }\end{array}$} & \multirow{2}{*}{$\begin{array}{c}\text { Baseline } \\
\text { (Year) }\end{array}$} & \multirow{2}{*}{$\begin{array}{l}\text { Final } \\
\text { (Year) }\end{array}$} & \multicolumn{3}{|c|}{ Baseline vs. Final } \\
\hline & & & Difference ${ }^{2}$ & $\begin{array}{l}\text { Statistically } \\
\text { Significant }^{3}\end{array}$ & $\begin{array}{l}\text { Percent } \\
\text { Change }^{4}\end{array}$ \\
\hline 80.0 & $\begin{array}{c}150.0 \\
(1995-97)\end{array}$ & $\begin{array}{c}132.8 \\
(2005-07)\end{array}$ & -17.2 & No & $-11.5 \%$ \\
\hline 50.0 & $\begin{array}{c}71.1 \\
(1995-97)\end{array}$ & $\begin{array}{c}57.0 \\
(2005-07)\end{array}$ & -14.1 & Yes & $-19.8 \%$ \\
\hline 15.0 & $\begin{array}{c}29.5 \\
(1995-97)\end{array}$ & $\begin{array}{c}21.9 \\
(2005-07)\end{array}$ & -7.6 & No & $-25.8 \%$ \\
\hline $7 \%$ & $\begin{array}{c}14 \% \\
(2001)\end{array}$ & $\begin{array}{c}13 \% \\
(2008)\end{array}$ & -1 & No & $-7.1 \%$ \\
\hline 1.9 & $\begin{array}{c}5.8 \\
(2002)\end{array}$ & $\begin{array}{c}4.3 \\
(2008)\end{array}$ & -1.5 & No & $-25.9 \%$ \\
\hline $38 \%$ & $\begin{array}{c}13 \% \\
(2003)\end{array}$ & $\begin{array}{c}12 \% \\
(2008)\end{array}$ & -1 & No & $-7.7 \%$ \\
\hline $40 \%$ & $\begin{array}{c}33 \% \\
(2002)\end{array}$ & $\begin{array}{c}33 \% \\
(2008)\end{array}$ & 0 & No & $0.0 \%$ \\
\hline $98.8 \%$ & $\begin{array}{l}96.0 \% \\
(2003)\end{array}$ & $\begin{array}{l}95.9 \% \\
(2008)\end{array}$ & -0.1 & No & $-0.1 \%$ \\
\hline
\end{tabular}

Percent of targeted change achieved ${ }^{5}$ (continued) 
Figure 24-1. Progress Toward Target Attainment for Focus Area 24: Respiratory Diseases (continued)

Moved away

from target ${ }^{1}$
Moved toward

target
Met or exceeded

target

24-7. Persons with asthma receiving

c. Education on early signs, symptoms and responses to asthma episodes (age adjusted)

d. Medication regimens that prevent need for $>1$ beta agonist inhalation canister per month (age adjusted)

e. Long-term management care after hospitalization due to asthma (age adjusted)

f. Assistance in reducing exposure to environmental risk factors (age adjusted)

24-8. State-based asthma surveillance systems (no. States)

24-9. Activity limitations due to chronic lung and breathing problems (age adjusted, 45+ years)

24-10. Deaths from chronic obstructive pulmonary disease (COPD, excluding asthma) (age adjusted, per 100,000 population, $45+$ years)

24-12. Drivers involved in fatal motor vehicle crashes due to excessive sleepiness

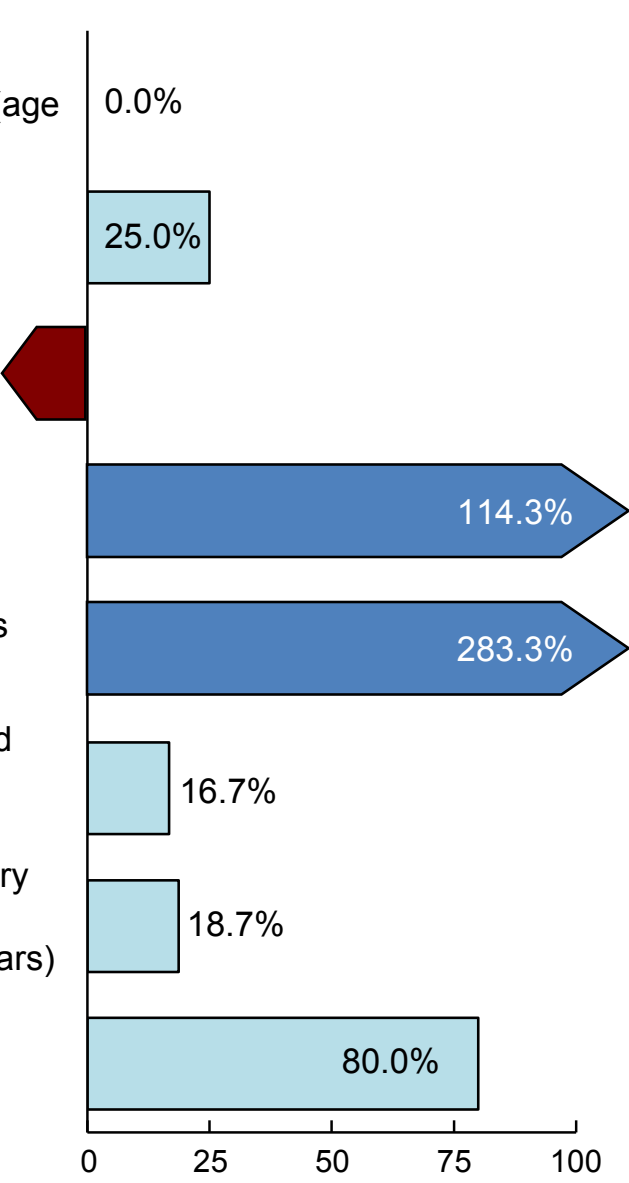

Percent of targeted change achieved 5

\begin{tabular}{|c|c|c|c|c|c|}
\hline \multirow{2}{*}{$\begin{array}{l}2010 \\
\text { Target }\end{array}$} & \multirow{2}{*}{$\begin{array}{c}\text { Baseline } \\
\text { (Year) }\end{array}$} & \multirow{2}{*}{$\begin{array}{l}\text { Final } \\
\text { (Year) }\end{array}$} & \multicolumn{3}{|c|}{ Baseline vs. Final } \\
\hline & & & Difference $^{2}$ & $\begin{array}{l}\text { Statistically } \\
\text { Significant }^{3}\end{array}$ & $\begin{array}{c}\text { Percent } \\
\text { Change }^{4}\end{array}$ \\
\hline $68 \%$ & $\begin{array}{c}65 \% \\
(2003)\end{array}$ & $\begin{array}{c}65 \% \\
(2008)\end{array}$ & 0 & No & $0.0 \%$ \\
\hline $94 \%$ & $\begin{array}{c}86 \% \\
(2003)\end{array}$ & $\begin{array}{c}88 \% \\
(2008)\end{array}$ & 2 & No & $2.3 \%$ \\
\hline $87 \%$ & $\begin{array}{c}76 \% \\
(2003)\end{array}$ & $\begin{array}{c}69 \% \\
(2008)\end{array}$ & -7 & No & $-9.2 \%$ \\
\hline $50 \%$ & $\begin{array}{c}43 \% \\
(2002)\end{array}$ & $\begin{array}{c}51 \% \\
(2008)\end{array}$ & 8 & Yes & $18.6 \%$ \\
\hline 25 & $\begin{array}{c}19 \\
(2003)\end{array}$ & $\begin{array}{c}36 \\
(2009)\end{array}$ & 17 & $\begin{array}{l}\text { Not } \\
\text { tested }\end{array}$ & $89.5 \%$ \\
\hline $1.9 \%$ & $\begin{array}{c}2.5 \% \\
(1997)\end{array}$ & $\begin{array}{l}2.4 \% \\
(2008)\end{array}$ & -0.1 & No & $-4.0 \%$ \\
\hline 62.3 & $\begin{array}{l}123.9 \\
(1999)\end{array}$ & $\begin{array}{l}112.4 \\
(2007)\end{array}$ & -11.5 & Yes & $-9.3 \%$ \\
\hline $1.4 \%$ & $\begin{array}{l}2.4 \% \\
(2000)\end{array}$ & $\begin{array}{c}1.6 \% \\
(2009)\end{array}$ & -0.8 & $\begin{array}{c}\text { Not } \\
\text { tested }\end{array}$ & $-33.3 \%$ \\
\hline
\end{tabular}

(continued) 


\section{Figure 24-1. Progress Toward Target Attainment for Focus Area 24: Respiratory Diseases (continued)}

\section{NOTES}

See the Reader's Guide for more information on how to read this figure. See DATA2010 at http://wonder.cdc.gov/data2010 for all Healthy People 2010 tracking data. Tracking data are not available for objectives 24-11a and 24-11b.

\section{FOOTNOTES}

${ }^{1}$ Movement away from target is not quantified using the percent of targeted change achieved. See Technical Appendix for more information.

${ }^{2}$ Difference $=$ Final value - Baseline value. Differences between percents $(\%)$ are measured in percentage points.

${ }^{3}$ When estimates of variability are available, the statistical significance of the difference between the final value and the baseline value is assessed at the 0.05 level. See Technical Appendix for more information.

\footnotetext{
${ }^{4}$ Percent change $=\frac{\text { Final value }- \text { Baseline value }}{\text { Baseline value }} \times 100$

Final value - Baseline value

Healthy People 2010 target - Baseline value $\times 100$
}

\section{DATA SOURCES}

24-1a-e. National Vital Statistics System-Mortality (NVSS-M), CDC, NCHS.

24-2a-c. National Hospital Discharge Survey (NHDS), CDC, NCHS.

24-3a-c. National Hospital Ambulatory Medical Care Survey (NHAMCS), CDC, NCHS.

24-4-24-6. National Health Interview Survey (NHIS), CDC, NCHS.

24-7a-f. National Health Interview Survey (NHIS), CDC, NCHS

24-8. Behavioral Risk Factor Surveillance System (BRFSS), CDC, NCCDPHP.

24-9. National Health Interview Survey (NHIS), CDC, NCHS.

24-10. National Vital Statistics System-Mortality (NVSS-M), CDC, NCHS.

24-12. Fatality Analysis Reporting System (FARS), Department of Transportation (DOT) 
Figure 24-2. Health Disparities Table for Focus Area 24: Respiratory Diseases

Disparities from the best group rate for each characteristic at the most recent data point and changes in disparity

24-3a. Emergency department visits for asthma-Children $<5$ years (per 10,000 pop.) (1995-97, 2005-07)

b. Emergency department visits for asthma-Children and adults 5-64 years (per 10,000 pop.) (1995-97, 2005-07)

c. Emergency department visits for asthma-Older adults $65+$ years (per 10,000 pop.) (1995-97, 2005-07)

24-4. Activity limitations among persons with asthma (age adjusted) $(2001,2008)$

24-5. School or work days missed by persons with asthma, due to asthma (5-64 years) $(2002,2008)^{2}$

24-6. Patient education among persons with asthma (age adjusted) $(2003,2008)$

24-7a. Persons with asthma receiving written asthma plans from health care provider (age adjusted) (2002 2008)

b. Persons with asthma receiving proper-use instructions with prescribed inhalers (age adjusted) (2003, 2008)

c. Persons with asthma receiving education on early signs, symptoms and responses to asthma episodes (age adjusted) $(2003,2008)$

d. Persons with asthma receiving medication regimens that prevent need for $>1$ beta agonist inhalation canister per month (age adjusted) $(2003,2008)$

e. Persons with asthma receiving long-term management care after hospitalization due to asthma (age adjusted) $(2003,2008)^{2}$

f. Persons with asthma receiving assistance in reducing exposure to environmental risk factors (age adjusted) $(2002,2008)$

24-9. Activity limitations due to chronic lung and breathing problems (age adjusted, 45+ years) $(1997,2008)^{3}$

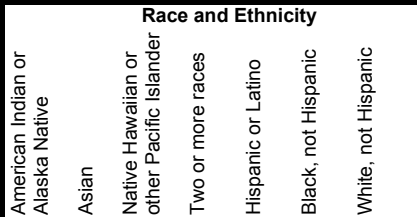

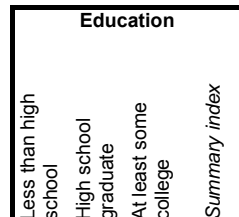

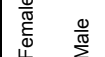

b

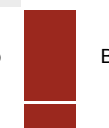

$b^{i}$
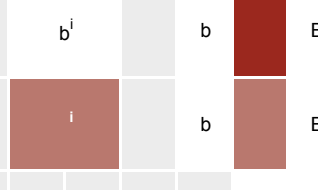

B

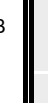

|

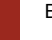

|

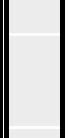 from the baseline to the most recent data point.
Population-based objectives
24-1a. Deaths from asthma-Children $<5$ years [per million population (pop.)] $(1999,2007)$
b. Deaths from asthma-Children and adolescents 5-14 years (per million pop.) (1999, 2007)
c. Deaths from asthma-Adolescents and adults 15-34 years (per million pop.) $(1999,2007)^{1}$
d. Deaths from asthma-Adults 35-64 years (per million pop.) (1999, 2007)
e. Deaths from asthma-Older adults $65+$ years (per million pop.) (1999, 2007)
24-2a. Hospitalizations for asthma-Children $<5$ years (per 10,000 pop.) $(1998,2007)$
b. Hospitalizations for asthma-Children and adults 5-64 years (age adjusted, per 10,000 pop.) (1998, 2007)
c. Hospitalizations for asthma-Older adults $65+$ years (age adjusted, per 10,000 pop.) $(1998,2007)$

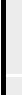


Characteristics and Groups

Deaths from chronic obstructive pulmonary disease (COPD, excluding asthma) (age adjusted, per 100,000 pop., $45+$ years) $(1999,2007)^{1}$

24-11a. Medical evaluation for persons with symptoms of obstructive sleep apnea (age adjusted, 20+ years) (2005-08)
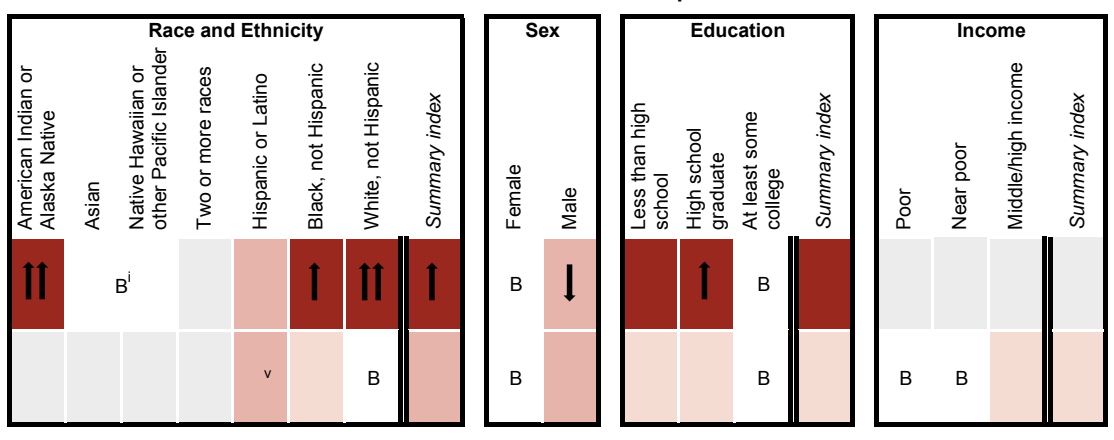

NOTES

See DATA2010 at http://wonder.cdc.gov/data2010 for all Healthy People 2010 tracking data. Disparity data are either unavailable or not applicable for objectives $24-$ 8, 24-11b, and 24-12.

Years in parentheses represent the baseline and most recent data years (if available).

Disparity from the best group rate is defined as the percent difference between the best group rate and each of the other group rates for a characteristic (e.g., race and ethnicity). The summary index is the average of these percent differences for a characteristic. Change in disparity is estimated by subtracting the disparity at baseline from the disparity at the most recent data point. Change in the summary index is estimated by subtracting the summary index at baseline from the summary index at the most recent data point. See Technical Appendix for more information.

Measures of variability were available for all objectives in this table. Thus, the variability of best group rates was assessed, and statistical significance was tested. Disparities of $10 \%$ or more are displayed when the differences from the best group rate are statistically significant at the 0.05 level. Changes in disparities over time are indicated by arrows when the changes are greater than or equal to 10 percentage points and are statistically significant at the 0.05 level. See Technical Appendix.

LEGEND

$\begin{aligned} & \text { The "best" group rate at the most recent } \\ & \text { data point. }\end{aligned}$
$\begin{aligned} & \text { Disparity from the best group rate at the } \\ & \text { most recent data point. }\end{aligned}$

\begin{tabular}{|l|l|l|} 
Availability of Data & Data not available. & Characteristic not selected for this objective. \\
\hline
\end{tabular}

\section{FOOTNOTES}

1 Most recent data by education level is for 2002 .

2 Most recent data by sex is for 2003.

3 Baseline data by race and ethnicity are for 1999 .

i Data are for Asian or Pacific Islander.

ii Data include persons of Hispanic origin.

iii The group with the best rate at the most recent data point is different from the group with the best rate at baseline. Both rates met the reliability criterion. See Technical Appendix.

iv Change in the summary index cannot be assessed. See Technical Appendix.

$\checkmark$ Data are for Mexican American.

DATA SOURCES

24-1a-e. National Vital Statistics System-Mortality (NVSS-M), CDC, NCHS.

24-2a-c. National Hospital Discharge Survey (NHDS), CDC, NCHS.

24-3a-c. National Hospital Ambulatory Medical Care Survey (NHAMCS), CDC, NCHS.

-24-6. National Health Interview Survey (NHIS), CDC, NCHS

24-7a-f. National Health Interview Survey (NHIS), CDC, NCHS.

24-9. National Health Interview Survey (NHIS), CDC, NCHS

24-10. National Vital Statistics System-Mortality (NVSS-M), CDC, NCHS.

24-11a. National Health and Nutrition Examination Survey (NHANES), CDC, NCHS.

Respiratory Diseases

Page 24-17 
Figure 24-3. Deaths from Chronic Obstructive Pulmonary Disease

(Excluding Asthma, 45+ Years) 2005-07

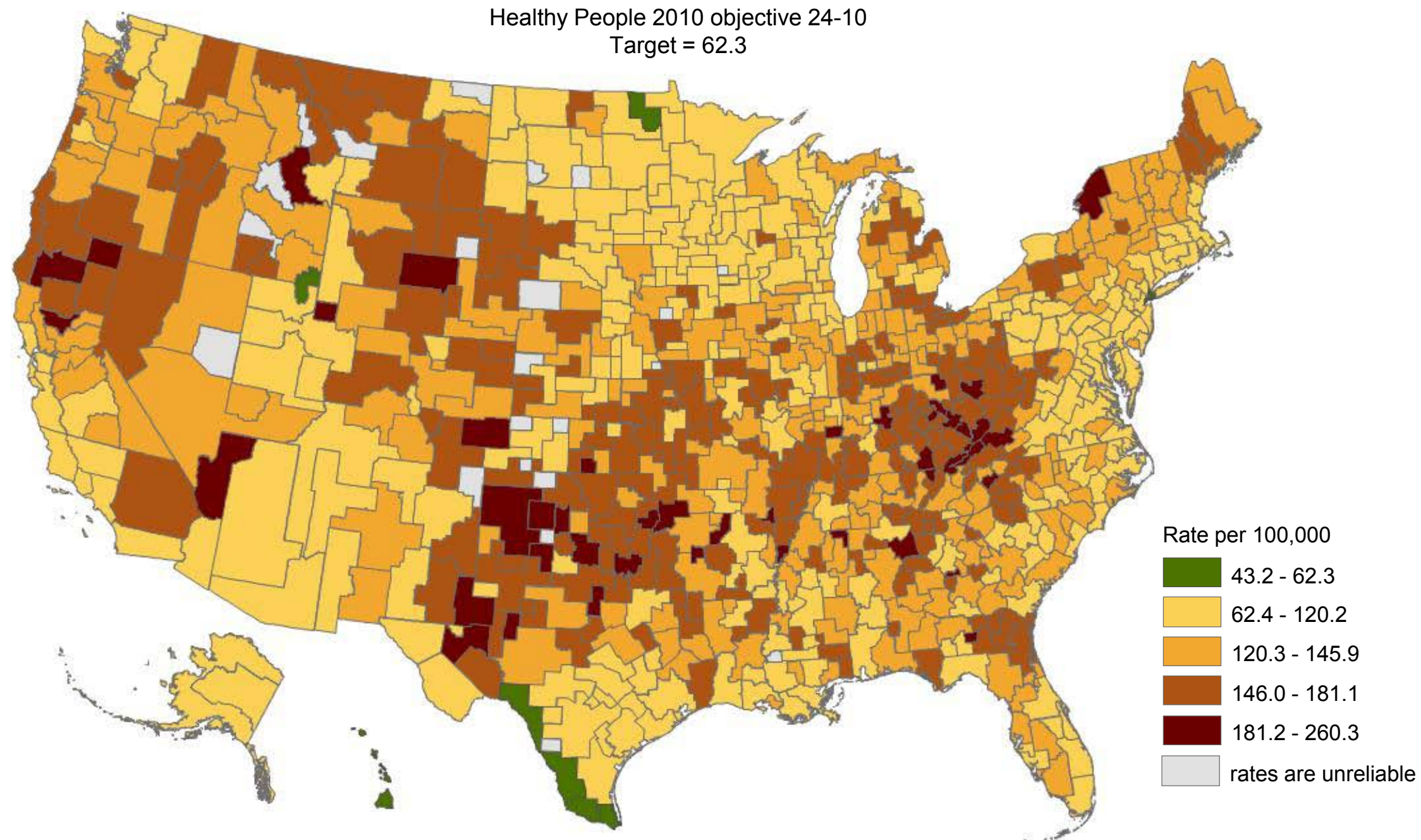

lowest category (green) shows health service areas that met target

NOTES: Data are for ICD-10 codes J40-J44 reported as underlying cause, for ages 45 and older. Rates are per 100,000 U. S. Population age-adjusted to the 2000 standard population. Rates are displayed by a modified Jenks classification for U.S. health service area.

SOURCE: National Vital Statistics System - Mortality, CDC, NCHS. 


\section{Final Review}

\section{Sexually Transmitted Diseases}

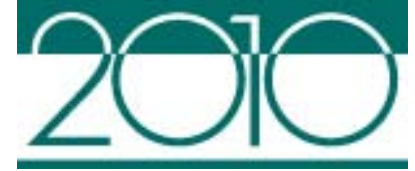

25

\section{Lead Agency}

Centers for Disease Control and Prevention

\section{Contents}

Goal.

Highlights.

Summary of Progress.

Transition to Healthy People 2020

Data Considerations

Notes

Comprehensive Summary of Objectives

Progress Chart

Health Disparities Table

Chlamydia infections-Map.

Gonorrhea-Map

Domestic transmission of primary and secondary syphilis-Map 


\section{Goal: Promote responsible sexual behaviors, strengthen community capacity, and increase accessibility to quality services to prevent sexually transmitted diseases (STDs) and their complications}

Sexually transmitted diseases (STDs) refer to the more than 25 infectious organisms that are transmitted primarily through sexual activity. This chapter includes objectives that monitor cases of STD, responsible sexual behavior among adolescents, and the availability of screening programs for genital Chlamydia.

All Healthy People tracking data quoted in this chapter, along with technical information and operational definitions for each objective, can be found in the Healthy People 2010 database, DATA2010, available from http://wonder.cdc.gov/data2010/.

More information about this Focus Area can be found in the following publications:

- Healthy People 2010: Understanding and Improving Health, available from http://www.healthypeople.gov/2010/Document/tableofcontents.htm\#under.

- Healthy People 2010 Midcourse Review, available from http://www.healthypeople.gov/2010/data/midcourse/html/default.htm\#FocusAreas.

\section{Highlights}

- Substantial progress was achieved in objectives for this Focus Area during the past decade [1]. Almost two thirds (63\%) of the STD objectives with data to measure progress moved toward or achieved their Healthy People 2010 targets (Figure 10-1). However, health disparities of $50 \%$ or more among racial and ethnic populations, as well as by sex, were observed (Figure 10-2), as highlighted below [2].

- Chlamydia infections (objectives 25-1a through d) increased, moving away from the Healthy People 2010 targets [3]. Infections among females aged 15-24 years attending family planning clinics (objective 25-1a) increased by 62\% between 1997 and 2009, from $5.0 \%$ to $8.1 \%$. Similarly, for persons attending STD clinics, infections among females (objective $25-1$ b) increased by $34.4 \%$ between 1997 and 2009 , from $12.2 \%$ to $16.4 \%$, whereas infections among males (objective 25-1c) increased by $52.9 \%$ between 1997 and 2009 , from $15.7 \%$ to $24.0 \%$. Each of these three objectives had a 2010 target of $3.0 \%$. Chlamydia infections among females aged 24 years and under who were enrolled in National Job Training Programs (objective 25-1d) increased by $15.8 \%$ between 2002 and 2009 , from $10.1 \%$ to $11.7 \%$, moving away from the 2010 target of $6.8 \%$. Health disparities among racial and ethnic groups were observed for all four of these objectives. For example: 
- In 2009, white non-Hispanic women attending family planning clinics, STD clinics, or enrolled in National Job Training Programs had the lowest (best) rates of Chlamydia infection among racial and ethnic groups: 5.4\%, 12.1\%, and 5.9\%, respectively. The rate for black non-Hispanic women attending family planning clinics, $14.8 \%$ in 2009, was more than two and a half times the best rate (that for white non-Hispanic women), while the rate for black non-Hispanic women enrolled in National Job Training Programs, $14.8 \%$ in 2009 , was twice the best rate [2].

- Asian men attending STD clinics had the lowest (best) rate of Chlamydia infection among racial and ethnic populations, $14.4 \%$ in 2009 , whereas black non-Hispanic men had a rate of $29.4 \%$ in 2009 , about twice the best rate [2].

- Chlamydia infection varied by geographic area. In 2009, the states of Idaho, Maine, New Hampshire, Utah, Vermont, and West Virginia had the lowest rates. Rates were highest in Alaska and Mississippi (Figure 25-3).

- The incidence of gonorrhea (objective 25-2a) declined by 18.9\% between 1997 and 2009, from 122 to 99 new cases per 100,000 population, moving toward the 2010 target of 19 new cases per 100,000 population. New cases of gonorrhea among females aged 15-44 years (objective 25-2b) declined by $8.6 \%$ between 2002 and 2009, from 279 to 255 per 100,000 population, moving toward the target of 42 new cases per 100,000 population.

- Among racial and ethnic groups, the Asian or Pacific Islander population had the lowest (best) rates of new cases of gonorrhea (objective 25-2a), 19 new cases per 100,000 population in 1997 and 18 new cases per 100,000 in 2009. The Hispanic or Latino population had rates of 65 per 100,000 in 1997 and 59 in 2009; the American Indian or Alaska Native population had rates of 97 per 100,000 in 1997 and 113 in 2009; and the black non-Hispanic population had rates of 809 per 100,000 in 1997 and 556 in 2009.

- In 2009, the rate for the Hispanic or Latino population was almost three and a half times the best group rate (that for the Asian or Pacific Islander population); the rate for the American Indian or Alaska Native was almost six and a half times the best rate; and the rate for the black non-Hispanic population was almost 31 times the best rate [2].

- Between 1997 and 2009, the disparity between the American Indian or Alaska Native population and the Asian or Pacific Islander population (group with the best rate) increased by 117 percentage points, while the disparity between the black non-Hispanic population and the Asian or Pacific Islander population decreased by 1,169 percentage points [4].

- Racial and ethnic disparities in the incidence of gonorrhea among females aged 1544 years (objective 25-2b) were similar to those observed in the total population.

- The Asian or Pacific Islander population had the lowest (best) rate, 37 per 100,000 population in 2009. The rates for the white non-Hispanic, Hispanic or Latino, American Indian or Alaska Native, and black non-Hispanic populations were $83,128,311$, and 1,198 per 100,000 in 2009, respectively.

- The rate for the white non-Hispanic population was more than twice the best group rate (that for the Asian or Pacific Islander population); the rate for the Hispanic or Latino population was about three and a half times the best rate; the rate for the American Indian or Alaska Native population was almost eight and a half times the best rate; and the rate for the black nonHispanic population was over 32 times the best rate [2]. 
- The incidence of gonorrhea among females aged 15-44 years (objective 25-2b) for the Asian or Pacific Islander population was 43 new cases per 100,000 population in 1997 and 37 per 100,000 in 2009, whereas the rates for the American Indian or Alaska Native populations were 304 per 100,000 in 1997 and 311 in 2009.

- Between 1997 and 2009, the disparity between the American Indian or Alaska Native population and the Asian or Pacific Islander population (group with the best rate) increased by 134 percentage points [4].

- Gonorrhea incidence varied by geographic region. In 2009, incidence was lower in the West, Midwest, and Northeast. Seven states, including Idaho, Montana, and Utah in the West, and Maine, New Hampshire, and Vermont in New England, achieved the Healthy People 2010 target. The District of Columbia had the highest incidence of gonorrhea (Figure 25-4).

- Domestic transmission of primary and secondary syphilis (objective 25-3) increased by $43.7 \%$ between 1997 and 2009, from 3.2 new cases per 100,000 population to 4.6 new cases per 100,000, moving away from the Healthy People 2010 target of 0.2 new cases per 100,000 population.

- Among racial and ethnic groups, the Asian or Pacific Islander population had the lowest (best) rates of new cases of syphilis: 0.3 new cases per 100,000 population in 1997 and 1.6 in 2009. Rates for the American Indian or Alaska Native population were 2.0 per 100,000 in 1997 and 2.4 in 2009; rates for the Hispanic or Latino population were 1.6 per 100,000 in 1997 and 4.5 in 2009; and rates for the black non-Hispanic population were 22.0 per 100,000 in 1997 and 19.2 in 2009.

- In 2009, the rate for the Hispanic or Latino population was almost three times the best group rate (that for the Asian or Pacific Islander population), while the rate for the black non-Hispanic population was 12 times the best rate [2].

- Between 1997 and 2009, the disparity between the American Indian or Alaska Native population and the Asian or Pacific Islander population (group with the best rate) declined by 517 percentage points; while the disparity between the Hispanic or Latino population and the Asian or Pacific Islander population declined by 252 percentage points; and the disparity between the black non-Hispanic population and the Asian or Pacific Islander population declined by 6,133 percentage points [4].

- Females had lower (better) rates of new cases of syphilis than males: 2.9 new cases per 100,000 population in 1997, and 1.4 in 2009. The rates for males were $3.6 \mathrm{new}$ cases per 100,000 in 1997 and 7.8 in 2009 . The 2009 rate for males was more than five and a half times the rate for females [2]. Between 1997 and 2009, the disparity between males and females increased by 433 percentage points [4].

- Domestic transmission of primary and secondary syphilis varied by geographic area. Four states achieved the Healthy People 2010 target: Alaska, Idaho, South Dakota, and Vermont. In 2009, Louisiana had the highest incidence of domestic transmission of primary and secondary syphilis (Figure 25-5).

- The incidence of congenital syphilis (objective 25-9) declined by $64.3 \%$ between 1997 and 2009, from 28 new cases per 100,000 live births to 10 new cases per 100,000 live births, moving toward the 2010 target of 1 new case per 100,000 population. 
- Among racial and ethnic groups, the white non-Hispanic population had the lowest (best) rates of new cases of congenital syphilis: 4 new cases per 100,000 live births in 1997 and 3 in 2009. The American Indian or Alaska Native population had rates of 11 new cases per 100,000 live births in 1997 and 12 in 2009; the Hispanic or Latino population had rates of 34 new cases per 100,000 live births in 1997 and 12 in 2009; and the black non-Hispanic population had rates of 123 new cases per 100,000 live births in 1997 and 35 in 2009.

- In 2009, the rates for the American Indian or Alaska native and the Hispanic or Latino populations were four times the best rate (that for the white nonHispanic population), while the rate for the black non-Hispanic population was almost 12 times the best rate [2].

- Between 1997 and 2009, the disparity between the American Indian or Alaska Native population and the white non-Hispanic population (group with the best rate) increased by 125 percentage points; while the disparity between the Hispanic or Latino and the white non-Hispanic population declined by 450 percentage points; and the disparity between the black nonHispanic population and the white non-Hispanic population declined by 1,908 percentage points [4].

- The proportion of persons aged 20-29 years with genital herpes infections (objective 25-4) declined by $35.3 \%$ between $1988-94$ and $2005-08$, from $17 \%$ to $11 \%$, exceeding the 2010 target of $14 \%$.

- The proportion of women aged 15-44 years who had ever required treatment for pelvic inflammatory disease (PID) (objective 25-6) declined by 50\% between 1995 and 2006-08, from $8 \%$ to $4 \%$, exceeding the 2010 target of $5 \%$.

\section{Summary of Progress}

- Figure 25-1 presents a quantitative assessment of progress in achieving the Healthy People 2010 objectives for STDs [1]. Data to measure progress toward target attainment were available for 16 objectives. Of these:

- Two objectives exceeded their 2010 targets (objectives 25-4 and 25-6).

- Eight objectives moved toward their targets. No statistically significant difference between the baseline and final data points was observed for one of these objectives (25-7). Data to test the significance of the difference were unavailable for seven objectives (25-2a and b; 25-9; 25-11a and c; and 25-16-a and b).

- Six objectives moved away from their targets (objectives 25-1a through d; 25-3; and 25-11b). Data to test the significance of the difference between the baseline and final data points were unavailable for all of these objectives.

- One objective remained developmental (objective 25-5) and one objective had no data available to measure progress (objective 25-13) [5]. One objective (25-8) was moved to the HIV Focus Area and seven were dropped during the decade (objectives 25-10, 25-12, 25-14, 25-15, 25-17, 25-18, and 25-19) [6]. 
- Figure 25-2 displays health disparities from the best group rate for each characteristic at the most recent data point [2]. It also displays changes in disparities from baseline to the most recent data point [4].

- Two objectives had statistically significant health disparities of $10 \%$ or more by race and ethnicity (objectives 25-4 and 25-7) and eight additional objectives with racial and ethnic disparities of $10 \%$ or more lacked data to assess statistical significance (objectives 25-1a through d; 25-2a and b; 25-3; and 25-9).

- Of these 10 objectives, the white non-Hispanic population had the best rate for six objectives (25-1a, b, and d; 25-2a; 25-9; 25-11a; and 25-11c). The Asian or Pacific Islander population had the best rate for three objectives (25-2a and b, and 25-3) and the Asian population had the best rate for one objective (25-1c).

- One objective had statistically significant disparities of $10 \%$ or more by sex (objective 25-11c) and two additional objectives with disparities of $10 \%$ or more by sex lacked data to assess statistical significance (objectives 25-2a and 25-3). Of these three objectives, males had better rates for two objectives (25-2a and 25-11c) and females had a better rate for one objective (25-3).

- Health disparities of $100 \%$ or more were observed among racial and ethnic populations, as well as by sex. Changes in disparity of 100 percentage points or more also were observed. These are discussed in the Highlights, above.

\section{Transition to Healthy People 2020}

For Healthy People 2020, the Sexually Transmitted Disease Topic Area has a smaller set of objectives than were included in Healthy People 2010. See HealthyPeople.gov for a complete list of Healthy People 2020 topics and objectives.

The Healthy People 2020 objectives can be grouped into several sections:

- Bacterial STD illness and disability

- Viral STD illness and disability

- STD complications affecting females

- STD complications affecting fetuses and newborns

- Personal health services.

The differences between the Healthy People 2010 and Healthy People 2020 objectives are summarized below:

- The Healthy People 2020 Sexually Transmitted Diseases Topic Area has 18 objectives, whereas the Healthy People 2010 Focus Area had 26 objectives.

- Six Healthy People 2010 objectives, were retained "as is" [7]. These objectives include:

- Chlamydia infections among females aged 15-24 years attending family planning clinics (objective 25-1a)

- Chlamydia infections among females aged 24 years and under enrolled in a National Job Training Program (objective 25-1d) 
- Gonorrhea infections among females aged 15-44 years (objective 25-2b)

- Young adults with genital herpes infection due to herpes simplex, type 2 (objective 25-4)

- Females aged 15-44 years who have ever required treatment for pelvic inflammatory disease (PID) (objective 25-6)

- Congenital syphilis (objective 25-9).

- Four Healthy People 2010 objectives (25-3, 25-5, and 25-16a and b) were modified to create nine Healthy People 2020 objectives [8].

- Sustained domestic transmission of primary and secondary syphilis (objective 25-3) was divided into two objectives: domestic transmission of primary and secondary syphilis among males, and domestic transmission of primary and secondary syphilis among females.

- Sexually active females aged 24 years and under enrolled in commercial health insurance plans who are screened for genital Chlamydia infections during the measurement year (objective 25-16a) was split into two objectives: 1) sexually active females aged 16-20 years enrolled in commercial health insurance plans who are screened for genital Chlamydia infections during the measurement year; and 2) sexually active females aged 21-24 years enrolled in commercial health insurance plans who are screened for genital Chlamydia infections during the measurement year.

- Sexually active females aged 24 years and under enrolled in Medicaid plans who are screened for genital Chlamydia infections during the measurement year (objective 25-16b) was divided into two objectives: 1 ) sexually active females aged 16-20 years enrolled in Medicaid plans who are screened for genital Chlamydia infections during the measurement year; and 2) sexually active females aged 21-24 years enrolled in Medicaid plans who are screened for genital Chlamydia infections during the measurement year.

- Human papillomavirus (HPV) infections among females aged 14-49 years (objective 25-5) was split into three objectives depicting the different HPV types: HPV types 6 and 11; HPV types 16 and 18; and all other HPV types.

- One developmental Healthy People 2010 objective was moved to the HIV Focus Area: heterosexually transmitted HIV infections in women aged 13-24 years (objective 25-8) [5].

- Seven Healthy People 2010 objectives were dropped during the decade due to lack of nationally representative data sources: neonatal STDs (objective 25-10); responsible sexual behavior messages on television (objective 25-12); screening for STDs in jails (objective 2514); contracts with managed care providers to treat partners of STD patients (objective 2515); STD screening of pregnant women during prenatal visits (objective 25-17); primary care provider compliance with STD treatment standards (objective 25-18); and provider referral services for partners of STD patients (objective 25-19).

- Eight objectives were archived [9].

- Two objectives, Chlamydia infections among females and males aged 15-24 years attending STD clinics (objectives 25-1b and c), were archived because they were not deemed accurate measures of the community burden associated with Chlamydia since they focused on persons who had sought care for a suspected STD. 
- One objective, new cases of gonorrhea (objective 25-2a), was archived because it was redundant with the Healthy People 2020 gonorrhea objectives.

- One objective, fertility problems among childless females with an STD or PID (objective 25-7), was archived because it did not differentiate between STDs and PID and hence could not be used to assess STD-associated infertility.

- Three objectives, measuring responsible sexual behavior among students in grades 9-12 (objectives 25-11a through c), were archived because they overlapped with the Family Planning objectives.

- One objective, Hepatitis B vaccines offered in tribal, state, and local STD clinics (objective 25-13), was archived because it lacked a viable data source.

- Three new objectives were added to the Healthy People 2020 Sexually Transmitted Diseases Topic Area:

- Gonorrhea infections among males aged 15-44 years

- Chlamydia infections among females aged 15-44 years

- Chlamydia infections among males aged 24 years and under who enrolled in a National Job Training Program.

Appendix D, “A Crosswalk Between Objectives From Healthy People 2010 to Healthy People 2020," summarizes the changes between the two decades of objectives, reflecting new knowledge and direction for this area.

\section{Data Considerations}

Figures 25-3 (Chlamydia), 25-4 (Gonorrhea) and 25-5 (Primary and Secondary Syphilis) present state-level data from state and local health department STD control programs. National data for these objectives come from the STD Surveillance System (STDSS) and are the basis for setting the targets. The state level data may not be comparable to the National data from the STDSS.

Education and income are the primary measures of socioeconomic status (SES) in Healthy People 2010. Most data systems used in Healthy People 2010 define income as a family's income before taxes. In order to facilitate comparisons among groups and over time, while adjusting for family size and for inflation, Healthy People 2010 categorizes income using the poverty thresholds developed by the U.S. Census Bureau. Thus, the three categories of family income that are primarily used are:

- Poor-below the Federal poverty level

- Near poor-100\% to $199 \%$ of the Federal poverty level

- Middle/high income-200\% or more of the Federal poverty level.

These categories may be overridden by considerations specific to the data system, in which case they are modified as appropriate. See Healthy People 2010: General Data Issues, referenced below.

In general, data on educational attainment are presented for persons aged 25 years and over, consistent with guidance given by the U.S. Bureau of the Census. However, because of the 
requirements of the different data systems, the age groups used to calculate educational attainment for any specific objective may differ from the age groups used to report the data for other Healthy People 2010 objectives, as well as from select populations within the same objective. Therefore, the reader is urged to exercise caution in interpreting the data by educational attainment shown in the Health Disparities Table. See Healthy People 2010: General Data Issues, referenced below.

Additional information on data issues is available from the following sources:

- All Healthy People 2010 tracking data can be found in the Healthy People 2010 database, DATA2010, available from http://wonder.cdc.gov/data2010/.

- Detailed information about the data and data sources used to support these objectives can be found in the Operational Definitions on the DATA2010 website, available from http://wonder.cdc.gov/data2010/focusod.htm.

- More information on statistical issues related to Healthy People tracking and measurement can be found in the Technical Appendix and in Healthy People 2010: General Data Issues, which is available in the Data Issues section of the NCHS Healthy People website under Healthy People 2010..

\section{Notes}

1. Displayed in the Progress Chart (Figure 25-1), the percent of targeted change achieved expresses the difference between the baseline and the final value relative to the initial difference between the baseline and the Healthy People 2010 target. As such, it is a relative measure of progress toward attaining the Healthy People 2010 target. See the Reader's Guide for more information. When standard errors were available, the difference between the baseline and the final value was tested at the 0.05 level of significance. See the Figure 25-1 footnotes, as well as the Technical Appendix, for more detail.

2. Information about disparities among select populations is shown in the Health Disparities Table (Figure 25-2). Disparity from the best group rate is defined as the percent difference between the best group rate and each of the other group rates for a characteristic. For example, racial and ethnic health disparities are measured as the percent difference between the best racial and ethnic group rate and each of the other racial and ethnic group rates. Similarly, disparities by sex are measured as the percent difference between the better group rate (e.g., female) and the rate for the other group (e.g., male). Some objectives are expressed in terms of favorable events or conditions that are to be increased, while others are expressed in terms of adverse events or conditions that are to be reduced. In order to facilitate comparison of health disparities across different objectives, disparity is measured only in terms of adverse events or conditions. For comparability across objectives, objectives that are expressed in terms of favorable events or conditions are re-expressed using the adverse event or condition for the purpose of computing disparity, but they are not otherwise restated or changed. For example, objective 1-1, to increase the proportion of persons with health insurance (e.g., $72 \%$ of the American Indian or Alaska Native population aged under 65 years had some form of health insurance in 2008), is expressed in terms of the percentage of persons without health insurance (e.g., $100 \%-72 \%=28 \%$ of the American Indian or Alaska Native population aged under 65 years did not have any form of health insurance in 2008) when the disparity from the best group rate is calculated. See the Reader's Guide for more information. When standard errors were available, the difference between the best group rate and each of the other group rates was tested at the 0.05 level of significance. See the Figure 25-2 footnotes, as well as the Technical Appendix, for more detail.

3. Most of the observed increases in Chlamydia infections (objectives 25-1a through d) were due to increases in test sensitivity, which resulted in previously undiagnosed infections being detected. In 
addition, the Chlamydia infection rates tracked in objectives 25-1a through d have not been adjusted for increases in screening rates and efforts to target screening to persons most at risk of infection. Therefore, the reader is urged to exercise caution in interpreting the observed increases in Chlamydia infections.

4. The change in disparity is estimated by subtracting the disparity at baseline from the disparity at the most recent data point and, therefore, is expressed as a change in percentage points. See the Reader's Guide for more information. When standard errors were available, the change in disparity was tested at the 0.05 level of significance. See the Figure 25-2 footnotes, as well as the Technical Appendix, for more detail.

5. To be included in Healthy People 2010, an objective must have a national data source that provides a baseline and at least one additional data point for tracking progress. Some objectives lacked baseline data at the time of their development but had a potential data source and were considered of sufficient national importance to be included in Healthy People. These are called "developmental" objectives. When data become available, a developmental objective is moved to measurable status and a Healthy People target can be set.

6. Dropped objectives were not carried forward into Healthy People 2020. These objectives were either developmental or deleted at the Healthy People 2010 Midcourse Review or at another time in Healthy People 2010.

7. Retained "as is" objectives have no change in the numerator definition or in the denominator definition between the Healthy People 2010 and Healthy People 2020 objectives. These include objectives that were developmental in Healthy People 2010 and are developmental in Healthy People 2020 and for which no numerator or denominator information was available.

8. Modified objectives have some change in the numerator definition or in the denominator definition between the Healthy People 2010 and Healthy People 2020 objectives. These include objectives that went from developmental in Healthy People 2010 to measurable in Healthy People 2020 or vice versa.

9. Archived objectives had at least one data point in Healthy People 2010 but were not carried forward into Healthy People 2020. 


\section{Comprehensive Summary of Objectives: Sexually Transmitted Diseases}

\begin{tabular}{|c|c|c|}
\hline Objective & Description & Data Source or Objective Status \\
\hline $25-1 \mathrm{a}$ & $\begin{array}{l}\text { Chlamydia infections-Females } 15-24 \text { years attending family planning } \\
\text { clinics }\end{array}$ & STD Surveillance System (STDSS), CDC, NCHHSTP. \\
\hline $25-1 b$ & Chlamydia infections-Females $15-24$ years attending STD clinics & STD Surveillance System (STDSS), CDC, NCHHSTP. \\
\hline $25-1 c$ & Chlamydia infections-Males 15-24 years attending STD clinics & STD Surveillance System (STDSS), CDC, NCHHSTP. \\
\hline $25-1 d$ & $\begin{array}{l}\text { Chlamydia infections-Females } \leq 24 \text { years enrolled in National Job } \\
\text { Training Program }\end{array}$ & $\begin{array}{l}\text { STD Surveillance System (STDSS), CDC, NCHHSTP; National Job } \\
\text { Training Program. }\end{array}$ \\
\hline $25-2 a$ & Gonorrhea-New cases per 100,000 population & STD Surveillance System (STDSS), CDC, NCHHSTP. \\
\hline $25-2 b$ & $\begin{array}{l}\text { Gonorrhea-New cases per } 100,000 \text { population among females } 15-44 \\
\text { years }\end{array}$ & STD Surveillance System (STDSS), CDC, NCHHSTP. \\
\hline $25-3$ & $\begin{array}{l}\text { Domestic transmission of primary and secondary syphilis (new cases } \\
\text { per } 100,000 \text { population) }\end{array}$ & STD Surveillance System (STDSS), CDC, NCHHSTP. \\
\hline $25-4$ & Genital herpes infection among adults $20-29$ years & $\begin{array}{l}\text { National Health and Nutrition Examination Survey (NHANES), CDC, } \\
\text { NCHS. }\end{array}$ \\
\hline $25-5$ & Human papillomavirus (HPV) infection-females $14-49$ years & Developmental \\
\hline $25-6$ & $\begin{array}{l}\text { Treatment for pelvic inflammatory disease (PID) among females (15- } \\
44 \text { years) }\end{array}$ & National Survey of Family Growth (NSFG), CDC, NCHS. \\
\hline $25-7$ & $\begin{array}{l}\text { Fertility problems among childless females with an STD or PID (15-44 } \\
\text { years) }\end{array}$ & National Survey of Family Growth (NSFG), CDC, NCHS. \\
\hline $25-9$ & Congenital syphilis (new cases per 100,000 live births) & $\begin{array}{l}\text { STD Surveillance System (STDSS), CDC, NCHHSTP; National Vital } \\
\text { Statistics System-Natality (NVSS-N), CDC, NCHS. }\end{array}$ \\
\hline $25-10$ & Neonatal STDs & Dropped \\
\hline $25-11 a$ & Students who never had sexual intercourse (grades 9-12) & Youth Risk Behavior Surveillance System (YRBSS), CDC, NCCDPHP. \\
\hline $25-11 b$ & $\begin{array}{l}\text { Students who had sexual intercourse, but not in the past } 3 \text { months } \\
\text { (grades 9-12) }\end{array}$ & Youth Risk Behavior Surveillance System (YRBSS), CDC, NCCDPHP. \\
\hline $25-11 c$ & Students who used condoms at last intercourse (grades 9-12) & Youth Risk Behavior Surveillance System (YRBSS), CDC, NCCDPHP. \\
\hline $25-12$ & Responsible sexual behavior messages on television & Dropped \\
\hline $25-13$ & Hepatitis B vaccines offered in STD clinics-Tribal, State and local & $\begin{array}{l}\text { Survey of STD Programs, National Coalition of STD Directors (NCSD); } \\
\text { HIS. }\end{array}$ \\
\hline
\end{tabular}




\begin{tabular}{|c|c|c|}
\hline Objective & Description & Data Source or Objective Status \\
\hline $25-14$ & $\begin{array}{l}\text { Screening for sexually transmitted diseases in detention facilities and } \\
\text { jails }\end{array}$ & Dropped \\
\hline $25-15$ & $\begin{array}{l}\text { Contracts with managed care providers to treat non-plan partners of } \\
\text { STD patients }\end{array}$ & Dropped \\
\hline $25-16 a$ & $\begin{array}{l}\text { Annual screening for genital Chlamydia among females } \leq 25 \text { years- } \\
\text { Enrolled in commercial managed care organizations (MCOs) }\end{array}$ & $\begin{array}{l}\text { Healthcare Effectiveness Data and Information Set (HEDIS), National } \\
\text { Committee for Quality Assurance (NCQA). }\end{array}$ \\
\hline $25-16 b$ & $\begin{array}{l}\text { Annual screening for genital Chlamydia among females } \leq 25 \text { years- } \\
\text { Enrolled in Medicaid managed care organizations (MCOs) }\end{array}$ & $\begin{array}{l}\text { Healthcare Effectiveness Data and Information Set (HEDIS), National } \\
\text { Committee for Quality Assurance (NCQA). }\end{array}$ \\
\hline $25-17$ & STD screening of pregnant women during prenatal healthcare visits & Dropped \\
\hline $25-18$ & $\begin{array}{l}\text { Primary care provider compliance with recognized STD treatment } \\
\text { standards }\end{array}$ & Dropped \\
\hline $25-19$ & Provider referral services for partners of STD patients & Dropped \\
\hline
\end{tabular}


Figure 25-1. Progress Toward Target Attainment for Focus Area 25: Sexually Transmitted Diseases

Moved away

from target ${ }^{1}$

Moved toward

target

Met or exceeded

target

25-1. Chlamydia infections

a. Females 15-24 years attending family planning clinics

b. Females 15-24 years attending STD clinics

c. Males 15-24 years attending STD clinics

d. Females $\leq 24$ years enrolled in National Job Training Program

25-2. Gonorrhea

a. New cases per 100,000 population

b. New cases per 100,000 population among females $15-44$ years

25-3. Domestic transmission of primary and secondary syphilis (new cases per 100,000 population)

25-4. Genital herpes infection among adults 2029 years
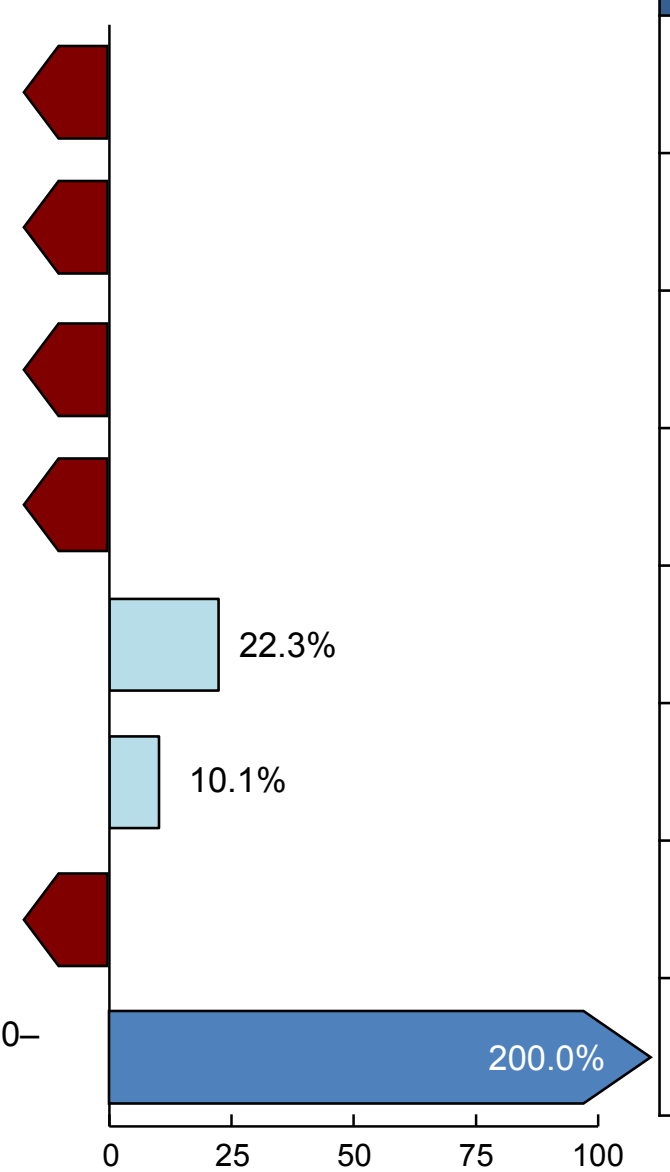

Percent of targeted change achieved ${ }^{5}$

\begin{tabular}{|c|c|c|c|c|c|}
\hline \multirow{2}{*}{$\begin{array}{c}2010 \\
\text { Target }\end{array}$} & \multirow{2}{*}{$\begin{array}{c}\text { Baseline } \\
\text { (Year) }\end{array}$} & \multirow{2}{*}{$\begin{array}{l}\text { Final } \\
\text { (Year) }\end{array}$} & \multicolumn{3}{|c|}{ Baseline vs. Final } \\
\hline & & & Difference ${ }^{2}$ & $\begin{array}{l}\text { Statistically } \\
\text { Significant }^{3}\end{array}$ & $\begin{array}{l}\text { Percent } \\
\text { Change }^{4}\end{array}$ \\
\hline $3.0 \%$ & $\begin{array}{c}5.0 \% \\
(1997)\end{array}$ & $\begin{array}{l}8.1 \% \\
(2009)\end{array}$ & 3.1 & $\begin{array}{c}\text { Not } \\
\text { tested }\end{array}$ & $62.0 \%$ \\
\hline $3.0 \%$ & $\begin{array}{l}12.2 \% \\
(1997)\end{array}$ & $\begin{array}{l}16.4 \% \\
(2009)\end{array}$ & 4.2 & $\begin{array}{l}\text { Not } \\
\text { tested }\end{array}$ & $34.4 \%$ \\
\hline $3.0 \%$ & $\begin{array}{l}15.7 \% \\
(1997)\end{array}$ & $\begin{array}{l}24.0 \% \\
(2009)\end{array}$ & 8.3 & $\begin{array}{l}\text { Not } \\
\text { tested }\end{array}$ & $52.9 \%$ \\
\hline $6.8 \%$ & $\begin{array}{l}10.1 \% \\
(2002)\end{array}$ & $\begin{array}{l}11.7 \% \\
(2009)\end{array}$ & 1.6 & $\begin{array}{c}\text { Not } \\
\text { tested }\end{array}$ & $15.8 \%$ \\
\hline 19 & $\begin{array}{c}122 \\
(1997)\end{array}$ & $\begin{array}{c}99 \\
(2009)\end{array}$ & -23 & $\begin{array}{l}\text { Not } \\
\text { tested }\end{array}$ & $-18.9 \%$ \\
\hline 42 & $\begin{array}{c}279 \\
(2002)\end{array}$ & $\begin{array}{c}255 \\
(2009)\end{array}$ & -24 & $\begin{array}{l}\text { Not } \\
\text { tested }\end{array}$ & $-8.6 \%$ \\
\hline 0.2 & $\begin{array}{c}3.2 \\
(1997)\end{array}$ & $\begin{array}{c}4.6 \\
(2009)\end{array}$ & 1.4 & $\begin{array}{l}\text { Not } \\
\text { tested }\end{array}$ & $43.7 \%$ \\
\hline $14 \%$ & $\begin{array}{c}17 \% \\
(1988-94)\end{array}$ & $\begin{array}{c}11 \% \\
(2005-08)\end{array}$ & -6 & Yes & $-35.3 \%$ \\
\hline
\end{tabular}

(continued) 
Figure 25-1. Progress Toward Target Attainment for Focus Area 25: Sexually Transmitted Diseases (continued)

Moved away from target ${ }^{1}$
Moved toward target

25-6. Treatment for pelvic inflammatory disease (PID) among females (15-44 years)

25-7. Fertility problems among childless females with an STD or PID (15-44 years)

25-9. Congenital syphilis (new cases per 100,000 live births)

25-11a. Students who never had sexual intercourse (grades 9-12)

25-11b. Students who had sexual intercourse, but not in the past 3 months (grades 9-12)

25-11c. Students who used condoms at last intercourse (grades 9-12)

25-16. Annual screening for genital chlamydia among females $\leq 25$ years

a. Enrolled in commercial managed care organizations (MCOs)

b. Enrolled in Medicaid managed care organizations (MCOs)

Met or exceeded target
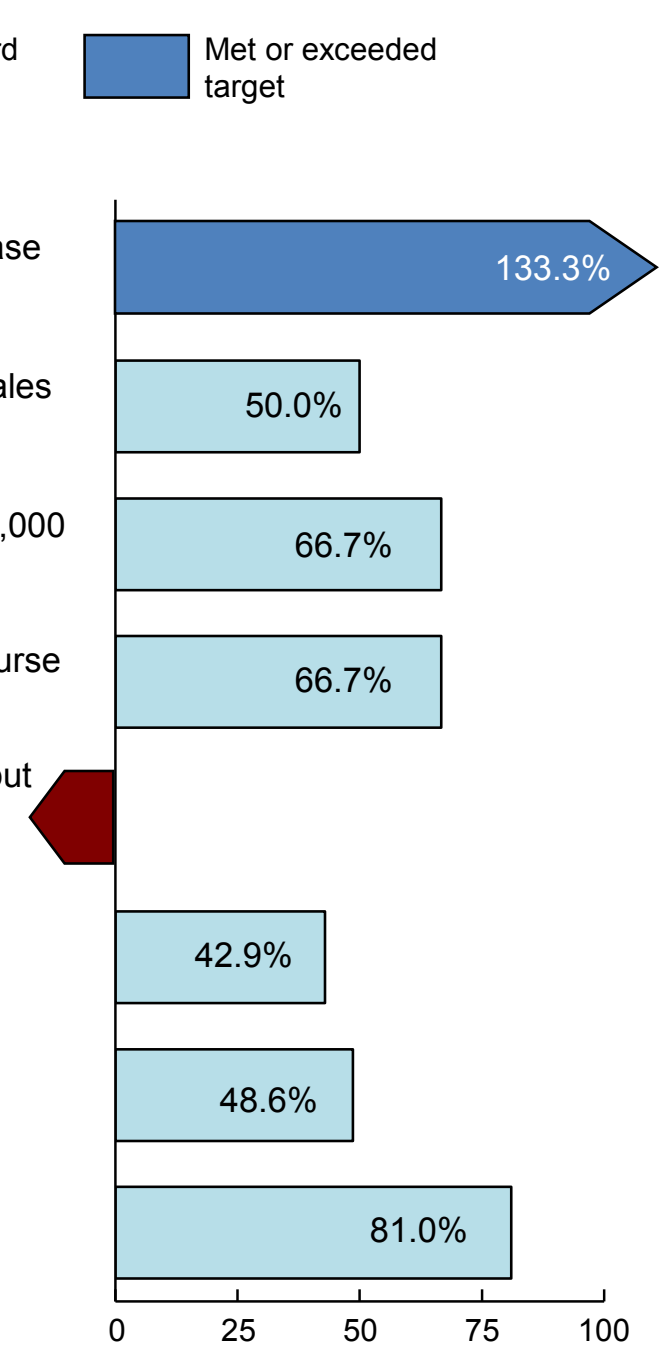

Percent of targeted change achieved ${ }^{5}$

\begin{tabular}{|c|c|c|c|c|c|}
\hline \multirow{2}{*}{$\begin{array}{l}2010 \\
\text { Target }\end{array}$} & \multirow{2}{*}{$\begin{array}{c}\text { Baseline } \\
\text { (Year) }\end{array}$} & \multirow{2}{*}{$\begin{array}{l}\text { Final } \\
\text { (Year) }\end{array}$} & \multicolumn{3}{|c|}{ Baseline vs. Final } \\
\hline & & & Difference $^{2}$ & $\begin{array}{l}\text { Statistically } \\
\text { Significant }^{3}\end{array}$ & $\begin{array}{l}\text { Percent } \\
\text { Change }^{4}\end{array}$ \\
\hline $5 \%$ & $\begin{array}{c}8 \% \\
(1995)\end{array}$ & $\begin{array}{c}4 \% \\
(2006-08)\end{array}$ & -4 & $\begin{array}{c}\text { Not } \\
\text { tested }\end{array}$ & $-50.0 \%$ \\
\hline $15 \%$ & $\begin{array}{c}27 \% \\
(1995)\end{array}$ & $\begin{array}{c}21 \% \\
(2006-08)\end{array}$ & -6 & No & $-22.2 \%$ \\
\hline 1 & $\begin{array}{c}28 \\
(1997)\end{array}$ & $\begin{array}{c}10 \\
(2009)\end{array}$ & -18 & $\begin{array}{c}\text { Not } \\
\text { tested }\end{array}$ & $-64.3 \%$ \\
\hline $56 \%$ & $\begin{array}{c}50 \% \\
\text { (1999) }\end{array}$ & $\begin{array}{c}54 \% \\
(2009)\end{array}$ & 4 & $\begin{array}{c}\text { Not } \\
\text { tested }\end{array}$ & $8.0 \%$ \\
\hline $30 \%$ & $\begin{array}{c}27 \% \\
\text { (1999) }\end{array}$ & $\begin{array}{c}26 \% \\
\text { (2009) }\end{array}$ & -1 & $\begin{array}{c}\text { Not } \\
\text { tested }\end{array}$ & $-3.7 \%$ \\
\hline $65 \%$ & $\begin{array}{c}58 \% \\
\text { (1999) }\end{array}$ & $\begin{array}{c}61 \% \\
(2009)\end{array}$ & 3 & $\begin{array}{c}\text { Not } \\
\text { tested }\end{array}$ & $5.2 \%$ \\
\hline $62 \%$ & $\begin{array}{c}25 \% \\
(2002)\end{array}$ & $\begin{array}{c}43 \% \\
(2009)\end{array}$ & 18 & $\begin{array}{c}\text { Not } \\
\text { tested }\end{array}$ & $72.0 \%$ \\
\hline $62 \%$ & $\begin{array}{c}41 \% \\
(2002)\end{array}$ & $\begin{array}{c}58 \% \\
\text { (2009) }\end{array}$ & 17 & $\begin{array}{l}\text { Not } \\
\text { tested }\end{array}$ & $41.5 \%$ \\
\hline
\end{tabular}

(continued) 


\section{Figure 25-1. Progress Toward Target Attainment for Focus Area 25: Sexually Transmitted Diseases (continued)}

\section{NOTES}

See the Reader's Guide for more information on how to read this figure. See DATA2010 at http://wonder.cdc.gov/data2010 for all Healthy People 2010 tracking data. Tracking data are not available for objectives 25-5 and 25-13. Objective 25-8 has been moved to Focus Area 13; see objective 13-18. Objectives 25-10, 25-12, 25-14, 25-15, 25-17, 25-18, and 25-19 were deleted at the Midcourse Review.

\section{FOOTNOTES}

${ }^{1}$ Movement away from target is not quantified using the percent of targeted change achieved. See Technical Appendix for more information.

${ }^{2}$ Difference $=$ Final value - Baseline value. Differences between percents (\%) are measured in percentage points.

${ }^{3}$ When estimates of variability are available, the statistical significance of the difference between the final value and the baseline value is assessed at the 0.05 level. See Technical Appendix for more information.

${ }^{4}$ Percent change $=\frac{\text { Final value }- \text { Baseline value }}{\text { Baseline value }} \times 100$

${ }^{5}$ Percent of targeted change achieved $=\frac{\text { Final value }- \text { Baseline value }}{\text { Healthy People } 2010 \text { target }- \text { Baseline value }} \times 100$.

\section{DATA SOURCES}

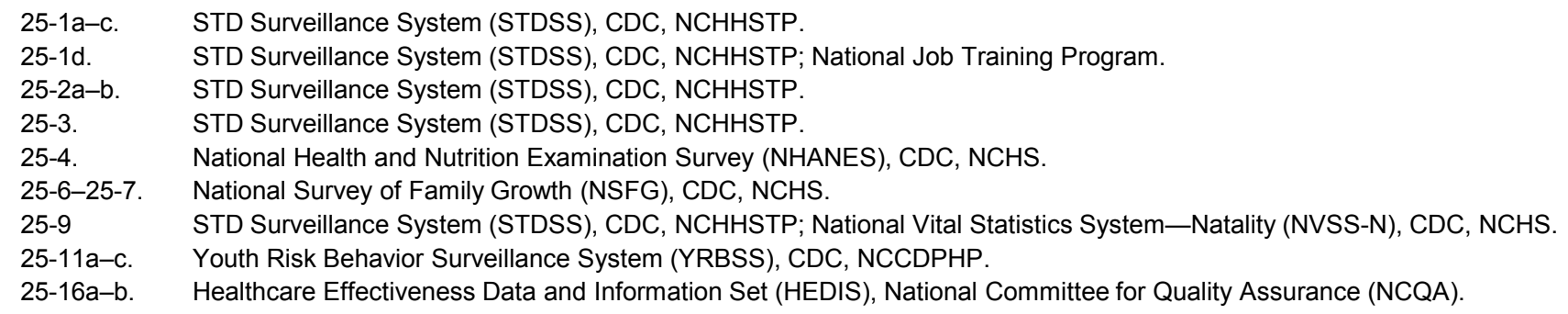


Figure 25-2. Health Disparities Table for Focus Area 25: Sexually Transmitted Diseases Disparities from the best group rate for each characteristic at the most recent data point and changes in disparity from the baseline to the most recent data point.

\begin{abstract}
Population-based objectives
25-1a. Chlamydia infections-Females 15-24 years attending family planning clinics $(1997,2009) \dagger$

b. Chlamydia infections-Females $15-24$ years attending STD clinics $(1997,2009) \dagger$

c. Chlamydia infections-Males 15-24 years attending STD clinics $(1997,2009) \dagger$

d. Chlamydia infections-Females $\leq 24$ years enrolled in National Job Training Program (2002, 2009)†

25-2a. Gonorrhea-New cases per 100,000 population (1997, 2009)†

b. Gonorrhea-New cases per 100,000 population among females $15-44$ years $(2002,2009) \dagger$

25-3. Domestic transmission of primary and secondary syphilis (new cases per 100,000 population) (1997, 2009)†

25-4. Genital herpes infection among adults 20-29 years (1988-94, 2005-08)*

25-6. Treatment for pelvic inflammatory disease (PID) among females (15-44 years) $(1995,2006-08) \ddagger$

25-7. Fertility problems among childless females with an STD or PID (15-44 years) $(1995,2006-08)^{*}$

25-9. Congenital syphilis (new cases per 100,000 live births) $(1997,2009) \dagger$

25-11a. Students who never had sexual intercourse (grades 9-12) $(1999,2009) \ddagger$

25-11b. Students who had sexual intercourse, but not in the past 3 months (grades 9-12) $(1999,2009) \ddagger$

25-11c. Students who used condoms at last intercourse (grades 9-12) (1999, 2009)‡
\end{abstract}

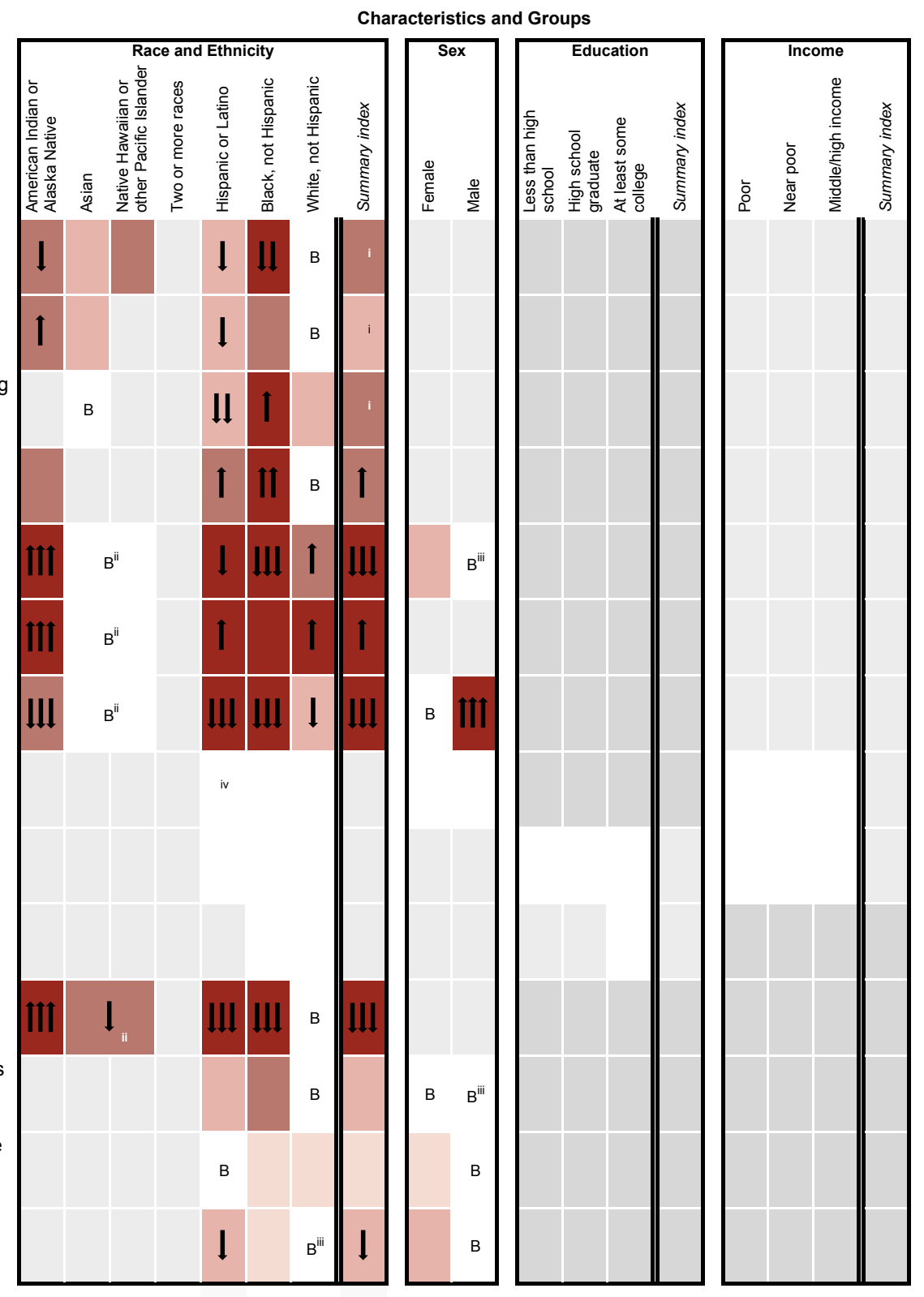

(continued) 


\section{Figure 25-2. Health Disparities Table for Focus Area 25: Sexually Transmitted Diseases (continued)}

NOTES

See DATA2010 at http://wonder.cdc.gov/data2010 for all Healthy People 2010 tracking data. Disparity data are either unavailable or not applicable for objectives 25-5, 25-8, 25-13, and 25-16a and b. Objectives 25-10, 25-12, 25-14, 25-15, and 25-17 through 25-19, were deleted at Midcourse Review.

Years in parentheses represent the baseline and most recent data years (if available).

Disparity from the best group rate is defined as the percent difference between the best group rate and each of the other group rates for a characteristic (e.g., race and ethnicity). The summary index is the average of these percent differences for a characteristic. Change in disparity is estimated by subtracting the disparity at baseline from the disparity at the most recent data point. Change in the summary index is estimated by subtracting the summary index at baseline from the summary index at the most recent data point. See Technical Appendix for more information.

LEGEND

\begin{tabular}{|c|c|c|c|c|c|}
\hline $\begin{array}{l}\text { The "best" group rate at the most recent } \\
\text { data point. }\end{array}$ & B & $\begin{array}{l}\text { The group with the best rate for } \\
\text { specified characteristic. }\end{array}$ & b & $\begin{array}{l}\text { Most favorable group rate for specified } \\
\text { characteristic, but reliability criterion not met. }\end{array}$ & $\begin{array}{l}\text { Reliability criterion for best } \\
\text { group rate not met, or data } \\
\text { available for only one group. }\end{array}$ \\
\hline & \multicolumn{5}{|c|}{ Percent difference from the best group rate } \\
\hline $\begin{array}{l}\text { Disparity from the best group rate at the } \\
\text { most recent data point. }\end{array}$ & & $\begin{array}{l}\text { Less than } 10 \% \text {, or difference not } \\
\text { statistically significant (when } \\
\text { estimates of variability are available). }\end{array}$ & & $10 \%-49 \%$ & $100 \%$ or more \\
\hline
\end{tabular}

Changes in disparity over time are shown when:

(a) disparities data are available at both baseline and most recent time points;

(b) data are not for the group(s) indicated by "B" or "b" at either time point; and

(c) the change is greater than or equal to 10 percentage points and statistically

significant, or when the change is greater than or equal to 10 percentage

points and estimates of variability were not available. See Technical Appendix.

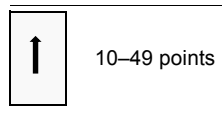

Increase in disparity (percentage points)

等

Availability of Data

Data not available.

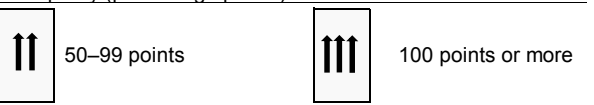

Decrease in disparity (percentage points)

I 10-49 points $\mathbb{\text { II }}$ 50-99 points $\quad$ III 100 points or more

FOOTNOTES

* Measures of variability were available. Thus, the variability of best group rates was assessed, and statistical significance was tested. Disparities of $10 \%$ or more are displayed when the differences from the best group rate are statistically significant at the 0.05 level. Changes in disparities over time are indicated by arrows when the changes are greater than or equal to 10 percentage points and are statistically significant at the 0.05 level. See Technical Appendix.

$†$ Measures of variability were not available. Thus, the variability of best group rates was not assessed, and statistical significance could not be tested. Nonetheless, disparities and changes in disparities over time are displayed according to their magnitude. See Technical Appendix.

$\ddagger$ Measures of variability were available only for the most recent data. Thus, the variability of best group rates was assessed only for the most recent data, and statistical significance was tested only for the most recent data. Disparities of $10 \%$ or more are displayed when the differences from the best group rate are statistically significant at the 0.05 level. Changes in disparities over time are displayed according to their magnitude, since measures of variability were not available at baseline and therefore statistical significance of changes in disparity could not be tested. See Technical Appendix.

i Change in the summary index cannot be assessed. See Technical Appendix.

ii Data are for Asian or Pacific Islander.

iii The group with the best rate at the most recent data point is different from the group with the best rate at baseline. Both rates met the reliability criterion. See Technical Appendix.

iv Data are for Mexican American.

\section{DATA SOURCES}

25-1a-c. STD Surveillance System (STDSS), CDC, NCHHSTP.

25-1d. STD Surveillance System (STDSS), CDC, NCHHSTP; National Job Training Program.

25-2a-b. STD Surveillance System (STDSS), CDC, NCHHSTP.

25-3. STD Surveillance System (STDSS), CDC, NCHHSTP.

25-4. National Health and Nutrition Examination Survey (NHANES), CDC, NCHS.

25-6-25-7. National Survey of Family Growth (NSFG), CDC, NCHS.

25-9. STD Surveillance System (STDSS), CDC, NCHHSTP; National Vital Statistics System—Natality (NVSS-N), CDC, NCHS.

25-11a-c. Youth Risk Behavior Surveillance System (YRBSS), CDC, NCCDPHP. 
Figure 25-3. Chlamydia Infections - (New Cases Per 100,000 Population) 2009

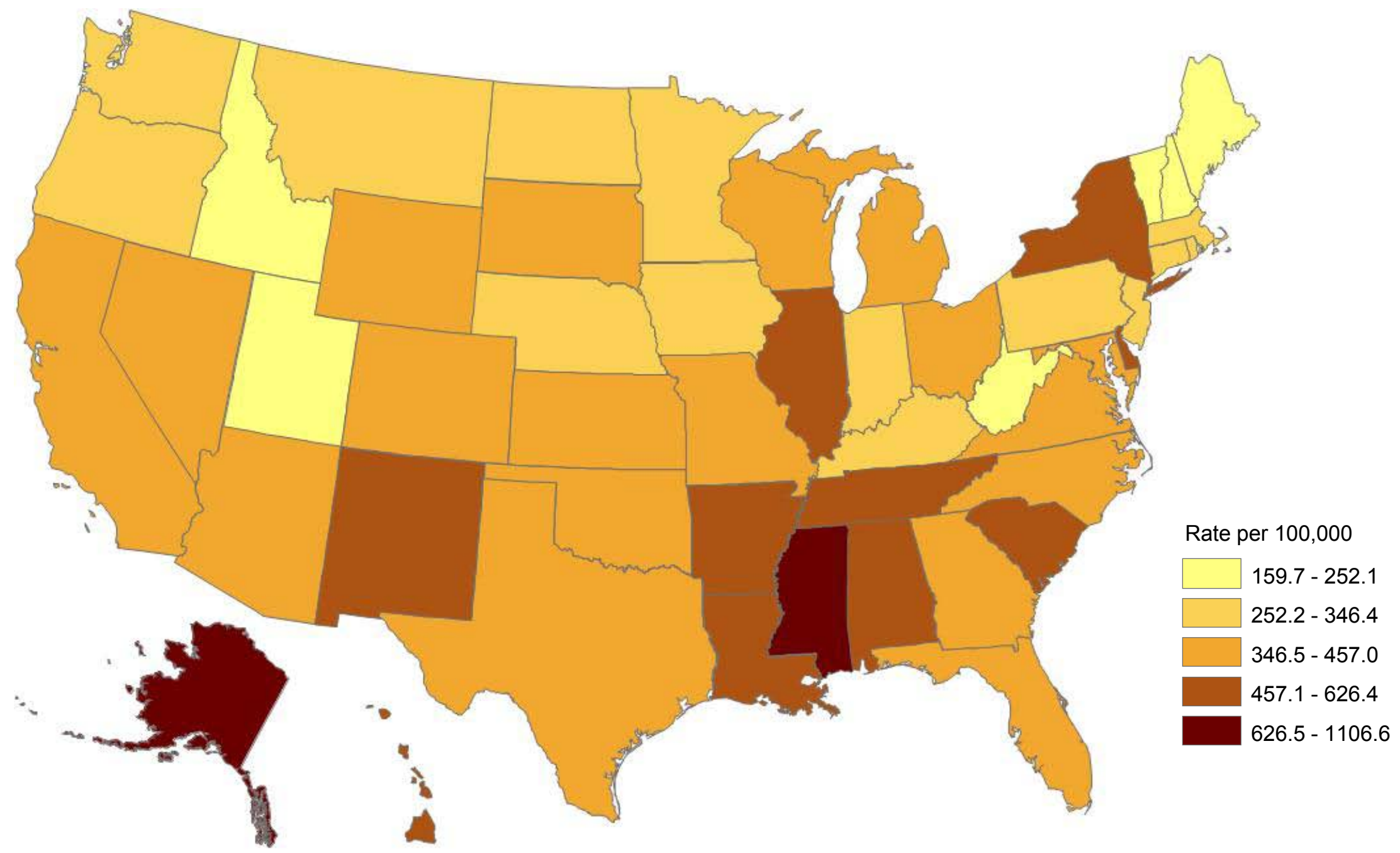

Notes: Data are crude rates, not age-adjusted to the 2000 standard population. Rates are displayed by a modified Jenks classification for U.S. states. National data for the objective come from the STD Surveillance System (STDSS) and is the basis for setting the target. State data from State and local Health Department STD Control Programs may not be comparable to the national data from the STDSS. Both US rates are shown for comparison purposes. SOURCE: STD Surveillance System (STDSS), CDC, NCHHSTP. 
Figure 25-4. Gonorrhea - (New Cases Per 100,000 Population) 2009

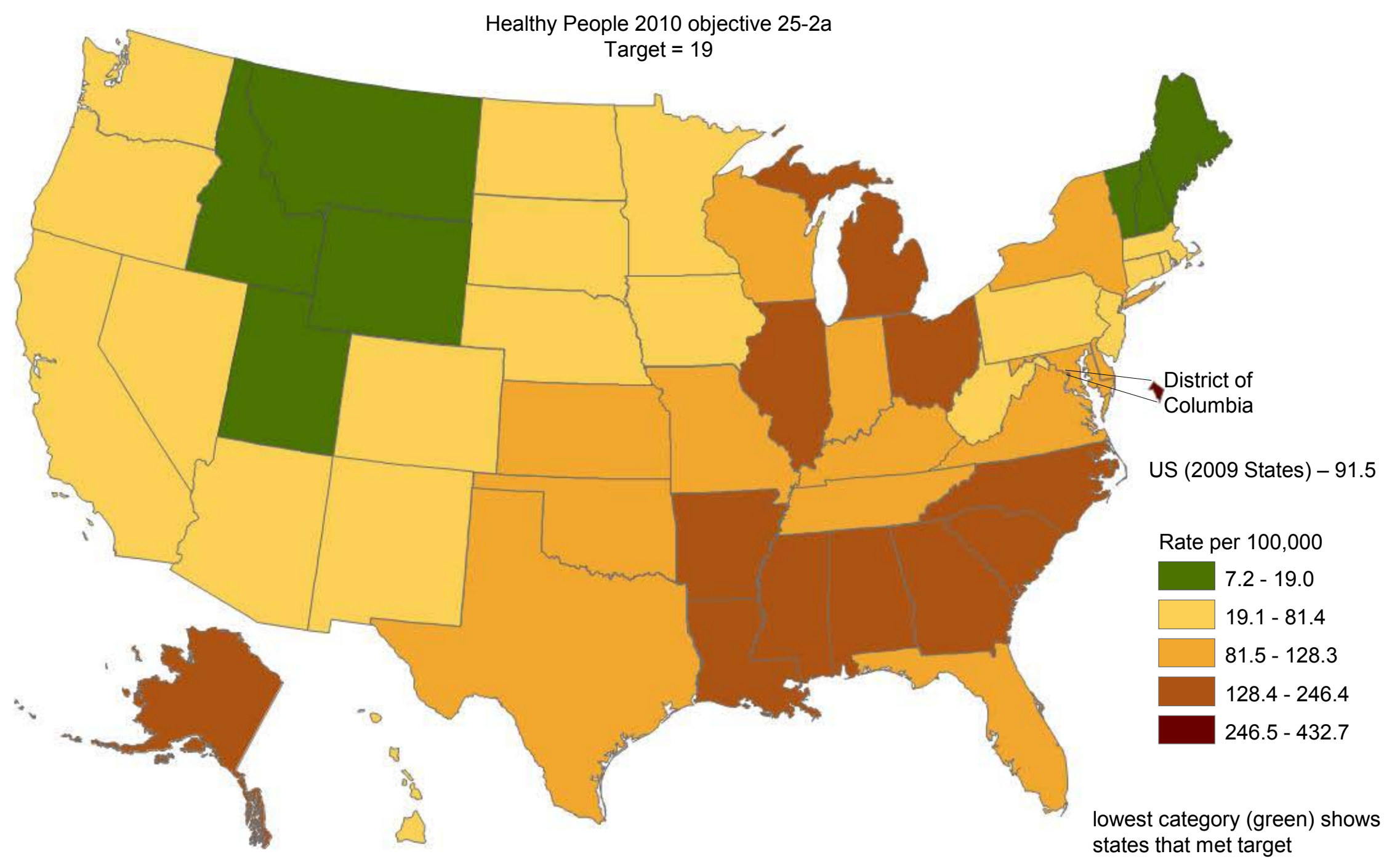

Notes: Data are crude rates, not age-adjusted to the 2000 standard population. Rates are displayed by a modified Jenks classification for U.S. states. National data for the objective come from the STD Surveillance System (STDSS) and is the basis for setting the target. State data from State and local Health Department STD Control Programs may not be comparable to the national data from the STDSS. Both US rates are shown for comparison purposes. SOURCE: STD Surveillance System (STDSS), CDC, NCHHSTP. 
Figure 25-5. Domestic Transmission of Primary and Secondary Syphilis

(New Cases Per 100,000 Population) 2009

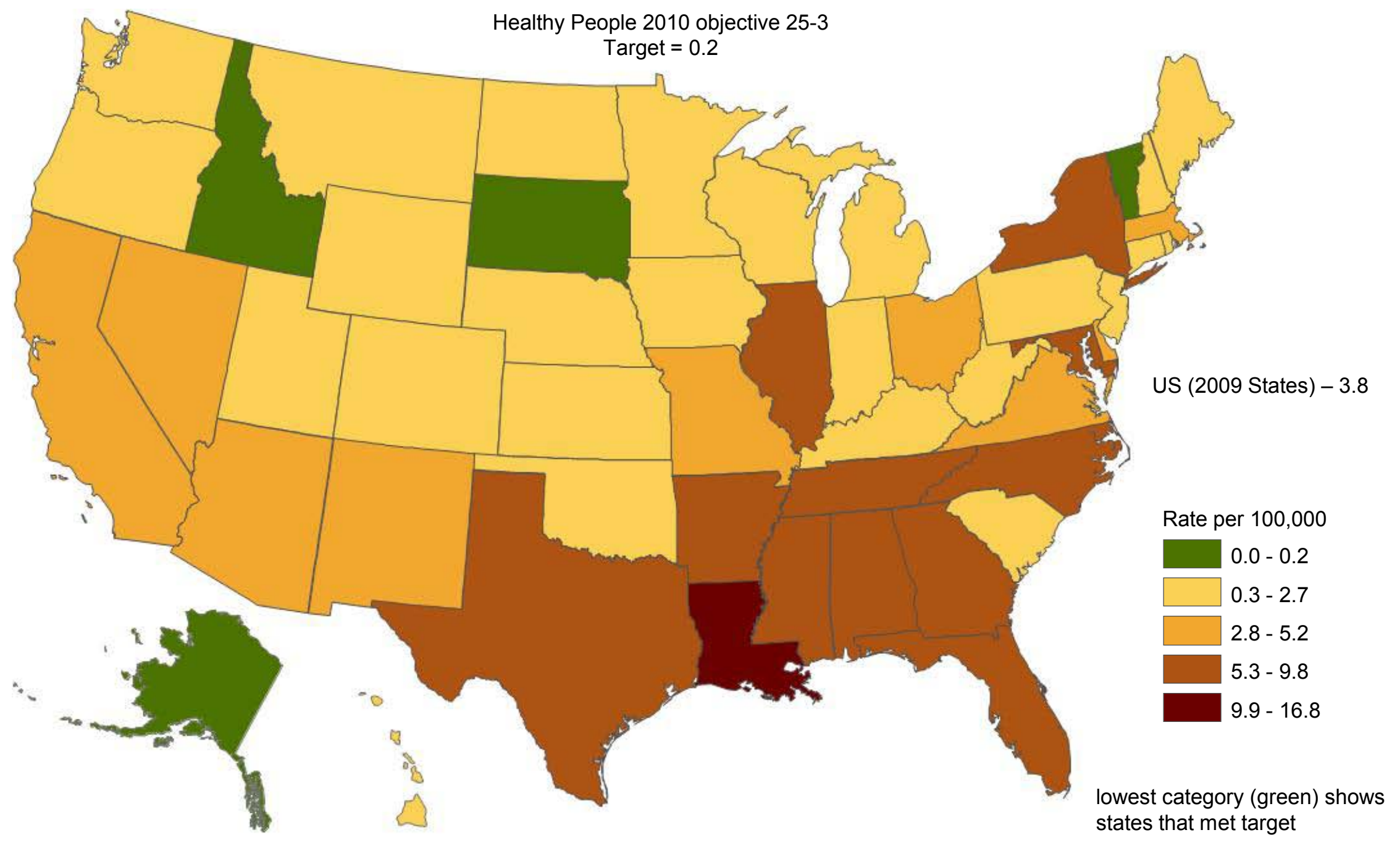

Notes: Data are crude rates, not age-adjusted to the 2000 standard population. Rates are displayed by a modified Jenks classification for U.S. states. National data for the objective come from the STD Surveillance System (STDSS) and is the basis for setting the target. State data from State and local Health Department STD Control Programs may not be comparable to the national data from the STDSS. Both US rates are shown for comparison purposes. SOURCE: STD Surveillance System (STDSS), CDC, NCHHSTP. 


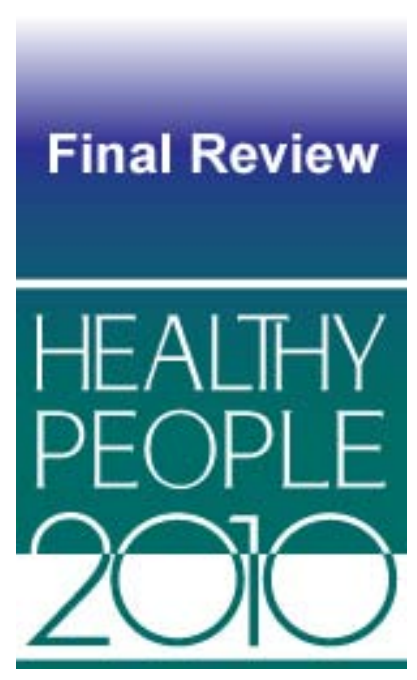

\section{Substance Abuse}

\section{Lead Agency}

National Institutes of Health

Substance Abuse and Mental Health Services Administration

\section{Contents}

Goal

Highlights.

Summary of Progress.

Transition to Healthy People 2020.

Data Considerations

References and Notes

Comprehensive Summary of Objectives

Progress Chart

Health Disparities Table

Cirrhosis Deaths-Map 


\section{Goal: Reduce substance abuse to protect the health, safety, and quality of life for all, especially children}

This chapter includes objectives that track alcohol and drug-related deaths, the use of alcohol and illicit drugs by adolescents and young adults, adolescent attitudes toward alcohol and/or drug use, and state laws addressing driving under the influence of alcohol (DUI).

All Healthy People tracking data quoted in this chapter, along with technical information and operational definitions for each objective, can be found in the Healthy People 2010 database, DATA2010, available from http://wonder.cdc.gov/data2010/.

More information about this focus area can be found in the following publications:

- Healthy People 2010: Understanding and Improving Health, available from http://www.healthypeople.gov/2010/Document/tableofcontents.htm\#under.

- Healthy People 2010 Midcourse Review, available from http://www.healthypeople.gov/2010/data/midcourse/html/default.htm\#FocusAreas.

\section{Highlights}

- Substantial progress was achieved in objectives for this Focus Area during the past decade [1]. Almost two thirds (63\%) of the Substance Abuse objectives with data to measure progress moved toward or achieved their Healthy People 2010 targets (Figure 26-1). However, health disparities of $10 \%$ or more were observed among racial and ethnic populations, as well as by sex, education level, and income (Figure 26-2), some of which are highlighted below [2].

- The rate of alcohol-related motor vehicle crash deaths (objective $26-1 \mathrm{a}$ ) declined by $24.5 \%$ between 1998 and 2009, from 5.3 to 4.0 deaths per 100,000 population, exceeding the Healthy People 2010 target of 4.8 .

- Among racial and ethnic populations, the Asian population had the lowest (best) rate of alcohol-related motor vehicle crash deaths, 0.6 per 100,000 population in 2008. The Hispanic or Latino, white non-Hispanic, black non-Hispanic, Native Hawaiian or Other Pacific Islander, and American Indian or Alaska Native populations had rates of $3.3,3.6,3.9,3.9$ and 10.9 per 100,000 population in 2008 , respectively.

- The rates for the Hispanic or Latino, white non-Hispanic, black non-Hispanic, and Native Hawaiian or Other Pacific Islander populations were five and a half to six and a half times the best rate (that for the Asian population). The rate for the American Indian or Alaska Native population was more than 18 times the best rate [2].

- Between 2000 and 2008, the disparities between these populations and the Asian population increased by at least 100 percentage points [3].

- Females had a lower (better) rate of motor vehicle crash deaths than males, 1.7 per 100,000 population in 2009 . The rate for males, 6.3 in 2009 , was more than three and a half times the rate for females [2]. 
- The cirrhosis death rate (objective 26-2) declined by 5.2\% between 1999 and 2007, from 9.6 to 9.1 deaths per 100,000 population (age adjusted), moving toward the target of 3.2.

- Among racial and ethnic groups, the Asian or Pacific Islander population had the lowest (best) cirrhosis death rate, 3.3 deaths per 100,000 population (age adjusted) in 2007. The white non-Hispanic, black non-Hispanic, Hispanic or Latino, and American Indian or Alaska Native populations had rates of 7.5, 8.7, 13.8, and 24.8 deaths per 100,000 (age adjusted) in 2007, respectively.

- The rate for the white non-Hispanic population was almost two and a half times the best rate (that for the Asian or Pacific Islander population); the rate for the black non-Hispanic population was more than two and a half times the best rate; the rate for the Hispanic or Latino population was more than four times the best rate; and the rate for the American Indian or Alaska Native population was about seven and a half times the best rate [2].

- Females had a better cirrhosis death rate than males, 5.9 vs. 12.7 deaths per 100,000 population (age adjusted) in 2007 . The rate for males was more than twice the rate for females.

- Among education groups, persons aged 25-64 years with at least some college education had the lowest (best) cirrhosis death rate, 5.6 deaths per 100,000 population (age adjusted), in 2002. High school graduates had a rate of 15.2, and persons with less than a high school education had a rate of 20.9. The rate for high school graduates was more than two and a half times the best group rate, while the rate for persons with less than a high school education was more than three and a half times the best group rate [2].

- Cirrhosis death rates varied by geographical area. In 2005-07, the rates were highest in areas of the Southwest and West (Figure 26-3).

- The rate of drug-induced deaths (objective 26-3) increased by $85.3 \%$ between 1999 and 2007 , from 6.8 deaths per 100,000 population (age adjusted) to 12.6, moving away from the 2010 target of 1.2 .

- Among racial and ethnic groups, the Asian or Pacific Islander population had the lowest (best) rates of drug-induced deaths: 1.4 per 100,000 population (age adjusted) in 1999 and 2.0 in 2007. The Hispanic or Latino population had rates of 6.5 per 100,000 (age adjusted) in 1999 and 6.5 in 2007; the black non-Hispanic population had rates of 9.4 per 100,000 (age adjusted) in 1999 and 11.4 in 2007; the American Indian or Alaska Native population had rates of 6.1 per 100,000 (age adjusted) in 1999 and 12.1 in 2007; and the white non-Hispanic population had rates of 6.8 per 100,000 (age adjusted) in 1999 and 15.1 in 2007.

- In 2007, the rate for the Hispanic or Latino population was almost three and a half times the best rate (that for the Asian or Pacific Islander population); the rates for the black non-Hispanic and American Indian or Alaska Native population were about six times the best rate; the rate for the white nonHispanic population was more than seven and a half times the best rate [2].

- Between 1999 and 2007, the disparity between the American Indian or Alaska Native population and the Asian or Pacific Islander population (the group with the best rate) increased by 169.3 percentage points, while the disparity between the white non-Hispanic and the Asian or Pacific Islander populations increased by 269.3 percentage points. 
- During the same period, the disparity between the Hispanic or Latino population and the Asian or Pacific Islander population decreased by 132 percentage points [3].

- Among education groups, persons aged 25-64 years with at least some college education had the lowest (best) rate of drug induced deaths, 7.4 per 100,000 population (age adjusted), in 2002. High school graduates had a rate of 22.4, about three times the best group rate. Persons with less than a high school education had a rate of 27.3, more than three and a half times the best group rate [2].

- Drug-related hospital emergency department visits (objective 26-4) increased by $27.9 \%$ between 2004 and 2009, from 1,619.05 (thousands) to 2,070.44, moving away from the 2010 target of 1,044.46 (thousands).

- The proportion of students in grades 9-12 who reported riding with a driver who had been drinking alcohol within the past 30 days (objective 26-6) decreased by $15.2 \%$ between 1999 and 2009 , from $33 \%$ to $28 \%$, exceeding the 2010 target of $30 \%$.

- The proportion of high school seniors who never consumed alcohol (objective 26-9c) increased by $47.4 \%$ between 1998 and 2009, from $19 \%$ to $28 \%$, moving toward the 2010 target of 29\%. During the same period, the proportion of high school seniors who never used illicit drugs (objective $26-9 \mathrm{~d}$ ) increased by $15.2 \%$, from $46 \%$ to $53 \%$, moving toward the 2010 target of $56 \%$.

- Between 1998 and 2009, steroid use among students in eighth, tenth, and twelfth grades (objectives $26-14$ a through c) increased by $8.3 \%, 8.3 \%$ and $29.4 \%$ respectively, from $1.2 \%$ to $1.3 \%, 1.2 \%$ to $1.3 \%$, and $1.7 \%$ to $2.2 \%$ respectively, moving away from the 2010 targets of $0.4 \%$ each.

- The number of states and the District of Columbia with laws restricting the legal operation of motor vehicles for drivers who had been drinking alcohol to a maximum blood alcohol concentration of 0.08 (objective 26-25) increased from 15 in 1998 to 51 in 2006, meeting the 2010 target of 51.

\section{Summary of Progress}

- Figure 26-1 presents a quantitative assessment of progress in achieving the Healthy People 2010 objectives for Substance Abuse [1]. Data to measure progress toward target attainment were available for 38 objectives. Of these:

- Four objectives (26-1a, 26-6, 26-16d, and 26-25) met or exceeded their 2010 targets.

- Twenty objectives moved toward their targets. A statistically significant difference between the baseline and the final data points was observed for 14 of these objectives (26-2, 26-9a through d, 26-10a and b, 26-11a and d, 26-15, 26-16b and e, and $26-17 \mathrm{a}$ and $\mathrm{b}$ ). No significant differences were observed for three objectives (26-16a, c, and f); and data to test the significance of the difference were unavailable for three objectives (26-13a and b, and 26-20).

- Three objectives (26-10c, 26-18b, and 26-24) showed no change. 
- Eleven objectives moved away from their targets. A statistically significant difference between the baseline and final data points was observed for two of these objectives (26-3 and 26-4). No significant differences were observed for seven objectives (26-11c, 26-14a through c, 26-17c, 26-18a, and 26-21); and data to test the significance of the difference were unavailable for two objectives $(26-11 \mathrm{~b}$ and 26-12).

- Five objectives (26-5, 26-7, 26-19, 26-22, and 26-23) remained developmental and two objectives (26-8a and b) had no data available to measure progress [4]. Three objectives (26-1b through d) were dropped during the decade [5].

- Figure 26-2 displays health disparities in Substance Abuse from the best group rate for each characteristic at the most recent data point [2]. It also displays changes in disparities from baseline to the most recent data point [3].

- Twenty-three objectives had statistically significant racial and ethnic health disparities of $10 \%$ or more (objectives $26-2$ through $26-4$; 26-6; 26-9a through d; 26-10a through c; 26-11a, c, and d; 26-14a; 26-16a c, d, and f; 26-17a through c; and 26-21). Three additional objectives had racial and ethnic health disparities of $10 \%$ or more but lacked data to assess statistical significance (objectives 26-1a, and 26$13 \mathrm{a}$ and $\mathrm{b})$. Of these 26 objectives, the black non-Hispanic population had the best rate for nine objectives (26-4, 26-9c and d, 26-10a and b, 26-11a and c, 26-17c, and 26-21). The Asian population had the best rate for six objectives (26-1a, 26-9a, 26$13 \mathrm{a}$ and $\mathrm{b}$, and $26-17 \mathrm{a}$ and $\mathrm{b}$ ). The white non-Hispanic population had the best rate for five objectives (26-6, 26-9b, 26-14a, and 26-16a and d); the Hispanic or Latino population had the best rate for four objectives (26-10c, 26-11d, and 26-16c and f); and the Asian or Pacific Islander population had the best rate for two objectives (262 and 26-3).

- Sixteen objectives had statistically significant health disparities of $10 \%$ or more by sex (objectives 26-2 through 26-4, 26-9d, 26-10b and c, 26-11a through c, 26-16a through $\mathrm{f}$, and 26-17a). One additional objective had health disparities of $10 \%$ or more by sex but no data to assess statistical significance (objective 26-1a). Females had the better group rate for all 17 of these objectives.

- Three objectives had statistically significant health disparities of $10 \%$ or more by education level (objectives 26-2, 26-3, and 26-10c) and one objective had health disparities of $10 \%$ or more by education level but no data to assess statistical significance (objectives 26-13b). Persons with at least some college education had the best group rate for all four of these objectives.

- Persons in the poor population had the best group rate for four of the six objectives with statistically significant health disparities of $10 \%$ or more by income (objectives 26-10a, 26-11d, and 26-18a and b). Persons in the middle/high income population had the best group rate for the remaining two objectives (26-9b and 26-15).

- Racial and ethnic health disparities of $100 \%$ or more were observed for several objectives, as were health disparities of $100 \%$ or more by sex and education level. Changes in disparity between the baseline and most recent data points also were observed. Many of these disparities are discussed in the Highlights, above. 


\section{Transition to Healthy People 2020}

For Healthy People 2020, the focus of the Substance Abuse topic area continues to address a wide range of health behaviors and interventions. Specific objectives are targeted to protect the health, safety, and quality of life for all, especially children.

The Healthy People 2020 Substance Abuse topic area can be grouped into three sections:

- Policy and prevention

- Screening and treatment

- Epidemiology and surveillance.

The differences between the Healthy People 2010 and Healthy People 2020 objectives are summarized below:

- The Healthy People 2020 Topic Area has 44 objectives whereas the Healthy People 2010 Substance Abuse Focus Area had 48 objectives.

- Twenty-seven Healthy People 2010 objectives were retained "as is" [6]. These include: nine objectives that target perceptions about and disapproval of substance use and abuse (objectives 26-16a through f and 26-17a through c), eight drug use/abstinence objectives (26-9d, 26-10b and c, 26-12, 26-14a through c, and 26-15), four treatment objectives (2618a and b, 26-20 and 26-21), three alcohol use/abstinence objectives (26-9c, and 26-11a and b), two mortality objectives (26-2 and 26-3) and an objective that targets riding with a driver who has been drinking alcohol (objective 26-6).

- Five Healthy People 2010 objectives were archived [7].

- Statistics to track lost productivity due to alcohol and drug abuse have not been calculated at the national level since baseline year data were obtained. As a result, these two objectives (26-8a and b) could not be retained in Healthy People 2020.

- During the course of the tracking period for Healthy People 2010, all states and the District of Columbia had enacted laws restricting the legal operation of motor vehicles for drivers, aged 21 years and over, who had been drinking alcohol to a maximum blood alcohol concentration of 0.08 . Due to the success experienced in the past decade, this objective (26-25) was archived in Healthy People 2020.

- Drug-related emergency department visits were tracked with data obtained from the Drug Abuse Warning Network (DAWN). Because all data collection activity will end once 2010 data have been collected, this objective (26-3) will be archived in Healthy People 2020.

- The Healthy People 2010 objective (26-24) that tracked administrative license revocation laws for persons under the influence of intoxicants also was archived in Healthy People 2020.

- Eight Healthy People 2010 objectives were dropped [5]. Five of these had remained developmental, and three were dropped during the decade [4].

- The dropped objectives include:

- Drug-related motor vehicle crash deaths (objective 26-1c)

- Drug-related motor vehicle crash injuries (objective 26-1d)

- Alcohol-related motor vehicle crash injuries (objective 26-1b). 
- The data systems proposed to measure five of the Substance Abuse objectives were unable to produce reliable estimates; therefore, the following objectives were not included in Healthy People 2020:

- Alcohol-related emergency department visits (objective 26-5)

- Intentional injuries from alcohol and drug-related violence (objective 26-7)

- Treatment in correctional institutions (objective 26-19)

- Emergency department referrals for alcohol or drug problems and suicide attempts (objective 26-22)

- Community partnerships and coalitions to prevent substance abuse (objective 26-23).

- Eight Healthy People 2010 objectives were modified to create seven Healthy People 2020 objectives [8].

- Alcohol-related motor vehicle crash deaths (objective 26-1a) were tracked in Healthy People 2010 as a rate per 100,000 population. In Healthy People 2020, the rate of death will be tracked per vehicle miles traveled (VMT).

- The Healthy People 2010 objectives that tracked the average age at first use of adolescents who used alcohol (objective 26-9a) and marijuana (objective 26-9b) for the first time in the previous year were modified. The two revised objectives will track the proportion of at risk adolescents who used alcohol and marijuana for the first time in the previous year.

- The proportion of adolescents who did not use alcohol or illicit drugs in the past 30 days (objective 26-10a) was tracked in Healthy People 2010. In Healthy People 2020 , the complement of this objective will be monitored (i.e., the proportion of adolescents who did use alcohol or illicit drugs in the past 30 days).

- In 2002, the National Institute on Alcohol Abuse and Alcoholism revised the definition of binge drinking for women from drinking five or more alcoholic beverages at the same time or within a couple of hours of each other to four or more alcoholic beverages [9]. For the Healthy People 2010, binge drinking for adolescents and adults (objectives 26-11c and d) was tracked with the original definition. Healthy People 2020 will track binge drinking with the revised definition.

- Male and female adults who exceeded guidelines for low-risk drinking (objectives 26-13a and b) were tracked separately in Healthy People 2010. In Healthy People 2020, the focus was modified slightly to track excessive drinking and the two objectives were combined into one.

- Ten new objectives, two of which are developmental, were added to the Healthy People 2020 Topic Area:

- Five objectives track past-year use of prescription drugs:

- Pain relievers

- Tranquilizers

- Stimulants

- Sedatives

- Any psychotherapeutic drug (including any noted above).

- Two new infrastructure-related objectives were added: one will track drug, driving while intoxicated (DWI) and other specialty courts, and the other will track states with mandatory ignition interlock laws for DWI offenders. 
- Two new treatment-related objectives were added: one will track referrals and follow-up of emergency department patients treated for alcohol and/or drug problems, and the other will track medical facilities that implement alcohol Screening and Brief Intervention (SBI).

- One new objective will track the number of deaths attributable to alcohol use.

Appendix D, "A Crosswalk Between Objectives From Healthy People 2010 to Healthy People 2020," summarizes the changes between the two decades of objectives, reflecting new knowledge and direction for this area.

\section{Data Considerations}

Education and income are the primary measures of socioeconomic status (SES) in Healthy People 2010. Most data systems used in Healthy People 2010 define income as a family's income before taxes. In order to facilitate comparisons among groups and over time, while adjusting for family size and for inflation, Healthy People 2010 categorizes income using the poverty thresholds developed by the U.S. Census Bureau. Thus, the three categories of family income that are primarily used are:

- Poor-below the Federal poverty level

- Near poor-100\% to $199 \%$ of the Federal poverty level

- Middle/high income-200\% or more of the Federal poverty level.

These categories may be overridden by considerations specific to the data system, in which case they are modified as appropriate. See Healthy People 2010: General Data Issues, referenced below.

Beginning in 2003, education data for cirrhosis and drug-induced deaths (objectives 26-2 and 26-3) from the National Vital Statistics System were suppressed. The educational attainment item was changed in the new U.S. Standard Certificate of Death in 2003 to be consistent with the U.S. Census Bureau data and to improve the ability to identify specific types of educational degrees. Many states, however, are still using the 1989 version of the U.S. Standard Certificate of Death, which focuses on highest school grade completed. As a result, educational attainment data collected using the 2003 version are not comparable with data collected using the 1989 version [10].

In general, data on educational attainment are presented for persons aged 25 years and over, consistent with guidance given by the U.S. Bureau of the Census. However, because of the requirements of the different data systems, the age groups used to calculate educational attainment for any specific objective may differ from the age groups used to report the data for other Healthy People 2010 objectives, as well as from select populations within the same objective. Therefore, the reader is urged to exercise caution in interpreting the data by educational attainment shown in the Health Disparities Table. See Healthy People 2010: General Data Issues, referenced below. 
Additional information on data issues is available from the following sources:

- All Healthy People 2010 tracking data can be found in the Healthy People 2010 database, DATA2010, available from http://wonder.cdc.gov/data2010/.

- Detailed information about the data and data sources used to support these objectives can be found in the Operational Definitions on the DATA2010 website, available from http://wonder.cdc.gov/data2010/focusod.htm.

- More information on statistical issues related to Healthy People tracking and measurement can be found in the Technical Appendix and in Healthy People 2010: General Data Issues, which is available in the Data Issues section of the NCHS Healthy People website under Healthy People 2010.

\section{References and Notes}

1. Displayed in the Progress Chart (Figure 26-1), the percent of targeted change achieved expresses the difference between the baseline and the final value relative to the initial difference between the baseline and the Healthy People 2010 target. As such, it is a relative measure of progress toward attaining the Healthy People 2010 target. See the Reader's Guide for more information. When standard errors were available, the difference between the baseline and the final value was tested at the 0.05 level of significance. See the Figure 26-1 footnotes, as well as the Technical Appendix, for more detail.

2. Information about disparities among select populations is shown in the Health Disparities Table (Figure 26-2). Disparity from the best group rate is defined as the percent difference between the best group rate and each of the other group rates for a characteristic. For example, racial and ethnic health disparities are measured as the percent difference between the best racial and ethnic group rate and each of the other racial and ethnic group rates. Similarly, disparities by sex are measured as the percent difference between the better group rate (e.g., female) and the rate for the other group (e.g., male). Some objectives are expressed in terms of favorable events or conditions that are to be increased, while others are expressed in terms of adverse events or conditions that are to be reduced. In order to facilitate comparison of health disparities across different objectives, disparity is measured only in terms of adverse events or conditions. For comparability across objectives, objectives that are expressed in terms of favorable events or conditions are re-expressed using the adverse event or condition for the purpose of computing disparity, but they are not otherwise restated or changed. For example, objective 1-1, to increase the proportion of persons with health insurance (e.g., $72 \%$ of the American Indian or Alaska Native population aged under 65 years had some form of health insurance in 2008), is expressed in terms of the percentage of persons without health insurance (e.g., $100 \%-72 \%=28 \%$ of the American Indian or Alaska Native population aged under 65 years did not have any form of health insurance in 2008) when the disparity from the best group rate is calculated. See the Reader's Guide for more information. When standard errors were available, the difference between the best group rate and each of the other group rates was tested at the 0.05 level of significance. See the Figure 26-2 footnotes, as well as the Technical Appendix, for more detail.

3. The change in disparity is estimated by subtracting the disparity at baseline from the disparity at the most recent data point and, therefore, is expressed as a change in percentage points. See the Reader's Guide for more information. When standard errors were available, the change in disparity was tested at the 0.05 level of significance. See the Figure 26-2 footnotes, as well as the Technical Appendix, for more detail. 
4. To be included in Healthy People 2010, an objective must have a national data source that provides a baseline and at least one additional data point for tracking progress. Some objectives lacked baseline data at the time of their development but had a potential data source and were considered of sufficient national importance to be included in Healthy People. These are called "developmental" objectives. When data become available, a developmental objective is moved to measurable status and a Healthy People target can be set.

5. Dropped objectives were not carried forward into Healthy People 2020. These objectives were either developmental or deleted at the Healthy People 2010 Midcourse Review or at another time in Healthy People 2010.

6. Retained "as is" objectives have no change in the numerator definition or in the denominator definition between the Healthy People 2010 and Healthy People 2020 objectives. These include objectives that were developmental in Healthy People 2010 and are developmental in Healthy People 2020 and for which no numerator or denominator information was available.

7. Archived objectives had at least one data point in Healthy People 2010 but were not carried forward into Healthy People 2020.

8. Modified objectives have some change in the numerator definition or in the denominator definition between the Healthy People 2010 and Healthy People 2020 objectives. These include objectives that went from developmental in Healthy People 2010 to measurable in Healthy People 2020 or vice versa.

9. NIAAA Newsletter, NIH Publication No. 04-5346. Available from: http://pubs.niaaa.nih.gov/publications/Newsletter/winter2004/Newsletter Number3.pdf

10. Xu JQ, Kochanek KD, Murphy SL, Tejada-Vera B. Deaths: Final data for 2007. National vital statistics reports; vol 58 no 19. Hyattsville, MD: National Center for Health Statistics. 2010. Available from: http://www.cdc.gov/nchs/data/nvsr/nvsr58/nvsr58 19.pdf.. 


\section{Comprehensive Summary of Objectives: Substance Abuse}

\begin{tabular}{|c|c|c|}
\hline Objective & Description & Data Sources or Objective Status \\
\hline $26-1 \mathrm{a}$ & Alcohol-related motor vehicle crash deaths (per 100,000 population) & $\begin{array}{l}\text { Fatality Analysis Reporting System (FARS), Department of } \\
\text { Transportation (DOT). }\end{array}$ \\
\hline $26-1 b$ & Alcohol-related motor vehicle crash injuries (per 100,000 population) & Dropped \\
\hline $26-1 c$ & Drug-related motor vehicle crash deaths (per 100,000 population) & Dropped \\
\hline $26-1 d$ & Drug-related motor vehicle crash injuries (per 100,000 population) & Dropped \\
\hline $26-2$ & Cirrhosis deaths (age adjusted, per 100,000 population) & National Vital Statistics System-Mortality (NVSS-M), CDC, NCHS. \\
\hline $26-3$ & Drug-induced deaths (age adjusted, per 100,000 population) & National Vital Statistics System-Mortality (NVSS-M), CDC, NCHS. \\
\hline $26-4$ & Drug-related hospital emergency department visits (thousands) & Drug Abuse Warning Network (DAWN), SAMHSA. \\
\hline $26-5$ & Alcohol-related hospital emergency department visits & Developmental \\
\hline $26-6$ & $\begin{array}{l}\text { Students who rode with a driver who had been drinking alcohol } \\
\text { (grades 9-12) }\end{array}$ & Youth Risk Behavior Surveillance System (YRBSS), CDC, NCCDPHP. \\
\hline $26-7$ & Intentional injuries from alcohol and drug-related violence & Developmental \\
\hline $26-8 \mathrm{a}$ & Lost productivity due to alcohol abuse (Loss in dollars per capita) & NIH, NIAAA. \\
\hline $26-8 b$ & Lost productivity due to drug abuse (Loss in dollars per capita) & Office of National Drug Control Policy (ONPCP). \\
\hline $26-9 a$ & $\begin{array}{l}\text { Average age at first use among adolescents who used alcohol for the } \\
\text { first time in past year (12-17 years) }\end{array}$ & National Survey on Drug Use and Health (NSDUH), SAMHSA. \\
\hline $26-9 b$ & $\begin{array}{l}\text { Average age at first use among adolescents who used marijuana for the } \\
\text { first time in past year (12-17 years) }\end{array}$ & National Survey on Drug Use and Health (NSDUH), SAMHSA. \\
\hline $26-9 c$ & High school seniors never consuming alcoholic beverages & Monitoring the Future Study (MTF), NIH, NIDA. \\
\hline $26-9 d$ & High school seniors never using illicit drugs & Monitoring the Future Study (MTF), NIH, NIDA. \\
\hline $26-10 a$ & $\begin{array}{l}\text { Adolescents not using alcohol or illicit drugs in past } 30 \text { days (12-17 } \\
\text { years) }\end{array}$ & National Survey on Drug Use and Health (NSDUH), SAMHSA. \\
\hline $26-10 b$ & Adolescents using marijuana in past 30 days (12-17 years) & National Survey on Drug Use and Health (NSDUH), SAMHSA. \\
\hline $26-10 c$ & Adults using illicit drugs in past 30 days (18+ years) & National Survey on Drug Use and Health (NSDUH), SAMHSA. \\
\hline $26-11 a$ & Binge drinking in the past 2 weeks-High school seniors & Monitoring the Future Study (MTF), NIH, NIDA. \\
\hline $26-11 b$ & Binge drinking in the past 2 weeks-College students & Monitoring the Future Study (MTF), NIH, NIDA. \\
\hline $26-11 c$ & Binge drinking in the past month-Adults $(18+$ years $)$ & National Survey on Drug Use and Health (NSDUH), SAMHSA. \\
\hline
\end{tabular}




\begin{tabular}{|c|c|c|}
\hline Objective & Description & Data Sources or Objective Status \\
\hline 26-11d & Binge drinking in the past month-Adolescents (12-17 years) & National Survey on Drug Use and Health (NSDUH), SAMHSA. \\
\hline $26-12$ & Average annual alcohol consumption (gallons per person, $14+$ years) & Alcohol Epidemiologic Data System (AEDS), NIH, NIAAA. \\
\hline $26-13 a$ & $\begin{array}{l}\text { Adults who exceed guidelines for low-risk drinking-Females (21+ } \\
\text { years) }\end{array}$ & $\begin{array}{l}\text { National Epidemiologic Survey on Alcohol and Related Condit } \\
\text { (NESARC), NIH, NIAAA. }\end{array}$ \\
\hline $26-13 b$ & Adults who exceed guidelines for low-risk drinking-Males (21+ years) & $\begin{array}{l}\text { National Epidemiologic Survey on Alcohol and Related Condit } \\
\text { (NESARC), NIH, NIAAA. }\end{array}$ \\
\hline $26-14 a$ & Steroid use among students $-8^{\text {th }}$ graders & Monitoring the Future Study (MTF), NIH, NIDA. \\
\hline $26-14 b$ & Steroid use among students $-10^{\text {th }}$ graders & Monitoring the Future Study (MTF), NIH, NIDA. \\
\hline $26-14 c$ & Steroid use among students $-12^{\text {th }}$ graders & Monitoring the Future Study (MTF), NIH, NIDA. \\
\hline $26-15$ & Inhalant use among adolescents (12-17 years) & National Survey on Drug Use and Health (NSDUH), SAMHSA. \\
\hline $26-16 a$ & $\begin{array}{l}\text { Disapproval of people who take 1-2 drinks a day of alcohol-8th } \\
\text { graders }\end{array}$ & Monitoring the Future Study (MTF), NIH, NIDA. \\
\hline $26-16 b$ & $\begin{array}{l}\text { Disapproval of people who take 1-2 drinks a day of alcohol-10th } \\
\text { graders }\end{array}$ & Monitoring the Future Study (MTF), NIH, NIDA. \\
\hline $26-16 c$ & $\begin{array}{l}\text { Disapproval of people who take 1-2 drinks a day of alcohol-12th } \\
\text { graders }\end{array}$ & Monitoring the Future Study (MTF), NIH, NIDA. \\
\hline $26-16 d$ & $\begin{array}{l}\text { Disapproval of people who try marijuana or hashish once or twice-8th } \\
\text { graders }\end{array}$ & Monitoring the Future Study (MTF), NIH, NIDA. \\
\hline $26-16 e$ & $\begin{array}{l}\text { Disapproval of people who try marijuana or hashish once or twice- } \\
\text { 10th graders }\end{array}$ & Monitoring the Future Study (MTF), NIH, NIDA. \\
\hline $26-16 f$ & $\begin{array}{l}\text { Disapproval of people who try marijuana or hashish once or twice- } \\
\text { 12th graders }\end{array}$ & Monitoring the Future Study (MTF), NIH, NIDA. \\
\hline $26-17 a$ & $\begin{array}{l}\text { Adolescents' perception of risk (12-17 years) }-5+\text { alcoholic drinks, } \\
\text { once or twice per week }\end{array}$ & National Survey on Drug Use and Health (NSDUH), SAMHSA. \\
\hline $26-17 b$ & $\begin{array}{l}\text { Adolescents' perception of risk (12-17 years)—Smoking marijuana } \\
\text { once a month }\end{array}$ & National Survey on Drug Use and Health (NSDUH), SAMHSA. \\
\hline $26-17 c$ & $\begin{array}{l}\text { Adolescents' perception of risk (12-17 years)—Cocaine use once a } \\
\text { month }\end{array}$ & National Survey on Drug Use and Health (NSDUH), SAMHSA. \\
\hline $26-18 a$ & Treatment for illicit drugs (12+ years) & National Survey on Drug Use and Health (NSDUH), SAMHSA. \\
\hline $26-18 b$ & Treatment for alcohol and/or drugs (12+ years) & National Survey on Drug Use and Health (NSDUH), SAMHSA. \\
\hline $26-19$ & Substance abuse treatment in correctional institutions & Developmental \\
\hline
\end{tabular}




\begin{tabular}{|c|c|c|}
\hline Objective & Description & Data Sources or Objective Status \\
\hline $26-20$ & Admissions for treatment for injection drug use (thousands) & Treatment Episodes Data System (TEDS), SAMHSA. \\
\hline $26-21$ & Treatment for alcohol abuse or dependence $(12+$ years $)$ & National Survey on Drug Use and Health (NSDUH), SAMHSA. \\
\hline $26-22$ & $\begin{array}{l}\text { Hospital emergency department referrals for alcohol or drug problems } \\
\text { and suicide attempts }\end{array}$ & Developmental \\
\hline $26-23$ & Community partnerships and coalitions to prevent substance abuse & Developmental \\
\hline $26-24$ & Administrative license revocation laws for DUI (no. states and D.C.) & $\begin{array}{l}\text { Department of Transportation (DOT), National Highway Traffic Safety } \\
\text { Administration (NHTSA). }\end{array}$ \\
\hline $26-25$ & $\begin{array}{l}\text { Maximum blood alcohol concentration of } 0.08 \text { for motor vehicle drivers } \\
(21+\text { years, no. states and D.C. })\end{array}$ & $\begin{array}{l}\text { Department of Transportation (DOT), National Highway Traffic Safety } \\
\text { Administration (NHTSA). }\end{array}$ \\
\hline
\end{tabular}


Figure 26-1. Progress Toward Target Attainment for Focus Area 26: Substance Abuse

Moved away from target ${ }^{1}$
Moved toward target

26-1a. Alcohol-related motor vehicle crash deaths (per 100,000 population)

26-2. Cirrhosis deaths (age adjusted, per 100,000 population)

26-3. Drug-induced deaths (age adjusted, per 100,000 population)

26-4. Drug-related hospital emergency department visits (thousands)

26-6. Students who rode with a driver who had been drinking alcohol (grades 9-12)

26-9a. Average age at first use among adolescents who used alcohol for the first time in past year (12-17 years)

26-9b. Average age at first use among adolescents who used marijuana for the first time in past year (12-17 years)

26-9c. High school seniors never consuming alcoholic beverages

Met or exceeded target

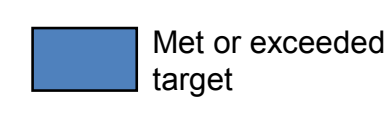

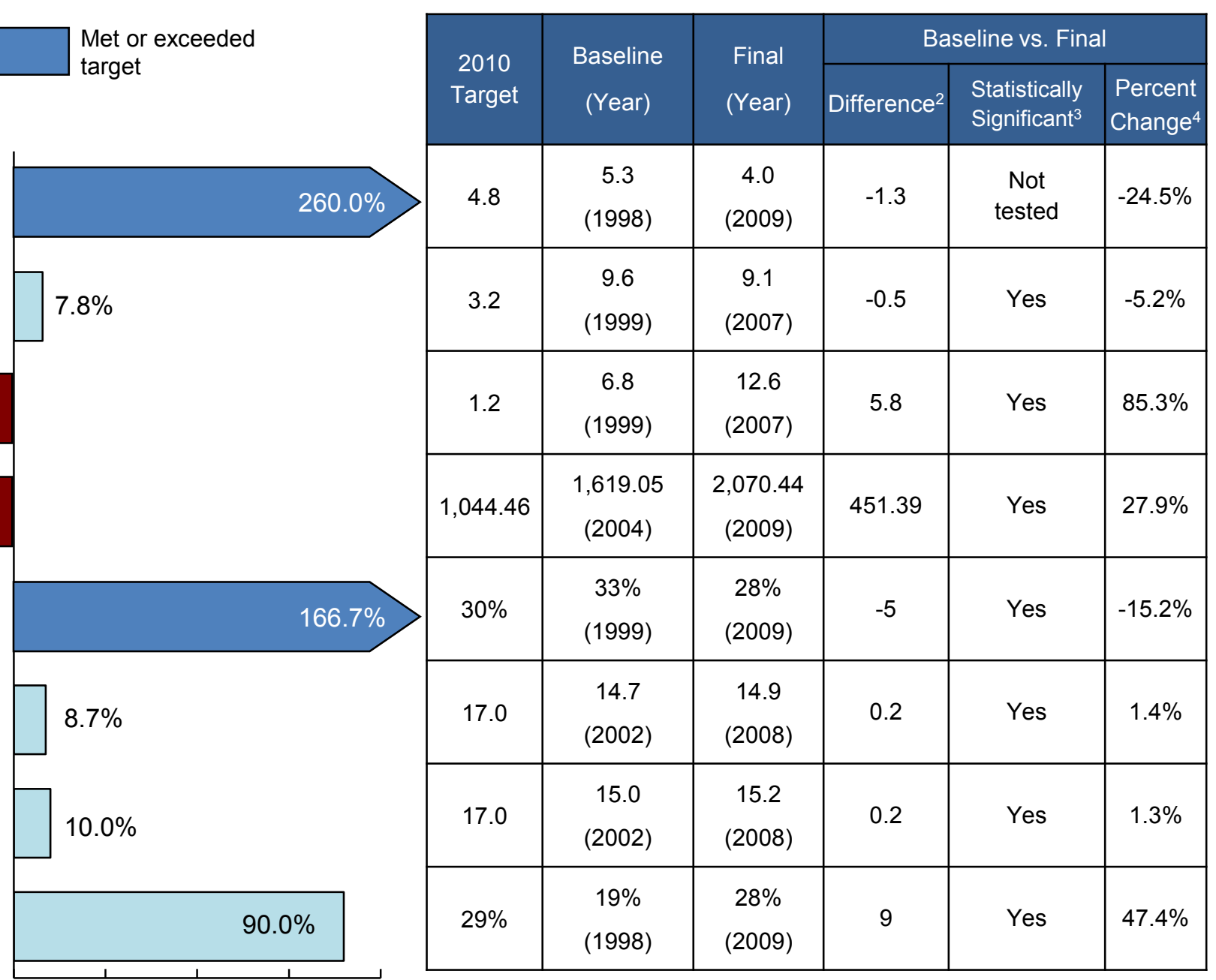

Percent of targeted change achieved ${ }^{5}$

(continued) 
Figure 26-1. Progress Toward Target Attainment for Focus Area 26: Substance Abuse (continued)

Moved away

from target ${ }^{1}$

Moved toward

target

Met or exceeded

target

26-9d. High school seniors never using illicit drugs

26-10a. Adolescents not using alcohol or illicit drugs in past 30 days (12-17 years)

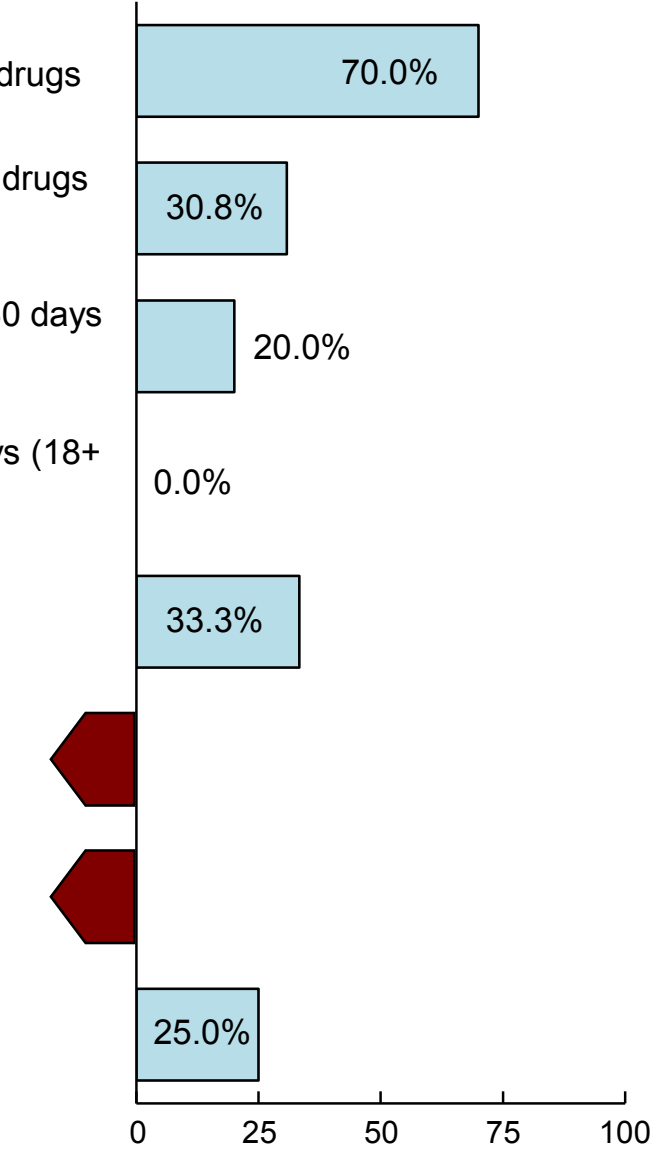

\begin{tabular}{|c|c|c|c|c|c|}
\hline \multirow{2}{*}{$\begin{array}{l}2010 \\
\text { Target }\end{array}$} & \multirow{2}{*}{$\begin{array}{c}\text { Baseline } \\
\text { (Year) }\end{array}$} & \multirow{2}{*}{$\begin{array}{l}\text { Final } \\
\text { (Year) }\end{array}$} & \multicolumn{3}{|c|}{ Baseline vs. Final } \\
\hline & & & Difference $^{2}$ & $\begin{array}{l}\text { Statistically } \\
\text { Significant }^{3}\end{array}$ & $\begin{array}{l}\text { Percent } \\
\text { Change }^{4}\end{array}$ \\
\hline $56 \%$ & $\begin{array}{c}46 \% \\
(1998)\end{array}$ & $\begin{array}{c}53 \% \\
(2009)\end{array}$ & 7 & Yes & $15.2 \%$ \\
\hline $91 \%$ & $\begin{array}{c}78 \% \\
(2002)\end{array}$ & $\begin{array}{c}82 \% \\
(2008)\end{array}$ & 4 & Yes & $5.1 \%$ \\
\hline $0.7 \%$ & $\begin{array}{l}8.2 \% \\
(2002)\end{array}$ & $\begin{array}{c}6.7 \% \\
(2008)\end{array}$ & -1.5 & Yes & $-18.3 \%$ \\
\hline $3.2 \%$ & $\begin{array}{c}7.9 \% \\
(2002)\end{array}$ & $\begin{array}{c}7.9 \% \\
(2008)\end{array}$ & 0 & No & $0.0 \%$ \\
\hline $11 \%$ & $\begin{array}{c}32 \% \\
(1998)\end{array}$ & $\begin{array}{c}25 \% \\
(2009)\end{array}$ & -7 & Yes & $-21.9 \%$ \\
\hline $20 \%$ & $\begin{array}{c}39 \% \\
(1998)\end{array}$ & $\begin{array}{c}40 \% \\
(2009)\end{array}$ & 1 & $\begin{array}{l}\text { Not } \\
\text { tested }\end{array}$ & $2.6 \%$ \\
\hline $13.4 \%$ & $\begin{array}{l}24.3 \% \\
(2002)\end{array}$ & $\begin{array}{l}24.9 \% \\
(2008)\end{array}$ & 0.6 & No & $2.5 \%$ \\
\hline $3.1 \%$ & $\begin{array}{l}10.7 \% \\
(2002)\end{array}$ & $\begin{array}{l}8.8 \% \\
(2008)\end{array}$ & -1.9 & Yes & $-17.8 \%$ \\
\hline
\end{tabular}

Percent of targeted change achieved ${ }^{5}$

(continued)

Binge drinking in the past month

C. Adults $(18+$ years $)$

d. Adolescents (12-17 years)

(continued) 
Figure 26-1. Progress Toward Target Attainment for Focus Area 26: Substance Abuse (continued)

Moved away

from target ${ }^{1}$

Moved toward

target

Met or exceeded

target

26-12. Average annual alcohol consumption (gallons per person, 14+ years)

26-13. Adults who exceed guidelines for low-risk drinking $(21+$ years $)$

a. Females

b. Males

26-14. Steroid use among students

a. 8th graders

b. 10th graders

c. 12th graders

26-15. Inhalant use among adolescents (12-17 years)

26-16. Disapproval of people who take 1-2 drinks a day of alcohol

a. 8th graders

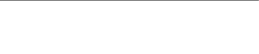

\begin{tabular}{|c|c|c|c|c|c|}
\hline \multirow{2}{*}{$\begin{array}{l}2010 \\
\text { Target }\end{array}$} & \multirow{2}{*}{$\begin{array}{c}\text { Baseline } \\
\text { (Year) }\end{array}$} & \multirow{2}{*}{$\begin{array}{l}\text { Final } \\
\text { (Year) }\end{array}$} & \multicolumn{3}{|c|}{ Baseline vs. Final } \\
\hline & & & Difference ${ }^{2}$ & $\begin{array}{l}\text { Statistically } \\
\text { Significant }^{3}\end{array}$ & $\begin{array}{l}\text { Percent } \\
\text { Change }^{4}\end{array}$ \\
\hline 1.96 & $\begin{array}{c}2.14 \\
(1997)\end{array}$ & $\begin{array}{c}2.31 \\
(2007)\end{array}$ & 0.2 & $\begin{array}{l}\text { Not } \\
\text { tested }\end{array}$ & $7.9 \%$ \\
\hline $50 \%$ & $\begin{array}{c}72 \% \\
(1992)\end{array}$ & $\begin{array}{c}55 \% \\
(2001-02)\end{array}$ & -17 & $\begin{array}{l}\text { Not } \\
\text { tested }\end{array}$ & $-23.6 \%$ \\
\hline $50 \%$ & $\begin{array}{c}74 \% \\
(1992)\end{array}$ & $\begin{array}{c}61 \% \\
(2001-02)\end{array}$ & -13 & $\begin{array}{c}\text { Not } \\
\text { tested }\end{array}$ & $-17.6 \%$ \\
\hline $0.4 \%$ & $\begin{array}{c}1.2 \% \\
(1998)\end{array}$ & $\begin{array}{c}1.3 \% \\
(2009)\end{array}$ & 0.1 & No & $8.3 \%$ \\
\hline $0.4 \%$ & $\begin{array}{c}1.2 \% \\
(1998)\end{array}$ & $\begin{array}{l}1.3 \% \\
(2009)\end{array}$ & 0.1 & No & $8.3 \%$ \\
\hline $0.4 \%$ & $\begin{array}{c}1.7 \% \\
(1998)\end{array}$ & $\begin{array}{l}2.2 \% \\
(2009)\end{array}$ & 0.5 & No & $29.4 \%$ \\
\hline $2.2 \%$ & $\begin{array}{l}4.4 \% \\
(2002)\end{array}$ & $\begin{array}{l}3.9 \% \\
(2008)\end{array}$ & -0.5 & Yes & $-11.4 \%$ \\
\hline $83 \%$ & $\begin{array}{c}77 \% \\
(1998)\end{array}$ & $\begin{array}{c}78 \% \\
(2009)\end{array}$ & 1 & No & $1.3 \%$ \\
\hline
\end{tabular}

(continued)

Percent of targeted change achieved 5

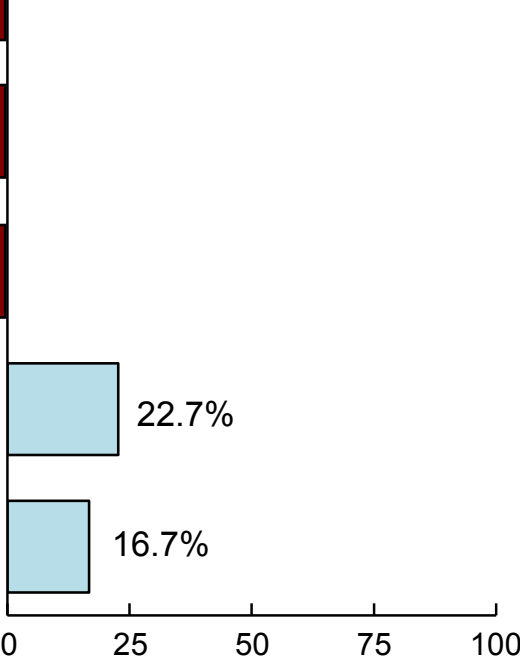

$77.3 \%$

$54.2 \%$
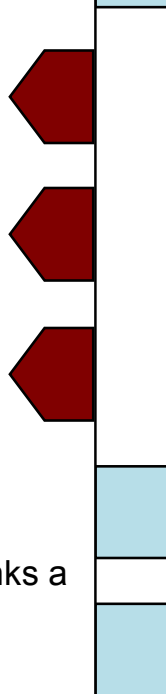
Figure 26-1. Progress Toward Target Attainment for Focus Area 26: Substance Abuse (continued)

Moved away

from target ${ }^{1}$

Moved toward

target

Met or exceeded

target

26-16. Disapproval of people who take 1-2 drinks a day of alcohol

b. 10th graders

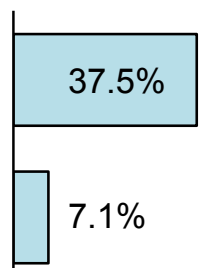

c. 12th graders

Disapproval of people who try marijuana or hashish once or twice

d. 8th graders

e. 10th graders

f. 12th graders

26-17 Adolescents' perception of risk (12-17 years)

a. 5+ alcoholic drinks, once or twice per week

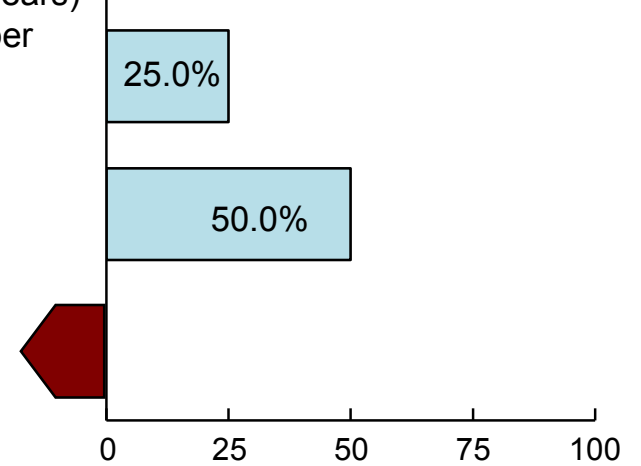

\begin{tabular}{|c|c|c|c|c|c|}
\hline \multirow{2}{*}{$\begin{array}{l}2010 \\
\text { Target }\end{array}$} & \multirow{2}{*}{$\begin{array}{c}\text { Baseline } \\
\text { (Year) }\end{array}$} & \multirow{2}{*}{$\begin{array}{l}\text { Final } \\
\text { (Year) }\end{array}$} & \multicolumn{3}{|c|}{ Baseline vs. Final } \\
\hline & & & Difference ${ }^{2}$ & $\begin{array}{l}\text { Statistically } \\
\text { Significant }^{3}\end{array}$ & $\begin{array}{l}\text { Percent } \\
\text { Change }^{4}\end{array}$ \\
\hline $83 \%$ & $\begin{array}{c}75 \% \\
(1998)\end{array}$ & $\begin{array}{c}78 \% \\
(2009)\end{array}$ & 3 & Yes & $4.0 \%$ \\
\hline $83 \%$ & $\begin{array}{c}69 \% \\
(1998)\end{array}$ & $\begin{array}{c}70 \% \\
(2009)\end{array}$ & 1 & No & $1.4 \%$ \\
\hline $72 \%$ & $\begin{array}{c}69 \% \\
(1998)\end{array}$ & $\begin{array}{c}75 \% \\
(2009)\end{array}$ & 6 & Yes & $8.7 \%$ \\
\hline $72 \%$ & $\begin{array}{c}56 \% \\
(1998)\end{array}$ & $\begin{array}{c}60 \% \\
(2009)\end{array}$ & 4 & Yes & $7.1 \%$ \\
\hline $72 \%$ & $\begin{array}{c}52 \% \\
(1998)\end{array}$ & $\begin{array}{c}55 \% \\
(2009)\end{array}$ & 3 & No & $5.8 \%$ \\
\hline $50 \%$ & $\begin{array}{c}38 \% \\
(2002)\end{array}$ & $\begin{array}{c}41 \% \\
(2008)\end{array}$ & 3 & Yes & $7.9 \%$ \\
\hline $36 \%$ & $\begin{array}{c}32 \% \\
(2002)\end{array}$ & $\begin{array}{c}34 \% \\
(2008)\end{array}$ & 2 & Yes & $6.3 \%$ \\
\hline $57 \%$ & $\begin{array}{c}51 \% \\
(2002)\end{array}$ & $\begin{array}{c}50 \% \\
(2008)\end{array}$ & -1 & No & $-2.0 \%$ \\
\hline
\end{tabular}

Percent of targeted change achieved ${ }^{5}$

(continued) 
Figure 26-1. Progress Toward Target Attainment for Focus Area 26: Substance Abuse (continued)

Moved away

from target ${ }^{1}$

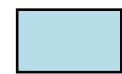

Moved toward

target

Met or exceeded

target

26-18a. Treatment for illicit drugs (12+ years)

26-18b. Treatment for alcohol and/or drugs (12+ years)

26-20. Admissions for treatment for injection drug use (thousands)

26-21. Treatment for alcohol abuse or dependence $(12+$ years $)$

26-24. Administrative license revocation laws for DUI (no. States and D.C.)

26-25. Maximum blood alcohol concentration of 0.08 for motor vehicle drivers $(21+$ years, no. States and D.C.)

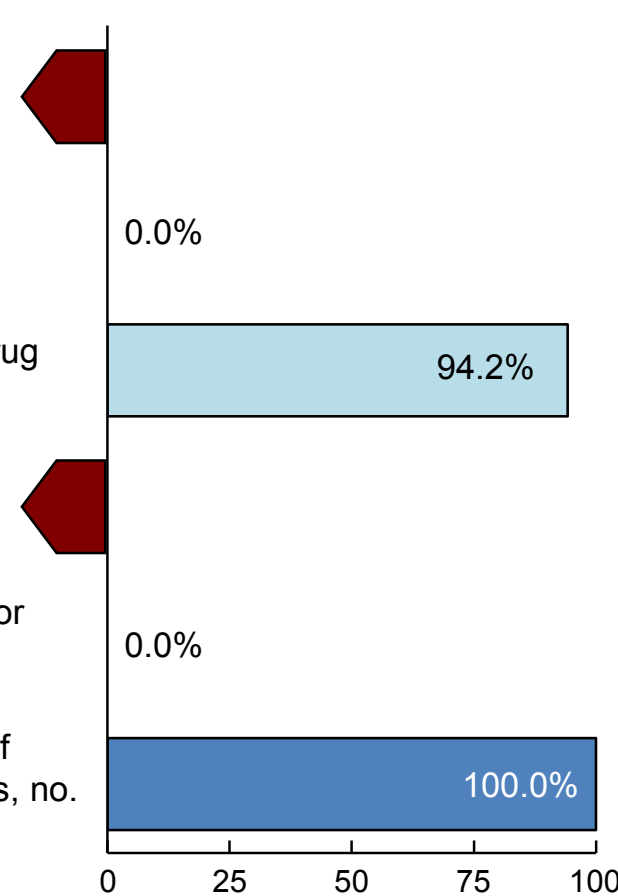

\begin{tabular}{|c|c|c|c|c|c|}
\hline \multirow{2}{*}{$\begin{array}{l}2010 \\
\text { Target }\end{array}$} & \multirow{2}{*}{$\begin{array}{c}\text { Baseline } \\
\text { (Year) }\end{array}$} & \multirow{2}{*}{$\begin{array}{l}\text { Final } \\
\text { (Year) }\end{array}$} & \multicolumn{3}{|c|}{ Baseline vs. Final } \\
\hline & & & Difference ${ }^{2}$ & $\begin{array}{l}\text { Statistically } \\
\text { Significant }^{3}\end{array}$ & $\begin{array}{l}\text { Percent } \\
\text { Change }^{4}\end{array}$ \\
\hline $24 \%$ & $\begin{array}{c}18 \% \\
(2002)\end{array}$ & $\begin{array}{c}16 \% \\
(2008)\end{array}$ & -2 & No & $-11.1 \%$ \\
\hline $16 \%$ & $\begin{array}{c}10 \% \\
(2002)\end{array}$ & $\begin{array}{c}10 \% \\
(2008)\end{array}$ & 0 & No & $0.0 \%$ \\
\hline 256.680 & $\begin{array}{c}215.560 \\
(1997)\end{array}$ & $\begin{array}{c}254.278 \\
(2008)\end{array}$ & 38.718 & $\begin{array}{c}\text { Not } \\
\text { tested }\end{array}$ & $18.0 \%$ \\
\hline $11.9 \%$ & $\begin{array}{c}8.3 \% \\
(2002)\end{array}$ & $\begin{array}{l}8.2 \% \\
(2008)\end{array}$ & -0.1 & No & $-1.2 \%$ \\
\hline 51 & $\begin{array}{c}42 \\
(1998)\end{array}$ & $\begin{array}{c}42 \\
(2007)\end{array}$ & 0 & $\begin{array}{c}\text { Not } \\
\text { tested }\end{array}$ & $0.0 \%$ \\
\hline 51 & $\begin{array}{c}15 \\
(1998)\end{array}$ & $\begin{array}{c}51 \\
(2006)\end{array}$ & 36 & $\begin{array}{c}\text { Not } \\
\text { tested }\end{array}$ & $240.0 \%$ \\
\hline
\end{tabular}

Percent of targeted change achieved ${ }^{5}$ 


\section{Figure 26-1. Progress Toward Target Attainment for Focus Area 26: Substance Abuse (continued)}

\section{NOTES}

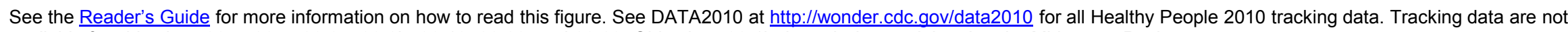
available for objectives 26-5, 26-7, 26-8a, 26-8b, 26-19, 26-22, and 26-23. Objectives 26-1b through d were deleted at the Midcourse Review.

\section{FOOTNOTES}

${ }^{1}$ Movement away from target is not quantified using the percent of targeted change achieved. See Technical Appendix for more information.

${ }^{2}$ Difference $=$ Final value - Baseline value. Differences between percents (\%) are measured in percentage points.

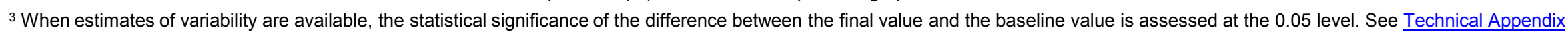
for more information.

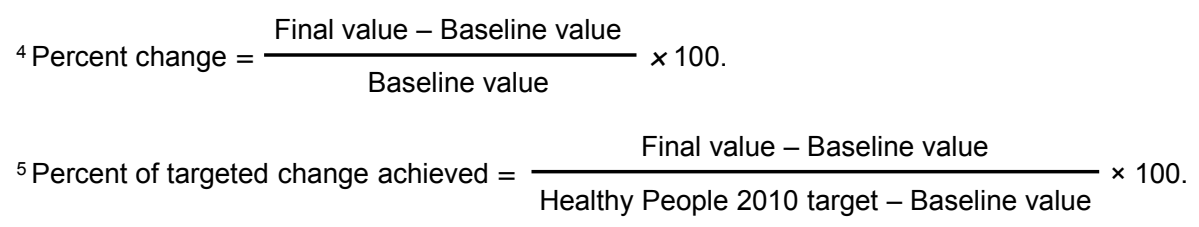

\section{DATA SOURCES}

26-1a. $\quad$ Fatality Analysis Reporting System (FARS), Department of Transportation (DOT).

26-2-26-3. National Vital Statistics System-Mortality (NVSS-M), CDC, NCHS.

26-4. $\quad$ Drug Abuse Warning Network (DAWN), SAMHSA.

26-6. Youth Risk Behavior Surveillance System (YRBSS), CDC, NCCDPHP.

26-9a-b. National Survey on Drug Use and Health (NSDUH), SAMHSA.

26-9c-d. Monitoring the Future Study (MTF), NIH, NIDA.

26-10a-c. National Survey on Drug Use and Health (NSDUH), SAMHSA.

26-11a-b. Monitoring the Future Study (MTF), NIH, NIDA.

26-11c-d. National Survey on Drug Use and Health (NSDUH), SAMHSA.

26-12. Alcohol Epidemiologic Data System (AEDS), NIH, NIAAA.

26-13a-b. National Epidemiologic Survey on Alcohol and Related Conditions (NESARC), NIH, NIAAA.

26-14a-c. Monitoring the Future Study (MTF), NIH, NIDA.

26-15. National Survey on Drug Use and Health (NSDUH), SAMHSA.

26-16a-f. Monitoring the Future Study (MTF), NIH, NIDA.

26-17a-c. National Survey on Drug Use and Health (NSDUH), SAMHSA.

26-18a-b. National Survey on Drug Use and Health (NSDUH), SAMHSA.

26-20. Treatment Episodes Data System (TEDS), SAMHSA

26-21. National Survey on Drug Use and Health (NSDUH), SAMHSA.

26-24-26-25. Department of Transportation (DOT), National Highway Traffic Safety Administration (NHTSA). 
Figure 26-2. Health Disparities Table for Focus Area 26: Substance Abuse

Disparities from the best group rate for each characteristic at the most recent data point and changes in disparity from the baseline to the most recent data point.

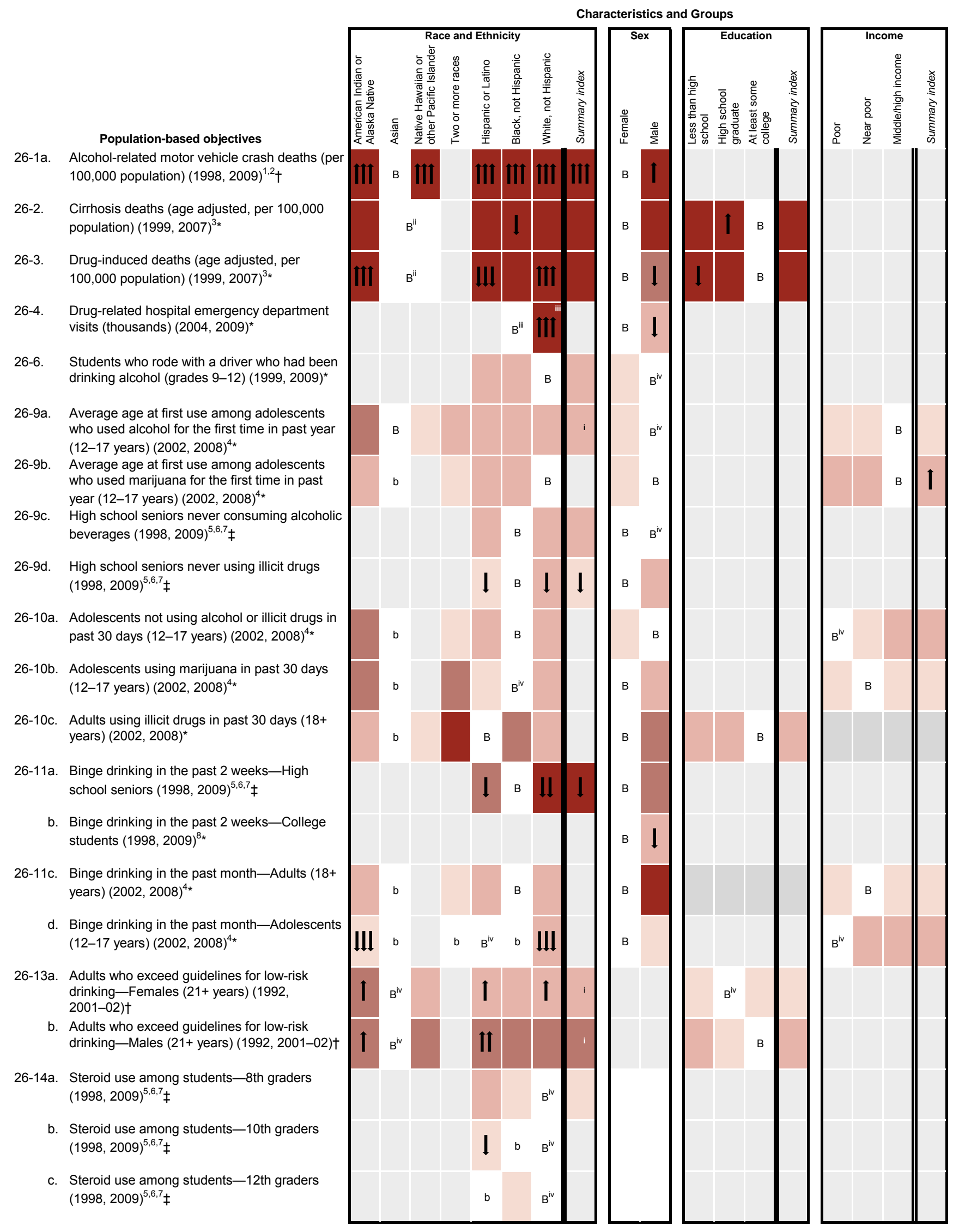

(continued) 


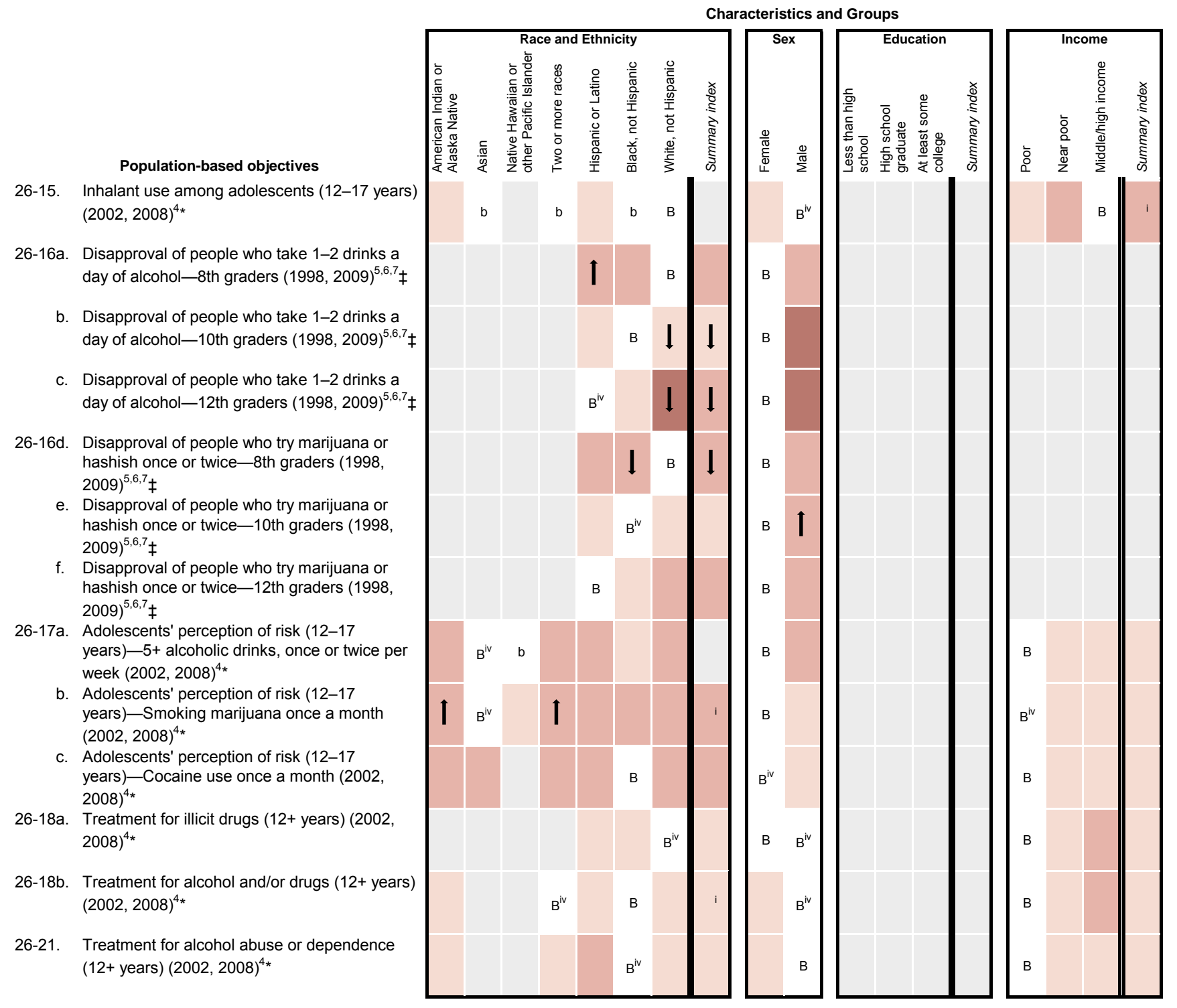

(continued) 


\section{Figure 26-2. Health Disparities Table for Focus Area 26: Substance Abuse (continued)}

NOTES

See DATA2010 at http://wonder.cdc.gov/data2010 for all Healthy People 2010 tracking data. Disparity data are either unavailable or not applicable for objectives 26-5, 26-7, 26-8a and b, 26-12, 26-19, 26-20, and 26-22 through 26-25. Objectives 26-1b through d were deleted at Midcourse Review.

Years in parentheses represent the baseline and most recent data years (if available).

Disparity from the best group rate is defined as the percent difference between the best group rate and each of the other group rates for a characteristic (e.g., race and ethnicity). The summary index is the average of these percent differences for a characteristic. Change in disparity is estimated by subtracting the disparity at baseline from the disparity at the most recent data point. Change in the summary index is estimated by subtracting the summary index at baseline from the summary index at the most recent data point. See Technical Appendix for more information.

LEGEND

The "best" group rate at the most recent data point.

B The group with the best rate for specified characteristic.

Most favorable group rate for specified

characteristic, but reliability criterion not met.

\begin{tabular}{|c|c|c|c|c|}
\hline \multirow[b]{2}{*}{$\begin{array}{l}\text { Disparity from the best group rate at the } \\
\text { most recent data point. }\end{array}$} & \multicolumn{4}{|c|}{ Percent difference from the best group rate } \\
\hline & $\begin{array}{l}\text { Less than } 10 \% \text {, or difference not } \\
\text { statistically significant (when } \\
\text { estimates of variability are available). }\end{array}$ & $10 \%-49 \%$ & $50 \%-99 \%$ & $100 \%$ or more \\
\hline
\end{tabular}

(in disparity over time are shown when:

(a) disparities data are available at both baseline and most recent time points;

(b) data are not for the group(s) indicated by "B" or "b" at either time point; and

(c) the change is greater than or equal to 10 percentage points and statistically

significant, or when the change is greater than or equal to 10 percentage

points and estimates of variability were not available. See Technical Appendix.

Increase in disparity (percentage points)

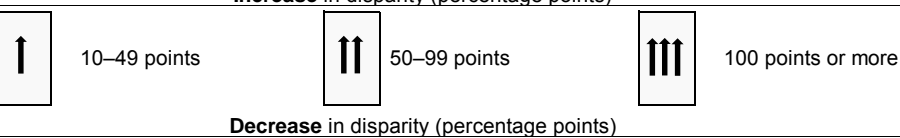

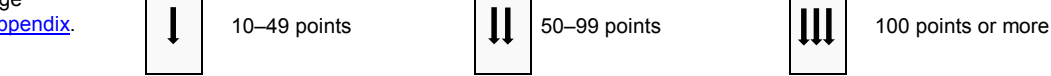

Availability of Data

Data not available.

Characteristic not selected for this objective.

FOOTNOTES

* Measures of variability were available. Thus, the variability of best group rates was assessed, and statistical significance was tested. Disparities of $10 \%$ or more are displayed when the differences from the best group rate are statistically significant at the 0.05 level. Changes in disparities over time are indicated by arrows when the changes are greater than or equal to 10 percentage points and are statistically significant at the 0.05 level. See Technical Appendix.

† Measures of variability were not available. Thus, the variability of best group rates was not assessed, and statistical significance could not be tested.

Nonetheless, disparities and changes in disparities over time are displayed according to their magnitude. See Technical Appendix.

¥ Measures of variability for data by race and ethnicity were available only for the most recent data. Thus, the variability of best group rates was assessed only for the most recent data, and statistical significance was tested only for the most recent data. Disparities of $10 \%$ or more are displayed when the differences from the best group rate are statistically significant at the 0.05 level. Changes in disparities over time are displayed according to their magnitude, since measures of variability were not available at baseline and therefore statistical significance of changes in disparity could not be tested. See Technical Appendix.

1 Baseline data by race and ethnicity are for 2000 .

2 Most recent data by race and ethnicity are for 2008.

3 Data by education level are for persons aged 25-64 years. Most recent data by education level are for 2002.

4 Baseline data by income are for 2005.

5 Baseline data by race and ethnicity are for 2004-05.

6 Most recent data by race and ethnicity are for 2008-09.

7 Measures of variability were available for data by sex. See footnote * above.

8 Most recent data by sex are for 2007.

i Change in the summary index cannot be assessed. See Technical Appendix.

ii Data are for Asian or Pacific Islander.

iii Data include persons of Hispanic origin.

iv The group with the best rate at the most recent data point is different from the group with the best rate at baseline. Both rates met the reliability criterion. See Technical Appendix.

\section{DATA SOURCES}

26-1a. Fatality Analysis Reporting System (FARS), Department of Transportation (DOT).

26-2-26-3. National Vital Statistics System-Mortality (NVSS-M), CDC, NCHS.

26-4. Drug Abuse Warning Network (DAWN), SAMHSA.

26-6. Youth Risk Behavior Surveillance System (YRBSS), CDC, NCCDPHP.

26-9a-b. National Survey on Drug Use and Health (NSDUH), SAMHSA.

26-9c-d. Monitoring the Future Study (MTF), NIH, NIDA.

26-10a-c. National Survey on Drug Use and Health (NSDUH), SAMHSA.

26-11a-b. Monitoring the Future Study (MTF), NIH, NIDA.

26-11c-d. National Survey on Drug Use and Health (NSDUH), SAMHSA.

26-13a-b. National Epidemiologic Survey on Alcohol and Related Conditions (NESARC), NIH, NIAAA.

26-14a-c. Monitoring the Future Study (MTF), NIH, NIDA.

26-15. National Survey on Drug Use and Health (NSDUH), SAMHSA.

26-16a-f. Monitoring the Future Study (MTF), NIH, NIDA

26-17a-c. National Survey on Drug Use and Health (NSDUH), SAMHSA.

26-18a-b. National Survey on Drug Use and Health (NSDUH), SAMHSA.

26-18b. National Survey on Drug Use and Health (NSDUH), SAMHSA

26-21. National Survey on Drug Use and Health (NSDUH), SAMHSA.

Substance Abuse

Page 26-22 
Figure 26-3. Cirrhosis Deaths 2005-07

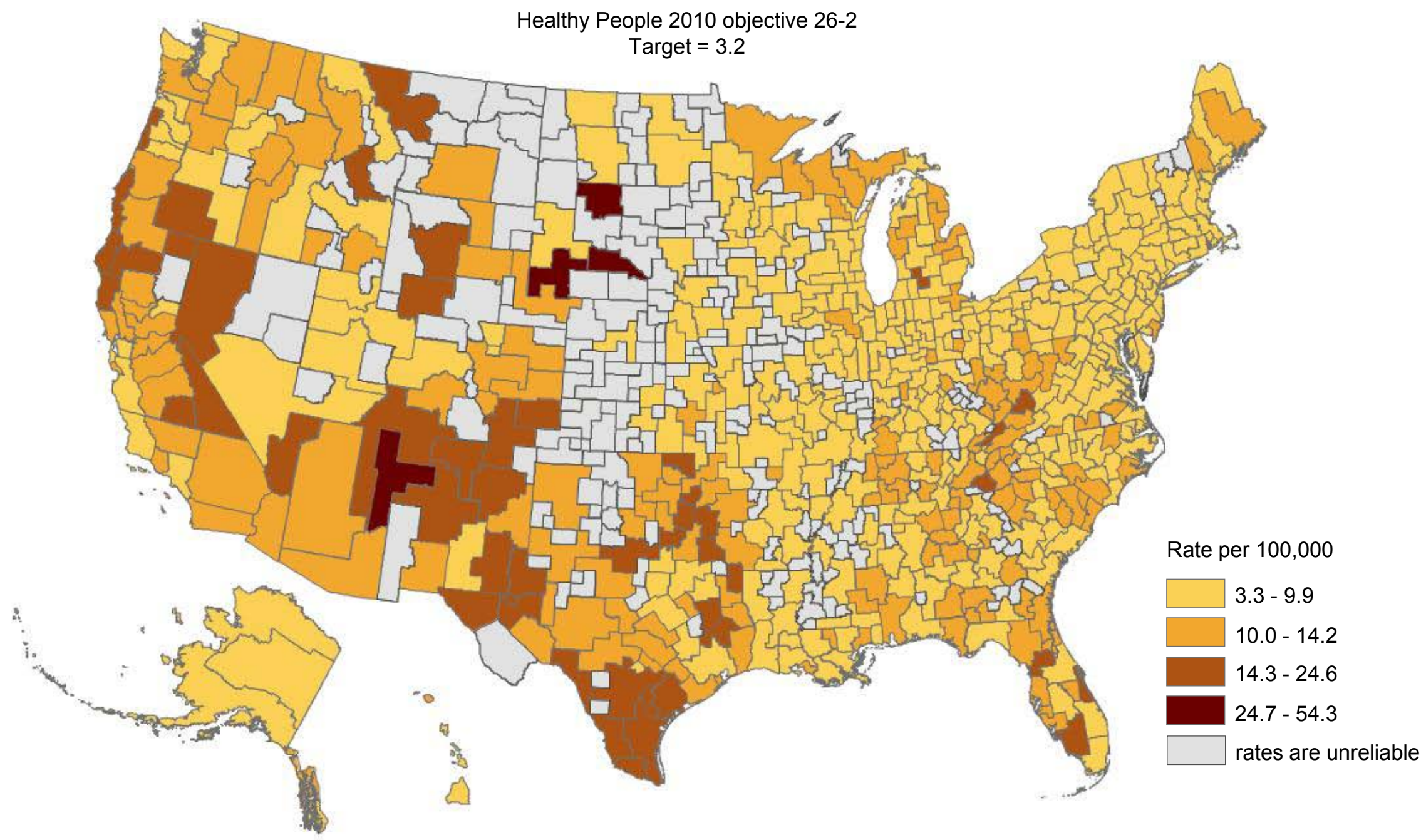

no health service areas with a reliable rate met the target

NOTES: Data are for ICD-10 codes K70, K73-K74 reported as underlying cause. Rates are per 100,000 U. S. Population age-adjusted to the 2000 standard population. Rates are displayed by a manual classification for U.S. health service area. SOURCE: National Vital Statistics System - Mortality, CDC, NCHS. 


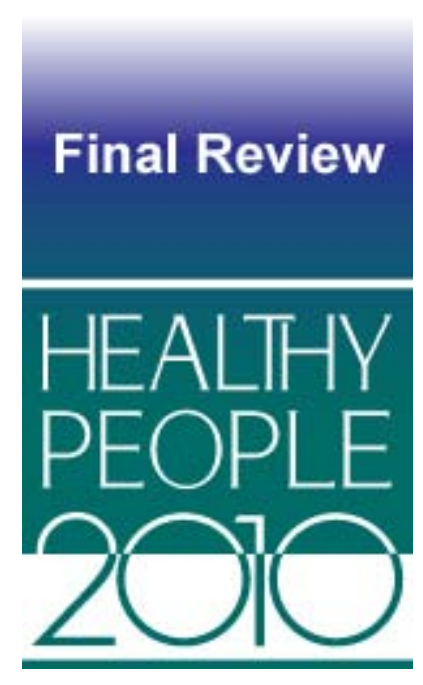

\section{Tobacco Use}

27

\section{Lead Agency}

Centers for Disease Control and Prevention

\section{Contents}

Goal.

Highlights.

Summary of Progress.

Transition to Healthy People 2020 .

Data Considerations

Notes

Comprehensive Summary of Objectives

Progress Chart

Health Disparities Table

Tobacco Use by Adults-Cigarettes-Map

Comprehensive Smoke Free Laws-Map

Excise Taxes on Retail Price of a Standard Pack of Cigarettes-Map. 


\section{Goal: Reduce illness, disability, and death related to tobacco use and exposure to secondhand smoke}

The objectives in this chapter monitor tobacco use, smoking cessation and the availability of treatment programs, environmental exposure to tobacco smoke, adolescent attitudes toward smoking, and tobacco control laws.

All Healthy People tracking data quoted in this chapter, along with technical information and operational definitions for each objective, can be found in the Healthy People 2010 database, DATA2010, available from http://wonder.cdc.gov/data2010/.

More information about this Focus Area can be found in the following publications:

- Healthy People 2010: Understanding and Improving Health, available from http://www.healthypeople.gov/2010/Document/tableofcontents.htm\#under.

- Healthy People 2010 Midcourse Review, available from http://www.healthypeople.gov/2010/data/midcourse/html/default.htm\#FocusAreas.

\section{Highlights}

- Substantial progress was achieved in objectives for this Focus Area during the past decade [1]. Eighty-five percent of the Tobacco Use objectives with data to measure progress moved toward or achieved their Healthy People 2010 targets (Figure 27-1). However, statistically significant health disparities were observed among racial and ethnic populations, as well as by sex, education level, income, and disability status (Figure 27-2), as discussed below [2].

- The percentage of adults aged 18 years and over who were current cigarette smokers (objective 27-1a) decreased by $12.5 \%$ between 1998 and 2008, from $24 \%$ to $21 \%$ (age adjusted), moving toward the Healthy People 2010 target of $12 \%$. However, from 2004 to 2008, the proportion of U.S. adults who were current cigarette smokers did not noticeably change. Disparities were observed for a number of populations, for example:

- Among educational groups, adults aged 25 years and over with at least some college education had the lowest (best) current cigarette smoking rate, 15\% (age adjusted) in 2008. Adults aged 25 years and over with less than a high school education had a rate of $30 \%$ (age adjusted) in 2008, twice the best group rate [2].

- Current cigarette smoking varied by geographic area. Utah was the only state to exceed the 2010 target, with an adult smoking rate of 9.2\% in 2008. Indiana, Kentucky, Missouri, Oklahoma, and West Virginia had the highest smoking rates (Figure 27-3). 
- The percentage of students in grades 9-12 who used tobacco products in the last month declined between 1999 and 2009. Student use of all tobacco products (which includes use of cigarettes, chewing tobacco, snuff, or cigars) (objective 27-2a) decreased by $35.0 \%$, from $40 \%$ in 1999 to $26 \%$ in 2009 , moving toward the 2010 target of $21 \%$. Student cigarette smoking (objective $27-2$ b) decreased by $45.7 \%$, from $35 \%$ in 1999 to $19 \%$ in 2009 , moving toward the 2010 target of $16 \%$; whereas cigar use (objective $27-2 \mathrm{~d}$ ) declined by $22.2 \%$, from $18 \%$ to $14 \%$, moving toward the 2010 target of $8 \%$. Student bidi use (objective $27-2 \mathrm{e}$ ) declined by $41.5 \%$ between 2000 and 2009 , from $4.1 \%$ to $2.4 \%$, meeting the target of $2.4 \%$.

- Female students had a lower (better) rate of cigar use in the past month than male students, $19 \%$ vs. $9 \%$ in 2009 . The rate for male students was more than twice that of female students [2].

- The percentage of children aged six years and under exposed to tobacco smoke at home (objective $27-9$ ) decreased by $70.4 \%$ between 1994 and 2005 , from $27 \%$ to 8\%, exceeding the Healthy People 2010 target of 10\%. Disparities were observed among a number of population groups, for example: among income groups, children aged six years and under living in middle/high income households had the lowest (best) rates of exposure to tobacco smoke at home, $5 \%$ in 2005, whereas children living in poor or near-poor households had rates of $15 \%$ and $12 \%$ in 2005 , respectively. The rate for children living in poor households was three times the best group rate, while the rate for children living in near-poor households was almost two and a half times the best group rate [2].

- Children living in poor households had rates of exposure to tobacco smoke of $38 \%$ in 1994 and 15\% in 2005; those living in near-poor households had rates of 33\% in 1994 and $12 \%$ in 2005; whereas those living in middle/high income households had rates of $19 \%$ in 1994 and 5\% in 2005. The disparity between children living in poor households and those living in middle/high income households increased by 100 percentage points between 1994 and 2005. During the same period, the disparity between children living in near-poor households and those living in middle/high income households increased by 66 percentage points [3].

- The percentage of nonsmokers aged four years and over exposed to environmental tobacco smoke (objective $27-10$ ) declined by $51.2 \%$ between $1988-94$ and $2005-08$, from $84 \%$ to $41 \%$ (age adjusted), exceeding the 2010 target of 56\%.

- The number of states with smoke-free indoor air laws (objectives 27-13a through $\mathrm{f}$, and i) increased between 1998 and 2009, moving toward the 2010 targets of 51 (all 50 states plus the District of Columbia). The number of states that had laws prohibiting smoking in private workplaces increased from zero states in 1998 to 30 (29 states plus the District of Columbia) in 2009 (objective 27-13a). The number of states that prohibited smoking in restaurants increased from one in 1998 to 28 (27 states plus the District of Columbia) in 2009 (objective 27-13c). And the number of states that prohibited smoking in bars increased from zero states in 1998 to 22 (21 states plus the District of Columbia) in 2009 (objective 27-13i).

- Twenty-one states had laws prohibiting smoking in private workplaces, restaurants, and bars in 2009. Nineteen states, including large-population states like California and Texas, had no such laws (Figure 27-4).

- Exposure to tobacco advertising on the Internet among students in grades 6-12 (objective $27-16$ a) increased by $32.1 \%$ between 2000 and 2009 , from $28 \%$ to $37 \%$, moving away from the 2010 target of $25 \%$. 
- The average combined Federal and State excise taxes on a standard pack of cigarettes (objective 27-21a) increased nearly four-fold, from \$0.63 in 1998 to $\$ 2.35$ in 2009, exceeding the 2010 target of $\$ 2.00$. (The 2009 figure includes an increase in the Federal cigarette tax to $\$ 1.01$.)

- Twenty-nine states had cigarette taxes of at least $\$ 2.00$ per pack in 2009 , achieving the target. South Carolina had the lowest combined tax rate: $\$ 1.08$ per pack (Figure 27-5).

\section{Summary of Progress}

- Figure 27-1 presents a quantitative assessment of progress in achieving the Healthy People 2010 objectives for Tobacco Use [1]. Data to measure progress toward target attainment were available for 40 objectives. Of these:

- Six objectives (27-2e, 27-9, 27-10, 27-16b, 27-20a, and 27-21a) met or exceeded their Healthy People 2010 targets.

- Twenty-eight objectives moved toward their 2010 targets. A statistically significant difference between the baseline and the final data points was observed for 11 of these objectives (27-1a, 27-2a and b, 27-2d, 27-4a, 27-5, 27-11, 27-12, and 27-17a through c). No significant differences were observed for three objectives (27-1b and c, and 27-3a); and data to test the significance of the difference were unavailable for 14 objectives (27-13a through f, 27-13i, 27-14a and b, 27-15, 27-19, 27-20a through c, and 27-21a and b).

- Six objectives moved away from their 2010 targets. A statistically significant difference between the baseline and final data points was observed for two objectives (27-3b and 27-16a); no significant differences were observed for four objectives (27-2c, 27-4b, 27-6, and 27-7).

- Four objectives remained developmental (objectives 27-13g and $\mathrm{h}$, and 27-18b and d), and three had no data available to measure progress (objectives 27-8a and b, and 27-18a) [4]. Two objectives were dropped during the decade (objectives 27-1d and 27-8c) [5].

- Figure 27-2 displays health disparities in Tobacco Use from the best group rate for each characteristic at the most recent data point [2]. It also displays changes in disparities from baseline to the most recent data point [3].

- Of the ten objectives with statistically significant health disparities of $10 \%$ or more by race and ethnicity, the Asian population had the best rate for three objectives (27-12, and 27-16a and b); the black non-Hispanic population had the best rate for two objectives (27-2a and b); the Hispanic or Latino population had the best rate for two objectives (27-1a and 27-10); the white non-Hispanic population had the best rate for two objectives (27-9 and 27-17a); and the white non-Hispanic and black non-Hispanic populations were tied for the best rate for one objective (27-4a).

- Females had better rates than males for 10 of the 12 objectives with statistically significant health disparities of $10 \%$ or more by sex (objectives $27-1 \mathrm{a}, 27-2 \mathrm{a}$ and $d$, 27-3b, 24-5, 27-7, 27-10, 27-12, and 27-17a and b). Males had better rates than females for two objectives (27-3a and 27-4a). 
- Persons with at least some college education had the best rates for all five of the objectives with statistically significant health disparities of $10 \%$ or more by education level (27-1a and b, 27-5, 27-10, and 27-12).

- Persons with middle/high incomes had the best rates for the four objectives with statistically significant health disparities of $10 \%$ or more by income (objectives 27 1, 27-4a, 27-9, and 27-12).

- Persons without disabilities had the better rates for both objectives with statistically significant health disparities of $10 \%$ or more by disability status (objectives $27-1$ a and c).

\section{Transition to Healthy People 2020}

The focus of the Healthy People 2020 Tobacco Use objectives remains similar to that of Healthy People 2010. There have been some changes in organization and some objectives have been expanded. See HealthyPeople.gov for a complete list of Healthy People 2020 topics and objectives.

The Healthy People 2020 objectives can be grouped into several sections:

- The Tobacco Use Prevalence section focuses on reducing tobacco use and initiation among youth and adults.

- The Health System Changes section addresses policies and strategies to increase access, affordability, and use of smoking cessation services and treatments.

- The Social and Environmental Changes section monitors policies to reduce exposure to secondhand smoke, increase the cost of tobacco, restrict tobacco advertising, and reduce illegal sales to minors.

The differences between the Healthy People 2010 and Healthy People 2020 objectives are summarized below:

- The Healthy People 2020 Tobacco Use Topic Area has a total of 70 objectives, 19 of which are developmental, whereas the Healthy People 2010 Tobacco Use Focus Area had 49 objectives, two of which were deleted at Midcourse Review [4,5].

- Twenty-two Healthy People 2010 objectives were retained "as is" [6]. These include: adult and adolescent tobacco use (objectives 27-1a through c, and 27-2a through d); initiation of cigarette use (objectives 27-3a and b); smoking cessation attempts for adults (objective 275), adolescents (objective 27-7), and during pregnancy (objective 27-6); indoor worksite policies (objective 27-12); smoke-free indoor air laws in private and public workplaces (objectives 27-13a and b), restaurants (objective 27-13c), public transportation (objective 27-13d), and bars (objective 27-13i); enforcement of illegal tobacco sales to minors (objectives 27-14a and b); and adolescent exposure to tobacco advertising and promotion on the Internet or in magazines and newspapers (objectives 27-16a and b). 
- Seven Healthy People 2010 objectives were modified and expanded into 14 objectives [7].

- Medicaid program coverage for treatment of nicotine dependency (objective 27-8b) will be measurable in Healthy People 2020 using a different data source.

- Exposure to environmental tobacco smoke (objective 27-10) was split into three age-group objectives including 3-11 years, 12-17 years, and 18 years and over.

- The smoke-free and tobacco-free schools objective (27-11) was modified to tobacco-free schools and has been split into three objectives including junior high, middle school, and high school.

- The objective on smoke-free indoor air laws in day care centers (objective 27-13e) was divided into two objectives for commercial and home based centers.

- The preemptive tobacco control laws objective (27-19) was expanded to include objectives for preemption in smoke-free indoor air, advertising, and youth access.

- Two objectives that monitor increases in Federal and State tax on cigarettes (objective 27-21a) and smokeless tobacco products (objective 27-21b) will be measured differently in Healthy People 2020.

- Three Healthy People 2010 objectives, evidence based tobacco control programs for states, territories, and for tribes, were retained as developmental due to lack of baseline data (objectives 27-18a through c) [4].

- Thirteen Healthy People 2010 objectives were archived [8]. These include: adolescent use of bidis (objective 27-2e); average age at first use of tobacco (objectives 27-4a and b); managed care organization coverage for treatment of nicotine dependency (objective 278a); exposure to tobacco smoke at home (objective 27-9); smoke-free indoor air laws for retail stores (objective 27-13f); retail license suspension for sales to minors (objective 2715); adolescent disapproval of smoking for eighth (objective 27-17a), tenth (objective 27$17 \mathrm{~b}$ ) and twelfth (objective 27-17c) graders; sales-weighted average tobacco specific nitrosamines (objective 27-20a); and polyaromatic hydrocarbon compounds (objective 27$20 \mathrm{~b}$ ) and volatile organic compounds (objective 27-20c) in cigarette smoke.

- While objective 27-9, to reduce the proportion of children who are regularly exposed to tobacco smoke at home, was archived, modified Healthy People 2020 objective TU-11.1 measures the exposure to second hand smoke among children aged 3-11 years using measured serum cotinine levels and is thought to measure children's exposure more accurately than self-report data.

- Two objectives were dropped at the Midcourse Review due to lack of data: adult use of other tobacco (objective 27-1d); and insurance coverage for treatment of nicotine dependency (objective 27-8c) [5]. Two additional objectives were dropped during the transition to Healthy People 2020 due to lack of data: smoke free indoor air laws for tribes (objective 27-13g) and territories (objective 27-13h).

- Thirty one new objectives were added to the Healthy People 2020 Tobacco Use Topic Area:

- Six new objectives address age at first use for all tobacco products, smokeless tobacco, and cigars for both the 12-17 years and the 18-25 years age groups.

- Three new objectives monitor smoking cessation success and smoking cessation using evidence based strategies.

- Eight objectives address screening and counseling in office based ambulatory care settings, hospital ambulatory care settings, dental care settings, and substance abuse care settings. 
- Ten new smoke-free indoor air objectives track indoor air in gaming halls, hotels and motels, multi-unit housing, vehicles with children, prisons and correctional facilities, substance abuse treatment facilities, mental health treatment facilities, entrances and exits of all public places, hospital campuses, and college and university campuses.

- An objective was added to include Head Start on the list of smoke-free and tobaccofree schools.

- One new objective will monitor increases in Federal and state tax on "other" smoked tobacco products.

- Two new objectives address adolescent exposure to tobacco advertising at the movies and at point of purchase.

Appendix D, "A Crosswalk Between Objectives from Healthy People 2010 to Healthy People 2020," summarizes the changes between the two decades of objectives, reflecting new knowledge and direction for this area.

\section{Data Considerations}

Figure 27-3 (Tobacco Use by Adults-Cigarettes) presents state-level data from the Behavioral Risk Factor Surveillance System (BRFSS). National data for these objectives come from the National Health Interview Survey (NHIS) and are the basis for setting the targets. BRFSS data may not be comparable to the National data from the NHIS.

Education and income are the primary measures of socioeconomic status (SES) in Healthy People 2010. Most data systems used in Healthy People 2010 define income as a family's income before taxes. In order to facilitate comparisons among groups and over time, while adjusting for family size and for inflation, Healthy People 2010 categorizes income using the poverty thresholds developed by the U.S. Census Bureau. Thus, the three categories of family income that are primarily used are:

- Poor-below the Federal poverty level

- Near poor-100\% to $199 \%$ of the Federal poverty level

- Middle/high income-200\% or more of the Federal poverty level.

These categories may be overridden by considerations specific to the data system, in which case they are modified as appropriate. See Healthy People 2010: General Data Issues, referenced below.

In general, data on educational attainment are presented for persons aged 25 years and over, consistent with guidance given by the U.S. Bureau of the Census. However, because of the requirements of the different data systems, the age groups used to calculate educational attainment for any specific objective may differ from the age groups used to report the data for other Healthy People 2010 objectives, as well as from select populations within the same objective. Therefore, the reader is urged to exercise caution in interpreting the data by educational attainment shown in the Health Disparities Table. See Healthy People 2010: General Data Issues, referenced below. 
Additional information on data issues is available from the following sources:

- All Healthy People 2010 tracking data can be found in the Healthy People 2010 database, DATA2010, available from http://wonder.cdc.gov/data2010/.

- Detailed information about the data and data sources used to support these objectives can be found in the Operational Definitions on the DATA2010 website, available from http://wonder.cdc.gov/data2010/focusod.htm.

- More information on statistical issues related to Healthy People tracking and measurement can be found in the Technical Appendix and in Healthy People 2010: General Data Issues, which is available in the Data Issues section of the NCHS Healthy People website under Healthy People 2010.

\section{Notes}

1. Displayed in the Progress Chart (Figure 27-1), the percent of targeted change achieved expresses the difference between the baseline and the final value relative to the initial difference between the baseline and the Healthy People 2010 target. As such, it is a relative measure of progress toward attaining the Healthy People 2010 target. See the Reader's Guide for more information. When standard errors were available, the difference between the baseline and the final value was tested at the 0.05 level of significance. See the Figure 27-1 footnotes, as well as the Technical Appendix, for more detail.

2. Information about disparities among select populations is shown in the Health Disparities Table (Figure 27-2). Disparity from the best group rate is defined as the percent difference between the best group rate and each of the other group rates for a characteristic. For example, racial and ethnic health disparities are measured as the percent difference between the best racial and ethnic group rate and each of the other racial and ethnic group rates. Similarly, disparities by sex are measured as the percent difference between the better group rate (e.g., female) and the rate for the other group (e.g., male). Some objectives are expressed in terms of favorable events or conditions that are to be increased, while others are expressed in terms of adverse events or conditions that are to be reduced. In order to facilitate comparison of health disparities across different objectives, disparity is measured only in terms of adverse events or conditions. For comparability across objectives, objectives that are expressed in terms of favorable events or conditions are re-expressed using the adverse event or condition for the purpose of computing disparity, but they are not otherwise restated or changed. For example, objective 1-1, to increase the proportion of persons with health insurance (e.g., $72 \%$ of the American Indian or Alaska Native population aged under 65 years had some form of health insurance in 2008), is expressed in terms of the percentage of persons without health insurance (e.g., $100 \%-72 \%=28 \%$ of the American Indian or Alaska Native population aged under 65 years did not have any form of health insurance in 2008) when the disparity from the best group rate is calculated. See the Reader's Guide for more information. When standard errors were available, the difference between the best group rate and each of the other group rates was tested at the 0.05 level of significance. See the Figure 27-2 footnotes, as well as the Technical Appendix, for more detail.

3. The change in disparity is estimated by subtracting the disparity at baseline from the disparity at the most recent data point and, therefore, is expressed as a change in percentage points. See the Reader's Guide for more information. When standard errors were available, the change in disparity was tested at the 0.05 level of significance. See the Figure 27-2 footnotes, as well as the Technical Appendix, for more detail. 
4. To be included in Healthy People 2010, an objective must have a national data source that provides a baseline and at least one additional data point for tracking progress. Some objectives lacked baseline data at the time of their development but had a potential data source and were considered of sufficient national importance to be included in Healthy People. These are called "developmental" objectives. When data become available, a developmental objective is moved to measurable status and a Healthy People target can be set.

5. Dropped objectives were not carried forward into Healthy People 2020. These objectives were either developmental or deleted at the Healthy People 2010 Midcourse Review or at another time in Healthy People 2010.

6. Retained "as is" objectives have no change in the numerator definition or in the denominator definition between the Healthy People 2010 and Healthy People 2020 objectives. These include objectives that were developmental in Healthy People 2010 and are developmental in Healthy People 2020 and for which no numerator or denominator information was available.

7. Modified objectives have some change in the numerator definition or in the denominator definition between the Healthy People 2010 and Healthy People 2020 objectives. These include objectives that went from developmental in Healthy People 2010 to measurable in Healthy People 2020 or vice versa.

8. Archived objectives had at least one data point in Healthy People 2010 but were not carried forward into Healthy People 2020. 


\section{Comprehensive Summary of Objectives: Tobacco Use}

\begin{tabular}{|c|c|c|}
\hline Objective & Description & Data Source or Objective Status \\
\hline $27-1 \mathrm{a}$ & Tobacco use by adults-Cigarettes (age adjusted, $18+$ years) & National Health Interview Survey (NHIS), CDC, NCHS. \\
\hline $27-1 b$ & Tobacco use by adults-Spit tobacco (age adjusted, $18+$ years) & National Health Interview Survey (NHIS), CDC, NCHS. \\
\hline $27-1 c$ & Tobacco use by adults-Cigars (age adjusted, 18+ years) & National Health Interview Survey (NHIS), CDC, NCHS. \\
\hline $27-1 d$ & Tobacco use by adults-Other (age adjusted, $18+$ years) & Dropped \\
\hline $27-2 \mathrm{a}$ & $\begin{array}{l}\text { Tobacco use in past month by students-Tobacco products (grades } \\
9-12 \text { ) }\end{array}$ & Youth Risk Behavior Surveillance System (YRBSS), CDC, NCCDPHP. \\
\hline $27-2 b$ & Tobacco use in past month by students-Cigarettes (grades 9-12) & Youth Risk Behavior Surveillance System (YRBSS), CDC, NCCDPHP. \\
\hline $27-2 c$ & Tobacco use in past month by students-Spit tobacco (grades 9-12) & Youth Risk Behavior Surveillance System (YRBSS), CDC, NCCDPHP. \\
\hline $27-2 d$ & Tobacco use in past month by students-Cigars (grades 9-12) & Youth Risk Behavior Surveillance System (YRBSS), CDC, NCCDPHP. \\
\hline $27-2 \mathrm{e}$ & Tobacco use in past month by students-Bidis (grades 9-12) & $\begin{array}{l}\text { National Youth Tobacco Survey: American Legacy Foundation; CDC, } \\
\text { NCCDPHP. }\end{array}$ \\
\hline $27-3 a$ & $\begin{array}{l}\text { Initiation of cigarette use-Adolescents } 12-17 \text { years (percent at } \\
\text { risk) }\end{array}$ & National Survey on Drug Use and Health (NSDUH), SAMHSA. \\
\hline $27-3 b$ & $\begin{array}{l}\text { Initiation of cigarette use-Young adults 18-25 years (percent at } \\
\text { risk) }\end{array}$ & National Survey on Drug Use and Health (NSDUH), SAMHSA. \\
\hline $27-4 a$ & Average age at first tobacco use-Adolescents $12-17$ years & National Survey on Drug Use and Health (NSDUH), SAMHSA. \\
\hline $27-4 b$ & Average age at first tobacco use-Young adults $18-25$ years & National Survey on Drug Use and Health (NSDUH), SAMHSA. \\
\hline $27-5$ & Smoking cessation attempts by adults (age adjusted, $18+$ years) & National Health Interview Survey (NHIS), CDC, NCHS. \\
\hline $27-6$ & $\begin{array}{l}\text { Smoking cessation in first trimester and for remainder of pregnancy } \\
\text { (females, } 18-49 \text { years) }\end{array}$ & National Health Interview Survey (NHIS), CDC, NCHS. \\
\hline $27-7$ & Smoking cessation attempts by students (grades 9-12) & Youth Risk Behavior Surveillance System (YRBSS), CDC, NCCDPHP. \\
\hline $27-8 \mathrm{a}$ & $\begin{array}{l}\text { Managed care organization coverage for treatment of nicotine } \\
\text { dependency }\end{array}$ & $\begin{array}{l}\text { Addressing Tobacco in Managed Care Survey, Robert Wood Johnson } \\
\text { Foundation. }\end{array}$ \\
\hline $27-8 b$ & $\begin{array}{l}\text { Medicaid program coverage for treatment of nicotine dependency } \\
\text { (no. States and D.C.) }\end{array}$ & $\begin{array}{l}\text { Health Policy Tracking Service, National Conference of State } \\
\text { Legislators. }\end{array}$ \\
\hline $27-8 c$ & Insurance coverage for treatment of nicotine dependency & Dropped \\
\hline $27-9$ & Exposure to tobacco smoke at home among children ( $\leq 6$ years) & National Health Interview Survey (NHIS), CDC, NCHS. \\
\hline
\end{tabular}




\begin{tabular}{|c|c|c|}
\hline Objective & Description & Data Source or Objective Status \\
\hline $27-10$ & $\begin{array}{l}\text { Exposure to environmental tobacco smoke among nonsmokers (age } \\
\text { adjusted, 4+ years) }\end{array}$ & $\begin{array}{l}\text { National Health and Nutrition Examination Survey (NHANES), CDC, } \\
\text { NCHS. }\end{array}$ \\
\hline $27-11$ & Smoke-free and tobacco-free schools & School Health Policies and Programs Study (SHPPS), CDC, NCCDPHP. \\
\hline $27-12$ & Indoor worksite policies that prohibit smoking & $\begin{array}{l}\text { Current Population Survey (CPS): Department of Commerce, Census } \\
\text { Bureau; Department of Labor, Bureau of Labor Statistics. }\end{array}$ \\
\hline $27-13 a$ & $\begin{array}{l}\text { Smoke-free indoor air laws-Private workplaces (no. States and } \\
\text { D.C.) }\end{array}$ & $\begin{array}{l}\text { State Tobacco Activities Tracking and Evaluation System (STATE), } \\
\text { CDC, NCCDPHP. }\end{array}$ \\
\hline $27-13 b$ & Smoke-free indoor air laws-Public workplaces (no. States and D.C.) & $\begin{array}{l}\text { State Tobacco Activities Tracking and Evaluation System (STATE), } \\
\text { CDC, NCCDPHP. }\end{array}$ \\
\hline $27-13 c$ & Smoke-free indoor air laws-Restaurants (no. States and D.C.) & $\begin{array}{l}\text { State Tobacco Activities Tracking and Evaluation System (STATE), } \\
\text { CDC, NCCDPHP. }\end{array}$ \\
\hline $27-13 d$ & $\begin{array}{l}\text { Smoke-free indoor air laws-Public transportation (no. States and } \\
\text { D.C.) }\end{array}$ & $\begin{array}{l}\text { State Tobacco Activities Tracking and Evaluation System (STATE), } \\
\text { CDC, NCCDPHP. }\end{array}$ \\
\hline $27-13 e$ & Smoke-free indoor air laws-Day care centers (no. States and D.C.) & $\begin{array}{l}\text { State Tobacco Activities Tracking and Evaluation System (STATE), } \\
\text { CDC, NCCDPHP. }\end{array}$ \\
\hline $27-13 f$ & Smoke-free indoor air laws-Retail stores (no. States and D.C.) & $\begin{array}{l}\text { State Tobacco Activities Tracking and Evaluation System (STATE), } \\
\text { CDC, NCCDPHP. }\end{array}$ \\
\hline $27-13 g$ & Smoke-free indoor air laws-Tribes (number) & Developmental \\
\hline $27-13 h$ & Smoke-free indoor air laws-Territories (number) & Developmental \\
\hline $27-13 i$ & Smoke-free indoor air laws-Bars (no. States and D.C.) & $\begin{array}{l}\text { State Tobacco Activities Tracking and Evaluation System (STATE), } \\
\text { CDC, NCCDPHP. }\end{array}$ \\
\hline $27-14 a$ & $\begin{array}{l}\text { Jurisdictions with } \leq 5 \% \text { illegal tobacco buy rate among minors- } \\
\text { States and D.C. }\end{array}$ & State Synar Enforcement Reporting, SAMHSA. \\
\hline $27-14 b$ & $\begin{array}{l}\text { Jurisdictions with } \leq 5 \% \text { illegal tobacco buy rate among minors- } \\
\text { Territories }\end{array}$ & State Synar Enforcement Reporting, SAMHSA. \\
\hline $27-15$ & Retail license suspension for sales to minors (no. States and D.C.) & $\begin{array}{l}\text { State Tobacco Activities Tracking and Evaluation System (STATE), } \\
\text { CDC, NCCDPHP. }\end{array}$ \\
\hline $27-16 a$ & $\begin{array}{l}\text { Exposure to tobacco advertising and promotions among students- } \\
\text { Internet (grades 6-12) }\end{array}$ & $\begin{array}{l}\text { National Youth Tobacco Survey: American Legacy Foundation; CDC, } \\
\text { NCCDPHP. }\end{array}$ \\
\hline $27-16 b$ & $\begin{array}{l}\text { Exposure to tobacco advertising and promotions among students- } \\
\text { Magazines and newspapers (grades 6-12) }\end{array}$ & $\begin{array}{l}\text { National Youth Tobacco Survey: American Legacy Foundation; CDC, } \\
\text { NCCDPHP. }\end{array}$ \\
\hline
\end{tabular}




\begin{tabular}{|c|c|c|}
\hline Objective & Description & Data Source or Objective Status \\
\hline $27-17 a$ & $\begin{array}{l}\text { Student disapproval of smoking } 1+\text { pack of cigarettes per day-8th } \\
\text { graders }\end{array}$ & Monitoring the Future Study (MTF), NIH, NIDA. \\
\hline $27-17 b$ & $\begin{array}{l}\text { Student disapproval of smoking } 1+\text { pack of cigarettes per day-10th } \\
\text { graders }\end{array}$ & Monitoring the Future Study (MTF), NIH, NIDA. \\
\hline $27-17 c$ & $\begin{array}{l}\text { Student disapproval of smoking } 1+\text { pack of cigarettes per day }-12 \text { th } \\
\text { graders }\end{array}$ & Monitoring the Future Study (MTF), NIH, NIDA. \\
\hline $27-18 a$ & Evidence-bases tobacco control programs (no. States and D.C.) & $\begin{array}{l}\text { State Tobacco Activities Tracking and Evaluation System (STATE), } \\
\text { CDC, NCCDPHP, OSH. }\end{array}$ \\
\hline $27-18 b$ & Evidence-based tobacco control programs (no. Territories) & Developmental \\
\hline $27-18 c$ & Evidence-based tobacco control programs (no. Tribes) & Developmental \\
\hline $27-19$ & Preemptive tobacco control laws (no. States and D.C.) & $\begin{array}{l}\text { State Tobacco Activities Tracking and Evaluation System (STATE), } \\
\text { CDC, NCCDPHP. }\end{array}$ \\
\hline $27-20 \mathrm{a}$ & $\begin{array}{l}\text { Sales-weighted average tobacco-specific nitrosamines in cigarette } \\
\text { smoke (ng per cigarette) }\end{array}$ & $\begin{array}{l}\text { Office on Smoking and Health, CDC, NCCDPHP; Division of } \\
\text { Laboratory Sciences, CDC, NCEH. }\end{array}$ \\
\hline $27-20 b$ & $\begin{array}{l}\text { Sales-weighted average polyaromatic hydrocarbon compounds in } \\
\text { cigarette smoke (ng per cigarette) }\end{array}$ & $\begin{array}{l}\text { Office on Smoking and Health, CDC, NCCDPHP; Division of } \\
\text { Laboratory Sciences, CDC, NCEH. }\end{array}$ \\
\hline $27-20 c$ & $\begin{array}{l}\text { Sales-weighted average volatile organic compounds in cigarette } \\
\text { smoke ( } \mu \text { g per cigarette) }\end{array}$ & $\begin{array}{l}\text { Office on Smoking and Health, CDC, NCCDPHP; Division of } \\
\text { Laboratory Sciences, CDC, NCEH. }\end{array}$ \\
\hline $27-21 \mathrm{a}$ & $\begin{array}{l}\text { Average combined Federal and State excise taxes on retail price of a } \\
\text { standard pack of cigarettes in all } 50 \text { States and D.C. }\end{array}$ & $\begin{array}{l}\text { State Tobacco Activities Tracking and Evaluation System (STATE), } \\
\text { CDC, NCCDPHP. }\end{array}$ \\
\hline $27-21 b$ & Increased taxes on smokeless tobacco (no. States and D.C.) & $\begin{array}{l}\text { State Tobacco Activities Tracking and Evaluation System (STATE), } \\
\text { CDC, NCCDPHP. }\end{array}$ \\
\hline
\end{tabular}


Figure 27-1. Progress Toward Target Attainment for Focus Area 27: Tobacco Use

Moved away

from target ${ }^{1}$

Moved toward

target

Met or exceeded

target

27-1. Tobacco use by adults (age adjusted, 18+ years)
a. Cigarettes
b. Spit tobacco
c. Cigars

27-2. Tobacco use in past month by students (grades 9-12)

a. Tobacco products

b. Cigarettes

c. Spit tobacco

d. Cigars

e. Bidis

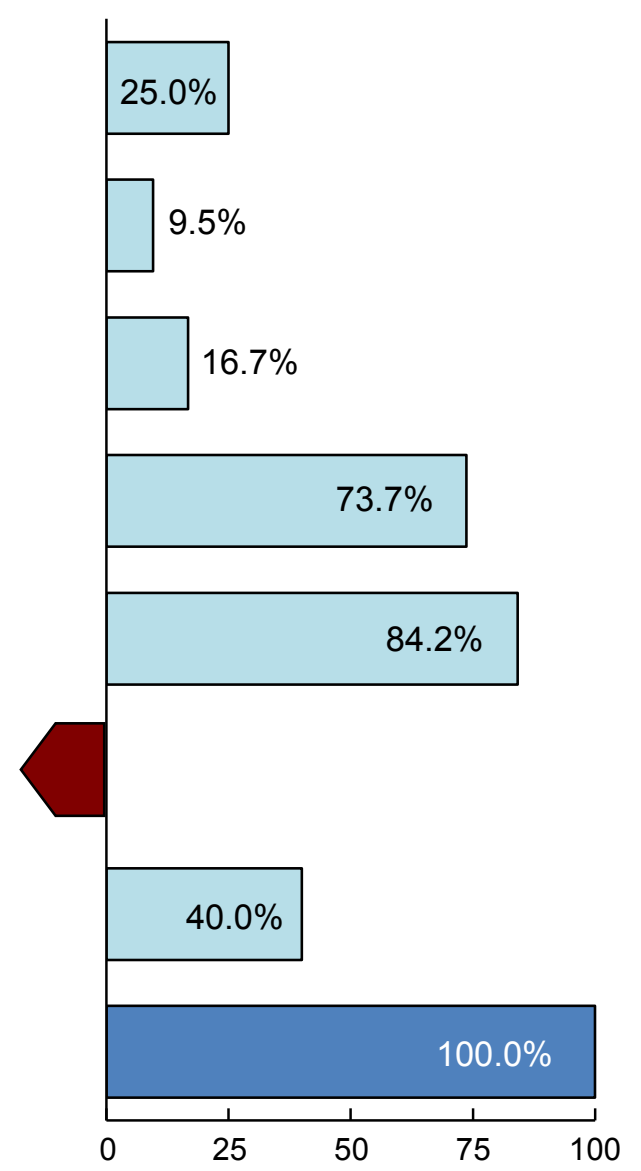

\begin{tabular}{|c|c|c|c|c|c|}
\hline \multirow{2}{*}{$\begin{array}{l}2010 \\
\text { Target }\end{array}$} & \multirow{2}{*}{$\begin{array}{c}\text { Baseline } \\
\text { (Year) }\end{array}$} & \multirow{2}{*}{$\begin{array}{l}\text { Final } \\
\text { (Year) }\end{array}$} & \multicolumn{3}{|c|}{ Baseline vs. Final } \\
\hline & & & Difference ${ }^{2}$ & $\begin{array}{l}\text { Statistically } \\
\text { Significant }^{3}\end{array}$ & $\begin{array}{l}\text { Percent } \\
\text { Change }^{4}\end{array}$ \\
\hline $12 \%$ & $\begin{array}{c}24 \% \\
(1998)\end{array}$ & $\begin{array}{c}21 \% \\
(2008)\end{array}$ & -3 & Yes & $-12.5 \%$ \\
\hline $0.4 \%$ & $\begin{array}{c}2.5 \% \\
(1998)\end{array}$ & $\begin{array}{c}2.3 \% \\
(2005)\end{array}$ & -0.2 & No & $-8.0 \%$ \\
\hline $1.2 \%$ & $\begin{array}{c}2.4 \% \\
(1998)\end{array}$ & $\begin{array}{c}2.2 \% \\
(2005)\end{array}$ & -0.2 & No & $-8.3 \%$ \\
\hline $21 \%$ & $\begin{array}{c}40 \% \\
(1999)\end{array}$ & $\begin{array}{c}26 \% \\
(2009)\end{array}$ & -14 & Yes & $-35.0 \%$ \\
\hline $16 \%$ & $\begin{array}{c}35 \% \\
(1999)\end{array}$ & $\begin{array}{c}19 \% \\
(2009)\end{array}$ & -16 & Yes & $-45.7 \%$ \\
\hline $1 \%$ & $\begin{array}{c}8 \% \\
(1999)\end{array}$ & $\begin{array}{c}9 \% \\
(2009)\end{array}$ & 1 & No & $12.5 \%$ \\
\hline $8 \%$ & $\begin{array}{c}18 \% \\
(1999)\end{array}$ & $\begin{array}{c}14 \% \\
(2009)\end{array}$ & -4 & Yes & $-22.2 \%$ \\
\hline $2.4 \%$ & $\begin{array}{l}4.1 \% \\
(2000)\end{array}$ & $\begin{array}{c}2.4 \% \\
(2009)\end{array}$ & -1.7 & Yes & $-41.5 \%$ \\
\hline
\end{tabular}

Percent of targeted change achieved ${ }^{5}$ (continued) 
Figure 27-1. Progress Toward Target Attainment for Focus Area 27: Tobacco Use (continued)

Moved away

from target ${ }^{1}$

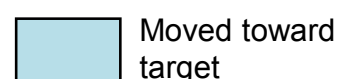

target

Met or exceeded

target

27-3. Initiation of cigarette use (percent at risk)

a. Adolescents $12-17$ years

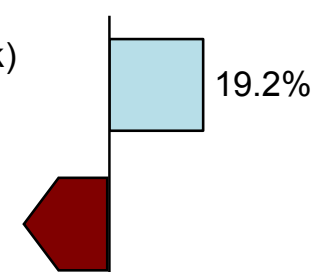

27-4. Average age at first tobacco use

a. Adolescents $12-17$ years

b. Young adults $18-25$ years

27-5. Smoking cessation attempts by adults (age

adjusted, $18+$ years)
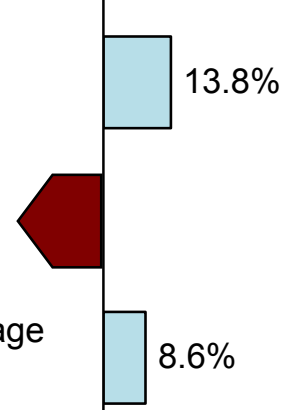

27-6. Smoking cessation in first trimester and for remainder of pregnancy (females, 18-49 years)

27-7. Smoking cessation attempts by students (grades 9-12)

27-9. Exposure to tobacco smoke at home among children ( $\leq 6$ years)

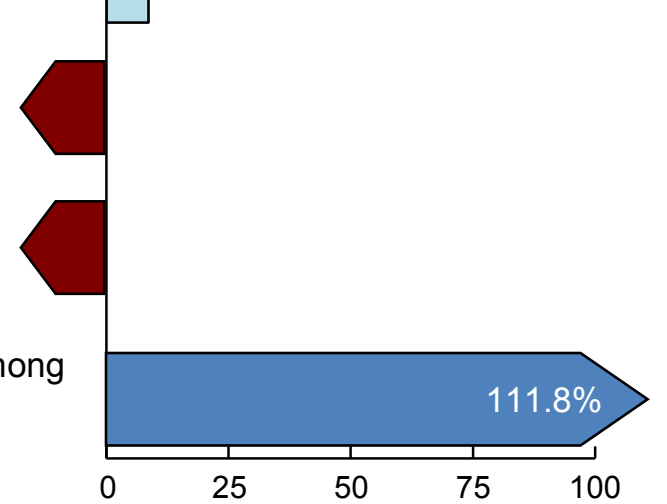

Percent of targeted change achieved ${ }^{5}$

\begin{tabular}{|c|c|c|c|c|c|}
\hline \multirow{2}{*}{$\begin{array}{c}2010 \\
\text { Target }\end{array}$} & \multirow{2}{*}{$\begin{array}{c}\text { Baseline } \\
\text { (Year) }\end{array}$} & \multirow{2}{*}{$\begin{array}{l}\text { Final } \\
\text { (Year) }\end{array}$} & \multicolumn{3}{|c|}{ Baseline vs. Final } \\
\hline & & & Difference $^{2}$ & $\begin{array}{l}\text { Statistically } \\
\text { Significant }^{3}\end{array}$ & $\begin{array}{c}\text { Percent } \\
\text { Change }^{4}\end{array}$ \\
\hline $4.1 \%$ & $\begin{array}{c}6.7 \% \\
(2002)\end{array}$ & $\begin{array}{c}6.2 \% \\
(2008)\end{array}$ & -0.5 & No & $-7.5 \%$ \\
\hline $4.4 \%$ & $\begin{array}{c}6.7 \% \\
(2002)\end{array}$ & $\begin{array}{l}8.3 \% \\
(2008)\end{array}$ & 1.6 & Yes & $23.9 \%$ \\
\hline 17.6 & $\begin{array}{c}14.7 \\
(2002)\end{array}$ & $\begin{array}{c}15.1 \\
(2008)\end{array}$ & 0.4 & Yes & $2.7 \%$ \\
\hline 20.9 & $\begin{array}{c}19.0 \\
(2002)\end{array}$ & $\begin{array}{c}18.9 \\
(2008)\end{array}$ & -0.1 & No & $-0.5 \%$ \\
\hline $80 \%$ & $\begin{array}{c}45 \% \\
(1998)\end{array}$ & $\begin{array}{c}48 \% \\
(2008)\end{array}$ & 3 & Yes & $6.7 \%$ \\
\hline $30 \%$ & $\begin{array}{c}14 \% \\
(1998)\end{array}$ & $\begin{array}{c}11 \% \\
(2005)\end{array}$ & -3 & No & $-21.4 \%$ \\
\hline $64 \%$ & $\begin{array}{c}61 \% \\
(2001)\end{array}$ & $\begin{array}{c}59 \% \\
(2009)\end{array}$ & -2 & No & $-3.3 \%$ \\
\hline $10 \%$ & $\begin{array}{c}27 \% \\
(1994)\end{array}$ & $\begin{array}{c}8 \% \\
(2005)\end{array}$ & -19 & Yes & $-70.4 \%$ \\
\hline
\end{tabular}

(continued) 
Figure 27-1. Progress Toward Target Attainment for Focus Area 27: Tobacco Use (continued)

Moved away

from target ${ }^{1}$

Moved toward

target

Met or exceeded

target

27-10. Exposure to environmental tobacco smoke among nonsmokers (age adjusted, 4+ years)

27-11. Smoke-free and tobacco-free schools

27-12. Indoor worksite policies that prohibit smoking

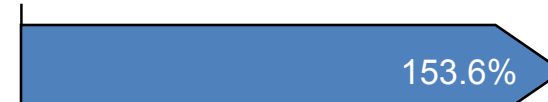

\begin{tabular}{|c|c|c|c|c|c|}
\hline \multirow{2}{*}{$\begin{array}{l}2010 \\
\text { Target }\end{array}$} & \multirow{2}{*}{$\begin{array}{c}\text { Baseline } \\
\text { (Year) }\end{array}$} & \multirow{2}{*}{$\begin{array}{l}\text { Final } \\
\text { (Year) }\end{array}$} & \multicolumn{3}{|c|}{ Baseline vs. Final } \\
\hline & & & Difference & $\begin{array}{l}\text { Statistically } \\
\text { Significant }^{3}\end{array}$ & $\begin{array}{l}\text { Percent } \\
\text { Change }^{4}\end{array}$ \\
\hline $56 \%$ & $\begin{array}{c}84 \% \\
(1988-94)\end{array}$ & $\begin{array}{c}41 \% \\
(2005-08)\end{array}$ & -43 & Yes & $-51.2 \%$ \\
\hline $100 \%$ & $\begin{array}{c}38 \% \\
(1994)\end{array}$ & $\begin{array}{c}64 \% \\
(2006)\end{array}$ & 26 & Yes & $68.4 \%$ \\
\hline $100 \%$ & $\begin{array}{c}69 \% \\
(1998-99)\end{array}$ & $\begin{array}{c}75 \% \\
(2006-07)\end{array}$ & 6 & Yes & $8.7 \%$ \\
\hline 51 & $\begin{array}{c}0 \\
(1998)\end{array}$ & $\begin{array}{c}30 \\
(2009)\end{array}$ & 30 & $\begin{array}{l}\text { Not } \\
\text { tested }\end{array}$ & * \\
\hline 51 & $\begin{array}{c}10 \\
(1998)\end{array}$ & $\begin{array}{c}34 \\
(2009)\end{array}$ & 24 & $\begin{array}{l}\text { Not } \\
\text { tested }\end{array}$ & $240.0 \%$ \\
\hline 51 & $\begin{array}{c}1 \\
(1998)\end{array}$ & $\begin{array}{c}28 \\
(2009)\end{array}$ & 27 & $\begin{array}{l}\text { Not } \\
\text { tested }\end{array}$ & $2,700.0 \%$ \\
\hline 51 & $\begin{array}{c}16 \\
(1998)\end{array}$ & $\begin{array}{c}38 \\
(2009)\end{array}$ & 22 & $\begin{array}{l}\text { Not } \\
\text { tested }\end{array}$ & $137.5 \%$ \\
\hline 51 & $\begin{array}{c}21 \\
(1998)\end{array}$ & $\begin{array}{c}40 \\
(2009)\end{array}$ & 19 & $\begin{array}{c}\text { Not } \\
\text { tested }\end{array}$ & $90.5 \%$ \\
\hline
\end{tabular}

e. Day care centers

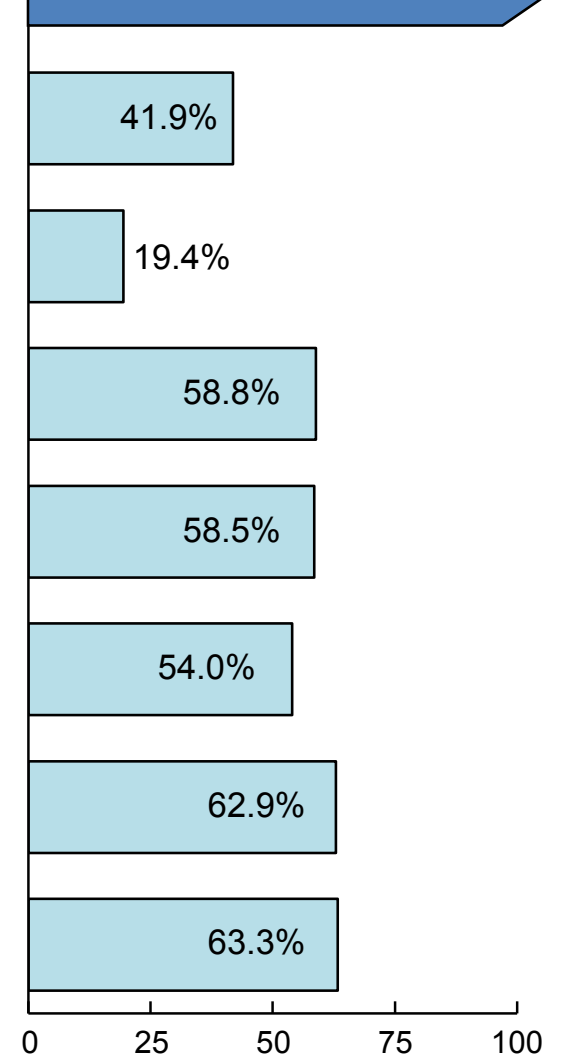

D.C.)

a. Private workplaces

b. Public workplaces

c. Restaurants

d. Public transportation

Percent of targeted change achieved ${ }^{5}$

(continued) 
Figure 27-1. Progress Toward Target Attainment for Focus Area 27: Tobacco Use (continued)

Moved away

from target ${ }^{1}$

Moved toward

target

Met or exceeded

target

27-13. Smoke-free indoor air laws (no. States and D.C.)

f. Retail stores

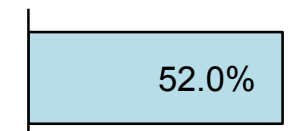

i. Bars

27-14. Jurisdictions with $\leq 5 \%$ illegal tobacco buy rate among minors

a. States and D.C.

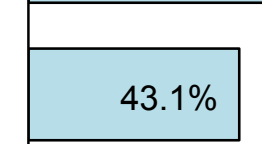

27-15. Retail license suspension for sales to minors (no. States and D.C.)

27-16. Exposure to tobacco advertising and promotions among students (grades 6-12) a. Internet

b. Magazines and newspapers

27-17. Student disapproval of smoking $1+$ pack of cigarettes per day

a. 8th graders

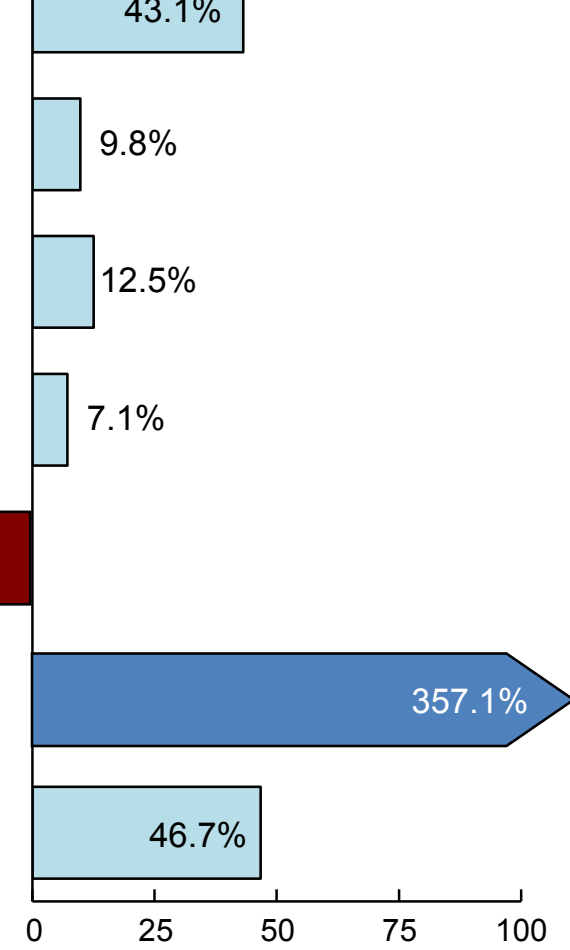

\begin{tabular}{|c|c|c|c|c|c|}
\hline \multirow{2}{*}{$\begin{array}{c}2010 \\
\text { Target }\end{array}$} & \multirow{2}{*}{$\begin{array}{c}\text { Baseline } \\
\text { (Year) }\end{array}$} & \multirow{2}{*}{$\begin{array}{l}\text { Final } \\
\text { (Year) }\end{array}$} & \multicolumn{3}{|c|}{ Baseline vs. Final } \\
\hline & & & Difference ${ }^{2}$ & $\begin{array}{l}\text { Statistically } \\
\text { Significant }^{3}\end{array}$ & $\begin{array}{l}\text { Percent } \\
\text { Change }^{4}\end{array}$ \\
\hline 51 & $\begin{array}{c}1 \\
(1998)\end{array}$ & $\begin{array}{c}27 \\
(2009)\end{array}$ & 26 & $\begin{array}{c}\text { Not } \\
\text { tested }\end{array}$ & $2,600.0 \%$ \\
\hline 51 & $\begin{array}{c}0 \\
(1998)\end{array}$ & $\begin{array}{c}22 \\
(2009)\end{array}$ & 22 & $\begin{array}{c}\text { Not } \\
\text { tested }\end{array}$ & * \\
\hline 51 & $\begin{array}{c}0 \\
(1998)\end{array}$ & $\begin{array}{c}5 \\
(2009)\end{array}$ & 5 & $\begin{array}{c}\text { Not } \\
\text { tested }\end{array}$ & * \\
\hline 8 & $\begin{array}{c}0 \\
(1998)\end{array}$ & $\begin{array}{c}1 \\
(2009)\end{array}$ & 1 & $\begin{array}{l}\text { Not } \\
\text { tested }\end{array}$ & * \\
\hline 51 & $\begin{array}{c}23 \\
(1998)\end{array}$ & $\begin{array}{c}25 \\
(2009)\end{array}$ & 2 & $\begin{array}{c}\text { Not } \\
\text { tested }\end{array}$ & $8.7 \%$ \\
\hline $25 \%$ & $\begin{array}{c}28 \% \\
(2000)\end{array}$ & $\begin{array}{c}37 \% \\
(2009)\end{array}$ & 9 & Yes & $32.1 \%$ \\
\hline $67 \%$ & $\begin{array}{c}74 \% \\
(2000)\end{array}$ & $\begin{array}{c}49 \% \\
(2009)\end{array}$ & -25 & Yes & $-33.8 \%$ \\
\hline $95 \%$ & $\begin{array}{c}80 \% \\
(1998)\end{array}$ & $\begin{array}{c}87 \% \\
(2009)\end{array}$ & 7 & Yes & $8.8 \%$ \\
\hline
\end{tabular}

Percent of targeted change achieved ${ }^{5}$

(continued) 
Figure 27-1. Progress Toward Target Attainment for Focus Area 27: Tobacco Use (continued)

Moved away

from target ${ }^{1}$

Moved toward

target

Met or exceeded

target

27-17. Student disapproval of smoking 1+ pack of cigarettes per day

b. 10th graders

c. 12th graders

27-19. Preemptive tobacco control laws (no. States and D.C.)

27-20a. Sales-weighted average tobacco-specific nitrosamines in cigarette smoke (ng per cigarette)

27-20b. Sales-weighted average polyaromatic hydrocarbon compounds in cigarette smoke (ng per cigarette)

27-20c. Sales-weighted average volatile organic compounds in cigarette smoke ( $\mu \mathrm{g}$ per cigarette)

27-21a. Average combined Federal and state excise taxes on retail price of a standard pack of cigarettes in all 50 States and D.C.

27-21b. Increased taxes on smokeless tobacco (no. States and D.C.)
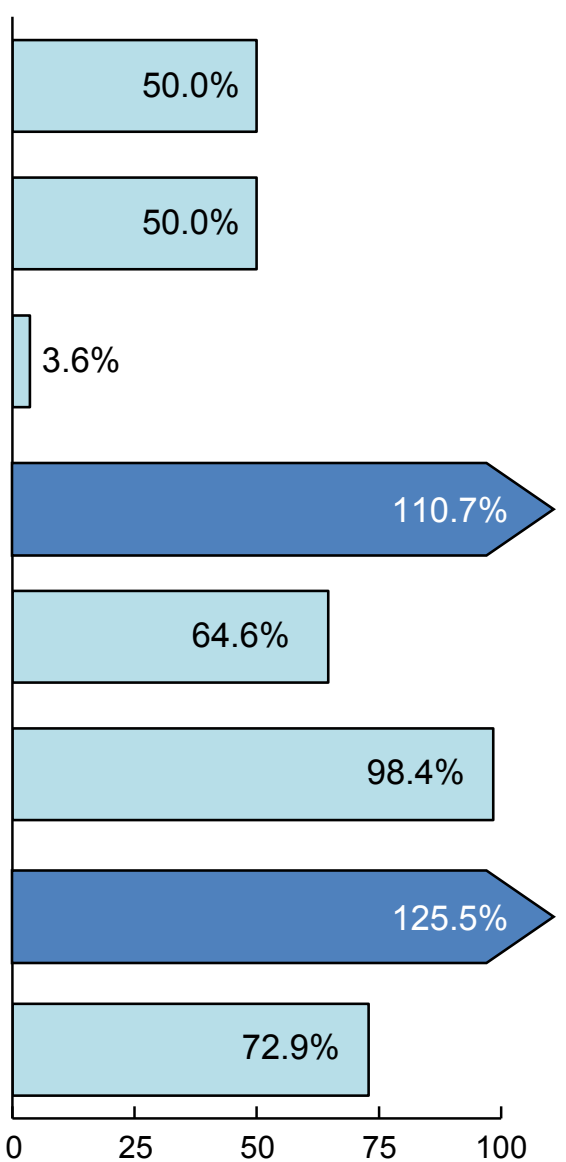

Percent of targeted change achieved ${ }^{5}$

\begin{tabular}{|c|c|c|c|c|c|}
\hline \multirow{2}{*}{$\begin{array}{c}2010 \\
\text { Target }\end{array}$} & \multirow{2}{*}{$\begin{array}{c}\text { Baseline } \\
\text { (Year) }\end{array}$} & \multirow{2}{*}{$\begin{array}{l}\text { Final } \\
\text { (Year) }\end{array}$} & \multicolumn{3}{|c|}{ Baseline vs. Final } \\
\hline & & & Difference $^{2}$ & $\begin{array}{l}\text { Statistically } \\
\text { Significant }^{3}\end{array}$ & $\begin{array}{l}\text { Percent } \\
\text { Change }^{4}\end{array}$ \\
\hline $95 \%$ & $\begin{array}{c}75 \% \\
(1998)\end{array}$ & $\begin{array}{c}85 \% \\
(2009)\end{array}$ & 10 & Yes & $13.3 \%$ \\
\hline $95 \%$ & $\begin{array}{c}69 \% \\
(1998)\end{array}$ & $\begin{array}{c}82 \% \\
(2009)\end{array}$ & 13 & Yes & $18.8 \%$ \\
\hline 0 & $\begin{array}{c}28 \\
(1998)\end{array}$ & $\begin{array}{c}27 \\
(2009)\end{array}$ & -1 & $\begin{array}{l}\text { Not } \\
\text { tested }\end{array}$ & $-3.6 \%$ \\
\hline 109.4 & $\begin{array}{c}121.5 \\
(2003-04)\end{array}$ & $\begin{array}{l}108.1 \\
(2007)\end{array}$ & -13.4 & $\begin{array}{l}\text { Not } \\
\text { tested }\end{array}$ & $-11.0 \%$ \\
\hline 894.3 & $\begin{array}{c}993.7 \\
(2003-04)\end{array}$ & $\begin{array}{l}929.5 \\
(2007)\end{array}$ & -64.2 & $\begin{array}{l}\text { Not } \\
\text { tested }\end{array}$ & $-6.5 \%$ \\
\hline 636.3 & $\begin{array}{l}707.0 \\
(2002)\end{array}$ & $\begin{array}{l}637.4 \\
(2007)\end{array}$ & -69.6 & $\begin{array}{l}\text { Not } \\
\text { tested }\end{array}$ & $-9.8 \%$ \\
\hline$\$ 2.00$ & $\begin{array}{l}\$ 0.63 \\
(1998)\end{array}$ & $\begin{array}{l}\$ 2.35 \\
(2009)\end{array}$ & $\$ 1.72$ & $\begin{array}{l}\text { Not } \\
\text { tested }\end{array}$ & $273.0 \%$ \\
\hline 51 & $\begin{array}{c}3 \\
(2000)\end{array}$ & $\begin{array}{c}38 \\
(2009)\end{array}$ & 35 & $\begin{array}{l}\text { Not } \\
\text { tested }\end{array}$ & $1,166.7 \%$ \\
\hline
\end{tabular}

(continued) 


\section{Figure 27-1. Progress Toward Target Attainment for Focus Area 27: Tobacco Use (continued)}

\section{NOTES}

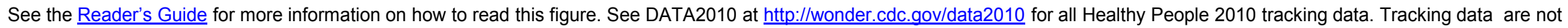
available for objectives 27-8a, 27-8b, 27-13g, 27-13h, and 27-18a through c. Objectives 27-1d and 27-8c were deleted at the Midcourse Review.

\section{FOOTNOTES}

${ }^{1}$ Movement away from target is not quantified using the percent of targeted change achieved. See Technical Appendix for more information.

${ }^{2}$ Difference $=$ Final value - Baseline value. Differences between percents $(\%)$ are measured in percentage points.

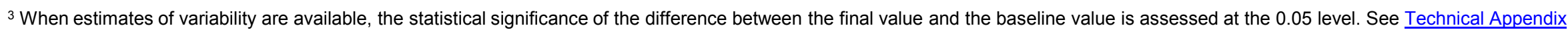
for more information.

${ }^{4}$ Percent change $=\frac{\text { Final value }- \text { Baseline value }}{\text { Baseline value }} \times 100$.

${ }^{5}$ Percent of targeted change achieved $=\frac{\text { Final value }- \text { Baseline value }}{\text { Healthy People } 2010 \text { target }- \text { Baseline value }} \times 100$.

* Percent change cannot be calculated. See Technical Appendix for more information.

\section{DATA SOURCES}

27-1a-c. National Health Interview Survey (NHIS), CDC, NCHS.

27-2a-d. Youth Risk Behavior Surveillance System (YRBSS), CDC, NCCDPHP.

27-2e. National Youth Tobacco Survey: American Legacy Foundation; CDC, NCCDPHP

27-3a-b. National Survey on Drug Use and Health (NSDUH), SAMHSA.

27-4a-b. National Survey on Drug Use and Health (NSDUH), SAMHSA.

27-5-27-6. National Health Interview Survey (NHIS), CDC, NCHS.

27-7. Youth Risk Behavior Surveillance System (YRBSS), CDC, NCCDPHP.

27-9. National Health Interview Survey (NHIS), CDC, NCHS.

27-10. National Health and Nutrition Examination Survey (NHANES), CDC, NCHS

27-11. School Health Policies and Programs Study (SHPPS), CDC, NCCDPHP.

27-12. Current Population Survey (CPS): Department of Commerce, Census Bureau; Department of Labor, Bureau of Labor Statistics.

27-13a-f. State Tobacco Activities Tracking and Evaluation System (STATE), CDC, NCCDPHP.

27-13i. State Tobacco Activities Tracking and Evaluation System (STATE), CDC, NCCDPHP.

27-14a-b. State Synar Enforcement Reporting, SAMHSA.

27-15. State Tobacco Activities Tracking and Evaluation System (STATE), CDC, NCCDPHP

27-16a-b. National Youth Tobacco Survey: American Legacy Foundation; CDC, NCCDPHP.

27-17a-c. Monitoring the Future Study (MTF), NIH, NIDA.

27-19. State Tobacco Activities Tracking and Evaluation System (STATE), CDC, NCCDPHP.

27-20a-c. Office on Smoking and Health, CDC, NCCDPHP; Division of Laboratory Sciences, CDC, NCEH.

27-21a-b. State Tobacco Activities Tracking and Evaluation System (STATE), CDC, NCCDPHP. 
Figure 27-2. Health Disparities Table for Focus Area 27: Tobacco Use

Disparities from the best group rate for each characteristic at the most recent data point and changes in disparity from the baseline to the most recent data point.

\begin{abstract}
Population-based objectives
27-1a. Tobacco use by adults-Cigarettes (age adjusted, $18+$ years $)(1998,2008)^{1 *}$

b. Tobacco use by adults-Spit tobacco (age adjusted, $18+$ years $)(1998,2005)^{2 *}$

c. Tobacco use by adults-Cigars (age adjusted, $18+$ years $)(1998,2005)^{2 \star}$

27-2a. Tobacco use in past month by students-Tobacco products (grades 9-12) $(1999,2009)^{\star}$

b. Tobacco use in past month by students-Cigarettes (grades 9-12) (1999, 2009)*

c. Tobacco use in past month by students-Spit tobacco (grades 9-12) $(1999,2009)^{*}$

d. Tobacco use in past month by students-Cigars (grades 9-12) $(1999,2009)^{*}$

e. Tobacco use in past month by students-Bidis (grades 9-12) $(2000,2009)^{*}$

27-3a. Initiation of cigarette use-Adolescents $12-17$ years (percent at risk) $(2002,2008)^{3 *}$

b. Initiation of cigarette use-Young adults $18-25$ years (percent at risk) $(2002,2008)^{3 *}$

27-4a. Average age at first tobacco use-Adolescents $12-17$ years $(2002,2008)^{3 *}$

b. Average age at first tobacco use-Young adults $18-25$ years $(2002,2008)^{3 *}$

27-5. Smoking cessation attempts by adults (age adjusted, $18+$ years $)(1998,2008)^{1 *}$

27-6. Smoking cessation in first trimester and for remainder of pregnancy (females, 18-49 years) $(1998,2005)^{4 *}$

27-7. Smoking cessation attempts by students (grades 9-12) $(2001,2009)^{*}$

27-9. Exposure to tobacco smoke at home among children $\left(\leq 6\right.$ years) $(1994,2005)^{4 *}$

27-10. Exposure to environmental tobacco smoke among nonsmokers (age adjusted, 4+ years) $(1988-94,2005-08)^{*}$

27-12. Indoor worksite policies that prohibit smoking $(1998-99,2006-07)^{5 *}$

27-16a. Exposure to tobacco advertising and promotions among students-Internet (grades 6-12) (2000, 2009)*

b. Exposure to tobacco advertising and promotions among students-Magazines and newspapers (grades 6-12) $(2000,2009)^{*}$
\end{abstract}

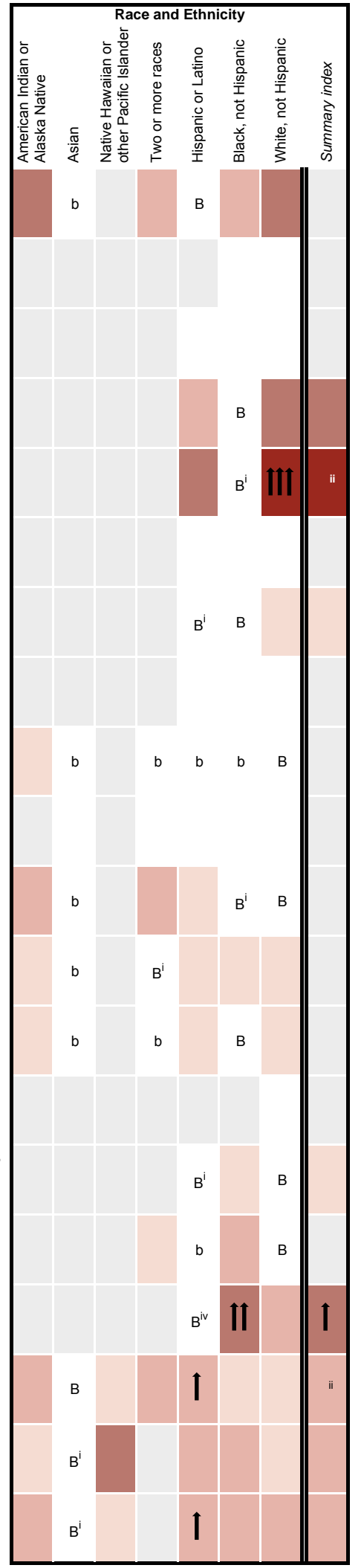

Characteristics and Groups

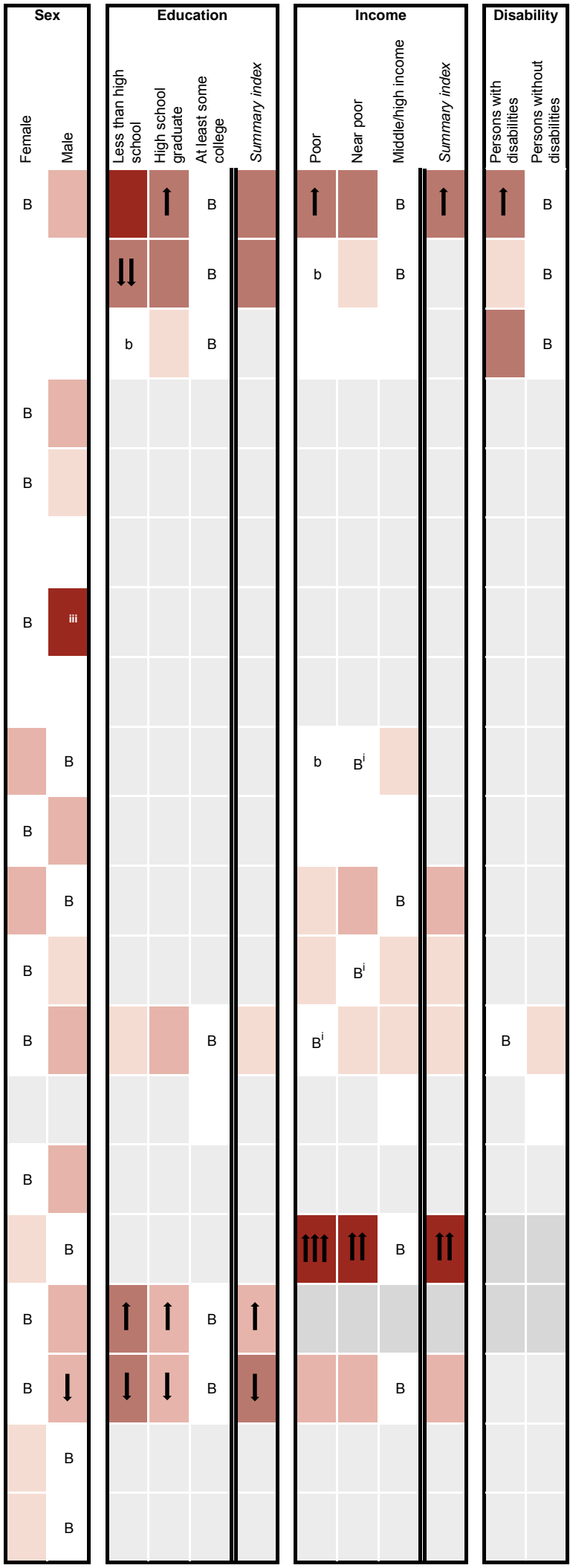

(continued) 

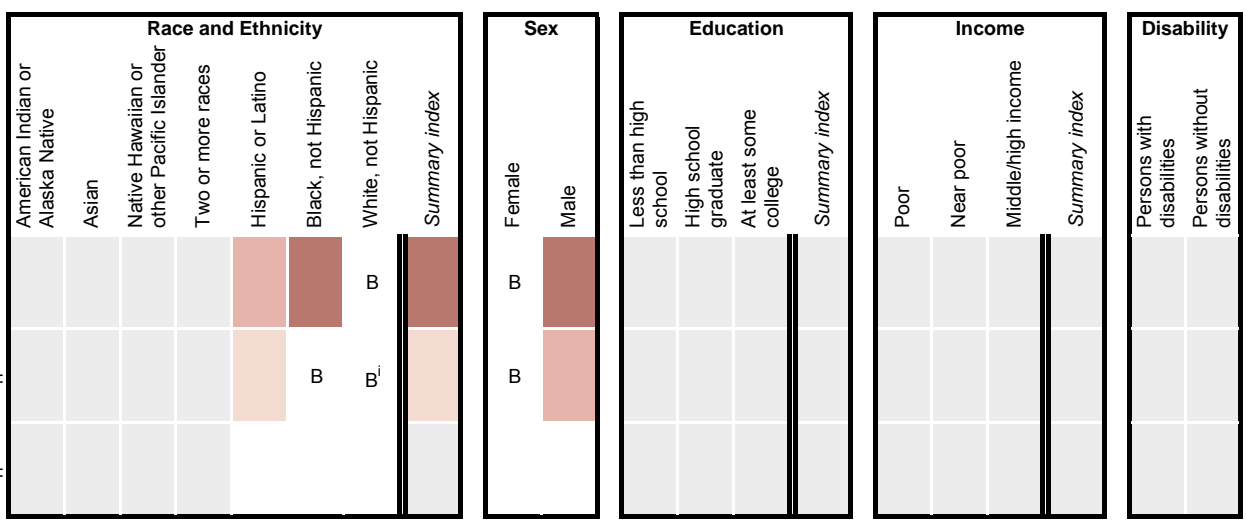

NOTES

See DATA2010 at http://wonder.cdc.gov/data2010 for all Healthy People 2010 tracking data. Disparity data are either unavailable or not applicable for objectives $27-$ $8 \mathrm{a}$ and b, 27-11, 27-13a through i, 27-14a and b, 27-15, 27-18a through c, 27-19, 27-20a through c, and 27-21a and b. Objectives 27-1d and 27-8c were deleted at Midcourse Review.

Years in parentheses represent the baseline and most recent data years (if available)

Disparity from the best group rate is defined as the percent difference between the best group rate and each of the other group rates for a characteristic (e.g., race and ethnicity). The summary index is the average of these percent differences for a characteristic. Change in disparity is estimated by subtracting the disparity at baseline from the disparity at the most recent data point. Change in the summary index is estimated by subtracting the summary index at baseline from the summary index at the most recent data point. See Technical Appendix for more information.

\begin{tabular}{l|l|l|l|l|l|l|l|l|l}
\cline { 2 - 3 } & The "best" group rate at the most recent data point. & B & $\begin{array}{l}\text { The group with the best rate for } \\
\text { specified characteristic. }\end{array}$ & b $\begin{array}{l}\text { Most favorable group rate for specified } \\
\text { characteristic, but reliability criterion not } \\
\text { met. }\end{array}$ & $\begin{array}{l}\text { Reliability criterion for best } \\
\text { group rate not met, or data } \\
\text { available for only one group. }\end{array}$ \\
\hline
\end{tabular}

\begin{tabular}{ll|l}
\hline $\begin{array}{l}\text { Disparity from the best group rate at the most recent } \\
\text { data point. }\end{array}$ & $\begin{array}{l}\text { Less than } 10 \% \text {, or not statistically } \\
\text { significant (when estimates of } \\
\text { variability are available). }\end{array}$ & $10 \%-49 \%$ \\
\hline
\end{tabular}

Changes in disparity over time are shown when:
(a) disparities data are available at both baseline and most recent time points;
(b) data are not for the group(s) indicated by "B" or "b" at either time point; and
(c) the change is greater than or equal to 10 percentage points and statistically significant,
or when the change is greater than or equal to 10 percentage points and estimates of
variability were not available. See Technical Appendix.

Availability of Data

Data not available.

* Measures of variability were available. Thus, the variability of best group rates was assessed, and statistical significance was tested. Disparities of $10 \%$ or more are displayed when the differences from the best group rate are statistically significant at the 0.05 level. Changes in disparities over time are indicated by arrows when the changes are greater than or equal to 10 percentage points and are statistically significant at the 0.05 level. See Technical Appendix.

‡ Measures of variability were available only for the most recent data. Thus, the variability of best group rates was assessed only for the most recent data, and statistical significance was tested only for the most recent data. Disparities of $10 \%$ or more are displayed when the differences from the best group rate are statistically significant at the 0.05 level. Changes in disparities over time are displayed according to their magnitude, since measures of variability were not available at baseline and therefore statistical significance of changes in disparity could not be tested. See Technical Appendix.

1 Baseline data by race and ethnicity are for 1999.

2 Baseline data by race and ethnicity are for 2000 .

3 Baseline data by income group are for 2005.

4 Baseline data by race and ethnicity are for 2005 .

5 Baseline data by race and ethnicity are for 2003.

6 Baseline data by race and ethnicity are for 2004-05, while most recent data by race and ethnicity are for 2008-09.

$i$ The group with the best rate at the most recent data point is different from the group with the best rate at baseline. Both rates met the reliability criterion. See Technical Appendix.

ii Change in the summary index cannot be assessed. See Technical Appendix.

iii Reliability criterion for best group rate not met, or data available for only one group, at baseline. Change in disparity cannot be assessed. See Technical Appendix.

iv Data are for Mexican American.

DATA SOURCES

27-1a-C. National Health Interview Survey (NHIS), CDC, NCHS

27-2a-d. Youth Risk Behavior Surveillance System (YRBSS), CDC, NCCDPHP.

27-2e. National Youth Tobacco Survey, American Legacy Foundation and CDC.

27-3a-b. National Survey on Drug Use and Health (NSDUH), SAMHSA.

27-4a-b. National Survey on Drug Use and Health (NSDUH), SAMHSA.

27-5-27-6. National Health Interview Survey (NHIS), CDC, NCHS.

27-7. Youth Risk Behavior Surveillance System (YRBSS), CDC, NCCDPHP.

27-9. National Health Interview Survey (NHIS), CDC, NCHS

27-10. National Health and Nutrition Examination Survey (NHANES), CDC, NCHS.

27-12. Current Population Survey (CPS): Department of Commerce, Census Bureau; Department of Labor, Bureau of Labor Statistics.

27-16a-b. National Youth Tobacco Survey, American Legacy Foundation and CDC, NCCDPHP.

27-17a-c. Monitoring the Future Study (MTF), NIH, NIDA.

Tobacco Use

Page 27-20 
Figure 27-3. Tobacco Use by Adults - Cigarettes (18+ Years) 2008

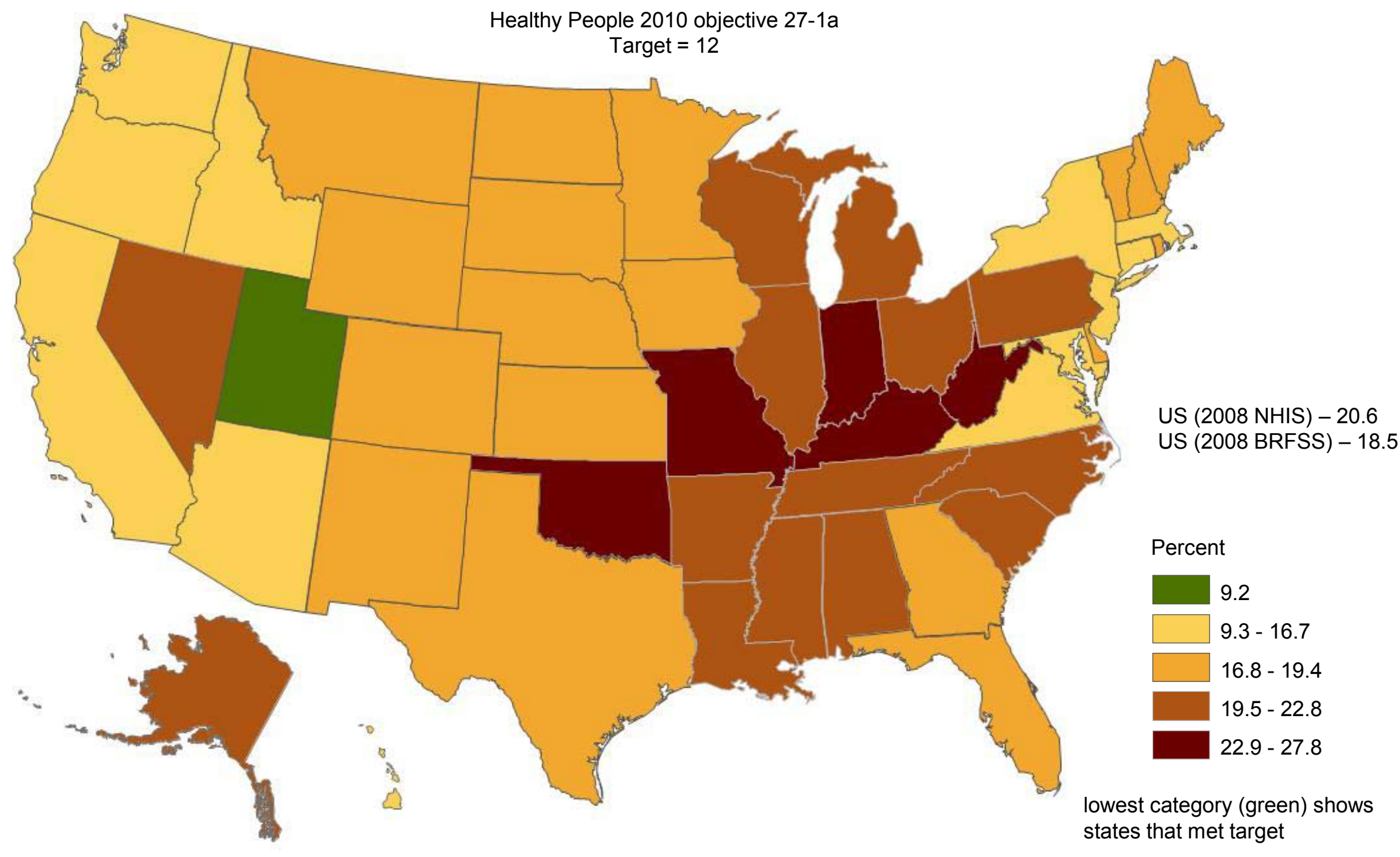

NOTES: Data are age-adjusted to the 2000 standard population. Data are for persons who have at least 100 cigarettes in their lifetime and currently report smoking everyday or some days. Rates are displayed by a modified Jenks classification for U.S. states. National data for the objective come from the National Health Interview Survey (NHIS) and is the basis for setting the target. State data from the BRFSS may not be comparable to the national data from the NHIS. Both US rates are shown for comparison purposes. BRFSS data displayed here may not BRFSS data elsewhere that is not age-adjusted. SOURCE: Behavioral Risk Factor Surveillance System (BRFSS), CDC. 
Figure 27-4. Comprehensive Smoke-free Laws (2009)

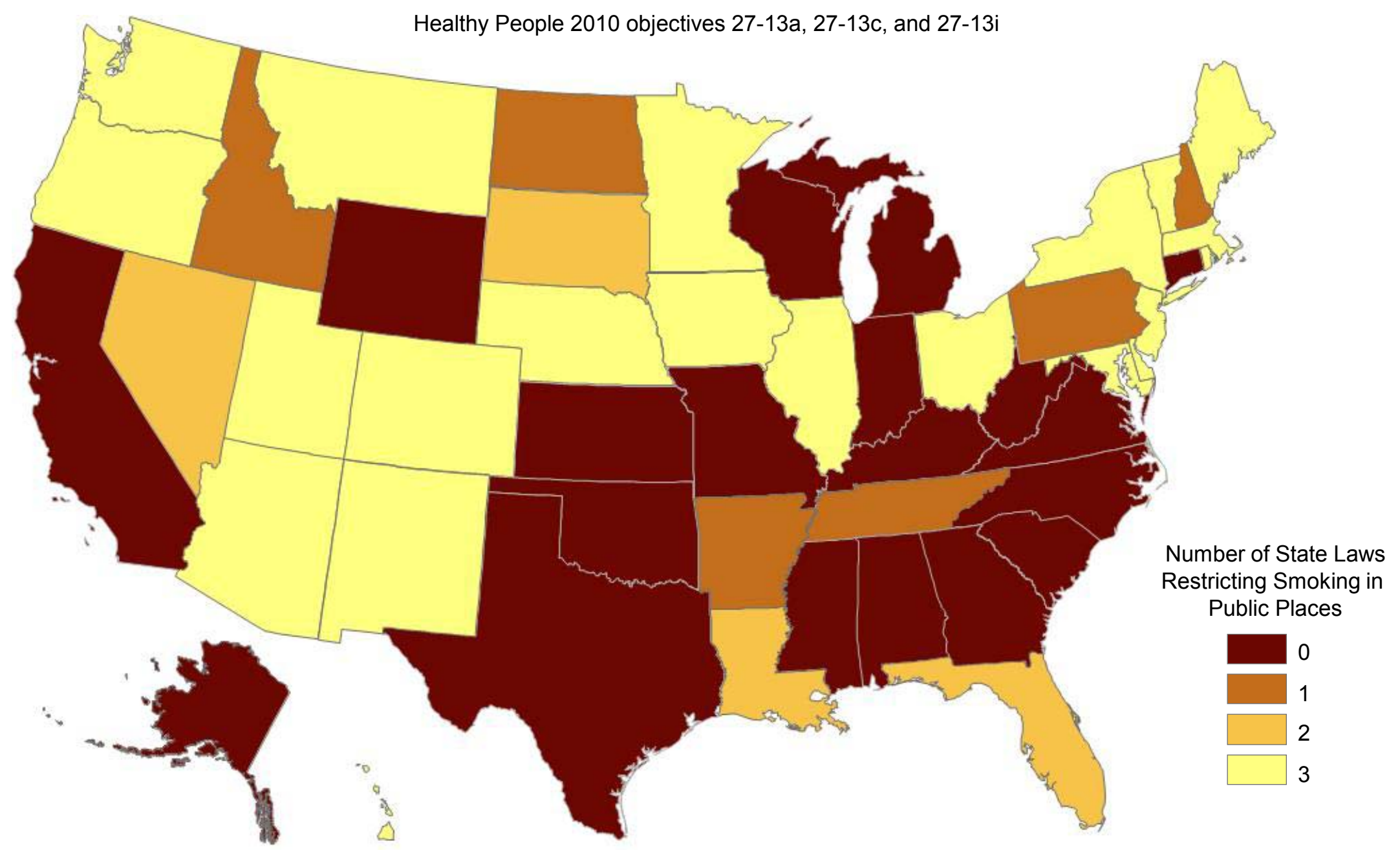

NOTES: Number of state laws restricting smoking in public places includes comprehensive state laws enacted banning smoking in: private worksites (27-13a), restaurants (27-13c), and bars (27-13i). Each measure has target of 51 states and the District of Columbia. SOURCE: State Tobacco Activities Tracking and Evaluation System (STATE), CDC, NCCDPHP, OSH, CDC. 


\section{Figure 27-5. Average Combined Federal and State Excise Taxes on Retail Price of a Standard Pack of Cigarettes in All 50 States and D.C. 2009}

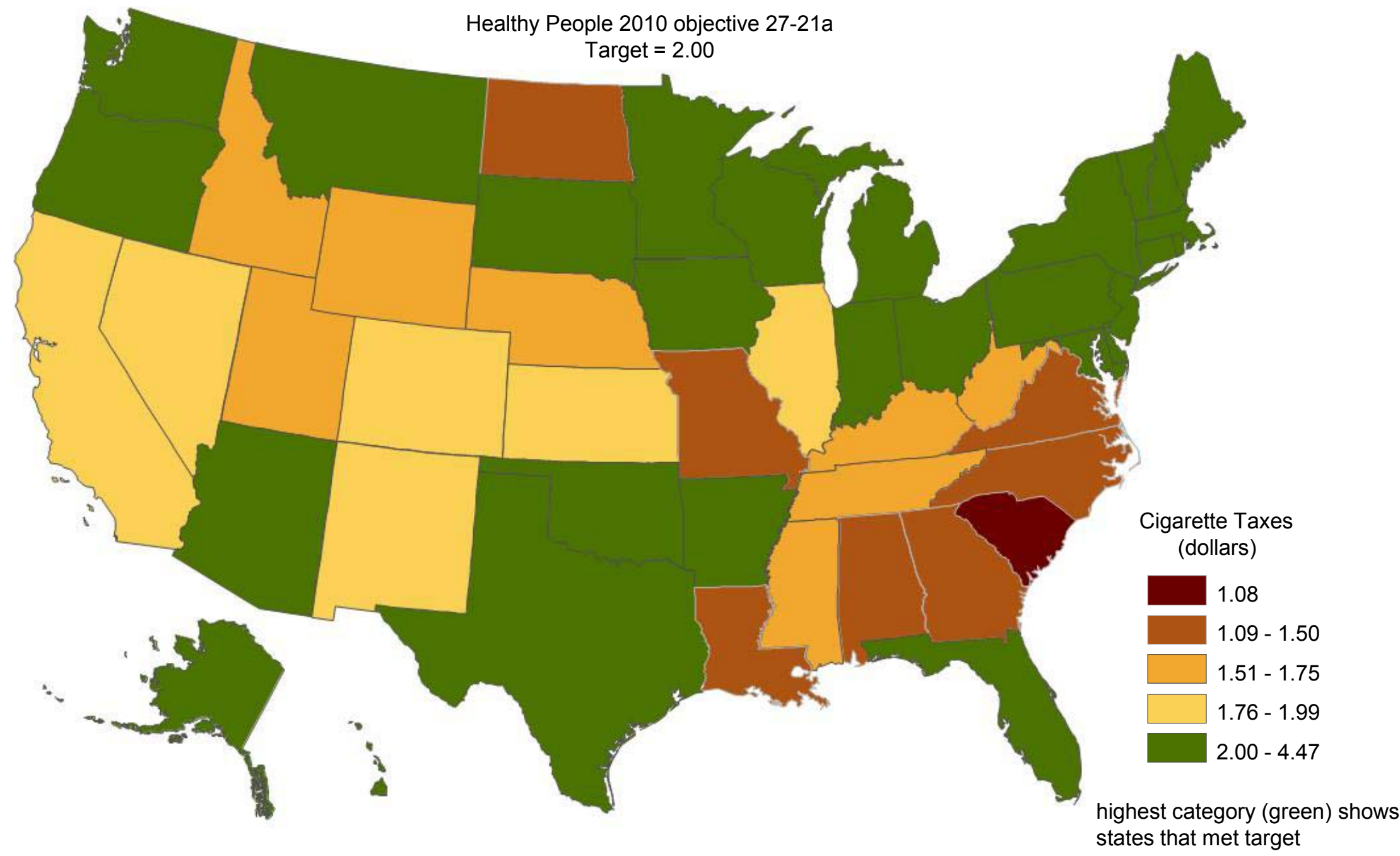

NOTES: Data are combined state and federal (\$1.01) cigarette excise taxes. Rates are displayed by a manual classification for U.S. states. SOURCE: State Tobacco Activities Tracking and Evaluation System (STATE), CDC, NCCDPHP, OSH, CDC. 


\section{Final Review}

\section{Vision and Hearing}

28

\section{Lead Agency}

National Institutes of Health

\section{Contents}

Goal.

Highlights

Summary of Progress.

Transition to Healthy People 2020

Data Considerations

Notes

Comprehensive Summary of Objectives 28-9

Progress Chart 28-11

Health Disparities Table 28-16 


\section{Goal: Improve the visual and hearing health of the Nation through prevention, early detection, treatment, and rehabilitation}

This chapter includes objectives that monitor progress in two major Healthy People areas:

- Vision. The objectives in this area track visual impairments, occupational eye injuries, eye examinations, visual rehabilitation services, and the use of protective equipment.

- Hearing. This area includes objectives that monitor hearing loss, newborn screening for hearing problems, the use of hearing aids, and hearing examinations.

All Healthy People tracking data quoted in this chapter, along with technical information and operational definitions for each objective, can be found in the Healthy People 2010 database, DATA2010, available from http://wonder.cdc.gov/data2010/.

More information about this Focus Area can be found in the following publications:

- Healthy People 2010: Understanding and Improving Health, available from http://www.healthypeople.gov/2010/Document/tableofcontents.htm\#under.

- Healthy People 2010 Midcourse Review, available from http://www.healthypeople.gov/2010/data/midcourse/html/default.htm\#FocusAreas.

\section{Highlights}

- Substantial progress was made in the Vision and Hearing Focus Area objectives during the past decade (Figure 28-1) [1]. Two thirds of the Vision objectives and over half of the Hearing objectives with data available to measure progress moved toward or achieved their Healthy People 2010 targets. Health disparities were observed for some objectives among select population groups (Figure 28-2), as highlighted below [2].

- Several Vision objectives exceeded their Healthy People 2010 targets:

- Visual impairment due to diabetic retinopathy among adults aged 18 years and over with diabetes (objective 28-5) declined, decreasing by 40.0\% between 2002 and 2008, from 45.8 to 27.5 per 1,000 population (age adjusted), exceeding the 2010 target of 40.9 .

- Occupational eye injuries declined. A decrease of 39.6\% was observed for injuries resulting in lost work days (objective 28-2a) between 2002 and 2008, from 4.8 to 2.9 per 10,000 full time workers in private industry, exceeding the 2010 target of 3.4. The rate of eye injuries treated in emergency departments (objective 28-2b) also decreased by $38.6 \%$ between 1999 and 2008, from 21.0 to 12.9 per 10,000 fulltime workers, exceeding the target of 14.7.

- Females had a lower (better) rate of occupational eye injuries resulting in lost work days, 1.3 per 10,000 full time workers in private industry in 2008. The rate for males, 4.0 per 10,000 full-time workers in private industry in 2008, was more than three times the rate for females [2]. 
- Adult use of protective eyewear at home (objective $28-9 \mathrm{~b}$ ) increased by $21.2 \%$ between 2002 and 2008, from 33\% to 40\% (age adjusted), exceeding the Healthy People 2010 target of $37 \%$.

- The use of vision rehabilitation services by visually impaired persons (objective 2810a) more than doubled during the same period, increasing from 14.0 to 30.1 per 1,000 visually impaired adults aged 18 years and over (age adjusted), exceeding the 2010 target of 15.5 .

- A statistically significant disparity was observed in blindness and visual impairment among children and adolescents (objective 28-4). Persons without disabilities had lower (better) rates of blindness and visual impairment in both 1997 and 2008, 19 and 24 per 1,000 population aged 17 years and under, respectively. The rates for persons with disabilities, were 92 per 1,000 population in 1997 and 68 in 2008. In 2008, the rate for children and adolescents with disabilities was almost three times the rate for those without disabilities. The disparity between children and adolescents without disabilities and those with disabilities declined by more than 100 percentage points between 1997 and 2008 [2,3].

- Several Hearing objectives, including the following, exceeded the 2010 targets:

- Otitis media in children and adolescents (objective 28-12) declined by almost 30\% between 1997 and 2007, from 344.7 to 246.6 per 1,000 population aged under 18 years, exceeding the target of 294.

- The use of cochlear implants by deaf or very hard of hearing persons (objective 2813b) increased between 2001 and 2006, from 57 to 92 per 10,000 population, exceeding the target of 63 .

- The use of hearing aids by adults with hearing loss (objectives 28-13c) also increased between 2001 and 2007, from 255.2 to 289.1 per 1,000 population aged 70 years and over, exceeding the target of 280.7 .

\section{Summary of Progress}

- Figure 28-1 presents a quantitative assessment of progress in achieving the Healthy People 2010 objectives for Vision and Hearing. Data to measure progress toward target attainment were available for 25 objectives [1]. Of these:

- Nine objectives (28-5, 28-8a and b, 28-9b, 28-10a, 28-12, 28-13b and c, and 28-14b) met or exceeded their Healthy People 2010 targets.

- Six objectives moved toward their targets. A statistically significant difference between the baseline and the final data points was observed for one of these objectives (28-2). No significant differences were observed for three objectives (28$7,28-9 \mathrm{~b}$, and 28-17); and data to test the significance of the difference were unavailable for two objectives (28-11a and b).

- One objective (28-1) showed no change.

- Nine objectives moved away from their targets. A statistically significant difference between the baseline and the final data points was observed for two of these objectives (28-3 and 28-10b). No significant differences were observed for six objectives (28-4, 28-6, 28-13a, 28-14a, 28-16a, and 28-18); and data to test the significance of the difference were unavailable for one objective (28-11c). 
- Four objectives (28-13d, 28-14c, 28-15, and 28-16b) had no data available to measure progress.

- Figure 28-2 displays health disparities in Vision and Hearing from the best group rate for each characteristic at the most recent data point [2]. It also displays changes in disparities from baseline to the most recent data point [3].

- Of the 10 objectives with statistically significant racial and ethnic health disparities of $10 \%$ or more, the white non-Hispanic population had the best rate for five objectives (28-3, 28-9b, 28-13c, 28-15, and 28-16b); the black non-Hispanic population had the best rate for two objectives (28-14a and b), as did the population of persons of two or more races (28-1 and 28-2); and the Hispanic or Latino population had the best rate for one objective (28-16a).

- For seven objectives, statistically significant health disparities of $10 \%$ or more were observed between females and males (objectives 28-1, 28-7, 28-9b, 28-13c, 28-14a, and $28-16 a$ and $b$ ). In addition, one objective exhibited a health disparity of $100 \%$ or more (see Highlights, above), but lacked data to assess statistical significance (objective 28-8a). Males had better rates than females for six of these eight objectives (28-7, 28-9b, 28-13c, 28-14a, and 28-16a and b). Females had better rates for two objectives (28-1 and 28-8a).

- Persons with at least some college education had the best group rate for three of the four objectives with statistically significant health disparities of $10 \%$ or more by education level (28-1, and 28-13a and c). High school graduates had the best group rate for one objective (28-7)

- Persons with middle/high incomes had the best group rate for three of the four objectives with statistically significant health disparities of $10 \%$ or more by income (objectives 28-3, 28-13-d, and 28-16a). Near-poor persons had the best group rate for the fourth objective (28-9a).

- Persons with disabilities had better rates than persons without disabilities for two of the three objectives with statistically significant health disparities of $10 \%$ or more by disability status (objectives 28-14b and 28-15). Persons without disabilities had the better rate for one objective (28-4).

- Health disparities of $100 \%$ or more were observed for two objectives: blindness and visual impairment in children and adolescents (objective 28-4), and occupational eye injuries resulting in lost work days (objective 28-8a). A statistically significant decline in disparity also was observed for blindness and visual impairment in children and adolescents; see Highlights, above.

\section{Transition to Healthy People 2020}

To emphasize the individual importance of Vision and Hearing, the Healthy People 2010 Vision and Hearing Focus Area was divided into two separate Topic Areas for Healthy People 2020: 1) Vision, and 2) Hearing and Other Sensory or Communication Disorders. Other sensory or communication disorders include disorders of the ear, nose, throat, and conditions associated with voice, speech, and language (ENT-VSL). See HealthyPeople.gov for a complete list of Healthy People 2020 topics and objectives. 
The Healthy People 2020 Vision Topic Area covers:

- Eye examinations among adults and vision screening among children

- Visual impairment due to selected eye diseases

- Occupational eye injuries

- Use of protective eyewear at home and during recreational activities

- Use of vision rehabilitation services and visual adaptive devices

- Comprehensive vision health service provided in Federally Qualified Health Centers.

The Healthy People 2020 Hearing and Other Sensory or Communication Disorders Topic Area includes new objectives in addition to Healthy People 2010 hearing objectives. The Topic Area can be grouped into several sections:

- Newborn hearing screening

- Ear infections (otitis media)

- Hearing

- Tinnitus

- Balance and dizziness

- Smell and taste (chemosenses)

- Voice, speech, and language

- Internet health care resources for ENT-VSL.

The differences between the objectives for Healthy People 2010 and Healthy People 2020 are summarized below:

- The Healthy People 2010 Vision and Hearing Focus Area had a total of 29 objectives, including 13 vision and 16 hearing objectives. For Healthy People 2020, the Vision Topic Area has 15 objectives and the Hearing and Other Sensory or Communication Disorders Topic Area has 36 objectives.

\section{Vision}

- Ten Healthy People 2010 Vision objectives (28-1 through 28-4, 28-8a and b, 28-9a and b, and $28-10 \mathrm{a}$ and b) were retained "as is" [4].

- Three Vision objectives (28-5, 28-6, and 28-7) on reduction of visual impairment caused by age-related eye diseases were modified because of changes in survey methodology [5].

- Two new Vision objectives were added to the Healthy People 2020 Topic Area:

- The first addresses visual impairment due to age-related macular degeneration.

- The second measures the proportion of Federally Qualified Health Centers (FQHCs) that provide comprehensive vision health services. 


\section{Hearing and Other Sensory or Communication Disorders}

- All sixteen Healthy People 2010 Hearing objectives (28-11a through 28-18) were retained "as is" [4].

- Twenty new objectives were added to this Healthy People 2020 Topic Area:

- Three tinnitus objectives focus on adults bothered by tinnitus who have seen a health provider, audiologist or otolaryngologist and tried appropriate treatment.

- Twelve new objectives on balance, dizziness and smell or taste disorders will track use of health care services, referrals to health care specialist, treatment, negative or adverse outcomes, falls and injuries caused by balance and dizziness conditions, and impact of these conditions on general health status and quality of life.

- Four voice, speech, and language objectives were introduced to Healthy People 2020 to highlight the importance of timely evaluation, treatment, and use of rehabilitation services in improving the quality of life of patients with VSL conditions.

- One new objective on the use of Internet health care resources for ENT-VSL disorders was added to measure the number of people who used Internet for health care information, guidance, or advice.

Appendix D, “A Crosswalk Between Objectives From Healthy People 2010 to Healthy People 2020," summarizes the changes between the two decades of objectives, reflecting new knowledge and direction for this area.

\section{Data Considerations}

Education and income are the primary measures of socioeconomic status (SES) in Healthy People 2010. Most data systems used in Healthy People 2010 define income as a family's income before taxes. In order to facilitate comparisons among groups and over time, while adjusting for family size and for inflation, Healthy People 2010 categorizes income using the poverty thresholds developed by the U.S. Census Bureau. Thus, the three categories of family income that are primarily used are:

- Poor-below the Federal poverty level

- Near poor-100\% to $199 \%$ of the Federal poverty level

- Middle/high income-200\% or more of the Federal poverty level.

These categories may be overridden by considerations specific to the data system, in which case they are modified as appropriate. See Healthy People 2010: General Data Issues, referenced below.

In general, data on educational attainment are presented for persons aged 25 years and over, consistent with guidance given by the U.S. Bureau of the Census. However, because of the requirements of the different data systems, the age groups used to calculate educational attainment for any specific objective may differ from the age groups used to report the data for other Healthy People 2010 objectives, as well as from select populations within the same objective. Therefore, the reader is urged to exercise caution in interpreting the data by educational attainment shown in the Health Disparities Table. See Healthy People 2010: General Data Issues, referenced below. 
Additional information on data issues is available from the following sources:

- All Healthy People 2010 tracking data can be found in the Healthy People 2010 database, DATA2010, available from http://wonder.cdc.gov/data2010/.

- Detailed information about the data and data sources used to support these objectives can be found in the Operational Definitions on the DATA2010 website, available from http://wonder.cdc.gov/data2010/focusod.htm.

- More information on statistical issues related to Healthy People tracking and measurement can be found in the Technical Appendix and in Healthy People 2010: General Data Issues, which is available in the Data Issues section of the NCHS Healthy People website under Healthy People 2010.

\section{Notes}

1. Displayed in the Progress Chart (Figure 28-1), the percent of targeted change achieved expresses the difference between the baseline and the final value relative to the initial difference between the baseline and the Healthy People 2010 target. As such, it is a relative measure of progress toward attaining the Healthy People 2010 target. See the Reader's Guide for more information. When standard errors were available, the difference between the baseline and the final value was tested at the 0.05 level of significance. See the Figure 28-1 footnotes, as well as the Technical Appendix, for more detail.

2. Information about disparities among select populations is shown in the Health Disparities Table (Figure 28-2). Disparity from the best group rate is defined as the percent difference between the best group rate and each of the other group rates for a characteristic. For example, racial and ethnic health disparities are measured as the percent difference between the best racial and ethnic group rate and each of the other racial and ethnic group rates. Similarly, disparities by sex are measured as the percent difference between the better group rate (e.g., female) and the rate for the other group (e.g., male). Some objectives are expressed in terms of favorable events or conditions that are to be increased, while others are expressed in terms of adverse events or conditions that are to be reduced. In order to facilitate comparison of health disparities across different objectives, disparity is measured only in terms of adverse events or conditions. For comparability across objectives, objectives that are expressed in terms of favorable events or conditions are re-expressed using the adverse event or condition for the purpose of computing disparity, but they are not otherwise restated or changed. For example, objective 1-1, to increase the proportion of persons with health insurance (e.g., $72 \%$ of the American Indian or Alaska Native population aged under 65 years had some form of health insurance in 2008), is expressed in terms of the percentage of persons without health insurance (e.g., $100 \%-72 \%=28 \%$ of the American Indian or Alaska Native population aged under 65 years did not have any form of health insurance in 2008) when the disparity from the best group rate is calculated. See the Reader's Guide for more information. When standard errors were available, the difference between the best group rate and each of the other group rates was tested at the 0.05 level of significance. See the Figure 28-2 footnotes, as well as the Technical Appendix, for more detail.

3. The change in disparity is estimated by subtracting the disparity at baseline from the disparity at the most recent data point and, therefore, is expressed as a change in percentage points. See the Reader's Guide for more information. When standard errors were available, the change in disparity was tested at the 0.05 level of significance. See the Figure 28-2 footnotes, as well as the Technical Appendix, for more detail. 
4. Retained "as is" objectives have no change in the numerator definition or in the denominator definition between the Healthy People 2010 and Healthy People 2020 objectives. These include objectives that were developmental in Healthy People 2010 and are developmental in Healthy People 2020 and for which no numerator or denominator information was available.

5. Modified objectives have some change in the numerator definition or in the denominator definition between the Healthy People 2010 and Healthy People 2020 objectives. These include objectives that went from developmental in Healthy People 2010 to measurable in Healthy People 2020 or vice versa. 


\section{Comprehensive Summary of Objectives: Vision and Hearing}

\begin{tabular}{|c|c|c|}
\hline Objective & Description & Data Source \\
\hline $28-1$ & $\begin{array}{l}\text { Dilated eye examination within the past } 2 \text { years (age adjusted, } \\
18+\text { years) }\end{array}$ & National Health Interview Survey (NHIS), CDC, NCHS. \\
\hline $28-2$ & Vision screening for children $(<6$ years $)$ & National Health Interview Survey (NHIS), CDC, NCHS. \\
\hline $28-3$ & $\begin{array}{l}\text { Uncorrected visual impairment due to refractive errors (age } \\
\text { adjusted per 1,000 standard population, } 12+\text { years) }\end{array}$ & National Health and Nutrition Examination Survey (NHANES), CDC, NCHS. \\
\hline $28-4$ & $\begin{array}{l}\text { Blindness and visual impairment in children and adolescents (per } \\
1,000 \text { population, } \leq 17 \text { years) }\end{array}$ & National Health Interview Survey (NHIS), CDC, NCHS. \\
\hline $28-5$ & $\begin{array}{l}\text { Visual impairment due to diabetic retinopathy (age adjusted per } \\
1,000 \text { standard population, } 18+\text { years with diabetes) }\end{array}$ & National Health Interview Survey (NHIS), CDC, NCHS. \\
\hline 28-6 & $\begin{array}{l}\text { Visual impairment due to glaucoma (age adjusted per 1,000 } \\
\text { standard population, } 45+\text { years) }\end{array}$ & National Health Interview Survey (NHIS), CDC, NCHS. \\
\hline $28-7$ & $\begin{array}{l}\text { Visual impairment due to cataract (age adjusted per } 1,000 \\
\text { standard population, } 65+\text { years) }\end{array}$ & National Health Interview Survey (NHIS), CDC, NCHS. \\
\hline $28-8 \mathrm{a}$ & $\begin{array}{l}\text { Occupational eye injuries resulting in lost work days (per 10,000 } \\
\text { full-time workers in private industry) }\end{array}$ & $\begin{array}{l}\text { Survey of Occupational Injuries and Illnesses (SOII), Department of Labor } \\
\text { (DOL), Bureau of Labor Statistics (BLS). }\end{array}$ \\
\hline $28-8 b$ & $\begin{array}{l}\text { Occupational eye injuries treated in emergency departments (per } \\
10,000 \text { full-time workers) }\end{array}$ & $\begin{array}{l}\text { National Electronic Injury Surveillance System (NEISS), Consumer } \\
\text { Product Safety Commission (CPSC) and CDC, NIOSH. }\end{array}$ \\
\hline $28-9 a$ & $\begin{array}{l}\text { Use of protective eyewear at home-Children and adolescents 6- } \\
17 \text { years }\end{array}$ & National Health Interview Survey (NHIS), CDC, NCHS. \\
\hline $28-9 b$ & $\begin{array}{l}\text { Use of protective eyewear at home-Adults 18+ years (age } \\
\text { adjusted) }\end{array}$ & National Health Interview Survey (NHIS), CDC, NCHS. \\
\hline $28-10 \mathrm{a}$ & $\begin{array}{l}\text { Use of vision rehabilitation services by visually impaired persons } \\
\text { (age adjusted per } 1,000 \text { standard population, } 18+\text { years) }\end{array}$ & National Health Interview Survey (NHIS), CDC, NCHS. \\
\hline $28-10 b$ & $\begin{array}{l}\text { Use of visual adaptive devices by visually impaired persons (age } \\
\text { adjusted, 18+ years) }\end{array}$ & National Health Interview Survey (NHIS), CDC, NCHS. \\
\hline $28-11 \mathrm{a}$ & Newborns receiving hearing screening before age 1 month & $\begin{array}{l}\text { Baseline: Directors of Speech and Hearing Programs in State Health and } \\
\text { Welfare Agencies. Final: Early Hearing Detection and Intervention (EHDI) } \\
\text { Program, CDC, NCBDD; and/or specific State data. }\end{array}$ \\
\hline
\end{tabular}




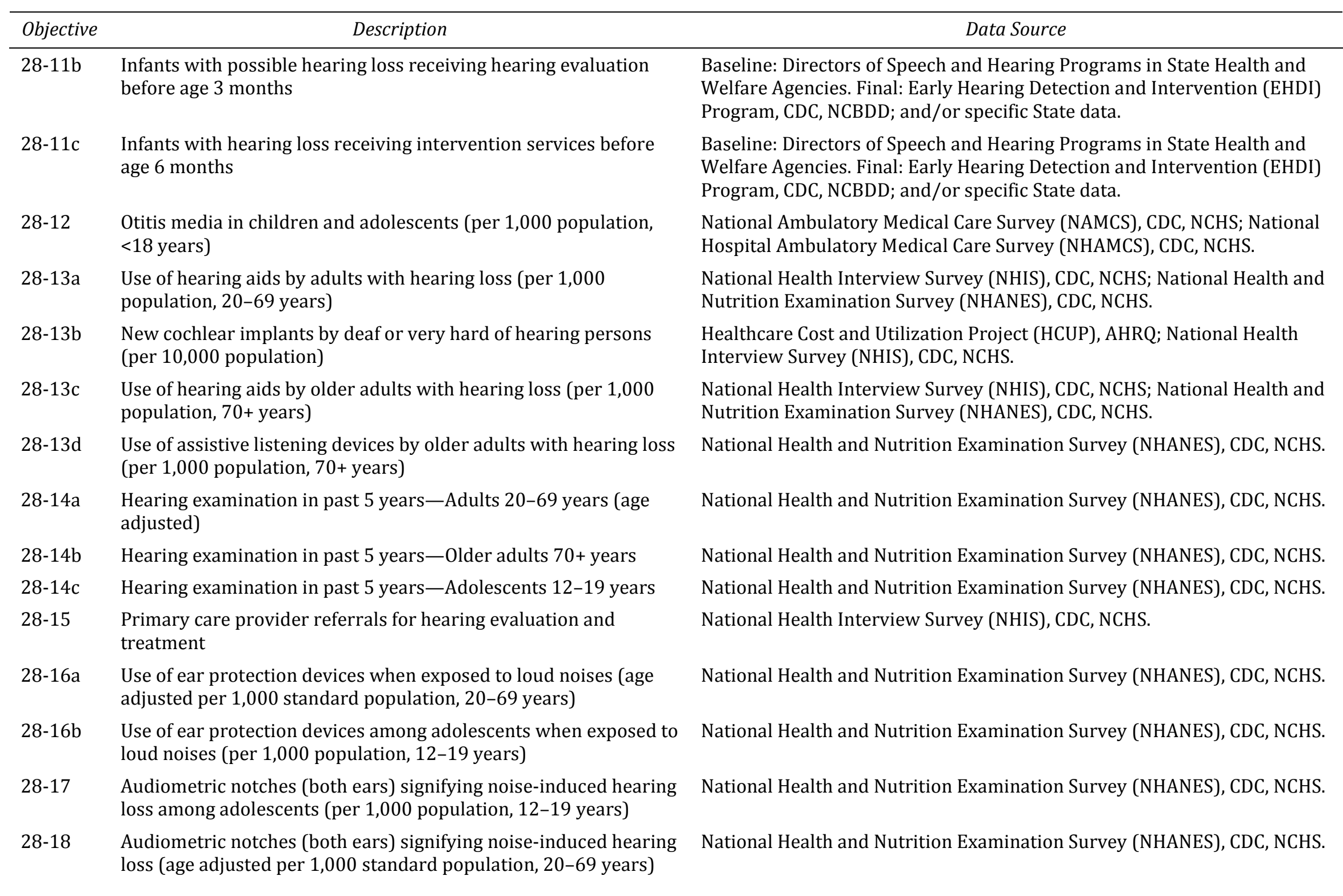


Figure 28-1. Progress Toward Target Attainment for Focus Area 28: Vision and Hearing

Moved away

from target ${ }^{1}$
Moved toward

target
Met or exceeded

target

28-1. Dilated eye examination within the past 2 years (age adjusted, 18+ years)

28-2. Vision screening for children ( $<6$ years)

28-3. Uncorrected visual impairment due to refractive errors (age adjusted, per 1,000 population, $12+$ years)

28-4. Blindness and visual impairment in children and adolescents (per 1,000 population, $\leq 17$ years)

28-5. Visual impairment due to diabetic retinopathy (age adjusted, per 1,000 population, 18+ years with diabetes)

28-6. Visual impairment due to glaucoma (age adjusted, per 1,000 population, $45+$ years)

28-7. Visual impairment due to cataract (age adjusted, per 1,000 population, $65+$ years)

28-8a. Occupational eye injuries resulting in lost work days (per 10,000 full-time workers in private industry)

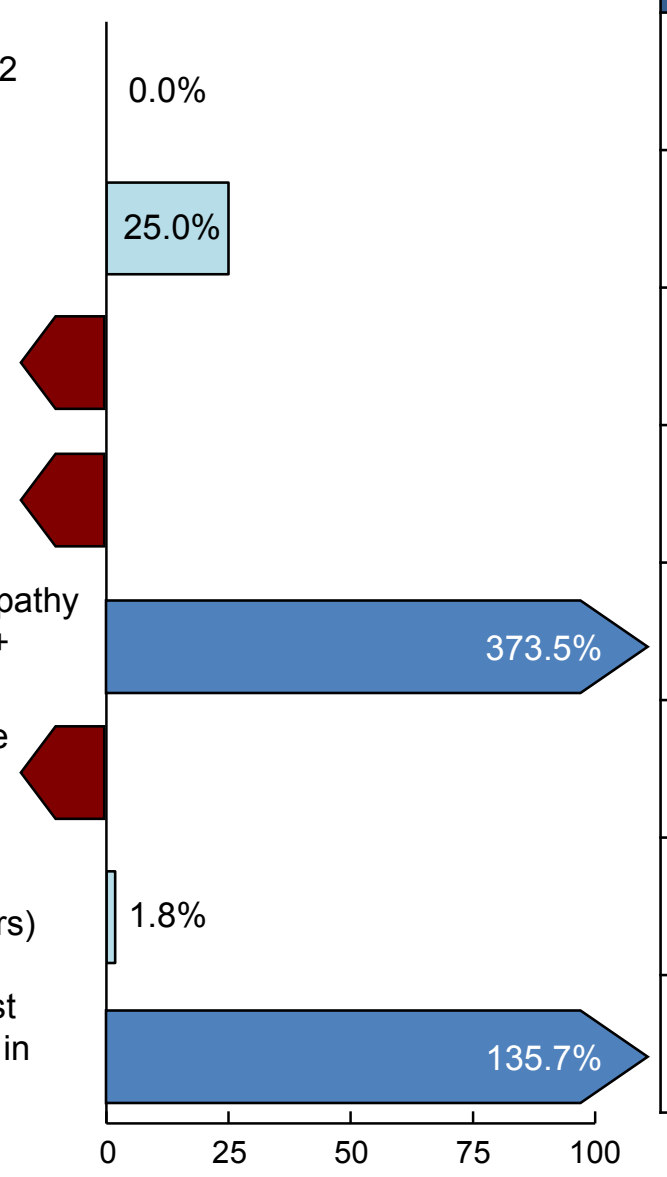

\begin{tabular}{|c|c|c|c|c|c|}
\hline \multirow{2}{*}{$\begin{array}{c}2010 \\
\text { Target }\end{array}$} & \multirow{2}{*}{$\begin{array}{c}\text { Baseline } \\
\text { (Year) }\end{array}$} & \multirow{2}{*}{$\begin{array}{l}\text { Final } \\
\text { (Year) }\end{array}$} & \multicolumn{3}{|c|}{ Baseline vs. Final } \\
\hline & & & Difference $^{2}$ & $\begin{array}{l}\text { Statistically } \\
\text { Significant }^{3}\end{array}$ & $\begin{array}{l}\text { Percent } \\
\text { Change }^{4}\end{array}$ \\
\hline $58 \%$ & $\begin{array}{c}55 \% \\
(2002)\end{array}$ & $\begin{array}{c}55 \% \\
(2008)\end{array}$ & 0 & No & $0.0 \%$ \\
\hline $52 \%$ & $\begin{array}{c}36 \% \\
(2002)\end{array}$ & $\begin{array}{c}40 \% \\
(2008)\end{array}$ & 4 & Yes & $11.1 \%$ \\
\hline 101.1 & $\begin{array}{c}118.0 \\
(1999-02)\end{array}$ & $\begin{array}{c}136.1 \\
(2005-08)\end{array}$ & 18.1 & Yes & $15.3 \%$ \\
\hline 18 & $\begin{array}{c}24 \\
(1997)\end{array}$ & $\begin{array}{c}28 \\
(2008)\end{array}$ & 4 & No & $16.7 \%$ \\
\hline 40.9 & $\begin{array}{c}45.8 \\
(2002)\end{array}$ & $\begin{array}{c}27.5 \\
(2008)\end{array}$ & -18.3 & Yes & $-40.0 \%$ \\
\hline 10.7 & $\begin{array}{c}13.5 \\
(2002)\end{array}$ & $\begin{array}{c}14.3 \\
(2008)\end{array}$ & 0.8 & No & $5.9 \%$ \\
\hline 91.4 & $\begin{array}{l}119.3 \\
(2002)\end{array}$ & $\begin{array}{l}118.8 \\
(2008)\end{array}$ & -0.5 & No & $-0.4 \%$ \\
\hline 3.4 & $\begin{array}{c}4.8 \\
(2002)\end{array}$ & $\begin{array}{c}2.9 \\
(2008)\end{array}$ & -1.9 & $\begin{array}{c}\text { Not } \\
\text { tested }\end{array}$ & $-39.6 \%$ \\
\hline
\end{tabular}

Percent of targeted change achieved ${ }^{5}$

(continued) 
Figure 28-1. Progress Toward Target Attainment for Focus Area 28: Vision and Hearing (continued)

Moved away from target ${ }^{1}$
Moved toward target
Met or exceeded target

28-8b. Occupational eye injuries treated in emergency departments (per 10,000 full-time workers)

28-9. Use of protective eyewear at home

a. Children and adolescents 6-17 years

b. Adults $18+$ years (age adjusted)

28-10a. Use of vision rehabilitation services by visually impaired persons (age adjusted, per 1,000 population, $18+$ years)

28-10b. Use of visual adaptive devices by visually impaired persons (age adjusted, $18+$ years)

28-11a. Newborns receiving hearing screening before age 1 month

28-11b. Infants with possible hearing loss receiving hearing evaluation before age 3 months

28-11c. Infants with hearing loss receiving intervention services before age 6 months

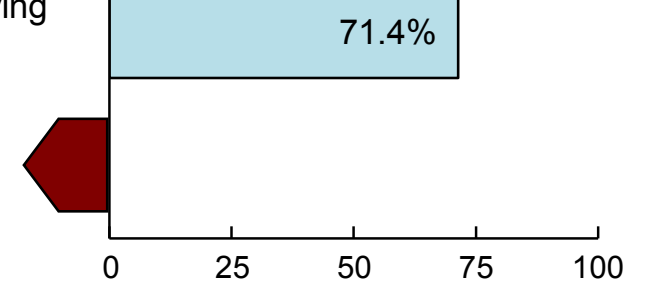

\begin{tabular}{|c|c|c|c|c|c|}
\hline \multirow{2}{*}{$\begin{array}{l}2010 \\
\text { Target }\end{array}$} & \multirow{2}{*}{$\begin{array}{c}\text { Baseline } \\
\text { (Year) }\end{array}$} & \multirow{2}{*}{$\begin{array}{l}\text { Final } \\
\text { (Year) }\end{array}$} & \multicolumn{3}{|c|}{ Baseline vs. Final } \\
\hline & & & Difference $^{2}$ & $\begin{array}{l}\text { Statistically } \\
\text { Significant }^{3}\end{array}$ & $\begin{array}{c}\text { Percent } \\
\text { Change }^{4}\end{array}$ \\
\hline 14.7 & $\begin{array}{c}21.0 \\
(1999)\end{array}$ & $\begin{array}{c}12.9 \\
(2008)\end{array}$ & -8.1 & Yes & $-38.6 \%$ \\
\hline $20 \%$ & $\begin{array}{c}15 \% \\
(2002)\end{array}$ & $\begin{array}{c}17 \% \\
(2008)\end{array}$ & 2 & No & $13.3 \%$ \\
\hline $37 \%$ & $\begin{array}{c}33 \% \\
(2002)\end{array}$ & $\begin{array}{c}40 \% \\
(2008)\end{array}$ & 7 & Yes & $21.2 \%$ \\
\hline 15.5 & $\begin{array}{c}14.0 \\
(2002)\end{array}$ & $\begin{array}{c}30.1 \\
(2008)\end{array}$ & 16.1 & Yes & $115.0 \%$ \\
\hline $26 \%$ & $\begin{array}{c}22 \% \\
(2002)\end{array}$ & $\begin{array}{c}11 \% \\
(2008)\end{array}$ & -11 & Yes & $-50.0 \%$ \\
\hline $90 \%$ & $\begin{array}{c}66 \% \\
(2001)\end{array}$ & $\begin{array}{c}82 \% \\
(2007)\end{array}$ & 16 & $\begin{array}{c}\text { Not } \\
\text { tested }\end{array}$ & $24.2 \%$ \\
\hline $70 \%$ & $\begin{array}{c}56 \% \\
(2001)\end{array}$ & $\begin{array}{c}66 \% \\
(2007)\end{array}$ & 10 & $\begin{array}{c}\text { Not } \\
\text { tested }\end{array}$ & $17.9 \%$ \\
\hline $85 \%$ & $\begin{array}{c}57 \% \\
(2001)\end{array}$ & $\begin{array}{c}50 \% \\
(2007)\end{array}$ & -7 & $\begin{array}{c}\text { Not } \\
\text { tested }\end{array}$ & $-12.3 \%$ \\
\hline
\end{tabular}

Percent of targeted change achieved ${ }^{5}$ 
Figure 28-1. Progress Toward Target Attainment for Focus Area 28: Vision and Hearing (continued)

Moved away

from target ${ }^{1}$

Moved toward

target

Met or exceeded

target

28-12. Otitis media in children and adolescents (per 1,000 population, $<18$ years)

28-13a. Use of hearing aids by adults with hearing loss (per 1,000 population, 20-69 years)

28-13b. New cochlear implants by deaf or very hard of hearing persons (per 10,000 population)

28-13c. Use of hearing aids by older adults with hearing loss (per 1,000 population, $70+$ years)

28-14. Hearing examination in past 5 years a. Adults 20-69 years (age adjusted)

b. Older adults $70+$ years

28-16a. Use of ear protection devices when exposed to loud noises (age adjusted, per 1,000 population, $20-69$ years)

28-17. Audiometric notches (both ears) signifying noise-induced hearing loss among adolescents (per 1,000 population, 12-19 years)

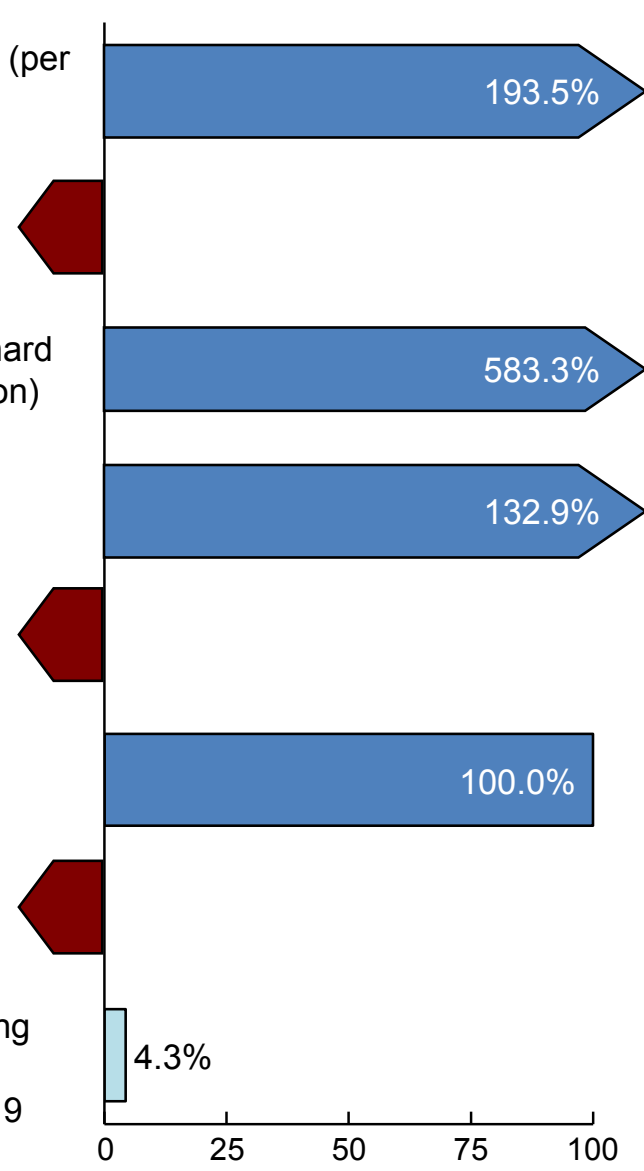

\begin{tabular}{|c|c|c|c|c|c|}
\hline \multirow{2}{*}{$\begin{array}{l}2010 \\
\text { Target }\end{array}$} & \multirow{2}{*}{$\begin{array}{c}\text { Baseline } \\
\text { (Year) }\end{array}$} & \multirow{2}{*}{$\begin{array}{l}\text { Final } \\
\text { (Year) }\end{array}$} & \multicolumn{3}{|c|}{ Baseline vs. Final } \\
\hline & & & Difference $^{2}$ & $\begin{array}{l}\text { Statistically } \\
\text { Significant }^{3}\end{array}$ & $\begin{array}{c}\text { Percent } \\
\text { Change }^{4}\end{array}$ \\
\hline 294 & $\begin{array}{l}344.7 \\
(1997)\end{array}$ & $\begin{array}{l}246.6 \\
(2007)\end{array}$ & -98.1 & Yes & $-28.5 \%$ \\
\hline 182.5 & $\begin{array}{l}165.9 \\
(2001)\end{array}$ & $\begin{array}{c}162.7 \\
(2006)\end{array}$ & -3.2 & No & $-1.9 \%$ \\
\hline 63 & $\begin{array}{c}57 \\
(2001)\end{array}$ & $\begin{array}{c}92 \\
(2006)\end{array}$ & 35 & Yes & $61.4 \%$ \\
\hline 280.7 & $\begin{array}{l}255.2 \\
(2001)\end{array}$ & $\begin{array}{l}289.1 \\
(2007)\end{array}$ & 33.9 & No & $13.3 \%$ \\
\hline $35 \%$ & $\begin{array}{c}30 \% \\
(1999-02)\end{array}$ & $\begin{array}{c}29 \% \\
(2003-04)\end{array}$ & -1 & No & $-3.3 \%$ \\
\hline $41 \%$ & $\begin{array}{c}38 \% \\
(1999-02)\end{array}$ & $\begin{array}{c}41 \% \\
(2003-06)\end{array}$ & 3 & No & $7.9 \%$ \\
\hline 514.5 & $\begin{array}{c}489.8 \\
(1999-02)\end{array}$ & $\begin{array}{c}483.0 \\
(2003-04)\end{array}$ & -6.8 & No & $-1.4 \%$ \\
\hline 34.7 & $\begin{array}{c}46.4 \\
(1988-94)\end{array}$ & $\begin{array}{c}45.9 \\
(2005-06)\end{array}$ & -0.5 & No & $-1.1 \%$ \\
\hline
\end{tabular}

Percent of targeted change achieved ${ }^{5}$

(continued) 
Figure 28-1. Progress Toward Target Attainment for Focus Area 28: Vision and Hearing (continued)

Moved away

from target ${ }^{1}$

Moved toward

target

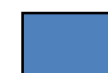

Met or exceeded

target

28-18. Audiometric notches (both ears) signifying noise-induced hearing loss (age adjusted, per 1,000 population, 20-69 years)

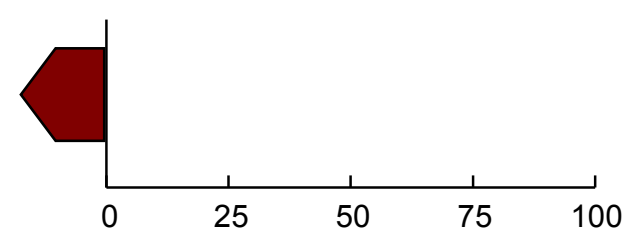

\begin{tabular}{|c|c|c|c|c|c|}
\hline \multirow{2}{*}{$\begin{array}{c}2010 \\
\text { Target }\end{array}$} & \multirow{2}{*}{$\begin{array}{c}\text { Baseline } \\
(\text { Year })\end{array}$} & Final & \multicolumn{3}{|c|}{ Baseline vs. Final } \\
\cline { 4 - 6 } & Year $)$ & Difference $^{2}$ & $\begin{array}{c}\text { Statistically } \\
\text { Significant }^{3}\end{array}$ & $\begin{array}{c}\text { Percent } \\
\text { Change }^{4}\end{array}$ \\
\hline 88.1 & $\begin{array}{c}119.0 \\
(1999-02)\end{array}$ & $\begin{array}{c}121.4 \\
(2003-04)\end{array}$ & 2.4 & No & $2.0 \%$ \\
\hline
\end{tabular}

\section{Percent of targeted change achieved ${ }^{5}$}

\section{NOTES}

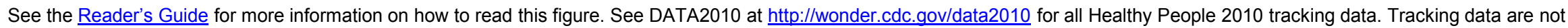
available for objectives $28-13 d, 28-14$ c, 28-15, and 28-16b.

\section{FOOTNOTES}

${ }^{1}$ Movement away from target is not quantified using the percent of targeted change achieved. See Technical Appendix for more information.

${ }^{2}$ Difference $=$ Final value - Baseline value. Differences between percents (\%) are measured in percentage points .

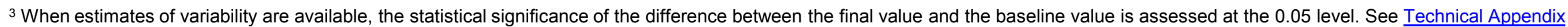
for more information.

${ }^{4}$ Percent change $=\frac{\text { Final value }- \text { Baseline value }}{\text { Baseline value }} \times 100$

${ }^{5}$ Percent of targeted change achieved $=\frac{\text { Final value }- \text { Baseline value }}{\text { Healthy People } 2010 \text { target }- \text { Baseline value }} \times 100$. 
Figure 28-1. Progress Toward Target Attainment for Focus Area 28: Vision and Hearing (continued)

\section{DATA SOURCES}

28-1-28-2. National Health Interview Survey (NHIS), CDC, NCHS.

28-3. National Health and Nutrition Examination Survey (NHANES), CDC, NCHS.

28-4-28-7. National Health Interview Survey, (NHIS), CDC, NCHS.

28-8a. Survey of Occupational Injuries and IIInesses (SOII), Department of Labor (DOL), Bureau of Labor Statistics (BLS).

28-8b. National Electronic Injury Surveillance System (NEISS), Consumer Product Safety Commission (CPSC) and CDC, NIOSH.

28-9a-b. $\quad$ National Health Interview Survey (NHIS), CDC, NCHS.

28-10a-b. National Health Interview Survey (NHIS), CDC, NCHS.

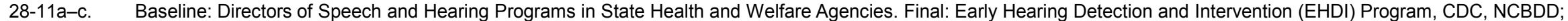
and/or specific State data.

28-12. National Ambulatory Medical Care Survey (NAMCS), CDC, NCHS; National Hospital Ambulatory Medical Care Survey (NHAMCS), CDC, NCHS.

28-13a. National Health Interview Survey (NHIS), CDC, NCHS; National Health and Nutrition Examination Survey (NHANES), CDC, NCHS.

28-13b. Healthcare Cost and Utilization Project (HCUP), AHRQ; National Health Interview Survey (NHIS), CDC, NCHS.

28-13c. National Health Interview Survey (NHIS), CDC, NCHS; National Health and Nutrition Examination Survey (NHANES), CDC, NCHS.

28-14a-b. National Health and Nutrition Examination Survey (NHANES), CDC, NCHS.

28-16a. National Health and Nutrition Examination Survey (NHANES), CDC, NCHS.

28-17-28-18. National Health and Nutrition Examination Survey (NHANES), CDC, NCHS. 
Figure 28-2. Health Disparities Table for Focus Area 28: Vision and Hearing

Disparities from the best group rate for each characteristic at the most recent data point and changes in disparity from the baseline to the most recent data point.

\section{Population-based objectives}

28-1. Dilated eye examination within the past 2 years (age adjusted, 18+ years) $(2002,2008)^{*}$

28-2. Vision screening for children $(<6$ years) $(2002$ 2008)*

28-3. Uncorrected visual impairment due to refractive errors (age adjusted, per 1,000 population, 12+ years) $(1999-02,2005-08)^{\star}$

28-4. Blindness and visual impairment in children and adolescents (per 1,000 population, $\leq 17$ years) $(1997,2008)^{1 *}$

28-5. Visual impairment due to diabetic retinopathy (age adjusted, per 1,000 population, 18+ years with diabetes) $(2002,2008)^{*}$

28-6. Visual impairment due to glaucoma (age adjusted, per 1,000 population, $45+$ years) $(2002,2008)^{*}$

28-7. Visual impairment due to cataract (age adjusted, per 1,000 population, $65+$ years) $(2002,2008)^{*}$

28-8a. Occupational eye injuries resulting in lost work days (per 10,000 full-time workers in private industry) $(2002,2008)^{2} \dagger$

28-8b. Occupational eye injuries treated in emergency departments (per 10,000 full-time workers) $(1999,2008)^{\star}$

28-9a. Use of protective eyewear at homeChildren and adolescents $6-17$ years (2002, 2008)*

b. Use of protective eyewear at homeAdults 18+ years (age adjusted) $(2002,2008)^{\star}$

28-10a. Use of vision rehabilitation services by visually impaired persons (age adjusted, per 1,000 population, $18+$ years) $(2002,2008)^{*}$

28-10b. Use of visual adaptive devices by visually impaired persons (age adjusted, 18+ years) $(2002,2008)^{\star}$

28-12. Otitis media in children and adolescents (per 1,000 population, $<18$ years) $(1997,2007)^{\star}$

28-13a. Use of hearing aids by adults with hearing loss (per 1,000 population, 20-69 years) (2001, 2006)*

28-13b. New cochlear implants by deaf or very hard of hearing persons (per 10,000 population) (2001, 2006)*

28-13c. Use of hearing aids by older adults with hearing loss (per 1,000 population, $70+$ years) (2001, 2007)*

28-13d. Use of assistive listening devices by older adults with hearing loss (per 1,000 population, $70+$ years) $(2005-06)^{*}$

28-14a. Hearing examination in past 5 yearsAdults 20-69 years (age adjusted) (1999-02 2003-04)*

b. Hearing examination in past 5 yearsOlder adults $70+$ years $(1999-02,2003-06)^{*}$

c. Hearing examination in past 5 yearsAdolescents $12-19$ years (2005-06)*
Characteristics and Groups

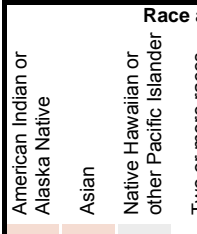
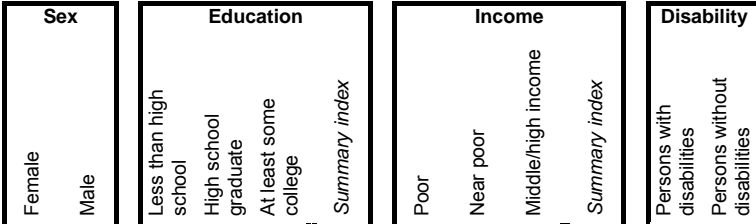

B

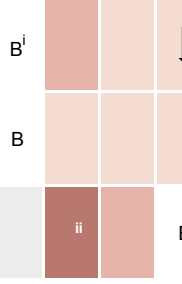

B

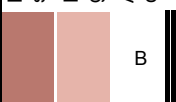

$B^{i}$

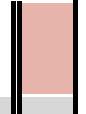

每
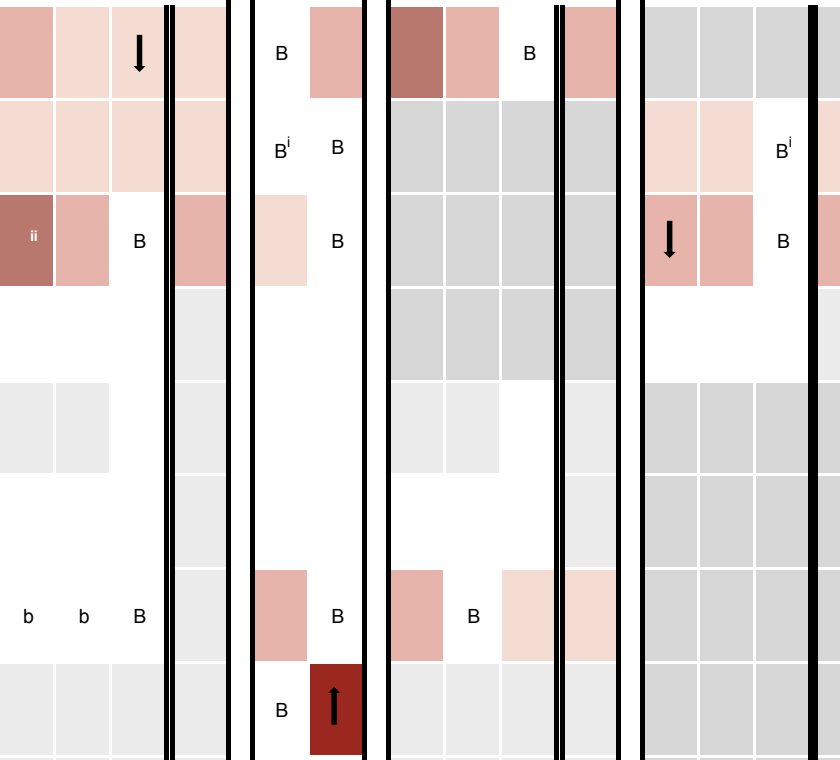

$B^{\mathrm{i}}$

$B^{\mathrm{i}}$

B

$B^{i}$

$B^{\mathrm{i}}$

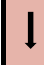

B

$B^{i}$

B

$B^{\mathrm{i}}$

$B^{\text {iv }}$

B

B

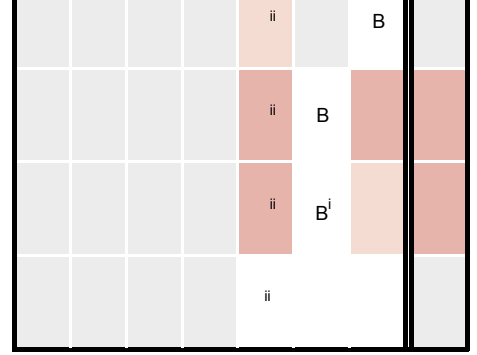

$B^{\mathrm{i}}$

B

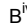

iv

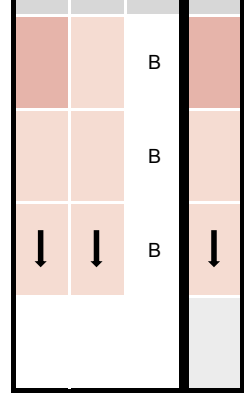

B

B

$\downarrow \mathrm{B}$

(continued) 


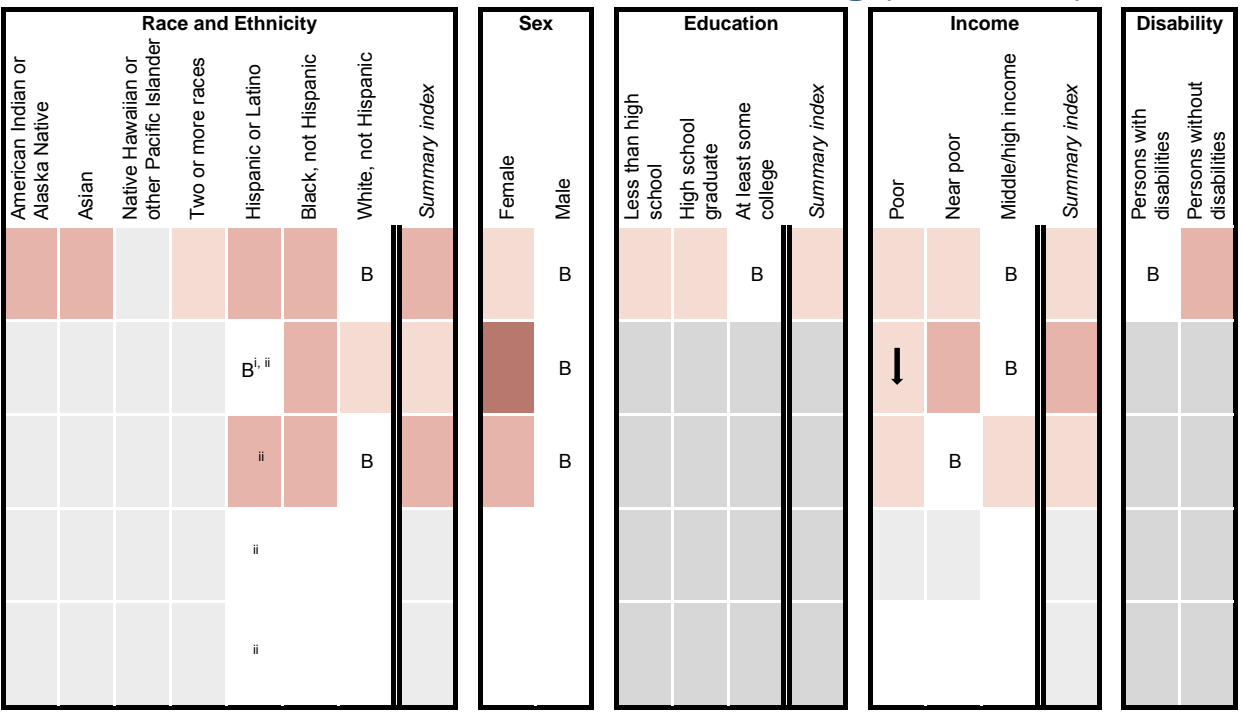

NOTES

Population-based objectives

28-15.

28-16a.

28-16b.

28-17.

28-18

c.

Years in parentheses represent the baseline and most recent data years (if available).

Disparity from the best group rate is defined as the percent difference between the best group rate and each of the other group rates for a characteristic (e.g., race and ethnicity). The summary index is the average of these percent differences for a characteristic. Change in disparity is estimated by subtracting the disparity at baseline from the disparity at the most recent data point. Change in the summary index is estimated by subtracting the summary index at baseline from the summary index at the most recent data point. See Technical Appendix for more information.

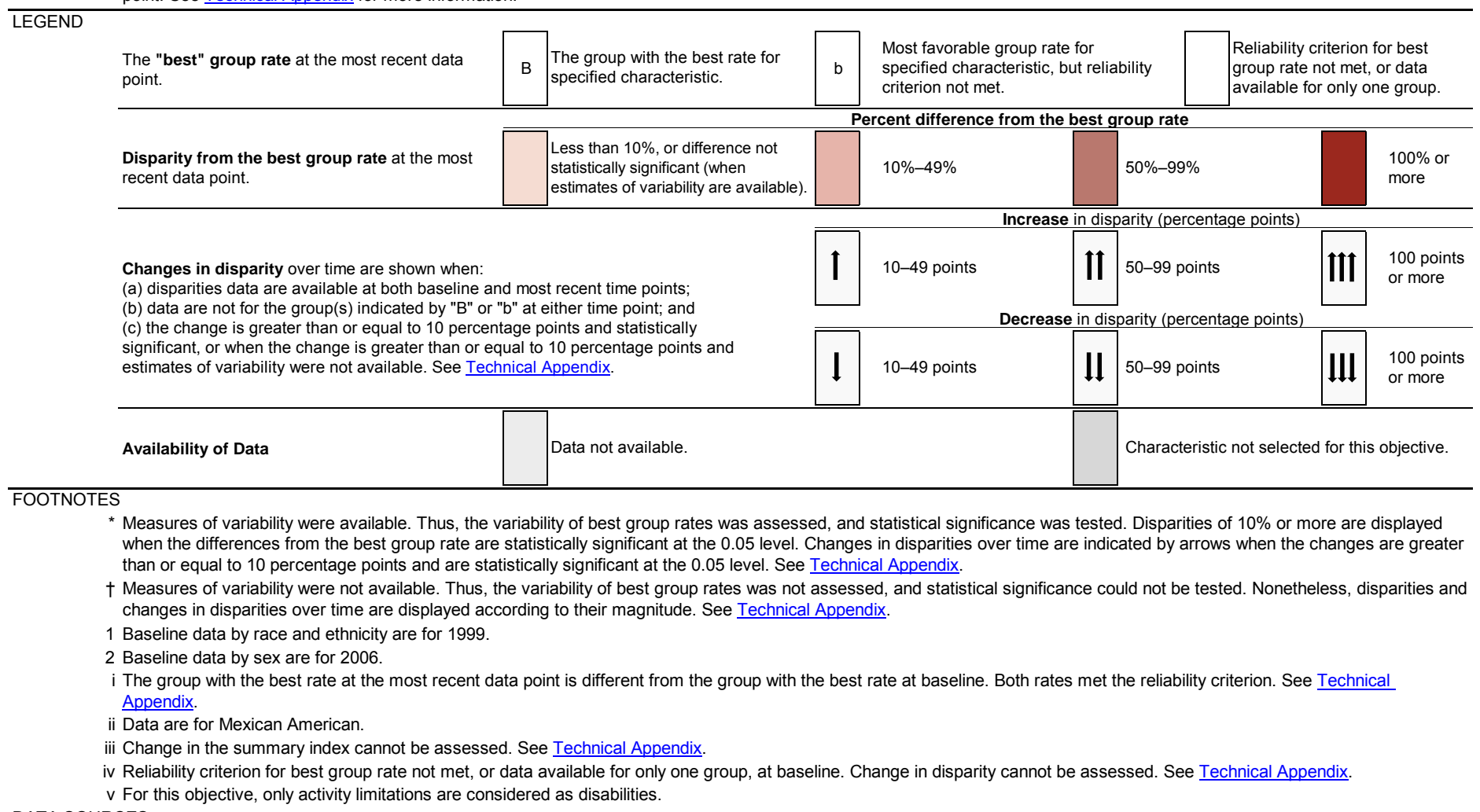
DATA SOURCES

28-1-28-2. National Health Interview Survey (NHIS), CDC, NCHS.

28-3. National Health and Nutrition Examination Survey (NHANES), CDC, NCHS

28-4-28-7. National Health Interview Survey (NHIS), CDC, NCHS.

28-8a. Survey of Occupational Injuries and Illnesses (SOII), Department of Labor (DOL), Bureau of Labor Statistics (BLS).

28-8b. National Electronic Injury Surveillance System (NEISS), Consumer Product Safety Commission (CPSC) and CDC, NIOSH

28-9a-b. National Health Interview Survey (NHIS), CDC, NCHS.

28-10a-b. National Health Interview Survey (NHIS), CDC, NCHS

28-12. National Ambulatory Medical Care Survey (NAMCS), CDC, NCHS; National Hospital Ambulatory Medical Care Survey (NHAMCS), CDC, NCHS.

28-13a. National Health Interview Survey (NHIS), CDC, NCHS; National Health and Nutrition Examination Survey (NHANES), CDC, NCHS.

28-13b. Healthcare Cost and Utilization Project (HCUP), AHRQ; National Health Interview Survey (NHIS), CDC, NCHS.

28-13c. National Health Interview Survey (NHIS), CDC, NCHS; National Health and Nutrition Examination Survey (NHANES), CDC, NCHS

28-13d. National Health and Nutrition Examination Survey (NHANES), CDC, NCHS

28-14a-c. National Health and Nutrition Examination Survey (NHANES), CDC, NCHS.

28-15. National Health Interview Survey (NHIS), CDC, NCHS.

28-16a-b. National Health and Nutrition Examination Survey (NHANES), CDC, NCHS

28-17-28-18. National Health and Nutrition Examination Survey (NHANES), CDC, NCHS

Vision and Hearing

Page 28-17 


\section{Final Review}

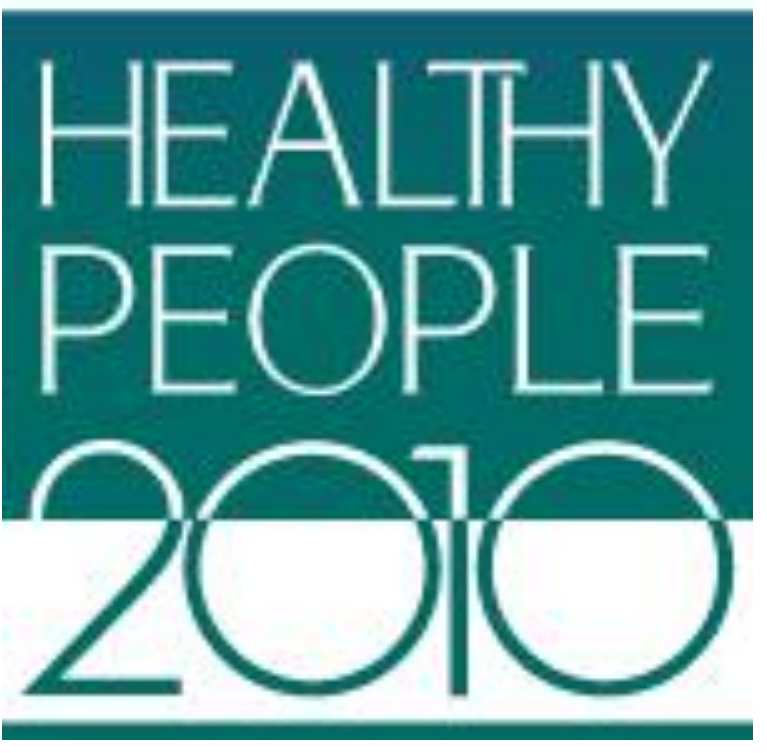

\section{Appendices}




\section{Appendix A: Technical Appendix}

This appendix provides additional information on a number of issues related to monitoring progress in Healthy People 2010.

- Measuring progress toward target attainment-procedures used to measure progress toward the targets for Healthy People 2010 objectives.

- Measuring quality and years of healthy life-procedures used to measure quality and years of healthy life in connection with the first goal of Healthy People 2010.

- Measuring health disparities-procedures used to measure and track health disparities among select population groups in connection with the second goal of Healthy People 2010.

- Mapping-procedures used for mapping select Healthy People 2010 objectives.

- DATA2010 - the online database for Healthy People 2010 objectives.

- General Data Issues - the guide to measurement issues in Healthy People 2010.

- Tracking Period-a note on how an objective's tracking period is defined in the Healthy People 2010 Final Review.

\section{Measuring Progress toward Target Attainment}

Progress toward the Healthy People 2010 targets at Final Review is shown in a Progress Chart (labeled Figure 1 in each Focus Area chapter). This chart displays the percent of targeted change that has been achieved for each objective.

\section{Percent of Targeted Change Achieved}

Targeted change is the difference between the baseline and the Healthy People 2010 (HP2010) target. The formula for the percent of targeted change achieved is as follows:

$$
\text { Percent of targeted change achieved }=\frac{\text { Final value }- \text { Baseline value }}{\text { HP2010 target }- \text { Baseline value }} \times 100 \text {. }
$$

The percent of targeted change achieved expresses the difference between the baseline and the final value as a percent of the initial difference between the baseline and the Healthy People 2010 target. As such, it is a relative measure of progress toward attaining the Healthy People 2010 target. In addition, the comparability of the percent of targeted change achieved does not depend on whether the underlying objective is expressed in terms of adverse or favorable events or conditions; see Measuring Health Disparities section, below. The percent of targeted change achieved was also used to monitor progress in Healthy People 2000 and was previously referred to as the progress quotient $[1,2]$. 
Baseline data values were published at the beginning of the decade for Healthy People 2010 objectives for which data were available [3]. Baseline data for additional objectives have become available since the publication of Healthy People 2010 [4]. Data beyond the baseline are available for about 76 percent of the objectives in Healthy People 2010.

\section{Example A-1}

School-based objective 7-2c in Healthy People 2010 called for an increase in the proportion of middle/junior and senior high schools that provide education to prevent violence, from a baseline of 58 percent in 1994 to a target of 80 percent by 2010. In 2006, 77 percent of schools provided education to prevent violence, see Figure 7-1 in the Focus Area 7 chapter. Using the formula above, 86.4 percent of the targeted change from the 1994 baseline to the Healthy People 2010 target was achieved in 2006. Indeed,

$$
\text { Percent of targeted change achieved }=\frac{77-58}{80-58} \times 100=\frac{19}{22} \times 100=86.4 \text { percent. }
$$

For population-based objectives, the percent of targeted change achieved also can be used to measure progress toward the Healthy People 2010 target for each population group with data beyond the baseline.

\section{Example A-2}

The Healthy People 2010 target for objective 16-1c was to reduce the infant death rate to 4.5 deaths per 1,000 live births by 2010 . For the total population, the 1998 baseline rate was 7.2 infant deaths per 1,000 live births, while that for the year 2006 was 6.7 infant deaths per 1,000 live births, see Figure 16-1 in the Focus Area 16 chapter. When the formula above is applied, 18.5 percent of the targeted change from the 1998 baseline to the Healthy People 2010 target was achieved in 2006:

$$
\text { Percent of targeted change achieved }=\frac{6.7-7.2}{4.5-7.2} \times 100=\frac{-0.5}{-2.7} \times 100=18.5 \text { percent. }
$$

In contrast, among infants of Asian or Pacific Islander mothers, the infant death rate declined from 5.5 deaths per 1,000 live births at baseline to 4.5 deaths per 1,000 live births in 2006. Using the formula above, 100 percent of the targeted change from the 1998 baseline to the Healthy People 2010 target was achieved in 2006:

$$
\text { Percent of targeted change achieved }=\frac{4.5-5.5}{4.5-5.5} \times 100=\frac{-1.0}{-1.0} \times 100=100 \text { percent. }
$$

Thus, the Healthy People 2010 target was met for the Asian or Pacific Islander group in 2006, while, overall, the population only achieved 18.5 percent of the targeted change. 


\section{Limitations}

In addition to assessing differentials in progress toward target attainment within the population, the percent of targeted change achieved may be used to compare how much of the targeted change was achieved for an objective relative to other objectives, though care must be exercised in its interpretation. Generally speaking, the reader is advised to keep the following points in mind:

- The percent of targeted change achieved is calculated using only the Healthy People 2010 target, baseline, and final data points. Fluctuations that may occur during the intervening years are not considered, even though they may be substantial.

- The number of years between the baseline and final data points for Healthy People 2010 might vary both between objectives and within objectives.

- Between objectives, differences in the number of years available to meet targets are a function of the data sources and any choices that were made regarding the most appropriate baseline year for each objective.

- To assist the reader in the interpretation of these comparisons, the baseline and final data years used for each objective are shown in parentheses following the short descriptions in the left-most panel of the Progress Chart for each Focus Area.

- Within objectives, differences in the number of years available to meet Healthy People 2010 targets for specific groups within the population can be affected by changes in the data templates used to classify the population (e.g., by race and ethnicity) during the tracking period.

- The period used to compute the percent of targeted change achieved will generally be consistent with that used to estimate disparities, see the Measuring Health Disparities section below for more details.

- The (absolute) value of the Healthy People 2010 targeted change from baseline might vary among select populations or across objectives with identical values for the percent of targeted change achieved. Therefore, two objectives may be identical in their percent of targeted change achieved, even though they differ in the magnitude of the change. See Example A-3 below.

Example A-3. Objective 7-4b in Healthy People 2010 called for 50 percent of senior high schools to attain a nurse-to-student ratio of at least 1:750 by year 2010, while objective 7$4 \mathrm{~d}$ called for 48 percent of elementary school to achieve that same nurse-to-student ratio by year 2010. The 1994 baseline data point for senior high schools was 26 percent, thus the absolute value of the targeted change for objective $7-4 \mathrm{~b}$ was 24 percentage points. On the other hand, the 2000 baseline data point for elementary schools was 42 percent, resulting in a targeted change of only 6 percentage points. In 2006, 38 percent of senior high schools and 45 percent of elementary schools had attained the desired nurse-to-student ratio. As a result, both objectives achieved 50 percent of their targeted change-12 of the targeted 24 percentage points for objective $7-4 \mathrm{~b}$, and 3 of the targeted 6 percentage points for objective 7-4d-even though they differed in the magnitude of the change. See Figure 7-1 in the Focus Area 7 chapter. 
In addition to the above limitations, there are a number of cases in which the percent of targeted change achieved cannot be calculated or does not adequately reflect change in an objective. Five hypothetical scenarios are presented below for the reader's consideration, further illustrating the care that must be exercised in the interpretation of the percent of targeted change achieved in Healthy People 2010.

\section{Scenario 1: Target met at baseline, movement in a positive direction}

Target $=5$; Baseline value $=5 ;$ Final value $=4$.

$$
\text { Percent of targeted change achieved }=\frac{4-5}{5-5} \times 100=\frac{-1}{0} \times 100=\text { undefined. }
$$

Cannot divide by 0 .

\section{Scenario 2: Target met at baseline, movement in a negative direction:}

Target $=0 ;$ Baseline value $=0 ;$ Final value $=2$.

$$
\text { Percent of targeted change achieved }=\frac{2-0}{0-0} \times 100=\frac{2}{0} \times 100=\text { undefined. }
$$

Cannot divide by 0 .

\section{Scenario 3: Target exceeded at baseline-further progress beyond target:}

Target $=30$; Baseline value $=35 ;$ Final value $=40$.

$$
\text { Percent of targeted change achieved }=\frac{40-35}{30-35} \times 100=\frac{5}{-5} \times 100=-100 \text { percent. }
$$

Here, progress has been made, but the percent of targeted change achieved appears to indicate movement away from the target.

\section{Scenario 4: Target exceeded at baseline-movement in a negative direction}

Target $=30$; Baseline value $=35$; Final value $=25$.

$$
\text { Percent of targeted change achieved }=\frac{25-35}{30-35} \times 100=\frac{-10}{-5} \times 100=200 \text { percent. }
$$

Here, progress has not been made, but the percent of targeted change achieved appears to indicate the target has been exceeded.

In the Progress Chart (Figure 1 for each Focus Area chapter), objectives as in scenarios 1 and 3 above are shown with arrows in the positive direction, with "100+\%" indicating that the target has been exceeded. Those as in scenarios 2 and 4 are shown with arrows in the negative direction. In all cases, footnotes indicate the precise amount cannot be calculated. 
Finally, when the targeted amount of change is small relative to the actual amount of observed change, the percent of targeted change achieved can have relatively large values that are difficult to interpret. Furthermore, the reader should be aware that target setting has a sizeable impact on the measure of progress toward target attainment used in Healthy People 2010. This phenomenon is illustrated in the following hypothetical scenario.

\section{Scenario 5: Target set closer to baseline-movement in a negative direction}

Consider: Target $=30 ;$ Baseline value $=50 ;$ Final value $=70$.

$$
\text { Percent of targeted change achieved }=\frac{70-50}{30-50} \times 100=\frac{20}{-20} \times 100=-100 \text { percent. }
$$

Compare to: Target $=40 ;$ Baseline value $=50 ;$ Final value $=70$.

$$
\text { Percent of targeted change achieved }=\frac{70-50}{40-50} \times 100=\frac{20}{-10} \times 100=-200 \text { percent. }
$$

In both cases, progress has not been made, the final value having exceeded the baseline value by 20 points. Yet, a target of 30 having been set closer to the baseline value than a target of 40, the percent of targeted change achieved appears to indicate a worse scenario in the second case than in the first, even though the difference between the baseline and final values remains unchanged.

In order to circumvent the difficulty in interpretation that arises for objectives like in scenario 5 above, movement away from the Healthy People 2010 target is not quantified using the percent of targeted change achieved in the Progress Chart (see footnote 1 for Figure 1 in each of the Focus Area chapters) for the Final Review. Instead, for such objectives, the reader is urged to examine the difference between the baseline and final values to assess progress.

\section{Testing for Trends}

As stated in the limitations section above, the percent of targeted change achieved is calculated using only the Healthy People 2010 target, baseline, and final data points. Fluctuations that may occur during the intervening years are not considered, even though they may be substantial. In addition, the number of years between the baseline and final data points for Healthy People 2010 might vary both between objectives and within objectives.

Nonetheless, the presence of a monotonic increasing or decreasing trend in the underlying measure can be tested with the nonparametric Mann-Kendall test, and the slope of a linear trend estimated with the nonparametric Sen's method [5].

The Mann-Kendall test is suitable for cases where the trend may be assumed to be monotonic, and thus where no seasonal or other cycle is present in the data. 
The Sen's method uses a linear model to estimate the slope of the trend when the variance of the residuals may be assumed constant in time. Missing values are allowed and the data need not conform to any particular distribution. Also, the Sen's method is not greatly affected by single data errors or outliers.

When the number of data points is less than 10, Sen's $S$ statistic can be used. When the number of data points is 10 or more, a normal approximation holds, and a $Z$ statistic can be used instead.

Results of the trend tests described above are used in the Highlights section of select Focus Area chapters-namely, chapters 6,10,13,15, and 20-in order to supplement findings on progress toward achieving Healthy People 2010 targets during the decade.

\section{Measuring Quality and Years of Healthy Life}

Goal 1 of Healthy People 2010 is to increase the quality and length of healthy life-years. This goal is tracked with three summary measures of health that belong to the family of measures called "healthy life expectancy." The three summary measures are:

1) Expected years of life in good or better health,

2) Expected years of life free of activity limitation, and

3) Expected years of life free of selected chronic diseases.

These healthy life expectancy measures are given in life-years, which indicate the average number of healthy years a person can expect to live if age-specific death rates and agespecific illness rates remain the same throughout his or her lifetime. Thus, healthy life expectancy is a "snapshot" of current death and illness patterns and can illustrate the longrange implications of the prevailing age-specific death and illness rates. The methods used to create the healthy life expectancy measures are described next.

\section{Methods}

The healthy life expectancies used in the Final Review publication are calculated using a double-decrement life table technique, based on the Sullivan method [6,7]. A traditional life table presents what would happen to a hypothetical cohort if it experienced exactly the same age-specific death rates during a given period of time [8]. A double-decrement life table analyzes what would happen to a hypothetical cohort if it experienced exactly the same age-specific death and age-specific illness rates during a given period of time. Although it is possible to create life tables based on single years of age, this analysis uses an abridged life table, with age intervals of five years.

To produce healthy life expectancies, age-specific death rates are combined with agespecific health prevalence rates to produce an estimate of overall healthy life expectancy [9]. 
The life table includes the following quantities:

- $q x$ - probability of dying - This column shows the probability of dying during the age interval. It is derived from death rates for a given year.

- $l x$-number surviving - This column shows the number of persons from birth surviving to the beginning of the next age interval. The life table typically begins with a population at birth of 100,000 , called the radix.

- $d x$-number dying - This column shows the number of deaths in each age interval out of the original 100,000 births. It is calculated by multiplying the $q x$ for the age interval by the $l x$ for the same age interval.

- Lx-person-years lived-This column shows the total time lived (in years) within the age interval by all of those who have survived to the beginning of the age interval.

- Tx-total number of person-years lived-This column shows the total number of person-years lived that would be lived after the beginning of the age interval.

- Ex-expectation of life-This column shows the average number of years remaining to be lived by those surviving to the age interval. It is derived by dividing the total number of person-years lived at the age interval and above by the number surviving to the beginning of the age interval $(T x / l x)$.

Life tables used to calculate healthy life expectancy include all of the quantities described above in addition to the following quantities regarding illness:

- Px-age-specific illness rate-This column shows the percentage of persons in the age interval in a given poor health state.

- $P x \times L x-H e a l t h y$ person-years lived-This column shows the number of healthy person-years lived during the age interval. This number is derived by multiplying the age-specific illness rate by the corresponding number of person-years lived during the age interval $(L x)$.

- THx-Total number of healthy person-years lived-This column shows the total number of healthy person-years that would be lived after the age interval.

- HLEx-Expectation of healthy life-The expectation of healthy life is the average number of years in good health remaining for those surviving to a given age with a given set of age-specific death rates and age-specific illness rates. It is derived by dividing the total healthy person-years that would be lived at age $x$ by the total number of persons who survived to that age interval $(\mathrm{THX} / \mathrm{l} \mathrm{x})$.

The use of healthy life expectancies enables comparisons across populations, as well as over long periods of time. The use of the Sullivan method for estimating healthy life expectancies is most appropriate for the cross-sectional data used to track Healthy People 2010 [10]. 


\section{Data Systems}

Analyses are based on 2000-01 (2002-03 for chronic conditions) and 2006-07 death data from the National Vital Statistics System (NVSS) and 2000-01 (2002-03 for chronic conditions) and 2006-07 health data from the National Health Interview Survey (NHIS). NHIS is a nationally representative continuing cross-sectional survey, which provides a "snapshot" of the health of the U.S. population. Approximately 35,000 households are interviewed each year. NVSS is a complete registration of all vital events and includes detailed data on all of the deaths that occur within the U.S.

These data systems are used for the study of healthy life expectancy because they contain detailed information on health and death. However, the institutionalized population is excluded from the NHIS sample. Because the institutionalized population is more likely to report poor health, the Healthy People 2010 healthy life expectancy measures might underestimate the effect of poor health on healthy life expectancies.

\section{Survey Questions}

Self-rated health status is measured by the single question from NHIS that asked respondents to rate their health as "excellent," "very good," "good," "fair," or "poor." For the purpose of determining Healthy People 2010 healthy life expectancy, a respondent was considered to be in poor health if he or she answered "fair" or "poor." This self-assessed health rating was shown to be a useful indicator of one's health for a variety of populations and allows for broad comparisons across different conditions and populations [11]. The measure also is included in the Behavioral Risk Factor Surveillance Survey, the National Health and Nutrition Examination Survey, and other health surveys.

Activity limitation is measured using questions about personal care needs, limitations of activities, and use of special equipment. Adults were asked whether they needed assistance with personal care needs, such as eating, bathing, dressing, or getting around inside the home; whether they needed assistance with routine care needs, such as household chores; and whether they had a mental or physical problem that kept them from working at a job or limits their activity in any way. They also were asked whether they had health problems that required the use of special equipment, such as a cane, wheelchair, or special telephone. If a respondent answered "yes" to any of these questions, he or she was classified as having activity limitations. Children were considered limited in activity if the proxy adult respondent responded "yes" to any of the limitation, special services, or special equipment questions that were specific to children. 
Selected chronic disease prevalence is measured by several questions that asked respondents whether a doctor had ever diagnosed them with a given disease. The list of selected chronic diseases represented those chronic diseases that were included in Healthy People 2010 and NHIS: heart disease, stroke, cancer, diabetes, hypertension, kidney disease, arthritis, and asthma. If a respondent answered "yes" to any of the selected diagnoses, he or she was classified as having a chronic disease. Ideally such a healthy life expectancy would adjust for severity of disease. However, NHIS does not collect data on the severity of the disease. The primary limitation of this measure is that it is restricted to diseases noted above. Thus, it underestimates the contribution of chronic disease to healthy life expectancy because other chronic conditions, especially chronic mental health conditions, are not included.

\section{Healthy People 2000}

The 2010 healthy life expectancy measures differ from the measure used for Goal 1 of Healthy People 2000. The Healthy People 2000 measure combined information about death, self-rated health, and activity limitations into a single measure known as years of healthy life [12]. For Healthy People 2010, these illness components have been separated into distinct measures. This strategy allows for greater ease in interpreting change and determining the mechanisms of change. The same double-decrement life table technique used in Healthy People 2000 is used to create the healthy life expectancy measures for Healthy People 2010.

\section{Limitations}

Healthy life expectancy is computed using the Sullivan method, the standard method for computing healthy life expectancy on a routine basis. Although the Sullivan method accurately depicts the current status of the population's health, it does not reflect the underlying transitions into and out of poor health states. In other words, the Sullivan method assumes that if a respondent reports an activity limitation at a given point in time, that respondent is limited in activities for the rest of his or her life. However, as the underlying disease processes have episodic fluctuations of deterioration and improvement over time, poor health states will also fluctuate. For example, a person diagnosed with functional limitations due to severe arthritis may take medication and experience better health states in the future, however, the Sullivan method does not account for future years of good health for such a person.

In addition, the Sullivan method can be biased when evaluating trends over a short period of time. Biases in trends of healthy life expectancy can occur if there are fluctuations in health over a short time period. The Sullivan method is less likely to give misleading estimates of trends in healthy life expectancy when changes in death rates and health status rates are smooth and relatively even. 


\section{Future Plans}

Goal 1 of Healthy People 2010 challenged the Nation increase quality and years of healthy life. Identifying the best approaches for measuring quality and years of healthy life is an evolving field. Future research will build upon these initial measures of healthy life expectancy. It would be desirable to include measures that account for the contribution of mental health status to quality of life and other health variables. In addition, the Healthy People 2010 healthy life expectancy measures are expected to be expanded to include expected years of life with good health behaviors in Healthy People 2020.

\section{Measuring Health Disparities}

The second overarching goal of Healthy People 2010 calls for eliminating health disparities among segments of the population, including differences that occur by race or ethnicity, sex, education or income, geographic location, disability, or sexual orientation [3]. These characteristics are applicable to objectives that measure aspects of the health of the population and do not apply to objectives that are based on schools, worksites, states, or other units of measures that are not population-based. The Health Disparities Table (Figure 2 in each of the Focus Area chapters) summarizes information about disparities from the best group rate for each of a selected set of population characteristics at the most recent data point, and changes in disparities from the baseline to the most recent data point.

The methods used to create the Health Disparities Table are described below. The rationale for methods employed in measuring disparity in Healthy People 2010 was provided in a previous report [13]. The "Goal 2: Eliminate Health Disparities" section in the Healthy People 2010 Final Review Executive Summary presents key findings concerning disparities.

\section{Measuring Objectives and Defining Groups}

Technical information (i.e., Operational Definitions) concerning the measurement of each objective and the classification of the population characteristics employed in monitoring the objectives is provided in Tracking Healthy People 2010 [14]. The original classification of racial and ethnic groups shown in Healthy People 2010 was altered based on revisions to the standards for the classification of Federal data on race and ethnicity that were published by the Office of Management and Budget in 1997 [3,15]. These standards permit each person to identify either with only one race or with more than one race. The race and ethnicity categories used to monitor the Healthy People 2010 population-based objectives were modified accordingly, resulting in the following categories:

- American Indian or Alaska Native

- Asian

- Native Hawaiian or other Pacific Islander

- Black or African American 
- White

- Two or more races

- American Indian or Alaska Native; White

- Black or African American; White

- Hispanic or Latino

- Not Hispanic or Latino

- Black or African American

- White

Federal data systems have been revising their collection and tabulation procedures to comply with the new standards on racial and ethnic identification. Some data systems began reporting data for calendar year 1999 using the new standards, and most of the remaining systems have adopted the new standards since then. However, some data systems are still in the process of adopting the revised standards, so the availability of comparable data for racial and ethnic groups varies by data source and across objectives.

In the Healthy People 2010 Final Review, seven racial and ethnic groups are shown in the Health Disparities Table (Figure 2 in each of the Focus Area chapters): American Indian or Alaska Native; Asian; Native Hawaiian or other Pacific Islander; two or more races; Hispanic; white, not Hispanic; and black, not Hispanic. The first four groups might also include small numbers of persons of Hispanic origin. The data systems used to track the population-based objectives in Healthy People 2010 might not provide data for all of these groups. Departures from the above categories are footnoted in the Health Disparities Table.

To maintain comparability of data by race and ethnicity over time for some objectives, a more recent data year might be used as the baseline because of the revised standards [15]. NHIS, for example, began reporting data according to the new racial and ethnic categories in 1999. Although the baseline year for objectives tracked with NHIS might be 1997 or 1998 , data for 1999 are employed as the baseline for measuring disparities for race and ethnicity data only. These departures are indicated by footnotes in the Health Disparities Table.

Education and income are the primary measures of socioeconomic status (SES) in Healthy People 2010. Most data systems used in Healthy People 2010 define income as a family's income before taxes. In order to facilitate comparisons among groups and over time, while adjusting for family size and for inflation, Healthy People 2010 categorizes income using the poverty thresholds developed by the U.S. Census Bureau. Thus, the three categories of family income that are primarily used are:

- Poor-below the Federal poverty level

- Near poor-100\% to $199 \%$ of the Federal poverty level

- Middle/high income-200\% or more of the Federal poverty level.

These categories may be overridden by considerations specific to the data system, in which case they are modified as appropriate. See Healthy People 2010: General Data Issues [16]. 


\section{Availability of Data}

The data used to monitor the Healthy People 2010 objectives come from a wide variety of data systems. Data for a particular population group might not be available because they are not collected by the data system, because they have been collected but not analyzed, or because they have been suppressed. Data are suppressed when the number of events is too small to produce statistically reliable estimates, when disclosure might violate confidentiality requirements, when the sample design does not produce representative estimates for a particular group, or when there is high item nonresponse or a large number of "unknown" entries. Criteria for data suppression for the data systems included in Healthy People 2010 are published in a previous report [17].

\section{Content of the Health Disparities Table}

The Health Disparities Table (Figure 2 in each of the Focus Area chapters) provides information about disparities between groups for the population-based objectives in each Focus Area. Short descriptions of the population-based objectives are listed along the leftmost column of the table. The baseline data year(s) is (are) shown in parentheses and, when more recent data are available, the most recent data year(s) is (are) also shown. The description of an objective generally also includes in parentheses any applicable information regarding the underlying measure (e.g., measurement unit) and/or the age of the targeted population.

Characteristics of the population (race and ethnicity, sex, education, income, geographic location, and disability status) are listed across the top of the Health Disparities Table. In general, characteristics applicable to each objective were designated in the original Healthy People 2010 document [3]. Race and ethnicity, sex, and education or income are available and included for most objectives; geographic location and disability status are included only if applicable and/or available.

Characteristics that were not designated for a particular objective are shaded in dark gray. When a characteristic is not applicable for any of the objectives in a Focus Area, it is omitted from the Health Disparities Table for that Focus Area. When data are not available for a particular population or for a particular characteristic, the corresponding boxes are shaded in light gray (see the fourth section of the legend reproduced in Figure A-1 below). If there are no characteristic-specific data available for an objective, or if it is not population-based, the objective is excluded from the table and annotated in the notes. In some cases, the data source for an objective provides data for groups that are defined in nonstandard ways. For example, some data sources provide data for the black and white populations that include persons of Hispanic origin. These departures from the standardized template used to monitor the Healthy People 2010 population-based objectives are indicated by footnotes in the Health Disparities Table. 


\section{Measuring Disparity from the Best Group Rate}

Definition. Disparity from the best group rate is defined as the percent difference between the best group rate and each of the other group rates for a characteristic.

For example, health disparities by race and ethnicity are measured as the percent difference between the best racial and ethnic group rate and each of the other racial and ethnic group rates. Similarly, disparities by sex are measured as the percent difference between the better group rate (e.g., female) and the rate for the other group (e.g., male).

Formula. The formula for disparity from the best group rate for a group $\mathrm{G}$ is as follows:

$$
\text { Disparity for group } \mathrm{G}=\frac{R_{\mathrm{G}}-R_{\mathrm{B}}}{R_{\mathrm{B}}} \times 100 \text {, }
$$

where $R_{B}$ is the best group rate and $R_{G}$ is the rate for group $\mathrm{G}$ for a particular characteristic.

Note. In computing disparities, the Healthy People 2010 Final Review uses the display values for rates, proportions, and other estimates in DATA2010. Those are typically rounded to the nearest whole number or to at most one decimal place, see section on DATA2010 below. As a result, the best group rate $R_{B}$ may in some rare instances be displayed as zero and subsequently treated as a zero in the above formula, resulting in an undefined division by zero. In order to avoid such an artificial situation, a small continuity correction is applied so as to always enable a meaningful calculation of disparities relative to the best group rate.

Some Healthy People 2010 objectives are expressed in terms of favorable events or conditions that are to be increased, while others are expressed in terms of adverse events or conditions that are to be reduced. In order to facilitate comparison of disparities across different objectives, disparity is measured only in terms of adverse events or conditions in Healthy People 2010 [1]. Those dichotomous objectives that are expressed in terms of favorable events or conditions are re-expressed using the adverse event or condition for the purpose of computing disparity $[12,18,19]$, but they are not otherwise restated or changed.

Example. Healthy People 2010 objective 1-1, to increase the proportion of persons with health insurance (e.g., $72 \%$ of the American Indian or Alaska Native population aged under 65 years had health insurance in 2008), is expressed in terms of the percentage of persons without health insurance (e.g., $100 \%-72 \%=28 \%$ of the American Indian or Alaska Native population aged under 65 years did not have health insurance in 2008) when the disparity from the best group rate is calculated. 
Special cases. Healthy People 2010 objectives 26-9a, 26-9b, and 27-4a, aim to increase the (average) age at first use of alcohol, marijuana, and tobacco, respectively, among adolescents aged 12-17 years. In order to facilitate comparison of disparities across different objectives, those three objectives are re-expressed using an adverse condition, as follows: decrease the (average) number of years between the (average) age at first use and age 18 years. Similarly, objective $27-4 \mathrm{~b}$ aims to increase the (average) age at first use of tobacco among young adults aged 18-25 years. This objective is re-expressed as follows: decrease the (average) number of years between the (average) age at first use and age 26 years. Finally, objective 16-16b aims to increase the median red blood cell (RBC) folate level among nonpregnant women aged 15-44 years. The underlying measure for this objective is a continuous measure which does not have a known upper limit. Nonetheless, an approximate upper limit is given by the $97.5^{\text {th }}$ percentile of RBC folate concentration among women aged 20-59 years, estimated at $596 \mathrm{ng} / \mathrm{mL}$ [20]. Thus, objective $16-16 \mathrm{~b}$ can be reexpressed using an adverse measure by subtracting the aggregate median RBC folate level for each population group from the value $596 \mathrm{ng} / \mathrm{mL}$. For the reader's reference, among the population groups considered in the Health Disparities Table, the population group with the highest median RBC folate level was the group with at least some college education, with an aggregate median RBC folate level of $267 \mathrm{ng} / \mathrm{mL}$ in 2005-06.

As a result of measuring disparity only in terms of adverse events or conditions, the group identified as having the best rate for a given characteristic in the Health Disparities Table is always the group with the least adverse event or condition. Thus, disparities defined by the above formula remain nonnegative quantities, equal zero only when the group $\mathrm{G}$ for which disparity is being assessed has rate equal to the best group rate.

In the few instances when two groups for a characteristic have identical best rates, both groups are identified by a "B." To ensure that disparity is measured from a reasonably stable data point, the most favorable group rate must have a relative standard error of less than 10 percent. When the relative standard error for the most favorable group rate is greater than or equal to 10 percent, a small letter " $b$ " is included in the cell and the next most favorable group rate with a relative standard error of less than 10 percent is identified as the reference group for that characteristic. Disparities are not calculated for cells identified by a small letter "b." When there is only one group with a relative standard error of less than 10 percent, a best group is not identified for purposes of measuring disparity, and the cells for all groups with data are blank, indicating that disparities could not be assessed. The first section of the legend for the Health Disparities Table (reproduced, here, in Figure A-1) addresses the identification of the best group rate for each characteristic.

When standard errors are not available, the best group is determined by the most favorable rate, see section on Estimates of Variability below. 
Figure A-1. Legend for the Health Disparities Table (Figure 2) in each of the Focus Area chapters

\begin{tabular}{|c|c|c|c|c|c|c|}
\hline \multicolumn{7}{|l|}{ LEGEND } \\
\hline $\begin{array}{l}\text { The "best" group rate at the most } \\
\text { recent data point. }\end{array}$ & B & $\begin{array}{l}\text { The group with the best rate for } \\
\text { specified characteristic. }\end{array}$ & $\mathrm{b}$ & \multicolumn{2}{|c|}{$\begin{array}{l}\text { Most favorable group rate for specified } \\
\text { characteristic, but reliability criterion not met. }\end{array}$} & $\begin{array}{l}\text { Reliability criterion for best } \\
\text { group rate not met, or data } \\
\text { available for only one group }\end{array}$ \\
\hline & \multicolumn{6}{|c|}{ Percent difference from the best group rate } \\
\hline $\begin{array}{l}\text { Disparity from the best group rate at } \\
\text { the most recent data point. }\end{array}$ & & $\begin{array}{l}\text { Less than } 10 \% \text {, or difference not } \\
\text { statistically significant (when } \\
\text { estimates of variability are available). }\end{array}$ & & $10 \%-49 \%$ & $50 \%-99 \%$ & $100 \%$ or more \\
\hline & & & & & parity (percent & \\
\hline \multirow{3}{*}{\multicolumn{3}{|c|}{$\begin{array}{l}\text { Changes in disparity over time are shown when: } \\
\text { (a) disparities data are available at both baseline and most recent time points; } \\
\text { (b) data are not for the group(s) indicated by "B" or "b" at either time point; and } \\
\text { (c) the change is greater than or equal to } 10 \text { percentage points and statistically } \\
\text { significant, or when the change is greater than or equal to } 10 \text { percentage points } \\
\text { and estimates of variability were not available. See Technical Appendix. }\end{array}$}} & & 10-49 points & 50-99 points & $\begin{array}{l}100 \text { points or } \\
\text { more }\end{array}$ \\
\hline & & & \multicolumn{4}{|c|}{ Decrease in disparity (percentage points) } \\
\hline & & & & 10-49 points & 50-99 points & $\begin{array}{l}100 \text { points or } \\
\text { more }\end{array}$ \\
\hline Availability of Data & & Data not available. & & & \multicolumn{2}{|c|}{ Characteristic not selected for this objective. } \\
\hline
\end{tabular}




\section{Representing the Size of Health Disparities by a Color Gradient}

In the Health Disparities Table, a color gradient is used to represent the size of the disparities (i.e., the percent differences between each group rate and the best group rate) at the most recent data point. In some cases, baseline data might be the only data available. The color gradient is shown in the second section of the legend, see Figure A-1.

When measures of variability (i.e., standard errors) are available, the variability of best group rates is assessed, and statistical significance is tested. For a group $\mathrm{G}$ within a given characteristic, a disparity of $10 \%$ or more is displayed when the (simple) difference from the best group rate (i.e., $R_{G}-R_{B}$ ) is statistically significant at the 0.05 level (see Figure A-1):

- The lightest color in the color gradient indicates a group with a disparity $<10 \%$ from the best group rate. When measures of variability are available, the lightest color in the color gradient also indicates disparities for which the difference $R_{G}-R_{B}$ is not statistically significant at the 0.05 level.

- The darkest color in the color gradient indicates a group with a disparity $\geq 100 \%$ and, when measures of variability are available, a difference $R_{G}-R_{B}$ from the best group rate that is statistically significant.

- The two intermediate colors in the color gradient indicate groups with a disparity of $10 \%-49 \%$ and groups with a disparity of $50 \%-99 \%$.

The statistical significance of the (simple) difference $R_{G}-R_{B}$ between groups can be assessed using the following Z-statistic:

$$
\mathrm{Z}=\frac{R_{\mathrm{G}}-R_{\mathrm{B}}}{\sqrt{\mathrm{SE}_{\mathrm{G}}^{2}+\mathrm{SE}_{\mathrm{B}}^{2}}},
$$

where $R_{G}$ is the rate for a group $\mathrm{G}$ of interest, $R_{B}$ is the rate for the best group, $\mathrm{SE}_{\mathrm{G}}$ is the standard error of the rate for group $\mathrm{G}$, and $\mathrm{SE}_{\mathrm{B}}$ is the standard error of the best group rate.

This formula assumes that the two groups are independent. Because, as mentioned earlier, the difference $R_{G}-R_{B}$ remains nonnegative, a one-tailed test is employed to assess statistical significance. When $Z \geq 1.645$, the difference $R_{G}-R_{B}$ between the two group rates is statistically significant at the 0.05 level. When the (simple) difference $R_{G}-R_{B}$ between the two group rates is significant, the disparity for group $G$ relative to the best group rate is considered significant. 


\section{Changes in Health Disparities over Time}

When data beyond the baseline are available, change in disparity over time is estimated by subtracting the disparity at baseline from the disparity at the most recent data point. The change is expressed in percentage points: positive differences represent an increase in disparity, and negative differences represent a decrease in disparity. See the third section of the legend reproduced in Figure A-1.

Changes in disparity over time are shown when:

a) Disparities data are available at both baseline and most recent time points;

b) Data are neither for the group(s) with the best rate for the specified characteristic, nor for the group(s) with the most favorable rate but for which the reliability criterion was not met, at either time point; and

c) The change is greater than or equal to 10 percentage points and statistically significant, or when the change is greater than or equal to 10 percentage points and estimates of variability are not available.

When standard errors are available for a data system, only statistically significant changes in disparities of 10 percentage points or more between the baseline and the most recent data points are indicated with arrows, see Figure A-1. Several steps are required to evaluate the statistical significance of a change in disparities over time.

Step 1. The disparity or percent difference (PD) from the best group rate at each time point is based on the ratio of the simple difference $\mathrm{SD}=R_{G}-R_{B}$ between the rate for the group of interest and the best group rate to the best group rate $R_{B}$ :

$$
\text { Disparity for group } \mathrm{G}=\frac{\mathrm{SD}}{R_{\mathrm{B}}} \times 100 \text {. }
$$

Step 2. The relative standard error (RSE) of the above ratio is computed based on the RSE of the numerator and the denominator. The RSE for the numerator SD is calculated as:

$$
\mathrm{RSE}_{\mathrm{SD}}=\frac{\sqrt{\mathrm{SE}_{\mathrm{G}}^{2}+\mathrm{SE}_{\mathrm{B}}^{2}}}{R_{\mathrm{G}}-R_{\mathrm{B}}},
$$

where $\mathrm{SE}_{\mathrm{G}}$ is the standard error of the rate for a group $\mathrm{G}$ of interest, $\mathrm{SE}_{\mathrm{B}}$ is the standard error for the best rate, $R_{G}$ is the rate for group $\mathrm{G}$, and $R_{B}$ is the best group rate.

Step 3. The RSE of the best group rate in the denominator of the above ratio is given by

$$
\mathrm{RSE}_{\mathrm{B}}=\frac{\mathrm{SE}_{\mathrm{B}}}{R_{\mathrm{B}}} .
$$

Step 4. An approximate relative standard error RSEPD for the disparity or percent difference (PD) is computed via the so-called "Delta Method"-a first-order Taylor series linearization of the variance of the ratio of two random variables [21] —using the numerator RSE (from step 2) and the denominator RSE (from step 3): 


$$
\mathrm{RSE}_{\mathrm{PD}}=\sqrt{\mathrm{RSE}_{\mathrm{SD}}^{2}+\mathrm{RSE}_{\mathrm{B}}^{2}}
$$

This first-order linearization assumes the simple difference $\mathrm{SD}=R_{G}-R_{B}$ between the rate $R_{G}$ for the group $\mathrm{G}$ of interest and the best group rate $R_{B}$ is independent of the best group rate.

Step 5. An approximate standard error $\mathrm{SE}_{\mathrm{PD}}$ for the percent difference (PD) is given by:

$$
\mathrm{SE}_{\mathrm{PD}}=\mathrm{RSE}_{\mathrm{PD}} \times \mathrm{PD} \text {. }
$$

Step 6. The statistical significance of a change in disparity or percent difference from the best group rate over time at the 0.05 level is assessed using the following Z-statistic:

$$
\mathrm{Z}=\frac{\mathrm{PD}_{1}-\mathrm{PD}_{0}}{\sqrt{\mathrm{SE}_{\mathrm{PD}, 1}^{2}+\mathrm{SE}_{\mathrm{PD}, 0}^{2}}},
$$

where $\mathrm{PD}_{1}$ is the percent difference at the most recent time point, $\mathrm{PD}_{0}$ is the percent difference at baseline, $\mathrm{SE}_{\mathrm{PD}, 1}$ is the standard error of the percent difference at the most recent time, and $S E_{P D, 0}$ is the standard error of the percent difference at baseline.

Note. Because of the various assumptions involved in deriving an approximate standard error $\mathrm{SE}_{\mathrm{PD}}$ for the percent difference in step 5 above, and because an alternative, more direct method for testing statistical significance is available for the simple difference $R_{G}-R_{B}$ between the two group rates, the standard error $\mathrm{SE}_{\mathrm{PD}}$ is not used for assessing the significance of disparities at each data point. As explained earlier, when the simple difference $R_{G}-R_{B}$ between the two group rates is statistically significant, the disparity for group $\mathrm{G}$ relative to the best group rate (i.e., the percent difference) is considered significant.

When measures of variability are not available, the variability of best group rates is not assessed, and statistical significance cannot be tested. Nonetheless, disparities and changes in disparities over time are displayed according to their magnitude. This is usually indicated in the footnotes of the Health Disparities Table by a $\uparrow$ footnote. See also the Estimates of Variability section below for more information.

When measures of variability are available only for the most recent data, the variability of best group rates is assessed only for the most recent data, and statistical significance is tested only for the most recent data. Changes in disparities of 10 percentage points or more over time are displayed according to their magnitude, since measures of variability are not available at baseline and therefore statistical significance of changes in disparity could not be tested. This is usually indicated in the footnotes of the Health Disparities Table by a $\neq$ footnote. See also the Estimates of Variability section below for more information. 


\section{Summary Measures}

Disparities are measured as percent differences between the best group rate and other group rates for a given population characteristic. When more than two groups are associated with that characteristic, such as race and ethnicity, income, and education, a summary index provides a way to determine whether, on average, disparities from the best group rate are increasing or decreasing. The formula for the summary index, also known as the index of disparity [22], is:

$$
\text { Summary index }=\frac{\sum_{\mathrm{G}=1}^{\mathrm{n}-1} \mathrm{PD}_{\mathrm{G}}}{\mathrm{n}-1} \text {. }
$$

where $\mathrm{PD}_{\mathrm{G}}$ is the nonnegative-possibly zero-disparity (i.e., percent difference) from the best group rate for each of the groups of interest $(G=1,2, \ldots n)$, and $n$ is the number of groups. Because the disparities are calculated with the best group rate as the reference point, the number of comparisons is equal to the number of groups minus one. These comparisons are made only when data are available for the same groups defined in the same way at the baseline and most recent data points.

Note. As explained previously, when the relative standard error for the most favorable group rate is greater than or equal to 10 percent, that group is flagged using a small letter "b" in the Health Disparities Table, and the next most favorable group rate with a relative standard error of less than 10 percent is identified as the reference group for that characteristic and flagged using a capital letter "B." As a result, the observed disparity or percent difference from the best group rate for a group that is flagged with a small letter " $b$ " becomes negative, since its observed rate is better than the best rate identified. Thus, all such groups with a "b" must be excluded from the calculation of the summary index, since the latter must remain nonnegative. However, in doing so, the summary index no longer accurately reflects the observed disparities in the population, since, by excluding the better rates, it necessarily underestimates the average disparity. For this reason, summary indices are not calculated for objectives where at least one group is identified with a small letter " $b$ " for a given characteristic. The corresponding cell in the Health Disparities Table is shaded in light gray to indicate that data are not available to accurately compute the summary index.

The statistical significance of a change in the summary index over time is assessed when standard errors for the rates on which the summary index is based are available. The magnitude and direction of changes are indicated by arrow symbols as described above. When standard errors are not available for the rates on which the summary index is based, changes are classified by size and direction without regard to statistical significance.

To obtain a standard error for the summary index, a type of resampling or "bootstrap" procedure is employed [23]. This procedure uses the rate and standard error for each group to reestimate each group rate 25,000 times assuming a random normal distribution. Based on these group rates, 25,000 estimates of the summary index of disparity are generated, and the distribution of these estimates is used to estimate the standard error of the summary index. 
The bootstrap procedure is used to estimate standard errors for the summary index at the most recent time and at baseline, in order to determine whether a change in the summary index over time is statistically significant. A $Z$ test for the change in the summary index can be computed as follows:

$$
\mathrm{Z}=\frac{\mathrm{ID}_{1}-\mathrm{ID}_{0}}{\sqrt{\mathrm{SE}_{\mathrm{ID}, 1}^{2}+\mathrm{SE}_{\mathrm{ID}, 0}^{2}}},
$$

where $\mathrm{ID}_{1}$ is the summary index at the most recent time point, $\mathrm{ID}_{0}$ is the summary index at baseline, $\mathrm{SE}_{\mathrm{ID}, 1}$ is the standard error of the summary index at the most recent time point, and $\mathrm{SE}_{\mathrm{PD}, 0}$ is the standard error of the summary index at baseline.

Because the value of the index could either increase or decrease, a two-tailed test is employed to assess statistical significance: a value of $|Z| \geq 1.96$ indicates that the change in the summary index is statistically significant at the 0.05 level.

\section{Estimates of Variability}

Estimates of variability (standard errors) are available for most of the population-based objectives in Healthy People 2010. When standard errors are available, they can be employed to assess the reliability of the best group rate as described above. This assessment is performed to ensure that the group chosen as the reference point is reasonably stable. Standard errors also are used to perform the tests of statistical significance described above. Generally speaking, these tests guard against the possibility that observed disparities or changes in disparities occur because of sampling error or other random sources of error.

When measures of variability are not available, the stability of best group rates is not assessed, and statistical significance of disparities and changes in disparities could not be tested. For such objectives, there is no quantifiable assurance that observed disparities and changes in disparities are not due to sampling error or other random sources of error. For such objectives, the reader is urged to exercise caution in interpreting disparities findings.

In the Health Disparities Table, objectives based on data for which estimates of variability are available and those for which estimates of variability are not available are designated by footnotes following the short description of each objective. These footnotes are as follows:

* Measures of variability were available. Thus, the variability of best group rates was assessed, and statistical significance was tested. Disparities of $10 \%$ or more are displayed when the differences from the best group rate are statistically significant at the 0.05 level. Changes in disparities over time are indicated by arrows when the changes are greater than or equal to 10 percentage points and are statistically significant at the 0.05 level. See Technical Appendix. 
$\dagger$ Measures of variability were not available. Thus, the variability of best group rates was not assessed, and statistical significance could not be tested. Nonetheless, disparities and changes in disparities over time are displayed according to their magnitude. See Technical Appendix.

₹ Measures of variability were available only for the most recent data. Thus, the variability of best group rates was assessed only for the most recent data, and statistical significance was tested only for the most recent data. Disparities of $10 \%$ or more are displayed when the differences from the best group rate are statistically significant at the 0.05 level. Changes in disparities over time are displayed according to their magnitude, since measures of variability were not available at baseline and therefore statistical significance of changes in disparity could not be tested. See Technical Appendix.

If a footnote applies to all objectives in a particular Health Disparities Table, then it is added to the notes and no footnote is inserted.

\section{Mapping}

When data are available at the sub-national level, selected objectives are mapped to display spatial variation in percents, rates, or counts. Sub-national data is presented either at the state or Health Service Area (HSA) level. HSAs are defined as "...one or more counties that are relatively self-contained with respect to the provision of routine hospital care" [24]. HSAs are contiguous but may span state boundaries. They frequently contain more than one county with an average of four and maximum of 20 counties. The current HSA classification system is based on the presence of at least one hospital in the HSA and patterns of travel between counties.

Maps are presented as simple chloropleths and use either a Jenks or modified Jenks classification [25]. A Jenks classification is a way to group ordered data in such a way that within group variance is minimized and between group variance is maximized. When geographic units (states or HSAs) have values that met the Healthy People 2010 target, the classification is modified by manually setting the best (lowest for objectives that seek to reduce events and highest for objectives that seek to increase events) cut-point to the Healthy People 2010 target. In some instances where the number of geographic units meeting the target is large a cut-point in the middle of the distribution is set to the target.

The Jenks classification is an iterative process whereby an arbitrary number of classes are created from an ordered set of data. For most maps presented here the default number of classes is five. The process proceeds by calculating the sum of the squared deviations between classes (SDBC), calculating the sum of squared deviations from the array mean (SDAM), subtract the SDBC from SDAM giving the squared deviation from class means (SDCM). Observations are iteratively moved from classes with larger SDBC to those with smaller SBDC until all SBDC are minimized. 
Mapping was done using ArcGIS ArcMap [26]. Maps are presented using a North American conic equidistant projection based on the 1983 North America geographic coordinate system. The states of Alaska and Hawaii retain these attributes but are not shown to scale or correct location, and were placed independently for greater ease of interpretation.

\section{DATA2010}

DATA2010 is an online, searchable database that contains baseline data, tracking data, and targets for all measurable objectives in Healthy People 2010 [4]. The database has been updated throughout the decade, generally quarterly, to provide the most accurate and upto-date data for tracking Healthy People 2010 objectives.

DATA2010 allows users to search the database for estimates by Focus Area, objective, data source, and keyword. In addition, users can access Healthy People 2010 Final Review data by downloading designated standard or statistical data spreadsheets in Excel format by Focus Area, accessible from http://wonder.cdc.gov/data2010/ftpselec.htm. Standard spreadsheets contain rounded estimates, whereas statistical spreadsheets contain rounded data as well as unrounded data and standard errors (both rounded to one decimal place), when available.

All of the data used to produce the Final Review Progress Charts and Disparity Tables are reflected in these static Final Review tables. Calculations on the Progress Charts and Disparities Tables are based on standard estimates and their associated unrounded standard errors, when available.

In addition, DATA2010 contains other technical information related to the Healthy People 2010 objectives, including Operational Definitions for each objective.

\section{General Data Issues}

Tracking Healthy People 2010 is a comprehensive guidebook on the statistics used for Healthy People 2010 [14]. It provides detailed information on how the data are derived and the major issues affecting the interpretation of the statistics. During the Healthy People 2010 Final Review, the General Data Issues section, Part A of Tracking Healthy People 2010, was updated as a standalone document titled Healthy People 2010: General Data Issues [16].

Healthy People 2010: General Data Issues discusses data-related topics that affect multiple objectives. Subjects covered include measuring years of healthy life; measuring health disparities; population estimates; the Healthy People 2010 population templates, including issues related to the revised Federal standards for classifying race and Hispanic origin; issues related to target setting and target adjustment; age adjustment, including implications of changes in the standard population for age adjustment; the ICD used for illness and death classification; state, local, and national data issues; and DATA2010. 


\section{Tracking Period}

In general, the tracking period for Healthy People 2010 was designed to cover a 10-year period. For most data systems, the final data year for Healthy People 2010 was selected to coincide with the baseline year used in Healthy People 2020 for those systems, even if more recent data were available when the Healthy People 2010 Final Review was being prepared. For example, the Healthy People 2010 final data point for most objectives based on data from the National Health Interview Survey (NHIS) was 2008, matching the baseline year for Healthy People 2020, although 2009 data were available. For objectives that were tracked from data sources that are not used in Healthy People 2020, the most recent data available were used as the baseline.

\section{References}

1. U.S. Department of Health and Human Services, Public Health Service. Healthy People 2000 Midcourse Review and 1995 Revisions. Washington, DC: U.S. Government Printing Office. 1995.

2. National Center for Health Statistics. Healthy People 2000 Final Review. Hyattsville, Maryland: 2001.

3. U.S. Department of Health and Human Services. Healthy People 2010. $2^{\text {nd }}$ ed. With Understanding and Improving Health and Objectives for Improving Health. 2 vols. Washington, D.C.: U.S. Government Printing Office. November 2000.

4. DATA2010. Available from http://wonder.cdc.gov/data2010.

5. Gilbert RO. Statistical Methods for Environmental Pollution Monitoring. New York, NY: Van Nostrand Reinhold. 1987.

6. Sullivan DF. A single index of mortality and morbidity. HSMHS Health Reports 86, 347-54. 1971.

7. Sullivan DF. Disability components for an index of health. National Center for Health Statistics. Vital Health Stat 2(42). 1971.

8. Anderson RN. Method for constructing complete annual U.S. life tables. National Center for Health Statistics. Vital Health Stat 2(129). 1999.

9. Molla MT, Madans JH, Wagener DK, Crimmins EM. Summary measures of population health: Report of findings on methodologic and data issues. National Center for Health Statistics. Hyattsville, Maryland. 2003.

10. Crimmins EM. What can we expect from summary indicators of population health? In Murray CJL, et al., eds. Summary Measures of Population Health. Geneva, Switzerland: World Health Organization. 2002.

11. Idler E, Benyamini Y. Self-rated health and mortality: A review of 28 studies. J Health Soc Behav 38(1), 21-37. 1997.

12. Erickson P, Wilson R, Shannon, I. Years of healthy life. Healthy People Statistical Notes, no. 7. Hyattsville, Maryland: National Center for Health Statistics. 1995.

13. Keppel KG, Pearcy JN, Klein RJ. Measuring progress in Healthy People 2010. Statistical Notes, no. 25. Hyattsville, Maryland: National Center for Health Statistics. September 2004. 
14. U.S. Department of Health and Human Services, Public Health Service. Tracking Healthy People 2010. Washington, DC: Government Printing Office. November 2000.

15. U.S. Office of Management and Budget. Revisions to the standards for the classification of Federal data on race and ethnicity. Federal Register 62/FR, 58781-90. 1997.

16. Healthy People 2010: General Data Issues. Available from: http://www.cdc.gov/nchs/healthy people.htm.

17. Klein RJ, Proctor SE, Boudreault MA, Turczyn KM. Healthy People 2010 criteria for data suppression. Statistical Notes, no. 24. Hyattsville, Maryland: National Center for Health Statistics. June 2002.

18. Keppel KG, Pamuk E, Lynch J, et al.Methodological issues in measuring health disparities. National Center for Health Statistics. Vital Health Stat 2(141). 2005.

19. Keppel KG, Pearcy JN. Measuring relative disparities in terms of adverse outcomes. J Public Health Manag Pract 11(6). 2005.

20. Pfeiffer CM, Johnson CL, Jain RB, Yetley EA, Picciano MF, Rader JI, et al. Trends in blood folate and vitamin B12 concentrations in the United Sates, 1988-2004. Am J Clin Nutr 86, 718-27. 2007.

21. Korn EL, Graubard BI. Analysis of Health Surveys. New York, NY: John Wiley \& Sons. 1999.

22. Pearcy JN, Keppel KG. A summary measure of health disparity. Public Health Rep 117, $273-$ 80. May-June 2002.

23. Efron B. The Jackknife, the Bootstrap, and Other Resampling Plans. Philadelphia, PA: SIAM Publishing Company. 1982.

24. Makuc DM, Haglund B, Ingram DD, et.al. Health Service Areas for the United States. National Center for Health Statistics. Vital Health Stat (2)112. 1991.

25. Coulson MR. In the matter of class intervals for chloropleth maps: with particular reference to the work of George F Jenks. Cartographica: The International Journal for Geographic Information and Geovisualization. 24(2), 16-39. 2006.

26. ArcGIS Desktop: ArcMap 9.3 (build 1770). Redlands, CA: Environmental Systems Research Institute. 


\section{Appendix B: Published Issues of Healthy People Statistical Notes}

This appendix provides a listing of published Healthy People statistical notes.

\begin{tabular}{|c|c|c|}
\hline Number & Title & Date \\
\hline 1 & Health Status Indicators for the Year 2000 & Fall 1991 \\
\hline 2 & Infant Mortality & Winter 1991 \\
\hline 3 & Health Status Indicators: Definitions and National Data & Spring 1992 \\
\hline 4 & Issues Related to Monitoring the Year 2000 Objectives & Summer 1993 \\
\hline 5 & Revisions to Healthy People 2000 Baselines & July 1993 \\
\hline 6 & Direct Standardization (Age-Adjusted Death Rates) & March 1995 \\
\hline 7 & Years of Healthy Life & April 1995 \\
\hline 8 & Evaluating Public Health Data Systems: A Practical Approach & June 1995 \\
\hline 9 & Monitoring Air Quality in Healthy People 2000 & September 1995 \\
\hline 10 & $\begin{array}{l}\text { Health Status Indicators: Differentials by Race and Hispanic } \\
\text { Origin }\end{array}$ & September 1995 \\
\hline 11 & $\begin{array}{l}\text { Operational Definitions for Year } 2000 \text { Objectives: Priority } \\
\text { Area 20, Immunization and Infectious Diseases }\end{array}$ & February 1997 \\
\hline 12 & $\begin{array}{l}\text { Operational Definitions for Year } 2000 \text { Objectives: Priority } \\
\text { Area 13, Oral Health }\end{array}$ & May 1997 \\
\hline 13 & Healthy People 2000 Midcourse Revisions: A Compendium & August 1997 \\
\hline 14 & $\begin{array}{l}\text { Operational Definitions for Year } 2000 \text { Objectives: Priority } \\
\text { Area 14, Maternal and Infant Health }\end{array}$ & December 1997 \\
\hline 15 & $\begin{array}{l}\text { Priority Data Needs: Sources of National, State, and Local- } \\
\text { level Data and Data Collection Systems }\end{array}$ & December 1997 \\
\hline 16 & $\begin{array}{l}\text { Operational Definitions for Year } 2000 \text { Objectives: Priority } \\
\text { Area 6, Mental Health and Mental Disorders }\end{array}$ & February 1998 \\
\hline 17 & $\begin{array}{l}\text { Operational Definitions for Year } 2000 \text { Objectives: Priority } \\
\text { Area 21, Clinical Preventive Services }\end{array}$ & December 1998 \\
\hline 18 & $\begin{array}{l}\text { Operational Definitions for Year } 2000 \text { Objectives: Priority } \\
\text { Area 1, Physical Activity and Fitness }\end{array}$ & December 1998 \\
\hline 19 & $\begin{array}{l}\text { Healthy People 2000: An Assessment Based on the Health } \\
\text { Status Indicators for the United States and Each State }\end{array}$ & November 2000 \\
\hline 20 & Age Adjustment Using the 2000 Projected U.S. Population & January 2001 \\
\hline 21 & $\begin{array}{l}\text { Summary Measures of Population Health: Methods for } \\
\text { Calculating Healthy Life Expectancy }\end{array}$ & August 2001 \\
\hline 22 & $\begin{array}{l}\text { Summary Measures of Population Health: Addressing the } \\
\text { First Goal of Healthy People 2010, Improving Health } \\
\text { Expectancy }\end{array}$ & September 2001 \\
\hline 23 & $\begin{array}{l}\text { Trends in Racial and Ethnic-Specific Rates for the Health } \\
\text { Status Indicators: United States, 1990-98 }\end{array}$ & January 2002 \\
\hline 24 & Healthy People 2010 Criteria for Data Suppression & July 2002 \\
\hline 25 & Measuring Progress in Healthy People 2010 & September 2004 \\
\hline 26 & $\begin{array}{l}\text { Comparing Racial and Ethnic Populations Based on Healthy } \\
\text { People } 2010 \text { Objectives }\end{array}$ & August 2008 \\
\hline
\end{tabular}




\section{Appendix C: Healthy People 2010 Workgroup Coordinators and Contributing Members}

\section{Access to Quality Health Services \\ Workgroup Coordinators}

Anne Dievler, PhD

Senior Public Health Analyst

Office of Research and Evaluation

Health Resources and Services Administration

Email: adievler@hrsa.gov

Claire A. Weschler, MSEd, CHES.

Agency for Healthcare Research and Quality

Center for Primary Care, Prevention, and

Clinical Partnerships

Email: claire.kendrick@ahrq.hhs.gov

\section{Contributing Members}

Samara Lorenz, MPA

(formerly Special Assistant

Office of Planning, Analysis and Evaluation

Health Resources and Services

Administration)

Lenee Simon, MPH

(formely Public Health Advisor

Office of Disease Prevention and Health

Promotion

Office of the Assistant Secretary for Health

U.S. Department of Health and Human

Services)

\section{Arthritis, Osteoporosis, and Chronic Back Conditions}

\section{Workgroup Coordinators}

Charles G. Helmick, MD

Medical Epidemiologist

Division of Adult and Community Health

National Center for Chronic Disease

Prevention and Health Promotion

Centers for Disease Control and Prevention

Email: cgh1@cdc.gov
Phil Tonkins

LCDR, USPHS

Health Scientist Administrator

Division of Skin and Rheumatic Diseases

National Institute of Arthritis and

Musculoskeletal and Skin Diseases (NIAMS)

National Institutes of Health (NIH)

Email: tonkinsw2@mail.nih.gov

\section{Contributing Members}

Joan A. McGowan, PhD

Director, Division of Musculoskeletal Diseases

NIAMS, NIH

One Democracy Plaza, MSC 4872

Email: joan mcgowan@nih.gov

James S. Panagis, MD MPH

CAPT, USPHS

Director, Orthopaedics Program

Division of Musculoskeletal Diseases

National Institute of Arthritis and

Musculoskeletal and Skin Diseases, NIH, DHHS

Email: panagisj@mail.nih.gov

Paul A. Scherr, PhD, DSc

Former workgroup coordinator

Supervisory Epidemiologist, retired

Division of Adult and Community Health

National Center for Chronic Disease

Prevention and Health Promotion

Centers for Disease Control and Prevention

Susana A. Serrate-Sztein, MD

Former workgroup coordinator

Director, Division of Skin and Rheumatic

Diseases

National Institute of Arthritis and

Musculoskeletal and Skin Diseases (NIAMS)

National Institutes of Health $(\mathrm{NIH})$

Email: szteins@mail.nih.gov

\section{Cancer}

Workgroup Coordinators

Kathy Cronin, PhD

Mathematical Statistician

Statistical Research and Applications Branch 
Division of Cancer Control and Population

Sciences

National Cancer Institute

National Institutes of Health

Email: cronink@mail.nih.gov

Lisa C. Richardson, MD, MPH

Associate Director for Science

Division of Cancer Prevention and Control

National Center for Chronic Disease

Prevention and Health Promotion

Centers for Disease Control and Prevention

Email: LRichardson@cdc.gov

Martina V. Taylor, MT (ASCP)

Senior Advisor for Implementation Science

Office of the Director

Division of Cancer Control and Population

Sciences

National Cancer Institute

National Institutes of Health

E-mail: martina.taylor@nih.gov

\section{Contributing Members}

Ralph J. Coates, PhD

Acting Associate Director for Science

Public Health Surveillance Program Office, OSELS

Centers for Disease Control \& Prevention

Email: rcoates@cdc.gov

Laurie Cynkin, MHS

Program Analyst

Office of the Director

Division of Cancer Control and Population

Sciences

National Cancer Institute

National Institutes of Health

E-mail: cynkinl@mail.nih.gov

Jon Kerner, PhD

(formerly Deputy Director for Research

Dissemination and Diffusion

Division of Cancer Control and Population

Sciences

National Cancer Institute

National Institutes of Health)

Cheryl C. Thomas, MPH

Deputy Associate Director for Science

Division of Cancer Prevention and Control

National Center for Chronic Disease

Prevention and Health Promotion

Centers for Disease Control and Prevention
Email: ccthomas@cdc.gov

\section{Chronic Kidney Disease}

Workgroup Coordinators

Lawrence Agodoa, MD, FACP

Director

Chronic Kidney Disease and End Stage Renal

Disease Programs

Office of Minority Health Research

Coordination

National Institute of Diabetes, Digestive and

Kidney Diseases

National Institutes of Health

Email: agodaoL@mail.nih.gov

Paul W. Eggers, PhD

Program Director for

Kidney and Urology Epidemiology

National Institute of Diabetes and

Digestive and Kidney Diseases

National Institutes of Health

Email: eggersp@extra.niddk.nih.gov

Desmond Williams, MD PhD

Assoc. Branch Chief

Team Lead for the CDC CKD Initiative

Epidemiology and Statistic Branch (ESB)

Division of Diabetes Translation

NCCDPHP

Centers for Disease Control \& Prevention

Email: dewilliams@cdc.gov

\section{Contributing Member}

Beth Forrest

USRDS Coordinating Center

Email: bforrest@usrds.org

\section{Diabetes}

\section{Workgroup Coordinators}

Lawrence Barker, PhD

Associate Director of Science

Division of Diabetes Translation

Centers for Disease Control and Prevention

Email: LBarker1@cdc.gov

Sanford Garfield, PhD

Senior Advisor

Biometry and Behavioral Research Program

Division of Diabetes, Endocrinology and

Metabolic Diseases

National Institute of Diabetes and 
Digestive and Kidney Diseases

National Institutes of Health

Email: garfields@ep.niddk.nih.gov

Edward Gregg, PhD

Chief, Epidemiology and Statistics Branch

Division of Diabetes Translation

Centers for Disease Control and Prevention

Email: EGregg@cdc.gov

\section{Disability and Secondary \\ Conditions}

Workgroup Coordinators

Donald Betts, MPA

Deputy Director

Division of Human Development and

Disability

National Center on Birth Defects and

Developmental Disabilities

Centers for Disease Control and Prevention

Email: dbetts@cdc.gov

David Keer, MA

Program Manager

National Institute on Disability and

Rehabilitation Research

U.S. Department of Education

Email: david.keer@ed.gov

Monica M. Manns

Public Health Analyst

Office of Policy, Planning and Evaluation

National Center on Birth Defects and

Developmental Disabilities

Centers for Disease Control and Prevention

E-mail: $\underline{\text { mmanns@cdc.gov }}$

Louis Quatrano, $\mathrm{PhD}$

Program Director, BSRE, NCMRR

Eunice Kennedy Shriver National Institute of

Child Health and Human Development

National Institutes of Health

Email: quatranol@mail.nih.gov

7. Educational and Community-Based Programs

Workgroup Coordinators

Chandak Ghosh, MD, MPH

Medical Consultant for Federal Policy

Office of Regional Operations

Health Resources and Services Administration
E-mail: cghosh@hrsa.gov

Audrey Williams

Public Health Advisor

Division of Adult and Community Health

Centers for Disease Control and Prevention

Email: akwilliams@cdc.gov

\section{Environmental Health}

\section{Workgroup Coordinators}

Whitney Neal, MSPH

Health Scientist

NCEH/ATSDR Office of Science

Centers for Disease Control and Prevention /

Agency for Toxic Substances and Disease

Registry

Email: WNeal@cdc.gov

Radha Pennotti, MPH

Public Health Analyst

NCEH/ATSDR Office of Policy Planning and

Evaluation

Centers for Disease Control and Prevention /

Agency for Toxic Substances and Disease

Registry

Email: $\underline{\text { RPennotti@cdc.gov }}$

\section{Family Planning}

\section{Workgroup Coordinators}

Vanessa A. White, MPH

Public Health Advisor

Office of Family Planning

Office of Population Affairs

U.S. Department of Health and Human

Services

Email: vanessa.white@hhs.gov

\section{Contributing Members}

Evelyn Kappeler (former coordinator)

Acting Director

Office of Adolescent Health

Office of the Assistant Secretary for Health

(ASH)

U.S. Department of Health and Human

Services

evelyn.kappeler@hhs.gov

Amy Margolis, MPH, CHES (former

coordinator)

Public Health Analyst

Office of Adolescent Health 
Office of the Assistant Secretary for Health (ASH) U.S. Department of Health and Human Services

amy.margolis@hhs.gov

Susan Moskosky, MS, RNC

Director

Office of Family Planning

Office of Population Affairs

Office of the Assistant Secretary for Health

U.S. Department of Health and Human

Services

susan.moskosky@hhs.gov

Gladys Martinez

Statistician

National Center for Health Statistics

Centers for Disease Control and Prevention

gmm7@CDC.GOV

William Mosher

Statistician

National Center for Health Statistics

Centers for Disease Control and Prevention wdm1@CDC.GOV

Stephanie Ventura

Chief, Reproductive Statistics Branch

National Center for Health Statistics

Centers for Disease Control and Prevention

siv1@CDC.GOV

\section{Food Safety}

\section{Workgroup Coordinators}

Elisa L. Elliot, PhD

Microbiologist

FDA, Center for Food Safety and Applied

Nutrition

OFDCER, Emergency Coordination and

Response Team HFS-15

Email: elisa.elliot@fda.hhs.gov

Delila Parham, DVM

Chief, Zoonoses and Food Hazards

Surveillance Branch

Applied Epidemiology Division

Office of Public Health Science

Food Safety and Inspection Service

U.S. Department of Agriculture
Aerospace Center

Email: delila.parham@fsis.usda.gov

\section{Health Communication}

\section{Workgroup Coordinators}

Cynthia Baur, PhD

Senior Advisor, Health Literacy

Office of the Associate Director for

Communication

Centers for Disease Control and Prevention

Email: cbaur@cdc.gov

Linda Harris, PhD

Public Health Advisor

Team Lead Health Communication and

eHealth

Office of Disease Prevention and Health

Promotion

Email: Linda.Harris@hhs.gov

\section{Heart Disease and Stroke}

\section{Workgroup Coordinators}

Yuling Hong, MD, PhD

Associate Director for Science

Division for Heart Disease and Stroke

Prevention

National Center for Chronic Disease

Prevention and Health Promotion

Centers for Disease Control and Prevention

Email: yhong@cdc.gov

Fleetwood Loustalot, $\mathrm{PhD}$

Commission Corps Officer

Division for Heart Disease and Stroke

Prevention

National Center for Chronic Disease

Prevention and Health Promotion

Centers for Disease Control and Prevention

Email: hgn1@cdc.gov

Joylene John-Sowah, MD, MPH

Medical Officer

Division for the Application of Research

Discoveries

National Heart and Lung and Blood Institute

National Institutes of Health

E-mail: johnsowahj@mail.nih.gov

Contributing Members

James I. Cleeman, MD 
Senior Medical Officer

Center for Quality Improvement and Patient Safety

Agency for Healthcare Research and Quality

E-mail: james.cleeman@ahrq.gov

Kurt Greenlund, PhD

Senior Epidemiologist

Division for Heart Disease and

Stroke Prevention

National Center for Chronic Disease

and Health Promotion

Centers for Disease Control and Prevention

E-mail: keg9@cdc.gov

Robinson Fulwood, PhD, MSPH.

Former workgroup coordinator,

Acting Deputy Director, retired

Division for the Application of Research

Discoveries

National Heart and Lung and Blood Institute

National Institutes of Health

Nora Keenan, PhD

Former workgroup coordinator

Epidemiologist, retired

Division for Heart Disease and Stroke

Prevention

National Center for Chronic Disease

Prevention and Health Promotion

Centers for Disease Control and Prevention

Darwin Labarthe, MD, MPH, PhD

Former workgroup coordinator

Director, retired

Division for Heart Disease and Stroke

Prevention

National Center for Chronic Disease

Prevention and Health Promotion

Centers for Disease Control and Prevention

Paul A. Scott, PhD

Director

Office of Science Policy and Planning

National Institute of Neurological Disorders

and Stroke

National Institutes of Health

Email: scottp@ninds.nih.gov

\section{HIV}

\section{Workgroup Coordinators}

J. Stan Lehman, MPH

Epidemiologist

Office of Policy and Planning

Division of HIV/AIDS Prevention

Centers for Disease Control and Prevention

Email: slehman@cdc.gov

Pamela Wilson, MSW, LCSW-C

Division of Science and Policy

Policy Development Branch

HIV/AIDS Bureau

Health Resources and Services Administration

Email:pwilson@hrsa.gov

\section{Immunization and Infectious}

Diseases

Workgroup Coordinators

Rebecca Gold, JD

Policy Analyst

National Center for Immunization and

Respiratory Diseases

Centers for Disease Control and Prevention

CORP Bldg 12 Room 5401

MS E05

E-mail: rgold@cdc.gov

C. Erik Williams

Office of Program Planning and Policy

Coordination

National Center for HIV/AIDS, Viral Hepatitis, STD, and TB Prevention

Coordinating Center for Infectious Diseases

Centers for Disease Control and Prevention

Email: gqu2@cdc.gov

\section{Contributing Members}

Bethany Anderson, MPH

Health Policy Analyst

Financial Management Office

Centers for Disease Control and Prevention

Email: bja3@cdc.gov

Lawrence Barker

Science Officer

National Center for Chronic Disease

Prevention and Health Promotion

Centers for Disease Control and Prevention

Email: 1sb8@cdc.gov 
Achal Bhatt, PhD

Health Communications Specialist

National Center for Immunization and

Respiratory Diseases

Centers for Disease Control and Prevention

Email: zvg8@cdc.gov

Shelly Bratton

Public Health Analyst

Center for Global Health

Centers for Disease Control and Prevention

Email: bwp8@cdc.gov

Jennifer Brooks

Public Health Analyst

Office of Public Health Preparedness and

Response

Centers for Disease Control and Prevention

Email: jlc9@cdc.gov

Sandra Cook

Management and Program Analyst

Office of Public Health Preparedness and

Response

Centers for Disease Control and Prevention

Email: sbc7@cdc.gov

Jonathan Edwards

Mathematician Statistician

National Center for Emerging Zoonotic and

Infectious Diseases

Centers for Disease Control and Prevention

Email: jde3@cdc.gov

Paulette Ford-Knights

Public Health Analyst

National Center for Emerging Zoonotic and

Infectious Diseases

Centers for Disease Control and Prevention

Email: pbf7@cdc.gov

Teresa Horan

Epidemiologist

National Center for Emerging Zoonotic and

Infectious Diseases

Centers for Disease Control and Prevention

Email: tch1@cdc.gov

Laurie Johnson

Deputy Director

National Center for Chronic Disease

Prevention and Health Promotion

Centers for Disease Control and Prevention

Email: laj1@cdc.gov
Peng-Jun Lu

Senior Service Fellow

National Center for Immunization and

Respiratory Diseases

Centers for Disease Control and Prevention

Email: $\underline{\operatorname{lhp} 8 @ c d c . g o v}$

Karen Mason, MPA

Public Health Analyst

National Center for Immunization and

Respiratory Diseases

Centers for Disease Control and Prevention

Email: bbx3@cdc.gov

Nancy Meissonier

Medical Officer

National Center for Immunization and

Respiratory Diseases

Centers for Disease Control and Prevention

Email: nar5@cdc.gov

Thomas Navin

Chief, Surveillance and Epidemiology Branch

National Center for Emerging Zoonotic and

Infectious Diseases

Centers for Disease Control and Prevention

Email: trn1@cdc.gov

Amy Pulver

Public Health Analyst

Office of the Director

Centers for Disease Control and Prevention

CDC/Washington

Email: cex3@cdc.gov

Daniel Riedford

Associate Director for Policy Planning and

Exter

National Center for Hepatitis, HIV and STD

Prevention

Centers for Disease Control and Prevention

Email: dgr0@cdc.gov

Valerie Robison

Dental Officer

National Center for Chronic Disease

Prevention and Health Promotion

Centers for Disease Control and Prevention

Email: vcr6@cdc.gov

Sandra Roush, MT, MPH

Epidemiologist

National Center for Immunization and

Respiratory Diseases

Centers for Disease Control and Prevention 
Email: swr1@cdc.gov

James Singleton

Branch Chief and Epidemiologist

National Center for Immunization and

Respiratory Diseases

Centers for Disease Control and Prevention xzs8@cdc.gov

Nicole Smith

Associate Director for Policy

Center for Global Health

Centers for Disease Control and Prevention

nba8@cdc.gov

Jeremy Sobel

Medical Officer

Center for Global Health

Centers for Disease Control and Prevention

Email: qzs3@cdc.gov

Carole Stanwyck

Epidemiologist

National Center for Immunization and

Respiratory Diseases

Centers for Disease Control and Prevention

Email: cea9@cdc.gov

Shannon Stokley

Lead Epidemiologist

National Center for Immunization and

Respiratory Diseases

Centers for Disease Control and Prevention

Email: zma2@cdc.gov

Susan Van Aacken, MSPP

Public Health Analyst

National Center for Chronic Disease and

Public Health Promotion

Centers for Disease Control and Prevention

zqi7@cdc.gov

Annemarie Wasley

Epidemiology

Center for Global Health

Centers for Disease Control and Prevention

Email: acw5@cdc.gov

Cindy Weinbaum

Medical Officer

National Center for Emerging Zoonotic and

Infectious Diseases

Centers for Disease Control and Prevention

Email: chw4@cdc.gov
Elizabeth Zell

Mathematical Statistician

National Center for Immunization and

Respiratory Diseases

Centers for Disease Control and Prevention

Email: ezr1@cdc.gov

\section{Injury and Violence Prevention}

\section{Workgroup Coordinator}

Arlene Greenspan, DrPH, MPH

Associate Director for Science

National Center for Injury Prevention and

Control

Centers for Disease Control and Prevention

Email: aig0@cdc.gov

\section{Contributing Members}

J. Lee Annest, PhD

Director

Office of Statistics and Programming

National Center for Injury Prevention and

Control

Centers for Disease Control and Prevention

Email: lannest@cdc.gov

Mick Ballesteros, $\mathrm{PhD}$

Deputy Associate Director for Science Division of Unintentional Injury Prevention National Center for Injury Prevention and Control

Centers for Disease Control and Prevention

Email: mballesteros@cdc.gov

Tad Haileyesus, MS

Mathematical Statistician

Office of Statistics and Programming

National Center for Injury Prevention and

Control

Centers for Disease Control and Prevention

Email: boq2@cdc.gov

Robin M. Ikeda, MD, MPH (Former workgroup coordinator)

Director

Office of Noncommunicable Diseases, Injury and Environmental Health

National Center for Injury Prevention and

Control

Centers for Disease Control and Prevention

Email: Rmi0@cdc.gov

Renee L. Johnson, RPT, MSPH

Epidemiologist 
Division of Injury Response

Centers for Disease Control and Prevention National Center for Injury Prevention and Control

Email: rjohnson1@cdc.gov

Caryll Rinehart

Public Health Analyst

Office of Policy, Planning, and Evaluation

National Center for Injury Prevention and

Control

Centers for Disease Control and Prevention

Email: cdr6@cdc.gov

George W. Ryan, PhD

Mathematical Statistician

Office of Statistics and Programming

National Center for Injury Prevention and

Control

Centers for Disease Control and Prevention

Email: gyr0@cdc.gov

Thomas R. Simon, PhD

Deputy Associate Director for Science

Division of Violence Prevention

National Center for Injury Prevention and

Control

Centers for Disease Control and Prevention

Email: tsimon@cdc.gov

David A. Sleet, PhD

Associate Director for Science

Division of Unintentional Injury Prevention

National Center for Injury Prevention and

Control

Centers for Disease Control and Prevention

Email: dds6@cdc.gov

Margarette Warner, $\mathrm{PhD}$

Health Statistician

Office of Analysis and Epidemiology

National Center for Health Statistics

Centers for Disease Control and Prevention

Email: MWarner@cdc.gov

Li-Hiu Chen, MS, PhD

Senior Service Fellow

Office of Analysis and Epidemiology

National Center for Health Statistics

Centers for Disease Control and Prevention

Email: LChen3@cdc.gov

\section{Maternal, Infant, and Child Health}

\section{Workgroup Coordinators}

Reem M. Ghandour, DrPH, MPA

Public Health Analyst, Office of Epidemiology, Policy and Evaluation Maternal and Child Health Bureau

Health Resources and Services Administration Email rghandour@hrsa.gov

Monica M. Manns

Public Health Analyst

Office of Policy, Planning and Evaluation

National Center on Birth Defects and

Developmental Disabilities

Centers for Disease Control and Prevention

E-mail: mmanns@cdc.gov

Mirna Perez, MA

Office of the Director, Division of

Reproductive Health

National Center for Chronic Disease

Prevention and Health Promotion

Centers for Disease Control and Prevention

Email: mperezrodriquez@cdc.gov

\section{Contributing Members}

William Byram Clayton

Public Health Analyst

Centers for Disease Control and Prevention

National Center for Chronic Disease

Prevention and Health Promotion

Office of the Director, Division of

Reproductive Health

Email: Bjc5@cdc.gov

Heather C. Hamner, PhD, MS, MPH

Health Scientist

Centers for Disease Control and Prevention

National Center on Birth Defects and

Developmental Disabilities

Division of Birth Defects and Developmental

Disabilities

Email: $\underline{\text { hfc2@cdc.gov }}$

Harriet Jett, JD; MPA

Division of Reproductive Health;

Associate Director for Partnership, Program

Development, Policy, and Evaluation

National Center for Chronic Disease

Prevention and Health Promotion

Centers for Disease Control and Prevention

Email: hjett@cdc.gov 
James Kucik, MPH

Health Scientist

Centers for Disease Control and Prevention

National Center on Birth Defects and

Developmental Disabilities

Division of Birth Defects and Developmental

Disabilities

Email: jkucik@cdc.gov

Cara Mai, MPH

Public Health Analyst

Centers for Disease Control and Prevention

National Center on Birth Defects and

Developmental Disabilities

Division of Birth Defects and Developmental

Disabilities

Email: mai@cdc.gov

Kim Van Naarden Braun, Ph.D

Epidemiologist

Centers for Disease Control and Prevention

National Center on Birth Defects and

Developmental Disabilities

Division of Birth Defects and Developmental

Disabilities

Email: Kvannaardenbraun@cdc.gov

Joe Mulinare, MD, MSPH

Medical Epidemiologist

Centers for Disease Control and Prevention

National Center on Birth Defects and

Developmental Disabilities

Division of Birth Defects and Developmental

Disabilities

Email: jmulinare@cdc.gov

Sarah Tinker, PhD, MPH

Epidemiologist

Centers for Disease Control and Prevention

National Center on Birth Defects and

Developmental Disabilities

Division of Birth Defects and Developmental

Disabilities

Email: stinker1@cdc.gov

\section{Medical Product Safety}

\section{Workgroup Coordinators}

Mary Ghods, RPh

Pharmacist

Office of the Center Director - Safe Use

Initiative

Center for Drug Evaluation and Research,

Food and Drug Administration
Email: Mary.ghods@fda.hhs.gov

Dale Slavin, PhD

Associate Director of

Programs/Program/Policy Analyst

Office of the Center Director - Safe Use

Initiative

Center for Drug Evaluation and Research,

Food and Drug Administration

Email: dale.slavin@fda.hhs.gov

\section{Mental Health and Mental \\ Disorders}

Workgroup Coordinators

Kevin J. Malone

Public Health Analyst

Office of Policy Planning and Innovation

Substance Abuse and Mental Health Services

Administration

Department of Health and Human Services

Email: kevin.malone@samhsa.hhs.gov

\section{Nutrition and Overweight}

\section{Workgroup Coordinators}

Nancy T. Crane, MS, MPH, RD

Expert Regulatory Review Scientist

Nutrition Programs and Labeling Staff (HFS-

830)

Office of Nutrition, Labeling and Dietary

Supplements

Food and Drug Administration

Email: nancy.crane@fda.hhs.gov

Deborah A. Galuska, MPH, PhD

Associate Director for Science

Division of Nutrition, Physical Activity, and

Obesity

Centers for Disease Control

Email: dgaluska@cdc.gov

Van S. Hubbard, MD, PhD

Rear Admiral, U.S. Public Health Service (ret.)

Director

NIH Division of Nutrition Research

Coordination

National Institutes of Health

Email: hubbardv@mail.nih.gov

Pamela E. Starke-Reed, PhD

Deputy Director

Division of Nutrition Research Coordination

National Institutes of Health 
Email: starkep@mail.nih.gov

\section{Contributing Members}

Mary E. Cogswell RN, DrPH

Epidemiologist

Division of Heart Disease and Stroke

Prevention

National Center for Chronic Disease

Prevention and Health Promotion

Centers for Disease Control and Prevention

Email: MCogswell@cdc.gov

Karen Dalenius, RD, MPH

Team Lead

Division of Nutrition, Physical Activity, and

Obesity

National Center for Chronic Disease

Prevention and Health Promotion

Centers for Disease Control and Prevention

Email: KDalenius@cdc.gov

Vincent DeJesus, MS

Nutritionist

Nutrition Programs and Labeling Staff

Office of Nutrition, Labeling and Dietary

Supplements

Food and Drug Administration

Email: vincent.dejesus@fda.hhs.gov

Kevin W. Dodd PhD

Mathematical Statistician

Division of Cancer Prevention

National Cancer Institute

National Institutes of Health

E-maildoddk@mail.nih.gov

Joseph D. Goldman MA

Mathematical Statistician

Food Surveys Research Group

Agricultural Research Service

U.S. Department of Agriculture

E-mail joe.goldman@ars.usda.gov

Laurence Grummer-Strawn, PhD

Branch Chief

Division of Nutrition, Physical Activity, and

Obesity

National Center for Chronic Disease

Prevention and Health Promotion

Centers for Disease Control and Prevention

Email: LGrummer-Strawn@cdc.gov

Anne C. Looker PhD

Distinguished Consultant
Office of Surveillance, Epidemiology and Laboratory Services

National Center for Health Statistics

Centers for Disease Control and Prevention

Email: ALooker@cdc.gov

Zuguo Mei, MD, MPH

Epidemiologist

Division of Nutrition, Physical Activity, and

Obesity

National Center for Chronic Disease

Prevention and Health Promotion

Centers for Disease Control and Prevention

Email: ZMei@cdc.gov

Crystal McDade-Ngutter, PhD

Health Program Specialist

Division of Nutrition Research Coordination

National Institutes of Health

e-mail: mcdadengutterc@mail.nih.gov

Mark Nord, PhD

Sociologist

Food Assistance Branch, Food Economics

Division

Economic Research Service

U.S. Department of Agriculture

E-mail marknord@ers.usda.gov

Christine Pfeiffer, PhD

Supervisory Research Chemist

Division of Laboratory Sciences

National Center for Environmental Health

Centers for Disease Control and Prevention

Email: CPfeiffer@cdc.gov

Barbara O. Schneeman, PhD

Director

Office of Nutrition, Labeling and Dietary

Supplements

Food and Drug Administration

Email: barbara.schneeman@fda.hhs.gov

Bettylou Sherry, PhD

Team Lead

Division of Nutrition, Physical Activity, and

Obesity

National Center for Chronic Disease

Prevention and Health Promotion

Centers for Disease Control and Prevention

Email: BSherry@cdc.gov 


\section{Occupational Safety and Health}

\section{Workgroup Coordinators}

Jessica Bilics, MPH

Public Health Analyst

Office of the Director

National Institute for Occupational Safety and

Health

Centers for Disease Control and Prevention

Email: \Bilics@cdc.gov

Lore Jackson Lee, MPH

Public Health Analyst

Office of the Director

National Institute for Occupational Safety and

Health

Centers for Disease Control and Prevention

Email: LJAcksonLee@cdc.gov

\section{Oral Health}

\section{Workgroup Coordinators}

Patrick Blahut, DDS, MPH, Diplomate ABDPH Deputy Director, Division of Oral Health, Indian Health Service

801 Thompson Ave., Rockville MD 20852

301-443-4323 (office)

301-256-7448 (cell)

patrick.blahut@ihs.gov

Amit Chattopadhyay

Office of Science Policy Analysis

NIH, National Institute of Dental and

Craniofacial Research

Email: chattopadhyaya@nidcr.nih.gov

Janet E. Leigh BDS, DMD,

Senior Dental Advisor

Office of Strategic Priorities, Office of Special

Health Affairs, HRSA

E-mail: jleigh@hrsa.gov

Hyewon Lee, DMD

Dental Consultant

Office of Strategic Priorities, Office of Special

Health Affairs, HRSA

Email: hlee@hrsa.gov

Gina Thornton-Evans, DDS, MPH

Dental Officer

Surveillance, Investigations and Research

Team

Centers for Disease Control and Prevention,

National Center for Chronic Disease
Prevention and Health Promotion,

Division of Oral Health

Email: gdt4@cdc.gov

\section{Contributing Member}

Bruce A. Dye, DDS, MPH

USPHS Dental Epidemiology Officer

Centers for Disease Control and Prevention

National Center for Health Statistics

Division of Health and Nutrition Examination

Statistics

Email bfd1@cdc.gov

\section{Physical Activity and Fitness}

\section{Workgroup Coordinators}

Janet Fulton

Epidemiologist

NCCDPHP/DNPAO/PAHB

Centers for Disease Control and Prevention

Email: janet.fulton@cdc.hhs.gov

Jane Wargo

Program Analyst

President's Council on Sports, Fitness and

Nutrition

Email: jane.wargo@hhs.gov

\section{Contributing Members}

Sandra Ham (Former workgroup lead)

Health Statistician

Physical Activity and Health Branch

Division of Nutrition, Physical Activity, and

Obesity

Centers for Disease Control and Prevention

Christine G. Spain

(Former workgroup lead - retired

Director)

Research, Planning, and Special Projects

President's Council on Physical Fitness and Sports

\section{Public Health Infrastructure}

\section{Workgroup Coordinators}

Liza Corso

Acting Branch Chief

Agency and Systems Improvement Branch

Division of Public Health Performance

Improvement 
Office of State, Tribal, Local, and Territorial Support

Centers for Disease Control and Prevention

Email: Lcorso@cdc.gov

Emily DeCoster, MPH

Healthy People Workgroup Coordinator

Health Resources and Services Administration

Office of Research and Evaluation

Office of Planning, Analysis and Evaluation

Email: edecoster@hrsa.gov

\section{Contributing Members}

Stephanie Bailey, MD (retired)

Centers for Disease Control and Prevention

Beverly Helene Smith, MHS, R.R.T.

Grants Management Specialist/ Public Health

Analyst

Health Resources and Services Administration

Office of Federal Assistance Management

Division of Grants Management Operations

Health Services Branch

Email: bsmith@hrsa.gov

\section{Respiratory Diseases}

\section{Workgroup Coordinators}

Joylene John-Sowah, MD, MPH

Medical Officer

Division for the Application of Research

Discoveries National Heart Lung and Blood

Institute

National Institutes of Health

Email: johnsowahj@mail.nih.gov

Jeanne Moorman, MS

Survey Statistician

Air Pollution and Respiratory Health Branch

National Center for Environmental Health

Centers for Disease Control and Prevention

Email: zva9@cdc.gov

\section{Contributing Members}

Lara Akinbami, MD

CDR, USPHS

Medical Officer III

Office of Analysis and Epidemiology

National Center for Health Statistics

Centers for Disease Control and Prevention

Email: LAkinbami@cdc.gov
M. Beth Benedict, DrPH, JD (retired)

Social Science Research Analyst

Division of State Program Research

Research and Evaluation Group

Office of Research, Development and

Information

Centers for Medicare \& Medicaid Services

Email: beth.benedict@cms.hhs.gov

Charles F. Dillon, MD, PhD, MPH

Medical Research Officer

Division of Health and Nutrition Examination

Statistics

National Center for Health Statistics

Centers for Disease Control and Prevention

Email: cid2@cdc.gov

Robinson Fulwood, PhD, MSPH. (former

colead, retired)

Acting Deputy Director

Division for the Application of Research

Discoveries

National Heart and Lung and Blood Institute

National Institutes of Health

Peter J. Gergen, MD, MPH

Division of Allergy, Immunology and

Transplantation

Asthma, Allergy, and Inflammation Branch

National Institute of Allergy and Infectious

Diseases

National Institutes of Health

Email: pgergen@niaid.nih.gov

Jeanette Guyton-Krishnan, PhD (former colead)

Acting Lead, Community Strategies Team

Office of Disease Prevention and Health

Promotion/OASH/OS

Email: \eanette.Guyton-Krishnan@hhs.gov

William Jirles, MPH

Program Analyst

Office of Policy, Planning, and Evaluation

National Institute of Environmental Health

Sciences

National Institutes of Health

Email: jirles@niehs.nih.gov

Elizabeth Lancet

Assistant Vice President, Research

American Lung Association

Email: elancet@lungusa.org

Daniel S. Lewin, PhD, D.ABSM 
Program Director

Sleep Disorders Medicine Program

National Center on Sleep Disorders Research

Division of Lung Diseases

National Heart Lung and Blood Institute

National Institutes of Health

Email: LewinDS@nhlbi.nih.gov

Antonello Punturieri, MD, PhD

Program Director

Division of Lung Diseases

National Heart, Lung, and Blood Institute

Email: punturieria@nhlbi.nih.gov

Stephen Reed, MD (former colead)

RADM, USPHS

Director

Influenza Coordination Unit

Office of Infectious Diseases

Centers for Disease Control and Prevention

Email: SRedd@cdc.gov

Diana Schmidt, MPH

Coordinator, (retired)

National Asthma Education and Prevention

Program

Division for the Application of Research

Discoveries

National Heart, Lung, and Blood Institute

National Institutes of Health

Michael Twery, PhD

Director

National Center on Sleep Disorders Research

Division of Lung Diseases, NHLBI, NIH

Email: Twery@nih.gov

\section{Sexually Transmitted Diseases \\ Workgroup Coordinator}

Rachel S. Wynn, MPH

Office of Policy, Planning, and External

Relations

Division of STD Prevention

National Center for HIV/AIDS, Viral Hepatitis,

STD and TB Prevention

Email: rwynn@cdc.gov

\section{Contributing Members}

Hillard Weinstock, MD, MPH

Medical Epidemiologist

National Center for HIV/AIDS, Viral Hepatitis,

STD, and TB Prevention (NCHHSTP)

Division of STD Prevention
Centers for Disease Control and Prevention HWeinstock@cdc.gov

John Douglas, MD

Chief Medical Officer

National Center for HIV/AIDS, Viral Hepatitis, STD, and TB Prevention (NCHHSTP)

Centers for Disease Control and Prevention

Łouglas@cdc.gov

Stuart Berman, MD, ScM

Senior Advisor to the Director

National Center for HIV/AIDS, Viral Hepatitis, STD, and TB Prevention (NCHHSTP)

Division of STD Prevention

Centers for Disease Control and Prevention

SBerman@cdc.gov

Amy Pulver, MA, MBA, MA

Policy Analyst

Office of the Associate Director For Policy

Centers for Disease Contol and Prevention

Washington Office

Amy.Pulver@cdc.hhs.gov

Jill Wasserman, MPH

Health Education Specialist

Department of Health and Human Services

Office of the Assistant Secretary for Health

Office on Women's Health

ఏill.Wasserman1@hhs.gov

\section{Substance Abuse}

\section{Workgroup Coordinators}

Ralph W. Hingson, ScD, MPH

Director

Division of Epidemiology and

Prevention Research

National Institute on Alcohol Abuse

and Alcoholism

National Institutes of Health

E-mail: rhingson@mail.nih.gov

Geoffrey Laredo

Senior Advisor to the Director

Office of Science Policy and Communications

National Institute on Drug Abuse

National Institutes of Health

E-mail: glaredo@nida.nih.gov

Kevin J. Malone

Public Health Analyst

Department of Health and Human Services 
Substance Abuse and Mental Health Services Administration

Office of Policy, Planning, and Innovation

Email: kevin.malone@samhsa.hhs.gov

\section{Contributing Members}

Nancy P. Brady

Special Assistant \&

Public Health Analyst

Substance Abuse \& Mental Health Services

Administration

Center for Behavioral Health Statistics \&

Quality

Nancy.brady@samhsa.hhs.gov

Bennett W. Fletcher, $\mathrm{PhD}$

CAPT, USPHS

Senior Research Psychologist

Services Research Branch

Division of Epidemiology, Services and

Prevention Research

National Instititute on Drug Abuse

National Institutes of Health

Andrea K. Kamargo, MSW

Senior Public Health Analyst

Office of Program Analysis and Coordination

Substance Abuse and Mental Health

Services Administration

E-mail: andrea.kamargo@samhsa.hhs.gov

Nancy J. Kennedy, DrPH

Senior Public Health Advisor

Division of Systems Development

E-mail: nancy.kennedy@samhsa.hhs.gov

Kari Kinnard

Highway Safety Specialist

Impaired Driving Division

NHTSA

Dorothy B. Lewis

Project Officer HIV/AIDS Team

Substance Abuse Mental Health Services

Administration

Center for Substance Abuse Treatment

Health Services Branch/Division of Services

Improvement

Dorothy.Lewis@samhsa.hhs.gov

Carter A. Roeber, $\mathrm{PhD}$

Senior Research Associate

LTG Associates, Inc.

\section{Tobacco Use}

\section{Workgroup Coordinators}

Allison MacNeil, MPH

Health Scientist

Centers for Disease Control and Prevention (CDC)

National Center for Chronic Disease

Prevention and Health Promotion

Office on Smoking and Health

Email: amacneil@cdc.gov

Gabbi Promoff, MA

Issues Management Team Lead

Centers for Disease Control and Prevention (CDC)

National Center for Chronic Disease

Prevention and Health Promotion

Office on Smoking and Health

GPromoff@cdc.gov

\section{Contributing Members}

Ralph Caraballo, $\mathrm{PhD}$

Epidemiology Branch Chief

Centers for Disease Control and Prevention

(CDC)

National Center for Chronic Disease

Prevention and Health Promotion

Office on Smoking and Health

RCaraballo@cdc.gov

Terry Pechacek, PhD

Associate Director for Science

Centers for Disease Control and Prevention

(CDC)

National Center for Chronic Disease

Prevention and Health Promotion

Office on Smoking and Health

TPechacek@cdc.gov

Dana Shelton, MPH

Associate Director for Policy

Centers for Disease Control and Prevention

(CDC)

National Center for Chronic Disease

Prevention and Health Promotion

Office on Smoking and Health

DShelton@cdc.gov

\section{Vision and Hearing}

\section{Workgroup Coordinators}

Rosemary Janiszewski, MS, CHES 
Associate Director for Communication, Health

Education, and Public Liaison

National Eye Institute

National Institutes of Health

Email: rjaniszewski@nei.nih.gov

Howard J. Hoffman

Program Director for Epidemiology and

Statistics

Division of Scientific Programs

National Institute on Deafness and Other

Communication Disorders

National Institutes of Health

Email: hoffmanh@nidcd.nih.gov

Lonnie L. Lisle

Office of Health Communication and Public

Liaison

National Institute on Deafness and Other

Communication Disorders

National Institutes of Health

Email: lislel@nidcd.nih.gov

\section{Adolescent Health}

Trina Anglin, MD, PhD

Chief, Office of Adolescent Health

Maternal and Child Health Bureau

Health Resources and Services Administration

Email: tanglin@hrsa.gov

Cherie Gray, MA

Public Health Analyst

Office of the Director

Division of Adolescent and School Health

Centers for Disease Control and Prevention

E-mail: cng2@cdc.gov

\section{Health of Racial/Ethnic Minority}

Populations

Myrtha Beadle

Acting Deputy Director

Office of Public Health Science

Office of Minority Health

Email: mbeadle@osophs.dhhs.gov

\section{Women's Health}

Suzanne Haynes, PhD

Senior Science Advisor

PHS Office of Women's Health

U.S. Department of Health and Human

Services shaynes@osophs.dhhs.gov

Health of People With Disabilities

Eileen Elias

Deputy Director

Office on Disability and Health

U.S. Department of Health and Human

Services

E-mail: eileen.elias@hhs.gov 


\section{Appendix D: A Crosswalk Between Objectives From Healthy People 2010 to Healthy People 2020}

\section{Definition of terms:}

- Retained "as is" objectives have no change in the numerator definition or in the denominator definition between the Healthy People (HP) 2010 and HP2020 objectives. These include objectives that were developmental in HP2010 and are developmental in HP2020 and for which no numerator information is available.

- Modified objectives have some change in the numerator definition or in the denominator definition between the HP2010 and HP2020 objectives. These include objectives that went from developmental in HP2010 to measurable in HP2020 or vice versa.

- Archived objectives had at least one data point in HP2010 but were not carried forward into HP2020.

- Dropped objectives were not carried forward into HP2020. These objectives were either developmental or deleted at the HP2010 Midcourse Review or at another time in HP2010.

\section{Legend:}

One objective in HP2010 was divided into two or more objectives in HP2020.

Two or more objectives in HP2010 were combined into one objective in HP2020.

\section{HP2010} Objectives

\section{HP2020 Objectives}

\begin{tabular}{l|l|l|l} 
Retained & Modified & Archived & Dropped
\end{tabular}

\section{Focus Area 1: Access to Quality Health Services}

\section{$1-1$}

$1-2$

$1-3 a$

$1-3 b$

$1-3 c$

$1-3 d$

$1-3 e$

1-3f

$1-3 \mathrm{~g}$

1-3h

$1-4 a$

$1-4 b$

1-4c ]

1-5

\section{1-6 ]}

$1-7 a$

1-7b

$1-7 \mathrm{c}$

1-7d

$1-7$ e

1-7f

$1-7 \mathrm{~g}$

$1-7 \mathrm{~h}$

1-8a

\section{AHS-1.1}

AHS-5.2

AHS-3

AHS-5.3

AHS-5.4

AHS-6. 1

AHS-6.2

AHS-6.3

AHS-6.4 


\begin{tabular}{|c|c|c|c|c|}
\hline \multirow{2}{*}{$\begin{array}{l}\text { HP2010 } \\
\text { Objectives }\end{array}$} & \multicolumn{4}{|c|}{ HP2020 Objectives } \\
\hline & Retained & Modified & Archived & Dropped \\
\hline $1-8 b$ & & & Archived & \\
\hline $1-8 c$ & & & Archived & \\
\hline $1-8 d$ & & & Archived & \\
\hline $1-8 e$ & & & Archived & \\
\hline $1-8 f$ & & & Archived & \\
\hline $1-8 g$ & & & Archived & \\
\hline $1-8 \mathrm{~h}$ & & & Archived & \\
\hline $1-8 i$ & & & Archived & \\
\hline $1-8 j$ & & & Archived & \\
\hline $1-8 \mathrm{k}$ & & & Archived & \\
\hline $1-8 \mid$ & & & Archived & \\
\hline $1-8 m$ & & & Archived & \\
\hline $1-8 n$ & & & Archived & \\
\hline $1-80$ & & & Archived & \\
\hline $1-8 p$ & & & Archived & \\
\hline $1-8 q$ & & & Archived & \\
\hline $1-8 r$ & & & Archived & \\
\hline $1-8 \mathrm{~s}$ & & & Archived & \\
\hline $1-8 t$ & & & Archived & \\
\hline $1-9 a$ & & & Archived & \\
\hline $1-9 b$ & & & Archived & \\
\hline $1-9 c$ & & & Archived & \\
\hline $1-10$ & & & Archived & \\
\hline $1-11 a$ & AHS-8.1 & & & \\
\hline $1-11 b$ & AHS-8.2 & & & \\
\hline $1-11 c$ & & & Archived & \\
\hline $1-11 d$ & & & Archived & \\
\hline $1-11 e$ & & & Archived & \\
\hline $1-11 f$ & & & Archived & \\
\hline $1-11 \mathrm{~g}$ & & & Archived & \\
\hline $1-12$ & & & Archived & \\
\hline $1-13 a$ & & & Archived & \\
\hline $1-13 b$ & & & Archived & \\
\hline $1-13 c$ & & & Archived & \\
\hline $1-13 d$ & & & Archived & \\
\hline $1-13 e$ & & & Archived & \\
\hline $1-13 f$ & & & Archived & \\
\hline $1-13 g$ & & & Archived & \\
\hline $1-13 h$ & & & Archived & \\
\hline $1-13 i$ & & & Archived & \\
\hline $1-14 a$ & & & Archived & \\
\hline $1-14 b$ & & & Archived & \\
\hline $1-15 a$ & & & Archived & \\
\hline $1-15 b$ & & & Archived & \\
\hline $1-15 c$ & & & Archived & \\
\hline $1-15 d$ & & & Archived & \\
\hline $1-16$ & & & Archived & \\
\hline
\end{tabular}

Focus Area 2: Arthritis, Osteoporosis and Chronic Back Conditions

\begin{tabular}{|l|}
\hline $2-1$ \\
\hline $2-2$ \\
\hline $2-3$ \\
\hline $2-4 a$ \\
\hline $2-4 b$ \\
\hline
\end{tabular}

AOCBC-1

AOCBC-2

AOCBC-4

AOCBC-7.1

AOCBC-7.2 


\begin{tabular}{|c|c|c|c|c|}
\hline \multirow{2}{*}{$\begin{array}{l}\text { HP2010 } \\
\text { Objectives }\end{array}$} & \multicolumn{4}{|c|}{ HP2020 Objectives } \\
\hline & Retained & Modified & Archived & Dropped \\
\hline $2-5 a$ & AOCBC-6.1 & & & \\
\hline $2-5 b$ & AOCBC-6.2 & & & \\
\hline $2-6$ & & & Archived & \\
\hline $2-7$ & AOCBC-9 & & & \\
\hline $2-8$ & AOCBC-8 & & & \\
\hline $2-9$ & AOCBC-10 & & & \\
\hline $2-10$ & & & Archived & \\
\hline $2-11$ & AOCBC-12 & & & \\
\hline
\end{tabular}

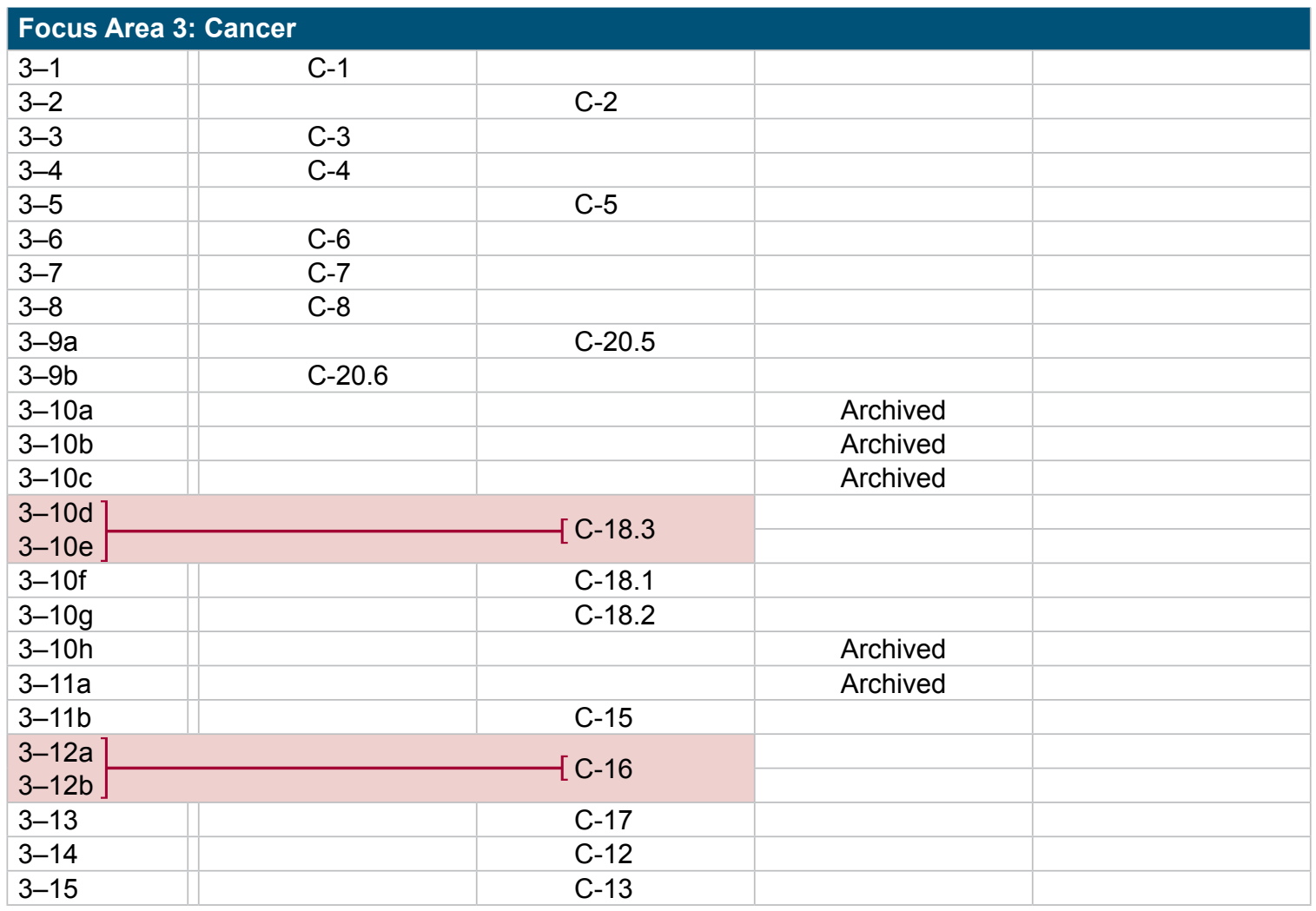

\section{Focus Area 4: Substance Abuse: Chronic Kidney Disease}

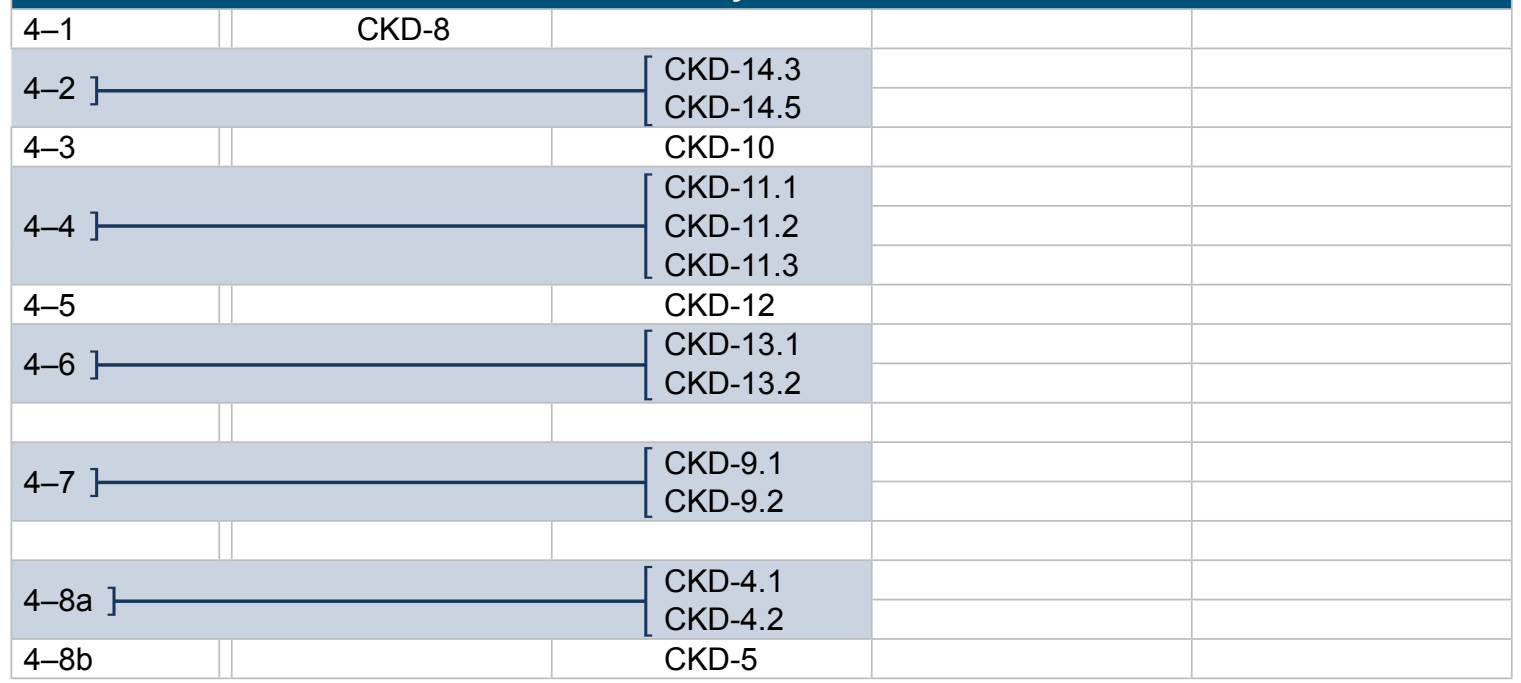




\begin{tabular}{|c|c|c|c|c|}
\hline \multirow{2}{*}{$\begin{array}{l}\text { HP2010 } \\
\text { Objectives }\end{array}$} & \multicolumn{4}{|c|}{ HP2020 Objectives } \\
\hline & Retained & Modified & Archived & Dropped \\
\hline \multicolumn{5}{|c|}{ Focus Area 5: Diabetes } \\
\hline $5-1$ & & $\mathrm{D}-14$ & & \\
\hline $5-2$ & $\mathrm{D}-1$ & & & \\
\hline $5-3$ & & & Archived & \\
\hline $5-4$ & & $\mathrm{D}-15$ & & \\
\hline $5-5$ & D-3 & & & \\
\hline $5-6$ & & & Archived & \\
\hline $5-7$ & & & Archived & \\
\hline $5-8$ & & & & Dropped \\
\hline $5-9$ & & & & Dropped \\
\hline $5-10$ & $\mathrm{D}-4$ & & & \\
\hline $5-11$ & $\mathrm{D}-12$ & & & \\
\hline $5-12$ & $\mathrm{D}-11$ & & & \\
\hline $5-13$ & $\mathrm{D}-10$ & & & \\
\hline $5-14$ & D-9 & & & \\
\hline $5-15$ & D-8 & & & \\
\hline $5-16$ & & & Archived & \\
\hline $5-17$ & $\mathrm{D}-13$ & & & \\
\hline
\end{tabular}

Focus Area 6: Disability and Secondary Conditions

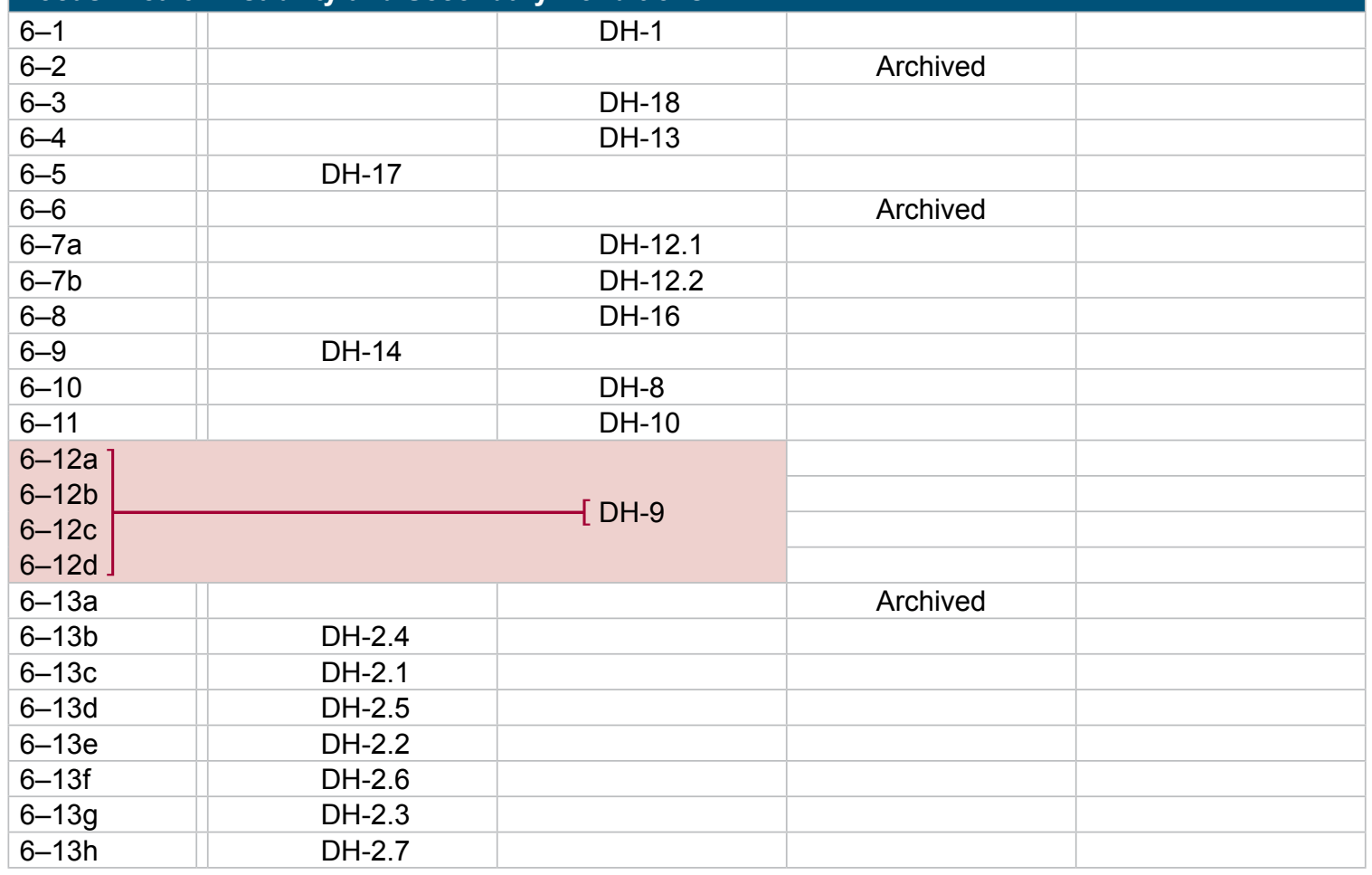

Focus Area 7: Educational and Community-Based Programs

\begin{tabular}{|l|l|l|}
\hline $7-1$ & ECBP-6 & \\
\hline $7-2 \mathrm{a}$ & & ECBP-2.1 \\
\hline $7-2 \mathrm{~b}$ & & ECBP-2.2 \\
\hline $7-2 \mathrm{c}$ & & ECBP-2.3 \\
\hline $7-2 \mathrm{~d}$ & & ECBP-2.4 \\
\hline $7-2 \mathrm{e}$ & & ECBP-2.5 \\
\hline $7-2 \mathrm{f}$ & & ECBP-2.6 \\
\hline $7-2 \mathrm{~g}$ & & ECBP-2.7 \\
\hline
\end{tabular}




\begin{tabular}{|c|c|c|c|c|}
\hline \multirow{2}{*}{$\begin{array}{l}\text { HP2010 } \\
\text { Objectives }\end{array}$} & \multicolumn{4}{|c|}{ HP2020 Objectives } \\
\hline & Retained & Modified & Archived & Dropped \\
\hline $7-2 \mathrm{~h}$ & & ECBP-2.8 & & \\
\hline $7-2 i$ & & ECBP-2.9 & & \\
\hline $7-2 \mathrm{j}$ & & & Archived & \\
\hline $7-3$ & & ECBP-7.1 & & \\
\hline $7-4 a$ & & ECBP-5.1 & & \\
\hline $7-4 b$ & ECBP-5.2 & & & \\
\hline $7-4 c$ & & ECBP-5.3 & & \\
\hline $7-4 d$ & ECBP-5.4 & & & \\
\hline $7-5 a$ & ECBP-8.1 & & & \\
\hline $7-5 b$ & & ECBP-8.2 & & \\
\hline $7-5 c$ & & ECBP-8.3 & & \\
\hline $7-5 d$ & & ECBP-5.4 & & \\
\hline $7-5 e$ & & ECBP-8.5 & & \\
\hline $7-5 f$ & & ECBP-8.6 & & \\
\hline $7-6$ & & ECBP-9 & & \\
\hline $7-7$ & & & & Dropped \\
\hline $7-8$ & & & & Dropped \\
\hline $7-9$ & & & & Dropped \\
\hline \multirow{9}{*}{$7-10]$} & & ECBP-10.1 & & \\
\hline & & ECBP-10.2 & & \\
\hline & & ECBP-10.3 & & \\
\hline & & ECBP-10.4 & & \\
\hline & & ECBP-10.5 & & \\
\hline & & ECBP-10.6 & & \\
\hline & & ECBP-10.7 & & \\
\hline & & ECBP-10.8 & & \\
\hline & & ECBP-10.9 & & \\
\hline $7-11 a$ & & & & Dropped \\
\hline $7-11 b$ & & & & Dropped \\
\hline $7-11 c$ & & & Archived & \\
\hline $7-11 d$ & & & & Dropped \\
\hline $7-11 e$ & & & & Dropped \\
\hline $7-11 f$ & & & & Dropped \\
\hline $7-11 \mathrm{~g}$ & & ECBP-11 & & \\
\hline $7-11 h$ & & & Archived & \\
\hline $7-11 i$ & & & Archived & \\
\hline $7-11 j$ & & & & Dropped \\
\hline $7-11 \mathrm{k}$ & & & & Dropped \\
\hline $7-11 \mid$ & & & & Dropped \\
\hline $7-11 m$ & & & Archived & \\
\hline $7-11 n$ & & & Archived & \\
\hline $7-110$ & & & Archived & \\
\hline $7-11 p$ & & & & Dropped \\
\hline $7-11 q$ & & & Archived & \\
\hline $7-11 r$ & & & Archived & \\
\hline $7-11 \mathrm{~s}$ & & & Archived & \\
\hline $7-11 t$ & & & Archived & \\
\hline $7-11 u$ & & & Archived & \\
\hline $7-11 v$ & & & Archived & \\
\hline $7-11 w$ & & & & Dropped \\
\hline $7-11 x$ & & & & Dropped \\
\hline $7-11 y$ & & & Archived & \\
\hline $7-11 z$ & & & Archived & \\
\hline
\end{tabular}




\begin{tabular}{|c|c|c|c|c|}
\hline \multirow{2}{*}{$\begin{array}{l}\text { HP2010 } \\
\text { Objectives }\end{array}$} & \multicolumn{4}{|c|}{ HP2020 Objectives } \\
\hline & Retained & Modified & Archived & Dropped \\
\hline $7-11 A$ & & & Archived & \\
\hline $7-11 \mathrm{~B}$ & & & & Dropped \\
\hline $7-12$ & & & Archived & \\
\hline \multicolumn{5}{|c|}{ Focus Area 8: Environmental Health } \\
\hline \multicolumn{5}{|c|}{$8-1 a]$} \\
\hline \multicolumn{5}{|l|}{$8-1 b$} \\
\hline \multicolumn{5}{|l|}{$8-1 c$} \\
\hline \multicolumn{5}{|c|}{$-[\mathrm{EH}-1$} \\
\hline \multicolumn{5}{|l|}{$8-1 e$} \\
\hline $8-1 f$ & & & & \\
\hline \multicolumn{5}{|l|}{$8-1 \mathrm{~g}$} \\
\hline $8-2 a$ & & $\mathrm{EH}-2.1$ & & \\
\hline $8-2 b$ & & $\mathrm{EH}-2.2$ & & \\
\hline $8-2 c$ & & EH-2.3 & & \\
\hline $8-2 d$ & & $\mathrm{EH}-2.4$ & & \\
\hline $8-3$ & & & Archived & \\
\hline \multirow{4}{*}{\multicolumn{3}{|c|}{$\begin{array}{l}\text { EH-3.1 } \\
\text { EH-3.2 } \\
\text { EH-3.3 }\end{array}$}} & & \\
\hline & & & & \\
\hline & & & & \\
\hline & & & & \\
\hline $8-6$ & $\mathrm{EH}-5$ & & & \\
\hline $8-7$ & & $\mathrm{EH}-6$ & & \\
\hline $8-8 a$ & & & Archived & \\
\hline $8-8 b$ & & & Archived & \\
\hline $8-9$ & & EH-7 & & \\
\hline $8-10 a$ & & & Archived & \\
\hline $8-10 b$ & & & Archived & \\
\hline $8-11$ & EH-8.1 & & & \\
\hline $8-12 a$ & EH-9 & & & \\
\hline $8-12 b$ & & & Archived & \\
\hline $8-12 c$ & & & Archived & \\
\hline $8-12 d$ & & & Archived & \\
\hline $8-13$ & $\mathrm{EH}-10$ & & & \\
\hline $8-14 a$ & & & & Dropped \\
\hline $8-14 b$ & & EH-11 & & \\
\hline $8-15$ & $\mathrm{EH}-12$ & & & \\
\hline $8-16 a$ & & & Archived & \\
\hline $8-16 b$ & & & Archived & \\
\hline $8-16 c$ & & $\mathrm{EH}-13.1$ & & \\
\hline $8-17$ & & & & Dropped \\
\hline $8-18$ & & & Archived & \\
\hline \multirow{2}{*}{\multicolumn{2}{|c|}{$8-19$}} & EH-14 & & \\
\hline & & EH-15 & & \\
\hline & & & & \\
\hline \multirow{9}{*}{$8-20]$} & & $\mathrm{EH}-16.1$ & & \\
\hline & & $\mathrm{EH}-16.2$ & & \\
\hline & & $\mathrm{EH}-16.3$ & & \\
\hline & & $\mathrm{EH}-16.4$ & & \\
\hline & & $\mathrm{EH}-16.5$ & & \\
\hline & & $\mathrm{EH}-16.6$ & & \\
\hline & & EH-16.7 & & \\
\hline & & EH-16.8 & & \\
\hline & & EH-16.9 & & \\
\hline
\end{tabular}




\begin{tabular}{|c|c|c|c|c|}
\hline \multirow{2}{*}{$\begin{array}{l}\text { HP2010 } \\
\text { Objectives }\end{array}$} & \multicolumn{4}{|c|}{ HP2020 Objectives } \\
\hline & Retained & Modified & Archived & Dropped \\
\hline $8-21$ & & & Archived & \\
\hline \multirow{3}{*}{\multicolumn{2}{|c|}{ 8-22 ] }} & \multirow{3}{*}{$\begin{array}{l}\text { EH-17.1 } \\
\text { EH-17.2 } \\
\text { EH-17.3 }\end{array}$} & & \\
\hline & & & & \\
\hline & & & & \\
\hline $8-23$ & EH-19 & & & \\
\hline $8-24 a$ & & & & Dropped \\
\hline $8-24 b$ & EH-20.9 & & & \\
\hline $8-24 c$ & $\mathrm{EH}-20.10$ & & & \\
\hline $8-24 d$ & & & Archived & \\
\hline $8-25 a$ & EH-20.1 & & & \\
\hline $8-25 b$ & EH-20.2 & & & \\
\hline $8-25 c$ & $\mathrm{EH}-20.3$ & & & \\
\hline $8-25 d$ & & & & Dropped \\
\hline $8-25 e$ & EH-20.4 & & & \\
\hline $8-25 f$ & & & Archived & \\
\hline $8-25 \mathrm{~g}$ & & & Archived & \\
\hline $8-25 h$ & EH-20.11 & & & \\
\hline $8-25 i$ & & & Archived & \\
\hline \multirow{2}{*}{\multicolumn{2}{|c|}{$8-25 \mathrm{j}$}} & \multirow{2}{*}{$\begin{array}{l}\text { EH-20.12 } \\
\text { EH-20.13 }\end{array}$} & & \\
\hline & & & & \\
\hline $8-25 \mathrm{k}$ & $\mathrm{EH}-20.14$ & & & \\
\hline $8-25 \mid$ & & & & Dropped \\
\hline $8-25 m$ & EH-20.6 & & & \\
\hline $8-25 n$ & & & Archived & \\
\hline $8-250$ & EH-20.7 & & & \\
\hline $8-25 p$ & EH-20.8 & & & \\
\hline $8-25 q$ & $\mathrm{EH}-20.5$ & & & \\
\hline $8-25 r$ & & & Archived & \\
\hline $8-25 s$ & & & Archived & \\
\hline $8-26$ & EH-21 & & & \\
\hline $8-27 a$ & $\mathrm{EH}-22.1$ & & & \\
\hline $8-27 b$ & EH-22.2 & & & \\
\hline $8-27 c$ & EH-22.3 & & & \\
\hline $8-27 d$ & EH-22.4 & & & \\
\hline $8-27 e$ & EH-22.5 & & & \\
\hline $8-27 f$ & & & Archived & \\
\hline $8-27 \mathrm{~g}$ & EH-22.6 & & & \\
\hline $8-27 \mathrm{~h}$ & $\mathrm{EH}-22.7$ & & & \\
\hline $8-27 i$ & & & Archived & \\
\hline $8-27 j$ & & & Archived & \\
\hline $8-27 k$ & & & Archived & \\
\hline $8-27 \mid$ & & & & Dropped \\
\hline $8-27 m$ & & & & Dropped \\
\hline $8-27 n$ & & & & Dropped \\
\hline $8-270$ & & & Archived & \\
\hline $8-28$ & & & & Dropped \\
\hline $8-29$ & & EH-24 & & \\
\hline $8-30 a$ & & & Archived & \\
\hline $8-30 b$ & & & Archived & \\
\hline $8-30 c$ & & & Archived & \\
\hline $8-30 d$ & & & Archived & \\
\hline $8-30 e$ & & & Archived & \\
\hline $8-30 f$ & & & Archived & \\
\hline
\end{tabular}




\begin{tabular}{|c|c|c|c|c|}
\hline \multirow{2}{*}{$\begin{array}{l}\text { HP2010 } \\
\text { Objectives }\end{array}$} & \multicolumn{4}{|c|}{ HP2020 Objectives } \\
\hline & Retained & Modified & Archived & Dropped \\
\hline $8-30 \mathrm{~g}$ & & & Archived & \\
\hline $8-30 \mathrm{~h}$ & & & Archived & \\
\hline $8-30 i$ & & & Archived & \\
\hline 8-30j & & & Archived & \\
\hline $8-30 k$ & & & Archived & \\
\hline $8-301$ & & & Archived & \\
\hline \multicolumn{5}{|c|}{ Focus Area 9: Family Planning } \\
\hline $9-1$ & FP-1 & & & \\
\hline $9-2$ & & FP-5 & & \\
\hline $9-3$ & & FP-6 & & \\
\hline $9-4$ & FP-2 & & & \\
\hline $9-5$ & & FP-3.2 & & \\
\hline $9-6 a$ & & & Archived & \\
\hline $9-6 b$ & & & Archived & \\
\hline $9-6 c$ & & & Archived & \\
\hline \multirow{2}{*}{\multicolumn{5}{|c|}{$9-7] \quad \square$ FP-8.1 }} \\
\hline & 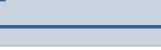 & {$[F P-8.2$} & & \\
\hline $9-8 a$ & & FP-9.3 & & \\
\hline $9-8 b$ & & FP-9.4 & & \\
\hline $9-9 a$ & FP-9.1 & & & \\
\hline $9-9 b$ & FP-9.2 & & & \\
\hline $9-10 a$ & & FP-10.1 & & \\
\hline $9-10 b$ & & FP-10.2 & & \\
\hline $9-10 c$ & & FP-11.1 & & \\
\hline $9-10 d$ & & FP-11.2 & & \\
\hline $9-10 e$ & & FP-10.3 & & \\
\hline $9-10 f$ & & FP-10.4 & & \\
\hline $9-10 \mathrm{~g}$ & & FP-11.3 & & \\
\hline $9-10 \mathrm{~h}$ & & FP-11.4 & & \\
\hline $9-11 a$ & FP-12.1 & & & \\
\hline $9-11 b$ & FP-12.2 & & & \\
\hline $9-11 c$ & FP-12.3 & & & \\
\hline $9-11 d$ & FP-12.4 & & & \\
\hline $9-11 e$ & FP-12.5 & & & \\
\hline $9-11 f$ & FP-12.6 & & & \\
\hline $9-11 \mathrm{~g}$ & FP-12.7 & & & \\
\hline $9-11 \mathrm{~h}$ & FP-12.8 & & & \\
\hline $9-11 \mathrm{i}$ & FP-13.1 & & & \\
\hline 9-11j & FP-13.2 & & & \\
\hline $9-11 \mathrm{k}$ & FP-13.3 & & & \\
\hline $9-11$ I & FP-13.4 & & & \\
\hline $9-11 \mathrm{~m}$ & FP-13.5 & & & \\
\hline $9-11 n$ & FP-13.6 & & & \\
\hline $9-110$ & FP-13.7 & & & \\
\hline $9-11 p$ & FP-13.8 & & & \\
\hline \multirow{2}{*}{\multicolumn{2}{|c|}{ 9-12 ] }} & $\mathrm{MICH}-17.1$ & & \\
\hline & & $\mathrm{MICH}-17.2$ & & \\
\hline $9-13$ & FP-4 & & & \\
\hline
\end{tabular}

Focus Area 10: Food Safety

10-1a

10-1b

10-1c

FS-1.1

FS-1.2

FS-1.3 


\begin{tabular}{|c|c|c|c|c|}
\hline \multirow{2}{*}{$\begin{array}{l}\text { HP2010 } \\
\text { Objectives }\end{array}$} & \multicolumn{4}{|c|}{ HP2020 Objectives } \\
\hline & Retained & Modified & Archived & Dropped \\
\hline $10-1 d$ & FS-1.4 & & & \\
\hline $10-1 e$ & & & & Dropped \\
\hline $10-1 f$ & FS-1.5 & & & \\
\hline $10-1 \mathrm{~g}$ & & & & Dropped \\
\hline $10-2 a$ & & & Archived & \\
\hline $10-2 b$ & & & Archived & \\
\hline $10-3 a$ & & FS-3.1 & & \\
\hline $10-3 b$ & & FS-3.2 & & \\
\hline $10-3 c$ & FS-3.3 & & & \\
\hline $10-3 d$ & FS-3.4 & & & \\
\hline $10-3 e$ & & & & Dropped \\
\hline $10-3 f$ & & & & Dropped \\
\hline $10-3 g$ & & & & Dropped \\
\hline $10-3 \mathrm{~h}$ & & & & Dropped \\
\hline $10-3 i$ & & & & Dropped \\
\hline $10-3 j$ & & & & Dropped \\
\hline $10-3 k$ & & & & Dropped \\
\hline $10-3 \mid$ & & & & Dropped \\
\hline $10-3 m$ & & & & Dropped \\
\hline $10-3 n$ & & & & Dropped \\
\hline $10-30$ & & & & Dropped \\
\hline $10-3 p$ & & & & Dropped \\
\hline $10-4 a$ & & & & Dropped \\
\hline $10-4 b$ & FS-4 & & & \\
\hline \multirow{4}{*}{$10-5]$} & & FS-5.1 & & \\
\hline & & FS-5.2 & & \\
\hline & & FS-5.3 & & \\
\hline & & FS-5.4 & & \\
\hline \multicolumn{5}{|l|}{$10-6 a$} \\
\hline \multicolumn{5}{|l|}{$10-6 b$} \\
\hline \multicolumn{5}{|l|}{$10-6 c$} \\
\hline \multicolumn{5}{|l|}{$10-6 d$} \\
\hline \multicolumn{5}{|l|}{$10-6 e$} \\
\hline \multicolumn{5}{|l|}{$10-6 f$} \\
\hline \multicolumn{5}{|l|}{$10-6 g$} \\
\hline \multicolumn{5}{|l|}{$10-6 h$} \\
\hline \multicolumn{5}{|l|}{$10-6 i$} \\
\hline $10-7$ & & & & Dropped \\
\hline
\end{tabular}

\section{Focus Area 11: Health Communication}

\begin{tabular}{|l||l|l|l|}
\hline $11-1$ & HCHIT-6.1 & \\
\hline $11-2 \mathrm{a}$ & & & Archived \\
\hline $11-2 \mathrm{~b}$ & & & Archived \\
\hline $11-3 \mathrm{a}$ & & Archived \\
\hline $11-3 \mathrm{~b}$ & & Archived \\
\hline $11-3 \mathrm{c}$ & & Archived \\
\hline $11-4 \mathrm{a}$ & & & \\
\hline $11-4 \mathrm{~b}$ & & & \\
\hline $11-4 \mathrm{c}$ & & & Archived \\
\hline $11-4 \mathrm{~d}$ & & & Archived \\
\hline $11-4 \mathrm{e}$ & & Archived \\
\hline $11-4 \mathrm{f}$ & & Archived \\
\hline $11-4 \mathrm{~g}$ & & Archived \\
\hline
\end{tabular}




\begin{tabular}{|c|c|c|c|c|}
\hline \multirow{2}{*}{$\begin{array}{l}\text { HP2010 } \\
\text { Objectives }\end{array}$} & \multicolumn{4}{|c|}{ HP2020 Objectives } \\
\hline & Retained & Modified & Archived & Dropped \\
\hline $11-5$ & & & Archived & \\
\hline $11-6 a$ & HCHIT-2.1 & & & \\
\hline $11-6 b$ & HCHIT-2.2 & & & \\
\hline $11-6 c$ & HCHIT-2.3 & & & \\
\hline $11-6 d$ & $\mathrm{HCHIT-2.4}$ & & & \\
\hline \multicolumn{5}{|c|}{ Focus Area 12: Heart Disease and Stroke } \\
\hline $12-1$ & HDS-2 & & & \\
\hline & HDS-16.1 & & & \\
\hline \multirow[t]{2}{*}{$12-2]$} & & - HDS-16.2 & & \\
\hline & & HDS-16.3 & & \\
\hline $12-3 a$ & & HDS-19.1 & & \\
\hline $12-3 b$ & & HDS-19.2 & & \\
\hline \multicolumn{5}{|l|}{$12-4]$} \\
\hline \multicolumn{5}{|l|}{$12-5$} \\
\hline $12-6 a$ & & HDS-24.1 & & \\
\hline $12-6 b$ & & HDS-24.2 & & \\
\hline $12-6 c$ & & HDS-24.3 & & \\
\hline $12-7$ & HDS-3 & & & \\
\hline $12-8$ & HDS-17.2 & & & \\
\hline $12-9$ & HDS-5.1 & & & \\
\hline $12-10$ & HDS-12 & & & \\
\hline \multirow{6}{*}{ 12-11] } & & HDS-10.1 & & \\
\hline & & HDS-10.2 & & \\
\hline & & HDS-10.3 & & \\
\hline & & HDS-10.4 & & \\
\hline & & HDS-10.5 & & \\
\hline & & HDS-11 & & \\
\hline $12-12$ & HDS-4 & & & \\
\hline $12-13$ & HDS-8 & & & \\
\hline $12-14$ & HDS-7 & & & \\
\hline $12-15$ & HDS-6 & & & \\
\hline $12-16$ & HDS-20.1 & & & \\
\hline \multicolumn{5}{|c|}{ Focus Area 13: HIV } \\
\hline $13-1$ & HIV-4 & & & \\
\hline $13-2$ & HIV-6 & & & \\
\hline $13-3$ & HIV-7 & & & \\
\hline $13-4$ & & & Archived & \\
\hline $13-5$ & HIV-1 & & & \\
\hline $13-6 a$ & & HIV-17.1 & & \\
\hline $13-6 b$ & & HIV-17.2 & & \\
\hline $13-7$ & & HIV-13 & & \\
\hline $13-8$ & HIV-16 & & & \\
\hline $13-9$ & & & & Dropped \\
\hline $13-10$ & & & & Dropped \\
\hline $13-11$ & HIV-15 & & & \\
\hline $13-12$ & & & & Dropped \\
\hline \multicolumn{5}{|l|}{ 13-13a] } \\
\hline \multicolumn{5}{|l|}{$13-13 b$} \\
\hline \\
\hline \multicolumn{5}{|l|}{$13-13 d$} \\
\hline \multicolumn{5}{|l|}{$13-13 e$} \\
\hline $13-13 f]$ & & & & \\
\hline
\end{tabular}




\begin{tabular}{|c|c|c|c|c|}
\hline \multirow{2}{*}{$\begin{array}{l}\text { HP2010 } \\
\text { Objectives }\end{array}$} & \multicolumn{4}{|c|}{ HP2020 Objectives } \\
\hline & Retained & Modified & Archived & Dropped \\
\hline $13-14$ & HIV-12 & & & \\
\hline $13-15$ & HIV-9 & & & \\
\hline $13-16$ & HIV-11 & & & \\
\hline $13-17 a$ & & HIV-8.1 & & \\
\hline $13-17 b$ & HIV-8.2 & & & \\
\hline $13-18$ & & & & Dropped \\
\hline
\end{tabular}

Focus Area 14: Immunizations and Infectious Diseases

14-1a

14-1b

$14-1 \mathrm{c}$

14-1d

14-1e

14-1f

14-1g

$14-1 \mathrm{~h}$

$14-1 \mathrm{i}$

$14-1 \mathrm{j}$

$14-1 \mathrm{k}$

$14-2$

14-3a

$14-3 b$

14-3c

14-3d

$14-3 e$

14-3f

14-3g

14-4

14-5a

14-5b

14-5c

14-5d

14-6

14-7

14-8

14-9

14-10

14-11

14-12

14-13

14-14

14-15

14-16

14-17

14-18

14-19

14-20a

14-20b

14-20c

14-20d

14-20e

14-21

14-22a

14-22b
IID-1.1

IID-1.4

IID-1.5

IID-1.8

IID-1.9

IID-1.10

IID-24 


\begin{tabular}{|c|c|c|c|c|}
\hline \multirow{2}{*}{$\begin{array}{l}\text { HP2010 } \\
\text { Objectives }\end{array}$} & \multicolumn{4}{|c|}{ HP2020 Objectives } \\
\hline & Retained & Modified & Archived & Dropped \\
\hline $14-22 c$ & IID-7.3 & & & \\
\hline $14-22 d$ & IID-7.4 & & & \\
\hline $14-22 e$ & IID-7.5 & & & \\
\hline $14-22 f$ & IID-7.6 & & & \\
\hline $14-22 g$ & IID-7.7 & & & \\
\hline $14-22 \mathrm{~h}$ & & IID-12.1 & & \\
\hline $14-23 a$ & & & Archived & \\
\hline $14-23 b$ & & & Archived & \\
\hline $14-23 c$ & & & Archived & \\
\hline $14-23 d$ & & & Archived & \\
\hline $14-23 e$ & & & Archived & \\
\hline $14-23 f$ & & IID-10.1 & & \\
\hline $14-23 g$ & & IID-10.2 & & \\
\hline $14-23 h$ & & IID-10.3 & & \\
\hline $14-23 i$ & & IID-10.4 & & \\
\hline 14-23j & & IID-10.5 & & \\
\hline $14-23 k$ & & & Archived & \\
\hline 14-23I & & & Archived & \\
\hline $14-24 a$ & & IID-8 & & \\
\hline $14-24 b$ & & & & Dropped \\
\hline $14-25 a$ & IID-17.1 & & & \\
\hline $14-25 b$ & IID-17.2 & & & \\
\hline $14-26$ & IID-18 & & & \\
\hline $14-27 a$ & & & Archived & \\
\hline $14-27 b$ & & & Archived & \\
\hline $14-27 c$ & & IID-11.1 & & \\
\hline $14-27 d$ & & IID-11.2 & & \\
\hline $14-28 a$ & & IID-15.1 & & \\
\hline $14-28 b$ & & IID-15.2 & & \\
\hline $14-28 c$ & IID-15.3 & & & \\
\hline $14-29 a$ & & IID-12.7 & & \\
\hline $14-29 b$ & IID-13.1 & & & \\
\hline $14-29 c$ & & IID-12.6 & & \\
\hline $14-29 d$ & IID-13.2 & & & \\
\hline $14-29 e$ & & IID-12.8 & & \\
\hline $14-29 f$ & & IID-13.3 & & \\
\hline $14-29 g$ & & IID-12.9 & & \\
\hline $14-30 a$ & & & Archived & \\
\hline 14-30b & & & Archived & \\
\hline 14-31a & & IID-16 & & \\
\hline 14-31b & & & Archived & \\
\hline
\end{tabular}

\begin{tabular}{|l||l|l|l|}
\hline Focus Area 15: Injury and Violence Prevention & \\
\hline $15-1$ & IVP-2.2 & & \\
\hline $15-2$ & IVP-3.2 & & \\
\hline $15-3$ & IVP-30 & & Archived \\
\hline $15-4$ & & & \\
\hline $15-5$ & IVP-31 & & \\
\hline $15-6$ & & IVP-4 & \\
\hline $15-7$ & & IVP-10 & \\
\hline
\end{tabular}




\begin{tabular}{|c|c|c|c|c|}
\hline \multirow{2}{*}{$\begin{array}{l}\text { HP2010 } \\
\text { Objectives }\end{array}$} & \multicolumn{4}{|c|}{ HP2020 Objectives } \\
\hline & Retained & Modified & Archived & Dropped \\
\hline \multicolumn{5}{|c|}{$[$ IVP-9.1 } \\
\hline \multicolumn{5}{|c|}{\begin{tabular}{l|l}
$15-8$ & IVP-9.2 \\
IVP-9.3
\end{tabular}} \\
\hline \multirow{2}{*}{\multicolumn{5}{|c|}{\begin{tabular}{l|l} 
IVI -9.4 \\
IVI
\end{tabular}}} \\
\hline & & & & \\
\hline \multirow{3}{*}{\multicolumn{2}{|c|}{$15-9]$}} & IVP-24.1 & & \\
\hline & & IVP-24.2 & & \\
\hline & & IVP-24.3 & & \\
\hline $15-10$ & & IVP-6 & & \\
\hline $15-11$ & & IVP-7 & & \\
\hline $15-12$ & & IVP-1.3 & & \\
\hline $15-13$ & IVP-11 & & & \\
\hline $15-14$ & IVP-12 & & & \\
\hline $15-15 a$ & IVP-13.1 & & & \\
\hline $15-15 b$ & IVP-13.2 & & & \\
\hline $15-16$ & IVP-18 & & & \\
\hline $15-17$ & IVP-14 & & & \\
\hline $15-18$ & IVP-19 & & & \\
\hline $15-19$ & IVP-15 & & & \\
\hline \multirow{3}{*}{\multicolumn{3}{|c|}{$\begin{array}{l}\text { IVP-16.1 } \\
\text { IVP-16.2 } \\
\text { IVP-16.3 } \\
\text { IVP-16.4 }\end{array}$}} & & \\
\hline & & & & \\
\hline & & & & \\
\hline $15-21$ & IVP-22 & & & \\
\hline $15-22$ & & IVP-17 & & \\
\hline $15-23 a$ & & & Archived & \\
\hline $15-23 b$ & & & Archived & \\
\hline $15-24$ & IVP-21 & & & \\
\hline $15-25$ & IVP-28 & & & \\
\hline $15-26 a$ & & & Archived & \\
\hline $15-26 b$ & & & Archived & \\
\hline \multirow{2}{*}{\multicolumn{5}{|c|}{$15-27] \quad[$ IVP-23.1 }} \\
\hline & & & & \\
\hline $15-28 a$ & AOCBC-11.1 & & & \\
\hline $15-28 b$ & AOCBC-11.2 & & & \\
\hline $15-29$ & IVP-25 & & & \\
\hline $15-30$ & & & Archived & \\
\hline $15-31 a$ & IVP-27.1 & & & \\
\hline $15-31 b$ & & & Archived & \\
\hline $15-31 c$ & IVP-27.2 & & & \\
\hline $15-32$ & IVP-29 & & & \\
\hline $15-33 a$ & & IVP-38 & & \\
\hline $15-33 b$ & & IVP-37 & & \\
\hline $15-34$ & & IVP-39.1 & & \\
\hline $15-35$ & & IVP-40.1 & & \\
\hline \multirow{2}{*}{\multicolumn{2}{|c|}{$15-36$}} & IVP-40.2 & & \\
\hline & & IVP-40.3 & & \\
\hline $15-37$ & IVP-33 & & & \\
\hline $15-38$ & IVP-34 & & & \\
\hline $15-39$ & IVP-36 & & & \\
\hline
\end{tabular}




\begin{tabular}{|c|c|c|c|c|}
\hline \multirow{2}{*}{$\begin{array}{l}\text { HP2010 } \\
\text { Objectives }\end{array}$} & \multicolumn{4}{|c|}{ HP2020 Objectives } \\
\hline & Retained & Modified & Archived & Dropped \\
\hline \multicolumn{5}{|c|}{ Focus Area 16: Maternal, Infant, and Child Health } \\
\hline $16-1 a$ & $\mathrm{MICH}-1.1$ & & & \\
\hline $16-1 b$ & $\mathrm{MICH}-1.2$ & & & \\
\hline $16-1 \mathrm{c}$ & $\mathrm{MICH}-1.3$ & & & \\
\hline $16-1 d$ & $\mathrm{MICH}-1.4$ & & & \\
\hline $16-1 \mathrm{e}$ & $\mathrm{MICH}-1.5$ & & & \\
\hline $16-1 f$ & $\mathrm{MICH}-1.6$ & & & \\
\hline $16-1 \mathrm{~g}$ & $\mathrm{MICH}-1.7$ & & & \\
\hline $16-1 \mathrm{~h}$ & $\mathrm{MICH}-1.8$ & & & \\
\hline $16-2 a$ & $\mathrm{MICH}-3.1$ & & & \\
\hline $16-2 b$ & $\mathrm{MICH}-3.2$ & & & \\
\hline $16-3 a$ & $\mathrm{MICH}-4.1$ & & & \\
\hline $16-3 b$ & $\mathrm{MICH}-4.2$ & & & \\
\hline $16-3 c$ & $\mathrm{MICH}-4.3$ & & & \\
\hline $16-4$ & & $\mathrm{MICH}-5$ & & \\
\hline $16-5 a$ & $\mathrm{MICH}-6$ & & & \\
\hline $16-5 b$ & & & & Dropped \\
\hline $16-5 c$ & & & & Dropped \\
\hline $16-6 a$ & & $\mathrm{MICH}-10.1$ & & \\
\hline $16-6 b$ & & $\mathrm{MICH}-10.2$ & & \\
\hline $16-7$ & & $\mathrm{MICH}-12$ & & \\
\hline $16-8$ & $\mathrm{MICH}-33$ & & & \\
\hline $16-9 a$ & $\mathrm{MICH}-7.1$ & & & \\
\hline $16-9 b$ & $\mathrm{MICH}-7.2$ & & & \\
\hline 16-10a & $\mathrm{MICH}-8.1$ & & & \\
\hline $16-10 b$ & $\mathrm{MICH}-8.2$ & & & \\
\hline $16-11 a$ & $\mathrm{MICH}-9.1$ & & & \\
\hline \multirow{2}{*}{\multicolumn{2}{|c|}{$16-11 b$}} & \multirow{2}{*}{$\begin{array}{l}\mathrm{MICH}-9.2 \\
\mathrm{MICH}-9.3\end{array}$} & & \\
\hline & & & & \\
\hline $16-11 c$ & $\mathrm{MICH}-9.4$ & & & \\
\hline $16-12$ & & $\mathrm{MICH}-13$ & & \\
\hline $16-13$ & & $\mathrm{MICH}-20$ & & \\
\hline $16-14 a$ & & & Archived & \\
\hline $16-14 b$ & & $\mathrm{MICH}-27$ & & \\
\hline \multirow{3}{*}{\multicolumn{2}{|c|}{$16-14 c]$}} & \multirow{4}{*}{$\begin{array}{l}\mathrm{MICH}-29.1 \\
\mathrm{MICH}-29.2 \\
\mathrm{MICH}-29.3\end{array}$} & & \\
\hline & & & & \\
\hline & & & & \\
\hline $16-14 d$ & & & & Dropped \\
\hline \multirow{2}{*}{\multicolumn{2}{|c|}{$16-15$}} & \multirow{2}{*}{$\begin{array}{l}\mathrm{MICH}-28.1 \\
\mathrm{MICH}-28.2\end{array}$} & & \\
\hline & & & & \\
\hline $16-16 a$ & $\mathrm{MICH}-14^{* *}$ & & & \\
\hline $16-16 b$ & & $\mathrm{MICH}-15^{\star *}$ & & \\
\hline $16-17 a$ & $\mathrm{MICH}-11.1$ & & & \\
\hline $16-17 b$ & & $\mathrm{MICH}-11.2$ & & \\
\hline $16-17 c$ & & MICH-11.3 & & \\
\hline $16-17 d$ & $\mathrm{MICH}-11.4$ & & & \\
\hline $16-18$ & & $\mathrm{MICH}-25$ & & \\
\hline $16-19 a$ & MICH-21.1 & & & \\
\hline $16-19 b$ & $\mathrm{MICH}-21.2$ & & & \\
\hline $16-19 c$ & $\mathrm{MICH}-21.3$ & & & \\
\hline $16-19 d$ & $\mathrm{MICH}-21.4$ & & & \\
\hline $16-19 e$ & $\mathrm{MICH}-21.5$ & & & \\
\hline $16-20 a$ & & $\mathrm{MICH}-32.1$ & & \\
\hline
\end{tabular}




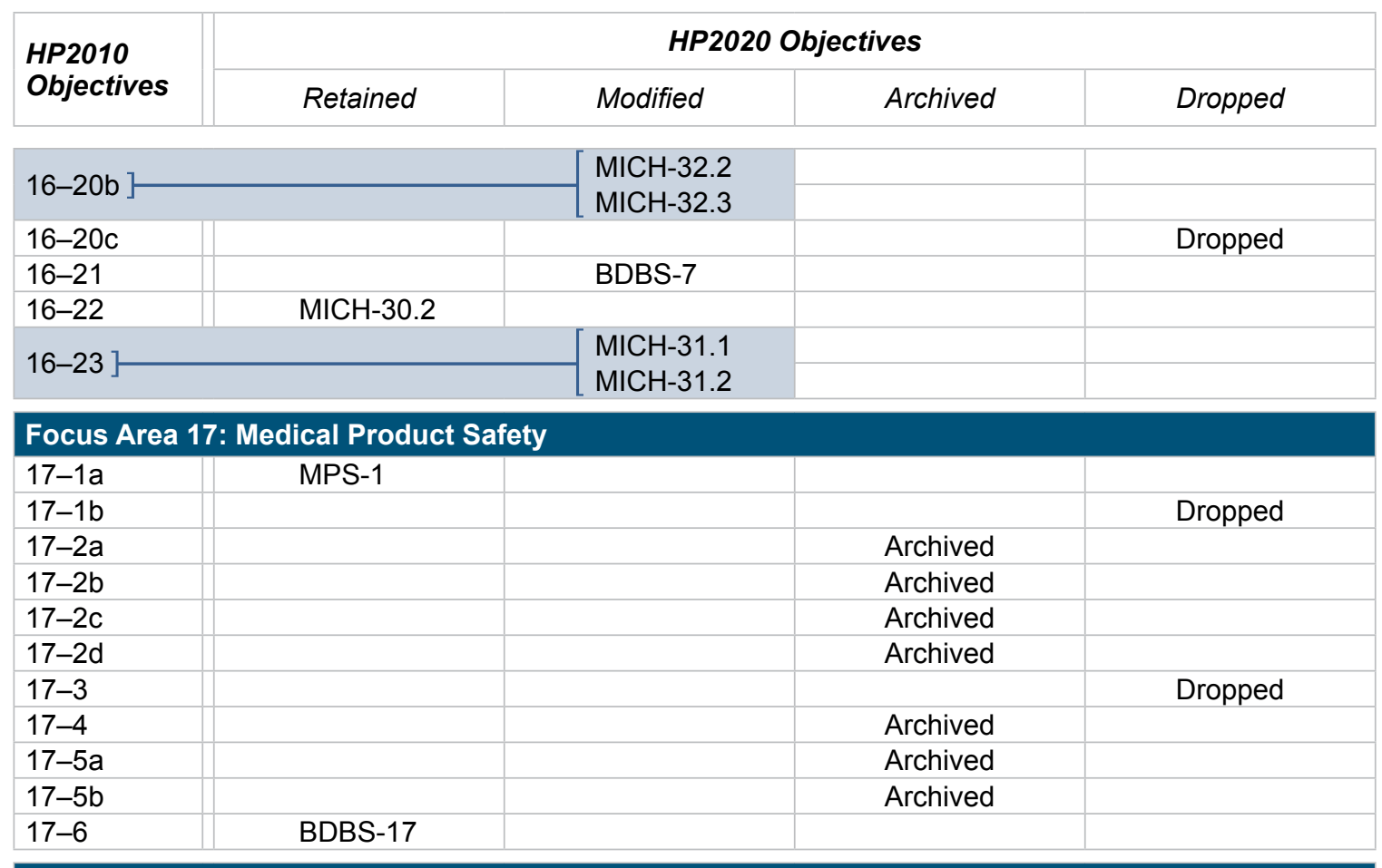

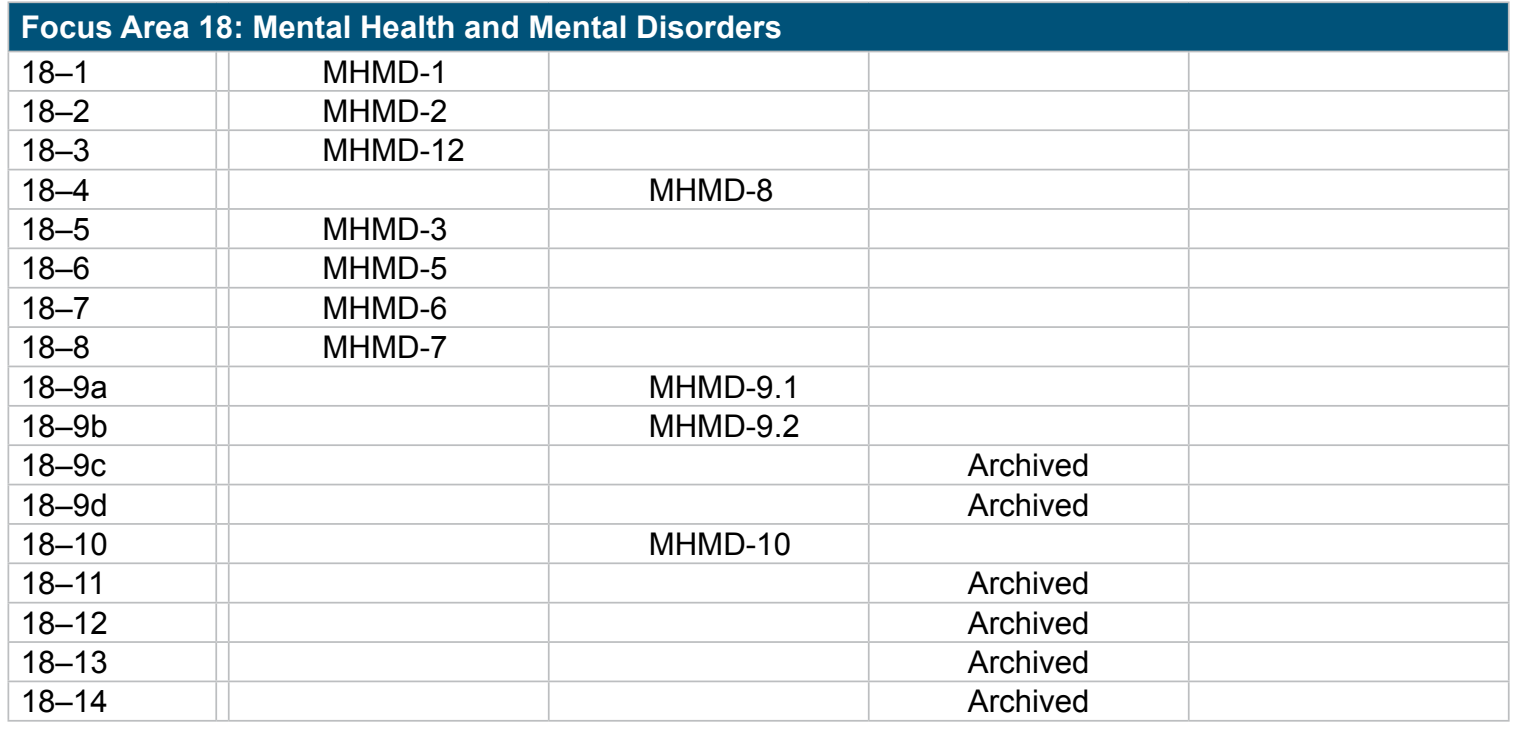

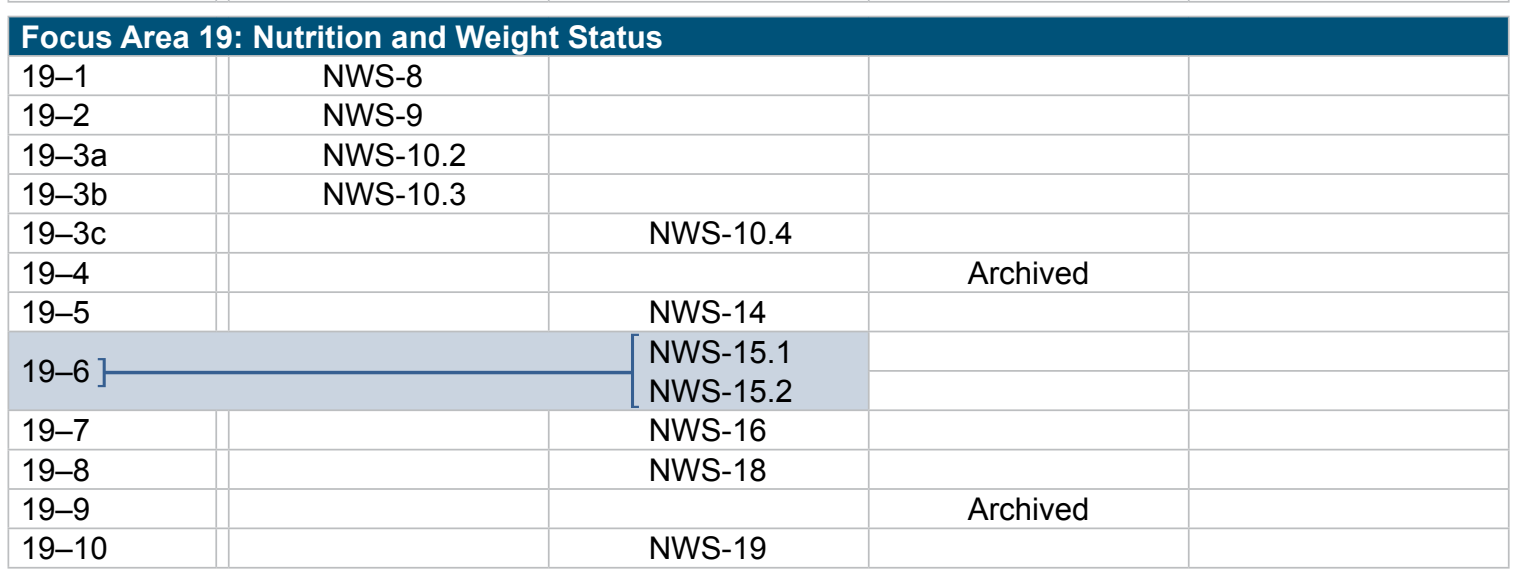




\begin{tabular}{|c|c|c|c|c|}
\hline \multirow{2}{*}{$\begin{array}{l}\text { HP2010 } \\
\text { Objectives }\end{array}$} & \multicolumn{4}{|c|}{ HP2020 Objectives } \\
\hline & Retained & Modified & Archived & Dropped \\
\hline $19-11$ & & NWS-20 & & \\
\hline $19-12 a$ & & NWS-21.1 & & \\
\hline $19-12 b$ & & NWS-21.2 & & \\
\hline $19-12 c$ & & NWS-21.3 & & \\
\hline $19-13$ & & & Archived & \\
\hline $19-14$ & NWS-22 & & & \\
\hline $19-15$ & & & & Dropped \\
\hline 19-16 & & NWS-7 & & \\
\hline $19-17$ & & NWS-6.1 & & \\
\hline $19-18$ & NWS-13* & & & \\
\hline
\end{tabular}

\section{Focus Area 20: Occupational Safety and Health}

\begin{tabular}{|l|l|l|l|l|}
\hline $20-1 \mathrm{a}$ & OSH-1.1 & & \\
\hline $20-1 \mathrm{~b}$ & OSH-1.2 & & \\
\hline $20-1 \mathrm{c}$ & OSH-1.3 & & \\
\hline $20-1 \mathrm{~d}$ & OSH-1.4 & & \\
\hline $20-1 \mathrm{e}$ & OSH-1.5 & & \\
\hline $20-2 \mathrm{a}$ & OSH-2.1 & & Archived & \\
\hline $20-2 \mathrm{c}$ & & & Archived & \\
\hline $20-2 \mathrm{~d}$ & & & Archived & \\
\hline $20-2 \mathrm{e}$ & & & Archived & \\
\hline $20-2 \mathrm{f}$ & & & \\
\hline $20-2 \mathrm{~g}$ & & & \\
\hline $20-2 \mathrm{~h}$ & & & \\
\hline $20-3$ & OSH-2.3 & & \\
\hline $20-4$ & OSH-4 & & \\
\hline $20-5$ & & & \\
\hline $20-6$ & & OSH-6 & \\
\hline $20-7$ & & & \\
\hline $20-8$ & & OSH-7 & \\
\hline $20-9$ & & & \\
\hline $20-10$ & & & \\
\hline $20-11$ & & & \\
\hline
\end{tabular}

\section{Focus Area 21: Oral Health}

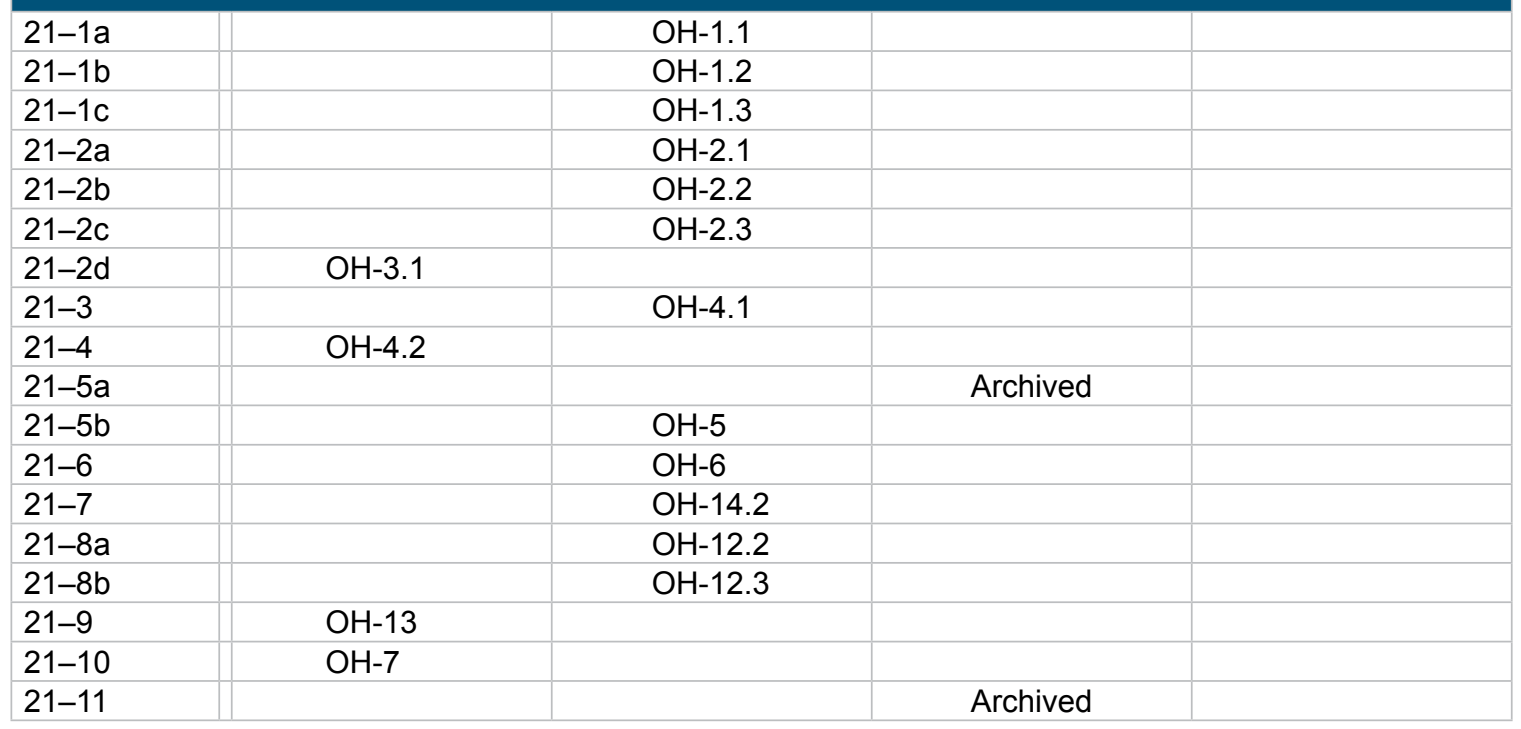




\begin{tabular}{|c|c|c|c|c|}
\hline \multirow{2}{*}{$\begin{array}{l}\text { HP2010 } \\
\text { Objectives }\end{array}$} & \multicolumn{4}{|c|}{ HP2020 Objectives } \\
\hline & Retained & Modified & Archived & Dropped \\
\hline $21-12$ & & $\mathrm{OH}-8$ & & \\
\hline $21-13 a$ & $\mathrm{OH}-9.1$ & & & \\
\hline $21-13 b$ & $\mathrm{OH}-9.2$ & & & \\
\hline $21-14$ & $\mathrm{OH}-10.1$ & & & \\
\hline \multirow{2}{*}{\multicolumn{2}{|c|}{$21-15]$}} & $\mathrm{OH}-15.1$ & & \\
\hline & & $\mathrm{OH}-15.2$ & & \\
\hline $21-16$ & & $\mathrm{OH}-16$ & & \\
\hline $21-17 a$ & & $\mathrm{OH}-17.1$ & & \\
\hline $21-17 b$ & & $\mathrm{OH}-17.2$ & & \\
\hline
\end{tabular}

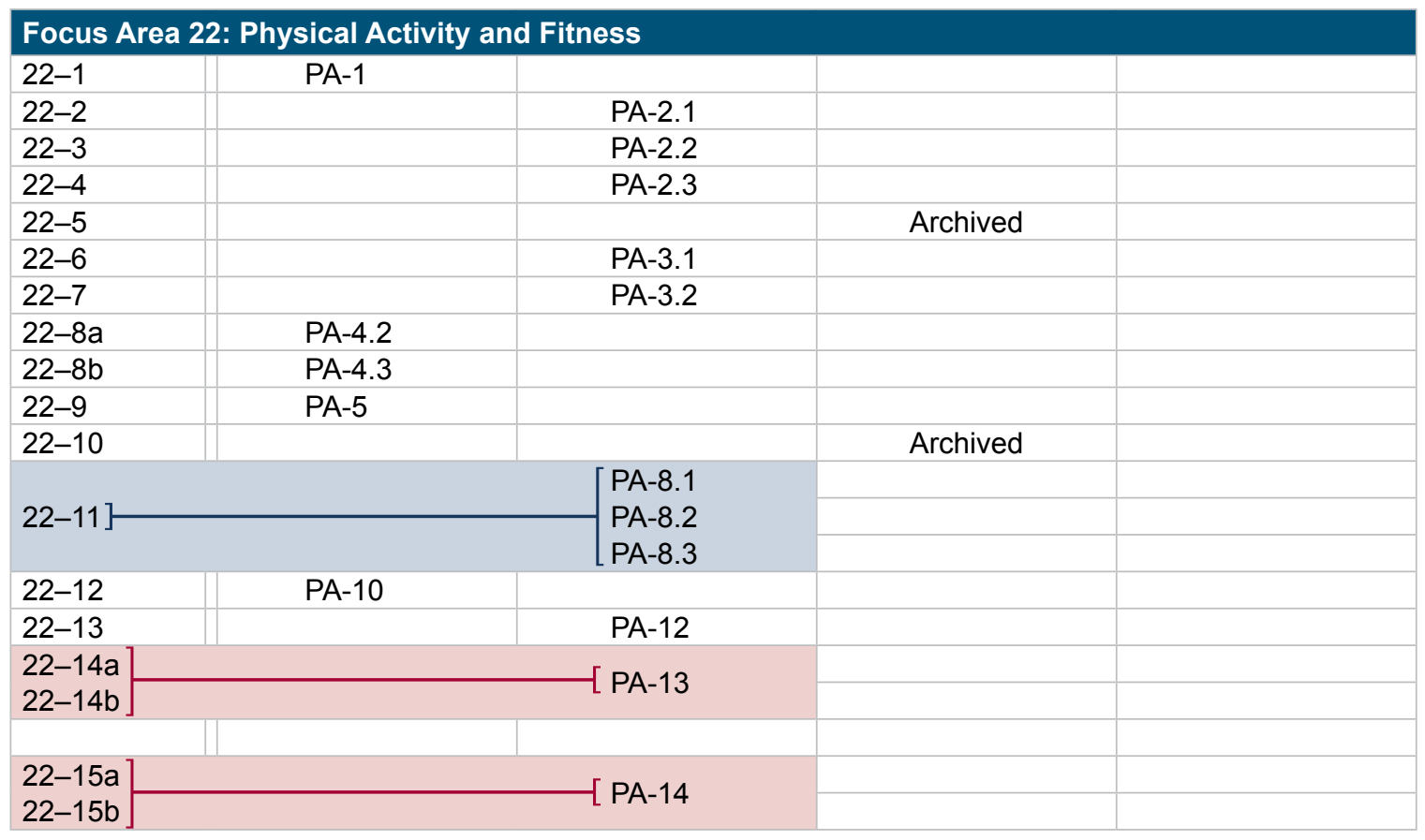

\section{Focus Area 23: Public Health Infrastructure}

\begin{tabular}{|c|c|c|c|c|}
\hline $23-1$ & & & & Dropped \\
\hline $23-2 a$ & & & Archived & \\
\hline $23-2 b$ & & & & Dropped \\
\hline $23-2 c$ & & & Archived & \\
\hline $23-2 d$ & & & Archived & \\
\hline $23-3$ & & & Archived & \\
\hline $23-4$ & & PHI-7 & & \\
\hline $23-5$ & & & & Dropped \\
\hline $23-6$ & & PHI-8.3 & & \\
\hline $23-7$ & & PHI-9 & & \\
\hline $23-8 a$ & PHI-1.1 & & & \\
\hline $23-8 b$ & PHI-1.2 & & & \\
\hline $23-9$ & PHI-3 & & & \\
\hline \multicolumn{5}{|l|}{ 23-10a } \\
\hline \multirow{2}{*}{\multicolumn{5}{|c|}{$\begin{array}{l}23-10 b \\
23-10 c\end{array}$}} \\
\hline & & $\{\mathrm{LHI}-2$ & & \\
\hline 23-11a & & PHI-14.1 & & \\
\hline $23-11 b$ & PHI-14.2 & & & \\
\hline $23-11 c$ & & & Archived & \\
\hline $23-11 d$ & & & Archived & \\
\hline
\end{tabular}




\begin{tabular}{|c|c|c|c|c|}
\hline \multirow{2}{*}{$\begin{array}{l}\text { HP2010 } \\
\text { Objectives }\end{array}$} & \multicolumn{4}{|c|}{ HP2020 Objectives } \\
\hline & Retained & Modified & Archived & Dropped \\
\hline $23-12 a$ & PHI-15.1 & & & \\
\hline $23-12 b$ & & PHI-15.2 & & \\
\hline $23-12 c$ & & PHI-15.3 & & \\
\hline $23-12 d$ & & PHI-15.4 & & \\
\hline $23-13 a$ & PHI-11.1 & & & \\
\hline $23-13 b$ & PHI-11.2 & & & \\
\hline $23-13 c$ & PHI-11.3 & & & \\
\hline $23-13 d$ & PHI-11.4 & & & \\
\hline $23-13 e$ & PHI-11.5 & & & \\
\hline $23-13 f$ & PHI-11.6 & & & \\
\hline $23-13 g$ & PHI-11.7 & & & \\
\hline $23-13 h$ & PHI-11.8 & & & \\
\hline $23-13 i$ & PHI-11.9 & & & \\
\hline 23-13j & PHI-11.10 & & & \\
\hline $23-13 k$ & PHI-11.11 & & & \\
\hline $23-14 a$ & PHI-13.1 & & & \\
\hline $23-14 b$ & PHI-13.2 & & & \\
\hline $23-14 c$ & & PHI-13.3 & & \\
\hline $23-14 d$ & & PHI-13.4 & & \\
\hline $23-15 a$ & & & Archived & \\
\hline $23-15 b$ & & & Archived & \\
\hline $23-16$ & & & & Dropped \\
\hline $23-17$ & & & & Dropped \\
\hline \multicolumn{5}{|c|}{ Focus Area 24: Respiratory Diseases } \\
\hline \multicolumn{5}{|c|}{$24-1 \mathrm{a}]$} \\
\hline \multirow{2}{*}{\multicolumn{5}{|c|}{$\begin{array}{l}24-1 b \\
24-1 c\end{array} \mid$}} \\
\hline & & & & \\
\hline $24-1 d$ & $\mathrm{RD}-1.2$ & & & \\
\hline $24-1 e$ & RD-1.3 & & & \\
\hline $24-2 a$ & RD-2.1 & & & \\
\hline $24-2 b$ & RD-2.2 & & & \\
\hline $24-2 c$ & RD-2.3 & & & \\
\hline $24-3 a$ & RD-3.1 & & & \\
\hline $24-3 b$ & RD-3.2 & & & \\
\hline $24-3 c$ & RD-3.3 & & & \\
\hline $24-4$ & RD-4 & & & \\
\hline \multirow{2}{*}{\multicolumn{2}{|c|}{ 24-5] }} & RD-5.1 & & \\
\hline & & RD-5.2 & & \\
\hline $24-6$ & RD-6 & & & \\
\hline $24-7 a$ & RD-7.1 & & & \\
\hline $24-7 b$ & RD-7.2 & & & \\
\hline $24-7 c$ & RD-7.3 & & & \\
\hline $24-7 d$ & RD-7.4 & & & \\
\hline $24-7 e$ & & & Archived & \\
\hline $24-7 f$ & RD-7.5 & & & \\
\hline $24-8$ & & RD-8 & & \\
\hline $24-9$ & & RD-9 & & \\
\hline $24-10$ & RD-10 & & & \\
\hline 24-11a & $\mathrm{SH}-1$ & & & \\
\hline 24-11b & & & & Dropped \\
\hline 24-12 & & $\mathrm{SH}-2$ & & \\
\hline
\end{tabular}




\begin{tabular}{|c|c|c|c|c|}
\hline \multirow{2}{*}{$\begin{array}{l}\text { HP2010 } \\
\text { Objectives }\end{array}$} & \multicolumn{4}{|c|}{ HP2020 Objectives } \\
\hline & Retained & Modified & Archived & Dropped \\
\hline \multicolumn{5}{|c|}{ Focus Area 25: Sexually Transmitted Diseases } \\
\hline $25-1 a$ & STD-1.1 & & & \\
\hline $25-1 b$ & & & Archived & \\
\hline $25-1 c$ & & & Archived & \\
\hline $25-1 d$ & STD-1.2 & & & \\
\hline $25-2 a$ & & & Archived & \\
\hline $25-2 b$ & STD-6.1 & & & \\
\hline $25-3$ & STD-7 & & & \\
\hline $25-4$ & STD-10 & & & \\
\hline $25-5$ & STD-9 & & & \\
\hline $25-6$ & STD-5 & & & \\
\hline $25-7$ & & & Archived & \\
\hline $25-8$ & & & & Dropped \\
\hline $25-9$ & STD-8 & & & \\
\hline $25-10$ & & & & Dropped \\
\hline 25-11a & & & Archived & \\
\hline $25-11 b$ & & & Archived & \\
\hline $25-11 c$ & & & Archived & \\
\hline 25-12 & & & & Dropped \\
\hline $25-13$ & & & Archived & \\
\hline $25-14$ & & & & Dropped \\
\hline 25-15 & & & & Dropped \\
\hline \multirow{2}{*}{\multicolumn{2}{|c|}{$25-16 a]$}} & \multirow{2}{*}{$\begin{array}{l}\text { STD-4.1 } \\
\text { STD-4.2 }\end{array}$} & & \\
\hline & & & & \\
\hline \multirow{2}{*}{\multicolumn{2}{|c|}{$25-16 b]$}} & \multirow{2}{*}{$\begin{array}{l}\text { STD-3.1 } \\
\text { STD-3.2 }\end{array}$} & 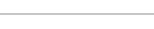 & \\
\hline & & & & \\
\hline $25-17$ & & & & Dropped \\
\hline $25-18$ & & & & Dropped \\
\hline $25-19$ & & & & Dropped \\
\hline \multicolumn{5}{|c|}{ Focus Area 26: Substance Abuse } \\
\hline $26-1 a$ & & SA-17 & & \\
\hline $26-1 b$ & & & & Dropped \\
\hline $26-1 c$ & & & & Dropped \\
\hline $26-1 d$ & & & & Dropped \\
\hline $26-2$ & SA-11 & & & \\
\hline $26-3$ & SA-12 & & & \\
\hline $26-4$ & & & Archived & \\
\hline $26-5$ & & & & Dropped \\
\hline $26-6$ & SA-1 & & & \\
\hline $26-7$ & & & & Dropped \\
\hline $26-8 a$ & & & Archived & \\
\hline $26-8 b$ & & & Archived & \\
\hline $26-9 a$ & & SA-2.1 & & \\
\hline $26-9 b$ & & SA-2.2 & & \\
\hline $26-9 c$ & SA-2.3 & & & \\
\hline $26-9 d$ & SA-2.4 & & & \\
\hline 26-10a & & SA-13.1 & & \\
\hline $26-10 b$ & SA-13.2 & & & \\
\hline $26-10 c$ & SA-13.3 & & & \\
\hline $26-11 a$ & SA-14.1 & & & \\
\hline $26-11 b$ & SA-14.2 & & & \\
\hline
\end{tabular}




\begin{tabular}{|c|c|c|c|c|}
\hline \multirow{2}{*}{$\begin{array}{l}\text { HP2010 } \\
\text { Objectives }\end{array}$} & \multicolumn{4}{|c|}{ HP2020 Objectives } \\
\hline & Retained & Modified & Archived & Dropped \\
\hline $26-11 c$ & & SA-14.3 & & \\
\hline $26-11 d$ & & SA-14.4 & & \\
\hline $26-12$ & SA-16 & & & \\
\hline \multicolumn{5}{|l|}{ 26-13a] } \\
\hline \multicolumn{5}{|l|}{$26-13 b]$} \\
\hline $26-14 a$ & SA-18.1 & & & \\
\hline $26-14 b$ & SA-18.2 & & & \\
\hline $26-14 c$ & SA-18.3 & & & \\
\hline $26-15$ & SA-21 & & & \\
\hline $26-16 a$ & SA-3.1 & & & \\
\hline $26-16 b$ & SA-3.2 & & & \\
\hline $26-16 c$ & SA-3.3 & & & \\
\hline $26-16 d$ & SA-3.4 & & & \\
\hline $26-16 e$ & SA-3.5 & & & \\
\hline $26-16 f$ & SA-3.6 & & & \\
\hline $26-17 a$ & SA-4.1 & & & \\
\hline $26-17 b$ & SA-4.2 & & & \\
\hline $26-17 c$ & SA-4.3 & & & \\
\hline $26-18 a$ & SA-8.1 & & & \\
\hline $26-18 b$ & SA-18.2 & & & \\
\hline $26-19$ & & & & Dropped \\
\hline $26-20$ & SA-7 & & & \\
\hline $26-21$ & SA-8.3 & & & \\
\hline $26-22$ & & & & Dropped \\
\hline $26-23$ & & & & Dropped \\
\hline $26-24$ & & & Archived & \\
\hline $26-25$ & & & Archived & \\
\hline \multicolumn{5}{|c|}{ Focus Area 27: Tobacco Use } \\
\hline $27-1 a$ & TU-1.1 & & & \\
\hline $27-1 b$ & TU-1.2 & & & \\
\hline $27-1 c$ & TU-1.3 & & & \\
\hline $27-1 d$ & & & & Dropped \\
\hline $27-2 a$ & TU-2.1 & & & \\
\hline $27-2 b$ & TU-2.2 & & & \\
\hline $27-2 c$ & TU-2.3 & & & \\
\hline $27-2 d$ & TU-2.4 & & & \\
\hline $27-2 e$ & & & Archived & \\
\hline $27-3 a$ & TU-3.2 & & & \\
\hline $27-3 b$ & TU-3.6 & & & \\
\hline $27-4 a$ & & & Archived & \\
\hline $27-4 b$ & & & Archived & \\
\hline $27-5$ & TU-4.1 & & & \\
\hline $27-6$ & TU-6 & & & \\
\hline $27-7$ & TU-7 & & & \\
\hline $27-8 a$ & & & Archived & \\
\hline $27-8 b$ & & TU-8 & & \\
\hline $27-8 c$ & & & & Dropped \\
\hline $27-9$ & & & Archived & \\
\hline \multirow{3}{*}{\multicolumn{2}{|c|}{$27-10$}} & {$[\mathrm{TU}-11.1$} & & \\
\hline & & TU-11.2 & & \\
\hline & & TU-11.3 & & \\
\hline
\end{tabular}




\begin{tabular}{|c|c|c|c|c|}
\hline \multirow{2}{*}{$\begin{array}{l}\text { HP2010 } \\
\text { Objectives }\end{array}$} & \multicolumn{4}{|c|}{ HP2020 Objectives } \\
\hline & Retained & Modified & Archived & Dropped \\
\hline \multirow{4}{*}{\multicolumn{2}{|c|}{$27-11$}} & TU-15.1 & & \\
\hline & & TU-15.2 & & \\
\hline & & TU-15.3 & & \\
\hline & & & & \\
\hline $27-12$ & TU-12 & & & \\
\hline $27-13 a$ & TU-13.1 & & & \\
\hline $27-13 b$ & TU-13.2 & & & \\
\hline $27-13 c$ & TU-13.3 & & & \\
\hline $27-13 d$ & TU-13.8 & & & \\
\hline \multirow{2}{*}{\multicolumn{2}{|c|}{$27-13 e]$}} & TU-13.6 & & \\
\hline & & TU-13.7 & & \\
\hline $27-13 f$ & & & Archived & \\
\hline $27-13 g$ & & & & Dropped \\
\hline $27-13 \mathrm{~h}$ & & & & Dropped \\
\hline $27-13 i$ & TU-13.4 & & & \\
\hline $27-14 a$ & TU-19.1 & & & \\
\hline $27-14 b$ & TU-19.2 & & & \\
\hline $27-15$ & & & Archived & \\
\hline $27-16 a$ & TU-18.1 & & & \\
\hline $27-16 b$ & TU-18.2 & & & \\
\hline $27-17 a$ & & & Archived & \\
\hline $27-17 b$ & & & Archived & \\
\hline $27-17 c$ & & & Archived & \\
\hline $27-18 a$ & & TU-20.1 & & \\
\hline $27-18 b$ & TU-20.2 & & & \\
\hline $27-18 c$ & TU-20.3 & & & \\
\hline \multirow{3}{*}{\multicolumn{2}{|c|}{$27-19$ - }} & TU-16.1 & & \\
\hline & & TU-16.2 & & \\
\hline & & TU-16.3 & & \\
\hline $27-20 a$ & & & Archived & \\
\hline $27-20 b$ & & & Archived & \\
\hline $27-20 c$ & & & Archived & \\
\hline $27-21 a$ & & TU-17.1 & & \\
\hline $27-21 b$ & & TU-17.2 & & \\
\hline \multicolumn{5}{|c|}{ Focus Area 28: Vision and Hearing } \\
\hline $28-1$ & $\mathrm{~V}-4$ & & & \\
\hline $28-2$ & $\mathrm{~V}-1$ & & & \\
\hline $28-3$ & $\mathrm{~V}-5.1$ & & & \\
\hline $28-4$ & $\mathrm{~V}-2$ & & & \\
\hline $28-5$ & & $V-5.2$ & & \\
\hline $28-6$ & & V-5.3 & & \\
\hline $28-7$ & & V-5.4 & & \\
\hline $28-8 a$ & V-3.1 & & & \\
\hline $28-8 b$ & V-3.2 & & & \\
\hline $28-9 a$ & V-6.1 & & & \\
\hline $28-9 b$ & $\mathrm{~V}-6.2$ & & & \\
\hline $28-10 a$ & $V-7.1$ & & & \\
\hline $28-10 b$ & V-7.2 & & & \\
\hline $28-11 a$ & ENT-VSL-1.1 & & & \\
\hline 28-11b & ENT-VSL-1.2 & & & \\
\hline $28-11 c$ & ENT-VSL-1.3 & & & \\
\hline $28-12$ & ENT-VSL-2 & & & \\
\hline
\end{tabular}




\begin{tabular}{|l||l|l|l|}
\hline \multirow{2}{*}{$\begin{array}{l}\text { HP2010 } \\
\text { Objectives }\end{array}$} & \multicolumn{3}{|c|}{ HP2020 Objectives } \\
\hline
\end{tabular}

* Has been changed to the complement (i.e., 100\%-'19-18'='NWS-13').

** Data for HP2010 are 2-year averages; HP2020 data are 4-year averages. 


\section{Appendix E: Evolution of Healthy People}

\begin{tabular}{|c|c|c|c|c|}
\hline Target Year & $\frac{1990}{190}$ & 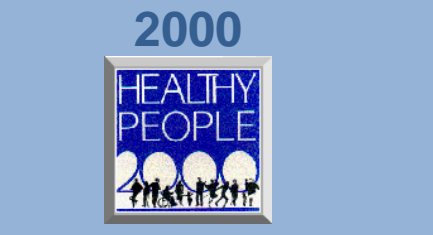 & $\begin{array}{l}2010 \\
\text { HEALIHY } \\
\text { PEOPLE } \\
\text { ZONO }\end{array}$ & $\begin{array}{c}2020 \\
\text { Healthy People } \\
2020 \\
\end{array}$ \\
\hline Overarching Goals & $\begin{array}{l}\text { - Decrease mortality: } \\
\text { infants-adults } \\
\text { - Increase } \\
\text { independence } \\
\text { among older adults }\end{array}$ & $\begin{array}{l}\text { - Increase span of } \\
\text { healthy life } \\
\text { - Reduce health } \\
\text { disparities } \\
\text { - Achieve access to } \\
\text { preventive services } \\
\text { for all }\end{array}$ & $\begin{array}{l}\text { - Increase quality } \\
\text { and years of } \\
\text { healthy life } \\
\text { - Eliminate health } \\
\text { disparities }\end{array}$ & $\begin{array}{l}\text { - Attain high-quality, } \\
\text { longer lives free of } \\
\text { preventable } \\
\text { disease, disability, } \\
\text { injury, and } \\
\text { premature death } \\
\text { - Achieve health } \\
\text { equity; eliminate } \\
\text { disparities } \\
\text { - Create social and } \\
\text { physical } \\
\text { environments that } \\
\text { promote good } \\
\text { health } \\
\text { - Promote quality of } \\
\text { life, healthy } \\
\text { development, } \\
\text { healthy behaviors } \\
\text { across life stages }\end{array}$ \\
\hline $\begin{array}{l}\text { Number of } \\
\text { Topic Areas }\end{array}$ & 15 & 22 & 28 & 42 \\
\hline $\begin{array}{l}\text { Number of } \\
\text { Objectives }\end{array}$ & 226 & 319 & 969 & 1,200 \\
\hline
\end{tabular}

